QA: QA

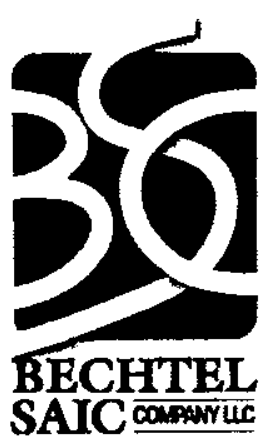

MDL-NBS-HS-000008 REV 02

October 2004

NOTICE OF OPEN CHANGE DOCUMENTS - THIS DOCUMENT IS IMPACTED BY THE LISTED CHANGE DOCUMENT AND CANNOT BE USED WITHOUT IT.

1) ACN-001, DATED 08/22/2005

\title{
Radionuclide Transport Models Under Ambient Conditions
}

Prepared for:

U.S. Department of Energy

Office of Civilian Radioactive Waste Management

Office of Repository Development

1551 Hillshire Drive

Las Vegas, Nevada 89134-6321

Prepared by:

Bechtel SAIC Company, LLC

1180 Town Center Drive

Las Vegas, Nevada 89144

Under Contract Number

DE-AC28-01RW12101 


\section{DISCLAIMER}

This report was prepared as an account of work sponsored by an agency of the United States Government. Neither the United States Government nor any agency thereof, nor any of their employees, nor any of their contractors, subcontractors or their employees, makes any warranty, express or implied, or assumes any legal liability or responsibility for the accuracy, completeness, or any third party's use or the results of such use of any information, apparatus, product, or process disclosed, or represents that its use would not infringe privately owned rights. Reference herein to any specific commercial product, process, or service by trade name, trademark, manufacturer, or otherwise, does not necessarily constitute or imply its endorsement, recommendation, or favoring by the United States Government or any agency thereof or its contractors or subcontractors. The views and opinions of authors expressed herein do not necessarily state or reflect those of the United States Government or any agency thereof. 
QA: QA

Radionuclide Transport Models Under Ambient Conditions

MDL-NBS-HS-000008 REV 02

October 2004 


\begin{tabular}{|l|l|l|}
\hline \multirow{2}{*}{ OCRWM } & Model Signature Page/Change History & Page iii \\
\cline { 3 - 3 } & & 1. Total Pages: 550 \\
\hline
\end{tabular}

2. Type of Mathematical Model

$\triangle$ Process Model $\square$ Model

System Model

Describe intended Use of Model

The purpose of this model report is to evaluate (by means of 2-D numerical and semi-analytical models and 3-D numerical models) the transport of radioactive solutes and colloids in the unsaturated zone under ambient conditions from the repository horizon to the water table at Yucca Mountain, Nevada.

\section{Title}

Radionuclide Transport Models Under Ambient Conditions

4. DI (including Rev. No., if applicable):

MDL-NBS-HS-000008 REV 02

\begin{tabular}{|l|l|}
\hline 5. Total Appendices & $\begin{array}{l}\text { 6. No. of Pages in Each Appendix } \\
\text { A-86, B-8, C-4, D-26, E-12, F-18, G-18, H-12, I-50, J-10, K-4, } \\
\text { L-8 }\end{array}$ \\
\hline
\end{tabular}

\begin{tabular}{|c|c|c|c|}
\hline & Printed Name & Signature & Date \\
\hline 7. Originator & Swen Magnuson & 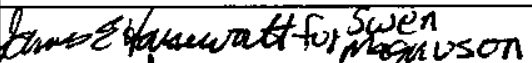 & \\
\hline $\begin{array}{ll}\text { 8. Independent Technical } \\
\text { Reviewer }\end{array}$ & Tito Bonano & & \\
\hline 9. Checker & Chunhong $\mathbf{L i}$ & 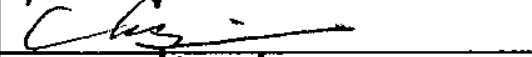 & \\
\hline 10. QER & Jim Graff & & \\
\hline $\begin{array}{l}\text { 11. Responsible } \\
\text { Manager/Lead }\end{array}$ & Jim Houseworth & sowrots & \\
\hline 12. Responsible Manager & Ming Zhu & & \\
\hline
\end{tabular}

Contributors to this model report are G. Moridis, A. Meijer, and J. Apps.

\begin{tabular}{|c|c|}
\hline \multicolumn{2}{|r|}{ Change History } \\
\hline 14. Revision No. & 15. Description of Change \\
\hline REV 00 & Initial Issue \\
\hline REV 00 ICN 01 & $\begin{array}{l}\text { - Update Section } 1 \text { to identify Technical Work Plan, ICN } 01 \text { initiated under (BSC } \\
\text { 2001), pg } 13 \text {. } \\
\text { Delete DTN LB991220140160.019 from Table } 4.1 \text { Input Data, pgs. 19-22. This } \\
\text { DTN is identified as corroborative information supporting assumption } 5.1 \text {. } \\
\text { - New assumptions added pg. 25-25A (5.1.7) to address various properties of } \\
\text { radionuclides. } \\
\text { Section } 8.1 \text { pages } 189-198 \text { updated to reflect most current TIC information, and } \\
\text { accession numbers. } \\
\text { - Added new reference in section } 8.1 \text { to reflect information about TWP. } \\
\text { - Updated Section } 8.3 \text { pgs. } 200-201 \text { to include most recent DTN information in } \\
\text { ATDT. }\end{array}$ \\
\hline
\end{tabular}




\begin{tabular}{|c|c|}
\hline & $\begin{array}{l}\text { - Updated Section } 8.4 \text { to identify submittal dates for output data DTNs, pages } 202- \\
203 \text {. } \\
\text { The following sections were revised: } 1.0,2.0,3.0,4.0,5.0,6.0,7.0,8.0 \text {, and } \\
\text { Attachment XIII. All changes are shown by change bars. } \\
\text { The following pages were revised: } 1,2,4,9,13,15,17,19-29,31,34,36-37,39- \\
42,44,46,51,63,65,69-70,74,77,84,95,99,102,104-109,111-112,115,134- \\
137,144,152,161-162,167-168,181,186-187,189-203 \text {, and Attachment XIII. } \\
\text { Attachment XI of this technical product contains documentation of single use } \\
\text { software routines or macros that were qualified under procedure AP-SI.1Q, Software } \\
\text { Management, prior to the release of Rev. } 3 \text { of said procedure. As the scope of this } \\
\text { ICN did not involve a change to the routine codes, and they have not been used to } \\
\text { develop additional quality affecting information in this technical product, these } \\
\text { single use software routines or macros will remain documented herein, in } \\
\text { accordance with AP-SI.1Q prior to the release of Revision } 3 \text {. }\end{array}$ \\
\hline REV 01 & $\begin{array}{l}\text { The entire model documentation was revised. Side bars are not used because the } \\
\text { changes were too extensive to use Step 5.8d)1) per AP-SIII.10Q, Rev. } 2 / \mathrm{ICN} 0 \text {. }\end{array}$ \\
\hline REV 02 & $\begin{array}{l}\text { Entire model document revised. Changes too extensive to use Step 5.8.f)1) per AP- } \\
\text { SIII. } 10 \text { Q, Rev. } 2 \text { /ICN } 7 \text {. }\end{array}$ \\
\hline
\end{tabular}




\section{CONTENTS}

Page

ACRONYMS AND ABBREVIATIONS ..................................................................... xxi

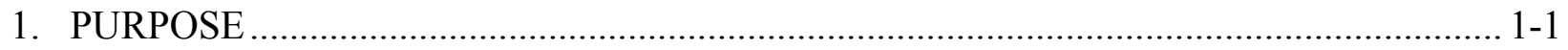

2. QUALITY ASSURANCE ................................................................................. 2-1

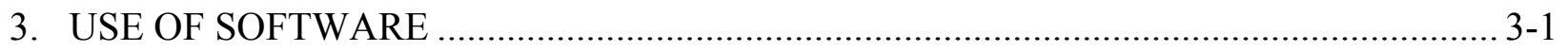

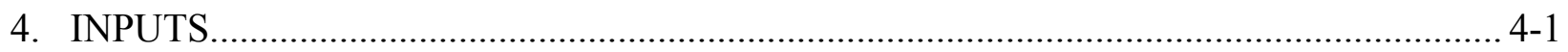

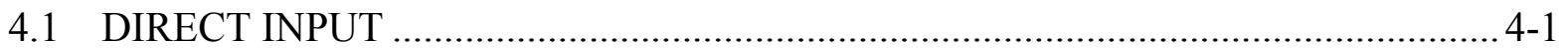

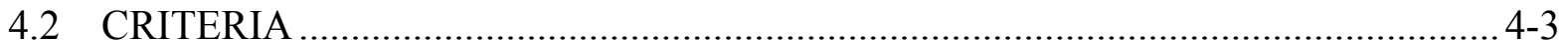

4.3 CODES, STANDARDS, AND REGULATIONS .............................................. 4-6

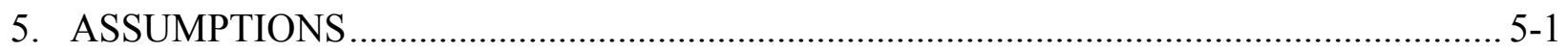

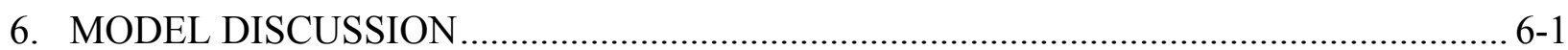

6.1 GEOLOGICAL MODEL AND PHYSICAL PROCESSES ................................... 6-5

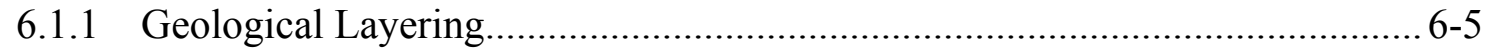

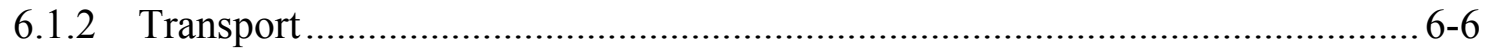

6.1.2.1 Advection ..................................................................... 6-6

6.1.2.2 Hydrodynamic Dispersion........................................................ 6-7

6.1.2.3 Sorption ......................................................................... 6-8

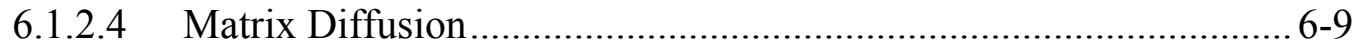

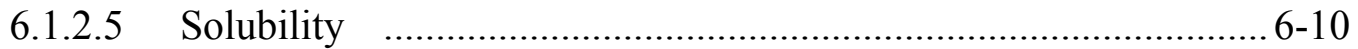

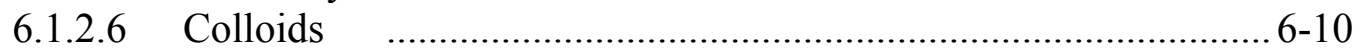

6.1.2.7 Perched Water ......................................................................... 6-10

6.1.2.8 Daughter Products .................................................................. 6-11

6.1.2.9 Effect of Heat on Transport..................................................... 6-11

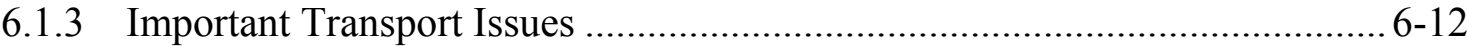

6.1.3.1 Measurements of $K_{d}$ Values ..................................................... 6-12

6.1.3.2 Solute Sorption Issues ........................................................... 6-13

6.1.3.2.1 Potential Sorption of Anions .................................. 6-13

6.1.3.2.2 Solute Size Effects ................................................. 6-15

6.1.3.3 Colloidal Behavior ........................................................... 6-15

6.1.3.3.1 Colloid Types and Classes ...................................... 6-15

6.1.3.3.2 Colloid Generation and Stability.............................. 6-16

6.1.3.3.3 Colloid Deposition ............................................. 6-16

6.1.3.3.4 Colloid-Contaminant-Matrix Interactions ............... 6-16

6.1.3.3.5 Colloid Migration in Macroporous and Fractured Systems ............................................................. 6-17

6.1.3.4 Colloidal Behavior in Unsaturated Media.................................... 6-18

6.1.3.4.1 Filtration as a Function of Water Saturation .............. 6-18

6.1.3.4.2 Gas-Water Interface ........................................... 6-18 


\section{CONTENTS (Continued)}

Page

6.1.3.5 Colloids at Yucca Mountain............................................................ 6-19

6.1.3.6 Impact of Colloids on Radionuclide Transport in Yucca

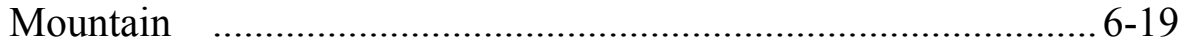

6.1.4 Important Points for Consideration in Colloid Transport ............................... 6-20

6.1.5 Modeling Stipulations ...................................................................... 6-21

6.1.5.1 Stipulations Involved in the Flow Component of the Transport

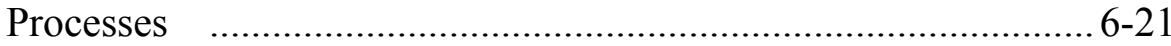

6.1.5.2 Stipulations/Approaches/Simplifications Involved in Transport Processes .................................................................... 6-23

6.1.5.3 Stipulations Involved in the Dual-Continuum Approach................ 6-25

6.1.5.4 Stipulations Involving Initial and Boundary Conditions................. 6-26

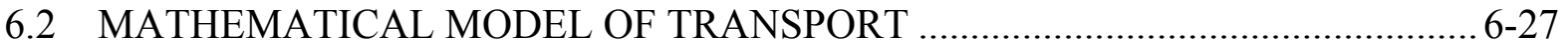

6.2.1 General Mass Balance Equations............................................................ 6-27

6.2.2 Accumulation Terms ......................................................................... 6-28

6.2.2.1 Equations of the Accumulation Terms ......................................... 6-28

6.2.2.2 Implications for Transport in the UZ .......................................... 6-29

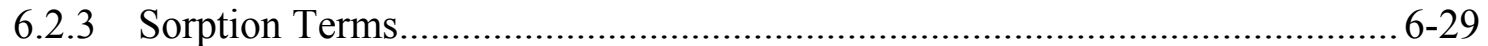

6.2.3.1 Equilibrium Physical Sorption .................................................. 6-29

6.2.3.2 Kinetic Physical Sorption......................................................... 6-30

6.2.3.3 Kinetic Chemical Sorption ............................................................ 6-31

6.2.3.4 Implications for Transport in the UZ ........................................... 6-31

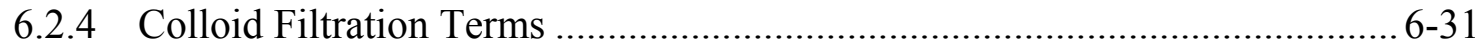

6.2.4.1 Equations of Colloid Filtration..................................................... 6-31

6.2.4.2 Implications for Transport in the UZ .......................................... 6-33

6.2.5 Colloid-Assisted Transport Terms .............................................................. 6-34

6.2.5.1 Equations of Colloid-Assisted Transport ....................................... 6-34

6.2.5.2 Implications for Transport in the UZ .......................................... 6-34

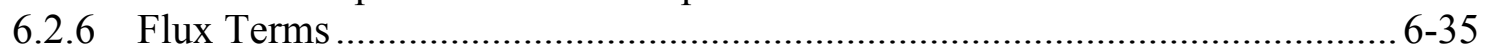

6.2.6.1 Equations of the Flux Terms ....................................................... 6-35

6.2.6.2 Application to Colloid Fluxes ..................................................... 6-35

6.2.6.3 Implications for Transport in the UZ .......................................... 6-36

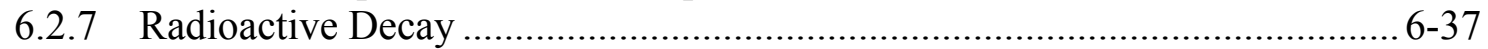

6.2.7.1 Equations of Radioactive Decay .............................................. 6-37

6.2.7.2 Implications for Transport in the UZ ........................................... 6-37

6.2.8 Daughter Products of Radioactive Decay ...................................................... 6-38

6.2.8.1 Transport Equations of Daughters............................................... 6-38

6.2.8.2 Implications for Transport in the UZ .......................................... 6-38

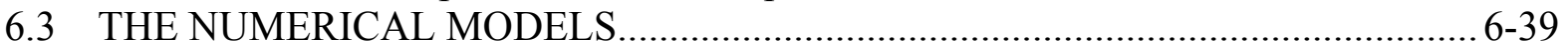

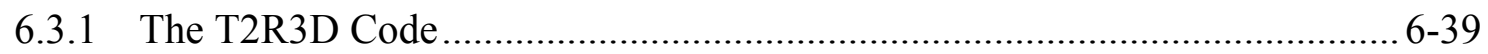

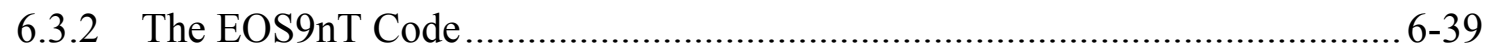

6.3.3 Other Computational Tools.................................................................... 6-40

6.4 FEATURES, EVENTS, AND PROCESSES ……............................................... 6-41

6.5 SORPTION MODEL, DIFFUSION, RADIOACTIVE DECAY, AND

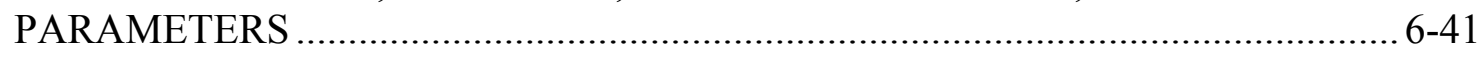




\section{CONTENTS (Continued)}

Page

6.6 A PRELIMINARY CONCEPTUAL MODEL OF TRANSPORT BASED ON

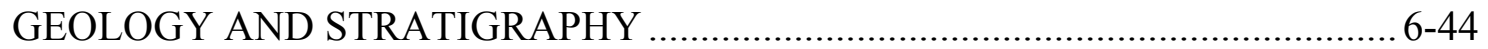

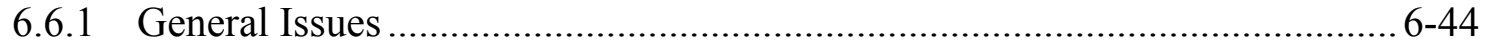

6.6.2 Hydrogeologic Profiles ....................................................................... 6-44

6.6.3 Conceptual Model of Transport in Cross Sections 1 and 2 ......................... 6-44

6.6.4 Conceptual Model of Transport in Cross Section 3 ………......................... 6-50

6.6.5 Synopsis and Discussion......................................................................... 6-50

6.7 THREE-DIMENSIONAL TRANSPORT SIMULATIONS …………………….... 6-52

6.7.1 Climatic Conditions ………………………........................................... 6-52

6.7.2 Conceptual Hydrologic Model, Grids, and Flow Simulations........................ 6-52



6.7.3.1 Instantaneous Release.............................................................. 6-54

6.7.3.2 Continuous Release .................................................................. 6-54

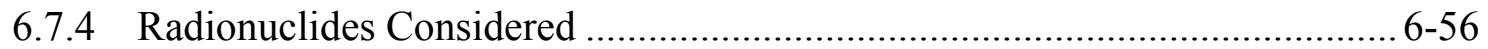

6.7.5 Important Geologic Features................................................................... 6-56

6.7.6 Transport Simulation Options.................................................................. 6-56

6.7.7 Basic Data Inputs and Outputs................................................................... 6-57

6.7.8 Review of the Approach and Basic Approximations in the

Three-Dimensional Site-Scale Radionuclide Transport Studies...................... 6-57

6.8 THREE-DIMENSIONAL SIMULATIONS OF ${ }^{99}$ TC TRANSPORT

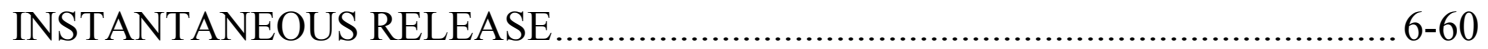

6.8.1 ${ }^{99}$ Tc Transport under Present-Day Infiltration............................................. 6-60

6.8.1.1 Breakthrough Curves............................................................. 6-61

6.8.1.2 Transport-Controlling Features and Flow Patterns ......................... 6-63

6.8.1.3 Evidence Supporting the Transport Patterns .................................. 6-65

6.8.2 ${ }^{99} \mathrm{Tc}$ Transport under Monsoon and Glacial-Transition Infiltrations............... 6-91

6.8.3 Uncertainties in ${ }^{99} \mathrm{Tc}$ Transport Predictions.............................................. 6-92

6.8.3.1 Uncertainties in the Estimates of Flow and Hydraulic

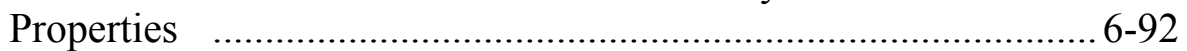

6.8.3.2 Uncertainties in the Retardation Processes ................................... 6-93

6.8.3.3 Climatic Uncertainties ................................................................ 6-94

6.9 THREE-DIMENSIONAL TRANSPORT OF ${ }^{237} \mathrm{NP}$ - INSTANTANEOUS

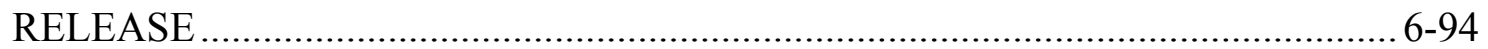

6.9.1 Transport of ${ }^{237} \mathrm{~Np}$ Under Present-Day Infiltration......................................... 6-94

6.9.1.1 Breakthrough Curves.................................................................... 6-94

6.9.1.2 Transport Mechanisms and Patterns.............................................. 6-95

6.9.1.3 Transport-Controlling Features ..................................................... 6-97

6.9.1.4 Comparison to the ${ }^{99} \mathrm{Tc}$ Transport ................................................ 6-97

6.9.1.5 Effect of Sorption in the Main Geologic Units on ${ }^{237} \mathrm{~Np}$

Transport ……..................................................................... 6-97

6.9.2 ${ }^{237} \mathrm{~Np}$ Transport under Monsoon and Glacial-Transition Infiltration ............. 6-99

6.9.3 Uncertainties in ${ }^{237} \mathrm{~Np}$ Transport Predictions ............................................ 6-100

6.9.3.1 Uncertainties in Matrix Diffusion ............................................... 6-100

6.9.3.2 Uncertainties in Sorption............................................................. 6-101 


\section{CONTENTS (Continued)}

6.10 THREE-DIMENSIONAL TRANSPORT OF ${ }^{239} \mathrm{PU}$ - INSTANTANEOUS

RELEASE

6.10.1 Transport of ${ }^{239} \mathrm{Pu}$ under Present-Day Infiltration

6-103

6.10.1.1 Breakthrough Curves 6-103

6.10.1.2 Transport Mechanisms and Patterns, and

Transport-Controlling Features

6.10.1.3 Comparison to the ${ }^{99} \mathrm{Tc}$ and ${ }^{237} \mathrm{~Np}$ Transport 6-103

6.10.1.4 Effect of Sorption in the Main Geologic Units on ${ }^{239} \mathrm{Pu}$ Transport 6-105

6.10.2 Uncertainties in ${ }^{239} \mathrm{Pu}$ Transport Predictions 6-107

6.10.3 Applicability of Uncertainty Studies to Other Radionuclides. 6-108

6.11 THREE-DIMENSIONAL TRANSPORT OF ${ }^{233} \mathrm{U}$ AND ${ }^{235} \mathrm{U}$

- INSTANTANEOUS RELEASE

6-108

6.11.1 Transport of ${ }^{233} \mathrm{U}$ Under Different Climatic Conditions 6-108

6.11.2 Transport of ${ }^{235} \mathrm{U}$ under Different Climatic Conditions. 6-108

6.12 THREE-DIMENSIONAL TRANSPORT OF ${ }^{241} \mathrm{AM}$ AND ${ }^{90} \mathrm{SR}$ - INSTANTANEOUS RELEASE

6.12.1 ${ }^{241}$ Am Transport.

6-111

6.12.2 ${ }^{90} \mathrm{Sr}$ Transport. 6-111

6.13 THREE-DIMENSIONAL TRANSPORT OF ${ }^{135} \mathrm{CS}$ - INSTANTANEOUS

RELEASE 6-111

6.14 THREE-DIMENSIONAL TRANSPORT OF ${ }^{226}$ RA, ${ }^{229} \mathrm{TH}$, AND ${ }^{231} \mathrm{PA}-$

INSTANTANEOUS RELEASE 6-114

6.15 THREE-DIMENSIONAL SIMULATIONS OF ${ }^{99} \mathrm{TC},{ }^{237} \mathrm{NP}$, AND ${ }^{239} \mathrm{PU}$ TRANSPORT - CONTINUOUS RELEASE 6-116

6.15.1 The Continuous-Release Scenario 6-120

6.15.2 The Definition of the Breakthrough Curves for Continuous Release

6-120

6.15.3 Transport of Continuously Released ${ }^{99} \mathrm{Tc},{ }^{237} \mathrm{~Np}$, and ${ }^{239} \mathrm{Pu}$ under

6.15.4 Transport Patterns of Continuously Released ${ }^{99} \mathrm{Tc},{ }^{237} \mathrm{~Np}$, and ${ }^{239} \mathrm{Pu}$ under

6-120

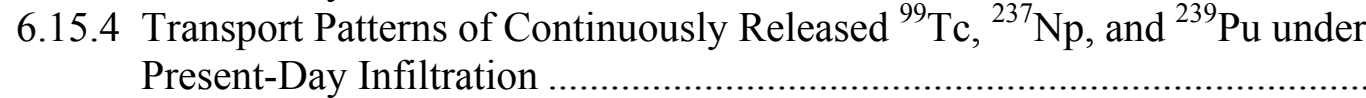

6.16 THREE-DIMENSIONAL TRANSPORT OF THE ${ }^{239} \mathrm{PU} \longrightarrow{ }^{235} \mathrm{U} \longrightarrow{ }^{231} \mathrm{PA}$ 6-121

CHAIN - CONTINUOUS RELEASE 6-123

6.17 THREE-DIMENSIONAL TRANSPORT OF THE ${ }^{241} \mathrm{AM} \longrightarrow{ }^{237} \mathrm{NP} \longrightarrow{ }^{233} \mathrm{U}$ $\longrightarrow{ }^{229}$ TH CHAIN - CONTINUOUS RELEASE ............................................ 6-126

6.18 THREE-DIMENSIONAL SITE-SCALE TRANSPORT OF ${ }^{239} \mathrm{PUO}_{2}$ COLLOIDS - CONTINUOUS RELEASE 6-128

6.18.1 Colloidal Forms and Properties 6-129

6.18.2 Colloid Filtration Model and Coefficients 6-129

6.18.3 True Colloid Transport Simulation Cases 6-130

6.18.4 Colloid Transport Case 1 (True Colloids) 6-131

6.18.5 Colloid Transport Case 2 (True Colloids) 6-132

6.18.5.1 Breakthrough Curves 6-132

6.18.5.2 Transport Mechanisms and Patterns of the $6 \mathrm{~nm}$ Colloid 6-132 


\section{CONTENTS (Continued)}

Page

6.18.5.3 Transport Mechanisms and Patterns of the $450 \mathrm{~nm}$ Colloid ........ 6-135

6.18.6 Colloid Transport Case 3 (True Colloids) ................................................ 6-135

6.18.7 Colloid Transport Case 4 (True Colloids) ............................................... 6-136

6.18.8 Transport of Wasteform Colloids (Case 2 and 3) ........................................ 6-136

6.18.9 Uncertainties and Limitations ............................................................. 6-137

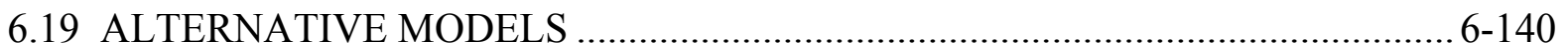

6.19.1 Alternative Representations and Methods ................................................ 6-140

6.19.2 Alternative Diffusion Model.................................................................... 6-141

6.19.2.1 Instantaneous Release ........................................................ 6-142

6.19.2.2 Continuous Release ............................................................. 6-144

6.20 BARRIER EVALUATION, UNCERTAINTIES, AND A NOTE OF CAUTION .6-146

6.20.1 Barrier Performance.............................................................................. 6-146

6.20.2 The Effect of Radionuclide Release Directly into the Fault Fractures ......... 6-151

6.20.3 Interpreting the Model Report Results............................................... 6-156

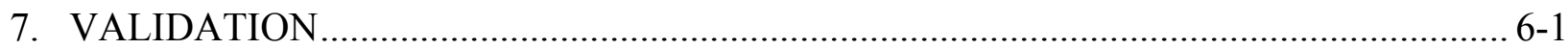

7.1 CONFIDENCE BUILDING DURING MODEL DEVELOPMENT TO

ESTABLISH SCIENTIFIC BASIS AND ACCURACY FOR INTENDED USE...... 7-1

7.2 POSTDEVELOPMENT MODEL VALIDATION TO SUPPORT THE SCIENTIFIC

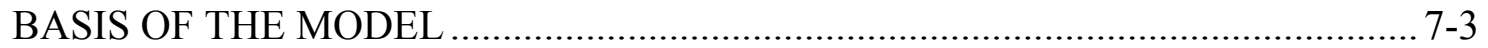

7.2.1 Stipulated Validation Requirements, Methods, and Criteria .......................... 7-3

7.2.2 Validation Approach and Criteria ............................................................. 7-4

7.2.3 Corroboration with Alternative Mathematical Models (Method 2).................. 7-6

7.2.3.1 Comparison of T2R3D and EOS9nT with linear equilibrium

Sorption During Isothermal Transport ........................................ 7-6

7.2.3.2 Test 1: Transport of a Nonsorbing, Nondecaying Tracer............... 7-7

7.2.3.3 Test 2: Transport of Sorbing Radioactive Tracers ......................... 7-8

7.2.3.4 Test 3: Transport of the ${ }^{234} \mathrm{U}->{ }^{230} \mathrm{Th}->{ }^{226} \mathrm{Ra}$ Chain..................... 7-9

7.2.3.5 Test 4: ${ }^{3} \mathrm{H}$ Transport in a Fracture-Matrix System ....................... 7-12

7.2.3.6 Test 5: Colloid and Colloid-Assisted Radionuclide Transport ..... 7-13

7.2.4 Corroboration with Experimental Data (Method 1).................................. 7-16

7.2.4.1 UZ Radionuclide Transport Model Postdevelopment

Validation Through Corroboration with the Busted Butte Field

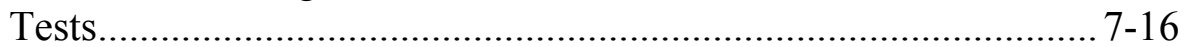

7.2.4.1.1 Calibration Versus Validation................................. 7-16

7.2.4.1.2 Phase 1A Test....................................................... 7-17

7.2.4.1.3 Phase 1B Test......................................................... 7-24

7.2.4.1.4 Phase 2C Test...................................................... 7-28

7.2.4.1.5 Summary of Postdevelopment Validation from

Busted Butte Field Tests ......................................... 7-35

7.2.4.2 Postdevelopment Validation Using Chloride and ${ }^{14} \mathrm{C}$ Field

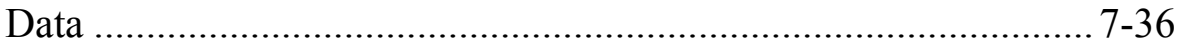




\section{CONTENTS (Continued)}

7.3 VALIDATION OF THE MATRIX DIFFUSION SUB-MODEL BY CORROBORATION WITH DATA FROM ALCOVE 8/NICHE 3

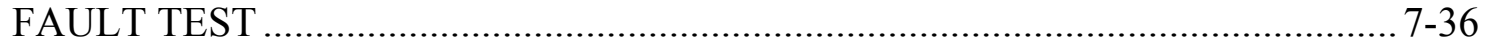

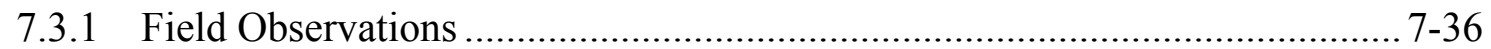

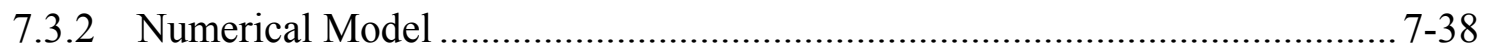

7.3.3 Model Simulations and Discussions …………........................................ 7-39

7.3.3.1 Calibration of Rock Properties Using Seepage-Rate Data and the Average Water-Travel-Velocity Data ………………………... 7-39

7.3.3.2 Prediction of Tracer Transport with Matrix Diffusion.................... 7-41

7.3.3.3 A Further Discussion of Matrix Diffusion Effects.......................... 7-42

7.4 VALIDATION OF THE SORPTION SUB-MODEL ........................................... 7-50

7.5 VALIDATION OF THE COLLOID TRANSPORT SUB-MODEL ......................... 7-51

7.6 VALIDATION OF THE ACTIVE FRACTURE SUB-MODEL WITH MATRIX

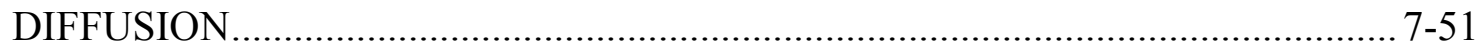

7.6.1 Confidence Building during Model Development.......................................... 7-52

7.6.2 Postdevelopment Validation ....................................................................... 7-53

7.7 PUBLICATION IN REFEREED PROFESSIONAL JOURNAL ……..................... 7-55

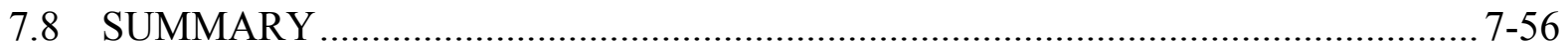

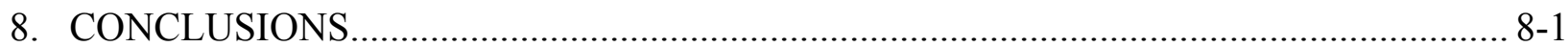

8.1 SUMMARY OF MODELING ACTIVITY .............................................................. 8-1

8.2. HOW THE APPLICABLE ACCEPTANCE CRITERIA ARE ADDRESSED ............ 8-1

8.2. MODEL REPORT CONCLUSIONS …………................................................ 8-4

8.2.1 General Conclusions from the Three-Dimensional Studies............................. 8-4

8.2.2 Conclusions from the Three-Dimensional Studies of Instantaneously

Released Radioactive Solutes …………………………………………...... 8-6

8.2.3 Conclusions from the Three-Dimensional Studies of Continuously

Released Radioactive Solutes ...................................................................... 8-7

8.2.4 Conclusions from the Three-Dimensional Studies of Continuously

Released Radioactive Colloids ..................................................................... 8-7

8.2.5 Conclusions from the Study of Alternative Conceptual Models ...................... 8-8

8.2.6 Conclusions from the Barrier Evaluation …………...................................... 8-8

8.3. MODEL OUTPUT, UNCERTAINTIES, AND LIMITATIONS............................... 8-9

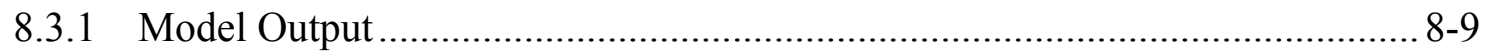

8.3.2 Model Uncertainties ............................................................................... 8-9

8.3.2.1 Uncertainties in the Flow Conceptual Model and the

Corresponding Parameters ………………………………….......... 8-9

8.3.2.2 Climatic Uncertainties .............................................................. 8-9

8.3.2.3 Uncertainties in Matrix Diffusion ................................................ 8-10

8.3.2.4 Uncertainties in Sorption......................................................... 8-10

8.3.2.5 Uncertainties in Filtration............................................................ 8-10

8.3.3 Limitations of Applicability ..................................................................... 8-10

9. INPUTS AND REFERENCES ................................................................................. 9-1 9.1 DOCUMENTS CITED ............................... ERROR! BOOKMARK NOT DEFINED. 


\section{CONTENTS (Continued)}

Page

9.2 CODES, STANDARDS, REGULATIONS, AND PROCEDURES ……………...... 9-20

9.3 SOURCE DATA, LISTED BY DATA TRACKING NUMBER ……......................9-21

9.4 OUTPUT DATA, LISTED BY DATA TRACKING NUMBER ………..................9-26

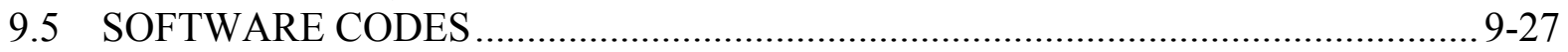

APPENDIX A - TECHNICAL BASIS FOR $K_{d}$ PROBABILITY DISTRIBUTION FUNCTIONS

APPENDIX B - CORRELATIONS FOR SAMPLING OF SORPTION COEFFICIENT PROBABILITY DISTRIBUTIONS

APPENDIX C - SUPPORTING GEOLOGICAL DATA FOR THE CONCEPTUAL MODEL

APPENDIX D - FIGURES FROM THE ${ }^{237}$ Np THREE-DIMENSIONAL TRANSPORT STUDIES (INSTANTANEOUS RELEASE, MEAN PRESENT-DAY INFILTRATION) Output DTN: LB0307MR0060R1.002

APPENDIX E - FIGURES FROM THE ${ }^{99}$ Tc THREE-DIMENSIONAL TRANSPORT STUDIES (CONTINUOUS RELEASE, MEAN PRESENT-DAY INFILTRATION)

APPENDIX F - FIGURES FROM THE THREE-DIMENSIONAL TRANSPORT STUDIES OF THE $6 \mathrm{~nm}^{239} \mathrm{PuO}_{2}$ COLLOID IN CASE 2 (CONTINUOUS RELEASE, MEAN PRESENT-DAY INFILTRATION) F-1

APPENDIX G - FIGURES FROM THE THREE-DIMENSIONAL TRANSPORT STUDIES OF THE $450 \mathrm{~nm}{ }^{239} \mathrm{PuO}_{2}$ COLLOID IN CASE 2 (CONTINUOUS RELEASE, MEAN PRESENT-DAY INFILTRATION) G-1

APPENDIX H - FIGURES FROM THE ${ }^{99}$ Tc THREE-DIMENSIONAL TRANSPORT STUDIES (INSTANTANEOUS RELEASE ONLY FROM NONFAULTED DOMAIN, MEAN PRESENT-DAY INFILTRATION) $\mathrm{H}-1$

APPENDIX I - TEMPERATURE EFFECT ON RADIONUCLIDE SORPTION

APPENDIX J - QUALIFICATION OF SORPTION DATA

DTNS: LA0305AM831341.001

AND LA0310AM831341.001

APPENDIX K - PLAN FOR QUALIFICATION OF SORPTION DATA DTNS: LA0305AM831341.001 AND LA0310AM831341.001 K-1

APPENDIX L - QUALIFICATION OF COMPOSITIONS OF WATERS FROM UE-25 J-13 AND UE-25 P\#1(CARBONATE) 


\section{INTENTIONALLY LEFT BLANK}




\section{FIGURES}

Page

6-1. Two-Dimensional (Plan View) of the Yucca Mountain Site Identifying the Locations of Boreholes USW SD-6, SD-12, and UZ-14, Representing Cross Sections 1, 2, and 3

6-2. Hydrogeologic Units (and their Layers) of the UZ Model Domain from the

Repository to the Groundwater in Cross Section 1 near the USW SD-6 Borehole....... 6-46

6-3. Hydrogeologic Units (and their Layers) of the UZ Model Domain from the Repository to the Groundwater Table in Cross Section 2 near the USW SD-12 Borehole.

6-4. Hydrogeologic Units (and their Layers) of the UZ Model Domain from the Repository to the Groundwater in Cross Section 3 near the USW UZ-14 Borehole..... 6-48

6-5. A Conceptual Model of Transport in Cross Section 1 .............................................. 6-49

6-6. A Conceptual Model of Transport in Cross Section 3 .............................................. 6-51

6-7. Two-Dimensional (Plan View) of the UZ Model Grid Design at the Repository Level

6-8. Cumulative Breakthrough of the ${ }^{99} \mathrm{Tc}$ Mass Fraction $\mathrm{R}_{\mathrm{M}}$ at the Water Table for Varying Present-Day Climatic Scenarios and Instantaneous Release.....

6-9. Distribution of the Relative Mass Fraction $X_{R}$ of ${ }^{99} \mathrm{Tc}$ in the Fractures of the tsw39 Layer at $t=10$ Years for Mean Present-Day Infiltration and Instantaneous Release.

6-10. Distribution of the Relative Mass Fraction $\mathrm{X}_{\mathrm{R}}$ of ${ }^{99} \mathrm{Tc}$ in the Matrix of the tsw39 Layer at $t=10$ Years for Mean Present-Day Infiltration and Instantaneous Release.... 6-67

6-11. Distribution of the Relative Mass Fraction $X_{R}$ of ${ }^{99} \mathrm{Tc}$ in the Fractures of the tsw39 Layer at $t=100$ Years for Mean Present-Day Infiltration and Instantaneous Release....

6-12. Distribution of the Relative Mass Fraction $\mathrm{X}_{\mathrm{R}}$ of ${ }^{99} \mathrm{Tc}$ in the Matrix of the tsw39 layer at $t=100$ Years for Mean Present-Day Infiltration and Instantaneous Release.....

6-13. Distribution of the Relative Mass Fraction $X_{R}$ of ${ }^{99} \mathrm{Tc}$ in the Fractures of the tsw39 Layer at $t=1,000$ Years for Mean Present-Day Infiltration and Instantaneous Release.

6-14. Distribution of the Relative Mass Fraction $X_{R}$ of ${ }^{99} \mathrm{Tc}$ in the Matrix of the tsw39 Layer at $t=1,000$ Years for Mean Present-Day Infiltration and Instantaneous Release.....

6-15. Distribution of the Relative Mass Fraction $X_{R}$ of ${ }^{99} \mathrm{Tc}$ in the Fractures of the tsw39 Layer at $t=10,000$ Years for Mean Present-Day Infiltration and Instantaneous Release

6-16. Distribution of the Relative Mass Fraction $X_{R}$ of ${ }^{99} \mathrm{Tc}$ in the Matrix of the tsw39

Layer at $t=10,000$ Years for Mean Present-Day Infiltration and Instantaneous Release....

6-17. Distribution of the Relative Mass Fraction $X_{R}$ of ${ }^{99} \mathrm{Tc}$ in the Fractures of the tsw39 Layer at $t=100,000$ Years for Mean Present-Day Infiltration and Instantaneous Release. 


\section{FIGURES (Continued)}

Page

6-18. Distribution of the Relative Mass Fraction $X_{R}$ of ${ }^{99} \mathrm{Tc}$ in the Matrix of the tsw39 Layer at $t=100,000$ Years for Mean Present-Day Infiltration and Instantaneous Release.

6-19. Distribution of the Relative Mass Fraction $X_{R}$ of ${ }^{99} \mathrm{Tc}$ in the Fractures Immediately above the Water Table at $t=10$ Years for Mean Present-Day Infiltration and Instantaneous Release....

6-20. Distribution of the Relative Mass Fraction $X_{R}$ of ${ }^{99} \mathrm{Tc}$ in the Matrix Immediately above the Water Table at $\mathrm{t}=10$ Years for a Mean Present-Day Infiltration and Instantaneous Release.

6-21. Distribution of the Relative Mass Fraction $X_{R}$ of ${ }^{99} \mathrm{Tc}$ in the Fractures Immediately above the Water Table at $\mathrm{t}=100$ Years for Mean Present-Day Infiltration and Instantaneous Release....

6-22. Distribution of the Relative Mass Fraction $X_{R}$ of ${ }^{99} \mathrm{Tc}$ in the Matrix Immediately above the Water Table at $\mathrm{t}=100$ Years for a Mean Present-Day Infiltration and Instantaneous Release.

6-23. Distribution of the Relative Mass Fraction $X_{R}$ of ${ }^{99} \mathrm{Tc}$ in the Fractures

Immediately above the Water Table at $t=1,000$ Years for Mean Present-Day Infiltration and Instantaneous Release....

6-24. Distribution of the Relative Mass Fraction $\mathrm{X}_{\mathrm{R}}$ of ${ }^{99} \mathrm{Tc}$ in the Matrix Immediately above the Water Table at $\mathrm{t}=1000$ years for a Mean Present-Day Infiltration and Instantaneous Release

6-25. Distribution of the Relative Mass Fraction $X_{R}$ of ${ }^{99} \mathrm{Tc}$ in the Fractures

Immediately above the Water Table at $t=10,000$ Years for Mean Present-Day Infiltration and Instantaneous Release....

6-26. Distribution of the Relative Mass Fraction $X_{R}$ of ${ }^{99} \mathrm{Tc}$ in the Matrix Immediately above the Water Table at $t=10,000$ Years for a Mean Present-Day Infiltration and Instantaneous Release

6-27. Distribution of the Relative Mass Fraction $\mathrm{X}_{\mathrm{R}}$ of ${ }^{99} \mathrm{Tc}$ in the Fractures Immediately above the Water Table at $t=100,000$ Years for Mean Present-Day Infiltration and Instantaneous Release....

6-28. Distribution of the Relative Mass Fraction $X_{R}$ of ${ }^{99} \mathrm{Tc}$ in the Matrix Immediately above the Water Table at $t=100,000$ Years for a Mean Present-Day Infiltration and Instantaneous Release

6-29a. Mineralogy Model Plots below the Repository ...................................................... 6-86

6-29b. Mineralogy Model Plots below the Repository ................................................... 6-87

6-30. Mean Present-Day Infiltration Rates at the Surface .......................................... 6-88

6-31. Percolation Fluxes at the Repository Level .......................................................... 6-89

6-32. Percolation Fluxes at the Water Table Level......................................................... 6-90

6-33. Cumulative Breakthrough of the ${ }^{99}$ Tc Mass Fraction $R_{M}$ at the Water Table for Varying Monsoon Climatic Scenarios and Instantaneous Release.....

6-34. Cumulative Breakthrough of the ${ }^{99} \mathrm{Tc}$ Mass Fraction $R_{M}$ at the Water Table for Varying Glacial-Transition Climatic Scenarios and Instantaneous Release. 


\section{FIGURES (Continued)}

Page

6-35. Effect of Uncertainty in $\mathrm{D}_{0}$ on the Cumulative Breakthrough of the ${ }^{99} \mathrm{Tc}$ Mass Fraction $R_{M}$ at the Water Table for Mean Present-Day Infiltration and Instantaneous Release.

6-36. Cumulative Breakthrough of the ${ }^{237} \mathrm{~Np}$ Mass Fraction $R_{M}$ at the Water Table for Varying Present-Day Climatic Scenarios and Instantaneous Release.....

6-37. The effect of sorption in TSw, $\mathrm{CHz}$, and $\mathrm{CHv}$ on the Cumulative Breakthrough of

${ }^{237} \mathrm{~Np}$ at the Water Table for Mean Present-Day Infiltration and Instantaneous

Release

6-38. Cumulative Breakthrough of the ${ }^{237} \mathrm{~Np}$ Mass Fraction $R_{M}$ at the Water Table for Varying Monsoon Climatic Scenarios and Instantaneous Release

6-39. Cumulative Breakthrough of the ${ }^{237} \mathrm{~Np}$ Mass Fraction $R_{M}$ at the Water Table for Varying Glacial-Transition Climatic Scenarios and Instantaneous Release.

6-40. Effect of Uncertainty in $D_{0}$ on ${ }^{237} \mathrm{~Np}$ Breakthrough at the Water Table for Mean Present-Day Infiltration and Instantaneous Release

6-41. Effect of Uncertainty in $\mathrm{K}_{\mathrm{d}}$ on ${ }^{237} \mathrm{~Np}$ Breakthrough at the Water Table for Mean Present-Day Infiltration and Instantaneous Release

6-42. Cumulative Breakthrough of the ${ }^{239} \mathrm{Pu}$ Mass Fraction $R_{M}$ at the Water Table for Varying Present-Day Climatic Scenarios and Instantaneous Release ....

6-43. Cumulative Breakthrough of the ${ }^{239} \mathrm{Pu}$ Mass Fraction $R_{M}$ at the Water Table for Instantaneous Release and (a) Monsoon and (b) Glacial-Transition Infiltration

6-44. The Effect of Sorption in TSw, CHz, and CHv on the Cumulative Breakthrough of ${ }^{239} \mathrm{Pu}$ at the Water Table for Mean Present-Day Infiltration and Instantaneous Release

6-45. Effect of Uncertainty in $D_{0}$ on the ${ }^{239} \mathrm{Pu}$ Breakthrough at the Water Table for Mean Present-Day Infiltration and Instantaneous Release

6-46. Cumulative Breakthrough of the ${ }^{233} \mathrm{U}$ Mass Fraction $R_{M}$ at the Water Table for Instantaneous Release for (a) Present-Day, (b) Monsoon, and (c) GlacialTransition Infiltration

6-47. Cumulative Breakthrough of the ${ }^{235} \mathrm{U}$ Mass Fraction $R_{M}$ at the Water Table for Instantaneous Release for (a) Present-Day, (b) Monsoon, and (c) GlacialTransition Infiltration

6-48. Cumulative Breakthrough of the ${ }^{241}$ Am Mass Fraction $R_{M}$ at the Water Table for Instantaneous Release for (a) Present-day, (b) Monsoon, and (c) GlacialTransition Infiltration

6-49. Cumulative Breakthrough of the ${ }^{90} \mathrm{Sr}$ Mass Fraction $R_{M}$ at the Water Table for Instantaneous Release for (a) Present-Day, (b) Monsoon, and (c) GlacialTransition Infiltration

6-50. Cumulative Breakthrough of the ${ }^{135}$ Cs Mass Fraction $R_{M}$ at the Water Table for Instantaneous Release for (a) Present-Day, (b) Monsoon, and (c) GlacialTransition Infiltration

6-51. Cumulative Breakthrough of the ${ }^{226} \mathrm{Ra}$ Mass Fraction $R_{M}$ at the Water Table for Instantaneous Release for (a) Present-Day, (b) Monsoon, and (c) GlacialTransition Infiltration 


\section{FIGURES (Continued)}

Page

6-52. Cumulative Breakthrough of the ${ }^{229} \mathrm{Th}$ Mass Fraction $R_{M}$ at the Water Table for Instantaneous Release for (a) Present-Day, (b) Monsoon, and (c) Glacial-

Transition Infiltration.

6-53. Cumulative Breakthrough of the ${ }^{231} \mathrm{~Pa}$ Mass Fraction $R_{M}$ at the Water Table for Instantaneous Release for (a) Present-Day, (b) Monsoon, and (c) GlacialTransition Infiltration.

6-54. Normalized Relative Release $R_{F}$ of ${ }^{99} \mathrm{Tc},{ }^{237} \mathrm{~Np}$, and ${ }^{239} \mathrm{Pu}$ at the Water Table for Continuous Release and Mean Present-Day Climatic Scenarios

6-55. Mass Fractions of Each Member of the ${ }^{239} \mathrm{Pu}$ Chain in the Release Stream at (a) the Repository and (b) the Water Table - Continuous Release Mean Present-Day Climatic Scenarios

6-56. Normalized Relative Release $R_{F}$ of the Sum of All Members of the ${ }^{239} \mathrm{Pu}$ Chain at the Water Table - Continuous Release Mean Present-Day Climatic Scenarios.

6-57. Mass Fractions of Each Member of the ${ }^{241} \mathrm{Am}$ Chain in the Release Stream at (a) the Repository and (b) the Water Table - Continuous Release Mean Present-Day Climatic Scenarios

6-58. Normalized Relative Release $R_{F}$ of the Sum of All Members of the ${ }^{241}$ Am Chain at the Water Table - Continuous Release Mean Present-Day Climatic Scenario

6-59. Normalized Release at the Water Table in (a) Case 1 and (b) Case 2 for True Colloid Transport for Mean Present-Day Infiltration (All the Continous Release Curves at the Repository Are Superimposed, Source Undergoes Decay).....

6-60. Normalized Release at the Water Table in (a) Case 3 and (b) Case 4 for Transport of True Colloids for Mean Present-Day Infiltration (All the Continuous Release Curves at the Repository Are Superimposed, Source Undergoes Decay).

6-61. Normalized Release at the Water Table in (a) Case 2 and (b) Case 3 for Transport of Wasteform Colloids with Embedded Radionuclides (Mean Present-Day Infiltration, Continuous Source with Decay)

6-62. Effect of (a) the MINC Concept and (b) Application of Particle-Tracking Approaches on Breakthrough Predictions at a Two-Dimensional Vertical Cross Section of the UZ for an Instantaneous Release under Mean, Present-Day Climate for a Nonsorbing, Non-decaying Tracer

6-63. Cumulative Breakthrough of Radioactive Solutes at the Water Table for the NoDiffusion Alternative Model (Mean Present-Day Infiltration, Instantaneous Release)

6-64. Relative Release Rate of Radioactive Solutes at the Water Table for the NoDiffusion Alternative Model (Mean Present-Day Infiltration, Continuous Release)

6-65. Comparison of Early Breakthrough at the Water Table for Mean GlacialTransition Climate and Instantaneous Release. Numbers immediately below element symbols are the matrix diffusion coefficients $\times 10^{-10}\left(\mathrm{~m}^{2} / \mathrm{s}\right)$.Numbers in second line below element symbols are the sorption coefficient values $(\mathrm{mL} / \mathrm{g})$ for devitrified, vitric, and zeolitic rock, respectively. DTN: LB0307MR0060R1.007

6-66. Cumulative Breakthrough of the ${ }^{99} \mathrm{Tc}$ and ${ }^{237} \mathrm{~Np}$ Mass Fractions $R_{M}$ at the Water Table (Instantaneous Release, Mean Present-Day Infiltration, No Fault Releases) .... 6-152 


\section{FIGURES (Continued)}

Page

6-67. Cumulative Breakthrough of the ${ }^{239} \mathrm{Pu}$ and ${ }^{235} \mathrm{U}$ Mass Fractions $R_{M}$ at the Water Table (Instantaneous Release, Mean Present-Day Infiltration, No Fault Releases) .... 6-153

6-68. Cumulative Breakthrough of the ${ }^{99} \mathrm{Tc}$ and ${ }^{237} \mathrm{~Np}$ Normalized Release Rates $R_{F}$ at the Water Table (Continuous Release of Decaying Source, Mean Present-Day Infiltration, No Fault Releases)

6-69. Cumulative Breakthrough of the ${ }^{239} \mathrm{Pu}$ and ${ }^{235} \mathrm{U}$ Normalized Release Rates $R_{F}$ at the Water Table (Continuous Release of Decaying Source, Mean Present-Day Infiltration, No Fault Releases)

7-1. Comparison between the Numerical Predictions of Breakthrough from TOUGH2 V1.11 Module EOS9nT V1.0 (LBNL 1999 [DIRS 113943]) and from T2R3D V1.4 (LBNL 1999 [DIRS 146654]).

7-2. Comparison between the Analytical Solution and the Numerical Predictions in Test 1

7-3. Comparison between the Numerical Predictions (TOUGH2 V1.11 Module EOS9nT V1.0 [LBNL 1999 (DIRS 113943)]; T2R3D V1.4 [LBNL 1999 (DIRS 146654)]) and the Analytical Solutions (Bear 1979 [DIRS 105038]) in Test 2

7-4. Comparison between the Analytical Solution and the TOUGH2 V1.11 Module EOS9nT V1.0 (LBNL 1999 [DIRS 113943]) Predictions in Test 3

7-5. Comparison between the Analytical Solution of Sudicky and Frind (1982 [DIRS 105043]) and the Numerical (TOUGH2 V1.11 Module EOS9nT V1.0 [LBNL 1999 (DIRS 113943) and T2R3D V1.4 [LBNL 1999 (DIRS 146654)]) Predictions in Test 4

7-6. Comparison between the Numerical (TOUGH2 V1.11 Module EOS9nT V1.0 (LBNL 1999 [DIRS 113943])) and the Analytical (de Marsily 1986 [DIRS 100439]) Solution of Colloid Transport in Test 5

7-7. Fluorescein Plume at the $\mathrm{y}=0.9 \mathrm{~m}$ Mineback Face at Borehole 3 .

7-8. Numerical Prediction of the Fluorescein Plume Using Calibrated Parameters (Busted Butte Test Phase 1A)

7-9. Field Measurements and Numerical Prediction of the Bromide Distribution in Busted Butte Test Phase 1A. The Solid Circles Indicate the Location of Measurements (DTN: LA9910WS831372.008 [DIRS 147156]), Which Appear in the Corresponding Boxes

7-10. Observed and Numerically Predicted (Calibrated) Breakthrough Curves of 2,6DFBA in the Busted Butte Phase 1B Test. Measurements from DTN: LA0201WS831372.008 [DIRS 162766]). Prediction from output DTN: LB0308MR0060R1.009.

7-11. Observed and Numerically Predicted (at Validation) Breakthrough Curves of $\mathrm{Br}$ in the Busted Butte Phase 1B Test. Measurements from DTN: LA0201WS831372.008 [DIRS 162766]), Prediction from output DTN: LB0308MR0060R1.009.

7-12. Observed and Numerically Predicted (Calibrated) Breakthrough Curves of Li in the Busted Butte Phase 2C Test 


\section{FIGURES (Continued)}

7-13. Observed and Numerically Predicted Breakthrough Curves of $\mathrm{Br}$ in the Busted Butte Phase 2C Test (at Validation).....

7-14. Infiltration Rate as a Function of Time $7-45$

7-15. Total Seepage Rate as a Function of Time. Data for 3/6/2001 through 1/28/2002 ..... 7-46

7-16. Water Travel Velocity Data at Two Boreholes $(9$ and 10) ....................................... 7-46

7-17. Observed Flux-Average Breakthrough Curve .......................................................... 7-47

7-18. A Cross Section View of Three-Dimensional Numerical Grid .................................. 7-47

7-19. A Comparison between Simulated Seepage Rates as a Function of Time (Run \#1) and Field Observations

7-20. A Comparison among Calculated Water Travel Velocities from Two Calibration Runs and the Velocity Data Observed from the Fault Test.....

7-21. A Comparison between Simulated Seepage Rates as a Function of Time (Run \#2) and Field Observations

7-22. Comparisons between Simulated Breakthrough Curves at the Niche for Two Different Fault-Matrix Interface Areas and the Observed Data.

7-23. Comparisons between Simulated Breakthrough Curves (Considering Dispersion) at the Niche for the Increased Fault-Matrix Interface Areas and the Observed Data.... 7-50

7-24. Comparison of (a) Simulated Matrix Liquid Saturation and (b) Water Potentials Using Calibrated Hydraulic Properties (Solid Line) with That Obtained Using a Smaller (Half) Value of $\gamma$ in the Units of Under Repository (Dashed Line) for Borehole USW SD-6 - From BSC 2004 [DIRS 169861], Section 6.8, Figure 6.8-2 ... 7-54

7-25. Comparison of a Simulated Breakthrough Curve of Relative Tracer Mass at the Groundwater Table Obtained for the Base Case (Using Calibrated Rock Hydraulic Properties, Red Solid Line), a Case Using a Smaller (Half) Value of $\gamma$ of the TSw Units (Blue Dash Line), and Another Case Using a Smaller (Half) Value of $\gamma$ of All Units below the Repository (Green Solid Line) - From BSC 2004 [DIRS 169861], Section 6.8, Figure 6.8-3 


\section{TABLES}

Page

3-1. Qualified Software Used in this Model Report........................................................... 3-1

4-1. Input Data................................................................................................... $4-1$

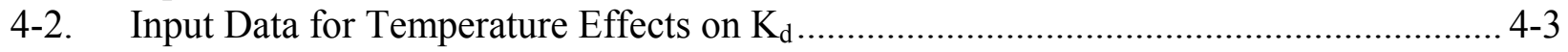

4-3. Project Requirements and YMRP Acceptance Criteria Applicable to this Model

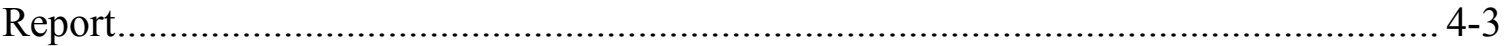

5-1. Main Data Assumptions Used in this Study ............................................................ 5-1

6-1. Scientific Notebooks Used for this Model Report..................................................... 6-4

6-2. FEPs Included Through This Model Report.......................................................... 6-41

6-3. $\quad \mathrm{K}_{\mathrm{d}}$ in the Rocks of the Unsaturated Zone............................................................... 6-42

6-4. Properties of Radionuclides in the Transport Simulations ......................................... 6-43

6-5. Percolation Fluxes (mm/year) for Different Climatic Regimes................................ 6-53

6-6. DTNs of Input and Output Files of the Three-Dimensional Site-Scale Transport

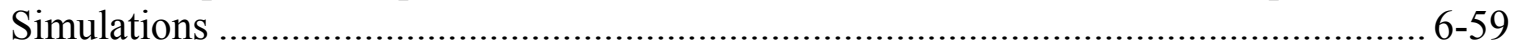

6-7. Input Parameters for the Three-Dimensional Site-Scale Simulations of Solute Transport (\#1 Perched-Water Model) .................................................................... 6-62

6-8. Properties of the Four Colloids in the EOS9nT Simulations .................................... 6-130

6-9. Input Parameters for the EOS9nT Three-Dimensional Site-Scale Simulations of Colloid Transport (\# 1 Perched-Water Model, Mean Present-Day Infiltration).......... 6-130

6-10. Radionuclide Transport Times to the Water Table (Instantaneous Release).............. 6-148

6-11. Radionuclide Transport Times to the Water Table under Continuous Release........... 6-149

6-12. Colloid Transport Times to the Water Table (Continuous Release).......................... 6-149

7-1. Parameters in Test 1 ..................................................................................... 7-11

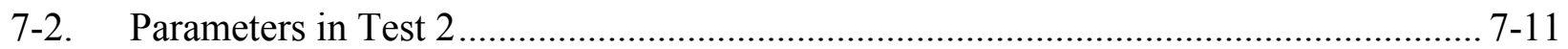

7-3. Parameters in Test 3 ........................................................................................ 7-11

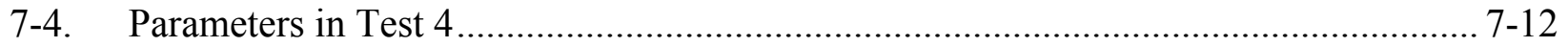

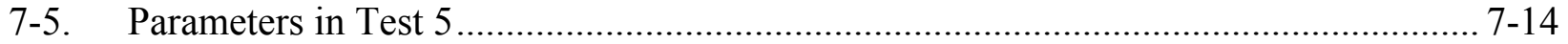

7-6. Model-Validation - Comparison of Numerical to Analytical Solutions in Tests 1

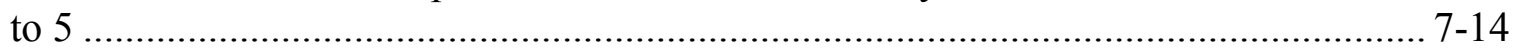

7-7. Operational, Transport, and Field Parameters in the Analysis of the Busted Butte Phase 1 Field Test ....................................................................................................... 7-19

7-8. Hydrological Parameters in the Analysis of the Busted Butte Phase 1A Field Test .... 7-20

7-9. Calibrated Parameters of Flow and Transport from the Analysis of the Busted Butte Phase 1A Field Test................................................................................... 7-21

7-10. Initial Parameters in the Analysis of the Busted Butte Phase 1B Field Test ............... 7-27

7-11. Calibrated Flow and Transport Parameters from the Analysis of the Busted Butte Phase 1B Field Test ................................................................................................ 7-28

7-12. Operational, Transport, and Field Parameters in the Analysis of the Busted Butte Phase 2 Field Test..... 


\section{TABLES (Continued)}

7-13. Hydrological and Transport Parameters in the Analysis of the Busted Butte Phase 2 Field Test

7-14. Calibrated Flow and Transport Parameters from the Analysis of the Busted Butte Phase 2C Field Test

7-15. Comparison of Numerical Solutions to Field Tests ................................................ 7-35

7-16. Uncalibrated Rock Properties ................................................................................ 7-44

7-17. Rock Properties Calibrated from Seepage Rate Data (Run \#1) .................................. 7-44

7-18. Rock Properties Calibrated from Both Seepage Rate and Water Travel Velocity Data (Run \#2)

8-1. Output Data and Data Tracking Numbers $8-11$ 


\section{ACRONYMS AND ABBREVIATIONS}

B.E.T. $\quad$ Brunauer, Emmett, Teller method

CHn Calico Hills nonwelded units

$\mathrm{CHv} \quad$ Calico Hills vitric units

$\mathrm{CHz} \quad$ Calico Hills zeolitic units

EDL electrical double layer

ESF Exploratory Studies Facility

FEP feature, event, and process

GRS Gesellschaft für Anlagen und Reaktorsicherheit

LA license application

MINC Multiple Interactive Continua

NTS Nevada Test Site

QA quality assurance

REE rare earth element

TDMS Technical Data Management System

TSPA- $\quad$ total system performance assessment

TSw Topopah Spring welded unit

UZ unsaturated zone

YMP Yucca Mountain Project

YMRP Yucca Mountain Review Plan, Final Report 


\section{INTENTIONALLY LEFT BLANK}




\section{PURPOSE}

The purpose of this model report is to document the unsaturated zone (UZ) radionuclide transport model, which evaluates, by means of three-dimensional numerical models, the transport of radioactive solutes and colloids in the UZ, under ambient conditions, from the repository horizon to the water table at Yucca Mountain, Nevada.

This model report also addresses comments made in the Model Validation Status Review (BSC 2003 [DIRS 170030]), validates the active fracture model with Matrix Diffusion, and justifies the values of sorption coefficients $\left(\mathrm{K}_{d} \mathrm{~s}\right)$. These activities are in accordance with the Technical Work Plan (TWP) for: Unsaturated Zone Transport Model Report Integration (BSC 2004 [DIRS 171282], Section 2.1.1). This model report used flow fields provided by model report UZ Flow Models and Submodels (BSC 2004 [DIRS 169861]).

The output of this model report is a set of breakthrough curves for use as benchmarks to validate another model, FEHM (LANL 2003 [DIRS 165741]), which is to be used for UZ transport calculations for the total system performance assessment (TSPA) for license application (LA). Validation of the FEHM-based model is documented in Particle Tracking Model and Abstraction of Transport Processes (BSC 2004 [DIRS 170041]). This validation is specified in Section 2.2.3 of the technical work plan (BSC 2004 [DIRS 171282]) and was also specified in Section I-2-2-2 of the technical work plan (BSC 2004 [DIRS 167969]) under the validation of the FEHM particle tracking model, where it states "Agreement with other codes will be demonstrated by running the same problem that has previously been run with DCPT, T2R3D, or TOUGH2." Transport calculations presented in this report using DCPT (LBNL 2002 [DIRS 154342]) and T2R3D (LBNL 1999 [DIRS 146654]) are intended to be used as part of the validation of the FEHM particle tracking model.

In the UZ radionuclide transport model simulations presented in this model report, radionuclides are considered to be uniformly distributed at time zero in the fractures throughout the repository elements, and their subsequent transport to the water table is simulated. The UZ radionuclide transport model does not consider the waste form, waste package, drip shield, or any other component of the Engineered Barrier System, and, therefore, the results are not to be construed as predictions of potential radionuclide transport after the repository closure, but only as examples of radionuclide transport calculations for this initial condition (i.e., corresponding to a time frame starting at the onset of radionuclide release).

This model report documents the UZ radionuclide transport model. This model considers:

- Transport of radionuclides through fractured tuffs

- Effects of changes in the intensity and characteristics of fracturing between different hydrogeologic units

- Colloid transport

- Physical and retardation processes 
- Effects of different conceptual representations of the matrix-fracture system

- Effects of different modeling approaches in the description of the transport problem.

The present model report documents the capabilities of the UZ radionuclide transport model, which can describe flow (saturated and/or unsaturated) and transport, and accounts for (a) advection, (b) molecular diffusion, (c) hydrodynamic dispersion (with full three-dimensional tensorial representation), (d) kinetic or equilibrium physical and/or chemical sorption (linear, Langmuir, Freundlich, or combined), (e) first-order linear chemical reaction, (f) radioactive decay and tracking of daughters, (f) colloid filtration (equilibrium, kinetic, or combined), and (g) colloid-assisted solute transport. The present model report also includes determination and discussion of the distribution coefficients $\mathrm{K}_{\mathrm{d}}$ of the various sorbing radioactive solutes in the UZ rocks, as well as a corresponding uncertainty analysis (see Appendix A, this model report).

Transport of radioactive solutes and colloids (incorporating the processes described above) from the repository horizon to the water table are simulated to support model development and studies for performance assessment. The results of the simulations are used to evaluate the transport of radioactive solutes and colloids, and to determine the processes, mechanisms, and geologic features that have a significant effect on transport. The contribution of radioactive-decay daughter products to the total radionuclide inventory transport and from the bottom of the repository to the water table are evaluated, as well as the effect on transport of different conceptual models for fracture-matrix systems. The effect of different modeling approaches on transport predictions are considered.

The primary uncertainty for using the modeling results documented in this report is that the input transport parameters were based on limited site data. For some input parameters, best estimates were used because no specific data were available. An additional uncertainty is that the radionuclide transport model is based on the conceptual models and numerical approaches used for developing flow fields and infiltration maps, and thus they share the same limitations (see BSC 2004 [DIRS 169861]).

The following analysis and model reports are the sources of DTNs that provide direct inputs to the TSPA-LA that are also used in this report:

- UZ Flow Models and Submodels (BSC 2004 [DIRS 169861])

- Site-Scale Saturated Zone Transport (BSC 2004 [DIRS 170036])

- Analysis of Hydrologic Properties Data (BSC 2003 [DIRS 161773])

The following analysis and model reports use output from this report:

- Features, Events, and Processes in UZ Flow and Transport

- EBS Radionuclide Transport Abstraction

- Particle Tracking Model and Abstraction of Transport Processes.

Compared to Technical Work Plan for: Performance Assessment Unsaturated Zone (BSC 2004 [DIRS 167969]) and Section 2.2.2 of Technical Work Plan for: Unsaturated Zone Transport Model Report Integration (BSC 2004 [DIRS 171282]), there are some variations from the 
criteria used in the validation component of this study. For the validation of the radionuclide transport model using the numerical codes, their simulation results are compared to relevant analytical solutions. The validation criterion used in this case is one or more of the following:

(a) Mass fractions agree within 5 percent when $C_{R}=C / C_{0} \leq 10^{-4}$

(b) The location of the tracer fronts agree within 5 percent when $C_{R}=C / C_{0} \leq 10^{-4}$

(c) The tracer mass balances agree within 5 percent.

The results presented in Section 7.2.3 show that this criterion was met in all instances. The criterion presented above is somewhat different from that discussed in the Technical Work Plan for: Performance Assessment Unsaturated Zone (BSC 2004 [DIRS 167969], Section I-2-1-1) and listed in Section 7.2.1 of this model report because the latter (a) is inadequate in that it captures only one of the three possible aspects for quantitative agreement, and (b) can be unrealistic near the tail end of the $C_{R}$ curve (where $C_{R}$ values are very low, and both analytical and numerical predictions may be unreliable because of round off error). For calibration and additional validation of the radionuclide transport model using numerical codes (through comparison of their predictions to field measurements-Method 1), the criterion used is the following:

- Overall agreement between predictions and field measurements are within 50 percent of each other.

- If this agreement is not possible, document the analysis and discussion of the reasons for the observed deviations.

Inability of the radionuclide transport model to match/predict field observations is not necessarily a sign of inability to validate the model if (1) the quality of the field measurements are suspect (i.e., the test design may be responsible for uncertainties in the measurement), (2) there is insufficient information to fully describe the field test, or (3) field data involve very steep gradients (involving very significant parameter changes over a short distance or time) that lead to measurements prone to uncertainties and inaccuracies.

The approach and criteria for the Method 1 validation in this model report is somewhat different from the one discussed in the Technical Work Plan for: Performance Assessment Unsaturated Zone (BSC 2004 [DIRS 167969], Section I-2-1-1) and listed in Section 7.2.1 of this model report. The technical work plan criterion is inadequate, because it addresses only the option of time-series data (i.e., breakthrough) without accounting for the possibility of data involving concentration distribution in space at a particular time. The validation criterion used addresses this shortcoming, is more inclusive, and is stricter. 


\section{INTENTIONALLY LEFT BLANK}




\section{QUALITY ASSURANCE}

Development of this model report and the supporting modeling activities have been determined to be subject to the Yucca Mountain Project's (YMP's) quality assurance (QA) program as indicated in Technical Work Plan for: Unsaturated Zone Transport Model Report Integration (BSC 2004 [DIRS 171282], Section 8.2, Work Package AUZM07). This report was prepared under AP-SIII.10Q, Models. Approved QA procedures identified in Section 4 of the technical work plan have been used to conduct and document the activities described in this model report. Section 8.4 of the technical work plan also identifies the methods used to control the electronic management of data during the modeling and documentation activities.

This model report discusses ambient radionuclide transport through hydrogeologic units, which are a natural barrier and are classified in the Q-List (BSC 2004 [DIRS 168361]) as "Safety Category" because it is important to waste isolation, as defined in AP-2.22Q, Classification Analyses and Maintenance of the Q-List. The results of this report are important to the demonstration of compliance with the postclosure performance objectives prescribed in 10 CFR 63.113 [DIRS 156605]. The report contributes to the analysis data used to support PA; the conclusions do not directly impact engineered features important to safety, as defined in AP-2.22Q. 


\section{INTENTIONALLY LEFT BLANK}




\section{USE OF SOFTWARE}

The software codes and routines used in this study are listed in Table 3-1. All the software items used in this study, that is, TOUGH2 V1.11MEOS9nTV1.0 (LBNL 1999 [DIRS 113943]), TOUGH2 V1.4 Module EOS9 V1.4 (LBNL 2000 [DIRS 146496]), TOUGH2 V1.6 Module EOS9 (LBNL 2003 [DIRS 160242]; LBNL 2003 [DIRS 161491]), T2R3D V1.4 (LBNL 1999 [DIRS 146654]), DCPT V2.0 (LBNL 2001 [DIRS 154342]), iTOUGH2 V4.0 (LBNL 1999 [DIRS 139918]), PHREEQC V2.3 (BSC 2001 [DIRS 155323]), Bkread.f V1.0 (LBNL 2002 [DIRS 162143]), XtractG.f90 V1.0 (LBNL 2003 [DIRS 162786]), and Smesh.f V1.0 (LBNL 2002 [DIRS 162142]), were appropriate for the intended application used only within the range of their software validation, and obtained from software configuration management per AP-SI.1Q, Software Management. The superceded procedure AP-SI.1Q, Software Management, was used because this procedure governing the use of software was in effect at the time Revision 01 was completed. There has been no additional use of software in the preparation of Revision 02.

Table 3-1. Qualified Software Used in this Model Report

\begin{tabular}{|l|l|l|l|l|}
\hline $\begin{array}{c}\text { Software } \\
\text { Name }\end{array}$ & \multicolumn{1}{|c|}{ Version } & \multicolumn{1}{|c|}{$\begin{array}{c}\text { Software Tracking } \\
\text { Number }\end{array}$} & $\begin{array}{c}\text { Platform / Operating } \\
\text { System }\end{array}$ & $\begin{array}{c}\text { Document Input } \\
\text { Reference } \\
\text { System Number }\end{array}$ \\
\hline iTOUGH2 & 4.0 & $10003-4.0-00$ & Dec Alpha / OSF1 V4.0 & [DIRS 139918] \\
\hline TOUGH2 & 1.11 MEOS9nTV1.0 & $10065-1.11$ MEOS9nTV1.0-00 & Dec Alpha / OSF1 V4.0 & [DIRS 113943] \\
\hline TOUGH2 & 1.4 & $10007-1.4-01$ & Dec Alpha / OSF1 V4.0 & [DIRS 146496] \\
\hline TOUGH2 & 1.6 & $10007-1.6-01$ & Dec Alpha / OSF1 V5.1 & [DIRS 161491] \\
\hline TOUGH2 & 1.6 & $10007-1.6-00$ & Dec Alpha / OSF1 V4.0 & [DIRS 160242] \\
\hline T2R3D & 1.4 & $10006-1.4-00$ & Dec Alpha / OSF1 V4.0 & [DIRS 146654] \\
\hline DCPT & 2.0 & $10078-2.0-00$ & PC / Windows NT V4.0 & [DIRS 154342] \\
\hline PHREEQC & 2.3 & $10068-2.3-00$ & PC / Windows 98 & [DIRS 155323] \\
\hline Bkread.f & 1.0 & $10894-1.0-00$ & Sun Sparc / SunOS 5.5.1 & [DIRS 162143] \\
\hline XtractG.f90 & 1.0 & $10930-1.0-00$ & Dec Alpha / OSF1 V5.1 & [DIRS 162786] \\
\hline Smesh.f & 1.0 & $10896-1.0-00$ & Sun Sparc / SunOS 5.5.1 & [DIRS 162142] \\
\hline
\end{tabular}

The software code TOUGH2 (V1.11 MEOS9nTV1.0, STN: 10065-1.11MEOS9NTV1.0-00) (LBNL 1999 [DIRS 113943]) simulates flow and the decoupled transport of multiple radioactive solutes and/or colloids (parents and daughters) in complex subsurface systems involving porous and/or fractured media. The transport equations account for advection, molecular/colloidal diffusion, hydrodynamic dispersion, kinetic or equilibrium physical and chemical sorption (linear, Langmuir, Freundlich, or combined), first-order linear chemical reaction, colloid filtration, and colloid-assisted solute transport.

The code T2R3D (V1.4, STN: 10006-1.4-00) (LBNL 1999 [DIRS 146654]) simulates flow and the coupled transport of a single radioactive solute tracer in complex subsurface systems involving porous and/or fractured media. The transport equations account for advection, molecular diffusion, hydrodynamic dispersion, and linear equilibrium sorption. 
The software codes TOUGH2 (V1.4, STN: 10007-1.4-01, Module EOS9 V1.4) (LBNL 2000 [DIRS 146496]), TOUGH2 (V1.6, STN: 10007-1.6-00 Module EOS9 V1.6) (LBNL 2002 [DIRS 160242]), and TOUGH2 (V1.6, STN: 10007-1.6-01) LBNL 2003 [DIRS 161491]) solve the Richards equation to simulate flow in complex subsurface systems (involving porous and/or fractured media).

The software code DCPT (V2.0, STN: 10078-2.0-00) (LBNL 2001 [DIRS 154342]) involves the particle-tracking method to simulate transport of a single radioactive solute tracer in complex subsurface systems involving porous and/or fractured media. The transport equations account for advection, molecular diffusion, hydrodynamic dispersion, and linear equilibrium sorption.

The software iTOUGH2 (V4.0, STN: 10003-4.0-00) (LBNL 1999 [DIRS 139918]) and Bkread.f (V1.0, STN: 10894-1.0-00) (LBNL 2002 [DIRS 162143]) are used for model calibration and prediction of Alcove 8/Niche 3 tests documented in Section 7.3. A numerical model, generated with a software routine Smesh.f (V1.0, STN: 10896-1.0-00) (LBNL 2002 [DIRS 162142]), was developed for the test site to compare the simulation results with the relevant field observations.

For elements that sorb primarily through surface complexation reactions, the experimental data are augmented with the results of modeling calculations using PHREEQC (V2.3, STN: 10068-2.3-00) (BSC 2001 [DIRS 155323]). The inputs for the modeling calculations include groundwater compositions, surface areas, binding constants for the elements of interest, and thermo-dynamic data for solution species. These modeling calculations provide a basis for interpolation and extrapolation of the experimentally derived sorption distribution coefficient dataset (see Appendix A).

The software XtractG.f90 (V1.0, STN: 10930-1.0-00) (LBNL 2003 [DIRS 162786]) was used to ready the mesh files for the EOS9nT simulations, for the preparation of the corresponding initial conditions files, and the extraction of desired data subsets for plotting.

Standard visual display graphics programs (i.e., IGOR Pro. 4, Tecplot V8.0, Adobe Illustrator V8.0) also were used to illustrate information but are not subject to software QA requirements, per Section 2.0 of AP-SI.1Q.

This model report documents the UZ radionuclide transport model. 


\section{INPUTS}

The input used in this model report consist of the following:

- Transport properties

- Calibrated fracture and matrix properties

- Base case flow fields

- Geochemical data

- Numerical grids.

\subsection{DIRECT INPUT}

Specific input data sets and their associated data tracking numbers and sources are listed in Table 4-1. The Q-status of these data is provided in the Document Input Reference System. Discussions of uncertainties in the input data and parameters, and detailed explanations about the selection of corresponding choice of values are addressed in Section 6.

The molecular diffusion coefficients and half-lives of radionuclides were obtained from accepted sources and have been qualified in the Technical Data Management System (TDMS). Sorption coefficients were measured on rock samples from Yucca Mountain and using approved procedures, rock properties, including matrix permeability and porosity, fracture permeability and spacing, were calibrated with field tests on Yucca Mountain. Further discussion of the suitability of the sorption data is presented in Appendix J. The grid for numerical simulation was based upon the geological framework model of Yucca Mountain. Therefore, these data are all appropriate for simulations of radionuclide transport.

Table 4-1. Input Data

\begin{tabular}{|c|c|c|}
\hline Description & Parameters & $\begin{array}{c}\text { Data Tracking Number / Technical } \\
\text { Information Source }\end{array}$ \\
\hline \multirow{6}{*}{$\begin{array}{l}\text { Properties of the main } \\
\text { radionuclides in the transport } \\
\text { simulations (Tc, } \mathrm{Np}, \mathrm{Pu}, \mathrm{U}, \mathrm{Pa} \text {, } \\
\mathrm{U}, \mathrm{Th}, \mathrm{Am}, \mathrm{Cs}, \mathrm{Ra}, \mathrm{Sr}) \text { (Section } \\
6.5 \text { to Section 6.18) }\end{array}$} & Molecular diffusion coefficient & $\begin{array}{l}\text { Lide } 1992 \text { [DIRS 166224], pp. 5-111 to } \\
5-112\end{array}$ \\
\hline & $\begin{array}{l}\text { Half-life for the first-order } \\
\text { radioactive decay } T_{1 / 2} \\
\end{array}$ & $\begin{array}{l}\text { Lide } 1992 \text { [DIRS 166224], pp. 11-13 to } \\
\text { 11-133 }\end{array}$ \\
\hline & Sorption distribution coefficient $K_{d}$ & $\begin{array}{l}\text { LA0305AM831341.001 [DIRS 163789] } \\
\text { LA0407AM831343.001 [DIRS 171009] } \\
\text { LA0407AM831341.001 [DIRS 170623] } \\
\text { LA0407AM831341.002 [DIRS 170621] } \\
\text { LA0407AM831341.004 [DIRS 170622] } \\
\text { LA0407AM831341.005 [DIRS 170625] } \\
\text { LA0407AM831341.003 [DIRS 170626] } \\
\text { LA0407AM831341.006 [DIRS 170628] } \\
\text { LA0310AM831341.001 [DIRS 165865] }\end{array}$ \\
\hline & $\begin{array}{l}\text { Solubilities of radioactive } \\
\text { elements }\end{array}$ & $\begin{array}{l}\text { MO0408SPADCLRE.000 [DIRS 171601] } \\
\text { LA0407AM831343.001 [DIRS 171009] }\end{array}$ \\
\hline & $\begin{array}{l}\text { Water compositions for UE-25 J- } \\
13 \text { and UE-25 p\#1 waters. }\end{array}$ & Ogard and Kerrisk 1984 [DIRS 100783] \\
\hline & Colloid density $\rho_{c}\left(\mathrm{PuO}_{2}\right)$ & Lide 1993 [DIRS 123032], p. 4-83 \\
\hline
\end{tabular}


Table 4-1. Input Data (Continued)

\begin{tabular}{|l|l|l|}
\hline \multicolumn{1}{|c|}{ Description } & \multicolumn{1}{|c|}{ Parameters } & \multicolumn{1}{c|}{$\begin{array}{c}\text { Data Tracking Number / Technical } \\
\text { Information Source }\end{array}$} \\
\hline $\begin{array}{l}\text { Data for 3-D site-scale } \\
\text { radionuclide (solute) transport } \\
\text { modeling (Section 6.5 to } \\
\text { Section 6.18) }\end{array}$ & $\begin{array}{l}\text { 3-D site-scale grid, hydraulic } \\
\text { properties/parameters of the } \\
\text { various hydrogeologic units, } \\
\text { pressures, water saturations, 3-D } \\
\text { flow fields for three scenarios } \\
\text { (mean, high, and low) for each of } \\
\text { three climatic conditions } \\
\text { (present-day, monsoon, and } \\
\text { glacial-transition) }\end{array}$ & $\begin{array}{l}\text { LB03013DSSCP3I.001 [DIRS 162379], } \\
\text { LB03023DSSCP9I.001 [DIRS 163044] }\end{array}$ \\
\cline { 2 - 3 } & $\begin{array}{l}\text { Porosity and grain density of the } \\
\text { various hydrogeologic units }\end{array}$ & LB0210THRMLPRP.001 [DIRS 160799] \\
\hline $\begin{array}{l}\text { EOS9nT 3-D site-scale } \\
\text { simulations of colloid transport } \\
\text { (perched-water model \#1, mean } \\
\text { present-day infiltration) (Section } \\
\text { 6.7) }\end{array}$ & $\begin{array}{l}\text { 3-D site-scale grid, hydraulic } \\
\text { properties/parameters of the } \\
\text { various hydrogeologic units, } \\
\text { pressures, water saturations, 3-D } \\
\text { flow fields }\end{array}$ & LB03013DSSCP3I.001 [DIRS 162379], \\
LB03023DSSCP9I.001 [DIRS 163044] & \\
\cline { 2 - 3 } & $\begin{array}{l}\text { Pore size data for pore size } \\
\text { exclusion estimation }\end{array}$ & $\begin{array}{l}\text { GS950608312231.008 [DIRS 144662] } \\
\text { GS980908312242.039 [DIRS 145272] }\end{array}$ \\
\hline $\begin{array}{l}\text { EOS9nT 3-D site-scale } \\
\text { simulations (radioactive solutes } \\
\text { and colloids, Section 6.15 to } \\
\text { Section 6.18) }\end{array}$ & 3-D grid used in the simulations & LB03023DSSCP9I.001 [DIRS 163044] \\
\hline
\end{tabular}

3-D = three-dimensional

DTN: LB0210THRMLPRP.001 (DIRS 160799) is used for porosity and grain density. Although errors were identified in DTN LB0210THRMLPRP.001 (DIRS 160799) and these errors have been corrected in DTN LB0402THRMLPRP.001 (DIRS 168481), there are four reasons why the original data set is being used for radionuclide transport:

- The corrected data set became available too late to be implemented in TSPA-LA.

- For most applications, other UZ models supporting TSPA-LA use the properties given in DTN LB0210THRMLPRP.001 (DIRS 160799). Therefore, these properties are needed for consistency with other models implemented in TSPA-LA. Those applications that do not use DTN LB0210THRMLPRP.001 (DIRS 160799) use DTN: LB0207REVUZPRP.002 (DIRS 159672) (not DTN LB0402THRMLPRP.001 [DIRS 168481]) because this data set contains additional statistical information (standard deviations) for hydrologic properties.

- The errors in DTN LB0210THRMLPRP.001 (DIRS 160799) are negligible. Profiles along three boreholes between the repository and the water table (Appendix C) were checked for total porosity (for nonsorbing radionuclides) and total "storage capacity" (porosity plus sorptive storage fraction) using properties from DTN: LB0210THRMLPRP.001 (DIRS 160799) and from DTN: LB0402THRMLPRP.001 (DIRS 168481). The differences were less than 5 percent in all but one case and less than 11 percent in the exceptional case, using a matrix water saturation of 0.9 and a sorption coefficient $\left(\mathrm{K}_{\mathrm{d}}\right)$ of $1 \mathrm{~mL} / \mathrm{g}$ for the sorbing 
cases. These differences may be compared with the order of magnitude uncertainties in transport times shown in Figures 6-8, 6-33, 6-34, 6-36, 6-38, and 6-39.

- The differences in all cases give smaller total porosities and sorptive storage fractions, leading to faster transport using DTN LB0210THRMLPRP.001 (DIRS 160799) instead of DTN LB0402THRMLPRP.001 (DIRS 168481).

Radionuclide sorption data for evaluating thermal influences are reported in the form of $\mathrm{K}_{\mathrm{d}}$ values. Sources of $\mathrm{K}_{\mathrm{d}}$ values for cesium, strontium, barium, and neptunium are listed in Table 4-2. These data are further discussed in Appendix A, together with data from other sources used as corroborative information. Note that data for other radionuclides were not considered because they did not include $\mathrm{K}_{\mathrm{d}}$ measurements as a function of temperature.

Table 4-2. Input Data for Temperature Effects on $\mathrm{K}_{\mathrm{d}}$

\begin{tabular}{|l|l|l|}
\hline \multicolumn{1}{|c|}{ Description } & \multicolumn{1}{|c|}{ Parameters } & \multicolumn{1}{c|}{$\begin{array}{c}\text { Data Tracking Number/Technical } \\
\text { Information Source }\end{array}$} \\
\hline $\mathrm{K}_{\mathrm{d}}$ for barium & Distribution coefficient & LA0010JC831341.001 [DIRS 162476] \\
\hline $\mathrm{K}_{\mathrm{d}}$ for cesium & Distribution coefficient & LA0010JC831341.002 [DIRS 153321] \\
\hline $\mathrm{K}_{\mathrm{d}}$ for strontium & Distribution coefficient & LA0010JC831341.003 [DIRS 153322] \\
\hline $\mathrm{K}_{\mathrm{d}}$ for neptunium & Distribution coefficient & LA0010JC831341.007 [DIRS 153319] \\
\hline
\end{tabular}

\subsection{CRITERIA}

The general requirements to be satisfied by the TSPA-LA are stated in 10 CFR 63.114 (DIRS 156605). Technical requirements to be satisfied by the TSPA-LA are identified in the Project Requirements Document (Canori and Leitner 2003 [DIRS 166275]). The acceptance criteria that will be used by the U.S. Nuclear Regulatory Commission to determine whether the technical requirements have been met are identified in the Yucca Mountain Review Plan, Final Report (YMRP) (NRC 2003 [DIRS 163274]). The pertinent requirements and criteria for this report are summarized in Table 4-3.

Table 4-3. Project Requirements and YMRP Acceptance Criteria Applicable to this Model Report

\begin{tabular}{|c|c|c|c|}
\hline $\begin{array}{l}\text { Requirement } \\
\text { Number }^{\mathrm{a}}\end{array}$ & Requirement Title $^{a}$ & 10 CFR 63 Link & $\begin{array}{c}\text { YMRP Acceptance } \\
\text { Criteria }^{b}\end{array}$ \\
\hline PRD -002/T-016 & Requirements for performance assessment & $\begin{array}{l}10 \text { CFR } 63.114 \\
\text { (a)-(c), (e), (g) } \\
\text { [DIRS 156605] }\end{array}$ & 2.2.1.3.7.3, criteria 1 to 4 \\
\hline
\end{tabular}

${ }^{a}$ From Canori and Leitner (2003 [DIRS 166275]).

${ }^{\mathrm{b}}$ From NRC (2003 [DIRS 163274]).

YMRP=Yucca Mountain Review Plan

In cases where subsidiary criteria are listed in the YMRP for a given criterion, only the subsidiary criteria addressed by this scientific analysis are listed. The acceptance criteria identified in Section 2.2.1.3.7.3 of the YMRP (NRC 2003 [DIRS 163274] are given 
below. Explanation of how this report meets the acceptance criteria below is given in Section 8.2.

\section{YMRP Section 2.2.1.3.7.3 Radionuclide Transport in the Unsaturated Zone}

\section{Acceptance Criterion 1: System Description and Model Integration Are Adequate.}

(1) Total system performance assessment adequately incorporates important design features, physical phenomena, and couplings, and uses consistent and appropriate assumptions throughout the radionuclide transport in the unsaturated zone abstraction process.

(2) The description of the aspects of hydrology, geology, geochemistry, design features, physical phenomena, and couplings, that may affect radionuclide transport in the unsaturated zone, is adequate. For example, the description includes changes in transport properties in the unsaturated zone, from water-rock interaction. Conditions and assumptions in the total system performance assessment abstraction of radionuclide transport in the unsaturated zone are readily identified, and consistent with the body of data presented in the description;

(3) The abstraction of radionuclide transport in the unsaturated zone uses assumptions, technical bases, data, and models that are appropriate and consistent with other related U.S. Department of Energy abstractions. For example, the assumptions used for radionuclide transport in the unsaturated zone are consistent with the abstractions of radionuclide release rates and solubility limits and flow paths in the unsaturated zone (Sections 2.2.1.3.4 and 2.2.1.3.6 of the Yucca Mountain Review Plan, respectively) (NRC 2003 [DIRS 163274]). The descriptions and technical bases provide transparent and traceable support for the abstraction of radionuclide transport in the unsaturated zone.

(5) Sufficient data and technical bases for the inclusion of features, events, and processes, related to radionuclide transport in the unsaturated zone in the total system performance assessment abstraction, are provided; and

(6) Guidance in NUREG-1297 and NUREG-1298 (Altman et al. 1988 [DIRS 103597]; [DIRS 103750]), or other acceptable approaches, is followed for peer review and data qualification.

\section{Acceptance Criterion 2: Data Are Sufficient for Model Justification.}

(1) Geological, hydrological, and geochemical values, used in the license application, are adequately justified (e.g., flow-path length, sorption coefficients, retardation factors, colloid concentrations, etc.). Adequate descriptions of how the data were used, interpreted, and appropriately synthesized into the parameters are provided;

(2) Sufficient data have been collected on the characteristics of the natural system to establish initial and boundary conditions for the total system performance assessment abstraction of radionuclide transport in the saturated zone; 
(3) Data on the geology, hydrology, and geochemistry of the unsaturated zone, including the influence of structural features, fracture distributions, fracture properties, and stratigraphy, used in the total system performance assessment abstraction are based on appropriate techniques. These techniques may include laboratory experiments, sitespecific field measurements, natural analog research, and process-level modeling studies. As appropriate, sensitivity or uncertainty analyses, used to support the U.S. Department of Energy total system performance assessment abstraction, are adequate to determine the possible need for additional data.

\section{Acceptance Criterion 3: Data Uncertainty Is Characterized and Propagated through the Model Abstraction.}

(1) Models use parameter values, assumed ranges, probability distributions, and bounding assumptions that are technically defensible, reasonably account for uncertainties and variabilities, and do not result in an under-representation of the risk estimate;

(2) For those radionuclides where the total system performance assessment abstraction indicates that transport in fractures and matrix in the unsaturated zone is important to waste isolation: (i) estimated flow and transport parameters are appropriate and valid, based on techniques that may include laboratory experiments, field measurements, natural research, and process-level modeling studies, conducted under conditions relevant to the unsaturated zone at Yucca Mountain; and (ii) models are demonstrated to adequately reproduce field transport test results. For example, if a sorption coefficient approach is used, the assumptions implicit in that approach are verified;

(4) Uncertainty is adequately represented in parameter development for conceptual models, process-level models, and alternative conceptual models, considered in developing the abstraction of radionuclide transport in the unsaturated zone. This may be done either through sensitivity analyses or use of conservative limits.

\section{Acceptance Criterion 4: Model Uncertainty Is Characterized and Propagated through the Model Abstraction.}

(1) Alternative modeling approaches of features, events, and processes are considered and are consistent with available data and current scientific understanding, and the results and limitations are appropriately considered in the abstraction;

(2) Conceptual model uncertainties are adequately defined and documented, and effects on conclusions regarding performance are properly assessed;

(3) Consideration of conceptual model uncertainty is consistent with available site characterization data, laboratory experiments, field measurements, natural analog information and process-level modeling studies; and the treatment of conceptual mode uncertainty does not result in an under-representation of the risk estimate; and 
(4) Appropriate alternative modeling approaches are consistent with available data and current scientific knowledge, and appropriately consider their results and limitations, using tests and analyses that are sensitive to the processes modeled. For example, for radionuclide transport through fractures, the U.S. Department of Energy adequately considers alternative modeling approaches, to develop its understanding of fracture distributions and ranges of fracture flow and transport properties in the unsaturated zone.

\subsection{CODES, STANDARDS, AND REGULATIONS}

No specific, formally established codes, standards, or regulations have been identified as applying to this model report. 


\section{ASSUMPTIONS}

This section discusses assumptions involved in the transport model made in the absence of supporting data, with a short discussion of supporting rationale. The assumed parameter values are listed in Table 5-1.

The transfer coefficient $K_{i}$ indicates the ratio of the concentration of dissolved species in immobile and mobile water. By setting $K_{i}=1$, it is assumed that immobile water is equilibrated instantaneously with mobile water. This assumption was also made by de Marsily (1986 [DIRS 100439], pp. 238 to 239) and is consistent with the assumption of linear adsorptive equilibrium. This topic is discussed further in Section 6.2.3.1; no further confirmation is required.

The kinetics of declogging (release of colloidal particles after being immobilized) is not well understood. Therefore, a wide range of values has been assumed (Section 6.18.3). Simulation results shown in Sections 6.18.4 through 6.18.7 show that transport is not sensitive to the declogging kinetic coefficient; the assumption is therefore adequate for predicting radionuclide transport, and no further confirmation is required.

Because a fracture is by definition devoid of matrix material, assigning a porosity of 1 to fractures is reasonable and requires no confirmation.

Table 5-1. Main Data Assumptions Used in this Study

\begin{tabular}{|l|l|l|l|}
\hline \multicolumn{1}{|c|}{ Parameter } & \multicolumn{1}{c|}{ Sections } & \multicolumn{1}{c|}{ Value } & \multicolumn{1}{c|}{ Justification } \\
\hline $\begin{array}{l}\text { Transfer coefficient } K_{i} \text { for } \\
\text { solutes }\end{array}$ & 6.8 to 6.20 & 1 & Conventionally used value \\
\hline $\begin{array}{l}\text { Backward (reverse) kinetic } \\
\text { filtration (declogging) } \\
\text { coefficient } \kappa^{-}\end{array}$ & 6.18 & $100 \kappa^{+}, 0.1 \kappa^{+}, 0$ & $\begin{array}{l}\text { Reasonable estimates } \\
\text { bracketing the range of } \kappa^{-} \\
\text {(ranges are sufficiently wide } \\
\text { to cover likely values) }\end{array}$ \\
\hline Fracture porosity $\phi$ & 6.6 to $6.17,6.20$ & 1 & $\begin{array}{l}\text { Reasonable estimate } \\
\text { (reasonable for open } \\
\text { fractures) }\end{array}$ \\
\hline
\end{tabular}

The immediately preceding upstream document to this model report is UZ Flow Models and Submodels (BSC 2004 [DIRS 169861]). No assumptions involving numerical values of input parameters were used in that report. However, several approximations and idealization were used for the model development of the UZ Flow Models and Submodels model report. 


\section{INTENTIONALLY LEFT BLANK}




\section{MODEL DISCUSSION}

The objective of this model report is to provide a defensible and credible model of radionuclide transport in individual vertical quasi two-dimensional slices of the UZ, and in large three-dimensional systems using the flow fields submitted for use in TSPA-LA calculations. This is in accordance with the Technical Work Plan for: Unsaturated Zone Transport Model Report Integration (BSC 2004 [DIRS 171282], Section 2.2.2) and with Technical Work Plan (TWP) for: Performance Assessment Unsaturated Zone (BSC 2004 [DIRS 167969], Section 1.11). The output/product of this model report is a set of breakthrough curves for use as benchmarks to validate the FEHM particle-tracking calculations performed for TSPA-LA (BSC 2004 [DIRS 170041]).

This section describes the development of the radionuclide transport model. The primary objectives of the radionuclide transport model are to:

- Integrate the available data for the development of a comprehensive model of radionuclide transport through the UZ of Yucca Mountain under a range of current and future climate conditions, using the comprehensive, calibrated three-dimensional model of the unsaturated flow developed in UZ Flow Models and Submodels (BSC 2004 [DIRS 169861], Section 6.2). Both the radionuclide transport model developed in this report and the FEHM particle-tracking model use this same calibrated three-dimensional unsaturated flow model to define the water velocity distribution.

- Identify the controlling transport processes and phenomena and evaluate the effectiveness of matrix diffusion and sorption as retardation processes.

- Identify the geologic features that are important to radionuclide transport.

- Estimate the migration of important radionuclide solutes and their daughter products from the repository toward the water table.

- Evaluate the effects of various climatic conditions on radionuclide transport.

- Estimate the migration of radioactive colloids from the repository toward the water table, and to determine the sensitivity of colloid transport to the kinetic coefficients of colloid filtration.

- Evaluate the effect of fracture spacing, intensity, and configuration on radionuclide transport and retardation through important hydrogeologic units.

The radionuclide transport model is a dual permeability model that uses steady state flow fields, and simulates transports of solutes and colloids advectively and by diffusion between fracture and matrix. The solutes and colloids also are subject to sorption and filtrations for the case of colloids. 
This section consists of the following subsections:

Section 6.1. Geological Model and Physical Processes. The focus is on (a) the geology and stratigraphy in the UZ of Yucca Mountain, the site for the repository, (b) the processes and phenomena involved in and affecting transport, and (c) important issues that may have an impact on predictions about and understanding of transport behavior of radioactive solutes and colloids in the UZ. Basic modeling approaches are listed.

Section 6.2. Mathematical Model of Transport. This section explains the mathematical basis and implications of the various processes and phenomena involved in the transport of solutes and colloids in the UZ.

Section 6.3. The Numerical Models. This section discusses the codes that implement the numerical models developed from the principles discussed in Section 6.2. These codes are T2R3D V1.4 (hereafter referred to as T2R3D; LBNL 1999 [DIRS 146654]) and TOUGH2 V1.11MEOS9nTV1.0 (hereafter referred to as EOS9nT; LBNL 1999 [DIRS 113943]), and were used for the simulations discussed in Sections 6.6 to 6.20, and in Section 7.

Section 6.4. Features, Events, and Processes. This section focuses on the discussion of selected features, events, and processes (FEPs) taken from the licensing application FEP List, and which are associated with the subject matter of this model report.

Section 6.5. Sorption Model and Parameters. This section focuses on the sorption behavior of the radionuclides of interest in the various UZ rocks, and discusses the determination of the sorption coefficients used in this model report.

Section 6.6. A Preliminary Conceptual Model of Transport Based on Geology and Stratigraphy. This section discusses the development of a conceptual model of radionuclide transport in vertical columns covering the geologic spectrum from the repository to the water table based on the geology and stratigraphy at three representative UZ locations.

Section 6.7. Three-Dimensional Transport Simulations. This section discusses the grids, climatic conditions, perched water model, radionuclides, flow fields, and general approach used in the ensuing three-dimensional site-scale simulations of transport through the UZ (Sections 6.8 to 6.18, and 6.20), as well as the conditions and general options used in these EOS9nT simulations.

Section 6.8. Three-Dimensional Simulations of ${ }^{99}$ Tc Transport-Instantaneous Release. This section discusses the migration of ${ }^{99} \mathrm{Tc}$ through the UZ following a single release event (instantaneous release); investigates the effects of various climatic scenarios on transport, as well as the impact of uncertainty in the contribution of diffusion (as quantified by the choice of the molecular diffusion coefficient) on transport; and identifies transport-controlling geologic features, transport patterns, and important retardation mechanisms. 
Section 6.9. Three-Dimensional Transport of ${ }^{237} \mathrm{~Np}$-Instantaneous Release. This section investigates the transport of ${ }^{237} \mathrm{~Np}$ through the UZ under various climatic scenarios following a single radionuclide release event; studies the sensitivity of transport to uncertainty in matrix diffusion; identifies transport-controlling geologic features and important retardation mechanisms; and compares the concentration distributions and the transport patterns of ${ }^{237} \mathrm{~Np}$ to those of ${ }^{99} \mathrm{Tc}$.

Section 6.10. Three-Dimensional Transport of ${ }^{239} \mathrm{Pu}$-Instantaneous Release. This section studies the transport of ${ }^{239} \mathrm{Pu}$ through the UZ under various climatic scenarios following a single radionuclide release event, and for various levels of matrix diffusion (as controlled by the choice of the diffusion coefficient); identifies transport-controlling geologic features and important retardation mechanisms; and compares the concentration distributions and the transport patterns of ${ }^{239} \mathrm{Pu}$ to those of ${ }^{99} \mathrm{Tc}$ and ${ }^{237} \mathrm{~Np}$.

Section 6.11. Three-Dimensional Transport of ${ }^{233} U$ and ${ }^{235} U$-Instantaneous Release. This section discusses the transport of ${ }^{233} \mathrm{U}$ and ${ }^{235} \mathrm{U}$ through the $\mathrm{UZ}$ under various climatic scenarios following a single radionuclide release event.

Section 6.12. Three-Dimensional Transport of ${ }^{241} \mathrm{Am}$ and ${ }^{90} \mathrm{Sr}$-Instantaneous Release. This section focuses on the transport of ${ }^{241} \mathrm{Am}$ and ${ }^{90} \mathrm{Sr}$ through the UZ under various climatic scenarios following a single radionuclide release event.

Section 6.13. Three-Dimensional Transport of ${ }^{135}$ Cs-Instantaneous Release. This section addresses the issue of transport of ${ }^{135} \mathrm{Cs}$ through the UZ under various climatic scenarios following a single radionuclide release event.

Section 6.14. Three-Dimensional Transport of ${ }^{226} \mathrm{Ra},{ }^{229} \mathrm{Th}$, and ${ }^{231} \mathrm{~Pa}$-Instantaneous Release. This section discusses the transport of ${ }^{226} \mathrm{Ra},{ }^{229} \mathrm{Th}$, and ${ }^{231} \mathrm{~Pa}$, all of which sorb very strongly onto the rocks at Yucca Mountain, and the effect of various climatic scenarios on transport following a single radionuclide release event.

Section 6.15. Three-Dimensional Simulations of ${ }^{99} \mathrm{Tc},{ }^{237} \mathrm{~Np}$, and ${ }^{239} \mathrm{Pu}$ TransportContinuous Release. This section describes the migration of ${ }^{99} \mathrm{Tc},{ }^{237} \mathrm{~Np}$, and ${ }^{239} \mathrm{Pu}$ through the $\mathrm{UZ}$ when the radioactive species are released continuously from a decaying source; identifies transport-controlling geologic features, transport patterns, and important retardation mechanisms; and discusses the differences from the instantaneous release scenario.

Section 6.16. Three-Dimensional Transport of the ${ }^{239} \mathrm{Pu} \longrightarrow{ }^{235} \mathrm{U} \longrightarrow{ }^{231} \mathrm{~Pa}$ ChainContinuous Release. This section studies the transport of the three-member ${ }^{239} \mathrm{Pu}$ chain through the UZ when the radioactive species are continuously released from a decaying source. The importance of accounting for the transport of the whole chain rather than individual species is discussed. 
Section 6.17. Three-Dimensional Transport of the ${ }^{241} \mathrm{Am} \longrightarrow{ }^{237} \mathrm{~Np} \longrightarrow{ }^{233} \mathrm{U} \longrightarrow{ }^{229 T h}$ Chain-Continuous Release. This section explores the transport of the four-member ${ }^{241} \mathrm{Am}$ chain through the UZ when the radioactive species are continuously released from a decaying source. The importance of accounting for the transport of the whole chain rather than individual species is discussed.

Section 6.18. Three-Dimensional Site-Scale Transport of ${ }^{239} \mathrm{PuO}_{2}$ Colloids-Continuous Release. This section explains the transport of four radioactive colloids through the UZ for a mean present-day infiltration; identifies transport-controlling geological features and important retardation mechanisms; and discusses the differences between the transport patterns of the four colloids.

Section 6.19. Alternative Models. This section discusses the following:

- Different representations of the matrix-fracture system (multiple interactive continua [MINC] versus dual-permeability systems)

- Different conceptual methods of describing the transport problem (particle tracking vs. conventional representation)

- Effect of these alternative approaches on predictions of transport through a two-dimensional vertical cross section of the UZ at Yucca Mountain.

Section 6.20. Barrier Evaluation, Uncertainties, and a Note of Caution. This section reviews the results of the analysis and the overall barrier performance of the geological setting underneath the repository; addresses the issue of uncertainty in the predictions and possible implications stemming from this analysis; and discusses the framework within which the results of this model report should be interpreted.

The scientific notebooks used for the activities in this model report are listed in Table 6-1.

Table 6-1. Scientific Notebooks Used for this Model Report

\begin{tabular}{|l|l|l|l|}
\hline \multicolumn{1}{|c|}{$\begin{array}{c}\text { LBNL Scientific } \\
\text { Notebook }\end{array}$} & $\begin{array}{c}\text { YMP M\&O Scientific } \\
\text { Notebook ID }\end{array}$ & \multicolumn{1}{c|}{ Page Numbers } & \multicolumn{1}{c|}{ Citation } \\
\hline YMP-LBNL-GJM-5 & SN-LBNL-SCl-099-V3 & $1-109$ & $\begin{array}{l}\text { Wang 2003 } \\
\text { [DIRS 164021] }\end{array}$ \\
\hline YMP-LBNL-GJM-YS-1 & SN-LBNL-SCI-230-V1 & $8-90$ & $\begin{array}{l}\text { Wang 2003 } \\
\text { [DIRS 164021] }\end{array}$ \\
\hline YMP-LBNL-GJM-GZ-1 & SN-LBNL-SCI-235-V1 & $9-37$ & $\begin{array}{l}\text { Wang 2003 } \\
\text { [DIRS 164021] }\end{array}$ \\
\hline YMP-LBNL-GSB-LHH-3 & SN-LBNL-SCI-215-V1 & $107-109,111-112,114-115$ & $\begin{array}{l}\text { Wang 2003 } \\
\text { [DIRS 164021] }\end{array}$ \\
\hline YMP-LBNL-GSB-QH-1 & SN-LBNL-SCI-168-V1 & $1-38$ & Hu 2001 [DIRS 163148] \\
\hline
\end{tabular}

LBNL=Lawrence Berkeley National Laboratory; $\mathrm{M} \& \mathrm{O}=$ management and operating contractor; YMP=Yucca Mountain Project 


\subsection{GEOLOGICAL MODEL AND PHYSICAL PROCESSES}

\subsubsection{Geological Layering}

The geological model used in this model report is based on the geological framework model (BSC 2004 [DIRS 170029]) and the rock properties model (BSC 2004 [DIRS 170032]. The subsurface formations at Yucca Mountain consist of heterogeneous layers of anisotropic, fractured volcanic rocks. There are alternating layers of welded and nonwelded ash flow and air fall tuffs. The cooling history of these volcanic rock units determines their mechanical and hydrological properties. Beginning from the land surface, the Yucca Mountain geologic units are the Tiva Canyon, Yucca Mountain, Pah Canyon, the Topopah Spring Tuffs, and interbedded tuffs of the Paintbrush Group. Underlying these are the Calico Hills Formation, and the Prow Pass, Bullfrog, and Tram Tuffs of the Crater Flat Group. These formations have been divided into major hydrogeologic units based roughly on the degree of welding. These are the Tiva Canyon welded units; the Paintbrush nonwelded units, consisting primarily of the Yucca Mountain and Pah Canyon members and the interbedded tuffs; the Topopah Spring welded units (TSw); the Calico Hills nonwelded units (CHn); and the Crater Flat undifferentiated hydrogeologic units (Scott and Bonk 1984 [DIRS 104181]; BSC 2004 [DIRS 169855], Section 6).

Conceptual models of flow and transport at Yucca Mountain are described in UZ Flow Models and Submodels (BSC 2004 [DIRS 169861], Section 6). The present model report focuses on the subject of radionuclide transport in the hydrogeologic units beneath the repository horizon. The repository will be sited in the TSw unit (CRWMS M\&O 1999 [DIRS 103773]), and more specifically the tsw34, tsw35, and tsw36 layers of the UZ, depending on the location. More information on these layers can be found in Development of Numerical Grids for UZ Flow and Transport Modeling (BSC 2004 [DIRS 169855], Section 6) and Analysis of Hydrologic Properties Data (BSC 2004 [DIRS 170038], Section 6). Unsaturated flow in the TSw is primarily through the fractures, because the matrix permeability in many of the TSw layers can support flows of only a few millimeters per year, and the average fracture spacing in the TSw layers is on the order of $0.5 \mathrm{~m}$ (BSC 2004 [DIRS 170038], Section 6).

The CHn unit and the Prow Pass unit (formally a part of $\mathrm{CHn}$, but studied separately in the present model report) below the repository horizon are complex geological systems with strongly heterogeneous distributions of fracture and matrix hydrological properties, which are expected to have pronounced effects on flow and transport of radionuclides in the UZ. The permeability of nonwelded tuffs is strongly dependent on the degree of alteration of the rock minerals into zeolites. Zeolitic alteration in the CHn (a common occurrence in its lower layers) can reduce the matrix permeability by orders of magnitude in relation to that of the welded tuffs (BSC 2004 [DIRS 170038], Section 6). In nonwelded vitric tuffs, the matrix and fracture permeabilities are on the same order of magnitude (BSC 2004 [DIRS 170038], Section 6). Thus, the nonzeolite layers behave as porous (rather than fractured) media, and flow is matrix dominated. This has important implications for transport, because the longer contact times in these nonwelded tuff units allow increased radionuclide sorption.

The CHn major hydrogeologic unit is composed of Calico Hills vitric (CHv) and Calico Hills zeolitic (CHz) units (BSC 2004 [DIRS 169855], Section 6). Typically, radionuclides are more 
strongly adsorbed onto zeolitic units than onto vitric units (see Appendix A and Table 6-3). For example, the $K_{d}$ (see Equation 6-9) of ${ }^{237} \mathrm{~Np}$ in the vitrified and the zeolitic tuffs is $0.5 \mathrm{~mL} / \mathrm{g}$ and $1 \mathrm{~mL} / \mathrm{g}$, respectively (output DTN: LA0408AM831341.001, see Section 6.5). Consequently, migration of ${ }^{237} \mathrm{~Np}$ is expected to be more retarded in the zeolitic than in the vitrified units, if the contact time is the same.

Flow in the $\mathrm{CHz}$ units is expected to be concentrated in the fractures because of the large permeability contrast between matrix and fractures (BSC 2004 [DIRS 170038], Section 6); the permeability in the fractures is about four orders of magnitude larger than in the matrix (DTNs: LB0205REVUZPRP.001 [DIRS 159525] and LB0207REVUZPRP.002 [DIRS 159672]). Fracture-dominated flow is associated with short contact times, limited radionuclide retardation through diffusion and sorption, and, thus, transport over longer distances.

The fracture and matrix permeability values of the zeolitized Calico Hills unit are based on product outputs from the report Calibrated Properties Model (BSC 2004 [DIRS 169857] and the report Analysis of Hydrological Properties Data (BSC 2004 [DIRS 170038]). These hydrological properties, derived by inverse modeling to match pneumatic responses through fracture network and water-content and potential values in the matrix, are regarded as reasonable representation of the actual fracture and matrix permeability values of the zeolitized Calico Hills unit.

\subsubsection{Transport}

Radioactive contaminants can escape from the wastes stored in the repository. These contaminants can migrate through the UZ as a dissolved molecular species or in colloidal form. Transport of these radioactive solutes or colloids involves advection, hydrodynamic dispersion, sorption (solutes) or filtration (colloids), matrix diffusion, and radioactive decay. The transport of radionuclides also is affected by such factors as solubility limits, the presence of perched water, and heating effects from the repository. The phenomena, processes, and factors affecting transport are discussed in the following sections.

\subsubsection{Advection}

Advection is the transport of dissolved or colloidal species by flowing water. In the Yucca Mountain UZ, flow is predominately downward (in response to gravitational differentials), and so is advective transport (DOE 1998 [DIRS 100550], p. 3-112). Some lateral advection is also expected in response to lateral flow diversion at the boundaries of hydrogeologic units with sharp contrast in their hydraulic properties. Such diversion occurs in the perched-water bodies of the UZ (BSC 2004 [DIRS 169861], Section 6.2.2; DOE 1998 [DIRS 100550], p. 3-112). Laterally diverted flow ultimately finds a pathway to the water table through other, more permeable zones (e.g., faults).

Advection is probably the most important transport process in this study, because it controls the speed at which radionuclides move through the UZ to reach the water table. Advection in the fractures is expected to be the dominant transport mechanism in many layers of the various hydrogeologic units. This is because the expected flow rates in the fractured rock mass exceed 
the matrix. This leads inevitably to flow focusing in the more permeable fractures. Advection is the dominant transport mechanism in the fractures because of high permeability, limited fracture pore volumes, limited contact area, and short contact times between the radionuclide-carrying liquid phase and the matrix (only at the fracture walls). In a few hydrogeologic units, such as the $\mathrm{CHv}$, matrix flow is dominant, resulting in much slower transport velocities (compared to those in the fractures of other units) and longer radionuclide-matrix contact times (DOE 1998 [DIRS 100550], p. 3-112).

\subsubsection{Hydrodynamic Dispersion}

Hydrodynamic dispersion combines mechanical dispersion, caused by localized velocity variations, with molecular diffusion, caused by Brownian motion, and is proportional to the concentration gradient. The dispersion of the radionuclides can occur both along and transverse to the average flow direction. Hydrodynamic dispersion leads to the smoothing of sharp concentration fronts and reduces the breakthrough time (which can be defined as the arrival time of the edge of the contaminant front) at the water table.

Little information exists on the values of the longitudinal $\left(\alpha_{L}\right)$ and transverse $\left(\alpha_{T}\right)$ dispersivity $([L])$ in the various hydrogeologic units of the UZ. In past simulations (DOE 1998 [DIRS 100550], p. 3-122), the longitudinal dispersivity $\left(\alpha_{L}\right)$ values for both the fractures and the matrix of all units had a mean of $20 \mathrm{~m}$ and a standard deviation of $5 \mathrm{~m}$. Analysis of the Northern Ghost Dance fault test (LeCain et al. 2000 [DIRS 144612], Tables 18 and 19) indicates an $\alpha_{L}$ in the $0.06 \mathrm{~m}$ to $2.63 \mathrm{~m}$ range, but the validity of these estimates is uncertain because they are based on gas (rather than liquid) phase transport, short transport times (less than $200 \mathrm{~min}$ ), and a small scale experiment (less than $10 \mathrm{~m}$ ). Analysis of the C-well transport test indicates that $\alpha_{L}$ ranged between 3.2 and $62.5 \mathrm{~m}$ (BSC 2004 [DIRS 170010], Table 6.3-3); however, these results corresponded to the saturated conditions in the deeper formations of Yucca Mountain. Note that dispersion is not expected to play a significant part in the transport of radionuclides in the fractures because of the predominant role of advection as the main transport mechanism and the limited water flow into the matrix.

Dispersion is affected by the scale of observation. The value of dispersivity appears to increase with the scale of observation (Gelhar et al. 1992 [DIRS 122808], p. 1955; Fetter 1993 [DIRS 102009], pp. 65 to 66). Thus, $\alpha_{L}$ increased from $10^{-2}$ to $10^{4} \mathrm{~m}$ when the observation scale increased from $10^{-1}$ to $10^{5} \mathrm{~m}$, and its value did not appear to be significantly affected by the texture (porous versus fractured) of the aquifer medium (Gelhar et al. 1992 [DIRS 122808], p. 1955).

The ratio $\alpha_{L} / \alpha_{T}$ controls the shape of a contaminant plume in multidimensional transport. There is limited data on the relationship between $\alpha_{L}$ and $\alpha_{T}$. Based on the available field data, Fetter (1993 [DIRS 102009], pp. 65 to 66) reported that $\alpha_{L} / \alpha_{T}$ ranged between 6 and 20. Gelhar et al. (1992 [DIRS 122808], p. 1955) indicated that vertical $\alpha_{T}$ is typically an order of magnitude smaller than the horizontal $\alpha_{L}$. 


\subsubsection{Sorption}

Sorption is a general term that describes a combination of chemical interactions between the dissolved radionuclides and the solid phases - that is, either the immobile rock matrix or colloids (mobile or immobile). In transport studies, the concept of sorption does not identify the specific underlying interactions, such as surface adsorption, mineral precipitation, ion exchange, and surface complexation. By removing a portion of the dissolved species from the mobile liquid phase and transferring it to the immobile solid phase, sorption reduces the rate of advance of (i.e., retards) the concentration front of a dissolved or suspended species.

In Yucca Mountain studies, the effective $K_{d}$ approach (see Equation 6-9) is employed to quantify the extent of radionuclide-rock interactions by measuring the overall partitioning between the aqueous and the solid phase. Three basic rock types (devitrified tuffs, vitric tuffs, and zeolitic tuffs) have been studied as having distinctively different (i.e., from each other) sorption interactions with the radionuclides (DOE 1998 [DIRS 100550], p. 3-118). The estimation of $K_{d}$ values for several radionuclides in each of these rock types is the subject of the detailed analysis in Appendix A. Note that sorption is not only a function of the sorptive strength (as quantified by the value of $K_{d}$ ), but also of the contact time of the radionuclides with the rock matrix during transport through the UZ.

The $K_{d}$ values used in the Yucca Mountain studies have been estimated from batch experiments using crushed tuffs with a particle size of 75-500 $\mu \mathrm{m}$ under saturated conditions (Appendix A, this model report). Under true linear equilibrium sorption conditions, the $K_{d}$ value is independent of concentration or of the time of contact. The effective $K_{d}$ value of a particular radionuclide sorbing onto a rock can vary depending on the estimation approach. Dependence of the effective $K_{d}$ on concentration indicates nonlinear sorption, and $K_{d}$ dependence on contact time indicates kinetic behavior. The importance of kinetic, nonlinear, and irreversible sorption may need to be evaluated against the linear equilibrium isotherm assumed in UZ transport studies.

The $K_{d}$ values obtained from experiments involving low concentration solutions tend to be higher than those from higher concentration ones because the most active sorption sites are immediately occupied by the dilute solutes (while denser solutes will involve less active ones, i.e., indicating nonlinear sorption isotherms such as Langmuir or Freundlich). The obvious implication is that $K_{d}$ values from experiments involving concentrations similar to those expected in the field should be used to avoid overestimation or underestimation. Lower $K_{d}$ values can be estimated if (a) the duration of the sorption experiments is short, and (b) a linear equilibrium sorption model is assumed but sorption is actually kinetically controlled. Note that the determination approach in the Yucca Mountain studies (Appendices A and B of present model report) tends to underestimate the $K_{d}$ values because (a) it involves an averaging of $K_{d}$ values obtained from experiments using both low and high solution concentrations, while (b) the release concentrations are expected to be low at the repository.

If kinetic behavior is not an issue, batch experiments with dilute concentrations may overestimate the $K_{d}$ values because of the aforementioned reasons, and because the prevailing conditions (involving saturated crushed rock samples in complete contact with a large amount of solute-bearing liquid relative to the sample mass) may not be representative of the UZ, which is 
characterized by unsaturated conditions, a limited contact area, and a limited amount of solute in the finite invading stream (see Section 6.1.3.1 for a more thorough discussion).

Sorption kinetics could be important, especially for radionuclides sorbing onto zeolitic tuffs. Column experiments of ${ }^{237} \mathrm{~Np}$ transport in tuffs (Viswanathan et al. 1998 [DIRS 134579], p. 267) indicate the existence of kinetic sorption under flowing conditions, possibly because of the slow diffusion of ${ }^{237} \mathrm{~Np}$ into the tuff pores. It is not possible to analyze these experimental data without considering a kinetic sorption model. Kinetic sorption can have a substantial effect on transport in fast fracture flow because it reduces sorption in the matrix, thus allowing larger radionuclide concentrations and migration over longer distances in the fractures. Note that, although there is an appreciation of the potential kinetic behavior, sorption is treated as a linear equilibrium process in this model report because (a) the kinetic effects are unlikely to persist over the long time frames (up to a million years) of this study, the duration of which strongly suggests an equilibrium process after a relatively short initial kinetic period, and (b) the generally low $\mathrm{K}_{\mathrm{d}}$ values used in this study account for the early kinetic effects.

\subsubsection{Matrix Diffusion}

Diffusion can play an important role in radionuclide exchange between the fractures and the rock matrix. This process transfers radionuclides into the matrix (where water flow is slow and sorption occurs), thus removing them from (and slowing the advance of their front in) the fast fracture flow. Diffusive flux across a given interface is a function of the concentration gradient, the temperature, the size of the dissolved species and its electric charge, the matrix pore structure, and the water saturation (DOE 1998 [DIRS 100550], pp. 3-116 to 3-117).

The effective matrix diffusion coefficient is a linear function of the tortuosity coefficient $\tau$, which describes the tortuous nature of the pore networks (Grathwohl 2000 [DIRS 141512], pp. 28 to 35), including dead-end pores and steric hindrance (in extremely narrow pores). The term $\tau$ is defined as the distance between any two given points in a porous medium over the actual travel path through the pores between these two points, that is, $\tau<1$. Because there are limited experimental data on the $\tau$ distribution in the various Yucca Mountain hydrogeologic units, $\tau$ is approximated by the value of porosity $\phi$ (Farrell and Reinhard 1994 [DIRS 122803], p. 64; Grathwohl 2000 [DIRS 141512], pp. 28 to 35).

This approach to define $\tau$ was confirmed experimentally from rock diffusion experiments using devitrified tuffs where the effective diffusion coefficient $D_{e}$ for tritiated water was obtained from six tuff samples. The effective diffusion coefficient $D_{e}=\phi \tau S_{w} D_{0}$, where $D_{0}$ is the molecular diffusion coefficient $\left[L^{2} T^{-1}\right]$ and $S_{w}$ is the water saturation based on Dynamics of Fluids in Porous Media (Bear 1972 [DIRS 156269], Eq. 4.8.29). Based on the literature value of $D_{0}$ for tritiated water (Hu and Brusseau 1995 [DIRS 122846]), a $\tau$ estimate of $0.0806 \pm 0.035$ was obtained for media in which the reported average porosity was $0.0767 \pm 0.019$ (Triay et al. 1993 [DIRS 145123], p. 1530 and SN-LBNL-SCI-168-V1 (Hu 2001 [DIRS 163148], p. 23)). The good match between these two numbers validates the approach of using the porosity value as the tortuosity coefficient.

Regarding the effect of $S_{w}$ on $D_{e}$, Porter et al. (1960 [DIRS 123115]) reported that the $\tau \phi$ $S_{w}$ product decreased consistently in medium- and fine-textured soils as the capillary pressure 
increased from 0.33 to $15 \mathrm{~atm}$. The effect of saturation on $D_{e}$ may be more complex than the linear relationship currently assumed. Supporting evidence was provided from experiments by Conca and Wright (1990 [DIRS 101582]), who determined $D_{e}$ values for $\mathrm{K}^{+}$ions for a variety of water contents and grain sizes on four types of angular crushed gravel. For volumetric water contents ranging from 0.5 percent to 6 percent, the $D_{e}$ ranged from $10^{-14} \mathrm{~m}^{2} / \mathrm{s}$ to $10^{-11} \mathrm{~m}^{2} / \mathrm{s}$, that is, the dependence was much stronger than what could be expected from the linear relationship between $D_{e}$ and $S_{w}$. The linear relationship assumed in this model report results in more conservative transport estimates because diffusion from the fractures into the matrix is reduced.

\subsubsection{Solubility}

The concentration of any radionuclide (released from the stored radioactive wastes at the repository) in the water cannot exceed the radionuclide solubility limit, unless suspended colloids are involved. Limitations to radionuclide solubility in the water infiltrating the repository constitute the first barrier to transport and can reduce the extent of radionuclide migration by limiting the available source.

The lower solubility of many radionuclides can lead to slower and continuous release over time at the outer boundaries of engineered barrier systems surrounding the radioactive wastes because the source is not exhausted. For example, the solubility of ${ }^{237} \mathrm{~Np}$ is sufficiently low to extend the period of its release to tens of thousands of years (Viswanathan et al. 1998 [DIRS 134579], p. 273). By using relative concentrations, radionuclide solubility is not explicitly accounted for in this model report.

\subsubsection{Colloids}

Colloids are fine particles, generally considered to be between 0.001 and $10 \mu \mathrm{m}$ in diameter, which become suspended and are transportable in a moving liquid. The generation and mobilization of colloids may be considered important issues in contaminant transport, particularly the transport of radioactive true (intrinsic) colloids (e.g., colloidal $\mathrm{Pu}(\mathrm{IV})$ and $\mathrm{Pu}(\mathrm{V})$ ) and the colloid-assisted transport of radioactive species (e.g., ${ }^{239} \mathrm{Pu},{ }^{243} \mathrm{Am},{ }^{231} \mathrm{~Pa},{ }^{137} \mathrm{Cs}$, and ${ }^{229} \mathrm{Th}$, see BSC 2004 [DIRS 170025]) sorbed on pseudocolloids (e.g., naturally occurring clay colloids). See Sections 6.1.3.3 to 6.1.3.6 for a more detailed discussion.

\subsubsection{Perched Water}

Perched water is defined as a saturated zone located at a higher elevation than the static water table, to which it is not directly connected. Perched water usually occurs where low permeability horizons do not permit the rapid downward flow of water. Such bodies have been reported at several locations in UZ boreholes (Wu et al. 1999 [DIRS 117167]). The presence of perched water has implications for the transport times and flow paths of water and radionuclides through the UZ.

The majority of the perched-water bodies detected in the UZ boreholes were observed in formations overlaying relatively impermeable matrix material, such as the TSw basal vitrophyre (a glassy cooling unit). Although the vitrophyre is extensively fractured, many of the fractures have been filled with zeolitic material, thus limiting flow. A portion of the Calico Hills formation has been extensively altered to zeolites, creating perched-water bodies (Bodvarsson 
et al. 1999 [DIRS 120055], pp. 14 to 15). The blockage of fracture flow, which occurs below these perched-water bodies, can lead to lateral diversion of radionuclide migration if the percolation flux is sufficiently large.

Three perched-water models, namely no perched water, perched-water model \#1 (flow through the perched water), and perched-water model \#2 (flow bypassing the perched water) have been proposed in UZ Flow Models and Submodel (BSC 2004 [DIRS 169861], Sections 6.2 .2 and 6.2.5). The effects of each of these perched-water models on radionuclide transport will be investigated in the three-dimensional site-scale simulations discussed in Section 6.15.

\subsubsection{Daughter Products}

Chain-decay adds another layer of complexity because of the need to account for the transport of the daughter products, that is, the new radionuclides created from the decay of a parent radionuclide. The daughter products may have significantly different transport behavior than the parent radionuclide. Thus, the migration and fate of all the important members of the decay chain must be considered rather than just the parent radionuclide.

\subsubsection{Effect of Heat on Transport}

Radionuclide transport may also be influenced by the heat generated by the decaying radioactive waste, which affects the ambient hydrological and chemical conditions and can thermally alter the rock near the repository. For example, if the zeolitic layers below the repository horizon are thermally altered, the sorption of radionuclides is reduced. In addition to the thermal effect on the flow field and radionuclide sorption coefficient, temperature also enhances diffusion by increasing Brownian motion. This effect is quantified by an increase in the $D_{0}$ of radionuclides. The temperature-dependent $D_{0}$ is described by the following equation (Robin et al. 1987 [DIRS 123119], pp. 1105 to 1106):

$$
\left(\frac{D_{0} \mu}{T}\right)_{T_{1}}=\left(\frac{D_{0} \mu}{T}\right)_{T_{2}}
$$

where

$$
\begin{array}{ll}
\mathrm{T} & =\text { the absolute temperature } \\
\mu & =\text { the viscosity of water }
\end{array}
$$

The effect of temperature on sorption is discussed in Appendix I. In general, an increase in temperature leads to increased sorption of cationic species and decreased sorption of anionic species, providing that the sorbing minerals are not thermally altered, which could happen in the case of zeolites. In addition to theoretical predictions, Appendix I includes a discussion of experimental data that show sorption increasing with increasing temperature.

Temperatures in the UZ increase naturally with depth because of the geothermal gradient. Additionally, heat from the stored waste is expected to lead to higher temperatures over a large volume of the UZ for as long as 100,000 years (BSC 2004 [DIRS 169856], Section 6.5). The combined effect of higher temperatures is increased retardation of the radionuclides because of 
(a) increased diffusion from the fractures into the matrix and (b) increased sorption. The assumption of isothermal $25^{\circ} \mathrm{C}$ conditions in the studies of the present model report reflects a conservative approach and yields results that describe the worst-case scenario.

\subsubsection{Important Transport Issues}

\subsubsection{Measurements of $K_{d}$ Values}

The sorption behavior of radionuclides is usually described by the distribution coefficients $\left(K_{d}\right)$, which quantifies the partitioning of radionuclides between the solid and aqueous phases under a linear equilibrium isotherm. Most of the $K_{d}$ values are obtained from batch experiments using crushed rock. Effective $K_{d}$ values obtained from batch experiments involving high concentration solutions will tend to underestimate the field $K_{d}$ values if (a) the expected field concentrations are low and (b) nonlinear and/or kinetic sorption are involved (see discussion in Section 6.1.2.3, this model report). Conversely, batch experiments using crushed rock samples may overestimate the $K_{d}$ values (compared to intact rock samples and for the same solute concentration) if kinetic effects are involved and the contact time is short; but the difference in the $K_{d}$ estimates from the two sample types vanish for sufficiently long contact times. Crushing of the material creates new contact surfaces and increases the radionuclide accessibility to pores that may not contribute to sorption to intact rocks. Comparison of the $K_{d}$ values obtained from batch sorption experiments and from through-diffusion experiments (which involve relatively large samples) show significant differences, which are attributed to differences in the surface area of the samples and the water/solid ratio.

A detailed discussion of sorption of important radionuclides onto various $\mathrm{UZ}$ rocks, and a listing of the corresponding $K_{d}$ values, can be found in Triay et al. (1997 [DIRS 100422], p. 136). The Triay et al. study (1997 [DIRS 100422], p. 181) involved analysis of laboratory data, a large portion of which are included in the $K_{d}$ determination discussed in this model report (see Appendices A, B, and J).

Bradbury and Stephen (1986 [DIRS 122792]) investigated the sorption of ${ }^{85} \mathrm{Sr},{ }^{85} \mathrm{Tc}$, ${ }^{125} \mathrm{I}$, and ${ }^{137} \mathrm{Cs}$ onto Darley Dale sandstone samples. Comparing $K_{d}$ values obtained from batch (using less than $0.1 \mathrm{~mm}$ and 1-2 $\mathrm{mm}$ ) and diffusion-sorption experiments (using disks of $25 \mathrm{~mm}$ in diameter and about $5 \mathrm{~mm}$ in thickness), they determined that crushed rock tests can overestimate sorption by as much as one or two orders of magnitude. They concluded that the magnitude of the difference in the value of $K_{d}$ depends on the radionuclide, its concentration, the rock, the water/solid ratio, and the particle size distribution in the batch tests.

Holtta et al. (1997 [DIRS 122832]) studied the effect of specific surface area on the sorption of ${ }^{22} \mathrm{Na},{ }^{45} \mathrm{Ca}$, and ${ }^{85} \mathrm{Sr}$ on crushed crystalline rocks of six size fractions $(0.071$ to $0.15,0.15$ to 0.20 , 0.2 to $0.3,0.3$ to $0.85,0.85$ to 1.25 , greater than $1.25 \mathrm{~mm}$ ). Sorption of ${ }^{22} \mathrm{Na},{ }^{45} \mathrm{Ca}$, and ${ }^{85} \mathrm{Sr}$ on unaltered tonalite and mica gneiss was slight and exhibited virtually no dependence on the fraction size. Considerably higher sorption of ${ }^{22} \mathrm{Na}$ and ${ }^{85} \mathrm{Sr}$ occurred on altered tonalites of smaller fractions because of their larger specific surface areas. $K_{d}$ values from thin sections ( $0.030 \mathrm{~mm}$ in thickness) were in good agreement with those obtained from batch experiments, possibly because the thin-section thickness is within the range of the crushed-rock size fractions. 
Johansson et al. (1997 [DIRS 123001]; 1998 [DIRS 123004]) conducted batch sorption tests of alkali- and alkaline earth metals $\left({ }^{22} \mathrm{Na},{ }^{137} \mathrm{Cs},{ }^{45} \mathrm{Ca},{ }^{85} \mathrm{Sr}\right.$, and $\left.{ }^{133} \mathrm{Ba}\right)$ using six crushed size fractions $(0.045-0.090,0.090-0.25,0.25-0.5,0.5-1,1-2,2-4 \mathrm{~mm})$ of medium-grained Aspo diorite and fine-grained granite. The $K_{d}$ increased with decreasing particle size and differed by approximately one order of magnitude between the largest and smallest particle (because of different specific surface areas). They also determined that the best agreement between the $K_{d}$ values from diffusion experiments (using $20 \mathrm{~mm}$ rock disks) and from batch experiments was observed for the largest size fractions $(2-4 \mathrm{~mm})$ in the batch studies. They explained this observation by suggesting that, unlike the smaller fractions, the largest size fraction involved a large number of whole mineral grains.

Tachi et al. (1998 [DIRS 134571]) studied the sorption and diffusion behavior of Se in Tono tuffs in batch experiments (using crushed rock with sample sizes ranging between 0.075 and $0.355 \mathrm{~mm}$ ) and in through-diffusion experiments (using samples $30 \mathrm{~mm}$ in diameter and $5 \mathrm{~mm}$ in thickness). The $K_{d}$ values from the diffusion experiments were one order of magnitude lower than those from the batch experiments. Correcting for the difference in the specific surface areas could not fully account for the $K_{d}$ discrepancy. Mercury porosimetry suggested that the differences were caused by sorption in microscopic pores (less than $20 \mathrm{~nm}$ in diameter) in the crushed rock samples. When the contribution of these pores to sorption was not considered, the $K_{d}$ values from the batch sorption experiments and from the diffusion experiments were consistent.

The effect of the specific area surface of ground rock samples on the sorption of ${ }^{137} \mathrm{Cs}$, ${ }^{85} \mathrm{Sr}$, and ${ }^{237} \mathrm{~Np}$ was studied in nine size fractions (less than $0.038,0.038-0.063,0.063-0.075,0.075-0.106$, $0.106-0.25,0.25-0.50,0.5-1,1-2,2-4 \mathrm{~mm}$ ) of devitrified Topopah Spring tuff and zeolitized Calico Hills tuff (Rogers and Meijer 1993 [DIRS 123127]). They showed that the grinding process does not influence the sorption behavior for particle sizes larger than about $63 \mu \mathrm{m}$. They also indicated that ground samples must be washed carefully to remove very fine particles generated during the grinding process, which could lead to irreproducible or anomalously high $K_{d}$ values.

\subsubsection{Potential Sorption of Anions}

Adsorption of negatively charged ionic species from the aqueous phase onto mineral substrates is likely to occur whenever the mineral surface exhibits a net positive surface charge. Under typical ambient conditions in shallow systems (saturated or unsaturated), most rock-forming alumino-silicate minerals have negatively charged surfaces. It is possible, however, for these surfaces to become positively charged in the presence of acids or if the surfaces dry out and only residual oligolayers of water remain.

The transition from negatively to positively charged surface occurs at the point of zero charge, where the surface charge is zero and is usually expressed in terms of $\mathrm{pH}$. The point of zero charge differs from one mineral to another, but for most rock-forming minerals, it is less than 4. However, in some materials (such as iron oxides, sulfides, and some organic matter), the point of zero charge can be as high as 8 or 9 . 
These minerals display a tendency to adsorb anionic species such as $\mathrm{I}^{-}$and $\mathrm{TcO}_{4}^{-}$under ambient water table conditions. For example, the extent of $\mathrm{AsO}_{4}^{3-}$ sorption on hydrous ferric oxide is a function of pH (Dzombak and Morel 1990 [DIRS 105483], pp. 200 to 204). The edge sites on clays are also reported to contain positive sites. Under low $\mathrm{pH}$ conditions (e.g., $\mathrm{pH}=5$ ), significant sorption of anionic tracers occurs in systems with high concentrations of iron oxides and kaolinite (Boggs and Adams 1992 [DIRS 122790]; Seaman 1998 [DIRS 134563]).

Despite the availability of positively charged adsorption sites, ${ }^{129} \mathrm{I}^{-}$and ${ }^{99} \mathrm{TcO}_{4}^{-}$are in competition with other anionic species in solution, such as $\mathrm{SO}_{4}^{2-}, \mathrm{NO}_{3}^{-}, \mathrm{OH}^{-}$, etc., which are usually present in concentrations an order of magnitude larger than either ${ }^{129} \mathrm{I}^{-}$or ${ }^{99} \mathrm{TcO}_{4}^{-}$. Because of competition, their effective sorption is minimal. There are, however, exceptions, and these should be taken into account when evaluating their retardation.

Kaplan and Serne (1995 [DIRS 123010]) determined that iodine sorbed on all Hanford sediments, and its $K_{d}$ ranged from 0.7 to $15 \mathrm{~mL} / \mathrm{g}$, with a median value of $7 \mathrm{~mL} / \mathrm{g}$. This finding could have significant implications for radionuclide transport. Positively charged adsorption sites may exist on the edges of 2:1 clays such as smectite and illite, on Al- and Fe-oxide surfaces, and on 1:1 clays such as kaolinite. Anions may sorb onto these locally positive-charged sites, even though their numbers are limited under typical $\mathrm{pH}$ conditions.

Note that the study of Kaplan and Serne (1995 [DIRS 123010]) involved extremely low iodine concentrations (approximately 12 parts per trillion (ppt)). Therefore, only a small number of positively charged sites would be needed for the sorption of a measurable portion of the dissolved iodine. Had an initially larger concentration been used in these experiments (e.g., $1 \mathrm{ppm}$ ), it is reasonable to expect that a small fraction of iodine would have sorbed onto the solid phase, resulting in practically undetectable iodine sorption. It must be pointed out that in a low-level radioactive waste plume ${ }^{129}$ I concentrations are expected to be in the ppt range (Kaplan and Serne 1995 [DIRS 123010]).

Similarly, significant sorption of technetium could occur in soils containing considerable amounts of natural organic matter, which tends to sorb anionic species and could reduce technetium to its +4 oxidation state, causing precipitation and/or sorption (Kaplan and Serne 1995 [DIRS 123010]). In the Yucca Mountain UZ, however, conditions are both oxidizing and deficient in organic matter. Therefore, significant sorption is unlikely to occur until more reducing conditions are encountered within the saturated zone.

Currently, there is no information on the occurrence and extent of localized positively charged sorption sites in Yucca Mountain tuffs. However, the existence of such sites cannot be ruled out. Clays and oxides can potentially contribute to positively charged sites. Smectite is a significant constituent of some unwelded tuff horizons at Yucca Mountain. Although hematite and illite are present in minor or trace amounts in the unwelded tuffs, hematite is widely distributed in the matrix of the devitrified units. Smectite and hematite also coat fracture walls in Yucca Mountain tuffs (BSC 2004 [DIRS 169734], Section 5.2.1.1). No estimate exists of the potential effects of such sites on the site-scale transport of radioactive anionic species. 


\subsubsection{Colloidal Behavior}

Radioactive true colloids or radionuclides adsorbed onto pseudocolloids can be transported over significant distances (McCarthy and Zachara 1989 [DIRS 100778], pp. 496 to 498). The significant migration of strongly sorbing Pu and Am (more than $30 \mathrm{~m}$ ) from a low-level nuclear waste site at Los Alamos National Laboratory through unsaturated tuff over a period of approximately 30 years is attributed to colloid and/or colloid assisted transport, a hypothesis confirmed by laboratory experiments (Buddemeier and Hunt 1988 [DIRS 100712], p. 536). Using the ${ }^{240} \mathrm{Pu} /{ }^{239} \mathrm{Pu}$ isotope ratio to fingerprint the source of $\mathrm{Pu}$ in the water table, Kersting et al. (1999 [DIRS 103282], pp. 56 to 59) recently demonstrated that the soluble (ionic) $\mathrm{Pu}$ is practically immobile in the subsurface of the Nevada Test Site (NTS) because of its strong sorption, but can be transported over significant distances (1.3 km over a 30 -year period) in a colloidal form.

Colloids are very fine particles (such as clay minerals, metal oxides, viruses, bacteria, and organic macromolecules) that range in size between 1 and 10,000 nm (McCarthy and Zachara 1989 [DIRS 100778], pp. 496 to 498) and have high specific surface areas (approximately $300 \mathrm{~m}^{2} / \mathrm{g}$ ). Their chemical behavior is dominated by surface processes (EPRI 1999 [DIRS 113923], p. 3-1), and can have a high sorptive capacity for contaminants. Colloids are deposited on porous and fractured media by surface filtration, straining filtration, and physical-chemical filtration. Colloid transport differs from solute transport because the colloidal particle interactions (e.g., flocculation), mechanical clogging effects, and surface reactions (e.g., deposition or attachment) are substantially different from solute processes and phenomena.

A complete description of colloid-facilitated radionuclide transport requires consideration of a large number of processes (EPRI 1999 [DIRS 113923], pp. 4-5 to 4-6), including advection, diffusion, colloid generation, colloid stability, colloid-solute-matrix interactions, affinity of colloids for the gas-water interface, colloid filtration (surface and straining), and kinetically controlled physical-chemical filtration.

\subsection{Colloid Types and Classes}

The analysis in Waste Form and In-Drift Colloids-Associated Radionuclide Concentrations: Abstraction and Summary (BSC 2004 [DIRS 170025], Section 6.3.1) identifies the following types of colloids: true (or intrinsic), waste form colloids, and psuedocolloids. In the TSPA-LA abstraction, these colloids are modeled based on the source of colloids, which are considered to be waste form colloids, corrosion product colloids, and groundwater colloids (BSC 2004 [DIRS 170025], Section 6.3.1). Waste form colloids are formed from the corrosion of defense high-level glass waste form. These colloids are composed of smectite (clay) and may have plutonium and americium embedded in the colloid structure (BSC 2004 [DIRS 170025], Section 6.3.1). Colloids with embedded radionuclides will be termed "irreversible colloids." Radionuclides can also sorb to the surfaces of these colloids. Radionuclides sorbed to the surfaces of colloids will be called "reversible colloids" because the radionuclides can attach and detach from the colloid. Corrosion product colloids are formed from the corrosion of steel components in the engineered barrier system. These colloids are composed of a mixture of hydrous ferric oxides that are modeled as hematite (BSC 2004 [DIRS 170025], Section 6.3.1). Radionuclides may sorb to the surfaces of these colloids. Natural groundwater colloids are the 
result of natural geochemical processes in the rock which transform rock minerals into clay minerals. For the purposes of modeling colloid transport, these clays are treated as smectite (BSC 2004 [DIRS 170025], Section 6.3.1). Radionuclides may sorb to the surfaces of these colloids.

More details on the mathematical treatment of the transport of colloids can be found in Section 6.2 (present model report).

\subsection{Colloid Generation and Stability}

The formation of mobile colloidal suspensions in the subsurface is attributed to a number of mechanisms: (1) matrix dissolution caused by changes in $\mathrm{pH}$ or redox conditions; (2) supersaturation with respect to the inorganic species; (3) disruption of the mineral matrix by large changes in the flow regimes due to injection, pumping, or large episodic rainfall infiltrations; (4) release and movement of viruses and bacteria; and (5) formation of micelles from the agglomeration of humic acids (Abdel-Salam and Chrysikopoulos 1995 [DIRS 146647], pp. 199 to 200). Buddemeier and Hunt (1988 [DIRS 100712], p. 536) indicate that submicrometer colloids can easily be released from mineral and glass surfaces that are chemically, hydrodynamically, or mechanically stressed.

Colloid stabilization/destabilization include steric stabilization by mechanisms such as organic coating of inorganic colloids, and the effects of $\mathrm{pH}$ and ionic strength on coagulation and precipitation. The stability of colloid suspension is very sensitive to changes in ionic strength (EPRI 1999 [DIRS 113923], p. 4-6).

\subsection{Colloid Deposition}

Colloid deposition (physical-chemical filtration) during saturated flow through a porous medium is commonly assumed to occur in two steps: (1) transport of colloids to matrix surfaces by Brownian diffusion, interception, or gravitational sedimentation (i.e., colloid-matrix collision) and (2) attachment of colloids to matrix surfaces. The attachment efficiency (i.e., the fraction of collisions resulting in attachment) is strongly influenced by interparticle forces between colloids and matrix surfaces, such as van der Waals and electric double-layer interactions, steric stabilization, and hydrodynamic forces (Kretzschmar et al. 1995 [DIRS 123019], p. 435). Kretzschmar et al. (1997 [DIRS 123029], p. 1,129) demonstrated that colloid deposition generally follows a first-order kinetic rate law, and experimentally determined the corresponding collision efficiencies.

\subsection{Colloid-Contaminant-Matrix Interactions}

Colloid attachment to the host rock is strongly dependent on electrostatic interactions. Once attached, colloid detachment (declogging) is generally slow to irreversible. Sorption of radionuclides on colloids is controlled by a range of chemical processes such as ion exchange, surface complexation, and organic complexation (EPRI 1999 [DIRS 113923], p. 4-9).

If sorption of metal ions onto colloids is assumed to follow an equilibrium isotherm, metals are stripped rapidly from the colloids when these enter a clean part of the porous medium. This 
approach was incapable of explaining the long transport distances observed in field experiments (van de Weerd and Leijnse 1997 [DIRS 109249], p. 246). The problem was addressed by assuming (a) kinetic sorption of radionuclides onto the humic colloids and (b) kinetic colloid deposition (van de Weerd and Leijnse 1997 [DIRS 109249], pp. 245, 255). The sorption of dissolved ionic $\mathrm{Pu}(\mathrm{V})$ onto hematite, montmorillonite, and silica colloids in both natural and synthetic water was reported to be fast (Lu et al. 2000 [DIRS 166315], p. 44). Under equilibrium conditions, the $K_{d}$ values of $\mathrm{Pu}(\mathrm{V})$ were about $100 \mathrm{~mL} / \mathrm{g}$ for hematite and montmorillonite colloids. These very large values indicate that iron oxide and clay colloids in the water can significantly enhance the transport of ${ }^{239} \mathrm{Pu}$. Very large $K_{d}$ values of actinide sorption are also confirmed in Waste Form and In-Drift Colloids-Associated Radionuclide Concentrations: Abstraction and Summary (BSC 2004 [DIRS 170025], Section 6.3.3.1).

\subsection{Colloid Migration in Macroporous and Fractured Systems}

In conducting infiltration experiments with intact structured sandy loam cores using two types of colloidal suspensions, Jacobsen et al. (1997 [DIRS 122995], pp. 185 to 186) observed significant transport of clay and silt colloid particles through the macropores. Macropores can enhance the transport of colloids because all types of filtration are less pronounced in large pores, and the large water velocities can lead to increased detachment (as the large hydrodynamic forces can overcome the colloid-grain bonding forces).

In a study of colloid-facilitated transport of radionuclides through fractured media, Smith and Degueldre (1993 [DIRS 144658], pp. 143, 162 to 163) determined that the assumption of fast, linear, and reversible radionuclide sorption onto colloids is nonconservative. They observed that the time for radionuclide desorption (days or weeks) significantly exceeded the time for sorption (seconds or minutes). Such radionuclide-laden colloids can migrate over long distances in macropores and fractures, and the larger colloids exhibit little retardation because their size prevents them from entering the wall-rock pores. The Smith and Degueldre (1993 [DIRS 144658], pp. 143, 162 to 163) study concluded that the transport of radionuclides sorbed irreversibly on to colloids depends strongly on the extent of colloid interaction with the fractures.

Vilks and Bachinski (1996 [DIRS 109252], pp. 269, 272 to 278) studied particle migration and conservative tracer transport in a natural fracture within a large granite block, with overall dimensions of $83 \times 90 \times 60 \mathrm{~cm}$. Flushing experiments showed that suspended particles as large as $40,000 \mathrm{~nm}$ could be mobilized from the fracture surface. The mobility of suspended particles with diameters of 1,000-40,000 nm was significantly less than that of colloids (less than $90 \mathrm{~nm}$ ). They observed that the conservative tracer lagged slightly behind the colloid front, but colloid mobility was significantly reduced when the average water velocity decreased. Compared to dissolved tracers, the migration of colloids was more affected by the flow path and flow direction. This tendency can have a significant effect in the fracture-dominated flow of the Yucca Mountain UZ.

In field-scale colloid migration experiment, Vilks et al. (1997 [DIRS 109254], pp. 203, 212 to 213) showed that silica colloids as small as $20 \mathrm{~nm}$ can migrate through open fractures over distances of $17 \mathrm{~m}$, when a colloidal suspension was injected at average flow velocities of 1.6 and $2.9 \mathrm{~m} / \mathrm{h}$ (orders of magnitude higher than the natural flow rates at the site). Based on the analysis of the results of the study, they also suggested that, although colloid migration appeared to 
behave conservatively, colloids may have followed different pathways than dissolved conservative tracers.

\subsubsection{Colloidal Behavior in Unsaturated Media}

Most of the available literature focuses on the behavior of colloids in saturated media. The behavior of colloids in unsaturated media (BSC 2004 [DIRS 170006]) is a relatively new area of study and has significant additional complexity.

\subsection{Filtration as a Function of Water Saturation}

Colloid straining or filtration is a physical process that can also affect the distribution and transport of colloids. Wan and Tokunaga (1997 [DIRS 108285], p. 2413) distinguish two types of straining: conventional straining (if the colloid is larger than the pore throat diameter or the fracture aperture) and film straining (if the colloid is larger than the thickness of the adsorbed water film coating the grains of the rock).

Wan and Tokunaga (1997 [DIRS 108285], pp. 2413, 2419) developed a conceptual model to describe colloid transport in unsaturated media as a function of water saturation $S_{w}$. If the rock $S_{w}$ exceeds a critical saturation value $S_{c}$, colloids move through the system entirely within the aqueous phase. For $S_{w}<S_{c}$, colloids can only move in the thin film of water that lines the grain boundaries, and colloid transport through the water film depends on two parameters: the ratio of the colloid size to the film thickness, and the flow velocity. Temporal variations in the $S_{w}$ in the subsurface profile and the infiltration rate can lead to strongly nonlinear colloid mobility near $S_{c}$.

McGraw and Kaplan (1997 [DIRS 123043], p. 5.2) investigated the effect of colloid size (from 52 to $1900 \mathrm{~nm}$ ) and $S_{w}$ (from 6 to 100 percent) on colloid transport through unsaturated media in Hanford sediments. They showed a very strong dependence of filtration on the colloid size under unsaturated conditions. At a volumetric water content of 6 percent (the expected water content in the Hanford vadose zone), colloid removal increased exponentially with colloid size. The decrease in colloid mobility at low volumetric contents was attributed to resistance from friction (as the colloids were dragged along the sand grains). Colloid retardation increased as the ratio between the water film thickness and colloid diameter decreased.

\subsection{Gas-Water Interface}

Under unsaturated conditions, colloid transport may be either inhibited or enhanced (compared to saturated conditions) because of the presence of the air-water interface. Wan and Wilson (1994 [DIRS 114430], pp. 857, 863) determined that retention of both hydrophilic and hydrophobic colloids increased with the gas content of the porous medium. They showed that colloids preferentially concentrate in the gas-water interface rather than on the matrix surface. This tendency increases with the colloid surface hydrophobicity or contact angle, with hydrophobic colloids having the strongest affinity for the gas-water interface.

The implications of this colloid behavior are important. Colloid affinity for the gas-water interface may retard their transport through the UZ. Conversely, if the subsurface conditions permit the stability and migration of bubbles, colloid transport may be enhanced. 


\subsubsection{Colloids at Yucca Mountain}

The available data on colloid occurrence and concentrations at the Yucca Mountain site are limited, and pertain to saturated zone studies. There is no published information on colloids in the UZ. The current state of understanding is that (a) the natural colloid concentrations in native waters at the Yucca Mountain site are in the $10^{6}-10^{10}$ particles/mL range studies (Kung 1999 [DIRS 146992], pp. L-I-5, L-8-3 to L-8-5), and (b) waste-form colloids will be the dominant colloidal species of concern to transport. This conclusion is supported by Waste Form and In-Drift Colloids-Associated Radionuclide Concentrations: Abstraction and Summary (BSC 2004 [DIRS 170025], Section 6.3.1), which includes the most recent information on colloid occurrence, generation, stability, properties, and transport behavior.

Additional information from nearby sites, however, cannot be ignored. Preliminary results from a survey of the water from the NTS have shown that, in the Pahute Mesa, drainage (both on and off the NTS) has colloidal particle (greater than $3 \mathrm{~nm}$ in diameter) loadings of $0.8-6.9 \mathrm{mg} / \mathrm{L}$. Such relatively high concentrations can explain the observed transport of strongly sorbing radionuclides over significant distances, particularly because the ionic composition of the NTS water is not expected to promote the coagulation of clay colloids (Buddemeier and Hunt 1988 [DIRS 100712], p. 537). This is because NTS water is oxygenated and low in organic matter, and a substantial fraction of the colloidal material is composed of stable natural minerals (Buddemeier and Hunt 1988 [DIRS 100712], pp. 543 to 544).

In addition to natural colloids, anthropogenic colloids may be created from the waste itself or from repository construction and sealing materials. Waste- and repository-derived colloids at Yucca Mountain are likely to include organic colloids, iron oxyhydroxides, and aluminosilicate colloids (EPRI 1999 [DIRS 113923], p. 2-2, 6-22). In a 50-month experiment involving simulated weathering of a high-level nuclear waste glass, spallation and nucleation were identified as the main mechanisms of colloid genesis (Bates et al. 1992 [DIRS 100704]). The created colloids were identified as inorganic. The same study determined that $\mathrm{Pu}$ and $\mathrm{Am}$ released from waste were predominantly in the colloidal, rather than in the dissolved, form.

\subsubsection{Impact of Colloids on Radionuclide Transport in Yucca Mountain}

EPRI (1999 [DIRS 113923], pp. xv to xvii, and 2-2) indicated that it is important for the YMP to consider the migration of radionuclides, and to assess the potential role of colloids on radionuclide transport, within both the saturated and the unsaturated systems. Furthermore, EPRI (1999 [DIRS 113923], pp. 4-6 to 4-7) identified the following key areas of uncertainty for further study:

1. Colloid-matrix interactions.

2. Thermo-dynamic data for certain radionuclides and kinetic data to describe virtually all rate-dependent reactions.

3. Representation of the hydrological system: colloid transport differs from the transport of conservative tracers and is influenced by the details of the permeability structure, such as fracture geometry and the hydraulic conditions within the fracture zones. 
4. The effects of partially saturated conditions on colloid transport.

\subsubsection{Important Points for Consideration in Colloid Transport}

The assessment of the potential role of colloids in the transport of radionuclides at Yucca Mountain is particularly challenging for the reasons discussed in the previous sections, and is further complicated by the following factors:

1. Different types of colloids (minerals, organics, microbes, and polymeric actinide colloids) are involved, with substantially different characteristics and properties (e.g., hydrophobicity and surface charges) that strongly affect their behavior in the subsurface.

2. Colloid generation, stability, size distribution, and concentration are dynamic. Knowledge about the temporal and spatial distributions of colloid populations in the different hydrogeologic units of the UZ is difficult to obtain. Changes in the ionic strength of the aqueous solution (e.g., when percolating water in an episodic infiltration event encounters resident pore water) can affect colloid stability. There are also uncertainties about the colloid stability in the aqueous phase of the UZ and about the colloid generation from mineral-coated fracture walls (given the predominance of fracture flow in the UZ).

3. Measurements of colloid concentration in the UZ are difficult. Because of the low concentrations and low saturations in the UZ, the determination of colloid concentration in pore water is challenging, and serious questions arise about sample representativity and integrity.

4. The concentration of waste-form colloids is a key uncertainty. There is evidence to suggest that the low concentration of natural colloids in the Yucca Mountain UZ will not lead to significant colloid-assisted transport, and that waste-form colloids will be the dominant colloidal transport problem (BSC 2004 [DIRS 170025], Section 6.3.1). Thus, there is a need for reliable estimates of the types and generation rates of waste-form colloids. Currently, there is considerable uncertainty on this subject (EPRI 1999 [DIRS 113923], p. 6-7).

5. Colloids diffuse more slowly than dissolved species because of their larger size (see Section 6.2.5, Equation 6-23). For the largest colloids, diffusion is approximately three orders of magnitude slower than that for molecular species (Nuttall et al. 1991 [DIRS 123103], p. 189). For example, the diffusion coefficient $\mathrm{D}_{0}$ of a $0.1 \mu \mathrm{m}$ colloid at $20^{\circ} \mathrm{C}$ is $4.29 \times 10^{-12} \mathrm{~m}^{2} / \mathrm{sec}$ (see Equation $6-23$ in the present model report), while the $\mathrm{D}_{0}$ of $\mathrm{Br}$ is $2.08 \times 10^{-9} \mathrm{~m}^{2} / \mathrm{sec}$ (Lide 1992 [DIRS 166224], p. 5-111).

6. Using mercury porosimetry, Roberts and Lin (1997 [DIRS 101710], pp. 577 to 578) determined that the average pore diameters of welded and densely welded TSw tuff samples were $53.1 \mathrm{~nm}$ and 19.7 to $21.4 \mathrm{~nm}$, respectively. These extremely small pores are certain to exclude a significant proportion of colloids. It is possible for colloids to accumulate on the fracture walls and, thus, clog the matrix pores open to the fracture. 
This can lead to reduction in the matrix permeability and in the colloid diffusion into the matrix. Pore exclusion is not expected to be significant in the fractures, and colloids can travel significant distances in the fractures (especially given the limited diffusion into the matrix).

7. The affinity of colloids for the air-water interface depends on their hydrophobicity and electrostatic charge. Hydrophilic colloids, such as mineral fragments, have a low affinity for the interface, in contrast to hydrophobic colloids (such as organic colloids and microbes). This affinity increases with the positive charge on the colloids (EPRI 1999 [DIRS 113923], p. 6-11). The flow and saturation conditions in the UZ will determine whether this will enhance or retard transport. Note that the potential impact of the air-water interfaces on colloid transport has not yet been quantified.

\subsubsection{Modeling Stipulations}

This section discusses the basic stipulations in the mathematical basis of the transport model and provides a short discussion of the supporting rationale. Section 6.1.5.1 addresses the general stipulations underlying the flow component of the transport model of the Yucca Mountain UZ. Section 6.1.5.2 lists the assumptions of the transport processes. The stipulations involved in the treatment and mathematical representation of the fractured rocks using the dual-continuum approach are discussed in Section 6.1.5.3. Stipulations related to the initial and boundary conditions of the model domain are presented in Section 6.1.5.4. Sections 6.1.5.1 to 6.1.5.4 address issues related to large-scale three-dimensional numerical simulations.

\subsubsection{Stipulations Involved in the Flow Component of the Transport Processes}

The transport phenomena and processes in the Yucca Mountain UZ have a flow component and a transport component, each of which are described by a set of governing equations. These conserve mass, energy, and momentum in the system under study, while quantifying the system response to external mass and energy inputs and interrelationships between the various processes involved.

The basic flow stipulations are consistent with those discussed in UZ Flow Models and Submodels (BSC 2004 [DIRS 169861], Sections 5 and 6). They share identical conceptual models, which are stated below:

1. The macroscopic-continuum approach is a valid concept for the description of the flow and transport processes in the fractured UZ rocks.

Justification: The rationale for this approach is provided in UZ Flow Models and Submodels (BSC 2004 [DIRS 169861], Section 6.1).

Applicability: This approach is used in all numerical (i.e., three-dimensional site-scale) simulations of flow and transport in this model report (Sections 6.8 to 6.20, and Section 7). No further confirmation is required for the purposes of this study.

2. Darcy's law is a valid model to describe the flow of gas and water in the matrix and fractures of the UZ. 
Justification: Given the applicability of the macroscopic continuum approach, the gaseous and aqueous flows under ambient conditions in the UZ are sufficiently slow to correspond to a Reynolds number $\leq 10$, that is, the upper limit of applicability of Darcy's law (Bear 1972 [DIRS 156269], p. 127).

Applicability: This approach is used in all three-dimensional site-scale simulations of flow and transport in this model report (Sections 6.8 to 6.20, and Section 7). No further confirmation is required for the purposes of this study.

3. Richards' equation (Richards 1931 [DIRS 104252]) is a valid model of unsaturated water flow in both the matrix and the fractures of the UZ.

Justification: Under ambient conditions, the gas-phase pressure in the UZ is atmospheric, corrected for elevation. The absence of gas pressurization makes possible the adoption of Richards' equation, because the gas phase can be neglected and the aqueous phase flow occurs in response to gravitational and capillary pressure differentials.

Applicability: This approach is used in all three-dimensional site-scale simulations of flow and transport in this model report (Sections 6.8 to 6.20, and Section 7). No further confirmation is required for the purposes of this study.

4. Relative permeabilities and capillary pressures follow the van Genuchten (1980 [DIRS 100610]) and Mualem (1976 [DIRS 100599]) model and are continuous functions of the effective liquid and gas saturations.

Justification: This model is consistent with the macroscopic continuum approach and is reasonable for the matrix at higher saturation ranges (BSC 2004 [DIRS 170038] Section 6.2.4) which occur under ambient conditions in the vadose zone at Yucca Mountain. This model is also reasonable for fractures at low saturation ranges, as are expected to occur under ambient conditions, based on simulation studies (BSC 2004 [DIRS 170035], Section 6.3.5).

Applicability: This approach is used in all three-dimensional site-scale simulations of flow and transport in this model report (Sections 6.8 to 6.20, and Section 7). No further confirmation is required for the purposes of this study.

5. The water flow is isothermal.

Justification: This is a reasonable approximation. The flow parameters affected by temperature are water density and water viscosity. Ambient temperature extremes at the domain boundaries are about $20^{\circ} \mathrm{C}$ at the top and about $30^{\circ} \mathrm{C}$ at the water table at the bottom (BSC 2004 [DIRS 169861], Section 6.3). Between $20^{\circ} \mathrm{C}$ and $30^{\circ} \mathrm{C}$, the water density decreases from $998.21 \mathrm{~kg} / \mathrm{m}^{3}$ to $995.65 \mathrm{~kg} / \mathrm{m}^{3}$ (Lide 1993 [DIRS 123032], p. 6-10), that is, the change is very small.

The effect on viscosity is more pronounced. Between $20^{\circ} \mathrm{C}$ and $30^{\circ} \mathrm{C}$, the water viscosity decreases from $1.002 \times 10^{-3} \mathrm{~Pa} \cdot \mathrm{s}$ to $0.7977 \times 10^{-3} \mathrm{~Pa} \cdot \mathrm{s}$ (Lide 1993 
[DIRS 123032], p. 6-10), that is, a reduction of about 20 percent. Although this variation is not excessive (given the uncertainty in the values of the UZ system hydraulic properties), its effects are minimized by conducting the isothermal flow simulations at $25^{\circ} \mathrm{C}$.

Isothermal flow may be a less valid approximation in the immediate vicinity of the waste package because of the heat generated by the radioactive decay process. The nonisothermal flow under these conditions is the subject of another report (BSC 2004 [DIRS 169856]).

Applicability: This approximation is used in all three-dimensional site-scale simulations of flow and transport in this model report (Sections 6.8 to 6.20, and Section 7). No further confirmation is required for the purposes of this study.

6. Water flow through the $\mathrm{UZ}$ in the numerical simulations of radionuclide transport is considered time-invariant (steady-state).

Justification: This is a reasonable approximation, given the very long simulation periods $(\geq 100,000$ years). This approach allows the determination of the transport-behavior envelope by considering nine different infiltration scenarios (and, consequently, flow fields), and is consistent with the flow regimes discussed in $U Z$ Flow Models and Submodels (BSC 2004 [DIRS 169861], Section 6).

Applicability: This approximation is used in all three-dimensional site-scale simulations of flow and transport in this model report (Sections 6.8 to 6.20, and Section 7). No further confirmation is required for the purposes of this study.

\subsubsection{Stipulations/Approaches/Simplifications Involved in Transport Processes}

7. The individual and combined effects of diffusion (molecular and/or colloidal), surface diffusion, and hydrodynamic dispersion follow a Fickian model.

Justification: Given the macroscopic continuum approach, this is a valid model (de Marsily 1986 [DIRS 100439], pp. 228 to 277).

Applicability: This model is used in all studies in this model report (Sections 6.8-6.20, and Section 7). No further confirmation is required for the purposes of this study.

8. Transport occurs isothermally at $25^{\circ} \mathrm{C}$.

Justification: Isothermal transport at the average ambient temperature of the UZ is consistent with the assumption of isothermal flow at the same temperature. The transport parameters affected by temperature are (a) the diffusion coefficient $D_{0}$ of the dissolved or colloidal species and (b) the sorption parameters of the dissolved species or the filtration parameters of the suspended colloid. Natural temperature differentials in the undisturbed UZ profile occur because of the geothermal gradient. Substantial 
temperature increases over the ambient are expected after radioactive waste emplacement in the repository (BSC 2004 [DIRS 169856], Section 6).

An increasing temperature leads to a higher $D_{0}$ value according to the relationship discussed in Section 6.1.2.9 of this model report. Based on this relationship, an increase in temperature from $20^{\circ} \mathrm{C}$ (at the top of the $\mathrm{UZ}$ domain) to $30^{\circ} \mathrm{C}$ (at the water table, i.e., the bottom of the UZ domain) leads to an increase of $D_{0}$ by about 30 percent.

The effect of temperature on sorption is less well-defined. However, as is explained in Appendix I, corrections for temperature effects would be small and differences in physico-chemical conditions between laboratory and the field would likely introduce uncertainties larger than corrections for temperature.

Colloid filtration (deposition) generally follows a kinetic process (see Sections 6.2.3 and 6.18.2 in this model report). Equation 6-31 in this model report indicates that an increase in temperature increases the forward filtration coefficient $\kappa^{+}$, indicating an increase in the filtration (deposition, clogging) rate. There is no information on the effect of temperature on the reverse filtration coefficient $\kappa^{-}$.

Thus, an increasing temperature in the UZ enhances diffusion (a particularly important mechanism in species mass transfer from the flow-dominating fractures to the matrix) and increases sorption and/or filtration. The cumulative effect is slower transport. The assumption of isothermal transport should not be viewed as an approximation of the prevailing conditions in the UZ, but rather as a condition that reflects a worst-case transport scenario and leads to conservative estimates of radionuclide transport times to the water table. Investigation of the effect of water-phase changes on transport may be included in future revisions of the present model report.

Applicability: This simplification is used in all studies in this model report (Sections 6.8 to 6.20, Section 7). No further confirmation is required for the purposes of this study.

9. The concentration of the radioactive solutes or colloids is at a tracer level, that is, too low to have any measurable effect on the flow regime.

Justification: Ambient tracers and radionuclides escaping from the repository are expected to occur at concentrations that are too low to affect the aqueous solution density (BSC 2004 [DIRS 169856], Section 6).

Applicability: This approximation is used in all studies in this model report (Sections 6.8 to 6.20 and Section 7). No further confirmation is required for the purposes of this study.

10. There is no phase change, that is, no water evaporation and condensation. 
Justification: The rationale for this simplification is covered by the discussion in Stipulation (5). Water evaporation and condensation resulting from the heat generated by the radioactive decay of the wastes is covered by Drift-Scale THC Seepage Model (BSC 2004 [DIRS 169856], Section 6), in which it is shown that phase changes are not expected to last longer than the first 10,000 years after waste emplacement (i.e., a rather short time compared to the 100,000 to 1,000,000 years covered by the studies in this model report) and are limited to a rather small volume in the immediate vicinity of the repository. However, investigation of the effect of water-phase changes on transport may be included in future revisions of the present model report.

Applicability: This simplification applies to all studies in this model report (Sections 6.8 to 6.20, and Section 7). No further confirmation is required for the purposes of this study.

11. Filtration of colloids is limited to deep filtration, that is, it does not affect the medium porosity and permeability.

Justification: Neglecting filtration of colloids and resulting reductions in porosity and permeability maintains water velocities in the vadose zone model. This is a conservative treatment since otherwise there would be reduction in water velocity that would increase transport times through the radionuclide transport model.

Applicability: This simplification applies to the colloid studies in this model report (Section 6.18). No further confirmation is required for the purposes of this study.

These stipulations allow decoupling of the flow and transport equations. Richards' equation is first solved in the flow component of transport, followed by the sequential solution of the $n$ independent tracer transport equations.

\subsubsection{Stipulations Involved in the Dual-Continuum Approach}

The treatment of fracture-matrix interactions is a critical issue in the simulation of flow and transport under the two-phase flow conditions of the fractured UZ rocks. This model report closely follows the approach of UZ Flow Models and Submodels (BSC 2004 [DIRS 169861], Section 6):

12. The dual-permeability model is a valid approximation for flow and transport simulations of the fractured UZ rocks.

Justification: In addition to its computational efficiency, this model has a strong conceptual basis because it describes the matrix and fractures as separate but interconnected gridblocks and permits flow and transport between matrix gridblocks, fracture gridblocks, and fractures and matrix. A more detailed discussion on the rationale for this assumption can be found in the report by BSC (2004 [DIRS 170035], Section 6.3). The basis and methodology for implementing the fracture and matrix geometry can be found in Development of Numerical Grids for UZ Flow and Transport Modeling (BSC 2004 [DIRS 169855]). 
Applicability: This approach applies to all three-dimensional site-scale studies in this model report (Sections 6.8 to 6.20). No further confirmation is required for the purposes of this study.

\subsubsection{Stipulations Involving Initial and Boundary Conditions}

13. In the numerical simulations, the top boundary of the UZ model domain is maintained at spatially variable conditions of:

(a) Temporally constant gas pressure and saturation

(b) Temporally constant temperature

(c) Temporally constant infiltration rates (steady state).

Justification: The spatial variations of the parameters reflect differences in elevation, climate, and topography, and represent realistic approximations of the prevailing conditions. For sufficiently long simulation periods, temporal variations in these parameters tend to diminish. Moreover, at a relatively short distance below the land surface, such temporal variations diminish rapidly.

Applicability: These boundary conditions are used in all three-dimensional site-scale studies in this model report (Sections 6.8 to 6.20). No further confirmation is required for the purposes of this study.

14. The bottom boundary of the UZ model domain in the three-dimensional simulations coincides with the water table. It is maintained at spatially variable conditions of:

(a) Temporally constant water pressure and saturation

(b) Temporally constant temperature.

Justification: This is a good representation of conditions in the saturated zone, for the reasons discussed in the rationale for Assumption 13.

Applicability: These boundaries conditions apply to all three-dimensional site-scale studies in this model report (Sections 6.8 to 6.20). No further confirmation is required for the purposes of this study.

15. In the numerical studies of radionuclide transport, the boundaries of the UZ domain through which flow and transport occur are the top and bottom boundaries (i.e., the ground surface and the groundwater, respectively). No lateral flow and/or transport occur across any other boundaries.

Justification: The flow and transport through the top and bottom boundaries is consistent with the patterns of rainfall-fed infiltration and gravity-driven flow and drainage. The distance between the repository and these boundaries is sufficiently large to justify the assumption of no lateral flow and/or transport. 
Applicability: These boundaries conditions apply to all three-dimensional site-scale studies in this model report (Sections 6.8 to 6.20). No further confirmation is required for the purposes of this study.

\subsection{MATHEMATICAL MODEL OF TRANSPORT}

The mathematical model of transport and the implications of the various processes for radionuclide transport are discussed in the eight subsections in Section 6.2. The basic mass-balance equation of solutes and colloids are discussed in Section 6.2.1. Section 6.2.2 focuses on the accumulation terms of the mass balance equation. The equations of sorption (for solutes), filtration (for colloids), and colloid-assisted transport of solutes are described in Sections 6.2.3, 6.2.4, and 6.2.5, respectively. The flux terms of the mass-balance equation (Section 6.2.1) are described mathematically in Section 6.2.6. Radioactive decay is discussed in Section 6.2.7, and Section 6.2.8 describes the equations of transport for the daughter products of radioactive decay.

The mathematical model of the flow component in this model report is thoroughly discussed in UZ Flow Models and Submodels (BSC 2004 [DIRS 169861], Section 6).

\subsubsection{General Mass Balance Equations}

Following Pruess (1987 [DIRS 100684]; 1991 [DIRS 100413]), mass balance considerations in a control volume dictates that

$$
\frac{d}{d t} \int_{V_{n}} M_{\kappa} d V=\int_{\Gamma_{n}} \boldsymbol{F}_{\kappa} \cdot n \cdot d \Gamma+\int_{V_{n}} q_{\kappa} d V
$$

where

$$
\begin{array}{ll}
V, V_{n} & =\text { volume, volume of subdomain } n\left[L^{3}\right] \\
M_{\kappa} & =\text { mass accumulation term of component (tracer) } \kappa\left[M L^{-3}\right] \\
\Gamma, \Gamma_{n} & =\text { surface area, surface area of subdomain } n\left[L^{2}\right] \\
\mathbf{F}_{\kappa} & =\text { Darcy flux vector of component } \kappa\left[M L^{-2} T^{-1}\right] \\
\mathbf{n} & =\text { inward unit normal vector }\left[L^{0}\right] \\
q_{\kappa} & =\text { source/sink term of tracer } \kappa\left[M L^{-3} T^{-1}\right] \\
t & =\text { time }[T] .
\end{array}
$$

The conservation of mass for any subdomain $n$ is given by Equation 6-1, which in space-discretized form assumes the form of the following ordinary differential equation:

$$
\left(\frac{d M_{\mathrm{\kappa}}}{d t}\right)_{n}=\frac{1}{V_{n}} \sum_{m} A_{n m}\left(F_{\mathrm{\kappa}}\right)_{n m}+\left(q_{\kappa}\right)_{n}
$$


where $A_{n m}$ is the surface segment between elements $n$ and $m\left[L^{2}\right],\left(F_{\kappa}\right)_{n m}$ is the mass flux of tracer $\kappa$ between elements $n$ and $m\left[M L^{-2} T^{-1}\right]$, and $\left(q_{i}\right)_{n}$ is the mass rate of the source/sink of tracer $\kappa$ in element $n\left[\mathrm{ML}^{-3} T^{-1}\right]$. Equation 6-2 is general, and applies to solutes and colloids.

\subsubsection{Accumulation Terms}

\subsubsection{Equations of the Accumulation Terms}

The accumulation term $M$ of a tracer $\kappa$ (solute or colloid) in a porous or fractured medium is given by

$$
M_{\kappa}= \begin{cases}M_{L, \kappa}+M_{A g, \kappa}+\delta_{c} M_{A c, \kappa} & \text { for solutes } \\ M_{L, \kappa}+M_{F, \kappa} & \text { for colloids }\end{cases}
$$

where

$$
\begin{array}{ll}
M_{L, \kappa} & =\text { the mass of tracer } \kappa \text { in the aqueous phase }\left[M L^{-3}\right] \\
M_{A g, \kappa} & =\text { the mass of solute tracer } \kappa \text { adsorbed onto the porous or fractured medium grains } \\
& {\left[M L^{-3}\right]} \\
M_{A c, \kappa} & =\text { the mass of solute tracer } \kappa \text { adsorbed onto colloidal particles }\left[M L^{-3}\right] \\
M_{F, \kappa} & =\text { the mass of filtered colloidal tracer } \kappa\left[M L^{-3}\right]
\end{array}
$$

and the parameter

$$
\delta_{C}=\left\{\begin{array}{ll}
1 & \text { for Class III colloid } \\
0 & \text { for solutes }
\end{array}\right\}
$$

A detailed discussion on the different colloid classes can be found in Section 6.1.3.3.1 of this model report. Omitting for simplicity the $\kappa$ subscript, $M_{L}$ is obtained from

$$
M_{L}=\phi\left(S_{w}-S_{r}\right) \rho X+\phi S_{r} \rho \mathrm{X}
$$

where

$$
\begin{array}{ll}
X & =\text { the mass fraction of the tracer in the mobile fraction of the aqueous phase } \\
& {[M / M]} \\
\mathrm{X} & =\text { the mass fraction of the tracer in the immobile fraction of the aqueous phase } \\
& {[M / M]} \\
S_{w} & =\text { the water saturation }\left[L^{3} / L^{3}\right] \\
S_{r} & =\text { the immobile water saturation (can be set equal to the irreducible) }\left[L^{3} / L^{3}\right] \\
\phi & =\text { the porosity (matrix or fracture) }\left[L^{3} / L^{3}\right] \\
\rho & =\text { the water density }\left[M L^{-3}\right] .
\end{array}
$$

Equation 6-5 reflects the fact that solute concentrations are different in the mobile and immobile water fractions. Because water is very strongly bound (in electric double layers) to the porous or 
fractured medium grain surface, Brownian motion is limited and solubility in the immobile water is lower than in the mobile water fraction. The importance of this boundary layer has been recognized by de Marsily (1986 [DIRS 100439], p. 234), who differentiates $X$ and X, and Moridis (1999 [DIRS 117241]), who used the mobile fraction of water in the analysis of diffusion experiments. Using the linear equilibrium relationship (Moridis 1999 [DIRS 117241]),

$$
\mathrm{X}=K_{i} X
$$

where $K_{i}$ is a dimensionless mass transfer coefficient for solutes with $1 \geq K_{i}>0$. Because of their double layers and their relatively large size (compared to solutes), colloids are expected to concentrate in the mobile water fraction and to be less abundant in the immobile water fraction. Thus, a good approximation for colloids is $K_{i}<1$, but there is no supporting information on the subject. However, as discussed in Section 5, $K_{i}$ is assumed to have the value of 1 .

Substitution into Equation 6-5 then leads to

$$
M_{L}=\phi h \rho X, \quad \text { where } \quad h=S_{w}-S_{r}+K_{i} S_{r}
$$

\subsubsection{Implications for Transport in the UZ}

For rocks with high irreducible water saturations at the Yucca Mountain UZ, $K_{i}<1$ can lead to smaller $h$ values, indicating lower accumulation in the liquid phase. Under conditions of steady-state release, this can result in faster breakthroughs and, consequently, shorter transport times to the water table.

\subsubsection{Sorption Terms}

The discussion in this section is limited to sorption onto the matrix and fractures. Sorption onto pseudocolloids will be discussed in Section 6.2.5.

\subsubsection{Equilibrium Physical Sorption}

Omitting again for simplicity the $\kappa$ subscript, the mass of a solute sorbed onto the porous or fractured medium grains and following a linear equilibrium isotherm is given by

$$
M_{A g}=(1-\phi) \rho_{S} F
$$

where

$$
\begin{array}{ll}
\rho_{s} & =\text { the grain density }\left[M L^{-3}\right] ; \\
F & =F_{p}+F_{c}, \text { the total sorbed mass of solute per unit mass of the porous or fractured } \\
& \text { medium, }[M / M] \\
F_{p} & =\text { the physically sorbed mass of solute per unit mass of the porous or fractured } \\
& \text { medium }[M / M] \\
F_{c} & =\text { the chemically sorbed mass of solute per unit mass of the porous or fractured } \\
& \text { medium }[M / M]
\end{array}
$$


Following the concepts of de Marsily (1986 [DIRS 100439], p. 234) and considering that sorption onto the soil grains occurs as the dissolved species diffuses through the immobile water fraction (Moridis 1999 [DIRS 117241]), the equilibrium physical sorption is described by the equation

$$
F_{p}= \begin{cases}K_{d} \rho K_{i} X & \text { for linear equilibrium(LE) sorption, } \\ K_{F}\left(\rho K_{i} X\right)^{\beta} & \text { for Freundlich equilibrium (FE) sorption, } \\ \frac{K_{1} \rho K_{i} X}{1+K_{2} \rho K_{i} X} & \text { for Langmuir equilibrium (LAE) sorption }\end{cases}
$$

where $K_{d}\left[M^{-1} L^{3}\right], K_{F}\left[M^{-\beta} L^{3 \beta}\right], \beta, K_{1}\left[M^{-1} L^{3}\right]$, and $K_{2}\left[M^{-1} L^{3}\right]$ are sorption parameters specific to each solute and rock type. Of particular interest is the parameter $K_{d}$, called the distribution coefficient, which is the constant slope of the linear equilibrium adsorption isotherm of a solute in relation to the medium. The sorption of the various radionuclides in this model report follows a linear equilibrium isotherm (see Section 6.1.5.2).

\subsubsection{Kinetic Physical Sorption}

If a kinetic isotherm is followed, then sorption is described in equations given by

$$
\frac{d F_{p}}{d t}= \begin{cases}k_{\ell}\left(K_{d} \rho K_{i} X-\delta_{p} F_{p}\right) & \text { for linear kinetic (LKP) sorption, } \\ k_{F}\left[K_{F}\left(\rho K_{i} X\right)^{\beta}-F_{p}\right] & \text { for Freundlich kinetic (FKP) sorption, } \\ k_{L}\left(\frac{K_{1} \rho K_{i} X}{1+K_{2} \rho K_{i} X}-F_{p}\right) & \text { for Langmuir kinetic (LAKP) sorption, }\end{cases}
$$

where

$$
\delta_{p}= \begin{cases}1 & \text { for linear kinetic physical (LKP) sorption; } \\ 0 & \text { for linear irreversible physical (LIP) sorption, }\end{cases}
$$

and $k_{l}, k_{F}$, and $k_{L}$ are the kinetic constants for linear, Freundlich and Langmuir sorption, respectively $\left[T^{-1}\right.$ ] (de Marsily (1986 [DIRS 100439]). For $\delta_{p}=0$, the linear kinetic expression in Equation 6-9 can also be used to describe the chemical process of salt precipitation. 


\subsubsection{Kinetic Chemical Sorption}

The first-order reversible chemical sorption is represented by the linear kinetic chemical model

$$
\frac{d F_{c}}{d t}=k_{c}^{+} \rho K_{i} X-k_{c}^{-} F_{c}
$$

where $k_{c}^{+}\left[M^{-1} L^{3} T^{-1}\right]$ and $k_{c}^{-} \quad\left[T^{-1}\right]$ are the forward and backward kinetic constants, respectively. Note that Equation 6-11 can be used in conjunction with the physical sorption equations to describe combined sorption (Cameron and Klute 1977 [DIRS 117172]), for example, physical and chemical sorption. Combined sorption accounts for the different rates at which a species is sorbed onto different porous or fractured medium constituents. Thus, sorption onto organic components may be instantaneous (linear equilibrium), while sorption onto mineral surfaces may be much slower and kinetically controlled (Cameron and Klute 1977 [DIRS 117172]).

\subsubsection{Implications for Transport in the UZ}

Sorption is the main mechanism of radionuclide mass retardation from the transporting liquid phase. Nonsorbing radionuclides (such as ${ }^{3} \mathrm{H}$ or ${ }^{99} \mathrm{Tc}$ ) will not be retarded. Strongly sorbing radionuclides (such as $\mathrm{Np}, \mathrm{Pu}, \mathrm{U}, \mathrm{Th}, \mathrm{Am}$ ) will exhibit much longer arrival times to the water table. The transfer coefficient $K_{i}$ is obviously quite important, because a $K_{i}<1$ reduces the radionuclide sorbed onto the particle surfaces. Thus, the larger $K_{i}$ is, the more the radionuclide is retarded. Although this may not be significant in the case of strong sorbers, it may be important in less-strongly sorbing tracers.

Also of interest is sorption onto the fracture surfaces, as opposed to the volume-base sorption in the matrix. Currently, no information exists on the subject. The conventional approach in fractures with $\phi=1$ results in zero sorption. It is possible to obtain an estimate of surface sorption by (a) assuming a reasonable grain size for a matrix, (b) computing the internal surface area (available for sorption) per unit volume of the matrix, and (c) computing the surface area of a corresponding fracture for the same grain size. This approach usually produces very small surface $K_{d}$ values.

\subsubsection{Colloid Filtration Terms}

\subsubsection{Equations of Colloid Filtration}

Colloidal particles moving through porous media are subject to filtration, the mechanisms of which have been the subject of several investigations (e.g., Herzig et al. 1970 [DIRS 117519]). The mass of filtered colloids is then given by

$$
M_{F, \kappa}=\rho_{c, \kappa} \sigma_{\kappa}
$$

where $\rho_{c, \kappa}$ is the density of the colloidal particles of colloid $\kappa\left[M L^{-3}\right]$ and $\sigma_{\kappa}$ is the filtered concentration of the colloid expressed as volume of colloids per volume of the porous medium. 
When colloid deposition is a relatively fast process compared to the water velocity, it is possible to describe colloid filtration as a linear equilibrium process (James and Chrysikopoulos 1999 [DIRS 109517]). Omitting the $\kappa$ subscript, linear equilibrium filtration is then described by

$$
\sigma=K_{\sigma} K_{i} \rho X
$$

where $K_{\sigma}$ is a distribution coefficient $\left[M^{-1} L^{3}\right]$.

Colloid filtration is more accurately described by a linear kinetic model (Çorapçioglu et al. 1987 [DIRS 117300], pp. 269 to 342), which can take the following form:

$$
\frac{d \sigma}{d t}=\kappa\left(K_{\sigma} K_{i} \rho X-\delta_{p} \sigma\right)=\kappa^{+} X-\kappa^{-} \sigma
$$

where $\kappa\left[T^{-1}\right]$ is a kinetic coefficient, and $\kappa^{+}$and $\kappa^{-}\left[T^{-1}\right]$ are the kinetic forward and reverse colloid deposition rates (clogging and declogging coefficients), respectively, which are specific to each colloid and rock type. The term $\kappa$ - is commonly assumed to be zero (Bowen and Epstein 1979 [DIRS 117219]), but there is insufficient evidence to support this. As will be seen in Section 6.18 of the present model report, colloid transport in the UZ is very sensitive to this parameter. The parameter $\delta_{p}$ is analogous to that for sorption in Equations 6-10 and 6-11, and describes the reversibility of filtration.

From the reports by de Marsily (1986 [DIRS 100439], p. 273) and Ibaraki and Sudicky (1995 [DIRS 109297], p. 2,948), the following expression for the $\kappa^{+}$coefficient can be derived:

$$
\kappa^{+}=\varepsilon f u G
$$

where $\varepsilon$ is the filter coefficient of the porous medium $\left[L^{-1}\right], f$ is a velocity modification factor, $u$ is the Darcy velocity $\left[L T^{-1}\right]$, and $G$ is a dynamic blocking function that describes the variation of the porous or fractured medium porosity and specific surface with $\sigma$ (James and Chrysikopoulos 1999 [DIRS 109517]). The factor $f(1 \leq f \leq 1.5)$ accounts for the velocity of the colloidal particle flow being larger than that of water (Ibaraki and Sudicky 1995 [DIRS 109297], p. 2,948). This results from the relatively large size of the colloids, which tends to concentrate them in the middle of the pores where the water velocity is larger than the bulk average velocity. The factor $f$ tends to increase with decreasing ionic strength, but cannot exceed 1.5 because colloids cannot move faster than the maximum water velocity, which occurs at the middle of the pores and is equal to 1.5 the average pore velocity (Ibaraki and Sudicky 1995 [DIRS 109297], p. 2,948).

For deep filtration (i.e., in the case of very dilute colloidal suspensions), there is no interaction among the colloidal particles and no effects on the medium porosity and permeability, that is, $\phi$ is constant, and $G=1$. Note that it is possible for EOS9nT to have combined filtration, in which two different types of filtration (e.g., equilibrium and kinetic, or two kinetic filtrations with different $\kappa^{+}$and $\kappa^{-}$) occur simultaneously. 


\subsubsection{Implications for Transport in the UZ}

Filtration is one of the main mechanisms of radioactive colloid removal from the transporting liquid phase, the others being chemical destabilization (e.g., because of $\mathrm{pH}$ changes) and flocculation. Three types of filtration mechanisms may affect the transport of colloids through the porous or fractured medium: surface filtration, straining filtration, and physical-chemical filtration. Surface filtration occurs when particles are larger than the pores, in which case a filter cake is formed.

Straining filtration is determined by the ratio $R_{d}=d_{g} / d_{c}$, where $d_{g}$ is the diameter of the grains of the porous medium and $d_{c}$ is the suspended particle diameter. Based on the experimental data of Clogging of a Granular Porous Medium by Sediment, Final Report (Sakthivadivel 1969 [DIRS 134556]), $R_{d} \leq 10$ leads to cake filtration, $10<R_{d} \leq 20$ corresponds to substantial straining filtration (permeability reductions by a factor of 7-15 and particles occupying $0.3 \phi$ ), and $R_{d}>$ 20 results in limited straining (only $2-5$ percent of $\phi$ occupied by particles and permeability reductions by 10-50 percent). Herzig et al. (1970 [DIRS 117519], p. 15) indicated that little straining was expected when $R_{d}>12$, and calculated that when $R_{d}=50$, only 0.053 percent of $\phi$ would be occupied by particles.

A detailed discussion of the physical-chemical filtration of colloids can be found in "Governing Equations for Particle Transport in Porous Media." (Çorapçioglu et al. 1987 [DIRS 117300], pp. 269 to 342). The physical-chemical colloidal filtration by the porous/fractured medium incorporates three mechanisms: (a) contact with the pore walls, (b) colloid fixation onto the walls, and (c) release of previously fixed colloids (Herzig et al. 1970 [DIRS 117519]; Çorapçioglu et al. 1987 [DIRS 117300]).

Contact with the pore walls and colloidal capture can be the result of sedimentation (caused by a density differential between the colloidal particle and the carrier liquid), inertia (deviation of colloidal trajectories from the liquid streamlines because of their mass), hydrodynamic effects (caused by a variation in the velocity field of the liquid), direct interception (caused by collisions with the pore walls at convergent areas), and diffusion (Brownian motion causing colloids to move toward pore walls or dead-end pores).

Fixation on the pore walls occurs at retention sites that include edges between two convex surfaces, pore throats smaller than the colloidal size, and dead-end pores or regions of near-zero liquid velocity. Fixation is caused by retentive forces, which include axial pressure of the fluid at constriction sites, friction forces, Van der Waals forces, electrical forces, and chemical forces (Herzig et al. 1970 [DIRS 117519], pp. 4, 11 to 17). Finally, remobilization of colloidal particles may be caused by a number of factors, including collision between a loosely held colloid with a moving particle, an increase in pressure as colloids constrict flow, and a change in external conditions.

All three mechanisms are expected to be present in the Yucca Mountain UZ. The EOS9nT simulations can account for surface filtration by using a particle size versus pore size criterion, and by not allowing colloidal entry into media that do not meet this criterion. Straining filtration (pore-size exclusion) is described by using appropriate colloid accessibility factors (see 
Section 6.18), and physical-chemical filtration is represented by using appropriate parameters in Equations 6-14 or 6-15.

Note that it is not possible to account for cake filtration or for the effects of filtration on permeability and porosity, as this would violate the linearity in the models. However, these scenarios would be unlikely in the Yucca Mountain UZ because (a) natural pseudocolloids (such as clays) under natural conditions occur in small concentrations; (b) it is expected that, owing to adverse chemical conditions (e.g., pH, ionic strength) in the immediate vicinity of the repository, true colloids will be released at low concentrations for a long time; and (c) the expected concentrations of the waste form colloids (BSC 2004 [DIRS 170025], Section 6.3.2) are insufficient to support cake filtration.

\subsubsection{Colloid-Assisted Transport Terms}

\subsubsection{Equations of Colloid-Assisted Transport}

The mass of a tracer $\kappa$ sorbed reversibly onto colloids is described by

$$
M_{A c, \kappa}=\sum_{j=1}^{N_{c}}\left(\rho_{j} \sigma_{j}+\rho X_{j}\right) \mathrm{F}_{\kappa, j}
$$

where $\mathrm{F}_{\kappa, j}$ denotes the sorbed mass of solute $\kappa$ per unit mass of the pseudocolloid $j[M / M]$ and $N_{c}$ is the total number of pseudocolloid species (Moridis et al. 1999 [DIRS 123093]). The first term in the sum inside the parenthesis of Equation 6-17 describes the filtered (deposited) colloid concentration, and the second describes the concentration of the suspended colloids in the liquid phase. $\mathrm{F}_{\mathrm{k}, j}$ is computed from any combination of Equations 6-9-6-12, with the appropriate sorption parameters corresponding to each colloid.

\subsubsection{Implications for Transport in the UZ}

The formulation of Equation 6-17 allows consideration of the whole size spectrum of a particular colloid, as each size would have different transport behavior (see Equation 6-16) and different sorption properties (resulting from different surface area).

Of particular interest in UZ radioactive transport models is the potential of colloid-assisted transport to significantly enhance the migration of tracers whose normally strong sorbing behavior would confine them to the vicinity of the release point. Sorption of such a radionuclide (e.g., $\mathrm{Pu}$ ) onto pseudocolloids renders the whole colloid radioactive. The transport of such a colloid is no longer dictated by the strong Pu sorption behavior, but by the kinetics of colloid filtration. Coupled with the fact that colloids can move at velocities that can be up to 50 percent higher than the Darcy velocity (and especially so in fractures, where most of the UZ flow occurs; see Equation 6-16), this can result in transport times to the water table orders of magnitude shorter than the ones predicted for the corresponding sorbing solute. Note that the higher colloid velocities are not a function of the water flow, but depend entirely on the colloid size (see discussion in Section 6.2.4.1). 


\subsubsection{Flux Terms}

\subsubsection{Equations of the Flux Terms}

The flux term has contributions from advective, diffusive, and dispersive transport processes and is given by

$$
\mathbf{F}_{\kappa}=\mathbf{F}_{\omega} X_{\kappa}-\rho \mathbf{D}_{\kappa} \nabla X_{\kappa}-\mathbf{F}_{S, \kappa}
$$

where $\mathbf{F}_{s, \kappa}$ is the flux due to surface diffusion and $\mathbf{D}_{\kappa}$ is the dispersion tensor of tracer $\kappa$, a second order symmetric tensor with a principal axis aligned with the Darcy flow vector. Omitting the $\kappa$ subscript, $\mathbf{D}_{\kappa}$ is described by the equations

$$
\begin{gathered}
\mathbf{D}=D^{T} \mathbf{I}+\frac{D^{L}-D^{T}}{u^{2}} \mathbf{u} \mathbf{u}, \\
D^{L}=\phi\left(S_{w}-S_{r}\right) \tau D_{0}+\phi S_{r} \tau D_{0}+\alpha_{L} u, \\
D^{T}=\phi\left(S_{w}-S_{r}\right) \tau D_{0}+\phi S_{r} \tau D_{0}+\alpha_{T} u,
\end{gathered}
$$

where

$$
\begin{array}{ll}
\mathbf{I} & =\text { the unit vector } \\
\tau & =\text { the tortuosity coefficient of the pore paths }[L / L] \\
D_{0} & =\text { the molecular diffusion coefficient of tracer } i \text { in water }\left[L^{2} T^{-1}\right] \\
\alpha_{L}, \alpha_{T} & =\text { longitudinal and transverse dispersivities, respectively }[\mathrm{L}] \\
\mathbf{u} & =\text { the Darcy velocity vector }\left[L T^{-1}\right]
\end{array}
$$

Equation 6-18 accounts for surface diffusion, which can be responsible for significant transport in strongly sorbing media (Moridis 1999 [DIRS 117241]; Cook 1989 [DIRS 117314]). The surface diffusion flux is given by Jahnke and Radke (1987 [DIRS 117398]) as

$$
\mathbf{F}_{S}=(1-\phi) \rho_{s} \tau_{s} D_{s} \nabla F_{p}
$$

where $\tau_{s}$ is the tortuosity coefficient of the surface path $\left[L^{0}\right], D_{s}$ is the surface diffusion coefficient $\left[L^{2} T^{-1}\right]$, and $F_{p}$ is computed from equations (9) or (10). There is theoretical justification for the relationship $\tau_{s}=\frac{2}{3} \tau$ (Cook 1989 [DIRS 117314], p. 10).

\subsubsection{Application to Colloid Fluxes}

Equations 6-17 through 6-20 apply to solutes, but need the following modifications to render them suitable to colloidal transport. More specifically:

1. $\mathbf{F}_{s}=0$ because surface diffusion does not occur in colloids. 
2. The flux $\mathbf{F}_{s}$ and the Darcy velocities $\mathbf{u}$ are multiplied by the factor $f$ (see Section 6.2.4.1).

3. The dispersivities $\alpha_{L}$ and $\alpha_{T}$ are generally different from those for solutes (Ibaraki and Sudicky 1995 [DIRS 109297]), and may be a function of the colloidal particle size.

4. The term $D_{0}$ is the colloidal diffusion coefficient in water $\left[L^{2} T^{-l}\right]$ and is described by the Stokes-Einstein equation, according to Bird et al. (1960 [DIRS 103524], p. 514), as

$$
D_{0}=\frac{k T}{3 \pi \mu_{w} d_{c}}
$$

where $k$ is the Boltzmann constant $\left(1.38 \times 10^{-23} \mathrm{JK}^{-1}\right.$ in SI units), $T$ is the absolute water temperature $[K], \mu_{w}$ is the dynamic viscosity of water $\left[M L^{-1} T^{-1}\right]$, and $d_{c}$ is the colloid diameter $[L]$.

5. The fluxes in Equation 6-18 are multiplied by the colloid accessibility factors $f_{c}\left(0 \leq f_{c} \leq\right)$ at the interface of different media. The $f_{c}$ factor describes the portion of the colloidal concentration in a medium that is allowed to enter an adjacent medium of different characteristics, and quantifies pore size exclusion (straining).

In the treatment of the general three-dimensional dispersion tensor, velocities are averaged by using the projected area weighting method (Wu et al. 1996 [DIRS 100649], p. 23), in which a velocity component $u_{j}(j \nexists x, y, z)$ of the vector $\mathbf{u}$ is determined by vectorial summation of the components of all local connection vectors in the same direction, weighted by the projected area in that direction. This approach allows the solution of the transport problem in irregularly shaped grids, in which the velocities normal to the interface areas are not aligned with the principal axes.

\subsubsection{Implications for Transport in the UZ}

Because it is directly proportional to the water fluxes, advection in the fractures is by far the dominant mechanism of transport in the UZ of Yucca Mountain. This is further enhanced by longitudinal dispersion, molecular diffusion, and (possibly) surface diffusion (in decreasing order). Coupled with the fracture orientation and gravitational differentials, the result of the fracture and flow characteristics is a mainly downward migration of the radionuclides. Note that advection also occurs in the matrix (and is accounted for in the simulations), albeit at significantly lower rates.

Lateral spreading of the contaminants can be achieved through transverse dispersion, molecular diffusion, and (possibly) surface diffusion. As sorption occurs from the liquid phase onto the solids, and fractures are by far the main conduits of water, retardation of sorbing radionuclides will be controlled by the rate of tracer movement from the fractures to the sorbing matrix. This is represented by the sum of diffusive, dispersive, and surface diffusion fluxes, and results in the lateral migration of the contaminants. 
When not ignored in the numerical simulations (justified by the relative magnitude of advection), reasonable $\alpha_{L}$ estimates are used, and $\alpha_{T}$ is usually set to zero (especially in the fractures) since field study estimates of $\alpha_{L}$ and $\alpha_{T}$ in the UZ are limited. In past simulations (DOE 1998 [DIRS 100550], p. 3-122), an estimate of $\alpha_{L}=20 \mathrm{~m}$ was used. From the Northern Ghost Dance fault test (LeCain et al. 2000 [DIRS 144612]), $\alpha_{L}$ estimates in the $0.4 \mathrm{~m}$ to $2.6 \mathrm{~m}$ range were obtained but their validity is uncertain because they are based on gas (rather than liquid) phase transport, short transport times (less than $200 \mathrm{~min}$ ), and a small scale experiment (less than $10 \mathrm{~m}$ ). Accurate values of $D_{0}$, coupled with reasonable tortuosity coefficients (shown from laboratory experiments to be approximated by the porosity, see Section 5, this model report), provide a representative estimate of diffusive fluxes in the UZ simulations.

No information exists on whether the UZ media support surface diffusion (a possibility in zeolites). Surface diffusion can be important in tracers that exhibit strong sorption (e.g., Pu). A larger $K_{d}$ clearly indicates stronger sorption, but this does not mean immobilization of the dissolved species when the porous/fractured medium supports surface diffusion. On the contrary, the stronger the sorption (i.e., the larger the $K_{d}$ ), the larger the diffusion rate, with practically all of it attributable to the surface process (Moridis 1999 [DIRS 117241], pp. 1,735 to 1,736$)$.

\subsubsection{Radioactive Decay}

\subsubsection{Equations of Radioactive Decay}

When a tracer $\kappa$ undergoes radioactive decay, the rate of mass change is described by the first-order decay law

$$
\frac{d M_{\kappa}}{d t}=-\lambda_{\kappa} M_{\kappa}, \quad \text { where } \quad \lambda_{\kappa}=\frac{\ln 2}{\left(T_{1 / 2}\right)_{\kappa}}
$$

and $\left(T_{1 / 2}\right)_{\kappa}$ is the half-life of tracer $\kappa$. Substitution of Equations 6-18 and 6-24 into Equation 6-2 yields (Moridis et al. 1999 [DIRS 123093] Eq. 32)

$\left(\frac{d M_{\kappa}}{d t}\right)_{n}+\lambda_{\kappa}\left(M_{\kappa}\right)_{n}=\frac{1}{V_{n}} \sum_{m} A_{n m}\left[F_{n m} X_{\kappa}-\rho D_{\kappa} \nabla X_{\kappa}-\mathrm{F}_{s, \kappa}\right]+\left(q_{\kappa}\right)_{n}$

\subsubsection{Implications for Transport in the UZ}

The decay of radioactive substance is completely predictable and well documented. Thus, the decay of radioactive substances in UZ simulations can be computed very reliably. 


\subsubsection{Daughter Products of Radioactive Decay}

\subsubsection{Transport Equations of Daughters}

If a radioactive tracer $\kappa$ is a daughter product of the decay of tracer $j$, then the mass accumulation terms are adjusted (de Marsily 1986 [DIRS 100439], pp. 265 to 266) to yield the equation of transport of the daughter products as

$$
\begin{gathered}
\left(\frac{d M_{\kappa}}{d t}\right)_{n}+\lambda_{\kappa}\left(M_{\kappa}\right)_{n}-\lambda_{j} m_{r}\left(M_{j}\right)_{n} \\
=\frac{1}{V_{n}} \sum_{m} A_{n m}\left[F_{n m} X_{\kappa}-\rho \mathbf{D}_{i} \nabla X_{\kappa}-\mathbf{F}_{S, \kappa}+\left(q_{k}\right)_{n}\right.
\end{gathered}
$$

where $m_{r}=W_{k} / W_{j}$, and $W_{\kappa}$ and $W_{j}$ are the molecular weights of the daughter and parent species. Equation 6-26 applies to radioactive solutes or radioactive true colloids.

For daughters following an isotherm other than linear equilibrium, Equations 6-9 and 6-10 need to account for the generation of daughter mass from the decay of the sorbed parent, and become

$$
\frac{\partial F_{\kappa}}{\partial t}+\lambda_{\kappa} F_{v}-\lambda_{\kappa-1} m_{r} \zeta_{\kappa} F_{\kappa-1}=k_{A} \rho X_{\nu}-k_{B}\left(F_{\kappa}+m_{r} \zeta_{\kappa} F_{\kappa-1}\right)
$$

where $F_{\kappa^{-1}}$ is the sorbed mass of the parent,

$k_{A}=\left\{\begin{array}{ll}k_{\ell} K_{d} K_{i} \text { for LKP/LIP sorption } \\ k_{c}^{+} K_{i} \text { for LKC sorption }\end{array} k_{B}= \begin{cases}k_{p} \delta_{p} & \text { for LKP/LIP sorption, } \\ k_{c}^{-} & \text {for LKC sorption, }\end{cases}\right.$

and $\zeta_{\kappa}$ is the fraction of the mass of the decayed sorbed parent that remains sorbed as a daughter $\left(0 \leq \zeta_{\kappa} \leq 1\right)$. The term $\zeta_{\kappa}$ is introduced to account for the different sorption behavior of parents and daughters, and the fact that daughters can be ejected from grain surfaces due to recoil (e.g., the ejection of ${ }^{234} \mathrm{Th}$ from grain surfaces during the alpha decay of ${ }^{238} \mathrm{U}$ ) (Faure 1977 [DIRS 122805], pp. 288 to 289).

\subsubsection{Implications for Transport in the UZ}

If decay results in radioactive daughters, UZ simulations must compute the total radioactivity distribution, that is, the sum of the concentrations of all the members of the radioactive chain. This is especially true if the daughters have long half-lives. Intermediate chain products with short half-lives relative to the simulation periods can be ignored with impunity.

Although the EOS9nT can theoretically obtain the transport scenario of any number of daughters in the radioactive chain, this is especially true for daughters with short half-lives. With longer half-lives (such as for ${ }^{237} \mathrm{~Np},{ }^{239} \mathrm{Pu}$, etc.), machine accuracy considerations and roundoff errors (with the daughter concentrations becoming increasingly smaller moving down the radioactive chain) limit their number to a maximum of four or five with comparable half-lives. 
The $\zeta$ factor is a function of the type of decay, as well as of the chemical form of the sorbed cation. In alpha decay (e.g., $\left.{ }^{237} \mathrm{~Np},{ }^{239} \mathrm{Pu}\right), \zeta=0$. There is no information on the behavior of $\zeta$ for other types of decay. The $\zeta$ factor can have significant implications for the transport behavior of daughters if (a) the large sorbed masses of strongly sorbing parents are ejected back into the aqueous phase after decay, (b) the daughter is a much weaker sorber, and (c) its sorption is kinetically controlled. For equilibrium isotherms, this is not an issue.

\subsection{THE NUMERICAL MODELS}

In mathematical simulations, the conventional meaning of the term model is the mathematical description of the physics governing the behavior of the simulated system, whereas the term code is the implementation of the mathematical model in a computer language. Because model and code are intertwined, these terms have historically been used interchangeably. In the context of T2R3D and EOS9nT applications in the present model report, when the word model is used, it is preceded by the qualifying words numerical or semianalytical.

\subsubsection{The T2R3D Code}

The code T2R3D V1.4 (LBNL 1999 [DIRS 146654]; Wu et al. 1996 [DIRS 100649]) simulates the flow (saturated and/or unsaturated) and coupled transport of a single radioactive solute tracer in complex subsurface systems involving porous and/or fractured media. The transport equations account for advection, molecular diffusion, hydrodynamic dispersion, and linear equilibrium sorption.

T2R3D can simulate problems involving the coupled (a) flow of subsurface liquids, (b) transport of a solute, and (c) the transport of heat in complex heterogeneous subsurface systems. When a flow system has reached a steady state, T2R3D allows the option of solving only the solute transport equation.

\subsubsection{The EOS9nT Code}

The code used for the three-dimensional site-scale transport simulations in the model report is TOUGH2 V1.11MEOS9nTV1.0 (LBNL 1999 [DIRS 113943]; Moridis et al. 1999 [DIRS 123093]; 2003 [DIRS 161902]), which is a member of the TOUGH2 family of codes (Pruess 1991 [DIRS 100413]). It can simulate flow and transport of an arbitrary number $n$ of nonvolatile tracers (solutes and/or colloids) in the subsurface. EOS9nT first solves the Richards equation, which describes saturated or unsaturated water flow in subsurface formations, and obtains the flow regime. The set of $n$ linearly independent transport equations (corresponding to the $n$ solutes/colloids) are then solved sequentially. The $n$ tracer transport equations account for (a) advection, (b) molecular diffusion, (c) hydrodynamic dispersion (with full three-dimensional tensorial representation), (d) kinetic or equilibrium physical and chemical sorption (linear, Langmuir, Freundlich, or combined), (e) first-order linear chemical reaction, (e) radioactive decay, (f) colloid filtration (equilibrium, kinetic, or combined), and (g) colloid assisted solute transport. A total of $n-1$ daughter products of radioactive decay (or of a linear, first-order reaction chain) can be tracked.

EOS9nT includes two types of Laplace transform formulations of the tracer equations, in addition to conventional timestepping. The Laplace transform is applicable to steady-state flow 
fields and allows a practically unlimited time-step size and more accurate solution (as numerical diffusion is significantly reduced). Additional information on the EOS9nT numerical model can be found in the report by BSC (2001 [DIRS 161340], Attachment I). For the simulation of isothermal transport of a tracer that follows a linear equilibrium isotherm, T2R3D and EOS9nT have been shown to produce identical results (Section 7.2.3.1, this model report; DTN: LB03093RADTRNS.001 [DIRS 166225]).

\subsubsection{Other Computational Tools}

In addition to T2R3D and EOS9nT, the following computational tools were used:

1. The software codes TOUGH2 V1.4 Module EOS9 (LBNL 2000 [DIRS 146496]) and TOUGH2 V1.6 Module EOS9 (LBNL 2002 [DIRS 160242]; LBNL 2003 [DIRS 161491]), which solve the Richards equation to simulate flow (saturated and/or unsaturated) in complex subsurface systems (involving porous and/or fractured media). These codes were used to obtain the flow fields for the various climatic scenarios discussed by BSC (2004 [DIRS 169861], Section 6).

2. The software code PHREEQC V2.3 (BSC 2001 [DIRS 155323]) to perform the computations for the estimations of the $K_{d}$ (Section 6.4).

3. The XtractG.f90 V1.0 routine (LBNL 2003 [DIRS 162786]), which was used for the EOS9nT runs for the following applications:

(a) To modify the grid system for use in the EOS9nT runs. The modifications involved (1) determining the grid elements corresponding to the repository and (2) moving them to the end of the element file where (3) the upper and lower boundary elements are also moved. This was necessitated by the fact that the scenario of continuous contaminant release was investigated in this model report. Thus the repository elements represent internal boundary points. After moving these elements to the bottom of the element file, the volume of the first element is set to zero or a negative number. Then, these elements are considered in the determination of fluxes but are not included in mass balance computations. Additionally, XtractG.f90 can be used to render inactive all the elements above a given height (e.g., the highest repository element) which do not contribute to transport, thus, significantly reducing the size of the problem and making possible the solution within the array sizes of EOS9nT.

(b) To obtain initial condition files (INCON) using the SAVE files from the EOS9 runs.

(c) To create steady state velocity profiles using the flow field output files from the EOS9 runs.

(d) To extract concentrations at desired grid elements within the domain. 


\subsection{FEATURES, EVENTS, AND PROCESSES}

Table 6-2 lists FEPs were taken from the LA FEP List (DTN: MO0407SEPFEPLA.000 [DIRS 170760]). The LA FEP List is a revision to the previous project FEP list [The Development of Information Catalogued in REV00 of the YMP FEP Database (BSC 2001 [DIRS 154365])] used to develop the list of FEPs in the Technical Work Plan for: Unsaturated Zone Transport Model Report Integration (BSC 2004 [DIRS 171282], Table 2.1.5-1). The selected FEPs are those taken from the LA FEP List associated with the subject matter of this report. Those FEPs in Table 6-2 are included in TSPA-LA through this report. The cross-reference for each FEP to the relevant section (or sections) of this report is also given below. Screening arguments and dispostions for both excluded and included FEPs, respectively, are summarized in Features, Events, and Processes in UZ Flow and Transport (BSC 2004 [DIRS 170012]).

Table 6-2. FEPs Included Through This Model Report

\begin{tabular}{|l|l|l|}
\hline LA FEP Number & \multicolumn{1}{|c|}{ FEP Name } & \multicolumn{1}{c|}{ Section Where FEP is Addressed } \\
\hline $2.2 .08 .01 .0 \mathrm{~B}$ & $\begin{array}{l}\text { Chemical } \\
\text { characteristics of } \\
\text { groundwater in } \\
\text { the UZ }\end{array}$ & $\begin{array}{l}\text { Appendix A, Appendix A4 discusses the } \\
\text { chemical characteristics of UZ groundwater. }\end{array}$ \\
\hline 2.2.08.06.0B & $\begin{array}{l}\text { Complexation in } \\
\text { the UZ }\end{array}$ & $\begin{array}{l}\text { Complexation on mobile complexing agents } \\
\text { such as humic and fulvic acids is treated as } \\
\text { part of colloid transport in Section 6.1.3 and } \\
6.18 . \text { Complexation on mineral surfaces is } \\
\text { treated as part of sorption in Appendix A, } \\
\text { Section A7. }\end{array}$ \\
\hline $2.2 .08 .09 .0 \mathrm{~B}$ & $\begin{array}{l}\text { Sorption in the } \\
\text { UZ }\end{array}$ & $\begin{array}{l}\text { The sorption model and the distributions of } \mathrm{K}_{\mathrm{d}} \\
\text { values are presented in 6.1 and Table 6-3. } \\
\text { Data supporting the Kd distributions are } \\
\text { presented in Appendix A and the associated } \\
\text { DTNs are listed in Table 4-1. Colloid } \\
\text { transport is addressed in Section 6.1.3 and } \\
\text { 6.2.5. The model for colloid filtration in the UZ } \\
\text { and simulation results are presented in } \\
\text { Section 6.18. }\end{array}$ \\
\hline $2.2 .09 .01 .0 \mathrm{~B}$ & $\begin{array}{l}\text { Microbial activity } \\
\text { in the UZ }\end{array}$ & $\begin{array}{l}\text { The effects of microbial activity on colloid } \\
\text { transport and on complexing agents are } \\
\text { addressed in Sections 6.1.3 and 6.1.4. The } \\
\text { presence of representative biota in sorption } \\
\text { experiments is addressed in Section A5. }\end{array}$ \\
\hline
\end{tabular}

$F E P=$ feature, event, or process; UZ=unsaturated zone

\subsection{SORPTION MODEL, DIFFUSION, RADIOACTIVE DECAY, AND PARAMETERS}

This section discusses the radionuclide sorption model. The diffusion coefficients and the decay parameters of the radionuclides considered in this model report are also discussed.

The analysis in Appendices A and B substantiates the arguments for the validity of the linear equilibrium sorption model assumed to govern sorption in the UZ. The corresponding distribution coefficients $K_{d}$ of the various radionuclides (describing sorption onto the UZ rocks) 
are listed in Table 6-3. A detailed discussion of the derivation process of these estimates and of the supporting laboratory data can be found in Appendix A. In this model report, all computations involving sorption are based on the linear equilibrium model and use $K_{d}$ estimates of Table 6-3.

Table 6-3. $\mathrm{K}_{\mathrm{d}}$ in the Rocks of the Unsaturated Zone

\begin{tabular}{|c|c|c|c|}
\hline Species & Unit/Analysis & Distributiona & Coefficients describing distributionb (mL/g) \\
\hline \multirow[t]{3}{*}{$U$} & Zeolitic & Cumulative & $\left(\mathrm{K}_{\mathrm{d}}\right.$ value, probability) $(0,0)(0.5,0.5)(30,1.0)$ \\
\hline & Devitrified & Cumulative & $\left(\mathrm{K}_{\mathrm{d}}\right.$ value, probability $)(0,0)(0.2,0.5)(4,1.0)$ \\
\hline & Vitric & Cumulative & (K $K_{d}$ value, probability) $(0,0)(\mathbf{0 . 2}, 0.5)(3,1.0)$ \\
\hline \multirow[t]{3}{*}{$\mathrm{Np}$} & Zeolitic & Cumulative & (K $K_{d}$ value, probability) $(0,0)(0.5,0.5)(6,1.0)$ \\
\hline & Devitrified & Cumulative & (K $K_{d}$ value, probability) $(0,0)(0.5,0.5)(6,1.0)$ \\
\hline & Vitric & Cumulative & $\left(K_{d}\right.$ value, probability) $(0,0)(\mathbf{1 . 0}, 0.5)(3,1.0)$ \\
\hline \multirow[t]{3}{*}{$\mathrm{Pu}$} & Zeolitic & Cumulative & $\left(\mathrm{K}_{\mathrm{d}}\right.$ value, probability $)(10,0)(100,0.5)(200,1.0)$ \\
\hline & Devitrified & Cumulative & $\left(\mathrm{K}_{\mathrm{d}}\right.$ value, probability $)(10,0)(70,0.5)(200,1.0)$ \\
\hline & Vitric & Cumulative & (K $\mathrm{K}_{d}$ value, probability) $(10,0)(100,0.5)(200,1.0)$ \\
\hline \multirow[t]{3}{*}{ Am } & Zeolitic & $\begin{array}{l}\text { Truncated } \\
\text { Normal }\end{array}$ & Range $=1000-10,000$ Mean $=5500$ Std Dev $=1500\left(500^{c}\right)$ \\
\hline & Devitrified & $\begin{array}{l}\text { Truncated } \\
\text { Normal }\end{array}$ & Range $=\mathbf{1 0 0 0}-10,000$ Mean=5500 Std Dev=1500 \\
\hline & Vitric & Cumulative & $\left(K_{d}\right.$ value, probability) $(100,0)(400,0.5)(1,000,1.0)$ \\
\hline \multirow[t]{3}{*}{$\mathrm{Pa}$} & Zeolitic & $\begin{array}{l}\text { Truncated } \\
\text { Normal }\end{array}$ & Range $=1000-\mathbf{1 0 , 0 0 0}$ Mean=5500 Std Dev=1500 \\
\hline & Devitrified & $\begin{array}{l}\text { Truncated } \\
\text { Normal }\end{array}$ & Range $=1000-\mathbf{1 0 , 0 0 0}$ Mean=5500 Std Dev=1500 \\
\hline & Vitric & $\begin{array}{l}\text { Truncated } \\
\text { Normal }\end{array}$ & Range $=1000-\mathbf{1 0 , 0 0 0}$ Mean=5500 Std Dev=1500 \\
\hline \multirow[t]{3}{*}{ Cs } & Zeolitic & Cumulative & $\left(\mathrm{K}_{\mathrm{d}}\right.$ value, probability) $(425,0)(5,000,0.5)(20,000,1.0)$ \\
\hline & Devitrified & Uniform & Range $=1-15(7.5)$ \\
\hline & Vitric & Cumulative & (K $\mathrm{K}_{d}$ value, probability) $(0,0)(2,0.5)(100,1.0)$ \\
\hline \multirow[t]{3}{*}{$\mathrm{Sr}$} & Zeolitic & Uniform & Range $=50-2000(\mathbf{1 0 0 0})$ \\
\hline & Devitrified & Uniform & Range $=10-70(40)$ \\
\hline & Vitric & Uniform & Range $=0-50(25)$ \\
\hline \multirow[t]{3}{*}{$\mathrm{Ra}$} & Zeolitic & Uniform & Range $=1000-5,000(2,500)$ \\
\hline & Devitrified & Uniform & Range $=100-1,000(\mathbf{5 0 0})$ \\
\hline & Vitric & Uniform & Range $=50-600(300)$ \\
\hline \multirow[t]{3}{*}{ Th } & Zeolitic & Uniform & Range $=1,000-30,000(\mathbf{1 5}, \mathbf{0 0 0})$ \\
\hline & Devitrified & Uniform & Range $=1,000-10,000(5,000)$ \\
\hline & Vitric & Uniform & Range $=1,000-10,000(5,000)$ \\
\hline
\end{tabular}

Output-DTN: LA0408AM831341.001.

a The term "cumulative" indicates a piecewise linear distribution.

$\mathrm{b}$ The numbers in boldface were used in the simulations.

${ }^{c}$ The value used in the simulation was outside and conservative compared to the final selected range.

The diffusion coefficients and the decay properties are listed in Table 6-4. Of the listed $D_{0}$, only the first ones were used for the base three-dimensional simulations, while the rest were used for uncertainty bounding and sensitivity analysis (Sections 6.8 and 6.9). 
Table 6-4. Properties of Radionuclides in the Transport Simulations

\begin{tabular}{|c|c|c|c|c|}
\hline Radionuclide & $D_{0}\left(m^{2 l} s\right)^{*}$ & $T_{1 / 2}$ (years) $^{\ddagger \neq}$ & $\lambda=\frac{\ln 2}{T_{1 / 2}}\left(s^{-1}\right)$ & Decay Mode ${ }^{\ddagger \ddagger}$ \\
\hline${ }^{99} \mathrm{Tc}$ & $\begin{array}{l}4.55 \times 10^{-10 \dagger} \\
4.55 \times 10^{-11 \dagger \dagger} \\
10^{-9 \dagger \dagger}\end{array}$ & $2.13 \times 10^{5}$ & $1.03 \times 10^{-13}$ & $\beta-$ \\
\hline${ }^{237} \mathrm{~Np}$ & $\begin{array}{l}1.65 \times 10^{-10 \ddagger} \\
7.12 \times 10^{-10 \dagger \dagger} \\
1.65 \times 10^{-11 \dagger \dagger} \\
10^{-9 \dagger \dagger}\end{array}$ & $2.14 \times 10^{6}$ & $1.03 \times 10^{-14}$ & $A$ \\
\hline${ }^{239} \mathrm{Pu}$ & $\begin{array}{l}4.81 \times 10^{-10 \S} \\
4.81 \times 10^{-11+\dagger} \\
6.08 \times 10^{-10 \dagger \dagger} \\
10^{-9 t \dagger}\end{array}$ & $2.41 \times 10^{4}$ & $9.11 \times 10^{-13}$ & $\alpha$ \\
\hline${ }^{241} \mathrm{Am}$ & $3.69 \times 10^{-10 \|} \|$ & $4.322 \times 10^{2}$ & $5.08 \times 10^{-11}$ & $\beta-$ \\
\hline${ }^{233} \mathrm{U}$ & $4.94 \times 10^{-10 \S}$ & $1.59 \times 10^{5}$ & $1.384 \times 10^{-13}$ & A \\
\hline${ }^{235} \mathrm{U}$ & $4.89 \times 10^{-10 \S}$ & $7.08 \times 10^{8} \S \S$ & $3.10 \times 10^{-17}$ & A \\
\hline${ }^{231} \mathrm{~Pa}$ & $4.98 \times 10^{-10 \S}$ & $3.25 \times 10^{4}$ & $6.76 \times 10^{-13}$ & A \\
\hline${ }^{229} \mathrm{Th}$ & $5.02 \times 10^{-10} \S$ & $7.90 \times 10^{3}$ & $2.78 \times 10^{-12}$ & A \\
\hline${ }^{226} \mathrm{Ra}$ & $8.89 \times 10^{-10 ~ \# ~}$ & $1.599 \times 10^{3}$ & $1.37 \times 10^{-11}$ & A \\
\hline${ }^{135} \mathrm{Cs}$ & $2.06 \times 10^{-9 \#}$ & $2.30 \times 10^{6}$ & $9.55 \times 10^{-15}$ & $\beta-$ \\
\hline${ }^{90} \mathrm{Sr}$ & $7.91 \times 10^{-10 \#}$ & $2.90 \times 10^{1}$ & $7.57 \times 10^{-10}$ & $\beta-$ \\
\hline
\end{tabular}

NOTE: Mass corrections consist of multiplying the analog diffusion coefficient by the ratio of the mass of the analog to the mass of the radionuclide.

* Free-water diffusion coefficient (see equations 6-20 and 6-21)

$\dagger$ DTN: LA000000000034.002 [DIRS 148603]; free-water diffusion computed from reported diffusion coefficient in rock divided by tortuosity, where tortuosity is equal to the porosity (see Section 6.1.2.4). Note that the value of $4.55 \times 10^{-10} \mathrm{~m}^{2} / \mathrm{s}$, although slightly larger than the mean value of the data, lies well within one standard deviation from the mean and is, therefore, an acceptable value for the sensitivity calculations presented in this report. Given a porosity in the range 0.0457 to 0.3541 in and below the repository (DTN: LB0210THRMLPRP.001 [DIRS 160799]), the range of matrix diffusion coefficients is $2 \times 10^{-11} \mathrm{~m}^{2} / \mathrm{s}$ to $2 \times 10^{-10} \mathrm{~m}^{2} / \mathrm{s}$. This lies within the range for matrix diffusion coefficients developed in

DTN: LB0307FMRADTRN.001 [DIRS 165451].

‡ Using $\mathrm{Mn}^{+2}$ as analogue, corrected for mass, see Lide (1992 [DIRS 166224], pp. 5-111, 5-112).

$\S$ Using $\mathrm{UO}^{+2}$ as analogue, corrected for mass, see Lide (1992 [DIRS 166224], pp. 5-111, 5-112).

$\|$ Using $\mathrm{Sm}^{+3}$ and $\mathrm{Ce}^{+3}$ as analogues, corrected for mass, see Lide (1992 [DIRS 166224], pp. 5-111, 5-112). " Directly used, see Lide (1992 [DIRS 166224], 5-111, 5-112).

t† Values used for sensitivity analysis, see Section 6.8.3.2.

护 From Lide (1992 [DIRS 166224], pp. 11-28 to 11-133).

$\S$ Value adjusted from $7.04 \times 10^{8}$ years given in Lide (1992 [DIRS 166224], 11-28 to 11-133). 


\subsection{A PRELIMINARY CONCEPTUAL MODEL OF TRANSPORT BASED ON GEOLOGY AND STRATIGRAPHY}

\subsubsection{General Issues}

This section focuses on the development of a conceptual model of radionuclide transport in composite vertical cross sections at select locations of the UZ. The conceptual model is based on the geology, stratigraphy, and transport properties of the geohydrologic units in the profiles from the repository horizon to the water table at these locations. The focus is on the expected difference of transport patterns at different UZ locations (because of different geologies and conditions), and on the relative importance of particular geohydrologic units on overall transport. The topic of model calibration is discussed in Section 7.1.

\subsubsection{Hydrogeologic Profiles}

Transport is considered in three hydrogeologic profiles (columns) called Cross Sections 1, 2, and 3. The three cross sections are located near the USW SD-6, SD-12, and UZ-14 boreholes, respectively. The locations of the three boreholes in the context of the UZ site-scale model are shown in Figure 6-1. Thus, Cross Section 3 is located in the northern part of the repository site, while Cross Sections 1 and 2 are located in the southern part. The geologic profiles in Cross Sections 1, 2, and 3, including the layers of all the hydrogeologic units involved in radionuclide transport, their elevations, and thicknesses, are shown in Figures 6-2 through 6-4. Additional information on the three profiles can be found in Appendix C.

\subsubsection{Conceptual Model of Transport in Cross Sections 1 and 2}

Figure 6-5 illustrates a conceptual model of transport in Cross Sections 1 and 2. Water-borne radionuclides enter the TSw formation through the bottom of the repository. Radionuclide transport in the TSw hydrogeologic unit occurs mostly in the fractures.

At this location the vitric layers in the underlying $\mathrm{CHn}$ unit (i.e., layers ch1v through ch5v) behave as porous (rather than fractured) media because of the parity of permeability in the matrix and in the fractures (BSC 2001 [DIRS 161340], Section 6.7.2). Thus, transport occurs in both the matrix and the fractures, and the contact times between the radionuclides and the media is longer. The vitric layers are effective transport barriers because of the increased sorption (for sorbing radionuclides) and retardation (see Section 6.5, this model report). Once the radionuclide reaches the zeolitic ch6z unit, flow becomes again fracture-dominated, and, consequently, so does transport.

Transport in the pp4 layer, the top layer in the Prow Pass hydrogeologic unit, is controlled by its zeolitic nature, which leads to fractured-dominated flow and transport. The next layer (i.e., pp3) is devitrified and behaves similarly to the vitric layers in the $\mathrm{CHn}$ unit. The characteristics of the next two units (devitrified pp2 and zeolitic pp1) lead to an intermediate behavior between fracture- and matrix-dominated transport. Transport is again matrix-dominated in the upper welded Bullfrog unit (bf3) and fracture-dominated in the lower nonwelded Bullfrog unit (bf2). 

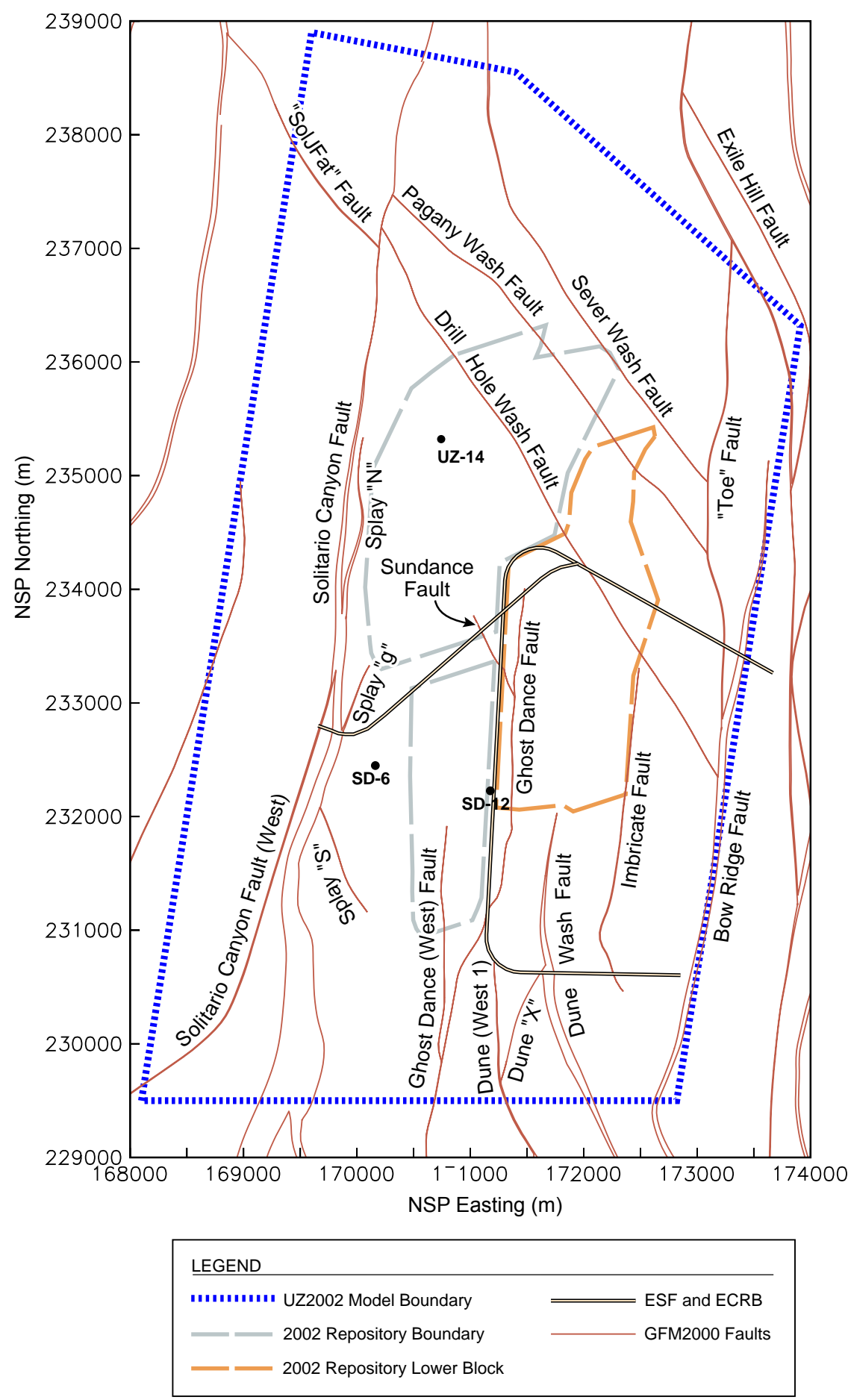

Source: Modified from BSC 2004 [DIRS 169855], Figure 6-1.

Figure 6-1. Two-Dimensional (Plan View) of the Yucca Mountain Site Identifying the Locations of Boreholes USW SD-6, SD-12, and UZ-14, Representing Cross Sections 1, 2, and 3 


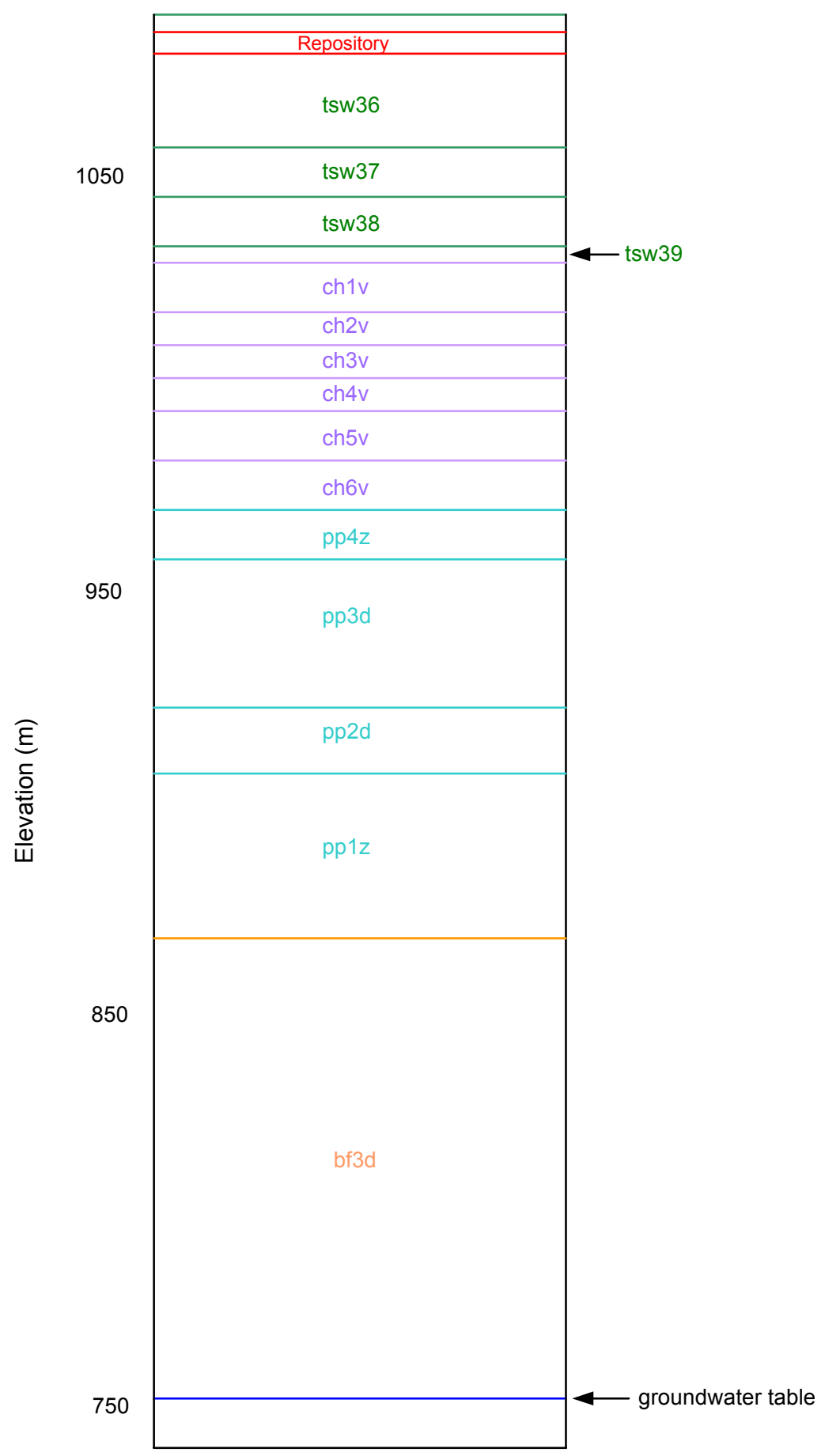

DTNs: MO9906GPS98410.000 [DIRS 109059]; LB03023DSSCP9I.001 [DIRS 163044].

Figure 6-2. Hydrogeologic Units (and their Layers) of the UZ Model Domain from the Repository to the Groundwater in Cross Section 1 near the USW SD-6 Borehole 


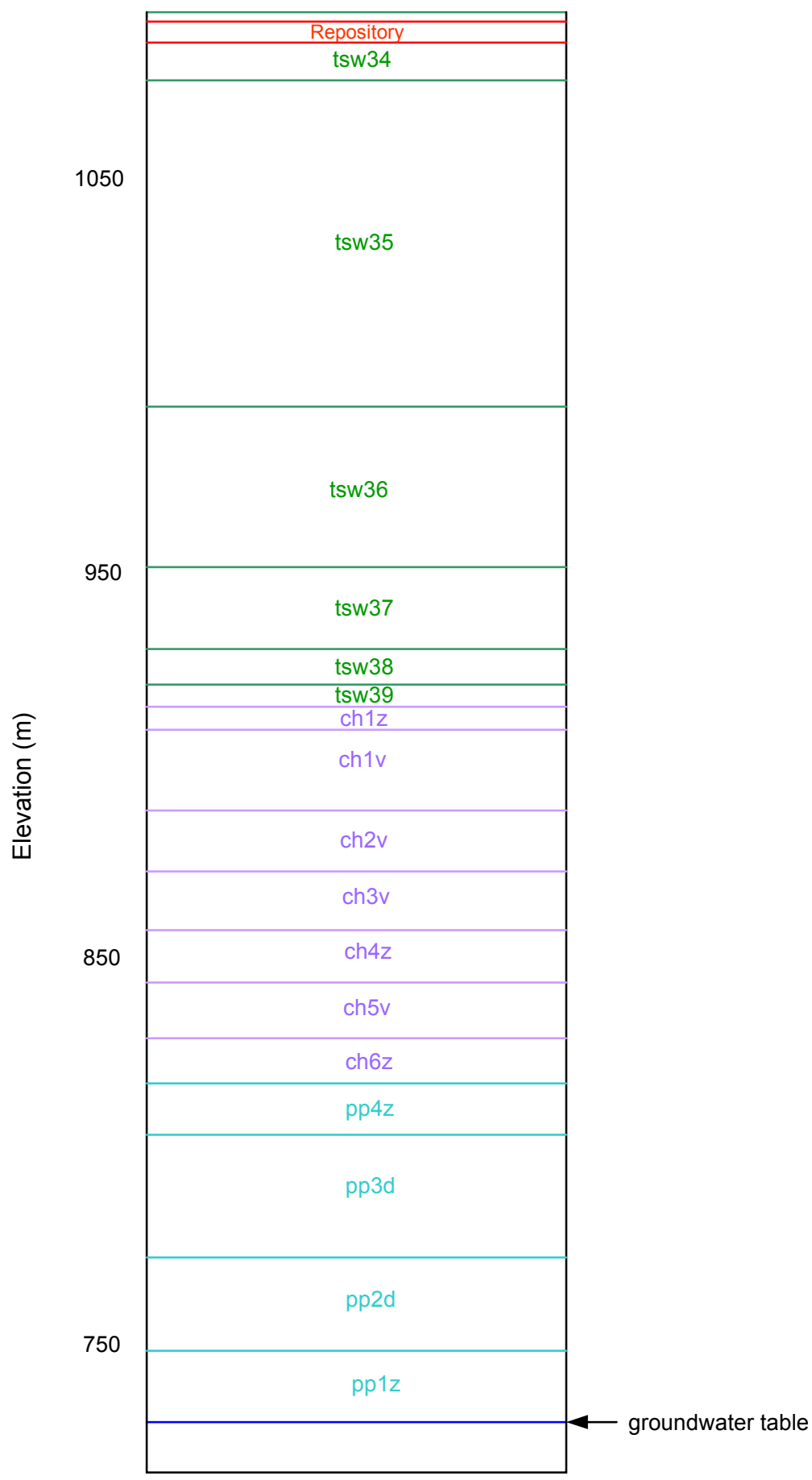

DTNs: MO9906GPS98410.000 [DIRS 109059]; LB03023DSSCP9I.001 [DIRS 163044].

Figure 6-3. Hydrogeologic Units (and their Layers) of the UZ Model Domain from the Repository to the Groundwater Table in Cross Section 2 near the USW SD-12 Borehole 


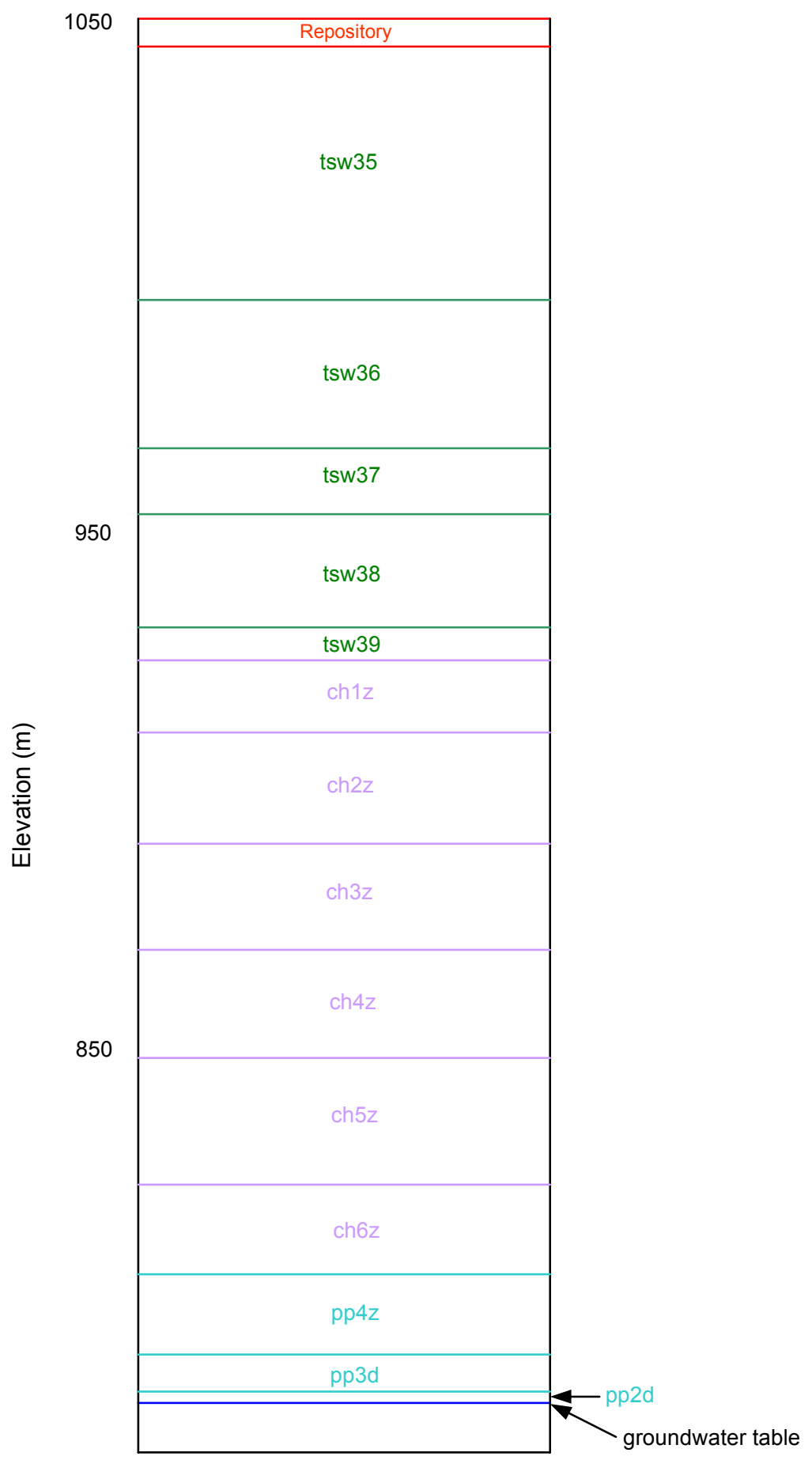

DTNs: MO9906GPS98410.000 [DIRS 109059]; LB03023DSSCP9I.001 [DIRS 163044].

Figure 6-4. Hydrogeologic Units (and their Layers) of the UZ Model Domain from the Repository to the Groundwater in Cross Section 3 near the USW UZ-14 Borehole 


\section{Repository}

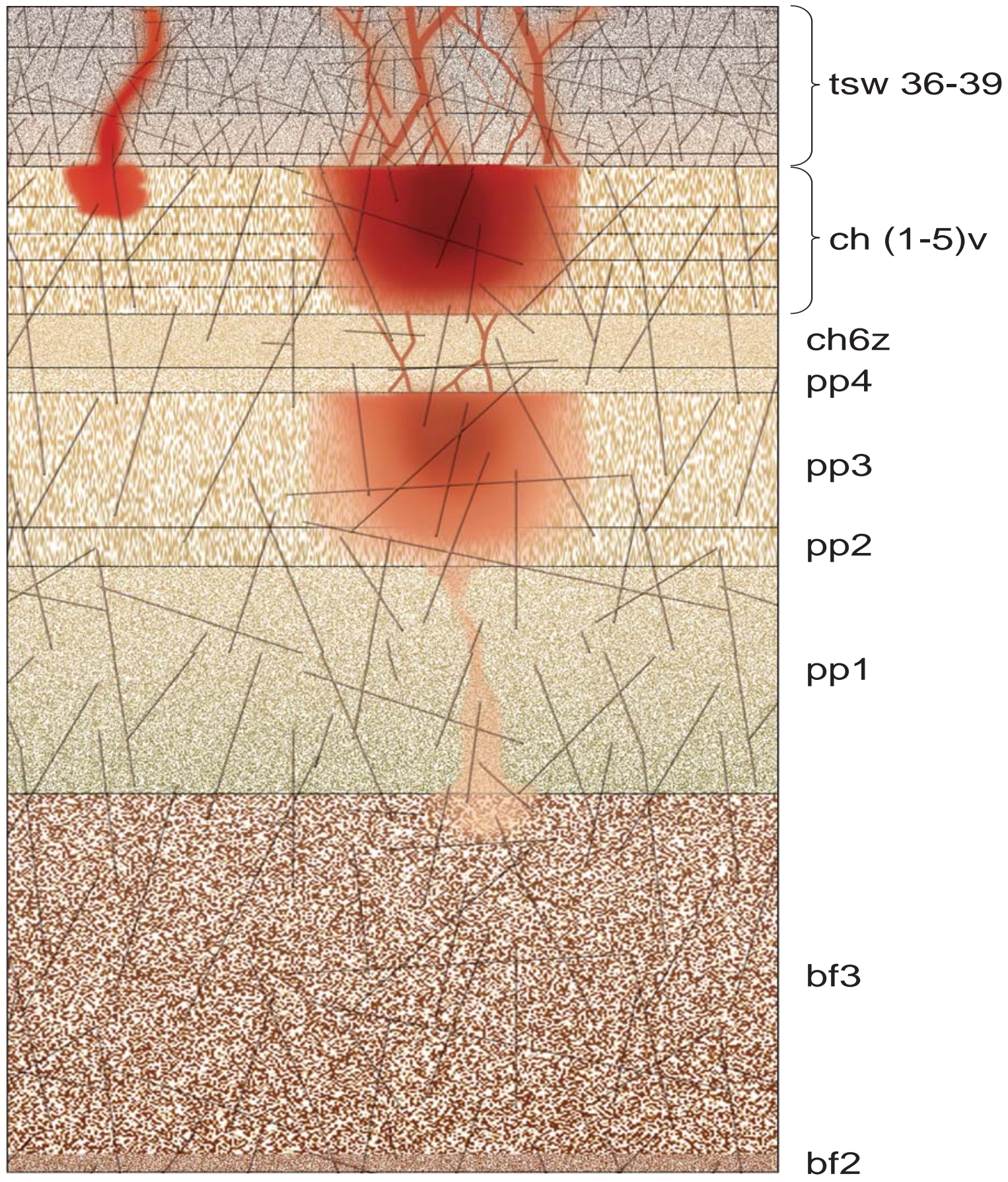

Figure 6-5. Schematic of Transport in Cross Section 1 


\subsubsection{Conceptual Model of Transport in Cross Section 3}

Figure 6-6 illustrates a conceptual model of transport in Cross Section 3. Water-borne radionuclides enter the TSw formation through the bottom of the repository. Radionuclide transport in the TSw hydrogeologic unit occurs mostly in the fractures. In contrast to Cross Section 1, Cross Section 3 is characterized by the absence of vitric layers in the underlying CHn. The zeolitic layers here have fracture spacing and fracture permeability similar to those in Cross Section 1, but the matrix permeability is about four orders of magnitude lower than that in the fractures (BSC 2004 [DIRS 169861]). The disparity in permeability directs practically all flow into the fractures, leading to fast transport.

The pp4 layer, the top layer in the Prow Pass hydrogeologic unit, behaves like a zeolitic layer, leading to fractured-dominated flow and transport. The next layer (i.e., pp3) is devitrified and behaves like porous media. The characteristics of the next two units (devitrified pp2 and zeolitic $\mathrm{pp} 1)$ lead to an intermediate behavior between fracture- and matrix-dominated transport.

\subsubsection{Synopsis and Discussion}

A review of the conceptual model in Section 6.6 indicates that while the geological profiles in Cross Sections 1 and 2 appear likely to afford good retardation of radionuclide transport (and especially of strongly sorbing radionuclides), this is unlikely to be the case in the domain of Cross Section 3, because of the preponderance of highly permeable (compared to the matrix) fractures of the zeolitic layers at this location. Based on general transport principles, a decreasing $K_{d}$ is expected to lead to faster transport to the water table, although strongly sorbing radionuclides (such as ${ }^{239} \mathrm{Pu},{ }^{231} \mathrm{~Pa},{ }^{229} \mathrm{Th}$ ) are unlikely to be significantly affected.

Increases in the infiltration rate, brought about by possible changes in climatic patterns, can have a substantial impact on breakthrough times, especially at the Cross Section 3 location. The effect of changes in infiltration is likely to become more pronounced with weaker-sorbing radionuclides.

The conceptual models supported by these results are consistent with the observations that were based on a simplified two-dimensional analysis included in the previous revision of this model report (BSC 2001 [DIRS 161340], Section 6). 


\section{Repository}

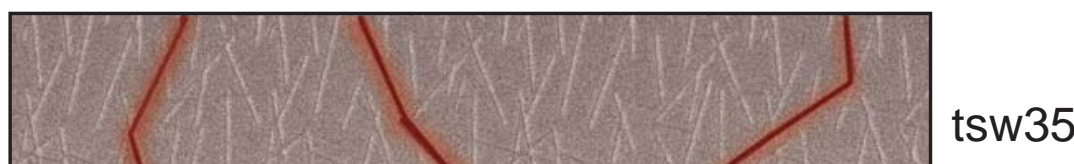

tsw36

tsw37

tsw38

tsw39

ch1z

$\operatorname{ch} 2 z$

$\operatorname{ch} 3 z$

$\operatorname{ch} 4 z$

$\operatorname{ch} 5 z$

$\operatorname{ch} 6 z$

pp4

pp3

pp2

pp1

Figure 6-6. Schematic of Transport in Cross Section 3 


\subsection{THREE-DIMENSIONAL TRANSPORT SIMULATIONS}

This section discusses the general issues involved in the three-dimensional radionuclide transport through the UZ of Yucca Mountain, that is, climatic conditions, hydrologic conceptual model, grids, flow systems, the radioactive species considered and the mode of their release, data inputs and outputs, in addition to the general approach and basic stipulations. All the three-dimensional numerical studies of radionuclide transport use a grid identical to that employed in the flow analysis documented in the model report UZ Flow Models and Submodels (BSC 2004 [DIRS 169861]). The development and features of the three-dimensional grids for this numerical modeling effort are documented in the Analysis Report Development of Numerical Grids for UZ Flow and Transport Modeling (BSC 2004 [DIRS 169855]) and further discussed by BSC (2004 [DIRS 169861], Section 6).

\subsubsection{Climatic Conditions}

The three climatic scenarios investigated here are identical to those discussed in UZ Flow Models and Submodels (BSC 2004 [DIRS 169861], Section 6): present-day, monsoon and glacial-transition (BSC 2004 [DIRS 170007], Section 6.9). For each climatic scenario, a high, mean, and low infiltration case was studied. The annual infiltration rates corresponding to each of the nine climatic cases are listed in Table 6-5.

\subsubsection{Conceptual Hydrologic Model, Grids, and Flow Simulations}

The grid, properties, and calibrated hydraulic parameters used in the transport simulations (DTN: LB03023DSSCP9I.001 [DIRS 163044]) were identical to those used for the analysis of flow in the report by BSC (2004 [DIRS 169861], Section 6), in the course in which they were derived. These correspond to the \#1 conceptual model of perched water (BSC 2004 [DIRS 169861], Section 6.2). This is the permeability barrier model, which uses the calibrated perched-water parameters for fractures and matrix in the northern part of the model domain, and modified property layers (including the tsw38, tsw39, $\mathrm{CH} 1 \mathrm{z}$ and $\mathrm{CH} 2 \mathrm{z}$ layers) where the lower basal vitrophyre of the TSw is above the zeolites of the CHn. A detailed discussion of this perched-water model can be found in the BSC report (2004 [DIRS 169861], Section 6.2).

A two-dimensional (plan view) of the grid at the repository level is shown in Figure 6-7. All three-dimensional transport simulations that involved the use of the T2R3D numerical model were conducted using this grid and the steady-state flow fields from the report by BSC (2004 [DIRS 169861], Section 6) for the 9 climatic scenarios (DTN: LB03023DSSCP9I.001 [DIRS 163044]). Table 6-5 provides averaged percolation values of all 9 climatic scenarios in order to show how there is more percolation flux under some climates than others.

For the three-dimensional EOS9nT simulations, the same grid was used, but its numbering was altered. This modification involved a very minor rearrangement of the element order number using the XtractG.f90 routine, which moved (a) the elements above the repository, (b) those corresponding to the location of the repository, and (c) the bottom boundary elements (corresponding to the water table) to the bottom of the element file. Moving these elements to the bottom of the element file made use of the ability of the TOUGH2 family of codes to treat them as inactive elements (i.e., elements that have time-invariant properties and conditions contribute to 
flow and transport as boundaries, and are not accounted for in mass and energy balances). To accomplish that, the only modification was making the volume of the first repository element zero or negative. In the TOUGH 2 convention, this rendered all the following elements inactive, thus, maintaining their properties and conditions constant throughout the simulations.

Making these elements inactive was possible because (a) the steady state of the flow fields is unaffected by making these elements inactive; (b) in the EOS9nT simulations the release rate was considered constant over time, a condition which necessitated treating the repository elements as internal boundary elements; and (c) the elements above the repository do not contribute to transport and can also be treated as boundary elements. This modification reduced the number of active elements by about 50 percent in the EOS9nT calculations, made the simulation possible within the array size of the code, and significantly reduced the execution times, with absolutely no effect on or compromise of the transport computations.

Note that all the properties and the attributes of the original grid were maintained, and the only change was the numbering sequence of the elements. Because the element names remained unaltered, the initial pressure and saturation conditions from the flow computations (BSC 2004 [DIRS 169861], Section 6) were applied unchanged, but the flow field could not be used as was provided by the simulations in the report by BSC (2004 [DIRS 169861], Section 6) because those did not reflect the changed element numbers.

Thus, an updated steady state flow field (involving the same information as the old one, but in a different sequence) was obtained from a single TOUGH 2 V1.4 (Module EOS9 V1.4) (LBNL 2000 [DIRS 146496]) simulation that used the modified grid and the initial pressure and saturation conditions from the flow computations (BSC 2004 [DIRS 169861], Section 6). Only a single run was needed because all the EOS9nT simulations were made assuming a mean present-day infiltration scenario (see Table 6-5). After the EOS9 simulation, the output file SAVE was compared to those from the simulations using the unmodified grids to confirm that they were identical. Note that EOS9nT (LBNL 1999 [DIRS 113943]) shares identical flow solution routines with EOS9, and confirmatory tests confirmed the identity of the flow solutions predicted by both EOS9 and EOS9nT. It was decided, however, to use the EOS9 module in the TOUGH2 flow simulations to ensure complete compatibility with the results in the report by BSC (2004 [DIRS 169861], Section 6).

The velocity distribution (stored in the input file VELOC for the EOS9nT simulations) was then extracted from the EOS9 output by using the XtractG.f90 routine. The DTN of the modified grid, the XtractG.f90 input and output, and the EOS9 input (modified) and output files are output DTN: LB0307MR0060R1.003.

Table 6-5. Percolation Fluxes (mm/year) for Different Climatic Regimes

\begin{tabular}{|l|c|c|c|}
\hline Value & Present-Day Infiltration & Monsoon Infiltration & $\begin{array}{c}\text { Glacial-Transition } \\
\text { Infiltration }\end{array}$ \\
\hline Lower & 1.25 & 4.43 & 2.35 \\
\hline Mean & 4.43 & 11.83 & 17.02 \\
\hline Upper & 10.74 & 19.23 & 31.69 \\
\hline
\end{tabular}

NOTE: Values averaged from DTN: GS000308311221.005 [DIRS 147613]. 


\subsubsection{Modes of Radionuclide Release}

In all the ensuing three-dimensional studies of site-scale radionuclide transport, the radioactive substances were released in the liquid phase in the fractures of the elements corresponding to the repository. The initial radionuclide concentration was spatially uniform in the area of release, which covered the entire footprint of the repository. Two modes of radionuclide release - instantaneous release and continuous release - are discussed here.

\subsubsection{Instantaneous Release}

An instantaneous release scenario corresponds to a single catastrophic event during which a finite mass of radionuclides is suddenly released in the liquid phase of the fractures of the repository. Study of the system performance following an instantaneous release provides the measure of the ability of the UZ to store and retard the migration of a limited mass of radioactive substances.

\subsubsection{Continuous Release}

Under a continuous release scenario, radionuclides are continuously released into the fractures of the elements corresponding to the repository. This is a realistic scenario, given the mass of the radioactive substances to be stored at the site, the expected deterioration of the canisters, and the planned closure of the repository after the storage of the radioactive materials (thus preventing access and repairs that might control continuous release).

The concentration of the released radionuclides changes over time because the released radionuclides undergo radioactive decay at the input points (boundaries). Such an approach involves the assumption that all the radionuclides were produced as a batch at the same time, and is valid for all but the shortest-lived isotopes. This is far more consistent with reality than an assumption of a constant-boundary concentration, which implies continuous radionuclide production at the release point.

Study of an instantaneous release scenario provides a criterion of absolute system performance and evaluates the time for a given radionuclide mass to migrate past the UZ boundaries. Such a measure of performance is inadequate and inapplicable under a continuous-release regime, the study of which necessitates a criterion of a relative system performance based on the relationship of inflow and outflows across the system boundaries. This provides a measure of the ability or capacity of the domain to accumulate radionuclide mass that enters its boundary at a given rate, and is just as important (if not more so) than the conventional breakthrough study that can only describe response to a finite mass release. Thus, under instantaneous releases, masses are compared, while in continuous release, the relationship of fluxes is established. For a complete evaluation of the repository, performance under both release scenarios must be evaluated. 

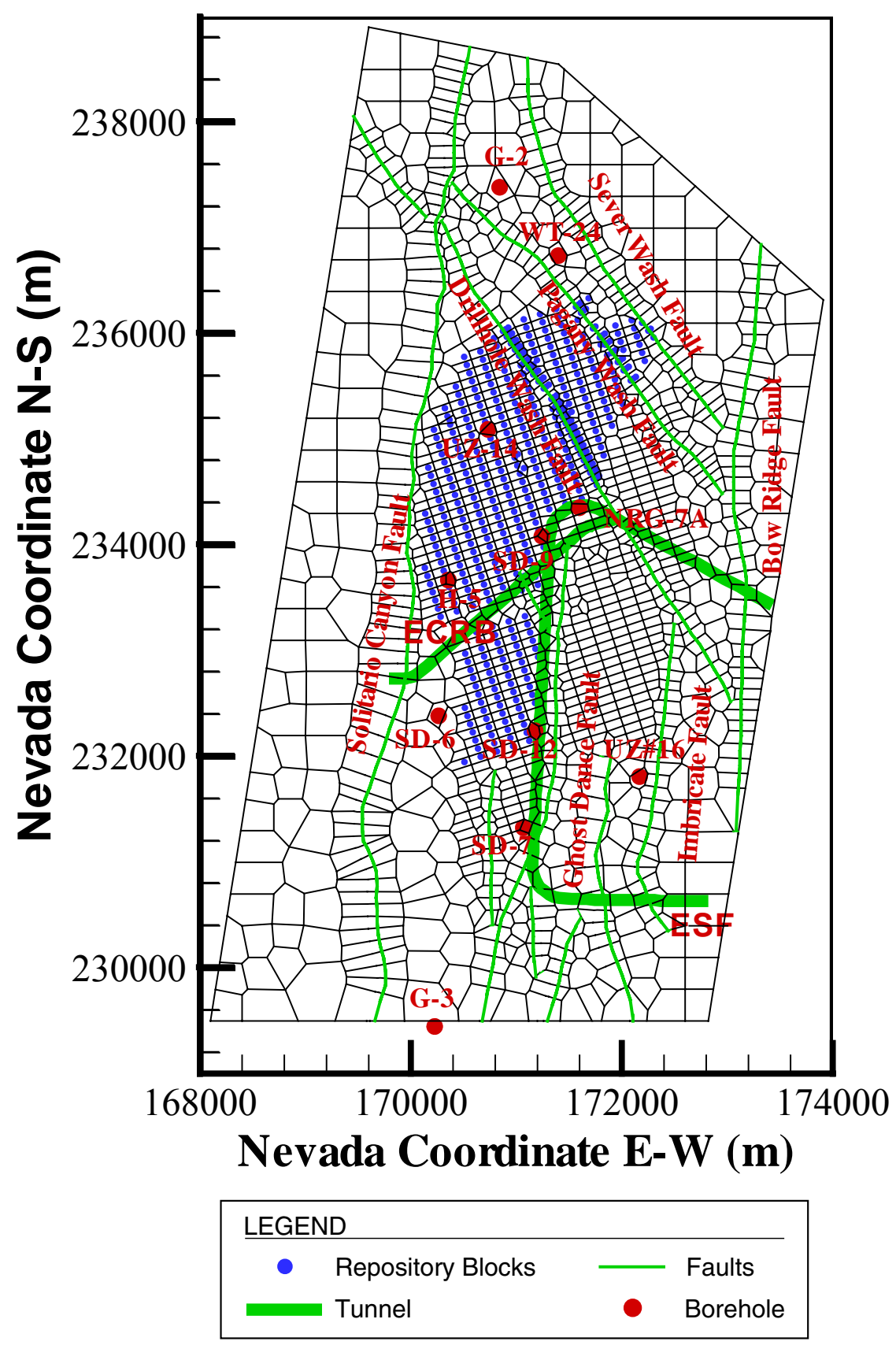

Source from BSC 2004 [DIRS 169861], Figure 6.1-1.

Figure 6-7. Two-Dimensional (Plan View) of the UZ Model Grid Design at the Repository Level 


\subsubsection{Radionuclides Considered}

The following radioactive solutes were considered:

(a) ${ }^{99} \mathrm{Tc}$ (nonsorbing),

(b) ${ }^{237} \mathrm{~Np},{ }^{235} \mathrm{U},{ }^{233} \mathrm{U}$ (moderately sorbing),

(c) ${ }^{241} \mathrm{Am},{ }^{239} \mathrm{Pu},{ }^{231} \mathrm{~Pa},{ }^{229} \mathrm{Th},{ }^{226} \mathrm{Ra},{ }^{90} \mathrm{Sr}$ (strongly sorbing).

Their properties are listed in Tables 6-3 and 6-4. The radionuclides are released at the gridblocks corresponding to the location of the repository. Additionally, for the three-dimensional EOS9nT simulations of continuous release, all the important members in the decay chains of ${ }^{241} \mathrm{Am}$ and ${ }^{239} \mathrm{Pu}$ were considered, according to the decay equations (Pigford et al. 1980 [DIRS 123113])

$$
\begin{gathered}
{ }^{241} \mathrm{Am} \rightarrow{ }^{237} \mathrm{~Np} \rightarrow{ }^{233} \mathrm{U} \rightarrow{ }^{229} \mathrm{Th} \\
{ }^{239} \mathrm{Pu} \rightarrow{ }^{235} \mathrm{U} \rightarrow{ }^{231} \mathrm{~Pa}
\end{gathered}
$$

Note that only the most important members of the radioactive chain are included in these decay equations, which omit daughters with short half-lives. Given that alpha decay is the decay mode of all the members in the ${ }^{237} \mathrm{~Np}$ and ${ }^{239} \mathrm{Pu}$ chains, $\zeta=0$ (Equation 6-27) in all the simulations reported in this report because the daughters are ejected from grain surfaces due to recoil (Faure 1977 [DIRS 122805], pp. 288 to 289).

The transport of radioactive colloids was also investigated. Spherical ${ }^{239} \mathrm{PuO}_{2}$ colloids with diameters of $6 \mathrm{~nm}, 100 \mathrm{~nm}, 200 \mathrm{~nm}$, and $450 \mathrm{~nm}$ were used in this study.

\subsubsection{Important Geologic Features}

As will be clearly shown in the ensuing Sections 6.7 through 6.17 , the role of faults in the three-dimensional site-scale transport model is very important because faults provide fast pathways for the radionuclide migration to the water table. In the EOS9, T2R3D, and EOS9nT simulations, the faults are represented as thin vertical domains characterized by large permeabilities. More detailed description of the faults, their properties, and their role in water flow can be found in Analysis of Hydrologic Properties Data (BSC 2004 [DIRS 170038], Section 6.4), Geologic Framework Model (GFM2000) (BSC 2004 [DIRS 170029], Sections 6.2, 6.4, and 6.5), and UZ Flow Models and Submodels (BSC 2004 [DIRS 169861], Section 6).

\subsubsection{Transport Simulation Options}

The three-dimensional simulations involving the instantaneous release of radionuclides were conducted using the T2R3D code with the steady-state flow field option. T2R3D employs conventional time-stepping.

All the three-dimensional simulations describing continuous release of solutes and colloids were conducted by using the EOS9nT code with the De Hoog et al. (1982 [DIRS 117308]) implementation of the Laplace transform formulation. This formulation was selected because of its speed and accuracy, its ability to provide information over the whole spectrum of the simulation period, and its ability to drastically reduce numerical diffusion. The simulations were 
very fast and efficient, requiring 1,800-2,200 seconds of execution time to cover a simulation period of 1,000,000 years.

EOS9nT allows the simultaneous solution of the concentration profiles of any number of radionuclides. Thus, all three parents $\left({ }^{99} \mathrm{Tc},{ }^{237} \mathrm{~Np},{ }^{239} \mathrm{Pu}\right)$ were considered simultaneously in the runs. Similarly, exploiting the fact that EOS9nT allows the tracking of any daughter products, the solutions for each of the parent radionuclides and its daughters in the decay Equations 6-29 and 6-30 were obtained simultaneously.

\subsubsection{Basic Data Inputs and Outputs}

All the three-dimensional T2R3D simulations for all radionuclides used the same grid, calibrated hydraulic parameters, and steady-state flow field. The initial condition files were different, as they reflected the nine different climatic conditions. All the corresponding data files were provided (and used without modification) from the flow analysis studies of BSC (2004 [DIRS 169861]). Input files were developed for the T2R3D simulations for the radionuclides studied in this model report.

The three-dimensional EOS9nT radionuclide simulations and the T2R3D simulations shared the same calibrated hydraulic parameters and initial flow conditions. The EOS9nT simulations used a slightly modified grid from that in T2R3D (Section 6.7.2) (composed of the same elements as the T2R3D grid, but with different ordering), and the corresponding flowfield for mean present-day infiltration. Using the latter, input files conforming to the EOS9nT requirements (including both flow and transport information for the various radionuclides under study) were created.

The DTNs of the input and output files used and created in the course of this study of radionuclide transport through the UZ are listed in Table 6-6.

\subsubsection{Review of the Approach and Basic Approximations in the Three-Dimensional Site-Scale Radionuclide Transport Studies}

For a better understanding and a more realistic interpretation of the results of the three-dimensional simulations, it is important to understand the basic approach and approximations employed in the study of transport in the UZ. Analysis of the simulation results without considering the extremely conservative approach and the succession of worst-case scenarios involved in the three-dimensional studies can lead to erroneous conclusions and a distorted view of the transport regimes through the UZ. To that effect, the note of caution discussed in Section 6.20.3 should be carefully reviewed.

The numerical simulations are based on the following approximations and approach (not all of which will be incorporated in the TSPA-LA model):

(a) No drip shields are considered, and flow through the canisters is assumed. Thus, water from the drift roof is allowed to fall onto, and then flow uninhibited through the waste package, carrying radionuclides as it emerges from the ruptured container. This is a worst-case scenario, and rather unrealistic. 
(b) All the radioactive packages in the entire repository rupture simultaneously, with all of them releasing their contents simultaneously at the beginning of the simulation time. This is an improbable (if not outright impossible) release scenario.

(c) The radionuclides are released directly into the fractures. The retardation effects of the invert (with porous media properties) underneath the waste packages are ignored. The presence of the invert can significantly delay the onset of radionuclide release into the underlying fractures.

(d) The effects of the shadow zone are ignored. However, the presence of the shadow zone can lead to significant retardation of radionuclide transport (BSC 2004 [DIRS 170040]). The presence of the shadow zone can lead to significant retardation of radioactive solute transport because of the generally lower saturations. These lead to drastically lower water velocities in the shadow zone (into which water moves mainly in response to capillary pressure differentials), and to significantly lower diffusive fluxes across the matrix-fracture interfaces. The effect of the shadow zone on the transport of colloids is expected to be far more severe because advective fluxes are extremely low, and diffusive fluxes (the only mechanism capable of transporting radionuclides past the boundaries of the shadow zone) are dramatically depressed (as the colloid diffusion coefficient is generally orders of magnitude lower than the molecular diffusion coefficient of solutes).

(e) The vertical fractures are modeled as continuous throughout the UZ from the repository to the water table. Additionally, all the vertical fractures are open (i.e., they are not even partially filled with a porous medium), and do not retard transport through solute sorption and/or colloid attachment onto the fracture walls. The implication of this approach/assumption of uninterrupted vertical fractures is fast and unimpeded advective transport of radionuclides from the repository to the water table. This worst-case scenario is highly unlikely.

(f) The horizontal fractures are modeled as interconnected, and are also connected (directly or indirectly) to the vertical fractures. This scenario leads to accelerated migration of radionuclides to the water table.

(g) The radioactive tracers (solutes or colloids) are stable, unaffected by the near field conditions (thermal, geochemical, physical), and are not subject to chemical immobilization (e.g., through irreversible sorption or precipitation) anywhere in the UZ.

(h) The distribution coefficients were estimated over longer concentration intervals using an approach that results in milder slopes and lower $\mathrm{K}_{\mathrm{d}}$ values. This results in lower overall sorption, and leads to more conservative estimates of transport that correspond to faster radionuclide arrivals at the water table. 
(i) For simulation of the instantaneous radionuclide release scenario, the relative concentration $\left(\mathrm{C}_{\mathrm{R}}=\mathrm{C} / \mathrm{C}_{0}\right)$ in the water saturation of all the elements corresponding to the fractures of the repository subdomain is set at an initial value of $C_{R}=1$, that is the initial release concentration is uniformly distributed in the repository fractures. Note that the water saturation in the fractures is that corresponding to the steady state flow field for the given infiltration regime.

(j) In this modeling report, the water table is always considered to be at its present-day elevation.

(k) Representative transport properties are used as discussed in Section 6.5.

Given these simplifications (each one of which can accelerate release by thousands of years), the simulation results reflect a rather unrealistically conservative radionuclide release scenario, and are relevant (though by no means representative) after the radioactive species escape the immediate vicinity of the repository in a stable form. The onset of such release can occur a long time (thousands to hundreds of thousands) of years after the initial placement of the radioactive wastes in the repository. Thus, the radionuclide arrival times discussed in the following sections are meaningful only after (a) the drip shield and the canisters fail completely, (b) the radionuclides escape the effects of near-field hydrology, chemistry, and hydrology, and (c) enter a fully interconnected fracture system.

Note that the transport model discussed here is intertwined with the UZ flow model discussed in the report by BSC (2004 [DIRS 169861]). As such, it shares the same conceptual strengths and weaknesses. Changes in the conceptual model of flow can and will have a significant effect on transport. In particular, if an alternative flow model (in which faults act as barriers rather than conduits to flow) is validated, dramatic changes in the transport performance of the UZ are not just possible but likely.

Table 6-6. DTNs of Input and Output Files of the Three-Dimensional Site-Scale Transport Simulations

\begin{tabular}{|l|l|}
\hline \multicolumn{1}{|c|}{ Item } & \multicolumn{1}{c|}{ DTN } \\
\hline 3-D grid for T2R3D runs & LB03023DSSCP9I.001 [DIRS 163044] \\
\hline Steady state 3-D flow fields for 9 climatic scenarios (Table 6-5) & LB03023DSSCP9I.001 [DIRS 163044] \\
\hline Calibrated rock properties, hydraulic parameters, flow parameters & $\begin{array}{l}\text { LB03023DSSCP9I.001 } \\
\text { [DIRS 163044], } \\
\text { LB0210THRMLPRP.001 } \\
\text { [DIRS 160799] }\end{array}$ \\
\hline $\begin{array}{l}\text { Input/Output files for the UZ radionuclide transport studies under } \\
\text { instantaneous release (11 radionuclides, 9 climatic scenarios, and } \\
\text { sensitivity analyses with direct radionuclide release into the fault } \\
\text { fracture) }\end{array}$ & Output DTN: LB0307MR0060R1.001 \\
\hline $\begin{array}{l}\text { Data summaries and plot files corresponding to output } \\
\text { DTN: LB0307MR0060R1.001 }\end{array}$ & Output DTN: LB0307MR0060R1.002 \\
\hline $\begin{array}{l}\text { Input/Output files for the UZ radionuclide transport studies under } \\
\text { mean present-day infiltration-continuous release (3 radioactive } \\
\text { parent species, a 3- and a 4- member radioactive chain, 4 } \\
\text { radioactive colloids, and sensitivity analyses with direct } \\
\text { radionuclide release into the fault fracture) }\end{array}$ & Output DTN: LB0307MR0060R1.003 \\
\hline
\end{tabular}


Table 6-6. DTNs of Input and Output Files of the Three-Dimensional Site-Scale Transport Simulations (Continued)

\begin{tabular}{|l|l|}
\hline \multicolumn{1}{|c|}{ Item } & \multicolumn{1}{|c|}{ DTN } \\
\hline $\begin{array}{l}\text { Data summaries and plot files corresponding to output } \\
\text { DTN: LB0307MR0060R1.003 }\end{array}$ & LB0307MR0060R1.004 \\
\hline $\begin{array}{l}\text { Input/Output files for UZ radionuclide transport studies without } \\
\text { direct releases in the faults-continuous and instantaneous release } \\
\text { (4 radionuclides, mean present-day infiltration) }\end{array}$ & LB0307MR0060R1.005 \\
\hline $\begin{array}{l}\text { Data summaries and plot files corresponding to } \\
\text { DTN: LB0307MR0060R1.005 }\end{array}$ & LB0307MR0060R1.006 \\
\hline $\begin{array}{l}\text { Data summaries and corresponding breakthrough curves for the } \\
\text { radionuclide transport scenarios in output DTNs: } \\
\text { LB0307MR0060R1.001; LB0307MR0060R1.003; and } \\
\text { LB0307MR0060R1.005 }\end{array}$ & LB0307MR0060R1.007 \\
\hline $\begin{array}{l}\text { Supplemental Radionuclide Transport Simulations: Input/Output } \\
\text { files (Influence of three main geologic units (TSw, CHv, and CHz), } \\
\text { irreversibly sorbed radionuclides onto colloids, transport of Tc, Np, } \\
\text { and Pu with no diffusion (instantaneous, continuous releases)) }\end{array}$ & LB0310MR0060R1.010 \\
\hline $\begin{array}{l}\text { Data summaries and plot files corresponding to } \\
\text { DTN: LB0310MR0060R1.010 }\end{array}$ & LB0310MR0060R1.011 \\
\hline
\end{tabular}

3-D=three-dimensional; $\mathrm{CHv}=$ Calico Hills vitric unit; $\mathrm{CHz}=$ Calico Hills zeolitic unit; $\mathrm{DTN}=$ data tracking number; TSw=Topopah Spring welded unit

\subsection{THREE-DIMENSIONAL SIMULATIONS OF ${ }^{99}$ Tc $\quad$ TRANSPORT INSTANTANEOUS RELEASE}

This section discusses the transport of ${ }^{99} \mathrm{Tc}$ by means of three-dimensional site-scale simulations representing the entire UZ system of Yucca Mountain. The study analyzes the migration of this finite radionuclide mass through the $\mathrm{UZ}$, and investigates the processes and phenomena that affect its arrival at the water table.

Section 6.8 includes three subsections. Section 6.8.1 discusses the transport of the nonsorbing ${ }^{99} \mathrm{Tc}$ for three levels of present-day infiltration. The results of this study are shown in Figures 6-8 to 6-32. Section 6.8.2 studies the transport of ${ }^{99} \mathrm{Tc}$ for three levels of (a) monsoon infiltration (Figure 6-33) and (b) glacial-transition infiltration (Figure 6-34). Finally, Section 6.8.3 discusses uncertainties involved in the predictions and addresses sensitivity analyses to develop the envelope of possible solutions (Figure 6-35).

\subsection{1 $\quad{ }^{99}$ Tc Transport under Present-Day Infiltration}

The DTNs of the input and output files for the three present-day infiltration scenarios are listed in Table 6-6. The input parameters used in the T2R3D simulations of three-dimensional transport of the nonsorbing ${ }^{99} \mathrm{Tc}$ are listed in Table 6-7. 


\subsubsection{Breakthrough Curves}

The release of a finite amount of radionuclide mass allows the use of the conventional concept of breakthrough. This is quantified by the evolution over time of the normalized cumulative breakthrough of the ${ }^{99} \mathrm{Tc}$ mass fraction arriving at the Yucca Mountain water table $R_{M}$, defined as

$$
R_{M}=\frac{M_{l}}{M_{0}},
$$

where $M_{0}$ is the instantaneously released initial mass of the radionuclide (at $t=0$ ) and $M_{l}$ is the cumulative radionuclide mass exiting the bottom of the domain of interest (i.e., out of the UZ) up to the time of observation. Note that $R_{\mathrm{M}}$ can tangentially approach a value less than 1.0 if the radionuclide undergoes radioactive decay. In the instantaneous release studies, only the mass of the parent radionuclide is considered, that is, the daughter products are not tracked. The $R_{M}$ of ${ }^{99} \mathrm{Tc}$ at the repository in Figure 6-8 shows a very strong dependence on the infiltration regime.

As the infiltration rate increases from low to mean, the $t_{10}$ time, defined as the time at which $R_{M}=0.1$, decreases from about 13,900 years to about 83 years. Thus, $t_{10}$ is an indicator of the effects of the most conductive geologic features (i.e., certain fractures) on transport. The $t_{50}$, defined as the time at which $R_{M}=0.5$, is not reached within 1,000,000 years for the low infiltration regime, but is at 6,640 years for mean infiltration regime. Further increase of infiltration to high present-day levels leads to a decrease of $t_{10}$ to about 6 years, and of $t_{50}$ to 230 years. The $t_{10}$ and $t_{50}$ of all the radionuclides discussed in this model report are summarized in tabular form in Tables 6-10,6-11, and 6-12. It should be kept in mind that the term $R_{M}$ is relative, and these findings are only important if the magnitude of the released mass at the repository becomes significant. Figure 6-8 also shows that the maximum attainable $R_{M}$ increases with the infiltration rate. This is expected because lower infiltration results in lower velocities and longer transport times through the UZ, and more radioactive decay occurs. 


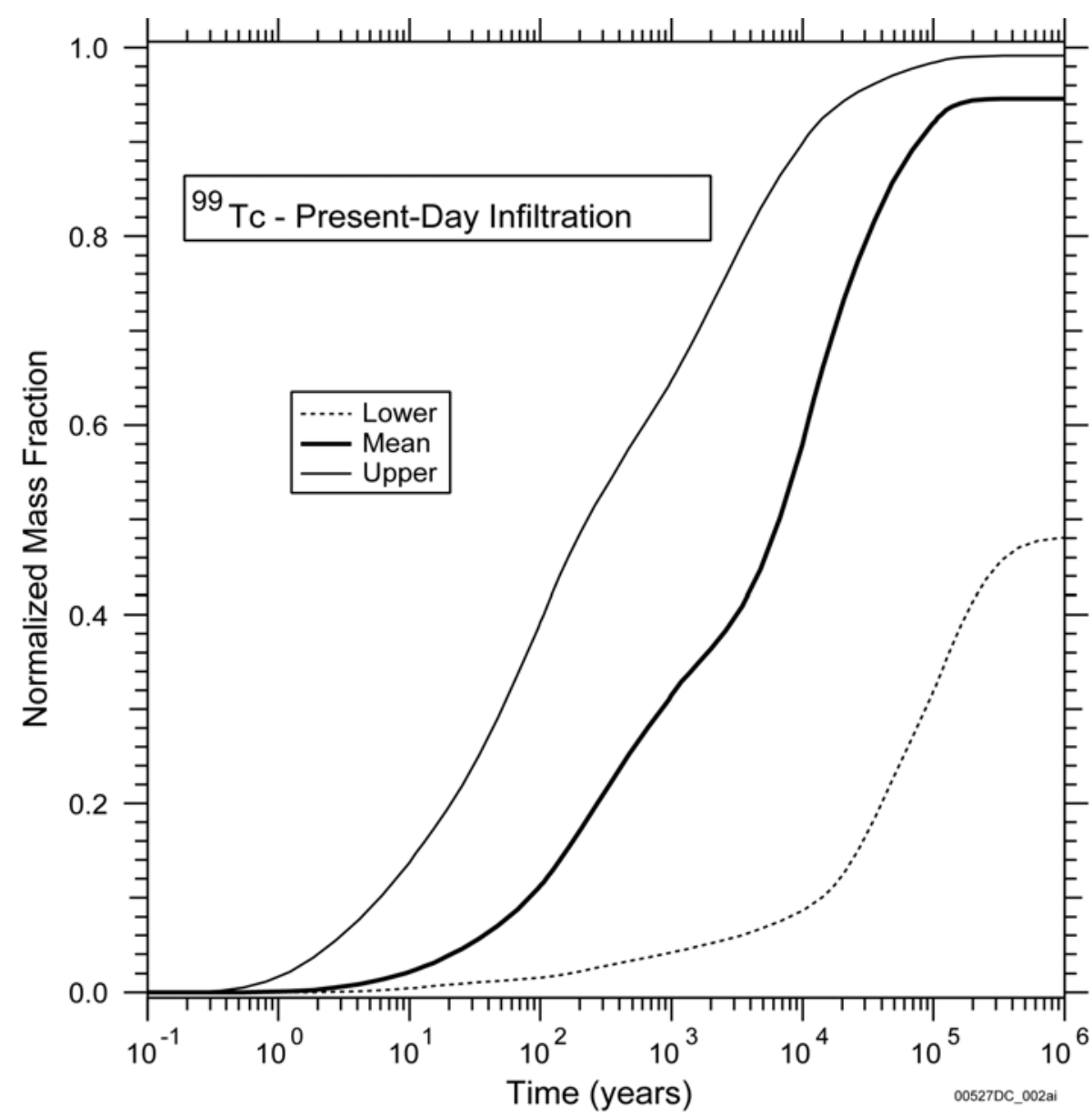

Output DTN: LB0307MR0060R1.007 data submitted with this model report.

Figure 6-8. Cumulative Breakthrough of the ${ }^{99}$ Tc Mass Fraction $R_{M}$ at the Water Table for Varying Present-Day Climatic Scenarios and Instantaneous Release

Table 6-7. Input Parameters for the Three-Dimensional Site-Scale Simulations of Solute Transport (\#1 Perched-Water Model)

\begin{tabular}{|l|l|}
\hline \multicolumn{1}{|c|}{ Parameters } & \multicolumn{1}{c|}{ Source } \\
\hline Tortuosity $\tau \approx \phi$ & $\begin{array}{l}\text { Grathwohl (2000 [DIRS 141512], pp. 28 to 35), } \\
\text { Farrell and Reinhard (1994 [DIRS 122803], p. 64) }\end{array}$ \\
\hline $\begin{array}{l}\text { Properties and characteristics of the geologic units, } \\
\text { steady-state pressures, water saturations, and flow } \\
\text { fields }\end{array}$ & $\begin{array}{l}\text { Table 6-6 (DTN: LB03023DSSCP9l.001 } \\
\text { [DIRS 163044], LB0210THRMLPRP.001 } \\
\text { [DIRS 160799]) }\end{array}$ \\
\hline Sorption distribution coefficients $K_{d}$ & 0.0- (see Appendix A6.) \\
\hline Molecular diffusion coefficient $D_{0}$ & Table 6-4 \\
\hline
\end{tabular}

NOTE: See Section 5, Table 5-1 for assumptions. 


\subsubsection{Transport-Controlling Features and Flow Patterns}

The study of radionuclide transport uses the relative mass fraction $X_{R}$, defined as

$$
X_{R}=\frac{X}{X_{0}}=\frac{C}{C_{0}}=C_{R}
$$

where $\mathrm{X}$ is the tracer mass fraction in the liquid phase $(M / M), \mathrm{C}$ is the tracer concentration in the liquid phase $\left(M / L^{3}\right)$, and the subscript 0 denotes the value of the subscripted parameter in the water released from the repository. For mean present-day infiltration, the distribution of the ${ }^{99} \mathrm{Tc}$ $X_{R}$ in the aqueous phase within fractures and the matrix in the gridblocks directly above the TSw-CHn interface (i.e., at the bottom of the TSw, corresponding to the tsw39 layer) is given in Figures 6-9 through 6-18 for $t=10,100,1,000,10,000$, and 100,000 years. Note that the $X_{R}$ distributions in Figures 6-9 through 6-18 do not correspond to a plan view of a horizontal cross section, but follow the uneven topography of the bottom of the tsw39 layer. The $X_{R}$ distributions of ${ }^{99} \mathrm{Tc}$ in the fractures and in the matrix immediately above the water table at the same times are given in Figures 6-19 through 6-28.

From the comparison of these figures with Figure 6-7, it becomes apparent that transport in the radionuclide transport model is both dominated and controlled by the faults. From the distribution of the fracture $X_{R}$ at the bottom of the TSw, it is evident that, immediately above the TSw-CHn interface (i.e., in the tsw39 layer), the Pagany Wash fault and the Drill Hole Wash fault in the northern part are the main transport-facilitating feature. As expected, radionuclide transport to that layer (immediately below the repository) is rapid. The impact of the faults on transport is evident as early as $t=10$ years, at which time the Pagany Wash fault appears to provide the fastest pathway (Figure 6-9), as indicated by the early appearance of radionuclides. Although ${ }^{99}$ Tc arrives later at the Drill Hole Wash fault, it appears to have a larger contribution than the Pagany Wash fault for $t \geq 100$ years. Both faults register a strong signature, and their presence is easily identified from the ${ }^{99} \mathrm{Tc}$ distributions (Figure 6-11). At the same time, it is noteworthy that the Sundance fault is beginning to cast a faint shadow on the fracture $X_{R}$ map.

The predominance of the Drill Hole Wash fault and the Pagany Wash fault as the main transport-facilitating geologic features at the tsw39 level is pervasive during the entire simulation period, as evidenced by Figures 6-13, 6-15, and 6-17. At later times ( $t \geq 10,000$ years), the Solitario Canyon fault becomes an important transport conduit. The northern reach of the Ghost Dance fault becomes visible at $t \geq 1,000$ years, but appears to be of secondary importance to transport to the tsw39 layer.

Review of the fracture $X_{R}$ distributions at the water table (Figures 6-19, 6-21, 6-23, 6-25, and 6-27) indicates that the Drill Hole Wash fault and the Pagany Wash fault continue to be the main transport conduits throughout the geologic profile, from the level of the repository to the water table. It is remarkable that these two faults show measurable radionuclide presence at the water table as early as $t=10$ years, and at concentrations that are higher and more widely distributed than those observed at the bottom of the TSw (Figures 6-11 and 6-13). In other words, transport through these two faults is so fast that the water table concentrations of ${ }^{99} \mathrm{Tc}$ exceed those immediately below the release point at $t=10$ years because the radionuclides released into the repository fractures have already reached the water table. 
In general, the appearance of ${ }^{99} \mathrm{Tc}$ at the water table appears to be earlier and more pronounced at the water table than at the bottom of the TSw. Additionally, more faults active in radionuclide transport are evident at the water table. Note the early and strong signature of the Sundance fault and of the Sever Wash fault (which appears to intersect the radionuclide-carrying fractures emanating from the Pagany Wash fault) at $t=10$ years (Figure 6-19).

The significance of the Drill Hole Wash fault as the dominant conduit of ${ }^{99} \mathrm{Tc}$ to the water table is further confirmed in Figure 6-21 ( $t=100$ years), where it is shown to have resulted in a large zone of high ${ }^{99} \mathrm{Tc}$ concentrations that extends outward from the fault axis. At this time, in addition to the Sundance fault, the northern portion of the Ghost Dance fault and the Solitario Canyon fault appear to be making significant contributions to transport.

Analysis of the matrix $X_{R}$ distributions at the bottom of the TSw and at the water table offers additional insights into the transport patterns. It is remarkable that, in tsw39, the matrix $X_{R}$ distributions at early times (Figures 6-10 and 6-12) show the highest concentrations in the southern part of the repository, in sharp contrast to the fractures that indicate transport exclusively in the northern part at the same times (Figures 6-9 and 6-11), and practically no sign of significant ${ }^{99} \mathrm{Tc}$ presence in the matrix next to the rapidly transporting fractures.

The reason for these vastly diverse transport patterns is the significantly different geology at the base of the TSw, which is dominated by the vitric $\mathrm{CHv}$ layers below the southern part of the repository, while the zeolitic $\mathrm{CHz}$ layers are predominant in the north. The permeabilities of the fractures and of the matrix of the vitric layers are similar in magnitude (BSC 2004 [DIRS 169857], Tables 4-2 and 4-3). This permeability parity, coupled with a large fracture spacing, result in a behavior similar to that of a nonfractured porous medium (BSC 2004 [DIRS 169857], Section 6.1.4). Thus, there is no early evidence of fracture flow in the southern part of the repository.

In contrast, although the zeolitic $\mathrm{CHz}$ layers in the northern part of the repository have fracture spacing and fracture permeability similar to the vitric $\mathrm{CHv}$ layers in the south, their matrix permeability is about four orders of magnitude lower than their fracture permeability (BSC 2004 [DIRS 169857], Tables 6-8, 6-9, and 6-10). Consequently, matrix flow in the zeolitic layers in the northern part of the repository is extremely slow and practically all the flow occurs in the fractures, leading to the fast transport observed in the fractures.

The eastward movement of ${ }^{99} \mathrm{Tc}$ in the matrix (Figures 6-12, 6-14 and 6-16) results from site geology. Once contamination reaches the interface, it moves primarily eastward, moving with the draining water that hugs the downward sloping (in this direction) low-permeability TSw-CHn interface. At later times, the matrix $X_{R}$ footprint shifts northward, as ${ }^{99}$ Tc diffusing from the fractures advances in matrix. This is evidenced by the transition apparent in Figures 6-12, 6-14, and 6-16).

The presence of ${ }^{99} \mathrm{Tc}$ in the matrix at the water table is significantly delayed, and a pattern similar to that at the bottom of the TSw at early times is not observed. Despite significant radionuclide concentrations in the fractures associated with the faults discussed earlier, the matrix shows scant evidence of ${ }^{99} \mathrm{Tc}$ presence (Figures 6-20 and 6-22). When it occurs, it follows the fault geometry and orientation, and is localized and limited to the immediate vicinity 
of the faults, indicating an origin consistent with diffusion from the fault fractures with no sign of downward migration through the matrix.

This matrix concentration pattern further attests to the preponderance of fractures as the main transport-facilitating features. A pervasive feature of the transport pattern at the water table is that the area affected by significant radionuclide concentration is significantly larger in the fracture system than in the matrix. This is the opposite of the observation at the bottom of the TSw, where the areal distribution of the matrix $X_{R}$ exceeds the footprint of the fracture $X_{R}$. The obvious conclusion is that the fractures are practically the exclusive source of radionuclides appearing in the matrix, and that the large water velocities in the fractures lead to the limited areal extent and penetration of ${ }^{99} \mathrm{Tc}$ into the matrix.

\subsubsection{Evidence Supporting the Transport Patterns}

Of particular interest is the emerging transport pattern, which indicates that radionuclide transport to the water table is faster in the northern part of the repository, where it is also areally concentrated. Based on the properties of the layers beneath the repository, this appears to be qualitatively consistent with expectations of transport in the vertical cross sections discussed in Section 6.6.

Thus, the general area of fastest, largest, and most extensive transport is in the northern part of the repository site, where the very low matrix permeability of the $\mathrm{CHz}$ directs practically all flow through the fractures. Figures 6-29a and 6-29b show the areally increasing extent of the fractured zeolitic tuffs with depth. As the distance from the repository increases, an increasing portion of the water flow occurs through the zeolites, in which the matrix permeability is very small and the fractured dominated flow (and, consequently, the advective transport) is fast.

In addition to geology, support for the observed transport pattern is provided by the infiltration and percolation distributions. A review of the infiltration pattern (Figure 6-30) at the surface, the percolation flux at the repository level (Figure 6-31), and the percolation flux at the groundwater level (Figure 6-32) indicates that they closely reflect the transport patterns in Figures 6-9 through 6-28. These figures indicate that the water flow pattern dictates the advective transport pattern.

The dominance of fracture flow (and, consequently, of advection-dominated transport) is supported by the relative magnitudes of hydraulic properties in the fractures and in the matrix. The permeability of the fractures in the faults can be as high as hundreds of darcies (BSC 2004 [DIRS 169857], Table 6-14; Bodvarsson et al. 1999 [DIRS 120055], p. 15), that is, orders of magnitude larger. The resulting fast advective transport is the reason for the transport pattern observed in Figures 6-9 through 6-28. 


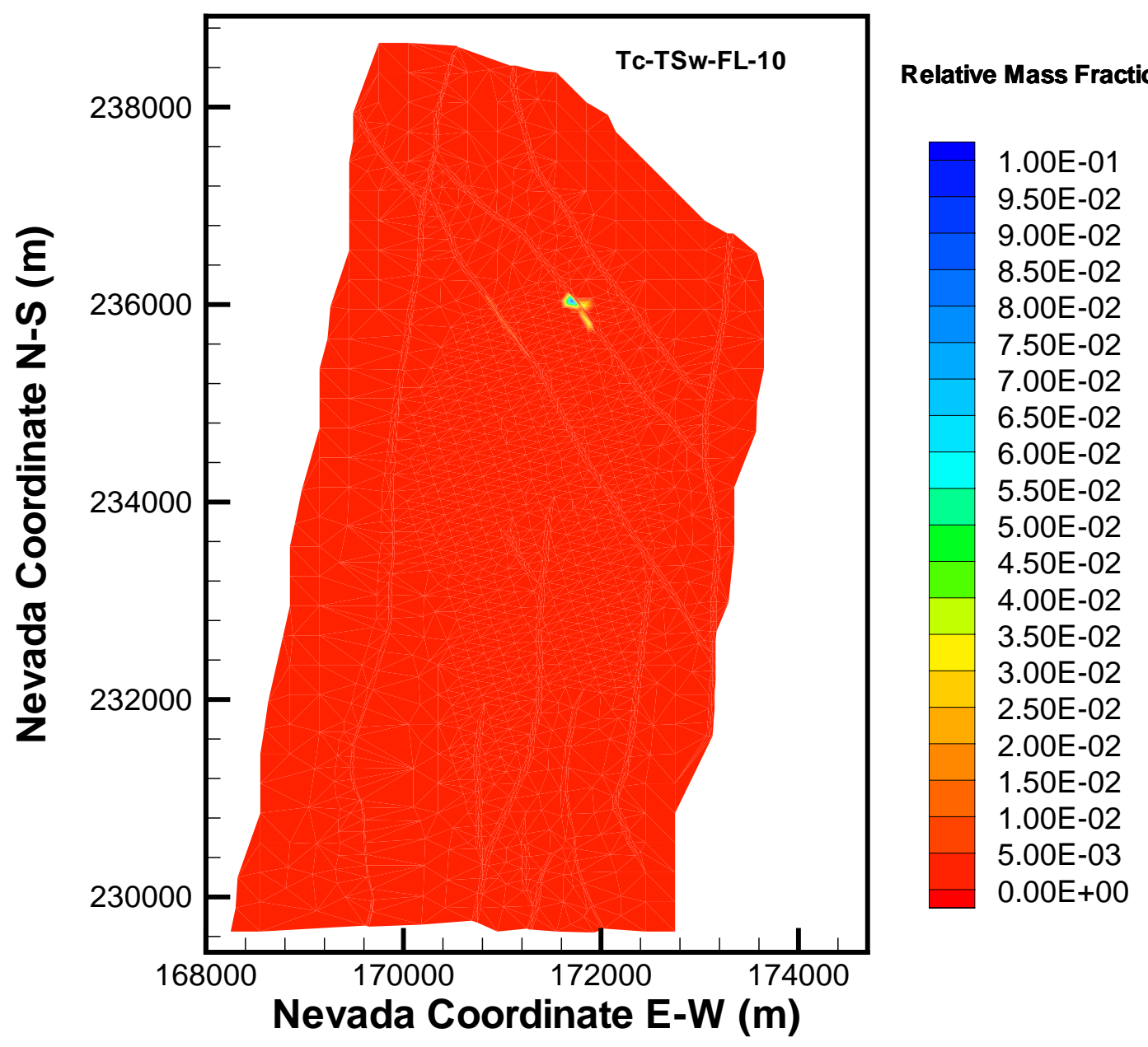

Output DTN: LB0307MR0060R1.002, data submitted with this model report.

Figure 6-9. Distribution of the Relative Mass Fraction $X_{R}$ of ${ }^{99} T c$ in the Fractures of the tsw39 Layer at $t=10$ Years for Mean Present-Day Infiltration and Instantaneous Release 


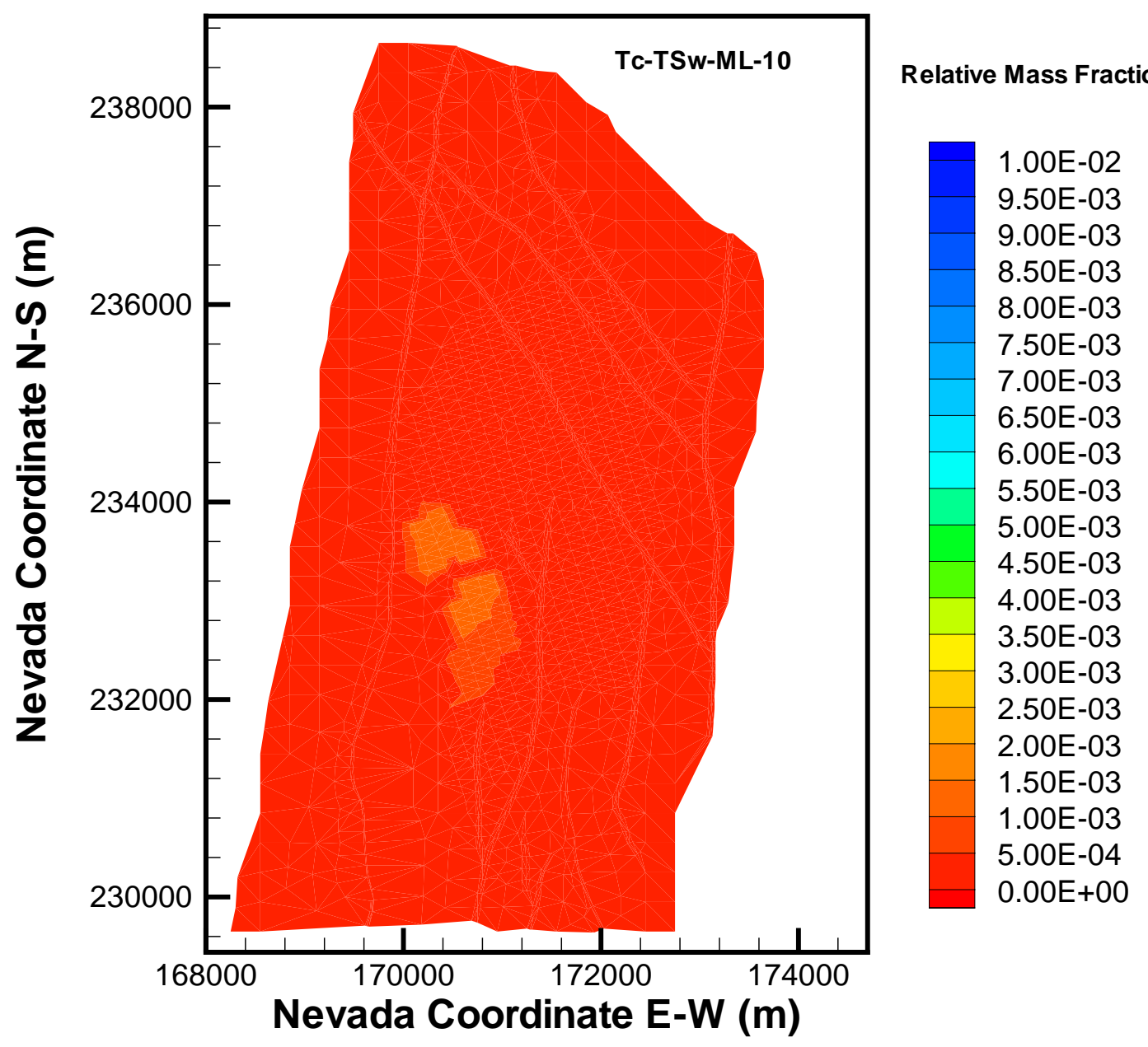

Output DTN: LB0307MR0060R1.002, data submitted with this model report.

Figure 6-10. Distribution of the Relative Mass Fraction XR of 99Tc in the Matrix of the tsw39 Layer at $\mathrm{t}=10$ Years for Mean Present-Day Infiltration and Instantaneous Release 


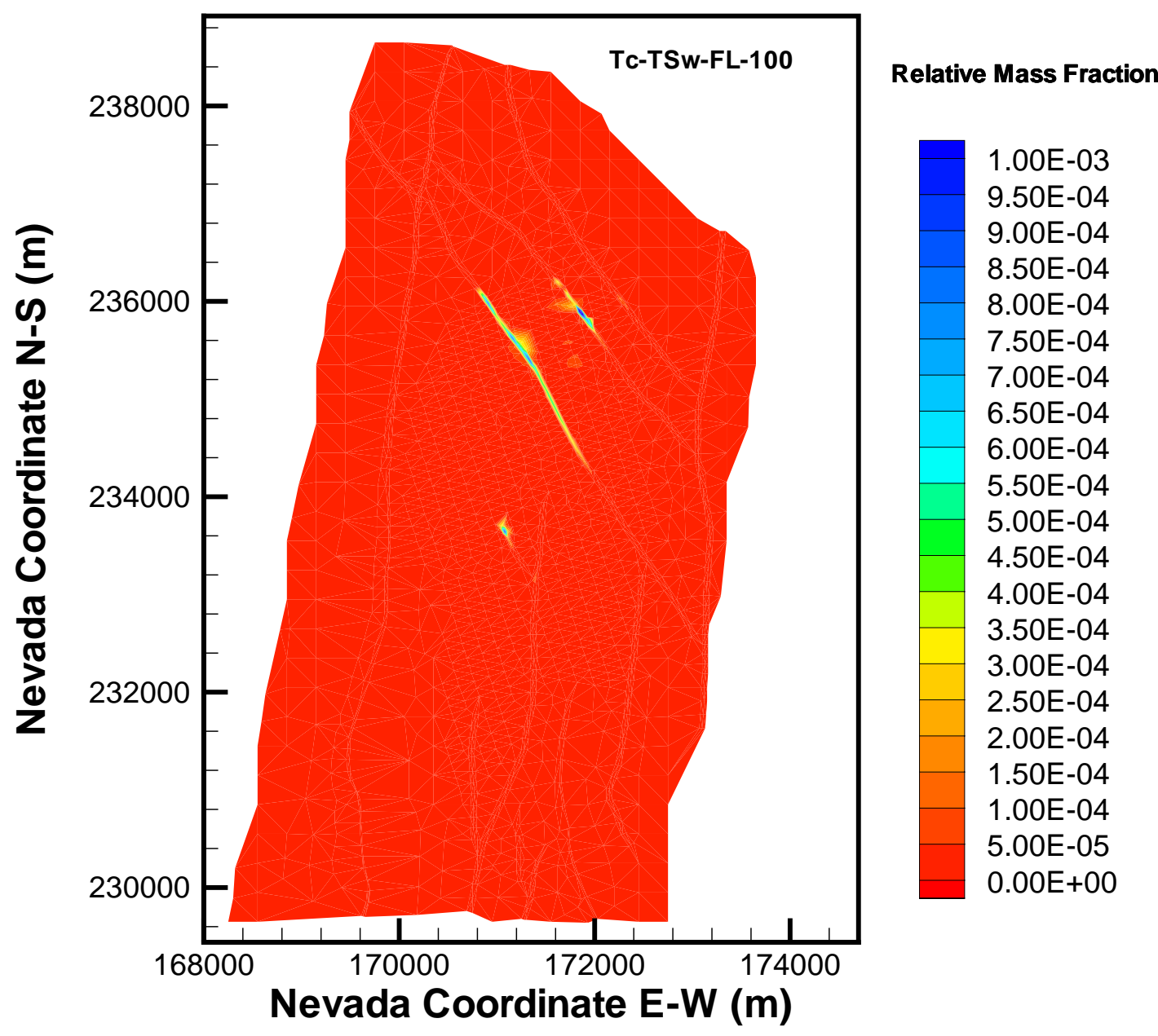

Output DTN: LB0307MR0060R1.002, data submitted with this model report.

Figure 6-11. Distribution of the Relative Mass Fraction $X_{R}$ of ${ }^{99} \mathrm{Tc}$ in the Fractures of the tsw39 Layer at $t=100$ Years for Mean Present-Day Infiltration and Instantaneous Release 


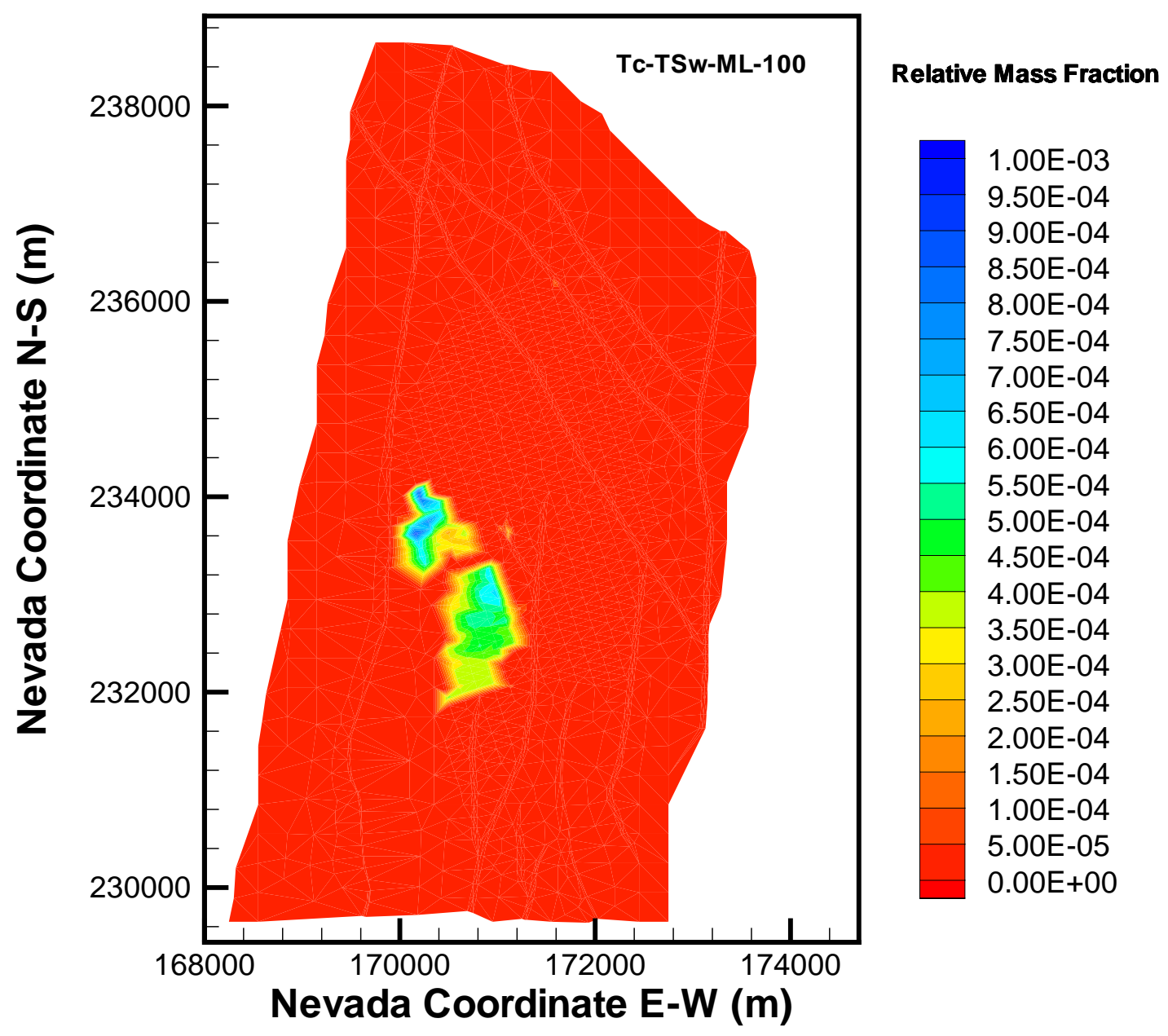

Output DTN: LB0307MR0060R1.002, data submitted with this model report.

Figure 6-12. Distribution of the Relative Mass Fraction $X_{R}$ of ${ }^{99} \mathrm{Tc}$ in the Matrix of the tsw39 layer at $t=100$ Years for Mean Present-Day Infiltration and Instantaneous Release 


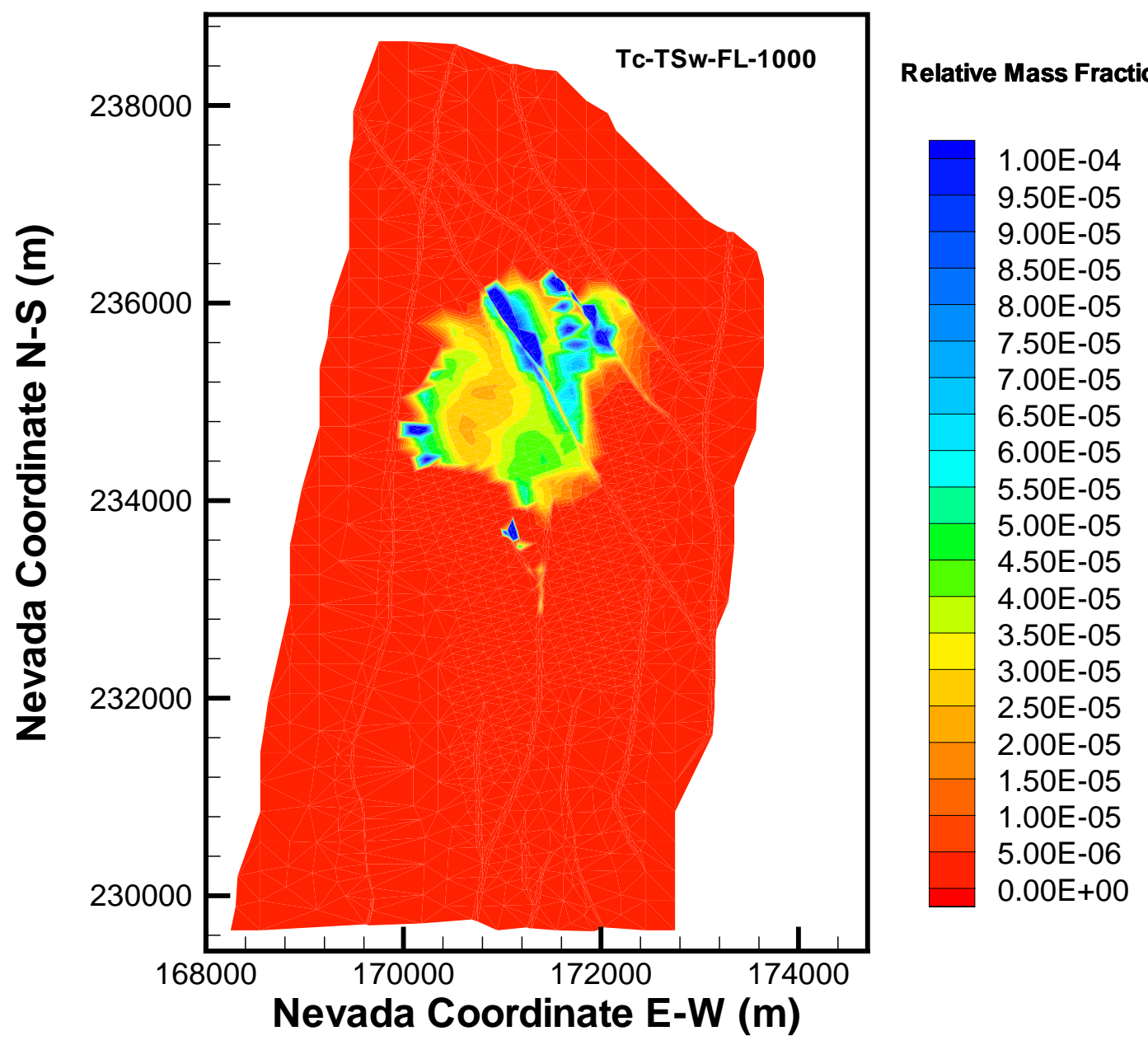

Output DTN: LB0307MR0060R1.002, data submitted with this model report.

Figure 6-13. Distribution of the Relative Mass Fraction $X_{R}$ of ${ }^{99} \mathrm{Tc}$ in the Fractures of the tsw39 Layer at $t=1,000$ Years for Mean Present-Day Infiltration and Instantaneous Release 


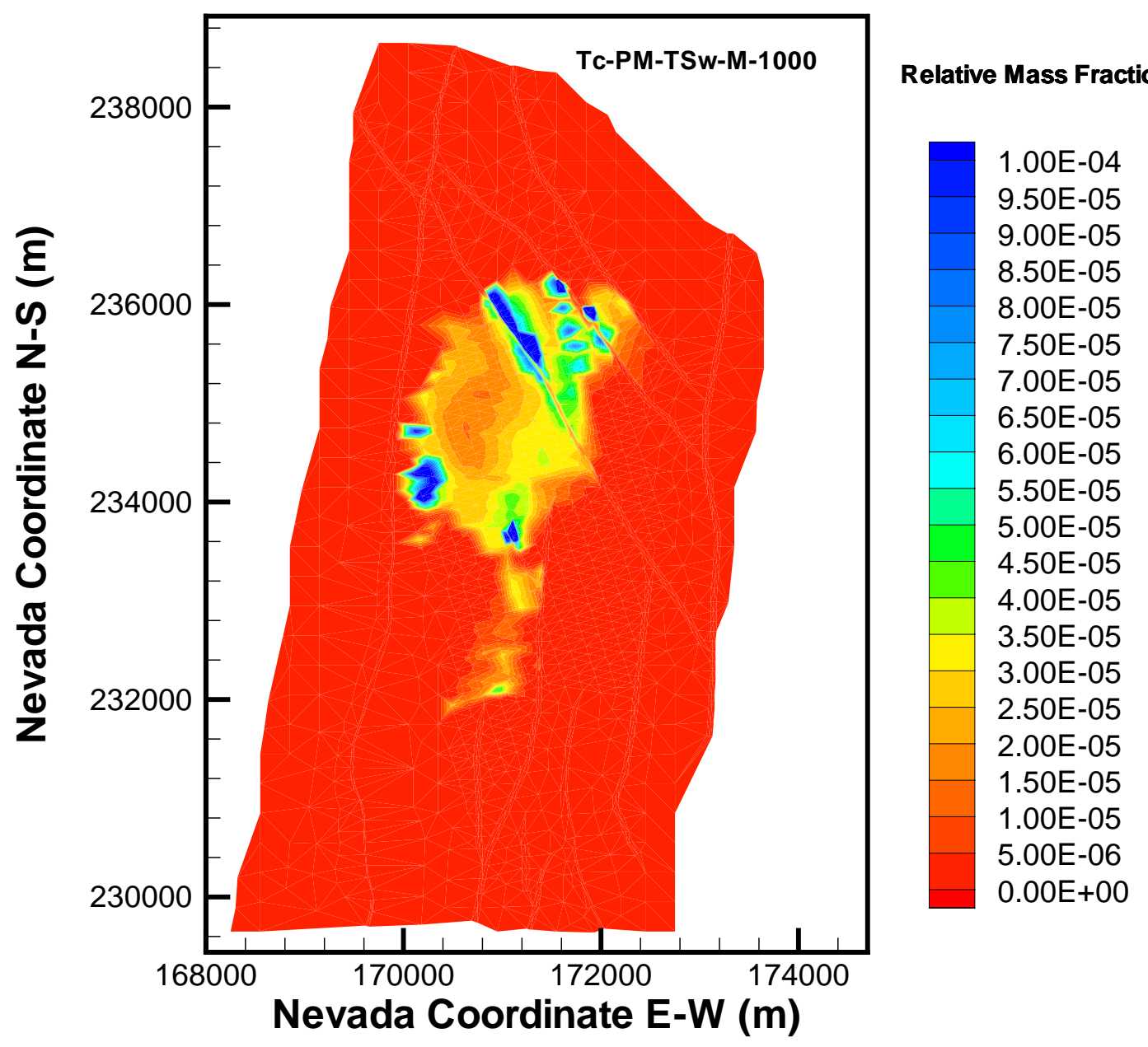

Output DTN: LB0307MR0060R1.002, data submitted with this model report.

Figure 6-14. Distribution of the Relative Mass Fraction $X_{R}$ of ${ }^{99} \mathrm{Tc}$ in the Matrix of the tsw39 Layer at $t=1,000$ Years for Mean Present-Day Infiltration and Instantaneous Release 


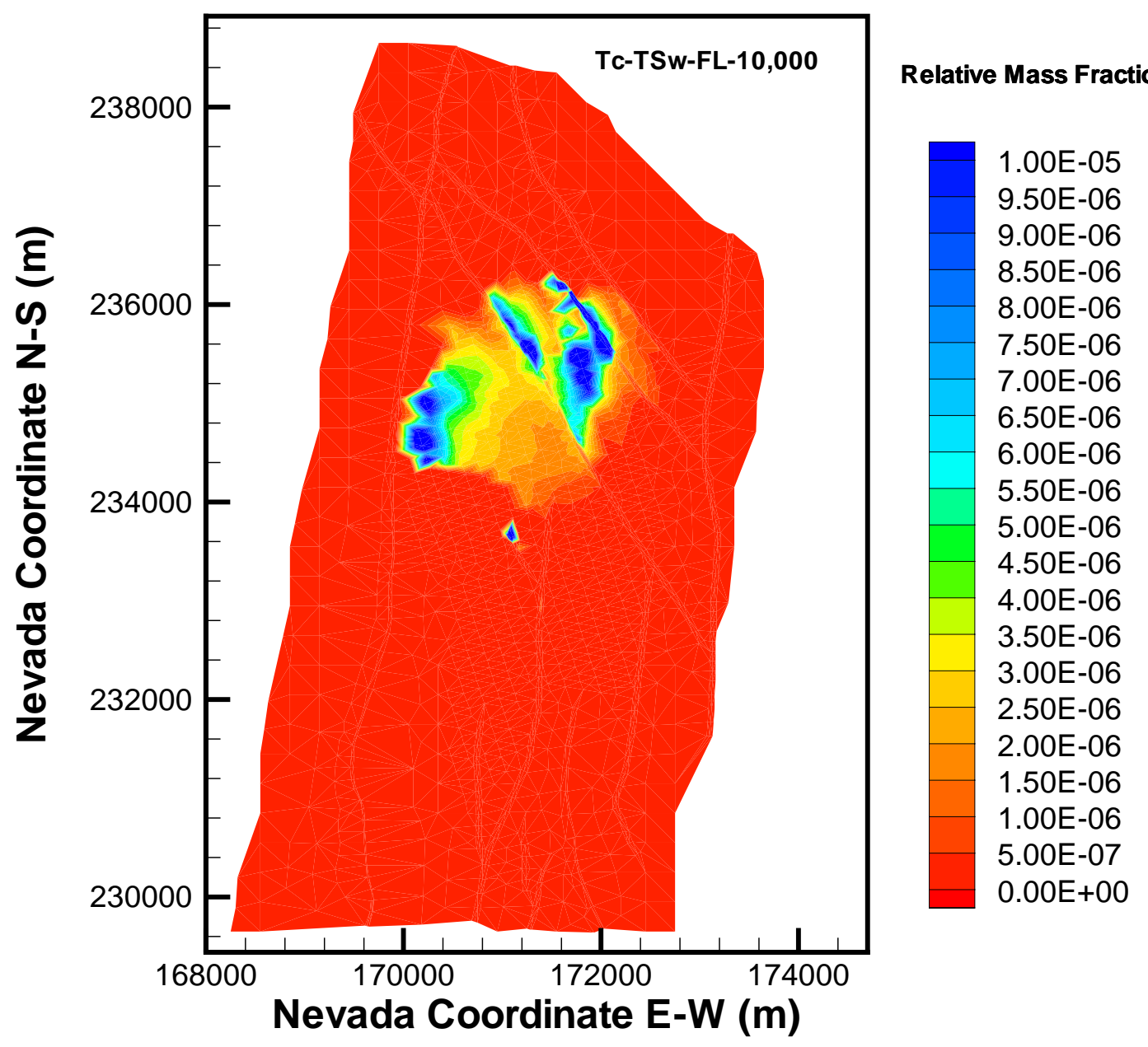

Output DTN: LB0307MR0060R1.002, data submitted with this model report.

Figure 6-15. Distribution of the Relative Mass Fraction $X_{R}$ of ${ }^{99} \mathrm{Tc}$ in the Fractures of the tsw39 Layer at $t=10,000$ Years for Mean Present-Day Infiltration and Instantaneous Release 


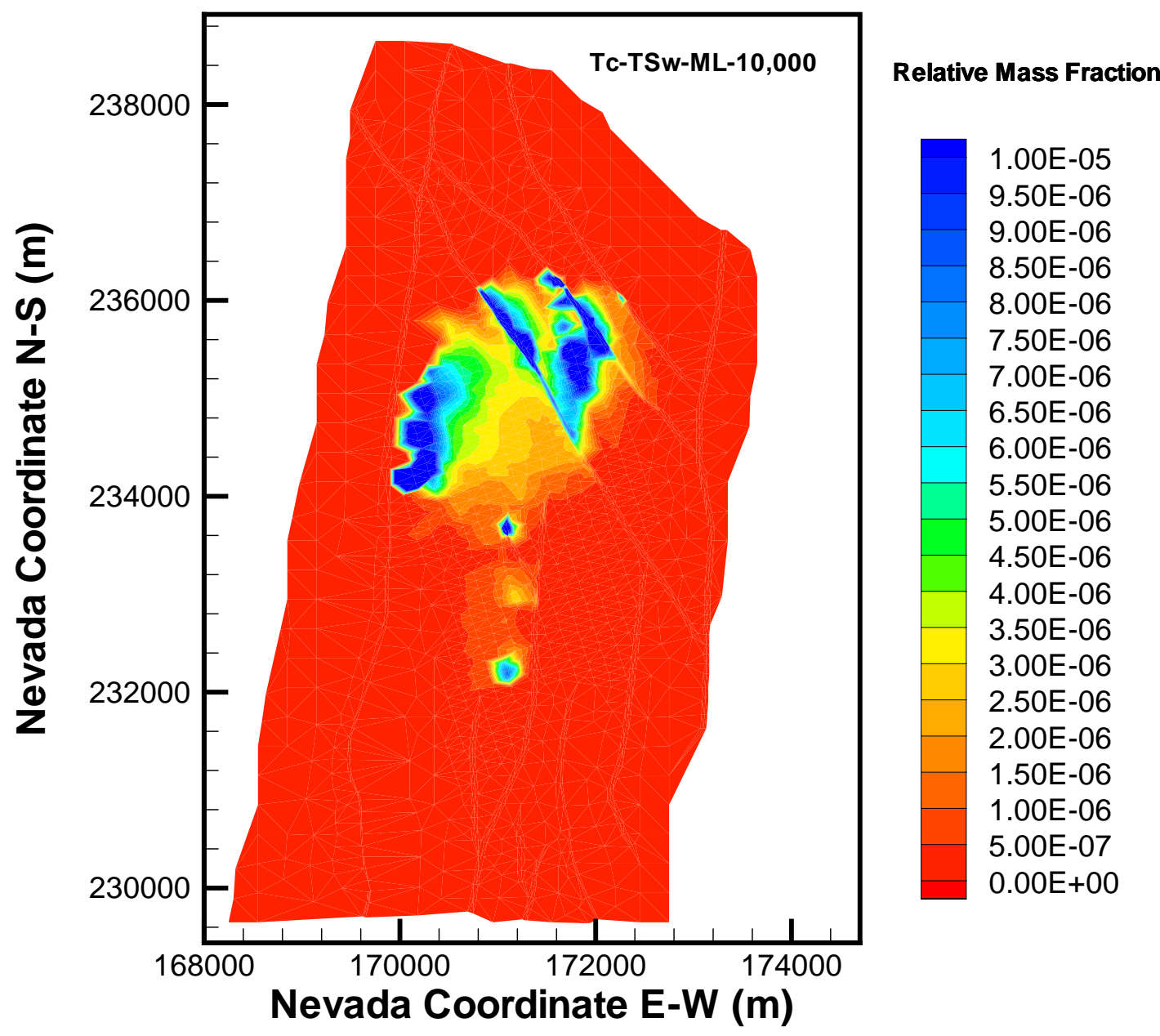

Output DTN: LB0307MR0060R1.002, data submitted with this model report.

Figure 6-16. Distribution of the Relative Mass Fraction $X_{R}$ of ${ }^{99} \mathrm{Tc}$ in the Matrix of the tsw39 Layer at $t=10,000$ Years for Mean Present-Day Infiltration and Instantaneous Release 


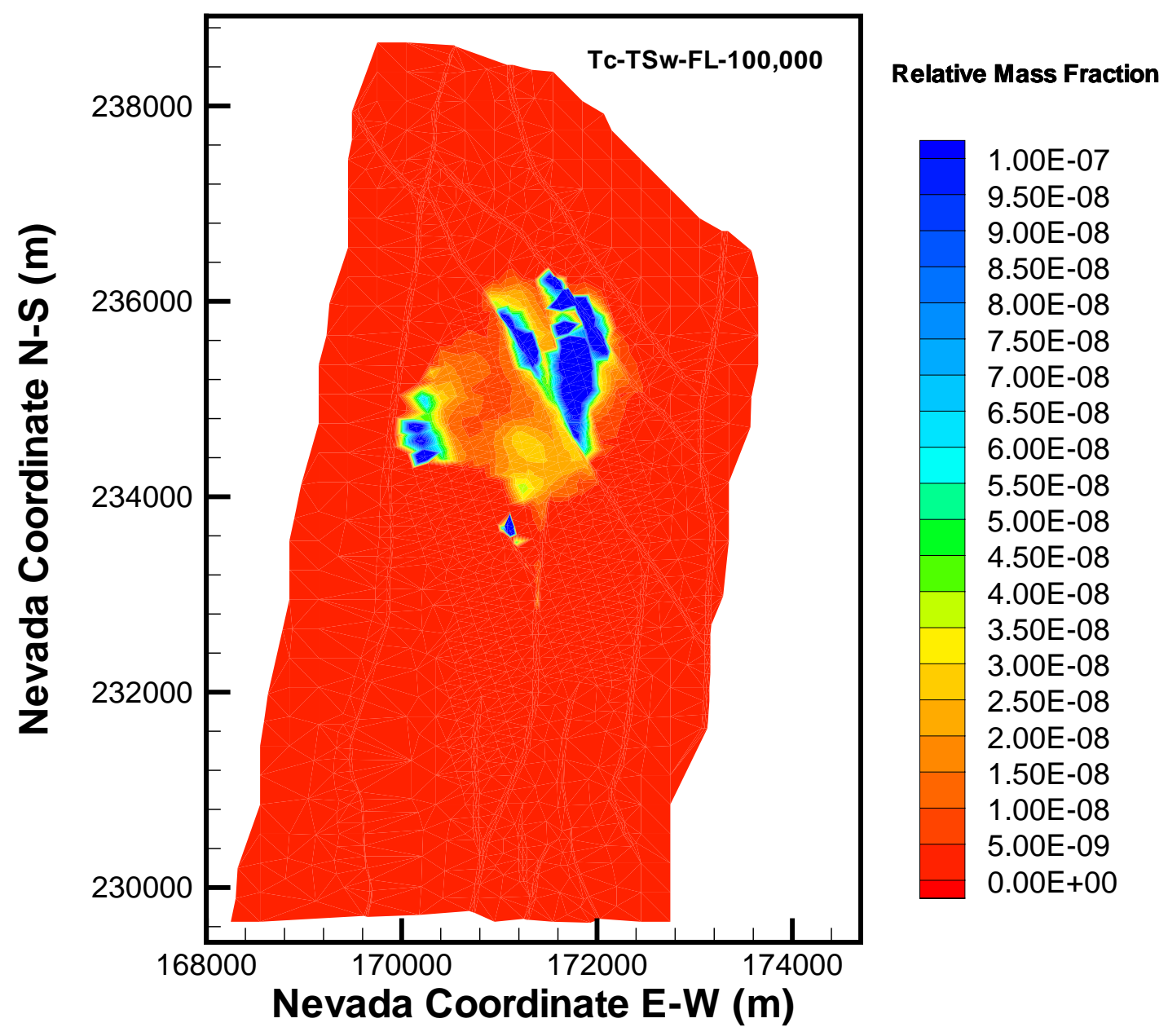

Output DTN: LB0307MR0060R1.002, data submitted with this model report.

Figure 6-17. Distribution of the Relative Mass Fraction $X_{R}$ of ${ }^{99} \mathrm{Tc}$ in the Fractures of the tsw39 Layer at $t=100,000$ Years for Mean Present-Day Infiltration and Instantaneous Release 


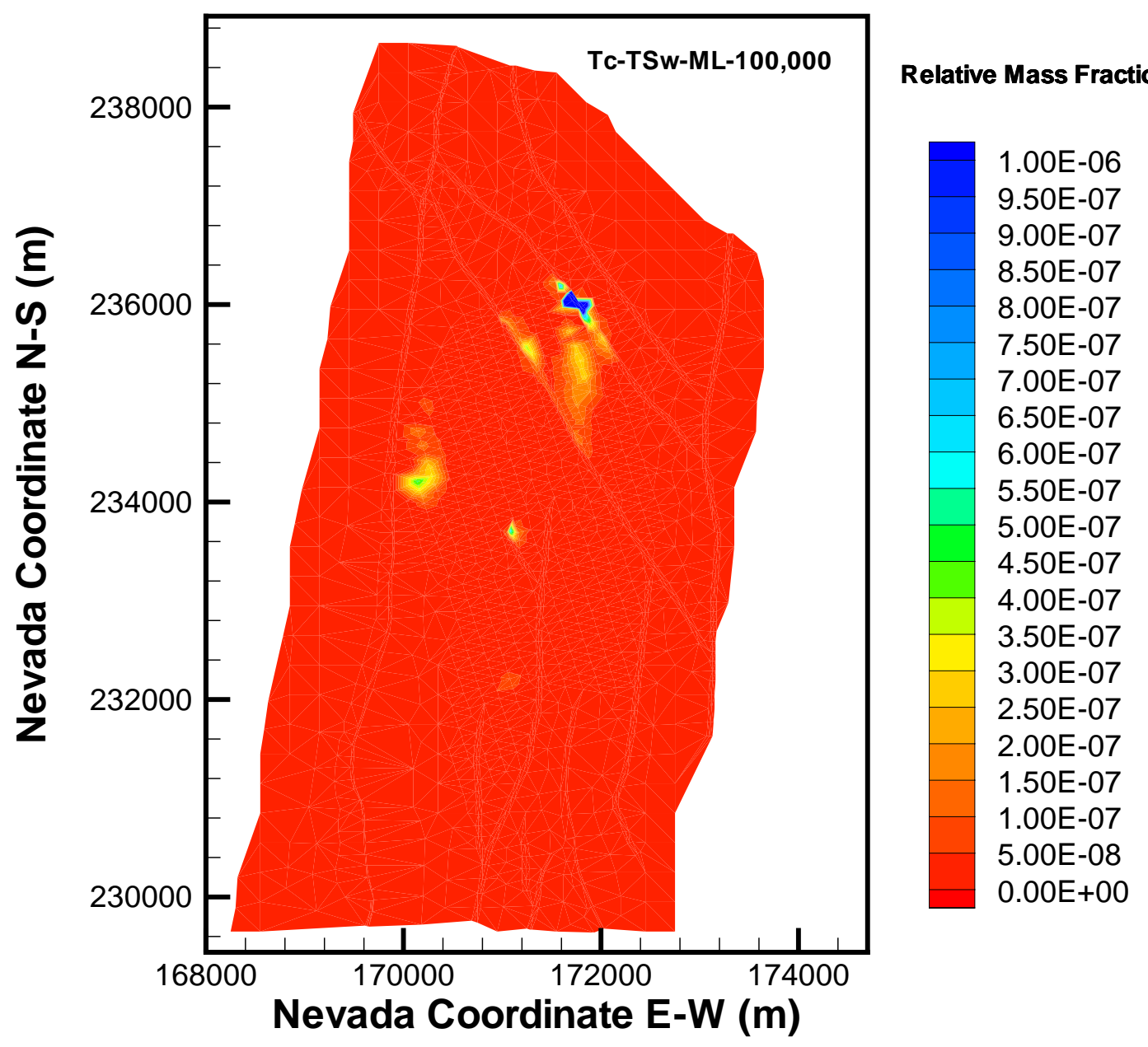

Output DTN: LB0307MR0060R1.002, data submitted with this model report.

Figure 6-18. Distribution of the Relative Mass Fraction $X_{R}$ of ${ }^{99} \mathrm{Tc}$ in the Matrix of the tsw39 Layer at $t=100,000$ Years for Mean Present-Day Infiltration and Instantaneous Release 


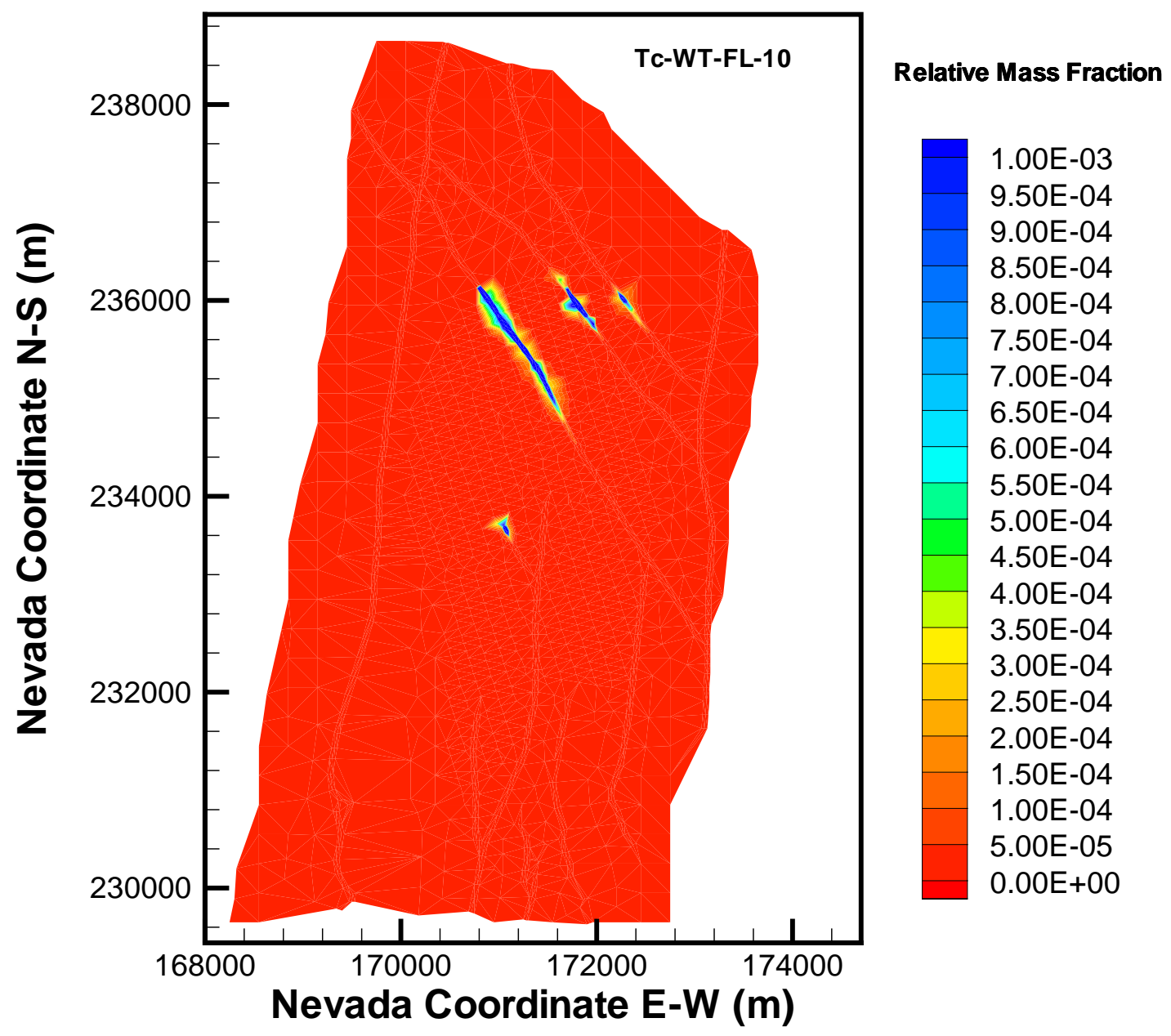

Output DTN: LB0307MR0060R1.002, data submitted with this model report.

Figure 6-19. Distribution of the Relative Mass Fraction $X_{R}$ of ${ }^{99} \mathrm{Tc}$ in the Fractures Immediately above the Water Table at $t=10$ Years for Mean Present-Day Infiltration and Instantaneous Release 


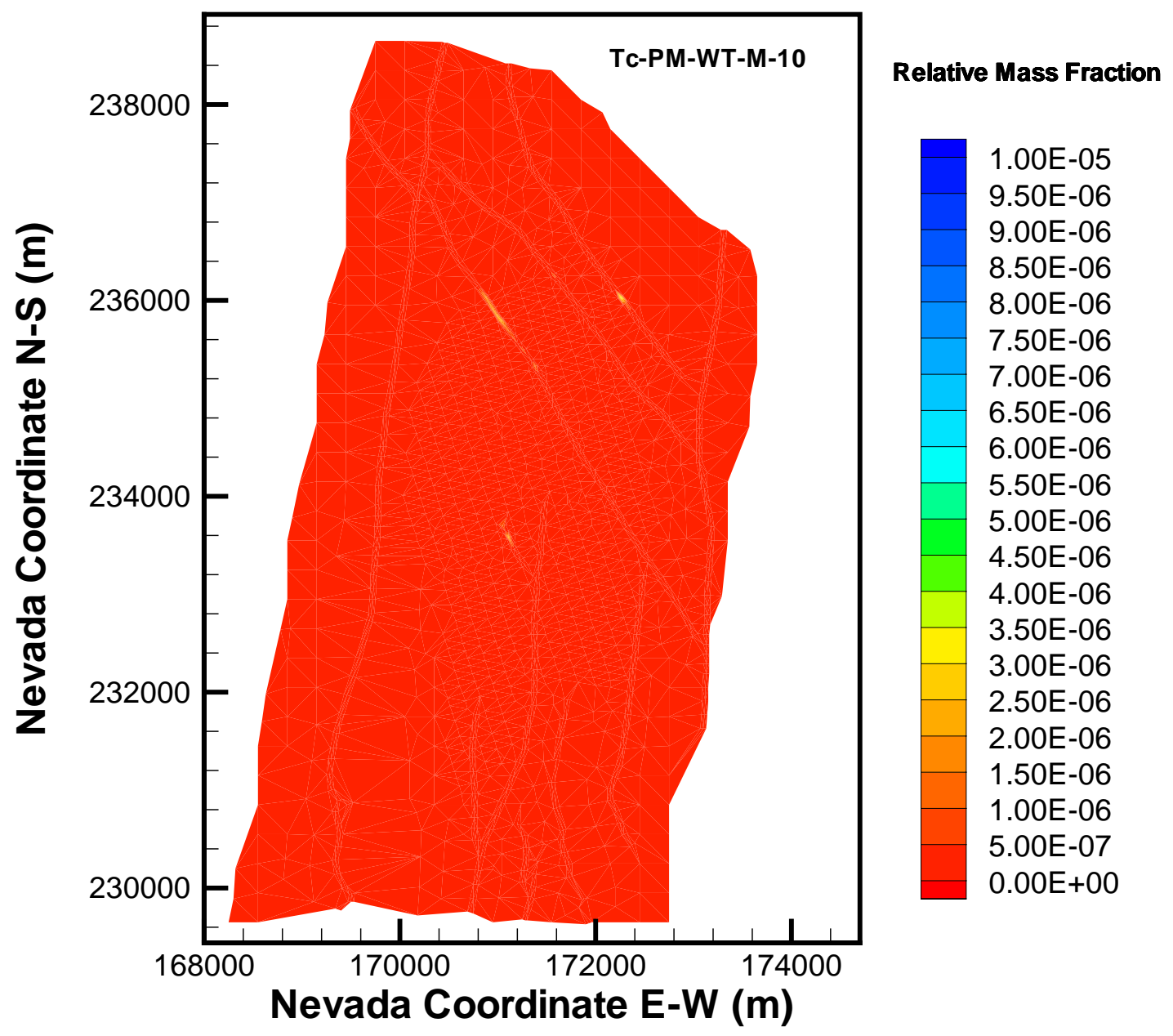

Output DTN: LB0307MR0060R1.002, data submitted with this model report.

Figure 6-20. Distribution of the Relative Mass Fraction $X_{R}$ of ${ }^{99} T c$ in the Matrix Immediately above the Water Table at $t=10$ Years for a Mean Present-Day Infiltration and Instantaneous Release 


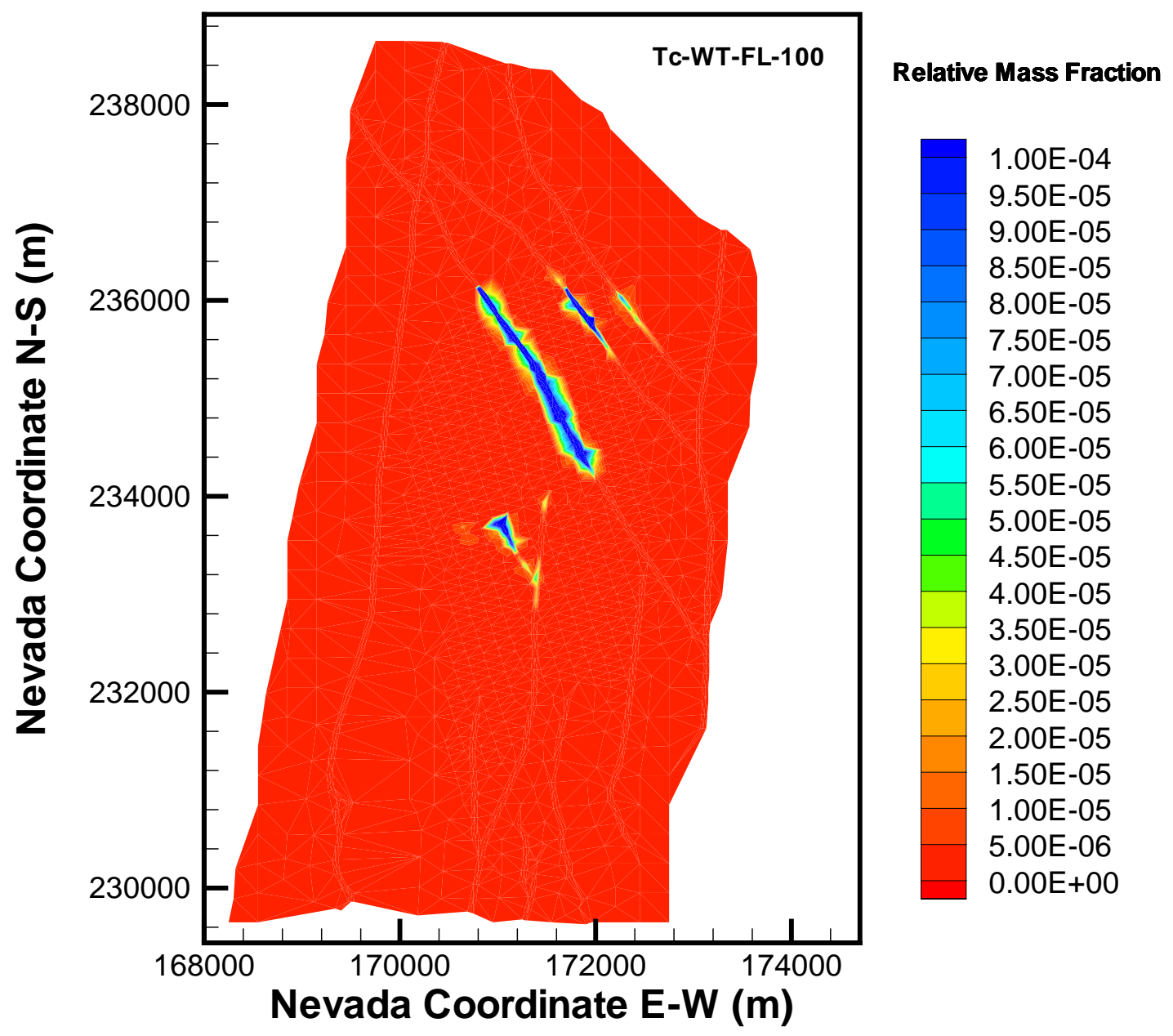

Output DTN: LB0307MR0060R1.002, data submitted with this model report.

Figure 6-21. Distribution of the Relative Mass Fraction $X_{R}$ of ${ }^{99} T c$ in the Fractures Immediately above the Water Table at $t=100$ Years for Mean Present-Day Infiltration and Instantaneous Release 


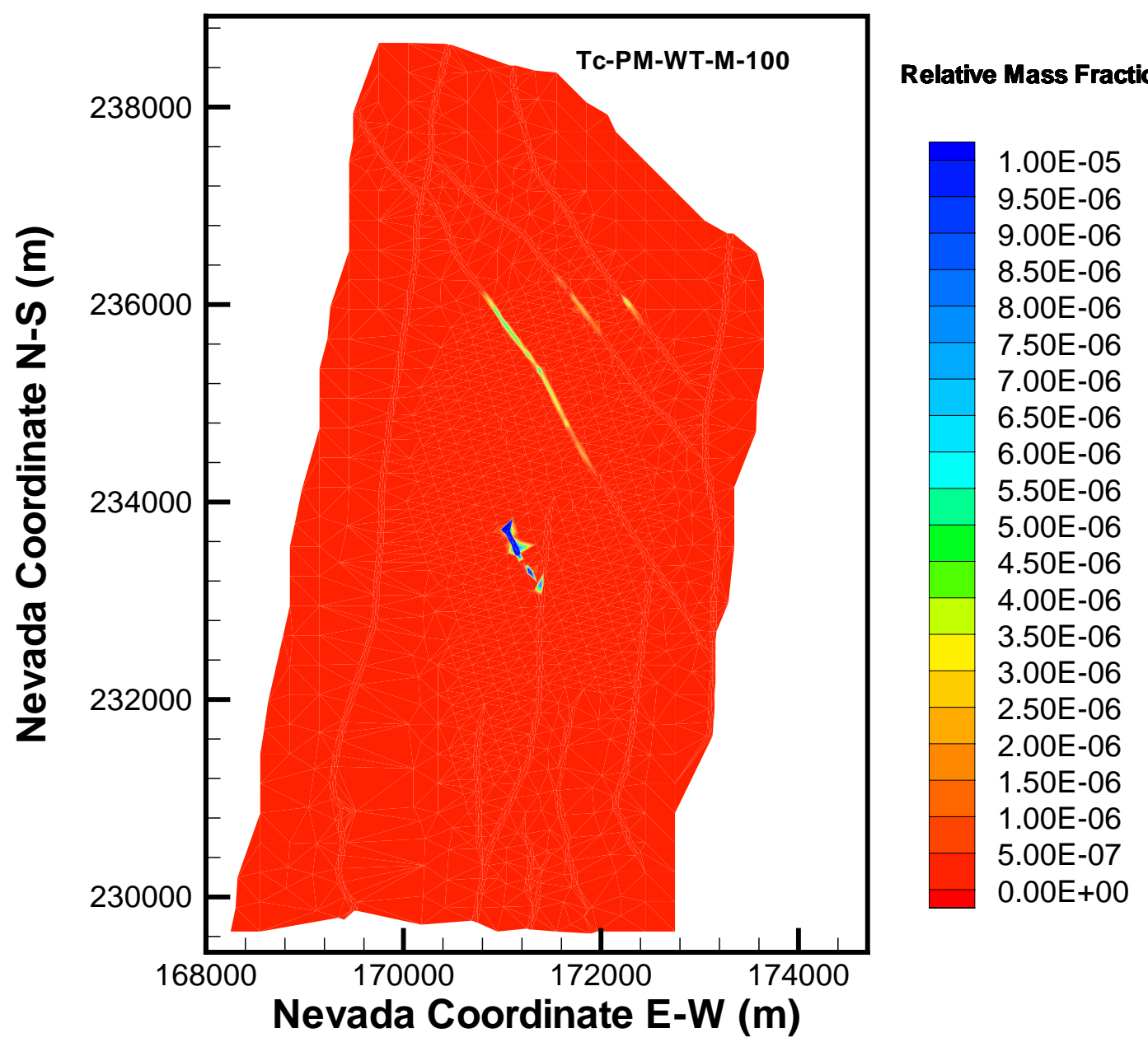

Output DTN: LB0307MR0060R1.002, data submitted with this model report.

Figure 6-22. Distribution of the Relative Mass Fraction $X_{R}$ of ${ }^{99} T c$ in the Matrix Immediately above the Water Table at $t=100$ Years for a Mean Present-Day Infiltration and Instantaneous Release 


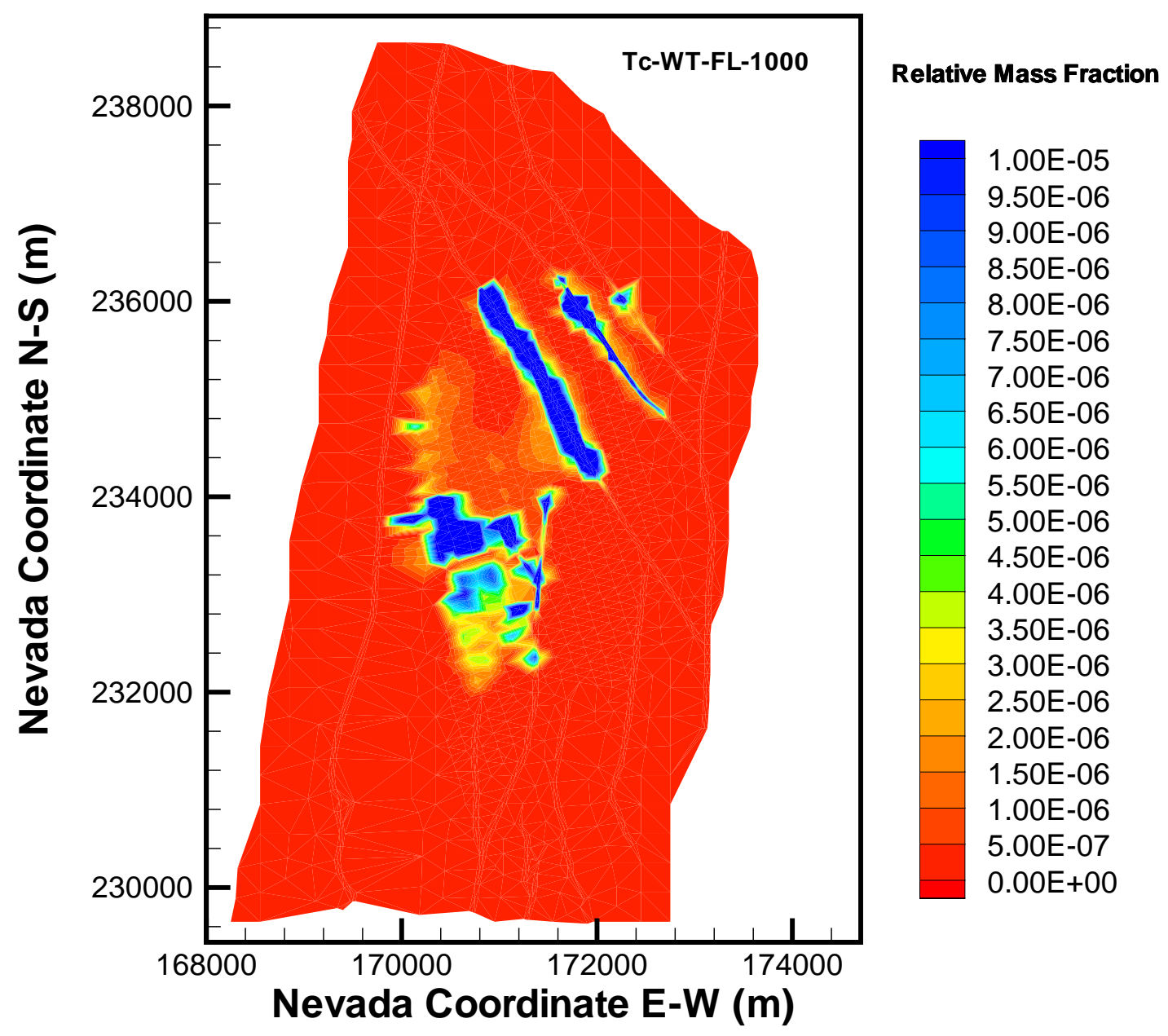

Output DTN: LB0307MR0060R1.002, data submitted with this model report.

Figure 6-23. Distribution of the Relative Mass Fraction $X_{R}$ of ${ }^{99} T c$ in the Fractures Immediately above the Water Table at $t=1,000$ Years for Mean Present-Day Infiltration and Instantaneous Release 


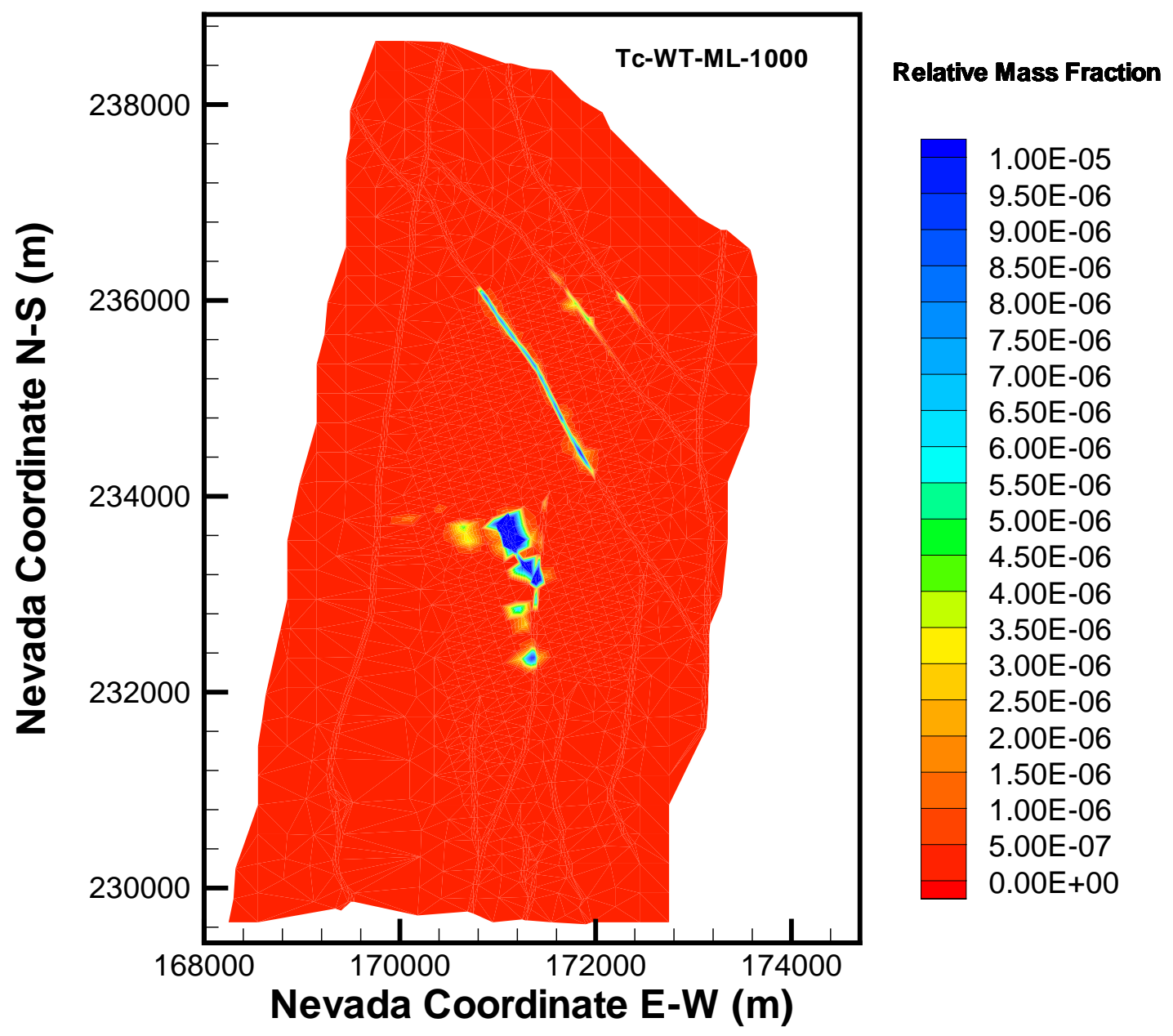

Output DTN: LB0307MR0060R1.002, data submitted with this model report.

Figure 6-24. Distribution of the Relative Mass Fraction $X_{R}$ of ${ }^{99} \mathrm{Tc}$ in the Matrix Immediately above the Water Table at $\mathrm{t}=1000$ years for a Mean Present-Day Infiltration and Instantaneous Release 


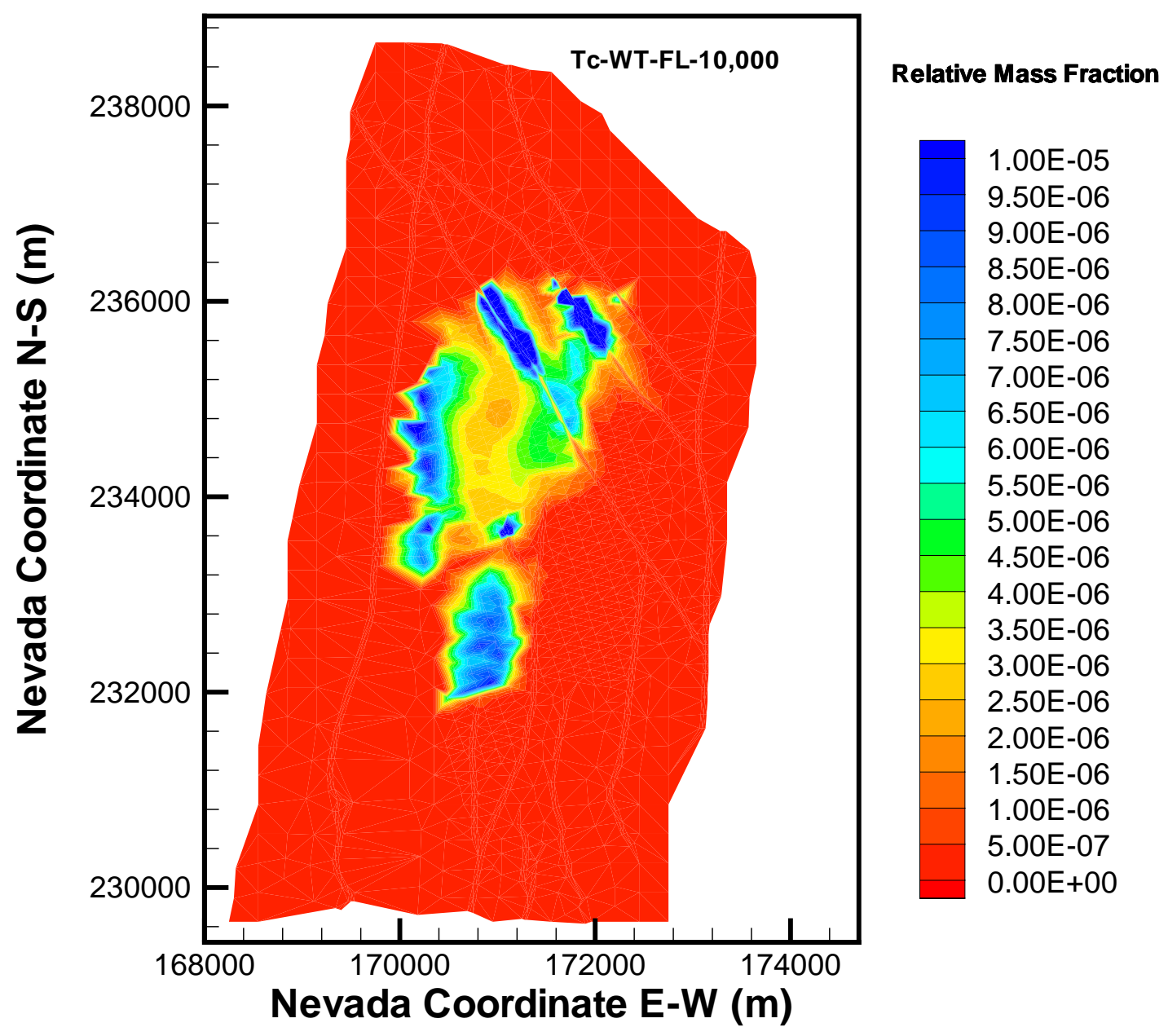

Output DTN: LB0307MR0060R1.002, data submitted with this model report.

Figure 6-25. Distribution of the Relative Mass Fraction $X_{R}$ of ${ }^{99} \mathrm{Tc}$ in the Fractures Immediately above the Water Table at $t=10,000$ Years for Mean Present-Day Infiltration and Instantaneous Release 


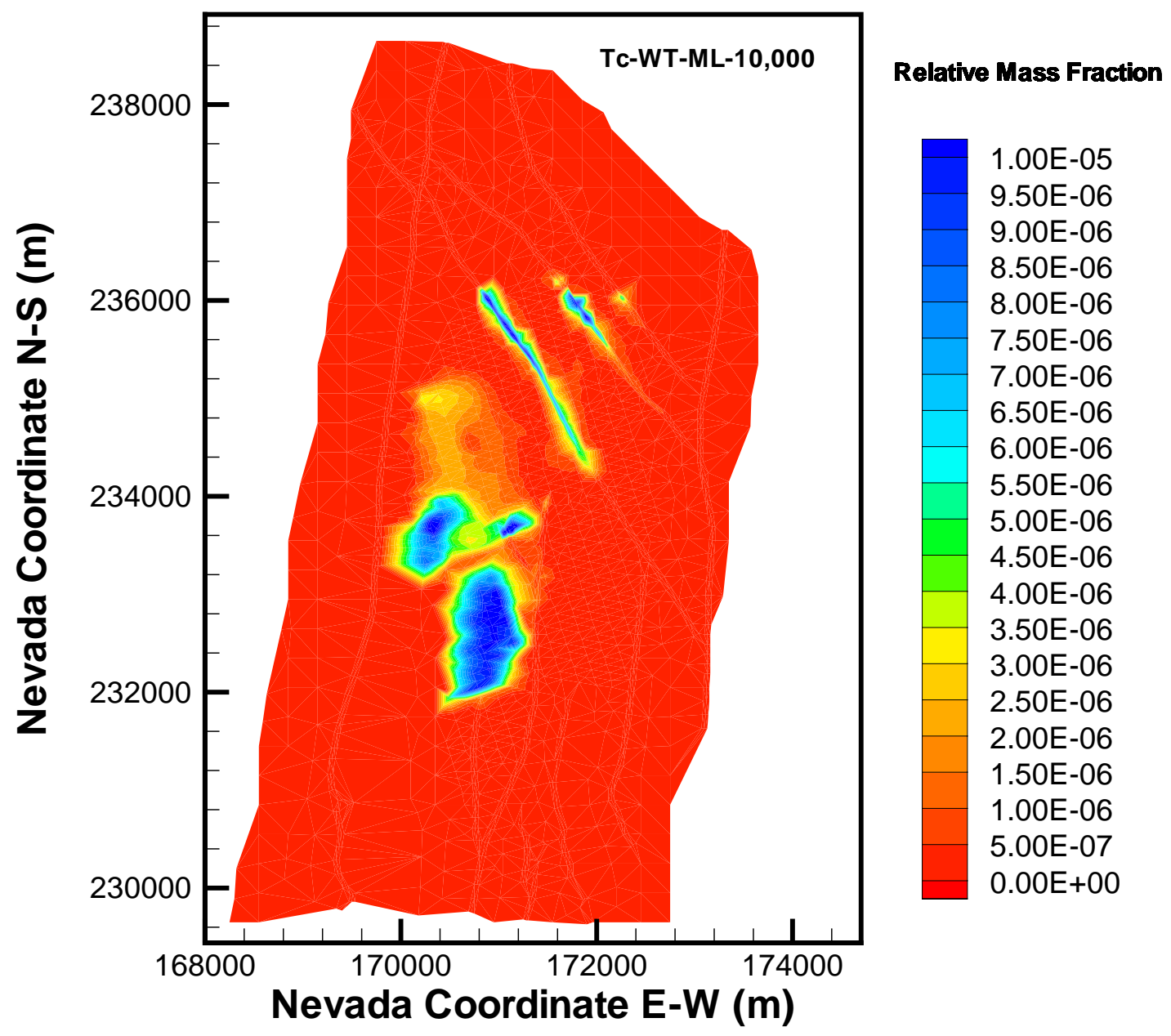

Output DTN: LB0307MR0060R1.002, data submitted with this model report.

Figure 6-26. Distribution of the Relative Mass Fraction $X_{R}$ of ${ }^{99} \mathrm{Tc}$ in the Matrix Immediately above the Water Table at $\mathrm{t}=10,000$ Years for a Mean Present-Day Infiltration and Instantaneous Release 


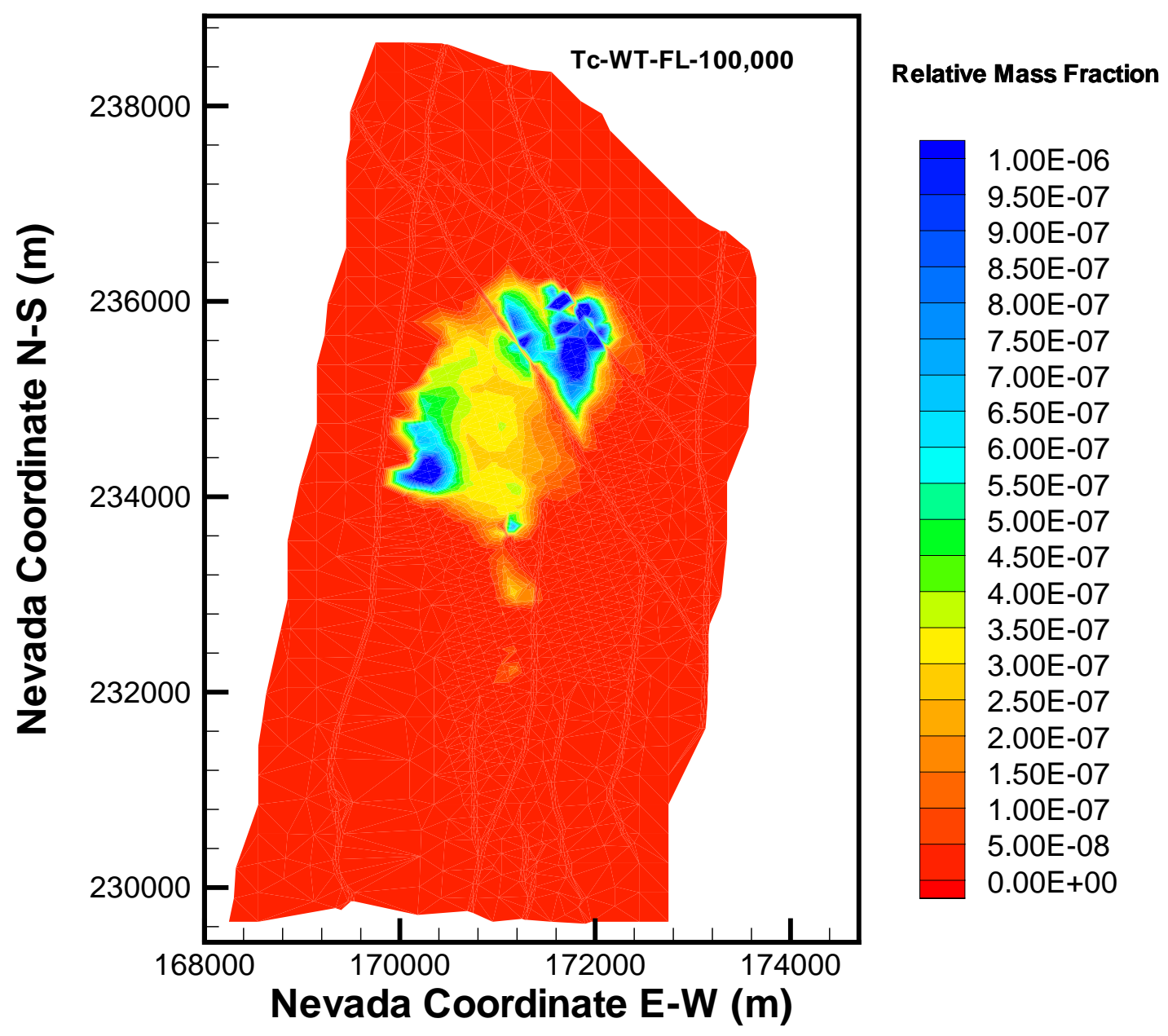

Output DTN: LB0307MR0060R1.002, data submitted with this model report.

Figure 6-27. Distribution of the Relative Mass Fraction $X_{R}$ of ${ }^{99} \mathrm{Tc}$ in the Fractures Immediately above the Water Table at $t=100,000$ Years for Mean Present-Day Infiltration and Instantaneous Release 


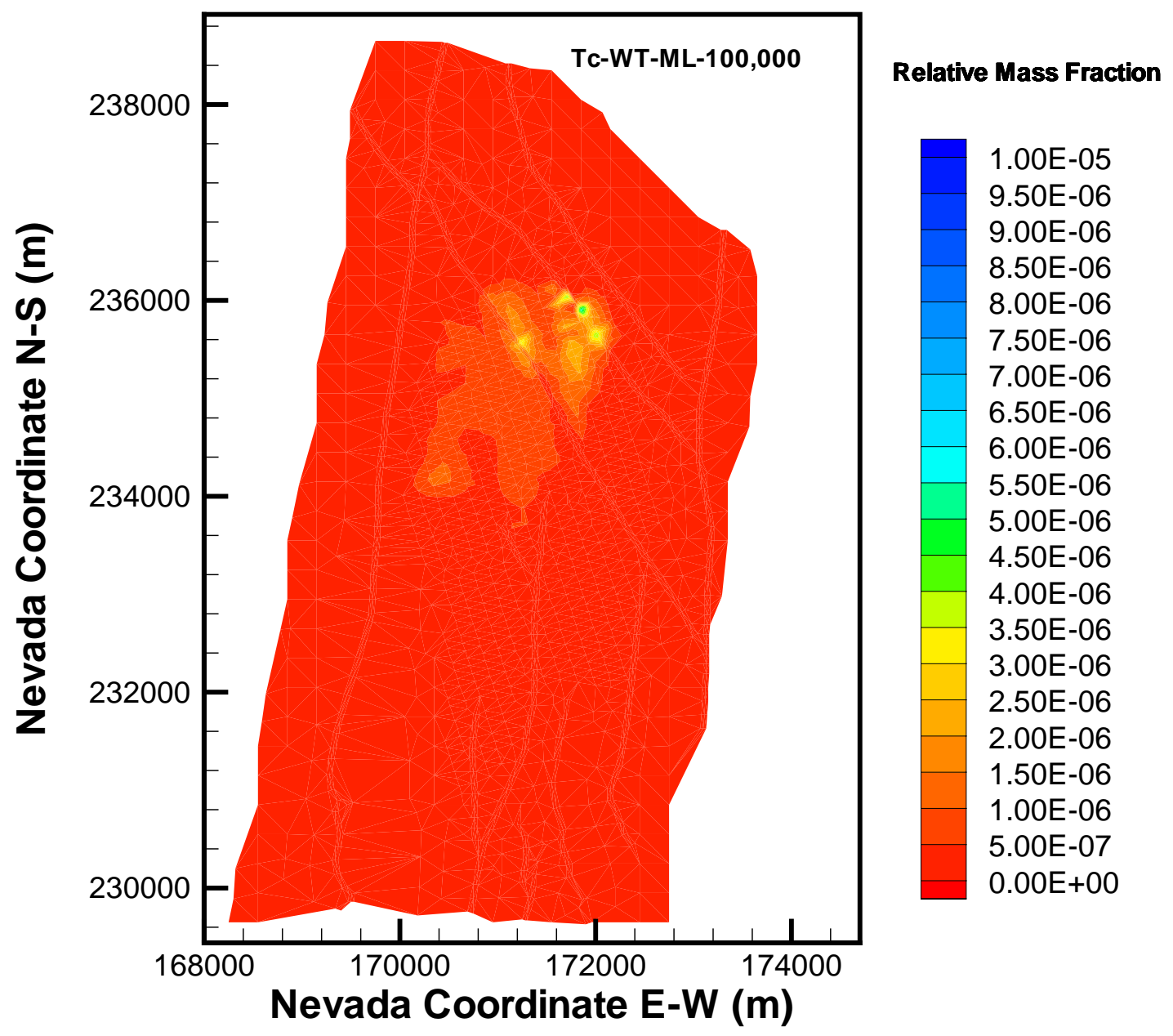

Output DTN: LB0307MR0060R1.002, data submitted with this model report.

Figure 6-28. Distribution of the Relative Mass Fraction $X_{R}$ of ${ }^{99} \mathrm{Tc}$ in the Matrix Immediately above the Water Table at $t=100,000$ Years for a Mean Present-Day Infiltration and Instantaneous Release 

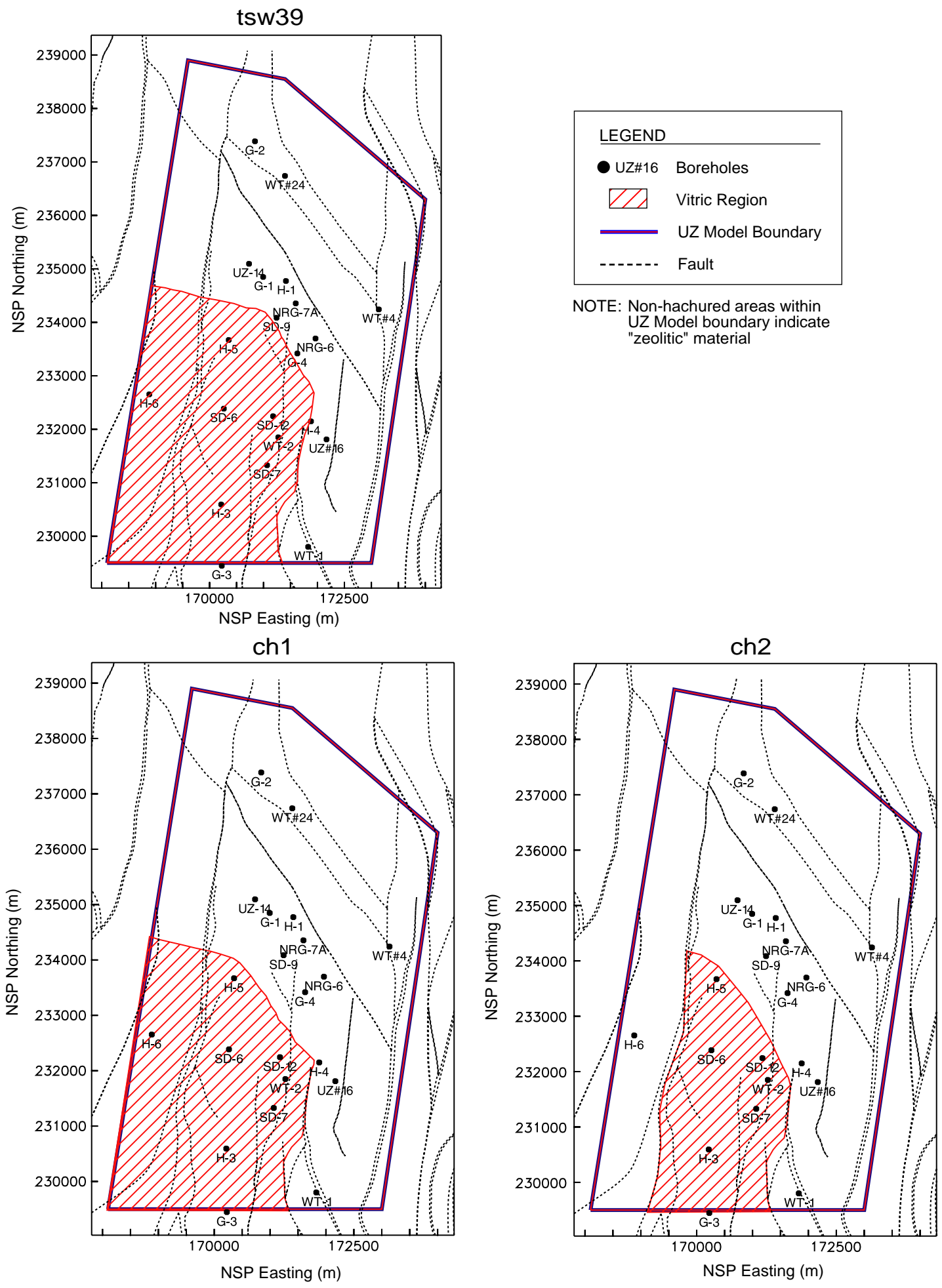

Source: BSC 2004 [DIRS 169855], Figure 6-7.

Figure 6-29a. Mineralogy Model Plots below the Repository 

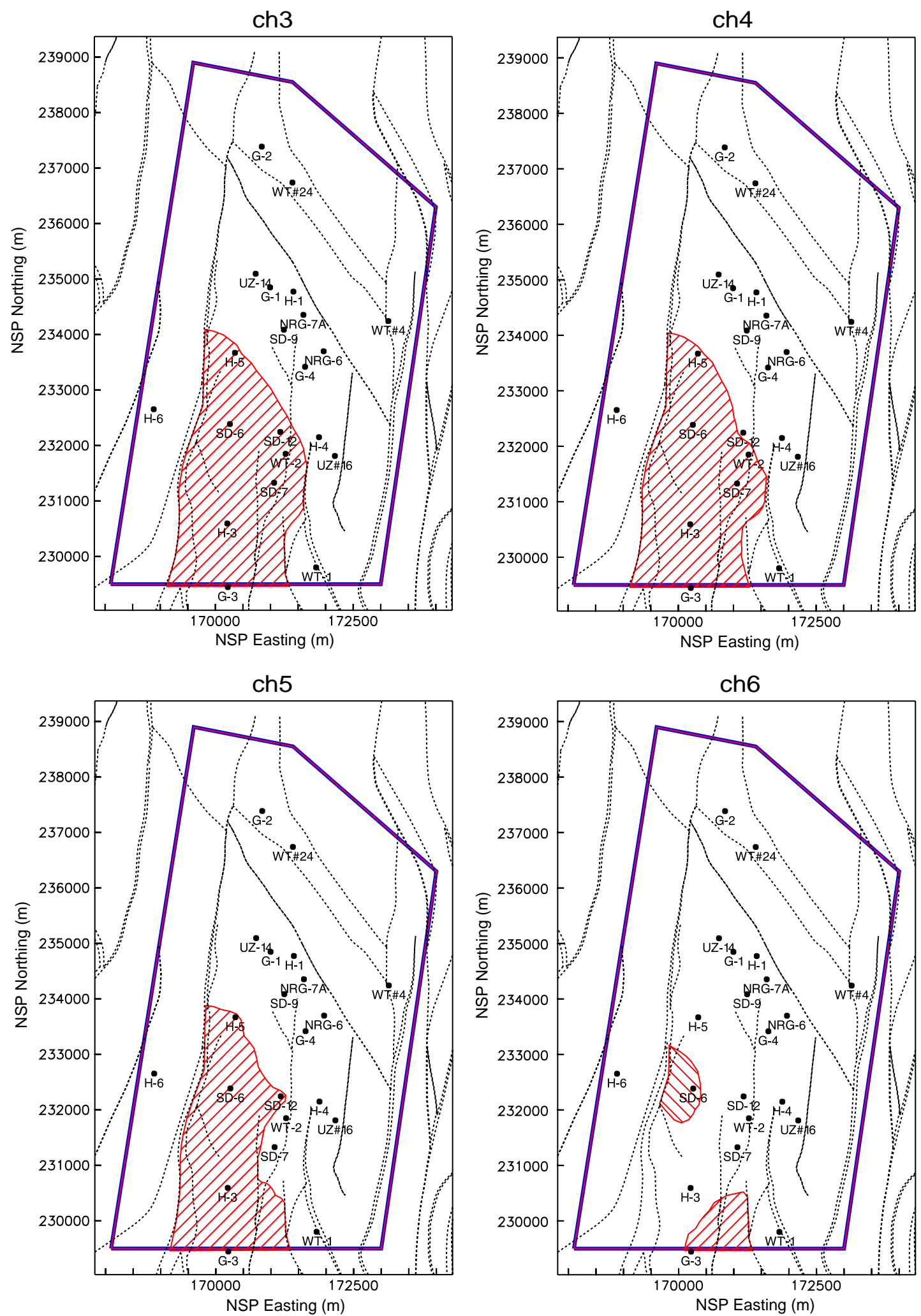

Source: BSC 2004 [DIRS 169855], Figure 6-8.

Figure 6-29b. Mineralogy Model Plots below the Repository 


\section{Present Day Infiltration (Mean)}

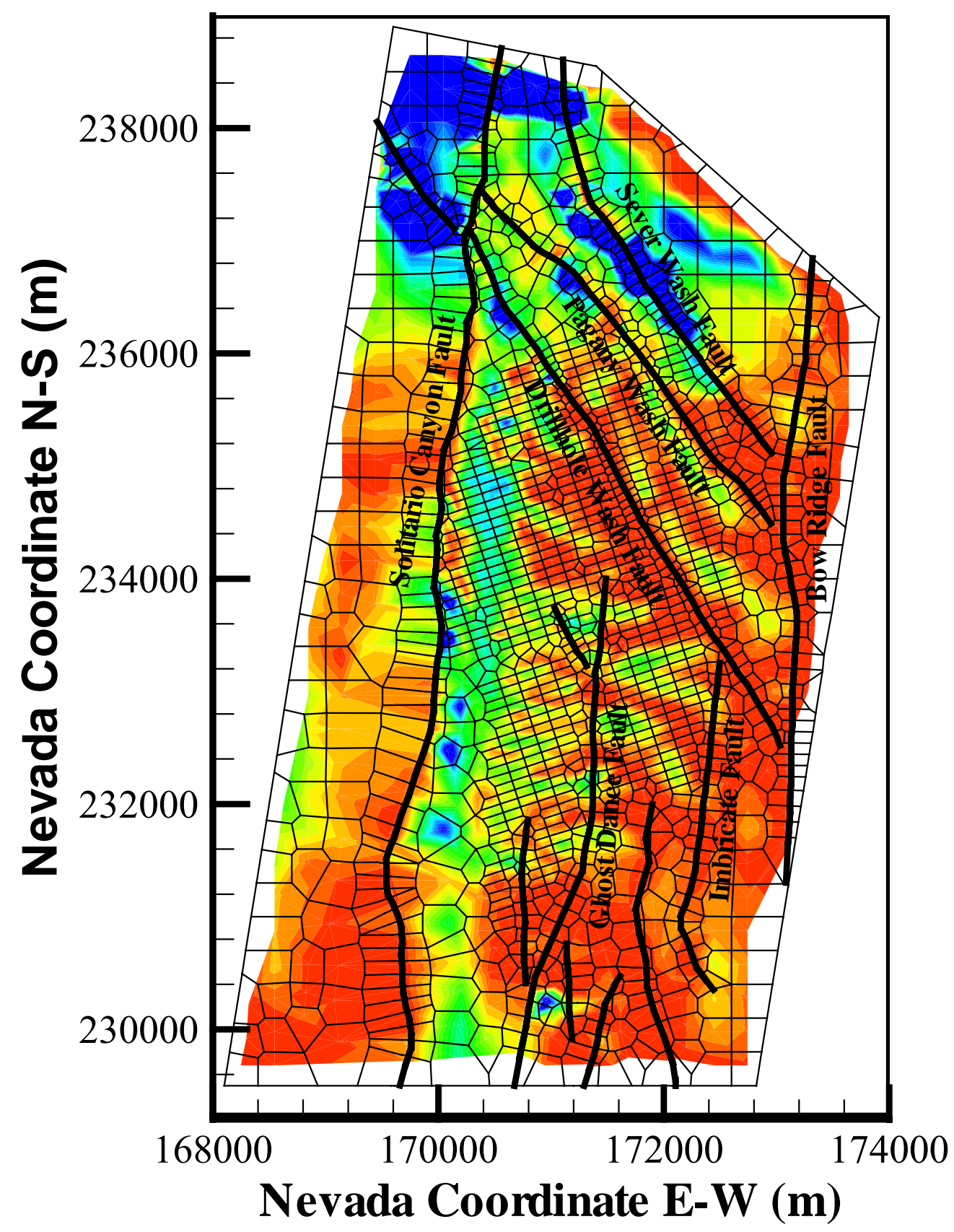

(mm/year)

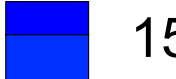

14.25

13.5

12.75

12

11.25

10.5

9.75

9

8.25

7.5

6.75

6

5.25

4.5

3.75

3

2.25

1.5

0.75

Source: BSC 2004 [DIRS 169861], Figure 6.1-2.

Figure 6-30. Mean Present-Day Infiltration Rates at the Surface 


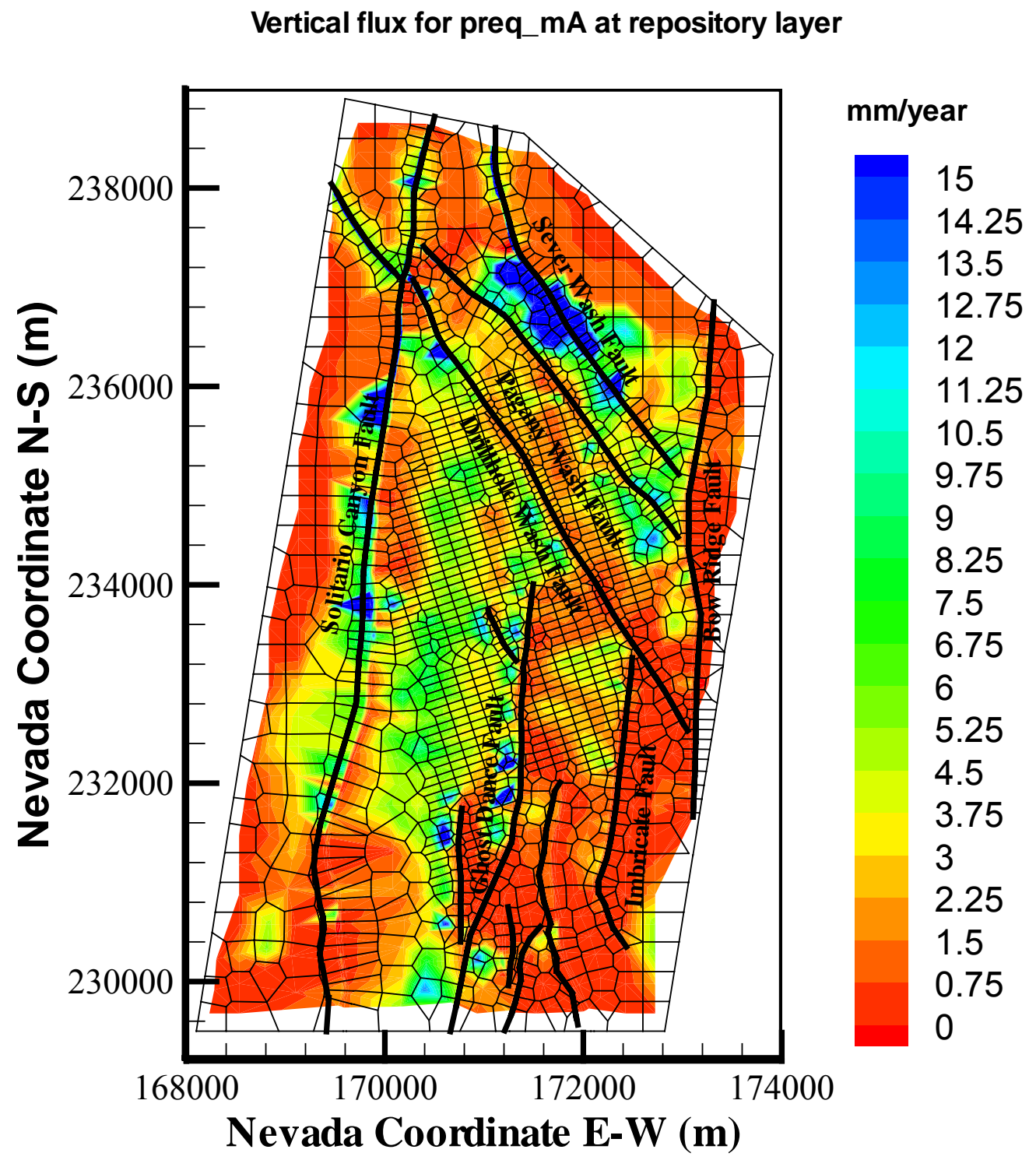

Source: BSC 2004 [DIRS 169861], Figure 6.6-1.

Figure 6-31. Percolation Fluxes at the Repository Level 


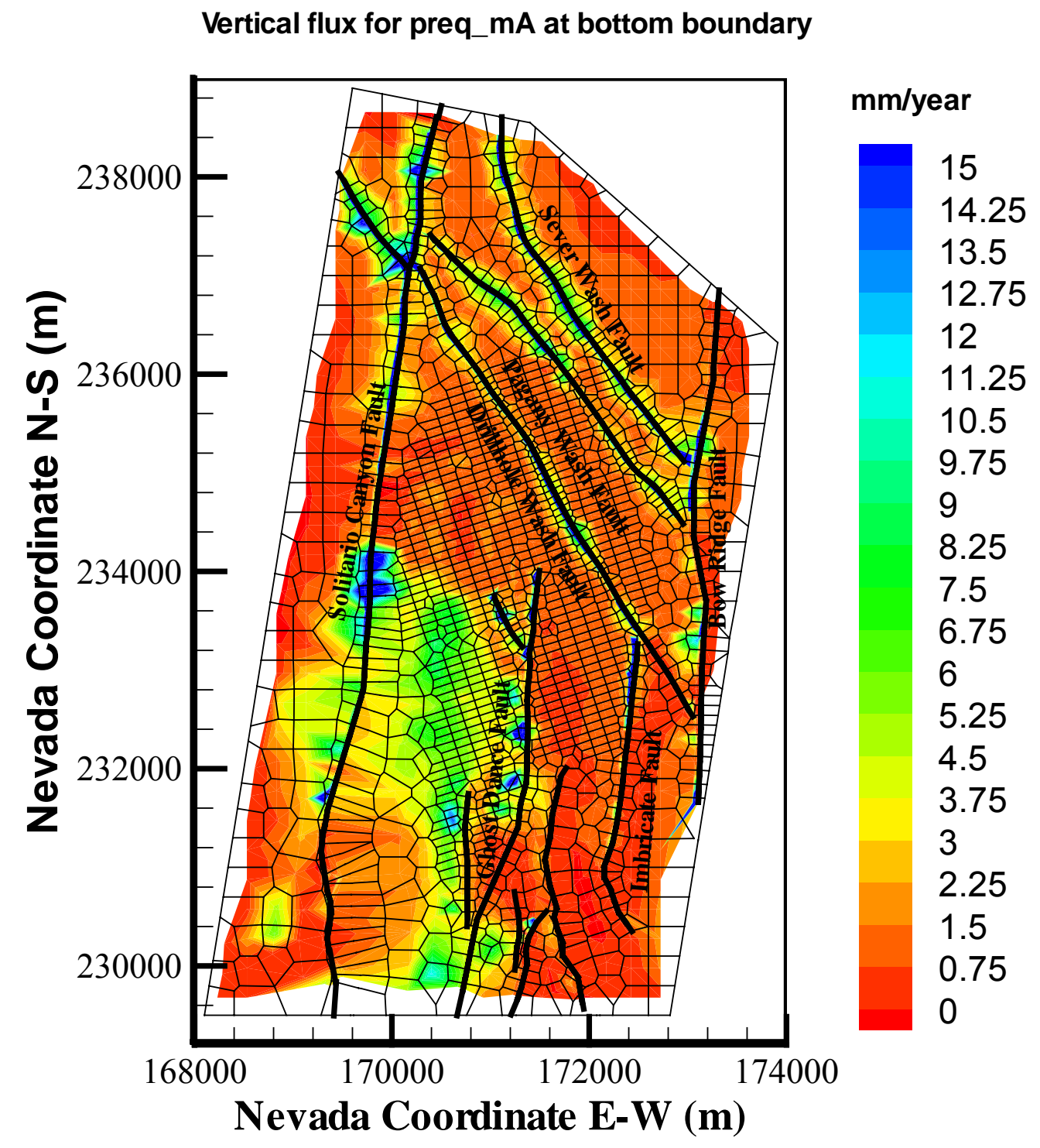

Source: BSC 2004 [DIRS 169861], Figure 6.6-6.

Figure 6-32. Percolation Fluxes at the Water Table Level 


\subsection{2 $\quad{ }^{99}$ Tc Transport under Monsoon and Glacial-Transition Infiltrations}

The DTNs of the input and output files for the three monsoon and the three glacial-transition infiltration scenarios are listed in Table 6-6. The monsoon and glacial-transition infiltration rate is higher than that for present-day infiltration of the same level (Table 6-5). The simulation results are consistent with the observations made in Section 6.8.1.

The higher infiltration rates result in lower $t_{10}$ and $t_{50}$ values than those for present-day infiltration. Thus, for low, mean, and high monsoon infiltration, the $t_{10}$ is $22,9.4$, and 2.4 years, respectively (Figure 6-33). For the same infiltration regimes, the corresponding $t_{50}$ is 1,$310 ; 417$; and 92.4 years. These times are significantly smaller than the ones for the present-day infiltration scenario (Figure 6-8), indicating faster transport through the system and earlier appearance of ${ }^{99} \mathrm{Tc}$ at the water table. Because transport is faster than under present-day infiltration, transport times to the water table are shorter and thus the amount of ${ }^{99} \mathrm{Tc}$ lost to radioactive decay is smaller. This is evident in Figure 6-33, in which the maximum attainable $R_{M}$ values are shown (a) to increase with the infiltration rate and (b) to be higher and closer to each other than those for present-day infiltration (Figure 6-8).

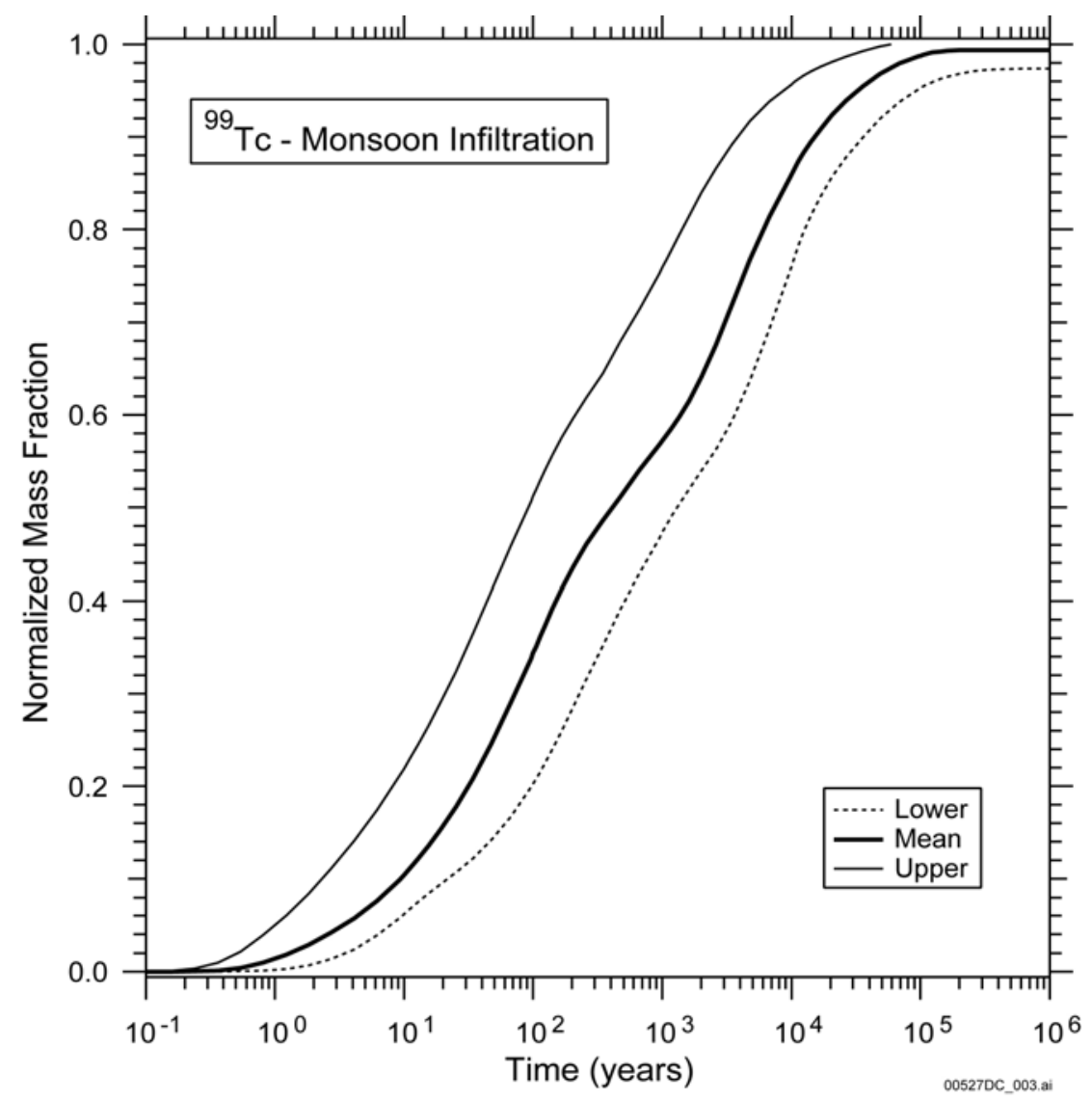

Output DTN: LB0307MR0060R1.007, data submitted with this model report.

Figure 6-33. Cumulative Breakthrough of the ${ }^{99} \mathrm{Tc}$ Mass Fraction $R_{M}$ at the Water Table for Varying Monsoon Climatic Scenarios and Instantaneous Release 


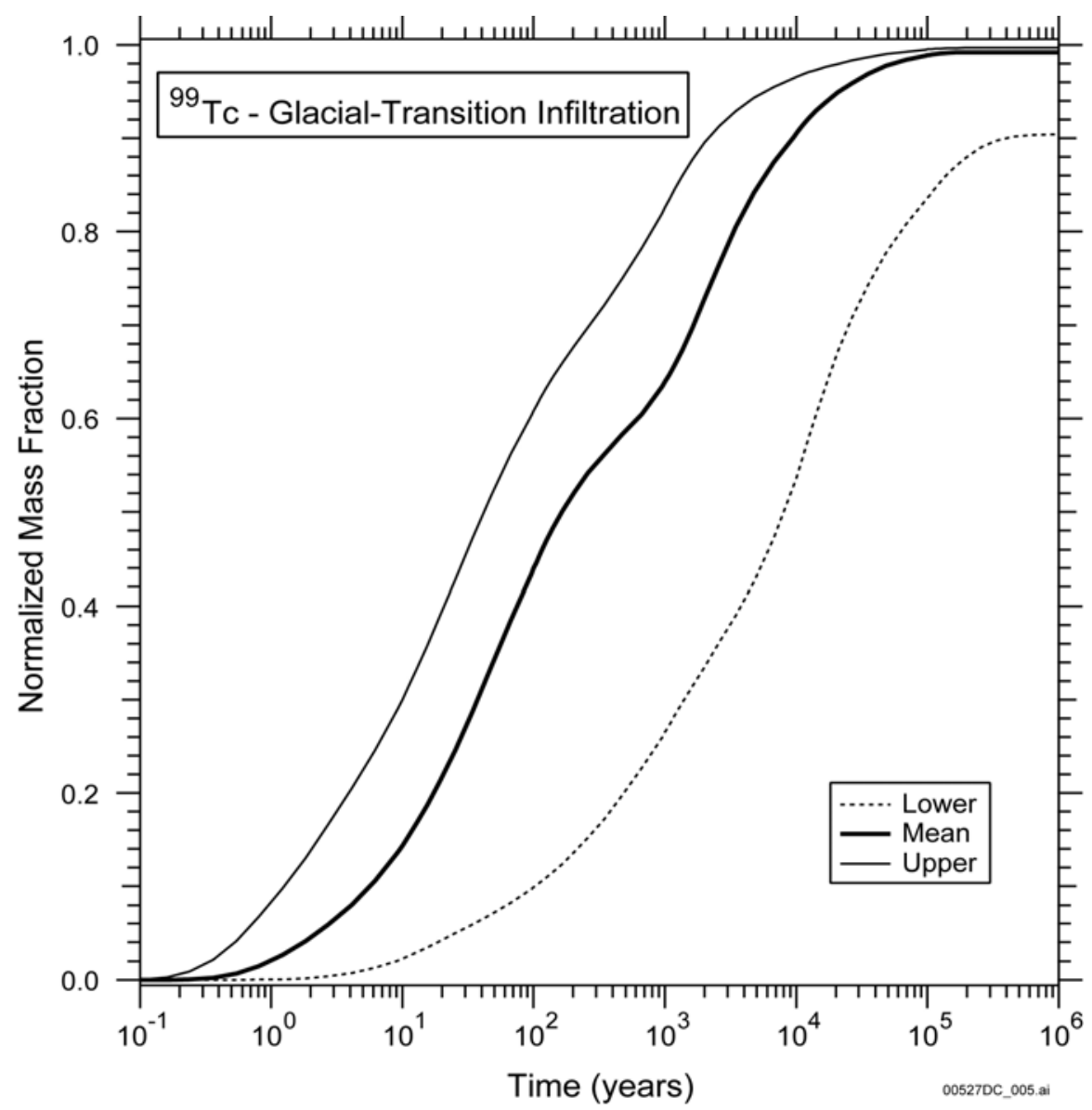

Output DTN: LB0307MR0060R1.007, data submitted with this model report.

Figure 6-34. Cumulative Breakthrough of the ${ }^{99}$ Tc Mass Fraction $R_{M}$ at the Water Table for Varying Glacial-Transition Climatic Scenarios and Instantaneous Release

For transport under glacial-transition infiltration, Figure 6-34 indicates very early arrivals at the water table. Thus, the $t_{10}$ for high, mean, and low glacial-transition infiltration are only 1.25 , 5.68, and 102 years, respectively, while the corresponding $t_{50}$ values are $42.3 ; 164$; and 8,140 years, respectively. As in the case of monsoon infiltration (Figure 6-33), the maximum attainable $R_{M}$ for high and mean glacial-transition infiltration is almost 1, indicating fast transport through the three-dimensional system.

\subsubsection{Uncertainties in ${ }^{99}$ Tc Transport Predictions}

The predictions of ${ }^{99} \mathrm{Tc}$ transport are subject to three general types of uncertainties.

\subsubsection{Uncertainties in the Estimates of Flow and Hydraulic Properties}

The impact of uncertainties in the estimate of flow and hydraulic properties is in the estimation of the magnitude and relative sizes of flows and saturations in the fractures, and in the matrices of the UZ. These directly affect both advective transport (and, consequently, transport times to the water table) and diffusive fluxes (see Equations 6-18 to 6-21). Although the importance of 
these uncertainties is readily recognized, addressing them is beyond the scope of this study, but rather the subject of the model report on the UZ flow model (BSC 2004 [DIRS 169861], Section 6). Because the radionuclide transport study is intertwined with UZ flow model (from which it derives its flow fields), it has the same flow-related limitations and uncertainties.

\subsubsection{Uncertainties in the Retardation Processes}

The only nondecay mechanisms of ${ }^{99} \mathrm{Tc}$ retardation in the process of transport through the UZ is matrix diffusion (including dispersion) because it is nonsorbing, and chemical immobilization is not considered. Uncertainties in matrix diffusion are reflected in the values of the diffusion coefficient $D_{0}$. Given the uncertainties in the estimation of the parameters in Equations 6-20 and 6-21, the choice of $D_{0}$ in general influences the effective diffusion coefficient.

To address this issue, the sensitivity of the ${ }^{99} \mathrm{Tc}$ transport through the UZ was investigated for the $D_{0}$ values shown in Table 6-4, which cover the possible $D_{0}$ range. The results are shown in Figure 6-35. As expected, $D_{0}$ has a significant impact on breakthrough predictions, resulting in faster arrival times for lower $D_{0}$ values.

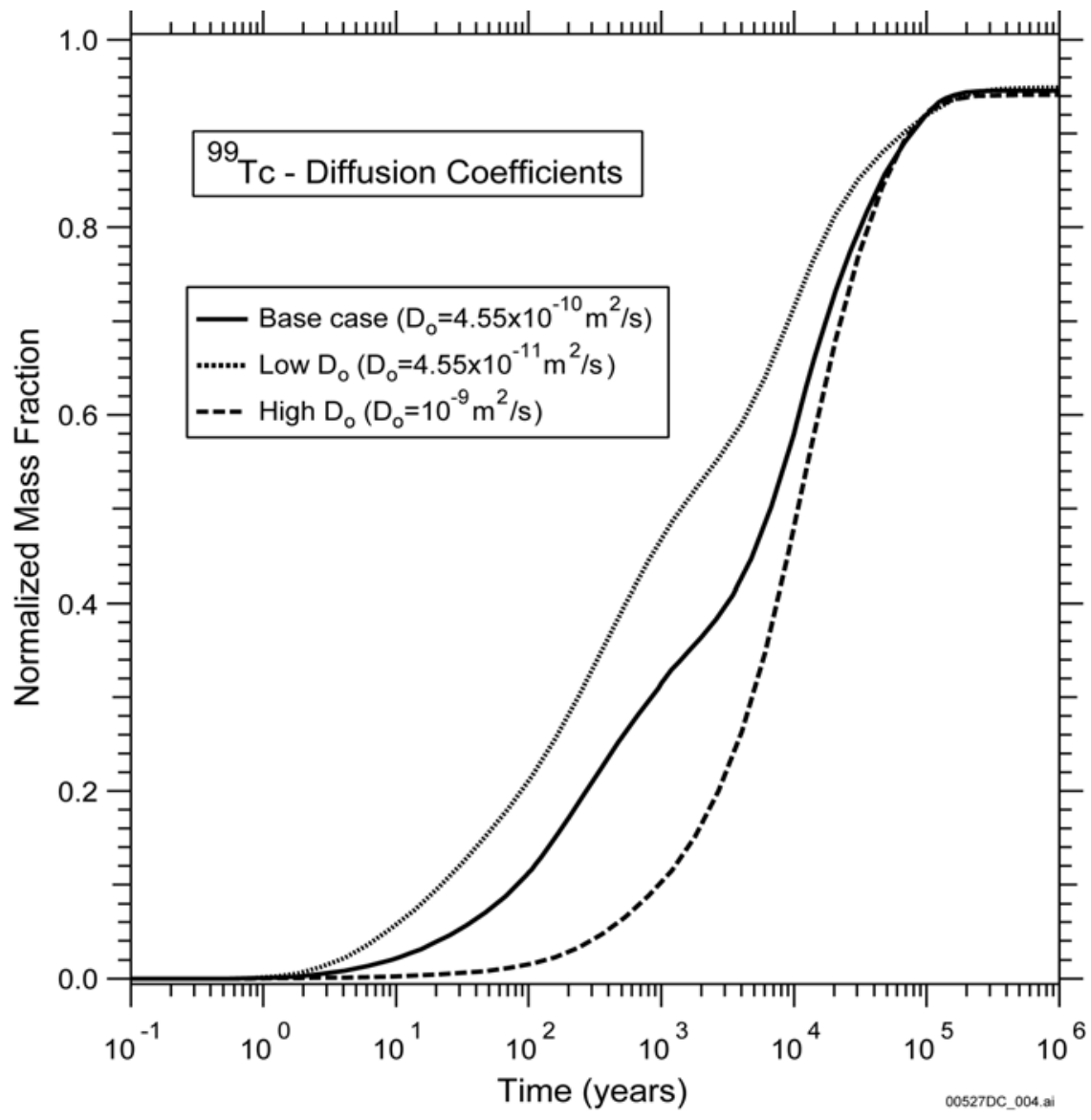

Output-DTN: LB0307MR0060R1.007, data submitted with this model report.

Figure 6-35. Effect of Uncertainty in $\mathrm{D}_{0}$ on the Cumulative Breakthrough of the ${ }^{99} \mathrm{Tc}$ Mass Fraction $R_{M}$ at the Water Table for Mean Present-Day Infiltration and Instantaneous Release 


\subsubsection{Climatic Uncertainties}

As the results in the previous sections on ${ }^{99} \mathrm{Tc}$ transport attest to, climatic changes affect the infiltration regime and can have a profound effect on transport. This uncertainty was addressed by estimating transport under all possible nine climatic scenarios of Table 6-5 (Figures 6-8, 6-33, and 6-34), thus bounding the possible solution.

\subsection{THREE-DIMENSIONAL TRANSPORT OF ${ }^{237} \mathrm{~Np}$ - INSTANTANEOUS RELEASE}

This section discusses the transport of ${ }^{237} \mathrm{~Np}$ through the UZ of Yucca Mountain following an instantaneous release (Section 6.7). The three-dimensional site-scale simulations are conducted by means of T2R3D.

Section 6.9 includes three subsections. Section 6.9.1 discusses the transport of the moderately sorbing ${ }^{237} \mathrm{~Np}$ for three levels of present-day infiltration. The results of this study are shown in Figure 6-36, and in Figures D-1 to D-24 of Appendix D. Section 6.9.2 provides a study of the transport of ${ }^{237} \mathrm{~Np}$ for three levels of (a) monsoon infiltration (Figure 6-38) and (b) glacial-transition infiltration (Figure 6-39). Section 6.9.3 addresses uncertainties involved in the predictions of ${ }^{237} \mathrm{~Np}$ transport, and involves sensitivity analyses to develop the envelope of possible solutions.

\subsubsection{Transport of ${ }^{237} \mathrm{~Np}$ Under Present-Day Infiltration}

The grids, flow fields, and conditions are as discussed in Section 6.7. The parameters used in the T2R3D simulations of three-dimensional transport of the moderately sorbing ${ }^{237} \mathrm{~Np}$ are listed in Tables 6-3, 6-4, 6-5, and 6-6. The DTN of the input and output files for the three present-day infiltration scenarios is output DTN: LB0307MR0060R1.001 (Table 6-6), and output DTN: LB0307MR0060R1.002.

\subsubsection{Breakthrough Curves}

The moderate sorption of ${ }^{237} \mathrm{~Np}$ results in retardation of its transport through the UZ system, as the cumulative breakthrough $R_{M}$ of the ${ }^{237} \mathrm{~Np}$ mass fraction at the water table indicates (Figure 6-36). Comparison to that of the nonsorbing ${ }^{99} \mathrm{Tc}$ (Figure 6-8) reveals a very different pattern. Thus, the moderate sorption of ${ }^{237} \mathrm{~Np}$ is sufficient to result in a large increase in $t_{10}$ and $t_{50}$ compared to those for the nonsorbing ${ }^{99} \mathrm{Tc}$.

Increased infiltration rate leads to faster transport and shorter transport times to the repository. When infiltration increases from mean to high, $t_{10}$ and $t_{50}$ are reduced from 410 and 25,400 to 4.5 and 1,600 years, respectively. Reduction of infiltration to the low present-day level results in analogous increase of $t_{10}$ to 33,800 , while $t_{50}$ is not reached until after $1,000,000$ years.

Because of significant retardation, the maximum attainable $R_{M}$ varies over a larger value range and does not necessarily reach a plateau within the simulation period. At $\mathrm{t}=1,000,000$ years, $R_{M}$ for mean and low infiltration is 0.96 and 0.48 , respectively, and the maximum possible values for either infiltration are not yet reached. At the same time, the $R_{M}$ for high infiltration is approaching its maximum at $R_{M}=0.99$. 


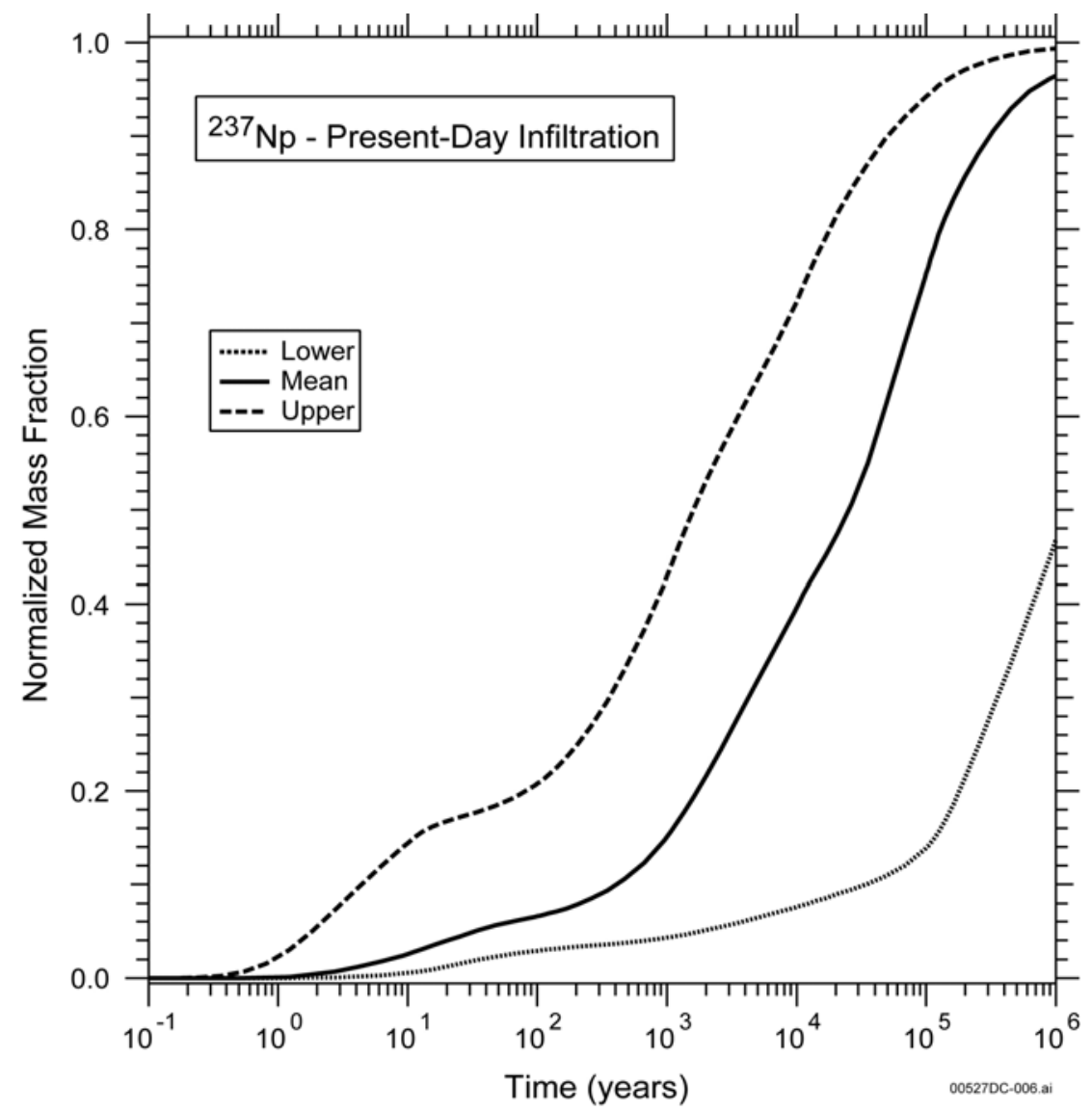

Output DTN: LB0307MR0060R1.007, data submitted with this model report.

Figure 6-36. Cumulative Breakthrough of the ${ }^{237} \mathrm{~Np}$ Mass Fraction $R_{M}$ at the Water Table for Varying Present-Day Climatic Scenarios and Instantaneous Release

\subsubsection{Transport Mechanisms and Patterns}

The importance of fractures on the ${ }^{237} \mathrm{~Np}$ transport becomes evident in Figure D-1 (DTN: LB0307MR0060R1.002), which shows the areal distribution of $X_{R}$ (see Section 6.9.2.2) in the aqueous phase in the fractures immediately above the TSw-CHn interface (i.e., in the tsw39 layer) at $t=10$ years (under mean present-day infiltration). As in the case of ${ }^{237} \mathrm{~Np}$, the Drill Hole Wash fault and Pagany Wash fault are clearly identified, as they define the extent of radionuclide presence and distribution at this level. The contrast with the distribution of $X_{R}$ in the aqueous phase of the matrix (Figure D-2), which shows no discernible difference from the background $X_{R}=0$ in the vicinity of these faults, is dramatic. Figure D-2 shows significant concentrations in the matrix in the southern part of the repository, where the permeability in the fractures is about the same as that in the matrix (see discussion in Sections 6.8.1.2 and 6.8.1.3).

Figures D-3 and D-4 show the fracture $X_{R}$ and the matrix $X_{R}$ distribution of ${ }^{237} \mathrm{~Np}$ in the tsw39 layer at $t=100$ years. Note the significant similarities to the corresponding distribution patterns in Figures D-1 and D-2. It is noteworthy that the fracture $X_{R}$ distribution shows significantly lower peak values, and that the contributions of the Sever Wash fault and the Sundance fault to transport to the tsw39 unit are becoming apparent at this time. 
The fracture $X_{R}$ and matrix $X_{R}$ at $\mathrm{t}=1,000$ years are shown in Figures D-5 and D-6, respectively. The magnitude of the fracture $X_{R}$ follows the downward trend identified in Figure D-3, although the areal distribution of ${ }^{237} \mathrm{~Np}$ occurrence in the fracture continuum expands, and the signatures of the main faults (Drill Hole Wash, Pagany Wash, Sever Wash, Sundance) become more prominent. The matrix $X_{R}$ in Figure D-6 shows the same footprint as those at $\mathrm{t}=10$ years and $\mathrm{t}=100$ years (Figures D-2 and D-4), but the maximum $X_{R}$ level is substantially lower. This indicates that the bulk of the mass of the instantaneously released ${ }^{237} \mathrm{~Np}$ has moved past the tsw39 level at $\mathrm{t}=100$ years in the southern part of the repository.

Figures D-7 and D-8 show the fracture $X_{R}$ and the matrix $X_{R}$ distribution of ${ }^{237} \mathrm{~Np}$ in the tsw39 layer at $t=10,000$ years, respectively, and reveal a change in the transport regimes. The areal extent of the fracture $X_{R}$ increases compared to that at earlier times. This is attributed to transport through fractures over a larger area, as ${ }^{237} \mathrm{~Np}$ arrives from the repository at the bottom of the TSw through less conductive fractures, and/or after the radionuclide reaches such fractures (following diffusion into the matrix from the main fast fractures). The matrix $X_{R}$ distribution in Figure D-8 confirms the earlier indication that the bulk of the ${ }^{237} \mathrm{~Np}$ mass has moved past the tsw39 layer because the extent and magnitude of the radionuclide signature in the southern part keeps decreasing. Conversely, the matrix in the vicinity of the main faults in the northern part of the repository is showing increasing evidence of ${ }^{237} \mathrm{~Np}$, the source of which is diffusion into the matrix through the fracture walls (since sufficient time has elapsed to allow it) and/or direct matrix flow.

At $\mathrm{t}=100,000$ years, the Solitario Canyon fault begins to contribute to the fracture $X_{R}$ (Figure D-9), although the matrix $X_{R}$ values continue to decrease as the radionuclide mass moves toward the water table and some of it sorbs onto the UZ rocks. This is further supported by the matrix $X_{R}$ distribution of ${ }^{237} \mathrm{~Np}$ at the same time (Figure D-10), which shows a faint signal practically everywhere as the bulk of the ${ }^{237} \mathrm{~Np}$ mass has already receded past the tsw39 layer. At $t=1,000,000$ years (Figures D-11 and D-12), there is only very faint evidence of ${ }^{237} \mathrm{~Np}$ in the vicinity of the Pagany Wash fault.

The $X_{R}$ distribution immediately above the water table provides another indication of the importance of fractures. For mean present-day infiltration, the fracture $X_{R}$ of ${ }^{237} \mathrm{~Np}$ immediately above the water table at $\mathrm{t}=10$ years (Figures D-13) clearly defines the Drill Hole Wash and Pagany Wash faults. The corresponding matrix $X_{R}$ (Figure D-14) shows a much weaker signature along the Drill Hole Wash fault. At $\mathrm{t}=100$ years, the fracture $X_{R}$ (Figure D-15) indicates concentration of the radionuclide along the main faults (i.e., the Drill Hole Wash and Pagany Wash faults, and, to a lesser extent, the Sever Wash and Sundance faults). It is remarkable that the matrix $X_{R}$ at the same time (Figure D-16) does not show the same pattern, but begins to show the effect of transport through the Sundance fault. Thus, the fast-conducting faults limit the extent of the ${ }^{237} \mathrm{~Np}$ lateral penetration into the matrix through the fracture walls because of the limited radionuclide mass, the high water velocity (which does not permit substantial diffusion), and the ${ }^{237} \mathrm{~Np}$ sorption onto the matrix.

At 1,000 and 10,000 years, the patterns described in Figures D-15 and D-16 persist. The Drill Hole Wash and Pagany Wash faults continue to dominate transport (Figures D-17 and D-19), while the vicinity of the Sundance fault is the location of significant matrix $X_{R}$ values (Figures D-18 and D-20 at $\mathrm{t}=1,000$ years and $\mathrm{t}=10,000$ years, respectively). The larger areal 
extent of fracture $X_{R}$ at $\mathrm{t}=10,000$ years results in the very low matrix $X_{R}$ signature of the Drill Hole Wash fault. The faults lead to relatively fast transport from the repository to the water table, resulting in short residence times and limited penetration into the matrix (and, consequently, limited retardation through sorption).

At longer times, the effects of limited radionuclide mass (a consequence of the instantaneous release scenario), sorption, and decay are demonstrated by the decreasing magnitude and areal extent of the fracture and matrix $X_{R}$ (Figures D-21 to D-24).

\subsubsection{Transport-Controlling Features}

Review of Figures D-1 through D-24 (see Appendix D), and comparison to the corresponding ${ }^{99}$ Tc figures (Figures 6-9 through 6-24) indicate that transport is both dominated and controlled by the same faults identified and discussed in Sections 6.8.1.2 and 6.8.1.3. Thus, the Drill Hole Wash fault and the Pagany Wash fault are the main transport-facilitating geologic features, and leads to the early appearance of ${ }^{237} \mathrm{~Np}$ in the tsw39 layer and at the water table. What is remarkable in the case of ${ }^{237} \mathrm{~Np}$ is that that the Drill Hole Wash fault and the Pagany Wash fault play an even more important role in transport, as they appear to convey a larger portion of the released mass and in a shorter time than in the case of ${ }^{99} \mathrm{Tc}$. These faults are very clearly identified as conduits of very fast transport to the water table. The Sever Wash fault and the Sundance fault are important at later times, but their contributions to transport are of lesser importance. The faults appear to act as barriers to the lateral migration of radionuclides.

As in the case of ${ }^{99} \mathrm{Tc}$ transport, radionuclide transport to the groundwater is much faster in the northern part of the repository, where it is also areally concentrated. This is caused by (a) the infiltration and percolation patterns; (b) the fast conduit to transport provided by the faults; (c) the permeability regimes (and their effect on flow and to transport) in the northern part; and (d) the distribution, fracture characteristics, and flow behavior of the $\mathrm{CHz}$ and $\mathrm{CHv}$ layers. A detailed discussion of transport patterns can be found in Section 6.9.2.3.

\subsubsection{Comparison to the ${ }^{99}$ Tc Transport}

The obvious reason for the difference between the ${ }^{99} \mathrm{Tc}$ and the ${ }^{237} \mathrm{~Np}$ transport behavior is sorption. This results in (a) the universally lower (than ${ }^{99} \mathrm{Tc}$ ) concentrations and the more limited areal extent (footprint) of ${ }^{237} \mathrm{~Np}$ in the fractures and in the matrix at the same times, (b) the persistence over a longer time of the area underneath the southern part of the repository in tsw39 as the location where the matrix $X_{R}$ is significant (Figures D-6 and D-8, vs. Figures 6-14 and 6-16), (c) the slower breakthroughs indicated by Figures D-12 through D-24 and Table 6-10, and (d) the more limited extent of the radionuclide penetration into the UZ.

\subsubsection{Effect of Sorption in the Main Geologic Units on ${ }^{237} \mathrm{~Np}$ Transport}

Of particular interest is the effect of the main hydrogeologic units (i.e., TSw, $\mathrm{CHv}$ and $\mathrm{CHz}$ ) on the transport and retardation of ${ }^{237} \mathrm{~Np}$. This was investigated by setting the corresponding distribution coefficients $K_{d}$ in all of the layers of each of these units to zero, and then comparing the resulting cumulative breakthrough curve to that for the base case. 
The results (for a mean present-day infiltration scenario) are shown in Figure 6-37, and indicate that $\mathrm{TSw}$ is by far the most important unit in the transport and retardation of ${ }^{237} \mathrm{~Np}$. By eliminating sorption in the all the TSw layers, water table arrivals of ${ }^{237} \mathrm{~Np}$ occur significantly earlier. The effect of no sorption in the $\mathrm{CHz}$ and $\mathrm{CHv}$ units are secondary (if not marginal), and become apparent only at later times. Given that no sorption occurs in the fractures, the lack of any appreciable retardation during advection through the $\mathrm{CHz}$ and $\mathrm{CHv}$ tends to further indicate that fracture flow is the overwhelmingly dominant mechanism of transport through these units, while advection through matrix flow does not play a significant role.

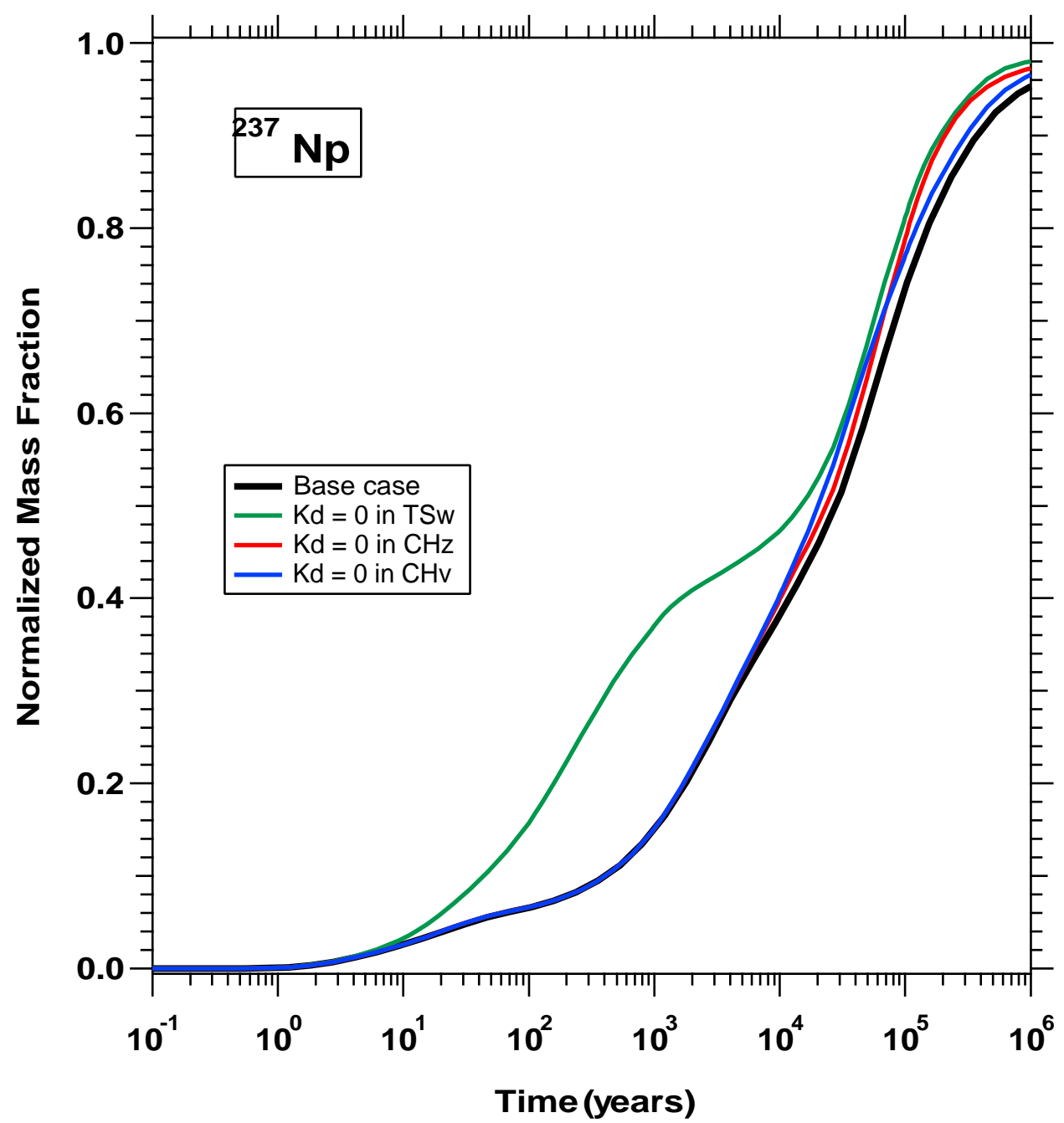

Output DTN: LB0310MR0060R1.010, data submitted with this model report.

Figure 6-37. The effect of sorption in $\mathrm{TSw}, \mathrm{CHz}$, and $\mathrm{CHv}$ on the Cumulative Breakthrough of ${ }^{237} \mathrm{~Np}$ at the Water Table for Mean Present-Day Infiltration and Instantaneous Release 


\subsection{2 $\quad{ }^{237} \mathrm{~Np}$ Transport under Monsoon and Glacial-Transition Infiltration}

The DTN of the input and output files for the three monsoon infiltration scenarios is output DTN：LB0307MR0060R1.001 (Table 6-6), output DTN: LB0307MR0060R1.002. The higher monsoon and glacial-transition infiltration rates (compared to those in present-day regimes, see Table 6-5) lead to the faster transport indicated in Figures 6-38 and 6-39 (when compared to that shown in Figure 6-36).

The $t_{10}$ for low, mean, and high monsoon infiltration is $14.8,8.5$, and 1.6 years, respectively. The corresponding $t_{50}$ are 6,$140 ; 2,120$; and 714 years, respectively. These times are significantly lower than the ones for the present-day infiltration scenario, as the faster transport through the system would warrant. Because transport is faster than under present-day infiltration, radioactive decay of the released ${ }^{237} \mathrm{~Np}$ has a less pronounced effect in the reduction of the $R_{M}$ at a given time.

The $t_{10}$ for low, mean, and high glacial-transition infiltration is 1830,4 , and 0.8 years, respectively. The corresponding $t_{50}$ are 34,$400 ; 1,070$; and 336 years, respectively. For high and mean glacial-transition infiltration, the $t_{10}$ and $t_{50}$ times are the shortest in the three infiltration regimes.

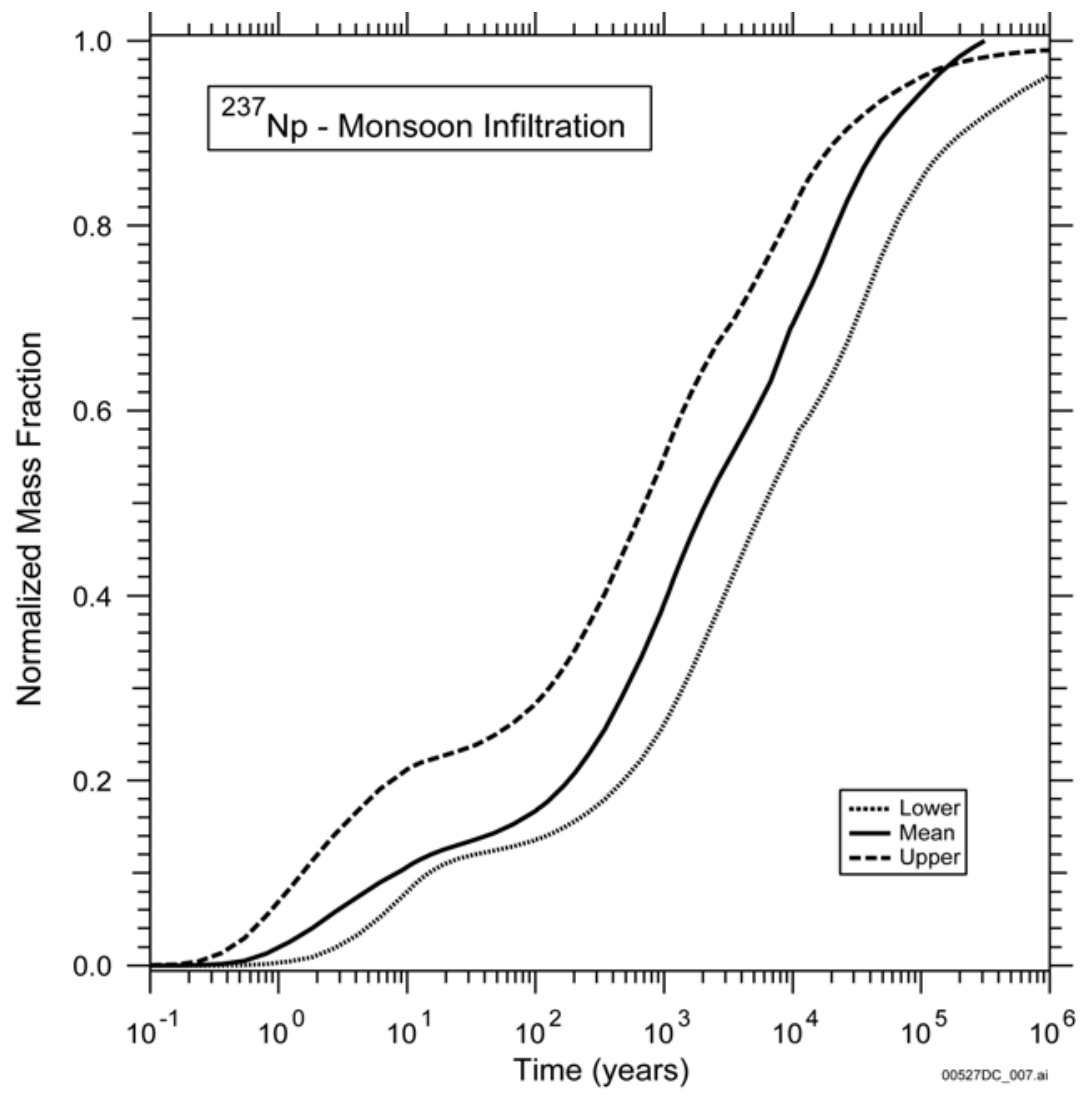

Output DTN: LB0307MR0060R1.007, data submitted with this model report.

Figure 6-38. Cumulative Breakthrough of the ${ }^{237} \mathrm{~Np}$ Mass Fraction $R_{M}$ at the Water Table for Varying Monsoon Climatic Scenarios and Instantaneous Release 


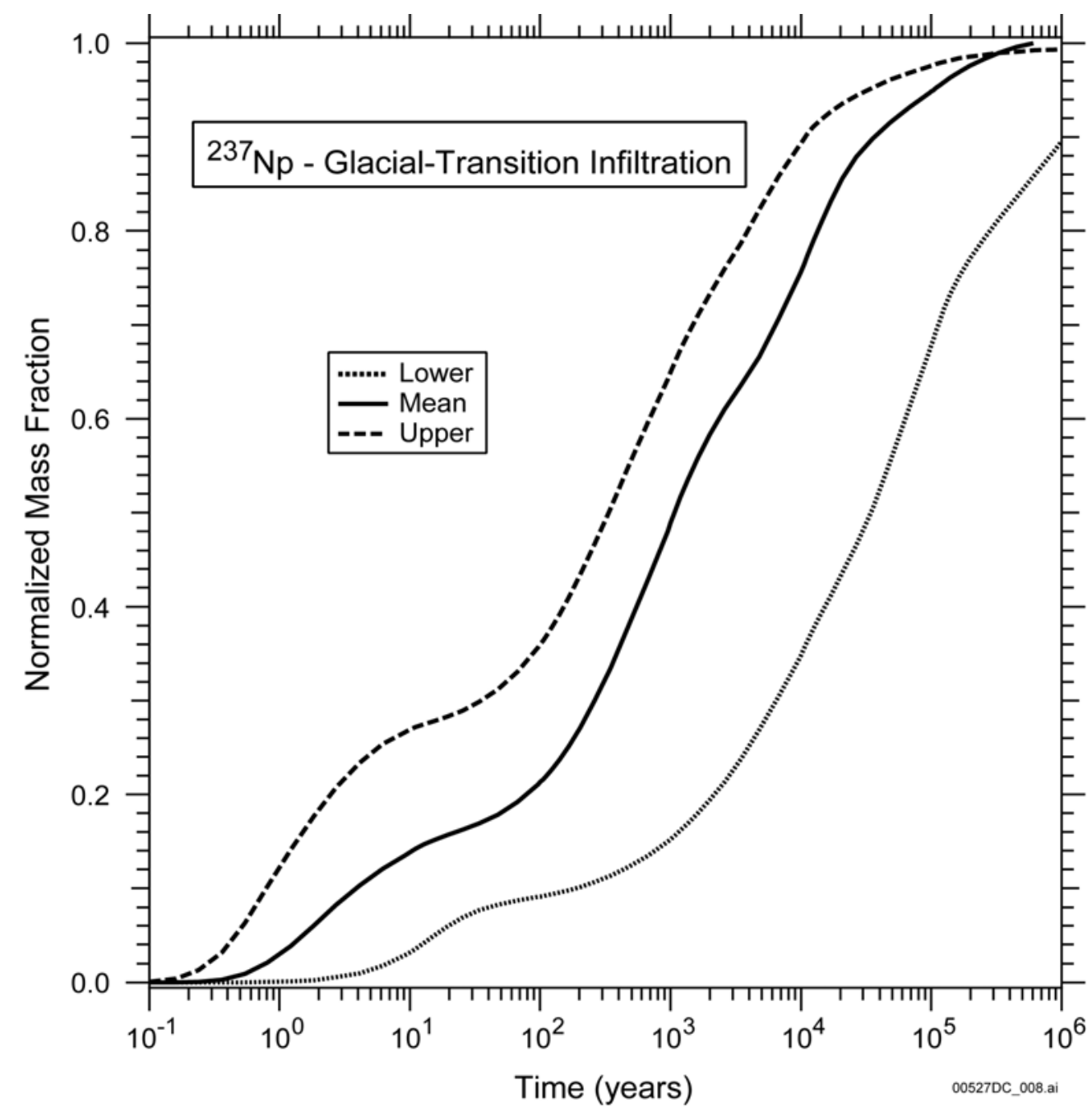

Output DTN: LB0307MR0060R1.007, data submitted with this model report.

Figure 6-39. Cumulative Breakthrough of the ${ }^{237} \mathrm{~Np}$ Mass Fraction $R_{M}$ at the Water Table for Varying Glacial-Transition Climatic Scenarios and Instantaneous Release

\subsubsection{Uncertainties in ${ }^{237} \mathrm{~Np}$ Transport Predictions}

The predictions of ${ }^{237} \mathrm{~Np}$ transport are subject to three general types of uncertainties discussed in Section 6.8.3. For the reasons discussed in that section, this section is limited to the issue of uncertainties in the transport retardation processes. Because ${ }^{237} \mathrm{~Np}$ is a sorbing radionuclide, uncertainties involve both diffusion and sorption issues.

\subsubsection{Uncertainties in Matrix Diffusion}

As previously indicated, uncertainties in matrix diffusion are reflected in the values of the diffusion coefficient $D_{0}$. To address this issue, the sensitivity of the ${ }^{237} \mathrm{~Np}$ transport through the UZ was investigated for the $D_{0}$ values shown in Table 6-4, which cover the possible $D_{0}$ range. The results are shown in Figure 6-40. 
As expected, $D_{0}$ has a significant impact on breakthrough predictions, resulting in faster arrival times for lower $D_{0}$ values.

The impact of uncertainty in the effective interfacial area between the fractures and matrix, which is related to the degree of flow focusing in the fractures, is not evaluated in this particular simulation. Insight into the impact of this uncertainty in the effective interfacial area can be seen in Section 7.6.1 where the sensitivity of mountain-scale results to the active fracture parameter $\gamma$ which modulates the effective interfacial area is evaluated.

\subsubsection{Uncertainties in Sorption}

Such uncertainties in sorption are reflected in the values of the distribution coefficient $K_{d}$. To address this issue, the sensitivity of the ${ }^{237} \mathrm{~Np}$ transport through the UZ was investigated for the $K_{d}$ values shown on Figure 6-41. These cover the range between zero (no sorption, in which case behavior similar to that of ${ }^{99} \mathrm{Tc}$ was expected) to the maximum values reported in Table 6-3. The results are shown in Figure 6-41.

As expected, $K_{d}$ has a significant impact on breakthrough predictions, resulting in faster arrival times for lower $K_{d}$ values. Note that the relative effect of $K_{d}$ uncertainty predicting transport of the sorbing ${ }^{237} \mathrm{~Np}$ appears to be much more pronounced than that for the $D_{0}$ uncertainty for the range tested here.

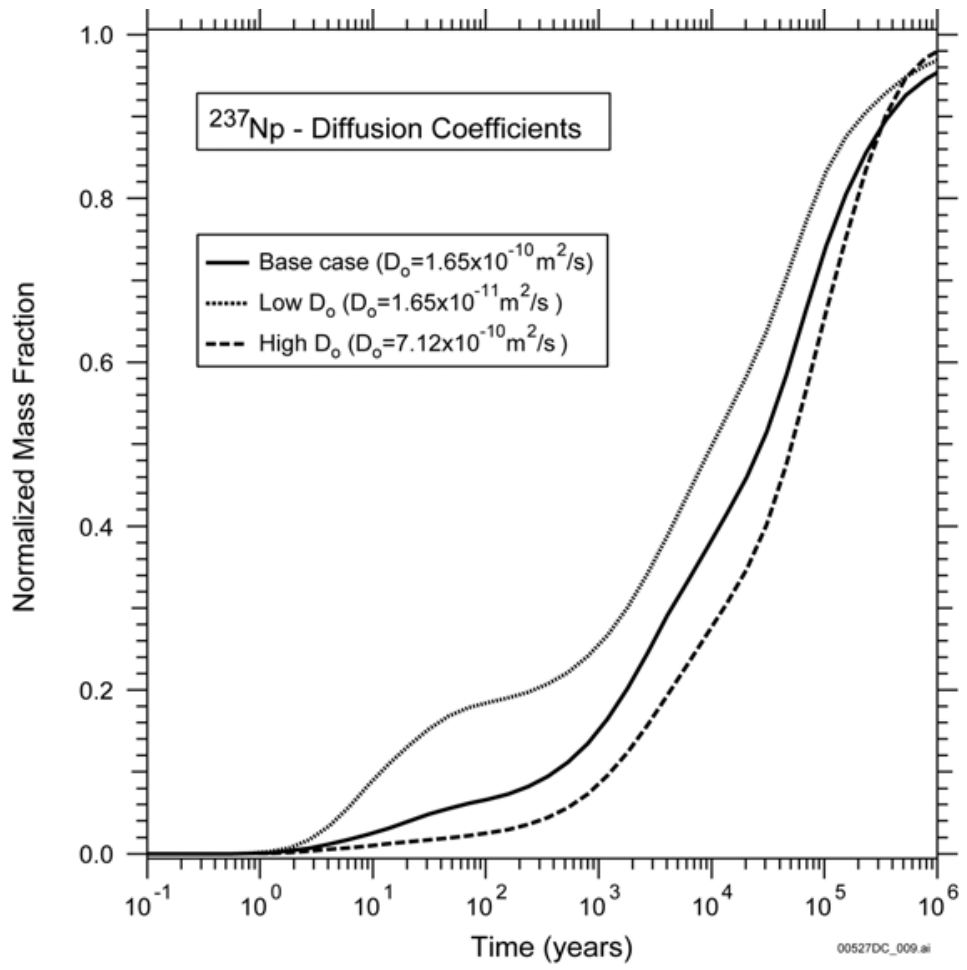

Output DTN: LB0307MR0060R1.007, data submitted with this model report.

Figure 6-40. Effect of Uncertainty in $D_{0}$ on ${ }^{237} \mathrm{~Np}$ Breakthrough at the Water Table for Mean Present-Day Infiltration and Instantaneous Release 


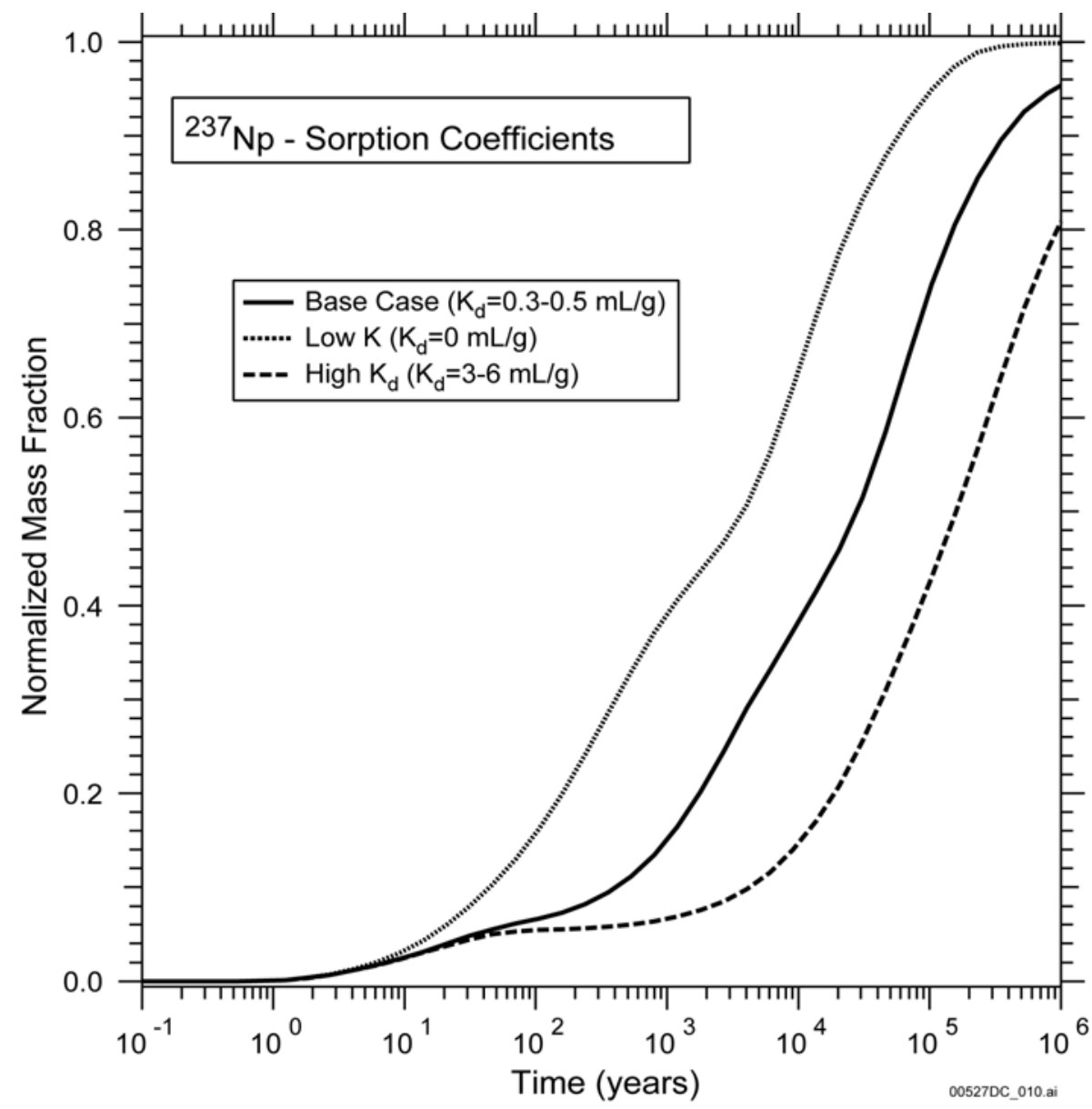

Output DTN: LB0307MR0060R1.007, data submitted with this model report.

Figure 6-41. Effect of Uncertainty in $\mathrm{K}_{d}$ on ${ }^{237} \mathrm{~Np}$ Breakthrough at the Water Table for Mean Present-Day Infiltration and Instantaneous Release

\subsection{THREE-DIMENSIONAL TRANSPORT OF ${ }^{239} \mathrm{Pu}$ - INSTANTANEOUS RELEASE}

This section addresses the transport of ${ }^{239} \mathrm{Pu}$ through the UZ of Yucca Mountain following an instantaneous release (Section 6.7). The three-dimensional site-scale simulations are conducted by using the T2R3D code.

Section 6.10.1 discusses the transport of the strongly sorbing ${ }^{239} \mathrm{Pu}$ for three levels of (a) present-day, (b) monsoon, and (c) glacial-transition infiltration. The results of this study are shown in Figures 6-42 and 6-43. Section 6.10.2 addresses the issue of uncertainty involved in the predictions of ${ }^{239} \mathrm{Pu}$ transport and conduct sensitivity analyses to develop the envelope of possible solutions (Figure 6-45). Section 6.10.3 discusses the applicability of the uncertainty studies conducted thus far on other radionuclides. 


\subsubsection{Transport of ${ }^{239}$ Pu under Present-Day Infiltration}

The grids, flow fields, and conditions discussed in Section 6.7 are used in the simulation of all infiltration regimes. The parameters used in the EOS9nT simulations of three-dimensional transport of the strongly sorbing ${ }^{239} \mathrm{Pu}$ are listed in Tables 6-3, 6-4, 6-5, and 6-6. The DTN of the input and output files for the three present-day infiltration scenarios is output DTN: LB0307MR0060R1.001 and output DTN: LB0307MR0060R1.002. The $t_{10}$ and $t_{50}$ of

${ }^{239} \mathrm{Pu}$ for the various infiltration regimes are listed in Table 6-10.

\subsubsection{Breakthrough Curves}

The cumulative breakthrough $R_{M}$ of the ${ }^{239} \mathrm{Pu}$ mass fraction at the water table for present-day infiltration is shown in Figure 6-42. If the contributions of the daughter products (i.e., ${ }^{235} \mathrm{U}$ and ${ }^{231} \mathrm{~Pa}$ ) are not accounted for, $R_{M}$ first reaches a plateau at the $R_{M}=0.1$ level (at $\mathrm{t}=20$ years, a clear indication of fracture flow), and then a second plateau at about 100,000 years (denoting matrix flow). This behavior results from the strong sorption of ${ }^{239} \mathrm{Pu}$.

The faster transport depicted in Figures 6-43 is the result of the higher monsoon and glacial-transition infiltration rates, respectively. The changes in climatic conditions lead to breakthrough curves that follow the same pattern established in the ${ }^{239} \mathrm{Pu}$ present-day transport. A higher infiltration results in two plateaus that are higher than those for present-day infiltration, but occurs at about the same times (denoting fracture and matrix transport). As in the cases of ${ }^{99} \mathrm{Tc}$ and ${ }^{237} \mathrm{~Np}$, a higher infiltration leads to earlier arrival of ${ }^{239} \mathrm{Pu}$ at the water table. However, the mass arriving at the water table represents a very small portion of the initial release because of strong sorption.

\subsubsection{Transport Mechanisms and Patterns, and Transport-Controlling Features}

The importance of fractures on the ${ }^{239} \mathrm{Pu}$ transport is very significant, as can be attested to by the contour plots available in the output submitted with this model report (output DTN: LB0307MR0060R1.002). Thus, the fracture $X_{R}$ at times as early as $\mathrm{t}=10$ years shows a sizable ${ }^{239} \mathrm{Pu}$ presence in tsw39 and a measurable presence at the water table. Review of the transport patterns of the fracture $X_{R}$ in tsw39 and at the water table confirms the earlier observations (from the ${ }^{99} \mathrm{Tc}$ and ${ }^{237} \mathrm{~Np}$ studies) that the Drill Hole Wash and the Pagany Wash faults are the main conduits of fast radionuclide transport through the UZ to the water table. The Sever Wash and the Sundance faults also contribute to transport at later times, but the Solitario Canyon fault appears to have no effect because of the strong sorption of ${ }^{239} \mathrm{Pu}$ onto the UZ rocks. The matrix $X_{R}$ distribution closely follows that of the fracture $X_{R}$, but has a more limited areal extent and substantially lower magnitude. This is consistent with a ${ }^{239} \mathrm{Pu}$ transport pattern dominated by the fault fractures, with practically no matrix-to-matrix advection, and with ${ }^{239} \mathrm{Pu}$ occurrence in the matrix due to diffusion from the fractures.

\subsubsection{Comparison to the ${ }^{99}$ Tc and ${ }^{237} \mathrm{~Np}$ Transport}

A comparison of the ${ }^{239} \mathrm{Pu}$ transport to the transport of ${ }^{99} \mathrm{Tc}$ (Section 6.8) and ${ }^{237} \mathrm{~Np}$ (Section 6.9 and Appendix D) clearly indicates the same general patterns. They all exhibit the same behavior, are affected by the same mechanisms, and are controlled by the same geologic features, but the magnitude of the observed fracture and matrix $X_{R}$ varies considerably because of the very 
different sorption affinity of these radionuclides onto the various UZ rocks. Increasing sorption affinity leads to lower concentrations (especially in the fractures), and consequently more limited areal distributions of mobile radionuclides.

Thus, ${ }^{239} \mathrm{Pu}$ transport exhibits (a) an $X_{R}$ that is universally lower than in the mildly sorbing ${ }^{237} \mathrm{~Np}$, and even more so than in the nonsorbing ${ }^{99} \mathrm{Tc}$, and a more limited areal extent (footprint) of ${ }^{239} \mathrm{Pu}$ in the fractures and in the matrix at the same times; (b) persistence over a longer time of the area underneath the southern part of the repository in tsw39 as the location where the matrix $X_{R}$ is significant; (c) slower breakthroughs; and (d) a more limited extent of the radionuclide penetration into the UZ.

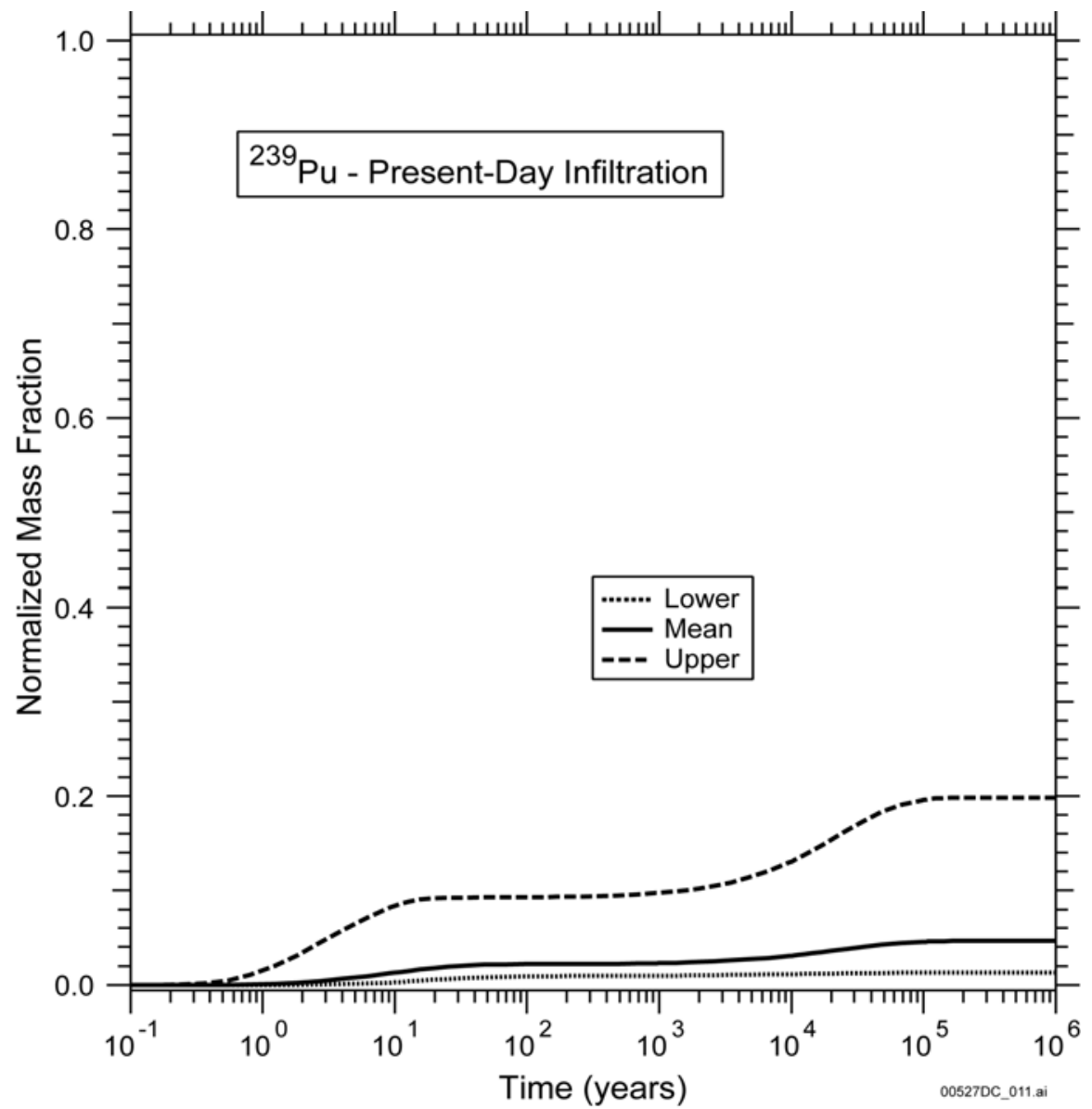

Output DTN: LB0307MR0060R1.007, data submitted with this model report.

Figure 6-42. Cumulative Breakthrough of the ${ }^{239} \mathrm{Pu}$ Mass Fraction $R_{M}$ at the Water Table for Varying Present-Day Climatic Scenarios and Instantaneous Release 
(a)

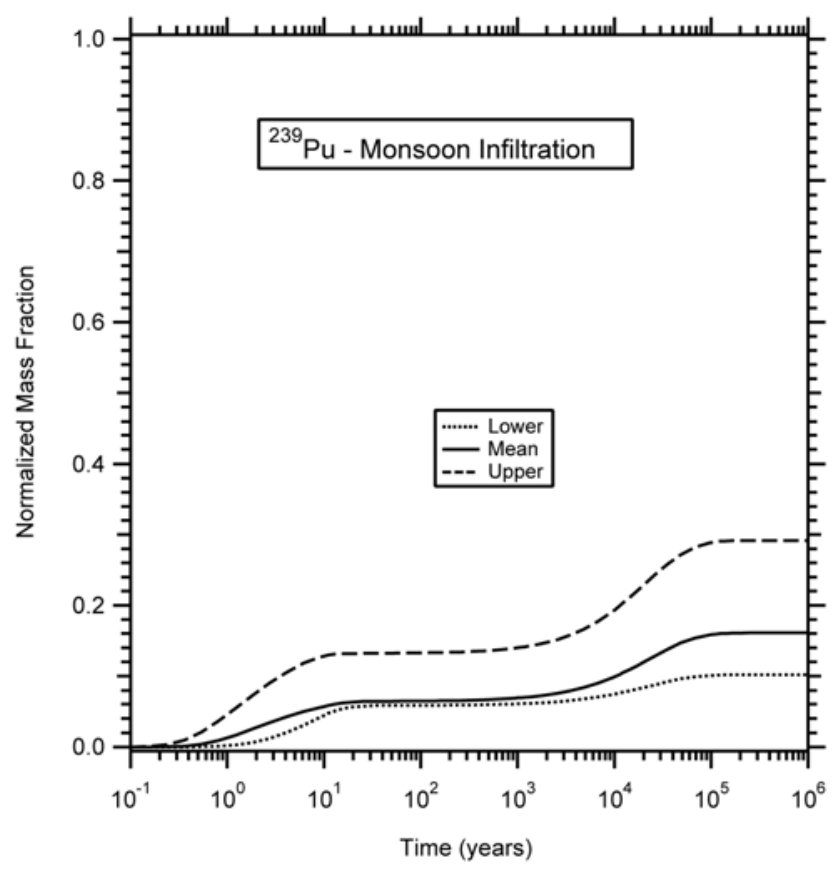

(b)

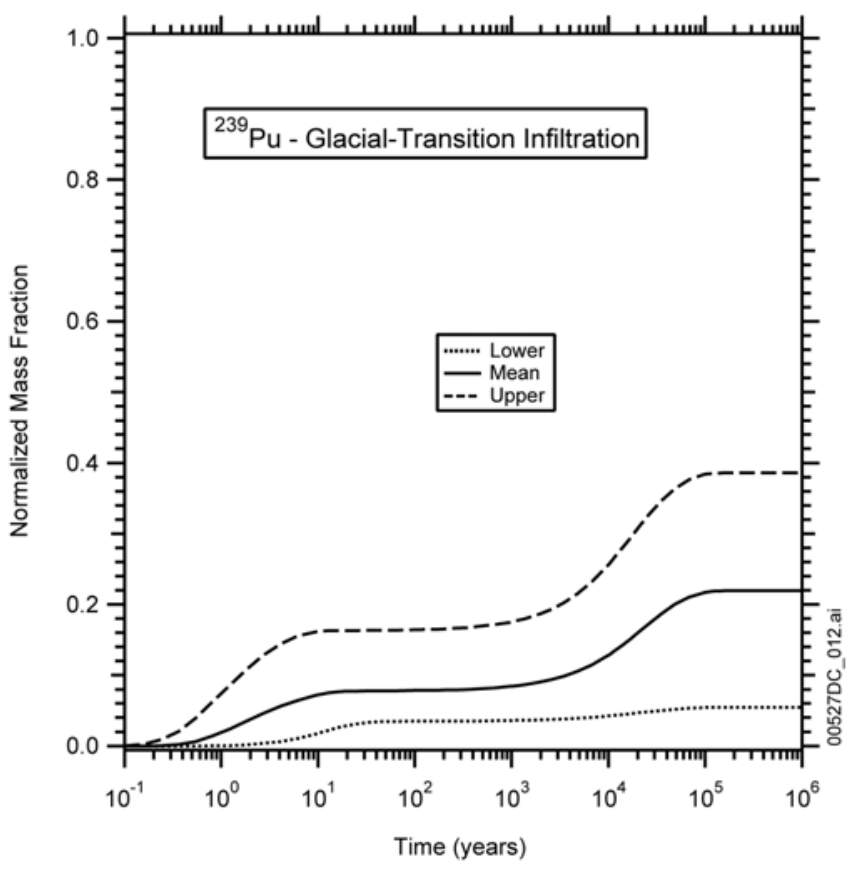

Output DTN: LB0307MR0060R1.007, data submitted with this model report.

Figure 6-43. Cumulative Breakthrough of the ${ }^{239} \mathrm{Pu}$ Mass Fraction $R_{M}$ at the Water Table for Instantaneous Release and (a) Monsoon and (b) Glacial-Transition Infiltration

\subsubsection{Effect of Sorption in the Main Geologic Units on ${ }^{239} \mathrm{Pu}$ Transport}

The effect of the main hydrogeologic units (i.e., TSw, $\mathrm{CHv}$, and $\mathrm{CHz}$ ) on the transport and retardation of ${ }^{239} \mathrm{Pu}$ was also investigated. The cumulative breakthrough curves corresponding to a zero $K_{d}$ for the TSw, $\mathrm{CHv}$, and $\mathrm{CHz}$ units are shown in Figure 6-44. 
The results (for a mean present-day infiltration scenario) are analogous to, but far more pronounced than, those discussed in the case of ${ }^{237} \mathrm{~Np}$ transport (see Section 6.9.1.5, this model report). By setting the $K_{d}$ to zero in the all the TSw layers, larger amounts of ${ }^{239} \mathrm{Pu}$ arrive earlier at the water table, and a much higher $R_{M}$ value is attained, indicating that much larger amounts of ${ }^{239} \mathrm{Pu}$ can cross the UZ. The overall effect of no sorption in the $\mathrm{CHz}$ and $\mathrm{CHv}$ units is practically insignificant compared to the base case. As in the case of ${ }^{237} \mathrm{~Np}$ transport, these results further underscore the importance of the TSw unit on the transport and retardation of ${ }^{239} \mathrm{Pu}$, and illustrate the overwhelming dominance of fractures in UZ transport.

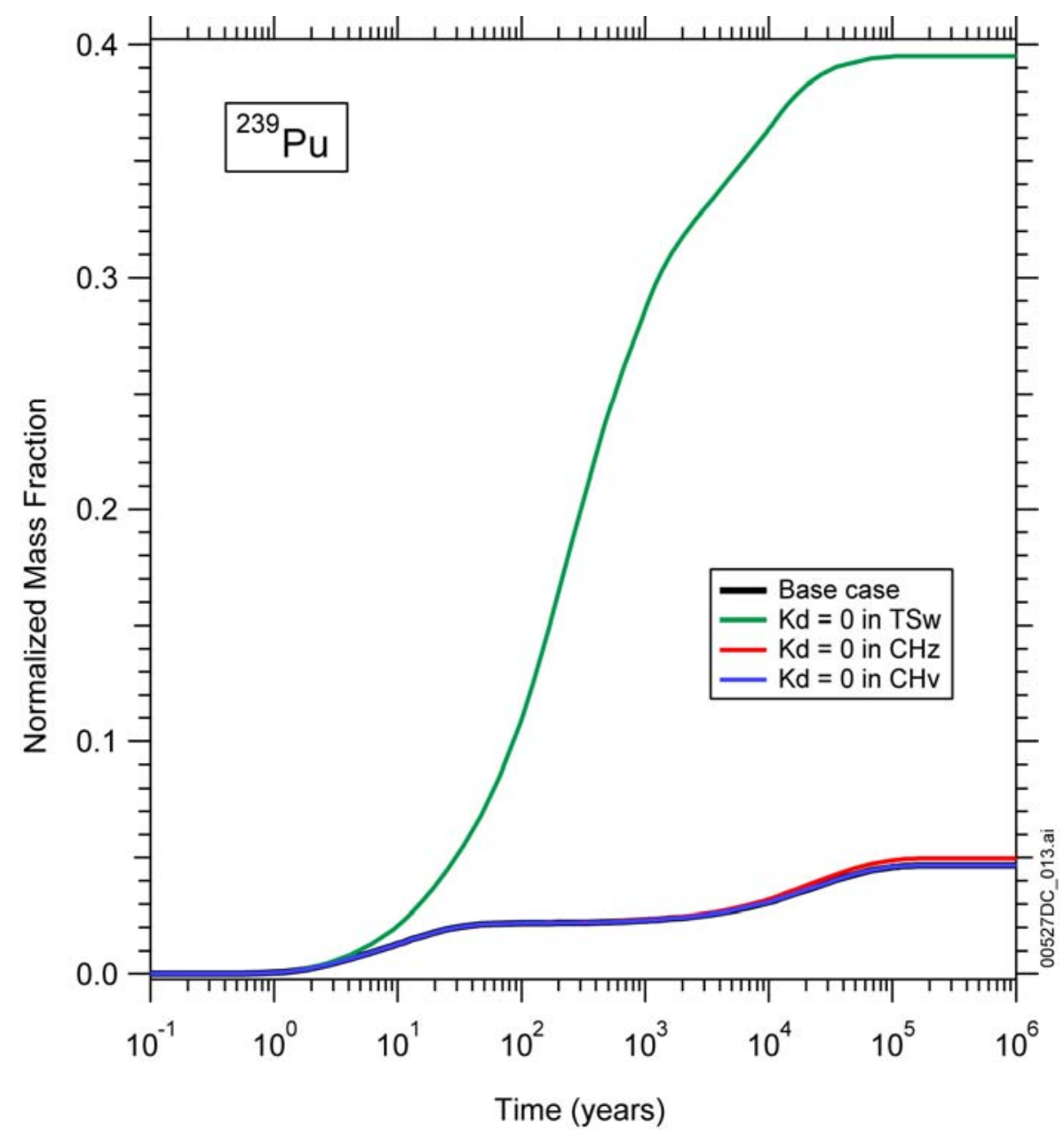

Output DTN: LB0310MR0060R1.010, data submitted with this model report.

Figure 6-44. The Effect of Sorption in TSw, $\mathrm{CHz}$, and $\mathrm{CHv}$ on the Cumulative Breakthrough of ${ }^{239} \mathrm{Pu}$ at the Water Table for Mean Present-Day Infiltration and Instantaneous Release 


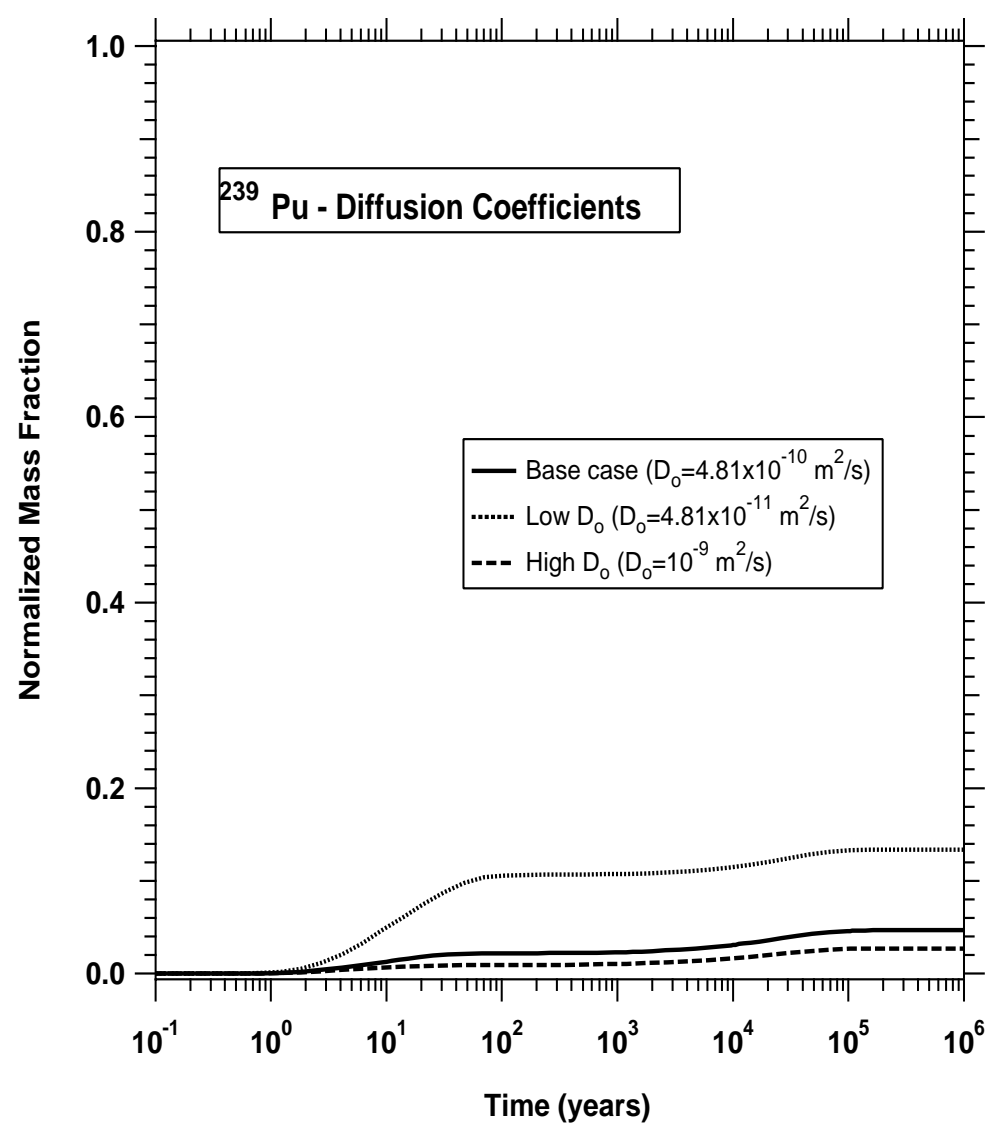

Output DTN: LB0307MR0060R1.007, data submitted with this model report.

Figure 6-45. Effect of Uncertainty in $D_{0}$ on the ${ }^{239} \mathrm{Pu}$ Breakthrough at the Water Table for Mean PresentDay Infiltration and Instantaneous Release

\subsubsection{Uncertainties in ${ }^{239} \mathrm{Pu}$ Transport Predictions}

The predictions of ${ }^{239} \mathrm{Pu}$ transport are subject to three general types of uncertainties discussed in Sections 6.8.3 and 6.9.3. For the reasons discussed in these sections, discussion is limited to the issue of uncertainties in the transport retardation processes. Although ${ }^{239} \mathrm{Pu}$ is a sorbing radionuclide subject to the impact of uncertainties in diffusion and sorption, only uncertainty in diffusion is addressed here. The reason for this approach is that ${ }^{239} \mathrm{Pu}$ exhibits sorbing behavior that is so exceptionally strong it would be physically unrealistic to reduce $K_{d}$ to levels that may have an impact on transport. Even order-of-magnitude reduction in $K_{d}$ is insufficient to significantly affect ${ }^{239} \mathrm{Pu}$ transport behavior.

The study of uncertainties in diffusion follows the pattern discussed in Sections 6.8.3 and 6.9.3. Uncertainties in matrix diffusion are reflected in the values of the diffusion coefficient $D_{0}$. The sensitivity of the ${ }^{239} \mathrm{Pu}$ transport through the UZ was investigated for the $D_{0}$ values shown in Table 6-4, which cover the possible $D_{0}$ range. The results are shown in Figure 6-45.

As expected, $D_{0}$ has an impact on breakthrough predictions, resulting in faster arrival times for lower $D_{0}$ values. However, the relative effect of $D_{0}$ uncertainty in predicting transport of the strongly sorbing ${ }^{239} \mathrm{Pu}$ is comparatively less pronounced than that of the mildly sorbing ${ }^{237} \mathrm{~Np}$. 
That is, the impact of $D_{0}$ uncertainty on radionuclide breakthrough predictions appears to be mitigated by sorption.

\subsubsection{Applicability of Uncertainty Studies to Other Radionuclides.}

The insights obtained from the analysis of uncertainty in the cases of ${ }^{99} \mathrm{Tc},{ }^{237} \mathrm{~Np}$, and ${ }^{239} \mathrm{Pu}$ are directly applicable to all the other radionuclides studied in this model report. By covering the spectrum of sorption behavior and diffusion in the uncertainty study, it is possible to develop general rules to broadly predict the effect of uncertainty. Thus, the effect of uncertainties in the breakthrough of nonsorbing radionuclides will be akin to that of ${ }^{99} \mathrm{Tc}$. Mildly sorbing radionuclides can be described by using the ${ }^{237} \mathrm{~Np}$ analogue, while strong sorbers will be described by the ${ }^{239} \mathrm{Pu}$ behavior.

\subsection{THREE-DIMENSIONAL TRANSPORT OF ${ }^{233} \mathrm{U}$ AND ${ }^{235} \mathrm{U}$ - INSTANTANEOUS RELEASE}

This section discusses the transport of ${ }^{233} \mathrm{U}$ and ${ }^{235} \mathrm{U}$ through the Yucca Mountain UZ system after an instantaneous release scenario (Section 6.7). The two isotopes share the same physical properties (e.g., diffusion and sorption behavior), but differ in their half-lives (Table 6-4).

The grids, flow fields, and conditions are as discussed in Section 6.7. The parameters used in these T2R3D simulations of three-dimensional transport of the moderately sorbing ${ }^{233} \mathrm{U}$ and ${ }^{235} \mathrm{U}$ are listed in Tables 6-3, 6-4, 6-5, and 6-6. The DTN of the input and output files for the nine present-day infiltration scenarios is output DTN: LB0307MR0060R1.001, and output DTN: LB0307MR0060R1.002 (Table 6-6).

\subsubsection{Transport of ${ }^{233}$ U Under Different Climatic Conditions}

The breakthrough curves of ${ }^{233} \mathrm{U}$ (a mildly sorbing species; see Table 6-3) transport through the UZ are shown in Figure 6-46(a), (b), and (c), for mean present-day, monsoon and glacial-transition infiltration regimes, respectively. A comparison of these figures to those of ${ }^{237} \mathrm{~Np}$ (Figure 6-36, 6-38, and 6-39) indicates that their behavior is analogous at early times, but deviates at later times because of the larger $T_{1 / 2}$ of ${ }^{233} \mathrm{U}$ (leading to different maximum attainable $R_{M}$ values). The $t_{10}$ and $t_{50}$ of ${ }^{233} \mathrm{U}$ for the various infiltration regimes are listed in Table 6-10.

\subsubsection{Transport of ${ }^{235} \mathrm{U}$ under Different Climatic Conditions}

The breakthrough curves of ${ }^{235} U$ transport through the UZ are shown in Figures 6-47(a), (b), and (c) for mean present-day, monsoon, and glacial-transition infiltration regimes, respectively. Compared to the equivalent figures for ${ }^{233} \mathrm{U}$, the breakthrough curves show differences at later times (e.g., in the maximum attainable $R_{M}$ level), reflecting the differences in $T_{1 / 2}$ (Table 6-4). Because of the isotopic relationship to ${ }^{233} \mathrm{U}$, the behavior ${ }^{235} \mathrm{U}$ (a mildly sorbing species, see Table 6-3) is analogous to that ${ }^{237} \mathrm{~Np}$. The $t_{10}$ and $t_{50}$ of ${ }^{235} \mathrm{U}$ for the various infiltration regimes are listed in Table 6-10.

A review of the figures in this section confirms the well-established pattern observed thus far: increasing infiltration enhances transport by resulting in a shorter transport time through the UZ, and leads to a higher maximum attainable $R_{M}$. 
(a)

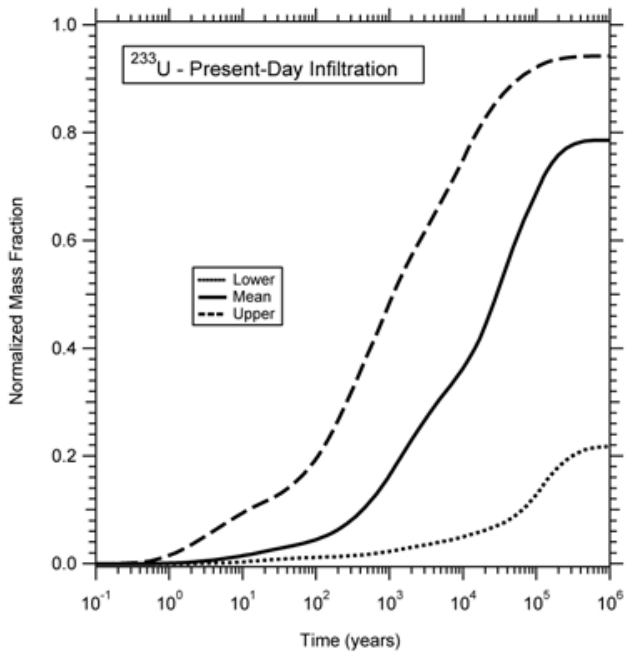

(b)



(c)

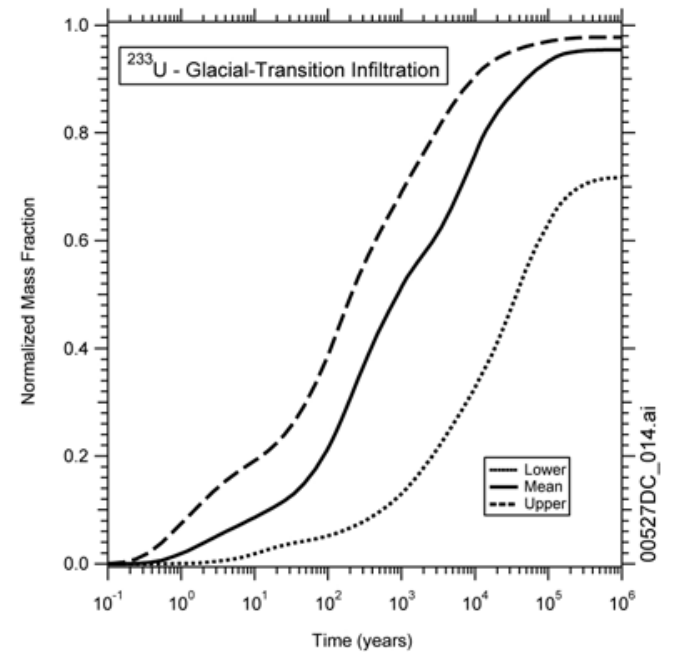

Output DTN: LB0307MR0060R1.007, data submitted with this model report.

Figure 6-46. Cumulative Breakthrough of the ${ }^{233} \mathrm{U}$ Mass Fraction $R_{M}$ at the Water Table for Instantaneous Release for (a) Present-Day, (b) Monsoon, and (c) Glacial-Transition Infiltration 
(a)

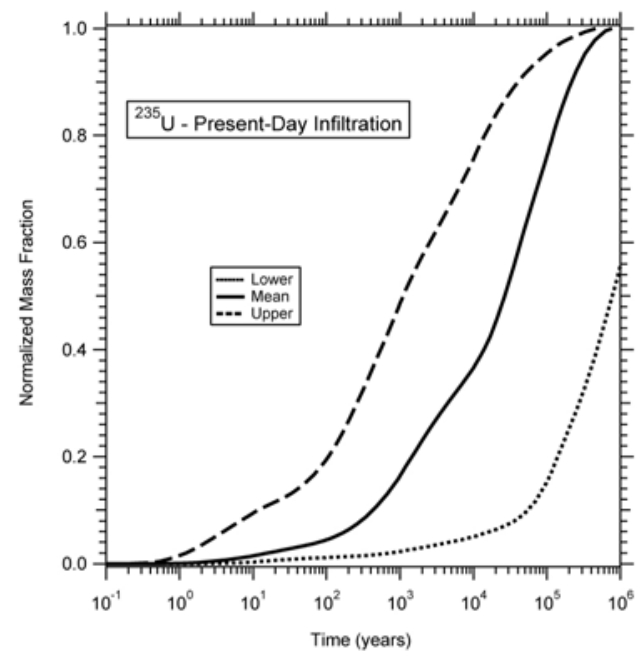

(b)

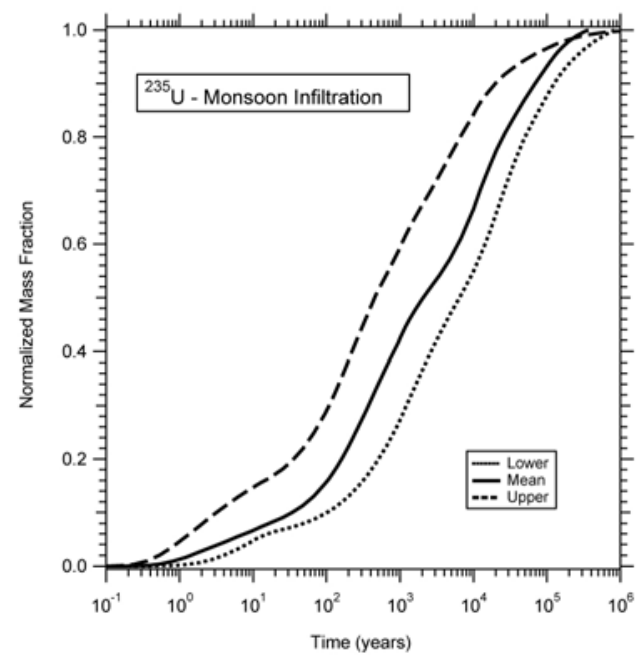

(c)

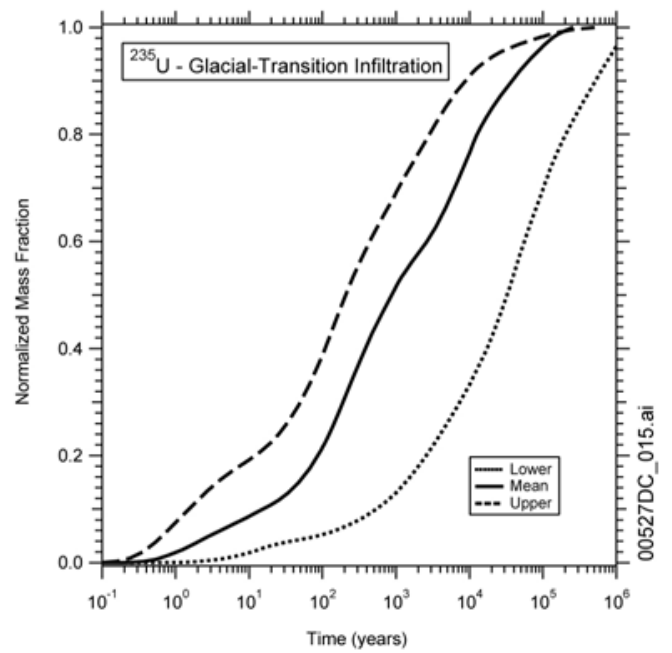

Output DTN: LB0307MR0060R1.007, data submitted with this model report.

Figure 6-47. Cumulative Breakthrough of the ${ }^{235} \mathrm{U}$ Mass Fraction $R_{M}$ at the Water Table for Instantaneous Release for (a) Present-Day, (b) Monsoon, and (c) Glacial-Transition Infiltration 


\subsection{THREE-DIMENSIONAL TRANSPORT OF ${ }^{241} \mathrm{Am}$ AND ${ }^{90} \mathrm{Sr}$ - INSTANTANEOUS RELEASE}

This section analyzes the transport of ${ }^{241} \mathrm{Am}$ and ${ }^{90} \mathrm{Sr}$ through the Yucca Mountain UZ after an instantaneous release scenario (Section 6.7). Both ${ }^{241} \mathrm{Am}$ and ${ }^{90} \mathrm{Sr}$ are very strong sorbers (even stronger than $\mathrm{Pu}$; see Table 6-3), but have the two shortest $T_{1 / 2}$ among the radionuclides studied in this model report (Table 6-4).

The grids, flow fields, and conditions are as discussed in Section 6.7.2. The parameters used in these T2R3D simulations are listed in Tables 6-3, 6-4, 6-5, and 6-6. The DTN of input and output files for nine infiltration scenarios is output DTN: LB0307MR0060R1.001 (Table 6-6).

\subsection{1 ${ }^{241}$ Am Transport}

The breakthrough curves of ${ }^{241}$ Am transport through the UZ are shown in Figures 6-48(a), (b), and (c) for mean present-day, monsoon, and glacial-transition infiltration regimes, respectively. These figures indicate that the maximum attainable $R_{M}$ is quite low, and that, although an increasing infiltration results invariably in a larger $R_{M}$, the increase is not significant. As in all radionuclides in this model report, the breakthrough curves are characterized by early arrival at the water table and an early single plateau at a low $R_{M}$ level (below 0.2 ), denoting fracture transport. A second plateau (denoting matrix transport, as observed in the case of the ${ }^{239} \mathrm{Pu}$ transport) does not materialize because of (a) the much stronger sorption of ${ }^{241} \mathrm{Am}$ onto the matrix (thus reducing the radionuclide available for matrix transport) and (b) its rather short halflife $\left(4.322 \times 10^{2}\right.$ years, Table 6-4). The $t_{10}$ and $t_{50}$ of ${ }^{241} \mathrm{Am}$ for the various infiltration regimes are listed in Table 6-10.

\subsection{2 ${ }^{90}$ Sr Transport}

The breakthrough curves of ${ }^{90} \mathrm{Sr}$ transport through the UZ are shown in Figures 6-49(a), (b), and (c) for mean present-day, monsoon, and glacial-transition infiltration regimes, respectively. As expected because of the similarity to ${ }^{241} \mathrm{Am}$, the maximum attainable $R_{M}$ is quite low and does not increase significantly with an increasing infiltration. The breakthrough curves are characterized by early arrival at the water table and an early single plateau at a low $R_{M}$ level (below 0.15), denoting fracture transport. The second plateau (denoting matrix transport) observed in the case of ${ }^{239} \mathrm{Pu}$ transport (Section 6.10) does not materialize because of (a) the much stronger sorption of ${ }^{90} \mathrm{Sr}$ onto the matrix (thus reducing the radionuclide available for matrix transport) and (b) its very short half-life $\left(2.9 \times 10^{1}\right.$ years, Table 6-4). The $t_{10}$ and $t_{50}$ of ${ }^{90} \mathrm{Sr}$ for the various infiltration regimes are listed in Table 6-10. 
(a)

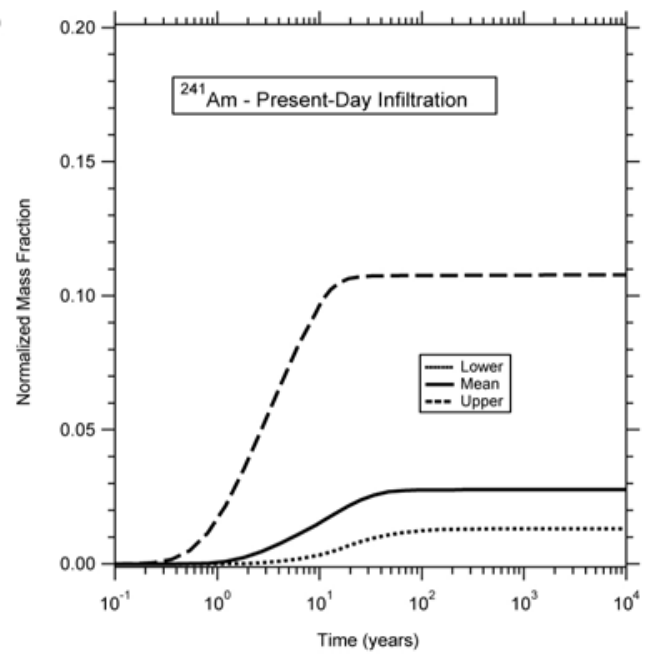

(b)

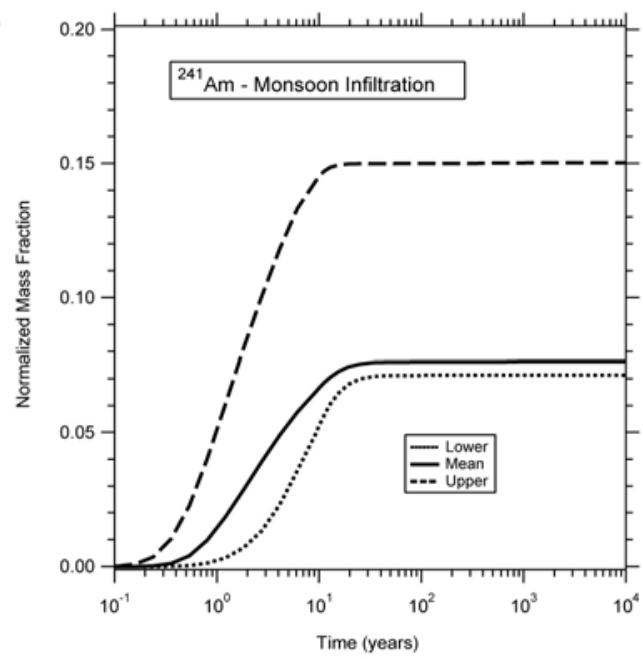

(c)

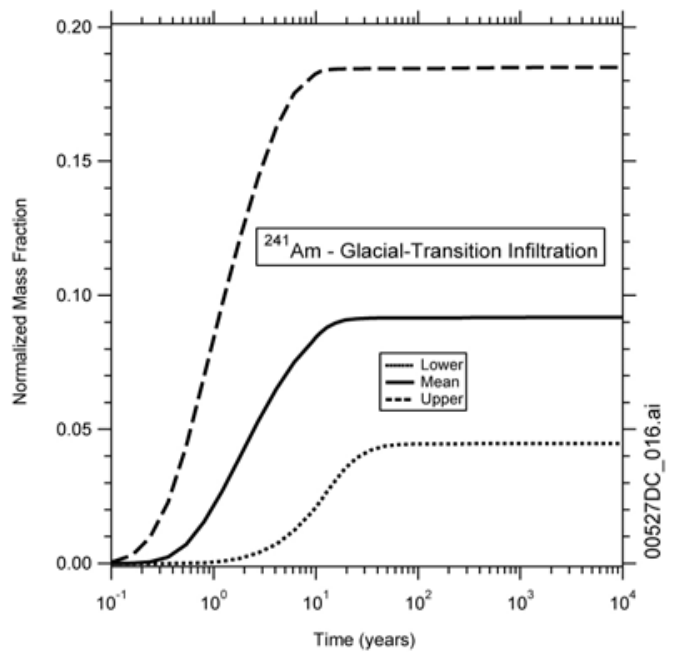

Output DTN: LB0307MR0060R1.007, data submitted with this model report.

Figure 6-48. Cumulative Breakthrough of the ${ }^{241} \mathrm{Am}$ Mass Fraction $R_{M}$ at the Water Table for Instantaneous Release for (a) Present-day, (b) Monsoon, and (c) Glacial-Transition Infiltration 

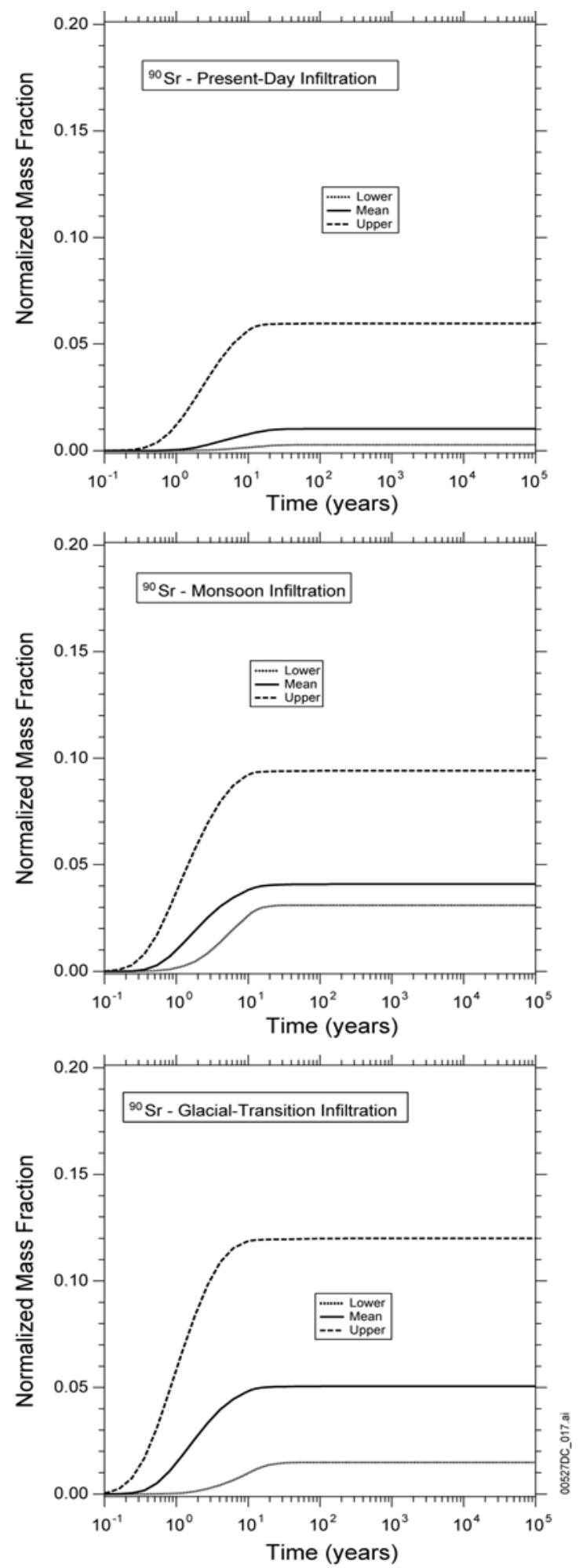

Output DTN: LB0307MR0060R1.007, data submitted with this model report.

Figure 6-49. Cumulative Breakthrough of the ${ }^{90} \mathrm{Sr}$ Mass Fraction $R_{M}$ at the Water Table for Instantaneous Release for (a) Present-Day, (b) Monsoon, and (c) Glacial-Transition Infiltration 


\subsection{THREE-DIMENSIONAL TRANSPORT OF ${ }^{135} \mathrm{Cs}$ - INSTANTANEOUS RELEASE}

This section analyzes the transport of ${ }^{135} \mathrm{Cs}$ through the Yucca Mountain UZ following an instantaneous release event. ${ }^{135} \mathrm{Cs}$ is a very strong sorber (even stronger than $\mathrm{Pu}$ on zeolitic rocks, but less on vitric and devitrified; see Table 6-3), but is also among the most diffusive species (Table 6-4).

The grids, flow fields, and conditions are as discussed in Section 6.7. The parameters used in the T2R3D simulations of three-dimensional transport of ${ }^{135} \mathrm{Cs}$ are listed in Tables 6-3, 6-4, 6-5, and 6-6. The DTN of the input and output files for all nine scenarios is output DTN: LB0307MR0060R1.001 (Table 6-6).

The breakthrough curves of ${ }^{135} \mathrm{Cs}$ transport through the UZ are shown in Figures 6-50(a), (b), and (c), for mean present-day, monsoon, and glacial-transition infiltration regimes, respectively. In all climatic scenarios, the very strong sorption of ${ }^{135} \mathrm{Cs}$ demonstrates itself by the very low $R_{M}$ level, which persists for a very long time. The very strong diffusion of ${ }^{135} \mathrm{Cs}$ into the matrix prevents its early arrival at the water table through advective transport in the fractures, taking about 1,000 years for concentrations at the water table to register an upward trend. After this initial delay (during which the effect of the infiltration rates do not substantially affect transport), significant $R_{M}$ values are attainable, and breakthrough begins to be affected by the climatic regime. The $t_{10}$ and $t_{50}$ of ${ }^{135} \mathrm{Cs}$ for the various infiltration regimes are listed in Table 6-10. 

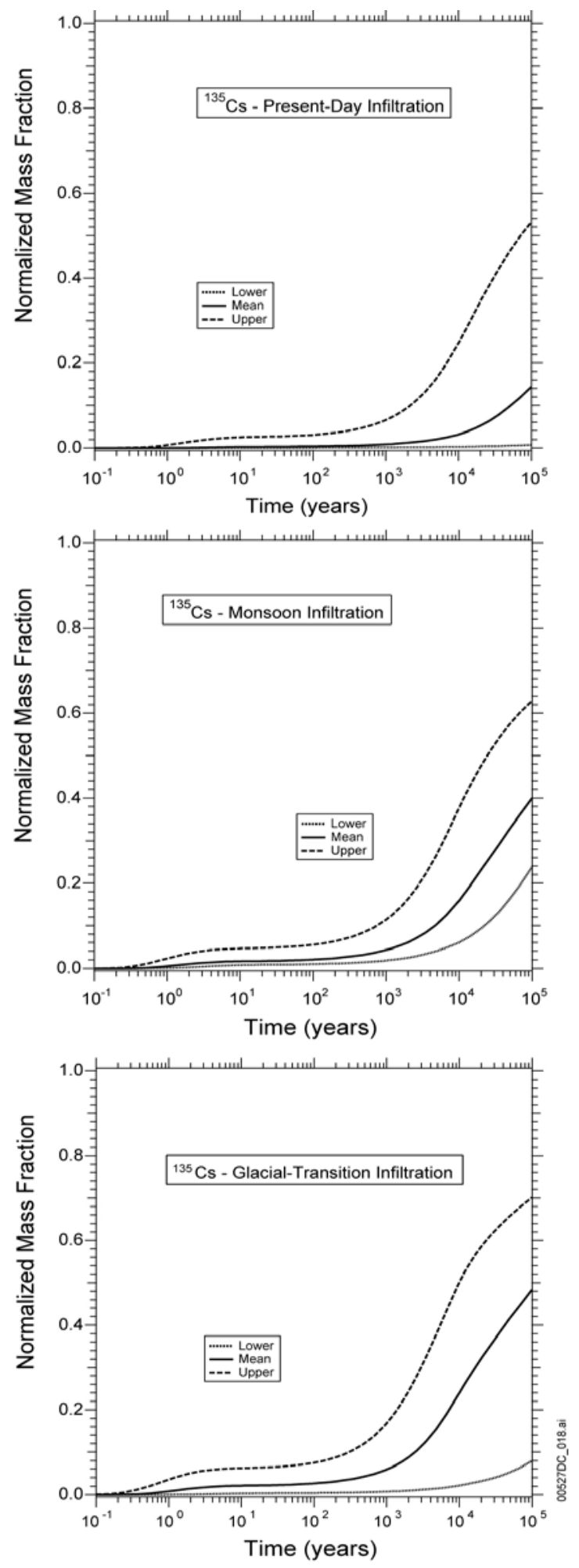

Output DTN: LB0307MR0060R1.007, data submitted with this model report.

Figure 6-50. Cumulative Breakthrough of the ${ }^{135} \mathrm{Cs}$ Mass Fraction $R_{M}$ at the Water Table for Instantaneous Release for (a) Present-Day, (b) Monsoon, and (c) Glacial-Transition Infiltration 


\subsection{THREE-DIMENSIONAL TRANSPORT OF ${ }^{226} \mathrm{Ra},{ }^{229} \mathrm{Th}, \mathrm{AND}{ }^{231} \mathrm{~Pa}-$ INSTANTANEOUS RELEASE}

This section analyzes the transport ${ }^{226} \mathrm{Ra},{ }^{229} \mathrm{Th}$, and ${ }^{231} \mathrm{~Pa}$, three radionuclides that are among the insoluble species and the strongest known sorbers. These are instantaneously released at the repository level, and their migration through the UZ zone and breakthrough at the water table is studied. These three radionuclides have medium half-lives, ranging from a few thousand to a few tens of thousands of years (Table 6-4).

The grids, flow fields, and conditions are as discussed in Section 6.7. The parameters used in these T2R3D three-dimensional simulations are listed in Tables 6-3, 6-4, 6-5, and 6-6. The DTN of the input and output files for the all nine scenarios is output DTN: LB0307MR0060R1.001 (Table 6-6).

Breakthrough curves of ${ }^{226} \mathrm{Ra}$ transport through the UZ are shown in Figures 6-51(a), (b), and (c), for mean present day, monsoon, and glacial-transition infiltration regimes, respectively. The corresponding figures for the ${ }^{229} \mathrm{Th}$ breakthrough are 6-52(a), (b), and (c), and those figures for ${ }^{231} \mathrm{~Pa}$ are 6-53(a), (b), and (c), respectively. As expected, these three radionuclides exhibit very similar behavior, and similar to those of ${ }^{241} \mathrm{Am}$ and ${ }^{90} \mathrm{Sr}$. Their maximum attainable $R_{M}$ is quite low, and although larger infiltration results invariably in a larger $R_{M}$, the increase is not significant. Breakthrough curves are characterized by early arrival at the water table and an early plateau at a low $R_{M}$ level (below 0.2), denoting fracture transport. The second plateau (denoting matrix transport) that was observed in the case of the ${ }^{239} \mathrm{Pu}$ transport does not materialize because of (a) their much stronger sorption onto the matrix that does not permit transport through it, and (b) their medium half-life (dwarfed by the 1,000,000-year-long period of observation depicted in the figures; see Table 6-4). The $t_{10}$ and $t_{50}$ of ${ }^{226} \mathrm{Ra},{ }^{229} \mathrm{Th}$, and ${ }^{231} \mathrm{~Pa}$ for the various infiltration regimes are listed in Table 6-10. 

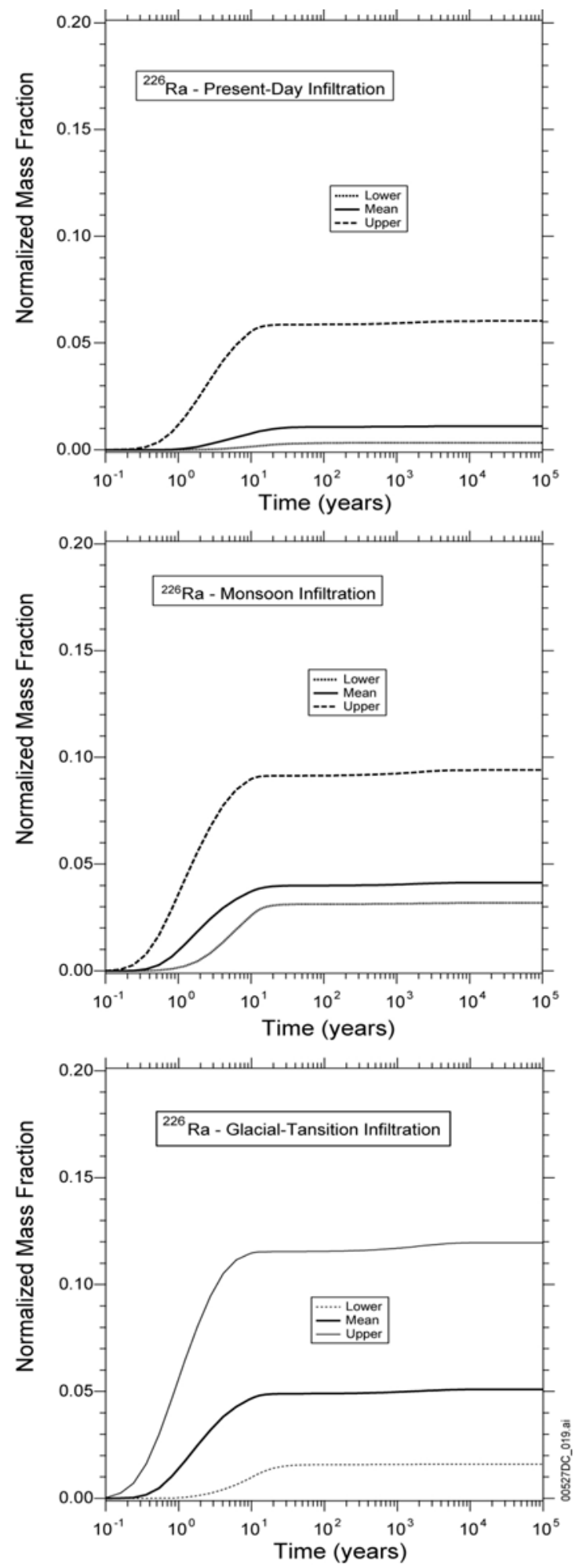

Output DTN: LB0307MR0060R1.007, data submitted with this model report.

Figure 6-51. Cumulative Breakthrough of the ${ }^{226} \mathrm{Ra}$ Mass Fraction $R_{M}$ at the Water Table for Instantaneous Release for (a) Present-Day, (b) Monsoon, and (c) Glacial-Transition Infiltration 

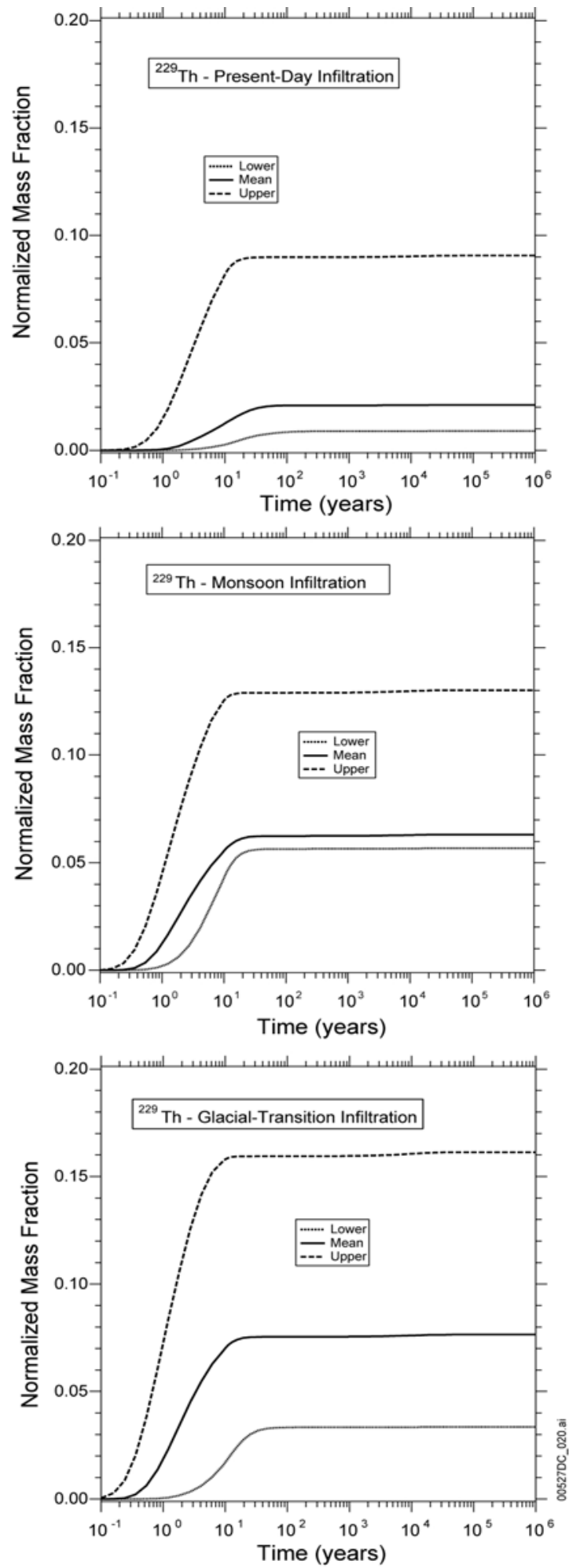

Output DTN: LB0307MR0060R1.007, data submitted with this model report.

Figure 6-52. Cumulative Breakthrough of the ${ }^{229}$ Th Mass Fraction $R_{M}$ at the Water Table for Instantaneous Release for (a) Present-Day, (b) Monsoon, and (c) Glacial-Transition Infiltration 
(a)

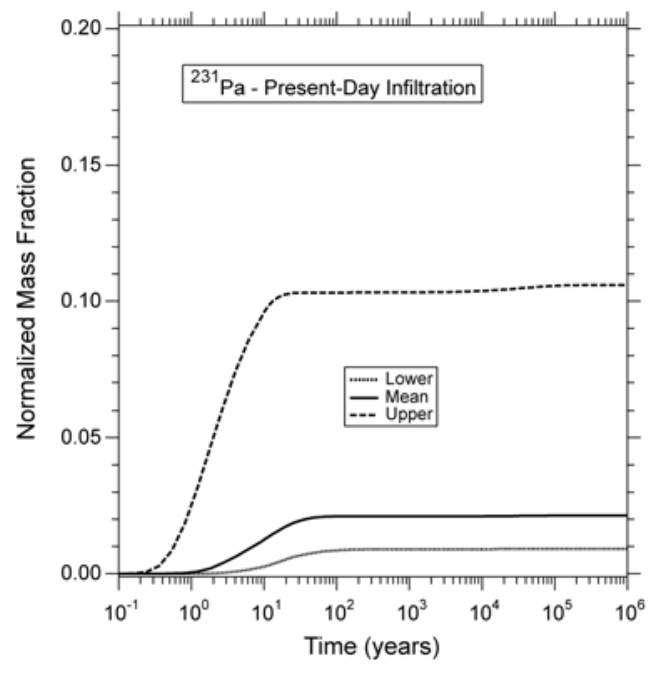

(b)

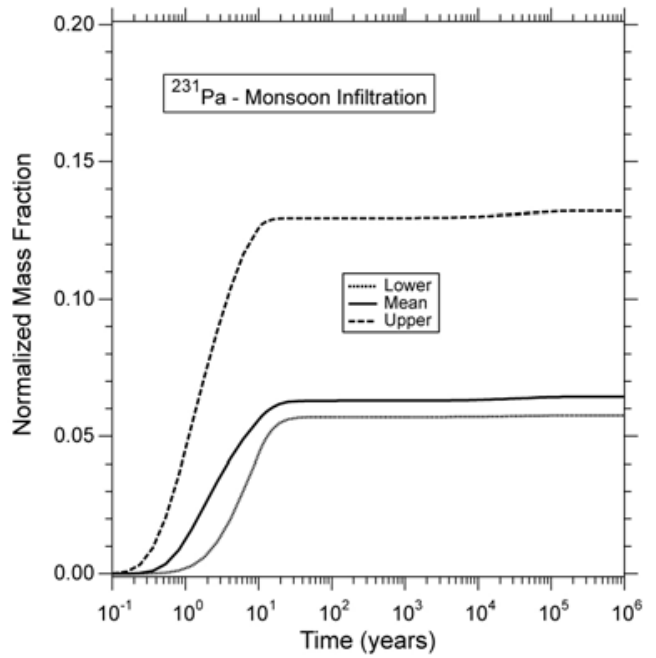

(c)

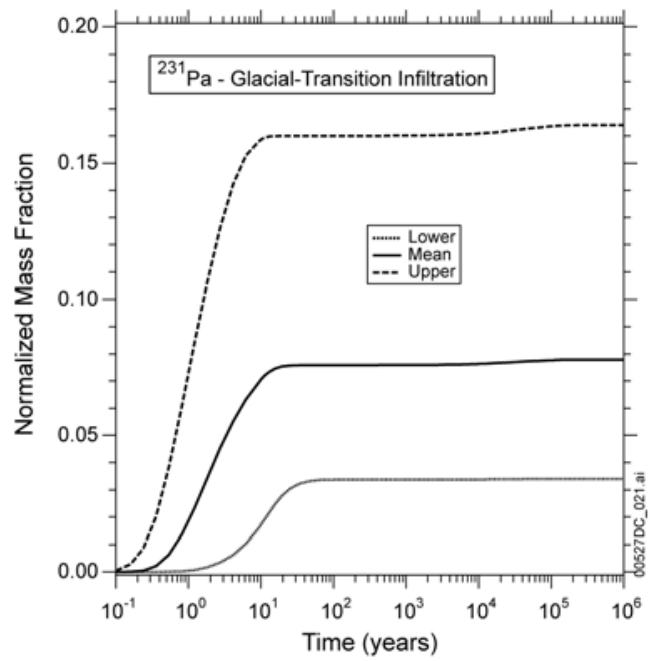

Output DTN: LB0307MR0060R1.007, data submitted with this model report.

Figure 6-53. Cumulative Breakthrough of the ${ }^{231} \mathrm{~Pa}$ Mass Fraction $R_{M}$ at the Water Table for Instantaneous Release for (a) Present-Day, (b) Monsoon, and (c) Glacial-Transition Infiltration 


\subsection{THREE-DIMENSIONAL SIMULATIONS OF ${ }^{99} \mathrm{Tc},{ }^{237} \mathrm{~Np}$, AND ${ }^{239} \mathrm{Pu}$ TRANSPORT - CONTINUOUS RELEASE}

This section describes the three radionuclides discussed in Sections 6.8 to 6.10 under a scenario of continuous release.

\subsubsection{The Continuous-Release Scenario}

The concept of the continuous-release scenario investigated in these simulations was discussed in Section 6.7.3.2. Only mean present-day infiltration was considered in these simulations. The grids, flow fields, and conditions are as discussed in Section 6.7. The parameters used in these EOS9nT simulations of three-dimensional transport of ${ }^{99} \mathrm{Tc},{ }^{237} \mathrm{~Np}$, and ${ }^{239} \mathrm{Pu}$ are listed in Tables 6-3, 6-4, 6-5, and 6-6. The DTN of the input and output files for the three present-day infiltration scenarios is output DTN: LB0307MR0060R1.003 (Table 6-6).

\subsubsection{The Definition of the Breakthrough Curves for Continuous Release}

As the radionuclide concentration in the repository and in the bottom boundary elements (corresponding to the water table) remain constant over time, the breakthrough concept is based on the normalized or relative release rate $R_{F}$, which is defined as

$$
R_{F}=\frac{\text { Mass release rate at the groundwater boundary }\left[M T^{-1}\right]}{\text { Mass release rate at the proposed repository }\left[M T^{-1}\right]}
$$

Thus, $R_{F}$ involves a ratio of rates, while the conventional $R_{M}$ is a ratio of masses. As in $R_{M}$, $t_{10}$ and $t_{50}$ are defined as the times at which $R_{F}=0.1$ and $R_{F}=0.5$, respectively. For radioactive chains, the quantity in the denominator reflects the sum of the fluxes of all members in the release stream. For decaying release concentration, the denominator reflects the original radionuclide release rate from repository elements.

Of particular interest is the distribution of the relative sorbed or filtered concentration $F_{R}\left[M L^{-3}\right]$, defined as

$$
F_{R}=\frac{F}{X_{0}}
$$

where $\mathrm{F}$ is as defined in Equation 6-8, and $X_{0}$ is the species mass fraction in the water released from the repository. This is important in the evaluation of sorbing radionuclides such as ${ }^{237} \mathrm{~Np}$ and ${ }^{239} \mathrm{Pu}$.

\subsubsection{Transport of Continuously Released ${ }^{99} \mathrm{Tc},{ }^{237} \mathrm{~Np}$, and ${ }^{239} \mathrm{Pu}$ under Present-Day Infiltration}

The breakthrough curves for continuously released ${ }^{99} \mathrm{Tc},{ }^{237} \mathrm{~Np}$, and ${ }^{239} \mathrm{Pu}$ are shown in Figure 6-54, which also shows the decaying fluxes (reflecting decaying concentrations, as the water fluxes remain time-invariant) for reference. The $t_{10}$ and $t_{50}$ of ${ }^{99} \mathrm{Tc},{ }^{237} \mathrm{~Np}$, and ${ }^{239} \mathrm{Pu}$ under a continuous release scenario for a mean present-day infiltration are listed in Table 6-11. 
Comparison of the $t_{10}$ and $t_{50}$ from Figure 6-54 to those from the instantaneous-release scenarios (Sections 6.8, 6.9, and 6.10) shows that, in this case, they are generally consistent. Note, however, that, in general, the two can be very different. Of interest is the shape of the $R_{F}$ curves, which begin to show a decline after reaching a maximum before the point where the influx and outflow curves coincide. The existence of such a maximum is a direct consequence of considering a decaying source. $R_{F}$, the ratio of outflow to initial inflow, begins increasing as radionuclides reach the water table in increasing quantities. However, the increase can only proceed up to a point, beyond which there is a balance between influx and outflow (indicated by the coincidence of the two curves in Figure 6-54), which involves ever-declining radionuclide concentrations.

\subsubsection{Transport Patterns of Continuously Released ${ }^{99} \mathrm{Tc},{ }^{237} \mathrm{~Np}$, and ${ }^{239} \mathrm{Pu}$ under Present-Day Infiltration}

The distribution of ${ }^{99} \mathrm{Tc}$ fracture and matrix $X_{R}$ in tsw39 and at the watertable at various times after the radionuclide release are shown in Appendix E. Under continuous release, it is expected that the radionuclide distributions at early times would be similar to those from instantaneous release, but the two would diverge at later times because of the different masses involved in each scenario.

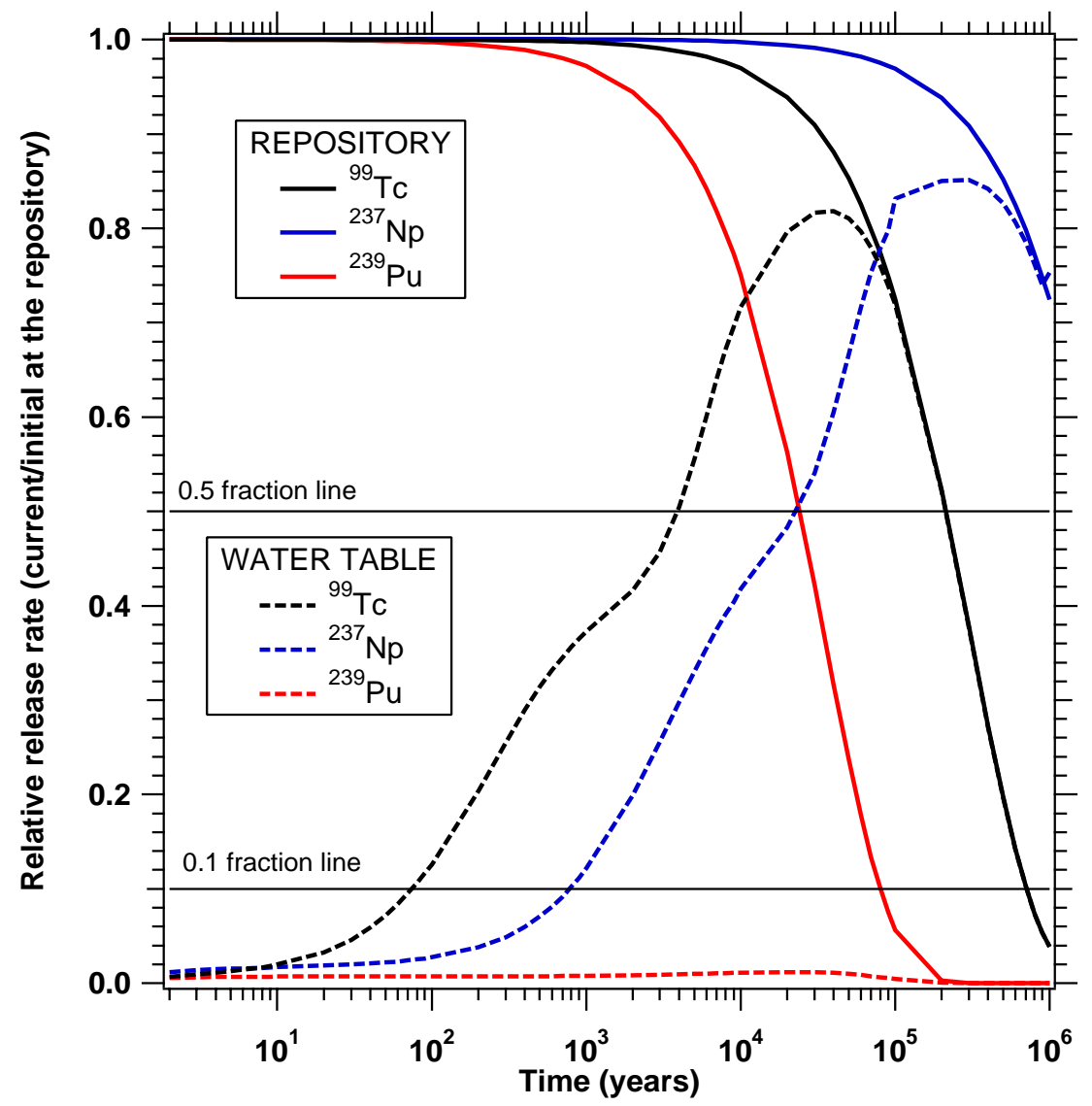

Output DTN: LB0307MR0060R1.007, data submitted with this model report.

Figure 6-54. Normalized Relative Release $R_{F}$ of ${ }^{99} \mathrm{Tc},{ }^{237} \mathrm{~Np}$, and ${ }^{239} \mathrm{Pu}$ at the Water Table for Continuous Release and Mean Present-Day Climatic Scenarios 
One of the objectives of this study was to determine whether transport under continuous release is dominated by different geologic features than those controlling instantaneous release. Comparison of Figures E-1 through E-10 to Figures 6-9 through 6-18 indicate that at early times, $(t \leq 100$ years) the concentration distributions in tsw39 show the same patterns and are in line with expectations. The magnitude of concentrations is different, but this was entirely expected because the total radionuclide mass in the UZ is substantially larger in a continuous release scenario. Differences are observed for $t \geq 1,000$ years, which show increasing fracture and matrix concentrations over time. The Drill Hole Wash and Pagany Wash faults are the main geologic features responsible for fast transport to the bottom of the TSw and the water table. Note that the shift of the matrix concentrations from underneath the southern part toward the northern part of the repository (Figures 6-14, 6-16, 6-18) does not occur under continuous release, and the southern part continues to exhibit the largest concentrations. The areal extent of radionuclide distribution in both the fractures and the matrix appears to reach a maximum at $t=1,000$ years (Figures E-7 and-E.8). Although these footprints appear constant after this time, the concentrations change over time and reach a maximum at $t=10,000$ years (Figures E-9 and E-10), after which they decline because of radioactive decay. An important observation is the large footprint and the corresponding high values of the matrix $X_{R}$ under continuous release. Thus, the amount of ${ }^{99} \mathrm{Tc}$ in the mobile liquid in the matrix is greater and over a larger area under continuous release.

Comparison of Figures E-11 through E-20 to Figures 6-19 through 6-28 indicates that the concentration distributions above the water table show the same general patterns. For obvious reasons, the magnitude of concentrations is different, but the areal distributions are remarkably similar. Significant differences are observed at $t=100,000$ years, when both the areal extent and the magnitude of the ${ }^{99} \mathrm{Tc}$ concentrations are significantly larger under continuous release. This is entirely consistent with the much larger total radionuclide mass released in the UZ.

The corresponding distributions of ${ }^{237} \mathrm{~Np}$ and ${ }^{239} \mathrm{Pu}$, respectively, in tsw39 and at the water table under continuous release for a mean present-day infiltration are included in output DTN: LB0307MR0060R1.004, which also include figures with the $F_{R}$ distributions. Comparison to the figures for ${ }^{237} \mathrm{~Np}$ and ${ }^{239} \mathrm{Pu}$ to those for ${ }^{99} \mathrm{Tc}$ in Appendix E indicate that the same general patterns persist. The differences in the magnitude and areal extent of concentrations are mainly due to the different sorbing behavior of the radionuclides. Thus, with an increasing sorption affinity to the UZ rocks from ${ }^{99} \mathrm{Tc}$ to ${ }^{237} \mathrm{~Np}$ to ${ }^{239} \mathrm{Pu}$, the areal extent of the radionuclide occurrence decreases and the corresponding maximum $X_{R}$ in the fractures decrease, the maximum $X_{R}$ in the matrix decreases even more severely, and the matrix $X_{R}$ increases. Note that at $\mathrm{t}=100,000$ years, significant amounts of ${ }^{237} \mathrm{~Np}$ and ${ }^{239} \mathrm{Pu}$ are sorbed over areas exceeding the footprint of the repository at both the tsw39 level and at the water table. The implication is that these sorbed radionuclides can become sources for long-term release to the water table, even if releases from the repository cease. 


\subsection{THREE-DIMENSIONAL TRANSPORT OF THE ${ }^{239} \mathrm{Pu} \longrightarrow{ }^{235} \mathrm{U} \longrightarrow{ }^{231} \mathrm{~Pa}$ CHAIN - CONTINUOUS RELEASE}

This section discusses the transport of the three-member ${ }^{239} \mathrm{Pu} \longrightarrow{ }^{235} \mathrm{U} \longrightarrow{ }^{231} \mathrm{~Pa}$ chain, continuously released from the repository, by means of EOS9nT three-dimensional site-scale simulations of the entire UZ system of Yucca Mountain.

Only mean present-day infiltration was considered in these simulations. The grids, flow fields, and conditions are as discussed in Section 6.7, and the parameters used in these EOS9nT simulations are listed in Tables 6-3, 6-4, 6-5, and 6-6. The DTN of the input and output files for the three present-day infiltration scenarios is output DTN: LB0307MR0060R1.003 (Table 6-6).

The evolution of the mass fractions $R_{F}$ in the release stream at the repository and at the water table are shown in Figures 6-55(a) and (b). Figure 6-55(a) reflects decay and generation of each of the members of the ${ }^{239} \mathrm{Pu}$ chain, and, being a boundary condition, is unaffected by flow or transport conditions in the UZ. Note that the released stream becomes depleted in ${ }^{239} \mathrm{Pu}$ due to decay after about 100,000 years, at which time ${ }^{235} \mathrm{U}$ is practically the only species entering the $\mathrm{UZ}$ from the repository.

The mass fractions at the water table in Figure 6-55(b) incorporate the cumulative effects of migration through the UZ and reflect the effects of matrix diffusion and sorption on the effluent concentration. Comparison of Figures 6-55(a) and (b) reveals a very important difference. The radionuclide stream at the water table shows a much earlier enrichment in ${ }^{235} \mathrm{U}$, and ${ }^{235} \mathrm{U}$ is the increasingly dominant species after $t=2000$ years. The reason for the difference is the much stronger sorption of ${ }^{239} \mathrm{Pu}$ in the UZ, coupled with the relatively weak sorption of ${ }^{235} \mathrm{U}$. The contribution of ${ }^{231} \mathrm{~Pa}$ appears practically insignificant in the first 1,000,000 years because of the long half-life of ${ }^{235} \mathrm{U}$ and its strong affinity for sorption (Section 6.11).

Figures 6-55(a) and (b) reflect relative concentrations of radionuclides in the inflow and outflow streams, but provide no information on the actual inflow and outflow relationship. This is addressed by the relative release rates at the water table in Figure 6-56, computed as the ratio of the total outflow rates (i.e., sum of individual member fluxes) versus the total inflow rates at the repository. A comparison of Figure 6-56 to that for the ${ }^{239} \mathrm{Pu}$ in Figure 6-55(a) (obtained under the same conditions and in the same domain) indicates the importance of considering all the members of the chain in the computations, and the significant error that will be introduced if only the parents are considered. The $t_{10}$ and $t_{50}$ of this chain under a continuous release scenario for a mean present-day infiltration are listed in Table 6-11. Given the long half-life of ${ }^{239} \mathrm{Pu}$ and the much longer half-life of ${ }^{235} \mathrm{U}$ (see Table 6-4), the daughter contributions are very important and cannot be neglected. 
(a)
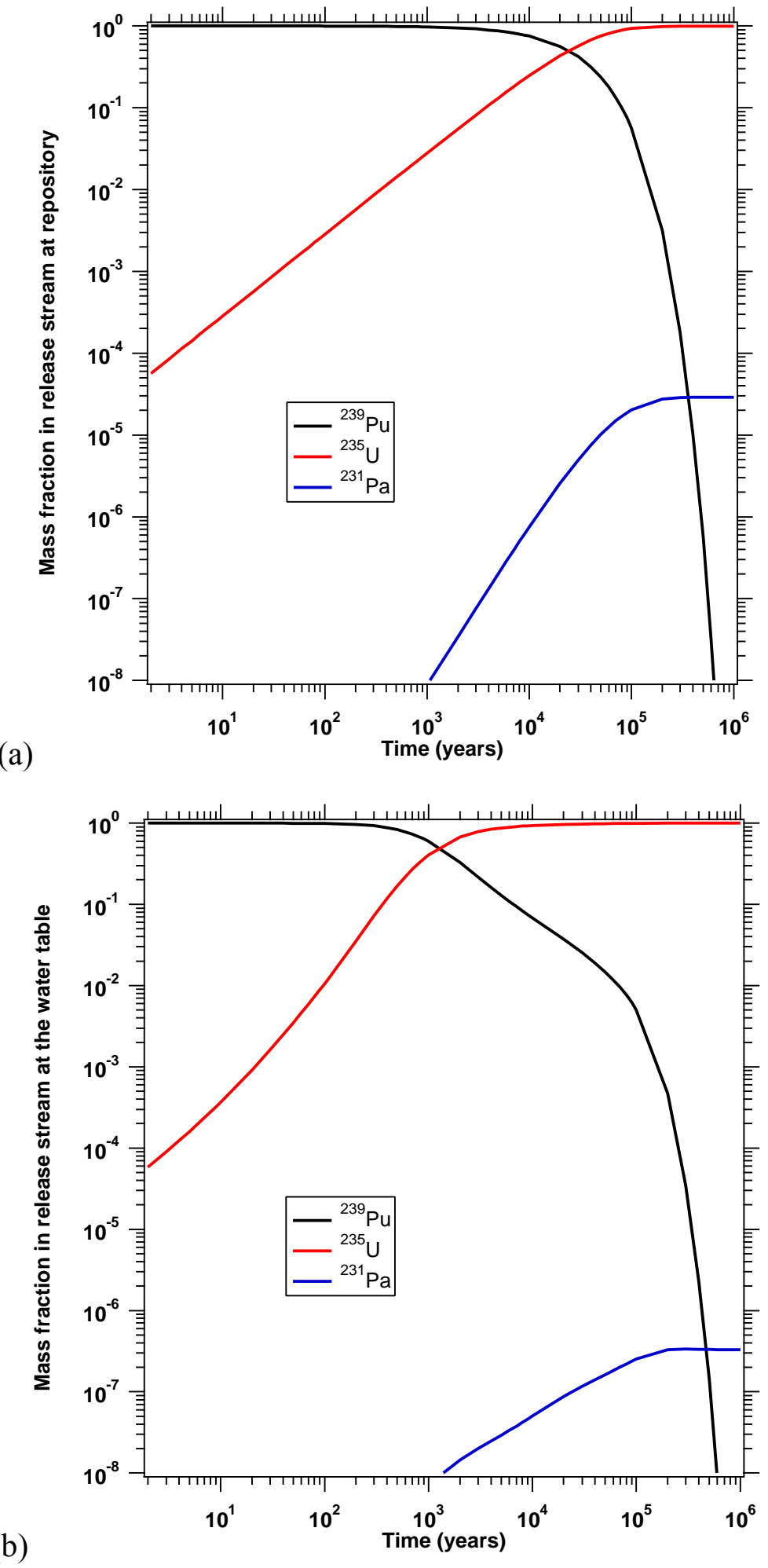

Output DTN: LB0307MR0060R1.007, data submitted with this model report.

Figure 6-55. Mass Fractions of Each Member of the ${ }^{239} \mathrm{Pu}$ Chain in the Release Stream at (a) the Repository and (b) the Water Table - Continuous Release Mean Present-Day Climatic Scenarios 


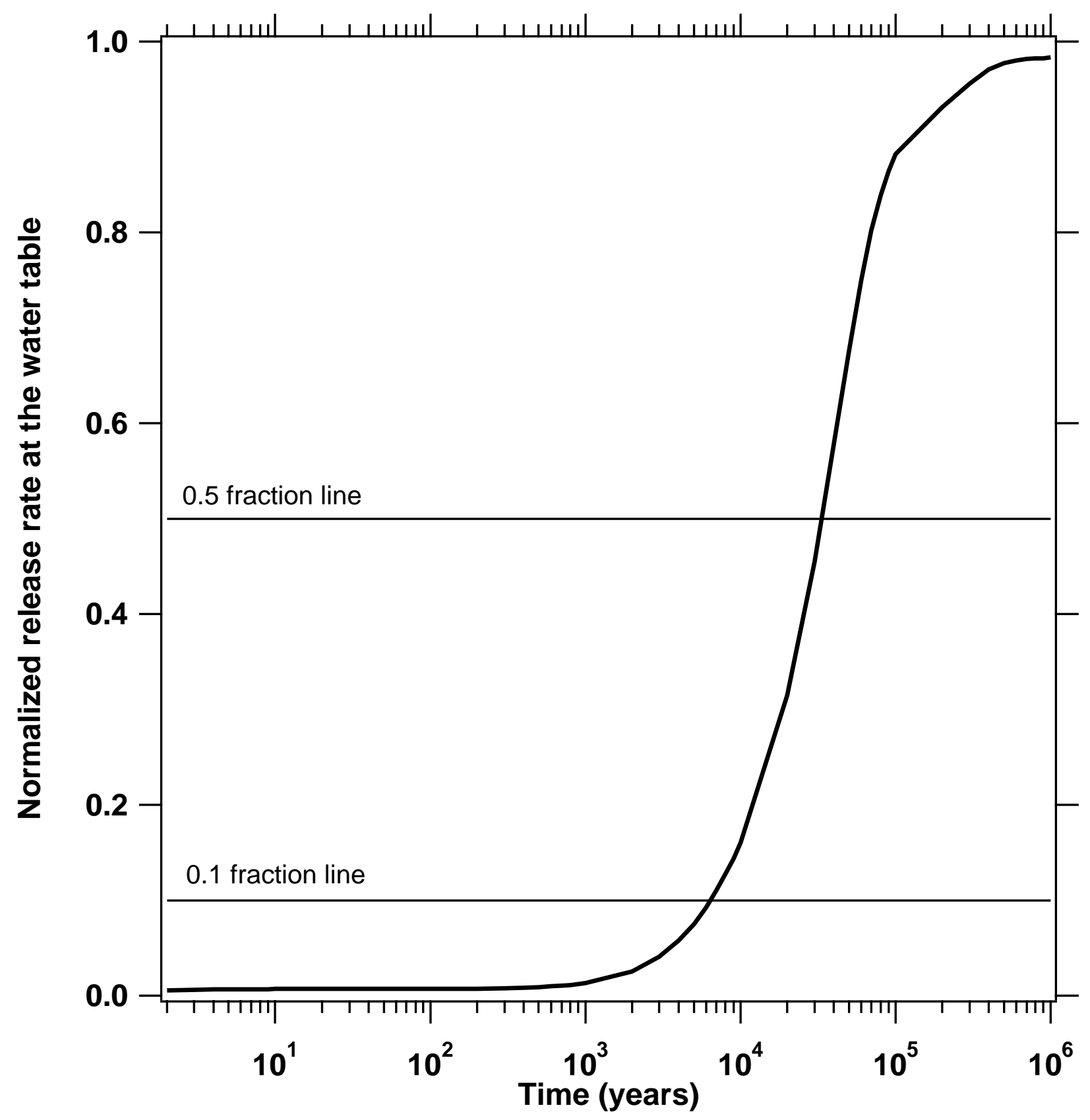

Output DTN: LB0307MR0060R1.007, data submitted with this model report.

Figure 6-56. Normalized Relative Release $R_{F}$ of the Sum of All Members of the ${ }^{239} \mathrm{Pu} C h a i n$ at the Water Table - Continuous Release Mean Present-Day Climatic Scenarios 


\subsection{THREE-DIMENSIONAL TRANSPORT OF THE ${ }^{241}$ Am $\longrightarrow{ }^{237} \mathbf{N p}^{\longrightarrow}{ }^{233} \mathrm{U}$ $\longrightarrow{ }^{229}$ Th CHAIN - CONTINUOUS RELEASE}

This section addresses the transport of the four-member ${ }^{241} \mathrm{Am} \longrightarrow{ }^{237} \mathrm{~Np} \longrightarrow{ }^{233} \mathrm{U} \longrightarrow{ }^{229} \mathrm{Th}$ chain continuously released from the repository. Each member of the chain has its own distinct transport behavior. Only mean present-day infiltration was considered in these simulations. The grids, flow fields, and conditions are as discussed in Section 6.7. The parameters used in these EOS9nT simulations are listed in Tables 6-3, 6-4, 6-5, and 6-6. The DTN of the input and output files for the three present-day infiltration scenarios is output DTN: LB0307MR0060R1.003 (Table 6-6).

The evolution of the mass fractions $R_{F}$ in the release stream at the repository and at the water table is shown in Figures 6-57(a) and (b). Figure 6-57(a) is a straightforward representation of decay and generation for each member of the ${ }^{241} \mathrm{Am}$ chain, and is unaffected by flow or transport conditions in the UZ because it reflects boundary conditions. Because of the short half-life of ${ }^{241} \mathrm{Am}$, its concentration in the release stream falls to practically zero after about 3,000 years, at which time ${ }^{237} \mathrm{~Np}$ is overwhelmingly the dominant species leaving the repository.

The mass fractions at the water table in Figure 6-57(b) reflect the cumulative effects of passage through the UZ, that is, the effects of matrix diffusion and sorption on the radionuclide concentration. Comparison of Figures 6-57(a) and (b) reveals that, as in the ${ }^{239} \mathrm{Pu}$ chain case, the effluent at the water table is enriched in ${ }^{237} \mathrm{~Np}$ much earlier (about $t=500$ years). This behavior is caused by the much stronger sorption of ${ }^{241} \mathrm{Am}$ in the UZ, coupled with the relatively weak sorption of ${ }^{237} \mathrm{~Np}$ (Table 6-3 and Section 6.9). The contribution of ${ }^{233} \mathrm{U}$ exceeds only 1 percent after about 1,000,000 years, while the long cumulative half-lives of its parents make the contribution of ${ }^{229}$ Th negligible.

The relative release rate at the water table is computed as the ratio of the total outflow rates (i.e., sum of individual member fluxes) versus the total inflow rates at the repository, and is shown in Figure 6-58. For the reasons discussed in Section 6.15, it is important to consider all the members of the chain in the computations of transport. Given the long half-life of ${ }^{237} \mathrm{~Np}$ (much longer than that of its ${ }^{241} \mathrm{Am}$ parent; see Table 6-4), the daughter contributions cannot be neglected with impunity. The daughter contributions are even more important under wetter infiltration scenarios. The $t_{10}$ and $t_{50}$ of this chain under a continuous release scenario for a mean present-day infiltration are listed in Table 6-11. 


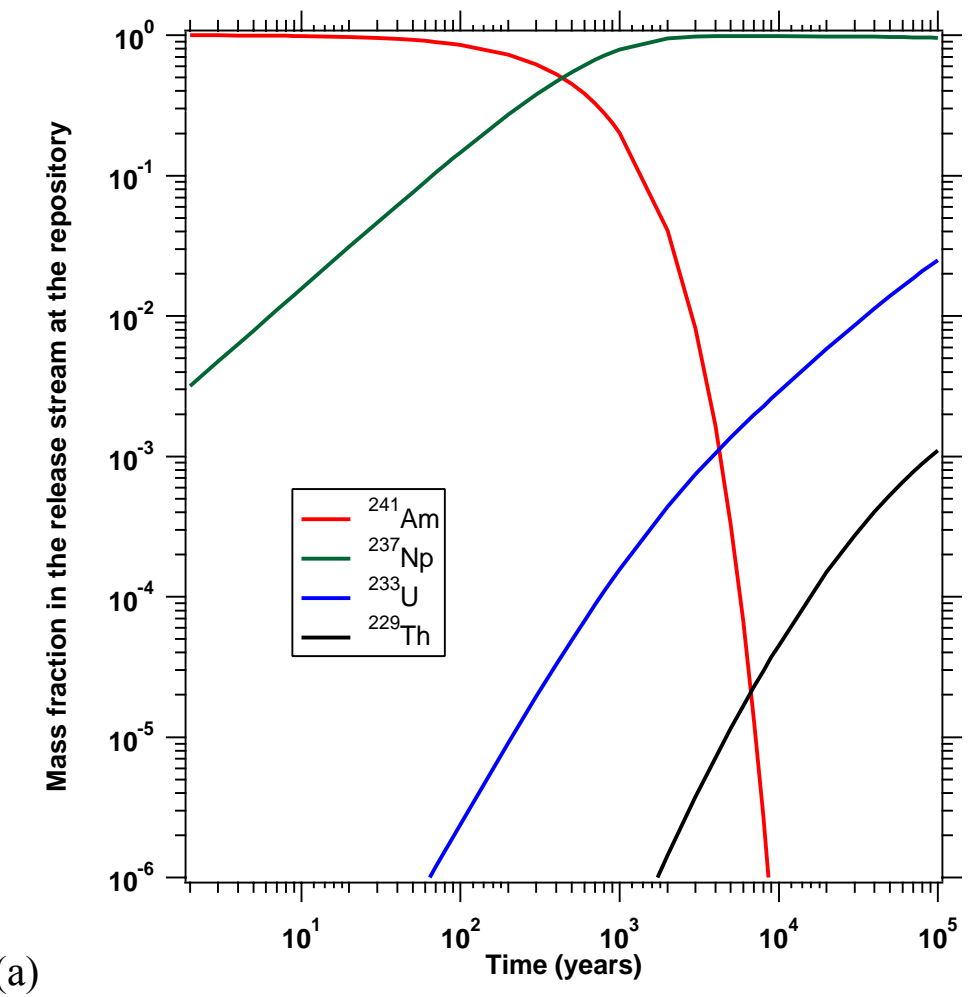

(a)

(b)

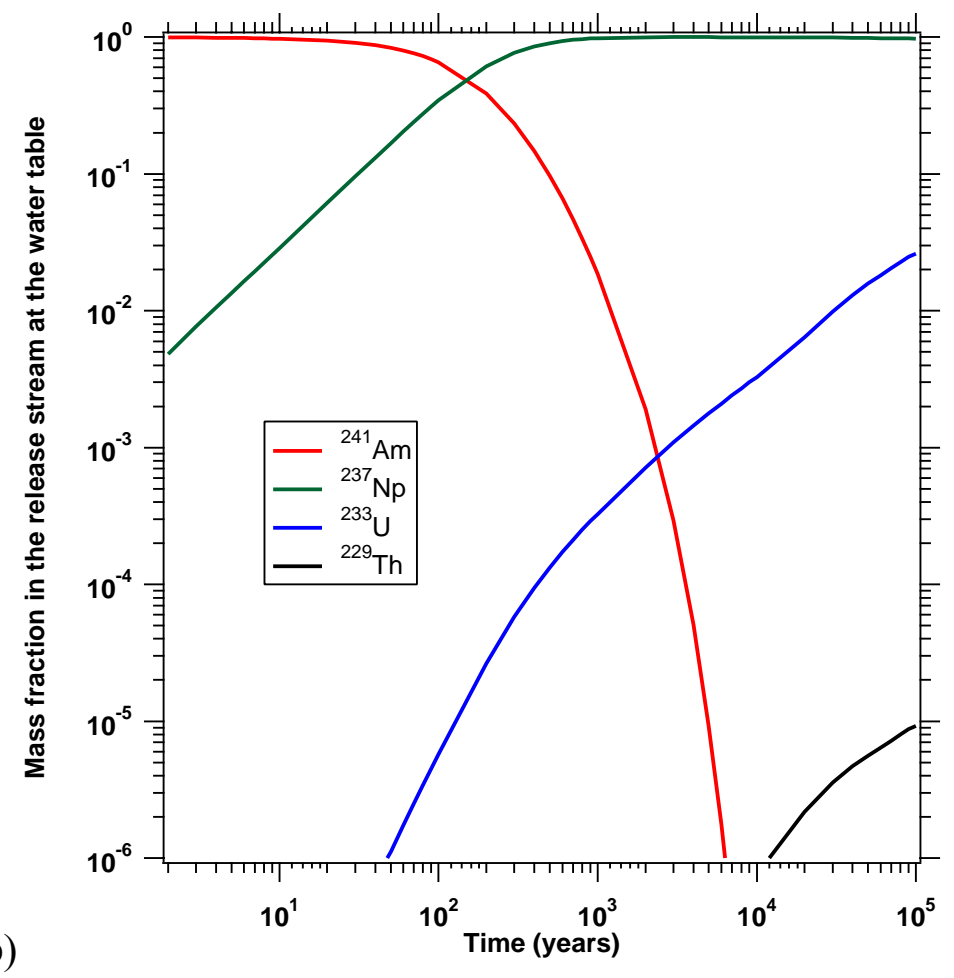

Output DTN: LB0307MR0060R1.007, data submitted with this model report.

Figure 6-57. Mass Fractions of Each Member of the ${ }^{241} \mathrm{Am}$ Chain in the Release Stream at (a) the Repository and (b) the Water Table - Continuous Release Mean Present-Day Climatic Scenarios 


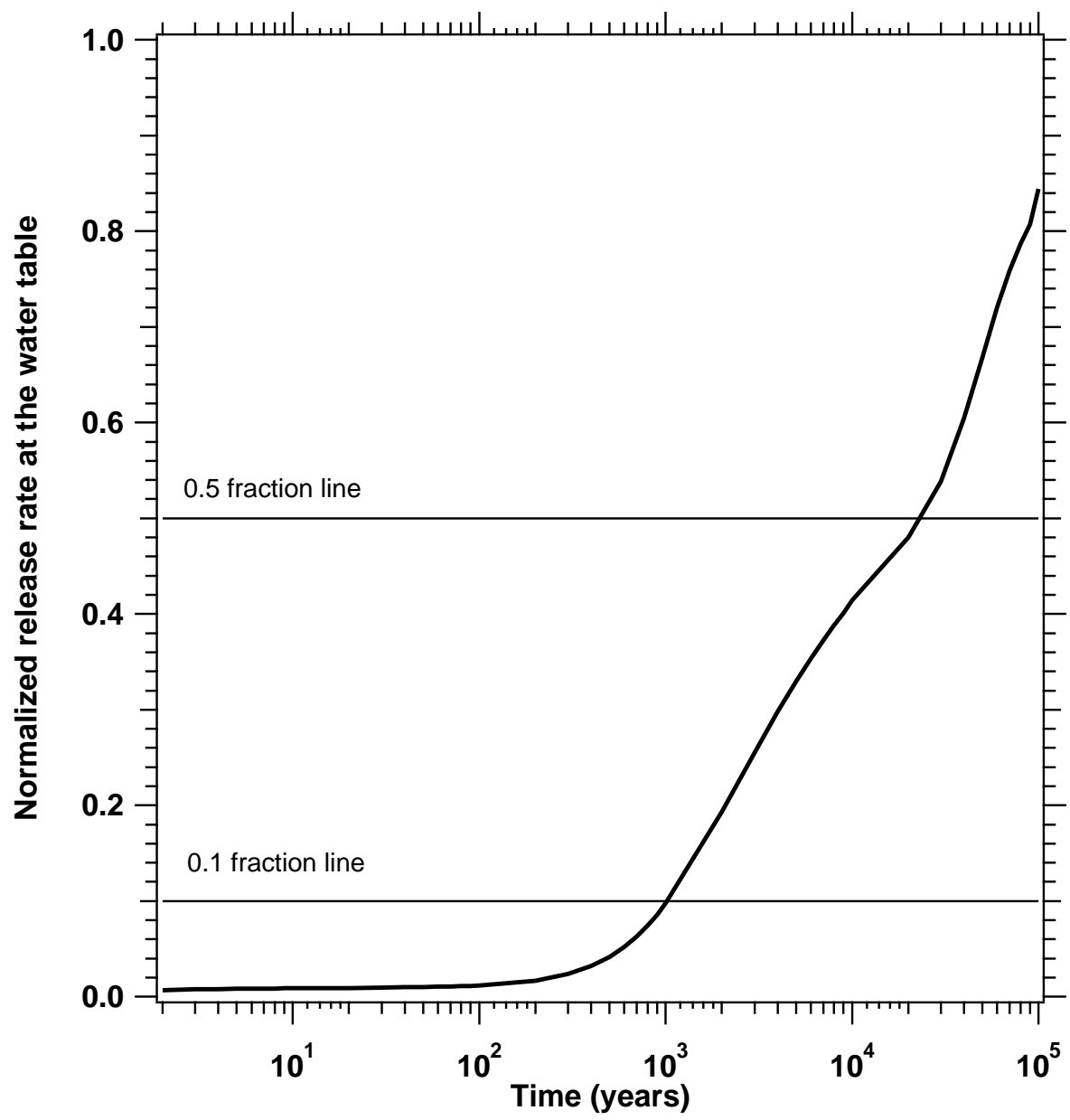

Output DTN: LB0307MR0060R1.007, data submitted with this model report.

Figure 6-58. Normalized Relative Release $R_{F}$ of the Sum of All Members of the ${ }^{241} \mathrm{Am}$ Chain at the Water Table - Continuous Release Mean Present-Day Climatic Scenario

\subsection{THREE-DIMENSIONAL SITE-SCALE TRANSPORT OF ${ }^{239} \mathrm{PuO}_{2}$ COLLOIDS - CONTINUOUS RELEASE}

This section addresses the large-scale three-dimensional site-scale transport of true colloids in the Yucca Mountain UZ for mean present-day infiltration. The flow field and the three-dimensional grid are identical to the ones discussed in Sections 6.14 to 6.17. Radioactive colloids undergoing decay at the source were continuously released into the fractures of the elements corresponding to the repository.

Section 6.18 includes eight subsections. Section 6.18.1 discusses the colloidal forms and properties. Section 6.18.2 presents the mathematical model of filtration used in EOS9nT and discusses its parameters for the four colloids considered in the simulations. Section 6.18.3 describes the four cases of colloid transport investigated in this model report, and Sections 6.18.4 to 6.18.8 discuss the simulation results for each case. 


\subsubsection{Colloidal Forms and Properties}

True colloids were considered first in this study (see Section 6.1.3.3.1). True colloids are colloids in which the entire nonaqueous component of the colloid is composed of the radioactive substance. These colloids were taken to have the properties of ${ }^{239} \mathrm{PuO}_{2}$ and are subject to radioactive decay. Additionally, the colloid size and density were considered invariable during the simulation. This results in more conservative predictions because (a) matrix diffusion does not increase in response to a smaller colloid diameter, and (b) colloid filtration (the parameters of which are based on the actinide mass per unit volume) does not decrease due to lower concentrations. This type of colloid was not included in the TSPA, but is presented here as a sensitivity case.

Waste form colloids (i.e., pseudocolloids in which radionuclides are irreversibly embedded) were considered next. Computation of the transport of these colloids involves solution of the transport equation of the underlying nonradioactive pseudocolloid. The radioactivity concentration is computed from

$$
C_{r}=C_{c} X_{r 0} f_{t}
$$

where $C_{r}$ is the actinide concentration $\left[M L^{-3}\right], C_{c}$ is the underlying nonradioactive psuedocolloid particle concentration $\left[\mathrm{ML}^{-3}\right], X_{r 0}$ is the initial mass fraction (i.e., at the time of release) of the embedded radionuclide in the colloidal particle, and $f_{t}$ is the remaining radionuclide mass fraction at time $t$. For a parent radionuclide, $f_{t}=\exp (-\lambda t)$. In this study, the embedded radionuclide in the waste form colloid was assumed to be ${ }^{239} \mathrm{Pu}$.

In both true and waste form colloidals considered in this study, four different colloid sizes were considered. Their sizes and their accessibility factors were calculated based on DTNs: GS950608312231.008 [DIRS 144662] and GS980908312242.039 [DIRS 145272] and are shown in Table 6-8. The parameters used in these EOS9nT simulations of three-dimensional transport of the colloids are listed in Table 6-5, 6-6, 6-8, and 6-9. The DTN of the input and output files for the three present-day infiltration scenarios is output DTN: LB0307MR0060R1.003 (Table 6-6).

\subsubsection{Colloid Filtration Model and Coefficients}

Pore-size exclusion (straining filtration) was described using the accessibility factors shown in Table 6-8. The linear kinetic model of colloid filtration described by Equation 6-15 was used in the simulations. The forward kinetic coefficient $\kappa^{+}$was computed from Equation 6-16, in which the velocity coefficients are as shown in Table 6-8, and the filter coefficient $\varepsilon$ is computed using Equations 2 and 6 of Harvey and Garabedian's report (1991 [DIRS 109256]). In our nomenclature, this is

$$
\varepsilon=1.5 \frac{1-\phi}{d_{m}} \alpha_{c} \eta_{c}
$$


where $d_{m}$ is the particle size of the medium grains or the fracture aperture $[L], \alpha_{c}$ is the collision efficiency factor, and $\eta$ is the single collector efficiency; and

$$
\eta_{c}=0.9\left(\frac{k_{B} T}{\mu_{w} d_{c} d_{m} u}\right)^{2 / 3}+1.5\left(\frac{d_{c}}{d_{m}}\right)^{2}+\left(\rho_{c}-\rho\right) \frac{g d_{c}^{2}}{18 \mu_{w} u}
$$

in which $k_{B}$ is the Boltzmann constant, $d_{c}$ is the colloid diameter [L], $T$ is the absolute temperature, and all other terms remain as previously defined. The clogging (forward) kinetic coefficients $\kappa^{+}$are computed internally in EOS9nT.

No information exists on the kinetic declogging (reverse) coefficient $\kappa^{-}$. To alleviate the problem, $\kappa^{-}$in the EOS9nT coefficients was entered as a fraction of $\kappa^{+}$(see Table 5-1). This approach was used because the kinetic coefficient $\kappa^{+}$is a linear function of the flow velocity.

Table 6-8. Properties of the Four Colloids in the EOS9nT Simulations

\begin{tabular}{|l|l|l|l|l|}
\hline \multicolumn{1}{|c|}{ Parameter } & \multicolumn{1}{c|}{$\mathbf{4 5 0} \mathbf{~ n m}$} & \multicolumn{1}{c|}{$\mathbf{2 0 0} \mathbf{~ n m}$} & \multicolumn{1}{c|}{$\mathbf{1 0 0} \mathbf{~ n m}$} & \multicolumn{1}{c|}{$\mathbf{~ n m}$} \\
\hline Diffusion coefficient $D_{0}\left(\mathrm{~m}^{2} / \mathrm{s}\right){ }^{*}$ & $9.53 \times 10^{-13}$ & $2.15 \times 10^{-12}$ & $4.29 \times 10^{-12}$ & $7.15 \times 10^{-11}$ \\
\hline Velocity modification factor $f \dagger$ & 1.5 & 1.2 & 1.1 & 1.0 \\
\hline Accessibility factor $f_{c}$ in the TSw $\ddagger$ & 0.05 & 0.10 & 0.20 & 0.65 \\
\hline Accessibility factor $f_{c}$ in the $\mathrm{CHv} \ddagger$ & 0.45 & 0.50 & 0.55 & 0.80 \\
\hline Accessibility factor $f_{c}$ in the $\mathrm{CHz} \ddagger$ & 0.20 & 0.25 & 0.25 & 0.65 \\
\hline
\end{tabular}

* From Grathwohl 2000 [DIRS 141512].

† Reasonable estimates, see Section 6.2.4.1.

† DTN: GS950608312231.008 [DIRS 144662], GS980908312242.039 [DIRS 145272] (indirect input). Accessibility factors were developed froom the water retention data using the pore size distribution methodology given in Marshall and Holmes (1979 [DIRS 102532], Section 8.5)

Table 6-9. Input Parameters for the EOS9nT Three-Dimensional Site-Scale Simulations of Colloid Transport (\# 1 Perched-Water Model, Mean Present-Day Infiltration)

\begin{tabular}{|l|l|}
\hline \multicolumn{1}{|c|}{ Parameters } & \multicolumn{1}{c|}{ Source } \\
\hline Tortuosity $\tau \simeq \phi^{\text {a }}$ & Farrell and Reinhard (1994 [DIRS 122803], p. 64) \\
\hline $\begin{array}{l}\text { Properties and characteristics of the geologic units, } \\
\text { steady-state pressures, water saturations and flow } \\
\text { fields }\end{array}$ & $\begin{array}{l}\text { DTN: LB0210THRMLPRP.001 [DIRS 160799], } \\
\text { LB03023DSSCP9I.001 [DIRS 163044] (Table 6-7) }\end{array}$ \\
\hline Colloid density $\rho_{c}=11,460 \mathrm{~kg} / \mathrm{m}^{3}\left(\mathrm{PuO}_{2}\right)$ & Lide (1993 [DIRS 123032], p. 4-83) \\
\hline $\begin{array}{l}\text { Forward kinetic filtration (clogging) coefficient } \mathrm{\kappa}+: \text { from } \\
\text { Equations 6-16, 6-32, and 6-33, parameters computed } \\
\text { at } 25^{\circ} \mathrm{C}\end{array}$ & $\begin{array}{l}\text { Harvey and Garabedian 1991 [DIRS 109256], } \\
\text { p. 180, Equation 6 }\end{array}$ \\
\hline
\end{tabular}

a See Section 6.1.2.4 for reasonable estimation of $\tau$.

\subsubsection{True Colloid Transport Simulation Cases}

The following four cases were investigated: 
Case 1. $\kappa^{-}=0$. This corresponds to a case of no declogging, in which colloids, once filtered, do not detach from the pore/fracture walls.

Case $2 . \kappa^{-} / \kappa^{+}=100$. This corresponds to strong kinetic declogging and will provide an estimate of maximum colloidal transport.

Case 3. $\kappa^{-} / \kappa^{+}=0.1$. This corresponds to weak kinetic declogging and approaches equilibrium filtration behavior.

Case 4. Same as in Case 2, but the fractures are assumed to have the same colloidal transport properties as the corresponding matrix. This study provides an estimate of the importance of fractures in the transport of colloids. Note that the change in the filtration properties affects only 1 percent of the fracture pore volume (because $\phi=0.99$ in the fractures; see Equation 6-30).

There is no filtration (attachment) of colloids onto the fracture walls in Cases 1 to 3 . The $t_{10}$ and $t_{50}$ of the ${ }^{239} \mathrm{PuO}_{2}$ colloid transport under a continuous release scenario and for a mean present-day infiltration are listed in Table 6-12.

\subsubsection{Colloid Transport Case 1 (True Colloids)}

This simulation assumes that no declogging occurs once colloids are attached to the matrix. Figure 6-59(a) shows the relative release rate $R_{F}$ of the four colloid sizes at the water table. For reference, the decaying release rate of the colloids at the repository (the same for all

${ }^{239} \mathrm{Pu}$ colloids) is included in the figure. Two observations appear particularly important. The first is the very fast breakthrough of the larger colloids (characterized by a rapid rise of the breakthrough curves). The fast breakthrough is about the same for all the larger colloids. The $t_{10}$ for the three larger colloids is only about 5 years. After the fast rise, $t_{50}$ is not reached for any colloid, and the $R_{F}$ begins declining as decay affects concentrations. The second observation is that, contrary to expectations, the smallest colloid $(6 \mathrm{~nm})$ exhibits the slowest breakthrough. It is really noteworthy that this colloid never reaches the 0.1 fraction level, indicating that it is sufficiently small to enter the matrix and get attached to the fracture walls. This behavior results from a combination of the following factors:

1. The faster transport velocity of larger colloids, which, by virtue of their size, can only move in the center of fractures where velocities are larger than the average water velocity. In this case, the $450 \mathrm{~nm}$ colloid moves at 1.5 times the water velocity (i.e., $f=1.5$; see Equation 6-16 in Section 6.2.4.1).

2. Larger colloids are unable to penetrate the matrix from the fractures because of size exclusion. Thus, the colloid mass in the fractures is not reduced through colloidal diffusion and/or hydrodynamic dispersion, and practically all of it moves exclusively in the fractures. The $6 \mathrm{~nm}$ colloid can diffuse into the matrix, a process that in Figure 6-59(a) manifests itself by the substantially slower breakthrough, the slow and mild rise of the curve, and the low maximum $R_{F}$ value. 
After the initial rapid rise, the slope of the $R_{F}$ curve in the larger colloids becomes much milder, and is then followed by a short upward trend again, before it begins to decline at a point that coincides with the beginning of the rapid decline in concentration due to decay. This second upward trend is associated with the beginning of matrix flow of colloids. A similar trend, but much delayed, is observed in the $6 \mathrm{~nm}$ colloid.

\subsubsection{Colloid Transport Case 2 (True Colloids)}

\subsubsection{Breakthrough Curves}

Figure 6-59(b) shows the relative release rate $R_{F}$ of the four colloid sizes at the water table. The differences between Case 1 and Case 2 are very small, and are most prominent in the case of the $6 \mathrm{~nm}$ colloid. The $F_{R}$ is higher in Case 2 because the few colloids that manage to enter the matrix are not irreversibly attached as in Case 1, but declog rather rapidly. The relative insensitivity to the clogging model in the matrix indicates the dominant role of the fractures in the three-dimensional site scale system, with the matrix appearing to have a minuscule contribution. Because of the similarity between Figures 6-59(a) and (b), the comments made in Case 1 apply here too.

\subsubsection{Transport Mechanisms and Patterns of the $6 \mathrm{~nm}$ Colloid}

The importance of fractures on the transport of the $6 \mathrm{~nm}$ colloid (Co0060) is evident in Figure F-1 (see Appendix F), which shows the areal distribution of $X_{R}$ in the aqueous phase in the fractures in the tsw39 layer at $t=10$ years. Despite the very early time, the colloid concentration in the fracture is substantial. The reasons for the significant presence (in terms of areal extent and level of concentration) of the $6 \mathrm{~nm}$ colloid are that (a) diffusion into the matrix is limited because of its relatively large size (compared to solutes) and pore exclusion, and (b) the tsw39 layer is above the TSw-CHn interface, where significant filtration occurs. Note that the distribution pattern of the fracture $X_{R}$ in Figure F-1 indicates that the colloids accumulate at the TSw-CHn interface and, unable to cross it, move along the sloping interface in an easterly direction. This transport is more pronounced in the $6 \mathrm{~nm}$ colloid than in the solutes.

In contrast to Figure F-1, the matrix $X_{R}$ in Figure F-2 shows no difference from the background at the same time. In Figure F-3, the distribution of matrix $F_{R}$ at $t=10$ years shows a sizable presence of the $6 \mathrm{~nm}$ colloid filtered onto the matrix of the tsw39 layer. As in the case of sorbing solutes, the area of nonzero $F_{R}$ (Figure F-3) is larger than that of the fracture $X_{R}$ footprint (Figure F-3) at the same time, and shows the presence of filtered $6 \mathrm{~nm}$ colloids at locations where there appear to be no colloids suspended in the aqueous phase in the fractures. 
At $t=100$ years, the fracture $X_{R}$ in Figure F-4 is significant over a large area (larger than that for solutes at the same time), and is at its maximum value over a large portion of this area. Although the fracture $X_{R}$ (Figure F-5) does not register a measurable difference from the UZ background, the matrix $F_{R}$ (Figure F-6) shows a significant presence of the $6 \mathrm{~nm}$ colloid over an area larger than that covered by the fracture $X_{R}$ (Figure F-4). Note that the matrix $F_{R}$ distribution indicates more filtration in the northern part of the repository. This is consistent with the permeability barriers that result in the perched-water bodies at this location.

A comparison of the matrix $F_{R}$ of the $6 \mathrm{~nm}$ colloid (Figure F-6) to that of the dissolved ${ }^{239} \mathrm{Pu}$ (output DTN: LB0307MR0060R1.004) shows a drastically different pattern. While the matrix $F_{R}$ distribution of ${ }^{239} \mathrm{Pu}$ shows concentration of sorption in the southern part of the repository, the $F_{R}$ of the $6 \mathrm{~nm}$ colloid shows filtration in both the northern and the southern part, that is, colloids and sorbing radioactive solutes (Sections 6.12 to 6.15) are not similarly affected under the \#1 perched-water model. The reason for the different behavior is attributed to (a) the lower diffusion of the colloid into (and the lack of filtration onto) the matrix (thus the faster transport of colloids in larger amounts than solutes to the TSw-CHn interface), (b) the lack of sorption, and (c) the combined effects of pore-size exclusion and filtration at the interface quantitatively larger than the effects of sorption of the limited dissolved ${ }^{239} \mathrm{Pu}$ amounts.

The fracture $X_{R}$, matrix $X_{R}$, and matrix $F_{R}$ distributions at $\mathrm{t}=1,000$ years and $\mathrm{t}=10,000$ years are shown in Figures F-7 to F-12, and they follow the same pattern. The matrix $X_{R}$ registers measurable deviations from background at $\mathrm{t}=1,000$ years (Figure F-8), and it shows extensive areas of relatively high concentrations at $t=10,000$ years (Figure F-11). At $t=10,000$ years, the concentration in the fractures exceeds that in the water released from the repository (i.e., $X_{R}=1.07$ in tsw39). This is caused by pore-exclusion (straining) at the TSw-CHn interface, which leads to the accumulation of the $6 \mathrm{~nm}$ colloid in the tsw39.

Transport at the water table is shown in Figures F-16 to F-30, and follows patterns analogous to those in the tsw39 layer. The high concentrations at the water table at early times $(\mathrm{t} \leq 100$ years $)$ are noteworthy. Matrix $X_{R}$ begins to show signs of colloid presence at $\mathrm{t}=1,000$ years, and demonstrates highly localized areas of large concentration at $\mathrm{t}=10,000$ years. The nonzero area of the matrix $F_{R}$ is consistently larger than that for fracture $X_{R}$. 


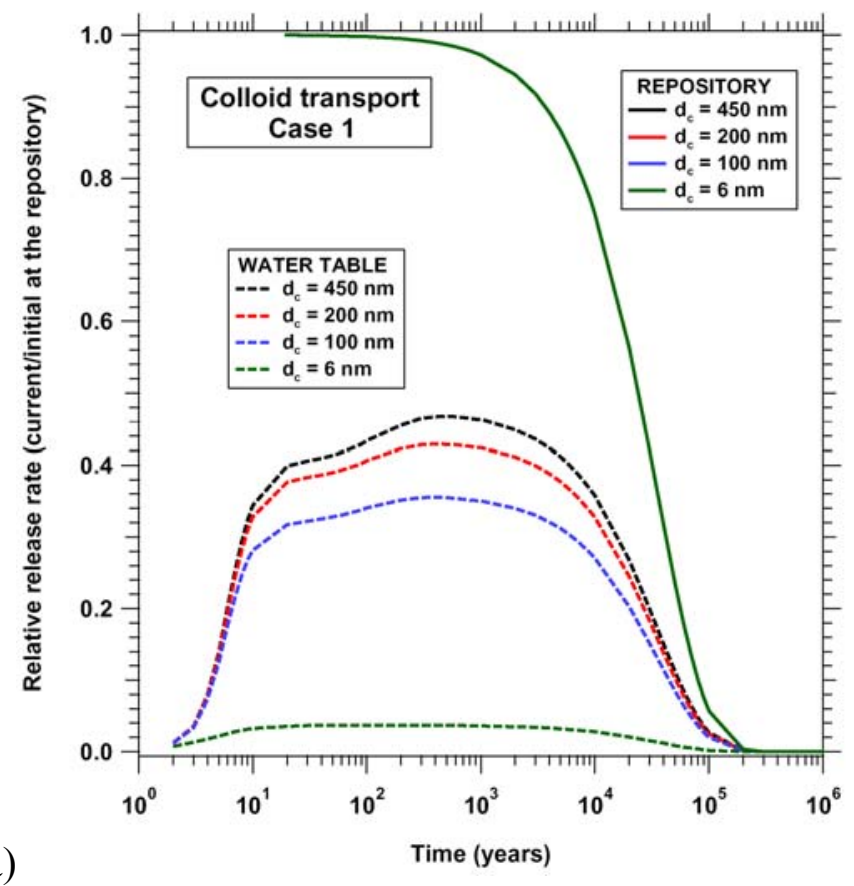

(a)

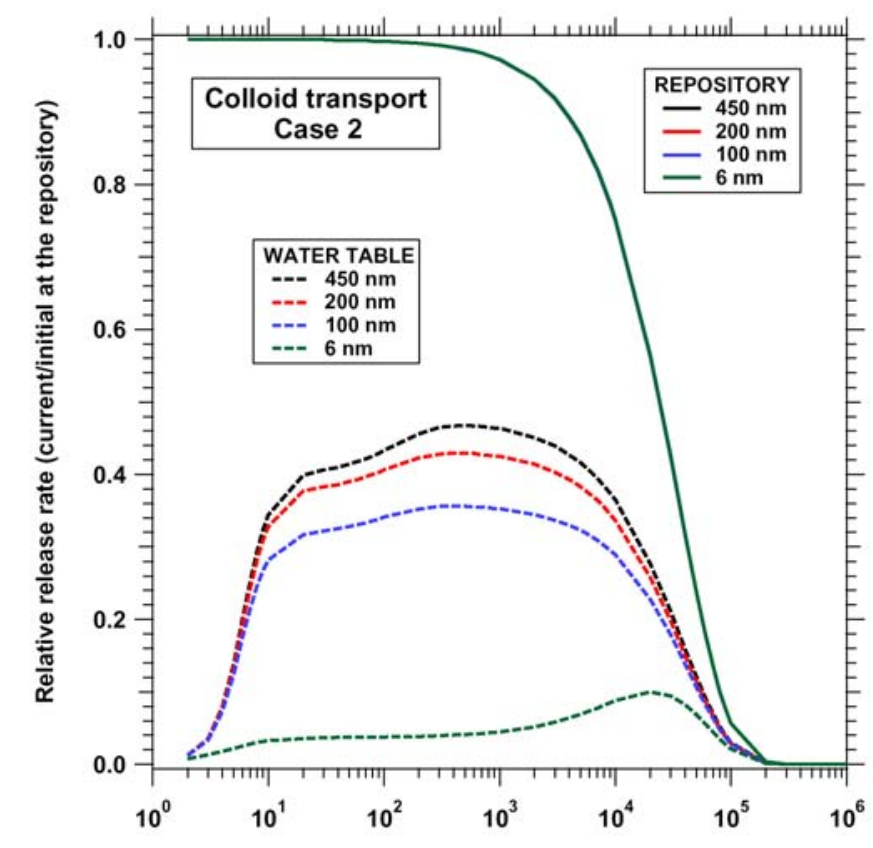

(b) Time (year)

Output DTN: LB0307MR0060R1.007, data submitted with this model report.

Figure 6-59. Normalized Release at the Water Table in (a) Case 1 and (b) Case 2 for True Colloid Transport for Mean Present-Day Infiltration (All the Continous Release Curves at the Repository Are Superimposed, Source Undergoes Decay) 
Note that the fracture $X_{R}$ above the water table exceeds that in the water released from the repository, and the differences between the two increases with time. At $\mathrm{t}=10,000$ years, the maximum fracture $X_{R}>2$, that is, the concentration of the $6 \mathrm{~nm}$ colloid in the fractures above the water table is more than double that in the water released at the repository. This occurs because of the fast transport of the colloids in the fractures (where limited attachment occurs because of the large fracture porosity, that is, $\varphi=1.0$ ) and their accumulation. This accumulation is caused by the very significant pore-size exclusion and kinetic (physical-chemical) filtration at the water table, which prevent colloids from entering the saturated zone while allowing water to flow into it. This occurs because the saturated zone behaves as a porous (rather than a fractured) medium with the properties of the TSw matrix.

Review of Figures F-1 through F-30 indicates that transport of the $6 \mathrm{~nm}$ colloid is dominated by the faults identified and discussed in Sections 6.10 to 6.12. Diffusion from the fractures into the matrix is delayed for the reasons discussed earlier, but the areal extent and the magnitude of the filtered concentration (indicated by the $F_{R}$ distribution) indicates that its effect is sizable.

\subsubsection{Transport Mechanisms and Patterns of the $450 \mathrm{~nm}$ Colloid}

The fracture $X_{R}$, matrix $X_{R}$, and matrix $F_{R}$ distributions of the $450 \mathrm{~nm}$ colloid (Co450) in the tsw39 and above the water table are shown in Figures G-1 through G-30 (see Appendix G). These figures correspond to the same times discussed in the $6 \mathrm{~nm}$ colloid.

Comparison of the figures in Appendix $\mathrm{G}$ to those for the $6 \mathrm{~nm}$ colloid (Appendix $\mathrm{F}$ ) indicates that fractures are far more important to the transport of the $450 \mathrm{~nm}$ colloid. Supporting evidence is provided by (a) the larger areal extent and values of the fracture $X_{R}$, (b) the smaller areal extent and lower values of the matrix $F_{R}$, and (c) the substantial delay in the appearance of $450 \mathrm{~nm}$ colloids in the matrix aqueous phase, when compared to the $6 \mathrm{~nm}$ case.

Thus, the fracture $X_{R}$ in Figure $\mathrm{G}-4(\mathrm{t}=100$ years) indicates contributions from the whole area (at least) of the repository, and shows a much larger footprint than the one corresponding to the $6 \mathrm{~nm}$ colloid. At the water table and at early times (less than 10,000 years), the maximum fracture $X_{R}$ exceeds $1(=1.50)$ because of straining (pore-size exclusion). Because of its larger size, the straining is more pronounced for the $450 \mathrm{~nm}$ colloid than in the $6 \mathrm{~nm}$ colloid, and leads to higher concentrations at earlier times behind the interface. An $X_{R}>1$ does not necessarily mean clogging and decrease in permeability (at least for a long time) if the concentrations of the colloid waste form are sufficiently low.

Compared to the $6 \mathrm{~nm}$ colloid, the $450 \mathrm{~nm}$ colloid exhibits consistently lower matrix $F_{R}$ values over smaller areas (see Figures G-3, G-6, G-9, G-12, G-15, G-18, G-21, and G-24). This is consistent with the expectation of lower diffusion from the fractures into the matrix and increased straining because of its larger size.

\subsubsection{Colloid Transport Case 3 (True Colloids)}

The change in the magnitude of the reverse kinetic filtration coefficient $\kappa^{-}$(as a fraction of $\kappa^{+}$) has a very small effect on colloid transport (Figure 6-60(a)), attesting to the fact that the role of advection through fracture flow (the same in Cases 1 through 3 ) is by far the dominant mechanism of colloid transport. Although this case is closer to equilibrium filtration, the effect 
on transport is apparent only in the case of the $6 \mathrm{~nm}$ colloid (which can enter the pores, and thus be subject to filtration), which shows an $R_{F}$ even lower than that of Case 2.

The insensitivity of transport of the larger colloids to the sorption model in the matrix indicates that the matrix has practically no participation in the retardation, which is attributed to straining at matrix/fracture interfaces. The pattern that emerges is the same as the one discussed in Case 1, and all the comments made and conclusions reached therein apply.

\subsubsection{Colloid Transport Case 4 (True Colloids)}

By assigning a porosity of 1 percent to the fractures, and setting the fracture filtration properties equal to those of the matrix in Case 2, a system is created in which the fractures are partially filled with a porous medium. The simulation results are shown in Figure 6-60(b). The effect of limited diffusion on transport (because of pore-size exclusion and filtration) becomes more obvious in this case. While the more-freely diffusing $6 \mathrm{~nm}$ colloids exhibit a behavior similar to the one in Cases 1 through 4, the effect on the larger colloids is more dramatic. The occurrence of even a minor fillup retards the colloid transport, increasing $t_{10}$ to about 30 years, and registering a $t_{50}$ of about 100 (for the $450 \mathrm{~nm}$ colloid) to 150 years (for the $200 \mathrm{~nm}$ colloid). The maximum $R_{F}$ exceeds that in Case 2 because of temporary colloid storage in (and subsequent release from) the porous medium that partially fills the fractures in Case 4.

Additionally, the breakthrough curve assumes a decidedly different shape in the larger colloids, marked by a sudden and dramatic reversal that is consistent in all the three larger colloids. As the occurrence of this phenomenon coincides with the onset of rapid decline in concentrations due to radioactive decay, it is assumed that the kinetic filtration model is very sensitive to minute changes in concentration at this point-hence the sudden slope reversal. However, this issue is not well understood and will require further investigation.

Note that the significant differences among Figures 6-60(b) and 6-59(a), 6-59(b), and 6-60(a) result from assigning matrix filtration properties to only 1 percent of the pore volume of the fractures (by no means an unphysical or unlikely scenario). The significant retardation of the colloids gives a measure of the importance of fractures and their relative contribution to the total colloid transport through the UZ system.

\subsubsection{Transport of Wasteform Colloids (Case 2 and 3)}

The transport simulations were repeated for wasteform colloids with the filtration behavior of Cases 2 and 3 (see Section 6.18.3). As discussed earlier, radioactive concentrations (and fluxes) were obtained by first computing the transport of the nonradioactive wasteform colloid, and then multiplying it by the remaining radionuclide mass fraction $f_{t}$ (see Eq. 6-31, Section 6.18.1).

Given the approximation of constant colloid size in the true colloid simulations, the $R_{F}$ results of the wasteform colloid simulations were not expected to be very different. Although the radioactive concentrations are much lower in the transport of wasteform colloids than in the transport of true colloids (of the same size and colloid population per unit volume) because of the radioactive component constitutes a small fraction of the total mass, the relative release rate $R_{F}$ (with respect to the initial release rate at the repository) in both cases are practically identical. This is explained by the fact that, mathematically speaking, there is very little difference in the 
transport equations between these two classes of colloids of the same sizes when the same colloid filtration parameters are assumed. Realistic differences between these two colloid types are certain to exist in the properties of these two very different (physically and chemically) colloid classes, which will affect their filtration behavior and the corresponding filtration parameters. However, information on the subject is scant.

Figure 6-61 confirms the expectation discussed above. A comparison of the $R_{F}$ curves over time shows practically no difference from the corresponding results for the true colloids in Figures 6-59 and 6-60.

\subsubsection{Uncertainties and Limitations}

While the results in this section provide some elucidation of colloid transport, caution should be exercised in the interpretation of the simulation results. The reason for this caution is the realization of knowledge gaps that hamper the study of colloid behavior. These results should be viewed as indicative and qualitative, rather than quantitative and predictive.

It is acknowledged that basic knowledge about the kinetic clogging and declogging coefficients $\kappa^{+}$and $\kappa^{-}$is in early stages of development, thus limiting our predictive capabilities. Despite the insensitivity of transport to the filtration parameters in Cases 1 through 3 , the significant change in transport behavior when 1 percent of the fracture volume is given the attributes of the matrix should provide grounds for caution.

There are significant uncertainties in colloid modeling. It is not known whether the applicability limits of the currently available kinetic models, developed from theoretical principles (Herzig et al. 1970 [DIRS 117519]) and tested in uniform sandy laboratory experiments (van de Weerd and Leijnse 1997 [DIRS 109249]) or small-scale field tests (Harvey and Garabedian 1991 [DIRS 109256]), are breached under the UZ conditions at Yucca Mountain. The C-Well experiments (BSC 2004 [DIRS 170006]) addressed some of the colloid-related uncertainties under realistic field conditions. However, the applicability of the information gleaned from those tests (conducted in the saturated zone) to the UZ has not been established. The affinity of colloids for air-water interfaces can have a significant effect on their transport. The limitations in the equations for the prediction of the forward kinetic filtration (clogging coefficient) have not been tested under the UZ conditions, and the subject of the kinetic declogging coefficient has not been studied. Additionally, it is unclear how representative the current size-exclusion (straining filtration) models are. Additional challenges and uncertainties are discussed in detail in Section 6.1.3.

These limitations are not simply limitations of studies undertaken to investigate radionuclide transport at Yucca Mountain but are limitations in the general state of knowledge on the subject in the industry and academia. It should be noted that these uncertainties and limitations likewise apply to the downstream studies in the particle tracking abstraction (BSC 2004 [DIRS 170041]) and the TSPA-LA. However, the specific treatment in this study has no impact on the downstream studies because the results of these colloid simulations are not used in the downstream studies. 


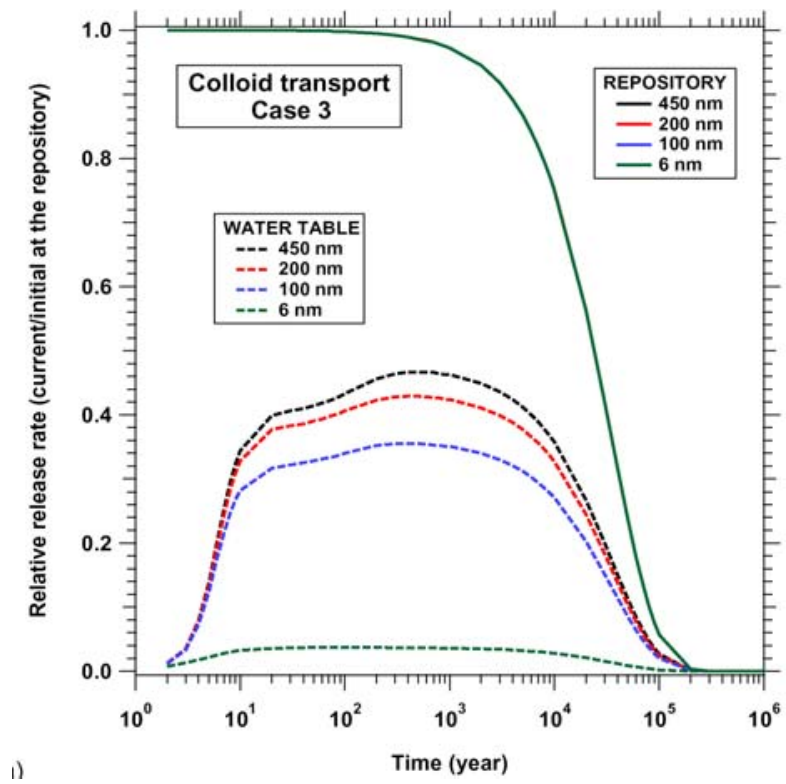

(a)

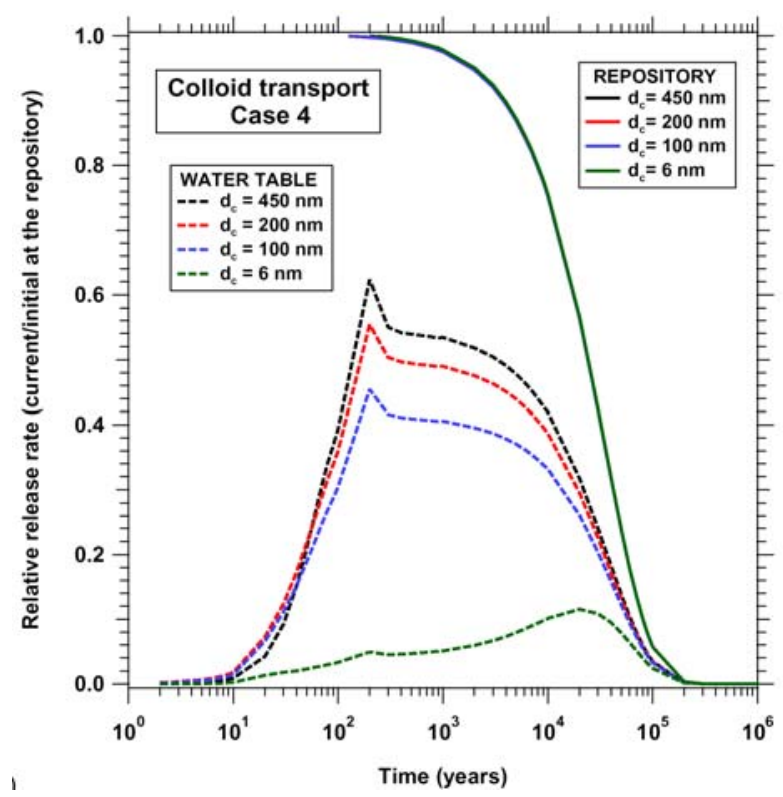

(b)

Output DTN: LB0307MR0060R1.007, data submitted with this model report.

Figure 6-60. Normalized Release at the Water Table in (a) Case 3 and (b) Case 4 for Transport of True Colloids for Mean Present-Day Infiltration (All the Continuous Release Curves at the Repository Are Superimposed, Source Undergoes Decay) 


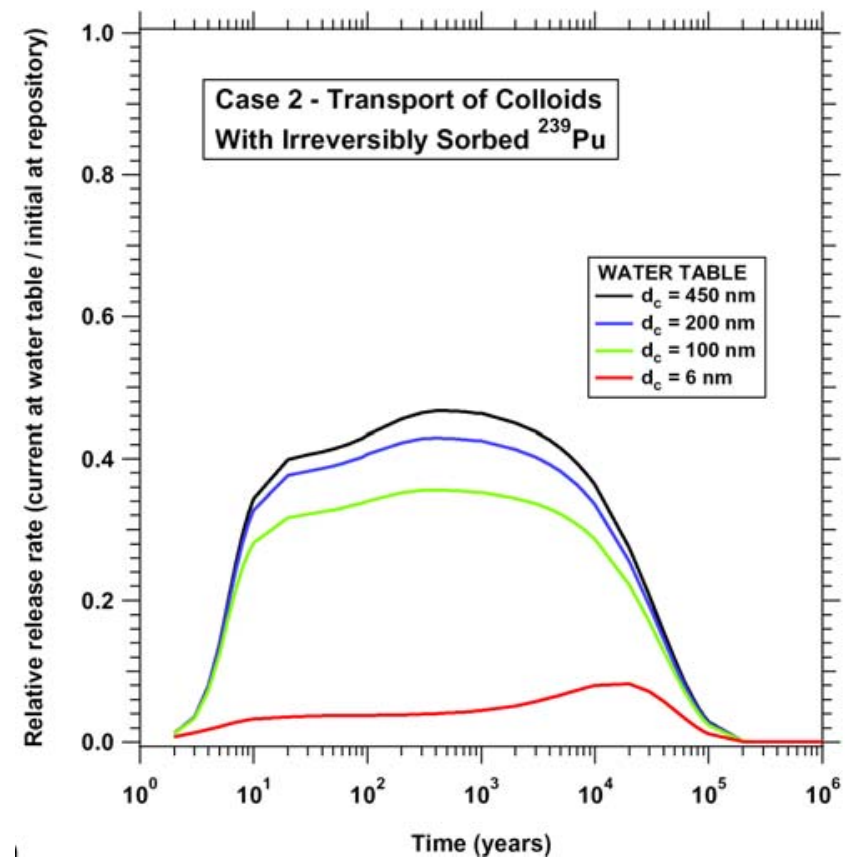

(a)

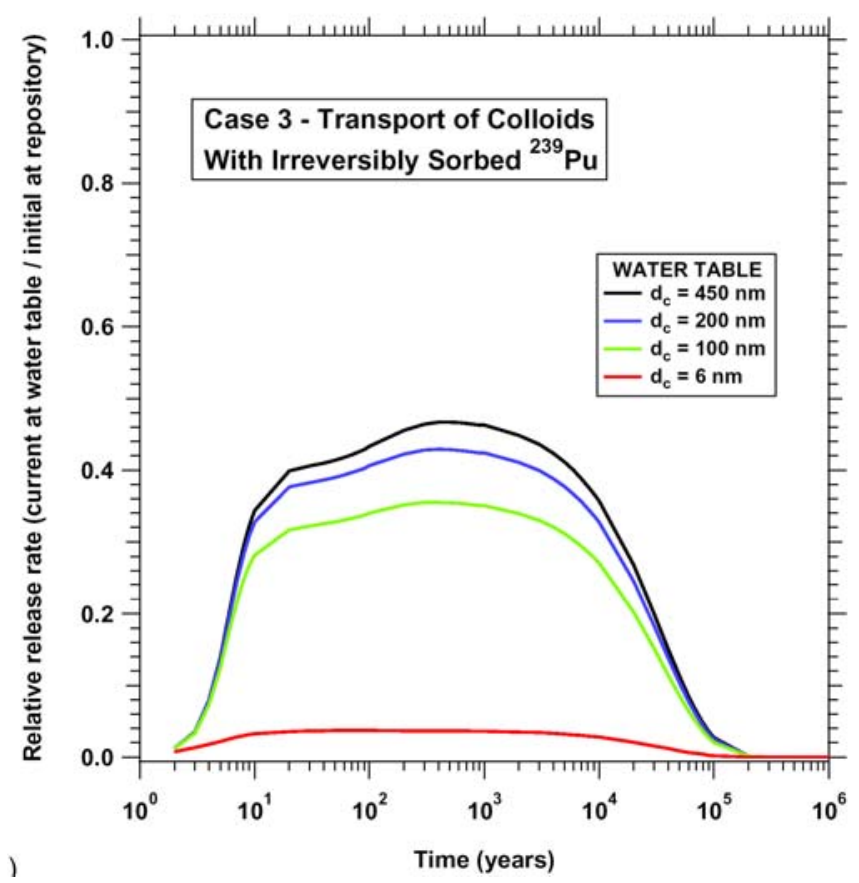

(b)

Output DTN: LB0310MR0060R1.010, data submitted with this model report.

Figure 6-61. Normalized Release at the Water Table in (a) Case 2 and (b) Case 3 for Transport of Wasteform Colloids with Embedded Radionuclides (Mean Present-Day Infiltration, Continuous Source with Decay) 


\subsection{ALTERNATIVE MODELS}

\subsubsection{Alternative Representations and Methods}

This section discusses alternative conceptual models involving:

(a) Different representations of the matrix-fracture system (MINC versus dual-permeability systems)

(b) Different conceptual methods of describing the transport problem (particle tracking versus conventional representation).

This section describes the effects of employing the concept of MINC (as implemented by the development of a different grid system in TOUGH2 simulations) (Pruess and Narasimhan 1985 [DIRS 101707]) on transport predictions related to the radionuclide transport model. In the MINC method, the steep gradients at the matrix fracture surface are resolved by subgridding of the matrix blocks in an appropriate number of shells. The MINC concept is based on the notion that changes at the fracture-matrix interface will propagate rapidly through the fracture system, while invading the tight matrix comparatively slowly. Therefore, changes in the matrix conditions are controlled locally by the distance from the fractures, leading to the creation of a system of nested subdomains. The MINC behavior is expected to result in slower breakthrough curves (as the enhanced fracture-matrix interaction allows for increased diffusion), longer contact times, and more effective sorption (in sorbing media/solute systems). As members of the TOUGH2 family of codes, both T2R3D and EOS9nT are capable of handling MINC grids.

Also investigated was the potential of analyzing the transport problem using particle-trackingbased numerical models (DCPT V2.0; LBNL 2002 [DIRS 154342]) that appear attractive in terms of execution and storage requirements compared to the conventional models (e.g., T2R3D and EOS9nT). The curve labeled "DCPT V1.0" was computed using DCPT V2.0 (LBNL 2002 [DIRS 154342]) by setting fracture-matrix interaction to the original, simpler algorithm used by DCPT V1.0 (LBNL 2000 [DIRS 132448]). DCPT V2.0 accounts for the changes in the diffusion gradient length scale into the matrix with time, and, in this respect, is analogous to the MINC method. DCPT V1.0 uses a method with a fixed diffusion length into the matrix analogous to a dual-permeability method.

The grid that described transport of a conservative tracer through a two-dimensional vertical cross section (slice) of the UZ was provided by DTN: LB0308AMRU0185.001 [DIRS 165172]. The input and output files and data for this study are in DTNs: LB03093RADTRNS.001 [DIRS 166225] and LB03093RADTRNS.002 [DIRS 166071].

The results of the simulation are shown in Figure 6-62. Predictions from T2R3D and EOS9nT with a conventional dual-permeability grid and a MINC grid are in good agreement. The result with the MINC grid conforms to expectations, resulting in slower breakthrough curves. Note that despite its scientific and conceptual appeal, the application of the MINC concept to the three-dimensional UZ site-scale model is challenging because it necessitates replacement of the single matrix block in the current dual permeability system with several MINC subdomains. This increases the already large size of the problem, the memory requirements, and the execution times. 
The DCPT V1.0 and DCPT V2.0 (LBNL 2000 [DIRS 132448]; 2002 [DIRS 154342]) particle-tracking code could provide an alternative, because it enjoys the advantages of low memory requirements and fast execution. Figure 6-62 shows that the DCPT V1.0 and V2.0 (LBNL 2000 [DIRS 132448]; 2002 [DIRS 154342]) solutions are close to the predictions from the MINC grid (although somewhat more diffusive).

\subsubsection{Alternative Diffusion Model}

As shown in Sections 6.8 to 6.18 , the main mechanism of radionuclide retardation in the $\mathrm{UZ}$ is diffusion from the fractures into the matrix. This process transfers radionuclides from the fractures (the pathways of fast flow and the main conduits of transport) to the matrix, where their transport is retarded because of the much slower water velocities and matrix sorption.

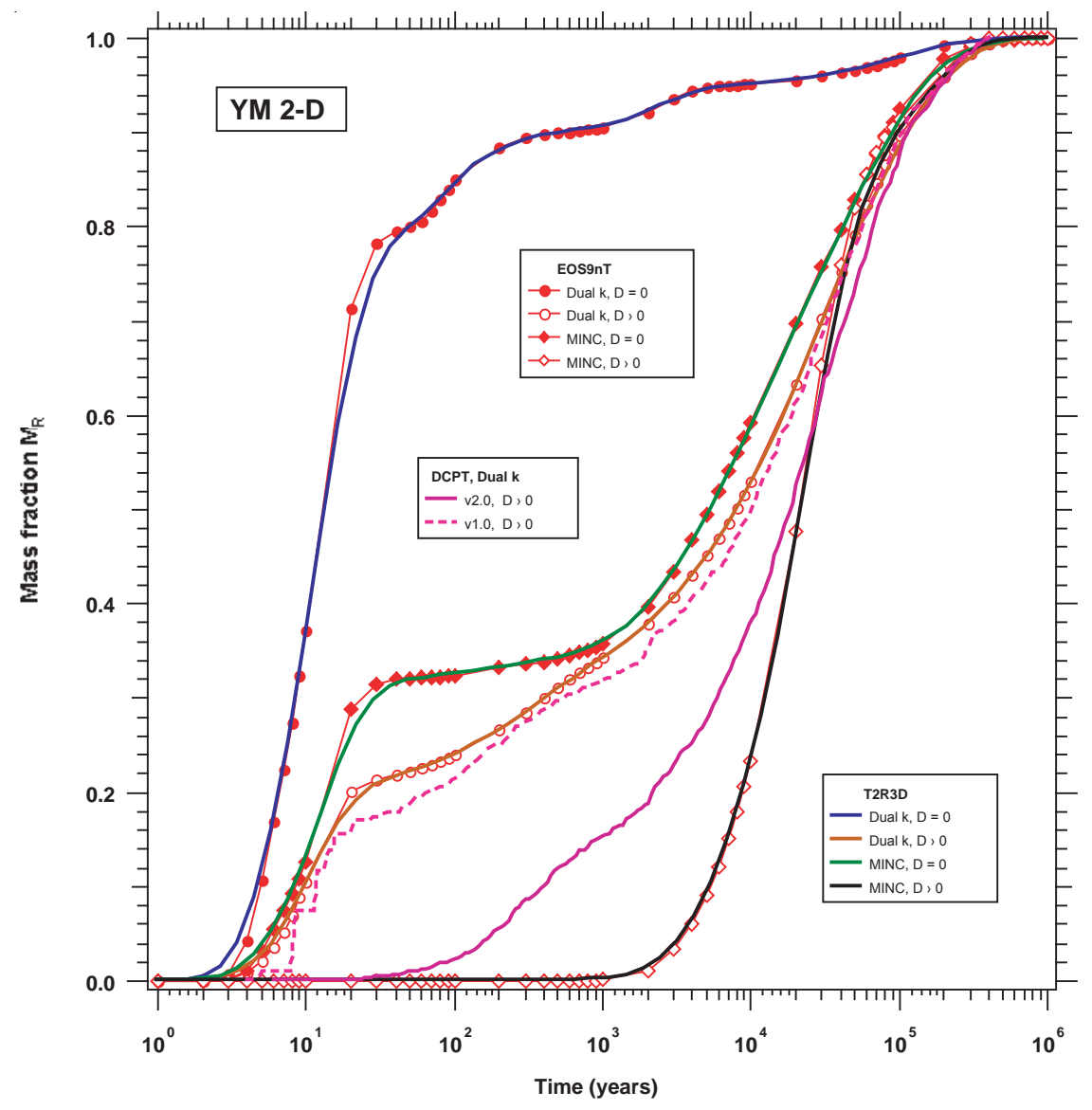

DTNs: LB03093RADTRNS.001 [DIRS 166225]; LB03093RADTRNS.002 [DIRS 166071].

Figure 6-62. Effect of (a) the MINC Concept and (b) Application of Particle-Tracking Approaches on Breakthrough Predictions at a Two-Dimensional Vertical Cross Section of the UZ for an Instantaneous Release under Mean, Present-Day Climate for a Nonsorbing, Non-decaying Tracer 
This section explores the effect on transport of an alternative conceptual model that does not consider matrix diffusion. In other words, transport in this alternative model is limited to advection in the matrix and in the fractures. Thus, there is no mechanism for radionuclide removal from the fractures other than (a) the relatively small sorption on the fracture walls, where the majority in fracture flow (the dominant portion of flow); and (b) sorption in the matrix, where only a small fraction of flow occurs. For non- and moderately sorbing radionuclides, this means that there is no means of retardation, and the contaminants in the fractures are transported virtually unhindered to the groundwater.

Note that this alternative model is far more relevant to solutes than to colloids. Because of the very small diffusion coefficient of colloids, transport is advection dominated and diffusive fluxes for the larger colloids (100 $\mathrm{nm}$ to $450 \mathrm{~nm}$ colloids) are minuscule, as attested to by the practical coincidence of the early portions of the $R_{F}$ curves (see Section 6.18, this model report).

\subsubsection{Instantaneous Release}

The grids, flow fields, and conditions are identical to those in Section 6.7. The parameters used in these T2R3D simulations are listed in Tables 6-3, 6-5, and 6-6. The input and output files for these simulations are included in output DTNs: LB0310MR0060R1.010 and LB0310MR0060R1.011, respectively (Table 6-6).

For instantaneous release and a mean present-day infiltration, the cumulative breakthrough curves of the normalized mass fraction $R_{M}$ in Figure 6-63 confirm the expectation of virtually unimpeded transport to the groundwater. The contrast to the $R_{M}$ evolution when matrix diffusion is considered (also included in Figure 6-63) is dramatic. Despite their very different sorption affinity to the UZ rocks, all three radionuclides (the nonsorbing ${ }^{99} \mathrm{Tc}$, the moderately sorbing ${ }^{237} \mathrm{~Np}$, and the strongly sorbing ${ }^{239} \mathrm{Pu}$ ) move unhindered in the fractures in the absence of matrix diffusion, and their breakthrough curves to $t=100$ years coincide. Transport in this case is extremely fast. The $t_{10}$ and $t_{50}$ of all three radionuclides are about 5 years and 20 years, respectively.

The very fast early transport and the coincidence of the $R_{M}$ curves are the result of advection (linked to the to rapid fracture flow) and the lack of matrix diffusion. This result is consistent with expectation because there is no other mechanism that can affect transport during this early stage. Note that by setting $\phi=1$ in the fractures, sorption onto the fracture walls is not considered in these simulations.

After the very fast rise of $R_{M}$ (indicated by the steep slope for $\mathrm{t}>100$ years in figure 6-63), the $R_{M}$ slope becomes much flatter and the three breakthrough curves begin to differentiate because of contributions of matrix flow (and advection) to transport. The change of the slope indicates exhaustion of the radionuclide supply in the fractures (from the initial from the instantaneous release). The breakthrough curve of ${ }^{239} \mathrm{Pu}$ daughters shows a plateau after the initial steep rise portion. This is caused by the very strong ${ }^{239} \mathrm{Pu}$ sorption onto the matrix of the UZ rocks, which does not allow ${ }^{239} \mathrm{Pu}$ in the matrix flow to reach the water table. Thus, all the ${ }^{239} \mathrm{Pu}$ in the fractures reaches the water table in a very short time, and all ${ }^{239} \mathrm{Pu}$ in the matrix is trapped by sorption. 
Because of its moderate sorption affinity to the UZ rocks, the ${ }^{237} \mathrm{~Np}$ breakthrough shows a very mild increase from $t=100$ years to $t=20000$ years, after which time it registers a fast rise. This is attributed to arrival at the water table (through matrix flow and advection) of ${ }^{237} \mathrm{~Np}$ that had sorbed onto and traveled through the matrix, into which it had been transported by fracture-to-matrix flow. Because of lack of sorption, the ${ }^{99} \mathrm{Tc}$ breakthrough curve is marked by a shorter period of the mild slope (up to about $t=3,000$ years) and an earlier arrival of ${ }^{99} \mathrm{Tc}$ through matrix advection.

The results of this study emphasize the importance of diffusion from the fractures into the matrix as the main mechanism for retardation of radionuclide transport. When this process is not considered in the simulations, radionuclides reach the water table in significant quantities in less than 5 years.

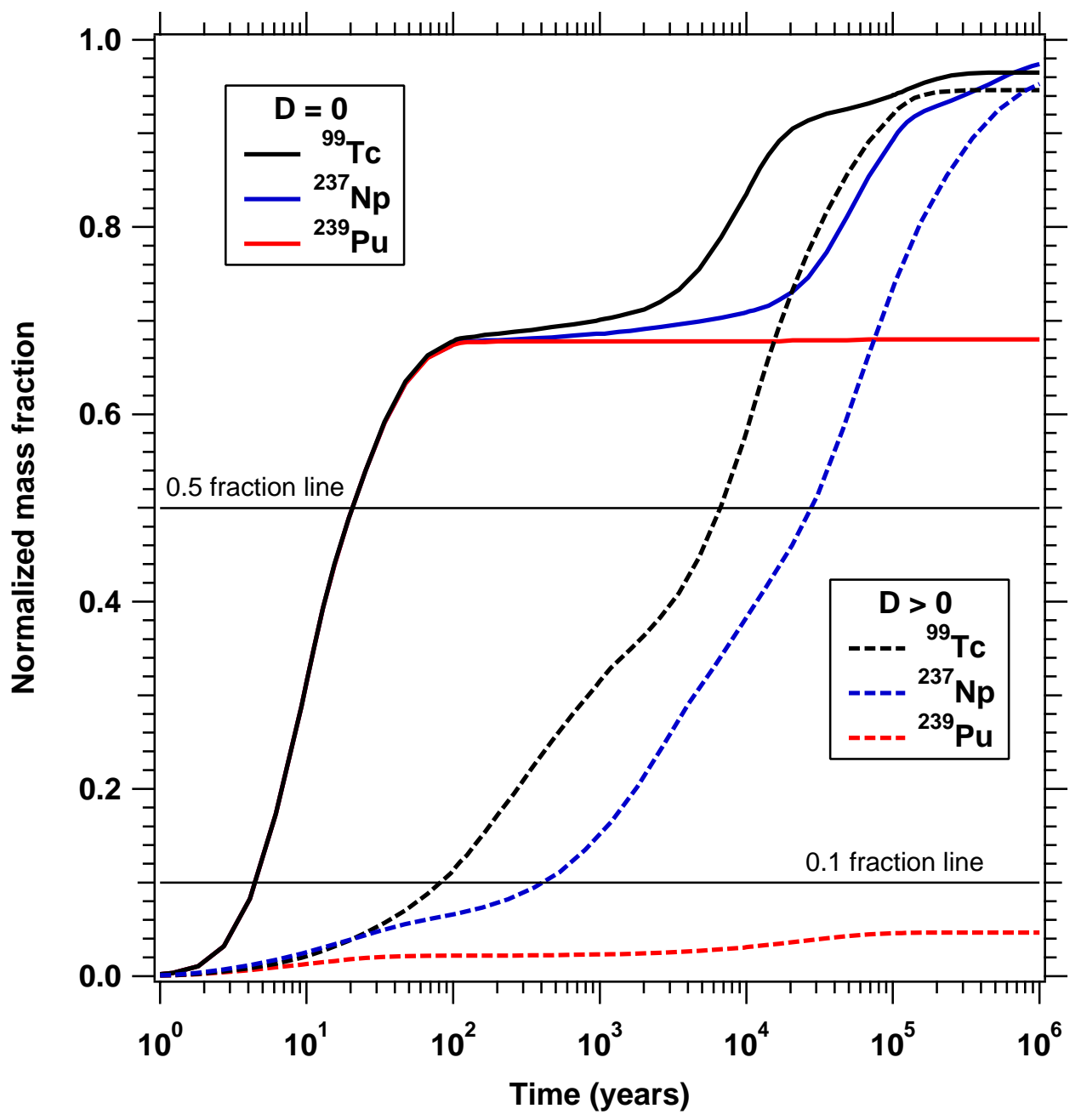

Output DTNs: LB0310MR0060R1.010, LB0310MR0060R1.011.

Figure 6-63. Cumulative Breakthrough of Radioactive Solutes at the Water Table for the No-Diffusion Alternative Model (Mean Present-Day Infiltration, Instantaneous Release) 


\subsubsection{Continuous Release}

The grids, flow fields, and conditions are identical to those in Section 6.15. The parameters used in these EOS9nT simulations are listed in Tables 6-3, 6-5, and 6-6. The input and output files for these simulations are included in output DTNs: LB0310MR0060R1.010 and LB0310MR0060R1.011, respectively (Table 6-6).

For continuous release and a mean present-day infiltration, the relative release rate $R_{F}$ curves in Figure 6-64 confirm the expectation of virtually unimpeded transport to the groundwater. For reference, the relative release rates of the decaying parents at the repository are also included. The contrast to the $R_{F}$ evolution when matrix diffusion is considered (see Figure 6-54, this model report) is remarkable. Despite their very different sorption affinity to the UZ rocks, all three radionuclides (the nonsorbing ${ }^{99} \mathrm{Tc}$, the moderately sorbing ${ }^{237} \mathrm{~Np}$, and the strongly sorbing ${ }^{239} \mathrm{Pu}$ ) move unhindered in the fractures in the absence of matrix diffusion, and their breakthrough curves to $\mathrm{t}=70$ years practically coincide. Transport in this case is extremely fast. The $t_{10}$ of all three radionuclides is about 2.5 years, while $t_{50}$ ranges from 70 (for ${ }^{99} \mathrm{Tc}$ ) to 80 (for ${ }^{237} \mathrm{~Np}$ ) to 85 years (for ${ }^{239} \mathrm{Pu}$ ). The early behavior reflects fracture transport exclusively, and the identity of the $R_{F}$ curves of the three radionuclides results from the absence of diffusion into the matrix. Note that, by setting $\phi=1$ in the fractures, sorption onto the fracture walls is not considered in these simulations.

The $R_{F}$ curves show a very mildly sloping region (almost a plateau) at $\mathrm{t}=30$ years. The near-flat portion of the $R_{F}$ curves indicates a roughly constant radionuclide release rate at the water table, all of which is attributable to fracture flow. The matrix effects begin to become evident for $\mathrm{t}>70$ years when matrix flow (and the contaminants it transports) begins to arrive at the water table, at which time the $R_{F}$ curves of the three radionuclides begin to diverge. The arrival of matrix flow is indicated by the rising (steeper) portion of the $R_{F}$ curve after the first plateau (associated with fracture-based advection).

After $\mathrm{t}=100$ years, the nonsorbing ${ }^{99} \mathrm{Tc}$ is not retarded during matrix flow and arrives at the water table earlier (indicated by higher $R_{F}$ values) than the moderately sorbing ${ }^{237} \mathrm{~Np}$, while the ${ }^{239} \mathrm{Pu}$ arrival at the water table is the slowest of the three and consistent with its strong sorption affinity to the UZ rocks. After attaining maxima at different times (consistent with the different half-lives of the actinides), $R_{F}$ curves begin to decline because of radioactive decay

Accounting for the ${ }^{239} \mathrm{Pu}$ daughters leads to a somewhat different pattern. A plateau in $R_{F}$ is observed from $\mathrm{t}=1,000$ to $\mathrm{t}=10,000$ years (denoting stability of the ratio of repository and water table fluxes), followed by a sharp increase. This is attributed, almost exclusively, to the arrival at the water table of larger amounts of the ${ }^{235} \mathrm{U}$ daughter, which sorbs less strongly and has a longer half-life than ${ }^{239} \mathrm{Pu}$. The ${ }^{235} \mathrm{U}$ arriving at the water table originates from direct ${ }^{235} \mathrm{U}$ source releases at the repository (transported mostly by fracture advection), and from the decay of both free and sorbed ${ }^{239} \mathrm{Pu}$ in the matrix of the UZ rocks. The difference in the $R_{F}$ patterns and the much higher levels of $R_{F}$ attained in this case underscore the importance of accounting for daughters in radionuclide transport studies (see Sections 6.16 and 6.17). 
The results of this study confirm the observations made in the case of instantaneous release on the importance of diffusion from the fractures into the matrix as the main mechanism for retardation of radionuclide transport. When this process is not considered in the simulations, very fast transport times are observed, and radionuclides reach the water table in less than 5 years.

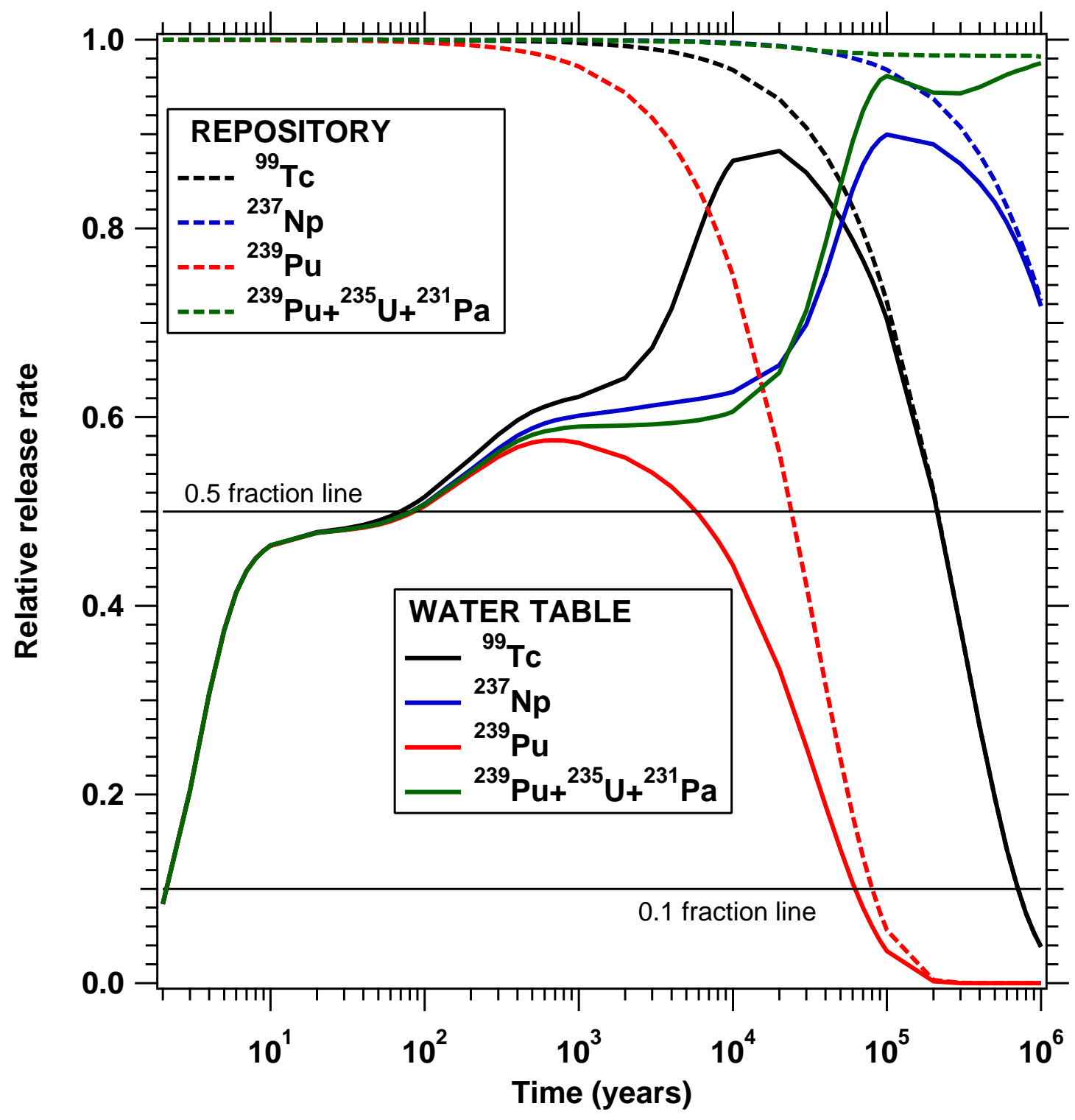

Output DTNs: LB0310MR0060R1.010, LB0310MR0060R1.011.

Figure 6-64. Relative Release Rate of Radioactive Solutes at the Water Table for the No-Diffusion Alternative Model (Mean Present-Day Infiltration, Continuous Release) 


\subsection{BARRIER EVALUATION, UNCERTAINTIES, AND A NOTE OF CAUTION}

In this section, the results presented in Sections 6.6 to 6.20 are distilled into an overall evaluation of the performance of the Yucca Mountain UZ as a barrier. Other issues are uncertainty and the effect it may have on predictions of barrier performance, and the framework within which the results and predictions in the model report should be understood and interpreted.

\subsubsection{Barrier Performance}

The $t_{10}$ and $t_{50}$ values for all the radionuclides, all the climatic scenarios, and the two release scenarios (instantaneous and continuous) are listed in Tables 6-10 to 6-12. A long $t_{10}$ and a long $t_{50}$ are desirable because they indicate desirable barrier performance. Of the two, $t_{10}$ provides a measure of influence for the most conductive fractures on the UZ barrier behavior, while $t_{50}$ accounts for the effects of both fractures and matrix. A short $t_{10}$ and a short $t_{50}$ could be an indication of inadequate barrier performance. A short $t_{10}$ but an adequately long $t_{50}$ is an indication of a system with strong early arrivals at the water table because of fracture-dominated flow (and, consequently, transport), but with an adequate overall barrier performance in the long run.

A review of the results in Tables 6-10 to 6-12 indicates that, in conformance with expectations, the barrier performance of the $\mathrm{UZ}$ is a function of the radionuclide under investigation. Additionally, there is an almost universal early arrival of a small portion of the total released mass (usually less than or equal to 10 percent) at the water table. The early arrivals are caused by the permeable fractures at the Drill Hole Wash fault and the Pagany Wash fault, and, to a much lesser extent, the Sundance fault, which lead to fast advective transport. Thus, by having the repository footprint straddling important faults, and by following an approach that releases radionuclides throughout the repository, the barrier of the UZ is breached, once the waste package and EBS systems are breached.

Based on the flow model described in the report by BSC (2004 [DIRS 169861], Section 6) and incorporated into this transport study (and despite the adverse release patterns and approaches discussed above), the UZ appears to be an effective barrier in the transport of the following radionuclides:

a) ${ }^{90} \mathrm{Sr}$ and ${ }^{226} \mathrm{Ra}$ : In the 1,000,000-year study period, their migration through the UZ registers arrivals of less than 10 percent of the original mass (under instantaneous release) at the water table, even under the most humid of the present-day and monsoon infiltration regimes, and for low and mean glacial-transition infiltration. This is caused by an early arrival (related to fracture flow and the related advection) of a small portion of the total mass (greater than 10 percent) only for an upper glacial-transition infiltration. Early arrivals are caused by the permeable fractures at the faults discussed earlier. The low fraction of the total mass reaching the water table is caused by diffusion into the matrix, strong sorption in the matrix, and the relatively short half-lives of these radionuclides combining to prevent any matrix-associated radionuclide advection to the water table. 
b) ${ }^{229} \mathrm{Th}$ : With the exception of the upper monsoon and glacial-transition infiltrations, less than 10 percent of the initially released mass reaches the water table. Although a short $t_{10}$ is observed for the most humid of the glacial-transition and monsoon infiltration regimes, only a small portion of the total mass (less than 20 percent) arrives at the water table in the 1,000,000-year study period. This is attributed to the strong sorption after diffusion into the matrix, and the relatively short half-life of ${ }^{229} \mathrm{Th}$, which eliminate any matrix-related ${ }^{229}$ Th advection to the water table.

c) ${ }^{241} \mathrm{Am}$ and ${ }^{231} \mathrm{~Pa}$ : With the exception of the most humid (upper) conditions of all three climatic scenarios (present-day, monsoon, and glacial-transition), less than 10 percent of the initially released mass reaches the water table. As appears to be the case with all radionuclides, there is an early arrival, but only a small portion of the total mass (less than 20 percent) arrives at the water table in the 1,000,000-year study period. This is caused by diffusion into the matrix, strong sorption in the matrix, and the relatively short half-lives of these radionuclides. Thus, radionuclide arrivals are limited to early advection due to fracture flow, with no apparent contributions of matrix transport to water table arrivals.

d) ${ }^{239} \mathrm{Pu}$ : The transport of ${ }^{239} \mathrm{Pu}$ is characterized by the early arrival (common to all radionuclides at the $\mathrm{UZ}$ ) of a small portion of the total released mass. As in the case of ${ }^{241} \mathrm{Am}$ and ${ }^{231} \mathrm{~Pa}$, with the exception of the most humid (upper) conditions of all three climatic scenarios (present-day, monsoon, and glacial-transition), less than 10 percent of the initially released mass reaches the water table in this early arrival. Although ${ }^{239} \mathrm{Pu}$ sorbs strongly onto the matrix after diffusion from the fractures (the overwhelmingly dominant transport features), the breakthrough curves in Figures 6.10 .1 to 6.10 .3 indicate contributions of matrix-related advection to transport (indicated by the second plateau in the figures). This is possible because of the long half-life of ${ }^{239} \mathrm{Pu}$, which exceeds the transport time of matrix flow to the water table. However, the fraction of the total mass that arrives at the water table over the 1,000,000-year study period does not exceed the 50 percent level under any climatic conditions.

The effects of having (a) the repository straddle important faults and releasing radionuclides directly into the fault fractures (the approach followed in this study) and (b) the improbably conservative (if not practically impossible) approach described in Section 6.7.8 are apparent in the UZ barrier performance in the transport of the remaining radionuclides considered in this study. By allowing these conditions, the barrier appears far less effective, as indicated by the breakthrough curves of the variably sorbing ${ }^{135} \mathrm{Cs}$ (strongly on zeolites, much less on other rocks) the mildly sorbing ${ }^{233} \mathrm{U},{ }^{235} \mathrm{U},{ }^{237} \mathrm{~Np}$; and the nonsorbing ${ }^{99} \mathrm{Tc}$. All these radionuclides have long half-lives (indicating matrix-based advective contributions to transport) and show substantial arrivals at the water table at times shorter than their half-lives. 
Table 6-10. Radionuclide Transport Times to the Water Table (Instantaneous Release)

\begin{tabular}{|c|c|c|c|c|c|c|c|}
\hline \multirow[b]{2}{*}{ Radionuclide } & \multirow{2}{*}{$\begin{array}{c}\text { Infiltration } \\
\text { Scenario }\end{array}$} & \multicolumn{2}{|c|}{ Present-Day } & \multicolumn{2}{|c|}{ Monsoon } & \multicolumn{2}{|c|}{ Glacial-Transition } \\
\hline & & $t_{10}$ (years) & $t_{50}$ (years) & $t_{10}$ (years) & $t_{50}$ (years) & $t_{10}$ (years) & $t_{50}$ (years) \\
\hline \multirow[t]{3}{*}{${ }^{241} \mathrm{Am}$} & Lower & - & - & - & - & - & - \\
\hline & Mean & - & - & - & - & - & - \\
\hline & Upper & 12 & - & 3 & - & 1 & - \\
\hline \multirow[t]{3}{*}{${ }^{237} \mathrm{~Np}$} & Lower & 33,800 & $>1,000,000$ & 15 & 6,160 & 185 & 34,400 \\
\hline & Mean & 410 & 25,400 & 8 & 2,120 & 4 & 1,070 \\
\hline & Upper & 4 & 1,600 & 2 & 714 & 1 & 336 \\
\hline \multirow[t]{3}{*}{${ }^{231} \mathrm{~Pa}$} & Lower & - & - & - & - & - & - \\
\hline & Mean & - & - & - & - & - & - \\
\hline & Upper & 13 & - & 4 & - & 2 & - \\
\hline \multirow[t]{3}{*}{${ }^{239} \mathrm{Pu}$} & Lower & - & - & 86,000 & - & - & - \\
\hline & Mean & - & - & 10,400 & - & 3,710 & - \\
\hline & Upper & 1,530 & - & 4 & - & 2 & - \\
\hline \multirow[t]{3}{*}{${ }^{226} \mathrm{Ra}$} & Lower & - & - & - & - & - & - \\
\hline & Mean & - & - & - & - & - & - \\
\hline & Upper & - & - & - & - & 3 & - \\
\hline \multirow[t]{3}{*}{${ }^{90} \mathrm{Sr}$} & Lower & - & - & - & - & - & - \\
\hline & Mean & - & - & - & - & - & - \\
\hline & Upper & - & - & - & - & 3 & - \\
\hline \multirow[t]{3}{*}{${ }^{99} \mathrm{Tc}$} & Lower & 13,900 & $>1,000,000$ & 22 & 1,310 & 102 & 8,140 \\
\hline & Mean & 83 & 6,640 & 9 & 417 & 6 & 164 \\
\hline & Upper & 6 & 230 & 2 & 92 & 1 & 42 \\
\hline \multirow[t]{3}{*}{${ }^{229} \mathrm{Th}$} & Lower & - & - & - & - & - & - \\
\hline & Mean & - & - & - & - & - & - \\
\hline & Upper & - & - & 4 & - & 2 & - \\
\hline \multirow[t]{3}{*}{${ }^{233} \mathrm{U}$} & Lower & 65,200 & $>1,000,000$ & 103 & 6,730 & 549 & 36900 \\
\hline & Mean & 433 & 29,100 & 34 & 2,130 & 16 & 893 \\
\hline & Upper & 12 & 1,120 & 3 & 458 & 2 & 208 \\
\hline \multirow[t]{3}{*}{${ }^{235} \mathrm{U}$} & Lower & 55,300 & 784,800 & 101 & 6480 & 540 & 32,600 \\
\hline & Mean & 430 & 26,500 & 34 & 2080 & 15 & 882 \\
\hline & Upper & 12 & 1,100 & 3 & 450 & 2 & 206 \\
\hline \multirow[t]{3}{*}{${ }^{135} \mathrm{Cs}$} & Lower & $>1,000,000$ & $>1,000,000$ & 22,400 & $>1,000,000$ & 150,000 & $>1,000,000$ \\
\hline & Mean & 52,500 & $>1,000,000$ & 4,690 & 309,000 & 2,460 & 120,000 \\
\hline & Upper & 2,170 & 71,200 & 753 & 24,500 & 305 & 990 \\
\hline
\end{tabular}

Output DTN: LB0307MR0060R1.007, table values derived from individual breakthrough curves.

NOTES: (-): $t_{10}$ or $t_{50}$ has never been reached because of radioactive decay.

$(>1,000,000)$ : Simulations only up to $1,000,000$ years. The $t_{10}$ or $t_{50}$ can be reached after $1,000,000$ years. 
Table 6-11. Radionuclide Transport Times to the Water Table under Continuous Release

\begin{tabular}{|l|l|l|l|}
\hline \multicolumn{1}{|c|}{ Case } & \multicolumn{1}{|c|}{ Species } & \multicolumn{1}{|c|}{$\boldsymbol{t}_{10}$ (years) } & $\boldsymbol{t}_{50}$ (years) \\
\hline \multirow{3}{*}{ Three-Parents } & ${ }^{99} \mathrm{Tc}$ & 74 & 3,901 \\
\cline { 2 - 4 } & ${ }^{237} \mathrm{~Np}$ & 781 & 22,940 \\
\cline { 2 - 4 } & ${ }^{239} \mathrm{Pu}$ & - & - \\
\hline${ }^{239} \mathrm{Pu}-$ Chain & ${ }^{239} \mathrm{Pu}+{ }^{235} \mathrm{U}+{ }^{231} \mathrm{~Pa}$ & $6,419^{\mathrm{a}}$ & $33,660^{\mathrm{a}}$ \\
\hline${ }^{241} \mathrm{Am}-$ Chain & ${ }^{241} \mathrm{Am}+{ }^{237} \mathrm{~Np}+{ }^{233} \mathrm{U}+{ }^{229} \mathrm{Th}$ & $1,027^{\mathrm{a}}$ & $23,450^{\mathrm{a}}$ \\
\hline
\end{tabular}

Output DTN: LB0307MR0060R1.007, table values derived from individual breakthrough curves.

NOTE: (-): $t_{10}$ or $t_{50}$ has never been reached because of radioactive decay.

a Corresponds to the sum of the chain members.

Table 6-12. Colloid Transport Times to the Water Table (Continuous Release)

\begin{tabular}{|c|c|c|c|}
\hline Case $^{a}$ & $\begin{array}{c}\text { Colloid Size } \\
\text { (nm) }\end{array}$ & $t_{10}$ (years) & $\begin{array}{c}t_{50} \\
\text { (years) }\end{array}$ \\
\hline \multirow[t]{4}{*}{1} & 450 & 4.35 & - \\
\hline & 200 & 4.39 & - \\
\hline & 100 & 4.53 & - \\
\hline & 6 & - & - \\
\hline \multirow[t]{4}{*}{2} & 450 & 4.35 & - \\
\hline & 200 & 4.39 & - \\
\hline & 100 & 4.53 & - \\
\hline & 6 & - & - \\
\hline \multirow[t]{4}{*}{3} & 450 & 4.35 & - \\
\hline & 200 & 4.39 & - \\
\hline & 100 & 4.52 & - \\
\hline & 6 & - & - \\
\hline \multirow[t]{4}{*}{4} & 450 & 32.4 & 243 \\
\hline & 200 & 27.8 & 251 \\
\hline & 100 & 27.6 & - \\
\hline & 6 & - & - \\
\hline
\end{tabular}

Output DTN: LB0307MR0060R1.007, table values derived from individual breakthrough curves.

NOTE: (-): $t_{10}$ or $t_{50}$ has never been reached because of radioactive decay.

${ }^{a}$ Cases are defined in Section 6.18.3.

Figure 6-65 shows the early breakthrough behavior for the different elements. These curves are for an instantaneous, uniform release of mass throughout the repository at time zero under a mean glacial-transition climate. The variations in early breakthrough (less than about 3 years) for all elements are shown to be primarily a function of the matrix diffusion coefficient and are relatively insenstive to the sorption coefficient. The figure also shows that increasing sorption beyond a level of approximately $100 \mathrm{~mL} / \mathrm{g}$ has only a negligible effect on the breakthrough behavior. Stronger sorbing elements such as Th-229 and Pa-231 have more significant breakthrough than Sr-90 or Ra-226 as a result of lower diffusion coefficients for Th-229 and $\mathrm{Pa}-231$. The threshold level beyond which sorption becomes insensitive to the magnitude of the coefficient may be seen to be about $100 \mathrm{~mL} / \mathrm{g}$ in the $\mathrm{Pu}-239$ breakthrough curve. The rising 
breakthrough tail for Pu-239 approaching 10,000 years is indicative of a threshold value of sorption below which the magnitude of the sorption coefficient begins to play a more significant role. Sorption coefficients beyond the $100 \mathrm{~mL} / \mathrm{g}$ level appear to have little influence on transport behavior. The behavior of Cs-135 is a result of low sorption coefficients in the devitrified and vitric rock, very strong sorption in the zeolitic rock, as well as a large matrix diffusion coefficient. The rising breakthrough for Cs-135 approaching 10,000 years is attributed to releases that do not encounter zeolitic rock. The sorption coefficients for Sr-90 might suggest that this radionuclide would have some rise in the breakthrough approaching 10,000 years, but the short half-life of this radionuclide, 29 years, prevents any long-term breakthrough. The half-lives of Am-241 (430 years), Ra-226 (1600 years), and Th-229 (7900 years) also potentially impact the breakthrough behavior.

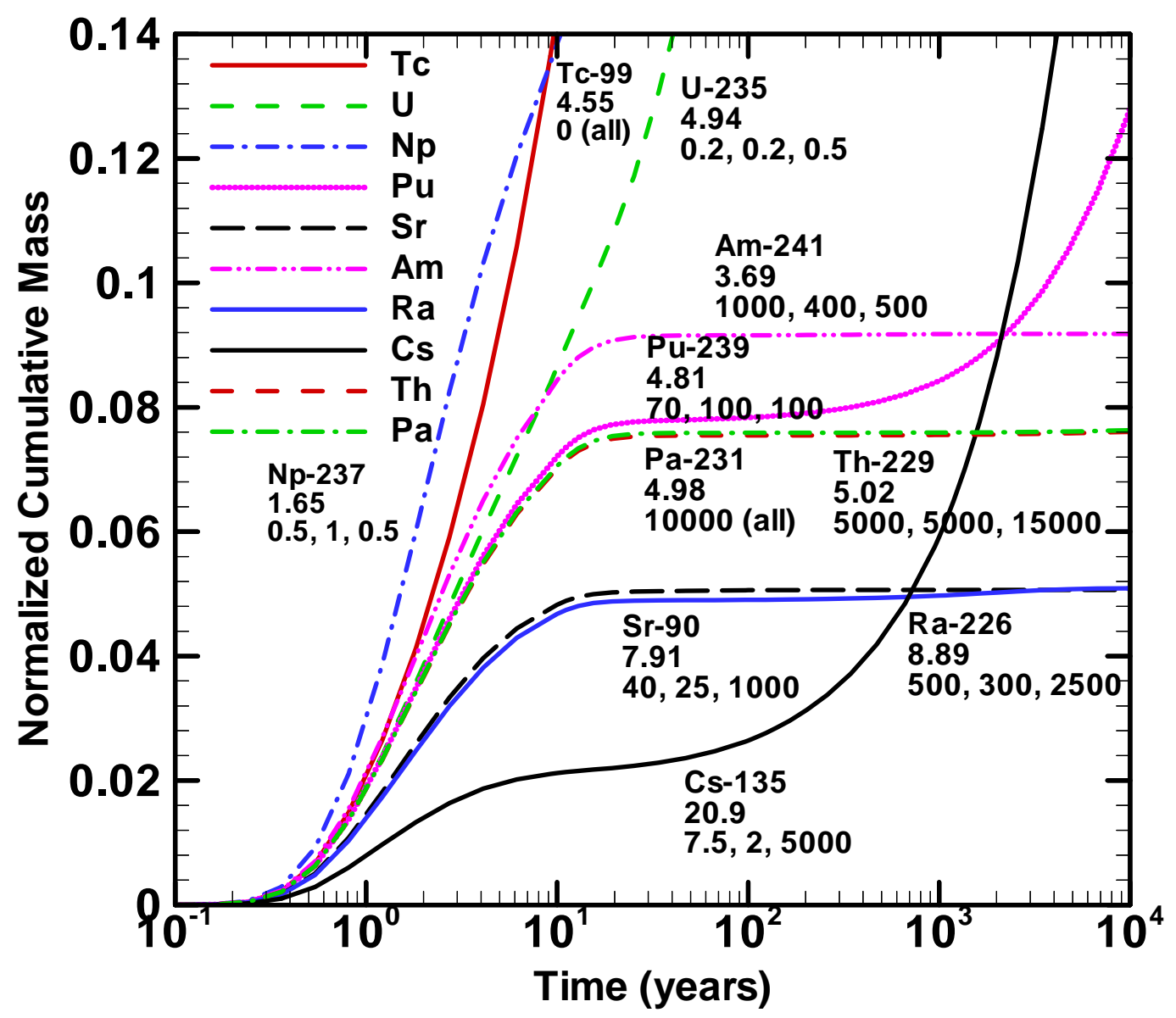

DTN: LB0307MR0060R1.007

Figure 6-65. Comparison of Early Breakthrough at the Water Table for Mean Glacial-Transition Climate and Instantaneous Release. Numbers immediately below element symbols are the matrix diffusion coefficients $\times 10^{-10}\left(\mathrm{~m}^{2} / \mathrm{s}\right)$. Numbers in second line below element symbols are the sorption coefficient values $(\mathrm{mL} / \mathrm{g})$ for devitrified, vitric, and zeolitic rock, respectively. 


\subsubsection{The Effect of Radionuclide Release Directly into the Fault Fractures}

Based on the breakthrough results and the transport patterns determined in Sections 6.8 to 6.19, the early arrival of radionuclides at the Yucca Mountain water table (indicated by the low $t_{10}$ values) was attributed to advective transport through the fractures associated with the Drill Hole Wash fault and the Pagany Wash fault. The concentration distributions in the fractures at both the bottom of the TSw and at the water table, even at very early times $(t=10$ years) and for both types of release (instantaneous and continuous), coincide with the fault outline. Thus, it was important to evaluate the impact of releasing radionuclides directly into the fractures of these faults.

This was accomplished by repeating select radionuclide transport simulations in which the initial radionuclide concentration in repository gridblocks (a) representing the fault or (b) being adjacent to it had been set to zero. In essence, radionuclide sources were removed from the faults and from gridblocks on either side of them. These simulations were limited to (a) mean-present day infiltration and (b) the radioactive solutes ${ }^{99} \mathrm{Tc},{ }^{237} \mathrm{~Np},{ }^{239} \mathrm{Pu}$, and ${ }^{235} \mathrm{U}$, which cover the spectrum of sorption behavior. Both release scenarios (instantaneous and continuous) were investigated.

The grids, flow fields, and conditions are as discussed in Section 6.7. The parameters used in three-dimensional simulations are listed in Tables 6-3, 6-4, 6-5, and 6-6. The initial condition files INCON were the only difference between the input files and those of the corresponding simulations discussed in Sections 6.8 to 6.11 and 6.15. The DTN of the input and output files for the three present-day infiltration scenarios is output DTN: LB0307MR0060R1.005 (Table 6-6).

Contrary to expectations, eliminating potential sources from the vicinity of the fault fractures appears to have only a small effect on transport and arrivals at the water table. For instantaneous release (when a finite radionuclide mass is involved), the $R_{M}$ breakthrough curves in Figures 6-66 and 6-67 show a small increase in $t_{10}$ (in line with expectations because there is no release into the fast-conducting fault fractures), but $t_{50}$ is practically unchanged. The $R_{F}$ breakthrough curves in Figures 6-68 and 6-69 for continuous release show practically no discernible difference from those that account for release into the faults (Section 6.15).

The reason for this is demonstrated in the contour plots of the ${ }^{99}$ Tc distribution of $X_{R}$ in Appendix H. The effects of eliminating releases from the immediate vicinity of the faults are obvious at the bottom of the TSw, at which level the radionuclide distribution shows significant retardation compared to that shown in Figures 6-9 to 6-18. This is particularly evident in the northern part of the repository, where the outlines of the Drill Hole Wash fault and of the Pagany Wash fault (which dominated transport when radionuclides were released throughout the area of the repository) are absent from the contour plots in Figures H-1 to H-10. Remarkably, the $X_{R}$ distribution at the water table (Figures $\mathrm{H}-11$ to H-20) is very similar to that with direct releases into the faults (Figures 6-19 to 6-28), and is dominated by transport through the Drill Hole Wash fault and the Pagany Wash fault. 


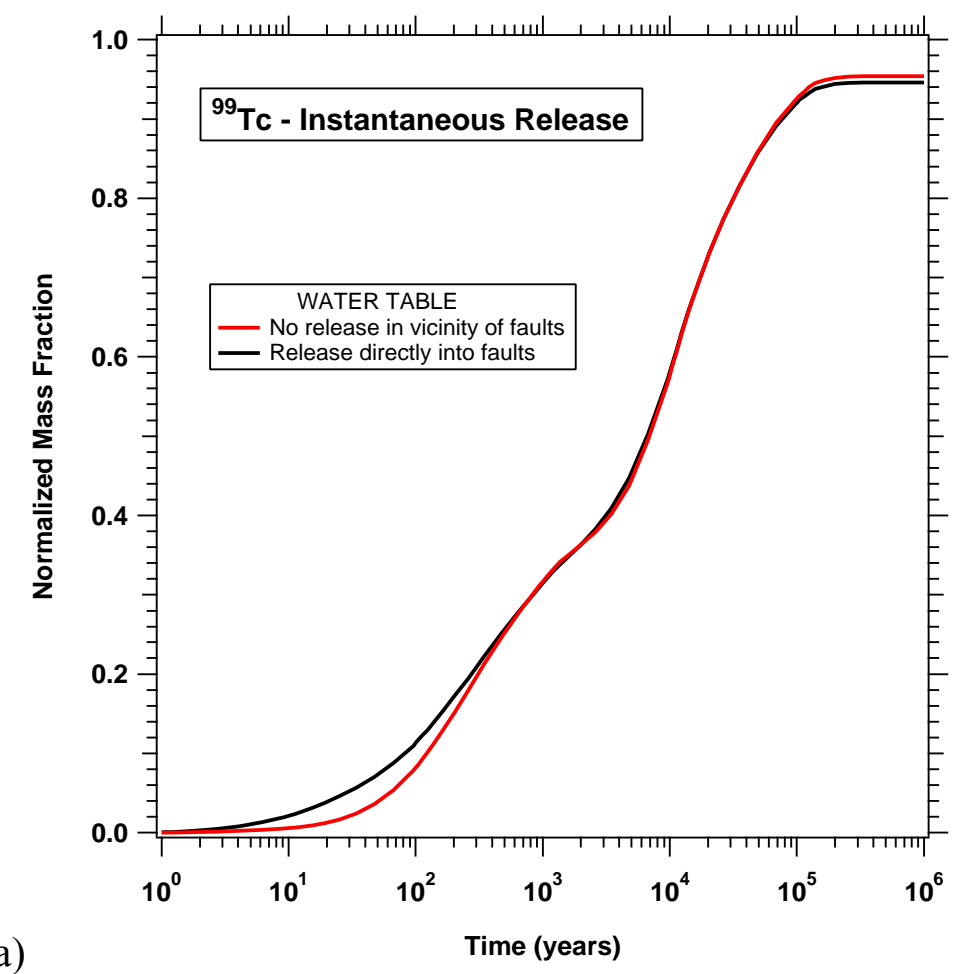

(a)

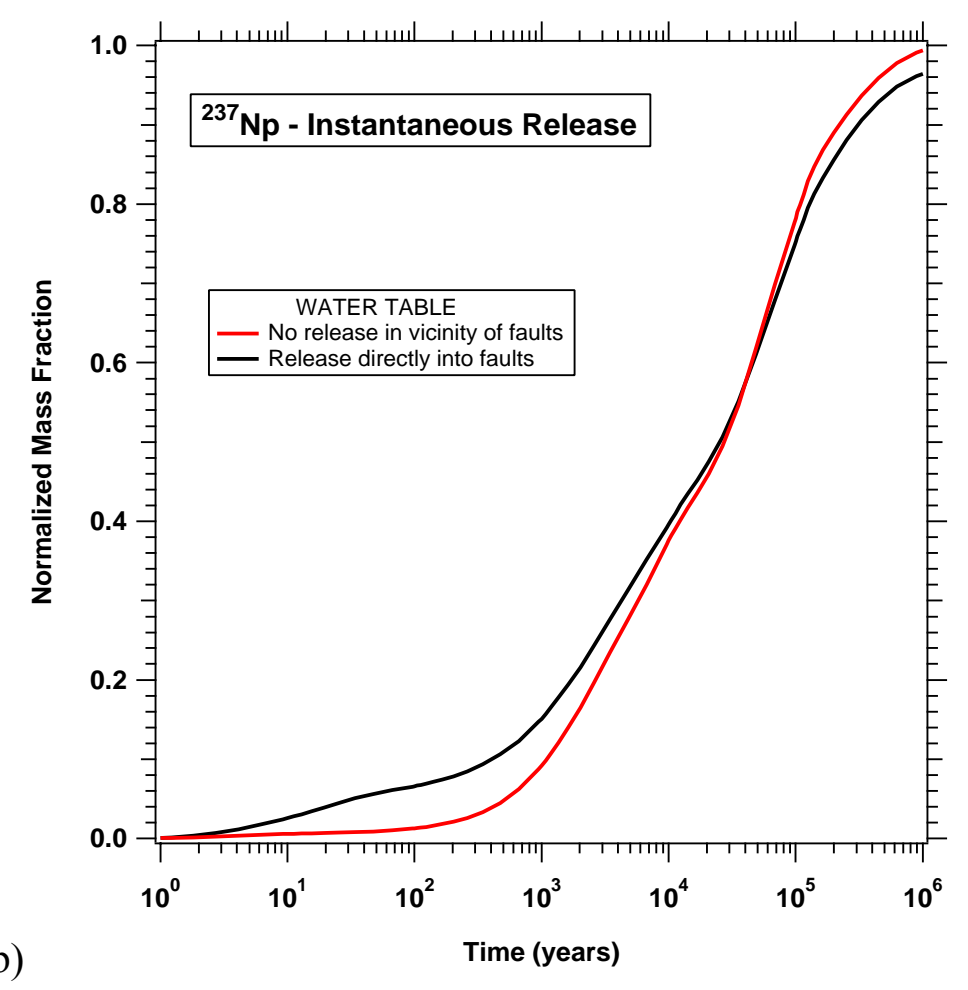

Output DTN: LB0307MR0060R1.007, data submitted with this model report.

Figure 6-66. Cumulative Breakthrough of the ${ }^{99} \mathrm{Tc}$ and ${ }^{237} \mathrm{~Np}$ Mass Fractions $R_{M}$ at the Water Table (Instantaneous Release, Mean Present-Day Infiltration, No Fault Releases) 


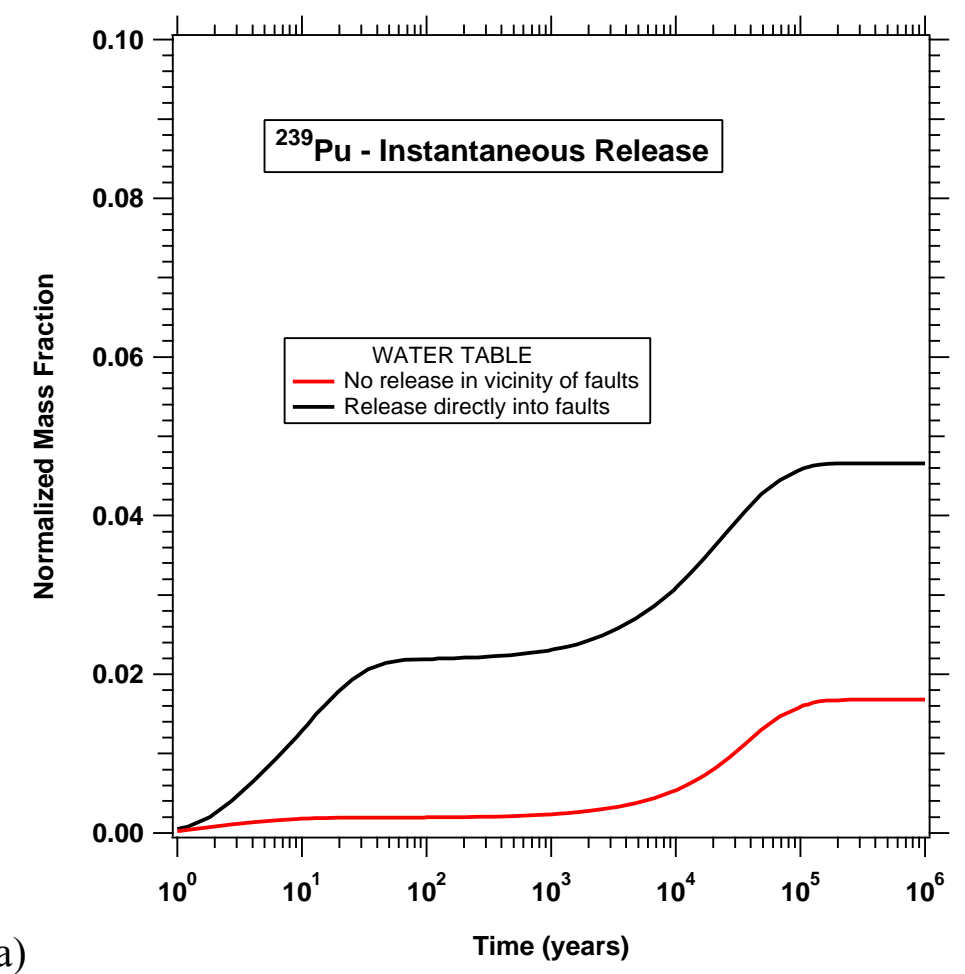

(a)

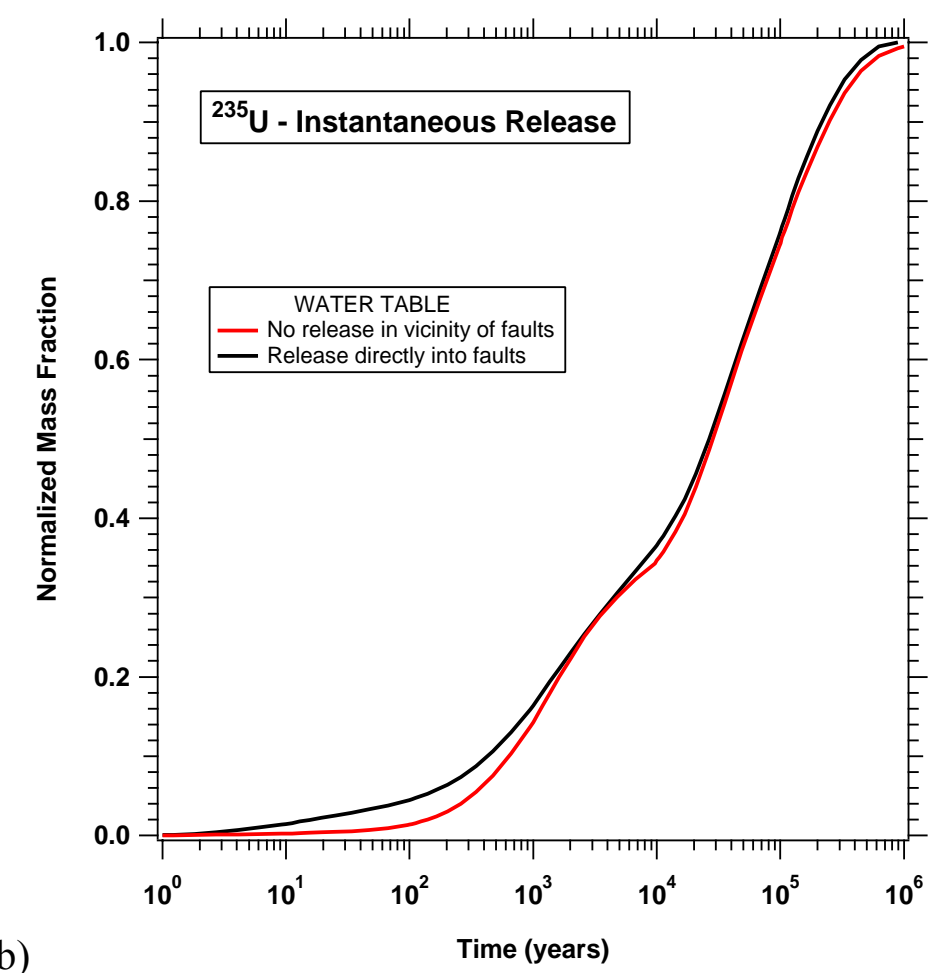

Output DTN: LB0307MR0060R1.007, data submitted with this model report.

Figure 6-67. Cumulative Breakthrough of the ${ }^{239} \mathrm{Pu}$ and ${ }^{235} \mathrm{U}$ Mass Fractions $R_{M}$ at the Water Table (Instantaneous Release, Mean Present-Day Infiltration, No Fault Releases) 


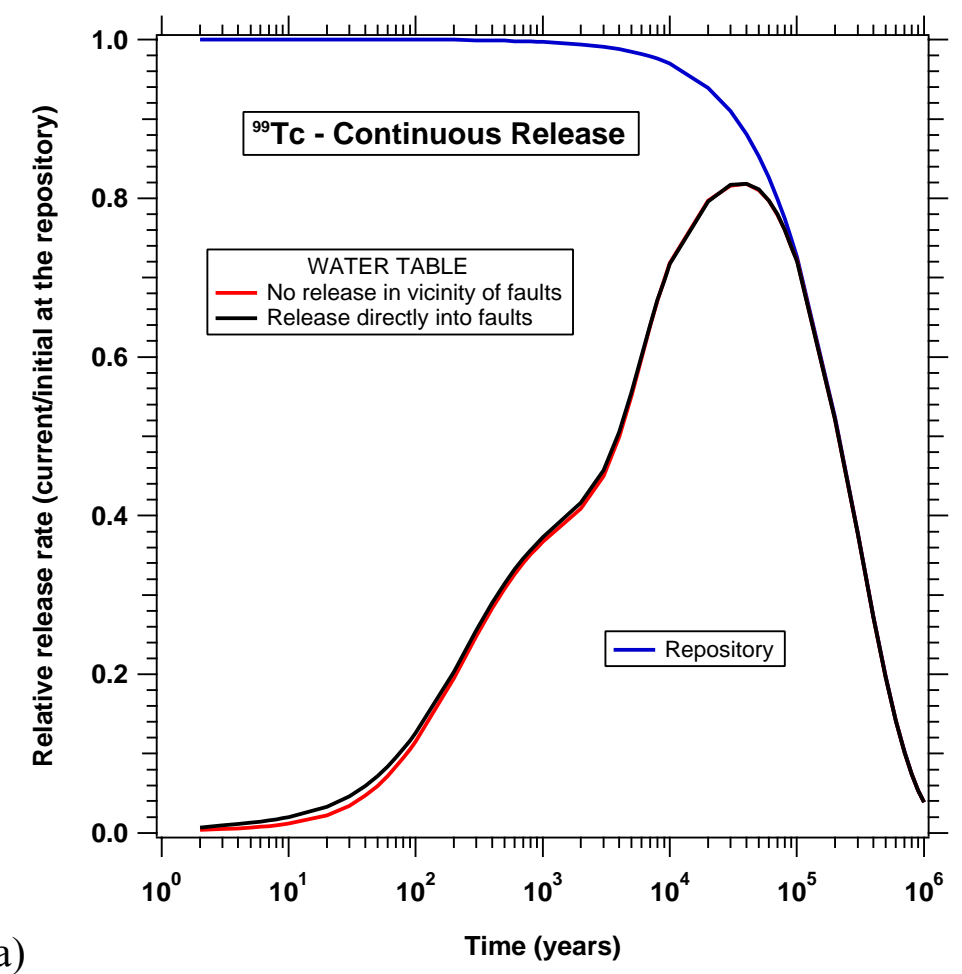

(a)

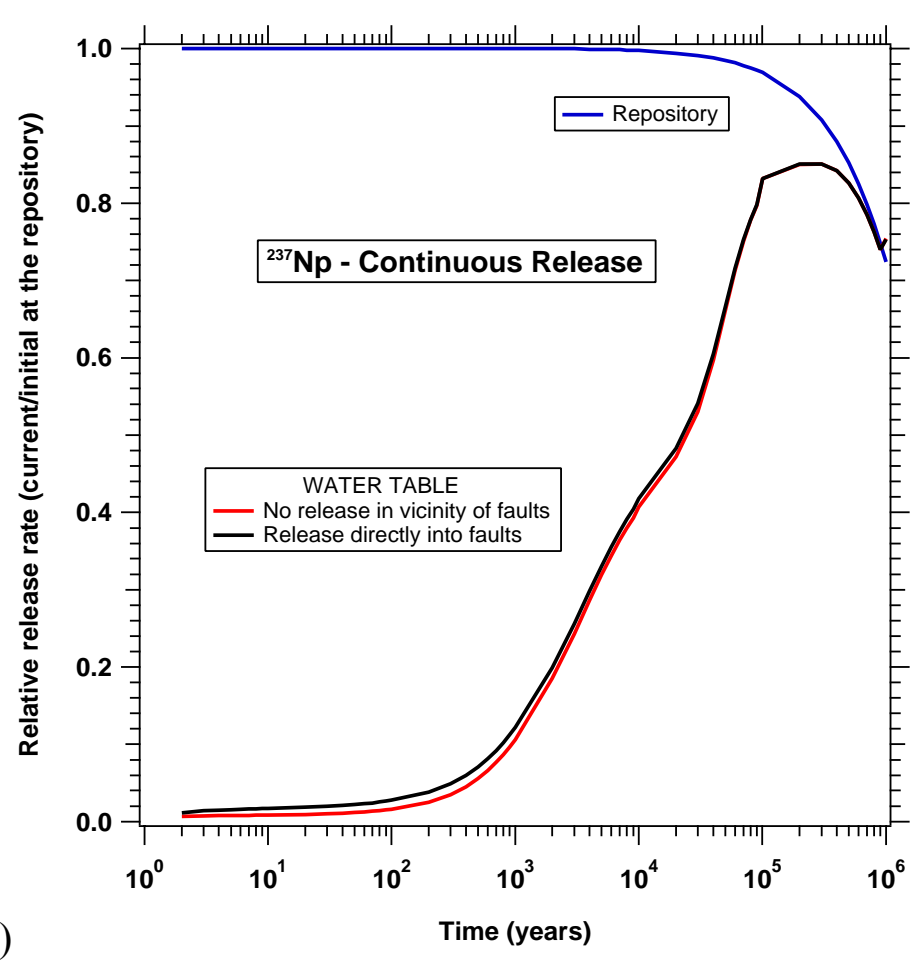

Output DTN: LB0307MR0060R1.007, data submitted with this model report.

Figure 6-68. Cumulative Breakthrough of the ${ }^{99} \mathrm{Tc}$ and ${ }^{237} \mathrm{~Np}$ Normalized Release Rates $R_{F}$ at the Water Table (Continuous Release of Decaying Source, Mean Present-Day Infiltration, No Fault Releases) 


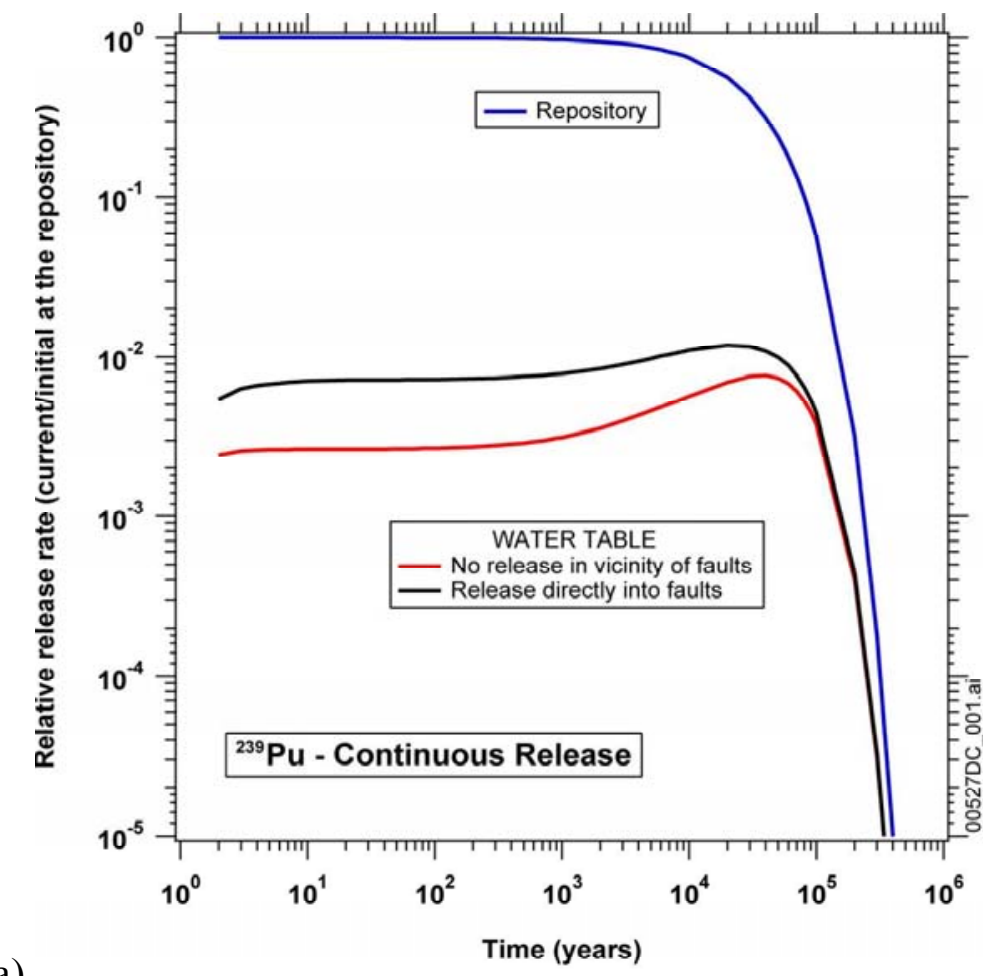

(a)

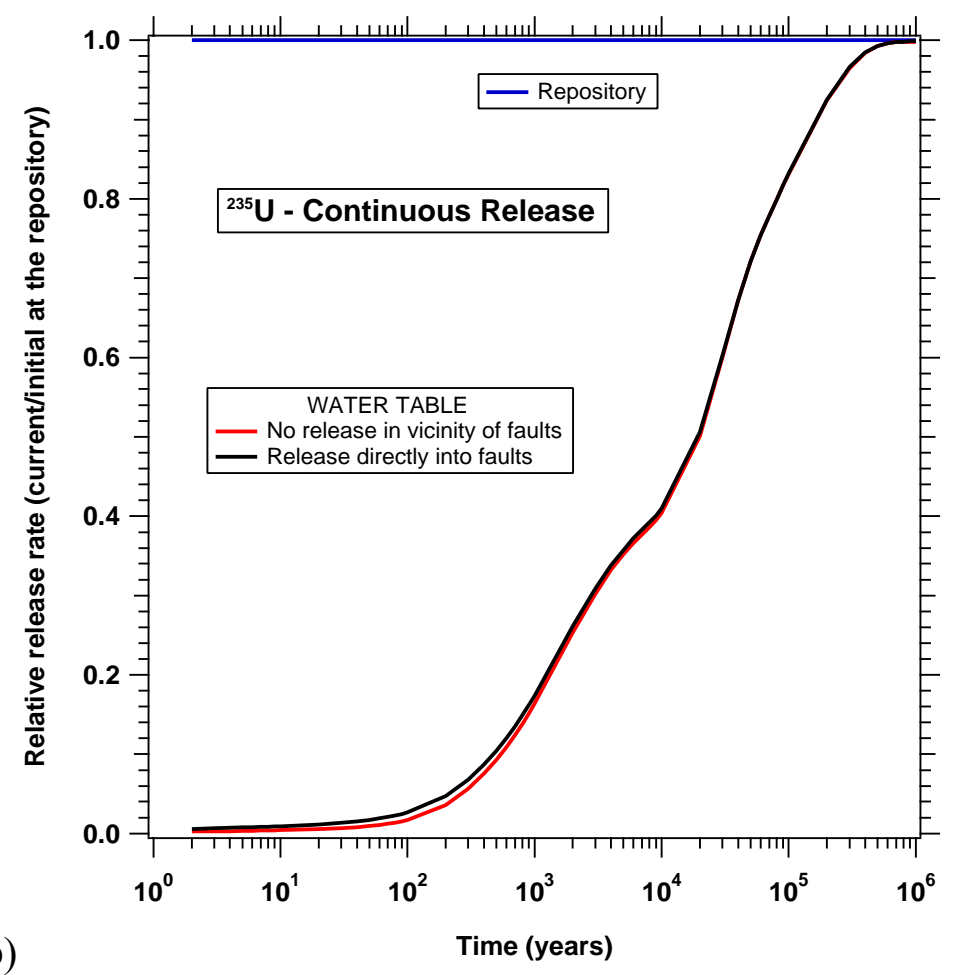

Output DTN: LB0307MR0060R1.007, data submitted with this model report.

Figure 6-69. Cumulative Breakthrough of the ${ }^{239} \mathrm{Pu}$ and ${ }^{235} \mathrm{U}$ Normalized Release Rates $R_{F}$ at the Water Table (Continuous Release of Decaying Source, Mean Present-Day Infiltration, No Fault Releases) 
This pattern indicates that (a) avoiding radionuclide releases directly into and in the vicinity of fractures does retard transport to shallow planes of reference such as the bottom of the TSw, but, (b) given a sufficient deep UZ, lateral transport through an interconnected (both vertically and horizontally) fracture network compensates for the lack of direct releases into the faults. Thus, the interconnected fracture system leads to radionuclide transport to, and emergence at, the water table through fault fractures into which no direct releases at the repository ever occurred. The same pattern is evident in the $X_{R}$ distribution of all other radionuclides and release scenarios investigated here (see output DTN: LB0307MR0060R1.006).

\subsubsection{Interpreting the Model Report Results}

While the results presented in this model report provide some elucidation of the issue of tracer (solute and colloid) transport in the UZ, caution should be exercised in their interpretation. This is because of the conceptual approach and assumptions of this study, and some knowledge gaps (especially in the area of colloid behavior).

It is important to keep in mind that this study uses relative quantities (with respect to the tracer concentration in the water released from the repository), and consequently, all the concentration predictions are relative in nature and presuppose the ability (by no means guaranteed) of the radioactive solutes and colloids to reach the underlying fractures. Thus, predictions of large relative concentrations may mean little unless and until the tracer release becomes possible, significant, and known. Moreover, the presented analysis assumes that conditions for the creation and stability of solute and colloidal species exist, and that the effects of the near-field chemical, physical, mineralogical, and thermal conditions on their creation and stability over time can be ignored. While these assumptions may provide a worst-case scenario, they may be unrealistically conservative.

Note that the three-dimensional site-scale simulations discussed here do not describe a realistic (expected-case) scenario: radioactive tracer release is modeled as occurring continuously, uniformly, even into faults, over the whole area of the repository. This presupposes the near-simultaneous rupture of all the waste-containing vessels, the ability of water dripping into the repository to focus exclusively on the ruptured vessels and to flow through them, the spatially and temporally constant contaminant release from each waste package, and the lack of any immobilization process (e.g., precipitation, colloid flocculation, etc.) before they enter the fractures. The geologic model used in the analysis involves continuous fracture-to-fracture flow in certain portions of the UZ, greatly facilitating transport from the repository to the groundwater. While these assumptions provide the upper limit of a worst-case scenario, this is an implausible (if not a virtually impossible) situation. In that respect, the results discussed here should be viewed as an attempt to identify and evaluate the mechanisms, processes, and geological features that control UZ transport using the largest possible input signal (i.e., the worst-case scenario), rather than as an effort to quantitatively predict the effects of a realistic radionuclide release regime. 
Finally, there are significant uncertainties in colloid modeling. The currently available kinetics models were developed from theoretical principles (Herzig et al. 1970 [DIRS 117519]; Yao et al. 1971 [DIRS 101169]) and were tested under saturated conditions, in uniform sandy laboratory experiments (van de Weerd and Leijnse 1997 [DIRS 109249]), and small-scale field tests (Harvey and Garabedian 1991 [DIRS 109256]) involving microspheres or bacteria. It is not known if these models are applicable to the unsaturated complex fracture-matrix system and the expected colloidal waste forms in the UZ of Yucca Mountain. The limitations of the equations predicting $\kappa^{+}$have not been tested under the UZ conditions, and the subject of $\kappa^{-}$has not been studied to an extent to allow confident modeling. 


\section{INTENTIONALLY LEFT BLANK}




\section{VALIDATION}

For the UZ radionuclide transport model, Level II validation is required (BSC 2004 [DIRS 171282] Section 2.2.2, BSC 2004 [DIRS 167969] Section I-2-1-1). Per the technical work plan, the required level of confidence required in the modeling is obtained through methods related to confidence building during model development (Section 7.1) and postdevelopment model validation activities (Section 7.2). Additionally, the technical work plan calls for validation of sub-models related to matrix diffusion (Section 7.3), sorption (Section 7.4), colloid transport (Section 7.5) and the active-fracture model (Section 7.6). Lastly, Section 7.7 provides a summary of the model validation.

\subsection{CONFIDENCE BUILDING DURING MODEL DEVELOPMENT TO ESTABLISH SCIENTIFIC BASIS AND ACCURACY FOR INTENDED USE}

For level II validation, Section I-2-1-1 in Technical Work Plan for: Performance Assessment Unsaturated Zone (BSC 2004) [DIRS 167969]) specifies steps for Confidence Building During Model Development. The development of the model should be documented in accordance with the requirements of Section 5.3.2(b) of AP-SIII.10Q and criteria of AP-2.27Q Attachment 3. The development of the UZ radionuclide transport model has been conducted according to these criteria as follows:

1. Selection of input parameters and/or input data, and a discussion of how the selection process builds confidence in the model [AP-SIII.10Q 5.3.2(b) (1) and AP-2.27Q Attachment 3 Level I (a)]

Confidence in the UZ radionuclide transport model was built during model development by selecting input data where possible that was collected from Yucca Mountain field characterization activities and were directly relevant to conditions at Yucca Mountain. These inputs are necessary to parameterize the relevant physical and chemical processes of advection, dispersion, diffusion, sorption, radioactive decay, and colloid-facilitated transport that are included in the model. In addition to hydrologic properties input via the UZ flow model and submodels (BSC 2004 [DIRS 169861]), direct inputs for transport are listed in Section 4.1. The molecular diffusion coefficients and half-lives of radionuclides were obtained from accepted sources and have been qualified in the Technical Data Management System (TDMS). Sorption coefficients were measured on rock samples from Yucca Mountain and using approved procedures, rock properties, including matrix permeability and porosity, fracture permeability and spacing, were calibrated with field tests on Yucca Mountain. Additional model inputs relating to specific applications used in this report are described in Sections 6.5, 6.7, 6.8, 6.18, 7.2, and 7.3. By using site-specific information where possible and qualified sources otherwise, confidence has been gained that the UZ radionuclide transport model represents transport through the UZ, based on current understanding of the physical and chemical processes governing transport. 
2. Description of calibration activities, and or initial boundary condition runs, and/or run convergences and a discussion of how the activity or activities build confidence in the model. Inclusion of a discussion of impacts of any-non convergence runs. [(AP-SIII.10Q 5.3.2(b)(2) and AP-2.27Q Attachment 3 Level 1 (e)]

The UZ radionuclide transport model relies on calibrated flow simulations and resultant flow fields from the UZ flow model (BSC 2004 [DIRS 169861]). Section 6.1.5 describes initial and boundary conditions. In brief, the prescribed infiltration conditions at the upper boundary in the flow simulation are varied to obtain present-day and future climatic scenarios. The bottom boundary is appropriately represented as a water table aquifer. The lateral boundaries are sufficiently far away so that no-flux conditions are appropriate.

For transport appropriate initial conditions are zero concentration everywhere in the domain since the interest in transport is on anthropogenic impacts and, with the exception of some uranium isotopes, there are no background concentrations present. These initial and boundary conditions are appropriate for transport simulation and provide confidence that the transport simulations are appropriate. As the transport solution for both T23RD and EOS9nT involves linear sets of equations describing advective-dispersive transport there were no runs for which convergence could not be obtained.

The mountain-scale unsaturated zone transport model is based on the unsaturated zone flow model that is calibrated to available data. The radionuclide transport model, however, has been developed in the absense of direct data on the mountain-scale against which to calibrate transport. Therefore, the unsaturated zone transport model development does not include formal, numerical model calibration on the mountain-scale to data beyond that used for calibration of the flow model. There were, however, postdevelopment model validation activities (see Section 7.2) conducted at lesser scales to improve confidence in transport. These included ensuring correct implementation of the governing equations, comparisons of the model against experimental data collected from the Busted Butte Field Tests (Section 7.2.4.1) and chloride and ${ }^{14} \mathrm{C}$ concentration profiles from Yucca Mountain boreholes (Section 7.2.4.2). Specific submodels were validated through additional model comparisons to the Alcove 8/Niche 3 Fault Test (Section 7.3) and the active fracture model with matrix diffusion (Section 7.6).

3. Discussion of the impacts of uncertainties to model results. [(AP-SIII.10 Q 5.3.2(b)(3) and AP-2.27Q Attachment 3 Level 1 (d) and (f)]

As discussed in Sections 6.1, 6.2, 6.8 to 6.10, 6.18, 7.3.3.3, and 8.3, model uncertainty is consistent with available site characterization data, laboratory experiments, field measurements, and process-level modeling studies. Although there are acknowledged uncertainties regarding implementation of the physical and chemical processes in the UZ radionuclide transport model, Sections 6.7.8 and 8.2.1 describe why the UZ radionuclide transport model is a conservative model and that the assumptions employed with it do not under-represent resulting doses from transport through the $\mathrm{UZ}$. 
4. Formulation of defensible assumptions and simplifications. [AP-2.27Q Attachment 3 Level I (b)]

Discussion of model assumptions and stipulations is provided in Section 5 and Section 6.1.5. Section 5 addresses assumptions for which there is no substantiating evidence and explains the basis by which values were assigned. Stipulations in Section 6.1.5 address simplifications used in the modeling process and the rationale for using them. These stipulations include those involved with the underlying flow component, the transport processes, the use of the dual-continuum approach, and initial and boundary conditions.

5. Consistency with physical principles, such as conservation of mass, energy, and momentum. [AP-2.27Q Attachment 3 Level I (c)]

The conceptual formation in Section 6.1 and the mathematical model description in Section 6.2 demonstrate consistency with physical principles of advection, dispersion, diffusion, radioactive decay, sorption, and colloid-facilitated transport. The transport phenomena and processes in the Yucca Mountain UZ have a flow component and a transport component, each of which are described by a set of governing equations. These govening equations and their implementation into the $\mathrm{UZ}$ radionuclide transport model conserve mass, energy, and momentum in the system under study, while quantifying the system response to external mass and energy inputs and interrelationships between the various processes involved. The basic flow stipulations are consistent with those discussed in UZ Flow Models and Submodels (BSC 2004 [DIRS 169861], Sections 5 and 6).

\subsection{POSTDEVELOPMENT MODEL VALIDATION TO SUPPORT THE SCIENTIFIC BASIS OF THE MODEL}

This section discusses postdevelopment activities to validate the UZ radionuclide transport model. Section I-2-1-1 in Technical Work Plan for: Performance Assessment Unsaturated Zone (BSC 2004 [DIRS 167969]) specifies methods and criteria for Post-Development Validation Activities. The methods used in this section are identified in AP-SIII.10Q, Section 5.4.1 (c).

\subsubsection{Stipulated Validation Requirements, Methods, and Criteria}

This section discusses the validation requirements for the radionuclide transport model and the corresponding criteria. The validation approaches and criteria are based on the technical work plan stipulations (BSC 2004 [DIRS 171282], Section 2.2.2; BSC 2004 [DIRS 167969], Section I-2-1-1), which indicate the following:

Quantitative, qualitative, and review criteria will be used for the model validation. One or more of following quantitative criteria will be used:

- Method 1-Corroboration with Experimental Data:

The Busted Butte unsaturated zone transport test will be simulated, and the predicted and experimental results (normalized concentration vs. time) will be compared. The validation criteria is: the time at which $\mathrm{UZ}$ radionuclide transport model predicts a value 
of normalized concentration between 0.03 and 0.97 will be less than twice as long, or any amount shorter than the time indicated by a smoothed breakthrough curve fit through the data.

The following section (Section 7.2.2) explains that Method 1 is not applicable to the analysis of Busted Butte test data. However, for purposes of postdevelopment, model validation for radionuclide transport model field measurements of pore-water chloride concentrations from the Exploratory Studies Facility (ESF) and of gas-phase ${ }^{14} \mathrm{C}$ ages from boreholes were used. The latter model validation activities are described in detail in the report by BSC (2004 [DIRS 169861], Section 7), and pointed to in Section 7.2.4.2.

- Method 2-Corroboration with Alternative Mathematical Models:

Comparison to analytical solutions (when available). This step is necessary, but not sufficient. This validation step will be considered successful if the difference between the model and the analytical solutions does not exceed 1 percent (value of concentration at a given location and time).

- Method 3-Corroboration with Data from Literature:

Comparison with the results of laboratory studies that have been described in peer-reviewed journals and to which the model can be applied. This validation step will be considered successful if the difference between the model and the laboratory data (value of normalized concentration at a given location and time) does not exceed 50 percent.

- Method 4-Independent Technical Review

Technical review conducted in accordance with the applicable technical work plans (BSC 2004 [DIRS 171282], Section 2.2.2; BSC 2004 [DIRS 167969], Sections 2.8 and I-2-1-1) is considered adequate if conducted by reviewers independent of the development, checking, and review of the model documentation and if the reviewers are proficient in UZ hydrology and numerical techniques.

\section{- Publication in a Refereed Professional Journal (for Corroboration)}

Publication in a refereed professional journal or review by an external agency, documented by the external agency, may be used to demonstrate additional confidence in the model, if publication is used in conjunction with one or more of the model validation activities described above. The model validation, including comparisons with corroborating data, must be included in the publication or review.

\subsubsection{Validation Approach and Criteria}

The UZ radionuclide transport model was validated using the numerical codes T2R3D and EOS9nT (see Sections 7.2.3 and 7.2.4). The validation process is based on a combination of (a) comparisons to known analytical solutions and models (Method 2); (b) scientific acceptance, as demonstrated by publications in refereed journals; and (c) model acceptance by other organizations involved in radionuclide transport studies (Method 5). 
Comparisons and model performance evaluation in the study of the Busted Butte field tests (or other field data) of tracer transport are also presented in Section 7.2.4.1 as a calibration and validation exercise conducted during model development to enhance confidence in the radionuclide transport model. In addition, field measurements of pore-water chloride concentrations from the ESF and of gas-phase ${ }^{14} \mathrm{C}$ ages from boreholes were used in postdevelopment model validation of the radionuclide transport model. The latter model validation activities are described in detail by BSC (2004 [DIRS 169861], Section 7), and pointed to in Section 7.2.4.2.

For the radionuclide transport model validation through predictions of the numerical (TOUGH2 V1.11 MEOS9nTV1.0 (LBNL 1999 [DIRS 113943]) and T2R3D V1.4 (LBNL 1999 [DIRS 146654])) codes, three conditions must be met:

- The relevant physical processes must be accounted for and accurately represented by the mathematics.

- The mathematical equations must be accurately solved by the numerical methods.

- The physical properties of the rocks must be correct.

For the validation of the radionuclide transport model using the numerical code, their simulation results are compared to relevant analytical solutions (Method 2). The validation criterion used in this case is one or more of the following:

(a) Mass fractions agree within 5 percent when $C_{R}=C / C_{0} \leq 10^{-4}$, or

(b) The location of the tracer fronts agree within 5 percent when $C_{R}=C / C_{0} \leq 10^{-4}$, or

(c) The tracer mass balances agree within 5 percent.

The results presented in Table 7-6 show that this criterion was met in all instances. The criterion here is somewhat different from the one discussed in the BSC report (BSC 2004 [DIRS 167969], Section I-2-1-1) and listed in Section 7.2.1 of this model report. The latter (a) is inadequate because it captures only one of the three possible aspects for quantitative agreement; (b) can be unrealistic near the tail end of the $C_{R}$ curve, where $C_{R}$ values are very low; and both analytical and numerical predictions may be unreliable because of roundoff error.

For validation through scientific acceptance, the criterion is publication, or acceptance for publication, in peer-reviewed journals in the fields of hydrology and contaminant transport. Similarly, the criterion through acceptance by other organizations is involvement of the organization in radionuclide transport studies, and reporting of the results of these studies in topical reports or peer-reviewed journals.

For additional validation of the radionuclide transport model using numerical codes through comparison of their predictions to field measurements, the criterion is:

- Overall agreement within 50 percent of each other

- If this is not possible, capturing the correct trend, accompanied by a documented analysis and discussion of the reasons for the observed deviations. 
Inability of the model to match/predict field observations is not necessarily a sign of inability to validate the model if (a) the quality of the measurements is suspect, (b) the test design may be responsible for uncertainties in the measurements, (c) there is insufficient information to fully describe the field test, or (d) the problem is demonstrated to involve very steep gradients (involving very significant parameter changes over a short distance or time) that lead to measurements prone to uncertainties and inaccuracies.

\subsubsection{Corroboration with Alternative Mathematical Models (Method 2)}

In this validation phase, several problems relevant to conditions and possible transport scenarios at Yucca Mountain, with known analytical solutions, were studied. The specifics of these problems and the code performance are discussed in detail below. This section also presents a comparison of simulation of isothermal transport of a tracer that follows a linear equilibrium isoterm for using T2R3D and EOS9nT. The latter is presented first, followed by the comparison to analytical solutions.

\subsubsection{Comparison of T2R3D and EOS9nT with linear equilibrium Sorption During Isothermal Transport}

The two numerical codes used for the transport studies in this model report are TOUGH2 V1.11 Module EOS9nT V1.0 (LBNL 1999 [DIRS 113943]) and T2R3D V1.4 (LBNL 1999 [DIRS 146654]). The software code TOUGH2 V1.11 Module EOS9nT V1.0 (LBNL 1999 [DIRS 113943]) simulates flow (saturated and/or unsaturated) and the decoupled transport of multiple radioactive solutes and/or colloids (parents and daughters) in complex subsurface systems involving porous and/or fractured media. The transport equations account for radioactive decay, advection, molecular/colloidal diffusion, hydrodynamic dispersion, kinetic or equilibrium physical and chemical sorption (linear, Langmuir, Freundlich, or combined), first-order linear chemical reaction, colloid filtration, and colloid-assisted solute transport. EOS9nT can employ conventional time stepping or two Laplace transform formulations.

The code T2R3D V1.4 (LBNL 1999 [DIRS 146654]) simulates flow (saturated and/or unsaturated) and the coupled transport of a single radioactive solute tracer in complex subsurface systems involving porous and/or fractured media. The transport equations account for radioactive decay, advection, molecular diffusion, hydrodynamic dispersion, and linear equilibrium sorption.

These two codes have been shown to produce identical results in simulations of transport involving (a) conventional time stepping and (b) nonsorbing tracers or tracers whose sorption follows an equilibrium linear sorption model. A comparison of the performance of the two codes is shown in Figure 7-1, which depicts predictions of transport of a conservative (nondecaying, nonsorbing) tracer along a vertical plane (slice) of Yucca Mountain. Figure 7-1 shows that the EOS9nT and T2R3D results practically coincide. Further evidence of the identity of the EOS9nT and T2R3D predictions is provided in the report by BSC (2001 [DIRS 161340], Figure 6.4.5) and in DTNs: LB03093RADTRNS.001 [DIRS 166225], LB03093RADTRNS.002 [DIRS 166071]. 


\subsubsection{Test 1: Transport of a Nonsorbing, Nondecaying Tracer}

This problem involved transport of a nonsorbing, nondecaying tracer through a porous medium. A family of analytical solutions to this problem were developed by Bear (1979 [DIRS 105038]). The properties and parameters for this problem are listed in Table 7-1, and the comparison of the analytical to the numerical solutions is listed in Table 7-6.

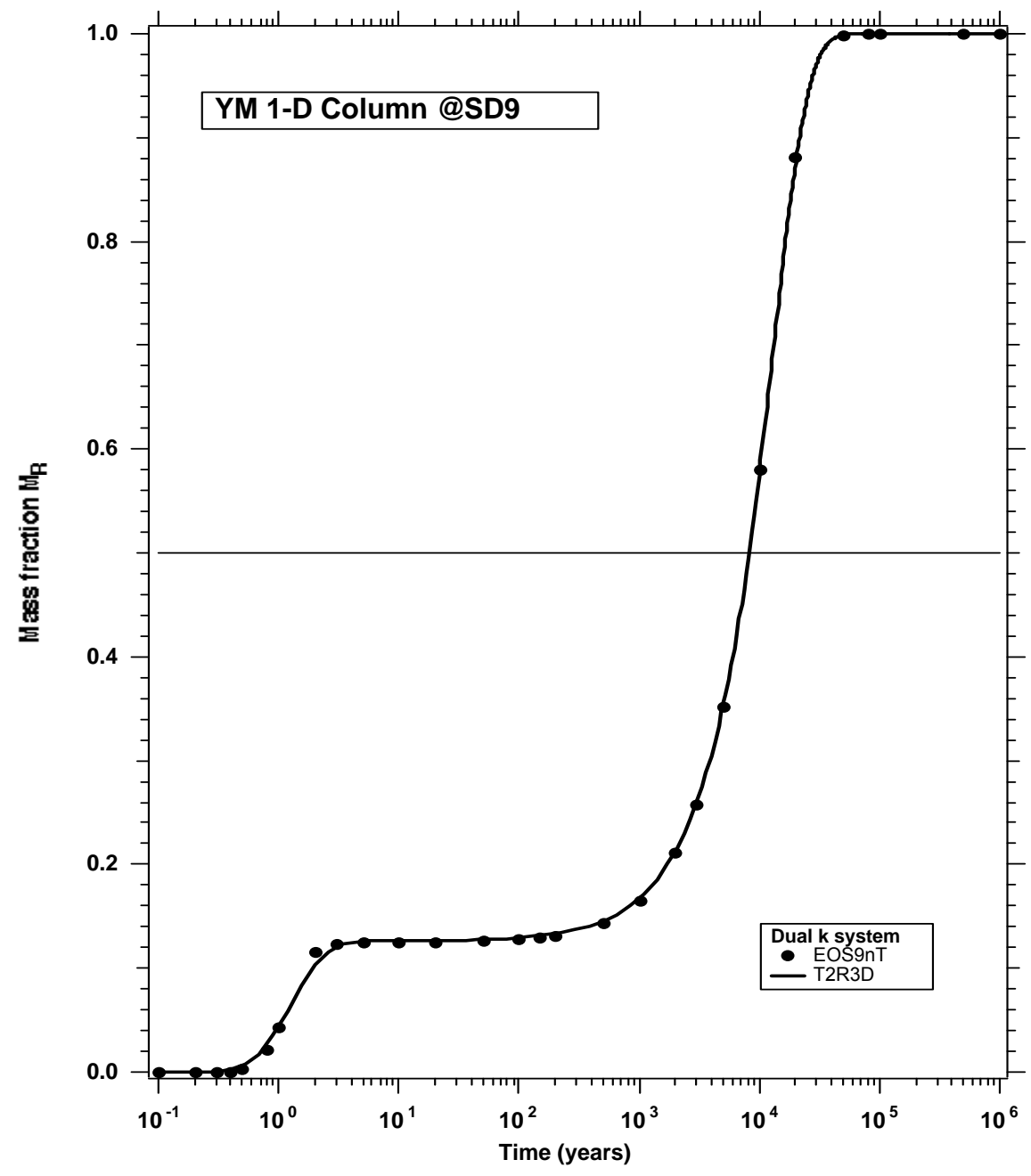

DTN: LB03093RADTRNS.001 [DIRS 166225] and LB03093RADTRNS.002 [DIRS 166071].

Figure 7-1. Comparison between the Numerical Predictions of Breakthrough from TOUGH2 V1.11 Module EOS9nT V1.0 (LBNL 1999 [DIRS 113943]) and from T2R3D V1.4 (LBNL 1999 [DIRS 146654])

Both T2R3D V1.4 (LBNL 1999 [DIRS 146654]) and TOUGH2 V1.11 Module EOS9nT V1.0 (LBNL 1999 [DIRS 113943]) were used in this test. Figure 7-2 shows a comparison between the analytical solution and the numerical model predictions, and includes solutions from three different time treatments, designated by $\mathrm{T}, \mathrm{H}, \mathrm{S}$ to indicate regular time stepping, De Hoog Laplace space solution and Stehfest Laplace space solution, respectively. Note that the results for regular time stepping (i.e., the T-solutions) reflect both the EOS9nT and T2R3D estimates, 
which are not shown separately because they are practically indistinguishable from each other and from the Laplace space formulation solutions.

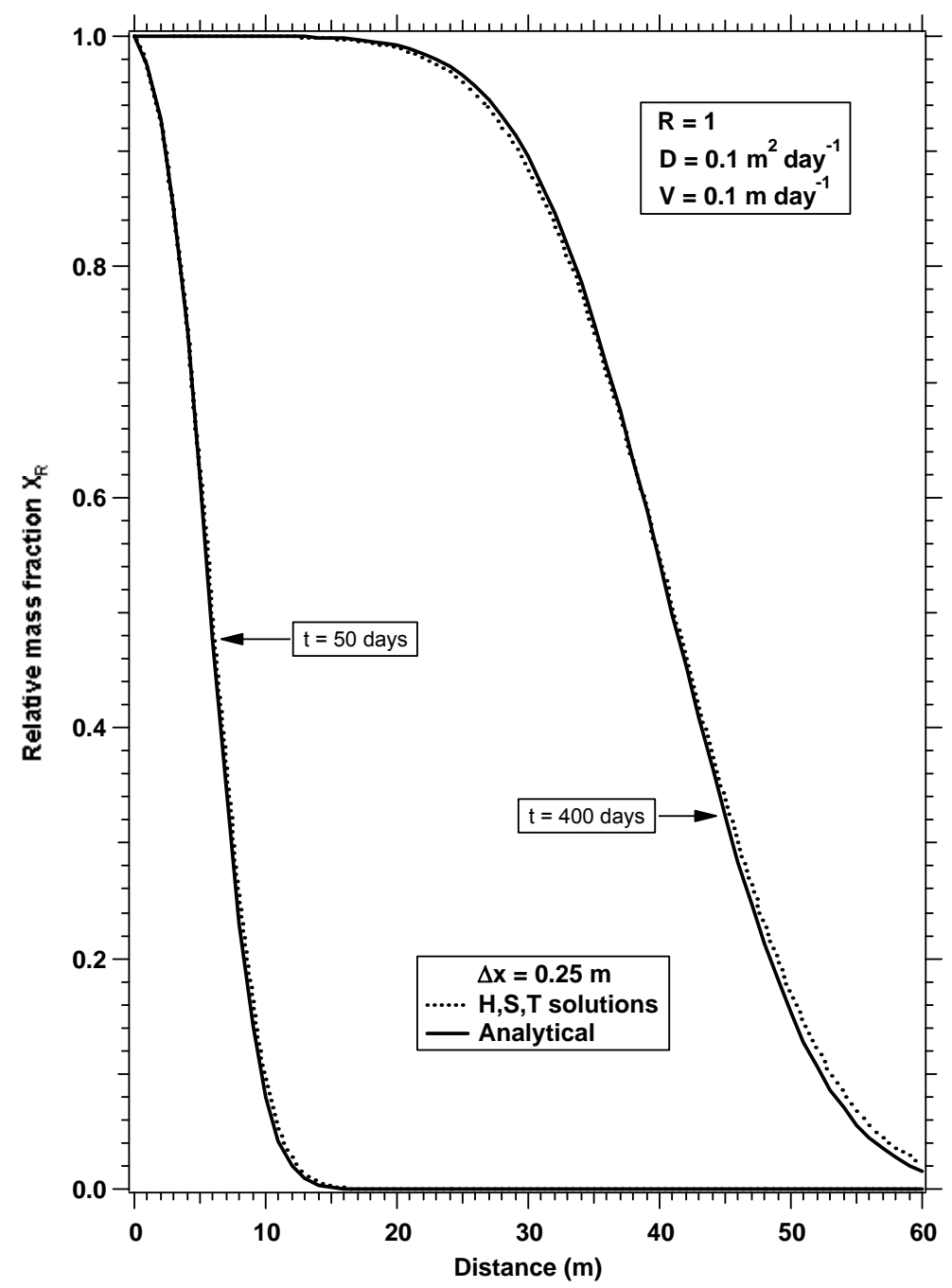

Output DTN: LB0408MR0060R2.001.

NOTE: The results from three different time treatments (designated by $\mathrm{T}, \mathrm{H}, \mathrm{S}$ to indicate regular time stepping, De Hoog Laplace space solution, and Stehfest Laplace space solution, respectively) are indistinguishable.

Figure 7-2. Comparison between the Analytical Solution and the Numerical Predictions in Test 1

\subsubsection{Test 2: Transport of Sorbing Radioactive Tracers}

The transport of three sorbing radioactive tracers is studied in this test. The analytical solutions to this problem were developed by Bear (1979 [DIRS 105038]). Properties and parameters for this problem are listed in Table 7-2, and the comparison of the analytical to the numerical solutions is listed in Table 7-6. The input and output files for this test are in output DTN: LB0408MR0060R2.001. 
Both T2R3D V1.4 (LBNL 1999 [DIRS 146654]) and TOUGH2 V1.11 Module EOS9nT V1.0 (LBNL 1999 [DIRS 113943]) were used in this test. Figure 7-3 shows a comparison between the analytical solution and the numerical solutions, and includes solutions from three different time treatments (i.e., the T, H, S solutions). As in Test 1, the results for regular time stepping reflect both the EOS9nT and T2R3D estimates. They are not shown separately because they are practically indistinguishable from each other and from the Laplace space formulation solutions.

\subsubsection{Test 3: Transport of the ${ }^{234} \mathrm{U}->{ }^{230} \mathrm{Th}->{ }^{226}$ Ra Chain}

This problem involved transport of the three-member radioactive chain ${ }^{234} \mathrm{U}->{ }^{230} \mathrm{Th}->{ }^{226} \mathrm{Ra}$ through a porous medium. All the members of this chain exist in species that sorb onto the porous medium. The analytical solution to this problem was developed by Pigford et al. (1980 [DIRS 123113]). The properties and parameters for this problem are listed in Table 7-3, and the comparison of the analytical to the numerical solutions is listed in Table 7-6. The input and output files for this test are in output DTN: LB0408MR0060R2.001.

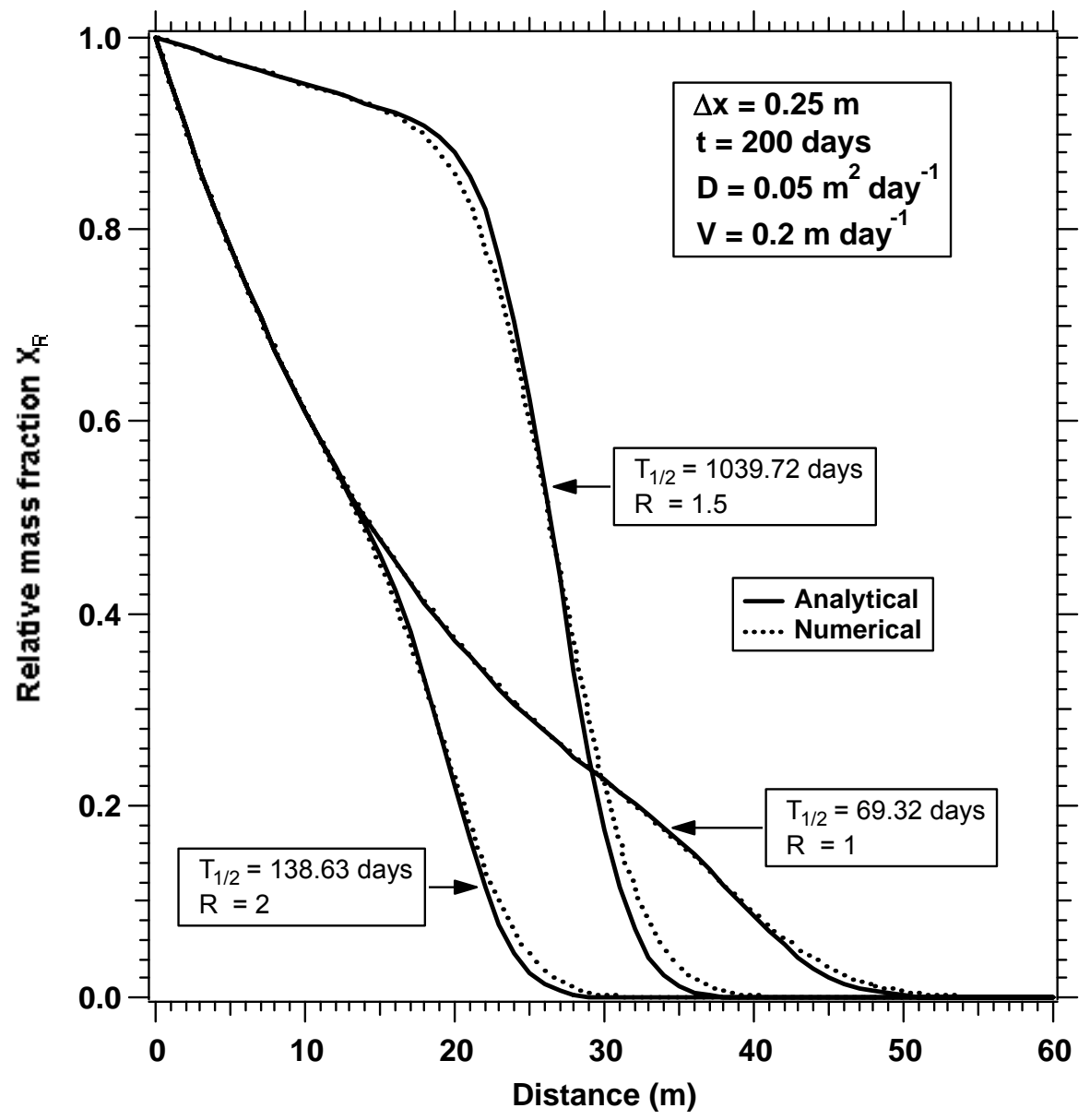

Output DTN: LB0408MR0060R2.001.

Figure 7-3. Comparison between the Numerical Predictions (TOUGH2 V1.11 Module EOS9nT V1.0 [LBNL 1999 (DIRS 113943)]; T2R3D V1.4 [LBNL 1999 (DIRS 146654)]) and the Analytical Solutions (Bear 1979 [DIRS 105038]) in Test 2 


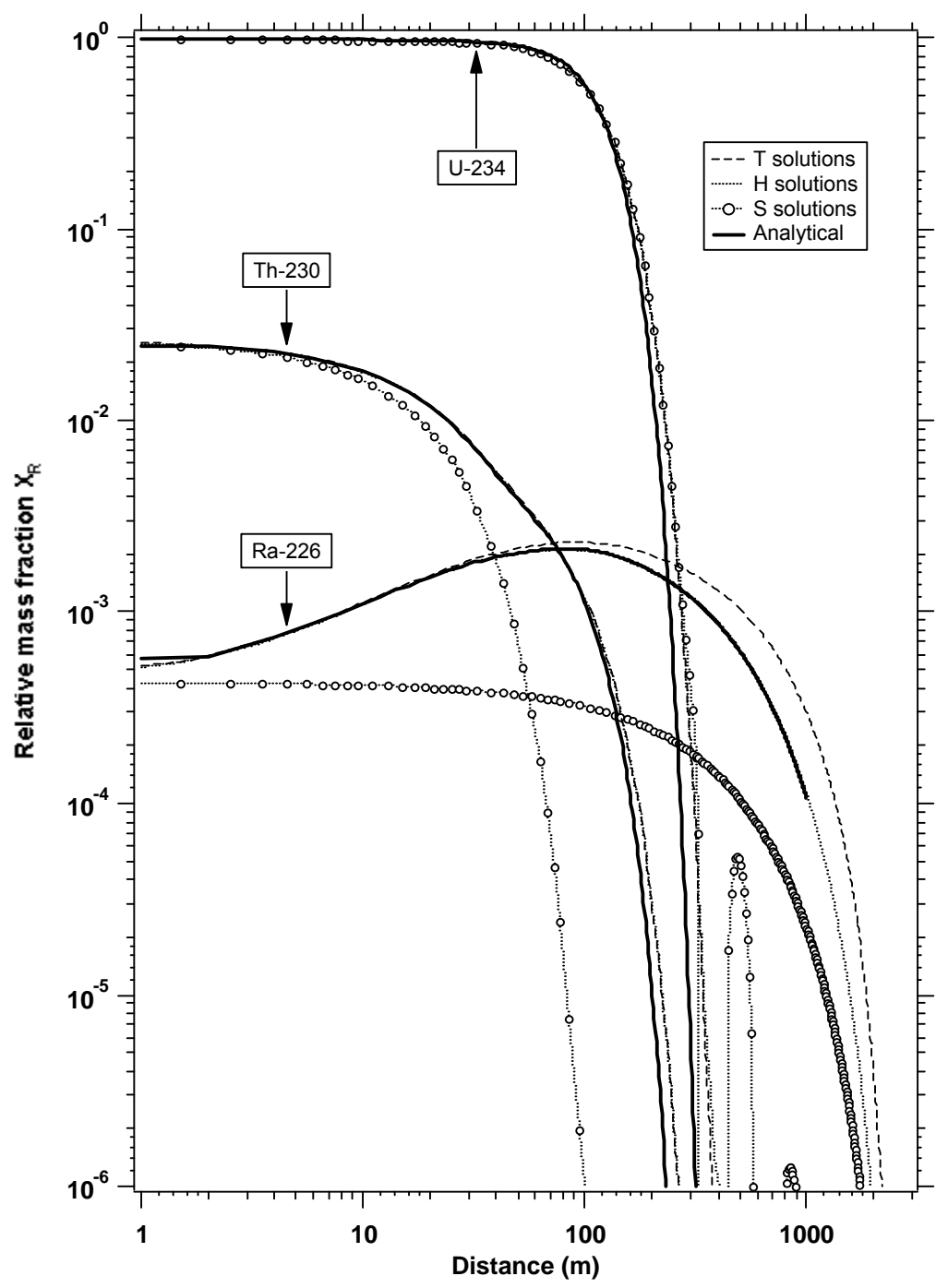

Output DTN: LB0408MR0060R2.001.

NOTE: While the results from regular timestepping and the De Hoog Laplace space solution (designated by $\mathrm{T}$ and $\mathrm{H}$, respectively) are in excellent agreement with the analytical solutions, the results from the Stehfest Laplace space solution for the daughters become increasingly inaccurate.

Figure 7-4. Comparison between the Analytical Solution and the TOUGH2 V1.11 Module EOS9nT V1.0 (LBNL 1999 [DIRS 113943]) Predictions in Test 3

Because it involves transport of parent and daughter species, T2R3D cannot be used for this study. The results in Figure 7-4 were obtained with EOS9nT, and show a practical coincidence of the $\mathrm{T}$ - and H-solutions with the analytical solution. When the Stehfest algorithm is implemented (S-solution), accurate estimates of the parent species are obtained, but significant deviations occur in the transport prediction of daughter species. Thus, the Stehfest formulation should not be used for transport studies of radioactive chains. 
Table 7-1. Parameters in Test 1

\begin{tabular}{|l|l|}
\hline \multicolumn{1}{|c|}{ Parameter } & \multicolumn{1}{c|}{ Value } \\
\hline$V(\mathrm{~m} /$ day $)$ & 0.1 \\
\hline$D\left(\mathrm{~m}^{2} /\right.$ day $)-$ Equation 20 & 0.1 \\
\hline$K_{d}\left(\mathrm{~m}^{3} / \mathrm{kg}\right)$ & 0 \\
\hline Water saturation $S_{w}$ & 1 \\
\hline$\tau$ & 1 \\
\hline
\end{tabular}

Output DTN: LB0408MR0060R2.001.

Table 7-2. Parameters in Test 2

\begin{tabular}{|l|l|}
\hline \multicolumn{1}{|c|}{ Parameter } & \multicolumn{1}{c|}{ Value } \\
\hline$V(\mathrm{~m} /$ day $)$ & 0.2 \\
\hline$D\left(\mathrm{~m}^{2} /\right.$ day $)$ - Equation 20 & 0.05 \\
\hline Water saturation $S_{\mathrm{w}}$ & 1 \\
\hline Tortuosity factor $\tau$ & 1 \\
\hline$\rho_{\mathrm{s}}\left(\mathrm{kg} / \mathrm{m}^{3}\right)$ & 2600 \\
\hline$K_{d}\left(\mathrm{~m}^{3} / \mathrm{kg}\right)$ & $0,2.14 \times 10^{-5}, 4.27 \times 10^{-5}$ \\
\hline $\mathrm{T} 1 / 2($ days $)$ & $69.32,1039.72,138.63$ \\
\hline
\end{tabular}

Output DTN: LB0408MR0060R2.001.

Table 7-3. Parameters in Test 3

\begin{tabular}{|l|l|}
\hline \multicolumn{1}{|c|}{ Parameter } & \multicolumn{1}{c|}{ Value } \\
\hline$V(\mathrm{~m} /$ day) & 0.274 \\
\hline $\mathrm{D}\left(\mathrm{m}^{2} /\right.$ day) - Equation 20 & 2.74 \\
\hline Water saturation $\mathrm{S}_{\mathrm{w}}$ & 1 \\
\hline Tortuosity factor $\tau$ & 1 \\
\hline$\rho_{\mathrm{s}}\left(\mathrm{kg} / \mathrm{m}^{3}\right)$ & 2600 \\
\hline$K_{d}\left(\mathrm{~m}^{3} / \mathrm{kg}\right)$ & $0,2.14 \times 10^{-5}, 4.27 \times 10^{-5}$ \\
\hline $\mathrm{T} 1 / 2($ days $)$ & $69.32,1039.72,138.63$ \\
\hline$K_{d}$ of ${ }^{234} \mathrm{U}\left(\mathrm{m}^{3} / \mathrm{kg}\right)$ & 1.65 \\
\hline$K_{d}$ of ${ }^{230} \mathrm{Th}\left(\mathrm{m}^{3} / \mathrm{kg}\right)$ & 8.24 \\
\hline$K_{d}$ of ${ }^{226} \mathrm{Ra}\left(\mathrm{m}^{3} / \mathrm{kg}\right)$ & 0.0823 \\
\hline $\mathrm{T} 1 / 2$ of ${ }^{234} \mathrm{U}\left(\right.$ years) ${ }^{\mathrm{a}}$ & $2.45 \times 10^{5}$ \\
\hline $\mathrm{T} 1 / 2$ of ${ }^{230} \mathrm{Th}($ years) & $7.54 \times 10^{4}$ \\
\hline $\mathrm{T} 1 / 2$ of ${ }^{226} \mathrm{Ra}$ (years) ${ }^{\mathrm{a}}$ & $1.60 \times 10^{3}$ \\
\hline
\end{tabular}

Output DTN: LB0408MR0060R2.001.

a From Pigford et al. 1980 (DIRS 123113). 


\subsubsection{Test 4: ${ }^{3} \mathrm{H}$ Transport in a Fracture-Matrix System}

This problem describes the transport of a radioactive substance in a system of parallel fractures. Advection, dispersion, and diffusion occur in the fractures, while the species diffuses through the fracture walls into the matrix. The analytical solution to this problem was developed by Sudicky and Frind (1982 [DIRS 105043]). Properties and parameters for this problem are identified in the report by Sudicky and Frind, and are listed in Table 7-4. The comparison of the analytical to the numerical solutions is listed in Table 7-6. The input and output files for this test are in output DTN: LB0408MR0060R2.001.

Both T2R3D V1.4 (LBNL 1999 [DIRS 146654]) and TOUGH2 V1.11 Module EOS9nT V1.0 (LBNL 1999 [DIRS 113943]) were used in this test. Figure 7-5 shows a comparison between the analytical solution and the numerical solutions, and includes solutions from three different time treatments (i.e., the T, H, S solutions). As in Tests 1 and 2, the results for regular time stepping reflect both the EOS9nT and T2R3D estimates. The numerical results are not reported separately because, at a minimum, they coincide in the first three decimal digits.

Table 7-4. Parameters in Test 4

\begin{tabular}{|c|c|}
\hline Parameter $^{a}$ & Value \\
\hline$\rho_{\mathrm{s}}\left(\mathrm{kg} / \mathrm{m}^{3}\right)$ & 2600 \\
\hline$D_{0}\left(\mathrm{~m}^{2} /\right.$ day $)$ & $1.6 \times 10^{-9}$ \\
\hline Fracture aperture $(\mathrm{m})$ & $10^{-4}$ \\
\hline Fracture $V$ (m/day) & 0.1 \\
\hline Fracture $S_{w}$ & 1 \\
\hline Fracture $\phi$ & 1 \\
\hline Fracture $\tau$ & 1 \\
\hline$\alpha_{L}(m)$ & 0.1 \\
\hline Matrix $\vee(\mathrm{m} /$ day $)$ & 0 \\
\hline Matrix block width $(\mathrm{m})$ & 0.5 \\
\hline Matrix $S_{w}$ & 1 \\
\hline Matrix $\phi$ & 1 \\
\hline Matrix $\tau$ & 1 \\
\hline$K_{d}\left(\mathrm{~m}^{3} / \mathrm{kg}\right)$ & 0 (in fractures and matrix) \\
\hline $\mathrm{T} 1 / 2$ (years) & 12.35 \\
\hline
\end{tabular}




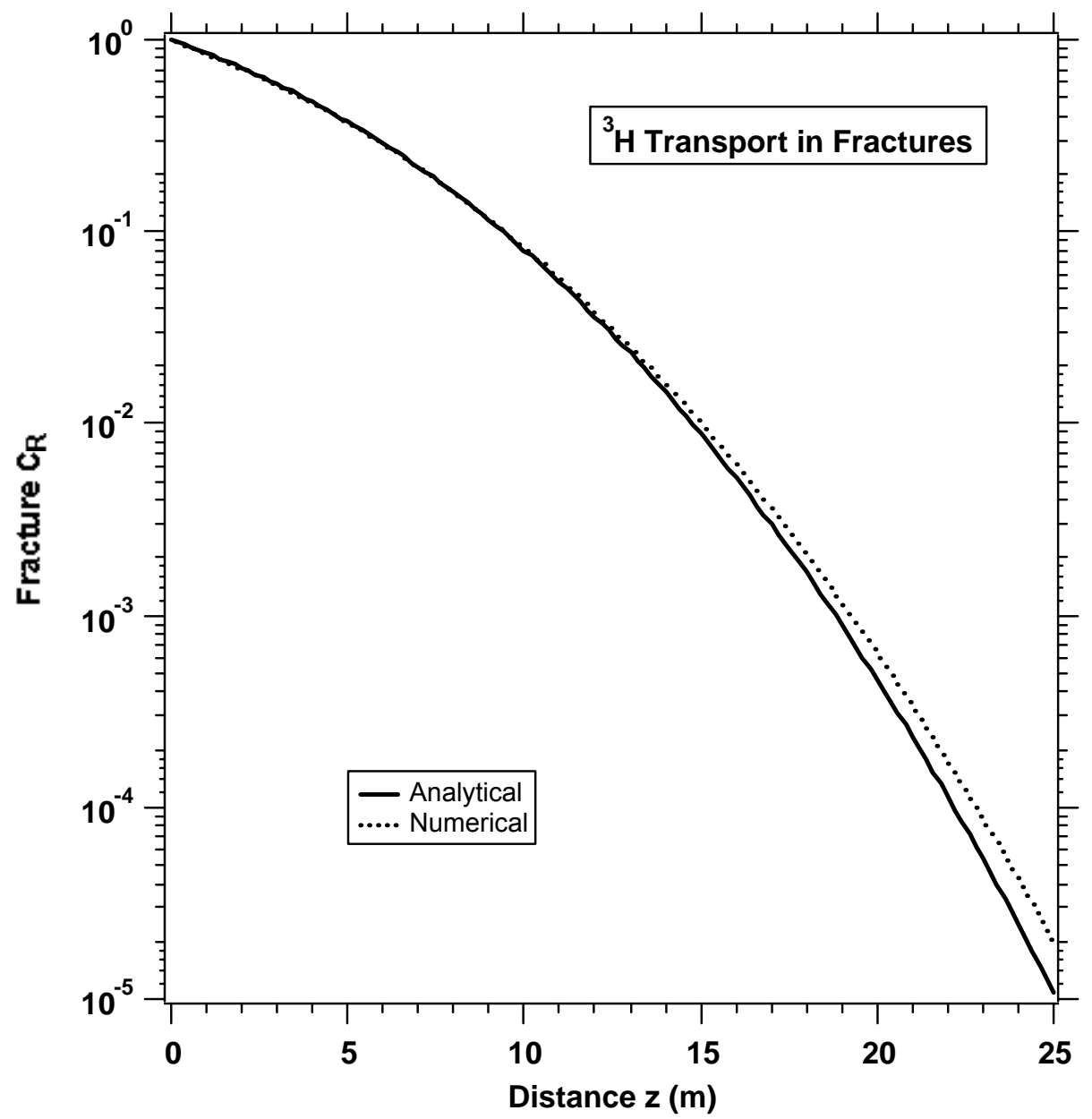

Output DTN: LB0408MR0060R2.001.

NOTE: The numerical results from the two codes and the three different time treatments are indistinguishable, and are indicated by the single curve.

Figure 7-5. Comparison between the Analytical Solution of Sudicky and Frind (1982 [DIRS 105043]) and the Numerical (TOUGH2 V1.11 Module EOS9nT V1.0 [LBNL 1999 (DIRS 113943) and T2R3D V1.4 [LBNL 1999 (DIRS 146654)]) Predictions in Test 4

\subsubsection{Test 5: Colloid and Colloid-Assisted Radionuclide Transport}

This transport of a colloid and a solute is described in this problem. The colloid filtration (attachment) is controlled by a kinetic process. The analytical solution to the problem of transport of colloids undergoing kinetic filtration is presented by de Marsily (1986 [DIRS 100439], pp. 273 to 274). The solute is radioactive and is irreversibly sorbed onto the colloid; thus, it cannot enter the liquid phase and is transported only by the mobile colloids. All the radionuclides (produced in a single batch at the same time) that enter the system (through the boundaries or through sources) are sorbed onto colloids. This being the case, the radionuclide decays uniformly, and its concentration is the same on all colloids. The radionuclide concentration on each colloid is controlled by radioactive decay, and by choosing an observation time equal to $T_{1 / 2}$, its relative concentration is 0.5 . 
Properties and parameters for this problem are listed in Table 7-5, and the comparison of the analytical to the numerical solutions is listed in Table 7-6. The input and output files are in output DTN: LB0408MR0060R2.001. Because it involves transport of a kinetically filtering colloid, T2R3D cannot be used for this study. The numerical results in Figure 7-6 were obtained with EOS9nT and show a practical coincidence with the analytical solution, while the radionuclide relative concentration on the colloids is confirmed to be a uniform 0.5 . This is validation by Method 2 .

Table 7-5. Parameters in Test 5

\begin{tabular}{|l|l|}
\hline \multicolumn{1}{|c|}{ Parameter } & \multicolumn{1}{c|}{ Value } \\
\hline$S_{w}$ & 1 \\
\hline$\rho_{\mathrm{s}}\left(\mathrm{kg} / \mathrm{m}^{3}\right)$ & 2600 \\
\hline$D_{0}\left(\mathrm{~m}^{2} /\right.$ day $)$ & $\begin{array}{l}\text { From Eq. 6-23 (Section } \\
6.2)\end{array}$ \\
\hline$\rho_{\mathrm{c}}\left(\mathrm{kg} / \mathrm{m}^{3}\right)$ & 1000 \\
\hline$U(\mathrm{~m} / \mathrm{day})$ & 2 \\
\hline$\phi$ & 0.3 \\
\hline$\tau$ & 1 \\
\hline$\alpha_{\mathrm{L}}(\mathrm{m})$ & 0.15 \\
\hline$\varepsilon(1 / \mathrm{m})$ & 30 \\
\hline
\end{tabular}

Output DTN: LB0408MR0060R2.001.

Table 7-6. Model-Validation - Comparison of Numerical to Analytical Solutions in Tests 1 to 5

\begin{tabular}{|l|l|l|}
\hline \multicolumn{1}{|c|}{ Test \# } & \multicolumn{1}{|c|}{ \% Difference } & \multicolumn{1}{|c|}{$\begin{array}{c}\text { Pass/Fail } \\
\text { Criterion }\end{array}$} \\
\hline 1 & 0.48 (max of T,S,H solutions) & Pass \\
\hline 2 & 0.93 (max of T,S,H solutions, 3 cases) & Pass \\
\hline 3 & $\begin{array}{l}{ }^{234} \mathrm{U}: 0.93 \text { (T solution), } 0.09 \text { (H-solution), } 0.97 \text { (H-solution) } \\
{ }^{230} \mathrm{Th:} 0.87 \text { (T solution), } 0.16 \text { (H-solution) } \\
\end{array}$ & Pass \\
\hline 4 & 0.83 (max of T,S,H solutions) 0.98 (T solution), 0.21 (H-solution) & \\
\hline 5 & 0.33 (max of T,S,H solutions) & Pass \\
\hline
\end{tabular}

Output DTN: LB0408MR0060R2.001.

$\mathrm{T}$ - regular time stepping solution

$\mathrm{H}$ - De Hoog Laplace space solution

S - Stehfest Laplace space solution 


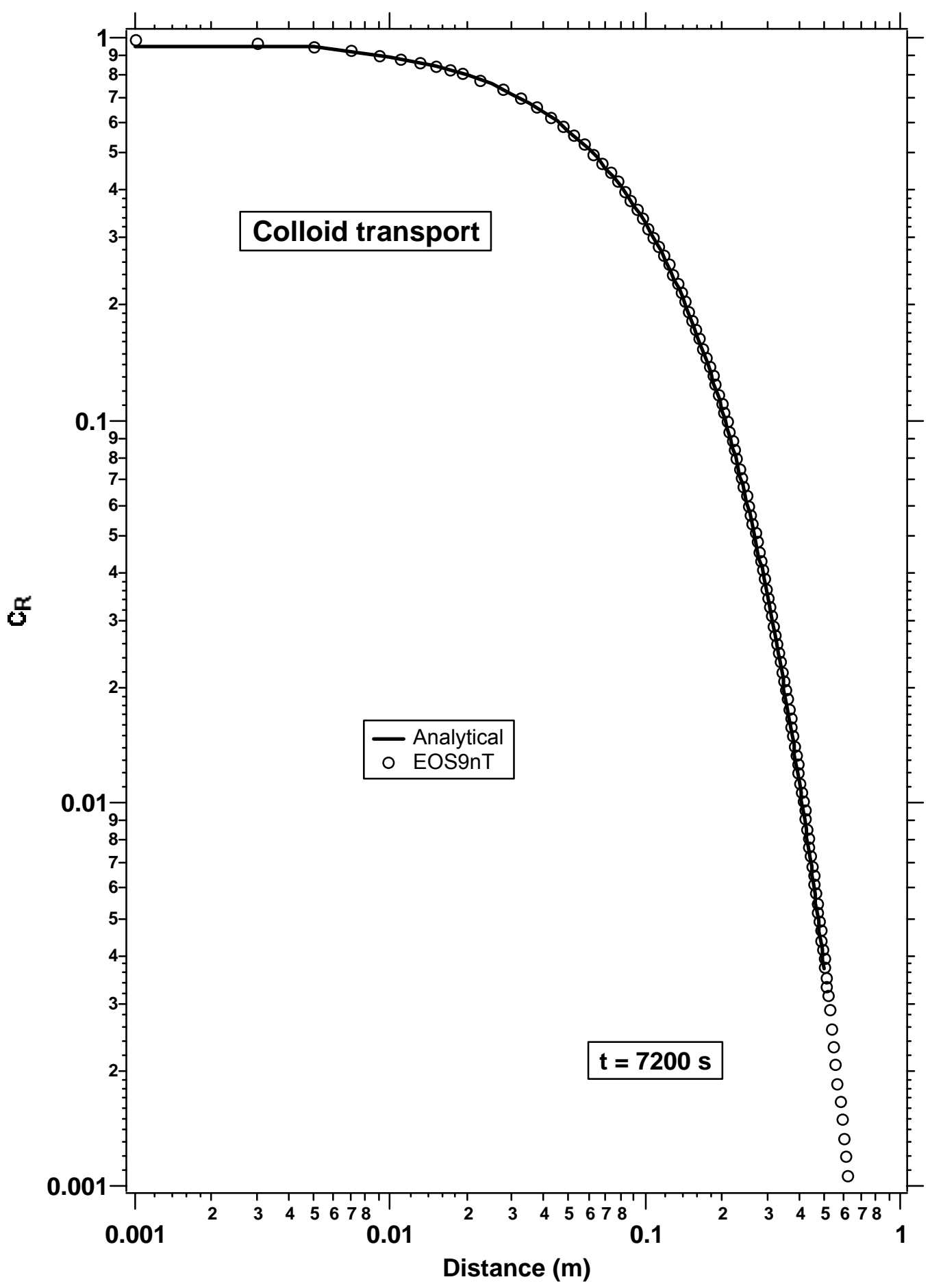

Output DTN: LB0408MR0060R2.001.

Figure 7-6. Comparison between the Numerical (TOUGH2 V1.11 Module EOS9nT V1.0 (LBNL 1999 [DIRS 113943])) and the Analytical (de Marsily 1986 [DIRS 100439]) Solution of Colloid Transport in Test 5 


\subsubsection{Corroboration with Experimental Data (Method 1)}

The Busted Butte studies analyzed in the model report are presented in Section 7.2.4.1.1 through 7.2.4.1.3 as a postdevelopment model validation exercise conducted to enhance confidence in the radionuclide transport model. Section 7.2.4.2 points to the postdevelopment validation of the radionuclide transport model using field measurements of pore-water chloride concentrations and gas-phase ${ }^{14} \mathrm{C}$ ages.

\subsubsection{UZ Radionuclide Transport Model Postdevelopment Validation Through Corroboration with the Busted Butte Field Tests}

The Busted Butte unsaturated zone transport test was a long-term experiment conducted in Busted Butte near Yucca Mountain (BSC 2004 [DIRS 170004], Section 6.13) to investigate flow and transport issues in the UZ site-process models for Yucca Mountain, including:

- Effect of heterogeneities on flow and transport under unsaturated conditions in the $\mathrm{CHn}$ hydrogeologic unit near the TSw-CHn interface, particularly fracture-matrix interactions and permeability contrast boundaries

- Migration behavior of colloids in the $\mathrm{CHn}$ layers

- Validation of laboratory sorption results

- Scaling effects (from laboratory to field to site) on three-dimensional, site-scale flow and transport simulations.

The site was selected because of the presence of a readily accessible interface of the TSw and $\mathrm{CHv}$ units, and the similarity of these layers to those beneath the repository horizon. The study of the TSw/CHv interface is important because of the significant role that the vitric layers of the $\mathrm{CHv}$ unit play in radionuclide retardation (see Section 6.10, this model report). The test proceeded in two phases that differ in design, purpose, and experimental scales, among other factors. A detailed description of the tests can be found in the BSC report (BSC 2004 [DIRS 170004], Section 6.13).

The Busted Butte studies analyzed in this model report are presented as a calibration and validation exercise conducted during model development to enhance confidence in the radionuclide transport model. This analysis and modeling includes portions of the data sets obtained during the main phases of the test, namely Phases 1A, 1B, and 2C. The reasons for the choice of tracers and of the data set segments used in this analysis are discussed in detail in the following sections.

\subsection{Calibration Versus Validation}

The expected spatial variability in hydraulic and transport parameters makes problematic the use of single parameters derived from large-scale inversion processes, such as the process involved in the determination of the hydraulic properties of the various hydrogeologic units in this model report, to describe the small-scale experiments at the Busted Butte site. Because detailed spatial characterizations of the hydraulic parameters were not available, it was necessary to calibrate the 
radionuclide transport model at each of the three Busted Butte test beds involved in the three phases of the field test (i.e., phases 1A, 1B, and 2C). These calibrations were intended to determine the main hydraulic parameters of the medium under consideration and account for (a) heterogeneity and anisotropy in hydraulic and transport parameters that are present even in small-scale systems, and (b) the effects of the size of the model grid cells (i.e., scale effects), an issue distinct from heterogeneity. The calibrated hydraulic parameters were then used in an attempt to validate and enhance confidence in the transport model using transport parameters available from other independent sources.

To accomplish the task, two separate data sets of tracer concentrations were used from each of the three test phases, of which one was used for calibration and the other for validation. This is a valid approach because the transport behavior of the two tracers used for calibration and validation was completely independent, that is, there is no sorption-related correlation. If a particular tracer was known or expected to have a sorbing affinity for the tested rock, the corresponding data set was used for the calibration effort, which included an estimation of the tracer's transport parameters (i.e., the $K_{d}$ distribution coefficient and tortuosity) in addition to the hydraulic parameters. The nonsorbing tracer, $\mathrm{Br}$ in all three of the test phases, was used for validation because (a) it is more demanding as a conservative species, and (b) it has a well-known (and independently determined) $K_{d}(=0)$ and a molecular diffusion coefficient $D_{0}\left(=2.080 \times 10^{-9} \mathrm{~m}^{2} / \mathrm{s}\right.$, see Table 7-7).

In Test Phases 1A and 1B, the tracer used for calibration was either nonsorbing (fluorescein in Test Phase 1A) or very mildly sorbing (2,6-DFBA in Test Phase 1B). Thus, the retardation of the calibration tracer was none or very small, and the time frame used for calibration and validation was the same. In the case of the Li tracer in Test Phase $2 \mathrm{C}$, its relatively strong sorption resulted inevitably in a significant retardation, leading to later appearance than the $\mathrm{Br}$ at the collection point. When $\mathrm{Li}$ began registering significant concentrations, the $\mathrm{Br}$ concentration was invariant at the collection point because it had already reached a maximum equal to the injection concentration. In this case, the Li data set corresponding to the later times was used for calibration, while the earlier $\mathrm{Br}$ data set was used for validation. This further strengthens validation because of the larger degree of separation between the $\mathrm{Li}$ and $\mathrm{Br}$ data sets.

\subsection{Phase 1A Test}

The purpose of this modeling study was to calibrate and validate the model through the reconciliation of field measurements and predictions of the concentration and water saturation, following the injection of nonreactive tracers into the ch1v, and the subsequent flow and transport in the ch1v and ch2v layers. Of the four tracers injected during the field experiment, only the transport of two, the nonsorbing $\mathrm{Br}$ and fluorescein, is analyzed here because of the poor quality and unreliability of the data from the other two tracers. In this analysis,

(a) the fluorescein data are used for calibration, while

(b) the $\mathrm{Br}$ data are used for validation.

Additional discussion on the calibration and validation approach can be found in Section 7.2.4.1.1 of this model report. 


\subsection{Procedure and Layout}

A schematic of the borehole layout in the Phase 1A test can be found in the report by BSC (2001 [DIRS 161340], Attachment VI), while more detailed information is provided by BSC (2004 [DIRS 170004], Section 6.13). Parameters describing the site conditions and test operation and the corresponding sources are listed in Table 7-7. Only injection into borehole 3, located in the ch1v layer of the $\mathrm{CHn}$ unit, was considered. Borehole 3 is located in the ch1v layer and is about $20 \mathrm{~cm}$ above the ch1v-ch2 $\mathrm{v}$ interface. The injection tests into Boreholes 1 , located in the ch1v layer, but is further removed from the interface; 2; and 4, located in the ch2v layer of the CHn unit, were not analyzed because of the poor quality of the data. An extensive discussion of the test can be found in the BSC report (BSC 2004 [DIRS 170004], Section 6.13.2).

Mineback of the Phase1A test block provided the field data for this analysis. During mineback, as successive vertical slices were being removed, digital photographs under visible and ultraviolet light were taken to record the distribution of moisture and fluorescein. This distribution near Borehole 3 is used for the calibration of the simulation results discussed in the present section because it is the largest fluorescein plume (in addition to being the most uniform). Additionally, rock samples were collected by augering, and the exposed plane was accurately surveyed. These samples were analyzed for tracer concentration and are the basis for the validation of the $\mathrm{Br}$ transport.

\subsection{Conceptual and Numerical Model}

For this three-dimensional numerical study, the underlying geologic model considered a homogeneous and anisotropic unfractured rock matrix with the properties of the ch1v and $\operatorname{ch} 2 \mathrm{v}$ layers. Because of the injection configuration (as described by BSC 2004 [DIRS 170004], Section 6.13), only half the domain (i.e., the portion of the domain to the right of the injection point; see Figure 7-7) was simulated using a grid consisting of 22,463 elements. The TOUGH2 V1.11MEOS9nTV1.0 (LBNL 1999 [DIRS 113943]) was used for the simulation.

The determination of the hydraulic parameters was a significant component of the calibration effort. Initial estimates for these parameters were provided by two different sources: the UZ99 calibrated flow parameter set (DTN: LB997141233129.001 [DIRS 104055]) and a data set based on hydraulic property measurements from field-collected samples (DTNs: GS990308312242.007 [DIRS 107185], GS990708312242.008 [DIRS 109822]). Initial (uncalibrated) data, as well as all relevant flow and transport parameters, are listed in Table 7-7, which also includes the DTN of the field data and measurements used in the validation, and in Table 7-8. The DTN of the input and output files of this simulation is output DTN: LB0308MR0060R1.008.

\subsection{Calibration Using the Fluorescein Data}

For all calibrations, the following parameters are adjusted: $\phi, k, \tau, K_{d}$ (for known sorbing species), $D_{0}$ (only if not available from Lide (1992 [DIRS 166224])). For validation, the relevant calibrated parameters are used unchanged, and only the fixed parameters that uniquely describe individual transport behavior (i.e., the known $D_{0}$ of $\mathrm{Br}$ ) are different. 
Figures 7-7 and 7-8 show the fluorescein distribution, as recorded digitally in the field using a UV light, and the corresponding numerically predicted distribution (calibrated) at the $y=0.90 \mathrm{~m}$ mineback face. The final (calibrated) transport properties that resulted in the distribution of Figure 7-8 are listed in Table 7-8.

A comparison of the two figures indicates good agreement between predictions and observations. The absence of "stringers" and other features indicative of fracture presence, coupled with the ellipsoidal size of the plume, indicate that the assumption of an unfractured porous medium is valid. (Visual evidence of local heterogeneity in Figure 7-7 notwithstanding, the plume is quite uniform at the scale of observation.)

Although the size of the numerically predicted horizontal axis is somewhat larger than the observed one, the compressed shape of the fluorescein plume is accurately captured, the compression of the lower half of the plume due to its vicinity with the less permeable ch2v is accurately rendered, and the size of the vertical axis of the ellipsoid fluorescein plume is in good agreement with that measured in Figure 7-7. However, it is not possible to further refine the calibration because critical information on the minimum concentration at which the tracer will fluoresce is unavailable, thus depriving the study of more reliable information on the extent of the outer boundaries of the plume. Additionally, the lack of scaling information in any of the other digital photographs from the mineback did not allow the use of a larger data set to more accurately determine the appropriate parameters (and the site heterogeneity distribution) and effectively constrain the solution.

Table 7-7. Operational, Transport, and Field Parameters in the Analysis of the Busted Butte Phase 1 Field Test

\begin{tabular}{|c|c|c|}
\hline Parameter & Value & Source \\
\hline $\begin{array}{l}\text { Phase 1A: } \\
\text { Average injection rate }(\mathrm{mL} / \mathrm{hr})\end{array}$ & 2 & LA0008WS831372.001 [DIRS 156582] \\
\hline $\begin{array}{l}\text { Phase 1A: } \\
\text { Injection duration (days) }\end{array}$ & 286 & LA0008WS831372.001 [DIRS 156582] \\
\hline $\begin{array}{l}\text { Phase 1B: } \\
\text { Average injection rate }(\mathrm{mL} / \mathrm{hr})\end{array}$ & 3 & LA0008WS831372.001 [DIRS 156582] \\
\hline $\begin{array}{l}\text { Phase 1B: } \\
\text { Injection duration (days) }\end{array}$ & 191 & LA0008WS831372.001 [DIRS 156582] \\
\hline$\alpha_{L}(m)-$ Ch1v and Ch2v & 0.1 & Reasonable estimate \\
\hline$\alpha_{T}(\mathrm{~m})-\mathrm{Ch} 1 \mathrm{v}$ and Ch2v & 0 & Reasonable estimate \\
\hline$D_{0}$ of $\mathrm{Br}\left(\mathrm{m}^{2} / \mathrm{s}\right)$ & $2.08 \times 10^{-9}$ & Lide (1992 [DIRS 166224]), p. 5-111 \\
\hline$D_{0}$ of Fluorescein $\left(\mathrm{m}^{2} / \mathrm{s}\right)$ & $8 \times 10^{-10}$ & Initial estimate \\
\hline$D_{0}$ of $2,6-$ DFBA $\left(\mathrm{m}^{2} / \mathrm{s}\right)$ & $7.6 \times 10^{-10}$ & Benson and Bowman (1994 [DIRS 122788]) \\
\hline$K_{d}$ of $\mathrm{Br}\left(\mathrm{m}^{3} / \mathrm{kg}\right)$ & 0 & BSC (2004 [DIRS 170004\}, Section 6.13.1.8) \\
\hline$K_{d}$ of Fluorescein $\left(\mathrm{m}^{3} / \mathrm{kg}\right)$ & 0 & BSC (2004 [DIRS 170004\}, Section 6.13.1.8) \\
\hline$K_{d}$ of $2,6-$ DFBA $\left(\mathrm{m}^{3} / \mathrm{kg}\right)$ & 0 & BSC (2004 [DIRS 170004\}, Section 6.13.1.8) \\
\hline $\begin{array}{l}\text { Phase 1A: } \\
\text { Br concentration measurements }\end{array}$ & & LA9910WS831372.008 [DIRS 147156] \\
\hline $\begin{array}{l}\text { Phase 1A: } \\
\text { Fluorescein distribution (plume images) in the rock }\end{array}$ & & LA0302WS831372.001 [DIRS 162765] \\
\hline $\begin{array}{l}\text { Phase 1B: } \\
\text { Br concentration measurements }\end{array}$ & & LA0201WS831372.008 [DIRS 162766] \\
\hline $\begin{array}{l}\text { Phase 1A: } \\
\text { 2,6-DFBA concentration measurements }\end{array}$ & & LA0201WS831372.008 [DIRS 162766] \\
\hline
\end{tabular}


Table 7-8. Hydrological Parameters in the Analysis of the Busted Butte Phase 1A Field Test

\begin{tabular}{|l|l|l|l|l|}
\hline \multicolumn{1}{|c|}{ Parameter $^{\mathbf{a}}$} & \multicolumn{1}{c|}{ Ch1v $^{\mathbf{a}}$} & \multicolumn{1}{c|}{ Ch2v $^{\mathbf{a}}$} & \multicolumn{1}{c|}{ Ch1v $^{\mathbf{b}}$} & \multicolumn{1}{c|}{ Ch2v $^{\mathbf{b}}$} \\
\hline$\phi$ & 0.273 & 0.345 & 0.320 & 0.360 \\
\hline$k_{x}=k_{y}=k_{z}\left(\mathrm{~m}^{2}\right)$ & $9.9 \times 10^{-13}$ & $9.27 \times 10^{-14}$ & $3.14 \times 10^{-13}$ & $1.82 \times 10^{-14}$ \\
\hline$\tau$ & 0.7 & 0.7 & 0.7 & 0.7 \\
\hline$\alpha(1 / \mathrm{m})^{c}$ & 0.140 & 0.503 & 0.471 & 0.741 \\
\hline$n^{c}$ & 1.538 & 1.427 & 1.332 & 1.200 \\
\hline$S_{r}$ & 0.03 & 0.07 & 0.07 & 0.07 \\
\hline Initial $S_{w}$ & 0.3 & 0.3 & 0.3 & 0.3 \\
\hline
\end{tabular}

a UZ99 calibrated properties, DTN: LB997141233129.001 [DIRS 104055].

${ }^{\mathrm{b}}$ Based on USGS measured properties, DTNs: GS990308312242.007 [DIRS 107185], GS990708312242.008 [DIRS 109822], as reported in DTN: LB991220140160.010 [DIRS 164858].

${ }^{c}$ van Genuchten (1980 [DIRS 100610]) model parameter.

\subsection{Validation of the Bromide Transport Model}

Figure 7-9 shows the numerically predicted distribution at the $\mathrm{y}=0.90 \mathrm{~m}$ mineback face at validation, as well as the field measurements of the $\mathrm{Br}$ relative concentration obtained from samples acquired during the mineback operations. The properties that resulted in the distribution of Figure 7-9 are those listed in Table 7-9, and were provided by the calibration of the fluorescein data (Section 7.2.4.1.2.3). For the prediction of $\mathrm{Br}$ transport, the simulations were conducted using the fluorescein-derived calibrated parameters, without any adjustment, and the known $D_{0}$ of $\mathrm{Br}$.

A comparison of the two concentration curves in Figure 7-9 indicates good agreement between predictions and observations. The model accurately reproduces the rather uniform concentrations along the $\mathrm{x}$-axis that pass by the injection point, as well as the magnitude of the observed concentrations.

Toward the outer extents of the horizontal axis of the plume, there appear to exist discrepancies between observations and predictions. Some of the discrepancies are consistent with the rather elongated shape of the predicted tracer plume. Part of this may be attributed to the fact that these occur in areas of steep gradients (i.e., in areas of condensation of the contour lines, when large differences occur within a short distance). An additional issue is uncertainty in the measurements, as reflected by a measured relative concentration of 2.77 (with respect to the injection concentration) in the center of the plume. While this high concentration is possible, if, for example, the sample was inadvertently allowed to dry, it may be indicative of some error in the measurements.

Based on these results, the Br transport model is validated because it was based on calibrated parameters from a different data set, that of fluorescein.

\subsection{Remarks and Observations}

A review of the parameters obtained in the process of calibration in Table 7-9 indicates that they are within a reasonable range and they are not in obvious conflict with the initial data listed in 
Table 7-8. While the possibility of a nonunique solution cannot be summarily dismissed, the calibrated solution (fluorescein) and the corresponding validated solution (of Br transport) are both mathematically correct and physically meaningful. A quantitative measure of the model validation, stemming from the comparison of field observations to model predictions, is provided in Table 7-15. Although there are some discrepancies, the agreement between observations and numerical predictions is sufficiently good to enhance confidence in the radionuclide transport model.

\section{$90 \mathrm{~cm}$}

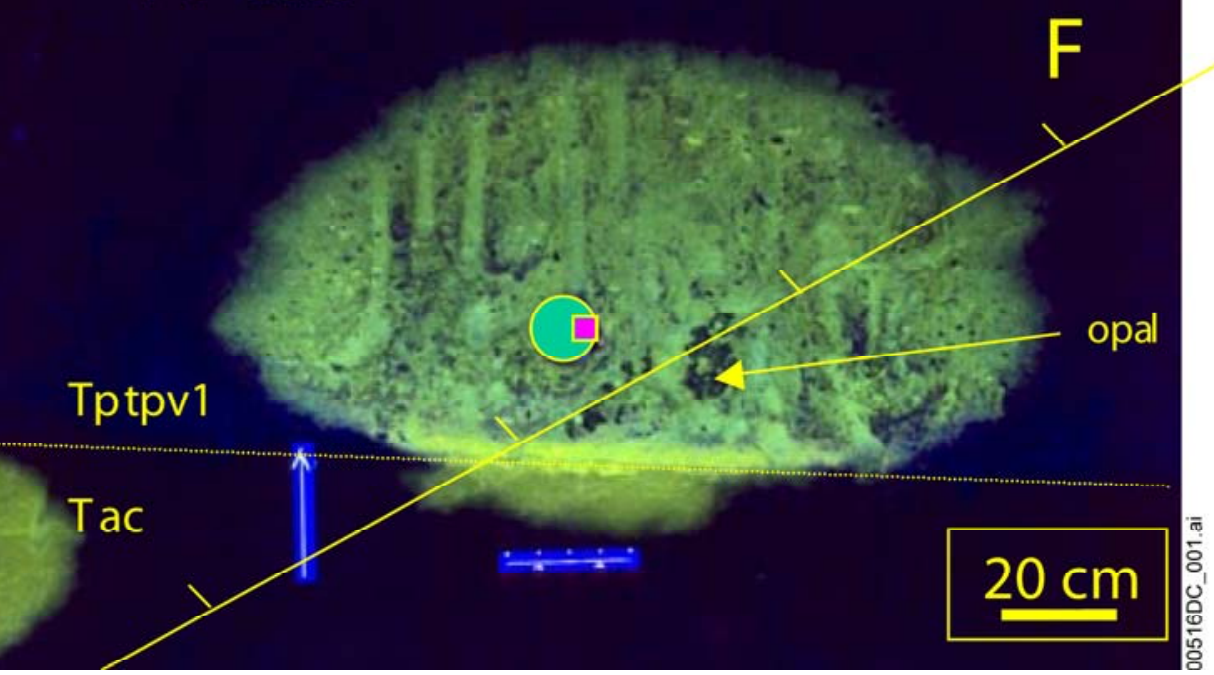

Source DTN: LA0302WS831372.001 [DIRS 162765] (scale corrected).

Figure 7-7. Fluorescein Plume at the $y=0.9 \mathrm{~m}$ Mineback Face at Borehole 3.

Table 7-9. Calibrated Parameters of Flow and Transport from the Analysis of the Busted Butte Phase 1A Field Test

\begin{tabular}{|l|l|l|}
\hline \multicolumn{1}{|c|}{ Parameter $^{\mathbf{a}}$} & \multicolumn{1}{c|}{ Ch1v } & \multicolumn{1}{c|}{ Ch2v } \\
\hline$\phi$ & 0.320 & 0.360 \\
\hline$k_{x}\left(\mathrm{~m}^{2}\right)$ & $2.14 \times 10^{-13}$ & $8.20 \times 10^{-13}$ \\
\hline$k_{y}\left(\mathrm{~m}^{2}\right)$ & $4.14 \times 10^{-13}$ & $2.82 \times 10^{-13}$ \\
\hline$k_{z}\left(\mathrm{~m}^{2}\right)$ & $6.28 \times 10^{-14}$ & $3.64 \times 10^{-14}$ \\
\hline$\tau$ & 0.22 & 0.12 \\
\hline$\alpha(1 / \mathrm{m})^{c}$ & 0.471 & 0.741 \\
\hline$n^{c}$ & 1.332 & 1.200 \\
\hline$S_{r}$ & 0.07 & 0.07 \\
\hline $\begin{array}{l}D_{o} \text { of } \\
\text { Fluorescein } \\
\left(\mathrm{m}^{2} / \mathrm{s}\right)\end{array}$ & $4 \times 10^{-10}$ & \\
\hline
\end{tabular}

Output DTN: LB0308MR0060R1.008.

a All other parameters remaining as in Tables 7-7 and $7-8$. 


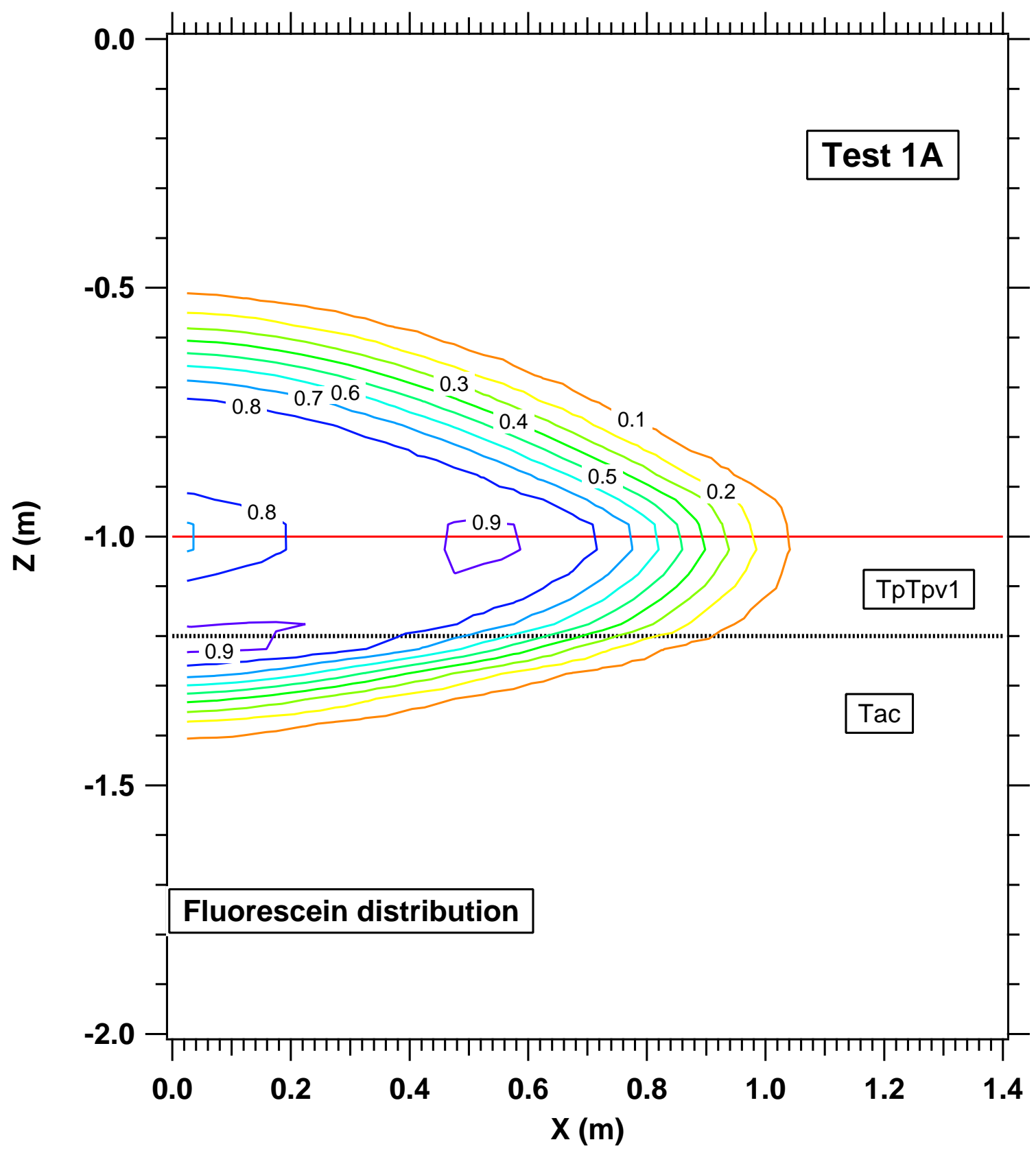

Output DTN: LB0308MR0060R1.009.

Figure 7-8. Numerical Prediction of the Fluorescein Plume Using Calibrated Parameters (Busted Butte Test Phase 1A) 


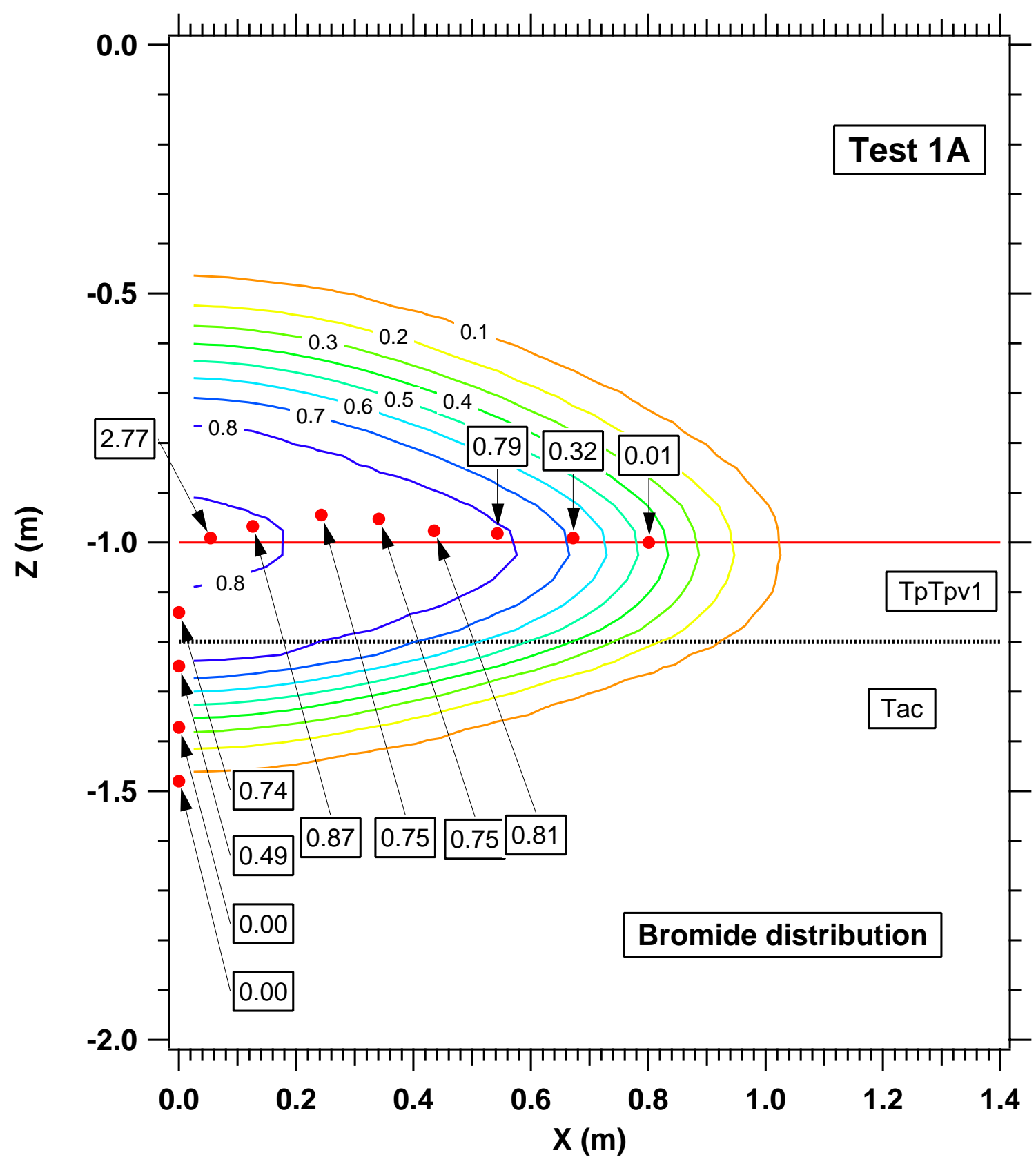

Output DTN: LB0308MR0060R1.009.

Figure 7-9. Field Measurements and Numerical Prediction of the Bromide Distribution in Busted Butte Test Phase 1A. The Solid Circles Indicate the Location of Measurements (DTN: LA9910WS831372.008 [DIRS 147156]), which Appear in the Corresponding Boxes 


\subsection{Phase 1B Test}

The purpose of this modeling study was to calibrate and validate the model through the reconciliation of field measurements and predictions for breakthrough curves of tracers injected into Borehole 5 (located in the tsw39 layer, see BSC 2004 [DIRS 170004], Section 6.13). Of the five tracers injected during Phase $1 \mathrm{~B}$ of the Busted Butte test, only $\mathrm{Br}$ (nonsorbing) and 2,6 -difluorobenzoic acid (2,6-DFBA, assumed to be nonsorbing) were used due to the poor quality of other tracer data. Of these, the 2,6-DFBA data were used for calibration, and the $\mathrm{Br}$ data set was used for validation. Additional discussion on the calibration and validation approach can be found in Section 7.2.4.1.1 of this model report.

\subsection{1 $\quad$ Procedure and Layout}

An extensive discussion of the Phase 1B test can be found in In Situ Field Testing of Processes (BSC 2004 [DIRS 170004], Section 6.13). Only a short description is presented here.

In the Phase 1B field test, the tracers were injected into the lower portion of the Topopah Spring basal vitrophyre (Tptpv2 in the lithostratigraphic units; tsw39 in the UZ99 layers of the hydrogeologic units), which is a relatively low-permeability fractured rock. The design, configuration, and important dimensions of the Phase 1B test are shown in Radionuclide Transport Models Under Ambient Conditions (BSC 2001 [DIRS 161340], Attachment VI), while more detailed information is provided in In Situ Field Testing of Processes) (BSC 2004 [DIRS 170004], Section 6.13), which depicts the injection/collection pair of Boreholes 5 and 6 . The tracer solution was injected at $\mathrm{y}=1.30 \mathrm{~m}$ from the rock face into Boreholes 5 . Water samples from boreholes were collected and analyzed regularly during the injection period.

The parameters describing the site conditions and test operation, and the corresponding sources, are listed in Table 7-7, which also includes the DTN of the data used for this validation effort. Although the Phase 1B test involved injection and collection into another set of boreholes (Boreholes 7 and 8, respectively), that portion of the test is not needed for this discussion.

The determination of the hydraulic parameters was a significant component of the calibration effort. Initial estimates for these parameters (listed in Table 7-9) were provided by two different sources: the UZ99 calibrated flow parameter set (DTN: LB997141233129.001 [DIRS 104055]) and a data set based on hydraulic property measurements from field-collected core samples (DTNs: GS990308312242.007 [DIRS 107185], GS990708312242.008 [DIRS 109822]).

\subsection{Conceptual and Numerical Model}

The initial uncalibrated input parameters of flow and transport for the simulation of the Phase 1B test are listed in Tables 7-7 and 7-10. For this three-dimensional numerical study, the geologic model treated the domain as a homogeneous and anisotropic unfractured rock matrix. Although this geologic layer is known to be fractured, the assumption of an unfractured rock matrix as the domain model in the simulation appears to be a valid one because the system behavior during the 
injections did not exhibit evidence of fracture flow. The same grid used in the simulation of Phase 1A was used here, but with the appropriate domain properties, that is, those of the Tptpv2 unit. The DTN of the input and output files of this simulation is output DTN: LB0308MR0060R1.008. The TOUGH2 V1.11MEOS9nTV1.0 (LBNL 1999 [DIRS 113943]) was used for the simulation.

For this calibration and validation process, only the data from the first 100 days of the Phase 1B test were used. The reason for this selective truncation of the data set was the existence of obvious inconsistencies and errors in the data. More specifically, measured concentrations of both $\mathrm{Br}$ and 2,6-DFBA exhibited pronounced fluctuations and appeared to drop to zero (undetectable) levels after 100 days. This occurred at a time when injection not only proceeded uninterrupted, but also continued to do so at the same injection levels for another 90 days. If sampling and measurement errors are not considered a possibility, then the only reasonable explanation for such a response would be a sudden and practically infinite dilution (e.g., if very large amounts of pure water reached the sample collection point), a scenario not supported by the facts. This being the case, the data beyond 100 days were deemed unreliable, and thus they were not included in the validation.

\subsection{Calibration Using the 2,6-DFBA Data}

The calibrated parameters obtained from the 2,6-DFBA transport model in Phase 1B of the Busted Butte test are listed in Table 7-11. A comparison of the measured and the numerically predicted breakthrough curves of 2,6-DFBA (based on the calibrated parameters) in Figure 7-10 shows a very good agreement. Note that the pattern and response of the breakthrough curves confirm the visual observation that the system did not exhibit fracture flow behavior during the Phase $1 \mathrm{~B}$ test, thus supporting the validity of the unfractured medium approach made in the simulations.

\subsection{Validation of the Br transport model}

The calibrated parameters, based on the 2,6-DFBA transport analysis, Table 7-11, were used for the prediction of the $\mathrm{Br}$ transport in Phase $1 \mathrm{~B}$ of the Busted Butte test. The known $D_{0}$ of $\mathrm{Br}$ and its $K_{d}=0$ value were used in the validation process. A comparison of the measured and the numerically predicted breakthrough curves of $\mathrm{Br}$ in Figure 7-11 shows a very good agreement.

A quantitative measure of the model validation stemming from the comparison of field observations to model predictions is provided in Table 7-15. The level of agreement between observations and numerical predictions enhances confidence in the radionuclide transport model. 


\subsection{Remarks and Observations}

A review of the calibrated parameters in Table 7-11 indicates that with the possible exception of permeability, they are within a reasonable range, and that they are not in obvious conflict with the initial data listed in Tables 7-7 and 7-8. The only possible deviation between initial estimates and the final parameters is the need for a nonzero (although small) $K_{d}$ for 2,6-DFBA. This appears to be in conflict with the conventional, though not fully supported, assumption that 2,6-DFBA is nonsorbing.

Careful consideration of the chemistry of the system indicates that it is entirely possible for an organic substance to sorb onto a rock. In this case, the very low permeability of the rock allows a long contact time, making such a sorption scenario plausible, especially given the fact that organic substances are generally considered to be slow sorbers (Cameron and Klute 1977 [DIRS 117172]). Additionally, the long residence time in the rock may allow a slow reaction of 2,6-DFBA (a weak acid) with minerals in the rock, a process that would result in a response consistent with an apparent sorption.

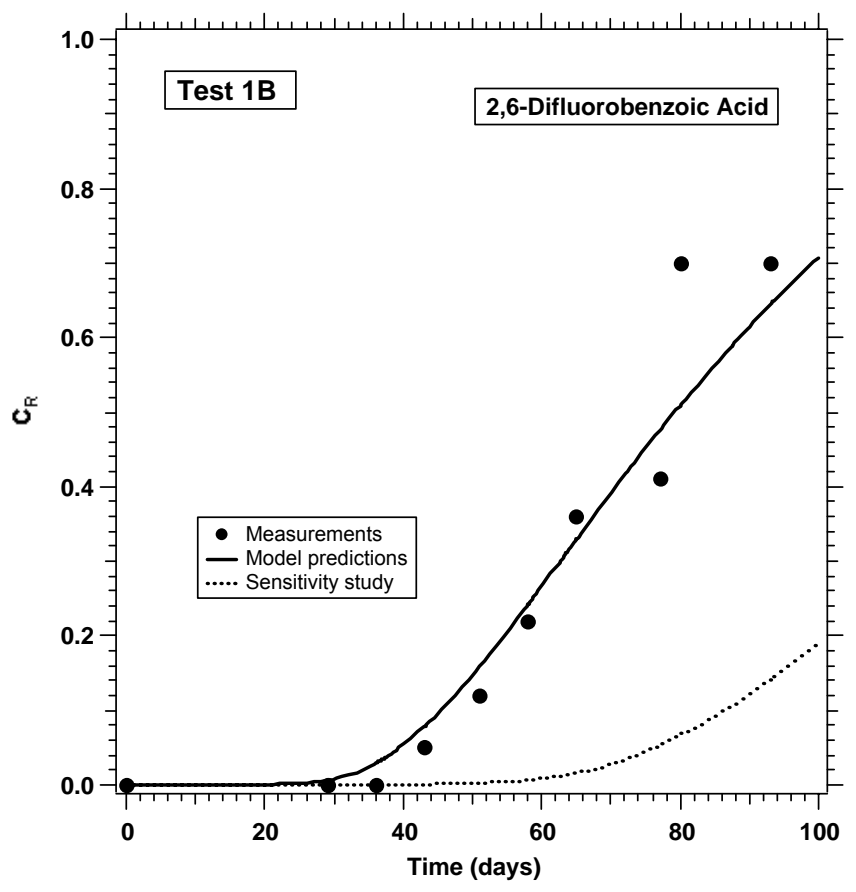

Figure 7-10. Observed and Numerically Predicted (Calibrated) Breakthrough Curves of 2,6-DFBA in the Busted Butte Phase 1B Test. Measurements from DTN: LA0201WS831372.008 [DIRS 162766]). Prediction from output DTN: LB0308MR0060R1.009. 


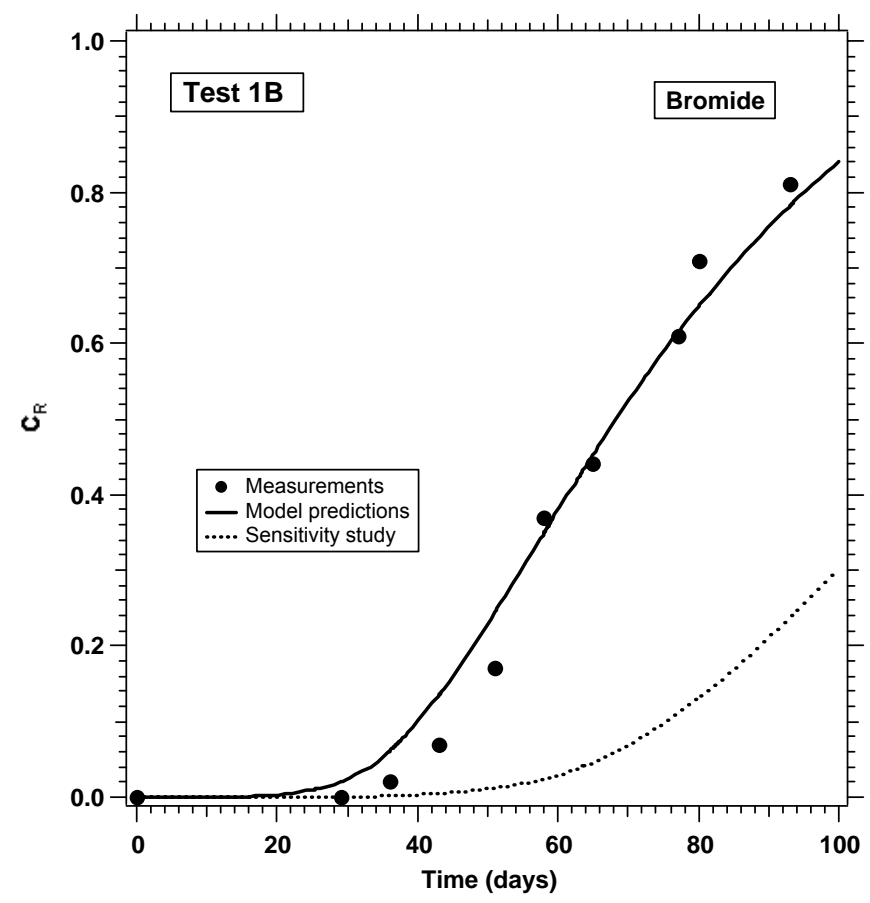

Figure 7-11. Observed and Numerically Predicted (at Validation) Breakthrough Curves of $\mathrm{Br}$ in the Busted Butte Phase 1B Test. Measurements from DTN: LA0201WS831372.008 [DIRS 162766]), Prediction from output DTN: LB0308MR0060R1.009

To investigate the system sensitivity to spatial resolution, Figures 7-10 and 7-11 include breakthrough curves at points $0.05 \mathrm{~m}$ below the collection point. It is obvious that concentrations in the vicinity of the collection points exhibit very steep gradients. This indicates that minor inaccuracies in the recording of the location of the collection point can register as a strong deviation. In light of this spatial sensitivity, the agreement between predictions and observations is deemed very good, and the observed small deviations can be easily explained.

Table 7-10. Initial Parameters in the Analysis of the Busted Butte Phase 1B Field Test

\begin{tabular}{|c|c|c|}
\hline Parameter $^{a}$ & $\mathrm{UZ99}^{\mathrm{a}}$ & USGS $^{b}$ \\
\hline$\phi$ & 0.173 & 0.370 \\
\hline$k_{x}=k_{y}=k_{z}\left(\mathrm{~m}^{2}\right)$ & $5.46 \times 10^{-17}$ & $3.06 \times 10^{-14}$ \\
\hline$\tau$ & 0.7 & 0.7 \\
\hline$\alpha(1 / m)^{c}$ & 0.225 & 0.225 \\
\hline$n^{c}$ & 1.612 & 1.612 \\
\hline $\mathrm{Sr}$ & 0.29 & 0.29 \\
\hline Initial $S_{w}$ & 0.3 & 0.3 \\
\hline$D_{0}$ of $2,6-$ DFBA $\left(\mathrm{m}^{2} / \mathrm{s}\right)$ & $7.6 \times 10^{-10}$ & \\
\hline \multicolumn{3}{|c|}{$\begin{array}{l}\text { a } \text { Based on UZ99 calibrated properties, DTN: LB997141233129.001 [DIRS 104055], as reported in DTN } \\
\text { LB991220140160.010 [DIRS 164858]. } \\
\text { b } \text { Based on USGS measured properties, DTNs: GS990308312242.007 [DIRS 107185], GS990708312242.008 } \\
\text { [DIRS 109822], as reported in DTN LB991220140160.010 [DIRS 164858]. } \\
\text { van Genuchten (1980 [DIRS 100610]) model parameter. }\end{array}$} \\
\hline \multicolumn{3}{|c|}{ USGS=U.S. Geological Survey } \\
\hline
\end{tabular}


Table 7-11. Calibrated Flow and Transport Parameters from the Analysis of the Busted Butte Phase 1B Field Test

\begin{tabular}{|l|l|}
\hline \multicolumn{1}{|c|}{ Parameter $^{\mathrm{a}}$} & \multicolumn{1}{c|}{ Value } \\
\hline$\phi$ & 0.270 \\
\hline$k_{x}\left(\mathrm{~m}^{2}\right)$ & $3.06 \times 10^{-17}$ \\
\hline$k_{y}\left(\mathrm{~m}^{2}\right)$ & $4.06 \times 10^{-17}$ \\
\hline$k_{z}\left(\mathrm{~m}^{2}\right)$ & $1.53 \times 10^{-17}$ \\
\hline$\tau$ & 0.07 \\
\hline$K_{d}$ of $2,6-$ DFBA $\left(\mathrm{m}^{3} / \mathrm{kg}\right)$ & $1.47 \times 10^{-5}$ \\
\hline
\end{tabular}

Output DTN: LB0308MR0060R1.008.

${ }^{\text {a }}$ All other parameters remaining as in Table 7-7 and 7-9.

\subsection{Phase 2C Test}

Phase 2 differs from Phase 1 in that the injection systems were designed to activate large volumes of the rock. A detailed description of this test phase can be found in In Situ Field Testing of Processes (BSC 2004 [DIRS 170004], Section 6.13.3). The injection points in this phase were distributed in horizontal parallel planes at locations designed to test the properties of the lower Topopah Spring Tuff (Tptpv2) and the underlying Calico Hills (Tptpv1) unit.

\subsection{Procedure and Layout}

Analysis is limited to Phase $2 \mathrm{C}$, which appears to have yielded the best quality data. In Phase $2 \mathrm{C}$, injection occurred in three horizontal boreholes (\#18, \#20, and \#21), each instrumented with nine injectors located at $0.61-\mathrm{m}$ intervals along the borehole. All three boreholes were located in the Tptpv2 unit. Because of the relatively short injection spacing and the long injection period of 695 days, the injection can be considered uniform along the three boreholes. While this assumption introduces an inaccuracy, it is rather small, is limited to the very early stages of injection when flow is spherical rather than the quasi-cylindrical at the later stages, and keeps decreasing over time. The implication of this uniformity is that it is possible to model the system using a two-dimensional grid, thus allowing higher spatial resolution.

After reviewing the tracer concentrations recorded at the various collection boreholes, this study concentrated in Borehole 16 because it is the closest to the horizontal plane of the three injection wells and, thus, has registered the strongest signals (in terms of tracer concentrations). Borehole 16 run was located on a horizontal plane (roughly coinciding with the Tptpv2- Tptpv1 interface) about $0.6 \mathrm{~m}$ below that of the injection boreholes and perpendicular to their main axis.

A rather large number of tracers were injected in the injection boreholes. After reviewing the concentration data, the analysis was limited to the transport of two tracers: $\mathrm{Br}$ and Li. This decision was reached because the data quality of the other tracers was rather poor and often devoid of any logical pattern, possibly attributed to the sample collection method that entailed removal and reintroduction of the same collection pads in the collection boreholes, replacement of pads with new ones, and difficulty in consistently placing the pads at their exact previous location following removal for sampling and testing. The nonsorbing $\mathrm{Br}$ and the sorbing $\mathrm{Li}$ had registered rather strong and generally, though not always, consistent signals in Borehole 16. In 
addition, having being injected as a solution of $\mathrm{LiBr}, \mathrm{Li}$ and $\mathrm{Br}$ were intertwined and offered an additional level of checking through their very different transport properties and the need to maintain mass balance.

The parameters describing the site conditions and test operation of Phase $2 \mathrm{C}$, and the corresponding sources, are listed in Table 7-12, which also includes the DTN of the data used for the exercise. In this analysis, the $\mathrm{Li}$ data were used for calibration, and the $\mathrm{Br}$ data for validation. Additional discussion on the calibration and validation approach can be found in Section 7.2.4.1.1 of this model report.

\subsection{Conceptual and Numerical Model}

Early exploratory studies indicated that, if the porous medium (unfractured rock) assumption were made, it was very difficult to reproduce the individual responses of $\mathrm{Br}$ or $\mathrm{Li}$, and impossible to obtain a consistent joint $\mathrm{Br}$ and $\mathrm{Li}$ behavior. Using just a consistent fracture domain (i.e., limiting the study to fracture flow and transport) produced a very fast breakthrough for $\mathrm{Br}$ and an impossibly fast one for the sorbing $\mathrm{Li}$, because of the inability to account for matrix diffusion and the lack of Li sorption. Thus, modeling the domain as a fracture-matrix system was the only alternative. A dual-permeability model was employed. The two-dimensional grid had a rather fine resolution $(\Delta x=\Delta y=0.05 \mathrm{~m})$. The initial (uncalibrated) input parameters of flow and transport for the simulation of the Phase $2 \mathrm{C}$ test are listed in Table 7-13. The DTN of the input and output files of this simulation is output DTN: LB0308MR0060R1.008. The TOUGH2 V1.11MEOS9nTV1.0 (LBNL 1999 [DIRS 113943]) was used for the simulation.

The initial steady-state flow field was obtained by setting an infiltration rate corresponding to $5 \mathrm{~mm} / \mathrm{year}$ at the top of the domain and running the flow simulation to steady state. Note that these runs were needed at every phase of the validation because the changes in the hydraulic parameters in the process of validation affected the flow field.

For the Li calibration process, only the data for $t=337$ days and $t=440$ days were used. Because of the sorbing behavior of $\mathrm{Li}$, the data for $\mathrm{t}<337$ days were marked by very low concentrations, significant variability, and the corresponding uncertainty. Concentration data for $t>440$ days showed rather inconsistent behavior attributed to issues discussed in Section 7.2.4.1.4.3.

For the $\mathrm{Br}$ validation process, only the data for $\mathrm{t}=125$ days and $\mathrm{t}=183$ days were used. The data for $t<125$ days were marked by very low concentrations and significant variability; inevitable because of the relatively large component of error, inaccuracy, and heterogeneity in the early data. Concentration data for $t>183$ days indicated a rather uniform distribution along the well bore (as the concentration peak had already been reached), differed only in the extent of the zone that had reached the maximum concentration (not a very reliable source of information for reasons explained later), and were not very useful. 
A quantitative measure of the model calibration and validation stemming from the comparison of field observations to model predictions is provided in Table 7-15.

Table 7-12. Operational, Transport, and Field Parameters in the Analysis of the Busted Butte Phase 2 Field Test

\begin{tabular}{|l|l|l|}
\hline \multicolumn{1}{|c|}{ Parameter } & \multicolumn{1}{|c|}{ Value } & \multicolumn{1}{c|}{ Source } \\
\hline Injection rate $(\mathrm{mL} / \mathrm{hr})$ & 120 & LA0008WS831372.001 [DIRS 156582] \\
\hline Injection duration (days) & 695 & LA0008WS831372.001 [DIRS 156582] \\
\hline$\alpha_{L}(\mathrm{~m})-$ Fractures & 0.1 & Reasonable estimate \\
\hline$\alpha_{L}(\mathrm{~m})-$ Matrix & 0.0 & Reasonable estimate \\
\hline$\alpha_{T}(\mathrm{~m})-$ Everywhere & 0 & Reasonable estimate \\
\hline$D_{0}$ of $\mathrm{Br}\left(\mathrm{m}^{2} / \mathrm{s}\right)$ & $2.08 \times 10^{-9}$ & Lide (1992 [DIRS 166224], p. 5-111) \\
\hline$D_{0}$ of $\mathrm{Li}\left(\mathrm{m}^{2} / \mathrm{s}\right)$ & $1.03 \times 10^{-9}$ & Lide (1992 [DIRS 166224], p. 5-111) \\
\hline Concentration measurements & & $\begin{array}{l}\text { LA0112WS831372.001 [DIRS 157100] LA0112WS831372.002 } \\
\text { [DIRS 157115] LA0112WS831372.003 [DIRS 157106] } \\
\text { LA0211WS831372.001 [DIRS 162763] }\end{array}$ \\
\hline
\end{tabular}

Table 7-13. Hydrological and Transport Parameters in the Analysis of the Busted Butte Phase 2 Field Test

\begin{tabular}{|l|l|l|l|l|}
\hline \multicolumn{1}{|c|}{ Parameter } & \multicolumn{1}{c|}{ F-TpTpv1 $^{\text {b }}$} & \multicolumn{1}{c|}{ M-TpTpv1 $^{\text {b }}$} & \multicolumn{1}{c|}{ F-TpTpv2 } & \multicolumn{1}{c|}{ M-TpTpv2 } \\
\hline$\phi^{\text {a }}$ & 1 & 0.354 & 1 & 0.0457 \\
\hline$K_{x}=K_{y}=K_{z}\left(m^{2}\right)^{a}$ & $2.2 \times 10^{-13}$ & $6.65 \times 10^{-13}$ & $8.1 \times 10^{-13}$ & $1.49 \times 10^{-13}$ \\
\hline$\tau^{\prime}$ & 1 & 0.7 & 1 & 0.7 \\
\hline$\alpha(1 / \mathrm{m})^{\mathrm{a}, \mathrm{c}}$ & 20.6 & 0.856 & 14.7 & 0.477 \\
\hline$N^{\mathrm{a}, \mathrm{c}}$ & 2.72 & 1.32 & 2.72 & 1.41 \\
\hline$S_{r}^{h}$ & 0.01 & 0.06 & 0.01 & 0.13 \\
\hline$K_{d}$ of $\mathrm{Br}(\mathrm{m} 3 / \mathrm{kg})^{\mathrm{d}}$ & - & 0 & - & 0 \\
\hline$K_{d}$ of $\mathrm{Br}(\mathrm{m})^{\mathrm{e}}$ & 0 & - & 0 & - \\
\hline$K_{d}$ of $\mathrm{Li}\left(\mathrm{m}^{3} / \mathrm{kg}\right)^{f}$ & - & $3.5 \times 10^{-4}$ & - & $8.8 \times 10^{-4}$ \\
\hline$K_{d}$ of $\mathrm{Li}(\mathrm{m})^{g}$ & 0 & - & 0 & - \\
\hline
\end{tabular}

${ }^{a}$ DTNs: LB03013DSSCP3I.001 [DIRS 162379], DTN: LB0205REVUZPRP.001 [DIRS 159525], LB0210THRMLPRP.001 [DIRS 160799].

${ }^{b} \mathrm{~F}$ : fracture properties, $\mathrm{M}$ : matrix properties.

${ }^{c}$ van Genuchten (1980 [DIRS 100610]) model parameter.

d DTN: LA0203WS831372.002 [DIRS 161526].

${ }^{\mathrm{e}} \mathrm{K}_{\mathrm{d}}$ denotes surface distribution coefficient - reasonable estimate.

f DTN: LA9912WS831372.001 [DIRS 156586], LA0203WS831372.002 [DIRS 161526].

g Initial estimate.

h DTN: LB0207REVUZPRP.002 [DIRS 159672].

I DTN: LB997141233129.001 [DIRS 104055] and Table 5-1 of this report. 


\subsection{Calibration Using the Li Data}

The parameters from the calibration of the Li transport model in Phase $2 \mathrm{C}$ of the Busted Butte test are listed in Table 7-14. Figure 7-12 shows the measured and the numerically predicted Li distributions along the collection borehole. The $K_{d}$ values in Table 7-14 are consistent with previously reported data (DTNs: LA9912WS831372.001 [DIRS 156586], LA0203WS831372.002 [DIRS 161526]). The data set used for the Li-based calibration corresponds to the later part of the field test because of the sorption-induced retardation (see discussion in Section 7.2.4.1.1 of this model report).

The $\mathrm{Li}$ distribution in Figure 7-12 shows a rather good match between predictions and measurements. The peaks, their rough locations, and the distributions are matched. For $t>440$ days, Li concentrations start decreasing, and then they resume increasing. This is consistent with concentrations in the pad being diluted by $\mathrm{Li}$-pore water until the main $\mathrm{Li}$ front arrives at the collection pad, at which time concentrations begin increasing again. Note that the analysis is complicated by lack of knowledge about the sorption behavior of Li in the pad (see discussion in Section 7.2.4.1.4.4).

\subsection{Validation of the Br Transport Model}

Figure 7-13 shows the measured and the numerically predicted $\mathrm{Br}$ distribution along the collection borehole. This distribution was obtained by using the calibrated properties (Table 7-14) from the analysis of the Li data, and the known $D_{0}$ and $K_{d}\left(=0 \mathrm{~m}^{3} / \mathrm{kg}\right)$ of Br. Compared to $\mathrm{Li}$, the $\mathrm{Br}$ distribution indicates a less diffusive behavior, attributed to the lack of sorption. Although different magnitudes are involved, the concentration distributions patterns (both observed and predicted) are consistent between the two tracers. Note that the data set used for the Br-based validation corresponds to the earlier part of the field test because of the reasons discussed in Section 7.2.4.1.1 of this model report.

A review of the $\mathrm{Br}$ predicted distribution reveals that the numerical solution accurately predicts the location and magnitude of the concentration peaks, but exhibits a narrower, more focused pattern and registers deviations away from these peaks. More specifically, the measured concentration of $\mathrm{Br}$ is more uniform along the collection borehole axis than what the numerical simulation predicts.

In other words, the measurements indicate a system that is more diffusive than the advective one indicated by the simulation. However, adjustment of permeabilities and diffusive fluxes through further calibration was not the correct approach, even disregarding the obvious danger that, to increase the diffusive component, one would have to reach rather unreasonable relations between the permeabilities of the fractures and the matrix. 


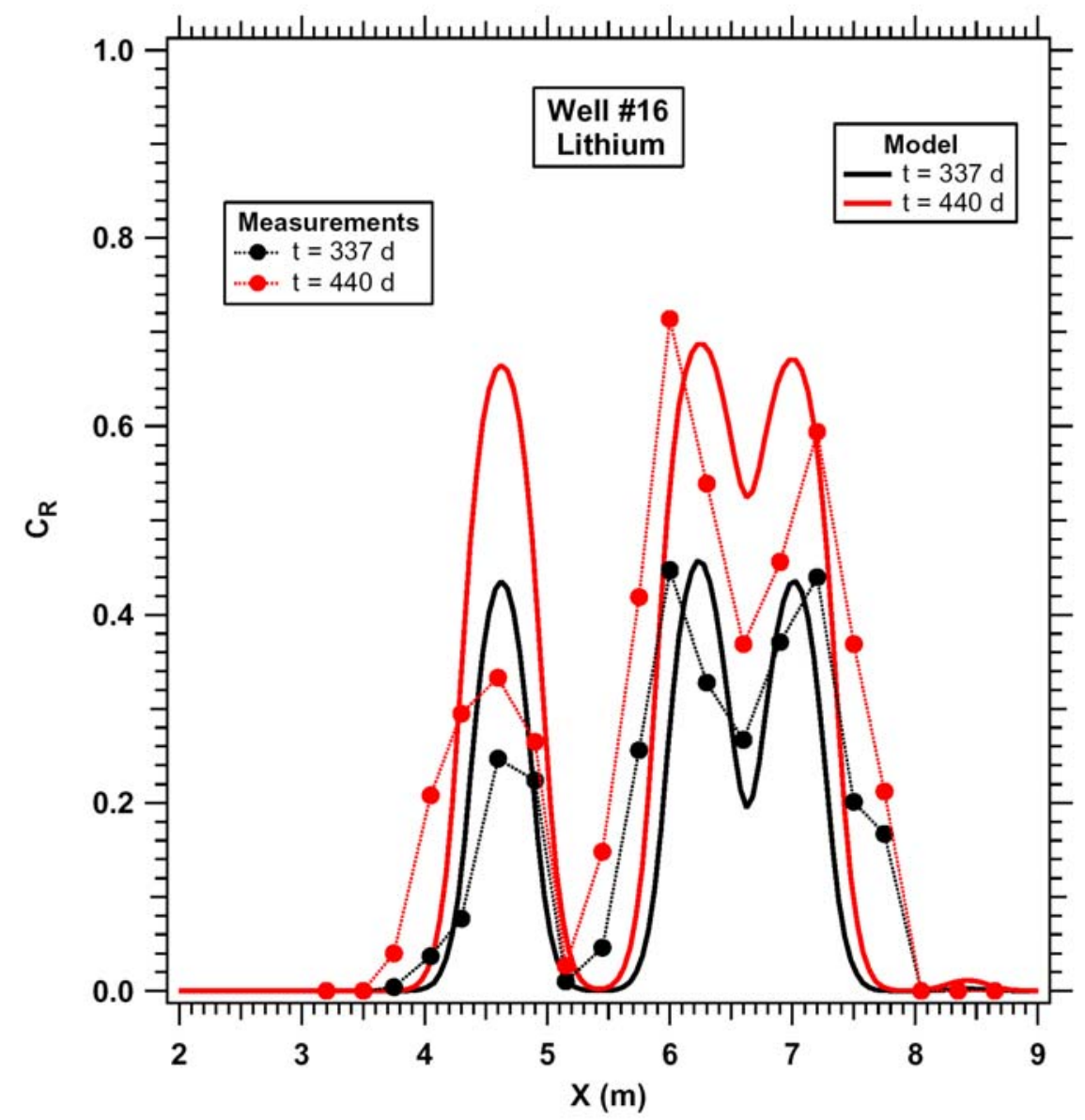

Observed Data from DTN: LA0201WS831372.007 [DIRS 164721], prediction from output DTN: LB0308MR0060R1.009.

Figure 7-12. Observed and Numerically Predicted (Calibrated) Breakthrough Curves of Li in the Busted Butte Phase 2C Test 


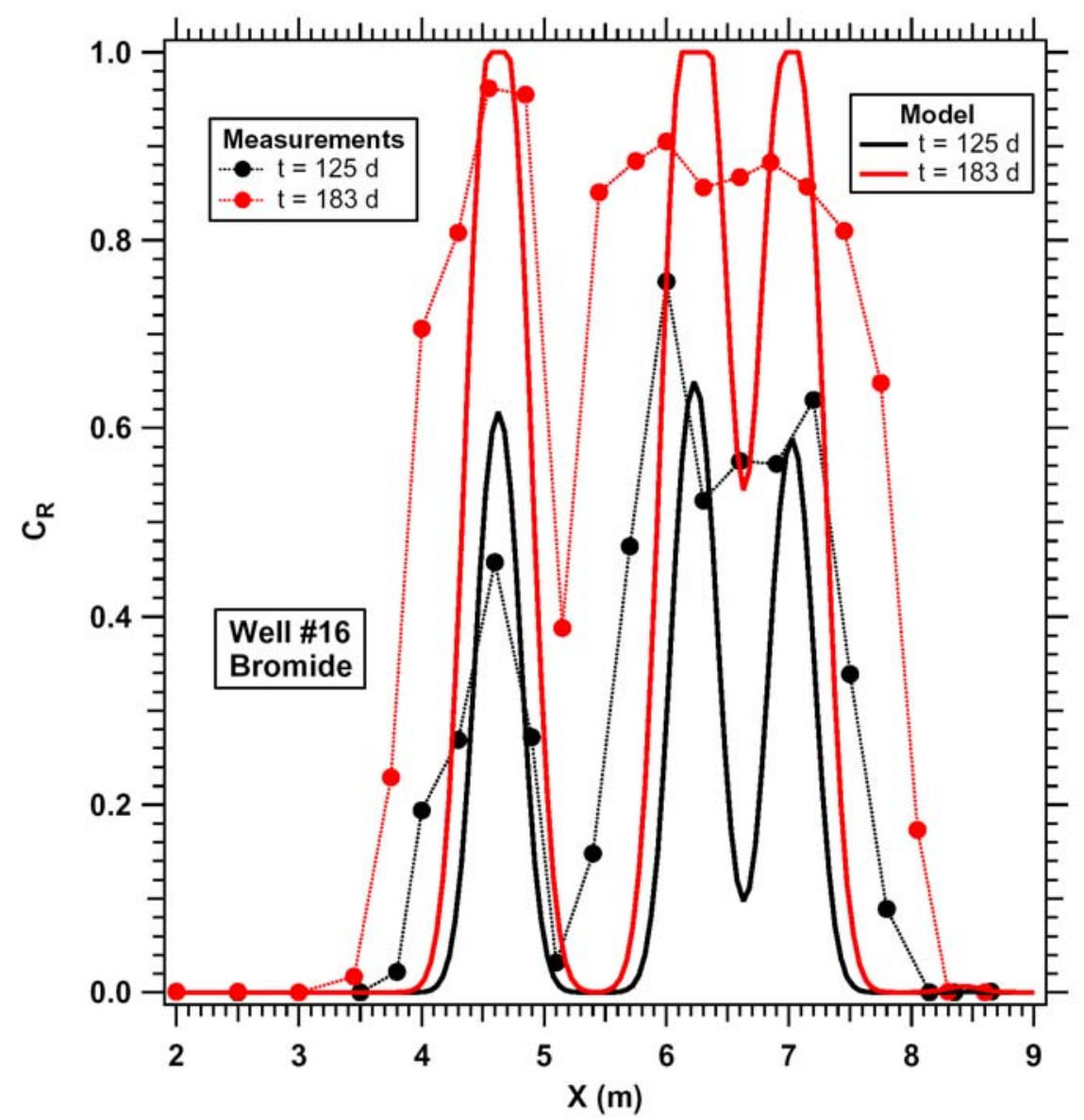

Observed Data from DTN: LA0112WS831372.003 [DIRS 157106], Prediction from output DTN: LB0308MR0060R1.009.

Figure 7-13. Observed and Numerically Predicted Breakthrough Curves of $\mathrm{Br}$ in the Busted Butte Phase 2C Test (at Validation)

The discrepancy problem had to be recast under the light of what the measurements meant. Use of the collection pad introduced a system with drastically different properties than the host rock, characterized by high permeability, porosity, irreducible water saturation, and capillary pressure. Tracer carrying fracture flow first reaching the collection pad arrives at distinct points (the points where the fractures intercept the borehole), and is quickly redistributed on the initially dry pad, thus leading to an apparently more uniform distribution than the one in the overlying rock. This is more evident on the left lobe of the distribution, corresponding to Borehole 18. Being relatively far from the other boreholes, it receives no cross contribution of fracture flow from injection into the other boreholes, and the distribution on the pad shows a more diffusive pattern than what the simulation predicts. In other words, the collection pads skew the data in a direction that indicates a more diffusive system. This being the case, it is expected that the most accurate data will be at early times and roughly under the injection boreholes, though not necessarily directly underneath, as the fracture density, distribution, and orientation are anything but known a priori. Thus, it is expected that matching the peaks is a sufficient indication of validation. Heterogeneity of fracture property and distribution is also a substantial issue at this 
small scale, and it is possible to create the appearance of enhanced diffusion if the fracture frequency is locally higher (e.g., on the left lobe of the curve).

If the effect of the pad is as described above, it is expected that it will lead to decreasing peak concentrations and more uniform spatial distribution as time advances. This is because after the arrival of tracers in the faster flowing fractures, the contributions of slower-flowing fractures, with more interactions with the matrix, and of the matrix itself release water of lower concentration mixed and redistributed on the pad, and leads to temporarily lower concentrations resulting from dilution. Of course, once the solute front crosses the matrix, concentrations are expected to rise again. A review of the $\mathrm{Br}$ measurement data indicates this exact pattern.

An attempt was made to simulate the effect of the pad. However, these simulations indicated that its behavior is sensitive to the initial conditions (whether it is an old one or a new replacement), its location (whether the old pad is placed at the exact same location from where it was removed), its wettability properties vis-à-vis the host rock, etc. This being the case, and given the uncertainties and the lack of an adequate description of the fracture-matrix system at the gridblock scale of this problem $(0.05 \mathrm{~m})$ (after all, the mathematical description of the matrix-fracture system is averaging even at this scale, and can be quite different from the actual system) no further attempt was made to refine the solution. The solution currently reflects concentration predictions in the overlying rock and not in the pad.

A quantitative measure of the model validation stemming from the comparison of field observations to model predictions is provided in Table 7-15. See also Section 7.2.2 concerning limitations of the comparisons for the edges of the plume. The level of agreement between observations and numerical predictions enhances confidence in the radionuclide transport model.

Table 7-14. Calibrated Flow and Transport Parameters from the Analysis of the Busted Butte Phase 2C Field Test

\begin{tabular}{|l|l|l|l|l|}
\hline \multicolumn{1}{|c|}{ Parameter $^{\text {a }}$} & F-TpTpv1 & M-TpTpv1 & F-TpTpv2 & M-TpTpv2 \\
\hline$\phi$ & 1 & 0.354 & 1 & 0.060 \\
\hline $\mathrm{k}_{\mathrm{x}}\left(\mathrm{m}^{2}\right)$ & $3 \times 10^{-13}$ & $1.3 \times 10^{-13}$ & $1.96 \times 10^{-12}$ & $1.2 \times 10^{-13}$ \\
\hline $\mathrm{k}_{\mathrm{z}}\left(\mathrm{m}^{2}\right)$ & $10^{-13}$ & $8 \times 10^{-14}$ & $7.1 \times 10^{-13}$ & $8 \times 10^{-14}$ \\
\hline$\tau$ & 2 & 0.9 & 2 & 0.654 \\
\hline$K_{d}$ of $\mathrm{Li}\left(\mathrm{m}^{3} / \mathrm{kg}\right)$ & - & $5.5 \times 10^{-4}$ & - & $9.3 \times 10^{-4}$ \\
\hline$K_{d}$ of $\mathrm{Li}\left(\mathrm{m}^{3} / \mathrm{kg}\right)^{\mathrm{b}}$ & $2.5 \times 10^{-6}$ & - & $4.3 \times 10^{-6}$ & - \\
\hline
\end{tabular}

Output DTN: LB0308MR0060R1.008.

${ }^{a}$ All other parameters remaining as in Tables 7-12 and 7-13.

${ }^{\mathrm{b}} \mathrm{K}_{\mathrm{d}}$ denotes surface distribution coefficient in fractures. 
Table 7-15. Comparison of Numerical Solutions to Field Tests

\begin{tabular}{l|l|}
\hline Field Test \# & \multicolumn{1}{c|}{ Difference* $^{*}$} \\
\hline 1A & Bromide $<50 \%$ (concentration, distribution) \\
\hline 1B & Bromide $<50 \%$ (breakthrough) \\
\hline 2C & Bromide $<50 \%$ (breakthrough) \\
\hline NOTE: *The 50 percent agreement refers to either concentration \\
comparison or arrival time comparison.
\end{tabular}

\subsection{Remarks and Observations}

A review of the calibration parameters in Table 7-14 indicates that they are within a reasonable range, and that they are not in obvious conflict with the initial data listed in Tables 7-12 and 7-13. Note that the distribution parameter $K_{d}$ of Li is within the experimentally determined range (DTN: LA9912WS831372.001 [DIRS 156586], LA0203WS831372.002 [DIRS 161526]). A surface sorption distribution coefficient had to be considered for the fractures-which is consistent with the rather strong sorbing behavior of Li. Given the degree of match between predictions and observations and the uncertainties involved in this study, the level of agreement between observations and numerical predictions is deemed acceptable and supports the claim of model validation.

Although it is true that the Phase $2 \mathrm{C}$ results could only be matched by a dual-permeability model and the TSPA-LA assumes that flow in the CHv is strictly in the matrix, it is important to note that the Phase $2 \mathrm{C}$ experiments were conducted primarily in the Tptpv1 lithostratigraphic subunit, with injection at the base of the Tptpv2 (basal vitrophyre of the TSw). The Tptpv1 part of the $\mathrm{CHv}$ at the local scale of the Phase $2 \mathrm{C}$ test is least likely to behave strictly as a single-continuum because it is immediately adjacent to the overlying fractured rock (Tptpv2) and the actual contact between the units may vary. However, in support of the TSPA-LA assumption of matrix flow in the $\mathrm{CHv}$ units, the $\mathrm{CHv}$ also includes a several hydrogeologic subunits that are part of the Tac lithostratigraphic subunit, which are much more likely to behave as a single-continuum due to the fact that matrix permeabilities in these units are about two orders of magnitude larger than in the Tptpv1.

\subsection{Summary of Postdevelopment Validation from Busted Butte Field Tests}

The simulations using the Busted Butte Field Tests demonstrated the dominance of matrix flow in the Calico Hills vitric formation. This validated a component of the UZ radionuclide transport model conceptual model (Section 6.6) that had matrix-dominated flow in the vitric portion of the Calico Hills formation beneath the repository. Although the Busted Butte Field Tests were conducted at a different site than immediately beneath the repository, at a scale smaller than that addressed by the UZ radionuclide transport model, and a dual-permeability model was necessary to match the Test $2 \mathrm{C}$ results, an explanation for the latter difference was plausible and offered in Section 7.2.4.1.4.5. As a result, confidence in the particular aspect of the UZ radionuclide transport model related to matrix-dominated flow in the Calico Hills vitric formation was gained through the simulation study using the Busted Butte Field Test results. 


\subsubsection{Postdevelopment Validation Using Chloride and ${ }^{14} \mathrm{C}$ Field Data}

Postdevelopment validation of the radionuclide transport model on a larger scale is provided by the analysis of field measurements of ${ }^{14} \mathrm{C}$ ages in the gas samples, and of pore water $\mathrm{Cl}^{-}$ concentrations in the ESF. The $14 \mathrm{C}$ validation primarily addresses the active fracture model and parameterization $(\gamma)$. The $\gamma$ parameter is considered part of the hydrologic properties. This parameter also affects UZ transport. BSC (2004 [DIRS 169861], Section 6.8.1) presents a solute transport sensitivity analysis which concludes that the $\gamma$ value used in TSPA conservatively underestimates radionulide transport times through the $\mathrm{UZ}$. The $\mathrm{Cl}^{-}$model primarily addresses issues concerning the spatial distribution of water flux and as such is more appropriately treated as a part of the flow model. These studies and the corresponding validation-supported analysis were conducted using the T2R3D numerical model, and are discussed in detail by BSC (2004 [DIRS 169861]), Sections 7.5 and 7.8, respectively.

\subsection{VALIDATION OF THE MATRIX DIFFUSION SUB-MODEL BY CORROBORATION WITH DATA FROM ALCOVE 8/NICHE 3 FAULT TEST}

Matrix diffusion can play an important role in radionuclide exchange between the fractures and the rock matrix (Moridis et al. 2003 [DIRS 161902], p. 255). This process transfers a radionuclide into the matrix (where water flow is slow), thus removing them from fast fracture flow. The diffusive flux is a function of the concentration gradient at the corresponding fracture-matrix interface, fracture interface area, and molecular diffusion coefficient (Section 6.2 of this report). The matrix diffusion model is an important component of the UZ transport model.

This section validates the matrix diffusion model by corroboration with data from the Alcove 8/Niche 3 fault test. The criteria for validation is that the predicted breakthrough curves collected at Niche 3 qualitatively agree with data, and predicted tracer transport time is not longer than observed (BSC 2004 [DIRS 167969], Section I-2-1-2, Attachment I, p. 26). As demonstrated in the subsections below discussing modeling results, the criteria are met. This modeling activity is also documented in a scientific notebook by Wang (2003 [DIRS 164021], SN-LBNL-SCI-215-V1, pp. 107 to 109).

The Alcove 8/Niche 3 fault test is described in Section 7.3.1. Data from this test include both hydrologic data and tracer data. The numerical model of the test is described in Section 7.3.2. Rock properties in the model were calibrated to the hydrologic data in two calibration runs, as described in Section 7.3.3.1. Tracer transport with matrix diffusion was then simulated, as described in Section 7.3.3.2, where it is demonstrated that the validation criteria are met.

\subsubsection{Field Observations}

This section highlights experimental observations used in this model analyses. The test was carried out in the upper lithophysal and middle nonlithophysal subunits in the Yucca Mountain UZ. These geological subunits correspond to model layers tsw33 and tsw34, respectively, in the site-scale model for the Yucca Mountain UZ. The tsw33 has some lithophysal cavities that may intersect fractures. Liquid water with and without tracers was released at the floor of an alcove along the fault (about $5 \mathrm{~m}$ long (DTN: GS020508312242.001 [DIRS 162129])) within tsw33. 
Seepage from the fault into a niche, and tracer concentrations of seeping liquid as a function of time were monitored. The niche is located within tsw34, about $20 \mathrm{~m}$ below the floor of the alcove; the interface between tsw33 and tsw34 is about $15 \mathrm{~m}$ below the floor of the alcove (DTN: LB0301N3SURDAT.001 [DIRS 162130]).

A water-pressure head of $2 \mathrm{~cm}$ was applied at an infiltration plot along the fault at the alcove. The plot consists of four trenches having different infiltration rates as a result of subsurface heterogeneity along the fault. Figure 7-14 shows the total infiltration rate as a function of time (DTNs: GS020508312242.001 [DIRS 162129] and GS020908312242.002 [DIRS 162141]). For simplicity, our model considers the uniformly distributed infiltration rate along the infiltration plot to be consistent with the use of the uniform property distribution in the site-scale model of the Yucca Mountain UZ. One consideration in our modeling study is to evaluate approaches used in the site-scale model. Considerable temporal variability of infiltration rate occurred during the test, as a result of infill materials within the fault just below the infiltration plot (Figure 7-14). In other words, the effective permeability of the fault just below the plot changed with time. It is also expected that most portions of the fault and the surrounding fractures away from the plot would still be unsaturated, although capillary pressure at the plot was positive during the test. Based on these observations, total infiltration rate, instead of a pressure head of $2 \mathrm{~cm}$, was used as the boundary condition in our model.

Seepage from the fault into the niche was measured during the test, with a number of trays used to cover the areas where seepage might occur. Seepage was found to be highly spatially variable. The total seepage rate as a function of time is given in Figure 7-15 (DTN: LB0303A8N3LIQR.001 [DIRS 162570]). Several boreholes were installed around the niche. Water arrival times at these boreholes were monitored through electrical resistivity probes. Figure 7-16 shows average water travel velocities determined from the arrival times from two boreholes just above the ceiling of the niche (DTN: LB0303A8N3LIQR.001 [DIRS 162570]). The fault is about $2 \mathrm{~m}$ from the borehole collars in Figure 7-16 (DTN: LB0303A8N3LIQR.001 [DIRS 162570]). Note that relatively uniform water-travel-velocity distribution within and near the fault was observed from these two boreholes.

After 209 days, two tracers with different molecular diffusion coefficients (Br and PFBA) were introduced into infiltrating water at the infiltration plot. Tracer concentrations in three of the trays at the niche capturing seeping water from the fault were measured (DTN: LB0303A8N3LIQR.001 [DIRS 162570]). For technical reasons, seepage rates corresponding to these three trays were not measured during the period of tracer concentration measurement. In this study, a flux-averaged breakthrough curve (concentration as a function of time) from these trays was used to represent average breakthrough curve for all trays at the niche where seepage was captured (Wang 2003 [DIRS 164021], SN-LBNL-SCI-215-V1, pp. 108 to 109). A constant flux value for each of the three trays was used for calculating the flux-averaged breakthrough curve shown in Figure 7-17. The constant flux values for the three trays were determined as the averaged value over 56 days before tracers were introduced. This flux-averaged breakthrough curve is comparable to simulation results. 


\subsubsection{Numerical Model}

A numerical model, generated with a software routine Smesh.f V1.0 (LBNL 2002 [DIRS 162142]), was developed for the fault test site to compare the simulation results with the relevant field observations. While comparison results will be presented below in Section 7.3.3, Section 7.3.2 focuses on schemes used for developing the numerical model.

A three-dimensional numerical grid was constructed for simulating the fault test (Figure 7-18). The fault was represented as a vertical fracture, and surrounding fractured rock is approximated as a dual-continuum system consisting of overlapped, interacting fracture and matrix continua. Global water flow and solute transport are allowed to occur in both continua. Figure 7-18 shows a cross section of the grid within the fault. The thickness of the grid in the direction perpendicular to fault walls is $3 \mathrm{~m}$ along each side of the fault. The fracture frequency used for generating the dual-continuum grid is $1.03 \mathrm{~m}^{-1}$ for tsw33 (determined from the fracture map at the alcove floor) and $1.5 \mathrm{~m}^{-1}$ for tsw34 (determined from the fracture map at the ceiling of the niche) (DTN: GS030108314224.001 [DIRS 162131]; Wang 2003 [DIRS 164021], SN-LBNL-SCI-215-V1, pp. 111 to 112). As shown in Figure 7-18, within a cross section of the grid along the fault, the grid spacing is $10 \mathrm{~cm}$ just above the ceiling of the niche, enabling the seepage process to be accurately simulated. Grid spacings in the direction perpendicular to the fault are $0.024 \mathrm{~m}, 0.168 \mathrm{~m}, 0.456 \mathrm{~m}, 0.756 \mathrm{~m}$, and $1.44 \mathrm{~m}$, respectively. The smallest spacing is adjacent to the fault, so that water imbibition and tracer diffusion into the fractured rock from the fault can be accurately captured. Cross sections parallel to the fault walls have identical grid meshes (Figure 7-18) for different distances from the fault. The niche is represented by an opening at the bottom of the grid (Figure 7-18), with the geometry of the opening determined from the survey data of the niche near the fault. Note that this is only an approximation of the geometry of the test site; a three-dimensional geometry of the niche with an underground tunnel connected to the niche is not incorporated into the model. However, since our main concern is flow and transport processes within the fault, this geometry representation should be adequate.

Temporally variable inflow rates are imposed on the top boundary, corresponding to the infiltration plot at the alcove floor. The side boundary corresponds to zero-flow (in the direction perpendicular to the simulation domain) conditions. The niche wall boundary is modeled by a zero capillary-pressure condition, representing capillary barrier effects (Birkholzer et al. 1999 [DIRS 105170]). The bottom boundary was assigned a constant matrix saturation of 0.85 , which is consistent with field observations under ambient conditions (Flint 1998 [DIRS 100033]). Also based on field observations of Flint (1998 [DIRS 100033]), matrix saturations are initially assigned to be 0.72 for tsw 33 and 0.85 for tsw34. Other initial conditions for the rock mass within the model domain are absence of solutes and low water saturation $(1.05 \mathrm{E}-2)$ in both the fractures and the fault. Rock properties used in model simulations are presented in the next section.

Model calibration was performed using an inverse modeling code iTOUGH2 V4.0 (LBNL 1999 [DIRS 139918]). The model calibration is defined herein as the adjustment of rock hydraulic parameters to make simulation results match the corresponding data. The goodness of match is measured using the standard least-squares approach, which minimizes the sum of the squared residuals weighted by the inverse of variance of the data. T2R3D V1.4 (LBNL 1999 [DIRS 146654]) was used for modeling tracer transport. 


\subsubsection{Model Simulations and Discussions}

The numerical model was first calibrated against the seepage and water-travel-velocity data to obtain the calibrated rock properties and the corresponding water flow field. Then, tracer transport simulations with different transport parameters were carried out to evaluate the effects of matrix diffusion and other related processes on solute transport in the fault.

\subsubsection{Calibration of Rock Properties Using Seepage-Rate Data and the Average Water-Travel-Velocity Data}

Both fracture and matrix properties were assumed to be homogeneous within each geological subunit (tsw33 and tsw34). Fault properties were assumed to be homogeneous within both units. This is based mainly on the following three considerations:

(1) Consideration of the heterogeneity within each subunit would introduce a large number of rock properties that need to be determined by more data than was available from the test site;

(2) As previously indicated, these treatments have been used by the site-scale model of the Yucca Mountain UZ. It is of interest to examine how well this simple representation of subsurface heterogeneity can be used to model the fault test.

(3) A recent study by Zhou et al. (2003 [DIRS 162133]) indicates that flow and transport in the Yucca Mountain UZ are mainly determined by large-scale heterogeneity, characterized by property differences between different geological units, rather than by property variability within a geological unit.

Rock hydraulic properties needed as inputs into the model include fracture and matrix permeabilities, fracture and matrix porosities, fault aperture and permeabilities, van Genuchten (1980 [DIRS 100610]) parameters (for matrix, fractures, and the fault), and the parameter of the active fracture model, $\gamma$, for fractures (Wang 2003 [DIRS 164021], SN-LBNL-SCI-215-V1, pp. 114 to 115). Because fracture van Genuchten parameters for tsw33 and tsw34 are similar, a simple average of these parameters was used as the corresponding parameters for the fault. The averaged $\mathrm{k} / \varphi$, where $\mathrm{k}$ is fracture permeability and $\varphi$ is the corresponding fracture porosity, was calculated as fault permeability. Note that in section 7.3.3, fracture porosity refers to the volume fraction of fractures in the entire rock volume, rather than the void space within the fracture. Also, note that because there is no matrix in the fault in our model (or $\varphi=1$ ), the weighted $\mathrm{k} / \varphi$, rather than weighted $\mathrm{k}$, is employed for estimating fault permeability. The aperture of the fault was estimated as the average of fracture apertures of the two subunits. Note that the active fracture model was developed for fracture networks rather than for a single fracture. Consequently, the active fracture model does not apply to the fault here. In fact, most of the parameter values mentioned above and given in Table 7-16 are not site specific for the fault test site. These values were used as initial guesses for model calibration against the seepage rate and water-travel-velocity data observed from the fault test. To reduce the number of variables in model calibration (or inverse modeling), parameters expected to significantly affect simulated water transport time and seepage rate were varied in the calibration, while other parameters were 
kept unchanged. The varied parameters were fracture and fault permeabilities, fracture porosity, fault aperture, and fracture and fault van Genuchten values.

Infiltration-seepage processes in the fault and the surrounding fractured rock were determined by several mechanisms. Liquid water applied at the alcove floor (Figure 7-18) flowed first into the fault and then into fractured networks connected to the fault. Matrix imbibition occurred at interfaces between fractures and the matrix, and between the fault and the matrix. When water arrived at the intersection between the fault and the niche, it could not immediately seep into the niche until the capillary pressure became zero because of capillary barrier effects (Philip et al. 1989 [DIRS 105743]; Birkholzer et al. 1999 [DIRS 105170]). The capillary barrier can divert flow away from the opening, resulting in only a portion of the water arriving at the niche ceiling actually seeping into the niche. Water transport time was determined by fracture porosity, fault aperture, and the matrix imbibition process.

Figure 7-19 shows a comparison between seepage-rate data and the simulation result from a model calibration (Run \#1) without considering the water-travel-velocity data. In this calibration run, fracture porosity and fault aperture were not varied. A fairly good match was obtained (Figure 7-19); however, water travel velocity is significantly overestimated (Figure 7-20). Water travel velocities were calculated from water arrival times at locations about $1 \mathrm{~m}$ above the middle of the opening in Figure 7-18. The transport time was defined as the time when fault or fracture saturation was increased from the initial value of $1.05 \mathrm{E}-2$ to $1.06 \mathrm{E}-2$. This comparison implies that seepage rate as a function of time may be mainly controlled by rock properties near seepage locations (influence zone of capillary barrier (Liu et al. 2002 [DIRS 160230])). On the other hand, water travel velocities are determined by rock properties from the infiltration plot to the locations where water travel velocities are monitored. Table 7-17 gives the calibrated properties obtained from Run \#1.

The overestimation of the water travel velocities may result from the following: (1) some cavities in tsw 33 are connected to fractures and could contribute to increasing the storage in the fracture continuum; (2) in reality, the fault is a zone rather than a single fracture. The effective aperture from this zone may be much larger than the assumed aperture value for the fault (Table 7-16). Neither of these factors was considered in Run \#1 (first calibration). Taking these factors into consideration, the new calibration (Run \#2) allowed both fault aperture and fracture porosity in tsw33 to be varied. The resultant values are $3 \mathrm{~cm}$ for fault aperture and 0.066 for fracture porosity of tsw33 (Table 7-18). While the actual width of the fault zone is unknown, the estimated equivalent fault aperture $(3 \mathrm{~cm})$ is considered to be acceptable. The estimated fracture porosity is consistent with those estimated from water release tests performed in the same geological unit (BSC 2004 [DIRS 170004], Section 6.11.3.1).

Figure 7-20 shows a comparison among calculated water travel velocities from two calibration runs and the velocity data observed from the fault test. The simulated water travel velocities from Run \#2 are much closer to the observed data than those from Run \#1 (especially near the fault). However, the water travel velocities away from the fault are still overestimated. One possible explanation is that matrix imbibition from fractures above the niche were underestimated because the dual-continuum approach considerably underestimates the pressure gradient near a fracture matrix interface during transient flow conditions (Pruess and Narasimhan 1985 [DIRS 101707]). While this problem could be resolved with the multiple 
interacting continua model of Pruess and Narasimhan (1985 [DIRS 101707]), the computational intensity of the inverse model problem under consideration would be significantly increased. Note that a model calibration involves a great number of forward simulation runs. Considering that (1) the transient flow effects would be considerably reduced at later time of the test and that (2) our focus here is on flow and transport within and near fault, simulated flow field and calibrated rock properties from Run \#2 were used for simulating tracer transport at the test site. Figure 7-21 also shows a comparison between simulated seepage rates as a function of time (Run \#2) and field observations. The match is reasonable.

\subsubsection{Prediction of Tracer Transport with Matrix Diffusion}

Tracer transport within the fault is controlled by several processes, including advection, diffusion into the matrix blocks (matrix diffusion), mass exchange between the fault and the surrounding fracture networks, and dispersion. Special attention in this study is given to evaluating the relative importance of matrix diffusion. The flow field obtained from Run \#2 was used to simulate tracer transport processes and compare simulation results with field observations (Figure 7-17). The breakthrough curve is obtained from the output of T2R3D V1.4 (LBNL 1999 [DIRS 146654]) using a software routine Bkread.f V1.0 (LBNL 2002 [DIRS 162143]).

Two conservative tracers with different molecular diffusion coefficients $\left(2.08 \mathrm{E}-9 \mathrm{~m}^{2} / \mathrm{s}\right.$ for $\mathrm{Br}$ (Lide 2002 [DIRS 160832], p. 5-96) and $7.60 \mathrm{E}-10 \mathrm{~m}^{2} / \mathrm{s}$ for PFBA (Benson and Bowman 1994 [DIRS 122788])) were used in the fault test. Based on analyses of the relevant diffusion experiment results for tuff matrix samples in the Yucca Mountain UZ, Moridis et al. (2003 [DIRS 161902], Table 1) reported that the tortuosity factor for the tuff matrix could be approximated by the corresponding matrix porosity. As a consequence, the average matrix porosity for tsw33 and tsw34 (0.13) was used as the tortuosity factor. The effective diffusion coefficient for the matrix diffusion process is the product of the molecular diffusion coefficient and tortuosity factor.

Figure 7-22 compares simulated breakthrough curves at the niche with the observed data. In this simulation, the dispersivity is assumed set to zero. (The relative importance of the dispersion will be discussed below.) Since the diffusive flux from the fault to the matrix is proportional to the product of the tortuosity factor and the fault-matrix interface area, changes in the interface area for a given tortuosity factor are equivalent to changes in tortuosity factor for a given interface area. For simplicity, the tortuosity factor value was changed in actual simulations, but the numerical grid defining the interface area was kept unchanged. Note that changes in the interface area should not significantly alter the flow field during the period of the tracer test. Tracers were introduced into infiltrating water at about 200 days after infiltration started, resulting in the matrix near the fault being almost saturated during the tracer test, and the matrix imbibition being insignificant. As shown in Figure 7-22, the simulated breakthrough curve exhibits a much larger concentration peak values but only slightly earlier arrival times for the tracer peaks. This meets the specified validation criteria. 


\subsubsection{A Further Discussion of Matrix Diffusion Effects}

Although the matrix diffusion model has been validated sufficiently for the purposes of license application, it is of interest to study its sensitivity to the interface area between fault and matrix. The need to increase interface areas between fractures (or faults) and the matrix in matching the field observations of tracer transport in fractured rock has been recently reported by several researchers. Shapiro (2001 [DIRS 162132]) reported an interpretation of concentration measurements for tritium and dichlorodifluoromethane collected from a glacial drift and fractured crystalline rock over $4 \mathrm{~km}^{2}$ in central New Hampshire. He found that the effective diffusion coefficient at the kilometer scale is at least three orders of magnitude greater than laboratory estimates of diffusion in crystalline rock. Neretnieks (2002 [DIRS 162140]) presented comparisons between several analytical solutions with tracer test results at the Äspö site, and reported a need for a factor 30-50 times larger for the fracture-matrix interface area than expected. He also indicated that nine other research groups reached a similar conclusion in their interpretation of the same test data set. Our results in this study are consistent with these previous findings.

Several mechanisms regarding the increase in the interface area have been reported in the literature. They include (1) advective mass exchanges from high-permeability fractures to low-permeability fractures (Shapiro 2001 [DIRS 162132]), (2) diffusion into stagnant water zones (Neretnieks 2002 [DIRS 162140]), and (3) enhanced fracture-matrix interface areas for fractures with small-trace lengths that do not contribute to global flow and are not considered in the survey data, and, therefore, in the numerical grid. In addition to these potential mechanisms, two other factors also contribute to the increase in the interface area. First, in the relevant analytical and numerical solutions to tracer transport, fracture walls are generally assumed to be flat. However, it is now well known that fracture walls are rough and characterized by fractal geometry (National Research Council 1996 [DIRS 139151]). Consequently, the actual interface areas between fractures and faults, and the matrix are larger than what are calculated using flat fracture walls. Second, a fault zone may include a great number of crushed matrix blocks that have smaller sizes than the fracture spacing in a nonfault zone. These crushed matrix blocks can make a significant contribution to the matrix diffusion within the fault, but are not considered in our numerical grid, where the fault is simply treated as a vertical fracture. To compensate for the effects of these mechanisms mentioned above, the model's fault-matrix interface areas obviously need to be increased.

Also shown in Figure 7-22 is a simulation with the interface area increased by a factor of 45, which is within the range reported by Neretnieks (2002 [DIRS 162140]). With this adjustment, the simulations match the data more closely. Although simulation results with the increased interface area reasonably match the observed data (Figure 7-22), the concentration difference at a given time for the two tracers is generally overestimated by the model. One plausible explanation is that the crushed matrix blocks within the fault zone have much smaller sizes than the fracture spacing. This, however, is not considered in our model, in which the matrix block size is characterized by fracture spacing. The smaller sizes correspond to shorter times needed by the equilibrating tracer concentration at the center and outer surface of a matrix block, reducing the difference between the effects of matrix diffusion on overall solute transport behavior for different molecular-diffusion coefficients. This can be further illustrated by an extreme case: an infinitely small block size within the fault and without mass exchange between 
the fault and nonfault fractured rock. In this case, the concentrations of the matrix block within the fault can be equilibrated simultaneously with those at the outer surface of the block for two tracers with different molecular diffusion coefficients. Consequently, although the existence of this kind of matrix block can still significantly retard tracer transport within the fault, identical breakthrough curves should be observed at Niche 3 for the two tracers. This issue was not further explored in the current modeling study because the matrix block-size distribution within the fault cannot be independently estimated or observed.

Compared with matrix diffusion, the macrodispersion process is not considered to be significant within the fault for this particular test. Field measurements indicate that water travel-velocity distribution is quite uniform within and near the fault (Figure 7-16), whereas macrodispersion results from variability in water velocity. This is further confirmed by observed breakthrough curves from three flow paths within the fault. They have arrival times similar to peak concentrations (DTN: LB0303A8N3L1QR.001 [DIRS 162570]). These experimental observations are consistent with the findings from our model analyses: the observed data were very difficult to match when a considerable degree of dispersion was included in the model. For example, Figure 7-23 shows simulated breakthrough curves with a longitudinal dispersivity value of $1 \mathrm{~m}$ and a transverse dispersivity value of $0.1 \mathrm{~m}$ (and with the increased fault-matrix interface area), compared to results in Figure 7-22 (without considering dispersion). Larger dispersivity values generally correspond to earlier arrival times of peak concentrations and to a larger difference between these peak concentrations for the two different tracers.

The Yucca Mountain site (where the fault test was carried out) is the proposed geological disposal site for high-level nuclear waste in the United States. Radionuclide transport from the repository horizon to the water table is a key factor affecting the performance of the repository. The study results herein have some important implications for the PA of the proposed site.

Matrix diffusion has been identified as a key mechanism for retarding the radionuclide transport in both unsaturated and saturated fractured rock (e.g., Bodvarsson et al. 2001 [DIRS 160133]; Neretnieks 2002 [DIRS 162140]). The enhancement of the fracture (fault)-matrix interface area (or effective matrix diffusion coefficient) seems to be common for matching field-scale solute transport observations, as suggested by this study and previous studies (Shapiro 2001 [DIRS 162132]; Neretnieks 2002 [DIRS 162140]). Some physical explanations for this enhancement are available, as discussed in Section 7.3.3.2. The current site-scale model for the Yucca Mountain UZ does not consider the effects of this enhancement. Consequently, the performance of the repository, estimated based on the site-scale model, may be underestimated.

The other related issue is the effects of cavities (existing in several geological layers at the Yucca Mountain site) on water flow and radionuclide transport processes. One may intuitively expect the cavities connected to fractures to act as capillary barriers under unsaturated conditions, because the cavity openings are much larger than fracture apertures. However, both this study and analyses of water-release tests performed in the related geological units at the Yucca Mountain site suggest that cavities are accessible by water within fracture networks, and, therefore, are retarding the downward water flow and radionuclide transport processes. This is also supported by field observation that mineral coatings exist in many cavities (BSC 2004 [DIRS 169734], Section 7.7). The coating is a signature for liquid-water flow paths. Although the cavity openings are larger than fracture apertures, the roughness of cavity walls could result 
in film flow (along cavity walls) from fractures to the cavities (Tokunaga and Wan 1997 [DIRS 139195]). The effects of cavities are also not considered in the site-scale model for the Yucca Mountain UZ. This omission would result in further underestimating the performance of the repository.

Table 7-16. Uncalibrated Rock Properties

\begin{tabular}{|c|c|c|c|c|c|}
\hline \multirow[b]{2}{*}{ Rock property } & \multirow[b]{2}{*}{ Fault } & \multicolumn{2}{|c|}{ tsw33 } & \multicolumn{2}{|c|}{ tsw34 } \\
\hline & & Fracture & Matrix & Fracture & Matrix \\
\hline Permeability $\left(\mathrm{m}^{2}\right)$ & $4.34 \times 10^{-11}$ & $5.5 \times 10^{-13}$ & $3.08 \times 10^{-17}$ & $0.35 \times 10^{-13}$ & $4.07 \times 10^{-18}$ \\
\hline Porosity & 1.00 & $6.6 \times 10^{-3}$ & 0.154 & 1. $\times 10^{-2}$ & 0.11 \\
\hline $\begin{array}{l}\text { Fracture frequency } \\
(1 / \mathrm{m})\end{array}$ & & 1.03 & & 1.5 & \\
\hline Fracture aperture $(\mathrm{m})$ & $1.12 \times 10^{-3}$ & $1.49 \times 10^{-3}$ & & $1.14 \times 10^{-3}$ & \\
\hline $\begin{array}{l}\text { Active fracture model } \\
\text { parameter } \gamma\end{array}$ & 0.0 & 0.41 & & 0.41 & \\
\hline $\begin{array}{l}\text { van Genuchten } \alpha \\
\left(\mathrm{Pa}^{-1}\right)\end{array}$ & $1.0 \times 10^{-3}$ & $1.46 \times 10^{-3}$ & $2.13 \times 10^{-5}$ & $5.16 \times 10^{-4}$ & $3.86 \times 10^{-6}$ \\
\hline van Genuchten m & 0.608 & 0.608 & 0.298 & 0.608 & 0.291 \\
\hline
\end{tabular}

DTNs: Wang 2003 [DIRS 164021], SN-LBNL-SCI-215-V1, pp. 114 to 115.

Table 7-17. Rock Properties Calibrated from Seepage Rate Data (Run \#1)

\begin{tabular}{|l|c|c|c|}
\hline \multicolumn{1}{|c|}{ Rock property } & Fault & tsw33 & tsw34 \\
\hline Fracture Permeability $\left(\mathrm{m}^{2}\right)$ & $6.67 \times 10^{-11}$ & $8.93 \times 10^{-13}$ & $3.16 \times 10^{-14}$ \\
\hline Fracture van Genuchten $\alpha\left(\mathrm{Pa}^{-1}\right)$ & $1.15 \times 10^{-3}$ & $1.67 \times 10^{-3}$ & $4.59 \times 10^{-4}$ \\
\hline
\end{tabular}

Source: DTN: LB0303A8N3MDLG.001 [DIRS 162773].

NOTE: All other rock properties are the same as those in Table 7-16. 
Table 7-18. Rock Properties Calibrated from Both Seepage Rate and Water Travel Velocity Data (Run \#2)

\begin{tabular}{|l|l|l|l|}
\hline \multicolumn{1}{|c|}{ Rock property } & & & \\
\hline Fracture Permeability $\left(\mathrm{m}^{2}\right)$ & \multicolumn{1}{c|}{ Fault } & \multicolumn{1}{c|}{ tsw33 } & \multicolumn{1}{c|}{ tsw34 } \\
\hline Fracture Porosity & $1.12 \times 10^{-10}$ & $1.23 \times 10^{-12}$ & $5.01 \times 10^{-13}$ \\
\hline Fracture aperture $(\mathrm{m})$ & & 0.066 & \\
\hline Fracture van Genuchten $\alpha\left(\mathrm{Pa}^{-1}\right)$ & 0.03 & & \\
\hline
\end{tabular}

Source: DTN: LB0303A8N3MDLG.001 [DIRS 162773].

NOTE: All other rock properties are the same as those in Table 7-16.

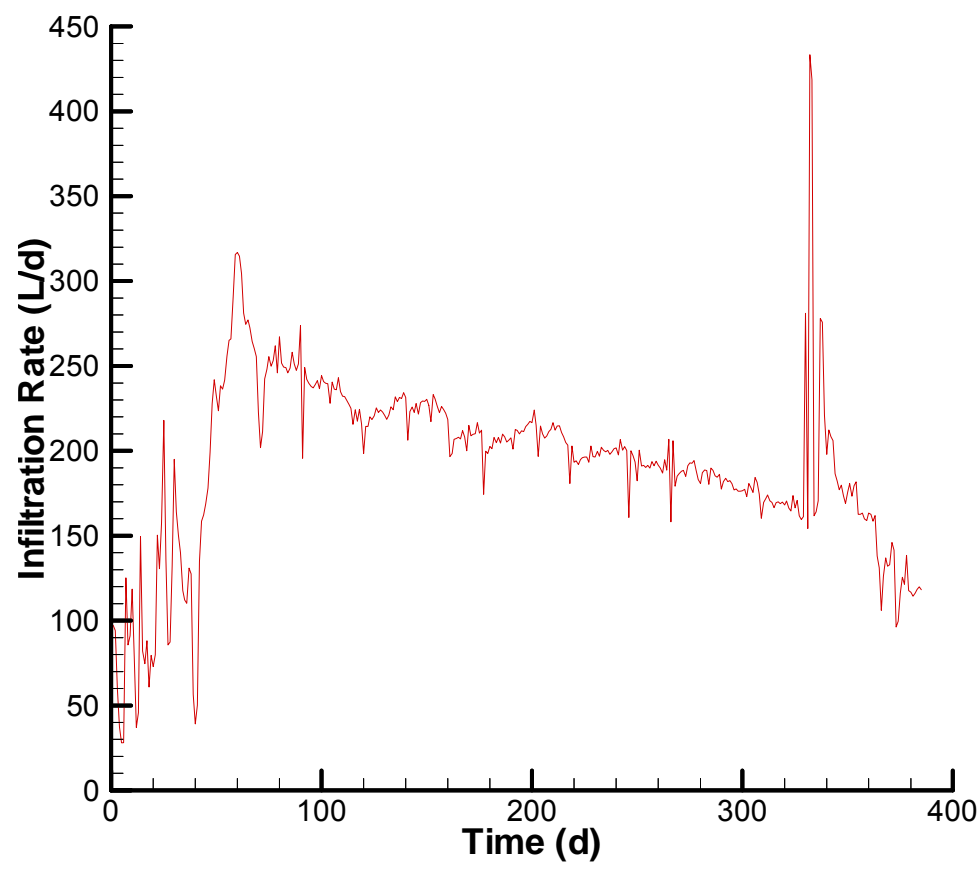

DTNs: GS020508312242.001 [DIRS 162129] and GS020908312242.002 [DIRS 162141].

Figure 7-14. Infiltration Rate as a Function of Time 


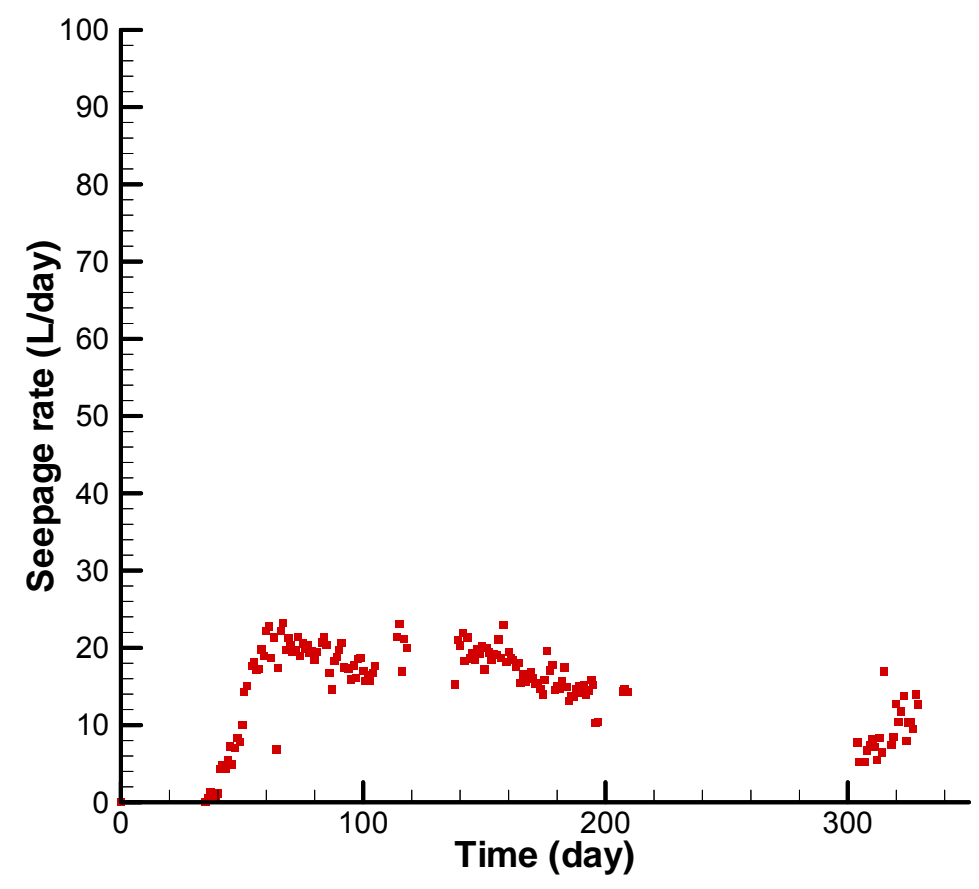

DTN: LB0303A8N3LIQR.001 [DIRS 162570].

Figure 7-15. Total Seepage Rate as a Function of Time. Data for 3/6/2001 through 1/28/2002

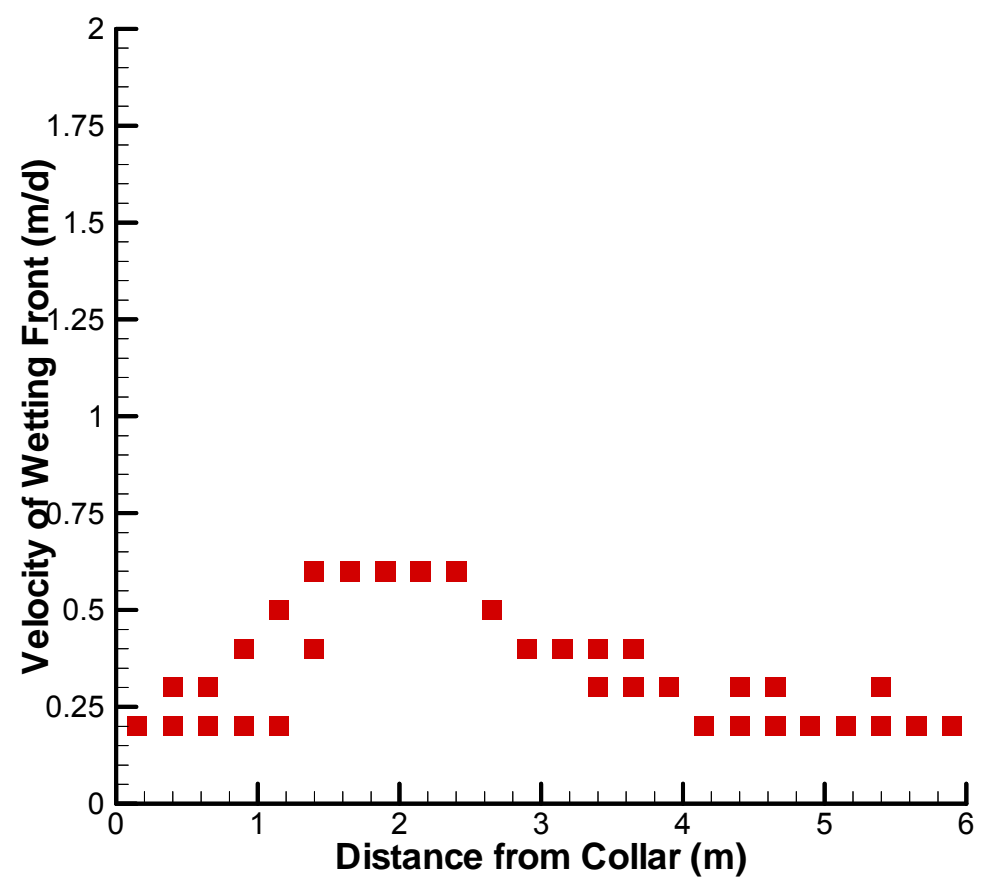

DTN: LB0303A8N3LIQR.001 [DIRS 162570].

Figure 7-16. Water Travel Velocity Data at Two Boreholes (9 and 10) 


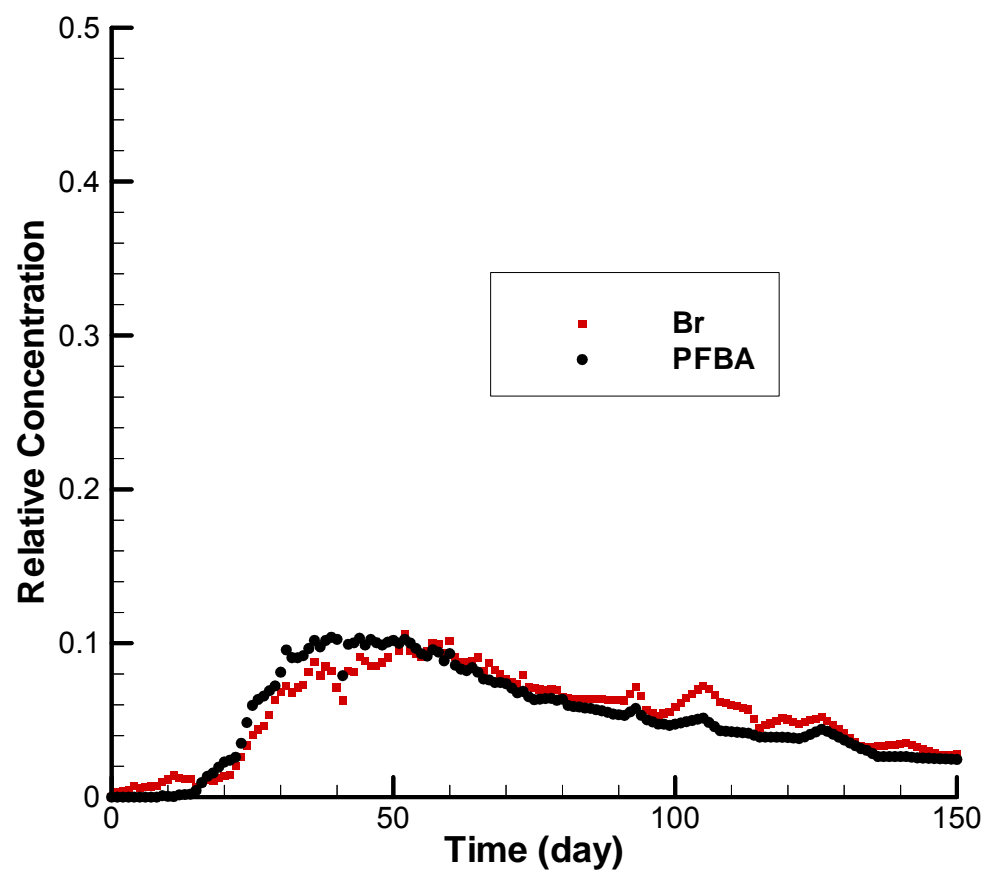

DTN: LB0303A8N3LIQR.001 [DIRS 162570] (Also see Wang 2003 [DIRS 164021], SN-LBNL-SCI-215-V1, P.108-109).

Figure 7-17. Observed Flux-Average Breakthrough Curve

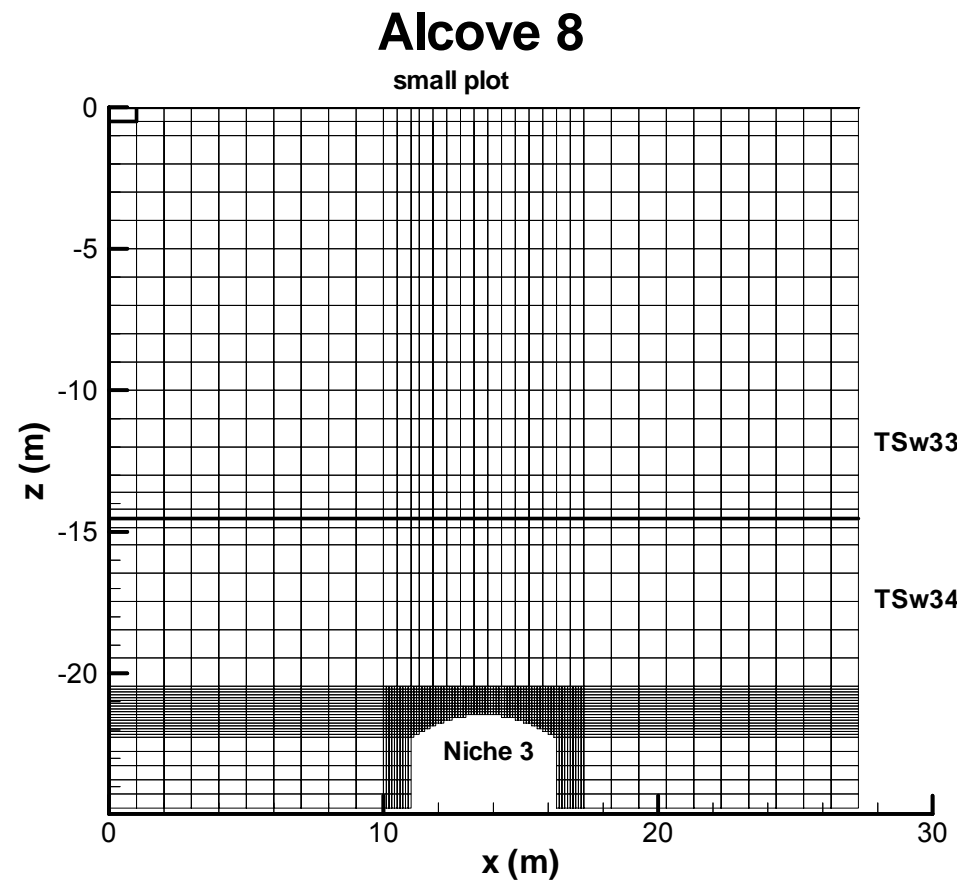

For illustration purposes only.

Figure 7-18. A Cross Section View of Three-Dimensional Numerical Grid 


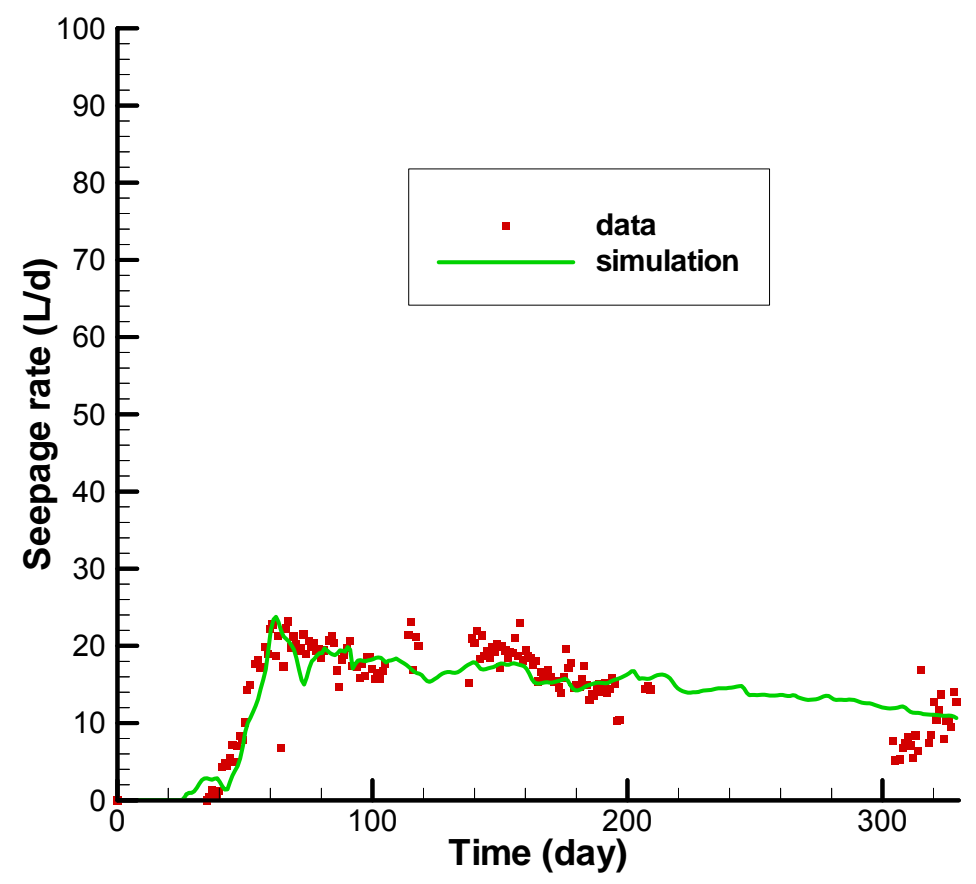

Source: DTN: LB0303A8N3MDLG.001 [DIRS 162773].

Figure 7-19. A Comparison between Simulated Seepage Rates as a Function of Time (Run \#1) and Field Observations

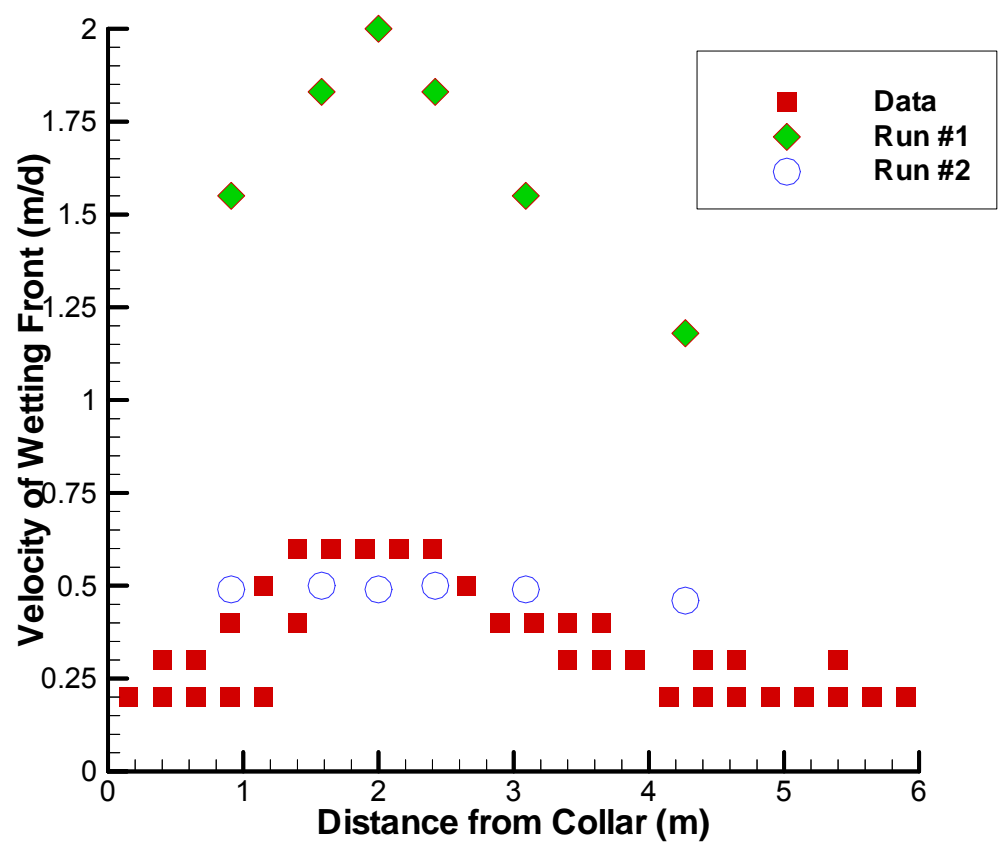

Source: DTN: LB0303A8N3MDLG.001 [DIRS 162773].

Figure 7-20. A Comparison among Calculated Water Travel Velocities from Two Calibration Runs and the Velocity Data Observed from the Fault Test 


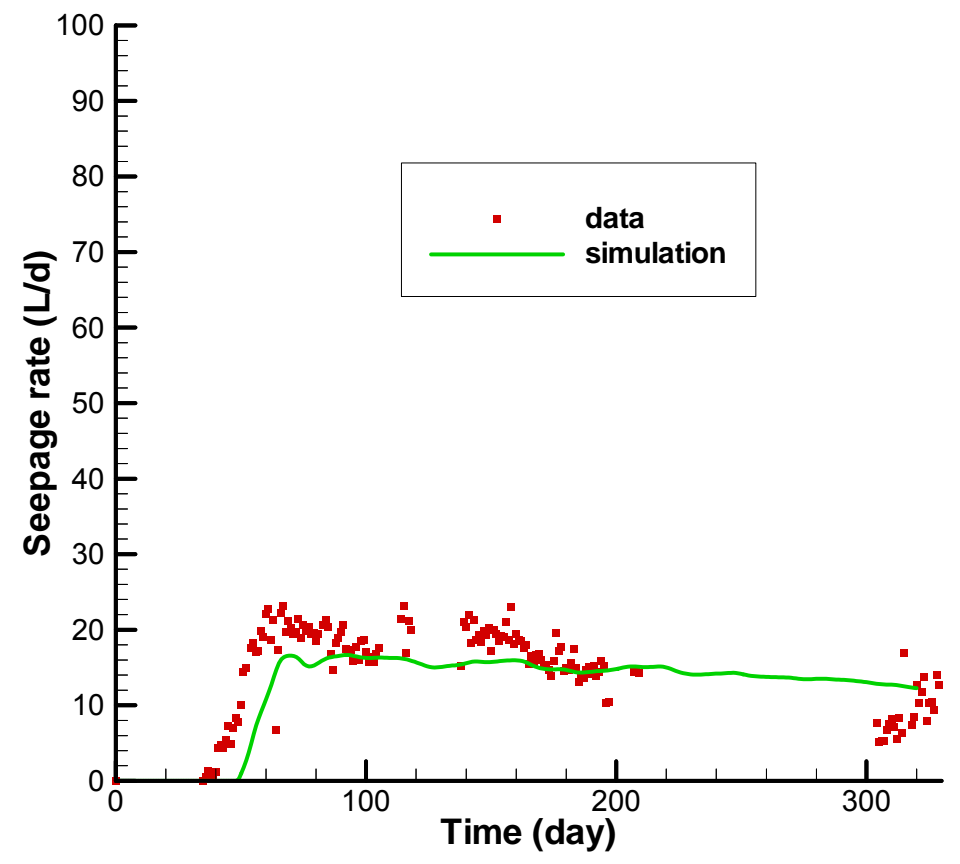

Source: DTN: LB0303A8N3MDLG.001 [DIRS 162773].

Figure 7-21. A Comparison between Simulated Seepage Rates as a Function of Time (Run \#2) and Field Observations

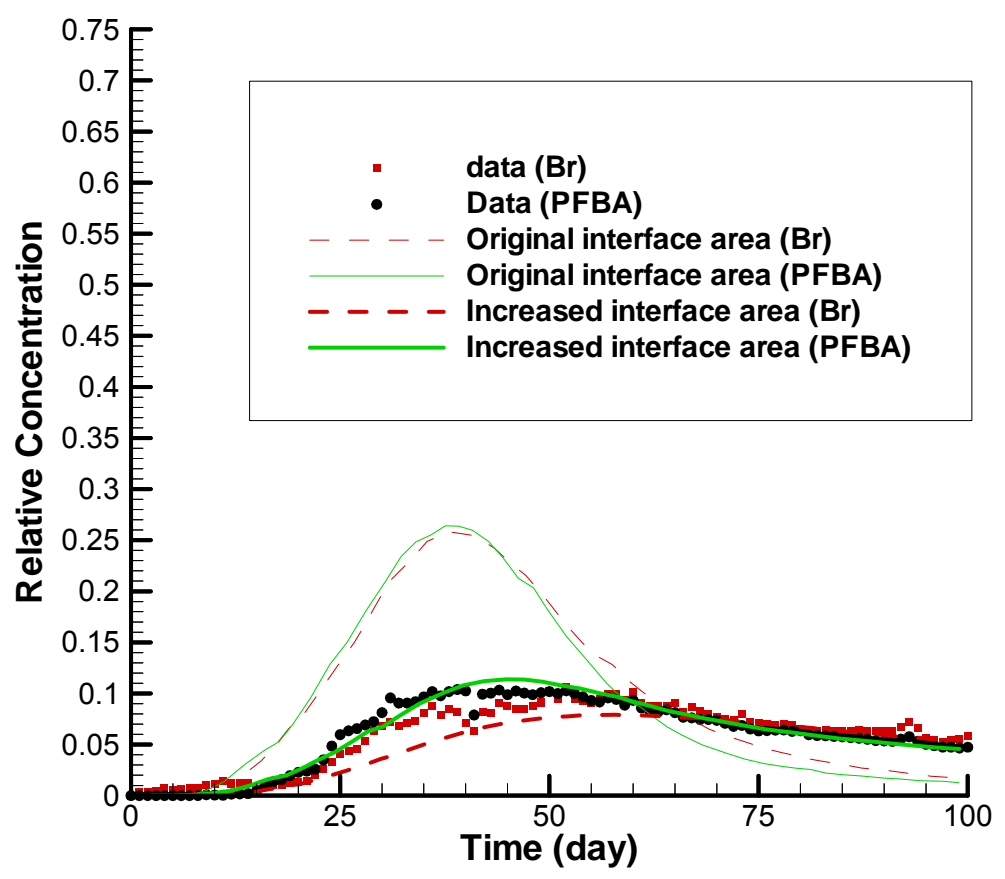

Source: DTN: LB0303A8N3MDLG.001 [DIRS 162773].

Figure 7-22. Comparisons between Simulated Breakthrough Curves at the Niche for Two Different Fault-Matrix Interface Areas and the Observed Data. 


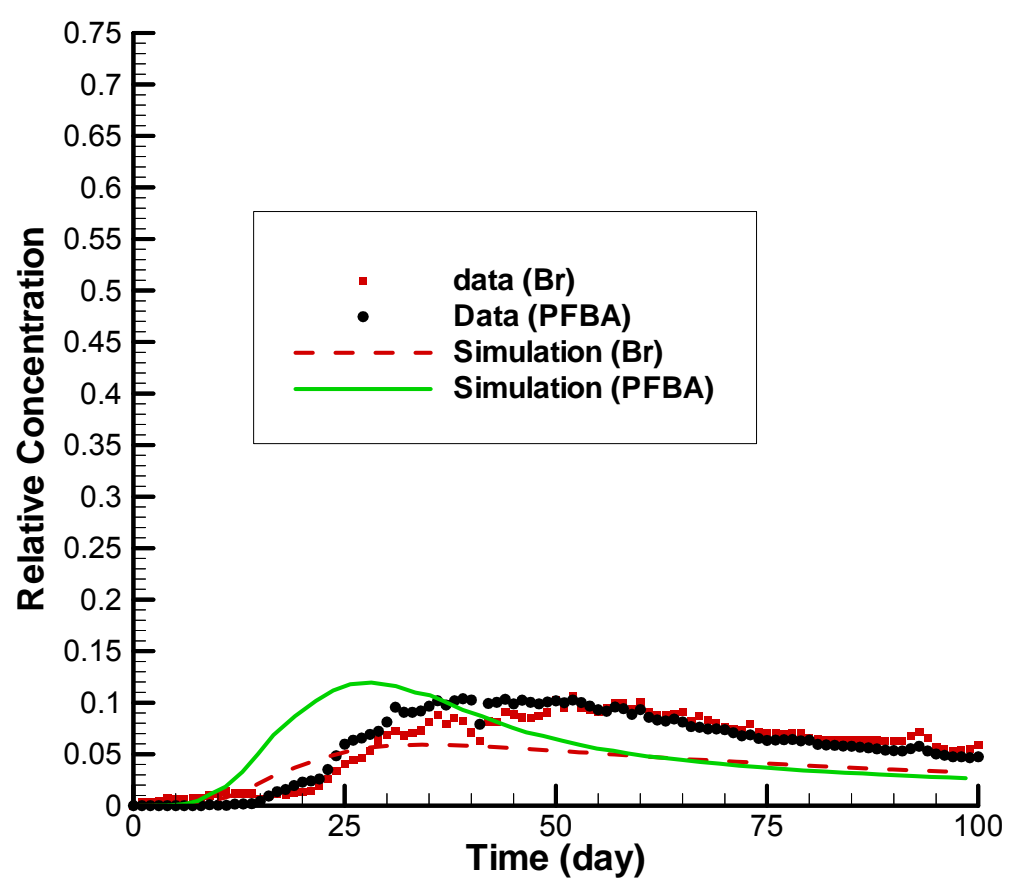

Source: DTN: LB0303A8N3MDLG.001 [DIRS 162773].

Figure 7-23. Comparisons between Simulated Breakthrough Curves (Considering Dispersion) at the Niche for the Increased Fault-Matrix Interface Areas and the Observed Data

\subsection{VALIDATION OF THE SORPTION SUB-MODEL}

The validation approach and criteria are discussed in the technical work plan (BSC 2004 [DIRS 171282], Section 2.2.2 and BSC 2004 [DIRS 167969], Section I-2-1-3), which stipulates that:

The sorption model is linear equilibrium, defined by $K_{d}$ values, between sorbed and aqueous species in the UZ. $K_{d}$ values will be justified by comparison with laboratory data from the TDMS, per the technical work plan (BSC 2004 [DIRS 167969] Section I-2-I-3). This comparison constitutes validation of the sorption model by Method 1, corroboration with experimental data. The technical work plan criterion for validation is that $K_{d}$ values calculated from experimental data be within a factor of 3 of the $K_{d}$ values used in the model.

The acceptance criterion, as stated in the technical work plan (BSC 2004 [DIRS 167969] Section I-2-I-3), is inadequate in this case because it presupposes estimation of a single $K_{d}$ from the analysis of the experimental data. Rather than estimating a single $K_{d}$ this model report estimates cumulative distribution probability distributions using available site-specific $K_{d}$ measurements. This approach is more thorough and aims to (a) identify the type and develop the parameters that describe the statistical distribution of $K_{d}$ based on data from a number of samples belonging to the same general hydrogeologic unit, and (b) provide representative $K_{d}$ estimates from these statistical distributions. 
Validation is provided by (a) confirmation that the analysis of the laboratory data (described in Appendices A and B) supports the linear equilibrium sorption model (onto which the $K_{d}$ concept is based) for a given radionuclide and rock, and (b) by using $K_{d}$ values that are within the range determined from these laboratory experiments and correspond to the expected values (as estimated from the statistical distributions).

Confirmation of the validity of the linear equilibrium sorption model is provided in Appendices A and B. Table 6-3 presents the $K_{d}$ range and the types of the $K_{d}$ probability distributions of the various radionuclides in the UZ rocks. The $K_{d}$ values used in the model (indicated by boldface numbers) are also listed in this table, and are shown to be within the measured range and to coincide with the median $K_{d}$ value from the statistical distributions. Thus, the sorption model is validated.

\subsection{VALIDATION OF THE COLLOID TRANSPORT SUB-MODEL}

The validation approach and criteria are discussed in the technical work plan (BSC 2004 [DIRS 171282], Section 2.2.2 and BSC 2004 [DIRS 167969], Section I -2 -1-4), which indicates that the colloid transport model was judged in the model validation status report (BSC 2003 [DIRS 170030], p. 113) to have been validated satisfactorily for its intended use. Therefore, no further validation of the colloid transport model is planned.

However, some additional validation was performed in the model report. This additional validation of the colloid transport model is included in the validation of the radionuclide transport model, and is discussed in Section 7.2.3.6 (Method 2) and Section 7.7 of this model report.

\subsection{VALIDATION OF THE ACTIVE FRACTURE SUB-MODEL WITH MATRIX DIFFUSION}

Validation of the active fracture model with matrix diffusion for transport was planned in the technical work plan (BSC 2004 [DIRS 171282], Section 2.2.2 and BSC 2004 [DIRS 167969], Section I-2-1-5). The technical work plan outlines the required level of confidence, validation approaches, and validation criteria.

The validation for the active fracture model with matrix diffusion combines two parts:

- The active fracture model, already validated for its intended use to represent site-scale flow, in a separate model report (BSC 2004 [DIRS 170035], Section 7).

- The matrix diffusion model, already validated for saturated or nearly saturated conditions in Section 7.3 of this report.

The resulting model for active fracture model with matrix diffusion is intended to represent transport of radionuclides in the UZ for in situ saturation conditions (high saturation in the rock matrix, and low saturation in the fractures).

The processes represented by the active fracture model and matrix diffusion are intertwined, and it is impossible to discriminate their separate effects (and the efficacy of the matrix diffusion 
part) from presently available UZ testing data. Instead, the validation strategy presented in this report draws from (1) separate validation of the active fracture model description of flow behavior, and (2) available evidence of matrix diffusion behavior from field testing in the UZ at Yucca Mountain.

Section 7.6 includes two subsections. Section 7.6.1 addresses the issue of confidence building during the development of the active fracture model with matrix diffusion (based on sensitivity analysis of the active fracture model for transport, aiming to ensure that the incorporation of UZ transport into the TSPA-LA is conservative for fracture-release cases), while Section 7.6.2 focuses on postdevelopment validation of the model.

\subsubsection{Confidence Building during Model Development}

In the active fracture model conceptualization (Liu et al. 1998 [DIRS 105729], pp. 2638 to 2641), only a portion of fracture networks are active (hydraulically conductive) under unsaturated conditions. The active portion is defined as a function of water saturation $\mathrm{S}$ to the power of the active fracture parameter $\gamma(0 \leq \gamma \leq 1)$ (Liu et al. 1998 [DIRS 105729], pp. 2638 to 2641). When $\gamma=0$ or $S=1$, (corresponding to a saturated condition) all fractures are active, while $\gamma=1$ indicates the smallest active fracture portion for a given saturation.

Using carbon-14 data (and incorporating the uncertainty involved in these data), the active fracture parameter $\gamma$ for tsw32-tsw38 were estimated to be 0.4 or less (BSC 2004 [DIRS 170035], Section 7.4.1). There is a discrepancy between these estimates and the $0.57-0.6$ values obtained through inverse modeling in the calibrated flow model (DTN: LB0208UZDSCPMI.002 [DIRS 161243]). Note that the calibrated values of $\gamma$ (approximately 0.6) were used in all three-dimensional site-scale simulations in this model report.

The impact of uncertainties in $\gamma$ on three-dimensional site-scale flow and transport through the UZ was investigated by BSC (2004 [DIRS 169861], in Section 6.8). The results of this sensitivity analysis indicated that (a) the percolation flux through the repository layers, and (b) the saturation and water potential distributions were not sensitive to large variations in the value $\gamma$, that is, reductions by 50 percent (Figure 7-24).

However, the sensitivity studies indicated that transport is more sensitive to uncertainty in the value of $\gamma$ than flow. Additionally, the effect is more pronounced at earlier times because diffusive fluxes become progressively smaller as the concentrations in the matrix and fractures approach equilibrium. The sensitivity of transport to $\gamma$ is demonstrated in Figure 7-25, which shows a substantial retardation in the arrival of a conservative tracer (with the diffusion coefficient of ${ }^{99} \mathrm{Tc}$ ) at the water table when $\gamma$ is reduced by 50 percent from its original calibrated value of approximately 0.6. In this case (which involved tracer release directly into the repository fractures), $t_{10}$ increased by over an order of magnitude from 40 to 650 years, while $t_{50}$ doubled from 3500 years to 7000 years.

This study demonstrates that the calibrated $\gamma$ value of approximately 0.6 for the hydrostratigraphic units underneath the repository horizon provided conservative estimates of transport times through the UZ (BSC 2004 [DIRS 169861] Section 6.8.2) when the tracers were 
released directly into the repository fractures. Note, however, that this value of $\gamma$ leads to an optimistic breakthrough estimate if the tracers are released into the matrix of the repository gridblocks (BSC 2004 [DIRS 170040] Section 6.3.3).

\subsubsection{Postdevelopment Validation}

The postdevelopment validation for the active fracture model with matrix diffusion combines the two parts described in the beginning of Section 7.6, that is, the active fracture model and the matrix diffusion model.

Evidence of matrix diffusion from the Alcove 8/Niche 3 Phase 1 Line-Release (fault) test is somewhat limited because the fracture saturation conditions along the transport pathway (i.e., at or near the fault) are believed to have been highly saturated (inferred from the $2 \mathrm{~cm}$ positive pressure head applied at the infiltration plot, see Section 7.3.1). Further testing in Yucca Mountain tuffs, under unsaturated conditions with multiple tracers, could significantly strengthen the validation arguments presented in this report. Two such tests are planned: the Alcove 8/Niche 3 Phase 2 large-plot test, and the cubic-meter-block laboratory test. Relevant unsaturated transport data are currently unavailable from either of these tests, a possibility that was foreseen in the technical work plan (BSC 2004 [DIRS 167969])). The technical work plan (BSC 2004 [DIRS 167969])) covering this model report selected independent technical review as a second method for postdevelopment model validation. However, the independent technical review was not utilized in this case. The independent technical review was not considered necessary for adequate validation given the independent validations conducted for the active fracture model (BSC 2004 [DIRS 170035], Section 7) and matrix diffusion models (see Section 7.3). Future confirmation testing of the UZ transport model is addressed in the Performance Confirmation Plan (Snell et al. 2004 [DIRS 169101], Section 3.1.3).

The validation approach for the active fracture model with matrix diffusion is based on the validation of the active fracture model flow model and the matrix diffusion model separately. It combines the active fracture model, which is validated in a separate model report (BSC 2004 [DIRS 169861]), with the matrix diffusion model, validated in Section 7.3 of this report. The validity of the active fracture model with matrix diffusion for transport model, for its intended use, is inferred from that of the two separate component models. Additional validation is not necessary because both the active fracture model validation and the matrix diffusion model validation have both active fracture (unsaturated flow through fractures) and matrix diffusion (diffusive exchange between fracture and matrix) processes represented in the validation data. 


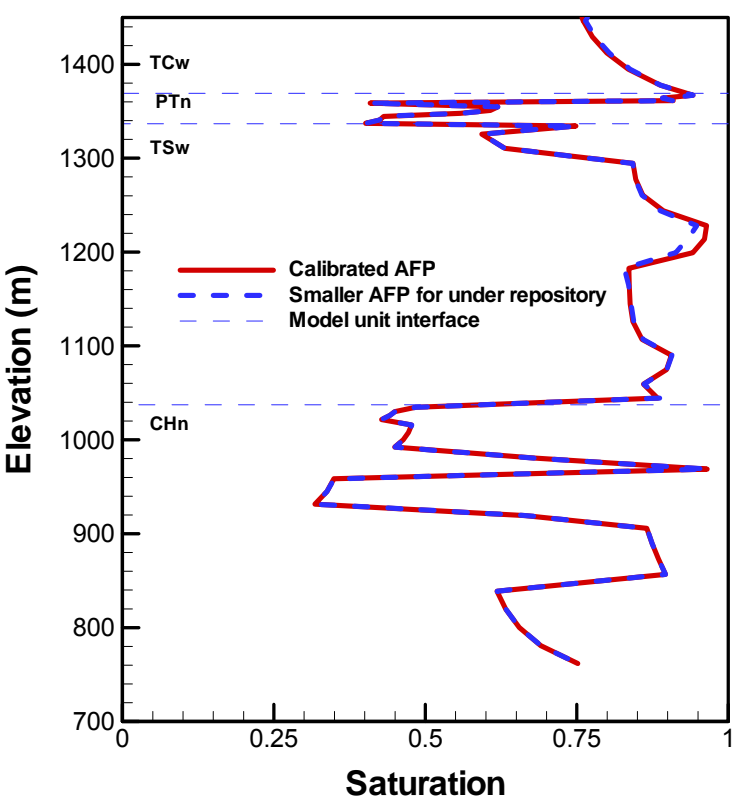

(a)

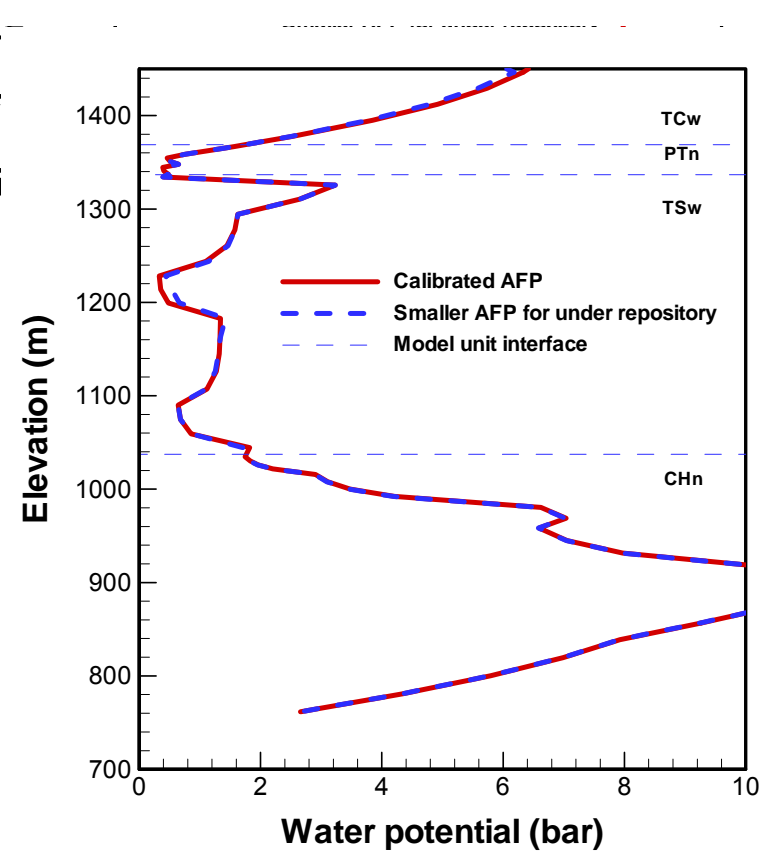

(b)

Model Results-DTN: LB0304RDTRNSNS.001 [DIRS 165992].

Figure 7-24. Comparison of (a) Simulated Matrix Liquid Saturation and (b) Water Potentials Using Calibrated Hydraulic Properties (Solid Line) with That Obtained Using a Smaller (Half) Value of $\gamma$ in the Units of Under Repository (Dashed Line) for Borehole USW SD-6 - From BSC 2004 [DIRS 169861], Section 6.8, Figure 6.8-2 


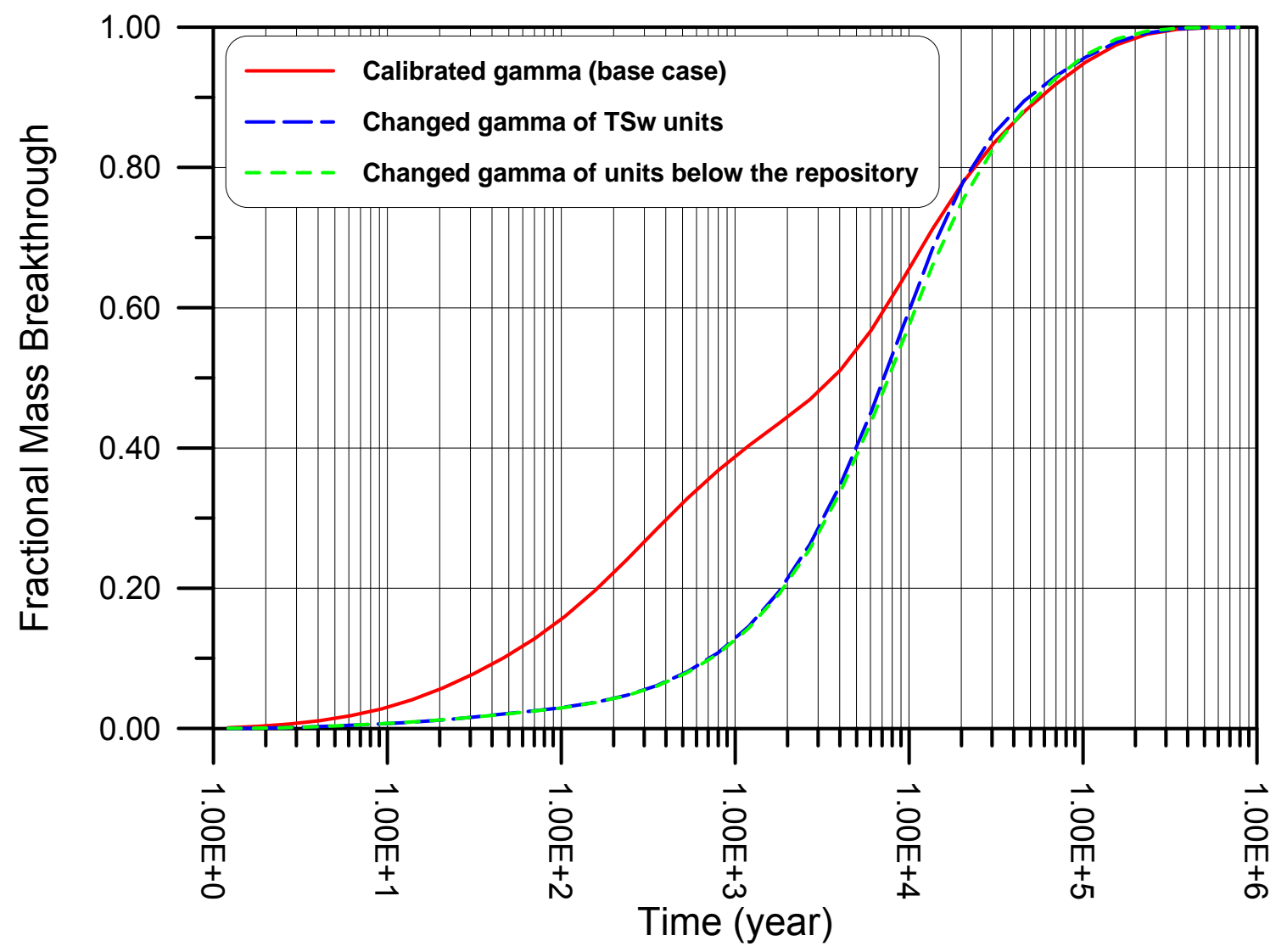

Model Results-DTN: LB0304RDTRNSNS.001 [DIRS 165992].

Figure 7-25. Comparison of a Simulated Breakthrough Curve of Relative Tracer Mass at the Groundwater Table Obtained for the Base Case (Using Calibrated Rock Hydraulic Properties, Red Solid Line), a Case Using a Smaller (Half) Value of $\gamma$ of the TSw Units (Blue Dash Line), and Another Case Using a Smaller (Half) Value of $\gamma$ of All Units below the Repository (Green Solid Line) - From BSC 2004 [DIRS 169861], Section 6.8, Figure 6.8-3

\subsection{PUBLICATION IN REFEREED PROFESSIONAL JOURNAL}

Publication in a referred professional journal is an additional postdevelopment validation method identified in Technical Work Plan for: Performance Assessment Unsaturated Zone (BSC 2004 [DIRS 167969] Section I-2-1-1). The citations provided in this section are included as an additional postdevelopment validation method.

Using T2R3D, Wu and Pruess (2000 [DIRS 153972]) studied the site-scale three-dimensional transport of radioactive solutes in the journal Advances in Water Resources. A scientific study by Moridis et al. (2003 [DIRS 161902]) of site-scale three-dimensional transport of radioactive colloids in the UZ of Yucca Mountain using EOS9nT was recently published in the Journal of Contaminant Hydrology. Both studies are based on essentially the same radionuclide transport model as the one discussed here, but it involved a three-dimensional grid from the previous revision of the relevant model reports (BSC 2001 [DIRS 161340]; BSC 2004 [DIRS 169861]). 
Additionally, EOS9nT is currently in use by Gesellschaft für Anlagen und Reaktorsicherheit (GRS) $\mathrm{mbH}$, a research and engineering organization owned by the Federal Government of Germany, the state of Bavaria, the state of North Rhine-Westphalia, the Technical Inspection Organization, and the Germanischer Lloyd. GRS has a substantial involvement in radioactivity issues, focusing on studies of the effects of potential radioactivity releases. The work of GRS using the radionuclide transport model embodied in EOS9nT is documented in two GRS reports (Javeri 2001 [DIRS 161966]; 2002 [DIRS 161967]).

\subsection{SUMMARY}

The UZ radionuclide transport model was developed to include the physical and chemical processes that could affect transport in the UZ at Yucca Mountain. These processes were advection, dispersion, diffusion, sorption, and colloid-facilitated transport. The model development included mathematical description of the governing equations (Section 6.2). Confidence building during model development and postdevelopment validation activities were conducted according to the technical work plan for this model report (BSC 2004 [DIRS 171282], Section 2.2.2; BSC 2004 [DIRS 167969], Section I-2-1.

The correct implementation of the governing equations was validated in postdevelopment activities (Section 7.2.3). Additional postdevelopment validation included comparisons of the model against experimental data collected from the Busted Butte Field Tests (Section 7.2.4.1) and chloride and ${ }^{14} \mathrm{C}$ concentration profiles from Yucca Mountain boreholes (Section 7.2.4.2). Specific submodels were validated through additional model comparisons to the Alcove $8 /$ Niche 3 Fault Test (Section 7.3) and the active fracture model with matrix diffusion (Section 7.6).

The spatial and temporal scale of the postdevelopment activities is obviously less than that over which transport may occur in the UZ beneath the repository. The spatial and temporal aspects of the $\mathrm{UZ}$ radionuclide transport model are captured at a proper scale, however, in the development, calibration, and validation of the UZ flow model (BSC 2002 [DIRS 169861]).

By these confidence building and validation activities, the UZ radionuclide transport model can be said to perform satisfactorily for its intended purpose of simulating transport under conditions of steady-state flow. The components of the UZ radionuclide transport model that are validated are the matrix diffusion submodel, the sorption submodel, the colloid transport submodel, and the active fracture with matrix diffusion submodel. 


\section{CONCLUSIONS}

Section 8 is composed of three subsections. Section 8.1 summarizes the modeling activity in the present model report. The main conclusions from the individual studies discussed in Section 6 are presented in Section 8.2, which includes conclusions from the two-dimensional studies, the three-dimensional site-scale studies for instantaneous and continuous release of solutes and colloids, and corresponding sensitivity analyses. Section 8.3 focuses on the model output, uncertainties, and limitations.

\subsection{SUMMARY OF MODELING ACTIVITY}

Modeling in Sections 6.8 through 6.14 involved the application of T2R3D V1.4 (LBNL 1999 [DIRS 146654]; Wu et al. 1996 [DIRS 100649]) to study the transport of important radionuclides ${ }^{99} \mathrm{Tc},{ }^{237} \mathrm{~Np},{ }^{239} \mathrm{Pu},{ }^{233} \mathrm{U},{ }^{235} \mathrm{U},{ }^{241} \mathrm{Am},{ }^{135} \mathrm{Cs},{ }^{90} \mathrm{Sr},{ }^{226} \mathrm{Ra},{ }^{229} \mathrm{Th}$, and $\left.{ }^{231} \mathrm{~Pa}\right)$ in the entire Yucca Mountain system, using large-scale three-dimensional grids and assuming an instantaneous (single event) release scenario.

Modeling in Section 6.15 involved the application of TOUGH2 V1.4 Module EOS9 V1.4 (LBNL 2000 [DIRS 146496]) to study the transport of ${ }^{99} \mathrm{Tc},{ }^{237} \mathrm{~Np}$, and ${ }^{239} \mathrm{Pu}$ in the entire Yucca Mountain system, using large-scale three-dimensional grids and assuming a continuous release scenario. In Section 6.16 and 6.17, TOUGH2 V1.4 Module EOS9 V1.4 (LBNL 2000 [DIRS 146496]) was used to study the three-dimensional site scale transport of the ${ }^{239} \mathrm{Pu} \longrightarrow{ }^{235} \mathrm{U}$

$\longrightarrow{ }^{231} \mathrm{~Pa}$ chain and of the ${ }^{241} \mathrm{Am} \longrightarrow{ }^{237} \mathrm{~Np} \longrightarrow{ }^{233} \mathrm{U} \longrightarrow{ }^{229} \mathrm{Th}$ chain, respectively, under a continuous release scenario.

The modeling activity in Section 6.18 involved the application of EOS9nT to study the transport of four true colloids of different sizes and properties in the entire Yucca Mountain system, using large-scale three-dimensional grids. Modeling using the T2R3D (LBNL 1999 [DIRS 146654]) and EOS9nT codes in Section 6.19 aimed at evaluating the Yucca Mountain UZ system performance under (a) an alternative conceptual model of representing the fracture-matrix system, and (b) an alternative modeling approach involving particle tracking. T2R3D and EOS9nT simulations in Section 6.20 evaluated the impact of the standard radionuclide release model (in which radionuclides are released simultaneously throughout the entire repository) by limiting releases to locations that are not in the immediate vicinity of permeable faults.

\subsection{HOW THE APPLICABLE ACCEPTANCE CRITERIA ARE ADDRESSED}

The following information describes how this analysis addresses the acceptance criteria in the Yucca Mountain Review Plan (NRC 2003 [DIRS 163274], Section 2.2.1.3.7.3). Only those acceptance criteria that are applicable to this report (see Section 4.2) are discussed. 


\section{Acceptance Criteria from Section 2.2.1.3.7.3, Radionuclide Transport in the Unsaturated Zone}

\section{Acceptance Criterion 1, System Description and Model Integration Are Adequate.}

Subcriterion (1): The methodology accounts for important design features and physical phenomena at a level consistent with the available data and uncertainty such that the radionuclide transport in the unsaturated zone is not underestimated. Section 6.1 adequately discusses the geological model and stratigraphy in the unsaturated zone of Yucca Mountain by addressing the phenomena, processes, and factors, which affect transport of radionuclides. Included are advection, hydrodynamic dispersion, sorption, matrix diffusion, solubility, colloid transport, formation of perched water, effects of repository-generated heat, and decay of radionuclides to daughter products. Section 6.4 adequately describes where the processes are incorporated in the document and provides a justification for each use of features, events, and processes (FEPs).

Subcriterion (2): Sections 6.1 and 6.5 provide an adequate discussion of the geological model and physical processes that are modeled. Section 6.1 adequately discusses the factors affecting transport of radionuclides in the unsaturated zone. It also covers important issues related transport behavior such as measurement of $\mathrm{K}_{d}$ values, solute sorption issues, colloidal behavior in saturated and unsaturated media, and important considerations in colloid transport. Section 6.1.5.1 analyzes the basic transport flow assumptions and stipulations and provides a statement of applicability and justification for use of each. Section 6.5 discusses the sorption model and the development of the diffusion coefficients $\left(\mathrm{K}_{\mathrm{d}}\right)$. Appendix $\mathrm{A}$ and Section 6.5 provides a detailed discussion of the derivation process and supporting laboratory data that form the bases for selecting $\mathrm{K}_{\mathrm{d}}$ distributions that are used in the TSPA.

Subcriterion (3): Transport relationships at the interface between the waste emplacement drift and the rock are accounted for in a manner that is consistent with the various flow and transport model abstractions used in the TSPA. Sections 6.1, 6.2, and 6.5 justify use of assumptions, technical bases, data and appropriate models. Section 6.2, in particular, provides the transport mathematical model, including the mass balance equations used, sorption terms affecting transport, colloid filtration terms, colloid-assisted transport terms, flux terms, and equations for radioactive decay and transport of daughter products. The descriptions and technical bases provide transparent and traceable support for the TSPA. As discussed in Subcriterion (2), Appendix A and Section 6.5 provides the bases for selecting $\mathrm{K}_{\mathrm{d}}$ distributions that are used in the TSPA.

Subcriterion (5): Section 4.1 lists those data that are direct inputs to TSPA-LA. Of these, only the data relating to sorption ultimately feed into the TSPA-LA. Appendix A provides the sorption experimental results that were used to develop cumulative probability distributions for TSPA-LA which are summarized in Table 6-3. Table 6-2 in Section 6.4 identifies those FEPS that were included in the TSPA-LA through this report. The table also provides a mapping of locations in the report where details of the FEPs are discussed. Section 6.1 describes the technical bases for including the various factors affecting transport in the unsaturated zone.

Subcriterion (6): This document has been developed under AP-SIII.10Q, Models.. 


\section{Acceptance Criterion 2: Data Are Sufficient for Model Justification.}

Subcriterion (1): Data on the geology and hydrology of the UZ, including fracture distributions, fracture properties and stratigraphy, used in the TSPA, are collected using appropriate techniques and are sufficient for describing the conceptual understanding of the UZ at Yucca Mountain because they are developed from comparable field sites. Sections 4.1 and 6.4 describe the sources of data and the locations in the document that the FEPs, which the data supports, are used, respectively. Adequate justifications for the data development and interpretation are provided in the supporting documents listed in Section 4.1. Detailed descriptions of how the data was interpreted and applied are provided, where applicable, throughout Section 6.

Subcriterion (2): Section 6.1.5.4 discusses initial and boundary conditions for the transport model that are based on the sufficient data (discussed in Section 4.1) and characteristics of the unsaturated zone. This model provides suitable estimates for the boundary conditions for the unsaturated zone mountain-scale transport abstraction in terms of the partitioning of radionuclide releases from waste emplacement drifts to the fractures and rock matrix supporting the inclusion of related FEPs.

Subcriterion (3): Yucca Mountain specific field tests, experiments, and geological data were developed by use of appropriate accepted techniques. Data sources described in Section 4.1 justify the acceptability of the data obtained from laboratory experiments, site-specific field measurements, and process-level modeling studies. Section 6.6 sufficiently discusses the geologic setting and influence of structural features, fracture distributions, fracture properties, and stratigraphy in developing the model for radiation transport in vertical columns based on three representative unsaturated zone locations. Section 7.2.4.2 points to a comparison of ${ }^{14} \mathrm{C}$ and pore water chloride concentration in the ESF that were not used as input to simulation for model validation.

\section{Acceptance Criterion 3: Data Uncertainty Is Characterized and Propagated through the Model Abstraction.}

Subcriterion (1): The models used in this report are described in Sections 6.1 to 6.3, 6.5 to 6.7, and validations of the models are presented in Sections 7.2 to 7.7. These models are based on and conservatively predict unsaturated zone transport at Yucca Mountain and are, therefore, technically defensible, account for uncertainties, and do not result in an under-representation of the risk. In fact, as discussed in Section 8.2.1, the underlying approach of the model is extremely conservative due to its involving successive levels of worst-case scenarios.

Subcriterion (2): Sections 6.8 to 6.18 examine radionuclide transport for individual radionuclides and decay chains. Sections 7.2-7.6 discuss validation of transport (7.2), diffusion (7.3), sorption (7.4), colloid transport (7.5) and active fracture (7.6) models by laboratory experiments, field measurements (7.2), and process-level modeling studies (7.2) that were conducted under conditions relevant to the unsaturated zone at Yucca Mountain. The models have been demonstrated to adequately and conservatively reproduce field transport test results through comparison with the Busted Butte test series and the Alcove 8-Niche 3 tests. 
Subcriterion (4): Sections 6.8 to $6.10,6.20$, and 8.3.2 adequately represent uncertainties in the various models and underlying data. Section 6.8 reviews varying climate scenarios that are used to analyze overall uncertainty in UZ transport for the TSPA. Section 6.9 discusses parametric uncertainties affecting sorption and matrix diffusion and Section 6.10 reviews matrix diffusion uncertainties. Section 8.3.2.4 discusses a sensitivity analysis of ${ }^{237} \mathrm{~Np}$ transport through the unsaturated zone for different $\mathrm{K}_{\mathrm{d}}$ values and related uncertainties.

Acceptance Criterion 4, Model Uncertainty Is Characterized and Propagated Through the Model Abstraction.

Subcriterion (1): Section 6.19 discusses alternative models considered. These models consisted of (1) a different representation of the matrix-fracture system and the interaction of solutes between these media and (2) different numerical methods to solve the transport equations. These models were developed to be consistent with available data and current scientific understanding.

Subcriterion (2): Sections 7.2 through 7.7 adequately document validation of the various conceptual models, model uncertainties, and affects on the conclusions regarding system performance. Section 6.7.8 summarizes the various conceptual model uncertainties and how each aspect of model implementation conservatively represents transport. For example, vertical fractures are modeled as continuous throughout the UZ and do not retard transport through solute sorption or colloid attachment onto the fracture walls.

Subcriterion (3): As discussed in Sections 6.1 to $6.7,7.2$ to 7.6, and 8.3, model uncertainty is consistent with available site characterization data, laboratory experiments, field measurements, and process-level modeling studies as shown in comparison of simulation results with the Busted Butte test series and the Alcove 8-Niche 3 tests. Sections 6.7.8 and 8.2.1 describes why this very conservative model and the assumptions employed with it do not under-represent the risk estimate.

Subcriterion (4): Section 6.19 discusses alternative modeling approaches consistent with available data and knowledge. Again, these models consisted of (1) a different representation of the matrix-fracture system and the interaction of solutes between these media and (2) different numerical methods to solve the transport equations. These alternative approaches are consistent with the current state of knowledge relating to alternative methods to represent transport in a porous fractured medium. In addition, Section 7.6 discusses validation of the active fracture model.

\subsection{MODEL REPORT CONCLUSIONS}

The following conclusions can be drawn from this study:

\subsubsection{General Conclusions from the Three-Dimensional Studies}

1. Three-dimensional site-scale simulations using EOS9nT indicate that, under the \#1 perched-water model, radionuclide transport from the bottom of the repository to the water table is both dominated and controlled by the faults. These provide fast pathways for downward migration to the water table, but also limit lateral transport across the fault walls into the formation. 
2. The three-dimensional site-scale T23RD and EOS9nT simulations for both an instantaneous and a continuous release regime indicate that radioactive solutes from the repository move faster and reach the water table earlier and over a larger area in the northern part of the repository. This appears to be consistent with the geologic model of the UZ, which is characterized by the presence of highly fractured zeolitic $\mathrm{CHz}$ layers in that area. The fractures in these layers are several orders of magnitude more conductive than the corresponding matrices. Thus, flow is fracture-dominated, and results in the transport pattern observed in this study.

3. The transport patterns follow the infiltration and percolation distributions. A review of the infiltration pattern at the surface, the percolation flux at the repository level, and the percolation flux at the groundwater level indicate that they closely reflect the transport patterns discussed in Conclusion 2 above. Thus, the water flow pattern dictates the advective transport pattern.

4. For both an instantaneous and a continuous release regime, the highly conductive Drill Hole Wash fault and Pagany Wash fault are the main pathways of transport (and related to the percolation patterns) for the dominance of the northern part of the repository. Radionuclides released directly into these faults or reaching them from adjacent areas move rapidly downward to reach the water table at an earlier time. Additionally, the Sundance fault, the Solitario fault, and the Sever Wash fault are transport-facilitating geological features, but their effect becomes significant at later times.

5. Fractures, including faults, are the main pathways of radionuclide transport. Diffusion from the fractures into the matrix is one of the main retardation processes in radionuclide transport. When radionuclides sorb onto the matrix into which they diffuse, their migration is further retarded. Sorption also leads to retardation in the fractures by resulting in larger concentration gradients (thus enhanced diffusion) between the liquid phases in the fractures and in the matrix.

6. Caution should be exercised in the analysis and interpretation of the three-dimensional simulation results because the underlying approach is extremely conservative, involves successive (and multiplicative) levels of worst-case scenarios, and is improbable (if not outright impossible). Thus, in this model report (a) all the waste packages are assumed to rupture at the same time throughout the repository; (b) water is allowed to seep into the repository by overcoming the capillary pressure barrier, to enter uninhibited the breached canisters, and to carry radionuclides directly into the fractures; (c) drip shields are not considered; (d) the retardation effects of the invert and of the shadow zone are ignored; (e) the vertical fractures are assumed to be open and continuous throughout the UZ from the repository to the water table; (f) the horizontal fractures are assumed to be interconnected, and are also connected (directly or indirectly) to the vertical fractures; and ( $\mathrm{g}$ ) the radioactive tracers (solutes or colloids) are stable, unaffected by the near field conditions (thermal, geochemical, physical), and are not subject to chemical immobilization (e.g., through irreversible sorption or precipitation) anywhere in the UZ. 
7. Note that the importance of faults and perched-water bodies in transport is directly dependent on the underlying geologic and perched-water conceptual models. It is reasonable to expect that changing geologic and perched-water models may lead to substantially different results given the sensitivity of transport to these geologic features. This realization underlines the importance of representative conceptual models.

\subsubsection{Conclusions from the Three-Dimensional Studies of Instantaneously Released Radioactive Solutes}

8. For present-day climatic conditions (Table 6-5) and instantaneously released radionuclides, the $t_{10}$ (see definition in Section 6.8) of the nonsorbing ${ }^{99} \mathrm{Tc}$ for low, mean, and high infiltration is 13,$900 ; 83$; and 6 years, and the $t_{50}$ (see definition in Section 6.8) is over 1,000,000; 6,640; and 230 years, respectively (Table 6-10). Under the same conditions, the $t_{10}$ of the moderately sorbing ${ }^{237} \mathrm{~Np}$ for low, mean, and high infiltration is 33,$800 ; 410$; and 4 years, and the corresponding $t_{50}$ is over $1,000,000$; 25,400; and 1,600 years, respectively. The strongly sorbing ${ }^{239} \mathrm{Pu}$ did not exhibit a sufficiently high breakthrough level at the water table for $t_{10}$ or $t_{50}$ to become meaningful in most cases (the only exception was a $t_{10}=1,530$ year for a high present-day infiltration.

9. For present-day climatic conditions (Table 6-5) and instantaneously released radionuclides, the $t_{10}$ (see definition in Section 6.8) of the moderately sorbing ${ }^{233} \mathrm{U}$ for low, mean, and high infiltration is 65,200; 433; and 12 years, and the $t_{50}$ (see definition in Section 6.8) is over 1,000,000; 29,100; and 1,120 years, respectively (Table 6-10). The $t_{10}$ and $t_{50}$ for ${ }^{235} \mathrm{U}$ are very close. These results indicate that the ${ }^{233} \mathrm{U}$ and ${ }^{235} \mathrm{U}$ behavior is similar to that of ${ }^{237} \mathrm{~Np}$. Under the same conditions, the $t_{10}$ of the variably sorbing ${ }^{135} \mathrm{Cs}$ (very different sorption affinity for different rocks, see Table 6-3) for low, mean, and high infiltration is 1,000,000; 52,500; and 2,170 years, and the corresponding $t_{50}$ is over 1,000,000; over 1,000,000; and 71,200 years, respectively.

10. For present-day climatic conditions (Table 6-5) and instantaneously released radionuclides, the cumulative breakthrough curves of ${ }^{241} \mathrm{Am},{ }^{231} \mathrm{~Pa},{ }^{229} \mathrm{Th},{ }^{226} \mathrm{Ra}$, and ${ }^{90} \mathrm{Sr}$ indicate very limited (if any) radionuclide arrivals at the water table (Table 6-10) because of very strong sorption (all) and short half-lives $\left({ }^{241} \mathrm{Am}\right.$ and $\left.{ }^{90} \mathrm{Sr}\right)$.

11. The results discussed in Conclusions 8 through 10 lead to the conclusion that transport to the water table is strongly dependent on the sorption affinity of the radionuclide to the geohydrologic units it encounters in the UZ. Generally speaking, lower $K_{d}$ values (quantifying weaker sorption) lead to faster radionuclide transport to the water table.

12. The three-dimensional site-scale simulations indicate that a change in the future climatic conditions can have a significant effect on transport. Increasing infiltration (as the climatic regime changes from present-day, to monsoon, to glacial-transition conditions, and its level changes from low to mean to high) leads to faster radionuclide transport to the water table. The acceleration in transport increases with decreasing sorption. 
13. Matrix diffusion (as quantified by the molecular diffusion coefficient $D_{0}$ ) has a significant impact on breakthrough predictions, resulting in faster arrival times for lower $D_{0}$ values. The relative effect of $D_{0}$ on breakthrough predictions appears to be mitigated by sorption, that is, the importance of $D_{0}$ in transport decreases with an increasing $K_{d}$.

\subsubsection{Conclusions from the Three-Dimensional Studies of Continuously Released Radioactive Solutes}

14. For mean present-day climatic conditions (Table 6-5) and continuously released radionuclides from a decaying source, the $t_{10}$ (see definition in Section 6.15 ) of ${ }^{99} \mathrm{Tc}$ and ${ }^{237} \mathrm{~Np}$ is 74 and 781 years, and the $t_{50}$ (see definition in Section 6.15) is 3,901 and 22,940 years, respectively (Table 6-11). In the case of the strongly sorbing ${ }^{239} \mathrm{Pu}, R_{F}$ did not reach the 0.1 level in the $1,000,000$ years of simulation.

15. In the simulation of three-dimensional transport of ${ }^{239} \mathrm{Pu}$, the members of the decay chain may have to be considered (i.e., ${ }^{239} \mathrm{Pu}{ }^{235} \mathrm{U}$, and ${ }^{231} \mathrm{~Pa}$ ) if the ${ }^{239} \mathrm{Pu}$ release rate at the repository is significant (Table 6-11). Although the $t_{10}$ for ${ }^{239} \mathrm{Pu}$ transport is always larger than $1,000,000$ years, ${ }^{235} \mathrm{U}$ becomes the main contributor in the radionuclide release at the water table after 10,000 years (at most) because of its weaker sorption onto the matrix and its longer half-life. The contributions of ${ }^{231} \mathrm{~Pa}$ are not important. Under mean present-day climatic conditions, the $t_{10}$ and the $t_{50}$ of the sum of the members of the ${ }^{239} \mathrm{Pu}$ chain are computed from the three-dimensional simulations as 6,419 and 33,660 years, respectively.

16. The analysis of three-dimensional transport of the members of the ${ }^{241} \mathrm{Am}$ chain indicates that only the ${ }^{237} \mathrm{~Np}$ daughter is significant in the 1,000,000-year time frame of this study. The strong sorption affinity and the short $T_{1 / 2}$ (432.2 years) of ${ }^{241} \mathrm{Am}$ precludes a significant role in the radionuclide stream arriving at the water table, while the long half-life of ${ }^{237} \mathrm{~Np}$ prevents its daughters from dominating the releases at the water table. Note that the $t_{10}$ and $t_{50}$ of the sum of the ${ }^{241} \mathrm{Am}$ chain members $(1,027$ and 23,450, respectively) are roughly the sum of $t_{10}$ and $t_{50}$ of the transport of pure

${ }^{237} \mathrm{~Np}$ (see Conclusion 17 and Table 6-11) and the $T_{1 / 2}$ (432.2 years) of ${ }^{241} \mathrm{Am}$.

\subsubsection{Conclusions from the Three-Dimensional Studies of Continuously Released Radioactive Colloids}

17. For continuous colloid release under a mean present-day infiltration regime and for a given kinetic clogging (forward filtration) coefficient $\kappa^{+}$, the transport of radioactive true colloids is not appreciably influenced by the kinetic declogging (reverse filtration) coefficient $\kappa^{-}$in larger colloids. This is caused by (a) the inability of the colloids to reach the matrix because of straining and a low $D_{0}$ (a result of their large size), and (b) the corresponding very limited filtration in the matrix. 
18. The colloid size has a significant effect on transport. Large colloids reach the water table faster. Because fractures are the main transport conduit at Yucca Mountain, the inability of larger colloids to diffuse into the matrix because of smaller $D_{0}$ values and size exclusion (straining) result in faster transport to the groundwater. Smaller colloidal particles can diffuse more easily into the matrix, and their transport is thus retarded.

19. When there is no filtration in the fractures (Cases 1 to 3 ), the results of the simulations of transport of ${ }^{239} \mathrm{PuO}_{2}$ colloids of four different sizes $(450 \mathrm{~nm}, 200 \mathrm{~nm}, 100 \mathrm{~nm}$, and $6 \mathrm{~nm}$ ) indicate that there is very limited retardation of the larger colloids, all of which have a very similar $t_{10}$ (Table 6-12). Note that $t_{10}$ increases with a decreasing size, but the change is very small (from 4.35 to 4.53 years when the colloid diameter decreases from $450 \mathrm{~nm}$ to $100 \mathrm{~nm}$ ). Although $R_{F}$ does not reach the 0.5 level, thus, $t_{50}$ cannot be defined, it rises rapidly and reaches 0.4 in about 20 years. Conversely, the retardation of the smallest colloid ( $6 \mathrm{~nm}$ ) is very significant, and $R_{F}$ is well below the 0.1 level.

20. When limited filtration in the fractures is considered (Case 4), the results of the simulations of transport of ${ }^{239} \mathrm{PuO}_{2}$ colloids of four different sizes $(450 \mathrm{~nm}, 200 \mathrm{~nm}$, $100 \mathrm{~nm}$, and $6 \mathrm{~nm}$ ) are similar to those for no filtration in the fractures. However, although $t_{10}$ is still low, it is significantly larger than that for no fracture filtration, and decreases from 32.4 to 27.6 years when the colloid diameter decreases from $450 \mathrm{~nm}$ to $100 \mathrm{~nm}$ (Table 6-12). In this case, $R_{F}$ rises rapidly and reaches 0.4 in about 100-150 years. As in the case for no fracture filtration, the retardation of the smallest colloid (6 $\mathrm{nm}$ ) is very significant, and $R_{F}$ remains well below 0.1 during the simulation period.

21. Size exclusion (straining) at the interfaces of different hydrogeologic layers leads to colloid concentrations immediately above the interface that can be higher than that in the water released from the repository. This phenomenon is more pronounced in larger colloids.

\subsubsection{Conclusions from the Study of Alternative Conceptual Models}

22. When the alternative conceptual model of MINC (to describe the fracture-matrix system) is employed, transport is delayed due to enhanced fracture-matrix interaction.

\subsubsection{Conclusions from the Barrier Evaluation}

23. Even under the improbably conservative approach involved in the three-dimensional site-scale studies (Section 6.7.8 and Conclusion 9), the UZ of Yucca Mountain appears to be an effective barrier to the transport of the strongly sorbing radionuclides $\left({ }^{90} \mathrm{Sr}\right.$, ${ }^{226} \mathrm{Ra},{ }^{229} \mathrm{Th},{ }^{241} \mathrm{Am},{ }^{231} \mathrm{~Pa}$, and ${ }^{239} \mathrm{Pu}$ ) to the water table.

24. The variably sorbing ${ }^{135} \mathrm{Cs}$ (strongly on zeolites, much less on other rocks), the mildly sorbing ${ }^{233} \mathrm{U},{ }^{235} \mathrm{U},{ }^{237} \mathrm{~Np}$, and the nonsorbing ${ }^{99} \mathrm{Tc}$ arrive at the water table at times that are fractions of their respective half-lives. However, this is not necessarily an indication of a breached or ineffective UZ barrier, but can be a direct consequence of the conceptual model of UZ flow and of the improbably conservative (if not practically impossible) approach described in Section 6.7.8. A more definitive 
evaluation of the barrier can be provided by using a more realistic scenario accounting for the successive retardation effects of the processes discussed in Section 6.7.8.

25. Eliminating potential sources from the vicinity of the fault fractures appears to have only a small effect on transport and arrivals at the water table. For instantaneous release (when a finite radionuclide mass is involved), the $R_{M}$ breakthrough curves in Figures 6-65 and 6-66 show a small increase in $t_{10}$ (in line with expectations because there is no release into the fast-conducting fault fractures), but $t_{50}$ is practically unchanged. The $R_{F}$ breakthrough curves in Figures 6-67 and 6-68 for continuous release show practically no discernible difference from those that account for release into the faults (Section 6.15).

\subsection{MODEL OUTPUT, UNCERTAINTIES, AND LIMITATIONS}

\subsubsection{Model Output}

As indicated in technical work plan (BSC 2004 [DIRS 171282], Section 2.1.1), the output/product of this model report is a set of breakthrough curves for use as benchmarks for the particle-tracking calculations performed for TSPA-LA (BSC 2004 [DIRS 170041]). All the breakthrough curves presented and discussed in this model report are included in output DTN: LB0307MR0060R1.007, which incorporates both the graphical representations and the model output used for their development. All output DTNs from this model report are summarized in Table 8-1.

\subsubsection{Model Uncertainties}

As indicated in Section 6, the predictions of transport are subject to three general types of uncertainties.

\subsubsection{Uncertainties in the Flow Conceptual Model and the Corresponding Parameters}

The impact of uncertainties in the flow conceptual model and the corresponding hydraulic parameters is in the estimation of the magnitude and relative sizes of flows and saturations in the fractures, and in the matrices of the UZ, which affect directly both advective transport (and, consequently, transport times to the water table following a radionuclide release) and diffusive fluxes (see Equations 18-21). Although the importance of these uncertainties is readily recognized, addressing them is beyond the scope of this study, but rather the subject of the model report on the UZ flow model (BSC 2004 [DIRS 169861], Section 6). Because the radionuclide transport study is intertwined with the UZ flow uncertainties (from which it derives its flow fields), it suffers from the same flow-related limitations and uncertainties.

\subsubsection{Climatic Uncertainties}

The impact of these uncertainties is assessed by estimating transport under all possible nine climatic scenarios of Table 6-5, thus, bounding the possible solution. From these simulations, the universal conclusion is that the transport time to the water table decreases, and overall transport increases, with an increasing infiltration. 


\subsubsection{Uncertainties in Matrix Diffusion}

Uncertainties in matrix diffusion are reflected in the values of the diffusion coefficient $D_{0}$. To address this issue, the sensitivity of transport through the UZ was investigated for the $D_{0}$ values shown in Table 6-4, which cover the possible $D_{0}$ range for all three possible types of radionuclides (strongly sorbing, mildly sorbing, and nonsorbing). The results of these studies indicated that the $D_{0}$ value has a significant impact on breakthrough predictions, resulting in faster arrival times for lower $D_{0}$ values. The impact increases as the sorption affinity of the radionuclides decreases.

\subsubsection{Uncertainties in Sorption}

Such uncertainties in sorption are reflected in the values of the distribution coefficient $K_{d}$ and are important only in the case of mildly sorbing radionuclides. (The effects for strongly sorbing radionuclides was not investigated, because the studies presented in the model report covered the whole range of possibilities and showed minimal effects). To address this issue, the sensitivity of ${ }^{237} \mathrm{~Np}$ transport through the UZ was investigated for the $K_{d}$ values shown on Figure 6-41. These cover the range between zero (no sorption, in which case behavior similar to that of ${ }^{99} \mathrm{Tc}$ was expected) to the maximum values reported in Table 6-3.

As expected, $K_{d}$ has a significant impact on breakthrough predictions, resulting in faster arrival times for lower $K_{d}$ values. The relative effect of $K_{d}$ uncertainty in the prediction of transport of the sorbing ${ }^{237} \mathrm{~Np}$ appears to be much more pronounced than that for the $D_{0}$ uncertainty for the range tested here.

\subsubsection{Uncertainties in Filtration}

The impact of these uncertainties is assessed by estimating (a) transport for a very wide range of the kinetic filtration parameters (clogging and declogging coefficients), and (b) the impact of filtration in the fractures (by considering minor fracture fillup), thus, bounding the possible solutions. The simulations indicate that the transport is not significantly affected by significantly varying the filtration parameters, transport time to the water table decreases with the colloid size, larger colloids show little retardation while very small ones are retarded significantly, and fracture filtration can have a substantial impact on transport.

\subsubsection{Limitations of Applicability}

The results reported in this model report should be viewed as representative of the upper bound of possible solutions when the approach discussed in Section 6.1.5 is employed. When viewed under this light, these results are applicable without limitations.

Generally speaking, the results and observations in the model report are not applicable to conditions that are not adequately described by the model report approach and underlying assumptions. This scenario could include a different conceptual model of UZ flow and/or considerations of the mitigating processes discussed in Sections 6.7.8 and 6.20.3, which are likely to lead to greater delay of radionuclide transport. Therefore, the model is appropriate for comparison with other transport models supporting TSPA-LA to ensure that radionuclide transport is not underestimated. 
Table 8-1. Output Data and Data Tracking Numbers

\begin{tabular}{|c|c|c|c|c|}
\hline \multirow[b]{2}{*}{ Output DTNs } & \multicolumn{3}{|c|}{ Location in this report } & \multirow[b]{2}{*}{ Remarks } \\
\hline & Text & Figure & Table & \\
\hline LA0408AM831341.001 & 6.1 .1 & & $6-3$ & $\begin{array}{l}\text { Unsaturated Zone Distribution } \\
\text { Coefficients }\left(K_{D}\right) \text { For U, Np, Pu, } \\
\text { Am, Pa, Cs, Sr, Ra, and Th. }\end{array}$ \\
\hline LA0311AM831341.001 & Appendix B & & $\begin{array}{l}\text { B-1 } \\
\text { B-2 }\end{array}$ & $\begin{array}{l}\text { Correlation Matrix for Sampling } \\
\text { of Sorption Coefficient Probability } \\
\text { Distributions. Submittal date: } \\
\text { 11/06/2003. }\end{array}$ \\
\hline LB0307MR0060R1.001 & $\begin{array}{l}6.9 .1 \\
6.9 .2 \\
6.10 .1 \\
6.11 \\
6.12 \\
6.13 \\
6.14 \\
\end{array}$ & & $6-6$ & $\begin{array}{l}\text { Ambient Radionuclide Transport } \\
\text { - Uniform Instantaneous } \\
\text { Release: Simulations } \\
\text { (Input/output files). }\end{array}$ \\
\hline LB0307MR0060R1.002 & $\begin{array}{l}6.8 .1 .3 \\
6.9 .1 \\
6.9 .1 .2 \\
6.9 .2 \\
6.10 .1 \\
6.10 .1 .2 \\
6.11 \\
\text { Appendix D } \\
\text { Appendix H }\end{array}$ & $\begin{array}{l}6-9 \text { through } 6-28 \\
\text { D-1 through D-24 } \\
\text { H-1 through } \mathrm{H}-20\end{array}$ & $6-6$ & $\begin{array}{l}\text { Ambient Radionuclide Transport } \\
\text { - Uniform Instantaneous } \\
\text { Release: Summaries. }\end{array}$ \\
\hline LB0307MR0060R1.003 & $\begin{array}{l}6.7 .2 \\
6.15 .1 \\
6.16 \\
6.17 \\
6.18 .1 \\
\end{array}$ & & $6-6$ & $\begin{array}{l}\text { Ambient Radionuclide Transport } \\
\text { - Uniform Continuous Release: } \\
\text { Simulations. (Input/output files) }\end{array}$ \\
\hline LB0307MR0060R1.004 & $\begin{array}{l}6.15 .4 \\
\text { Appendix E } \\
\text { Appendix F } \\
\text { Appendix G }\end{array}$ & $\begin{array}{l}\text { E-1 through E-20 } \\
\text { F-1 through F-30 } \\
\text { G-1 through G-30 }\end{array}$ & $6-6$ & $\begin{array}{l}\text { Ambient Radionuclide Transport } \\
\text { - Uniform Continuous Release: } \\
\text { Summaries. }\end{array}$ \\
\hline LB0307MR0060R1.005 & 6.20 .2 & & $6-6$ & $\begin{array}{l}\text { Ambient Radionuclide Transport } \\
\text { - No radionuclide releases in the } \\
\text { vicinity of major faults, } \\
\text { Instantaneous and Continuous } \\
\text { Release: Simulations } \\
\text { (Input/output files). }\end{array}$ \\
\hline LB0307MR0060R1.006 & 6.20 .2 & & $6-6$ & $\begin{array}{l}\text { Ambient Radionuclide Transport } \\
\text { - No radionuclide releases in the } \\
\text { vicinity of major faults, } \\
\text { Instantaneous and Continuous } \\
\text { Release: Summaries. }\end{array}$ \\
\hline
\end{tabular}


Table 8-1. Output Data and Data Tracking Numbers (Continued)

\begin{tabular}{|c|c|c|c|c|}
\hline \multirow[b]{2}{*}{ DTN } & \multicolumn{3}{|c|}{ Location in this report } & \multirow[b]{2}{*}{ Remarks } \\
\hline & Text & Figure & Table & \\
\hline LB0307MR0060R1.007 & 8.3 .1 & $\begin{array}{l}6-8 \\
6-33 \\
6-34 \\
6-35 \\
6-36 \\
6-38 \\
6-39 \\
6-40 \\
6-42 \\
6-43 \\
6-45 \\
6-46 \\
6-47 \\
6-48 \\
6-49 \\
6-50 \\
6-51 \\
6-52 \\
6-53 \\
6-54 \\
6-55 \\
6-56 \\
6-57 \\
6-58 \\
6-59 \\
6-60 \\
6-65 \\
6-66 \\
6-67 \\
6-68 \\
\end{array}$ & $\begin{array}{l}6-6 \\
6-10 \\
6-11 \\
6-12\end{array}$ & $\begin{array}{l}\text { Ambient Radionuclide Transport - } \\
\text { TSPA-LA Deliverable Extractions. }\end{array}$ \\
\hline LB0308MR0060R1.008 & $\begin{array}{l}\text { 7.2.3.3 } \\
7.2 .3 .4 \\
7.2 .3 .5 \\
7.2 .3 .6 \\
7.2 .4 .1 .2 .2 \\
7.2 .4 .1 .3 .2 \\
7.2 .4 .1 .4 .2\end{array}$ & & $\begin{array}{l}7-1 \\
7-2 \\
7-3 \\
7-4 \\
7-5 \\
7-9 \\
7-11\end{array}$ & $\begin{array}{l}\text { Ambient Radionuclide Transport - } \\
\text { Model Validation: Simulations } \\
\text { (Input/output files). }\end{array}$ \\
\hline LB0308MR0060R1.009 & & $\begin{array}{l}7-2 \\
7-3 \\
7-4 \\
7-5 \\
7-6 \\
7-8 \\
7-9 \\
7-10 \\
7-11 \\
7-12 \\
7-13 \\
\end{array}$ & $\begin{array}{l}7-6 \\
7-14\end{array}$ & $\begin{array}{l}\text { Ambient Radionuclide Transport - } \\
\text { Model Validation: Data } \\
\text { Summaries. }\end{array}$ \\
\hline LB0310MR0060R1.010 & $\begin{array}{l}6.19 .2 .1 \\
6.19 .2 .2\end{array}$ & $\begin{array}{l}6-37 \\
6-44 \\
6-61\end{array}$ & 6-6 & $\begin{array}{l}\text { Supplemental Radionuclide } \\
\text { Transport Simulations: } \\
\text { Simulations (Input/output files). }\end{array}$ \\
\hline LB0310MR0060R1.011 & $\begin{array}{l}6.19 .2 .1 \\
6.19 .2 .2\end{array}$ & & $6-6$ & $\begin{array}{l}\text { Supplemental Radionuclide } \\
\text { Transport Simulations: Data } \\
\text { Summaries. }\end{array}$ \\
\hline
\end{tabular}




\section{INPUTS AND REFERENCES}

The following is a list of the references cited in this document. Column 2 represents the unique six digit numerical identifier (the Document Input Reference System number), which is placed in the text following the reference callout (e.g., BSC 2004 [DIRS 161340]). The purpose of these numbers is to assist in locating a specific reference. Multiple sources by the same author (e.g., BSC 2004) are sorted alphabetically by title.

\subsection{DOCUMENTS CITED}

Abdel-Salam, A. and Chrysikopoulos, C.V. 1995. "Modeling of Colloid and 146647 Colloid-Facilitated Contaminant Transport in a Two-Dimensional Fracture with Spatially Variable Aperture." Transport in Porous Media, 20, (3), 197-221. Dordrecht, The Netherlands: Kluwer Academic Publishers. TIC: 247547.

Aksyuk, A.M.; Zhukovskaya, T.N.; and Tikhomirova, V.I. 1995. "Sorption of 165054 Strontium on Porphyrite at Room and Enhanced Temperature (Experimental Data)." Proceedings of the Fifth International Conference on Radioactive Waste Management and Environmental Remediation, ICEM '95, held in Berlin, Germany, September 3-7, 1995. Slate, S.; Baker, R.; Benda, G.; Creer, J. and Feizollahi, F., eds. Pages 691-694. New York, New York: American Society of Mechanical Engineers. TIC: 254875.

Allard, B. 1982. Sorption of Actinides in Granitic Rock. SKB TR-82-21. Stockholm, 104512 Sweden: Svensk Kärnbränsleförsörjning A.B. TIC: 205892.

Allard, B. and Beall, G.W. 1979. "Sorption of Americium on Geologic Media." Journal of Environmental Health, A14, (6), 507-518. New York, New York: Marcel Dekker. TIC: 224102.

Allard, B.; Beall, G.W.; and Krajewski, T. 1980. "The Sorption of Actinides in 104410 Igneous Rocks.” Nuclear Technology, 49, (3), 474-480. La Grange Park, Illinois: American Nuclear Society. TIC: 245772.

Allard, B.; Olofsson, U.; Torstenfelt, B.; and Kipatsi, H. 1983. Sorption Behaviour of 162982 Well-Defined Oxidation States. SKB TR-83-61. Stockholm, Sweden: Svensk Kärnbränsleförsörjning A.B. TIC: 206122.

Altman, W.D.; Donnelly, J.P.; and Kennedy, J.E. 1988. Qualification of Existing Data for High-Level Nuclear Waste Repositories: Generic Technical Position. NUREG-1298. Washington, D.C.: U.S. Nuclear Regulatory Commission. TIC: 200652.

Altman, W.D.; Donnelly, J.P.; and Kennedy, J.E. 1988. Peer Review for High-Level 103597 Nuclear Waste Repositories: Generic Technical Position. NUREG-1297. Washington, D.C.: U.S. Nuclear Regulatory Commission. TIC: 200651. 
Apps, J.A. 1992. Current Geochemical Models to Predict the Fate of Hazardous 165225

Wastes in the Injection Zones of Deep Disposal Wells. LBL-26007. Berkeley, California: Lawrence Berkeley Laboratory, Earth Sciences Division.

ACC: MOL.20030919.0119.

Apps, J.A.; Lucas, J.; Mathur, A.K.; and Tsao, L. 1977. Theoretical and Experimental 164864 Evaluation of Waste Transport in Selected Rocks: 1977 Annual Report of LBL Contract No. 45901AK. Waste Isolation Safety Assessment Program-Collection and Generation of Transport Data. LBL-7022. Berkeley, California: Lawrence Berkeley Laboratory. TIC: 210918.

Atun, G.; Bilgin, B.; and Mardinli, A. 1996. "Sorption of Cesium on Montmorillonite and Effects of Salt Concentration." Journal of Radioanalytical and Nuclear Chemistry, 211, (2), 435-442. New York, New York: Elsevier. TIC: 255302.

Bates, J.K.; Bradley, J.P.; Teetsov, A.; Bradley, C.R.; and Buchholtz ten Brink, M. 1992. "Colloid Formation During Waste Form Reaction: Implications for Nuclear Waste Disposal." Science, 256, 649-651. Washington, D.C.: American Association for the Advancement of Science. TIC: 239138.

Beall, G.W.; Lee, W.W.-L.; and Van Luik, A.E. 1986. "Americium Speciation and Distribution Coefficients in a Granitic Ground Water." Scientific Basis for Nuclear Waste Management IX, Symposium held September 9-11, 1985, Stockholm, Sweden. Werme, L.O., ed. 50, 501-508. Pittsburgh, Pennsylvania: Materials Research Society. TIC: 203664.

Bear, J. 1972. Dynamics of Fluids in Porous Media. Environmental Science Series. Biswas, A.K., ed. New York, New York: Elsevier. TIC: 217356.

Bear, J. 1979. Hydraulics of Groundwater. New York, New York: McGraw-Hill. TIC: 217574.

Benson, C.F. and Bowman, R.S. 1994. "Tri- and Tetrafluorobenzoates as Nonreactive 122788 Tracers in Soil and Groundwater." Soil Science Society of America Journal, 58, (4), 1123-1129. Madison, Wisconsin: Soil Science Society of America. TIC: 246741.

Berry, J.A.; Hobley, J.; Lane, S.A.; Littleboy, A.K.; Nash, M.J.; Oliver, P.; Smith144728 Briggs, J.L.; and Williams, S.J. 1989. "Solubility and Sorption of Protactinium in the Near-Field and Far-Field Environments of a Radioactive Waste Repository." Analyst, 114, 339-347. Cambridge, England: Royal Society of Chemistry. TIC: 247004.

Bethke, C.M. and Brady, P.V. 2000. "How the Kd Approach Undermines Ground 154437 Water Cleanup." Ground Water, 38, (3), 435-443. Westerville, Ohio: National Ground Water Association. TIC: 249781.

Bird, R.B.; Stewart, W.E.; and Lightfoot, E.N. 1960. Transport Phenomena.

103524

New York, New York: John Wiley \& Sons. TIC: 208957. 
Birkholzer, J.; Li, G.; Tsang, C-F.; and Tsang, Y. 1999. "Modeling Studies and

Analysis of Seepage into Drifts at Yucca Mountain." Journal of Contaminant

Hydrology, 38, (1-3), 349-384. New York, New York: Elsevier. TIC: 244160.

Bish, D.L. and Vaniman, D.T. 1985. Mineralogic Summary of Yucca Mountain,

101196

Nevada. LA-10543-MS. Los Alamos, New Mexico: Los Alamos National

Laboratory. ACC: MOL.19950412.0041.

Bodvarsson, G.S.; Boyle, W.; Patterson, R.; and Williams, D. 1999. "Overview of

120055

Scientific Investigations at Yucca Mountain-The Potential Repository for

High-Level Nuclear Waste." Journal of Contaminant Hydrology, 38, (1-3), 3-24.

New York, New York: Elsevier. TIC: 244160.

Bodvarsson, G.S.; Liu, H.H.; Ahlers, C.F.; Wu, Y-S.; and Sonnenthal, S. 2001.

160133

"Parameterization and Upscaling in Modeling Flow and Transport in the Unsaturated

Zone of Yucca Mountain." Chapter 11 of Conceptual Models of Flow and Transport in the Fractured Vadose Zone. Washington, D.C.: National Academy Press.

TIC: 252777.

Boggs, J.M. and Adams, E.E. 1992. "Field Study of Dispersion in a Heterogeneous

Aquifer, 4. Investigation of Adsorption and Sampling Bias." Water Resources

Research, 28, (12), 3325-3336. Washington, D.C.: American Geophysical Union.

TIC: 246740.

Bolt, G.H. 1991. "The Ionic Distribution in the Diffuse Double Layer." Chapter 1 of

165056

Soil Chemistry. Bolt, G.H., ed. B. Physico-Chemical Models. New York, New York:

Elsevier. TIC: 254878.

Bolt, G.H. and Van Riemsdijk, W.H. 1991. "Ion Adsorption on Inorganic Variable

Charge Constituents." Chapter 13 of Soil Chemistry. Bolt, G.H., ed. B. Physico-

Chemical Models. New York, New York: Elsevier. TIC: 254878.

Bowen, B.D. and Epstein, N. 1979. "Fine Particle Deposition in Smooth

Parallel-Plate Channels.” Journal of Colloid Interface Science, 72, (1), 81-97.

New York, New York: Academic Press. TIC: 224935.

Bradbury, M.H. and Stephen, I.G. 1986. "Diffusion and Permeability Based Sorption 122792 Measurements in Intact Rock Samples." Scientific Basis for Nuclear Waste Management IX, Symposium held September 9-11, 1985, Stockholm, Sweden. Werme, L.O., ed. 50, 81-90. Pittsburgh, Pennsylvania: Materials Research Society.

TIC: 203664.

Broxton, D.E.; Warren, R.G.; Hagan, R.C.; and Luedemann, G. 1986. Chemistry of

100023 Diagenetically Altered Tuffs at a Potential Nuclear Waste Repository, Yucca Mountain, Nye County, Nevada. LA-10802-MS. Los Alamos, New Mexico:

Los Alamos National Laboratory. ACC: MOL.19980527.0202. 
Brunauer, S.; Emmett, P.H.; and Teller, E. 1938. "Adsorption of Gases in 156646

Multimolecular Layers." Journal of American Chemical Society, 60, 309-319.

Washington, D.C.: American Chemical Society. TIC: 224534.

BSC 2001. Radionuclide Transport Models Under Ambient Conditions. MDL-NBS-

161340

HS-000008 REV 00 ICN 01. Las Vegas, Nevada: Bechtel SAIC Company.

ACC: MOL.20020409.0011.

BSC 2002. Radionuclide Screening. ANL-WIS-MD-000006 REV 01. Las Vegas,

160059

Nevada: Bechtel SAIC Company. ACC: MOL.20020923.0177.

BSC 2003. Analysis of Hydrologic Properties Data. MDL-NBS-HS-000014 REV 00.

161773

Las Vegas, Nevada: Bechtel SAIC Company. ACC: DOC.20030908.0001.

BSC 2003. Model Validation Status Review. TDR-WIS-MD-000005 REV 00 Errata

1. Las Vegas, Nevada: Bechtel SAIC Company. ACC: MOL.20011211.0095;

DOC.20031027.0004.

BSC 2004. Analysis of Hydrologic Properties Data. ANL-NBS-HS-000042 REV 00.

Las Vegas, Nevada: Bechtel SAIC Company. ACC: DOC.20041005.0004.

BSC 2004. Calibrated Properties Model. MDL-NBS-HS-000003 REV 02.

169857

Las Vegas, Nevada: Bechtel SAIC Company. ACC: DOC.20041006.0004.

BSC 2004. Conceptual Model and Numerical Approaches for Unsaturated Zone

170035

Flow and Transport. MDL-NBS-HS-000005 REV 01. Las Vegas, Nevada: Bechtel

SAIC Company. ACC: DOC.20040922.0006.

BSC 2004. Development of Numerical Grids for UZ Flow and Transport Modeling.

169855

ANL-NBS-HS-000015 REV 02. Las Vegas, Nevada: Bechtel SAIC Company.

ACC: DOC.20040901.0001.

BSC 2004. Dissolved Concentration Limits of Radioactive Elements. ANL-WIS-MD000010, Rev. 03. Las Vegas, Nevada: Bechtel SAIC Company.

BSC 2004. Drift-Scale Radionuclide Transport. MDL-NBS-HS-000016 REV 01.

Las Vegas, Nevada: Bechtel SAIC Company. ACC: DOC.20040927.0031.

BSC 2004. Drift-Scale THC Seepage Model. MDL-NBS-HS-000001, Rev. 03.

169856

Las Vegas, Nevada: Bechtel SAIC Company.

BSC 2004. Features, Events, and Processes in UZ Flow and Transport. ANL-NBS-

MD-000001, Rev. 03. Las Vegas, Nevada: Bechtel SAIC Company.

BSC 2004. Future Climate Analysis. ANL-NBS-GS-000008 REV 01. Las Vegas,

170002

Nevada: Bechtel SAIC Company. ACC: DOC.20040908.0005. 
BSC 2004. Geologic Framework Model (GFM2000). MDL-NBS-GS-000002 REV

170029

02. Las Vegas, Nevada: Bechtel SAIC Company. ACC: DOC.20040827.0008.

BSC 2004. In Situ Field Testing of Processes. ANL-NBS-HS-000005, Rev. 03.

170004

Las Vegas, Nevada: Bechtel SAIC Company.

BSC 2004. Particle Tracking Model and Abstraction of Transport Processes. MDL-

170041

NBS-HS-000020 REV 01. Las Vegas, Nevada: Bechtel SAIC Company.

ACC: DOC.20041020.0016.

BSC 2004. Q-List. 000-30R-MGR0-00500-000-000 REV 00. Las Vegas, Nevada:

168361

Bechtel SAIC Company. ACC: ENG.20040721.0007.

BSC 2004. Rock Properties Model. MDL-NBS-GS-000004 REV 01. Las Vegas,

170032

Nevada: Bechtel SAIC Company. ACC: DOC.20040915.0011.

BSC 2004. Saturated Zone Colloid Transport. ANL-NBS-HS-000031 REV 02.

Las Vegas, Nevada: Bechtel SAIC Company. ACC: DOC.20041008.0007.

BSC 2004. Saturated Zone In-Situ Testing. ANL-NBS-HS-000039, Rev. 01.

170010

Las Vegas, Nevada: Bechtel SAIC Company.

BSC 2004. Saturated Zone Site-Scale Flow Model. MDL-NBS-HS-000011, Rev. 02.

170037

Las Vegas, Nevada: Bechtel SAIC Company.

BSC 2004. Site-Scale Saturated Zone Transport. MDL-NBS-HS-000010, Rev. 02.

Las Vegas, Nevada: Bechtel SAIC Company.

BSC 2004. Technical Work Plan for: Performance Assessment Unsaturated Zone.

TWP-NBS-HS-000003 REV 02 Errata 001. Las Vegas, Nevada: Bechtel SAIC

Company. ACC: MOL.20030102.0108; DOC.20040121.0001.

BSC 2004. Technical Work Plan for: Unsaturated Zone Transport Model Report

171282

Integration. TWP-MGR-HS-000002 REV 00 ICN 01. Las Vegas, Nevada: Bechtel

SAIC Company. ACC: DOC.20040816.0001.

BSC 2004. UZ Flow Models and Submodels. MDL-NBS-HS-000006, Rev. 02.

169861

Las Vegas, Nevada: Bechtel SAIC Company.

BSC 2004. Waste Form and In-Drift Colloids-Associated Radionuclide

170025

Concentrations: Abstraction and Summary. MDL-EBS-PA-000004, Rev. 01.

Las Vegas, Nevada: Bechtel SAIC Company.

BSC 2004. Yucca Mountain Site Description. TDR-CRW-GS-000001 REV 02 ICN 01. Two volumes. Las Vegas, Nevada: Bechtel SAIC Company.

ACC: DOC.20040504.0008. 
Buddemeier, R.W. and Hunt, J.R. 1988. "Transport of Colloidal Contaminants in

100712

Groundwater: Radionuclide Migration at the Nevada Test Site." Applied

Geochemistry, 3, 535-548. Oxford, England: Pergamon Press. TIC: 224116.

Cameron, D.R. and Klute, A. 1977. "Convective-Dispersive Solute Transport with a

117172

Combined Equilibrium and Kinetic Adsorption Model." Water Resources Research,

13, (1), 183-188. Washington, D.C.: American Geophysical Union. TIC: 246265.

Canori, G.F. and Leitner, M.M. 2003. Project Requirements Document. TER-MGR-

MD-000001 REV 02. Las Vegas, Nevada: Bechtel SAIC Company.

ACC: DOC.20031222.0006.

Conca, J.L. and Wright, J. 1990. "Diffusion Coefficients in Gravel Under Unsaturated

101582

Conditions." Water Resources Research, 26, (5), 1055-1066. Washington, D.C.:

American Geophysical Union. TIC: 237421.

Cook, A.J. 1989. A Desk Study of Surface Diffusion and Mass Transport in Clay.

117314

EUR-12381EN. Brussels, Luxembourg: Commission of the European Communities.

TIC: 247584.

Corapcioglu, M.Y.; Abboud, N.M.; and Haridas, A. 1987. "Governing Equations for

Particle Transport in Porous Media." Proceedings of the NATO Advanced Study

Institute on "Fundamentals of Transport Phenomena in Porous Media," Newark,

Delaware, USA, July 14-23, 1985. Bear, J. and Corapcioglu, M.Y., eds. 271-342.

Boston, Massachusetts: Martinus Nijhoff Publishers. TIC: 247167.

Cotton, F.A. and Wilkinson, G. 1988. Advanced Inorganic Chemistry. 5th Edition.

105732

New York, New York: John Wiley \& Sons. TIC: 236867.

CRWMS (Civilian Radioactive Waste Management System) M\&O (Management and

Operating Contractor) 1999. Enhanced Design Alternative (EDA) II Repository

Layout for $10 \mathrm{~cm} / \mathrm{s}$ Ventilation Plan. Design Input Transmittal PA-SSR-99175.T.

Las Vegas, Nevada: CRWMS M\&O. ACC: MOL.19990409.0102.

Daniels, W.R.; Wolfsberg, K.; Rundberg, R.S.; Ogard, A.E.; Kerrisk, J.F.; Duffy,

C.J.; Newton, T.W.; Thompson, J.L.; Bayhurst, B.P.; Bish, D.L.; Blacic, J.D.; Crowe, B.M.; Erdal, B.R.; Griffith, J.F.; Knight, S.D.; Lawrence, F.O.; Rundberg, V.L.;

Skyes, M.L.; Thompson, G.M.; Travis, B.J.; Treher, E.N.; Vidale, R.J.; Walter, G.R.; Aguilar, R.D.; Cisneros, M.R.; Maestas, S.; Mitchell, A.J.; Oliver, P.Q.; Raybold, N.A.; and Wanek, P.L. 1982. Summary Report on the Geochemistry of Yucca Mountain and Environs. LA-9328-MS. Los Alamos, New Mexico: Los Alamos National Laboratory. ACC: NNA.19870406.0243.

Davis, J.A. and Kent, D.B. 1990. "Surface Complexation Modeling in Aqueous

Geochemistry." Mineral-Water Interface Geochemistry. Hochella, M.F., Jr. and

White, A.F., eds. Reviews in Mineralogy Volume 23. Pages 177-260.

Washington, D.C.: Mineralogical Society of America. TIC: 224085. 
Davis, J.A.; Coston, J.A.; Kent, D.B.; and Fuller, C.C. 1998. "Application of the 154436 Surface Complexation Concept to Complex Mineral Assemblages." Environmental Science \& Technology, 32, (19), 2820-2828. Washington, D.C.: American Chemical Society. TIC: 249656.

De Hoog, F.R.; Knight, J.H.; and Stokes, A.N. 1982. "An Improved Method for 117308 Numerical Inversion of Laplace Transforms." SIAM Journal on Scientific and Statistical Computing, 3, (3), 357-366. Philadelphia, Pennsylvania: Society for Industrial and Applied Mathematics. TIC: 246291.

de Marsily, G. 1986. Quantitative Hydrogeology: Groundwater Hydrology for Engineers. San Diego, California: Academic Press. TIC: 208450.

Dixit, S. and Van Cappellen, P. 2002. "Surface Chemistry and Reactivity of Biogenic Silica." Geochimica et Cosmochimica Acta, 66, (14), 2559-2568. New York, New York: Pergamon. TIC: 254531.

DOE (U.S. Department of Energy) 1998. Total System Performance Assessment. Volume 3 of Viability Assessment of a Repository at Yucca Mountain. DOE/RW-0508. Washington, D.C.: U.S. Department of Energy, Office of Civilian Radioactive Waste Management. ACC: MOL.19981007.0030.

Dzombak, D.A. and Morel, F.M.M. 1990. Surface Complexation Modeling, Hydrous Ferric Oxide. New York, New York: John Wiley \& Sons. TIC: 224089.

EPRI (Electric Power Research Institute) 1999. Colloids in Saturated and Partially-Saturated Porous Media, Approaches to the Treatment of Colloids in Yucca Mountain Total System Performance Assessment. EPRI TR-112135. Palo Alto, California: Electric Power Research Institute. TIC: 246964.

Erickson, K.L. 1979. "Radionuclide Adsorption Studies on Abyssal Red Clays." Chapter 15 of Radioactive Waste in Geologic Storage, Symposium Sponsored by the Division of Nuclear Chemistry and Technology at the 176th Meeting of the American Chemical Society, Miami Beach, Florida, September 11-15, 1978. Fried, S.; ed. ASC Symposium Series 100. Washington, D.C.: American Chemical Society. TIC: 213890.

Farrell, J. and Reinhard, M. 1994. "Desorption of Halogenated Organics from Model 122803 Solids, Sediments, and Soil Under Unsaturated Conditions. 2. Kinetics." Environmental Science \& Technology, 28, (1), 63-72. Washington, D.C.: American Chemical Society. TIC: 246770.

Faure, G. 1977. Principles of Isotope Geology. New York, New York: John Wiley \& Sons. TIC: 235628.

Fetter, C.W. 1993. Contaminant Hydrogeology. Upper Saddle River, New Jersey: 105483

164876 Prentice Hall. TIC: 240691. 
Flint, L.E. 1998. Characterization of Hydrogeologic Units Using Matrix Properties,

Yucca Mountain, Nevada. Water-Resources Investigations Report 97-4243. Denver, Colorado: U.S. Geological Survey. ACC: MOL.19980429.0512.

Freeze, G.A.; Brodsky, N.S.; and Swift, P.N. 2001. The Development of Information 154365 Catalogued in REV00 of the YMP FEP Database. TDR-WIS-MD-000003 REV 00 ICN 01. Las Vegas, Nevada: Bechtel SAIC Company. ACC: MOL.20010301.0237.

Freundlich, H. 1926. Colloid \& Capillary Chemistry. London, England: Methuen \& Company. TIC: 244810.

Gelhar, L.W.; Welty, C.; and Rehfeldt, K.R. 1992. "A Critical Review of Data on Field-Scale Dispersion in Aquifers." Water Resources Research, 28, (7), 1955-1974. Washington, D.C.: American Geophysical Union. TIC: 235780.

Grathwohl, P. 2000. Diffusion in Natural Porous Media: Contaminant Transport, Sorption/Desorption and Dissolution Kinetics. Boston, Massachusetts: Kluwer Academic Publishers. TIC: 247983.

Harvey, R.W. and Garabedian, S.P. 1991. "Use of Colloid Filtration Theory in Modeling Movement of Bacteria Through a Contaminated Sandy Aquifer." Environmental Science \& Technology, 25, (1), 178-185. Washington, D.C.: American Chemical Society. TIC: 245733.

Herzig, J.P.; Leclerc, D.M.; and Le Goff, P. 1970. "Flow of Suspension Through Porous Media - Application to Deep Filtration." Industrial \& Engineering Chemistry, 62, (5), 8-35. Washington, D.C.: American Chemical Society. TIC: 223585.

Holtta, P.; Siitari-Kauppi, M.; Huikuri, P.; Lindberg, A.; and Hautojarvi, A. 1997. "The Effect of Specific Surface Area on Radionuclide Sorption on Crushed Crystalline Rock." Scientific Basis for Nuclear Waste Management XX, Symposium held December 2-6, 1996, Boston, Massachusetts. Gray, W.J. and Triay, I.R., eds. 465, 789-796. Pittsburgh, Pennsylvania: Materials Research Society. TIC: 238884.

Honeyman, B.D. 1984. Cation and Anion Adsorption at the Oxide/Solution Interface in Systems Containing Binary Mixtures of Adsorbents: An Investigation of the Concept of Adsorptive Additivity. Ph.D. dissertation. Stanford, California: Stanford University, Department of Civil Engineering. TIC: 245390.

Hu, M.Q. 2001. Unsaturated Zone Flow and Transport Model. Scientific Notebook 163148 SN-LBNL-SCI-168-VI. ACC: MOL.20010905.0030.

Hu, Q. and Brusseau, M.L. 1995. "The Effect of Solute Size on Transport in 122846 Structured Porous Media." Water Resources Research, 31, (7), 1637-1646. Washington, D.C.: American Geophysical Union. TIC: 246800. 
Hummel, W.; Berner, U.; Curti, E.; Pearson, F.J.; and Thoenen, T. 2002. Nagra/PSI

161904

Chemical Thermodynamic Data Base 01/01. Parkland, Florida: Universal Publishers.

TIC: 253421.

Ibaraki, M. and Sudicky, E.A. 1995. "Colloid-Facilitated Contaminant Transport in

109297

Discretely Fractured Porous Media 1. Numerical Formulation and Sensitivity

Analysis." Water Resources Research, 31, (12), 2945-2960. Washington, D.C.:

American Geophysical Union. TIC: 245719.

Ishfaq, M.M.; Karim, H.M.A.; and Khan, M.A. 1997. "A Radiochemical Study on the 164879

Thermodynamics of Cesium Adsorption on Potassium Copper Nickel

Hexacyanoferrate(II) from Aqueous Solutions." Journal of Radioanalytical and

Nuclear Chemistry, 222, (1-2), 177-181. Amsterdam, The Netherlands: Elsevier.

TIC: 255231.

Jacobsen, O.H.; Moldrup, P.; Larsen, C.; Konnerup, L.; and Peterson, L.W. 1997.

"Particle Transport in Macropores of Undisturbed Soil Columns." Journal of

Hydrology, 196, (1-4), 185-203. Amsterdam, The Netherlands: Elsevier.

TIC: 246812.

Jahnke, F.M. and Radke, C.J. 1987. "Electrolyte Diffusion in Compacted Montmorillonite Engineered Barriers." Chapter 22 of Coupled Processes Associated with Nuclear Waste Repositories. Tsang, C-F., ed. Orlando, Florida: Academic Press. TIC: 200325.

James, S.C. and Chrysikopoulos, C.V. 1999. "Transport of Polydisperse Colloid

Suspensions in a Single Fracture." Water Resources Research, 35, (3), 707-718.

Washington, D.C.: American Geophysical Union. TIC: 245938.

Javeri, V. 2001. Analysen zum Transport Verschiedener Nuklidketten bei

Nichtlinearer Adsorption in einem Porösen Medium mit dem Rechenprogramm

TOUGH2 Abstract only. GRS-A-2923. Köln, Germany: Gessellschaft für

Anlagenund Reaktorsicherheit. TIC: 254975.

Javeri, V. 2002. Dreidimensionale Analysen zum Transport Verschiedener

Nuklidketten bei Nichtlinearer Adsorption in einem Porösen Medium mit dem

Rechenprogramm TOUGH2 Abstract only. GRS-A-3001. Köln, Germany:

Gessellschaft für Anlagenund Reaktorsicherheit. TIC: 255128.

Johansson, H.; Byegard, J.; Skarnemark, G.; and Skalberg, M. 1997. "Matrix

Diffusion of Some Alkali- and Alkaline Earth-Metals in Granitic Rock." Scientific

Basis for Nuclear Waste Management XX, Symposium held December 2-6, 1996,

Boston, Massachusetts. 465, 871-878. Pittsburgh, Pennsylvania: Materials Research

Society. TIC: 238884. 
Johansson, H.; Siitari-Kauppi, M.; Skalberg, M.; and Tullborg, E.L. 1998. "Diffusion

Pathways in Crystalline Rock - Examples from Aspo-Diorite and Fine-Grained Granite." Journal of Contaminant Hydrology, 35, 41-53. Amsterdam, The Netherlands: Elsevier. TIC: 246810.

Kaplan, D.I. and Serne, R.J. 1995. Distribution Coefficient Values Describing Iodine, 123010 Neptunium, Selenium, Technetium, and Uranium Sorption to Hanford Sediments. PNL-10379, Sup. 1. Richland, Washington: Pacific Northwest National Laboratory. TIC: 246721.

Kara, M.; Yuzer, H.; Sabah, E.; and Celik, M.S. 2003. “Adsorption of Cobalt from Aqueous Solutions onto Sepiolite." Water Research, 37, (1), 224-232. New York, New York: Pergamon. TIC: 254847.

Karickhoff, S.W. 1984. "Organic Pollutant Sorption in Aquatic Systems." Journal of 164881 Hydraulic Engineering, 110, (6), 707-735. New York, New York: American Society of Civil Engineers.. TIC: 255122.

Kaszuba J.P. and Runde W.H. 1999. "The Aqueous Geochemistry of Neptunium: Dynamic Control of Soluble Concentrations with Applications to Nuclear Waste Disposal." Environmental Science \& Technology, 33, (24), 4427-4433. Washington, D.C.: American Chemical Society. TIC: 246667.

Keeney-Kennicutt, W.L. and Morse, J.W. 1985. "The Redox Chemistry of Pu(V)O ${ }_{2}^{+}$ Interaction with Common Mineral Surfaces in Dilute Solutions and Seawater." Geochimica et Cosmochimica Acta, 49, (12), 2577-2588. New York, New York: Pergamon. TIC: 237000.

Kelmers, A.D. 1984. Review and Assessment of Radionuclide Sorption Information for the Basalt Waste Isolation Project Site (1979 through May, 1983).

NUREG/CR-3763. Washington, D.C.: U.S. Nuclear Regulatory Commission. TIC: 228866.

Kenna, B.T. 1981. "Temperature and pH Effects on Sorption Properties of Subseabed 165049 Clay." Scientific Basis for Nuclear Waste Management, Proceedings of the Third International Symposium, Boston, Massachusetts, November 17-20, 1980. Moore, J.G., ed. 3, 491-498. New York, New York: Plenum Press. TIC: 204407.

Kersting, A.B.; Efurd, D.W.; Finnegan, D.L.; Rokop, D.J.; Smith, D.K.; and 103282 Thompson, J.L. 1999. "Migration of Plutonium in Ground Water at the Nevada Test Site.” Nature, 397, (6714), 56-59. London, England: Macmillan Journals. TIC: 243597. 
Kieft, T.L.; Kovacik, W.P., Jr.; Ringelberg, D.B.; White, D.C.; Haldeman, D.L.;

Amy, P.S.; and Hersman, L.E. 1997. "Factors Limiting Microbial Growth and Activity at a Proposed High-Level Nuclear Repository, Yucca Mountain, Nevada." Applied and Environmental Microbiology, 63, (8), 3128-3133. Washington, D.C.: American Society for Microbiology. TIC: 236444.

Kilislioglu, A. and Bilgin, B. 2002. "Adsorption of Uranium on Halloysite." 165050 Radiochimica Acta, 90, (3), 155-160. München, Germany: R. Oldenbourg Verlag. TIC: 254872.

Kretzschmar, R.; Barmettler, K.; Grolimund, D.; Yan, Y.; Borkovec, M.; and Sticher, 123029 H. 1997. "Experimental Determination of Colloid Deposition Rates and Collision Efficiencies in Natural Porous Media." Water Resources Research, 33, (5), 1129-1137. Washington, D.C.: American Geophysical Union. TIC: 246817.

Kretzschmar, R.; Robarge, W.P.; and Amoozegar, A. 1995. "Influence of Natural 123019 Organic Matter on Colloid Transport Through Saprolite." Water Resources Research, 31, (3), 435-445. Washington, D.C.: American Geophysical Union. TIC: 246819.

Kung, K.S. 1999. Research and Development Notebook for Colloid Study Notebook. Scientific Notebook LA-CST-NBK-95-001, Volume II. ACC: MOL.19991206.0253.

Langmuir, D. and Herman, J.S. 1980. "The Mobility of Thorium in Natural Waters at Low Temperatures." Geochimica et Cosmochimica Acta, 44, 1753-1766. New York, New York: Pergamon Press. TIC: 237029.

Langmuir, I. 1918. "The Adsorption of Gases on Plane Surfaces of Glass, Mica and Platinum." Journal of the American Chemical Society, 40, 1361-1403.

Washington, D.C.: American Chemical Society. TIC: 223181.

LANL (Los Alamos National Laboratories) 1984. Quality Assurance Audit of 171444 Los Alamos National Laboratories (LANL) Nevada Nuclear Waste Storage Investigations (NNWSI) Project. Los Alamos, New Mexico: Los Alamos National Laboratories. ACC: NNA.19870317.0482.

LANL 1982. Quality Assurance Program Plan for Nevada Nuclear Waste 172031 Investigations. TWS-CMBQA-QP-01, Rev. 3. Los Alamos, New Mexico: Los Alamos National Laboratory. ACC: NNA.19891030.0029.

LeCain, G.D.; Anna, L.O.; and Fahy, M.F. 2000. Results from Geothermal Logging, 144612 Air and Core-Water Chemistry Sampling, Air-Injection Testing, and Tracer Testing in the Northern Ghost Dance Fault, Yucca Mountain, Nevada, November 1996 to August 1998. Water-Resources Investigations Report 99-4210. Denver, Colorado: U.S. Geological Survey. TIC: 247708.

Lide, D.R., ed. 1992. CRC Handbook of Chemistry and Physics. 73rd Edition. Boca Raton, Florida: CRC Press. TIC: 255239. 
Lide, D.R., ed. 1993. CRC Handbook of Chemistry and Physics. 74th Edition. Boca

123032

Raton, Florida: CRC Press. TIC: 209252.

Lide, D.R., ed. 2002. CRC Handbook of Chemistry and Physics. 83rd Edition. Boca

160832

Raton, Florida: CRC Press. TIC: 253582.

Liu, H.H.; Bodvarsson, G.S.; and Finsterle, S. 2002. "A Note on Unsaturated Flow in

160230

Two-Dimensional Fracture Networks." Water Resources Research, 38, (9), 15-1 to 15-9. Washington, D.C.: American Geophysical Union. TIC: 253307.

Liu, H.H.; Doughty, C.; and Bodvarsson, G.S. 1998. "An Active Fracture Model for Unsaturated Flow and Transport in Fractured Rocks." Water Resources Research, 34, (10), 2633-2646. Washington, D.C.: American Geophysical Union. TIC: 243012.

Los Alamos Scientific Laboratories 1979. Audit of Los Alamos Scientific

Laboratories Quality Assurance Program Plan for Nevada Nuclear Waste Storage

Investigations. Audit 79-5. Los Alamos, New Mexico: Los Alamos Scientific

Laboratories. ACC: HQZ.19880629.5531.

Lu, N.; Conca, J.; Parker, G.R.; Leonard, P.A.; Moore, B.; Strietelmeier, B.; and

Triay, I.R. 2000. Adsorption of Actinides onto Colloids as a Function of Time, Temperature, Ionic Strength, and Colloid Concentration, Waste Form Colloids Report for Yucca Mountain Program (Colloid Data Summary from 1999 to 2000 Research). LA-UR-00-5121. Los Alamos, New Mexico: Los Alamos National Laboratory. ACC: MOL.20031204.0108.

Marmier, N.; Delisée, A.; and Fromage, F. 1999. "Surface Complexation Modeling of 162986 $\mathrm{Yb}(\mathrm{III})$ and $\mathrm{Cs}(\mathrm{I})$ Sorption on Silica." Journal of Colloid and Interface Science, 212, (2), 228-233. New York, New York: Academic Press. TIC: 254530.

Marshall, T.J. and Holmes, J.W. 1979. Soil Physics. Melbourne, Australia:

102532 Cambridge University Press. TIC: 240992.

McCarthy, J.F. and Zachara, J.M. 1989. "Subsurface Transport of Contaminants." 100778 Environmental Science \& Technology, 23, (5), 496-502. Easton, Pennsylvania: American Chemical Society. TIC: 224876.

McGraw, M.A. and Kaplan, D.I. 1997. Colloid Suspension Stability and Transport Through Unsaturated Porous Media. PNNL-11565. Richland, Washington: Pacific Northwest National Laboratory. TIC: 246723.

Meyer, R.E.; Arnold, W.D.; Case, F.I.; O’Kelley, G.D.; and Land, J.F. 1990. Effects of Mineralogy on Sorption of Strontium and Cesium Onto Calico Hills Tuff. NUREG/CR-5463. Washington, D.C.: U.S. Nuclear Regulatory Commission. TIC: 254856. 
Moridis, G.J. 1999. "Semianalytical Solutions for Parameter Estimation in Diffusion

Cell Experiments." Water Resources Research, 35, (6), 1729-1740.

Washington, D.C.: American Geophysical Union. TIC: 246266.

Moridis, G.J.; Hu, Q.; Wu, Y-S.; and Bodvarsson, G.S. 2003. "Preliminary 3-D

161902

Site-Scale Studies of Radioactive Colloid Transport in the Unsaturated Zone at Yucca Mountain, Nevada." Journal of Contaminant Hydrology, 60, (3-4), 251-286.

New York, New York: Elsevier. TIC: 253921.

Moridis, G.J.; Wu, Y-S.; and Pruess, K. 1999. EOS9nT: A TOUGH2 Module for the

123093

Simulation of Water Flow and Solute/Colloid Transport in the Subsurface.

LBNL-42351. Berkeley, California: Lawrence Berkeley National Laboratory.

TIC: 246520.

Mualem, Y. 1976. "A New Model for Predicting the Hydraulic Conductivity of

Unsaturated Porous Media.” Water Resources Research, 12, (3), 513-522.

Washington, D.C.: American Geophysical Union. TIC: 217339.

Mustafa, S.; Safdar, M.; and Hussain, S.Y. 1991. "Temperature Effect on Heavy

165048

Metal Adsorption by (alpha)-Aluminium Hydroxide." Journal of Surface Science and

Technology, 7, (3), 273-281. Calcutta, India: Indian Society for Surface Science and

Technology. TIC: 254869.

National Research Council. 1996. Rock Fractures and Fluid Flow, Contemporary

139151

Understanding and Applications. Washington, D.C.: National Academy Press.

TIC: 235913.

Neretnieks, I. 2002. "A Stochastic Multi-Channel Model for Solute

Transport-Analysis of Tracer Tests in Fractured Rock." Journal of Contaminant Hydrology, 55, (3-4), 175-211. New York, New York: Elsevier. TIC: 253977.

Nitsche, H.; Gatti, R.C.; Standifer, E.M.; Lee, S.C.; Müller, A.; Prussin, T.;

155218

Deinhammer, R.S.; Maurer, H.; Becraft, K.; Leung, S.; and Carpenter, S.A. 1993.

Measured Solubilities and Speciations of Neptunium, Plutonium, and Americium in a Typical Groundwater (J-13) from the Yucca Mountain Region. LA-12562-MS.

Los Alamos, New Mexico: Los Alamos National Laboratory.

ACC: NNA.19930507.0136.

Nitsche, H.; Roberts, K.; Prussin, T.; Muller, A.; Becraft, K.; Keeney, D.; Carpenter, 144515 S.A.; and Gatti, R.C. 1994. Measured Solubilities and Speciations from Oversaturation Experiments of Neptunium, Plutonium, and Americium in UE-25P \#1 Well Water from the Yucca Mountain Region Milestone Report 3329-WBS1.2.3.4.1.3.1. LA-12563-MS. Los Alamos, New Mexico: Los Alamos National Laboratory. TIC: 210589. 
NRC (U.S. Nuclear Regulatory Commission) 2003. Yucca Mountain Review Plan,

Final Report. NUREG-1804, Rev. 2. Washington, D.C.: U.S. Nuclear Regulatory Commission, Office of Nuclear Material Safety and Safeguards. TIC: 254568.

Nuttall, H.E.; Jain, R.; and Fertelli,Y. 1991. "Radiocolloid Transport in Saturated and Unsaturated Fractures." High Level Radioactive Waste Management, Proceedings of the Second Annual International Conference, Las Vegas, Nevada, April 28-May 3, 1991. 1, 189-196. La Grange Park, Illinois: American Nuclear Society.

TIC: 204272.

Ogard, A.E. and Kerrisk, J.F. 1984. Groundwater Chemistry Along Flow Paths 100783 Between a Proposed Repository Site and the Accessible Environment. LA-10188-MS. Los Alamos, New Mexico: Los Alamos National Laboratory.

ACC: HQS.19880517.2031.

Pabalan, R.T.; Turner, D.R.; Bertetti, F.P.; and Prikryl, J.D. 1998. "Uranium ${ }^{\text {VI }}$ 162987 Sorption onto Selected Mineral Surfaces, Key Geochemical Parameters." Adsorption of Metals by Geomedia. Jenne, E.A., ed. Pages 99-130. New York, New York: Academic Press. TIC: 239504.

Paces, T. 1978. "Reversible Control of Aqueous Aluminum and Silica during the Irreversible Evolution of Natural Waters." Geochimica et Cosmochimica Acta, 42, (10), 1487-1493. New York, New York: Pergamon. TIC: 235309.

Parkhurst, D.L. 1995. User's Guide to PHREEQC-A Computer Program for Speciation, Reaction-Path, Advective-Transport, and Inverse Geochemical Calculations. Water-Resources Investigations Report 95-4227. Lakewood, Colorado: U.S. Geological Survey. TIC: 248314.

Parkhurst, D.L. and Appelo, C.A.J. 1999. User's Guide to PHREEQC (Version 2)-A Computer Program for Speciation, Batch-Reaction, One-Dimensional Transport, and Inverse Geochemical Calculations. Water-Resources Investigations Report 99-4259. Denver, Colorado: U.S. Geological Survey. TIC: 253046.

Parks, G.A. 1990. "Surface Energy and Adsorption at Mineral-Water Interfaces: An 165849 Introduction." Chapter 4 of Mineral-Water Interface Geochemistry. Hochella, M.F., Jr. and White, A.F., eds. Reviews in Mineralogy Volume 23. Washington, D.C.: Mineralogical Society of America. TIC: 240202.

Philip, J.R.; Knight, J.H.; and Waechter, R.T. 1989. "Unsaturated Seepage and 105743 Subterranean Holes: Conspectus, and Exclusion Problem for Circular Cylindrical Cavities." Water Resources Research, 25, (1), 16-28. Washington, D.C.: American Geophysical Union. TIC: 239117. 
Pigford, T.H.; Chambré, P.L.; Albert, M.; Foglia, M.; Harada, M.; Iwamoto, F.;

Kanki, T.; Leung, D.; Masuda, S.; Muraoka, S.; and Ting, D. 1980. Migration of Radionuclides Through Sorbing Media Analytical Solutions--II. LBL-11616. Two volumes. Berkeley, California: Lawrence Berkeley National Laboratory. TIC: 211541.

Porter, L.K.; Kemper, W.D.; Jackson, R.D.; and Stewart, B.A. 1960. "Chloride 123115 Diffusion in Soils as Influenced by Moisture Content." Soil Science Society of America Proceedings, 24, (5), 460-463. Danville, Illinois: Soil Science Society of America. TIC: 246854.

Pruess, K. 1987. TOUGH User's Guide. NUREG/CR-4645. Washington, D.C.: U.S. 100684 Nuclear Regulatory Commission. TIC: 217275.

Pruess, K. 1991. TOUGH2-A General-Purpose Numerical Simulator for Multiphase Fluid and Heat Flow. LBL-29400. Berkeley, California: Lawrence Berkeley Laboratory. ACC: NNA.19940202.0088.

Pruess, K. and Narasimhan, T.N. 1985. "A Practical Method for Modeling Fluid and Heat Flow in Fractured Porous Media." Society of Petroleum Engineers Journal, 25, (1), 14-26. Dallas, Texas: Society of Petroleum Engineers. TIC: 221917.

Rauf, N. and Tahir, S.S. 2000. "Thermodynamics of Fe(II) and Mn(II) Adsorption onto Bentonite from Aqueous Solutions." Journal of Chemical Thermodynamics, 32, (5), 651-658. New York, New York: Academic Press. TIC: 254910.

Reardon, E.J. 1981. “Kd's - Can They be Used to Describe Reversible Ion Sorption 154434 Reactions in Contaminant Migration?" Ground Water, 19, (3), 279-286. Worthington, Ohio: Water Well Journal Publishing Company. TIC: 249726.

Reed, M.H. 1982. "Calculation of Multicomponent Chemical Equilibria and Reaction 117901 Processes in Systems Involving Minerals, Gases and an Aqueous Phase." Geochimica et Cosmochimica Acta, 46, (4), 513-528. New York, New York: Pergamon Press. TIC: 224159.

Richards, L.A. 1931. "Capillary Conduction of Liquids Through Porous Mediums." Physics, 1, 318-333. New York, New York: American Physical Society.

TIC: 225383.

Roberts, J.J. and Lin, W. 1997. "Electrical Properties of Partially Saturated Topopah Spring Tuff: Water Distribution as a Function of Saturation." Water Resources Research, 33, (4), 577-587. Washington, D.C.: American Geophysical Union. TIC: 239736. 
Robin, M.J.L.; Gillham, R.W.; and Oscarson, D.W. 1987. "Diffusion of Strontium and Chloride in Compacted Clay-Based Materials." Soil Science Society of America Journal, 51, (5), 1102-1108. Madison, Wisconsin: Soil Science Society of America. TIC: 246867.

Rogers, P.S.Z. and Meijer, A. 1993. "Dependence of Radionuclide Sorption on Sample Grinding Surface Area, and Water Composition." High Level Radioactive Waste Management, Proceedings of the Fourth Annual International Conference, Las Vegas, Nevada, April 26-30, 1993. 2, 1509-1516. La Grange Park, Illinois: American Nuclear Society. TIC: 208542.

Rundberg, R.S.; Ogard, A.E.; and Vaniman, D.T., eds. 1985. Research and Development Related to the Nevada Nuclear Waste Storage Investigations, April 1-June 30, 1984. LA-10297-PR. Los Alamos, New Mexico: Los Alamos National Laboratory. ACC: NNA.19920922.0018.

Sabah, E.; Turan, M.; and Celik, M.S. 2002. "Adsorption Mechanism of Cationic Surfactants onto Acid- and Heat-Activated Sepiolites." Water Research, 36, (16), 3957-3964. New York, New York: Pergamon. TIC: 254848.

Sakthivadivel, R. 1969. Clogging of a Granular Porous Medium by Sediment, Final Report. HEL 15-7. Berkeley, California: University of California, College of Engineering, Hydraulic Engineering Laboratory. TIC: 250299.

Scott, R.B. and Bonk, J. 1984. Preliminary Geologic Map of Yucca Mountain, Nye 104181 County, Nevada, with Geologic Sections. Open-File Report 84-494. Denver, Colorado: U.S. Geological Survey. ACC: HQS.19880517.1443.

Seaman, J.C. 1998. "Retardation of Fluorobenzoate Tracers in Highly Weathered Soil 134563 and Groundwater Systems." Soil Science Society of America Journal, 62, (2), 354-361. Madison, Wisconsin: Soil Science Society of America. TIC: 246908.

Shapiro, A.M. 2001. "Effective Matrix Diffusion in Kilometer-Scale Transport in 162132 Fractured Crystalline Rock." Water Resources Research, 37, (3), 507-522.

Washington, D.C.: American Geophysical Union. TIC: 253979.

Smith, P.A. and Degueldre, C. 1993. "Colloid-Facilitated Transport of Radionuclides Through Fractured Media." Journal of Contaminant Hydrology, 13, 143-166. Amsterdam, The Netherlands: Elsevier. TIC: 224863.

Snell, R.D.; Appel, G.; and Smith, A. 2004. Performance Confirmation Plan. 169101 TDR-PCS-SE-000001 REV 03. Las Vegas, Nevada: Bechtel SAIC Company. ACC: DOC.20040412.0008.

Sposito, G. 1980. "Derivation of the Freundlich Equation for Ion Exchange Reactions 127235 in Soils.” Soil Science Society of America Journal, 44, (3), 652-654. Madison, Wisconsin: Soil Science Society of America. TIC: 223199. 
Sposito, G. 1981. "Single-Particle Motions in Liquid Water. II. The Hydrodynamic Model." Journal of Chemical Physics, 74, (12), 6943-6949. New York, New York: American Institute of Physics. TIC: 246772.

Sposito, G. 1984. The Surface Chemistry of Soils. New York, New York: Oxford 127253 University Press. TIC: 217687.

Spycher, N.F. and Reed, M.H. 1992. "Microcomputer-Based Modeling of Speciation and Water-Mineral-Gas Reactions Using Programs SOLVEQ and CHILLER." Water-Rock Interaction, Proceedings of the 7th International Symposium on Water-Rock Interaction, WRI-7, Park City, Utah, USA, 13-18 July 1992. Kharaka, Y.K. and Maest, A.S., eds. 2, 1087-1090. Brookfield, Vermont: A.A. Balkema. TIC: 208527.

Sudicky, E.A. and Frind, E.O. 1982. "Contaminant Transport in Fractured Porous Media: Analytical Solutions for a System of Parallel Fractures." Water Resources Research, 18, (6), 1634-1642. Washington, D.C.: American Geophysical Union. TIC: 217475.

Tachi, Y.; Shibutani, T.; Sato, H.; and Yui, M. 1998. "Sorption and Diffusion Behavior of Selenium in Tuff." Journal of Contaminant Hydrology, 35, (1-3), 77-89. Amsterdam, The Netherlands: Elsevier. TIC: 246891.

Thomas, K.W. 1987. Summary of Sorption Measurements Performed with Yucca Mountain, Nevada, Tuff Samples and Water from Well J-13. LA-10960-MS. Los Alamos, New Mexico: Los Alamos National Laboratory. ACC: NNA.19900604.0045.

Tokunaga, T.K. and Wan, J. 1997. "Water Film Flow Along Fracture Surfaces of 139195 Porous Rock." Water Resources Research, 33, (6), 1287-1295. Washington, D.C.: American Geophysical Union. TIC: 242739.

Triay, I.R.; Birdsell, K.H.; Mitchell, A.J.; and Ott, M.A. 1993. "Diffusion of Sorbing 145123 and Non-Sorbing Radionuclides." High Level Radioactive Waste Management, Proceedings of the Fourth Annual International Conference, Las Vegas, Nevada, April 26-30, 1993. 2, 1527-1532. La Grange Park, Illinois: American Nuclear Society. TIC: 208542.

Triay, I.R.; Cotter, C.R.; Huddleston, M.H.; Leonard, D.E.; Weaver, S.C.; Chipera, 101023 S.J.; Bish, D.L.; Meijer, A.; and Canepa, J.A. 1996. Batch Sorption Results for Neptunium Transport Through Yucca Mountain Tuffs. LA-12961-MS. Los Alamos, New Mexico: Los Alamos National Laboratory. ACC: MOL.19980924.0050. 
Triay, I.R.; Meijer, A.; Cisneros, M.R.; Miller, G.G.; Mitchell, A.J.; Ott, M.A.; Hobart, D.E.; Palmer, P.D.; Perrin, R.E.; and Aguilar, R.D. 1991. "Sorption of Americium in Tuff and Pure Minerals Using Synthetic and Natural Groundwaters." Radiochimica Acta, 52/53, 141-145. München, Germany: R. Oldenbourg Verlag. TIC: 222704.

Triay, I.R.; Meijer, A.; Conca, J.L.; Kung, K.S.; Rundberg, R.S.; Strietelmeier, B.A.; 100422 and Tait, C.D. 1997. Summary and Synthesis Report on Radionuclide Retardation for the Yucca Mountain Site Characterization Project. Eckhardt, R.C., ed. LA-13262MS. Los Alamos, New Mexico: Los Alamos National Laboratory. ACC: MOL.19971210.0177.

Turner, D.R.; Pabalan, R.T.; and Bertetti, F.P. 1998. "Neptunium(V) Sorption on Montmorillonite: An Experimental and Surface Complexation Modeling Study." Clays and Clay Minerals, 46, (3), 256-269. Boulder, Colorado: Clay Minerals Society. TIC: 254532.

van de Weerd, H. and Leijnse, A. 1997. "Assessment of the Effect of Kinetics on Colloid Facilitated Radionuclide Transport in Porous Media." Journal of Contaminant Hydrology, 26, 245-256. Amsterdam, The Netherlands: Elsevier. TIC: 245731.

van Geldermalsen, L.A. and Duursma, E.K. 1984. "Temperature Effects on Sorption of $\mathrm{Cd}, \mathrm{Eu}, \mathrm{Pu}$ and Am onto Estuarine and Marine Sediments." Proceedings of an International Symposium on the Behavior of Long-Lived Radionuclides in a Marine Environment ,1984. EUR 9214, Pages 83-95. Vienna, Austria: International Atomic Energy Agency. On Order

van Genuchten, M.T. 1980. "A Closed-Form Equation for Predicting the Hydraulic Conductivity of Unsaturated Soils." Soil Science Society of America Journal, 44, (5), 892-898. Madison, Wisconsin: Soil Science Society of America. TIC: 217327.

Vilks, P. and Bachinski, D.B. 1996. "Colloid and Suspended Particle Migration 109252 Experiments in a Granite Fracture." Journal of Contaminant Hydrology, 21, 269-279. Amsterdam, The Netherlands: Elsevier. TIC: 245730.

Vilks, P.; Frost, L.H.; and Bachinski, D.B. 1997. "Field-Scale Colloid Migration 165087 Experiments in a Granite Fracture." Journal of Contaminant Hydrology, 26, 203-214. Amsterdam, The Netherlands: Elsevier. TIC: 245732.

Viswanathan, H.S.; Robinson, B.A.; Valocchi, A.J.; and Triay, I.R. 1998. “A

Reactive Transport Model of Neptunium Migration from the Potential Repository at Yucca Mountain." Journal of Hydrology, 209, 251-280. Amsterdam, The Netherlands: Elsevier. TIC: 243441. 
Wan, J. and Tokunaga, T.K. 1997. "Film Straining on Colloids in Unsaturated Porous

108285

Media: Conceptual Model and Experimental Testing." Environmental Science \& Technology, 31, (8), 2413-2420. Washington, D.C.: American Chemical Society.

TIC: 234804.

Wan, J. and Wilson, J.L. 1994. "Colloid Transport in Unsaturated Porous Media."

Water Resources Research, 30, (4), 857-864. Washington, D.C.: American

Geophysical Union. TIC: 222359.

Wang, J.S. 2003. "Scientific Notebooks Referenced in Model Report N0125,

Abstraction of Drift-Scale Coupled Processes, MDL-NBS-HS-000018 REV 00."

Correspondence from J.S. Wang (BSC) to File, December 15, 2003, with

attachments. ACC: MOL.20031217.0389.

Wang, J.S. 2003. "Scientific Notebooks Referenced in Model Report U0060, Radionuclide Transport Models Under Ambient Conditions MDL-NBS-HS-000008 REV 01." Interoffice correspondence from J.S. Wang (BSC) to File, November 18, 2003, with attachments. ACC: MOL.20031118.0184.

Weber, W.J., Jr.; McGinley, P.M.; and Katz, L.E. 1992. “A Distributed Reactivity Model for Sorption by Soils and Sediments. 1. Conceptual Basis and Equilibrium Assessments.” Environmental Science \& Technology, 26, (10), 1955-1962.

Washington, D.C.: American Chemical Society. TIC: 255335.

Westall, J.C. 1987. "Adsorption Mechanisms in Aquatic Surface Chemistry.” Chapter 127323 1 of Aquatic Surface Chemistry: Chemical Processes at the Particle-Water Interface. A Volume in Environmental Science and Technology, A Wiley-Interscience Publication. Stumm, W., ed. New York, New York: John Wiley \& Sons.

TIC: 217674.

Wolery, T.J. 1983. EQ3NR, A Computer Program for Geochemical Aqueous 108808 Speciation-Solubility Calculations: User's Guide and Documentation. UCRL-53414. Livermore, California: Lawrence Livermore National Laboratory. TIC: 1148.

Wolery, T.J. and Daveler, S.A. 1992. EQ6, A Computer Program for Reaction Path Modeling of Aqueous Geochemical Systems: Theoretical Manual, User's Guide, and Related Documentation (Version 7.0). UCRL-MA-110662 PT IV. Livermore, California: Lawrence Livermore National Laboratory. ACC: MOL.19980701.0459.

Wu, Y-S. and Pruess, K. 2000. "Numerical Simulation of Non-Isothermal Multiphase Tracer Transport in Heterogeneous Fractured Porous Media." Advances in Water Resources, 23, (7), 699-723. New York, New York: Elsevier. TIC: 249626.

Wu, Y.S.; Ahlers, C.F.; Fraser, P.; Simmons, A.; and Pruess, K. 1996. Software Qualification of Selected TOUGH2 Modules. LBL-39490. Berkeley, California: Lawrence Berkeley National Laboratory. ACC: MOL.19970219.0104. 
Wu, Y.S.; Ritcey, A.C.; and Bodvarsson, G.S. 1999. "A Modeling Study of Perched

Water Phenomena in the Unsaturated Zone at Yucca Mountain.” Journal of Contaminant Hydrology, 38, (1-3), 157-184. New York, New York: Elsevier.

TIC: 244160.

Yao, K.M.; Habibian, M.T.; and O’Melia, C.R. 1971. "Water and Waste Water Filtration: Concepts and Applications." Environmental Science \& Technology, 5, (11), 1105-1112. Washington, D.C.: American Chemical Society. TIC: 239214.

Yehia, A.; Miller, J.D.; and Ateya, B.G. 1993. "Analysis of the Adsorption Behaviour 164922 of Oleate on Some Synthetic Apatites." Minerals Engineering, 6, (1), 79-86. New York, New York: Pergamon. TIC: 255300.

Zafar, U.; Khan, I.; Jamshaid, F.; and Naeem, S. 2002. "Adsorption Studies of Silver 165052 $(+1)$ on Bentonite." Journal of the Chemical Society of Pakistan, 24, (2), 92-97.

Karachi, Pakistan: Chemical Society of Pakistan. TIC: 254876.

Zhou, Q.; Liu, H-H.; Bodvarsson, G.S.; and Oldenburg, C.M. 2003. "Flow and 162133 Transport in Unsaturated Fractured Rock: Effects of Multiscale Heterogeneity of Hydrogeologic Properties.” Journal of Contaminant Hydrology, 60, (1-2), 1-30. New York, New York: Elsevier. TIC: 253978.

\subsection{CODES, STANDARDS, REGULATIONS, AND PROCEDURES}

10 CFR 50. Energy: Domestic Licensing of Production and Utilization Facilities. 107619 Readily available.

10 CFR 63. Energy: Disposal of High-Level Radioactive Wastes in a Geologic Repository at Yucca Mountain, Nevada. Readily available.

AP-2.22Q, Rev. 1, ICN 0. Classification Criteria and Maintenance of the Monitored Geologic Repository Q-List. Washington, D.C.: U.S. Department of Energy, Office of Civilian Radioactive Waste Management.

ACC: DOC.20030807.0002.

AP-2.27Q, Rev. 1, ICN 5. Planning for Science Activities. Washington, D.C.: U.S. Department of Energy, Office of Civilian Radioactive Waste Management. ACC: DOC.20041014.0001.

AP-SI.1Q, Rev. 05, ICN 2. Software Management. Washington, D.C.: U.S. Department of Energy, Office of Civilian Radioactive Waste Management. ACC: DOC.20030902.0003.

AP-SIII.2Q, Rev. 1, ICN 2. Qualification of Unqualified Data. Washington, D.C.: U.S. Department of Energy, Office of Civilian Radioactive Waste Management. ACC: DOC.20040127.0008. 
AP-SIII.10Q, Rev. 2, ICN 7. Models. Washington, D.C.: U.S. Department of Energy, Office of Civilian Radioactive Waste Management.

ACC: DOC.20040920.0002.

LA-9331-MS, Los Alamos National Laboratory Quality Assurance Plan And Procedures For The Nevada Nuclear Waste Storage Investigations, ACC: MOL.19980716.0258.

LANL-INC-DP-86, Sorption and Desorption Determinations by a Batch Sample Technique for the Dynamic Transport Task, NNA.19920416.0158.

Quality Assurance Program Index, MOL.19980924.0109 (R0), NNA.19891030.0032 (R1), NNA.19891030.0033 (R2), NNA.19891030.0034 (R3), and NNA.19891030.0035 (R4).

QMI 1-1, Quality Program Management, MOL.20040708.0327.

QMI 9-1, Acquisition and Calibration Control of Standards and Measuring and Testing Equipment, MOL.20040708.0329.

QMI 15-1, Quality Audits, MOL.20040708.0332.

QMR 1, Quality Assurance Management and Planning, MOL.20040708.0326.

QMR 9, Control and Calibration of Standards and Measuring and Testing Equipment, MOL.20040708.0328.

QMR 12, Corrective Action, MOL.20040708.0330.

TWS-CNC-DP-05, Tuff Experiments-Sorption Ratios and Migration Measurements, NNA.19900713.0290 (R0), NNA.19870430.0094 (R1), and NNA.19900809.0031 (R2).

TWS-CNC-WP-03, Tuff Experiments-Sorption Ratios and Migration Measurements, NNA.19891030.0062.

TWS-INC-DP-63, R1. Preparation of NTS Core Samples for Crushed Rock Experiments. Los Alamos, New Mexico: Los Alamos National Laboratory. ACC: HQX.19891002.0093.

\subsection{SOURCE DATA, LISTED BY DATA TRACKING NUMBER}

GS000308311221.005. Net Infiltration Modeling Results for 3 Climate Scenarios for 147613 FY99. Submittal date: 03/01/2000.

GS000608312271.001. Pore-Water Hydrochemistry and Isotopic Data for Boreholes 153407 USW NRG-6, USW NRG-7A, USW SD-7, USW SD-9, USW SD-12, USW UZ-14 and UE-25 UZ\#16 from 10/1/96 to 1/31/97. Submittal date: 06/23/2000. 
GS010608312272.001. Chemical Analysis of Pore Water from Boreholes USW

165860

UZ-7A, USW WT-24, USW SD-6, USW SD-7 and USW SD-12 During FY 1997

and FY 1998. Submittal date: 07/09/2001.

GS010708312272.002. Chemical Data for Pore Water from Tuff Cores of USW

156375

NRG-6, USW NRG-7/7A, USW UZ-14, USW UZ-N55 and UE-25 UZ\#16. Submittal date: $09 / 05 / 2001$.

GS011008312272.004. Analysis for Chemical Composition of Pore Water from Boreholes USW WT-24 and USW SD-6 During FY98 and FY99. Submittal date: 12/20/2001.

GS020408312272.003. Collection and Analysis of Pore Water Samples for the Period 160899 from April 2001 to February 2002. Submittal date: 04/24/2002.

GS020508312242.001. Trench Fault Infiltration in Alcove 8 Using Permeameters from March 5, 2001 to June 1, 2001. Submittal date: 05/22/2002.

GS020908312242.002. Trenched Fault Infiltration in Alcove 8 Using Permeameters from June 1, 2001 to March 26, 2002. Submittal date: 09/17/2002.

162141

GS030108314224.001. Geotechnical Data for Alcove 8 (ECRB) and Niche 3 (ESF): Full Periphery Geologic Map (Drawing OA-46-356). Submittal date: 02/05/2003.

GS950608312231.008. Moisture Retention Data from Boreholes USW UZ-N27 and UE-25 UZ\#16. Submittal date: 06/06/1995.

GS951208312272.004. Analysis for Chemical Composition of Perched-Water from Boreholes USW UZ-14, USW NRG-7A, USW SD-9, USW SD-7 and Groundwater from Boreholes UE-25 ONC\#1 and USW G-2 from 8/18/89 to 3/21/95. Submittal date: $09 / 12 / 2001$

GS980908312242.039. Unsaturated Water Retention Data for Lexan-Sealed Samples 145272 from USW SD-6 Measured Using a Centrifuge. Submittal date: 09/22/1998.

GS990208312272.001. Analysis for Chemical Composition of Pore Water from Borehole USW UZ-14 and UE-25 UZ\#16 and Groundwater from UE-25 UZ\#16. Submittal date: 02/23/1999.

GS990308312242.007. Laboratory and Centrifuge Measurements of Physical and 107185 Hydraulic Properties of Core Samples from Busted Butte Boreholes UZTT-BB-INJ1, UZTT-BB-INJ-3, UZTT-BB-INJ-4, UZTT-BB-INJ-6, UZTT-BB-COL-5 and UZTT-BB-COL-8. Submittal date: 03/22/1999.

GS990708312242.008. Physical and Hydraulic Properties of Core Samples from 109822 Busted Butte Boreholes. Submittal date: 07/01/1999. 
LA000000000034.002. Diffusion of Sorbing and Non-Sorbing Radionuclides.

148603

Submittal date: 06/22/1993.

LA0008WS831372.001. Calculated Daily Injection Rates for the Busted Butte

156582

Unsaturated Zone Transport Tests. Submittal date: 08/23/2000.

LA0010JC831341.001. Radionuclide Retardation Measurements of Sorption

162476

Distribution Coefficients for Barium. Submittal date: 10/19/2000.

LA0010JC831341.002. Radionuclide Retardation Measurements of Sorption

Distribution Coefficients for Cesium. Submittal date: 10/19/2000.

LA0010JC831341.003. Radionuclide Retardation Measurements of Sorption

153322

Distribution Coefficients for Strontium. Submittal date: 10/19/2000.

LA0010JC831341.007. Radionuclide Retardation Measurements of Sorption

153319

Distribution Coefficients for Neptunium. Submittal date: 10/19/2000.

LA0112WS831372.001. Busted Butte UZ Transport Test: Phase II Collection Pad

157100

Tracer Loading. Submittal date: 12/06/2001.

LA0112WS831372.002. Busted Butte UZ Transport Test: Phase II Collection Pad

157115

Tracer Concentrations. Submittal date: 12/06/2001.

LA0112WS831372.003. Busted Butte UZ Transport Test: Phase II Normalized

157106

Collection Pad Tracer Concentrations. Submittal date: 12/06/2001.

LA0201WS831372.007. Busted Butte UZ Transport Test: Phase II Normalized Collection Pad Metal Tracer Concentrations. Submittal date: 01/22/2002.

164721

LA0201WS831372.008. Busted Butte UZ Transport Test: Phase I Collection Pad

162766

Normalized Tracer Concentrations. Submittal date: 01/22/2002.

LA0203WS831372.002. Freundlich and Linear Isotherm Parameters for

161526

Radionuclides and Sorbing Tracers Used in the Busted Butte UZ Transport Test.

Submittal date: 03/20/2002.

LA0206AM831234.001. Eh-pH Field Measurements on Nye County EWDP Wells.

160051

Submittal date: 06/21/2002.

LA0206AM831234.002. Geochemical Field Measurements on Nye County EWDP 163852

Wells. Submittal date: 06/21/2002.

LA0211WS831372.001. Busted Butte Rock Extractions: Measured and Normalized Anion and FBA Tracer Concentrations. Submittal date: 11/19/2002.

162763

LA0302WS831372.001. Fluorescein Plume Images from the Phase 1A Mineback at Busted Butte. Submittal date: 02/26/2003.

162765 
LA0305AM831341.001. 1977 to 1987 Sorption Measurements of AM, BA, CS, NP, 163789 PU, PA, SR, TH, and U with Yucca Mountain Tuff Samples. Submittal date:

$05 / 21 / 2003$.

LA0310AM831341.001. Sorption/Desorption Measurements of Cesium on Yucca 165865 Mountain Tuff. Submittal date: 10/21/2003.

LA0407AM831341.001. Batch Sorption Coefficient Data for Barium on Yucca 170623 Mountain Tuffs in Representative Water Compositions. Submittal date: 07/12/2004.

LA0407AM831341.002. Batch Sorption Coefficient Data for Cesium on Yucca 170621 Mountain Tuffs in Representative Water Compositions. Submittal date: 07/12/2004.

LA0407AM831341.003. Batch Sorption Coefficient Data for Strontium on Yucca 170626 Mountain Tuffs in Representative Water Compositions. Submittal date: 07/12/2004.

LA0407AM831341.004. Batch Sorption Coefficient Data for Neptunium on Yucca 170622 Mountain Tuffs in Representative Water Compositions. Submittal date: 07/12/2004.

LA0407AM831341.005. Batch Sorption Coefficient Data for Plutonium on Yucca Mountain Tuffs in Representative Water Compositions. Submittal date: 07/12/2004.

LA0407AM831341.006. Batch Sorption Coefficient Data for Uranium on Yucca 170628 Mountain Tuffs in Representative Water Compositions. Submittal date: 07/12/2004.

LA0407AM831343.001. Modeling Calculations of Radionuclide Sorption via 171009 Surface-Complexation Reactions. Submittal date: 07/29/2004.

LA9910WS831372.008. Busted Butte UZ Transport Test: Gravimetric Moisture 147156 Content and Bromide Concentration in Selected Phase 1A Rock Samples. Submittal date: $11 / 03 / 1999$.

LA9912WS831372.001. Sorption of Fluorinated Benzoic Acids and Lithium on Rock Samples from Busted Butte, NV. Submittal date: 02/22/2000.

LB0205REVUZPRP.001. Fracture Properties for UZ Model Layers Developed from 159525 Field Data. Submittal date: 05/14/2002.

LB0207REVUZPRP.002. Matrix Properties for UZ Model Layers Developed from 159672 Field and Laboratory Data. Submittal date: 07/15/2002.

LB0208UZDSCPMI.002. Drift-Scale Calibrated Property Sets: Mean Infiltration 161243 Data Summary. Submittal date: 08/26/2002.

LB0210THRMLPRP.001. Thermal Properties of UZ Model Layers: Data Summary. 160799 Submittal date: 10/25/2002. 
LB03013DSSCP3I.001. 3-D Site Scale Calibrated Properties: Data Summaries.

162379

Submittal date: $01 / 27 / 2003$.

LB0301N3SURDAT.001. Niche 3107 Measurements and Elevations Used for Grid

162130

Generation. Submittal date: 01/29/2003.

LB03023DSSCP9I.001. 3-D Site Scale UZ Flow Field Simulations for 9 Infiltration

163044

Scenarios. Submittal date: 02/28/2003.

LB0303A8N3LIQR.001. Alcove 8 - Niche 3 Seepage Data Compilation. Submittal

162570 date: $03 / 19 / 2003$

LB0303A8N3MDLG.001. Alcove 8 - Niche 3 Seepage Modeling: Simulations.

162773

Submittal date: 03/31/2003.

LB0304RDTRNSNS.001. Supporting Files of 3D Flow and Transport Sensitivity

165992

Analyses. Submittal date: 04/29/2003.

LB0307FMRADTRN.001. Fracture-Matrix Partitioning Model: Spreadsheet

Calculations. Submittal date: 07/31/2003.

LB0308AMRU0185.001. Section 6.3.2 Matrix Block Discretization and its Effects on 165172 UZ Flow and Transport Simulations. Submittal date: 08/29/2003.

LB03093RADTRNS.001. Three Way Transport Model Comparison: Input/Output

166225

Files. Submittal date: 09/24/2003.

LB03093RADTRNS.002. Three Way Transport Model Comparison: Data

166071

Summaries. Submittal date: 09/24/2003.

LB0402THRMLPRP.001. Thermal Properties of UZ Model Layers: Data Summary.

168481

Submittal date: 02/20/2004.

LB991220140160.010. Model Prediction of Busted Butte Using T2R3D Input and

164858

Output Files. AMR U0060, "Radionuclide Transport Models Under Ambient

Conditions.". Submittal date: 03/14/2000.

LB997141233129.001. Calibrated Basecase Infiltration 1-D Parameter Set for the UZ 104055

Flow and Transport Model, FY99. Submittal date: 07/21/1999.

MO0006J13WTRCM.000. Recommended Mean Values of Major Constituents in

J-13 Well Water. Submittal date: 06/07/2000.

MO0007MAJIONPH.011. Major Ion Content of Groundwater from Selected Yucca Mountain Project Boreholes Extracted from ANL-NBS-HS-000021, Geochemical and Isotopic Constraints on Groundwater Flow Directions, Mixing and Recharge at Yucca Mountain, Nevada. Submittal date: 07/27/2000. 
MO0407SEPFEPLA.000. LA FEP List. Submittal date: 07/20/2004.

170760

MO0408SPADCLRE.000. Dissolved Concentration Limits of 14 Radioactive

171601

Elements for LA. Submittal date: 08/27/2004.

MO9906GPS98410.000. Yucca Mountain Project (YMP) Borehole Locations.

109059

Submittal date: 06/23/1999.

MO9910MWDISMMM.003. ISM3.1 Mineralogic Models. Submittal date:

119199 10/01/1999.

\subsection{OUTPUT DATA, LISTED BY DATA TRACKING NUMBER}

LA0408AM831341.001. Unsaturated Zone Distribution Coefficients (Kds) for U, $\mathrm{Np}, \mathrm{Pu}, \mathrm{Am}, \mathrm{Pa}, \mathrm{Cs}, \mathrm{Sr}, \mathrm{Ra}$, and Th. Submittal date: 08/24/2004.

LA0311AM831341.001. Correlation Matrix for Sampling of Sorption Coefficient Probability Distributions. Submittal date: 11/06/2003.

LB0307MR0060R1.001. Ambient Radionuclide Transport - Uniform Instantaneous Release. Submittal date: 07/19/2003.

LB0307MR0060R1.002. Ambient Radionuclide Transport - Uniform Instantaneous Release. Submittal date: 07/19/2003.

LB0307MR0060R1.003. Ambient Radionuclide Transport - Uniform Continuous Release. Submittal date: 07/19/2003.

LB0307MR0060R1.004. Ambient Radionuclide Transport - Uniform Continuous Release. Submittal date: 07/19/2003.

LB0307MR0060R1.005. Ambient Radionuclide Transport - Unfaulted Domain, Instantaneous and Continuous Release. Submittal date: 07/19/2003.

LB0307MR0060R1.006. Ambient Radionuclide Transport - Unfaulted Domain, Instantaneous and Continuous Release. Submittal date: 07/19/2003.

LB0307MR0060R1.007. Ambient Radionuclide Transport - TSPA Deliverable Extractions. Submittal date: 07/19/2003.

LB0308MR0060R1.008. Ambient Radionuclide Transport - Model Validation: Simulations. Submittal date: 08/29/2003.

LB0308MR0060R1.009. Ambient Radionuclide Transport - Model Validation: Data Summaries. Submittal date: 08/29/2003.

LB0310MR0060R1.010. Supplemental Radionuclide Transport Simulations: Input/Output files. Submittal date: 10/23/2003. 
LB0310MR0060R1.011. Supplemental Radionuclide Transport Simulations: Data Summaries. Submittal date: 10/23/2003.

LB0408MR0060R2.001. Radionuclide Transport Model Validation Through Comparison to Analytical Solutions. Submittal date: 08/17/2004.

\subsection{SOFTWARE CODES}

BSC 2001. Software Code: PHREEQC. V2.3. PC, LINUX, Windows 95/98/NT, 155323 Redhat 6.2. 10068-2.3-00.

LANL 2003. Software Code: FEHM. V2.21. SUN, SunOS 5.8; PC, Windows 2000 and Linux 7.1. 10086-2.21-00.

LBNL 1999. Software Code: iTOUGH2. V4.0. SUN, DEC. 10003-4.0-00.

139918

LBNL 1999. Software Code: T2R3D. V1.4. FORTRAN 77, SUN, DEC/ALPHA.

146654 10006-1.4-00.

LBNL 1999. Software Code: TOUGH2. V1.11 MEOS9nTV1.0. MAC, SUN, 113943 DEC/Alpha, PC. 10065-1.11MEOS9NTV1.0-00.

LBNL 2000. Software Code: DCPT. V1.0. PC. 10078-1.0-00.

132448

LBNL 2000. Software Code: TOUGH2. V1.4. Sun Ultra Sparc, SUN O.S. 5.5.2, and 146496 DEC/ALPHA, OSF 2 V4.0, OSF1 V5.1. 10007-1.4-01.

LBNL 2002. Software Code: Bkread.f. V1.0. SunOS 5.5.1. 10894-1.0-00.

162143

LBNL 2002. Software Code: DCPT. V2.0. PC, Windows. 10078-2.0-00.

154342

LBNL 2002. Software Code: Smesh.f. V1.0. SunOS 5.5.1. 10896-1.0-00.

162142

LBNL 2002. Software Code: TOUGH2. V1.6. PC/MS-DOS under Windows 98, Sun

160242

UltraSparc OS 5.5.1, DEC-Alpha OSF1 V4.0. 10007-1.6-00.

LBNL 2003. Software Code: TOUGH2. V1.6. PC/MS-DOS Windows 98, Sun

161491

UltraSparc/Sun OS 5.5.1, DEC-Alpha OSF1 V4.0. 10007-1.6-01.

LBNL 2003. Software Code: XtractG.f90. V1.0. MAC, OS 9.2; DEC Alpha, OSF1 V4.0 and OSF1 V5.1. 10930-1.0-00. 
APPENDIX A

TECHNICAL BASIS FOR $K_{d}$ PROBABILITY DISTRIBUTION FUNCTIONS 


\section{A1. INTRODUCTION}

This appendix provides the bases for the derivation of sorption coefficient probability distributions used in the unsaturated zone (UZ) transport calculations. The sorption coefficient data on which the distributions are based were obtained in laboratory experiments in which crushed rock samples from the Yucca Mountain site were contacted with groundwaters (or simulated groundwaters) representative of the site, spiked with one or more of the elements of interest. Sorption experiments have been carried out as a function of time, element concentration, atmospheric composition, particle size, and temperature. In some cases, the solids remaining from sorption experiments were contacted with unspiked groundwater in desorption experiments. The sorption and desorption experiments together provide information on the equilibration rates of the forward and backward sorption reactions. For elements that sorb primarily through surface complexation reactions, the experimental data are augmented with the results of modeling calculations using PHREEQC V2.3 (BSC 2001 [155323]). The inputs for the modeling calculations include groundwater compositions, surface areas, binding constants for the elements of interest, and thermo-dynamic data for solution species. These modeling calculations provide a basis for interpolation and extrapolation of the experimentally derived sorption coefficient dataset.

The primary controls on sorption behavior of the elements of interest in the UZ flow system include the detailed characteristics of mineral surfaces in the rock units through which water flows from the repository to the saturated zone. They also include the detailed chemistry of pore waters and perched waters in the UZ along this flow path, the sorption behavior of each element, and the concentrations of the various radionuclides in the groundwaters. These parameters will be discussed in the following sections.

This appendix provides probability distributions for the sorption coefficient of each element of interest, on the three major rock types (devitrified, zeolitic, and vitric) found in the UZ. The influence of expected variations in water chemistry, radionuclide concentrations, and variations in rock surface properties within one of the major rock types are incorporated into these probability distributions.

\section{A2. AQUIFER MATRIX COMPOSITIONS ALONG MOST PROBABLE TRANSPORT PATHWAYS}

Data on rock mineralogic compositions in the UZ are provided in DTN: MO9910MWDISMMM.003 [DIRS 119199]. These data have been incorporated into a site mineralogic model. There are three dominant rock types in the part of the UZ along potential flow paths from the repository to the saturated zone: devitrified tuff, zeolitic tuff, and vitric tuff. Devitrified tuff is composed primarily of silica (quartz and cristobalite) and alkali feldspar. It may also contain trace amounts of mica, hematite, calcite, tridymite, kaolinite, and hornblende, as well as minor amounts (less than 25 percent) of smectite and/or zeolite. For the purposes of this analysis, sorption coefficient distributions for devitrified tuff are based on data obtained on samples that are composed primarily of silica phases and feldspar with only trace amounts of other phases. Although devitrified tuff samples that contain significant amounts (greater than 5 percent) of clays or zeolites generally have higher sorption coefficients than samples that do not, the distribution of these types of altered rocks along the flow path is not 
established well enough to reliably incorporate these rock types into the sorption coefficient probability distributions.

Sorption coefficient distributions for zeolitic tuff exclusively use samples that contain more than 50 percent zeolite, with the balance made up of clay, silica phases, alkali feldspar, and/or glass. For vitric tuffs, sorption coefficient distributions are based on samples that contain more than 50 percent glass, with the remainder made up predominantly of feldspar, silica phases, zeolites, and clay.

The total number of samples for each rock type used in the analysis in this section included 38 devitrified tuffs, 34 zeolitic tuffs, and 7 vitric tuffs. Not all of these samples were obtained from the UZ. Nonetheless, these samples as a whole are taken to be representative of devitrified, zeolitic, and vitric tuffs in the Yucca Mountain UZ.

As explained below, surface areas for the tuffs are inputs to PHREEQC V2.3 calculations. Triay et al. (1996 [DIRS 101023], p. 62) presented surface-area analyses for 23 tuff samples. A table of the analyses with averages (omitting these outlying samples) is presented in Table A-1.

Table A-1. Surface Areas ${ }^{a}$ in $\mathrm{m}^{\mathrm{b}} / \mathrm{g}$ for Yucca Mountain Tuffs

\begin{tabular}{|c|c|c|c|c|c|c|}
\hline & & Not Ground & Dry-sieved2 & $\begin{array}{c}\text { Wet sieved2 } \\
\text { With J-13 water } \\
\text { trial } \# 1\end{array}$ & $\begin{array}{c}\text { Wet sieved2 } \\
\text { With J-13 water } \\
\text { trial \#2 }\end{array}$ & $\begin{array}{c}\text { Wet sieved2 } \\
\text { With UE-25 p\#1 } \\
\text { water }\end{array}$ \\
\hline USW G1-732 & Devitrified & 2.1 & 2.7 & 2.6 & 2.5 & 3.3 \\
\hline USW G1-1936 & Devitrified & 4.5 & 4.9 & 3.6 & 3.9 & 3.7 \\
\hline USW G4-270 & Devitrified & 2.0 & 6.4 & 5.1 & & 5.0 \\
\hline USW G4-275 & Devitrified & 2.9 & 4.5 & & & \\
\hline USW G4-2570 & Devitrified & 2.8 & 3.6 & 2.9 & 2.9 & 2.8 \\
\hline USW GU3-747 & Devitrified & 2.2 & 2.9 & 2.8 & 2.4 & 2.8 \\
\hline \multirow[t]{2}{*}{ USW GU3-2325 } & Devitrified & 1.8 & 2.5 & 2.2 & 1.8 & 2.5 \\
\hline & Average & 2.6 & 3.9 & 3.2 & 2.7 & 3.4 \\
\hline USW G1-1405 & Zeolitic & 32 & 28 & 26 & & 31 \\
\hline USW G2-1813 & Zeolitic & & & 34 & & \\
\hline USW G2-1951 & Zeolitic & & & 66 & & \\
\hline USW GU3-1992 & Zeolitic & & & 32 & & \\
\hline USW G4-1506 & Zeolitic & 22 & 30 & 27 & & 25 \\
\hline USW G4-1529 & Zeolitic & 37 & 21 & 22 & & 31 \\
\hline USW G4-1530 & Zeolitic & 40 & 41 & & & \\
\hline USW G4-1625 & Zeolitic & 28 & 27 & 28 & & 33 \\
\hline USW G4-1772 & Zeolitic & 23 & 22 & 23 & 23 & 23 \\
\hline \multirow[t]{2}{*}{ USW G4-2077 } & Zeolitic & 19 & 18 & & & \\
\hline & Average & 28.7 & 26.7 & 32.3 & 23 & 28.6 \\
\hline
\end{tabular}


Table A-1. Surface Areas ${ }^{a}$ in $\mathrm{m}^{\mathrm{b}} / \mathrm{g}$ for Yucca Mountain Tuffs (Continued)

\begin{tabular}{|l|l|l|l|l|l|l|}
\hline & & Not Ground & Dry-sieved2 & $\begin{array}{c}\text { Wet sieved2 } \\
\text { With J-13 water } \\
\text { trial \#1 }\end{array}$ & $\begin{array}{c}\text { Wet sieved2 } \\
\text { With J-13 water } \\
\text { trial \#2 }\end{array}$ & $\begin{array}{c}\text { Wet sieved2 } \\
\text { With UE-25 p\#1 } \\
\text { water }\end{array}$ \\
\hline USW G2-767 & Vitric & 0.89 & 1.1 & 0.62 & 0.71 & 0.87 \\
\hline USW GU3-1249 & Vitric & 0.52 & 0.92 & 0.99 & & 0.87 \\
\hline USW GU3-1407 & Vitric & 1.7 & 3.3 & 3.0 & & 3.2 \\
\hline & Average & $\mathbf{1 . 0}$ & $\mathbf{1 . 8}$ & $\mathbf{1 . 5}$ & $\mathbf{0 . 7 1}$ & $\mathbf{1 . 6}$ \\
\hline
\end{tabular}

Source: Triay et al. (1996 [DIRS 101023], pp. 5, 6, 8, 62, and Appendix C).

${ }^{a}$ BET method.

b $75-500 \mu \mathrm{m}$ fraction.

\section{A3. RADIONUCLIDES OF INTEREST AND THEIR POTENTIAL CONCENTRATION RANGES IN TRANSPORT SYSTEM}

The list of radionuclides for which sorption coefficient data are required was derived in Radionuclide Screening (BSC 2002 [DIRS 160059], Table 10). The list includes isotopes of $\mathrm{Am}, \mathrm{Cs}, \mathrm{Np}, \mathrm{Pa}, \mathrm{Pu}, \mathrm{Ra}, \mathrm{Sr}, \mathrm{Th}$, and U. Because different isotopes of a given (heavy) element behave the same in chemical reactions, this discussion will focus on this list of elements.

Sorption coefficients for the radionuclides-of-interest are often a function of the concentrations of the radionuclides present in solution (i.e., isotherms are often nonlinear). Thus, sorption experiments have been carried out as a function of radionuclide concentration. As the radionuclide concentrations are increased in the experiments, an upper limit is eventually reached for most elements-of-interest where a pure phase incorporating the element of interest may be precipitated out of solution. This concentration is known as the solubility limit for the element of interest in the experimental system. Because sorption experiments have been carried out by the Yucca Mountain Project (YMP) up to the solubility limit for most elements-of-interest, the ranges of sorption coefficients obtained reflect this dependency. The only element-of-interest for which the experimental concentrations did not approach a solubility limit was cesium. The solubility of cesium is very high (Section A-8.2). Thus, the sorption coefficient probability distributions for cesium must be calibrated to the cesium concentrations expected in the unsaturated zone.

A sorption experiment in which the final concentration of a radionuclide-of-interest in solution is in excess of a solubility limit should not be used to derive a sorption coefficient. The reason for this is that the concentration of the radionuclide-of-interest in the solid phase in this situation represents a combination of the amount sorbed onto the solid phase (i.e., rock) and the amount of the pure phase precipitated out of solution. A sorption coefficient is supposed to reflect only sorption reactions.

As part of the evaluation of the sorption coefficient data obtained by the Project for the various elements-of-interest and water/rock combinations, the final solution concentrations of the radionuclide-of-interest in each sorption experiment was compared against a solubility limit that reflected the experimental conditions (i.e., water composition, temperature, atmosphere). Experiments in which final solution concentrations of the radionuclide-of-interest exceeded these solubility limits were not used in the derivation of sorption coefficient probability distribution 
functions. The solubility limits used in this analysis were either obtained from solubility experiments carried out by YMP or from calculations using the geochemical code PHREEQC. For solubility limits obtained from solubility experiments using waters from the site (i.e., J-13

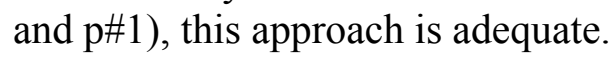

For the solubility limits not based on solubility experiments with site waters, a potential problem can arise with this approach. This problem results from the fact that the waters used in the sorption experiments (e.g., J-13, p\#1) contain natural concentrations of some of the elements-of-interest. For example, J-13 and p\#1 water contain background concentrations of the elements-of-interest barium, cesium, protactinium, strontium, and thorium. During sorption experiments, this background is sorbed to the solid phase along with the radioactive isotopes used in the experiments. Thus, the actual concentrations of these elements-of-interest were higher in these sorption experiments than the concentrations calculated only based on the activity of the radioactive isotopes used in the experiments. The problem arises mainly in sorption experiments carried out at final solution concentrations that were just below the solubility limit based only on the concentration of the radioactive isotope. When the natural element concentrations are added to the concentrations of the radioactive isotope, some of these solutions could actually have been oversaturated with a pure solid phase of the element-of-interest.

Normally, it would be expected that the precipitation of a pure phase would result in a higher sorption coefficient than would be the case of such a phase had not precipitated. However, as discussed below in the sections on individual elements, the experimental data do not reflect this expectation. In fact, experiments in which final solution concentrations exceeded solubility limits (i.e., over-saturated solutions) generally resulted in lower sorption coefficients than experiments with under-saturated solutions. A likely reason for this observed behavior is that the nucleation and/or precipitation rates of the appropriate pure solid phases were slow enough under the conditions used in the experiments so that no solid precipitates were formed. Alternatively, the pure-phase precipitates did form but were too fine-grained to be removed from solution by the techniques used to separate the solid and liquid phases at the end of each experiment.

There is another aspect of natural element "background" that is worth mention. The fact that those elements-of-interest that have a natural background generally had final solution concentrations in the sorption experiments carried out by YMP higher than those calculated only on the basis of the radioactive isotope concentration used in the experiments, even in under-saturated solutions. In those cases where the sorption isotherm for the element-of-interest is nonlinear, the presence of the natural background concentration in the sorption experiments would generally result in lower sorption coefficients than would be the case in the absence of the natural background concentrations.

Thus, in the derivation of sorption coefficient probability distributions for total system performance assessment, the inclusion of sorption coefficient data from experiments containing natural background concentrations generally results in an underestimation of retardation potential. 
Sorption coefficients for the radionuclides of interest can be a function of the concentrations of the radionuclides present in solution. Thus, experiments have been carried out as a function of radionuclide concentrations for most of the elements of interest. In most cases, experiments were carried out over a range of concentrations up to a solubility limit. Above the solubility limit, a solid phase incorporating the element of interest is precipitated out of solution. Therefore, the concentration of an element in solution cannot rise much higher than the solubility limit. Experiments in which the final solution concentrations for a given element of interest exceeded the solubility limit are not useful in this analysis and will be rejected.

Because experiments have been carried out at concentrations up to the solubility limit for most elements of interest, the experimental results and the probability distributions derived from them include this dependency. The only element for which the experimental concentrations did not approach a solubility limit was Cs. The solubility of cesium compounds is very high in Yucca Mountain waters (BSC 2004 [DIRS 169425], Section 6.17). Thus, the sorption coefficient probability distributions for cesium must be calibrated to the Cs concentrations expected in the UZ.

\section{A4. WATER COMPOSITIONAL RANGES ALONG TRANSPORT PATHWAYS}

The chemistry of pore waters and perched waters in the UZ along potential flow paths to the accessible environment is discussed in the Analysis of Geochemical Data for the Unsaturated Zone (BSC 2003-2004 [DIRS 168343169734], Section 5.2.2.4). In the UZ, two rather distinct water types exist in the ambient system. One is perched water and the other is pore water. Perched water is generally more dilute than pore water.

Table A-2. Composition of Yucca Mountain UZ Waters

\begin{tabular}{|c|c|c|c|c|c|c|}
\hline Element & Units & $\mathrm{J}-13^{\mathrm{a}}$ & $\begin{array}{c}\text { Range of } \\
\text { concentrations in } \\
\text { perched waters }\end{array}$ & $\begin{array}{c}\text { Range of concentrations } \\
\text { in pore waters within } \\
\text { and below repository } \\
\text { horizon }^{c}\end{array}$ & $\begin{array}{l}\text { UE } 25 \text { p\#1-c } \\
\text { Carbonate } \\
\text { aquifer }^{\mathrm{a}}\end{array}$ & $\begin{array}{c}\text { Syntheti } \\
\text { c p\#1 }\end{array}$ \\
\hline $\mathrm{Ca}$ & $\mathrm{mg} / \mathrm{L}$ & 11.5 & $2.9-45$ & $0.3-91.8$ & 87.8 & \\
\hline $\mathrm{Mg}$ & $\mathrm{mg} / \mathrm{L}$ & 1.76 & $0-4.1$ & $0-24.6$ & 31.9 & \\
\hline $\mathrm{Na}$ & $\mathrm{mg} / \mathrm{L}$ & 45 & $34-98$ & $3-207$ & 171 & 261 \\
\hline $\mathrm{K}$ & $\mathrm{mg} / \mathrm{L}$ & 5.3 & $3.6-5.8$ & $1.4-148.7$ & 13.4 & \\
\hline $\mathrm{SiO}_{2}$ & $\mathrm{mg} / \mathrm{L}$ & 64.2 & 7.7-64 & $5-352$ & 64.2 & \\
\hline $\mathrm{Cl}$ & $\mathrm{mg} / \mathrm{L}$ & 6.4 & $4.1-16$ & $6-130$ & 37 & \\
\hline $\mathrm{SO}_{4}$ & $\mathrm{mg} / \mathrm{L}$ & 18.1 & $4-223$ & $6-101.1$ & 129 & \\
\hline $\mathrm{HCO}_{3}$ & $\mathrm{mg} / \mathrm{L}$ & 128 & $112-197$ & $8-384$ & 666 & 691 \\
\hline $\mathrm{pH}$ & & 6.9 & $7.6-8.7$ & $6.7-9.7$ & 6.7 & \\
\hline
\end{tabular}

a DTN: Ogard and Kerrisk (1984 [DIRS 100783], Table 1).

b DTN: GS951208312272.004 [DIRS 165858].

c DTN: GS010708312272.002 [DIRS 156375]; GS011008312272.004 [DIRS 165859]; GS990208312272.001 [DIRS 146134]; GS010608312272.001 [DIRS 165860]; GS000608312271.001 [DIRS 153407]. 
The J-13 and UE p\#1-c waters were used in sorption experiments as end-member compositions intended to bracket the impact of water composition on sorption coefficients. Note that the "-c" designation for the UE $\mathrm{p \# 1}$ water indicates water taken from the section of the borehole in the carbonate aquifer. Because water from the UE $\mathrm{p} \# 1$ well was not available to the experimental program at all times, a synthetic $\mathrm{p} \# 1$ water was developed. This water (Table A-2) was primarily intended to have a bicarbonate concentration similar to that found in UE $\mathrm{p} \# 1$. It was used primarily in experiments with uranium, neptunium, and plutonium because the solution and sorption behavior of these elements is sensitive to the bicarbonate and carbonate concentrations in solution.

\section{A5. EXPERIMENTAL TECHNIQUES}

Most of the experimental data upon which the conclusions of this section are based were obtained at Los Alamos National Laboratories over a period of 25 years. Over this time period, there were minor changes in the experimental techniques used to obtain sorption coefficient data. However, the main technique was developed early on and was maintained through most of the 25-year time frame.

It is the intent of this section to provide an overview of the experimental techniques used to obtain sorption coefficients. The details of the various techniques used (e.g., radioactivity counting techniques) are provided in the quality assurance procedures developed for this purpose. These procedures can be obtained from the YMP.

The basic technique for the laboratory determination of sorption coefficients involved the contact of a groundwater sample, spiked with the radionuclide of interest, with a crushed sample of tuff or alluvium. The rock sample was generally obtained as a core sample. The rock and water samples were not sterilized and, therefore, contain representative microbial biota from the UZ. The effects of microbes on sorption are, therefore, included in the sorption coefficients measured in these experiments. The rock was crushed in a jaw crusher and subsequently sieved to a selected grain-size fraction. The sieving process was usually carried out under water. Initially, several different grain-size fractions were used in the experiments. With experience, it was concluded that the 75 to $500 \mu \mathrm{m}$ fraction was the most appropriate for use in these types of experiments. Results for samples, which included all sizes below a certain grain size (e.g., less than $35 \mu \mathrm{m}$ ), tended to produce higher sorption coefficient values than the 75 to $500 \mu \mathrm{m}$ fraction. This was thought to result from increased surface area, the creation of active surface sites, or mineral fractionation. Because the tuffs are very fine grained (i.e., crystal sizes on the order of $10-20 \mu \mathrm{m})$, crushing would not produce significant increases in mineral surface area. The idea that active sites were created by crushing the rock samples was negated by analysis of sorption coefficients over a range of grain sizes from a single rock sample. This analysis (Rogers and Meijer 1993 [DIRS 123127], pp. 1511 to 1512) showed that different size fractions do not necessarily produce different sorption coefficient values. This was thought to reflect the very fine-grained nature of the tuffs. Mineral fractionation is the process by which certain minerals such as clays become enriched in a very fine-grain-size fraction. This is the most likely explanation for the higher sorption coefficients obtained in experiments that included fines. 
One gram of crushed rock material was added to a test tube with $20 \mathrm{~mL}$ groundwater spiked with the radionuclide(s) of interest. The test tube was put on a shaker table for a predetermined period to allow reaction to occur. After the predetermined time had passed, the solution was separated from the solid phase by either centrifugation or filtration. Centrifugation was preferred for those elements thought to have an affinity for the filter medium. The separations were not always perfect, due to various experimental constraints. In some cases, the solid fraction was separately counted. A sorption coefficient was usually calculated from the difference between the initial and final solution concentrations. Corrections were generally made for sorption onto the surface of the test tube during the equilibration (shaking) period.

Some potential sources of errors and experimental artifacts that may pertain to the sorption coefficients obtained at Los Alamos include weighing errors, counting errors, errors resulting from solutions being oversaturated with the element of interest, errors from imperfect solid/liquid separations, errors from inaccurate correction for sorption onto container walls, recording errors, transcription errors, inadvertent laboratory errors, and calculation errors. These errors cannot be quantitatively assessed. However, their existence will become apparent in the scatter of the data on diagrams presented in a later section.

\section{A6. APPROACH TO THE DERIVATION OF KD RANGES FOR MAJOR ROCK TYPES IN THE YUCCA MOUNTAIN FLOW SYSTEM.}

The derivation of sorption coefficient probability distributions for the elements of interest on the major rock types in Yucca Mountain involves both an evaluation of available experimental data and sorption modeling. Experimental data will be used to evaluate the impact of variations in rock sorption properties within each of the rock types, radionuclide concentrations, sorption kinetics, and water chemistry on sorption coefficients for the elements of interest.

The radionuclides of interest are divided into three groups of radioelements. For the first group, including $\mathrm{Am}, \mathrm{Np}, \mathrm{Pa}, \mathrm{Pu}, \mathrm{Th}$, and $\mathrm{U}$, experimental data is used to evaluate the impact of radionuclide concentrations, sorption kinetics, and variations in water chemistry on sorption coefficients. Surface complexation modeling is used to further evaluate the impact of variations in water chemistry and surface area on sorption coefficients. The surface complexation models used in this analysis are based on the software code PHREEQC V2.3. The binding constants required for surface complexation modeling are either obtained from the literature or derived from experimental data involving sorption of the radioelement on quartz (see discussion in Section A7).

In the second group of elements, including $\mathrm{Cs}, \mathrm{Pa}, \mathrm{Ra}$, and $\mathrm{Sr}$, the ranges of sorption coefficient values for the major rock types are derived directly from the available experimental data and the ranges for environmental variables expected in the transport system. Although it would be preferable to have a theoretical model to evaluate /the impact of variations in water chemistry and rock chemistry on sorption coefficients for these radionuclides, sufficient data are not available to properly constrain such a model.

For the third group, including carbon, iodine, and technetium, the sorption coefficient is set to zero in volcanic rocks and in alluvium. 
The modeling of sorption in the total system performance assessment is based on the linear sorption model, which is characterized by the same lumped parameter for the sorption coefficient $K_{d}$ used in the process models. All aqueous radionuclides traveling in fractures are conservatively assumed not to be absorbed by the fracture walls (BSC 2004 [DIRS 170041], Section 6.5.8). For radionuclides that travel in the matrix, the partitioning of radionuclides between the solute and the matrix is described by the sorption coefficient for each radionuclide. The matrix sorption coefficients that have been developed for different rock types (zeolitic, devitrified, and vitric) are listed in Table 6-3 with their statistical distributions. These distributions are sampled to represent the uncertainty in sorption in the total system performance assessment. The influence of expected variations in water chemistry, radionuclide concentrations, and variations in rock surface properties within each of the major rock types were incorporated into the probability distributions. Effective $K_{d}$ values, obtained from batch experiments involving high-concentration solutions, will tend to underestimate the field $K_{d}$ values if the expected field concentrations are low and nonlinear sorption prevails (Section 6.1.2.3).

The distributions were derived from available project data combined with external data that indicate the functional dependencies of sorption on several independent variables. Many site-specific $K_{d}$ measurements have been made using Yucca Mountain rocks and representative groundwater. Project experimental data bounds the function for some, but not all, variables. Data from the literature (e.g., Allard and Beall 1979 [DIRS 147235]; Allard et al. 1980 [DIRS 104410]; Allard et al. 1983 [DIRS 162982]) provided further insights into how other variables affect sorption behavior. PHREEQC model results were used to assess how water chemistry and surface area affect sorption behavior. Chemical insights regarding the kinds of reactions involved were also used. These insights included potential impacts from variations in water compositions (e.g., Eh) outside those addressed in the experimental program, variations in surface areas, variations in mineralogical composition, and so on. In the case of mineralogical composition, an attempt was made to emphasize those experiments that used samples closest to the mean composition of the hydrologic units as defined in the transport model. All of this information was considered in the derivation of the final distribution for each radioelement on each rock type. To incorporate uncertainties in project and literature data and their application for this purpose, the results were conservatively biased by selecting values at the low ends of the ranges.

\section{A7. SURFACE COMPLEXATION MODELING}

The PHREEQC surface complexation model used in this analysis is a nonelectrostatic model (Parkhurst 1995 [DIRS 142177], p. 10-11). Inputs required for modeling include deprotonation constants, binding constants for elements of interest, total site concentration, water chemistry, and a thermo-dynamic database for solution species and solids. Because the tuffs contain up to 80 weight-percent silica (Broxton et al. 1986 [DIRS 100023], p. 39), a silica surface was used to represent the mineral surfaces in the tuffs. The surface complexation models included the effects of competition from common constituents in the rock such as $\mathrm{Ca}, \mathrm{Mg}, \mathrm{Na}, \mathrm{K}$, and $\mathrm{Al}$. Deprotonation constants for silica and binding constants for aluminum on silica were obtained from the report by Dixit and Van Cappellen (2002 [DIRS 162985], p. 2565). Binding constants were obtained from the report by Triay et al. (1997 [DIRS 100422], Table 22) for potassium and calcium on silica, and from the report by Marmier et al. (1999 [DIRS 162986], p. 228) for 
sodium on silica (the same value was used for potassium). The total site concentrations were obtained from surface areas reported in Batch Sorption Results for Neptunium Transport Through Yucca Mountain Tuffs (Triay et al. 1996 [DIRS 101023], p. 62) and a site density of 2.3 sites $/ \mathrm{nm}^{2}$ as recommended by Pabalan et al. (1998 [DIRS 162987], p. 124). The thermo-dynamic database used for the modeling was the LLNL.DAT database supplied with the baselined YMP version of the PHREEQC V2.3 code.

\section{A8. DISCUSSION AND ANALYSIS OF EXPERIMENTAL SORPTION COEFFICIENT DATA AND SURFACE COMPLEXATION MODELING}

The data and modeling results for each element are discussed in separate sections. The sections are arranged in alphabetical order.

\section{A8.1. AMERICIUM}

According to Nitsche et al. (1993 [DIRS 155218], pp. 78, 86), the solubility of americium in $\mathrm{J}-13$ water is controlled by $\mathrm{Am}(\mathrm{OH}) \mathrm{CO}_{3}$. At $\mathrm{pH}=8.5$, the solubility is $2.4(+/-1.9) \times$ $10^{-9} \mathrm{moles} / \mathrm{Liter}$ at $25^{\circ} \mathrm{C}$. At $\mathrm{pH}=7.0$, the solubility is $1.2(+/-0.3) \times 10^{-9} \mathrm{moles} / \mathrm{Liter}$ at $25^{\circ} \mathrm{C}$.

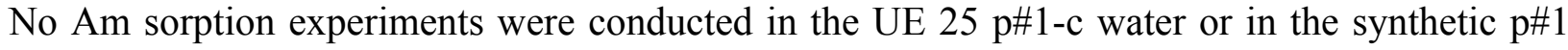
water.

The differences in the Am solubility values reported in Measured Solubilities and Speciations of Neptunium, Plutonium, and Americium in a Typical Groundwater (J-13) from the Yucca Mountain Region (Nitsche et al. 1993 [155218]) and in the report Dissolved Concentration Limits of Radioactive Elements (BSC 2004 [DIRS 169425], Section 6.9) could reflect the impact of coprecipitation reactions on the Nitsche et al. (1993) experiments. As noted by Nitsche et al. (1993 [DIRS 155218]), Am behaves very similarly to the lanthanide element neodynium. Neodynium is a member of the lanthanide series elements. The elements of this series behave similarly in chemical reactions. The lanthanide series (also called the rare earth elements) includes La, Ce, Pr, Nd, Pm, Sm, Eu, Gd, Tb, Dy, Ho, Er, Tm, and Yb. The chemistry of the element $\mathrm{Y}$ is very similar to the chemistry of the lanthanide series elements and is usually included in discussion of the series.

The experiments reported in Nitsche et al. (1993 [155218]) were carried out using natural J-13 water. This water would have contained some concentration of each of the lanthanide series elements and Y. The presence of a rare earth element (REE) and Y in this water could have lead to the coprecipitation of Am with REE and $\mathrm{Y}$ in a solid solution (i.e., Am-REE-YOHCO 3 ) during the experiments carried out by Nitsche et al (1993). This solid solution would have precipitated at lower Am concentrations than would have been the case if the REE and Y had not been present in J-13 water. In effect, some significant fraction of the concentrations of the REE and Y would be added to the concentration of Am in the solubility product.

The solubility calculations carried out in the BSC report (BSC 2004 [DIRS 169425], Section 6.9) did not consider the effects of trace elements, in particular the rare earth elements and Y. This may explain the differences in the solubility values obtained experimentally (Nitsche et al. 1993 [DIRS 155218]) and theoretically (BSC 2004 [DIRS 169425], Section 6.9). 
Furthermore, the results for transport of radionuclides given in Figure 6-65 show that transport is relatively insensitive to the value of the sorption coefficient in excess of $100 \mathrm{~mL} / \mathrm{g}$. In the case of Am, selecting the higher solubility limits given by BSC (2004 [DIRS 169425], Section 6.9) would lead to lower limits for $K_{d}$ on the order of $200 \mathrm{~mL} / \mathrm{g}$ instead of $1000 \mathrm{~mL} / \mathrm{g}$ for devitrified and zeolitic rock. Changes in $\mathrm{K}_{\mathrm{d}}$ over this range would have only a minor effect on aqueous transport of Am. Due to the high $\mathrm{K}_{\mathrm{d}}$ values for aqueous Am, the main transport mechanism for Am to reach the accessible environment is through colloid transport.

\section{A8.1.1 Devitrified Tuff}

Sorption coefficients measured on devitrified tuffs are plotted versus calculated final solution concentrations in Figure A-1. The points identified by the term "sorption" refer to experiments in which J-13 water spiked with americium was contacted with devitrified tuff. The points identified by the term "desorption" refer to experiments in which the solid remaining after the "sorption" step was contacted with unspiked J-13. The steep negative slopes evident at the higher concentrations for individual samples reflect a mass-balance constraint. That is, the final solution concentration is calculated using the starting solution concentration and the measured sorption coefficient. This forces a linear dependence of the sorption coefficient on the final solution concentration. The offset between the points for the various samples primarily reflects different starting concentrations.

The position of a sample along a slope reflects the degree of equilibration among various experiments on the same sample with the same starting concentration. When the sorption experiments are initiated, these trends start on the abscissa at the initial concentration. As experiment duration is increased, americium is sorbed to the solid phase, the solution concentration decreases correspondingly, and the $\mathrm{K}_{\mathrm{d}}$ increases. At equilibrium, the solution concentration remains constant with time, and the $\mathrm{K}_{\mathrm{d}}$ is at a maximum value. For desorption experiments, the trend is reversed. As the duration of a desorption experiment increases, the solution concentrations increase as americium is released from the solid, and the $\mathrm{K}_{\mathrm{d}}$ decreases. At equilibrium, the $\mathrm{K}_{\mathrm{d}}$ obtained from a desorption experiment should approach the $\mathrm{K}_{\mathrm{d}}$ value obtained from the associated sorption experiment, unless the isotherm is quite nonlinear (in which case the trends could be offset). The offset would be caused by a different total americium concentration in the sorption and desorption experiments. The $\mathrm{K}_{\mathrm{d}}$ value on which the two trends converge is considered the equilibrium value.

As shown in Figure $\mathrm{A}-1$, the calculated final solution concentrations were higher than the solubility of $\mathrm{Am}(\mathrm{OH}) \mathrm{CO}_{3}$ in numerous experiments with samples JA-32 and YM-54. Thus, the results for these experiments must be discounted. For the remaining experiments, the sorption coefficients range from 1,000 to more than $10,000 \mathrm{~mL} / \mathrm{g}$. In one sample (GU3-688), americium concentrations were analyzed by isotope dilution mass spectrometry (Triay et al. 1991 [DIRS 104129], p. 144). This technique has higher sensitivity than the (radioactivity) counting techniques normally used, thereby allowing the use of lower americium concentrations. The result obtained for this sample is in the range of the results obtained for the other samples. 
The sorption coefficients derived from desorption experiments are generally larger than those derived from the sorption experiments on a given sample (Figure A-1). This could result from slow desorption kinetics or a nonlinear isotherm.

Americium sorption coefficients are plotted versus duration of the sorption and desorption experiments in Figure A-2. The trend in the data (ignoring data from samples JA-32 and YM-54) suggests americium sorption coefficient values are not very sensitive to the duration of the experiments except in those experiments with very short durations. Thus, the kinetics of the americium sorption reactions appear to be relatively fast.

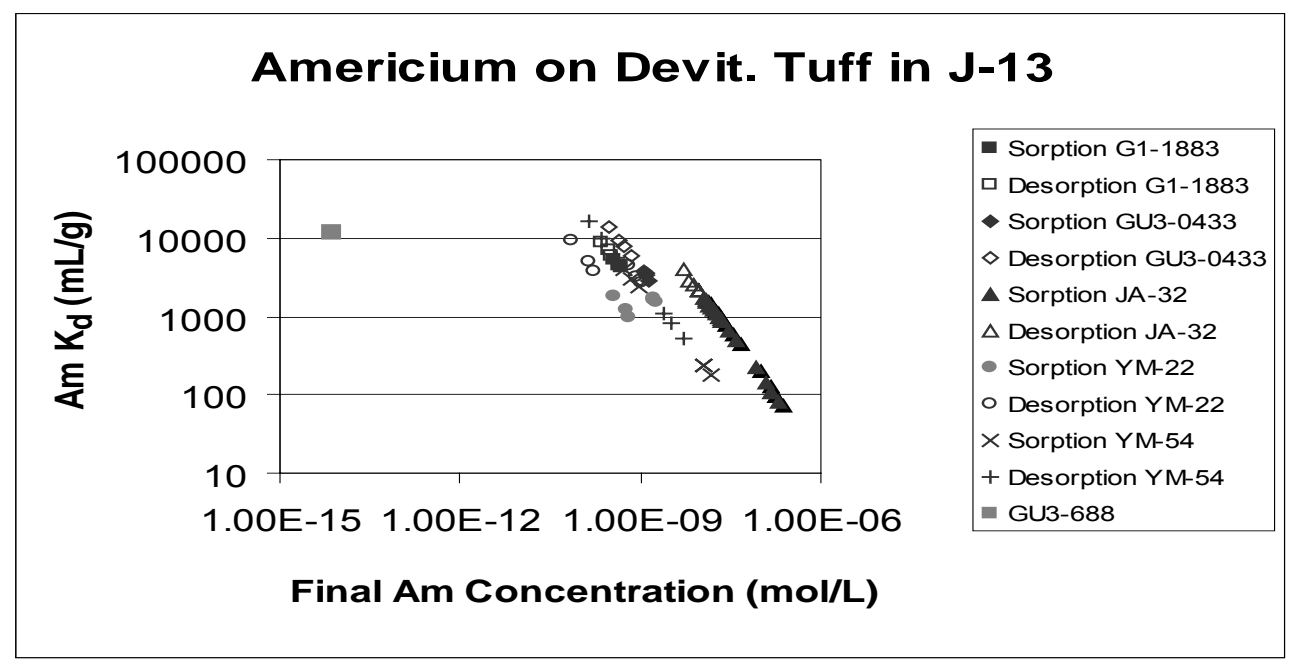

DTN: LA0305AM831341.001 [DIRS 163789].

Figure A-1. Americium Sorption Coefficients on Devitrified Tuff versus Calculated Final Cesium Concentration in Solution

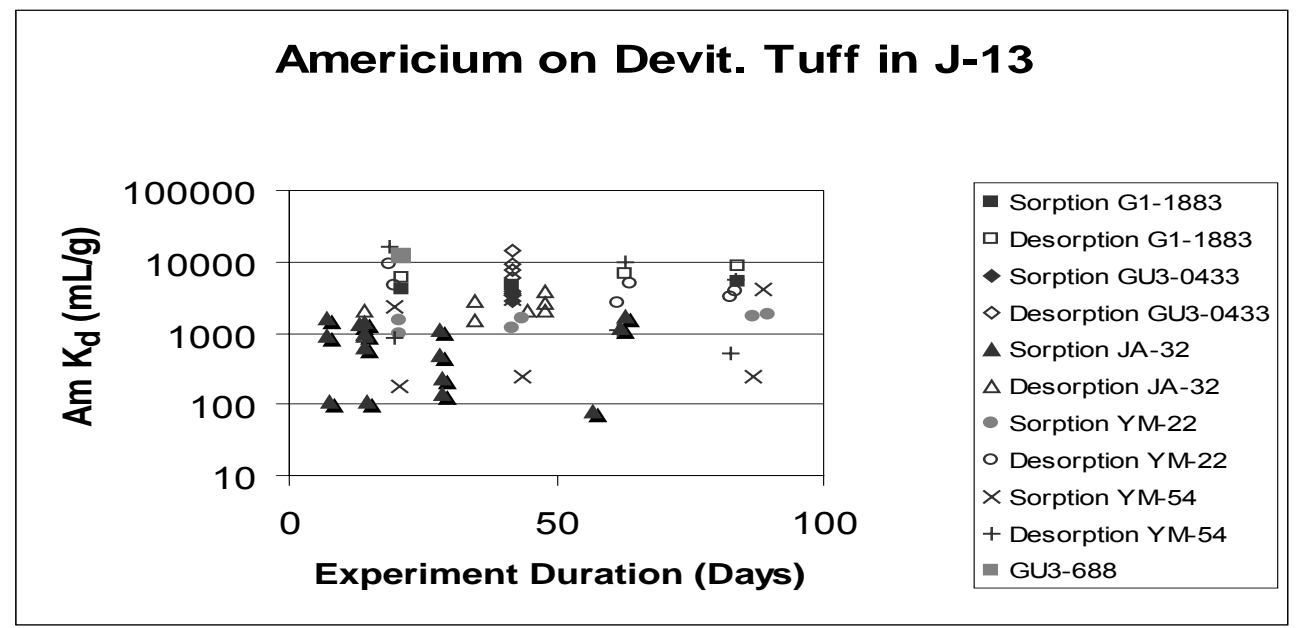

DTN: LA0305AM831341.001 [DIRS 163789].

Figure A-2. Americium Sorption Coefficients for Devitrified Tuff as a Function of Experiment Duration 
The effects of variations in water chemistry on americium sorption coefficients have been not been tested experimentally except for variations in final solution $\mathrm{pH}$. All the americium sorption and desorption experiments carried out with Yucca Mountain samples used J-13 water. Americium sorption and desorption coefficients in J-13 water are plotted versus $\mathrm{pH}$ in Figure A-3. It is evident that there is no clear trend among the data points plotted. The variations in sorption coefficients observed in multiple experiments with the same rock sample could reflect (radioactivity) counting statistics, long-term stability of the counting equipment, sorption kinetics, imperfect separation of the solid and liquid phases, and/or the consistency of adsorption of americium to the walls of the experimental containers. Counting statistics are generally a small percentage of the error in the measured sorption coefficient. Sorption kinetics may explain some of the scatter but not all of it. The other factors are likely significant, but they cannot be quantified with the available data.

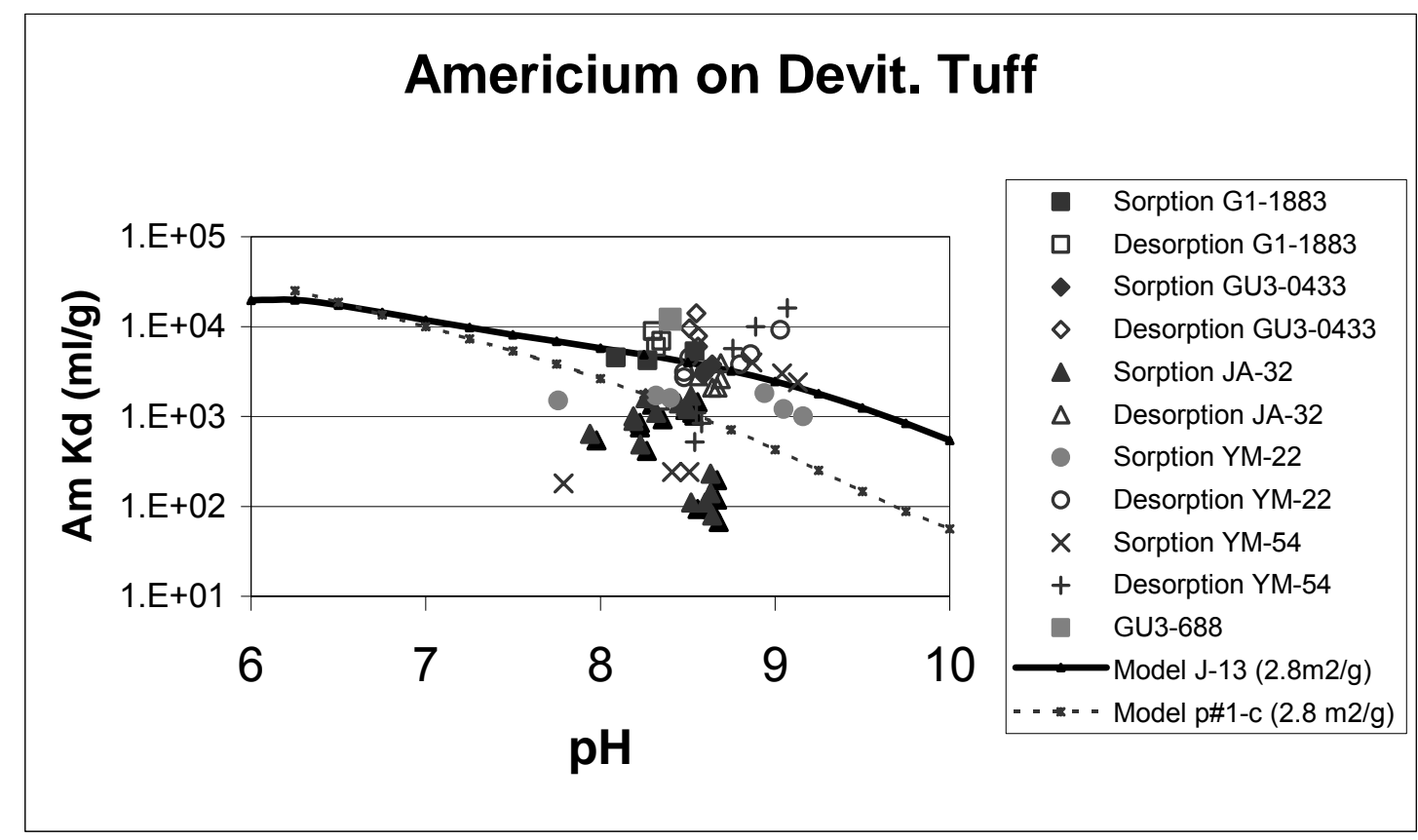

DTNs: LA0407AM831343.001 [DIRS 171009]; LA0305AM831341.001 [DIRS 163789].

Figure A-3. Americium Sorption Coefficients on Devitrified Tuff Versus pH of J-13 Water

To gauge the potential impact of variations in water chemistry on americium sorption coefficients, surface complexation modeling was carried out with PHREEQC. In the modeling, it was assumed all surface sites on devitrified tuff were equivalent to surface sites on silica. Binding constants for americium species on silica were derived by fitting data presented by Beall et al. (1986 [DIRS 162983], p. 502) for the sorption of americium onto quartz $\left(2.8 \mathrm{~m}^{2} / \mathrm{g}\right.$; Allard et al. 1980 [DIRS 104410], p. 478) in Oak Ridge National Laboratory Standard Water. This water is similar in composition to J-13. As shown in Figure A-4a, the sorption coefficients for americium on quartz (Beall et al. 1986 [DIRS 162983], p. 502) as a function of pH could be fit very well using two surface reactions involving an americium sulfate complex and an americium carbonate complex. These reactions are listed in Table A-3. 
Table A-3. Surface Complexation Reactions for Americium

\begin{tabular}{|ll|}
\hline $\mathrm{SiOH}+\mathrm{Am}^{3+}+\mathrm{SO}_{4}{ }^{2-} \leftrightarrow \mathrm{SiOAmSO}_{4}+\mathrm{H}^{+}$ & Log K$=5.5$ \\
\hline $\mathrm{SiOH}+\mathrm{Am}^{3+}+\mathrm{CO}_{3}{ }^{2-} \leftrightarrow \mathrm{SiOAmCO}_{3}+\mathrm{H}^{+}$ & Log $\mathrm{K}=6.5$ \\
\hline
\end{tabular}

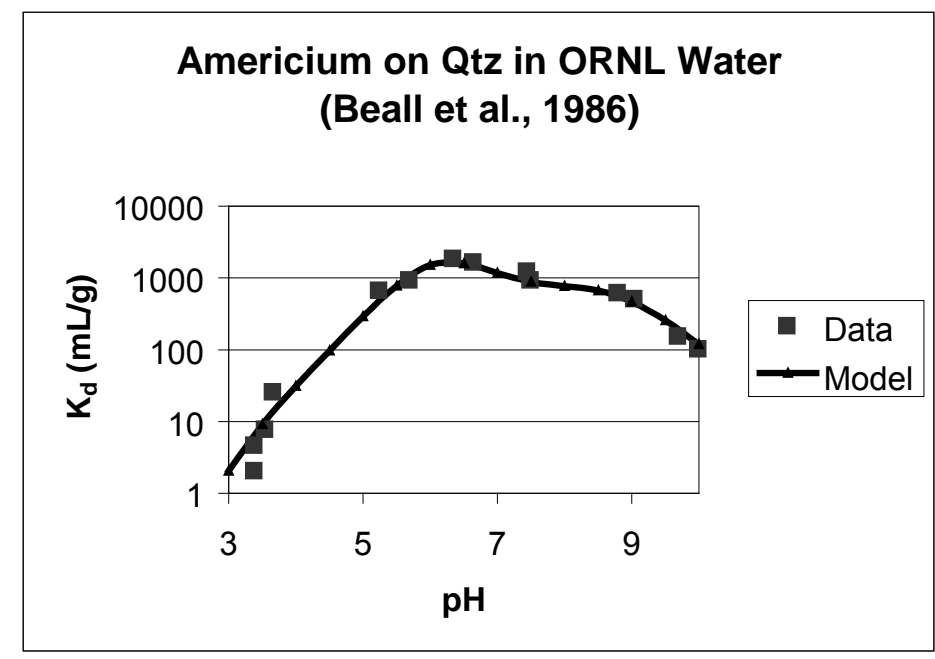

DTN: LA0407AM831343.001 [DIRS 171009]; LA0305AM831341.001 [DIRS 163789].

Figure A-4a. Americium Sorption Coefficients on Quartz from Beall et al. (1986 [DIRS 162983]) and Model Fit

Using a surface area of $2.8 \mathrm{~m}^{2} / \mathrm{g}$, americium sorption coefficients were calculated for "devitrified tuff" in J-13 as a function of $\mathrm{pH}$. The model curves are shown in Figure A-3. The fact that the model sorption coefficient for $\mathrm{J}-13$ at $\mathrm{pH}=8.5$ lies in the middle of the range of experimental values provides confidence that the model is reasonable. The model curves move up or down linearly with surface area. Thus, at least part of the range of experimental values could reflect variations in devitrified tuff surface areas.

The model curve shown in Figure A-3 indicates that sorption coefficients for americium will increase with decreasing $\mathrm{pH}$ in the $\mathrm{pH}$ range from 7.0 to 9.0. Thus, in terms of $\mathrm{pH}$, the experimentally derived sorption coefficients are at the low end of the range of coefficients to be expected in the unsaturated zone. The effect of variation in major ion chemistry of groundwater is shown by the curve calculated for $\mathrm{p} \# 1-\mathrm{c}$ groundwater. Americium sorption coefficients calculated using $\mathrm{p} \# 1-\mathrm{c}$ water are slightly lower in value compared to those calculated using J-13 water. This dependence on water chemistry was accounted for in the derivation for sorption coefficient probability distributions for americium.

Based on the experimental data and model curves plotted in Figure A-3, the range of americium sorption coefficients expected for devitrified tuffs in the unsaturated volcanic section at Yucca Mountain is selected as $1,000-10,000 \mathrm{~mL} / \mathrm{g}$. The probability distribution type selected is a truncated normal distribution with a mean of $5,500 \mathrm{~mL} / \mathrm{g}$ and a standard deviation of 
$1,500 \mathrm{~mL} / \mathrm{g}$. The data for JA- 32 and YM-54 were discounted in derivation of the distribution because the final solutions in the experiments with these samples were oversaturated with $\mathrm{Am}(\mathrm{OH}) \mathrm{CO}_{3}$. Figure $\mathrm{A}-4 \mathrm{~b}$ shows the empirical cumulative distribution for all measured americium sorption coefficients on devitrified tuff with the selected distribution superimposed. As discussed in Section A6., not all the empirical data was equally weighted in selecting the probability distribution as the influence of expected variations in water chemistry, radionuclide concentrations, and variations in rock surface properties within each major rock type were incorporated in making the selection.

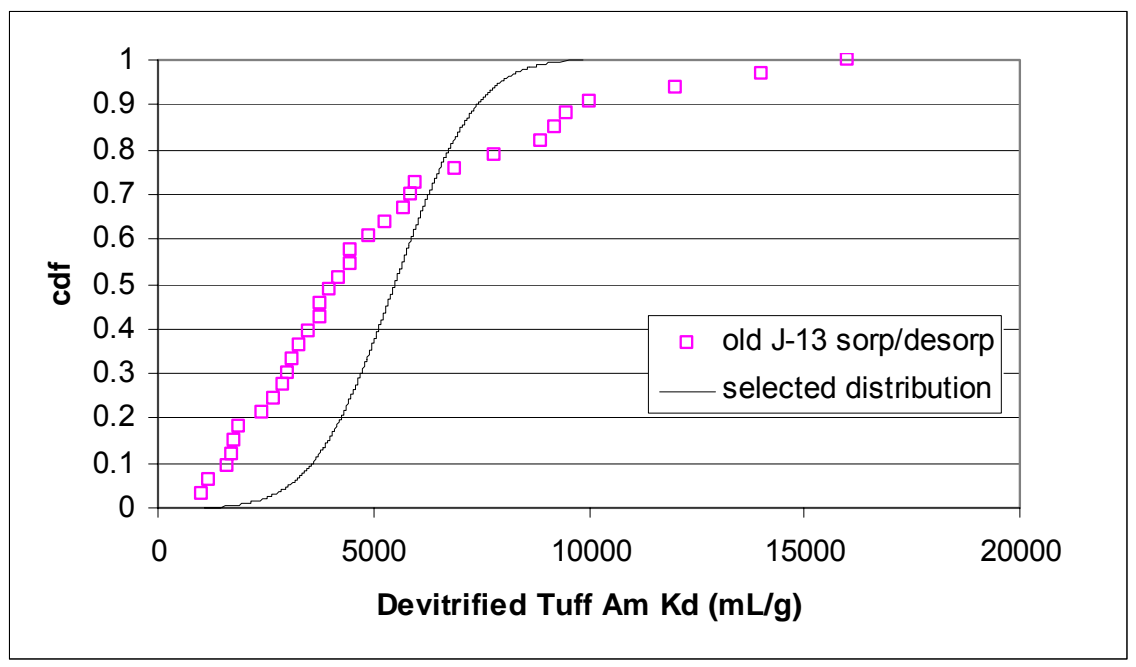

DTN: LA0305AM831341.001 [DIRS 163789].

Figure A-4b. Empirical Cumulative Distribution for Americium Sorption Coefficients on Devitrified Tuff

\section{A8.1.2 Zeolitic Tuff}

The measured sorption coefficients for zeolitic tuff are plotted versus calculated final solution concentrations in Figure A-4c. The calculated final solution concentrations were close to the solubility of $\mathrm{Am}(\mathrm{OH}) \mathrm{CO}_{3}$ only in sample G2-1952. The sorption coefficients obtained for this sample will be used in the derivation of the distribution. 


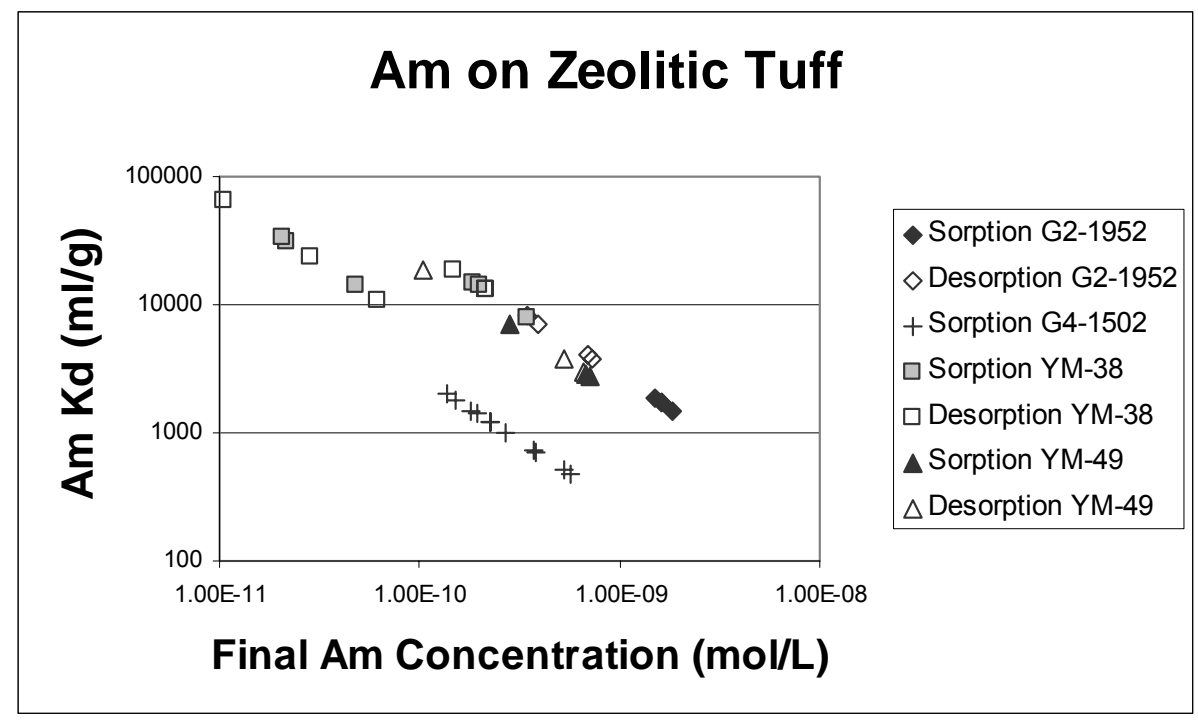

DTN: LA0305AM831341.001 [DIRS 163789].

Figure A-4c. Americium Sorption Coefficients on Zeolitic Tuff versus Calculated Final Americium Concentration in Solution

Americium sorption coefficients for zeolitic tuff are plotted versus duration of the sorption and desorption experiments in Figure A-5. As with the devitrified tuffs, the trend in the data (excluding sample JA-18) suggests americium sorption coefficient values obtained in experiments with solutions undersaturated with $\mathrm{Am}(\mathrm{OH}) \mathrm{CO}_{3}$ are not very sensitive (less than $10 \mathrm{X}$ ) to the duration of the experiments, except in those experiments with short durations (e.g., sample G4-1502). Thus, the kinetics of the americium sorption reactions on zeolitic tuff appear to be relatively fast.

The surface complexation model developed for zeolitic tuff is similar to the model developed for devitrified tuff, except that a surface area of $28 \mathrm{~m}^{2} / \mathrm{g}$ was used. The model sorption coefficient at $\mathrm{pH}=8.5$ lies at the high end of the range of experimental values (Figure A-6a). It might be expected that the sorption coefficients for zeolitic tuffs would be on the order of 10 times larger than those for devitrified tuffs to reflect the factor of 10 increase in surface area. The fact that the americium sorption coefficients obtained for zeolitic tuffs are not, on average, a factor of 10 larger than those measured in devitrified tuffs suggests not all the surface area in the zeolitic tuffs may be accessible to americium. Alternatively, the binding sites in the interior of zeolite crystals may not have a high affinity for americium species. 


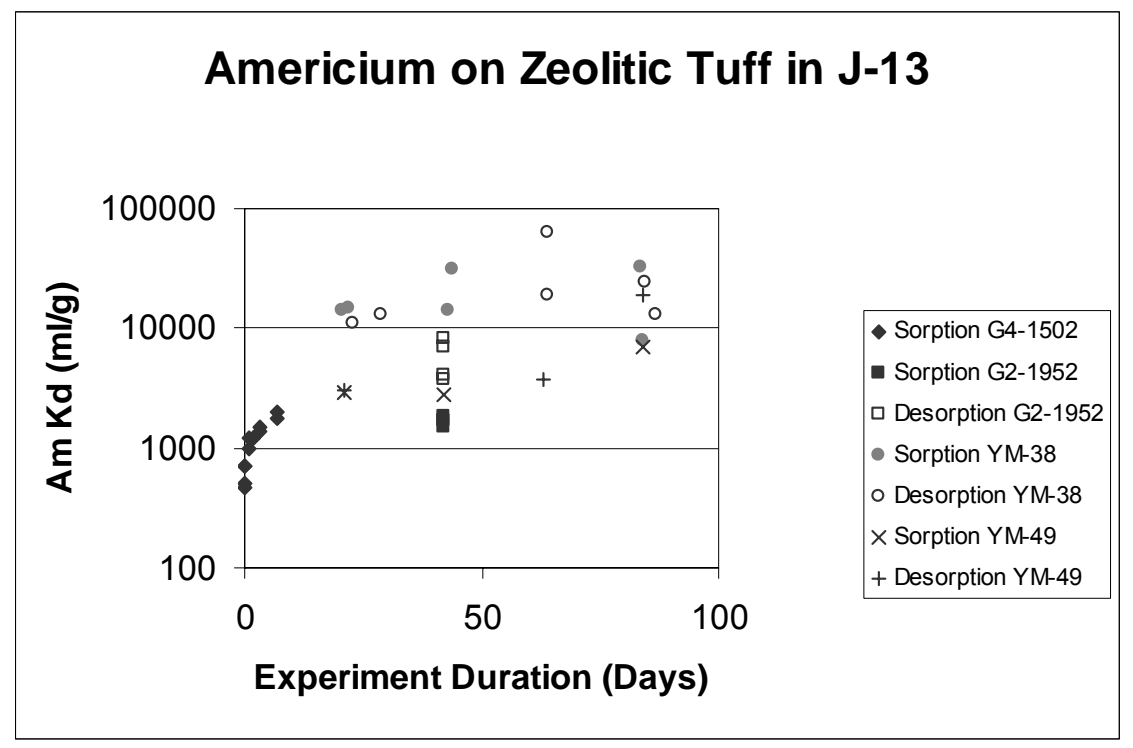

DTN: LA0305AM831341.001 [DIRS 163789].

Figure A-5. Americium Sorption Coefficients for Zeolitic Tuff as Function of Duration of Sorption and Desorption Experiments

As with devitrified tuff, the model curves for zeolitic tuffs shown in Figure A-6a indicate that sorption coefficients for americium will increase with decreasing $\mathrm{pH}$ in the $\mathrm{pH}$ range from 7.0 to 9.0. Thus, in terms of $\mathrm{pH}$, the experimentally derived sorption coefficients are at the low end of the range of coefficients to be expected in the unsaturated zone. The effect of variation in the major ion chemistry of groundwater is shown by the curve calculated for $\mathrm{p \# 1}$ groundwater. Americium sorption coefficients calculated using $\mathrm{p} \# 1$ water are similar to the coefficients calculated using J-13 water.

Based on the experimental data and model curves plotted in Figure A-6a, the range of americium sorption coefficients selected for zeolitic tuffs in the unsaturated volcanic section at Yucca Mountain is $1,000-10,000 \mathrm{~mL} / \mathrm{g}$. The probability distribution type selected is a truncated normal distribution with a mean of $5,500 \mathrm{~mL} / \mathrm{g}$ and a standard deviation of $1,500 \mathrm{~mL} / \mathrm{g}$. The upper end of this distribution could have been set at $100,000 \mathrm{~mL} / \mathrm{g}$ instead of $10,000 \mathrm{~mL} / \mathrm{g}$ based on the available data and modeling results. However, the $10,000 \mathrm{~mL} / \mathrm{g}$ value is large enough to effectively keep americium from being transported over the regulatory time frame. In addition, by using an upper limit of $10,000 \mathrm{~mL} / \mathrm{g}$, the same distribution can be used for devitrified and zeolitic tuffs. The data for JA-18 were discounted in derivation of the distribution because the final solutions in the experiments with this sample were oversaturated with $\mathrm{Am}(\mathrm{OH}) \mathrm{CO}_{3}$. Figure A-6b shows the empirical cumulative distribution for all the measured americium sorption coefficients on zeolitic tuff with the selected distribution superimposed. As discussed in Section A6., not all the empirical data was equally weighted in selecting the probability distribution as the influence of expected variations in water chemistry, radionuclide concentrations, and variations in rock surface properties within each major rock type were incorporated in making the selection. 


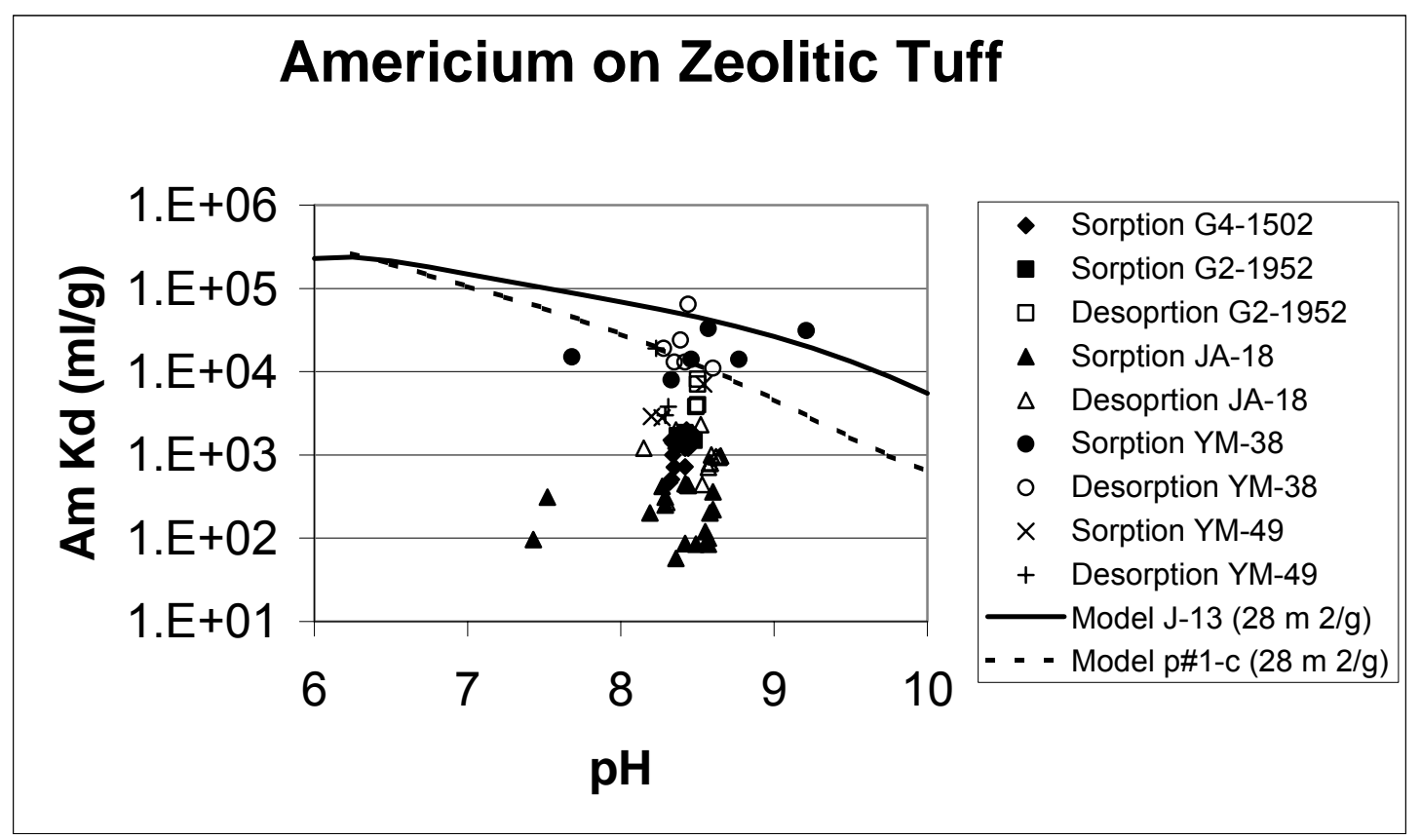

DTNs: LA0305AM831341.001 [DIRS 163789]; LA0407AM831343.001 [DIRS 171009].

Figure A-6a. Americium Sorption Coefficient Data and Modeling Results for Zeolitic Tuff as a Function of $\mathrm{pH}$

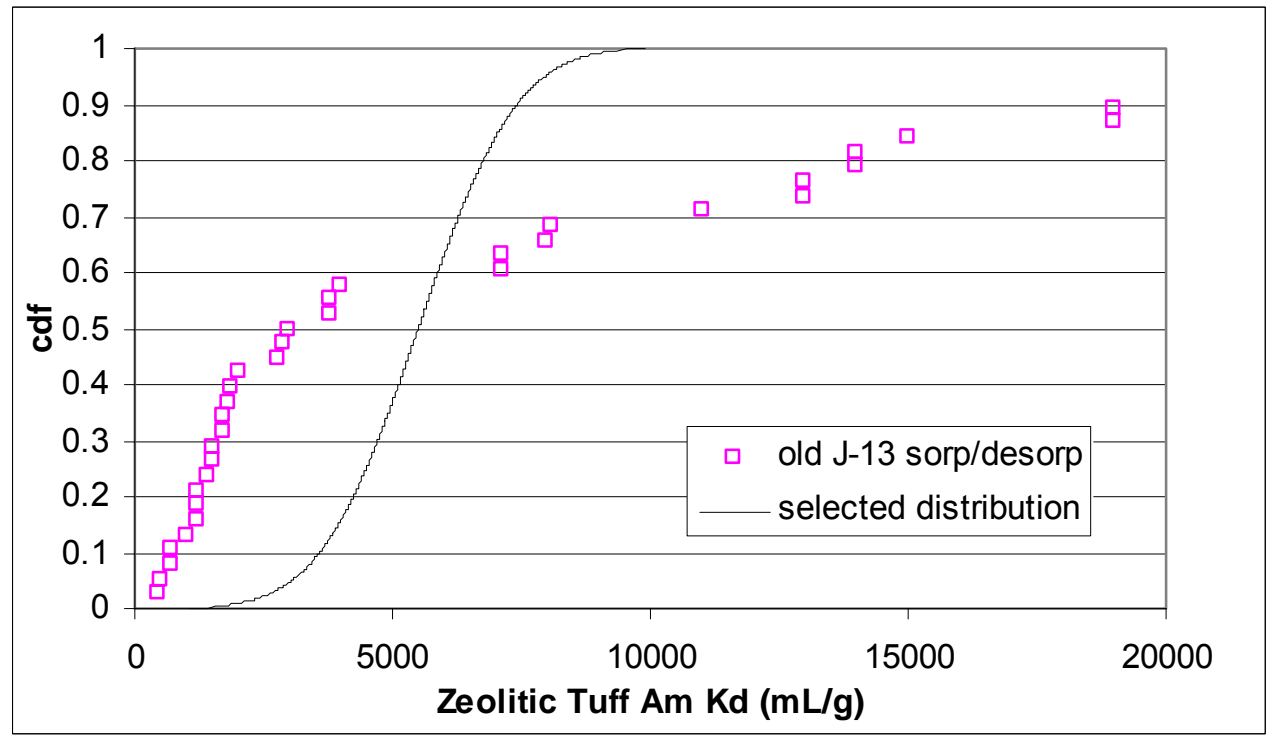

DTN: LA0305AM831341.001 [DIRS 163789].

Figure A-6b. Empirical Cumulative Distribution for Americium Sorption Coefficients on Zeolitic Tuff 


\section{A8.1.3 Vitric Tuff}

The measured sorption coefficients for vitric tuff are plotted versus calculated final solution concentrations in Figure A-7. The calculated final solution concentrations were close to $\mathrm{Am}(\mathrm{OH}) \mathrm{CO}_{3}$ solubility in essentially all the experiments with samples GU3-1203 and GU3-1301. Therefore, the sorption coefficients obtained for these samples are minimum values.

Americium sorption experiments on vitric tuffs were all carried out for a duration of 42 days. Thus, there is no information available on the kinetics of the americium sorption reactions on vitric tuff.

The dependence of Am sorption coefficients on water chemistry in vitric tuff will be very similar to the dependence calculated in devitrified tuff (Figure A-3). Thus, americium sorption coefficients will decrease with increasing $\mathrm{pH}$ for $\mathrm{pH}$ in the range 7-9. Since the sorption experiments with vitric tuffs were carried out at the high end of the $\mathrm{pH}$ range expected in the natural system, sorption coefficients in the natural system are expected to be larger than those experimentally observed.

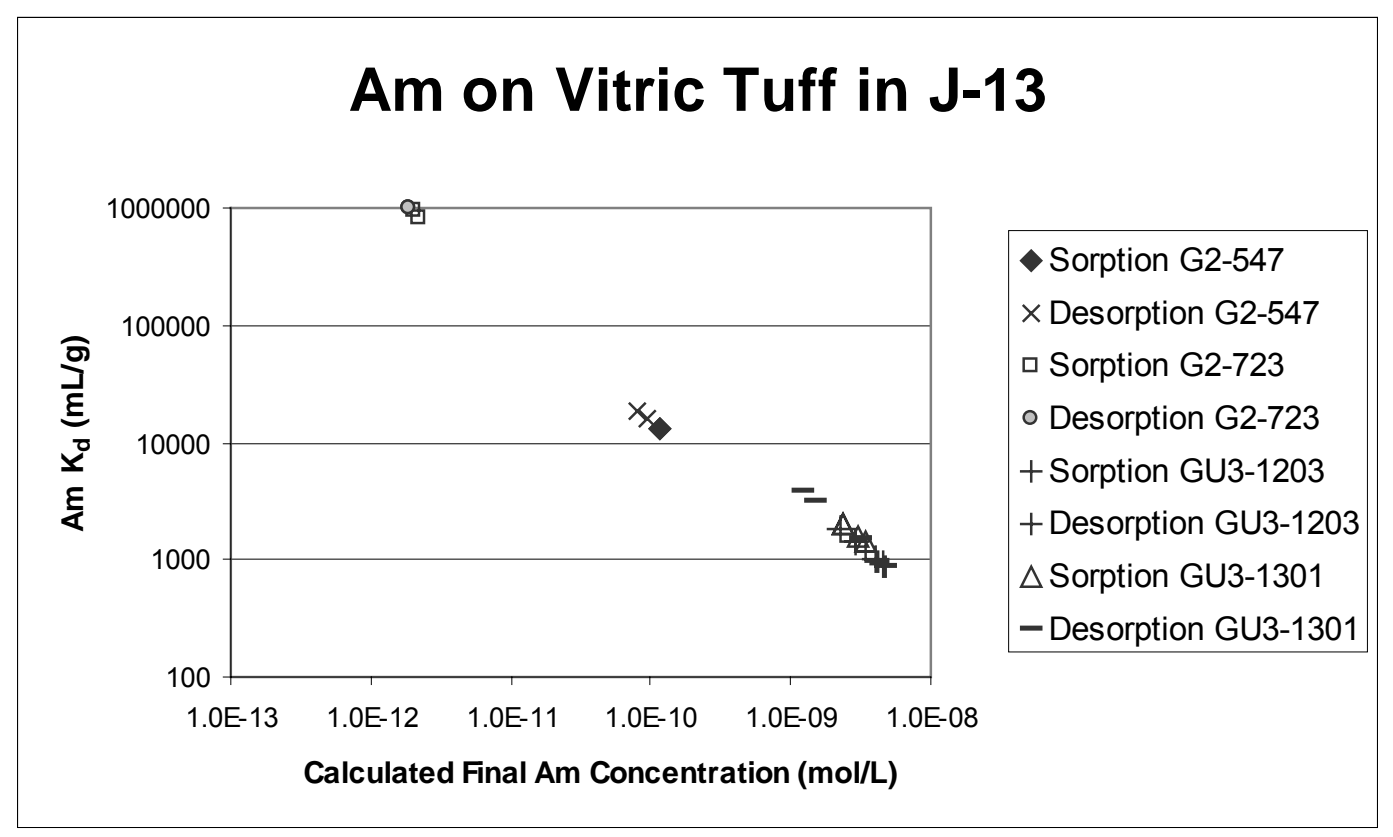

DTN: LA0305AM831341.001 [DIRS 163789].

Figure A-7. Americium Sorption Coefficients on Vitric Tuff versus Calculated Final Americium Concentration in Solution 


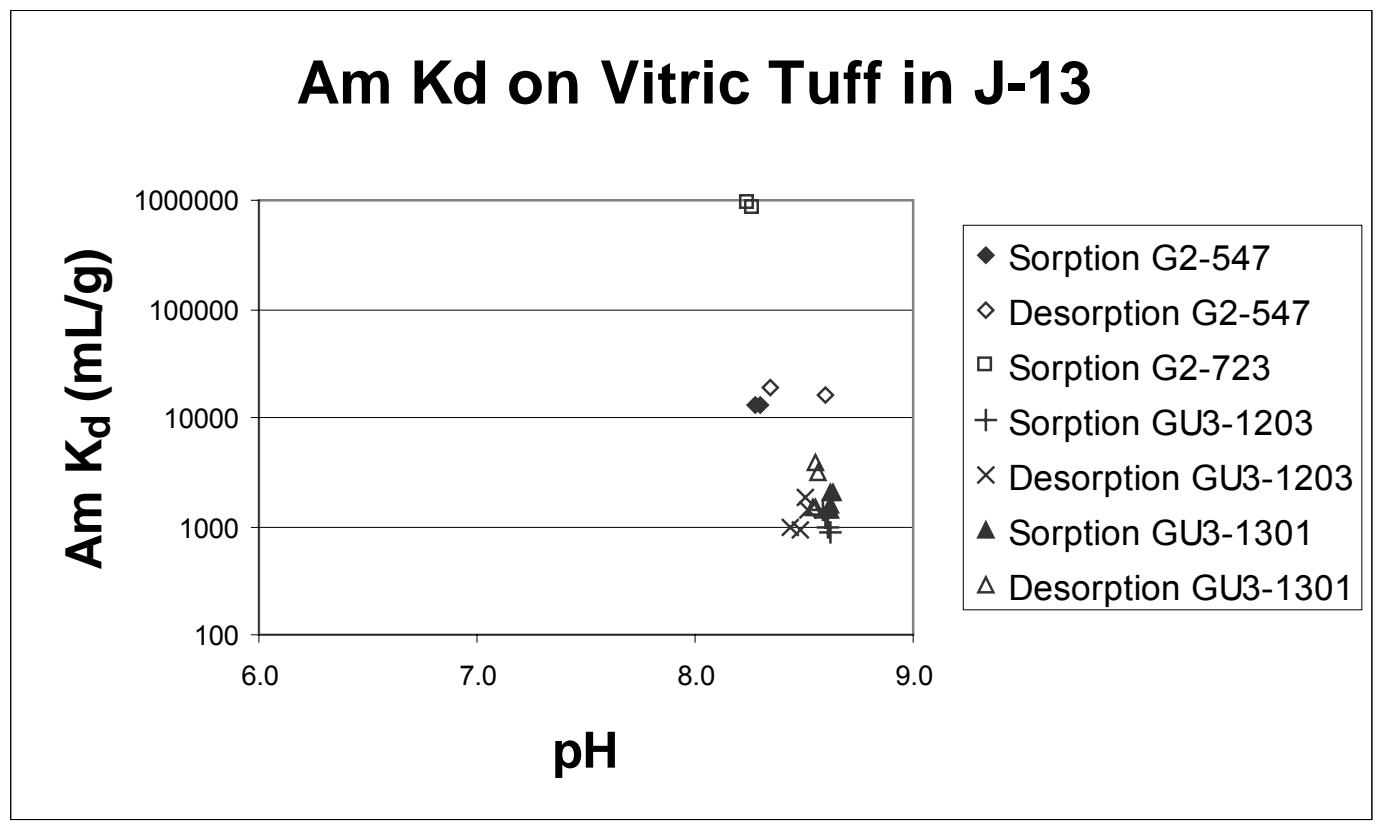

DTN: LA0305AM831341.001 [DIRS 163789].

Figure A-8a. Americium Sorption Coefficient Data and Modeling Results for Vitric Tuff as a Function of $\mathrm{pH}$

Based on the experimental data plotted in Figure A-8a and the inferred $\mathrm{pH}$ dependence from Figure A-3, the americium sorption-coefficient probability distribution selected for vitric tuff is a cumulative distribution with a value of $100 \mathrm{~mL} / \mathrm{g}$ at $0.0,400 \mathrm{~mL} / \mathrm{g}$ at 0.5 , and $1,000 \mathrm{~mL} / \mathrm{g}$ at 1.0 . The range of values in this distribution could have been set at between $1,000 \mathrm{~mL} / \mathrm{g}$ and $1,000,000 \mathrm{~mL} / \mathrm{g}$ based on the available data and modeling results. However, the lower values were selected to acknowledge the potential for vitric units to have smaller surface areas than those reported for vitric tuff in Table A-1. Surface areas of true vitrophyres are likely to be substantially lower than the surface areas listed in Table A-1 for vitric tuffs. The data for GU3-203 and GU3-1301 were rejected in derivation of the distribution because the final solutions in the experiments with this sample were oversaturated with $\mathrm{Am}(\mathrm{OH}) \mathrm{CO}_{3}$. Figure A-8b shows the empirical cumulative distribution for all the measured americium sorption coefficients on vitric tuff with the selected distribution superimposed. As discussed in Section A6, not all the empirical data was equally weighted in selecting the probability distribution as the influence of expected variations in water chemistry, radionuclide concentrations, and variations in rock surface properties within each major rock type were incorporated in making the selection. 


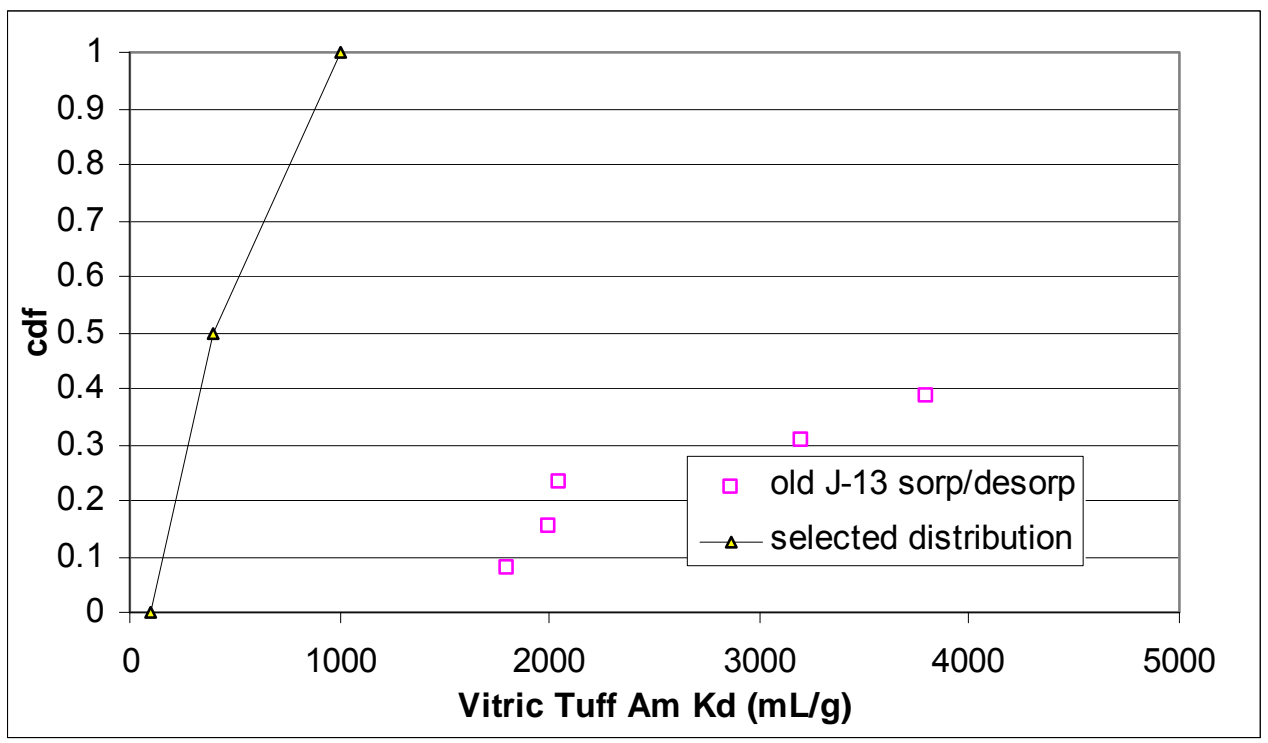

DTN: LA0305AM831341.001 [DIRS 163789].

Figure A-8b. Empirical Cumulative Distribution for Americium Sorption Coefficients on Vitric Tuff

\section{A8.2 CESIUM}

The solubility of cesium in $\mathrm{J}-13$ water at $25^{\circ} \mathrm{C}$ is very high. In fact, cesium concentrations in Yucca Mountain groundwaters will not have a solubility limitation (BSC 2004 [DIRS 169425], Section 6.17). 


\section{A8.2.1 Devitrified Tuff}

Experimentally derived sorption coefficients for cesium on devitrified tuff are plotted against the calculated final cesium concentrations of the experiments in Figure A-9. The data points are separated into groups on the basis of when the experiments were carried out (pre-1990 = "old" and post-1990 = "new"), water type, and whether the sorption coefficient was determined from a sorption or a desorption experiment. There are also data for sorption isotherms on samples YM-22 and G1-2840.

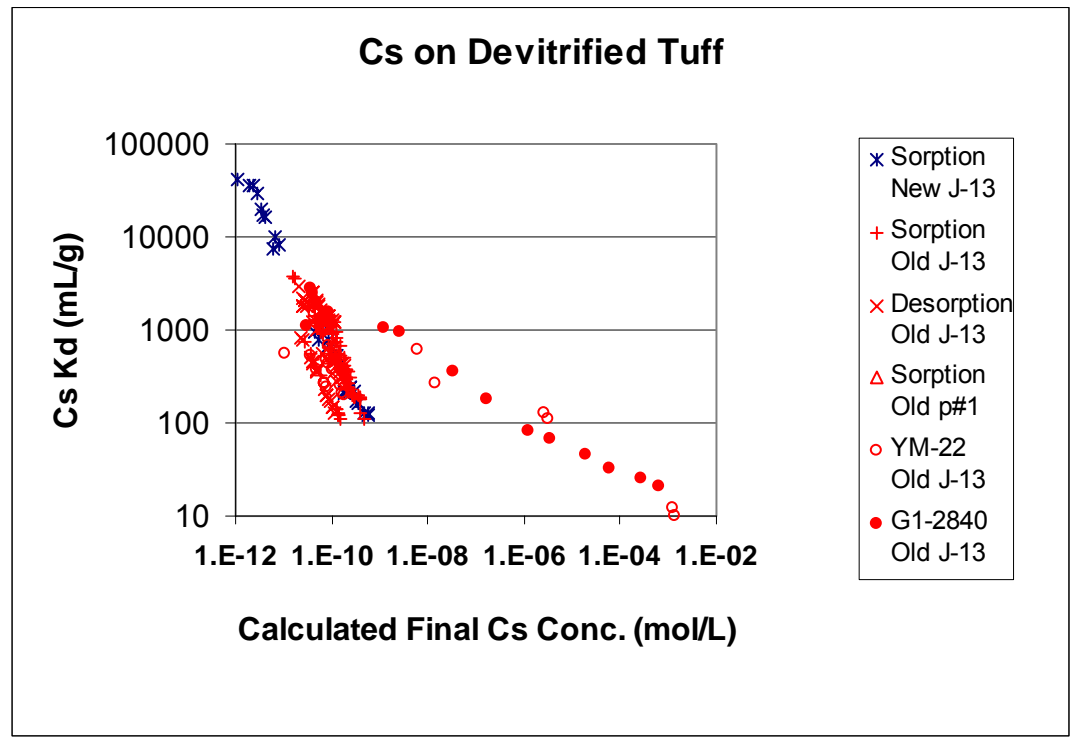

DTNs: New: LA0407AM831341.002 [DIRS 170621]; Old: LA0305AM831341.001 [DIRS 163789].

Figure A-9. Cesium Sorption Coefficients on Devitrified Tuff versus Calculated Final Cesium Concentration in Solution

Sorption coefficients obtained in "new" experiments lie within the range defined by the "old" experiments at similar cesium concentrations. Although most of the sorption experiments resulted in $\mathrm{K}_{\mathrm{d}}$ values greater than $100 \mathrm{~mL} / \mathrm{g}$, these experiments were carried out at cesium concentrations below $1.0 \times 10^{-6} \mathrm{~mol} / \mathrm{L}$. At higher cesium concentrations, the $\mathrm{K}_{\mathrm{d}}$ values obtained were between 10 and $100 \mathrm{~mL} / \mathrm{g}$. Nonlinear sorption isotherms were obtained for samples YM-22 and G1-2840 in J-13 water. The data for sample G1-2840 were fit using a Freundlich equation as shown in Figure A-10. Sorption coefficients obtained in experiments with $\mathrm{p \# 1}$ water fall in the middle of the cluster of points in Figure A-9 near $1 \times 10^{-10} \mathrm{~mol} / \mathrm{L}$. Thus, there is little or no impact caused by water-chemistry variations on cesium sorption coefficients in devitrified tuff. 


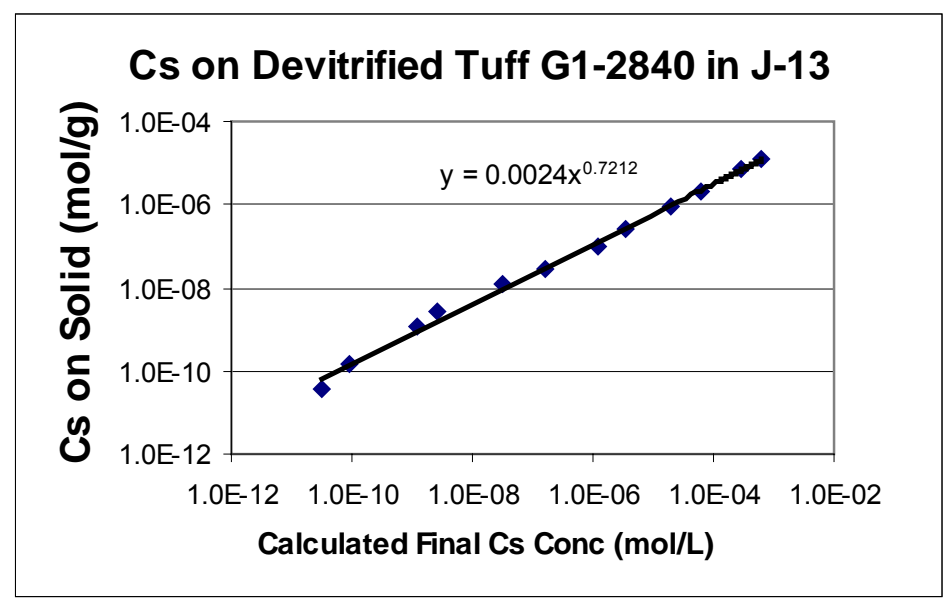

DTN: LA0305AM831341.001 [DIRS 163789].

Figure A-10. Freundlich Isotherm Fit to Sorption Coefficient Data for Cesium on Devitrified Tuff Sample G1-2840 in J-13 Water

The effects of experiment duration on the cesium sorption coefficients for devitrified tuff are shown in Figure A-11a. The large range in sorption coefficients obtained at a given duration (e.g., 21 days) mainly reflects variations in solution cesium concentrations, although variations in ion exchange capacities may also contribute to the range. The range of sorption coefficient values is fairly consistent with duration when the results for samples YM-22 and G1-2840 are excluded. This range extends from just above $100 \mathrm{~mL} / \mathrm{g}$ to above $1,000 \mathrm{~mL} / \mathrm{g}$. The consistency indicates cesium sorption reactions are fast.

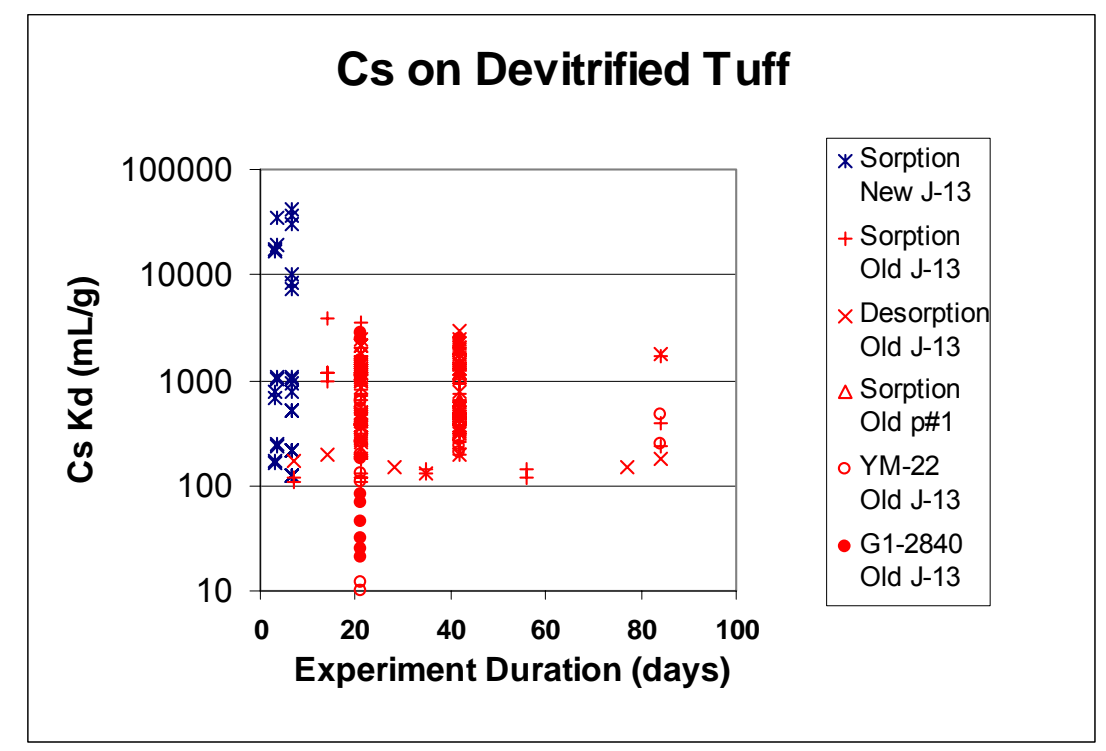

DTNs: New: LA0407AM831341.002 [DIRS 170621]; Old: LA0305AM831341.001 [DIRS 163789].

Figure A-11a. Cesium Sorption Coefficients on Devitrified Tuff versus Experiment Duration for Sorption (Forward) and Desorption (Backward) Experiments 
The cesium sorption-coefficient probability distribution derived for devitrified tuff in the UZ is a uniform distribution with a range of $1-15 \mathrm{~mL} / \mathrm{g}$. This distribution was chosen to acknowledge the potential for high cesium concentrations (e.g., greater than $10^{-3} \mathrm{~mol} / \mathrm{L}$ ) to be transported in the UZ. Figure A-11b shows the empirical cumulative distribution for all the measured cesium sorption coefficients on devitrified tuff in the UZ with the selected distribution superimposed. As discussed in Section A6., not all the empirical data was equally weighted in selecting the probability distribution as the influence of expected variations in water chemistry, radionuclide concentrations, and variations in rock surface properties within each major rock type were incorporated in making the selection.

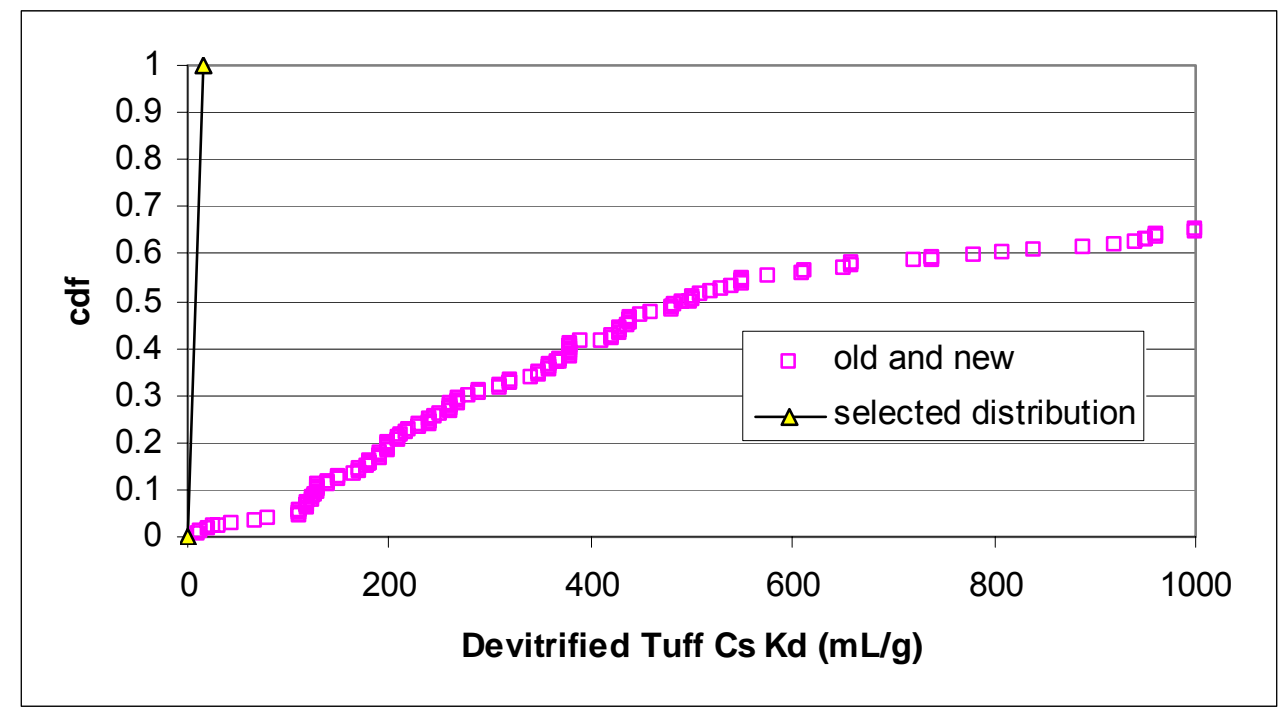

DTNs: New: LA0407AM831341.002 [DIRS 170621]; Old: LA0305AM831341.001 [DIRS 163789].

Figure A-11b. Empirical Cumulative Distribution for Cesium Sorption Coefficients on Devitrified Tuff

\section{A8.2.2 Zeolitic Tuff}

Experimentally derived sorption coefficients for cesium on zeolitic tuff are plotted against the calculated final cesium concentrations of the experiments in Figure A-12. The data points are separated into groups on the basis of when the experiments were carried out (pre-1990 = "old" and post-1990 = "new"), water type, and on whether the sorption coefficient was determined from a sorption or a desorption experiment. There are also data for a sorption isotherm on sample YM-38.

The range of measured cesium sorption coefficients for zeolitic tuffs is $3,500-72,000 \mathrm{~mL} / \mathrm{g}$. A sorption isotherm was obtained for sample YM-38 in J-13 water. As shown in Figure A-13, the isotherm is nearly linear. Sorption coefficients obtained in experiments with $\mathrm{p} \# 1$ water fall at the lower end of the range of values obtained for experiments with J-13 water (Figure A-12). Thus, there is some impact from variations in water chemistry on cesium sorption coefficients in zeolitic tuff, although this impact is minor. 


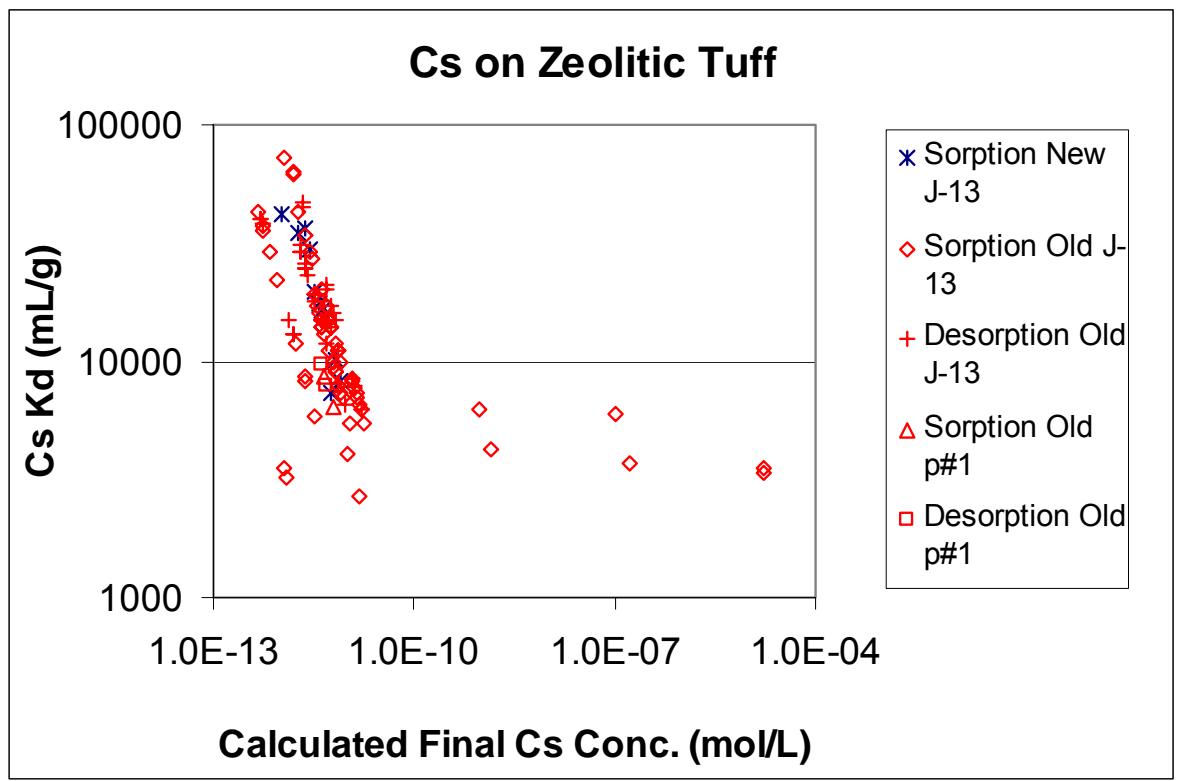

DTNs: LA0310AM831341.001[DIRS 165865].

Figure A-12. Cesium Sorption Coefficients on Zeolitic Tuff versus Calculated Final Cesium Concentration in Solution

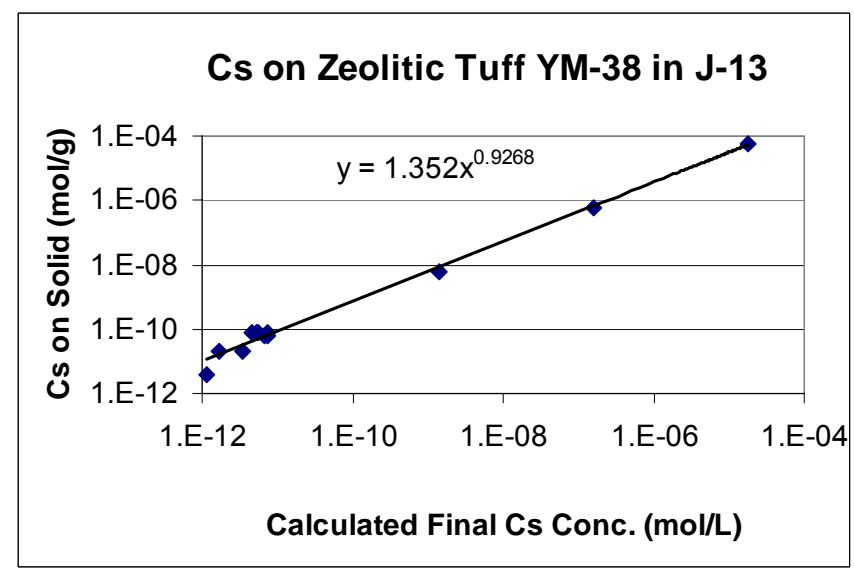

DTN: LA0310AM831341.001 [DIRS 165865].

Figure A-13. Freundlich Isotherm Fit to Sorption Coefficient Data for Sample YM-38 in J-13 Water 
The effects of experiment duration on cesium $\mathrm{K}_{\mathrm{d}}$ for zeolitic tuff are shown in Figure A-14a. The large range in sorption coefficients obtained at a given duration mainly reflects variations in cesium solution concentrations, although there must also be some contribution from variations in ion exchange capacities of the zeolitic tuff samples used in the experiments. The range of sorption coefficient values is fairly consistent with duration. For example, the range of sorption coefficient values for 3.6-day experiments is similar to the range for the 42-day experiments. This indicates the sorption reaction kinetics are fast.

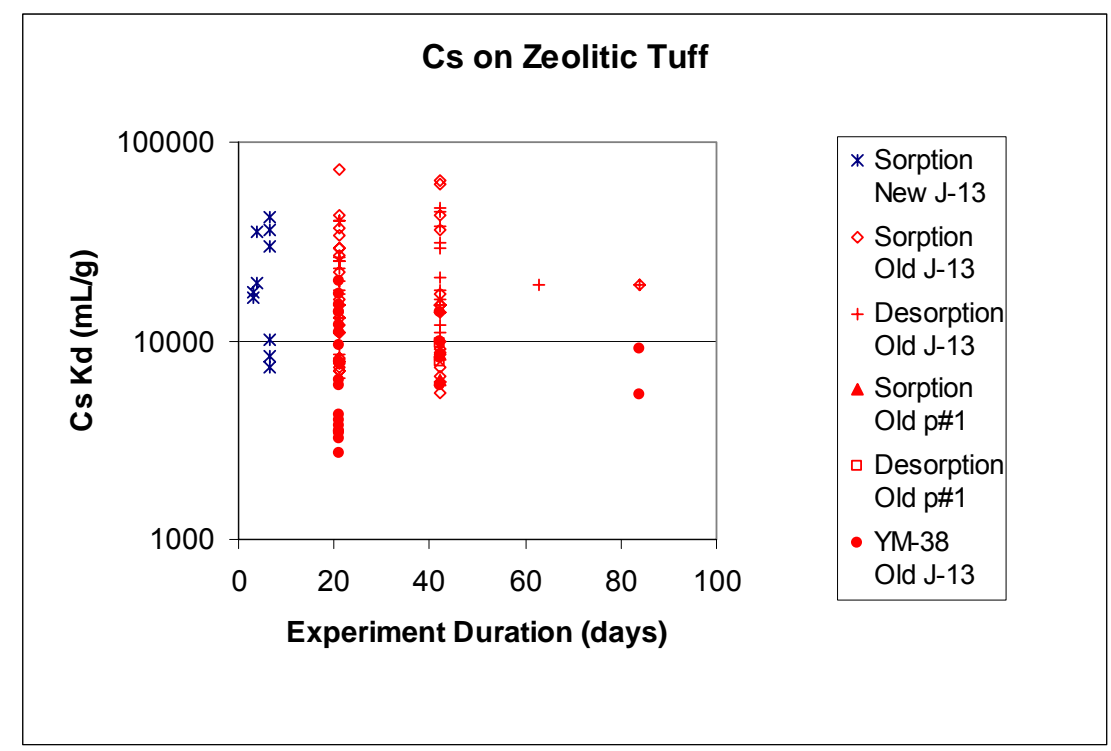

DTNs: New: LA0407AM831341.002 [DIRS 170621]; Old: LA0305AM831341.001 [DIRS 163789].

Figure A-14a. Cesium Sorption Coefficients on Zeolitic Tuff versus Experiment Duration for Sorption (Forward) and Desorption (Backward) Experiments

The cesium sorption-coefficient probability distribution selected for zeolitic tuff in the UZ is a cumulative distribution, starting with a value of $425 \mathrm{~mL} / \mathrm{g}$ at $0.0,5,000 \mathrm{~mL} / \mathrm{g}$ at 0.5 , and $20,000 \mathrm{~mL} / \mathrm{g}$ at 1.0 . The low end of this distribution was selected to acknowledge the potential for high cesium concentrations and lower-than-average ion exchange capacities during transport in the UZ. The middle value of the distribution was selected as a more representative value for cesium concentrations below $10^{-4} \mathrm{~mol} / \mathrm{L}$. The upper end of the range was selected as a minimum upper limit, given the potential impact of lower cesium solution concentrations and higher-than-average ion exchange capacities. Figure A-14b shows the empirical cumulative distribution for all the measured cesium sorption coefficients on zeolitic tuff in the UZ with the selected distribution superimposed. As discussed in Section A6., not all the empirical data was equally weighted in selecting the probability distribution as the influence of expected variations in water chemistry, radionuclide concentrations, and variations in rock surface properties within each major rock type were incorporated in making the selection. 


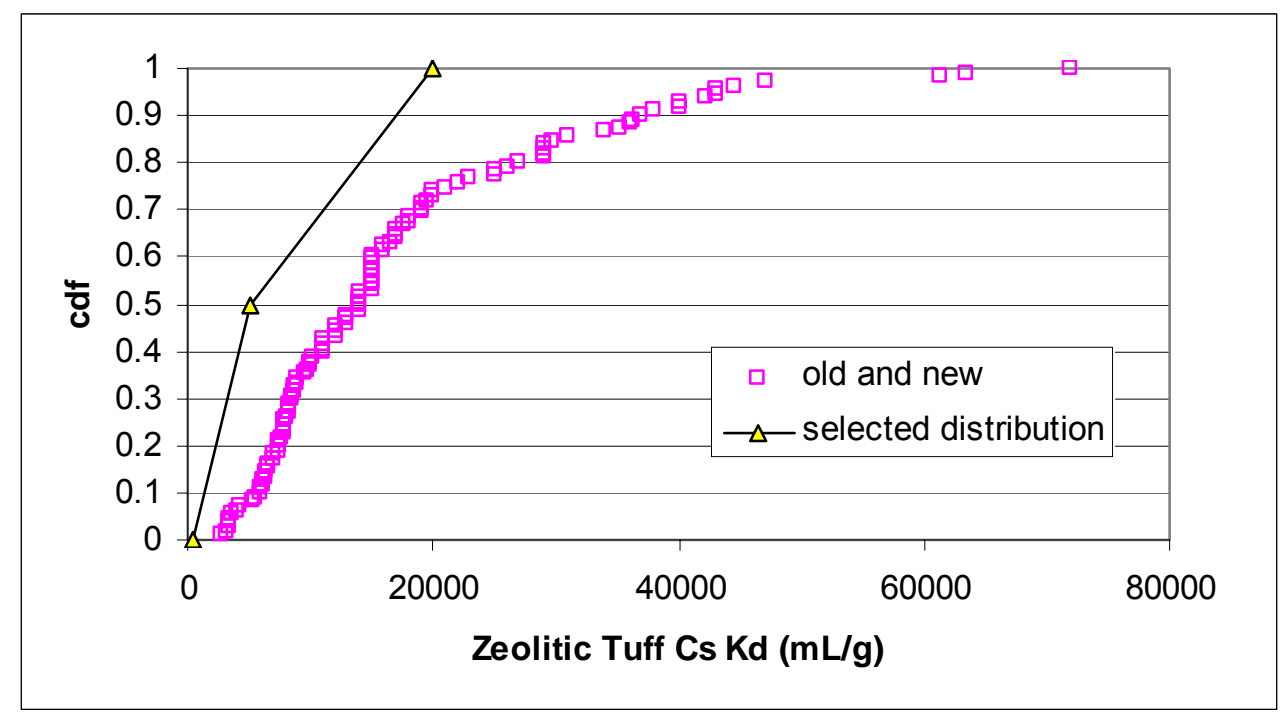

DTNs: New: LA0407AM831341.002 [DIRS 170621]; Old: LA0305AM831341.001 [DIRS 163789].

Figure A-14b. Empirical Cumulative Distribution for Cesium Sorption Coefficients on Zeolitic Tuff

\section{A8.2.3 Vitric Tuff}

Experimentally derived sorption coefficients for cesium on vitric tuff are plotted against the calculated final cesium concentrations of the experiments in Figure A-15. The data points are separated into groups on the basis of when the experiments were carried out (pre-1990 = "old" and post-1990 = "new"), water type, and on whether the sorption coefficient was determined from a sorption or a desorption experiment.

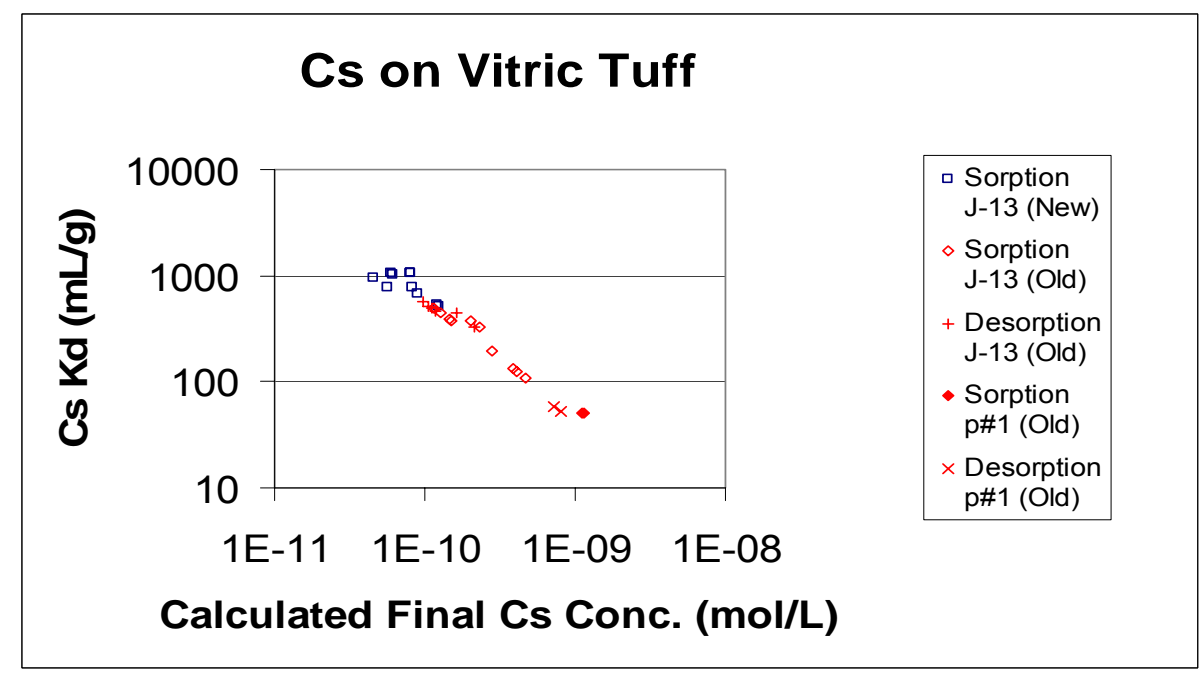

DTNs: New: LA0407AM831341.002 [DIRS 170621]; Old: LA0310AM831341.001[DIRS 165865].

Figure A-15. Cesium Sorption Coefficients on Vitric Tuff versus Calculated Final Cesium Concentration in Solution 
The range of measured cesium sorption coefficients for zeolitic tuffs is $50-1,000 \mathrm{~mL} / \mathrm{g}$. Sorption coefficients obtained in experiments with p\#1 water fall at the lower end of the range of values obtained for experiments with J-13 water (Figure A-15). However, the p\#1 experiments were performed with higher initial cesium solution concentrations than all the experiments performed with J-13 water. Thus, it is unclear to what extent water chemistry caused the sorption coefficients to be lower in $\mathrm{p} \# 1$ water compared to $\mathrm{J}-13$.

The effects of experiment duration on the cesium $K_{d}$ for vitric tuff are shown in Figure A-16a. That the sorption coefficient values obtained in short-term experiments exceed those obtained in longer-term experiments indicates that cesium sorption reactions on vitric tuff are fast.

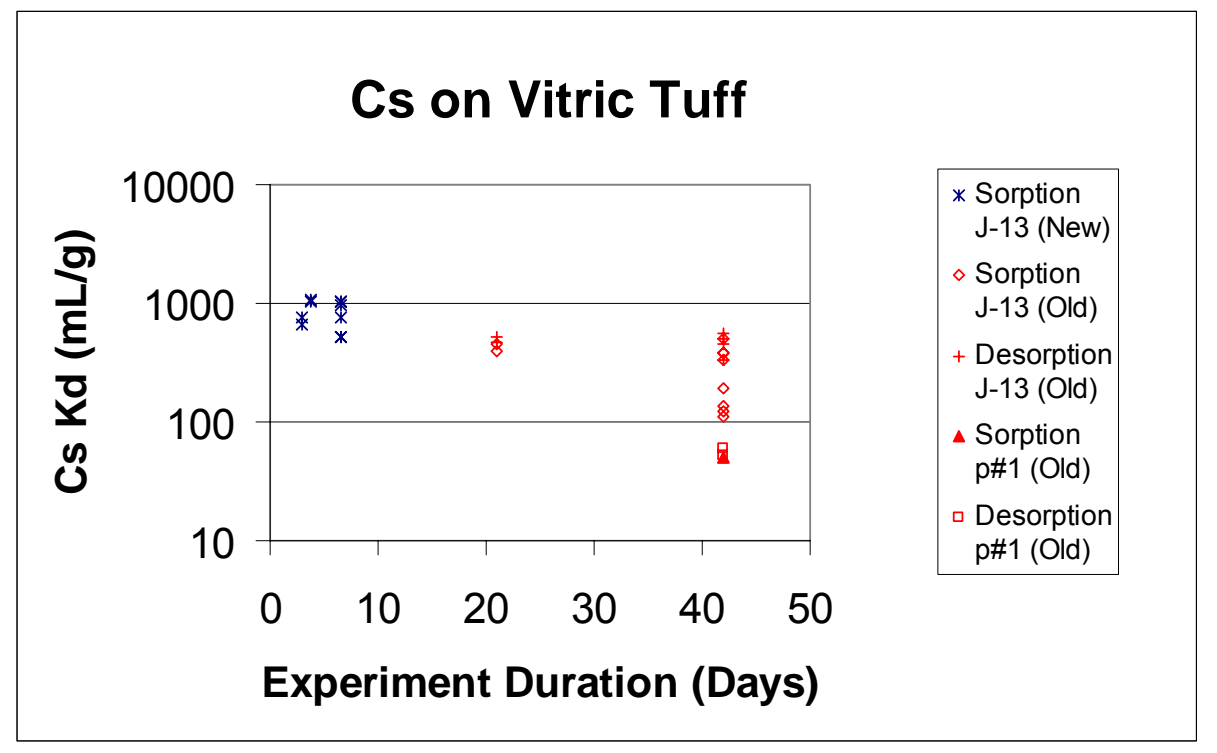

DTNs: New: LA0407AM831341.002 [DIRS 170621]; Old: LA0305AM831341.001 [DIRS 163789].

Figure A-16a. Cesium Sorption Coefficients on Vitric Tuff versus Experiment Duration for Sorption (Forward) and Desorption (Backward) Experiments

The cesium-sorption-coefficient probability distribution selected for vitric tuff in the UZ is a cumulative distribution, starting with a value of $0.0 \mathrm{~mL} / \mathrm{g}$ at 0.0 , rising to a value of $2.0 \mathrm{~mL} / \mathrm{g}$ at 0.5 , and ending at a value of $100 \mathrm{~mL} / \mathrm{g}$ at 1.0 . The low-end value was selected based on the possibility that cesium concentrations could be high in the UZ (i.e., higher than those plotted in Figure A-15), and the possibility that surface areas in vitrophyric zones could be lower than those listed in Table A-1. The middle value was selected to represent more representative surface areas, high cesium concentrations, and water chemistries like $p \# 1$. The upper end of the distribution was chosen as a minimum upper limit, given the potential impact of lower cesium solution concentrations (Figure A-15), higher surface areas in ash layers, and more dilute water chemistry (e.g., like J-13). Figure A-16b shows the empirical cumulative distribution for all the measured cesium sorption coefficients on vitric tuff in the UZ with the selected distribution superimposed. As discussed in Section A6., not all the empirical data was equally weighted in selecting the probability distribution as the influence of expected variations in water chemistry, radionuclide concentrations, and variations in rock surface properties within each major rock type were incorporated in making the selection. 


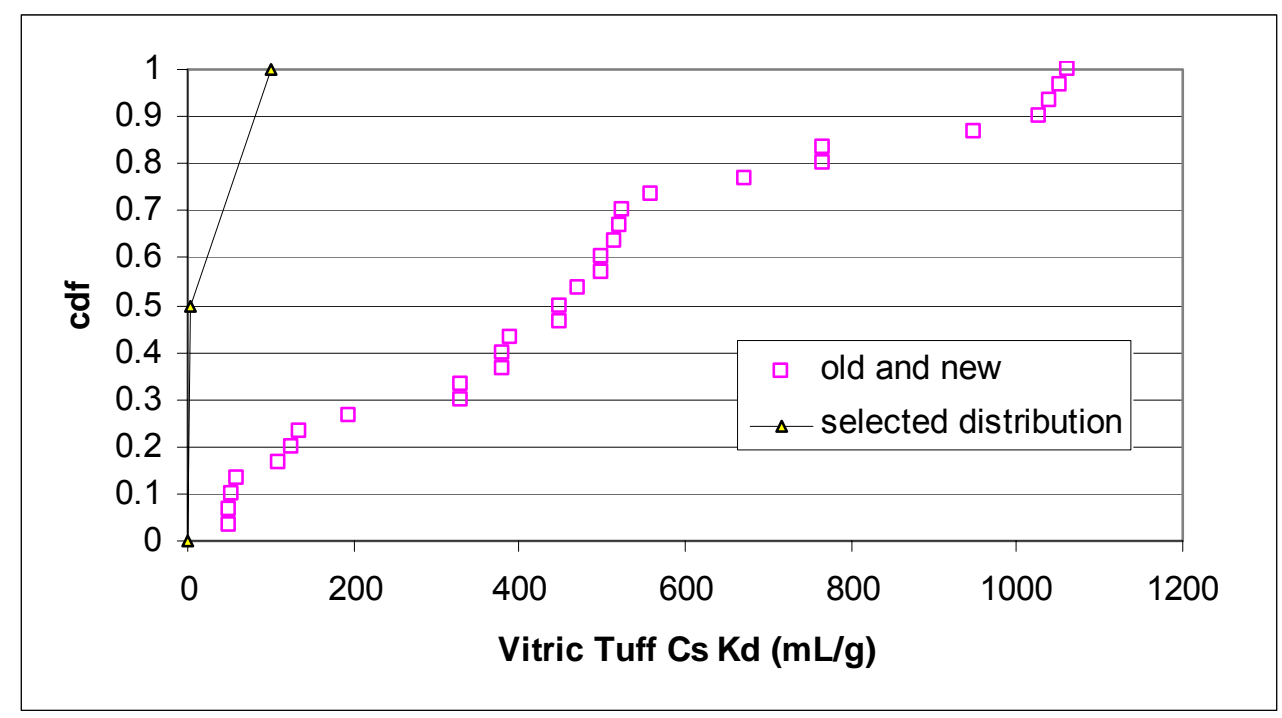

DTNs: New: LA0407AM831341.002 [DIRS 170621]; Old: LA0305AM831341.001 [DIRS 163789].

Figure A-16b. Empirical Cumulative Distribution for Cesium Sorption Coefficients on Vitric Tuff

\section{A8.3. NEPTUNIUM}

According to Kaszuba and Runde (1999 [DIRS 122379], Figure 3), the solubility of neptunium in $\mathrm{J}-13$ water at $25^{\circ} \mathrm{C}, \mathrm{pH}=8.5$, is $7.0 \times 10^{-6} \mathrm{~mol} / \mathrm{L}$ and the solubility controlling solid is $\mathrm{Np}_{2} \mathrm{O}_{5}$ under oxidizing conditions. At $\mathrm{pH}=7.0$, the solubility is higher, at $6.9 \times 10^{-5} \mathrm{~mol} / \mathrm{L}$ under oxidizing conditions. The solubility of neptunium in $\mathrm{p} \# 1$ water at $25^{\circ} \mathrm{C}, \mathrm{pH}=8.5$, is $2.5 \times 10^{-5} \mathrm{~mol} / \mathrm{L}$ and $7.3 \times 10^{-5} \mathrm{~mol} / \mathrm{L}$ at $25^{\circ} \mathrm{C}, \mathrm{pH}=7.0$, according to Kaszuba and Runde.

Solubilities for neptunium are also reported in Dissolved Concentration Limits of Radioactive Elements (BSC 2004 [DIRS 169425], Section 6.6). The solubilities are less than $8 \times 10^{-6} \mathrm{~mol} / \mathrm{L}$ for $\mathrm{pH}$ between 7.0 and 8.4 and $\log \mathrm{fCO}_{2}$ between -3.5 and -2.5 . Therefore, using the solubilities based on Kaszuba and Runde's report (1999 [DIRS 122379]), solubilities screen out fewer of the higher concentration results. Because neptunium sorption coefficients trend lower for higher solution concentrations, this leads to including more low-sorption results in the selected distributions.

\section{A8.3.1 Devitrified Tuff}

The results of sorption experiments with devitrified tuff are shown in Figure A-17. Some of the experiments with J-13 water had final neptunium concentrations above $7.0 \times 10^{-6} \mathrm{~mol} / \mathrm{L}$. Thus, the results for these experiments should be discounted because the experiments could have been oversaturated with $\mathrm{Np}_{2} \mathrm{O}_{5}$. All but four of the experiments with synthetic $\mathrm{p} \# 1$ water had final neptunium solution concentrations of less than $2.55 \times 10^{-5} \mathrm{~mol} / \mathrm{L}$. The results for the four experiments with over saturated final solutions will be discounted. The remaining data points, plotted in Figure A-18, suggest a dependence of the sorption coefficient on the final neptunium solution concentration. 


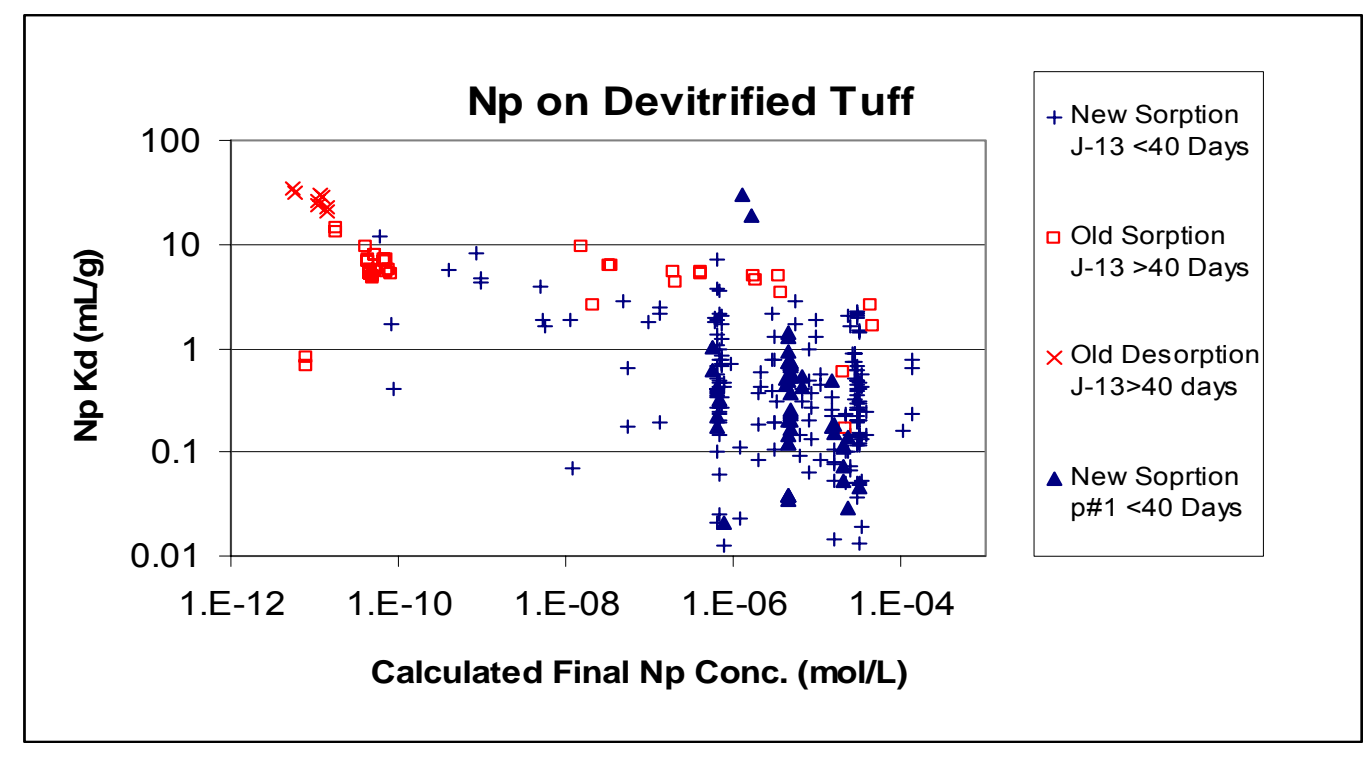

DTNs: New: LA0407AM831341.004 [DIRS 170622]; Old: LA0305AM831341.001 [DIRS 163789].

Figure A-17. Neptunium Sorption Coefficients on Devitrified Tuff versus Calculated Final Neptunium Concentration in Solution

Neptunium sorption experiments carried out as a function of experiment duration are shown in Figure A-19. A significant difference appears between the results for "old" and "new" experiments, with the "old" results generally having higher values than the "new" results. The most straightforward explanation is that the difference is caused by the "old" results representing experiments with longer durations than the "new" results. Within the "old" data points, the sorption coefficient values appear to reach a steady-state level after 42 days.

The impact of variations in $\mathrm{pH}$ on neptunium coefficients on devitrified tuffs is shown in Figure A-20a. There is a lot of scatter in the "new" data, and there do not appear to be clear positive or negative trends among these data points. Nor does there appear to be much difference between the J-13 and synthetic p\#1 results. The "old" data points are more consistent and show that the neptunium sorption coefficient depends very little on $\mathrm{pH}$, except at $\mathrm{pH}$ values less than 7.0. PHREEQC surface complexation model curves for "devitrified tuff" in J-13 and in p\#1 are also plotted. Neptunium binding constants on silica were obtained from the report by Turner et al. (1998 [DIRS 162989], p. 264). The J-13 curve lies between the "old" sorption and desorption points. This placement suggests that the curve may reflect the equilibrium values of neptunium sorption coefficients on devitrified tuff better than the experimental data. In this interpretation, the sorption data points reflect experiments that have not reached an equilibrium state. 


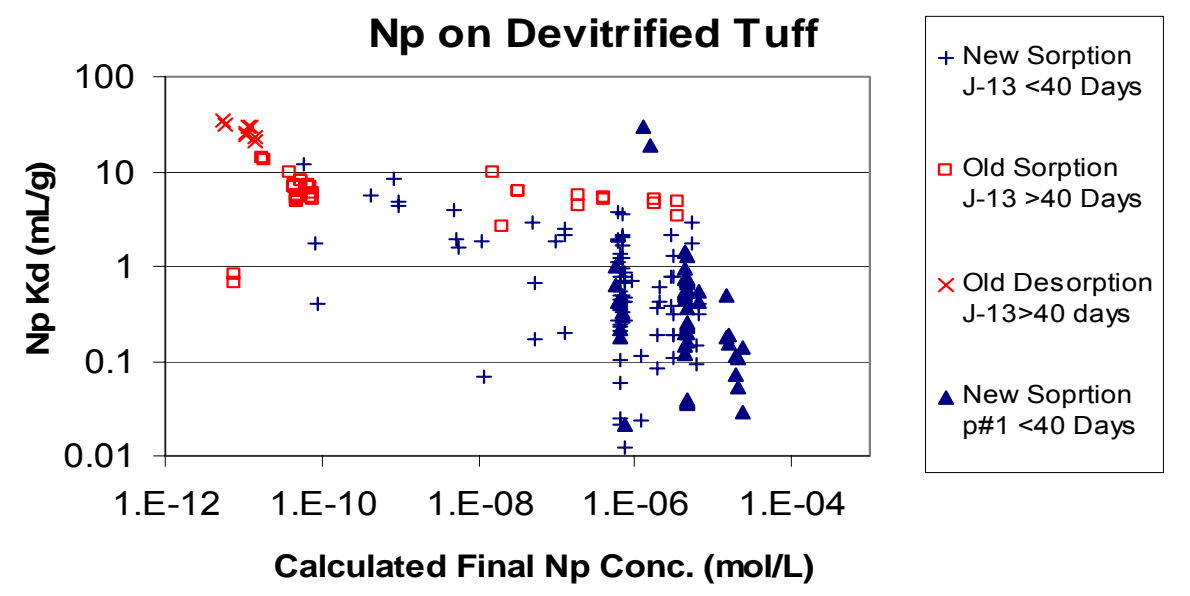

DTNs: New: LA0407AM831341.004 [DIRS 170622]; Old: LA0305AM831341.001 [DIRS 163789].

Figure A-18. Neptunium Sorption Coefficients on Devitrified Tuff versus Calculated Final Neptunium Concentration in Solution. Experiments Oversaturated with $\mathrm{Np}_{2} \mathrm{O}_{5}$ Have Been Omitted.

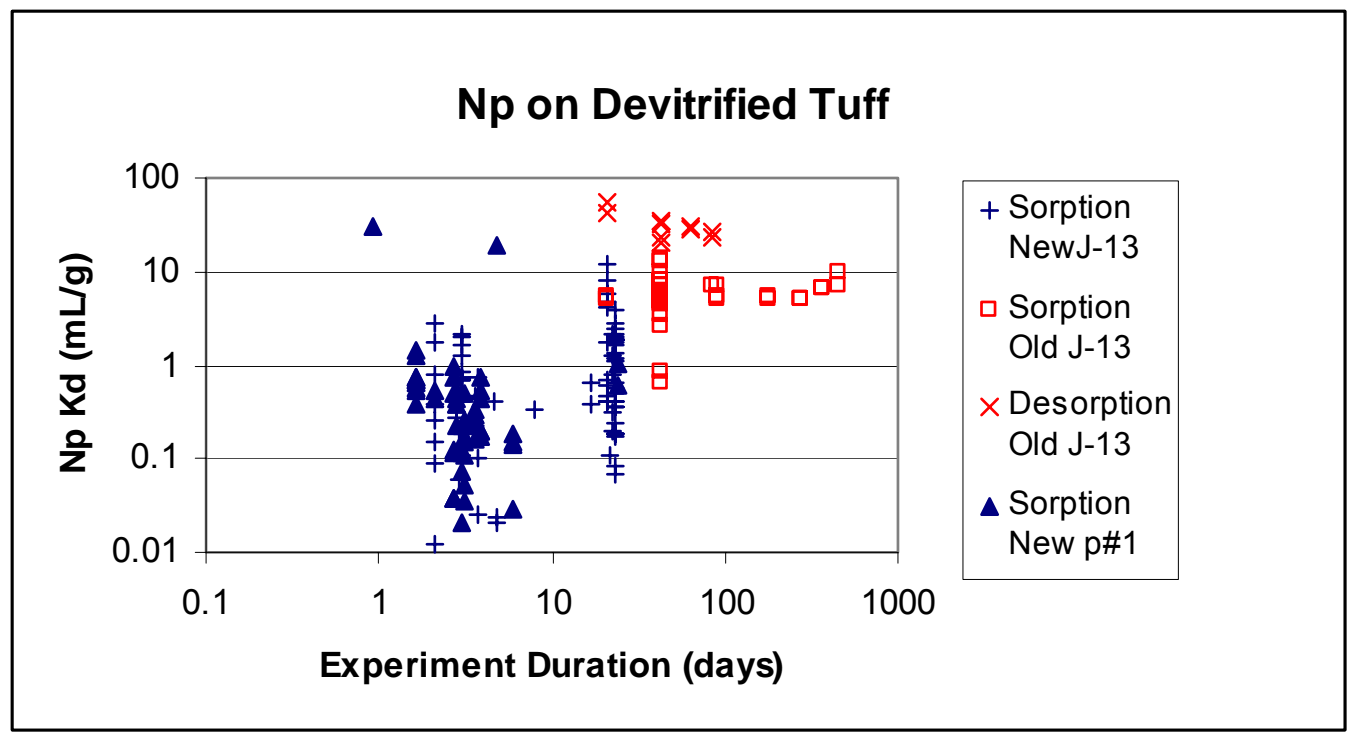

DTNs: New:LA0407AM831341.004 [DIRS 170622]; Old: LA0305AM831341.001 [DIRS 163789].

NOTE: Experiments oversaturated with $\mathrm{Np}_{2} \mathrm{O}_{5}$ have been omitted.

Figure A-19. Neptunium Sorption Coefficient on Devitrified Tuff versus Experiment Duration for Sorption (Forward) and Desorption (Backward) Experiments 


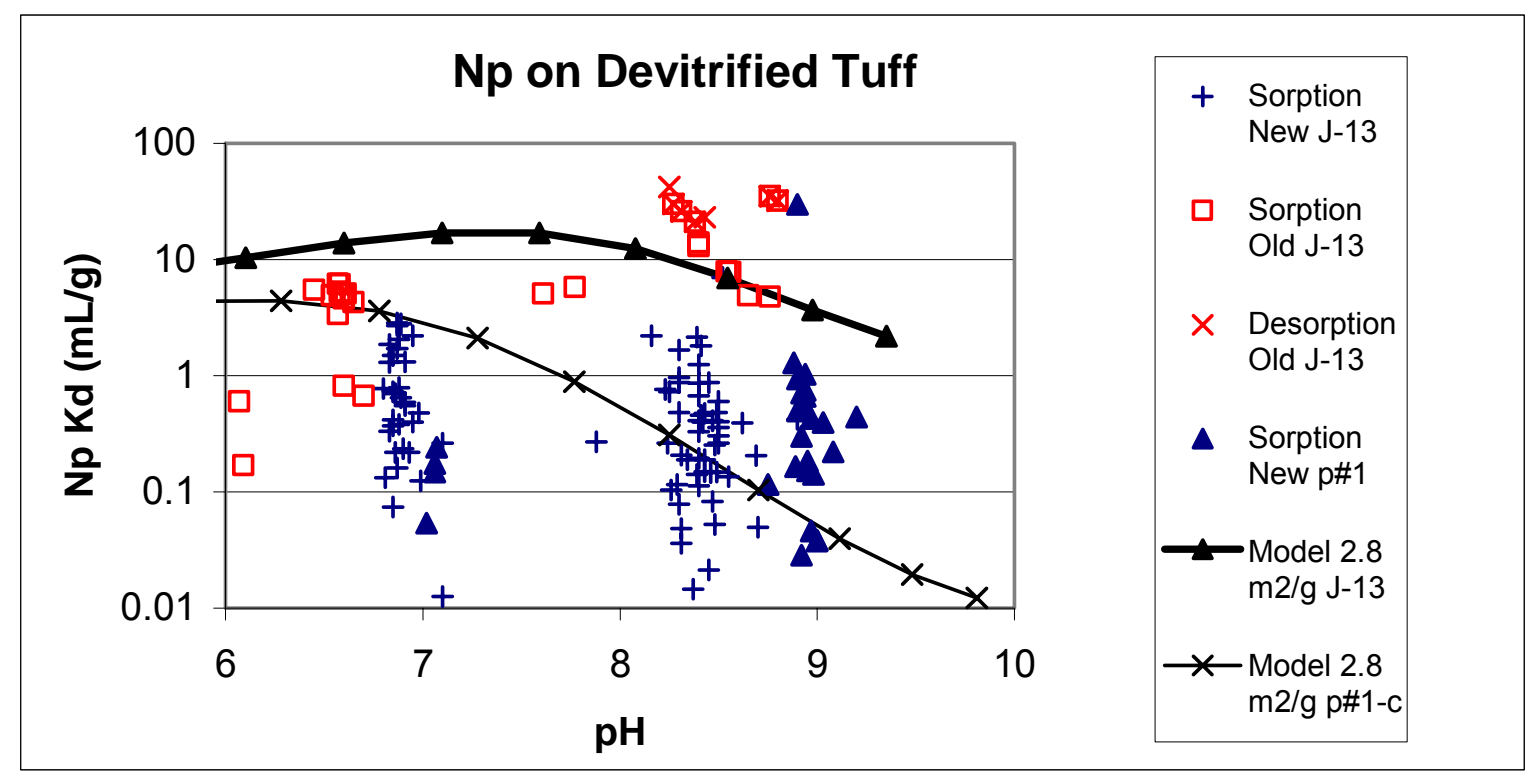

DTNs: New: LA0407AM831341.004 [DIRS 170622]; Old: LA0305AM831341.001 [DIRS 163789]; LA0407AM831343.001 [DIRS 171009].

NOTE: PHREEQC model results for $\mathrm{J}-13$ and $\mathrm{p \# 1}$ waters also are plotted.

Figure A-20a. Neptunium Sorption Coefficient on Devitrified Tuff in J-13 and Synthetic p\#1 versus Solution pH in Sorption (Forward) and Desorption (Backward) Experiments

The neptunium sorption-coefficient probability distribution selected for devitrified tuff in the $\mathrm{UZ}$ is a cumulative distribution, starting with a value of $0.0 \mathrm{~mL} / \mathrm{g}$ at 0.0 , increasing to a value of $0.5 \mathrm{~mL} / \mathrm{g}$ at 0.5 , and to a value of $6.0 \mathrm{~mL} / \mathrm{g}$ at 1.0 . The low end of the chosen range is selected based on the minimum value obtained in short-term experiments (up to 21 days). The upper end of the distribution was chosen as a minimum upper limit for a neptunium concentration near the solubility limit (Figure A-18), with emphasis on results from experiments with $\mathrm{p} \# 1$ water at $\mathrm{pH}$ near 7.0. It is acknowledged that a higher limit could be selected for the upper end of the distribution, based on the available data and modeling results. Figure A-20b shows the empirical cumulative distribution for all the measured neptunium sorption coefficients on devitrified tuff in the UZ with the selected distribution superimposed. As discussed in Section A6., not all the empirical data was equally weighted in selecting the probability distribution as the influence of expected variations in water chemistry, radionuclide concentrations, and variations in rock surface properties within each major rock type were incorporated in making the selection. 


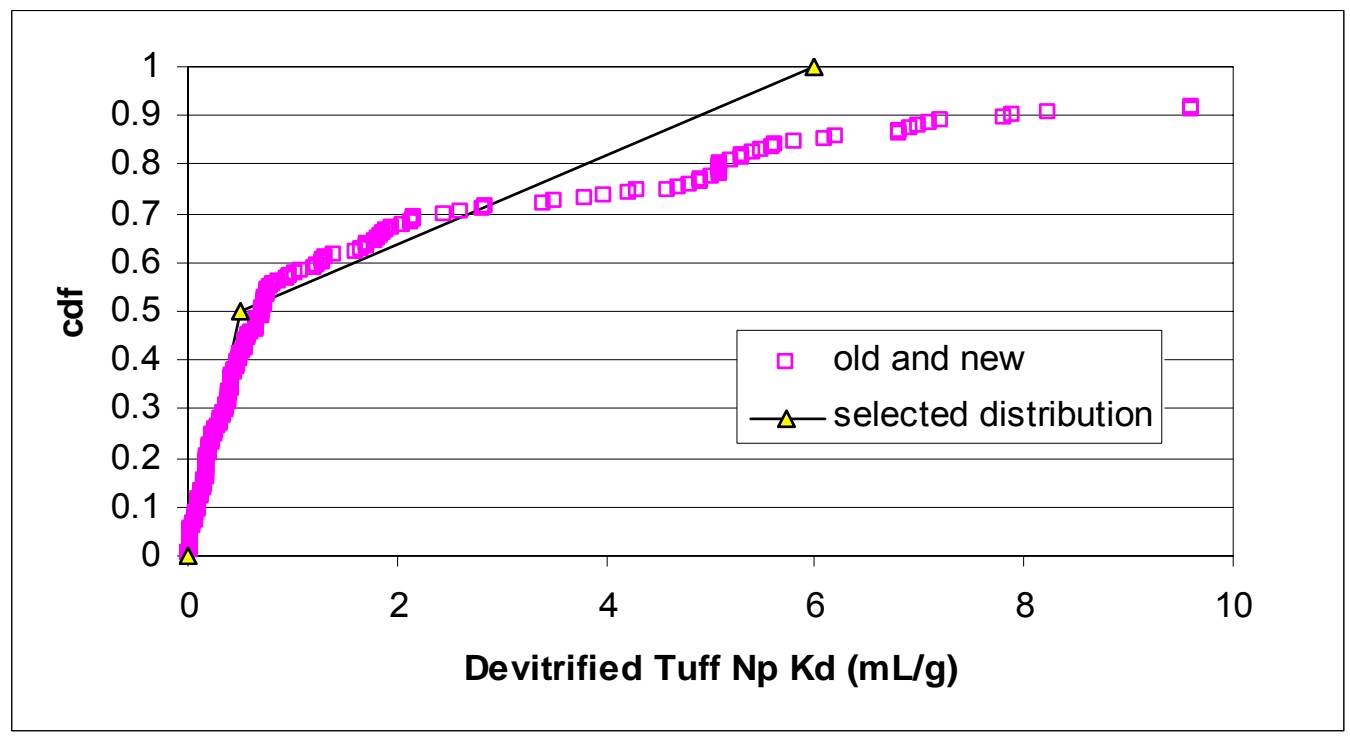

DTNs: New: LA0407AM831341.004 [DIRS 170622]; Old: LA0305AM831341.001 [DIRS 163789].

Figure A-20b. Empirical Cumulative Distribution for Neptunium Sorption Coefficients on Devitrified Tuff

\section{A8.3.2 Zeolitic Tuff}

Sorption coefficients on zeolitic tuff are shown as a function of calculated final solution concentration in Figure A-21. Many of the experiments with J-13 water had final neptunium concentrations above $7.0 \times 10^{-6} \mathrm{~mol} / \mathrm{L}$. Thus, the results for these experiments should be discounted because the experiments could have been oversaturated with $\mathrm{Np}_{2} \mathrm{O}_{5}$. Many of the experiments with synthetic $\mathrm{p} \# 1$ water had final neptunium solution concentrations close to or greater than $2.5 \times 10^{-5} \mathrm{~mol} / \mathrm{L}$. Results for these experiments must also be discounted.

Removal of the oversaturated experiments from the dataset results in Figure A-22. With the oversaturated data points removed, the sorption coefficient has virtually no dependence on the calculated final solution concentration for J-13 experiments. However, there is clearly a dependence of sorption coefficient on water chemistry in the short-term experiments. If the solubility of neptunium in synthetic $\mathrm{p \# 1}$ is less than the solubility in $\mathrm{J}-13$, this dependence on water chemistry may not be real. 

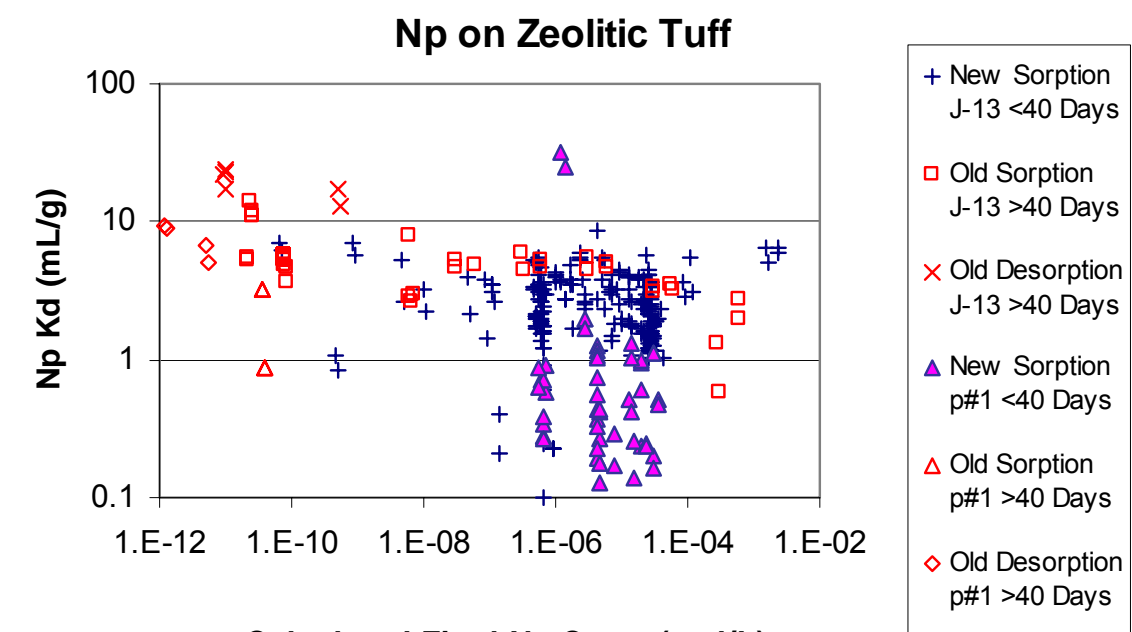

Calculated Final Np Conc. (mol/L)

DTNs: New: LA0407AM831341.004 [DIRS 170622]; Old: LA0305AM831341.001 [DIRS 163789].

Figure A-21. Neptunium Sorption Coefficients on Zeolitic Tuff versus Calculated Final Neptunium Concentration in Solution
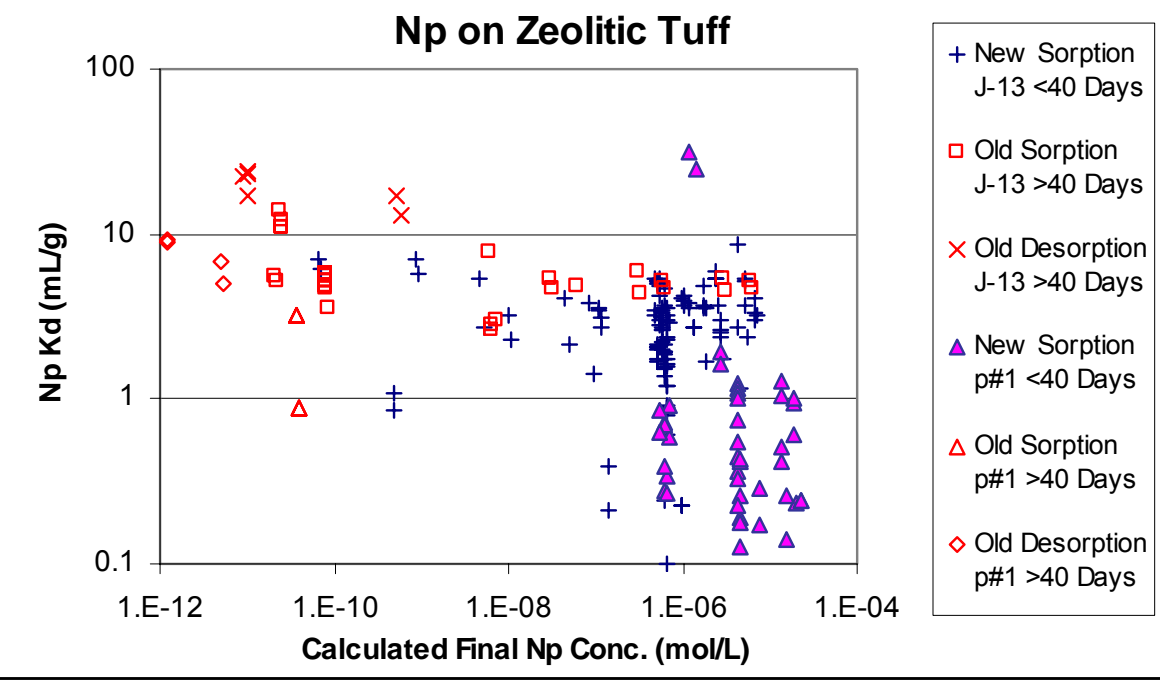

DTNs: New: LA0407AM831341.004 [DIRS 170622]; Old: LA0305AM831341.001 [DIRS 163789].

NOTE: Oversaturated experiments have been removed.

Figure A-22. Neptunium Sorption Coefficients on Zeolitic Tuff versus Calculated Final Neptunium Concentration in Solution 
Neptunium sorption experiments carried out as a function of experiment duration are shown in Figure A-23. There is a significant difference between the results for "old" and "new" experiments, with the "old" results generally having higher values than the "new" results. The most straightforward explanation is that the difference is caused by the "old" results representing experiments with longer durations than the "new" results. Within the "old" data points, the sorption coefficient values appear to reach a steady-state level after 42 days.

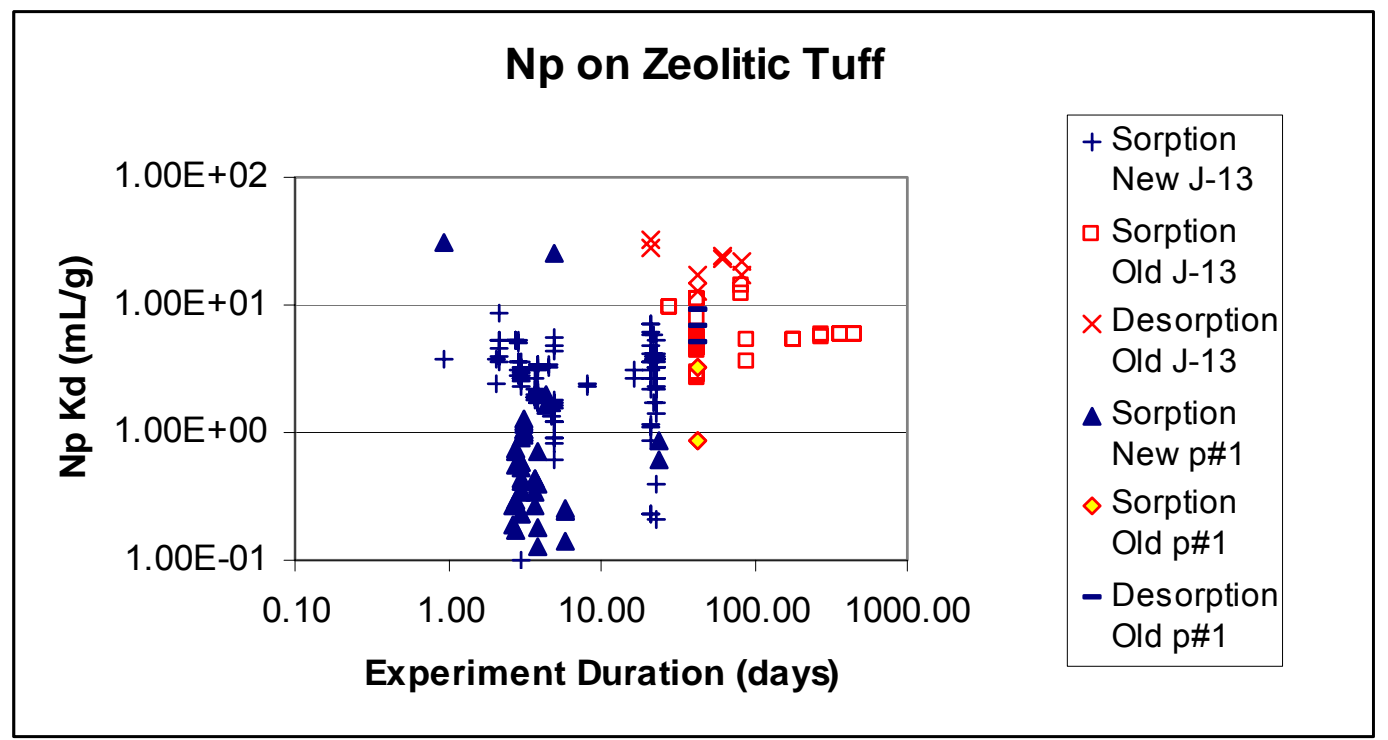

DTNs: New: LA0407AM831341.004 [DIRS 170622]; Old: LA0305AM831341.001 [DIRS 163789].

NOTE: Oversaturated experiments have been omitted.

Figure A-23. Neptunium Sorption Coefficient on Zeolitic Tuff versus Experiment Duration for Sorption (Forward) and Desorption (Backward) Experiments

The impact of variations in $\mathrm{pH}$ on neptunium coefficients for zeolitic tuffs is shown in Figure A-24a. There is a lot of scatter in the "new" data, and there do not appear to be clear positive or negative trends among these data points. Nor does there appear to be much difference between the J-13 and synthetic p\#1 results. The "old" data points are more consistent and show that the neptunium sorption coefficient has very little dependence on $\mathrm{pH}$, except at $\mathrm{pH}$ values less than 7.0. 

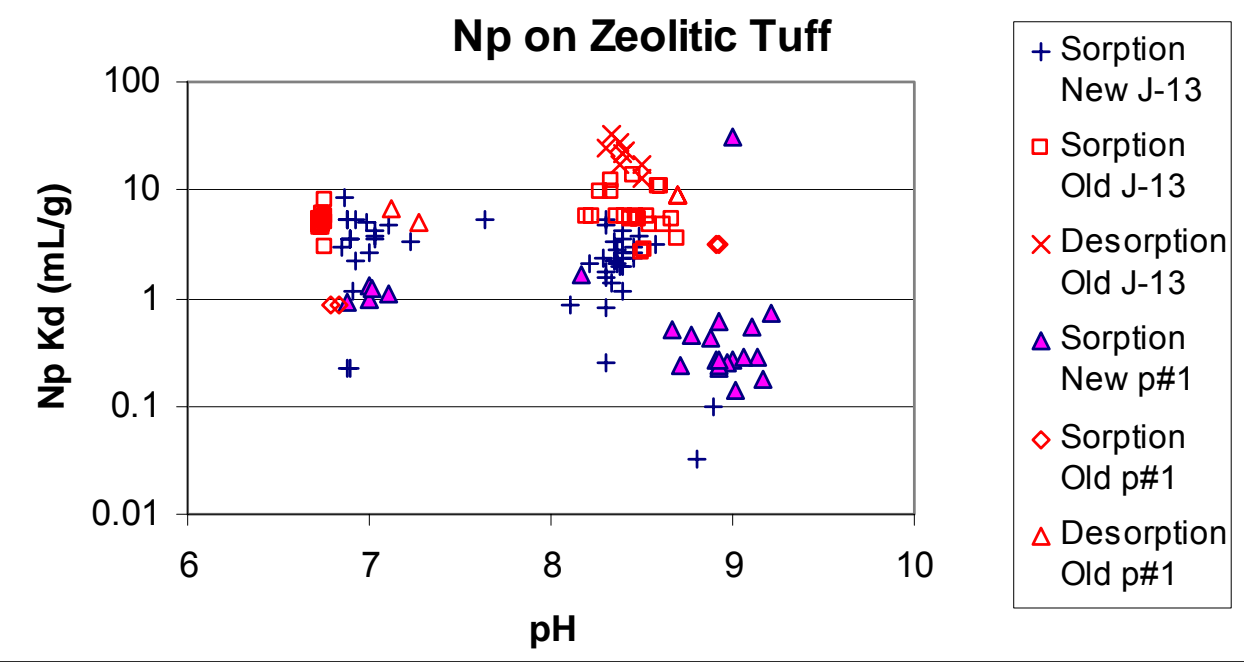

DTNs: New: LA0407AM831341.004 [DIRS 170622]; Old: LA0305AM831341.001 [DIRS 163789].

NOTE: Oversaturated experiments have been omitted.

Figure A-24a. Neptunium Sorption Coefficient on Devitrified Tuff in J-13 and Synthetic p\#1 versus Solution pH in Sorption (Forward) and Desorption (Backward) Experiments

The neptunium sorption-coefficient probability distribution selected for zeolitic tuff in the UZ is a cumulative distribution, starting at $0.0 \mathrm{~mL} / \mathrm{g}$ at 0.0 , increasing to a value of $0.5 \mathrm{~mL} / \mathrm{g}$ at 0.5 , and ending at a value of $6 \mathrm{~mL} / \mathrm{g}$ at 1.0. The low end of the chosen range is selected based on the minimum value obtained in short-term experiments (up to 21 days). The upper end of the distribution was chosen as a minimum upper limit for a neptunium concentration near the solubility limit (Figure A-21), with emphasis on results from experiments with $\mathrm{p \# 1}$ water at $\mathrm{pH}$ near 7.0. It is acknowledged that a higher limit could be selected for the upper end of the distribution, based on the available data. Figure A-24b shows the empirical cumulative distribution for all the measured neptunium sorption coefficients on zeolitic tuff in the UZ with the selected distribution superimposed. As discussed in Section A6., not all the empirical data was equally weighted in selecting the probability distribution as the influence of expected variations in water chemistry, radionuclide concentrations, and variations in rock surface properties within each major rock type were incorporated in making the selection.

\section{A8.3.3 Vitric Tuff}

Sorption coefficients on vitric tuff are shown as a function of calculated final solution concentration in Figure A-25. Many of the experiments had final neptunium concentrations above $7.0 \times 10^{-6} \mathrm{~mol} / \mathrm{L}$. Thus, the results for these experiments were omitted from the diagram because the experiments could have been oversaturated with $\mathrm{Np}_{2} \mathrm{O}_{5}$. Several of the experiments with synthetic p\#1 water had final neptunium solution concentrations close to or greater than $2.5 \times 10^{-5} \mathrm{~mol} / \mathrm{L}$. Results for these experiments were also omitted. The remaining data points show a clear dependence on final neptunium solution concentration. 


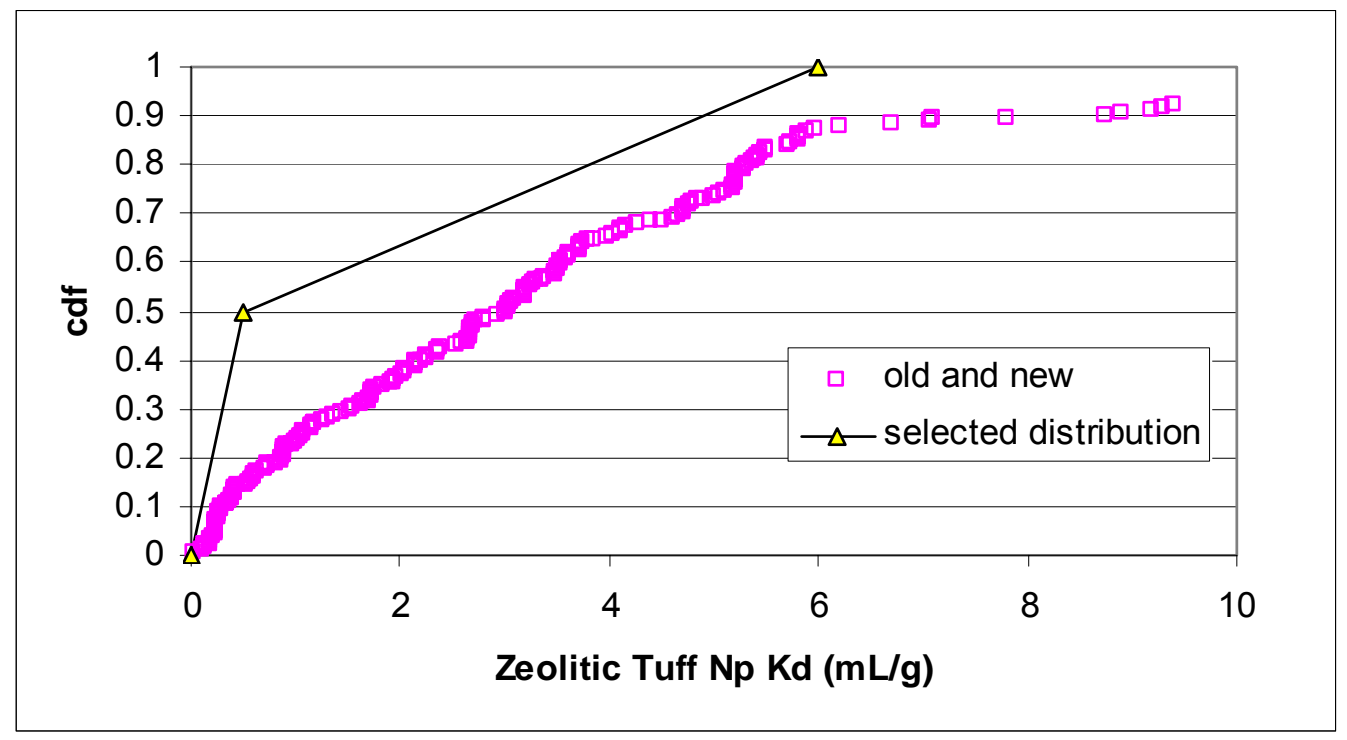

DTNs: New: LA0407AM831341.004 [DIRS 170622]; Old: LA0305AM831341.001 [DIRS 163789].

Figure A-24b. Empirical Cumulative Distribution for Neptunium Sorption Coefficients on Zeolitic Tuff

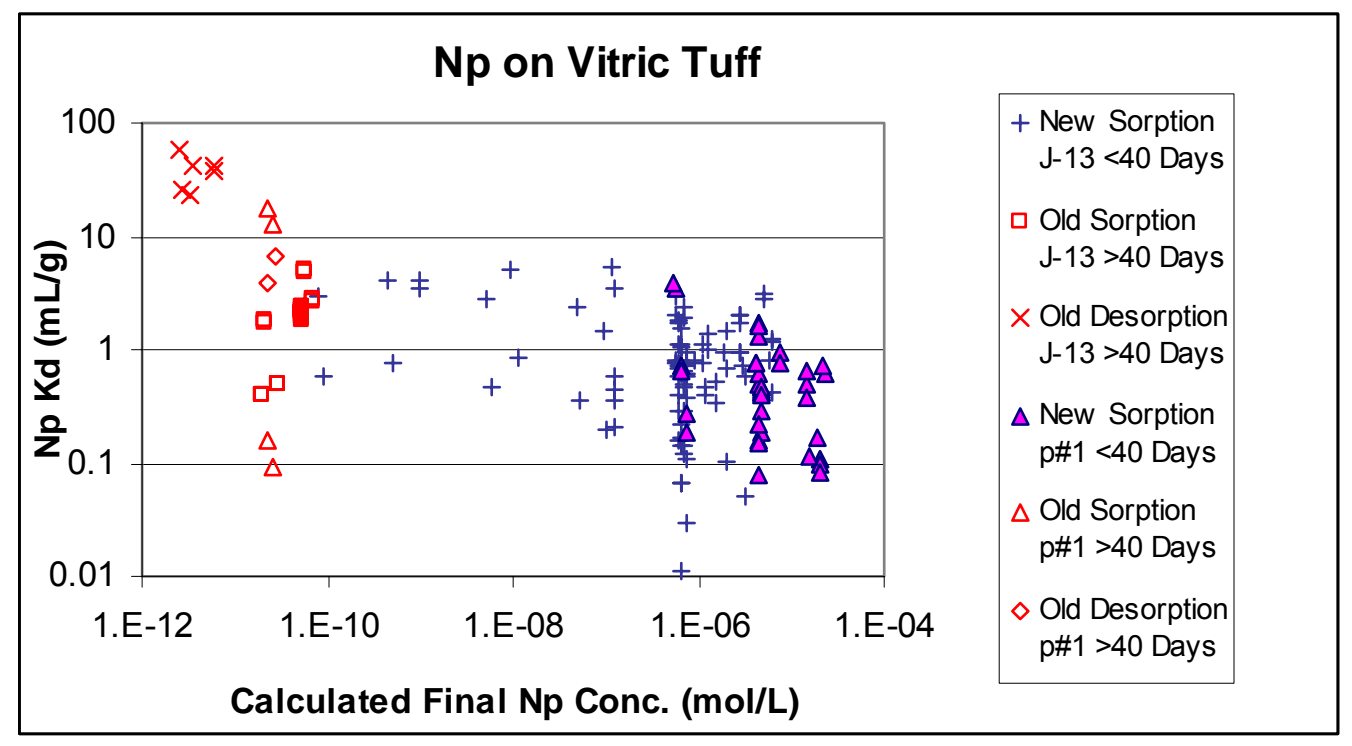

DTNs: New: LA0407AM831341.004 [DIRS 170622]; Old: LA0305AM831341.001 [DIRS 163789].

NOTE: Oversaturated experiments have been omitted.

Figure A-25. Neptunium Sorption Coefficients on Vitric Tuff versus Calculated Final Neptunium Concentration in Solution

Neptunium sorption experiments carried out as a function of experiment duration are shown in Figure A-26. There is a trend of increasing sorption coefficient with increasing duration. 


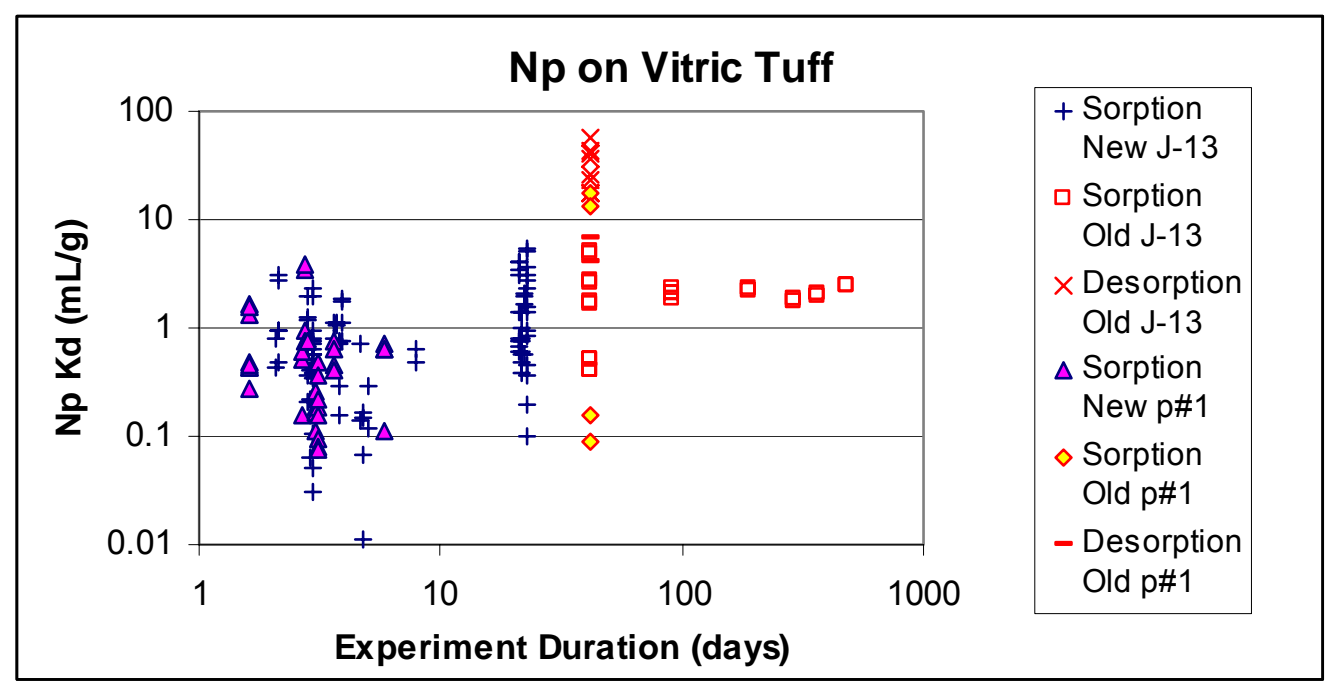

DTNs: New: LA0407AM831341.004 [DIRS 170622]; Old: LA0305AM831341.001 [DIRS 163789].

NOTE: Oversaturated experiments have been omitted.

Figure A-26. Neptunium Sorption Coefficient on Vitric Tuff versus Experiment Duration for Sorption (Forward) and Desorption (Backward) Experiments

The impact of variations in $\mathrm{pH}$ on neptunium coefficients on vitric tuffs is shown in Figure A-27a. There is a lot of scatter in the "new" data and there do not appear to be clear positive or negative trends among these data points. Nor does there appear to be much difference between the $\mathrm{J}-13$ and synthetic $\mathrm{p} \# 1$ results.

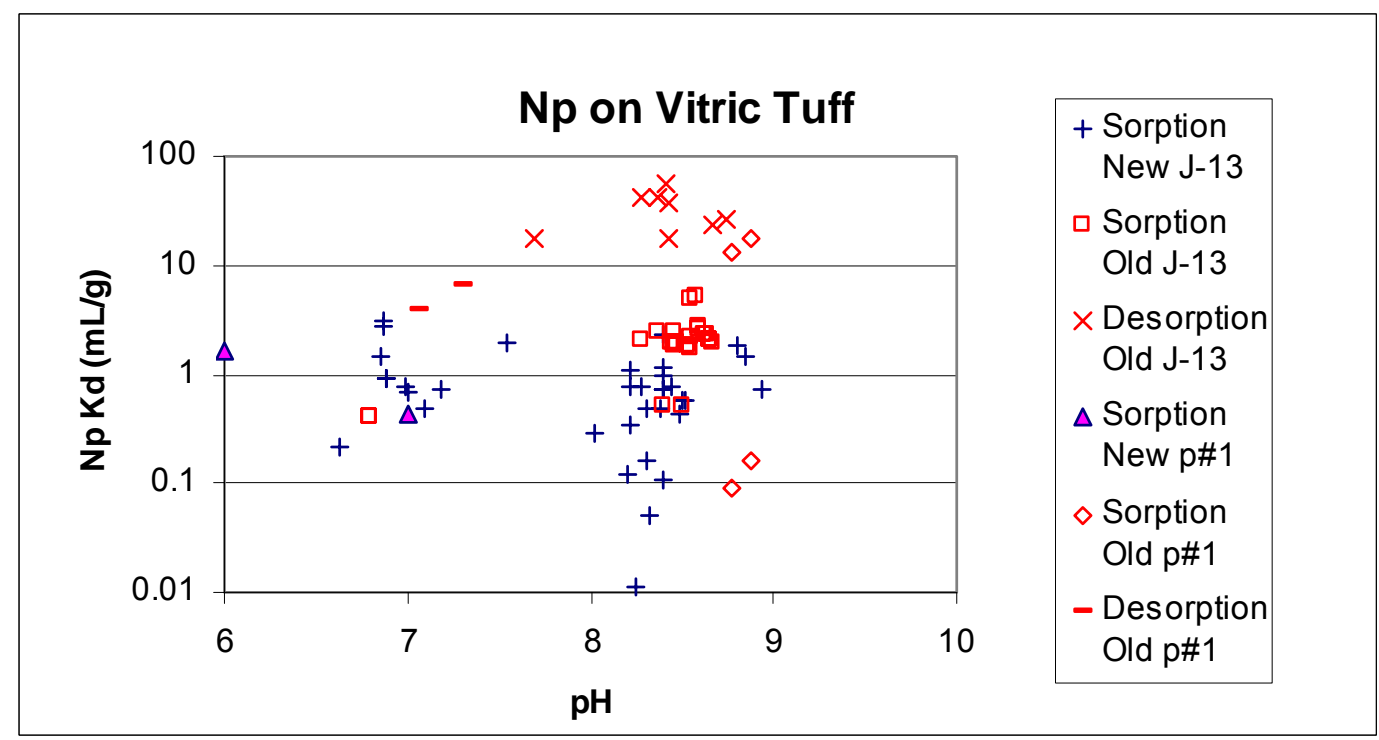

DTNs: New: LA0407AM831341.004 [DIRS 170622]; Old: LA0305AM831341.001 [DIRS 163789].

NOTE: Oversaturated experiments have been omitted.

Figure A-27a. Neptunium Sorption Coefficient on Devitrified Tuff in J-13 and Synthetic p\#1 versus Solution pH in Sorption (Forward) and Desorption (Backward) Experiments 
The neptunium sorption-coefficient probability distribution selected for vitric tuff in the UZ is a cumulative distribution starting at $0.0 \mathrm{~mL} / \mathrm{g}$ at 0.0 , increasing to a value of $1.0 \mathrm{~mL} / \mathrm{g}$ at 0.5 , and ending with a value of $3.0 \mathrm{~mL} / \mathrm{g}$ at 1.0 . The low end of the chosen range is selected based on the minimum value obtained in short-term experiments (up to 21 days). The upper end of the distribution was chosen as a minimum upper limit for a neptunium concentration near the solubility limit (Figure A-25), with emphasis on results from experiments with $\mathrm{p \# 1}$ water at $\mathrm{pH}$ near 7.0. It is acknowledged that a higher limit could be selected for the upper end of the distribution, based on the available data. Figure A-27b shows the empirical cumulative distribution for all the measured neptunium sorption coefficients on vitric tuff in the UZ with the selected distribution superimposed. As discussed in Section A6., not all the empirical data was equally weighted in selecting the probability distribution as the influence of expected variations in water chemistry, radionuclide concentrations, and variations in rock surface properties within each major rock type were incorporated in making the selection.

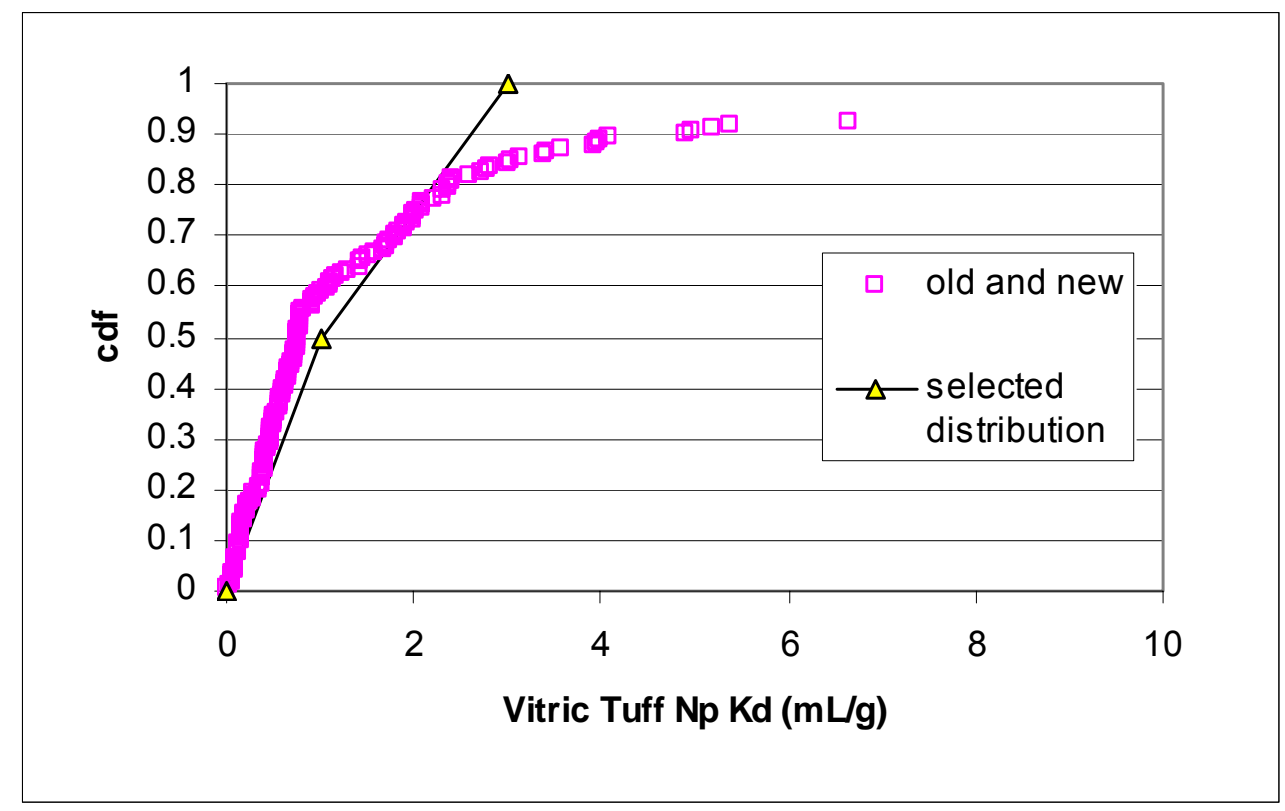

DTNs: New: LA0407AM831341.004 [DIRS 170622]; Old: LA0305AM831341.001 [DIRS 163789].

Figure A-27b. Empirical Cumulative Distribution for Neptunium Sorption Coefficients on Vitric Tuff

\section{A8.4 PLUTONIUM}

According to Nitsche et al. (1993 [DIRS 155218], p. 54), the solubility of plutonium in $\mathrm{J}-13$ water at $25^{\circ} \mathrm{C}, \mathrm{pH}=8.4$, is $2.9 \pm 0.8 \times 10^{-7} \mathrm{~mol} / \mathrm{L}$ and is not very sensitive to $\mathrm{pH}$ over the range from 7.0 to 8.4 . The solubility of plutonium in $\mathrm{p} \# 1$ water at $25^{\circ} \mathrm{C}, \mathrm{pH}=8.5$, is $1.0 \pm 0.1 \times$ $10^{-6} \mathrm{~mol} / \mathrm{L}$, and $4.5 \pm 0.4 \times 10^{-7} \mathrm{~mol} / \mathrm{L}$ at $25^{\circ} \mathrm{C}, \mathrm{pH}=7.0$ according to Nitsche et al. (1994 [DIRS 144515], p. 39). Thus, the solubility of plutonium in J-13 is somewhat lower than it is in $\mathrm{p} \# 1$. 
Solubilities for plutonium are also reported in Dissolved Concentration Limits of Radioactive Elements (BSC 2004 [DIRS 169425], Section 6.5; DTN: MO0408SPADCLRE.000 [DIRS 171601]). The solubilities are less than $1.5 \times 10^{-7} \mathrm{~mol} / \mathrm{L}$ for $\mathrm{pH}$ between 7.0 and 8.4 and $\log \mathrm{fCO}_{2}$ between -3.5 and -2.5 . Therefore, using the solubilities based on the reports by Nitsche et al. (1993 [DIRS 155218]; (1994 [DIRS 144515]), solubilities screen out fewer of the higher concentration results. Because plutonium sorption coefficients trend lower for higher solution concentrations, this leads including more low-sorption results in the selected distributions.

\section{A8.4.1 Devitrified Tuff}

The experimentally derived sorption coefficients for plutonium on devitrified tuff are plotted against the calculated final plutonium concentrations of the experiments in Figure A-28. The data points are separated into groups on the basis of when the experiments were carried out (pre-1990 = "old" and post-1990 = "new"), water chemistry, experiment duration, and whether the sorption coefficient was determined from a sorption or a desorption experiment. The plotted data indicate that the "new" and "old" data show similar ranges of $\mathrm{K}_{\mathrm{d}}$ values, that the longer-term experiments generally yield higher sorption coefficient values than the shorter term experiments, and that desorption experiments yield higher sorption coefficient values than sorption experiments. These points are discussed in greater detail below.

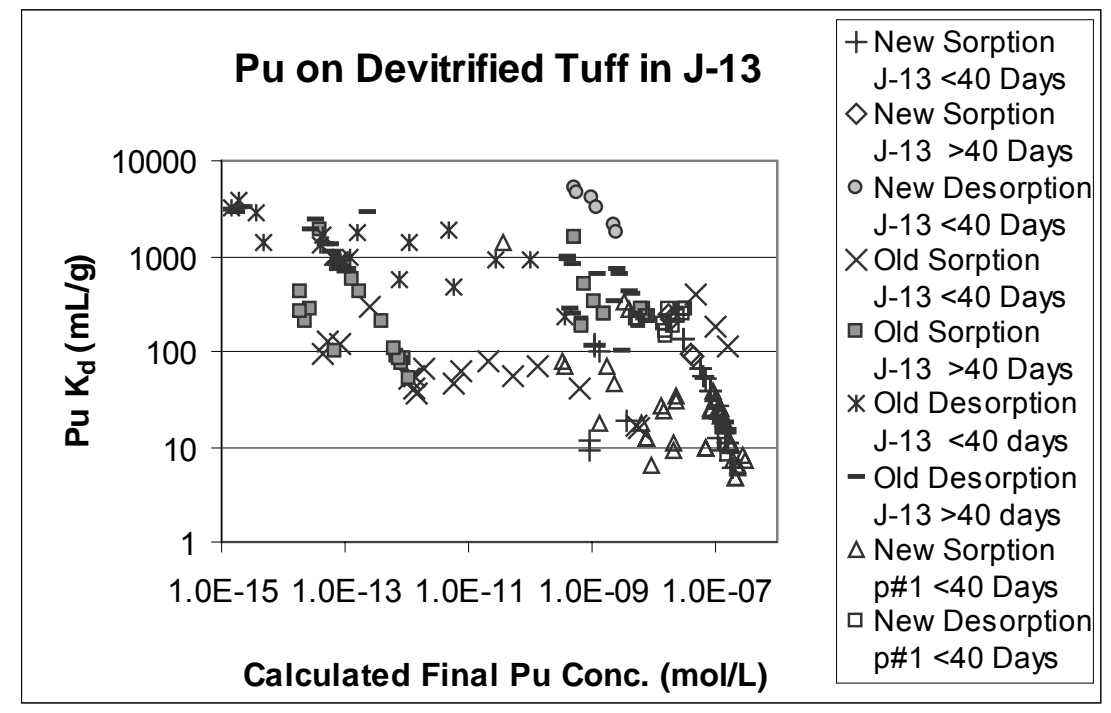

DTNs: New: LA0407AM831341.005 [DIRS 170625]; Old: LA0305AM831341.001 [DIRS 163789].

Figure A-28. Plutonium Sorption Coefficients on Devitrified Tuff versus Calculated Final Plutonium Concentration in Solution

The maximum calculated final plutonium concentration plotted in Figure A-28 is slightly less than the solubility determined by Nitsche et al. (1993 [DIRS 155218], p. 54) for plutonium in J-13. Thus, the plutonium sorption coefficients plotted in Figure A-28 reflect solutions that were undersaturated with respect to the solid plutonium phase precipitated in the experiments reported by Nitsche et al. (1993 [DIRS 155218], p. 54). 
The effect of the solution plutonium concentration on the sorption coefficient value obtained is shown more clearly in Figure A-29. Data are plotted for two devitrified tuff samples that contain only trace amounts of secondary phases (e.g., clays, zeolites). In addition, plotted data are restricted to 21-day experiment durations and $\mathrm{pH}$ between 7.2 and 9.2. As is evident in the figure, within the errors of the analyses, the measured plutonium sorption coefficients are nearly independent of the final plutonium solution concentration.

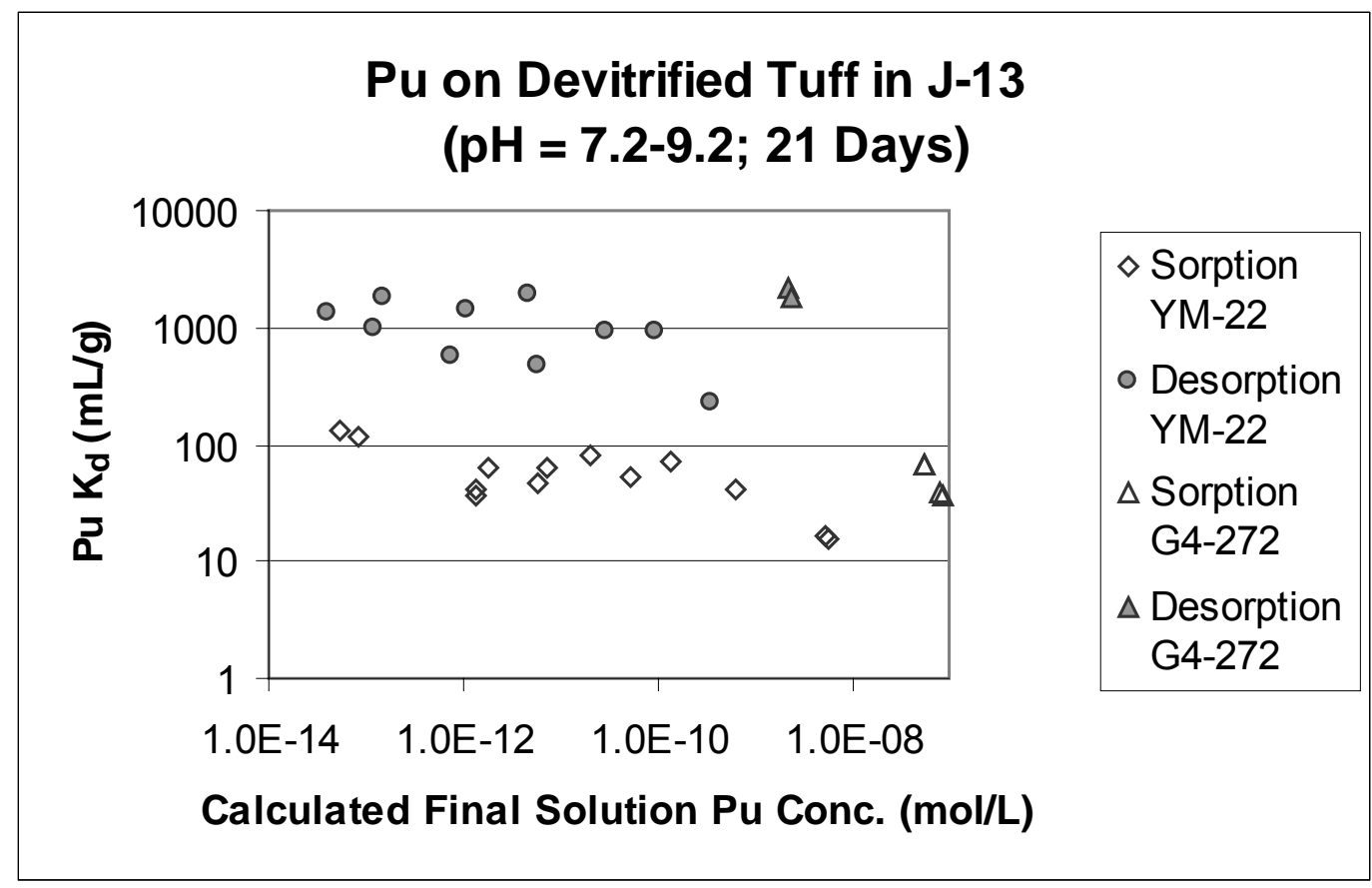

DTNs: New: LA0407AM831341.005 [DIRS 170625]; Old: LA0305AM831341.001 [DIRS 163789].

Figure A-29. Plutonium Sorption Coefficient versus Calculated Final Plutonium Solution Concentration in Moles/Liter for Experiments with Samples YM-22 and G4-272.

The effect of experiment duration on the plutonium $\mathrm{K}_{\mathrm{d}}$ is shown in Figure A-30. As expected, the $K_{d}$ values for sorption experiments increase with increasing duration and the $K_{d}$ values for desorption experiments decrease with increasing duration. However, the increase in the sorption values is much greater than the decrease in the desorption values over the time-frame of the experiments. This may reflect the reduction of plutonium +5 and/or +6 to plutonium +4 on the mineral surfaces present in devitrified tuff as discussed in greater detail below. The trends in the sorption and desorption data points suggest they would converge to values somewhere between 100 and $1,000 \mathrm{~mL} / \mathrm{g}$. Based on the data plotted, such convergence would require more than 100 days. Note that the "old" sorption data points exceeding $1,000 \mathrm{~mL} / \mathrm{g}$ are for samples that contain significant amounts of clay or zeolite. For this reason, these data are discounted in the derivation of the sorption coefficient probability distribution. 


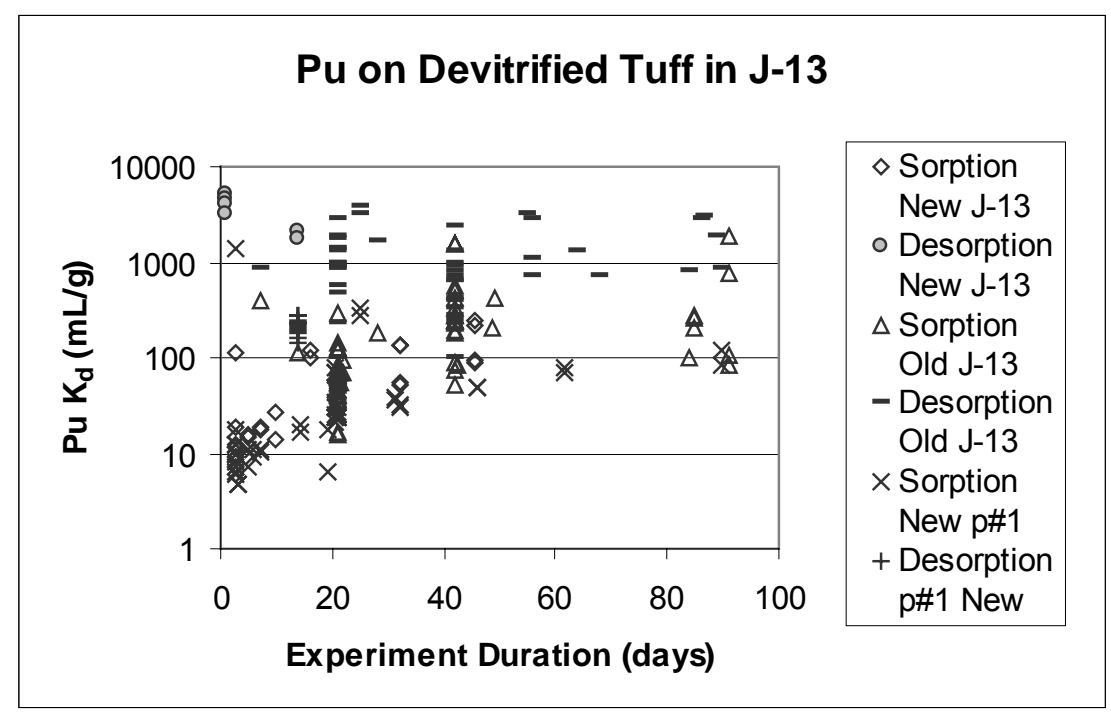

DTNs: New: LA0407AM831341.005 [DIRS 170625]; Old: LA0305AM831341.001 [DIRS 163789].

Figure A-30. Plutonium Sorption Coefficient on Devitrified Tuff versus Experiment Duration for Sorption (Forward) and Desorption (Backward) Experiments

The impact of variations in $\mathrm{pH}$ for plutonium sorption coefficients on devitrified tuffs is shown in Figure A-31. There is a lot of scatter in the data, and there do not appear to be clear positive or negative trends in any of the data groupings.

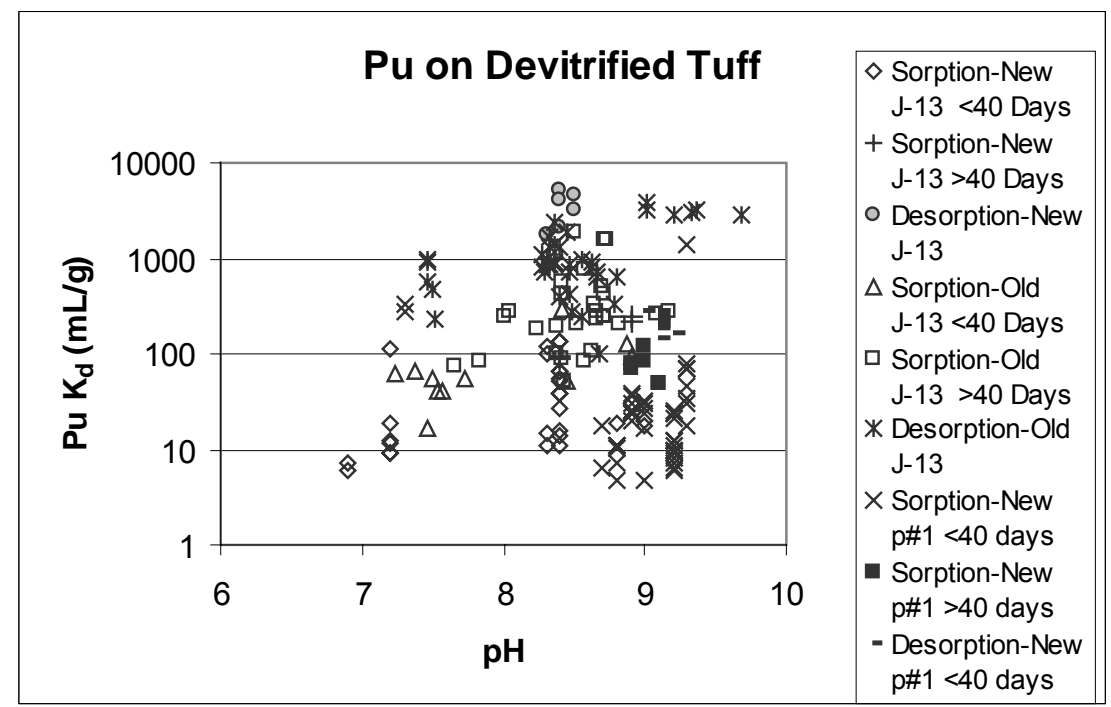

DTNs: New: LA0407AM831341.005 [DIRS 170625]; Old: LA0305AM831341.001 [DIRS 163789].

NOTE: Experiments lasting 40 days or more are plotted separately from experiments lasting less than 40 days.

Figure A-31. Plutonium Sorption Coefficient on Devitrified Tuff in J-13 and Synthetic p\#1 versus Solution $\mathrm{pH}$ in Sorption (Forward) and Desorption (Backward) Experiments 
The lack of clear trends is also evident when the results of short-term experiments (less than 40 days) are removed from the dataset. This is shown in Figure A-32, in which only the longer-term data are plotted.

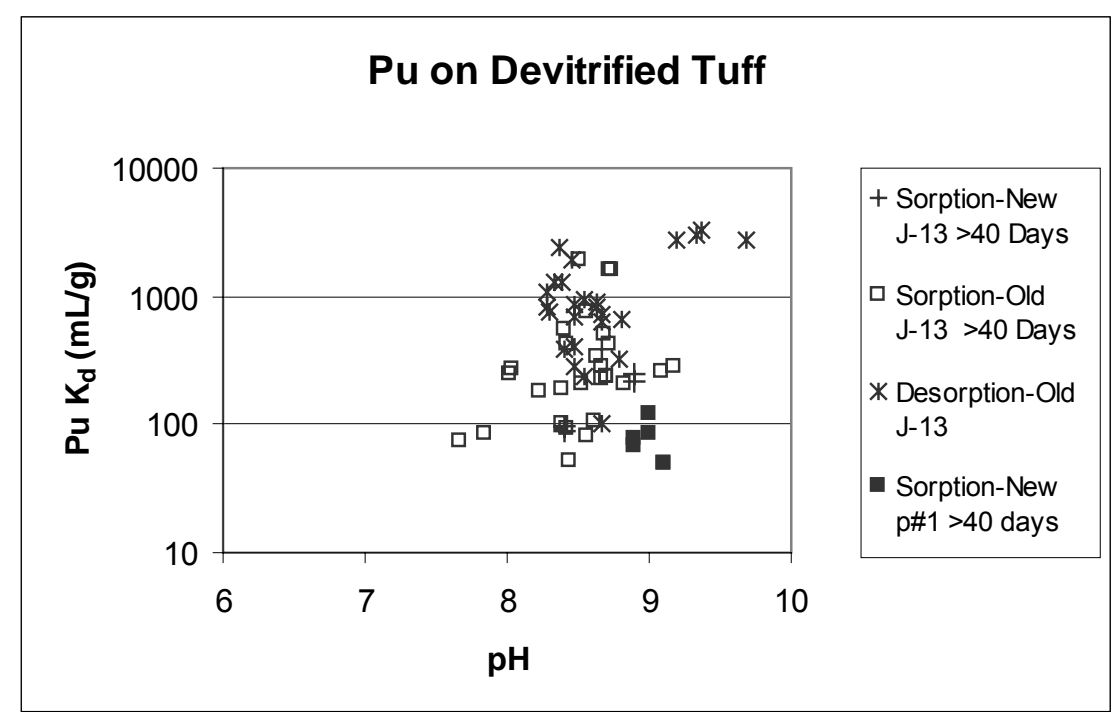

DTNs: New: LA0407AM831341.005 [DIRS 170625]; Old: LA0305AM831341.001 [DIRS 163789].

Figure A-32. Plutonium Sorption Coefficient on Devitrified Tuff in J-13 and Synthetic p\#1 versus Solution $\mathrm{pH}$ in Sorption (Forward) and Desorption (Backward) Experiments with Durations Greater than 40 Days

The impact of variations in the major ion composition of groundwaters are also shown in Figures A-30 through A-32. Although there is a limited set of experiments with synthetic p\#1 water, the results are within the range of the results obtained in experiments with J-13 water. Thus, there is no clear evidence of the impact of water chemistry variations on plutonium sorption coefficients in devitrified tuff.

A major factor not explicitly accounted for in the experimental program is the impact of variations in the Eh (oxidation/reduction potential normalized to the Standard Hydrogen Electrode) of Yucca Mountain groundwaters on plutonium sorption coefficients. The laboratory experiments upon which the data discussed in this section are based were invariably conducted under oxidizing conditions, because the waters used in the experiments contained dissolved oxygen and were in contact with the atmosphere. Nitsche et al. (1993 [DIRS 155218], pp. 60 to 61) found that plutonium dissolved in J-13 water is present predominantly in the +5 and +6 oxidation states. If plutonium in the +5 and +6 oxidation states behaves similarly to neptunium +5 and uranium +6 , respectively, as has been suggested by many investigators (e.g., Keeney-Kennicutt and Morse 1985 [DIRS 106313], pp. 2577 to 2578), then small values (less than $10 \mathrm{~mL} / \mathrm{g}$ ) would be expected for plutonium sorption coefficients under oxidizing conditions (see Neptunium and Uranium appendix sections). The fact that plutonium sorption coefficients measured under oxidizing conditions are up to 2-3 orders of magnitude larger than expected (Figure A-28) suggests that either plutonium +5 and +6 do not behave like neptunium +5 and uranium +6 in sorption reactions, or plutonium is reduced to the +4 oxidation state on 
rock/mineral surfaces. Data presented by Keeney-Kennicutt and Morse (1985 [DIRS 106313], p. 2577) support the latter alternative.

To further pursue the latter alternative, a PHREEQC model was developed to calculate plutonium sorption coefficients as a function of Eh. To develop this model, binding constants for neptunium +5 and uranium +6 species were used for plutonium +5 and plutonium +6 species, respectively. For plutonium +4 , binding constants were derived by fitting experimental data for plutonium sorption on quartz in Oak Ridge Reference Water published by Allard (1982 [DIRS 104512], p. 61). This water composition has somewhat reducing characteristics, so that plutonium +4 would be expected to be sorbed onto the solid phase. The sorption coefficients calculated with this model using J-13 water are plotted as a function of Eh in Figure A-33a. The curves plotted in Figure A-33a suggest that at Eh values less than $500 \mathrm{mv}$, plutonium is predominantly in the +4 oxidation state in the solution and on the solid phase. This Eh value is higher than the highest value measured in Yucca Mountain groundwater (Ogard and Kerrisk 1984 [DIRS 100783], pp. 10, 15; DTNs: LA0206AM831234.001 [DIRS 160051]; LA0206AM831234.002 [DIRS 163852]). This implies that plutonium +4 will be the dominant oxidation state sorbed in most Yucca Mountain groundwaters. At higher Eh values, plutonium in solution will become progressively oxidized to plutonium +5 and eventually to plutonium +6 . The end points of the curves (high Eh) plotted in Figure A-33a represent the Eh values expected when the water is in contact with the atmosphere. In the $\mathrm{p} \# 1$ water composition, the lower ends of the curves are shifted downward somewhat to lower $\mathrm{K}_{\mathrm{d}}$ values at a given Eh value (not shown).

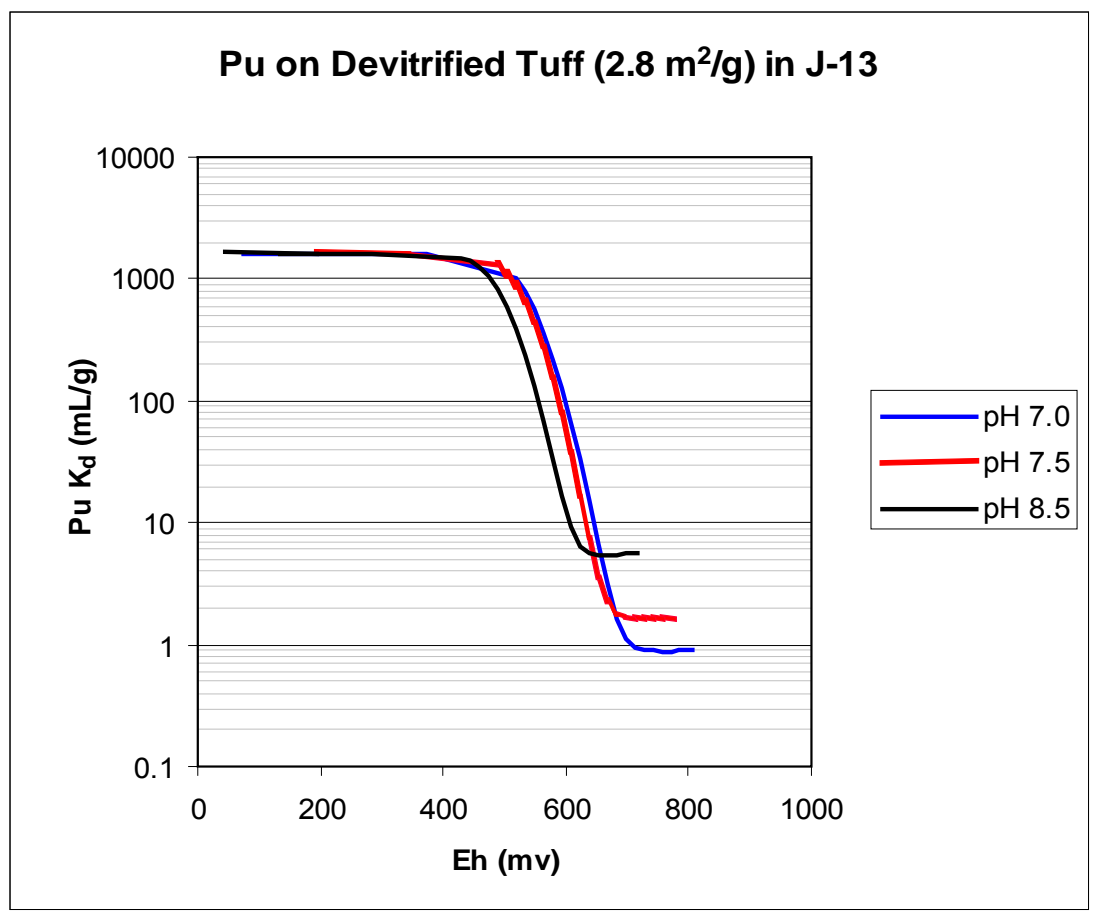

DTN: LA0407AM831343.001 [DIRS 171009].

Figure A-33a. Plutonium Sorption Coefficients versus Eh as Predicted by PHREEQC V2.3 Model. Separate Curves Are Shown for Different pH Values. 
The large range in plutonium sorption coefficients measured in devitrified tuffs (e.g., Figure A-28) could be explained if plutonium is present in more than one oxidation state on the rock/mineral surfaces in the devitrified tuffs. However, in the absence of definitive data for the oxidation state of plutonium on rock/mineral surfaces, the experimentally determined sorption coefficient values will be used to derive the probability distributions.

The plutonium sorption-coefficient probability distribution derived for devitrified tuff in the UZ is a cumulative distribution starting at $10 \mathrm{~mL} / \mathrm{g}$ at 0.0 , increasing to a value of $70 \mathrm{~mL} / \mathrm{g}$ at 0.5 , and ending at a value of $200 \mathrm{~mL} / \mathrm{g}$ at 1.0 . A cumulative distribution was chosen for the sake of simplicity given the available data set for $\mathrm{p} \# 1$ water. The low end of the chosen range is selected to allow for the possibility of relatively fast flow in the UZ. It also captures the potential impact of variations in surface areas among samples used in the experiments, variations in water chemistry, and variations in plutonium concentrations up to the solubility limit. The upper end of the distribution was chosen as a minimum upper limit given the potential impacts of sorption kinetics during potentially fast flow in the UZ. Figure A-33b shows the empirical cumulative distribution for all the measured plutonium sorption coefficients on devitrified tuff in the UZ with the selected distribution superimposed. As discussed in Section A6., not all the empirical data was equally weighted in selecting the probability distribution as the influence of expected variations in water chemistry, radionuclide concentrations, and variations in rock surface properties within each major rock type were incorporated in making the selection.

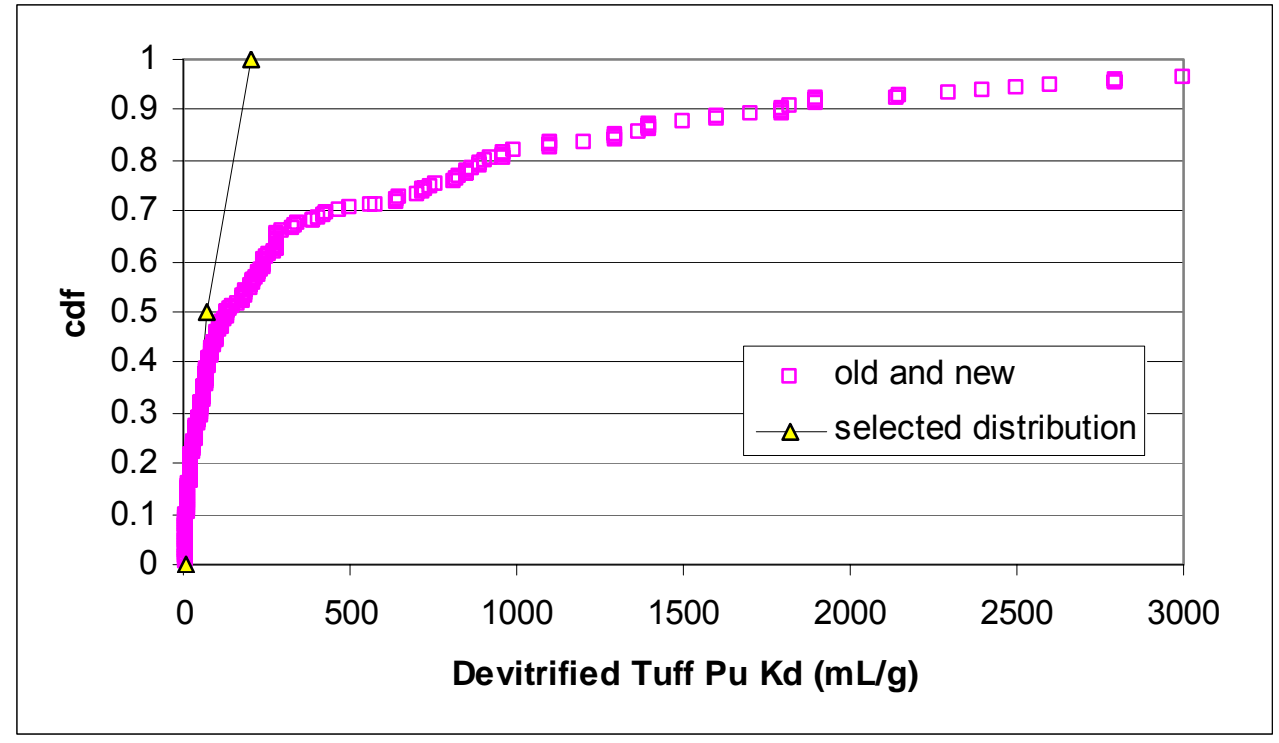

DTNs: New: LA0407AM831341.005 [DIRS 170625]; Old: LA0305AM831341.001 [DIRS 163789].

Figure A-33b. Empirical Cumulative Distribution for Plutonium Sorption Coefficients on Devitrified Tuff

\section{A8.4.2 Zeolitic Tuff}

The experimentally derived sorption coefficients for plutonium on zeolitic tuff are plotted against the calculated final plutonium concentrations in Figure A-34. As before, the data points are separated into groups on the basis of when the experiments were carried out (pre-1990 = "old" and post-1990 = "new"), water chemistry, experiment duration, and whether the sorption 
coefficient was determined from a sorption or a desorption experiment. The plotted data indicate that there is no clear trend of plutonium $\mathrm{K}_{d}$ with solution concentration. Most of the data from sorption experiments plot between sorption coefficient values of 100 and $1,000 \mathrm{~mL} / \mathrm{g}$. A series of "old" experiments at concentrations between $10^{-7}$ and $10^{-9} \mathrm{M} / \mathrm{L}$ yielded sorption coefficient values less than $100 \mathrm{~mL} / \mathrm{g}$. However, the same rock type when used in "new" experiments yielded a series of sorption coefficient values greater than $100 \mathrm{~mL} / \mathrm{g}$. Why there is a difference of almost a factor of ten between these two sets of data is not known with certainty. Part of the answer lies in the fact that, on average, the "old" data points represent shorter duration experiments than the "new" data points. However, other factors are likely involved. For example, the oxidation state of plutonium in the starting solution may play a part.

As with devitrified tuffs, the plutonium sorption coefficients for zeolitic tuff plotted in Figure A-34 reflect solutions that were undersaturated with the plutonium phase precipitated in the Nitsche et al. (1993 [DIRS 155218], p. 54; 1994 [DIRS 144515], p. 39) experiments.

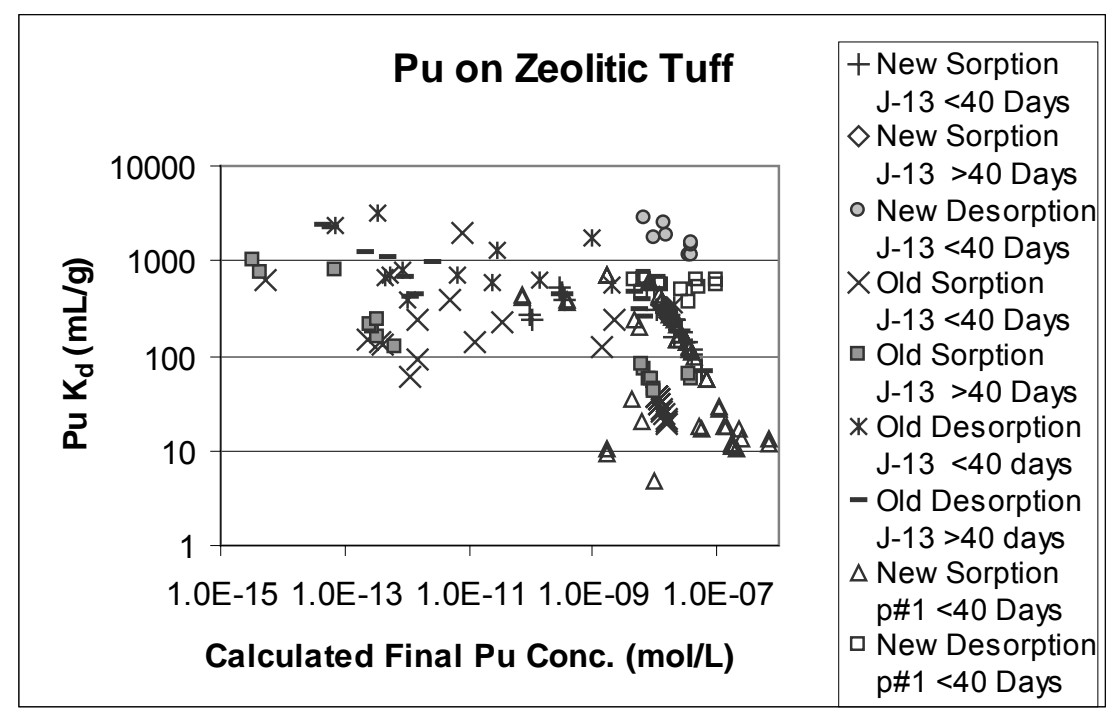

DTNs: New: LA0407AM831341.005 [DIRS 170625]; Old: LA0305AM831341.001 [DIRS 163789].

Figure A-34. Plutonium Sorption Coefficients on Zeolitic Tuff versus Calculated Final Plutonium Concentration in Solution

The effects of experiment duration on the plutonium $\mathrm{K}_{\mathrm{d}}$ for zeolitic tuff are shown in Figure A-35. As expected, the $K_{d}$ values for sorption experiments increase with increasing duration and the $\mathrm{K}_{\mathrm{d}}$ values for desorption experiments decrease with increasing duration, at least in short-term experiments. Interestingly, the decrease in longer-term desorption experiments is not very pronounced. The trends in the sorption and desorption data points suggest they would converge to values somewhere between 100 and $1,000 \mathrm{~mL} / \mathrm{g}$. This is similar to the range of values predicted for devitrified tuffs. Thus, the higher surface areas of zeolitic tuffs compared to devitrified tuffs (approximately 10 times) do not appear to result in higher sorption values. This effect was also observed by Pabalan et al. (1998 [DIRS 162987], p. 113) in experiments with uranium sorption on zeolite. The cause for the large range of values obtained at a given value for experiment duration is not known, but may largely result from variations in the oxidation state of 
plutonium in the starting solutions. However, variations in surface areas and surface chemistry among the samples used in the experiments must also contribute to the range observed.

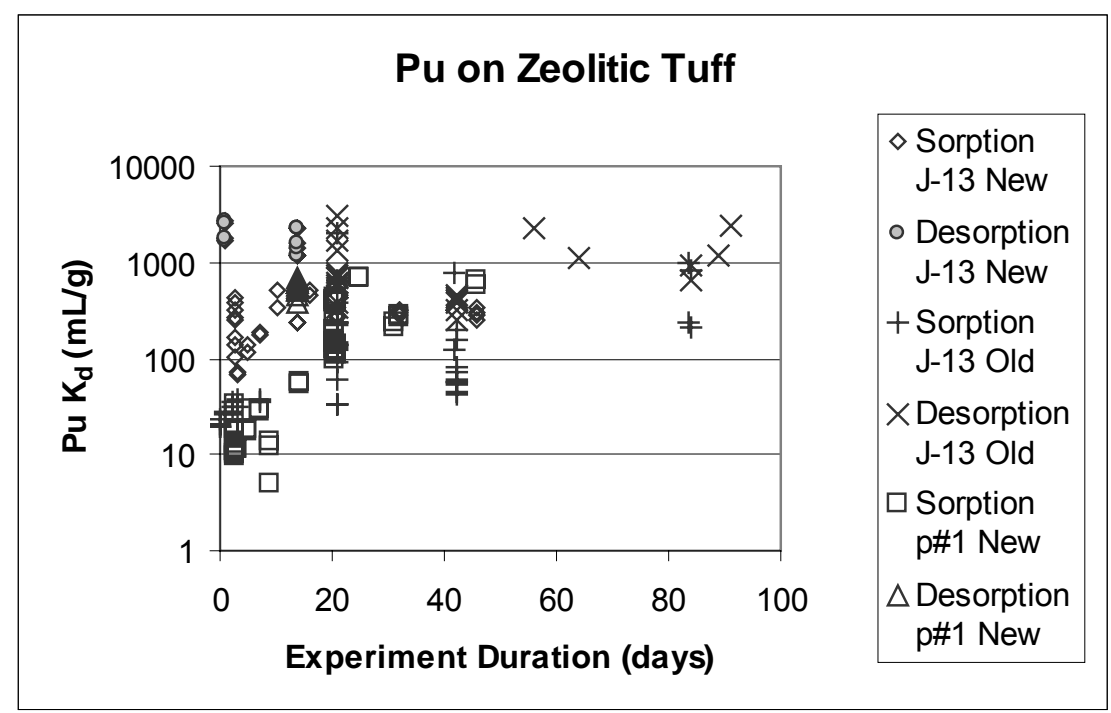

DTNs: New: LA0407AM831341.005 [DIRS 170625]; Old: LA0305AM831341.001 [DIRS 163789].

Figure A-35. Plutonium Sorption Coefficient on Zeolitic Tuff versus Experiment Duration for Sorption (Forward) and Desorption (Backward) Experiments

The impact of variations in $\mathrm{pH}$ on plutonium sorption coefficients on devitrified tuffs is shown in Figure A-36a. Although there is a lot of scatter in the data, there do not appear to be clear positive or negative trends among any of the data groupings.

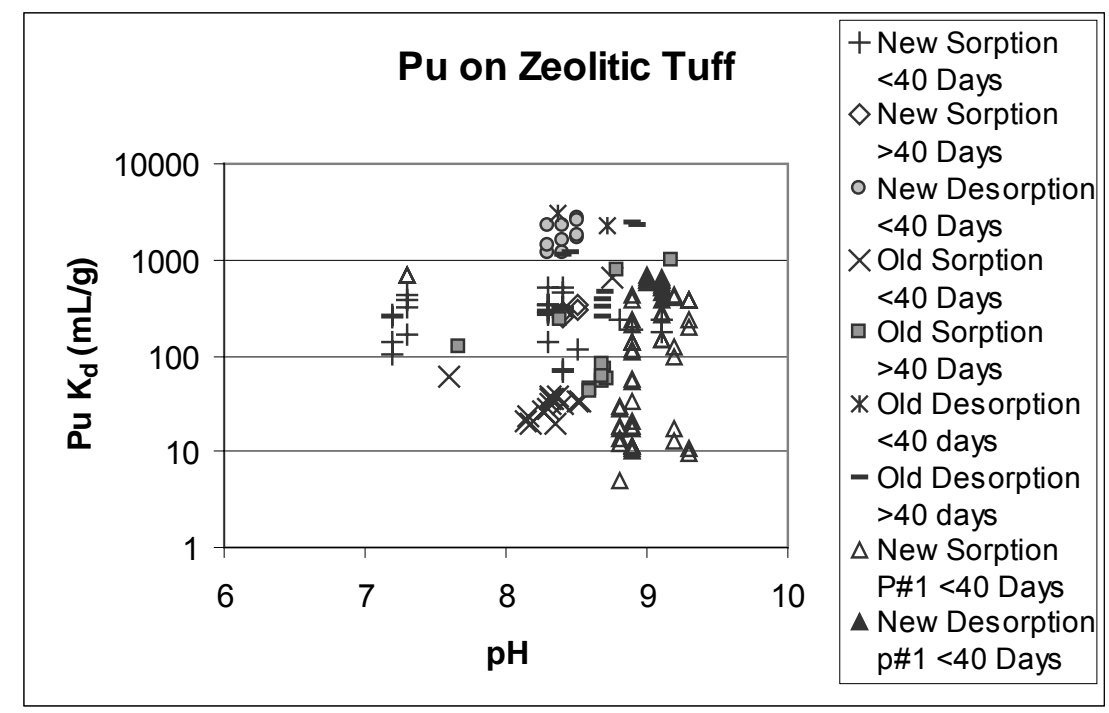

DTNs: New: LA0407AM831341.005 [DIRS 170625]; Old: LA0305AM831341.001 [DIRS 163789].

Figure A-36a. Plutonium Sorption Coefficient on Zeolitic Tuff in J-13 and Synthetic p\#1 versus Solution $\mathrm{pH}$ in Sorption (Forward) and Desorption (Backward) Experiments with Durations Greater than 40 Days 
The impact of variations in the major ion composition of groundwaters is also shown in Figure A-36a. Although there is a limited set of experiments with synthetic p\#1 water, the results are largely within the range of the results obtained in experiments with J-13 water. Thus, there is no clear evidence that water chemistry variations have an impact on plutonium sorption coefficients in devitrified tuff.

The plutonium sorption-coefficient probability distribution derived for zeolitic tuff in the UZ is a cumulative distribution, starting at $10 \mathrm{~mL} / \mathrm{g}$ at 0.0 with a value of $100 \mathrm{~mL} / \mathrm{g}$ at 0.5 and $200 \mathrm{~mL} / \mathrm{g}$ at 1.0 . The value at 0.5 was increased from $70 \mathrm{~mL} / \mathrm{g}$ for devitrified tuff to $100 \mathrm{~mL} / \mathrm{g}$ for zeolitic tuff to reflect the higher sorption coefficient values obtained with zeolitic tuff at lower $\mathrm{pH}$ values (7-7.5). Figure A-36b shows the empirical cumulative distribution for all the measured plutonium sorption coefficients on zeolitic tuff in the UZ with the selected distribution superimposed. As discussed in Section A6., not all the empirical data was equally weighted in selecting the probability distribution as the influence of expected variations in water chemistry, radionuclide concentrations, and variations in rock surface properties within each major rock type were incorporated in making the selection.

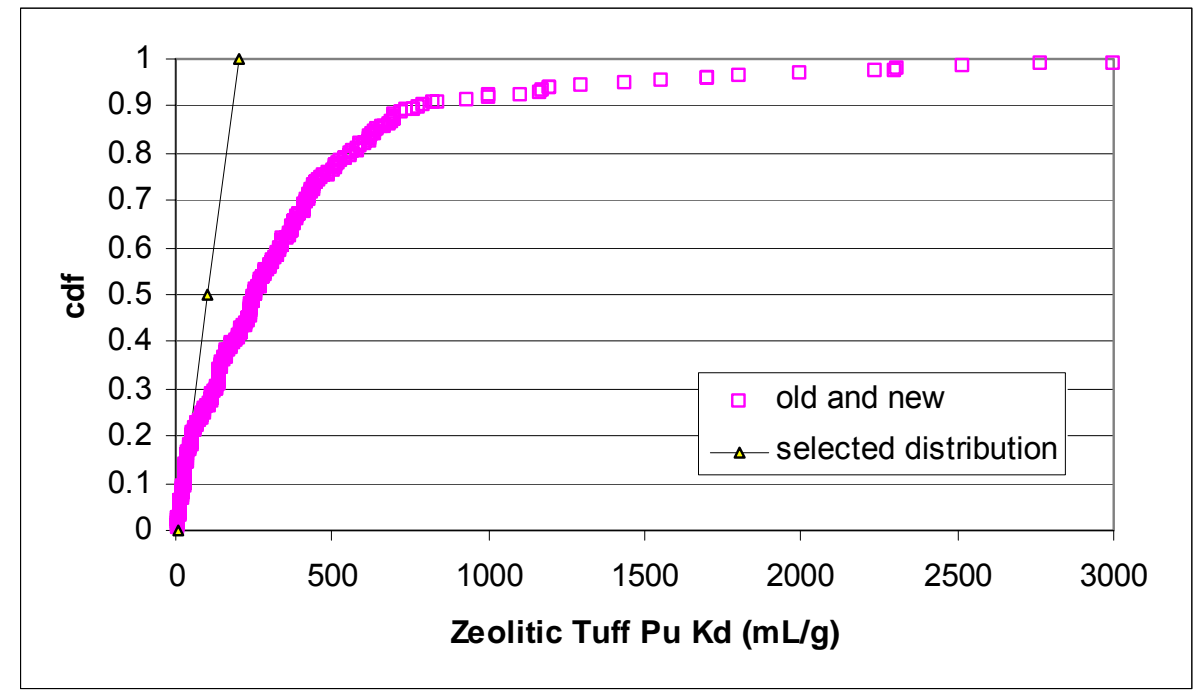

DTNs: New: LA0407AM831341.005 [DIRS 170625]; Old: LA0305AM831341.001 [DIRS 163789].

Figure A-36b. Empirical Cumulative Distribution for Plutonium Sorption Coefficients on Zeolitic Tuff

\section{A8.4.3 Vitric Tuff}

The experimentally derived sorption coefficients for plutonium on vitric tuff are plotted against the calculated final plutonium concentrations in Figure A-37. As before, the data points are separated into groups on the basis of when the experiments were carried out (pre-1990 = "old" and post-1990 = "new"), water chemistry, experiment duration, and on whether the sorption coefficient was determined from a sorption or a desorption experiment. The plotted data indicate that there is no clear trend of plutonium $\mathrm{K}_{d}$ with final solution concentration. As with devitrified tuffs, the plutonium sorption coefficients for vitric tuff plotted in Figure A-37 reflect solutions that were undersaturated with respect to the plutonium phase precipitated in the Nitsche et al. (1993 [DIRS 155218], p. 54; 1994 [DIRS 144515], p. 39) experiments. 


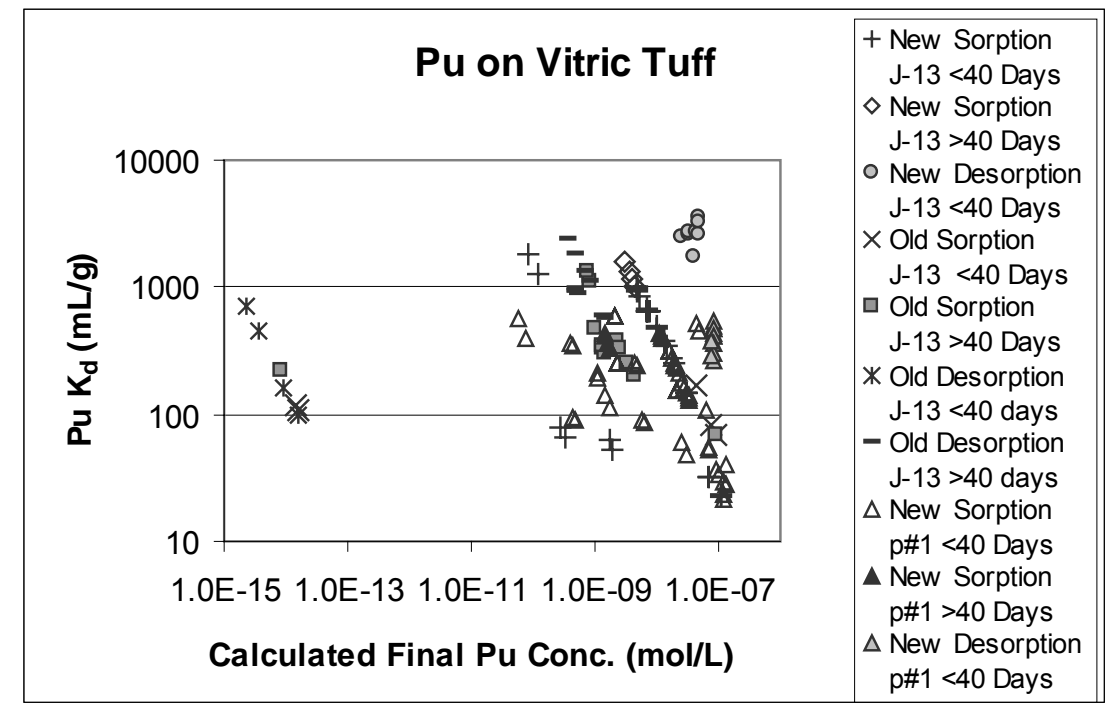

DTNs: New: LA0407AM831341.005 [DIRS 170625]; Old: LA0305AM831341.001 [DIRS 163789].

Figure A-37. Plutonium Sorption Coefficients on Vitric Tuff versus Calculated Final Plutonium Concentration in Solution

The data in Figure A-37 for experiments with synthetic p\#1 water plot in the middle of the data for experiments with J-13 water. Thus, there does not appear to be a significant impact of variations in water composition.

The effects of experiment duration on the plutonium $\mathrm{K}_{\mathrm{d}}$ for vitric tuff are shown in Figure A-38. As expected, the $K_{d}$ values for sorption experiments generally increase with increasing duration. The $\mathrm{K}_{\mathrm{d}}$ values for the "new" and "old" desorption experiments also increase with increasing duration, unlike the results for devitrified and zeolitic tuffs. However, as a group, the desorption results decrease with increasing duration. The trends in the sorption and desorption data points suggest they would converge to values somewhere between 100 and $1,000 \mathrm{~mL} / \mathrm{g}$. This is similar to the range of values predicted for devitrified and zeolitic tuffs. The cause for the large range of values obtained at a given value for experiment duration is not known, but may largely result from variations in the oxidation state of plutonium in the starting solutions in addition to analytical errors. Variations in surface areas and surface chemistry among the samples used in the experiments must also contribute to the range observed. 


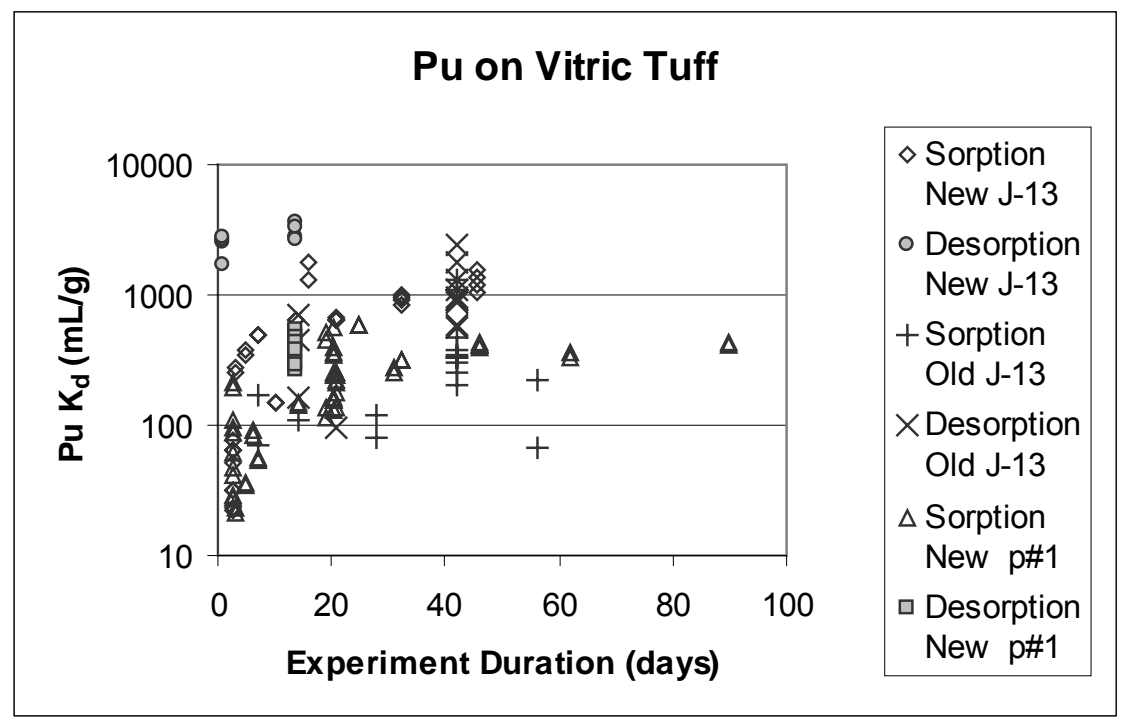

DTNs: New: LA0407AM831341.005 [DIRS 170625]; Old: LA0305AM831341.001 [DIRS 163789].

Figure A-38. Plutonium Sorption Coefficient on Vitric Tuff versus Experiment Duration for Sorption (Forward) and Desorption (Backward) Experiments

The impact that variations in $\mathrm{pH}$ have on plutonium sorption coefficients in vitric tuffs is shown in Figure A-39a. Although there is a lot of scatter in the data, there are no obvious trends among any of the data groupings.

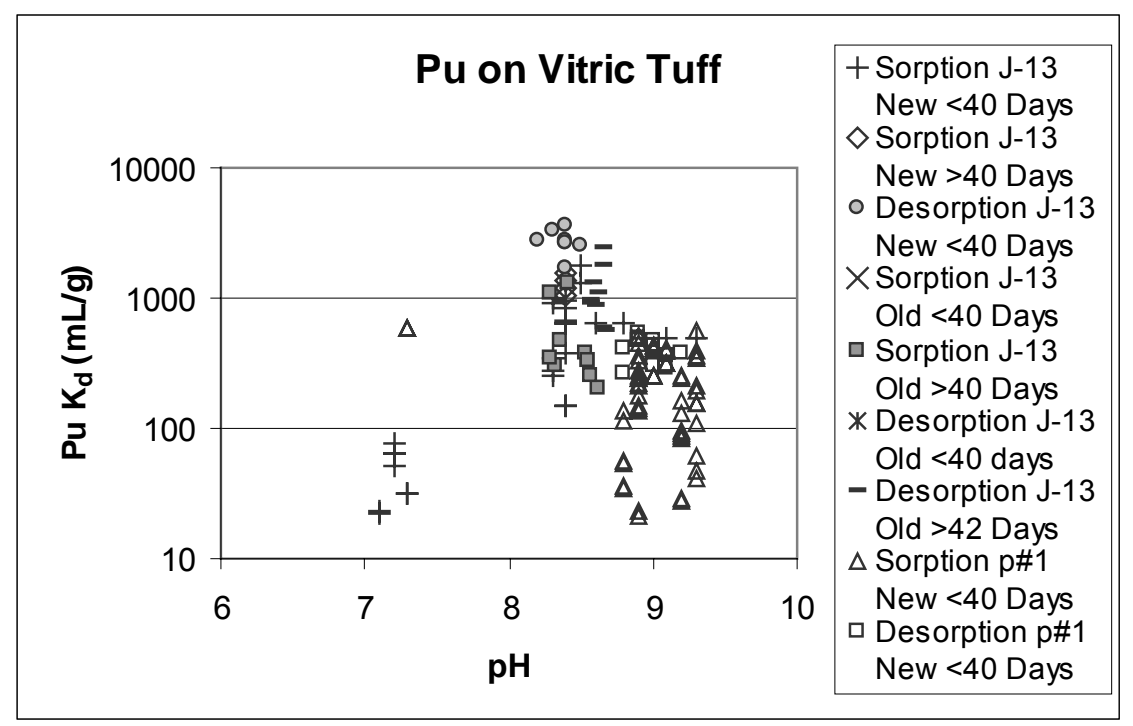

DTNs: New: LA0407AM831341.005 [DIRS 170625]; Old: LA0305AM831341.001 [DIRS 163789].

Figure A-39a. Plutonium Sorption Coefficient on Vitric Tuff in J-13 and Synthetic p\#1 versus Solution pH in Sorption (Forward) and Desorption (Backward) Experiments with Durations Greater than 40 Days 
The impact of variations in the major ion composition of groundwaters is also represented in Figures A-38 and A-39a. The available set of experiments with synthetic p\#1 water are within the range of the results obtained in experiments with J-13 water, although they tend to be at the lower end of this range.

The plutonium sorption-coefficient probability distribution selected for vitric tuff in the UZ is a cumulative distribution starting with a value of $10 \mathrm{~mL} / \mathrm{g}$ at 0.0 , increasing to a value of $100 \mathrm{~mL} / \mathrm{g}$ at 0.5 , and ending with a value of $200 \mathrm{~mL} / \mathrm{g}$ at 1.0 . The $10 \mathrm{~mL} / \mathrm{g}$ value is selected to compensate for the potential for fast transport through the UZ. The value of $100 \mathrm{~mL} / \mathrm{g}$ at 0.5 is intended to emphasize the median value among the sorption coefficients obtained in experiments with synthetic p\#1 water. The upper limit of $200 \mathrm{~mL} / \mathrm{g}$ was selected also to emphasize the $\mathrm{p \# 1}$ water chemistry. Figure A-39b shows the empirical cumulative distribution for all the measured plutonium sorption coefficients on vitric tuff in the UZ with the selected distribution superimposed. As discussed in Section A6., not all the empirical data was equally weighted in selecting the probability distribution as the influence of expected variations in water chemistry, radionuclide concentrations, and variations in rock surface properties within each major rock type were incorporated in making the selection.

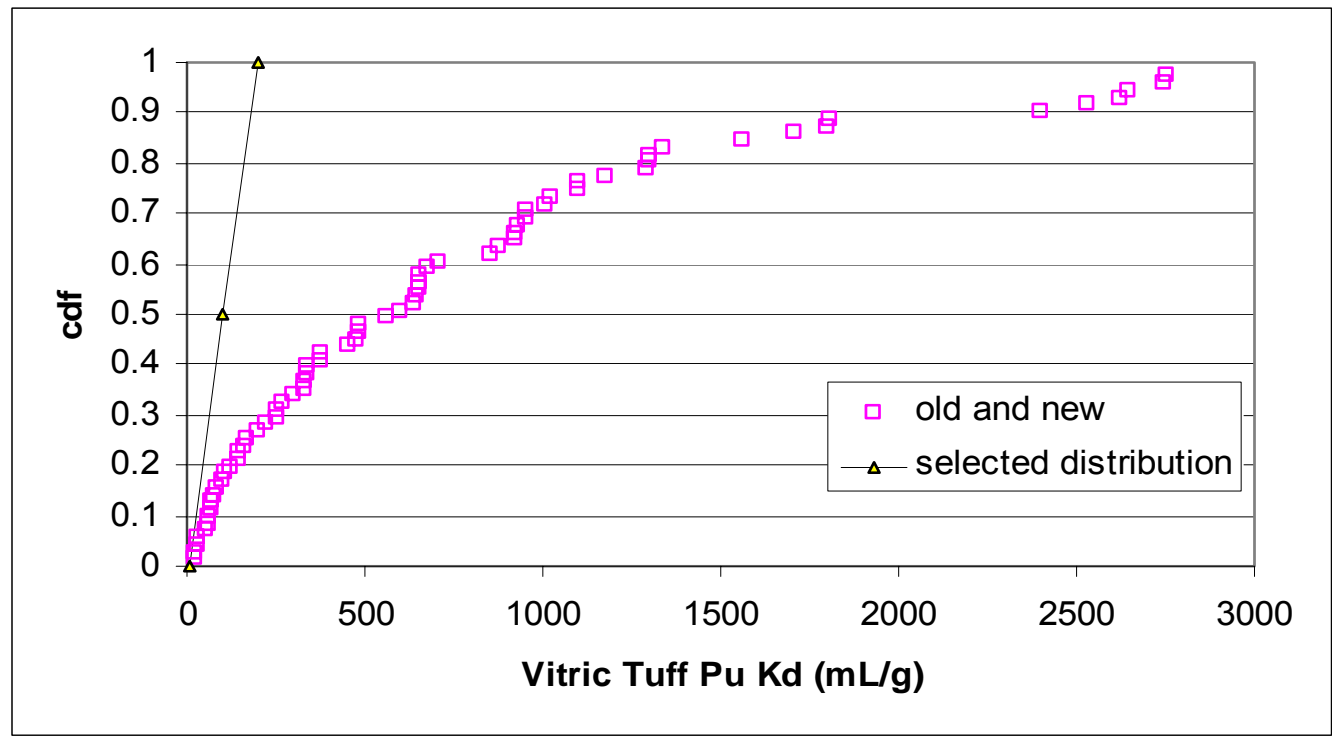

DTNs: New: LA0407AM831341.005 [DIRS 170625]; Old: LA0305AM831341.001 [DIRS 163789].

Figure A-39b. Empirical Cumulative Distribution for Plutonium Sorption Coefficients on Vitric Tuff

\section{A8.5 PROTACTINIUM}

The solubility for protactinium is very low in waters of the type found at Yucca Mountain. The best estimates of protactinium solubility in these waters range from $10^{-15}$ to $10^{-13} \mathrm{~mol} / \mathrm{L}$ (Berry et al. 1989 [DIRS 144728], p. 346). 


\section{A8.5.1 Devitrified Tuff}

The oxidation state of protactinium is +5 in groundwaters of the type found at Yucca Mountain (Cotton and Wilkinson 1988 [DIRS 105732], p. 1002). No sorption coefficient data have been obtained for protactinium on devitrified tuffs from Yucca Mountain. Allard et al. (1983 [DIRS 162982], p. 12) have reported protactinium sorption coefficient data for experiments with a silica sample having a surface area similar to that measured for devitrified tuffs. The solution composition used in the experiments was $0.01 \mathrm{M} \mathrm{NaClO}_{4}$. The initial protactinium concentration used in all experiments was $4.0 \times 10^{-12} \mathrm{~mol} / \mathrm{L}$. Allard's data indicate that protactinium sorption coefficients vary substantially (approximately 2 orders of magnitude) as a function of $\mathrm{pH}$ (as shown in Figure A-40). The cause for this variation in sorption coefficients with $\mathrm{pH}$ is unknown. Over the $\mathrm{pH}$ range expected in unsaturated zone waters at Yucca Mountain (7-8.5), the sorption coefficients reported by Allard et al. (1983 [DIRS 162982], p. 12) range from approximately 7,500 to $20,000 \mathrm{~mL} / \mathrm{g}$. The results for 6-hour experiments were similar to the results for experiments lasting up to 6 weeks. Thus, sorption kinetics for protactinium sorption reactions appear to be fast. Allard et al. (1983 [DIRS 162982], p. 12) reported results for alumina that were very similar to the results they reported for silica.

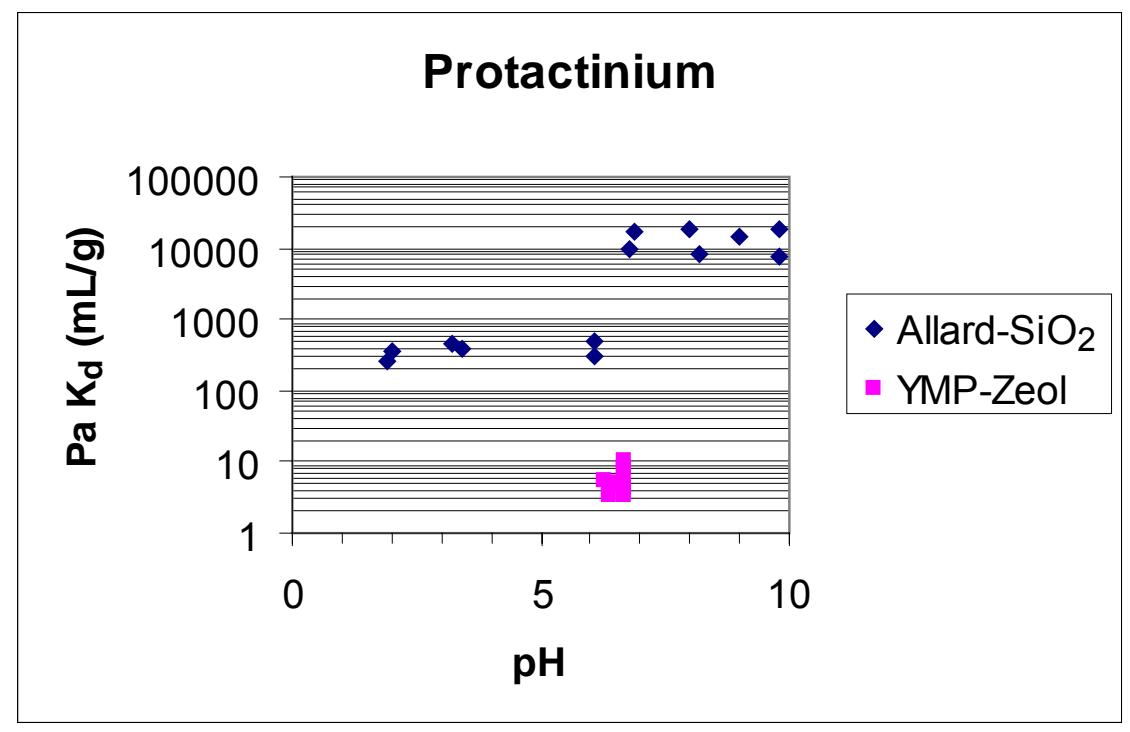

DTN: LA0305AM831341.001 [DIRS 163789].

Figure A-40. Protactinium Sorption Coefficients versus $\mathrm{pH}$

Hydrolysis reactions appear to dominate the solution chemistry of protactinium. The hydrolysis reactions of pentavalent protactinium in water are very complex even in relatively acidic solutions (Cotton and Wilkinson 1988 [DIRS 105732], p. 1002). In near-neutral solutions, the hydrolysis behavior of protactinium is essentially unknown. However, because hydrolysis reactions appear to dominate the solution behavior of protactinium, changes in the major ion chemistry of groundwaters are not expected to impact the sorption behavior of protactinium. Unfortunately, insufficient thermo-dynamic data are available to model protactinium sorption behavior using a surface complexation model. 
The sorption coefficient range for americium $(1,000$ to $10,000 \mathrm{~mL} / \mathrm{g})$ was used as a default for protactinium. This range is well within the range reported by Allard et al. (1983 [DIRS 162982], p. 12) for silica and alumina. As with americium sorption coefficients, a truncated normal distribution was selected. Corroboration of this range of sorption coefficient values is provided by protactinium sorption experiments performed by Berry et al. (1989 [DIRS 144728], p. 347). These authors report a range of 1,000 to $1,000,000 \mathrm{~mL} / \mathrm{g}$ in protactinium sorption coefficients for rock samples including sandstone, shale, granite, and clay in contact with natural groundwaters. Since there are no sorption coefficient data for protactinium on devitrified tuffs from Yucca Mountain, there is no figure included for an empirical cumulative distribution.

\section{A8.5.2 Zeolitic Tuff}

Sorption coefficients for protactinium on zeolitic tuffs from Yucca Mountain in J-13 water were reported by Rundberg et al. (1985 [DIRS 101355], p. 63). The reported sorption coefficients ranged from 3.3 to $10.1 \mathrm{~mL} / \mathrm{g}$ (Figure A-40). The initial solution concentrations in the experiments ranged from $1 \times 10^{-11}$ to $5 \times 10^{-14}$ molar. The initial solution concentration used by Allard et al. (1983 [DIRS 162982], p. 6) was in the low end of this range. Thus, initial solution concentration does not appear to explain the difference between the Allard et al. (1983 [DIRS 162982], p. 12) results and the results on Yucca Mountain samples. It is possible that the results on Yucca Mountain samples reflect oversaturation due to co-precipitation of protactinium with some other easily hydrolyzed species.

The $\mathrm{pH}$ values of the final solutions in the experiments on Yucca Mountain samples were in the range 6.3 to 6.7. These $\mathrm{pH}$ values are below the range expected in unsaturated zone waters at Yucca Mountain. Thus, the reported sorption coefficients do not directly apply to conditions in the Yucca Mountain UZ. As shown in Figure A-40, there appears to be an adsorption edge at a $\mathrm{pH}$ close to 6.8, and sorption coefficients increase by approximately 2 orders of magnitude at $\mathrm{pH}>6.8$. The cause for this increase is not known, but is likely related to hydrolysis reactions.

Because zeolitic tuffs have greater surface area than devitrified tuffs, the sorption coefficient distribution for devitrified tuff is used as a default for zeolitic tuff. This will lead to conservative predictions of protactinium transport rates. Since the available sorption coefficient data for protactinium on zeolitic tuffs were measured with final solution concentrations at $\mathrm{pH}$ values below a range expected to apply to Yucca Mountain, there is no figure included for an empirical cumulative distribution.

\section{A8.5.3 Vitric Tuff}

No sorption coefficient data is available for vitric tuffs. Because vitric tuffs have surface areas similar to the surface areas of devitrified tuffs, the sorption coefficient probability distribution for devitrified tuffs is used for vitric tuffs. Since there are no sorption coefficient data on vitric tuffs, there is no figure included for an empirical cumulative distribution 


\section{A8.6 RADIUM}

The chemistry of radium is very similar to that of barium (Cotton and Wilkinson 1988 [DIRS 105732], p. 144). Because barium has a radioactive isotope more readily measured by gamma counting than the radium isotopes, barium was used to measure sorption coefficients for radium. A limited number of experiments were performed with radium to confirm its sorption behavior relative to barium.

Barite is the solubility-controlling solid for barium in Yucca Mountain groundwaters. The solubility of barite (barium sulfate) in $\mathrm{J}-13$ water at $25^{\circ} \mathrm{C}$ is $9.0 \times 10^{-7} \mathrm{~mol} / \mathrm{L}$ (DTN: LA0407AM831343.001 [DIRS 171009]). The solubility of barite in p\#1 water at $25^{\circ} \mathrm{C}$ is $2.0 \times 10^{-7} \mathrm{~mol} / \mathrm{L}$. At $25^{\circ} \mathrm{C}$, the solubility of radium sulfate is $3.2 \times 10^{-7} \mathrm{~mol} / \mathrm{L}$ in J-13 water.

\section{A8.6.1 Devitrified Tuff}

The experimentally derived sorption coefficients for barium on devitrified tuff are plotted against the calculated final barium concentrations of the experiments in Figure A-41. The data points are separated into groups on the basis of when the experiments were carried out (pre-1990 = "old" and post-1990 = "new"), water chemistry, experiment duration, and whether the sorption coefficient was determined from a sorption or a desorption experiment.

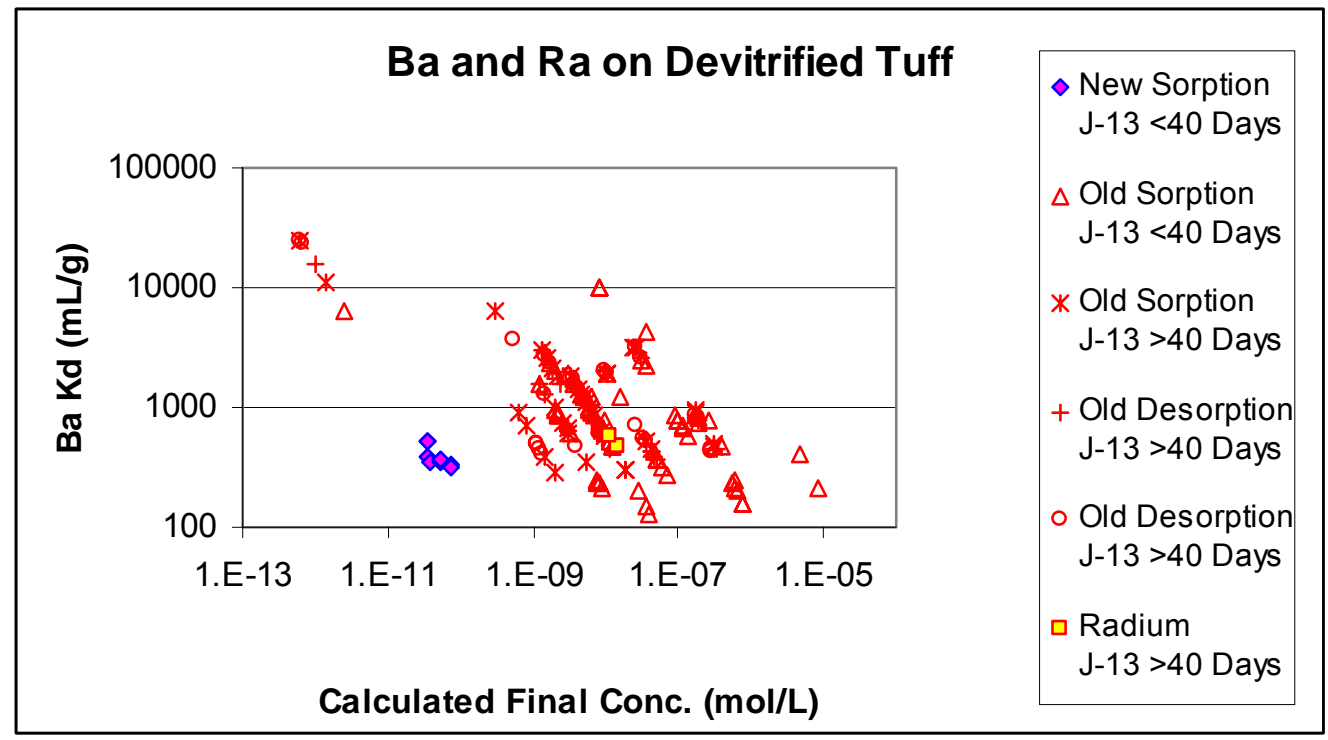

DTNs: New: LA0407AM831341.001 [DIRS 170623]; Old: LA0305AM831341.001 [DIRS 163789].

Figure A-41. Barium and Radium Sorption Coefficients on Devitrified Tuff versus Calculated Final Barium or Radium Concentrations in Solution

The calculated final concentration of barium in the experiments with $\mathrm{J}-13$ was below saturation with barite in all but one experiment (Figure A-41). The results for this one experiment will be omitted from further consideration. The radium solution concentrations were all lower than the radium sulfate saturation value in J-13. Thus, these experiments were undersaturated with radium sulfate. 
The data plotted in Figure A-41 show a general increase in sorption coefficient with decreasing concentration. This suggests that the isotherms for barium sorption onto devitrified tuff are nonlinear. The impact of this nonlinearity must be included in the sorption coefficient probability distribution.

The effects of experiment duration on the barium $K_{d}$ for devitrified tuff are shown in Figure A-42. The large range in sorption coefficients obtained at a given duration reflects variations in grain size of the crushed tuff samples used in the experiments, variations in solution concentrations, variations in surface chemistry, and analytical errors and artifacts. Experiments with crushed tuff samples that include the fines (e.g., less than $30 \mu \mathrm{m}$ ) often have sorption coefficients larger than samples from which the fines have been removed (e.g., 75 to $500 \mu \mathrm{m}$ ). This is partly due to the higher surface area of samples with fines and partly due to mineral fractionation. Mineral fractionation can occur during the sieving process and cause the preferential concentration of very fine grained minerals (e.g., clays) in the fine fraction.



DTNs: New: LA0407AM831341.001 [DIRS 170623]; Old: LA0305AM831341.001 [DIRS 163789].

Figure A-42. Barium and Radium Sorption Coefficients on Devitrified Tuff versus Experiment Duration for Sorption (Forward) and Desorption (Backward) Experiments

Figure A-43a shows the effects of experiment duration on sorption coefficients for samples with the fines removed. The few sorption coefficients with values near $10,000 \mathrm{~mL} / \mathrm{g}$ represent experiments with very low solution concentrations (Figure A-41). For the remaining experiments, the total range of sorption coefficient values is substantially reduced. Further, it appears likely the range would converge to a range between 100 and $1,000 \mathrm{~mL} / \mathrm{g}$ with increasing duration. The horizontal trend among data points at durations other than 21 and 42 days suggests that barium and radium sorption reactions are relatively fast. 


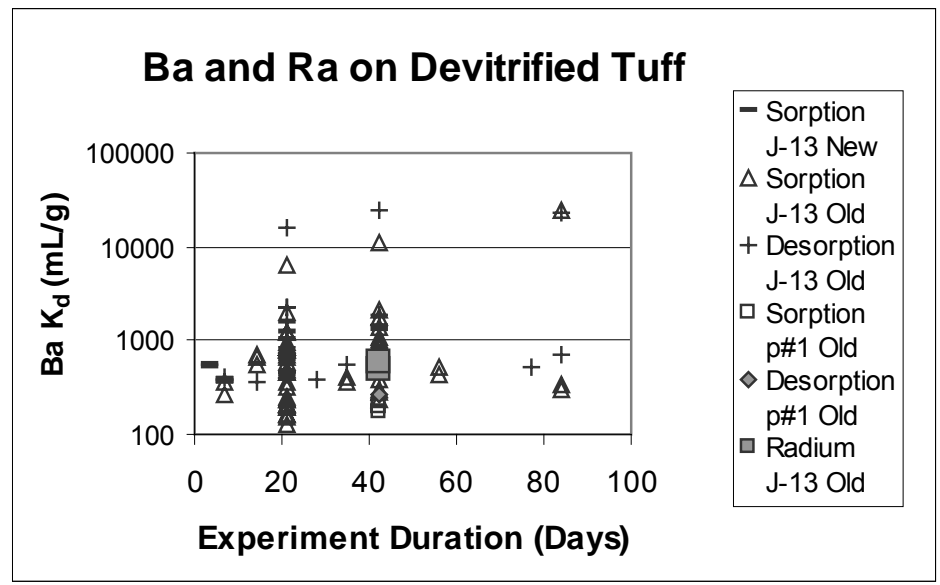

DTNs: New: LA0407AM831341.001 [DIRS 170623]; Old: LA0305AM831341.001 [DIRS 163789].

Figure A-43a. Barium and Radium Sorption Coefficients on Devitrified Tuff versus Experiment Duration for Sorption (Forward) and Desorption (Backward) Experiments

The impact of variations in the major ion composition of groundwaters is also shown in Figures A-42 and A-43a. Although there is a limited set of experiments with p\#1 water, the sorption coefficients obtained (at 42 days) fall at the low end of the range of results for J-13 water. Thus, there is some water-chemistry impact, although it is substantially less than an order of magnitude.

The radium sorption-coefficient probability distribution derived for devitrified tuff in the UZ is a uniform distribution with a range of $100-1,000 \mathrm{~mL} / \mathrm{g}$. The low end of the chosen range was selected based on the minimum value observed in long-term experiments (greater than 40 days), and the potential impact of variations in water chemistry and surface areas among devitrified tuffs at Yucca Mountain. Because there are experiments within the dataset that have solution concentrations close to saturation with a barium/radium sulfate, the effect of nonlinear isotherms is incorporated into the distribution. The upper end of the distribution was chosen as a minimum upper limit, given the potential impact of sorption kinetics, radium solution concentrations, and variation in surface areas. Figure A-43b shows the empirical cumulative distribution for all the measured barium sorption coefficients on devitrified tuff in the UZ with the selected distribution superimposed. As discussed in Section A6., not all the empirical data was equally weighted in selecting the probability distribution as the influence of expected variations in water chemistry, radionuclide concentrations, and variations in rock surface properties within each major rock type were incorporated in making the selection. 


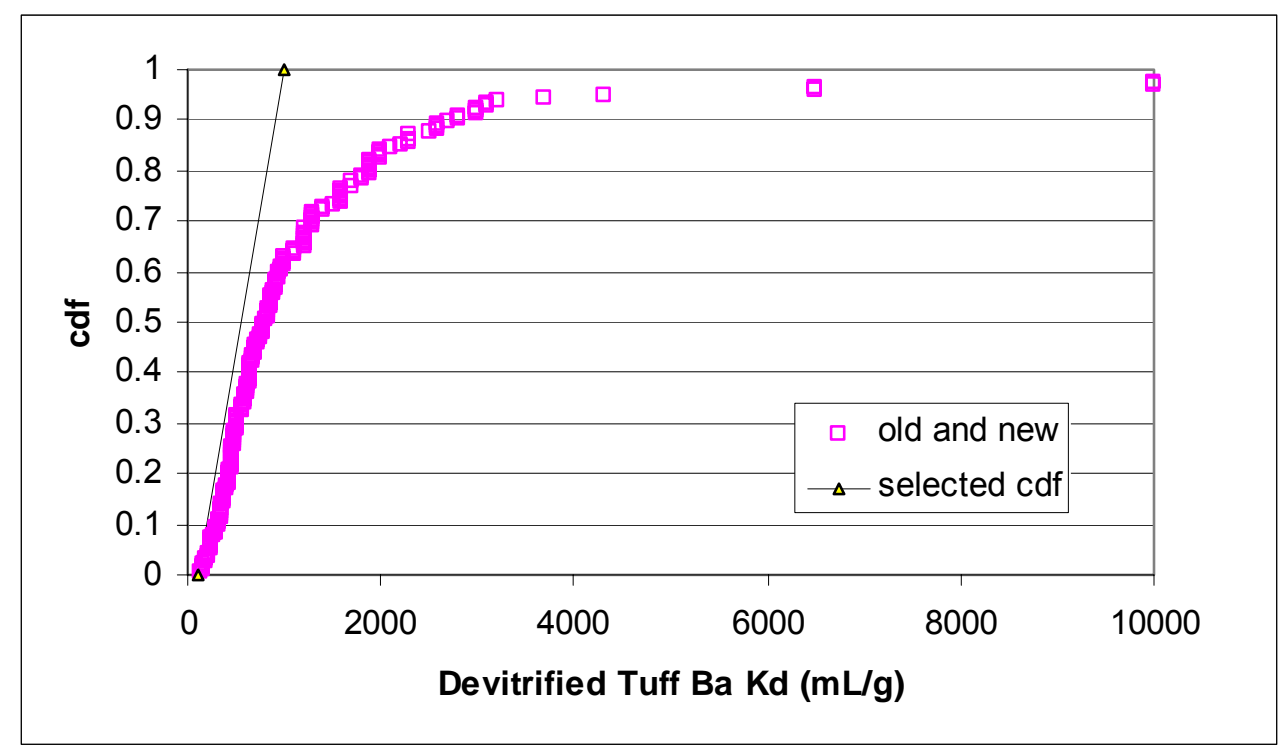

DTNs: New: LA0407AM831341.001 [DIRS 170623]; Old: LA0305AM831341.001 [DIRS 163789].

Figure A-43b. Empirical Cumulative Distribution for Barium Sorption Coefficients on Devitrified Tuff

\section{A8.6.2 Zeolitic Tuff}

The experimentally derived sorption coefficients for barium and radium on zeolitic tuff are plotted against the calculated final barium or radium concentrations in Figure A-44. As before, the data points are separated into groups on the basis of when the experiments were carried out (pre-1990 = "old" and post-1990 = "new"), experiment duration, and whether the sorption coefficient was determined from a sorption or a desorption experiment. The plotted data indicate that there is no clear trend of barium $\mathrm{K}_{\mathrm{d}}$ with solution concentration. Essentially all the sorption coefficients exceed a value of $10,000 \mathrm{~mL} / \mathrm{g}$, and some desorption experiments approach values of $1,000,000 \mathrm{~mL} / \mathrm{g}$. The radium results are in the range of the results for barium, confirming the similar sorption behavior of these two elements. As with devitrified tuffs, the barium and radium sorption coefficients for zeolitic tuff plotted in Figure A-44 reflect solutions that were undersaturated with barium and radium sulfate. The sorption coefficients obtained with $\mathrm{p} \# 1$ water are well within the range defined by the experiments with J-13 water. Thus, varying groundwater chemistry does not appear to have a clear impact. 


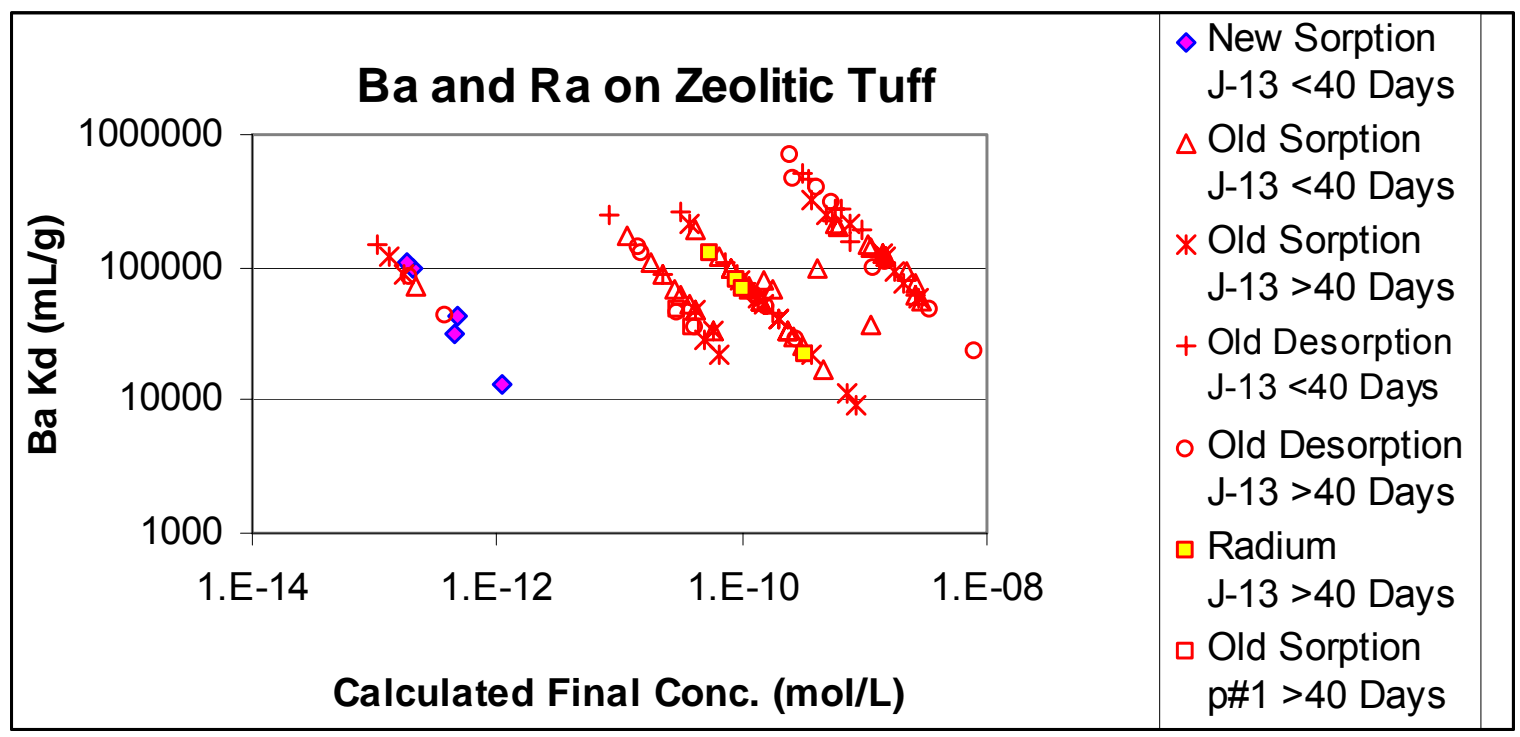

DTNs: New: LA0407AM831341.001 [DIRS 170623]; Old: LA0305AM831341.001 [DIRS 163789].

Figure A-44. Barium and Radium Sorption Coefficients on Zeolitic Tuff versus Calculated Final Barium or Radium Concentrations in Solution

Data plotted in Figure A-44 also indicate that there is no clear trend of barium $\mathrm{K}_{\mathrm{d}}$ with solution concentration. Data were obtained for a sorption isotherm on sample YM-38 (Figure A-45). As indicated by the exponent in the equation on the diagram, the calculated isotherm is essentially linear.

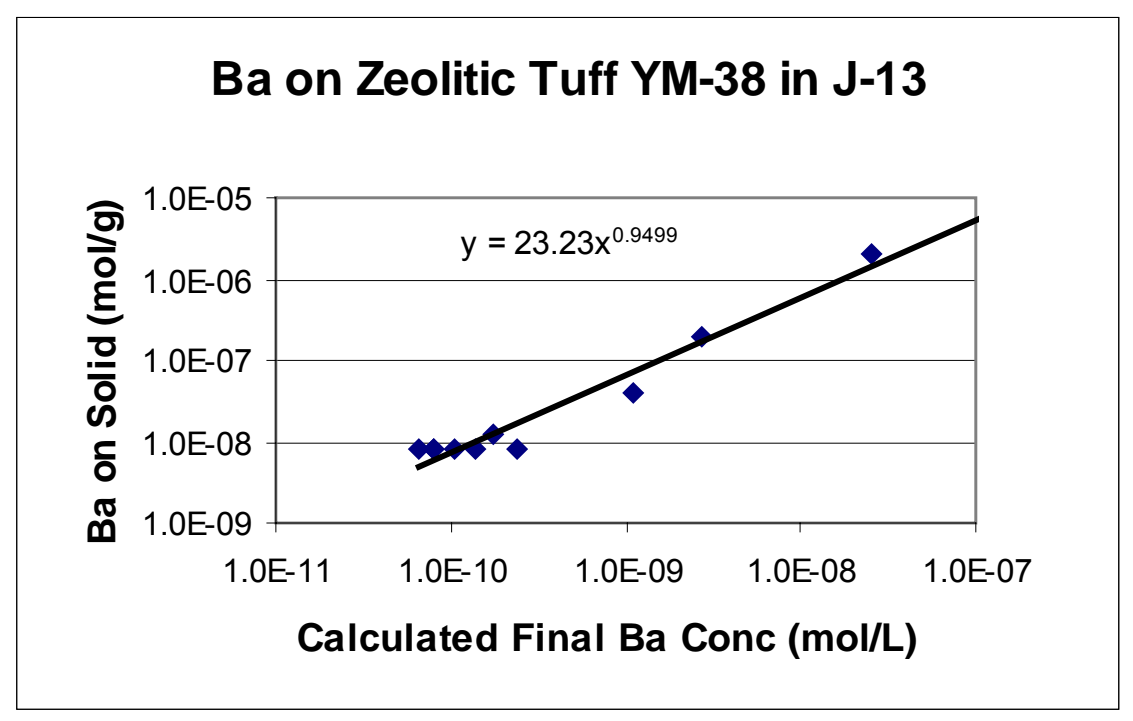

DTNs: Old: LA0305AM831341.001 [DIRS 163789].

Figure A-45. Isotherm Diagram for Ba Sorption on Zeolitic Tuff YM-38 in J-13 Water 
The effects of experiment duration on the barium $\mathrm{K}_{\mathrm{d}}$ for zeolitic tuff are shown in Figure A-46a. The barium and radium sorption reactions are quite fast, as indicated by the range of values obtained in 6-day experiments (Figure A-46a) being similar to the range of values obtained in 84 day experiments.

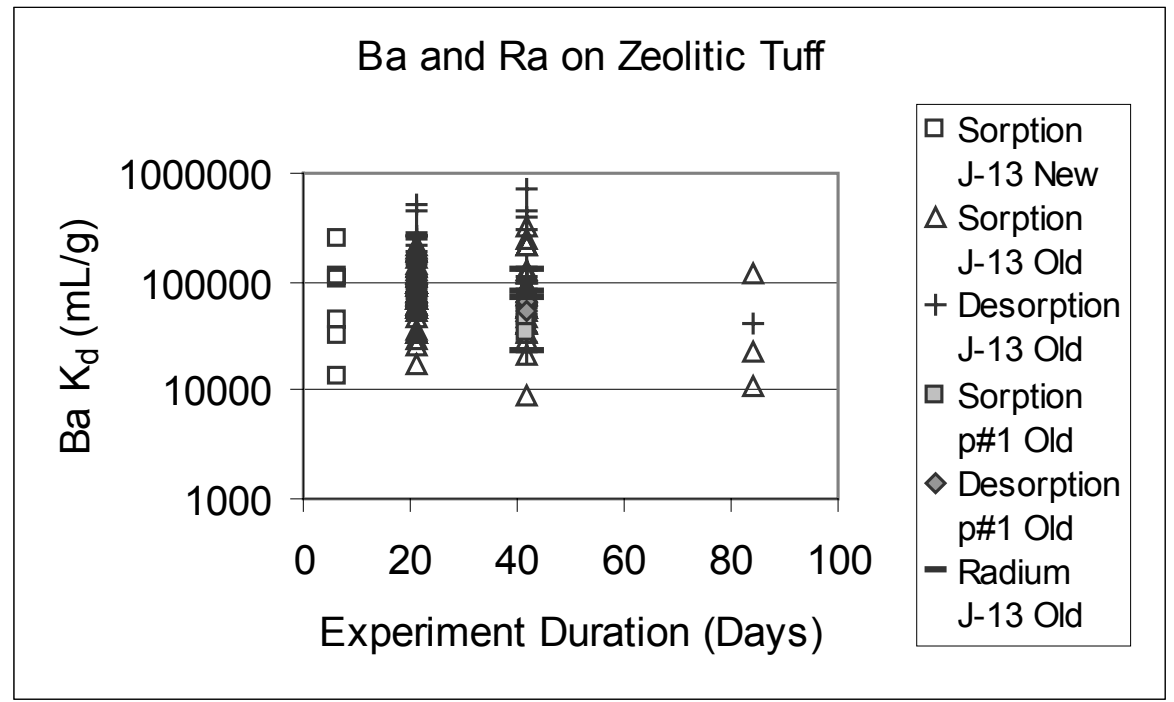

DTNs: New: LA0407AM831341.001 [DIRS 170623]; Old: LA0305AM831341.001 [DIRS 163789].

Figure A-46a. Barium and Radium Sorption Coefficient on Zeolitic Tuff in J-13 versus Experiment Duration for Sorption (Forward) and Desorption (Backward) Experiments

The impact of variations in the major ion composition of groundwaters is also shown in Figure A-46a. Although there is a limited set of experiments with synthetic p\#1 water, the results are largely within the range of the results obtained in experiments with J-13 water. Thus, there is no clear evidence of water-chemistry variations having an impact on barium/radium sorption coefficients in zeolitic tuff.

The radium sorption-coefficient probability distribution derived for zeolitic tuff in the unsaturated zone is a uniform distribution with a range of 1,000 to $5,000 \mathrm{~mL} / \mathrm{g}$. The low end of the chosen range was selected based on the possibility that at concentrations near the solubility limit, sorption coefficients may be lower than the minimum value observed in long-term experiments (i.e., isotherms may be nonlinear), and that rock chemistry $(\mathrm{Ca}+\mathrm{Mg} / \mathrm{Na}+\mathrm{K}$ in zeolites) may show greater variation than that represented among the samples used in the experiments. The upper end of the distribution was chosen as a minimum upper limit given the potential impacts of radium solution concentrations and variation in rock chemistry. Figure A-46b shows the empirical cumulative distribution for all the measured barium sorption coefficients on zeolitic tuff in the UZ with the selected distribution superimposed. As discussed in Section A6., not all the empirical data was equally weighted in selecting the probability distribution as the influence of expected variations in water chemistry, radionuclide concentrations, and variations in rock surface properties within each major rock type were incorporated in making the selection. 


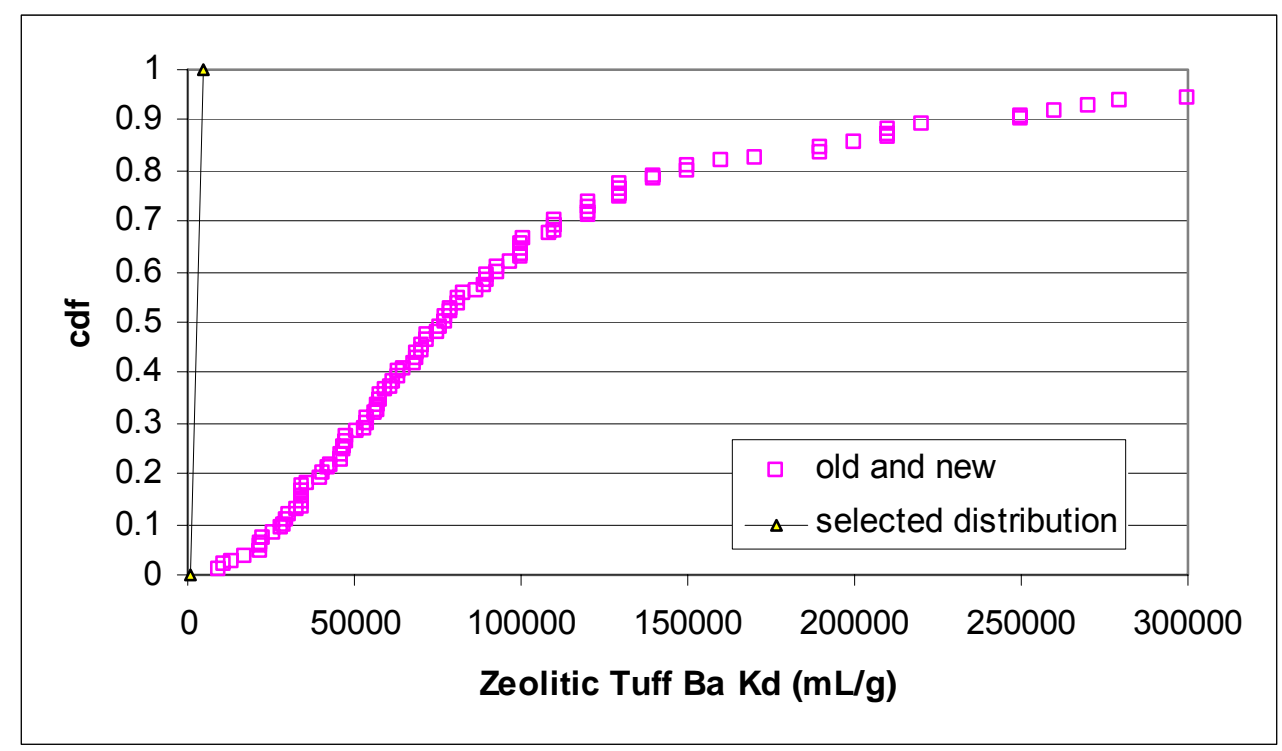

DTNs: New: LA0407AM831341.001 [DIRS 170623]; Old: LA0305AM831341.001 [DIRS 163789].

Figure A-46b. Empirical Cumulative Distribution for Barium Sorption Coefficients on Zeolitic Tuff

\section{A8.6.3 Vitric Tuff}

The experimentally derived sorption coefficients for barium and radium on vitric tuff are plotted against the calculated final barium or radium concentrations in Figure A-47. As before, the data points are separated into groups on the basis of when the experiments were carried out (pre-1990 = "old" and post-1990 = "new"), experiment duration, and on whether the sorption coefficient was determined from a sorption or a desorption experiment. Although there are a limited number of data points, the data plotted in Figure A-47 show a general increase in sorption coefficient with decreasing concentration. This suggests that the isotherms for barium sorption onto vitric tuff are nonlinear. The impact of this nonlinearity must be included in the sorption coefficient probability distribution.

The radium sorption coefficient values obtained are larger than the barium coefficients obtained at similar solution concentrations. This indicates that barium sorption coefficients provide a conservative estimate of radium sorption coefficients on vitric tuff. As with devitrified tuffs, the barium and radium sorption coefficients for vitric tuff plotted in Figure A-47 reflect solutions that were undersaturated with barium and radium sulfate. The sorption coefficients obtained with p\#1 water have values that are lower than those obtained in experiments with J-13 water. This indicates there is a groundwater composition effect for barium/radium sorption onto vitric tuff. 


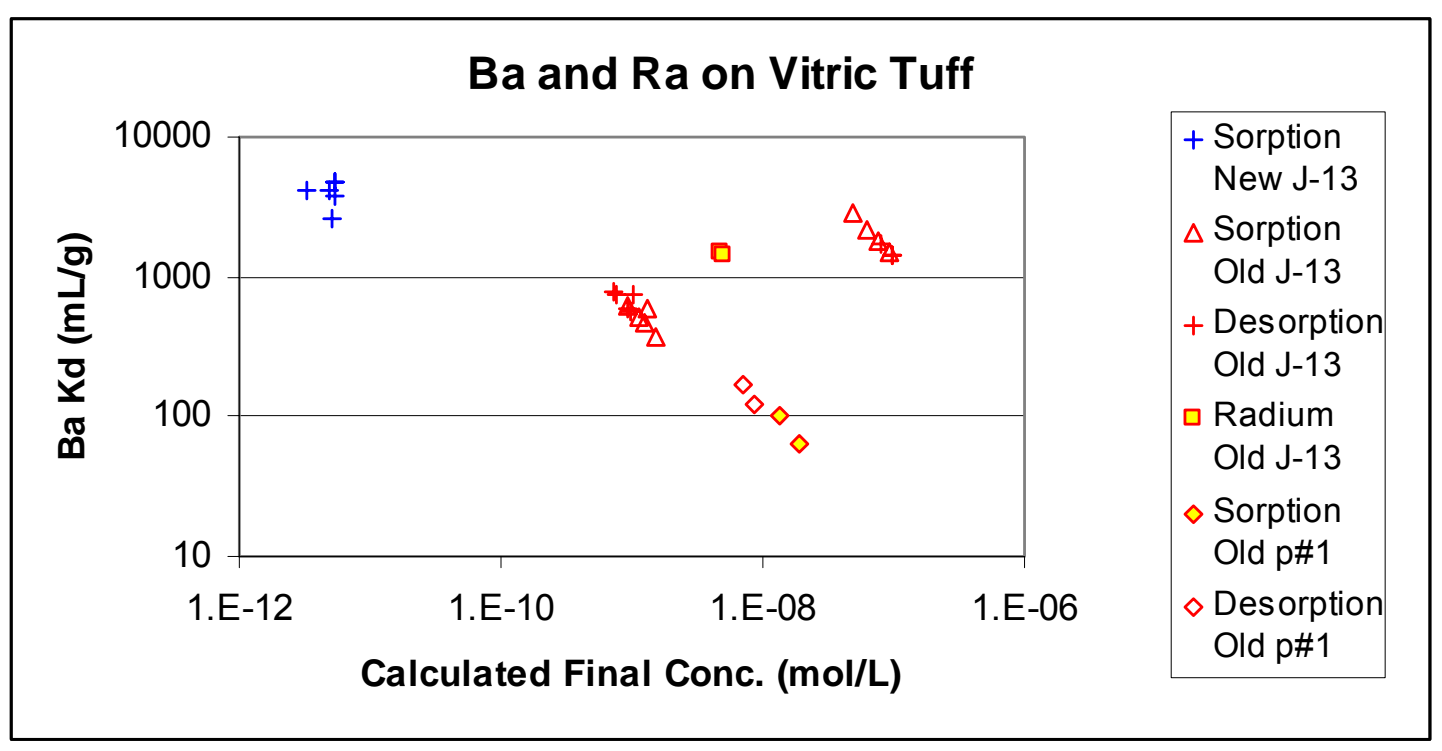

DTNs: New: LA0407AM831341.001 [DIRS 170623]; Old: LA0305AM831341.001 [DIRS 163789].

Figure A-47. Barium and Radium Sorption Coefficients on Vitric Tuff versus Calculated Final Barium or Radium Concentrations in Solution

The effects of experiment duration on the barium $\mathrm{K}_{\mathrm{d}}$ for vitric tuff are shown in Figure A-48a. The fact that the short-term experiments show higher sorption coefficient values than the longer-term experiments indicates that barium and radium sorption reactions on vitric tuff are fast.

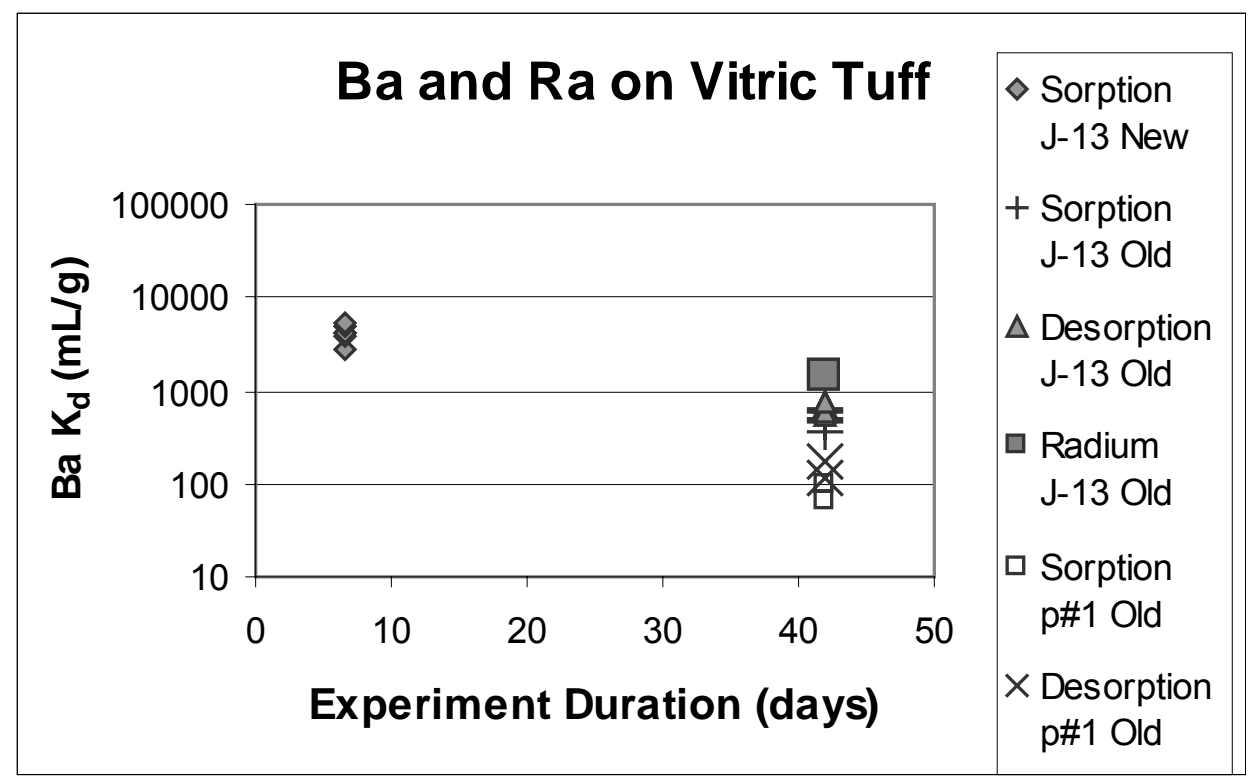

DTNs: New: LA0407AM831341.001 [DIRS 170623]; Old: LA0305AM831341.001 [DIRS 163789].

Figure A-48a. Barium and Radium Sorption Coefficient on Vitric Tuff versus Experiment Duration for Sorption (Forward) and Desorption (Backward) Experiments 
The impact of variations in the major ion composition of groundwaters are also shown in Figure A-48a. Although there is a limited set of experiments with synthetic p\#1 water, the sorption coefficient values obtained are clearly below the range of the values obtained in experiments with $\mathrm{J}-13$ water. Thus, there is evidence that water chemistry variations have an impact on barium sorption coefficients in devitrified tuff.

The radium sorption-coefficient probability distribution derived for vitric tuff in the UZ is a uniform distribution with a range from 50 to $600 \mathrm{~mL} / \mathrm{g}$. The value of $50 \mathrm{~mL} / \mathrm{g}$ was derived based on experiments with $\mathrm{p} \# 1$ water at final solution concentrations near saturation with barite. The value of $600 \mathrm{~mL} / \mathrm{g}$ was derived based on the potential impact of more dilute water chemistry (J-13-like) combined with the potential for radium concentrations near the solubility limit. Figure A-48b shows the empirical cumulative distribution for all the measured barium sorption coefficients on vitric tuff in the UZ with the selected distribution superimposed. As discussed in Section A6., not all the empirical data was equally weighted in selecting the probability distribution as the influence of expected variations in water chemistry, radionuclide concentrations, and variations in rock surface properties within each major rock type were incorporated in making the selection.

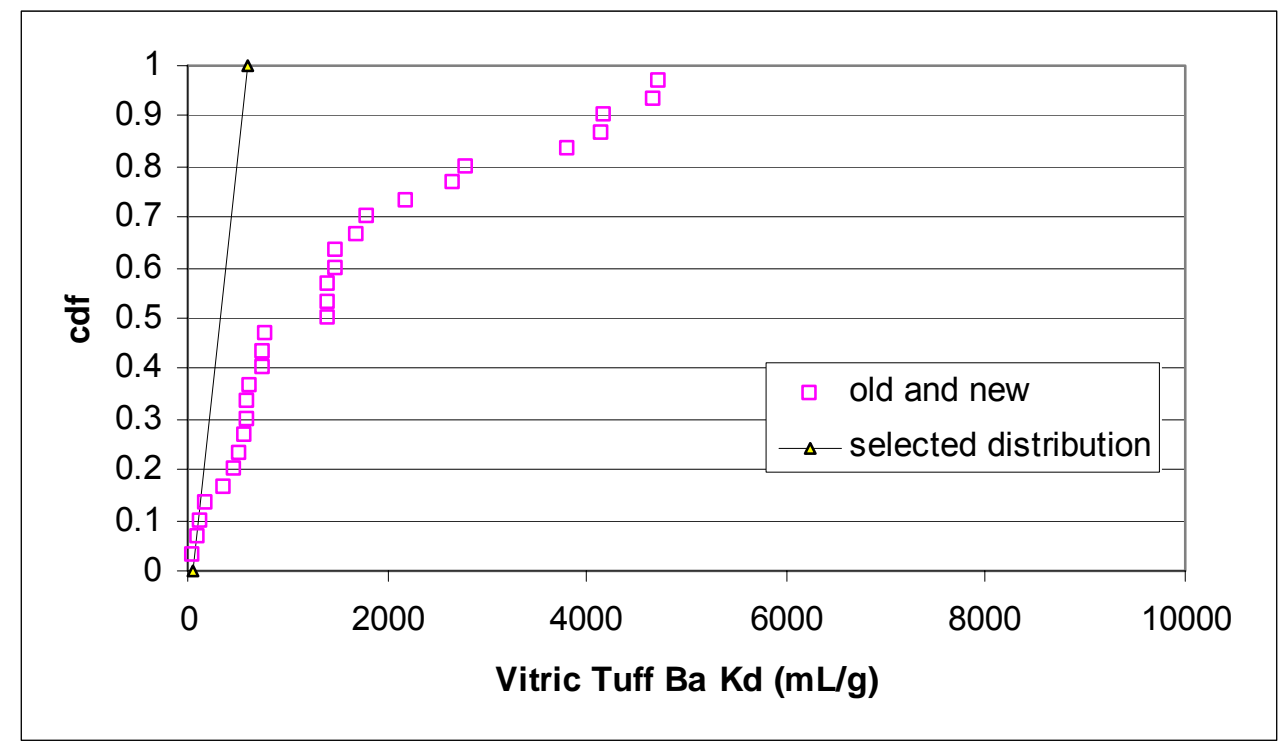

DTNs: New: LA0407AM831341.001 [DIRS 170623]; Old: LA0305AM831341.001 [DIRS 163789].

Figure A-48b. Empirical Cumulative Distribution for Barium Sorption Coefficients on Vitric Tuff

\section{A8.7 STRONTIUM}

The solubility of strontium in $\mathrm{J}-13$ water at $25^{\circ} \mathrm{C}$ ranges from $2.7 \times 10^{-5} \mathrm{~mol} / \mathrm{L}$ at $\mathrm{pH}=7.1$ to $1.4 \times 10^{-6} \mathrm{~mol} / \mathrm{L}$ at $\mathrm{pH}=8.5$ and the solubility-controlling phase is strontianite (strontium carbonate). The solubility of strontianite in $\mathrm{p} \# 1$ water at $25^{\circ} \mathrm{C}$ ranges from $2.4 \times 10^{-5} \mathrm{~mol} / \mathrm{L}$ at $\mathrm{pH}=6.9$ to $9.9 \times 10^{-7} \mathrm{~mol} / \mathrm{L}$ at $\mathrm{pH}=8.6$ (see DTN: LA0407AM831343.001 [DIRS 171009]). 


\section{A8.7.1 Devitrified Tuff}

The experimentally derived sorption coefficients for strontium on devitrified tuff are plotted against the calculated final strontium concentrations of the experiments in Figure A-49. The data points are separated into groups on the basis of when the experiments were carried out (pre-1990 = "old" and post-1990 = "new"), water type, and whether the sorption coefficient was determined from a sorption or a desorption experiment. There is also data for a sorption isotherm on sample G1-2840.

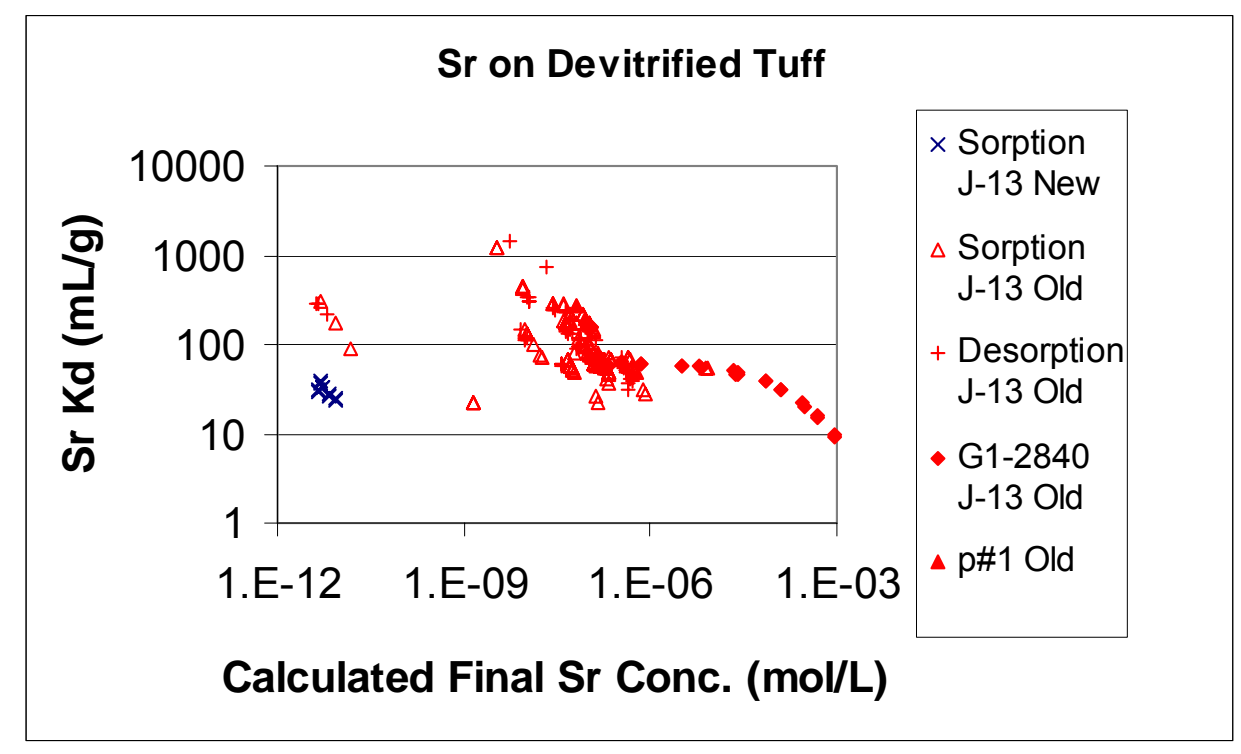

DTNs: New: LA0407AM831341.003 [DIRS 170626]; Old: LA0305AM831341.001 [DIRS 163789].

Figure A-49. Strontium Sorption Coefficients on Devitrified Tuff versus Calculated Final Strontium Concentration in Solution

Most of the calculated final solution concentrations for the sorption experiments are nearly all below saturation with strontianite in J-13. The data points with concentrations greater than $1.0 \times 10^{-6} \mathrm{~mol} / \mathrm{L}$ reflect experiments that were oversaturated with strontianite. Thus, the sorption coefficients obtained in these five experiments will not be used in the derivation of the strontium sorption coefficient probability distribution. The calculated final strontium concentrations used in experiments with $\mathrm{p} \# 1$ are all lower than the saturation value. Thus, oversaturation was not an issue in the strontium sorption experiments with $\mathrm{p} \# 1$ water.

A sorption isotherm was obtained for sample G1-2840 in J-13 water. The isotherm is linear below a strontium- 85 concentration approximately $5 \times 10^{-5} \mathrm{~mol} / \mathrm{L}$, as shown in Figure A-49. At higher concentrations, the final solutions were oversaturated with strontianite. Thus, these data points should not be used to construct an isotherm. Interestingly, if strontianite had precipitated in these experiments, it would be expected that the sorption coefficients would increase with increasing strontium concentrations. However, the data in Figure A-49 indicate sorption coefficients decreased with increasing solution concentrations. This suggests strontianite was not precipitated from solution for some reason. A likely explanation is that the nucleation or precipitation rates of strontianite are very slow relative to the duration of the experiments 
(21 days). Alternatively, strontianite was nucleated but was sufficiently fine grained so that it remained with the solution phase during the process used to separate the solid and liquid phases in the experiments.

The $\mathrm{K}_{\mathrm{d}}$ value in the linear portion of the isotherm in Figure A-49 is at the low end of the range of strontium sorption coefficients obtained with $\mathrm{J}-13$ and p\#1 waters. This suggests isotherms for the other samples plotted in Figure A-49 would lie at higher $\mathrm{K}_{d}$ values compared to the isotherm for G1-2840. The sorption coefficients obtained in experiments with $\mathrm{p} \# 1$ water fall in the middle of the range of values obtained for experiments with J-13 water. Thus, there is little or no impact of variations in water chemistry on strontium sorption coefficients on devitrified tuff.

The effects of experiment duration on the strontium $\mathrm{K}_{\mathrm{d}}$ for devitrified tuff are shown in Figure A-50. The large range in sorption coefficients obtained at a given duration reflects variations in grain size of the crushed tuff samples used in the experiments, variations in solution strontium concentrations, variations in surface chemistry, and analytical error and artifacts. Experiments with crushed tuff samples that include the fines (e.g., particle size less than $30 \mu \mathrm{m}$ ) usually have sorption coefficients that are larger than samples from which the fines have been removed (e.g., particle sizes 75 to $500 \mu \mathrm{m}$ ). This is partly caused by the higher surface area of samples with fines and partly by mineral fractionation. Mineral fractionation can occur during the sieving process and cause the preferential concentration of very fine grained minerals (e.g., clays) in the fine fraction.

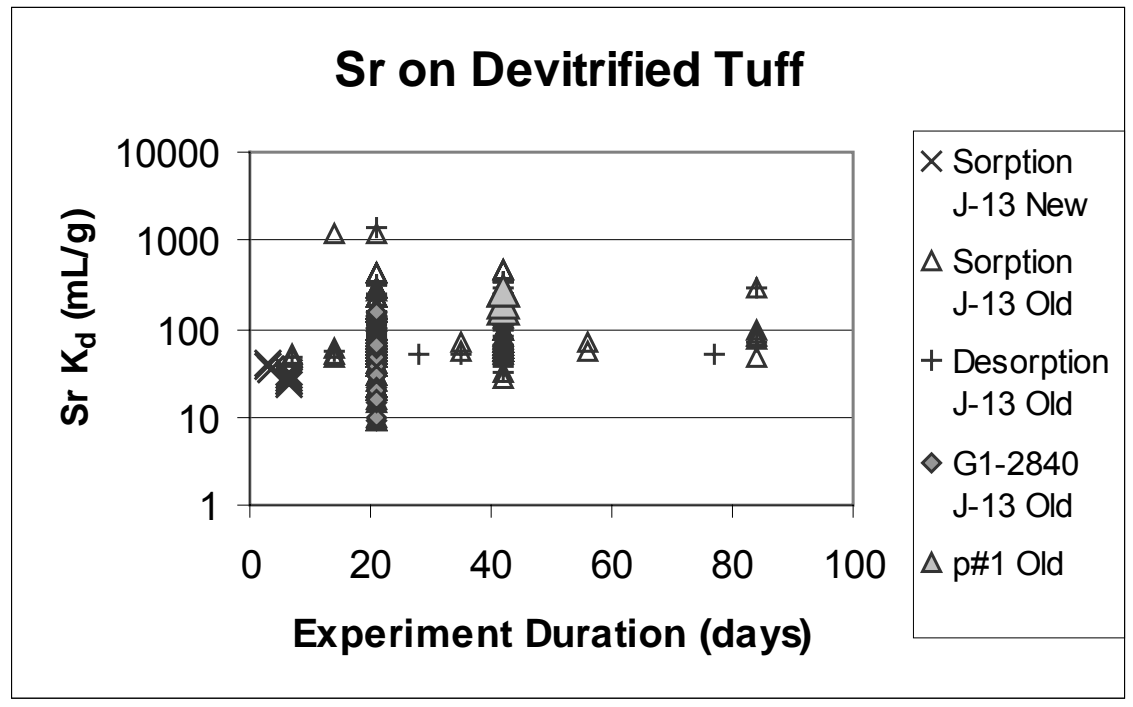

DTNs: New: LA0407AM831341.003 [DIRS 170626]; Old: LA0305AM831341.001 [DIRS 163789].

NOTE: Data points include experiments in which fines were not removed from the sample.

Figure A-50. Strontium Sorption Coefficients on Devitrified Tuff versus Experiment Duration for Sorption (Forward) and Desorption (Backward) Experiments 
Figure A-51a shows the effects of duration on sorption coefficients for crushed tuff samples with the fines removed. The total range of sorption coefficient values is reduced compared to Figure A-50. Further, it appears likely this range would converge to a range between 50 and $500 \mathrm{~mL} / \mathrm{g}$ with increasing duration. The horizontal trend among data points at durations other than 21 and 42 days suggests strontium sorption reactions are relatively fast.

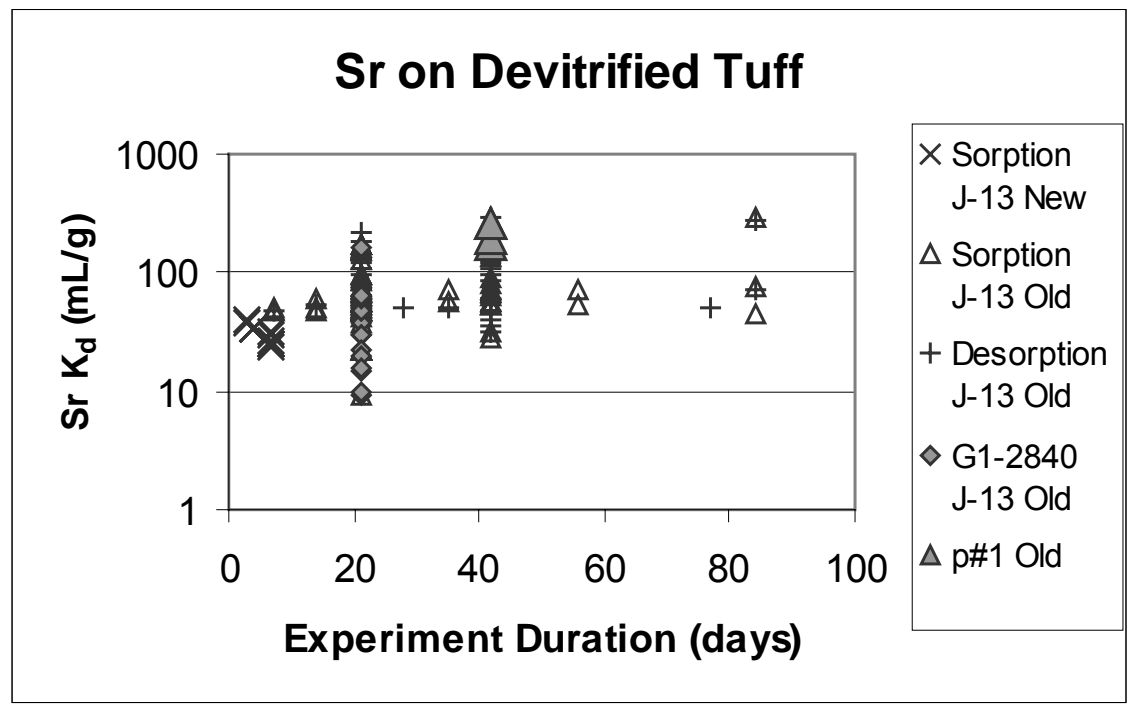

DTNs: New: LA0407AM831341.003 [DIRS 170626]; Old: LA0305AM831341.001 [DIRS 163789].

NOTE: Fines fraction is removed from all experiments.

Figure A-51a. Strontium Sorption Coefficients on Devitrified Tuff versus Experiment Duration for Sorption (Forward) and Desorption (Backward) Experiments

The strontium sorption coefficient probability distribution derived for devitrified tuff in the unsaturated zone is a uniform distribution with a range of $10-70 \mathrm{~mL} / \mathrm{g}$. The low end of the chosen range was selected based on the minimum value observed in isotherm experiments. Because the minimum value observed in isotherm experiments corresponds to solution concentrations close to saturation with a strontium carbonate, the effect of nonlinear isotherms is incorporated into the distribution. The upper end of the distribution was chosen as a minimum upper limit, given the potential impact of natural strontium in pore waters. Natural strontium concentrations measured in pore waters can be as high as $4.7 \times 10^{-5} \mathrm{~mol} / \mathrm{L}$ but the average is closer to $1.1 \times 10^{-5} \mathrm{~mol} / \mathrm{L}$ (DTN: GS020408312272.003 [DIRS 160899]). The isotherm shown in Figure A-49 would indicate a sorption coefficient of 50 to $70 \mathrm{~mL} / \mathrm{g}$ at this concentration. The high end of this range was chosen for the upper limit of the distribution. A uniform distribution was chosen to equally weight the sorption coefficient values in the selected range. Figure A-51b shows the empirical cumulative distribution for all the measured strontium sorption coefficients on devitrified tuff in the UZ with the selected distribution superimposed. As discussed in Section A6., not all the empirical data was equally weighted in selecting the probability distribution as the influence of expected variations in water chemistry, radionuclide concentrations, and variations in rock surface properties within each major rock type were incorporated in making the selection. 


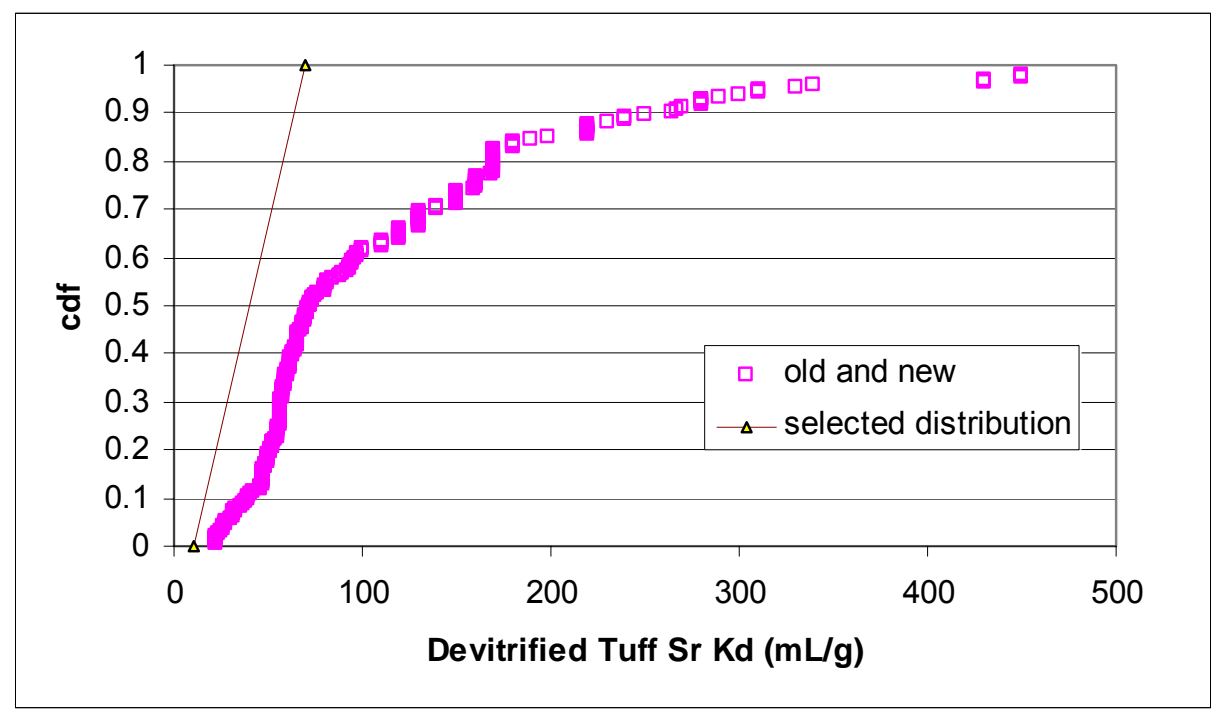

DTNs: New: LA0407AM831341.003 [DIRS 170626]; Old: LA0305AM831341.001 [DIRS 163789].

Figure A-51b. Empirical Cumulative Distribution for Strontium Sorption Coefficients on Devitrified Tuff

\section{A8.7.2 Zeolitic Tuff}

The experimentally derived sorption coefficients for strontium on zeolitic tuff are plotted against the calculated final strontium concentrations of the experiments in Figure A-52. The data points are separated into groups on the basis of water type, whether or not the solid phase included fines, and whether the sorption coefficient was determined from a sorption or a desorption experiment.



DTNs: New: LA0407AM831341.003 [DIRS 170626]; Old: LA0305AM831341.001 [DIRS 163789].

Figure A-52. Strontium Sorption Coefficients on Zeolitic Tuff versus Calculated Final Strontium Concentration in Solution 
All except two of the calculated final solution concentrations were below the saturation level with strontianite. The calculated final strontium concentrations used in experiments with $\mathrm{p} \# 1$ are all lower than the saturation value. Thus, oversaturation was not an issue in the strontium sorption experiments.

The few sorption coefficients obtained with $\mathrm{p} \# 1$ water tend to lie at the low end of the range of values shown in Figure A-52. This suggests variations in water composition will have an impact on the strontium sorption coefficient for zeolitic tuff.

Sorption experiments were carried out at a number of different starting concentrations to obtain an isotherm for sample YM-38. As shown in Figure A-53, the sorption coefficients obtained in these experiments are not consistent with a simple relationship between strontium concentration and sorption coefficient. Nonetheless, the sorption coefficients plotted in Figure A-52 were obtained over a range of strontium concentrations. Thus, the concentration dependence of the sorption coefficient is included in the dataset.

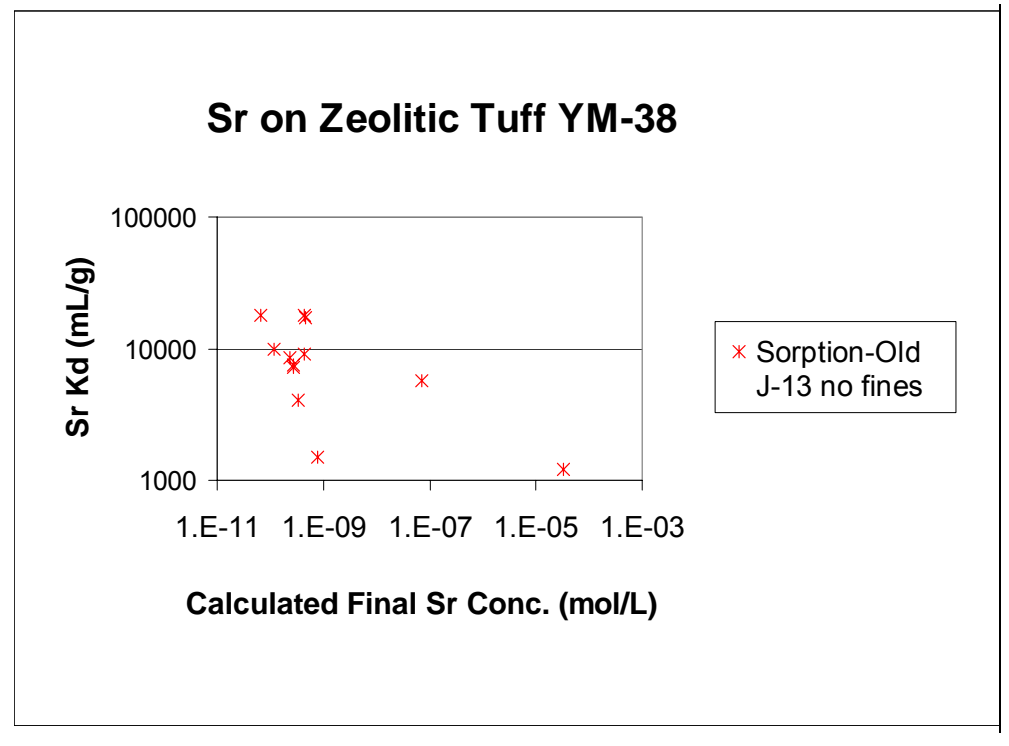

DTNs: New: LA0407AM831341.003 [DIRS 170626]; Old: LA0305AM831341.001 [DIRS 163789].

Figure A-53. Strontium Sorption Coefficients versus Calculated Final Solution Concentration in M/L for Sample YM-38 in J-13 Water

The effects of experiment duration on the strontium $\mathrm{K}_{\mathrm{d}}$ for zeolitic tuff are shown in Figure A-54. The large range in sorption coefficients obtained at a given duration reflects variations in the grain size of the crushed tuff samples used in the experiments, variations in solution strontium concentrations, variations in surface chemistry, and analytical errors and artifacts. Experiments with crushed tuff samples that include the fines (e.g., particle size less than $30 \mu \mathrm{m}$ ) usually have sorption coefficients larger than samples from which the fines have been removed (e.g., particle sizes 75 to $500 \mu \mathrm{m}$ ). This is partly a result of the higher surface area of samples with fines and partly due to mineral fractionation. Mineral fractionation can occur during the sieving process and cause the preferential concentration of very fine grained minerals (e.g., clays) in the fine fraction. 


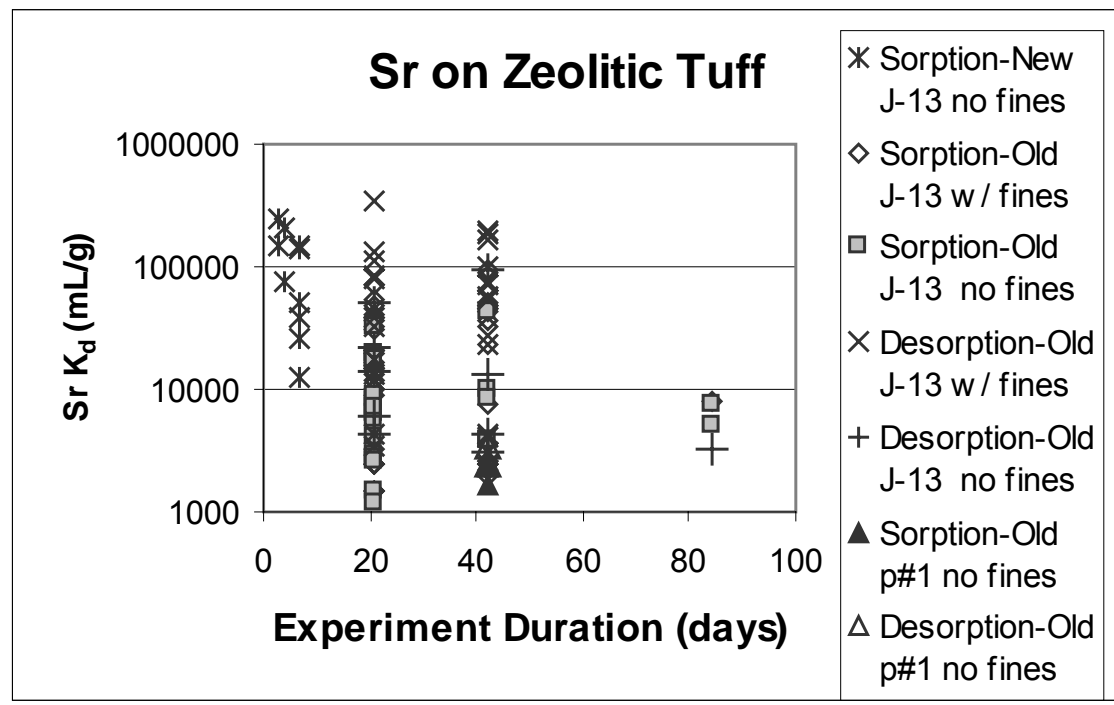

DTNs: New: LA0407AM831341.003 [DIRS 170626]; Old: LA0305AM831341.001 [DIRS 163789].

Figure A-54. Strontium Sorption Coefficients on Zeolitic Tuff versus Experiment Duration for Sorption (Forward) and Desorption (Backward) Experiments.

Figure A-55a shows the effects of duration on sorption coefficients for crushed tuff samples with the fines removed. The total range of sorption coefficient values is reduced slightly compared to Figure A-54. The rather limited range of values at 84 days is likely caused by the limited number of experiments carried at this duration. There is no clear trend of sorption coefficient value with duration. This likely reflects fast sorption kinetics.

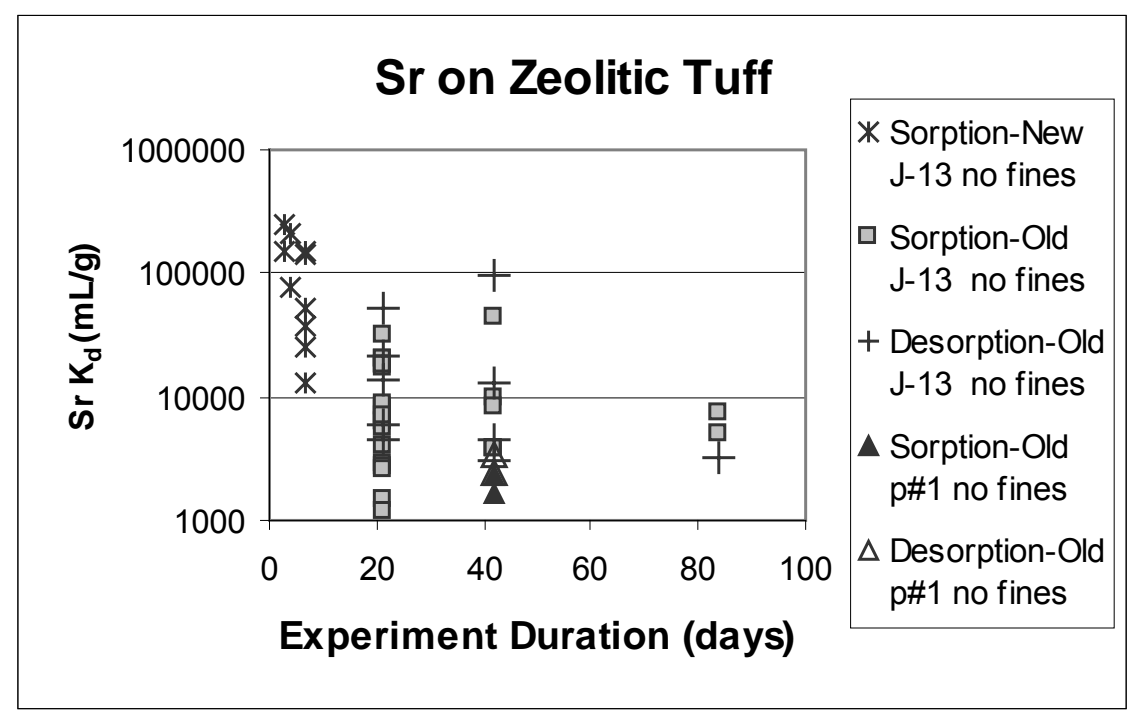

DTNs: New: LA0407AM831341.003 [DIRS 170626]; Old: LA0305AM831341.001 [DIRS 163789].

Figure A-55a. Strontium Sorption Coefficients on Zeolitic Tuff with Fine Fraction Removed versus Experiment Duration for Sorption (Forward) and Desorption (Backward) Experiments 
The strontium sorption coefficient probability distribution derived for zeolitic tuff in the UZ is a uniform distribution with a range of 50 to $2000 \mathrm{~mL} / \mathrm{g}$. The low end of the chosen range was selected based on the minimum value observed in isotherm experiments and the trend of lower sorption coefficients with increasing strontium concentration. When the trend is extended to the solubility limit for $\mathrm{p} \# 1$ water, the value obtained is well below $1,000 \mathrm{~mL} / \mathrm{g}$. A value of $50 \mathrm{~mL} / \mathrm{g}$ was chosen as a conservative value. The upper end of the distribution was chosen as a minimum upper limit, given the potential impact of natural strontium in pore waters. Natural strontium concentrations measured in pore waters can be as high as $4.7 \times 10^{-5} \mathrm{~mol} / \mathrm{L}$ but the average is closer to $1.1 \times 10^{-5} \mathrm{~mol} / \mathrm{L}$ (DTN: GS020408312272.003 [DIRS 160899]). The data shown in Figure A-52 would indicate a sorption coefficient of approximately $2,000 \mathrm{~mL} / \mathrm{g}$ at this concentration. A uniform distribution was chosen to equally weight the sorption coefficient values in the selected range. Figure A-55b shows the empirical cumulative distribution for all the measured strontium sorption coefficients on zeolitic tuff in the UZ with the selected distribution superimposed. As discussed in Section A6., not all the empirical data was equally weighted in selecting the probability distribution as the influence of expected variations in water chemistry, radionuclide concentrations, and variations in rock surface properties within each major rock type were incorporated in making the selection.

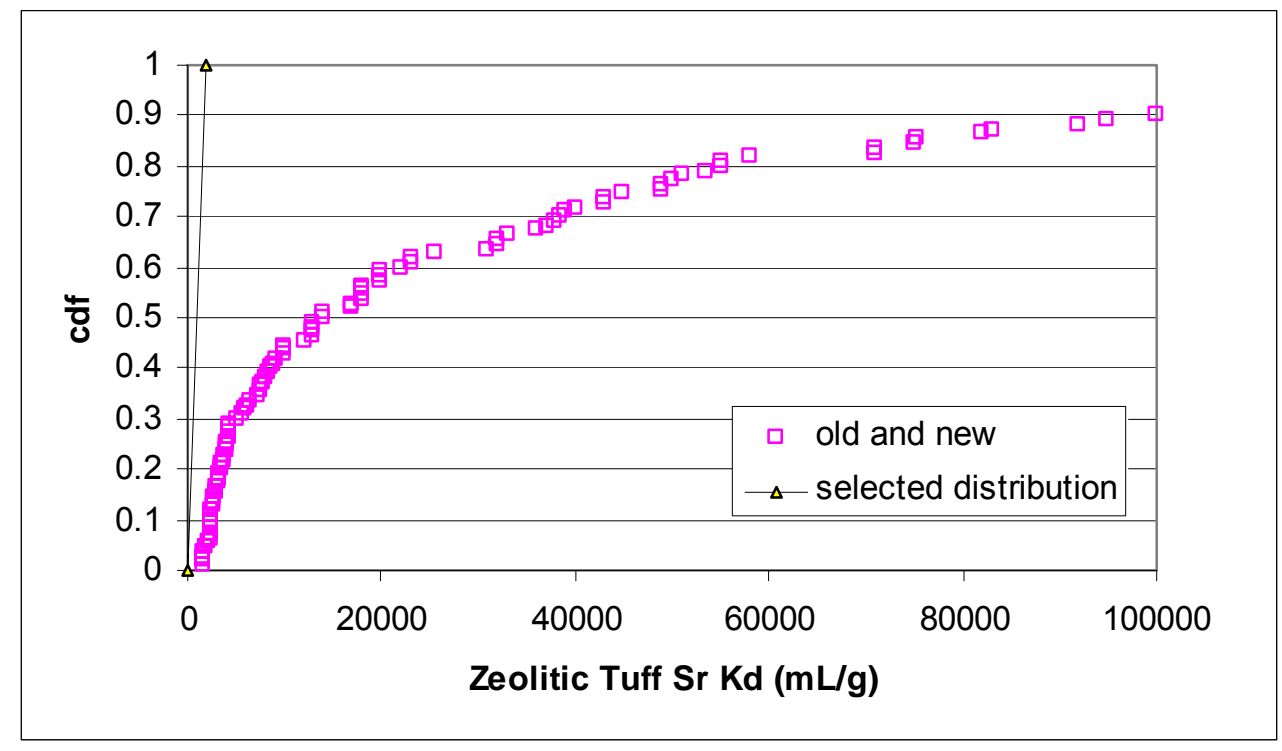

DTNs: New: LA0407AM831341.003 [DIRS 170626]; Old: LA0305AM831341.001 [DIRS 163789].

Figure A-55b. Empirical Cumulative Distribution for Strontium Sorption Coefficients on Zeolitic Tuff

\section{A8.7.3 Vitric Tuff}

The experimentally derived sorption coefficients for strontium on vitric tuff are plotted against the calculated final strontium concentrations of the experiments in Figure A-56. The data points are separated into groups on the basis of when the experiments were carried out (pre-1990 = "old" and post-1990 = "new"), water type, and whether the sorption coefficient was determined from a sorption or a desorption experiment. 


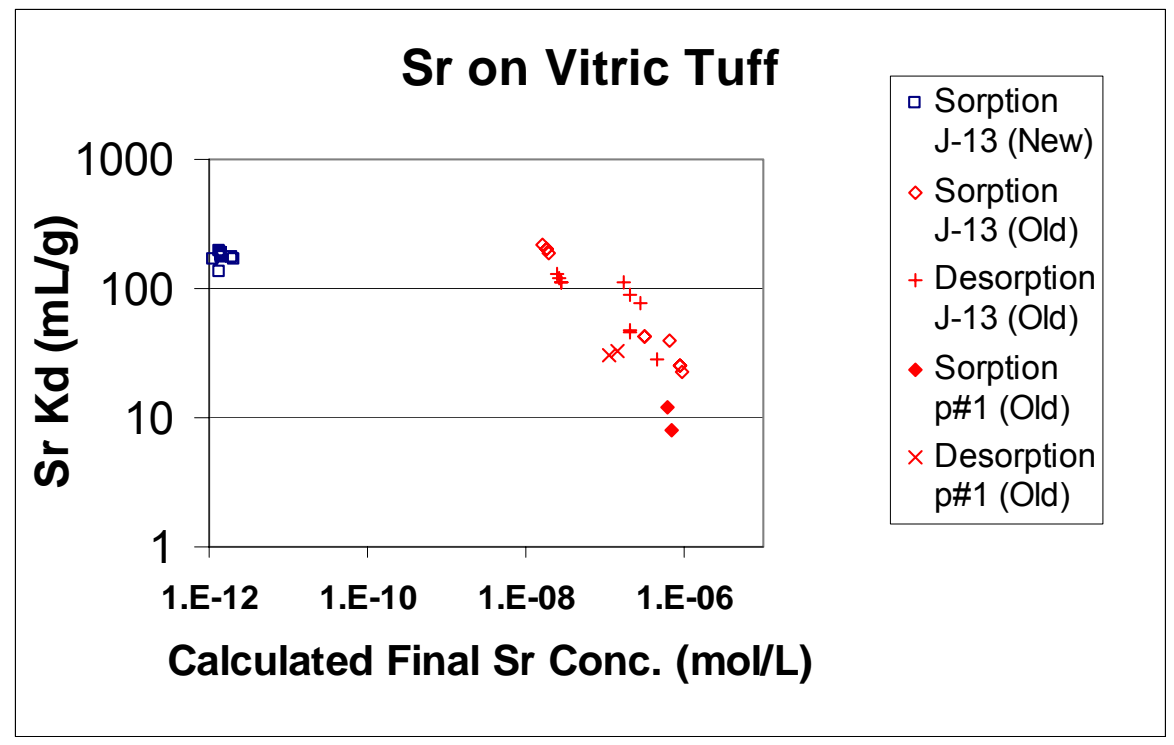

DTNs: New: LA0407AM831341.003 [DIRS 170626]; Old: LA0305AM831341.001 [DIRS 163789].

Figure A-56. Strontium Sorption Coefficients on Vitric Tuff versus Calculated Final Strontium Concentration in Solution

The calculated final solution concentrations for the sorption experiments are all below saturation with strontianite. Thus, oversaturation was not an issue in the strontium sorption experiments.

Sorption coefficients obtained in experiments with $\mathrm{p} \# 1$ water fall in the middle of the range of values obtained for experiments with J-13 water. Thus, variations in water chemistry have little or no impact on strontium sorption coefficients in devitrified tuff.

The effects of experiment duration on the strontium $K_{d}$ for devitrified tuff are shown in Figure A-57a. Because the short-term experiments have higher sorption coefficient values than the longer-term experiments, strontium sorption kinetics must be relatively fast.

The strontium sorption coefficient probability distribution derived for vitric tuff in the unsaturated zone is a uniform distribution with a range of 0 to $50 \mathrm{~mL} / \mathrm{g}$. The low end of the chosen range was selected based on the minimum value observed in experiments with $\mathrm{p} \# 1$ water and acknowledgment that the solution concentrations could get close to the saturation concentration $\left(8 \times 10^{-4} \mathrm{~mol} / \mathrm{L}\right)$ with the addition of natural strontium to the radioactive fraction released from the repository $\left(1.1-4.7 \times 10^{-5} \mathrm{~mol} / \mathrm{L} ; \mathrm{DTN}\right.$ : GS020408312272.003 [DIRS 160899]). The upper end of the distribution was chosen as a minimum upper limit in J-13 water with natural strontium. A uniform distribution was chosen to equally weight the sorption coefficient values in the selected range. Figure A-57b shows the empirical cumulative distribution for all the measured strontium sorption coefficients on vitric tuff in the UZ with the selected distribution superimposed. As discussed in Section A6., not all the empirical data was equally weighted in selecting the probability distribution as the influence of expected variations in water chemistry, radionuclide concentrations, and variations in rock surface properties within each major rock type were incorporated in making the selection. 


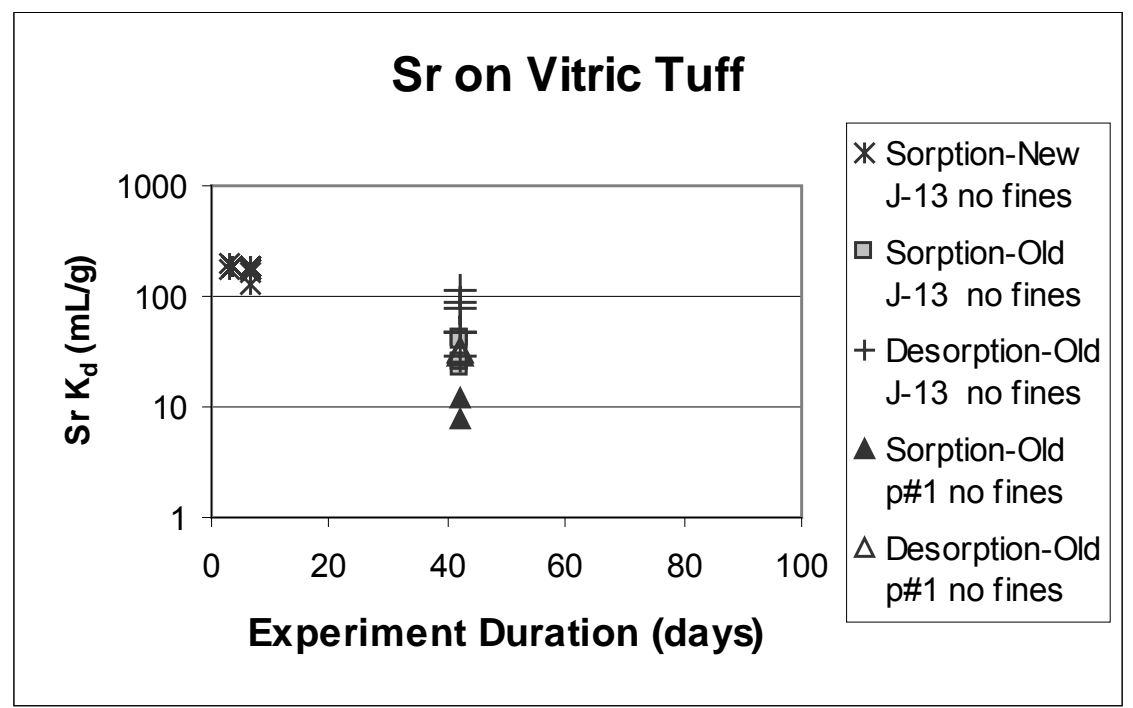

DTNs: New: LA0407AM831341.003 [DIRS 170626]; OId: LA0305AM831341.001 [DIRS 163789].

Figure A-57a. Strontium Sorption Coefficients on Vitric Tuff versus Experiment Duration for Sorption (Forward) and Desorption (Backward) Experiments

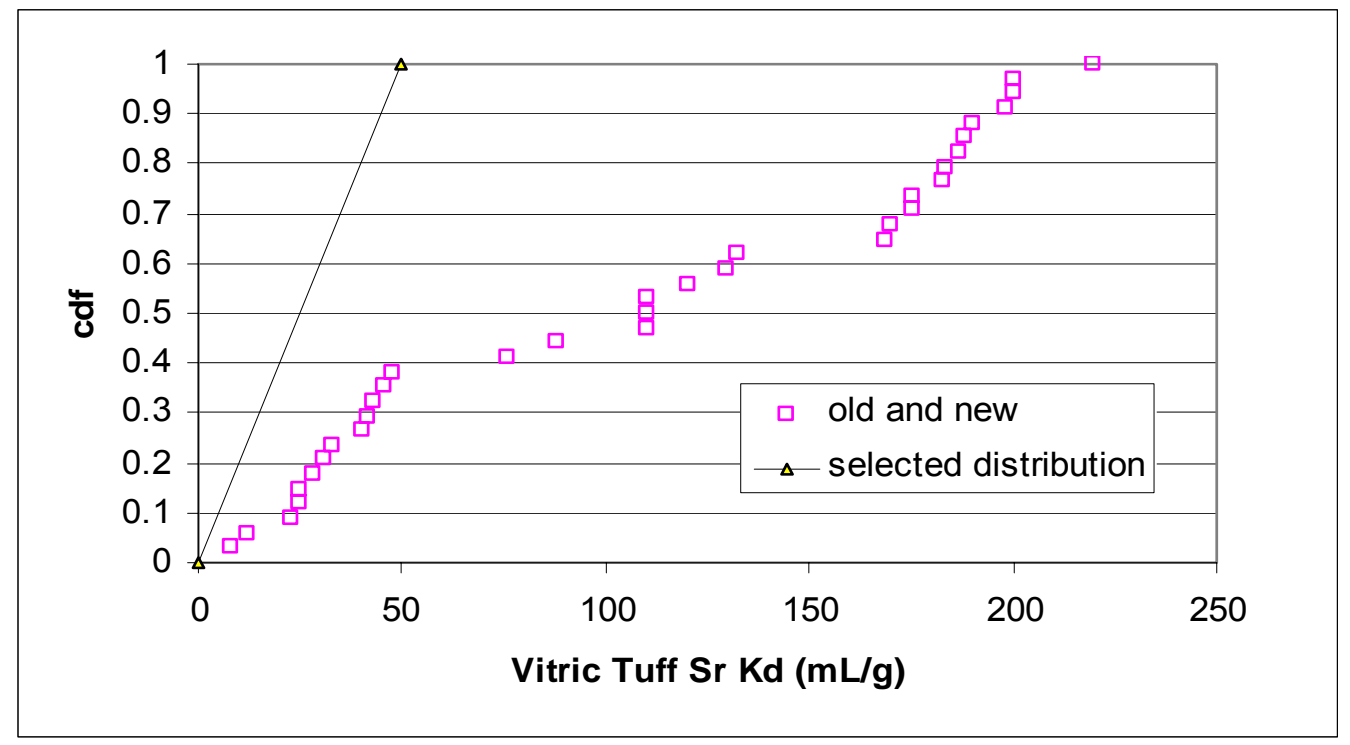

DTNs: New: LA0407AM831341.003 [DIRS 170626]; Old: LA0305AM831341.001 [DIRS 163789].

Figure A-57b. Empirical Cumulative Distribution for Strontium Sorption Coefficients on Vitric Tuff 


\section{A8.8 THORIUM}

The solubility of thorium dioxide in waters such as the UZ waters is estimated at $3.2 \times 10^{-9} \mathrm{~mol} / \mathrm{L}$ at $\mathrm{pH}>6.0$ (Hummel et al. 2002 [DIRS 161904], p. 377).

\section{A8.8.1 Devitrified Tuff}

Experiments with Yucca Mountain tuffs were carried out with initial concentrations in the $1.0 \times 10^{-7}$ to $6 \times 10^{-8} \mathrm{~mol} / \mathrm{L}$ range. Thus, the experiments were initially oversaturated with thorium dioxide. The calculated final thorium concentrations shown in Figure A-58 indicate that Th sorption onto the rock sample in some cases, though not all, brought the final solution concentrations below saturation with thorium dioxide. The results of experiments oversaturated with thorium dioxide are of questionable value. For the remaining experiments, the sorption coefficients range from 1,213 to $23,800 \mathrm{~mL} / \mathrm{g}$. There are no data available for the effect of experimental duration on sorption coefficient values for Yucca Mountain samples. However, Allard et al. (1983 [DIRS 162982], p. 10) reported that over experiment durations of 6 hours to 6 weeks, time had little influence on the measured sorption coefficients for Th on silica in $0.01 \mathrm{M} \mathrm{NaClO}$. Note that the starting concentrations reported by Allard et al. (1983 [DIRS 162982], p. 6) were below the saturation level for thorium dioxide.

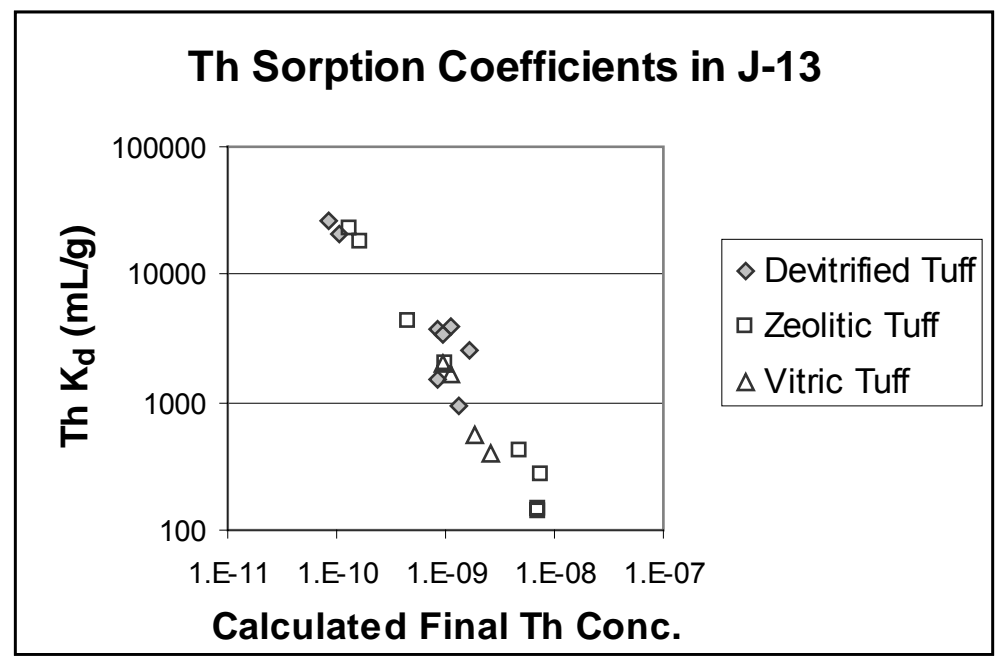

DTN: LA0305AM831341.001 [DIRS 163789].

Figure A-58. Thorium Sorption Coefficients on Tuff versus Calculated Final Thorium Concentration in Solution

There are no data available to evaluate the impact of variations in water chemistry on Th sorption coefficients. However, thorium forms primarily hydroxide complexes at near neutral $\mathrm{pH}$ (Langmuir and Herman 1980 [DIRS 147527], p. 1753). Therefore, water chemistry is expected to have very little influence on Th sorption coefficient values in Yucca Mountain groundwaters. However, water chemistry (i.e., $\mathrm{pH}$ ) does impact the solubility of thorium dioxide. This is the reason some of the experiments in Figure A-58 were oversaturated with thorium dioxide. As shown in Figure A-59a, the lowest sorption coefficients were obtained at near-neutral $\mathrm{pH}$ values where J-13 water was oversaturated with thorium dioxide. 


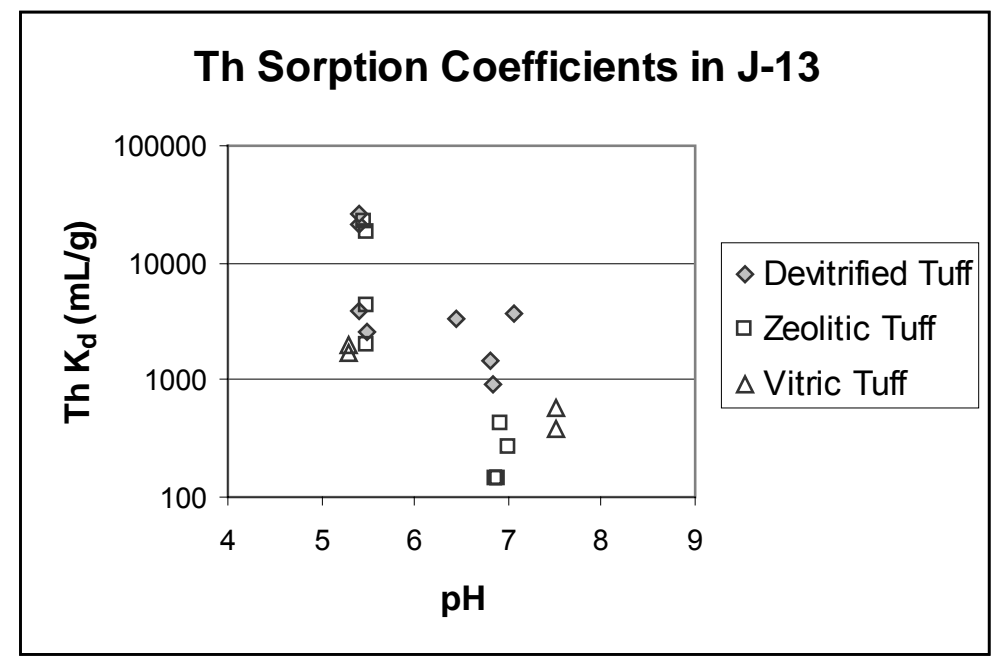

DTN: LA0305AM831341.001 [DIRS 163789].

Figure A-59a. Thorium Sorption Coefficients on Tuff versus pH

Based on the experimental data in Figures A-58 and A-59a, the range of thorium sorption coefficients expected for devitrified tuffs in the unsaturated volcanic section at Yucca Mountain is 1,000 to $10,000 \mathrm{~mL} / \mathrm{g}$. This range is intended to reflect the range in surface areas found in devitrified tuffs in the unsaturated zone and the range in thorium concentrations expected during unsaturated zone transport. The lower end of the range reflects sorption coefficients at thorium concentrations near the solubility limit. Given the sparseness of the available data, the probability distribution type selected is a uniform distribution. Figure A-59b shows the empirical cumulative distribution for all the measured thorium sorption coefficients on devitrified tuff in the UZ with the selected distribution superimposed. As discussed in Section A6., not all the empirical data was equally weighted in selecting the probability distribution as the influence of expected variations in water chemistry, radionuclide concentrations, and variations in rock surface properties within each major rock type were incorporated in making the selection.

\section{A8.8.2 Zeolitic Tuff}

Sorption coefficient data for zeolitic tuff are plotted in Figures A-58 and A-59a. Based on the available data, zeolitic tuffs have sorption coefficients for thorium that are similar to those obtained for devitrified tuffs.

Based on the data plotted in Figures A-58 and A-59a, the range of thorium sorption coefficients selected for zeolitic tuffs in the unsaturated volcanic section at Yucca Mountain is 1,000 to $30,000 \mathrm{~mL} / \mathrm{g}$. The upper end of this range was selected to reflect the higher surface areas of zeolitic tuffs relative to devitrified tuffs. A uniform distribution is selected for zeolitic tuffs. Figure A-59c shows the empirical cumulative distribution for the measured thorium sorption coefficients on zeolitic tuff in the UZ with the selected distribution superimposed. 


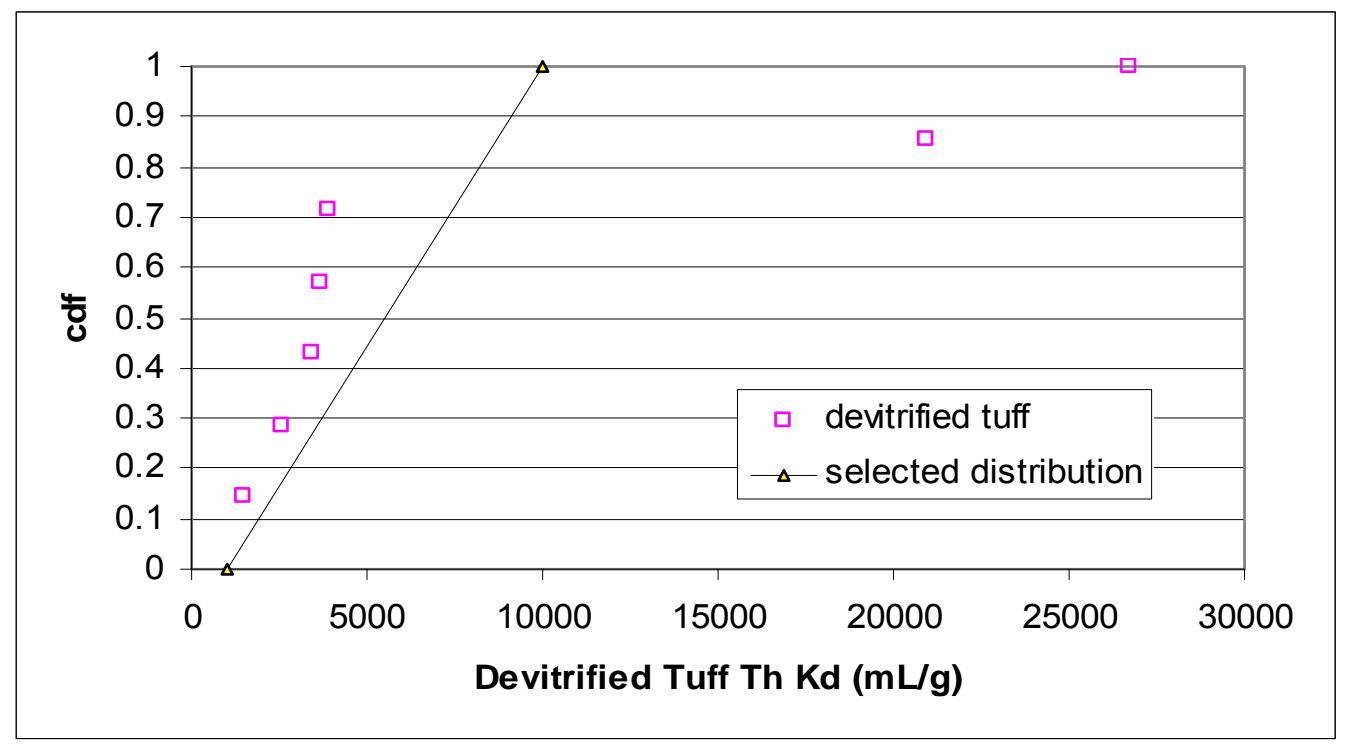

DTN: LA0305AM831341.001 [DIRS 163789].

Figure A-59b. Empirical Cumulative Distribution for Strontium Sorption Coefficients on Devitrified Tuff

\section{A8.8.3 Vitric Tuff}

Sorption coefficient data for vitric tuff are plotted in Figures A-58 and A-59a. Based on the available data, vitric tuffs have sorption coefficients for thorium in the range of those obtained for the other tuffs in those experiments that were undersaturated in thorium dioxide.

Based on the data plotted in Figures A-58 and A-59a, the range of thorium sorption coefficients selected for vitric tuffs in the unsaturated volcanic section at Yucca Mountain is 1,000 to $10,000 \mathrm{~mL} / \mathrm{g}$. A uniform distribution is selected for vitric tuffs. Figure A-59d shows the empirical cumulative distribution for all the measured thorium sorption coefficients on vitric tuff in the UZ with the selected distribution superimposed. As discussed in Section A6., not all the empirical data was equally weighted in selecting the probability distribution as the influence of expected variations in water chemistry, radionuclide concentrations, and variations in rock surface properties within each major rock type were incorporated in making the selection. 


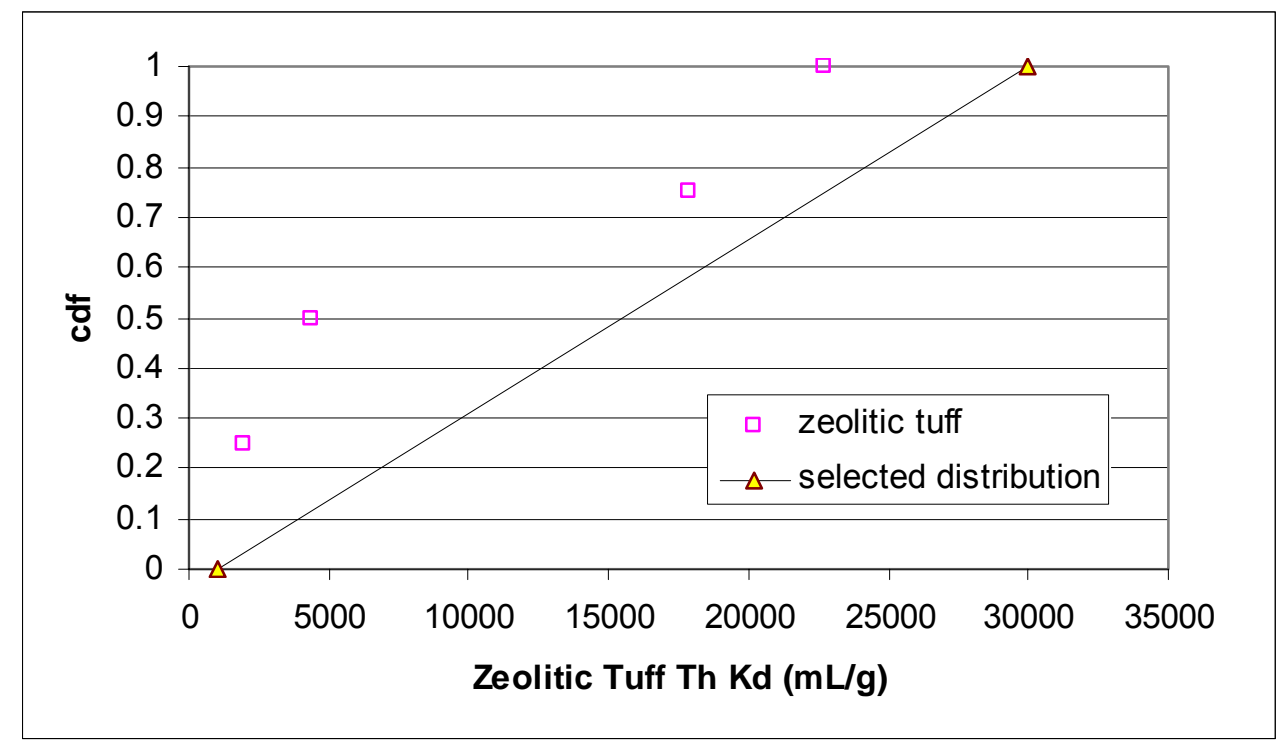

DTN: LA0305AM831341.001 [DIRS 163789].

Figure A-59c. Empirical Cumulative Distribution for Strontium Sorption Coefficients on Zeolitic Tuff

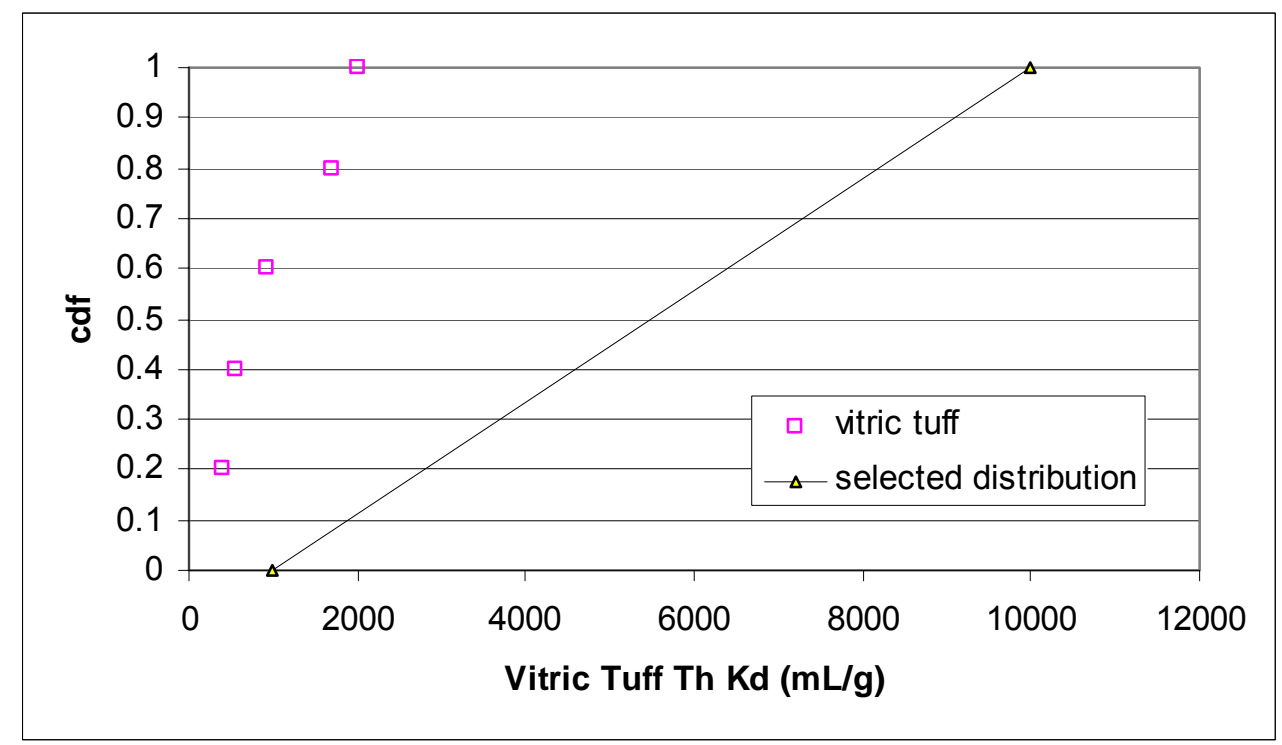

DTN: LA0305AM831341.001 [DIRS 163789].

Figure A-59d. Empirical Cumulative Distribution for Strontium Sorption Coefficients on Vitric Tuff 


\section{A8.9 URANIUM}

The solubility of uranium in J-13 water under oxidizing conditions ranges from $1.8 \times 10^{-4}$ at $\mathrm{pH}=7.1$ to $2.0 \times 10^{-4} \mathrm{~mol} / \mathrm{L}$ at $\mathrm{pH}=8.5$ (DTN: LA0407AM831343.001 [DIRS 171009]). The solubility of uranium in synthetic $\mathrm{p} \# 1$ water under oxidizing conditions ranges from $6.7 \times 10^{-4}$ at $\mathrm{pH}=6.9$ to $9.0 \times 10^{-4} \mathrm{~mol} / \mathrm{L}$ at $\mathrm{pH}=8.6$ (DTN: LA0407AM831343.001 [DIRS 171009]). The solubility-controlling solid in both waters is schoepite.

\section{A8.9.1 Devitrified Tuff}

As shown in Figure A-60, the calculated final uranium concentrations in the sorption experiments were generally below saturation with schoepite. The sorption coefficients obtained in sorption experiments with devitrified tuffs do not show a correlation with the calculated final uranium solution concentrations.

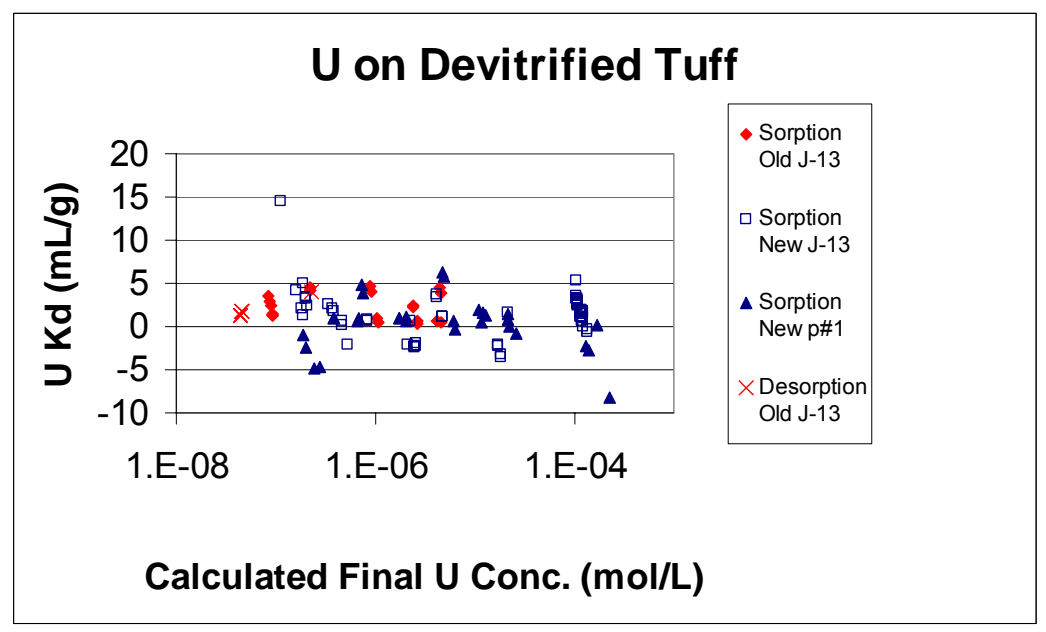

DTNs: New: LA0407AM831341.006 [DIRS 170628]; Old: LA0305AM831341.001 [DIRS 163789].

Figure A-60. Uranium Sorption Coefficients on Devitrified Tuff versus Calculated Final Uranium Concentration in Solution

Sorption experiments carried out as a function of time are shown in Figure A-61. Beyond approximately 3 days, there is no clear correlation between the sorption coefficients obtained and the duration of the experiments. The data imply that uranium sorption reactions on devitrified tuffs must be relatively fast (i.e., they reach steady state in a few days). The negative $\mathrm{K}_{\mathrm{d}}$ values shown in Figure A-61 and elsewhere in this section are not physical and reflect the analytical errors inherent in the experimental design. These experimental errors result from counting statistics, the stability of counters over time, corrections made for adsorption to container walls, and the $\mathrm{pH}$ of the tracer solution added to the experiment. 


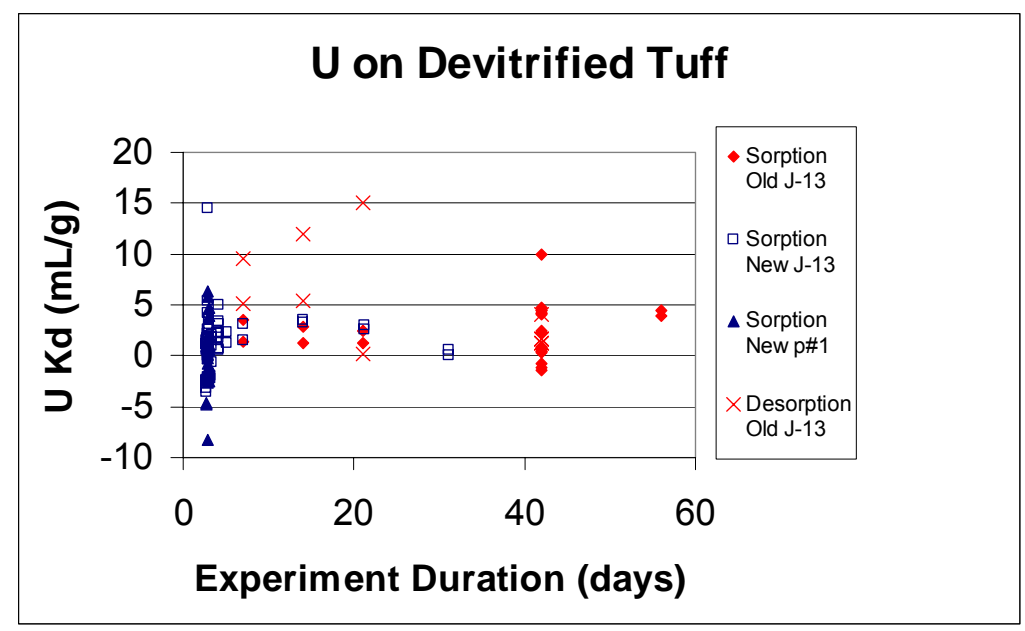

DTNs: New: LA0407AM831341.006 [DIRS 170628]; Old: LA0305AM831341.001 [DIRS 163789].

Figure A-61 Uranium Sorption Coefficients on Devitrified Tuff versus Experiment Duration for Sorption (Forward) and Desorption (Backward) Experiments

The dependence of the uranium sorption coefficient on water chemistry was tested with

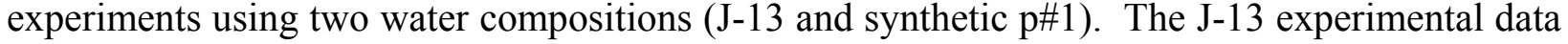
are shown as a function of $\mathrm{pH}$ in Figure A-62. The "old" data were obtained in the 1980s and the "new" data were obtained in the 1990s. The difference between them is not statistically significant. The range of values obtained at a given $\mathrm{pH}$ (e.g., 8.4) reflects experimental errors and natural variations in rock properties (e.g., surface area and mineral chemistry). It is not possible to discriminate between these possible causes with the available data. Based on the experimental data points, there does not appear to be a correlation between $\mathrm{K}_{\mathrm{d}}$ and $\mathrm{pH}$. However, surface complexation modeling with PHREEQC, using binding constants derived by Pabalan et al. (1998 [DIRS 162987], p. 124) for uranium on silica, points to a clear $\mathrm{pH}$ dependence as shown in Figure A-62. The two model curves reflect two different surface areas $\left(2.8\right.$ and $\left.5.6 \mathrm{~m}^{2} / \mathrm{g}\right)$. The $2.8 \mathrm{~m}^{2} / \mathrm{g}$ surface area is approximately an average value for devitrified tuffs at Yucca Mountain.

The sorption coefficients obtained in experiments with "synthetic p\#1" water are shown in Figure A-63a. Taken at face value, the experimental data suggest a trend of increasing $\mathrm{K}_{\mathrm{d}}$ with increasing $\mathrm{pH}$. However, the surface complexation modeling predicts a decrease in $\mathrm{K}_{\mathrm{d}}$ with increasing $\mathrm{pH}$, although the absolute $\mathrm{K}_{\mathrm{d}}$ values are rather small.

Based on the experimental data and model curves plotted in Figures A-62 and A-63a, a cumulative probability distribution type was selected for the sorption coefficient probability distribution for devitrified tuff, with a value of $0.0 \mathrm{~mL} / \mathrm{g}$ at $0.0,0.2 \mathrm{~mL} / \mathrm{g}$ at 0.5 , and $4.0 \mathrm{~mL} / \mathrm{g}$ at 1.0. Figure A-63b shows the empirical cumulative distribution for all the measured uranium sorption coefficients on devitrified tuff in the UZ with the selected distribution superimposed. As discussed in Section A6., not all the empirical data was equally weighted in selecting the probability distribution as the influence of expected variations in water chemistry, radionuclide concentrations, and variations in rock surface properties within each major rock type were incorporated in making the selection. 


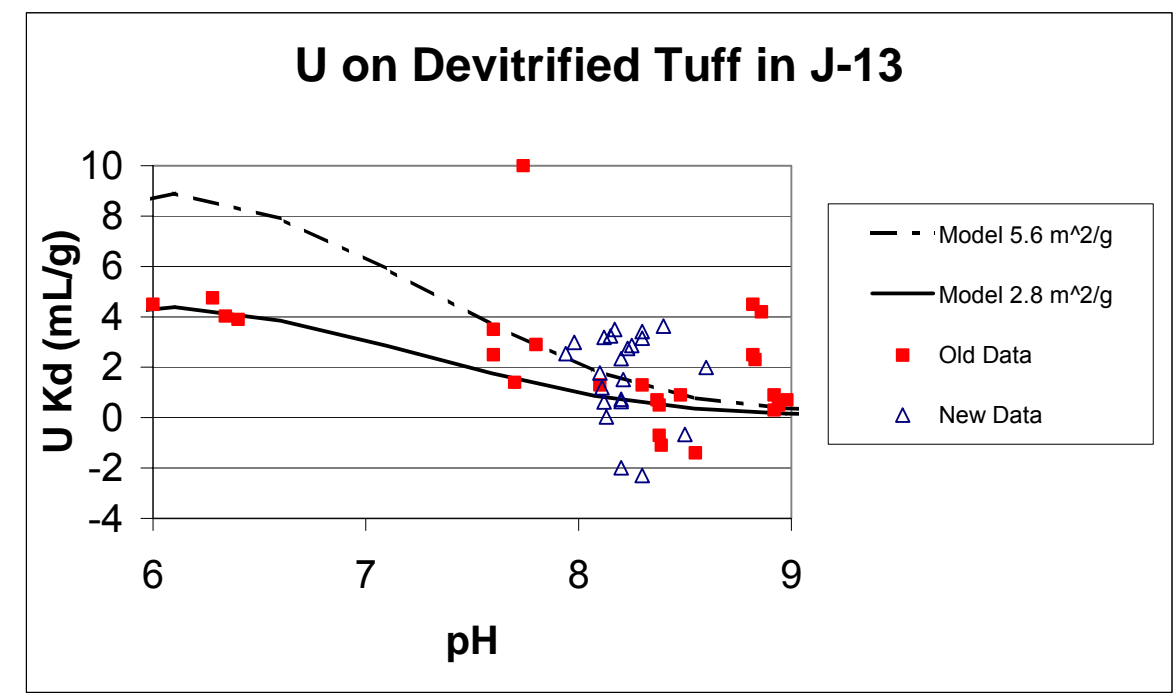

DTNs: New: LA0407AM831341.006 [DIRS 170628]; LA0407AM831343.001 [DIRS 171009]; OId: LA0305AM831341.001 [DIRS 163789].

NOTE: Model curves are from the PHREEQC surface complexation model.

Figure A-62. Uranium Sorption Coefficients on Devitrified Tuff versus pH

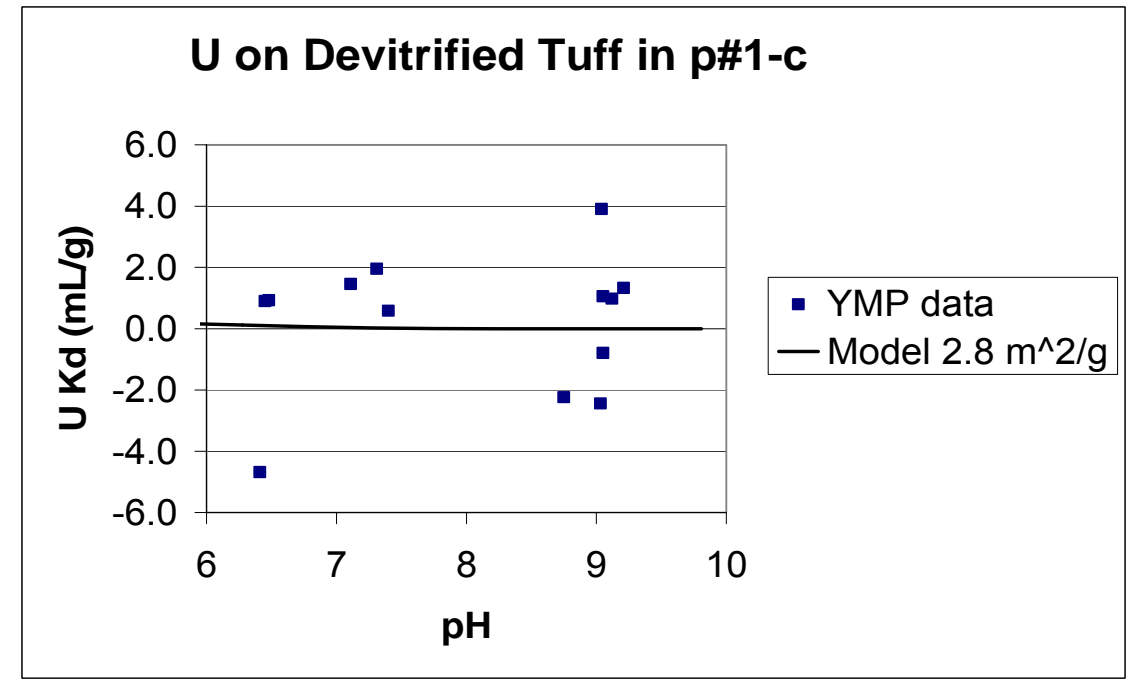

DTNs: New: LA0407AM831341.006 [DIRS 170628]; LA0407AM831343.001 [DIRS 171009]; Old: LA0305AM831341.001 [DIRS 163789].

NOTE: Model curve Is from the PHREEQC surface complexation model.

Figure A-63a. Uranium Sorption Coefficients on Devitrified Tuff in $\mathrm{p \# 1} \mathrm{Water} \mathrm{versus} \mathrm{pH}$ 


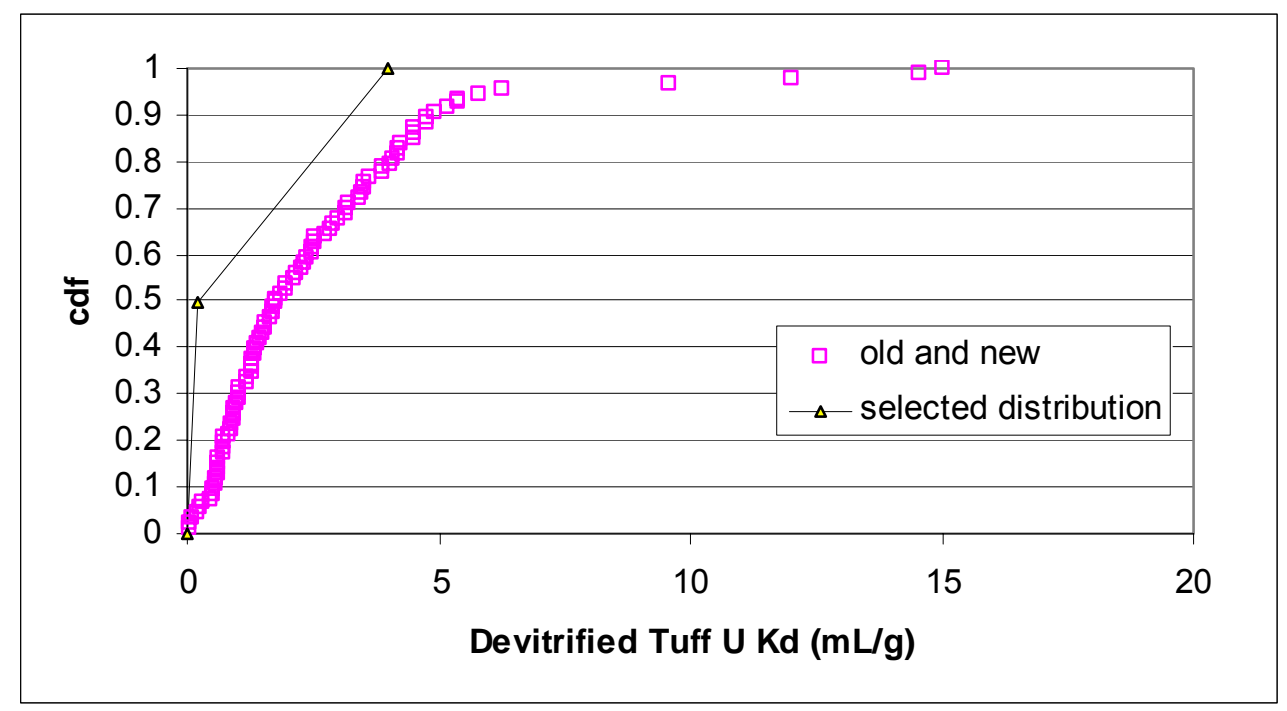

DTNs: New: LA0407AM831341.006 [DIRS 170628]; Old: LA0305AM831341.001 [DIRS 163789].

Figure A-63b. Empirical Cumulative Distribution for Uranium Sorption Coefficients on Devitrified Tuff

\section{A8.9.2 Zeolitic Tuff}

As shown in Figure A-64, the sorption coefficients obtained in sorption experiments with zeolitic tuffs do not show a clear correlation with the calculated final uranium solution concentrations. The high end of the concentrations plotted is below saturation with a solid uranium phase.



DTNs: New: LA0407AM831341.006 [DIRS 170628]; Old: LA0305AM831341.001 [DIRS 163789].

Figure A-64. Uranium Sorption Coefficients on Zeolitic Tuff versus Calculated Final Uranium Concentration in Solution 
Uranium sorption experiments on zeolitic tuffs carried out as a function of time are shown in Figure A-65. Beyond a period of approximately 3 days, there is no clear correlation between the sorption coefficients obtained and the duration of the experiments. These data imply that uranium sorption reactions on zeolitic tuffs are relatively fast (i.e., they reach steady state in a few days).

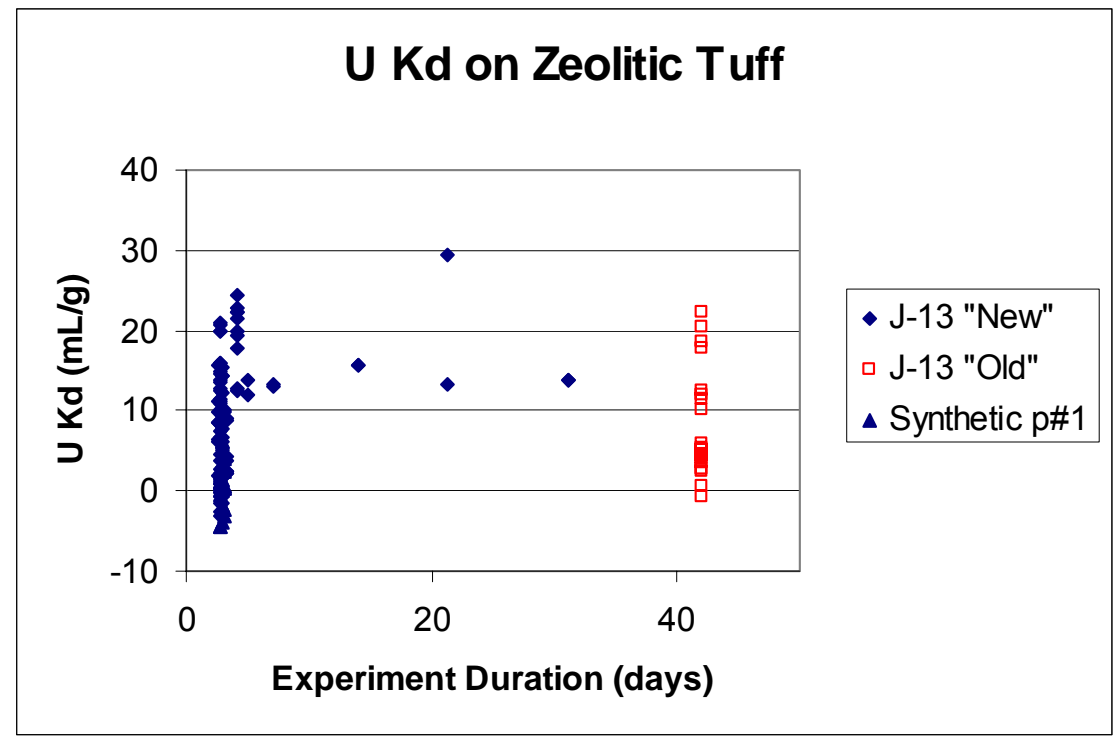

DTNs: New: LA0407AM831341.006 [DIRS 170628]; Old: LA0305AM831341.001 [DIRS 163789].

Figure A-65. Uranium Sorption Coefficients on Zeolitic Tuff as a Function of Experiment Duration

The dependence of the uranium sorption coefficient on water chemistry was tested with experiments using two water compositions (J-13 and synthetic p\#1). The J-13 data are shown as a function of $\mathrm{pH}$ in Figure A-66. The "old" data were obtained in the 1980s and the "new" data were obtained in the 1990s. The difference between them is not statistically significant. The range of values observed at a given $\mathrm{pH}$ (e.g., 8.4) reflects variations in rock properties and experimental errors. The experimental errors result from such things as counting statistics, the stability of counters over time, the accuracy of corrections for adsorption to container walls, and other experimental artifacts. In some cases, the $\mathrm{pH}$ of the tracer solution added to the experiment seems to have an effect. Some of these errors are random (e.g., counting errors) and others (e.g., adsorption to container walls) may have a nonrandom bias. It is not possible to separately evaluate these errors with the information available. Note that the distribution of data points in Figure A-66 does not indicate a strong correlation between $\mathrm{K}_{\mathrm{d}}$ and $\mathrm{pH}$.

Surface complexation modeling was carried out with PHREEQC to provide a framework in which to interpret the experimental data. Binding constants for uranium on silica derived by Pabalan et al. (1998 [DIRS 162987], p. 124) were used in the modeling. The modeling results show a clear $\mathrm{pH}$ dependence (Figure A-66) and the two model curves reflect two different surface areas. A surface area of $28 \mathrm{~m}^{2} / \mathrm{g}$ was used because it approximates an average value for zeolitic tuffs and because it is an order of magnitude larger than the average value used for modeling devitrified tuffs. A surface area of $14 \mathrm{~m}^{2} / \mathrm{g}$ was also used to show the impact of a factor of 2 change in surface area. 
The sorption coefficients obtained in experiments with "synthetic p\#1" water are shown in Figure A-67a. The magnitudes of the negative $K_{d}$ values plotted are similar to the magnitudes of the positive values plotted. Thus, the net values may be very close to zero. Taken at face value, the experimental data suggest a trend of increasing $\mathrm{K}_{\mathrm{d}}$ with increasing $\mathrm{pH}$. However, the surface complexation modeling predicts an increase in $\mathrm{K}_{\mathrm{d}}$ with decreasing $\mathrm{pH}$, although the absolute $\mathrm{K}_{\mathrm{d}}$ values are rather small, in agreement with the net values obtained from the experimental data.

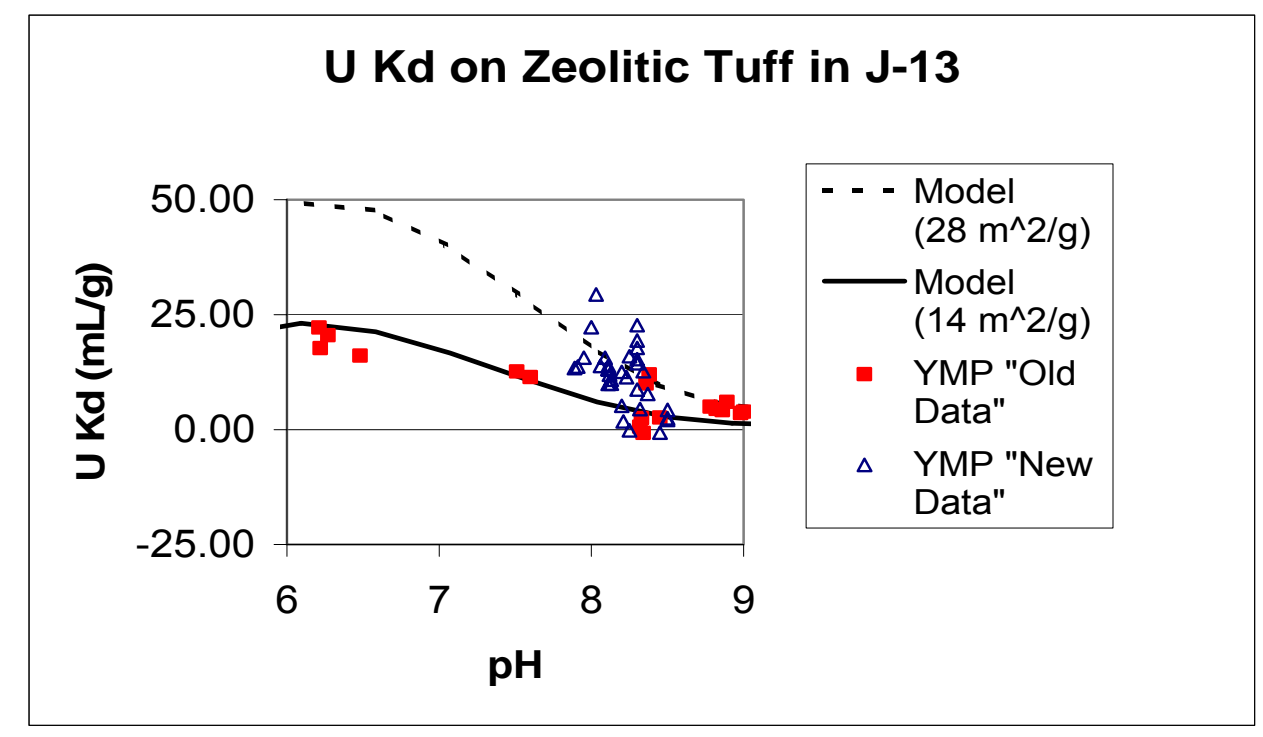

DTNs: New: LA0407AM831341.006 [DIRS 170628]; LA0407AM831343.001 [DIRS 171009];

Old: LA0305AM831341.001 [DIRS 163789].

NOTE: Model curves derived with PHREEQC surface complexation modeling also are shown.

Figure A-66. Uranium Sorption Coefficients for Zeolitic Tuff in J-13 Plotted as a Function of pH

Based on the experimental data and model curves plotted in Figures A-66 and A-67a, a cumulative probability distribution type was selected for the sorption coefficient probability distribution for zeolitic tuff with a value of $0.0 \mathrm{~mL} / \mathrm{g}$ at $0.0,0.5 \mathrm{~mL} / \mathrm{g}$ at 0.5 , and $30.0 \mathrm{~mL} / \mathrm{g}$ at 1.0. Figure A-67b shows the empirical cumulative distribution for all the measured uranium sorption coefficients on zeolitic tuff in the UZ with the selected distribution superimposed. As discussed in Section A6., not all the empirical data was equally weighted in selecting the probability distribution as the influence of expected variations in water chemistry, radionuclide concentrations, and variations in rock surface properties within each major rock type were incorporated in making the selection. 


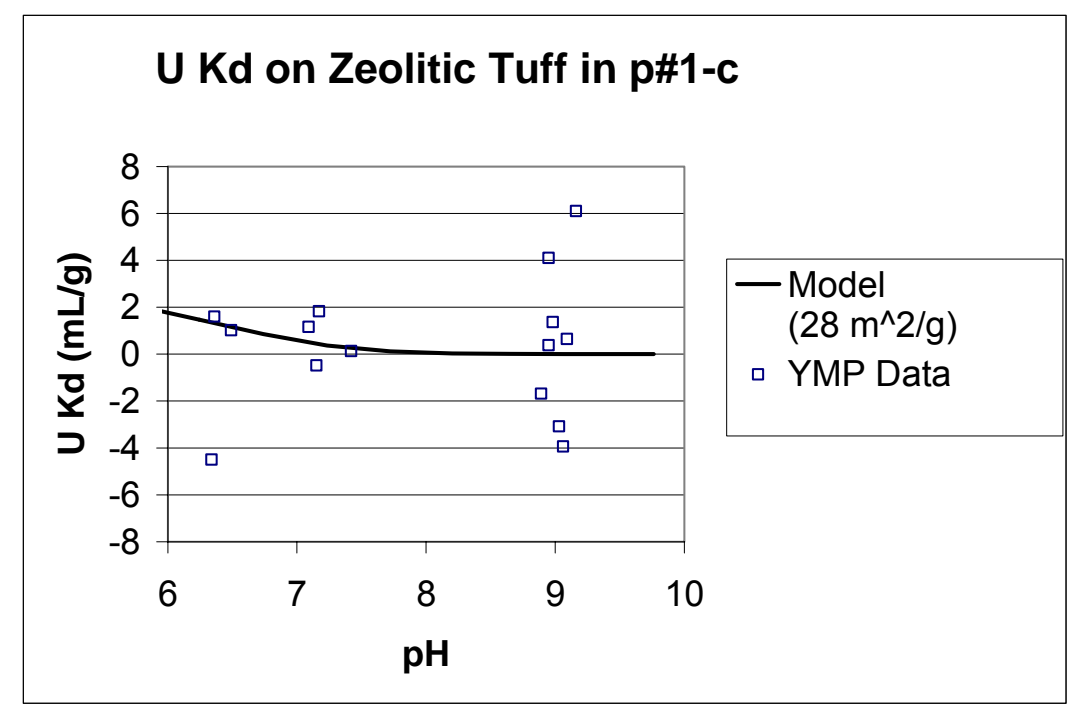

DTNs: New: LA0407AM831341.006 [DIRS 170628]; LA0407AM831343.001 [DIRS 171009]; Old: LA0305AM831341.001 [DIRS 163789].

NOTE: Model curves derived with PHREEQC surface complexation modeling also are shown.

Figure A-67a. Uranium Sorption Coefficients for Zeolitic Tuff in Synthetic p\#1 Plotted as a Function of $\mathrm{pH}$.

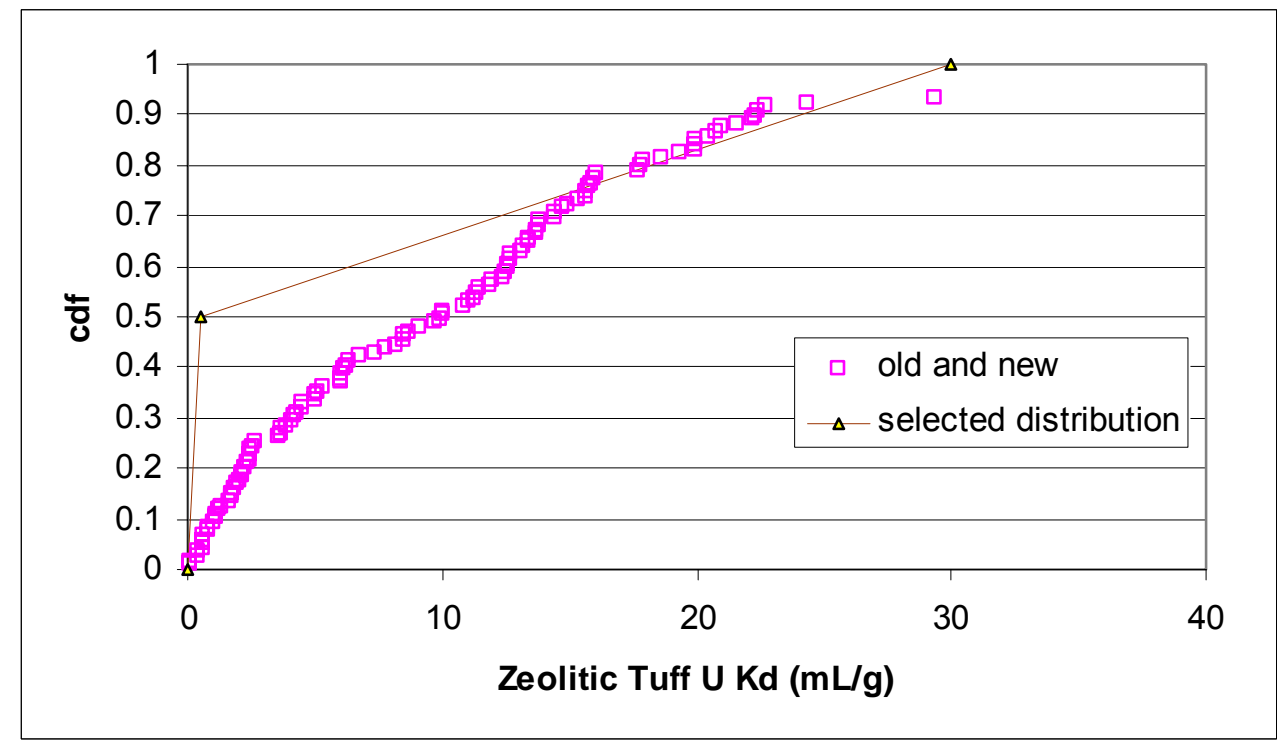

DTNs: New: LA0407AM831341.006 [DIRS 170628]; Old: LA0305AM831341.001 [DIRS 163789].

Figure A-67b. Empirical Cumulative Distribution for Uranium Sorption Coefficients on Zeolitic Tuff 


\section{A8.9.3 Vitric Tuff}

As shown in Figure A-68, the high end of the concentration range plotted is below saturation with schoepite. Thus, oversaturation was not a problem in sorption experiments with vitric tuffs.

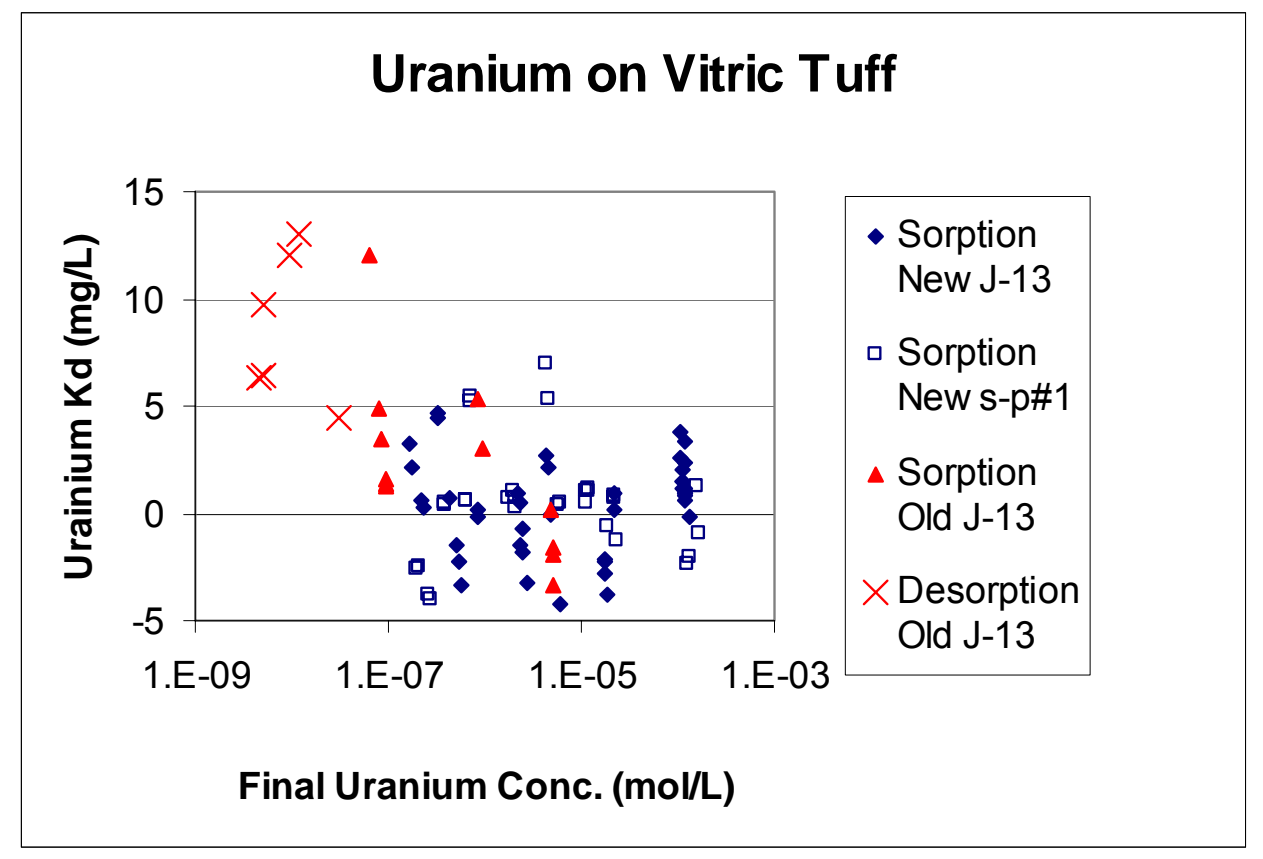

DTNs: New: LA0407AM831341.006 [DIRS 170628]; Old: LA0305AM831341.001 [DIRS 163789].

Figure A-68. Uranium Sorption Coefficients on Vitric Tuff versus Calculated Final Uranium Concentration in Solution

Uranium sorption experiments on vitric tuffs carried out as a function of time are shown in Figure A-69. Beyond a period of approximately 3 days, there is no clear correlation between the sorption coefficients obtained and the duration of the experiments. These data imply that uranium sorption reactions on vitric tuffs are relatively fast (i.e., they reach steady state in a few days). Sorption coefficients obtained from desorption experiments are larger, on average, than sorption coefficients obtained from sorption experiments. The desorption experiments were performed on a sample that contained 15 percent zeolite. Perhaps the presence of zeolite in this sample caused it to have a higher affinity for uranium. The point with the largest sorption coefficient value from sorption experiments $(12 \mathrm{~mL} / \mathrm{g})$ was also obtained on this particular sample. 


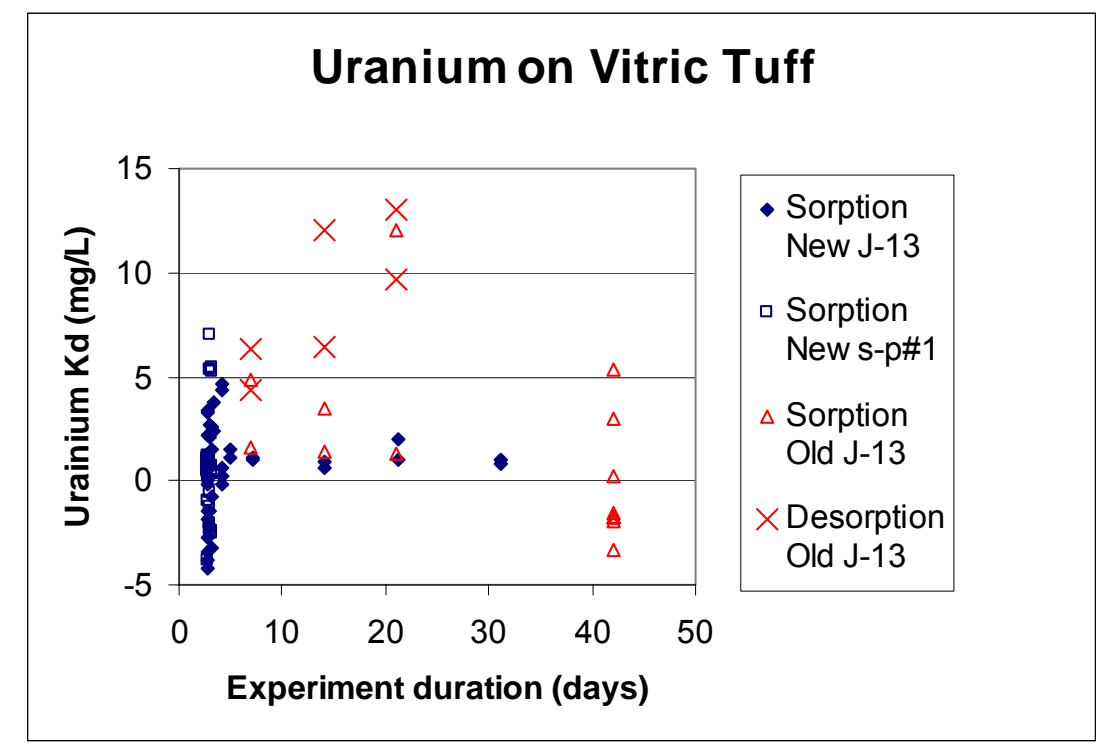

DTNs: New: LA0407AM831341.006 [DIRS 170628]; Old: LA0305AM831341.001 [DIRS 163789].

Figure A-69. Uranium Sorption Coefficients on Vitric Tuff as a Function of Experiment Duration

The dependence of the uranium sorption coefficient on water chemistry was tested with

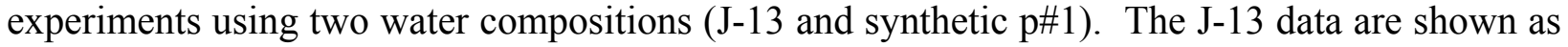
a function of $\mathrm{pH}$ in Figure A-70. The coefficients obtained in sorption experiments are smaller than the coefficients obtained in desorption experiments, as noted above. As before, the range of values observed at a given $\mathrm{pH}$ (e.g., 8.4) reflects variations in rock properties and experimental errors. The experimental errors result from such things as counting statistics, the stability of counters over time, the accuracy of corrections for adsorption to container walls, and other experimental artifacts. In some cases, the $\mathrm{pH}$ of the tracer solution added to the experiment seems to have an effect. Some of these errors are random (e.g., counting errors), and others (e.g., adsorption to container walls) may have a nonrandom bias. It is not possible to separately evaluate these errors with the information available. However, the magnitude of the negative values suggest error values of up to 100 percent.

Uranium sorption coefficients obtained with synthetic p\#1 water are plotted in Figure A-71. The average of the points plotted is close to zero.

Based on the experimental data plotted in Figures A-70 and A-71, a cumulative probability distribution type was selected for the sorption coefficient probability distribution for vitric tuff, with a value of $0.0 \mathrm{~mL} / \mathrm{g}$ at $0.0,0.2 \mathrm{~mL} / \mathrm{g}$ at 0.5 , and $3.0 \mathrm{~mL} / \mathrm{g}$ at 1.0 . Figure A-72 shows the empirical cumulative distribution for all the measured uranium sorption coefficients on vitric tuff in the UZ with the selected distribution superimposed. As discussed in Section A6., not all the empirical data was equally weighted in selecting the probability distribution as the influence of expected variations in water chemistry, radionuclide concentrations, and variations in rock surface properties within each major rock type were incorporated in making the selection. 


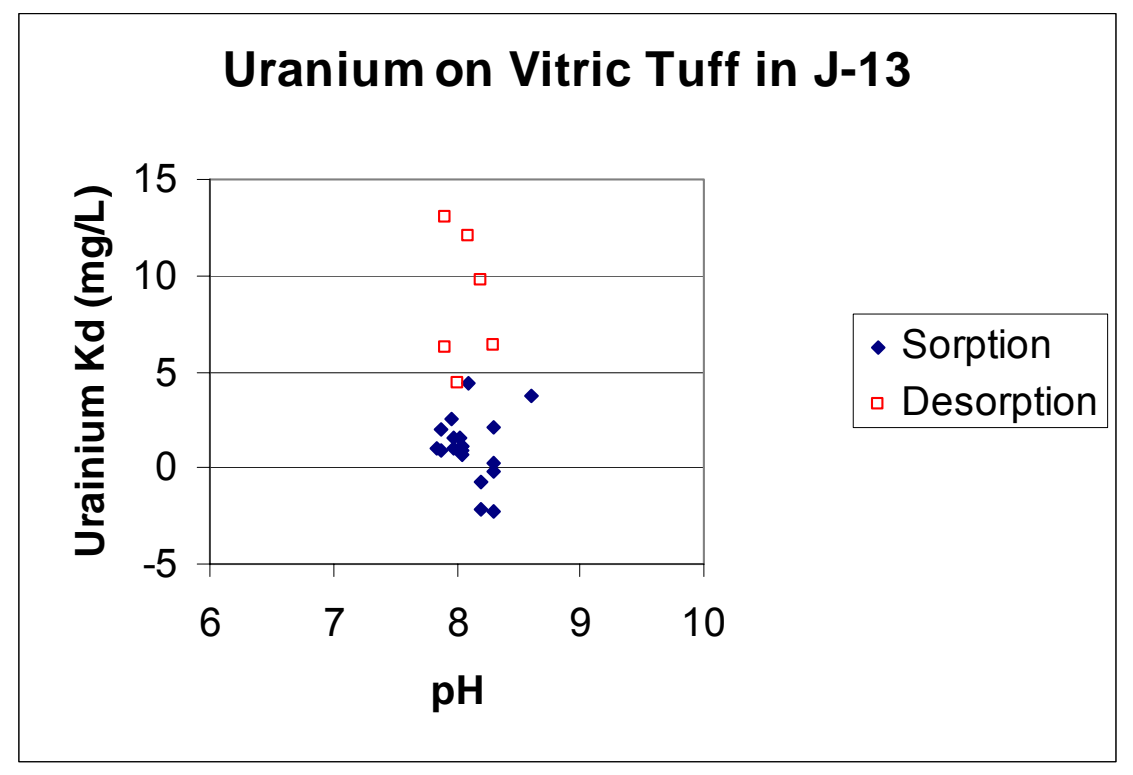

DTNs: New: LA0407AM831341.006 [DIRS 170628]; OId: LA0305AM831341.001 [DIRS 163789].

Figure A-70. Uranium Sorption Coefficients for Vitric Tuff in J-13 Plotted as a Function of $\mathrm{pH}$.

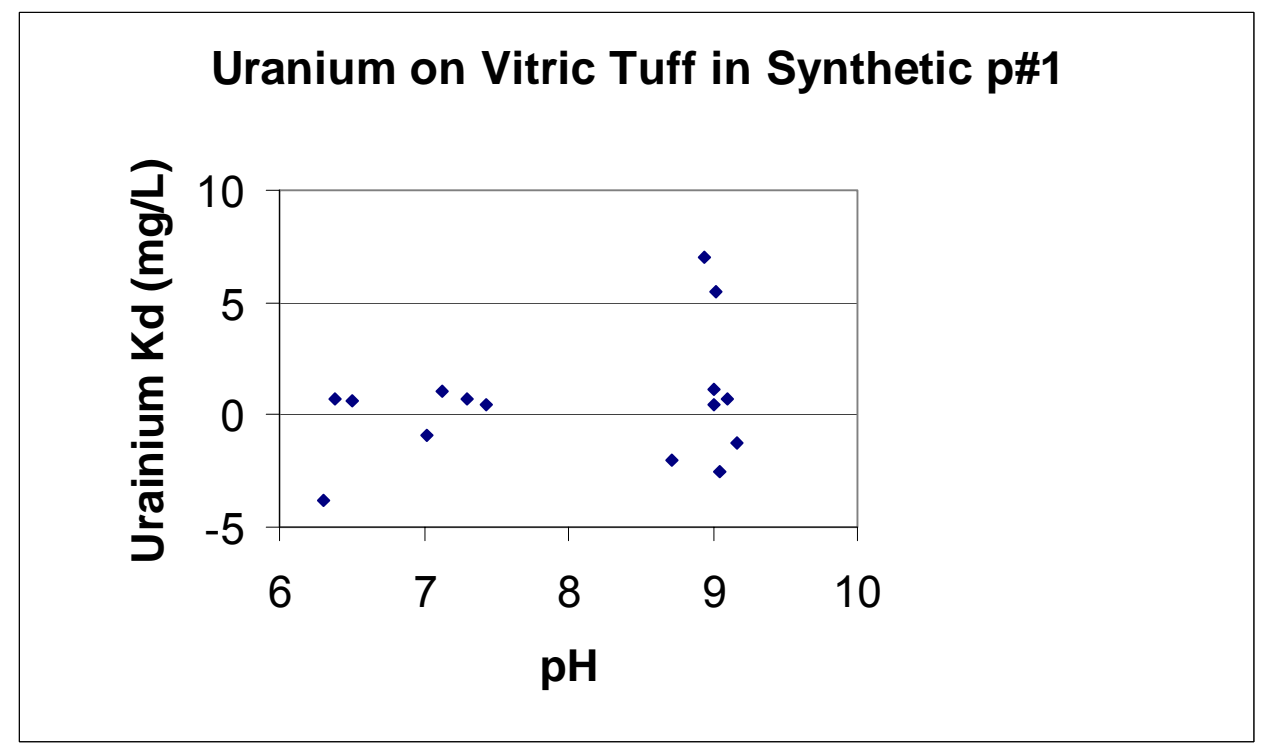

DTNs: New: LA0407AM831341.006 [DIRS 170628].

Figure A-71. Uranium Sorption Coefficients for Vitric Tuff in Synthetic p\#1 Plotted as a Function of $\mathrm{pH}$. 


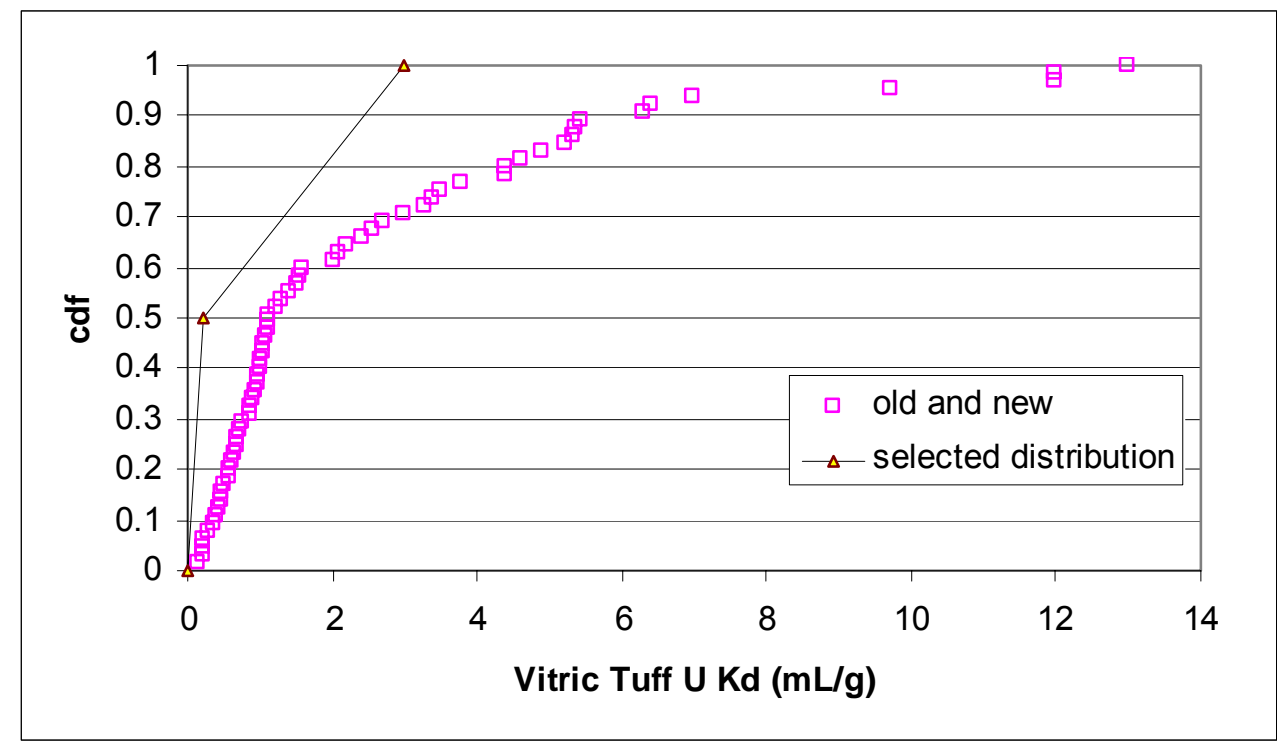

DTNs: New: LA0407AM831341.006 [DIRS 170628]; Old: LA0305AM831341.001 [DIRS 163789].

Figure A-72. Empirical Cumulative Distribution for Uranium Sorption Coefficients on Vitric Tuff

\section{A9. SUMMARY}

Sorption-coefficient probability distribution functions were derived for radionuclides of interest to be used in transport calculations. Experimental and modeling results were used to constrain the distributions. In general, the approach used in derivation of the distributions tended to underestimate the range and median or expected value. This was done to provide some conservatism in the derivation, given potential scaling uncertainties in the application of these distributions to transport calculations at the Yucca Mountain site. 


\section{APPENDIX B}

\section{CORRELATIONS FOR SAMPLING OF SORPTION COEFFICIENT PROBABILITY DISTRIBUTIONS}




\section{B1. CORRELATIONS FOR SAMPLING OF SORPTION COEFFICIENT PROBABILITY DISTRIBUTIONS}

In the total system performance assessment for license application, unsaturated zone (UZ) transport calculations are carried out separately for each radionuclide. The sorption-coefficient probability distribution for each radionuclide could be sampled independently in each transport calculation. However, such independent sampling could potentially lead to dose dilution. That is, independent sampling of the distributions could cause radionuclides to travel at independent rates, such that the calculated dose at the accessible environment would not be representative of maximum possible doses. Similarities in the chemical dependencies of sorption coefficients for the various radionuclides suggest transport rates in the UZ are likely to be correlated for some radionuclides.

Correlations for sampling sorption coefficient probability distributions have been derived for the elements $\mathrm{Am}, \mathrm{Cs}, \mathrm{Np}, \mathrm{Pa}, \mathrm{Pu}, \mathrm{Ra}, \mathrm{Sr}, \mathrm{Th}$, and $\mathrm{U}$. To derive the correlations, a rating system was first developed to rate the impact of six different variables on the sorption coefficient for a given element in each of the three major rocks types. The matrix containing the ratings is shown in Table B-1. The six variables are $\mathrm{pH}$, Eh, water chemistry, rock composition, rock surface area, and radionuclide concentration. Most of these parameters are self-explanatory. Water chemistry refers to the major ion concentrations and silica. Rock composition refers to both the mineralogic composition of the rocks and the chemical composition of the minerals (e.g., zeolite compositions).

The ratings are based on the sorption data and modeling presented in Section A5 of Appendix A, combined with professional judgment.

The rating system presented in Table B-1 was used to develop correlations between the sorption coefficient probability distributions for the elements of interest. Identical parameter ratings resulted in a correlation of 100 percent. If the two highest ratings were in the same parameters in the same order, a correlation of 75 percent was assigned. If the two highest ratings were in the same parameters but not in the same order, a correlation of 50 percent was assigned. If the three highest ratings were in the same parameters but not in the same order, a correlation of 25 percent was assigned. If the four highest ratings were in the same parameters but not in the same order, a correlation of 10 percent was assigned. If the three highest ratings were not in the same parameters, a correlation of zero percent was assigned. 
Table B-1. Ratings of Controls on Sorption Behavior

\begin{tabular}{|c|c|c|c|c|c|c|}
\hline Element & $\mathrm{pH}$ & Eh & $\begin{array}{c}\text { Water } \\
\text { Chemistry }\end{array}$ & $\begin{array}{l}\text { Rock } \\
\text { Comp. }\end{array}$ & $\begin{array}{c}\text { Surface } \\
\text { Area }\end{array}$ & RN Conc. \\
\hline Am (Devit.) & 2 & No & 3 & 4 & 1 & N/D \\
\hline Am (Zeol.) & 2 & No & 3 & 4 & 1 & N/D \\
\hline Am (Vitric) & 2 & No & 3 & 4 & 1 & $\mathrm{~N} / \mathrm{D}$ \\
\hline Cs (Devit.) & No & No & 3 & 4 & 2 & 1 \\
\hline Cs (Zeol.) & No & No & 2 & 1 & 3 & 4 \\
\hline Cs (Vitric) & No & No & 3 & 4 & 2 & 1 \\
\hline $\mathrm{Np}$ (Devit.) & 3 & No & 1 & 4 & 2 & 5 \\
\hline $\mathrm{Np} \quad$ (Zeol.) & 3 & No & 1 & 4 & 2 & 5 \\
\hline $\mathrm{Np}$ (Vitric) & 3 & No & 1 & 4 & 2 & 5 \\
\hline $\mathrm{Pa}$ (Devit.) & 2 & No & $N / D$ & $\mathrm{~N} / \mathrm{D}$ & 1 & N/D \\
\hline $\mathrm{Pa} \quad$ (Zeol.) & 2 & No & $N / D$ & N/D & 1 & N/D \\
\hline $\mathrm{Pa} \quad$ (Vitric) & 2 & No & $N / D$ & N/D & 1 & N/D \\
\hline $\mathrm{Pu} \quad$ (Devit.) & 4 & (No) & 2 & 1 & 3 & 5 \\
\hline $\mathrm{Pu} \quad$ (Zeol.) & 4 & (No) & 2 & 1 & 3 & 5 \\
\hline $\mathrm{Pu}$ (Vitric) & 4 & (No) & 2 & 1 & 3 & 5 \\
\hline $\mathrm{Ra}$ (Devit.) & No & No & 3 & 4 & 2 & 1 \\
\hline $\mathrm{Ra}$ (Zeol.) & No & No & 1 & 2 & 3 & 4 \\
\hline Ra (Vitric) & No & No & 3 & 4 & 2 & 1 \\
\hline Sr (Devit.) & No & No & 2 & 4 & 1 & 3 \\
\hline Sr (Zeol.) & No & No & 1 & 2 & 3 & 4 \\
\hline Sr (Vitric) & No & No & 2 & 4 & 1 & 3 \\
\hline Th (Devit.) & No & No & 2 & $\mathrm{~N} / \mathrm{D}$ & 1 & $\mathrm{~N} / \mathrm{D}$ \\
\hline Th (Zeol.) & No & No & 2 & N/D & 1 & $\mathrm{~N} / \mathrm{D}$ \\
\hline Th (Devit.) & No & No & 2 & N/D & 1 & N/D \\
\hline U (Devit.) & 2 & (No) & 1 & No & 4 & 3 \\
\hline$\cup \quad$ (Zeol.) & 2 & (No) & 1 & No & 4 & 3 \\
\hline$\cup \quad$ (Vitric) & 2 & (No) & 1 & No & 4 & 3 \\
\hline
\end{tabular}

Output DTN: LA0311AM831341.001.

NOTE: 1 = High impact, 5 = Low impact.

$(\mathrm{No})=$ No impact under oxidizing conditions. Note, however, that rock composition may provide locally reducing environments for plutonium to sorb as plutonium IV. For example, ferrous iron-bearing minerals may provide such local microenvironments.

$\mathrm{N} / \mathrm{D}=$ No data available. 
Table B-2. Correlations for Sampling Sorption Coefficient Probability Distributions

\begin{tabular}{|c|c|c|c|c|c|c|c|c|c|c|c|c|c|c|c|c|c|c|c|c|c|c|c|c|c|c|c|}
\hline Element & $\begin{array}{c}\mathrm{Am} \\
\text { (Devit). }\end{array}$ & $\begin{array}{c}\mathrm{Am} \\
\text { (Zeol.) }\end{array}$ & $\begin{array}{c}\mathrm{Am} \\
\text { (Vitric) }\end{array}$ & $\begin{array}{c}\text { Cs } \\
\text { (Devit). }\end{array}$ & $\begin{array}{c}\text { Cs } \\
\text { (Zeol.) }\end{array}$ & $\begin{array}{c}\mathrm{Cs} \\
\text { (Vitric) }\end{array}$ & $\begin{array}{c}\mathrm{Np} \\
\text { (Devit.) }\end{array}$ & $\begin{array}{c}\mathrm{Np} \\
\text { (Zeol.) }\end{array}$ & $\begin{array}{c}\mathrm{Np} \\
\text { (Vitric) }\end{array}$ & $\begin{array}{c}\mathrm{Pa} \\
\text { (Devit). }\end{array}$ & $\begin{array}{c}\mathrm{Pa} \\
\text { (Zeol.) }\end{array}$ & $\begin{array}{c}\mathrm{Pa} \\
\text { (Vitric) }\end{array}$ & $\begin{array}{c}\mathrm{Pu} \\
\text { (Devit). }\end{array}$ & $\begin{array}{c}\mathrm{Pu} \\
\text { (Zeool.) }\end{array}$ & $\begin{array}{c}\mathrm{Pu} \\
\text { (Vitric) }\end{array}$ & $\begin{array}{c}\mathrm{Ra} \\
\text { (Devit). }\end{array}$ & $\begin{array}{c}\mathrm{Ra} \\
\text { (Zeol.). }\end{array}$ & $\begin{array}{c}\mathrm{Ra} \\
\text { (Vitric) }\end{array}$ & $\begin{array}{c}\mathrm{Sr} \\
\text { (Devit.) }\end{array}$ & $\begin{array}{c}\mathrm{Sr} \\
\text { (Zeol.) }\end{array}$ & $\begin{array}{c}\mathrm{Sr} \\
\text { (vitric) }\end{array}$ & $\begin{array}{c}\mathrm{Th} \\
\text { (Devit). }\end{array}$ & $\begin{array}{c}\text { Th } \\
\text { (Zeool.) }\end{array}$ & $\begin{array}{c}\mathrm{Th} \\
\text { (Vitric) }\end{array}$ & $\begin{array}{c}u \\
\text { (Devit.) }\end{array}$ & $\begin{array}{c}\text { U } \\
\text { (zeol.) }\end{array}$ & $\underset{\text { (Vitric) }}{\mathrm{U}}$ \\
\hline 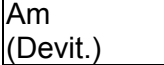 & 100 & & & & & & & & & & & & & & & & & & & & & & & & & & \\
\hline $\begin{array}{l}\text { Am } \\
\text { (Zeol.) }\end{array}$ & 100 & 100 & & & & & & & & & & & & & & & & & & & & & & & & & \\
\hline Amm & 100 & 100 & 100 & & & & & & & & & & & & & & & & & & & & & & & & \\
\hline $\mid \begin{array}{l}\text { Cs } \\
\text { (Devit.) }\end{array}$ & 0 & 0 & 0 & 100 & & & & & & & & & & & & & & & & & & & & & & & \\
\hline $\begin{array}{l}\text { Cos } \\
\text { (Zeol.) }\end{array}$ & 0 & 0 & 0 & 10 & 100 & & & & & & & & & & & & & & & & & & & & & & \\
\hline $\begin{array}{l}\text { CS } \\
\text { (Vitric) }\end{array}$ & 0 & 0 & 0 & 100 & 10 & 100 & & & & & & & & & & & & & & & & & & & & & \\
\hline Np (Devit.) & 25 & 25 & 25 & 0 & 0 & 0 & 100 & & & & & & & & & & & & & & & & & & & & \\
\hline $\begin{array}{l}\text { Nop } \\
\text { (Zeol.) }\end{array}$ & 25 & 25 & 25 & 0 & 0 & 0 & 100 & 100 & & & & & & & & & & & & & & & & & & & \\
\hline $\begin{array}{l}\text { No } \\
\text { (Vitric) }\end{array}$ & 25 & 25 & 25 & 0 & 0 & 0 & 100 & 100 & 100 & & & & & & & & & & & & & & & & & & \\
\hline $\begin{array}{l}\left(\begin{array}{l}\text { a } \\
\text { (Devit.) }\end{array}\right. \\
\text { (a) }\end{array}$ & 75 & 75 & 75 & 0 & 0 & 0 & 0 & 0 & 0 & 100 & & & & & & & & & & & & & & & & & \\
\hline $\begin{array}{l}\text { la } \\
\text { (zeol.) }\end{array}$ & 75 & 75 & 75 & 0 & 0 & 0 & 0 & 0 & 0 & 100 & 100 & & & & & & & & & & & & & & & & \\
\hline 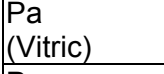 & 75 & 75 & 75 & 0 & 0 & 0 & 0 & 0 & 0 & 100 & 100 & 100 & & & & & & & & & & & & & & & \\
\hline $\begin{array}{l}\text { 保 } \\
\text { (Devit.) }\end{array}$ & 10 & 10 & 10 & 0 & 75 & 0 & 10 & 10 & 10 & 0 & 0 & 0 & 100 & & & & & & & & & & & & & & \\
\hline $\begin{array}{l}\text { Pu } \\
\text { (zeol.) }\end{array}$ & 10 & 10 & 10 & 0 & 75 & 0 & 10 & 10 & 10 & 0 & 0 & 0 & 100 & 100 & & & & & & & & & & & & & \\
\hline $\begin{array}{l}\text { Pu } \\
\text { (Vitric) }\end{array}$ & 10 & 10 & 10 & 0 & 75 & 0 & 10 & 10 & 10 & 0 & 0 & 0 & 100 & 100 & 100 & & & & & & & & & & & & \\
\hline $\begin{array}{l}\text { Ra } \\
\text { (Devit.) }\end{array}$ & 0 & 0 & 0 & 100 & 10 & 100 & 0 & 0 & 0 & 0 & 0 & 0 & 0 & 0 & 0 & 100 & & & & & & & & & & & \\
\hline $\begin{array}{l}\text { Ra } \\
\text { (Zeol.) }\end{array}$ & 0 & 0 & 0 & 10 & 50 & 10 & 0 & 0 & 0 & 0 & 0 & 0 & 50 & 50 & 50 & 10 & 100 & & & & & & & & & & \\
\hline $\begin{array}{l}\text { Ra } \\
\text { (vitric) }\end{array}$ & 0 & 0 & 0 & 100 & 10 & 100 & 0 & 0 & 0 & 0 & 0 & 0 & 0 & 0 & 0 & 100 & 10 & 100 & & & & & & & & & \\
\hline $\mid \begin{array}{l}\text { Sr } \\
\text { (Devit.) }\end{array}$ & 0 & 0 & 0 & 25 & 10 & 25 & 50 & 50 & 50 & 0 & 0 & 0 & 0 & 0 & 0 & 25 & 10 & 25 & 100 & & & & & & & & \\
\hline $\begin{array}{l}\text { Sr } \\
\text { (Zeol.) }\end{array}$ & 0 & 0 & 0 & 10 & 50 & 10 & 0 & 0 & 0 & 0 & 0 & 0 & 50 & 50 & 50 & 10 & 100 & 10 & 10 & 100 & & & & & & & \\
\hline 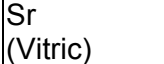 & 0 & 0 & 0 & 25 & 10 & 25 & 50 & 50 & 50 & 0 & 0 & 0 & 0 & 0 & 0 & 25 & 10 & 25 & 100 & 10 & 100 & & & & & & \\
\hline \begin{tabular}{|l|l} 
Th \\
(Devit).
\end{tabular} & 0 & 0 & 0 & 0 & 0 & 0 & 50 & 50 & 50 & 0 & 0 & 0 & 0 & 0 & 0 & 0 & 0 & 0 & 75 & 0 & 75 & 100 & & & & & \\
\hline $\begin{array}{l}\text { Th } \\
\text { (Zeol.) }\end{array}$ & 0 & 0 & 0 & 0 & 0 & 0 & 50 & 50 & 50 & 0 & 0 & 0 & 0 & 0 & 0 & 0 & 0 & 0 & 75 & 0 & 75 & 100 & 100 & & & & \\
\hline $\begin{array}{l}\text { Tht } \\
\text { (Vitric) }\end{array}$ & 0 & 0 & 0 & 0 & 0 & 0 & 50 & 50 & 50 & 0 & 0 & 0 & 0 & 0 & 0 & 0 & 0 & 0 & 75 & 0 & 75 & 100 & 100 & 100 & & & \\
\hline (Devit.) & 0 & 0 & 0 & 0 & 0 & 0 & 0 & 0 & 0 & 0 & 0 & 0 & 0 & 0 & 0 & 0 & 0 & 0 & 0 & 0 & 0 & 0 & 0 & 0 & 100 & & \\
\hline (Zeol.) & 0 & 0 & 0 & 0 & 0 & 0 & 0 & 0 & 0 & 0 & 0 & 0 & 0 & 0 & 0 & 0 & 0 & 0 & 0 & 0 & 0 & 0 & 0 & 0 & 100 & 100 & \\
\hline U U & 0 & 0 & 0 & 0 & 0 & 0 & 0 & 0 & 0 & 0 & 0 & 0 & 0 & 0 & 0 & 0 & 0 & 0 & 0 & 0 & 0 & 0 & 0 & 0 & 100 & 100 & 100 \\
\hline
\end{tabular}

Output DTN: LA0311AM831341.001.

NOTE: Numbers in table are percentages. 
INTENTIONALLY LEFT BLANK 


\section{B2. CORRELATION OF SAMPLING OF SORPTION-COEFFICIENT PROBABILITY DISTRIBUTIONS BETWEEN THE UNSATURATED ZONE AND SATURATED ZONE}

The sampling of sorption coefficient probability distributions for the UZ should not be correlated with sampling of sorption coefficient probability distributions for the saturated zone (SZ) except possibly for the elements radium and cesium in devitrified tuffs. This conclusion is based on an analysis of variations in the parameters that have the greatest impact on the sorption behavior of the radionuclides of interest (i.e., $\mathrm{pH}$, $\mathrm{Eh}$, water chemistry, rock composition, radionuclide concentration). This analysis is discussed in the following.

- Parameter: $\mathrm{pH}$

Beneath the repository footprint, variation in $\mathrm{pH}$ in $\mathrm{SZ}$ groundwater is limited (7.0 to 7.2). This is likely to be the case in any climate scenario, because the primary control on $\mathrm{pH}$ is elevated $P_{\mathrm{CO}_{2}}(\log =-2.0)$ maintained from below (i.e., Paleozoic aquifer).

Outside the repository footprint, $\mathrm{pH}$ shows somewhat greater variation (7.0 to 7.5). In the $\mathrm{UZ}$, the $\mathrm{pH}$ of pore water is more variable, particularly in zeolitic units. There is no evidence that the $\mathrm{pH}$ of SZ groundwaters are correlated with the $\mathrm{pH}$ of UZ pore waters or perched waters. Therefore, there is no reason to correlate the sorption-coefficient probability distributions based on $\mathrm{pH}$.

- Parameter: Eh

In the UZ, Eh will always be oxidizing. In the SZ, Eh can be reducing or oxidizing. Therefore, there is no reason to correlate the sorption-coefficient probability distributions based on Eh.

- Parameter: Water Chemistry (i.e., ionic strength, alkalinity)

SZ appears to have groundwater chemistry of glacial times. UZ pore water has chemistry of an arid climate. Further, UZ water chemistry appears to be layered, with variations mainly in the vertical direction. There is no evidence that UZ pore-water chemistry is areally correlated with SZ groundwater chemistry. Therefore, there is no reason to correlate the sorption-coefficient probability distributions based on water chemistry.

- Parameter: Rock Composition

Rock composition (e.g., zeolite compositions) is known to be variable horizontally and vertically in both the UZ and SZ. There is no evidence that rock compositions in the UZ are areally correlated with rock compositions in the SZ within the repository footprint. In fact, rock compositions tend to be layered, with variations primarily in the vertical direction. Therefore, there is no reason to correlate the sorption coefficient probability distributions based on rock composition. 
- Parameter: Surface Area

Rock surface area (see Table A-1 of Appendix A, this model report) will not change significantly over the regulatory time frame. There is no evidence that rock surface area in the UZ is correlated areally with rock surface area in the SZ. This also reflects the horizontal layering of rock compositions. Therefore, there is no reason to correlate the sorption coefficient probability distributions based on rock surface area.

- Parameter: Radionuclide Concentration

If the concentration of a radionuclide in the $\mathrm{UZ}$ is on the high end of the range, the concentration of the radionuclide in the SZ will also be on the high end of the range. Thus, there is a reason to correlate the sorption-coefficient probability distributions. However, radionuclide concentrations are the dominant control on radionuclide sorption behavior only for radium and cesium on devitrified and vitric tuffs (Table B-1). Thus, it would seem appropriate to correlate the sampling of sorption-coefficient distributions for these two elements in these two rock types. However, vitric tuffs are not a component of the saturated zone. Thus, only sorption-coefficient probability distributions for cesium and radium on devitrified tuff in the UZ and the saturated zone might need to be correlated. 
APPENDIX C

SUPPORTING GEOLOGICAL DATA FOR THE CONCEPTUAL MODEL 


\section{C1. SUPPORTING GEOLOGICAL DATA FOR THE CONCEPTUAL MODEL}

Table C-1. Thickness and Elevation of Hydrogeologic Units in the Vertical Column on the Location of USW SD-6 Borehole.

\begin{tabular}{|c|c|c|}
\hline Hydrogeologic unit & Thickness (meters) & Elevation (meters) \\
\hline tsw36 & 30.7 & 1090.0 \\
\hline tsw37 & 14.8 & 1059.3 \\
\hline tsw38 & 9.8 & 1044.5 \\
\hline tsw39 & 4.6 & 1034.7 \\
\hline ch1v & 14.4 & 1030.1 \\
\hline ch2v & 7.8 & 1015.7 \\
\hline ch3v & 7.9 & 1007.9 \\
\hline ch4v & 7.8 & 1000.0 \\
\hline ch5v & 11.7 & 992.2 \\
\hline ch6v & 11.6 & 980.5 \\
\hline$p p 4 z$ & 10.5 & 968.9 \\
\hline$p p 3 d$ & 39.1 & 958.4 \\
\hline$p p 2 d$ & 13.7 & 919.3 \\
\hline$p p 1 z$ & 66.8 & 905.7 \\
\hline bf3d & 86.9 (to water table) & 838.9 \\
\hline
\end{tabular}

Sources: DTN: MO9906GPS98410.000 [DIRS 109059]; DTN: LB03023DSSCP9I.001 [DIRS 163044].

Table C-2. Thickness and Elevation of Hydrogeologic Units in the Vertical Column on the Location of USW SD-12 Borehole.

\begin{tabular}{|c|c|c|}
\hline Hydrogeologic unit & Thickness (meters) & Elevation (meters) \\
\hline tsw34 & 17.9 & 1093.2 \\
\hline tsw35 & 83.7 & 1075.3 \\
\hline tsw36 & 41.4 & 991.6 \\
\hline tsw37 & 20.7 & 950.3 \\
\hline tsw38 & 9.1 & 929.6 \\
\hline tsw39 & 6.8 & 920.5 \\
\hline ch1z & 4.5 & 913.7 \\
\hline ch1v & 22.9 & 909.2 \\
\hline ch2v & 14.3 & 886.3 \\
\hline ch3v & 14.3 & 872.0 \\
\hline ch4z & 14.3 & 857.6 \\
\hline ch5v & 14.7 & 843.3 \\
\hline pp4z & 11.8 & 828.7 \\
\hline pp3d & 12.7 & 816.9 \\
\hline pp2d & 31.1 & 804.1 \\
\hline bf3d & 24.1 & 773.0 \\
\hline
\end{tabular}

Sources: DTN: MO9906GPS98410.000 DIRS 109059]; DTN: LB03023DSSCP9I.001 [DIRS 163044]. 
Table C-3. Thickness and Elevation of Hydrogeologic Units in the Vertical Column on the Location of USW UZ-14 Borehole.

\begin{tabular}{|l|l|l|}
\hline \multicolumn{1}{|c|}{ Hydrogeologic unit } & \multicolumn{1}{c|}{ Thickness (meters) } & \multicolumn{1}{c|}{ Elevation (meters) } \\
\hline tsw35 & 56.0 & 1050.0 \\
\hline tsw36 & 28.2 & 994.5 \\
\hline tsw37 & 13.0 & 966.3 \\
\hline tsw38 & 21.6 & 953.4 \\
\hline tsw39 & 5.7 & 931.7 \\
\hline ch1z & 15.0 & 926.0 \\
\hline ch2z & 21.1 & 911.0 \\
\hline ch3z & 21.0 & 890.0 \\
\hline ch4z & 21.1 & 868.9 \\
\hline ch5z & 24.4 & 847.9 \\
\hline ch6z & 18.5 & 823.5 \\
\hline pp4z & 15.3 & 805.0 \\
\hline pp3d & 7.4 & 789.6 \\
\hline pp2d & 1.9 (to water table) & 782.3 \\
\hline
\end{tabular}

Sources: DTN: MO9906GPS98410.000 [DIRS 109059]; DTN: LB03023DSSCP9I.001 [DIRS 163044]. 


\section{APPENDIX D}

FIGURES FROM THE ${ }^{237}$ Np THREE-DIMENSIONAL TRANSPORT STUDIES (INSTANTANEOUS RELEASE, MEAN PRESENT-DAY INFILTRATION)

OUTPUT DTN: LB0307MR0060R1.002 


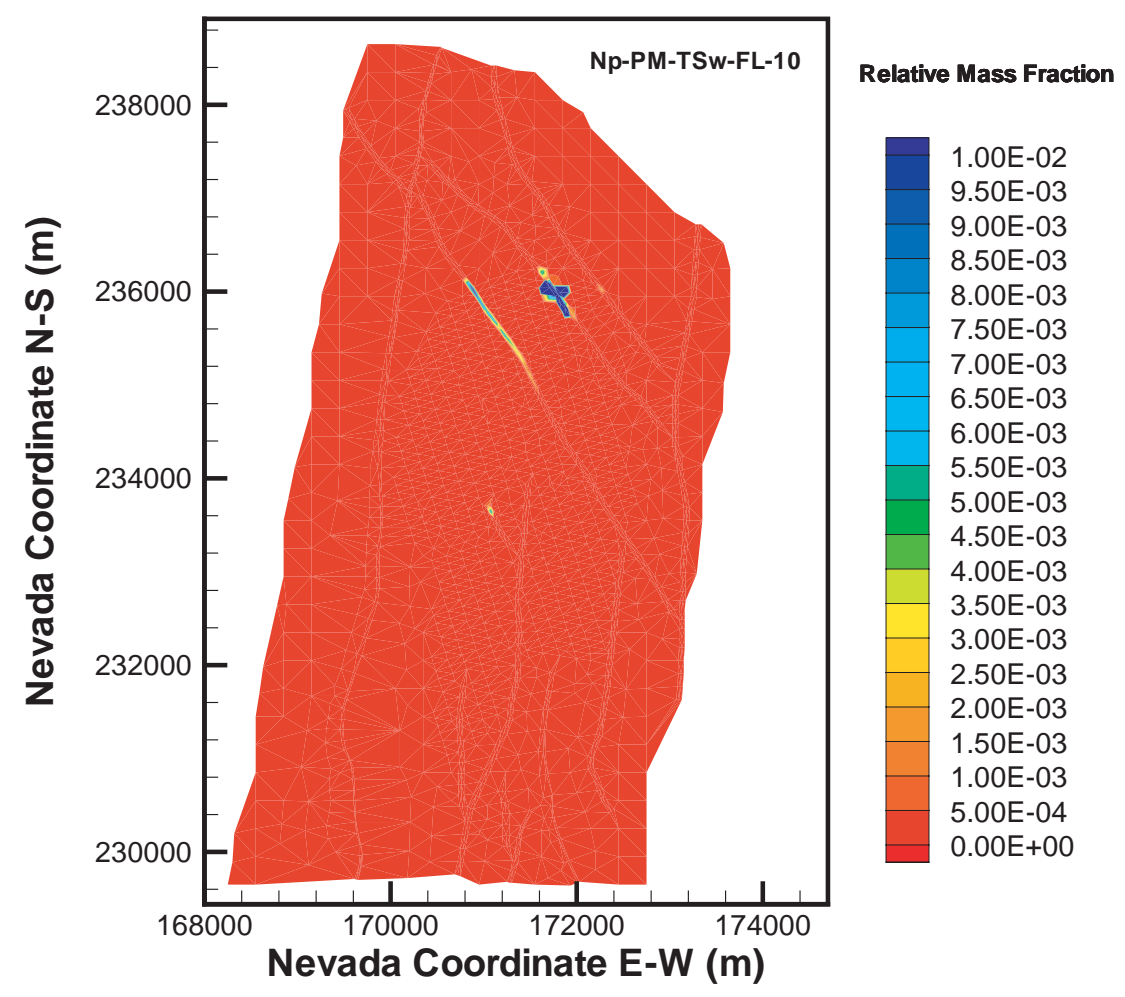

Output DTN: LB0307MR0060R1.002.

Figure D-1. Distribution of the Relative Mass Fraction $X_{R}$ of ${ }^{237} \mathrm{~Np}$ in the Fractures of the tsw39 Layer at $t=10$ Years for Mean Present-Day Infiltration and Instantaneous Release 


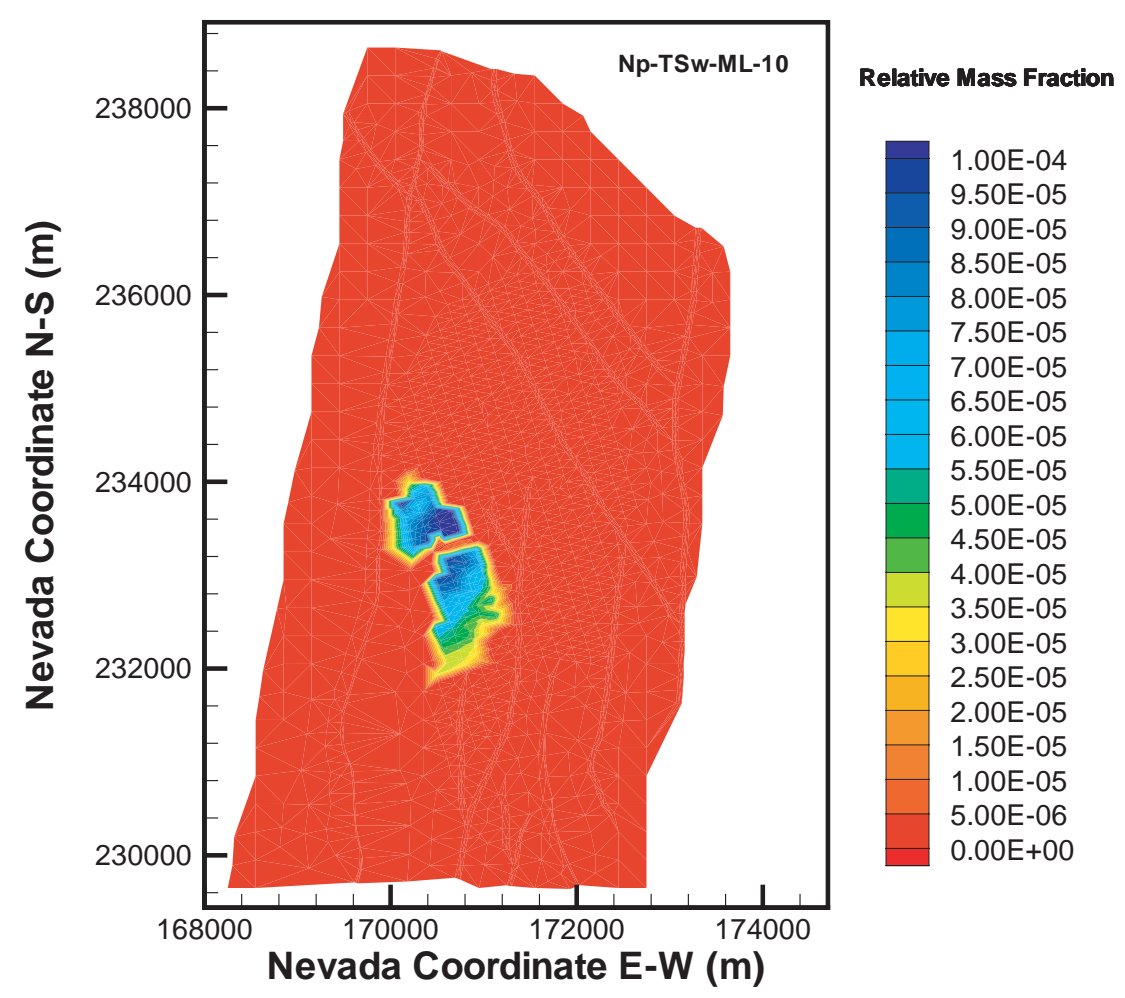

Output DTN: LB0307MR0060R1.002.

Figure D-2. Distribution of the Relative Mass Fraction $X_{R}$ of ${ }^{237} \mathrm{~Np}$ in the Matrix of the tsw39 layer at $t=$ 10 Years for Mean Present-Day Infiltration and Instantaneous Release 


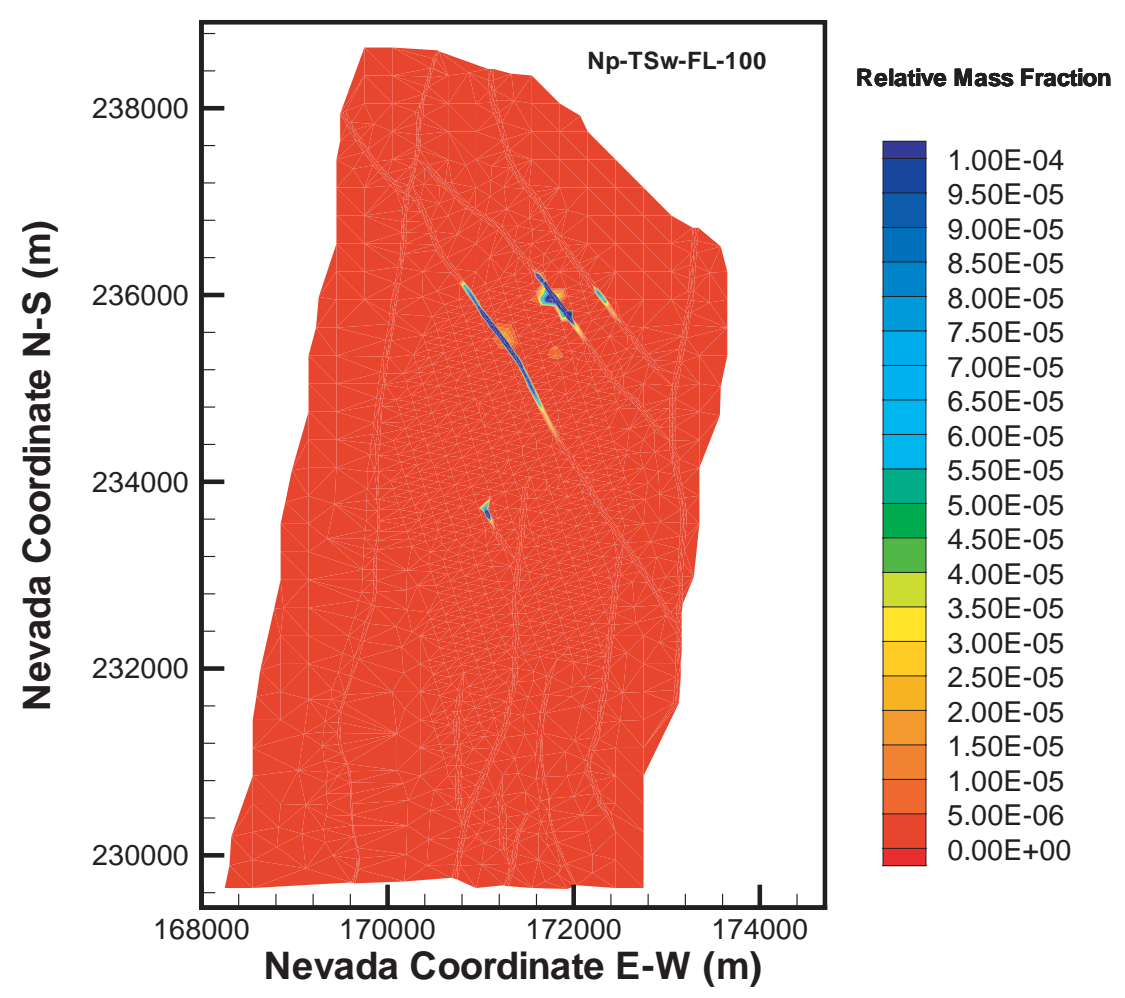

Output DTN: LB0307MR0060R1.002.

Figure D-3. Distribution of the Relative Mass Fraction $X_{R}$ of ${ }^{237} \mathrm{~Np}$ in the Fractures of the tsw39 Layer at $t=100$ years for Mean Present-Day Infiltration and Instantaneous Release 


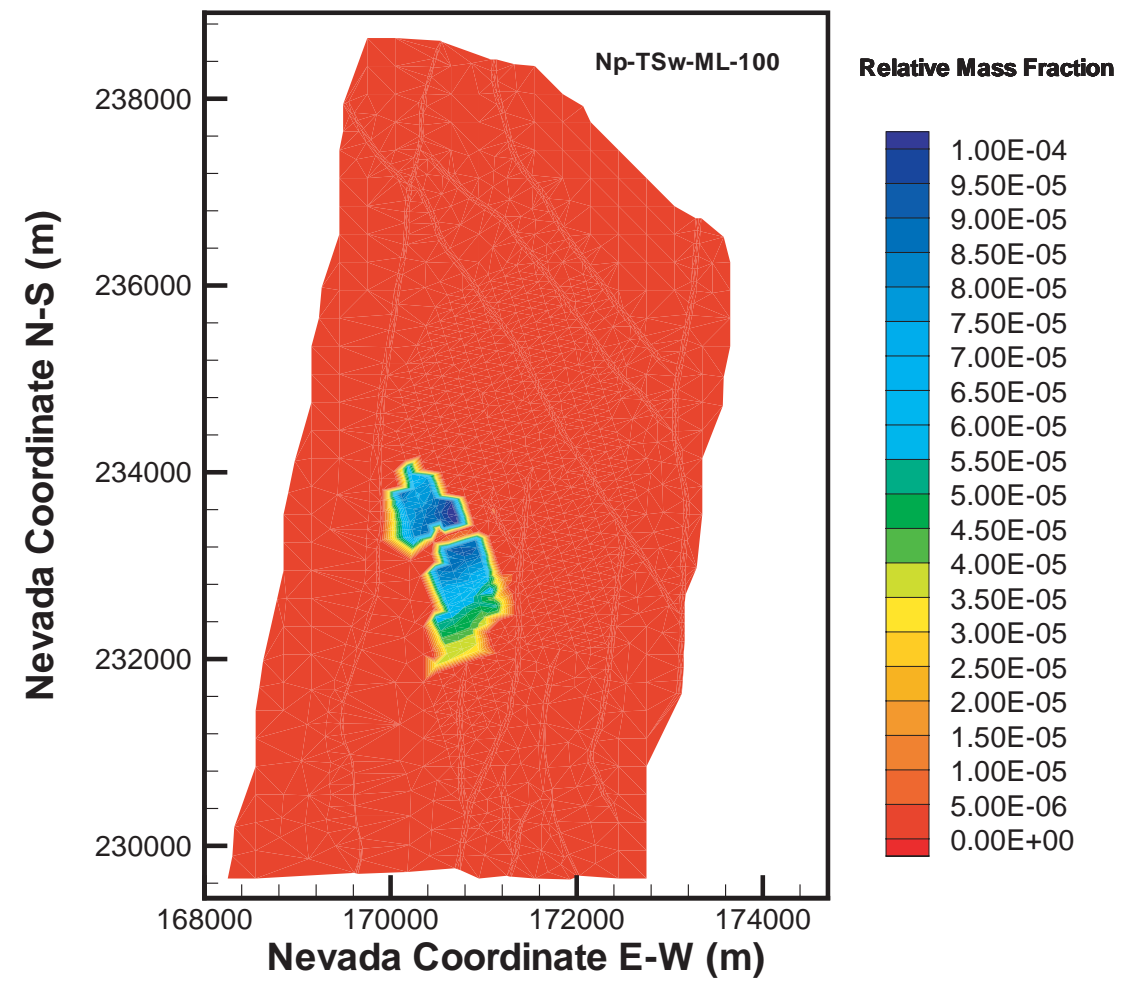

Output DTN: LB0307MR0060R1.002.

Figure D-4. Distribution of the Relative Mass Fraction $X_{R}$ of ${ }^{237} \mathrm{~Np}$ in the Matrix of the tsw39 Layer at $t=$ 100 years for Mean Present-Day Infiltration and Instantaneous Release 


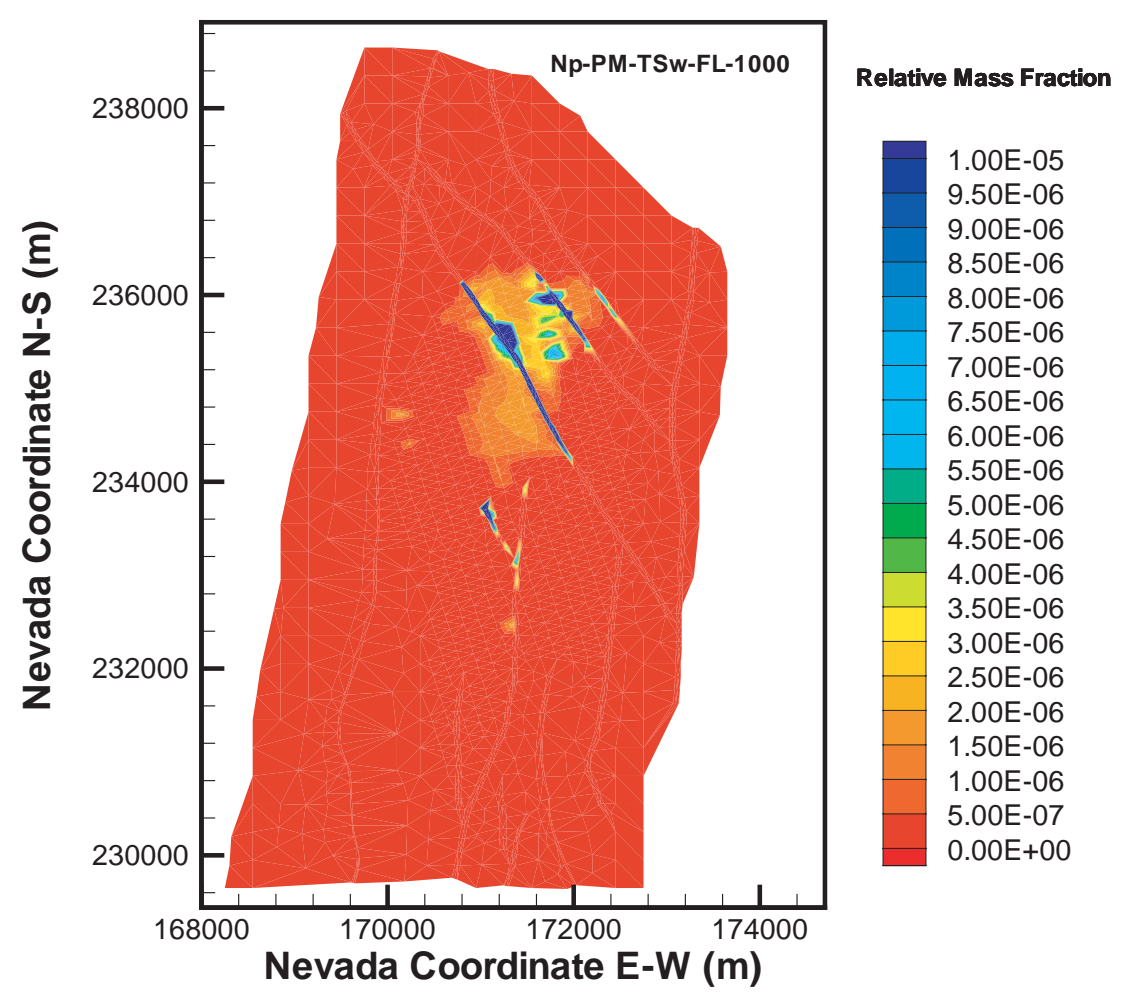

Output DTN: LB0307MR0060R1.002.

Figure D-5. Distribution of the Relative Mass Fraction $X_{R}$ of ${ }^{237} \mathrm{~Np}$ in the Fractures of the tsw39 layer at $t=1,000$ years for Mean Present-Day Infiltration and Instantaneous Release 


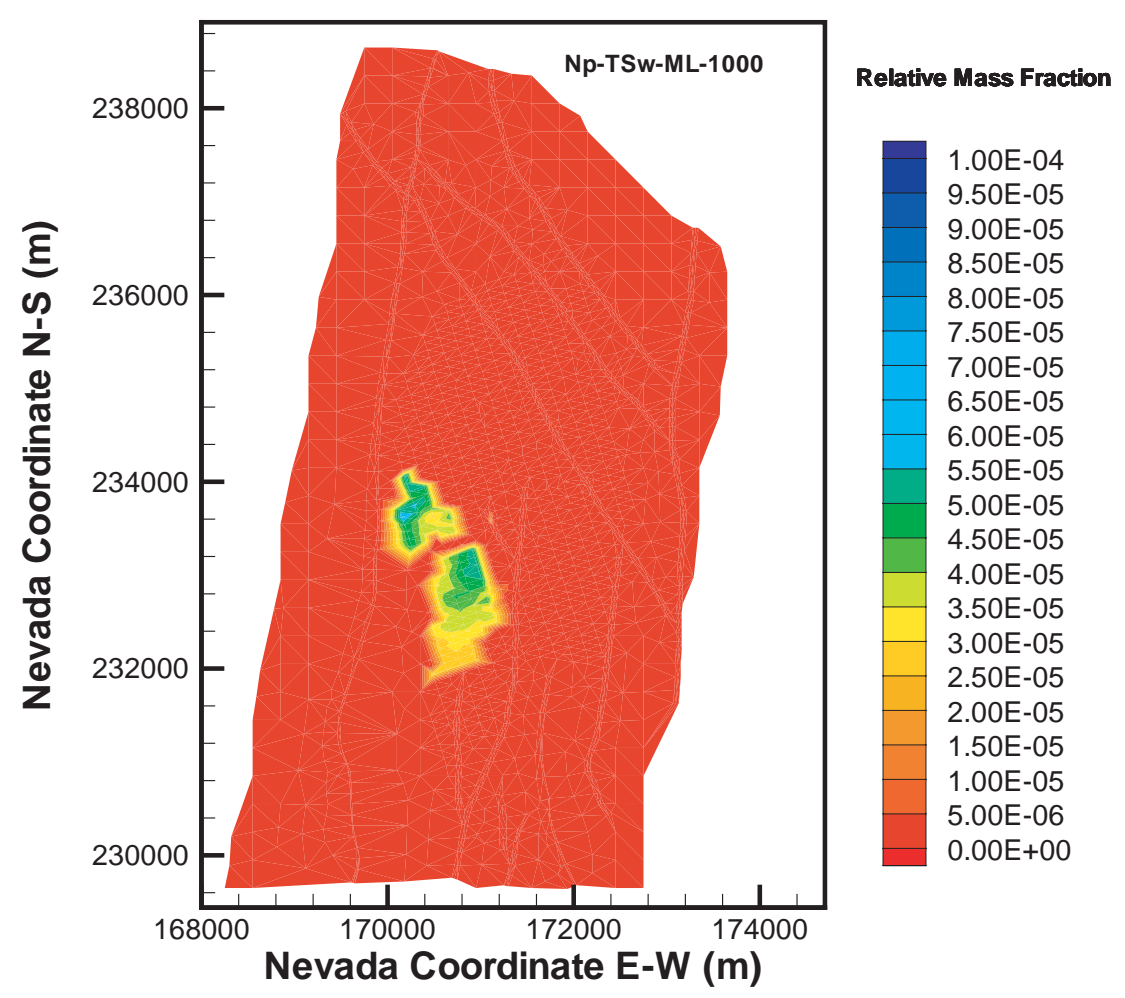

Output DTN: LB0307MR0060R1.002.

Figure D-6. Distribution of the Relative Mass Fraction $X_{R}$ of ${ }^{237} \mathrm{~Np}$ in the Matrix of the tsw39 Layer at $t=1,000$ Years for Mean Present-Day Infiltration and Instantaneous Release 


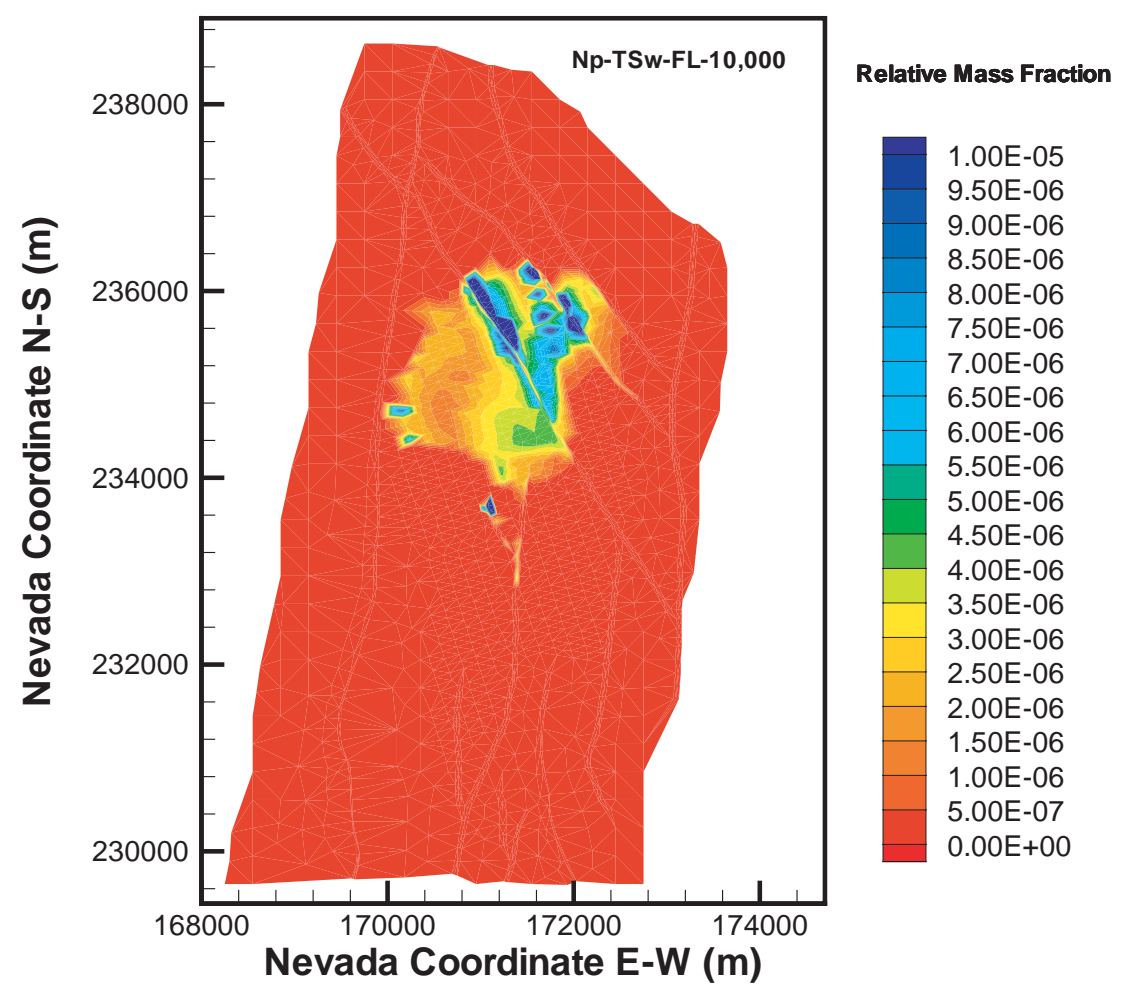

Output DTN: LB0307MR0060R1.002.

Figure D-7. Distribution of the Relative Mass Fraction $X_{R}$ of ${ }^{237} \mathrm{~Np}$ in the Fractures of the tsw39 Layer at $t=10,000$ Years for Mean Present-Day Infiltration and Instantaneous Release 


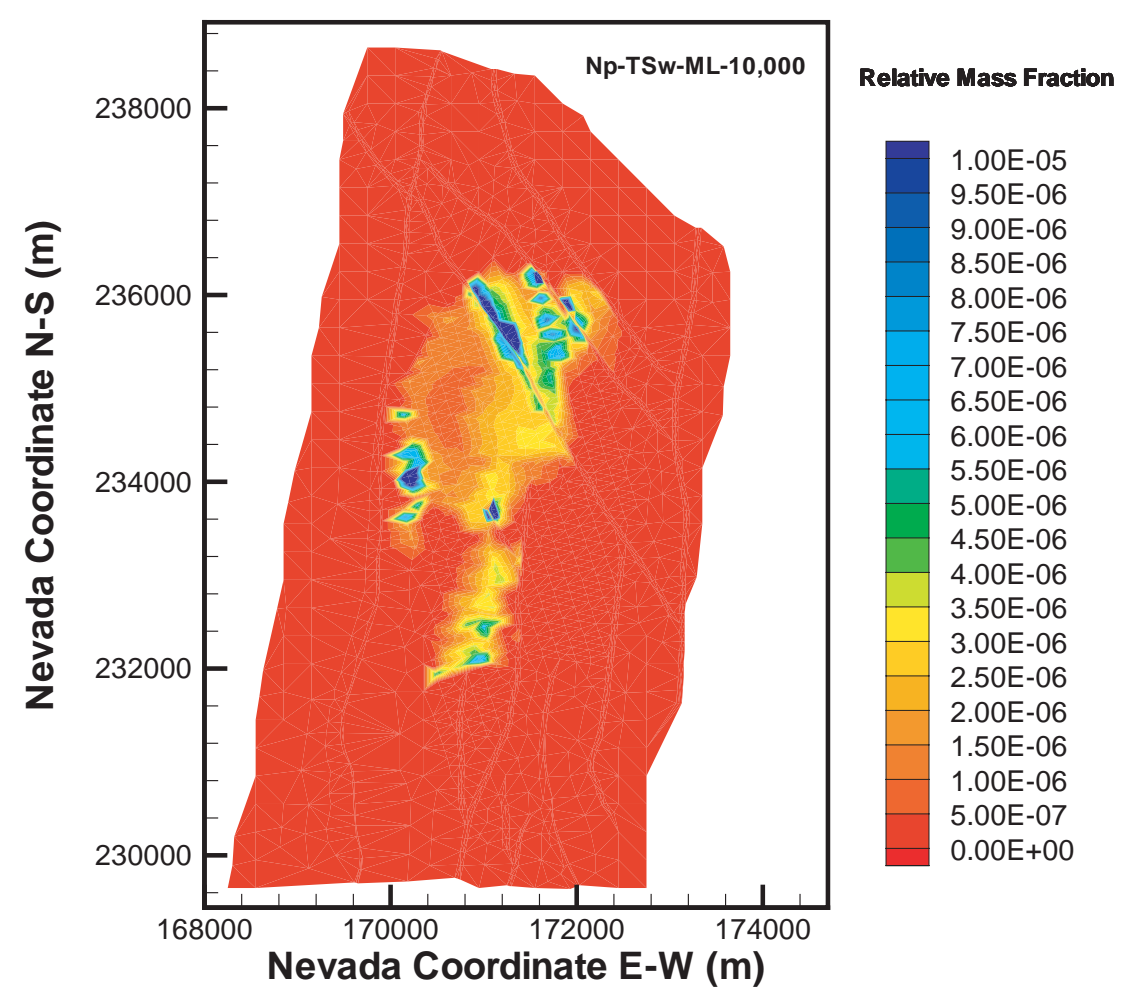

Output DTN: LB0307MR0060R1.002.

Figure D-8. Distribution of the Relative Mass Fraction $X_{R}$ of ${ }^{237} \mathrm{~Np}$ in the Matrix of the tsw39 Layer at $t=10,000$ Years for Mean Present-Day Infiltration and Instantaneous Release 


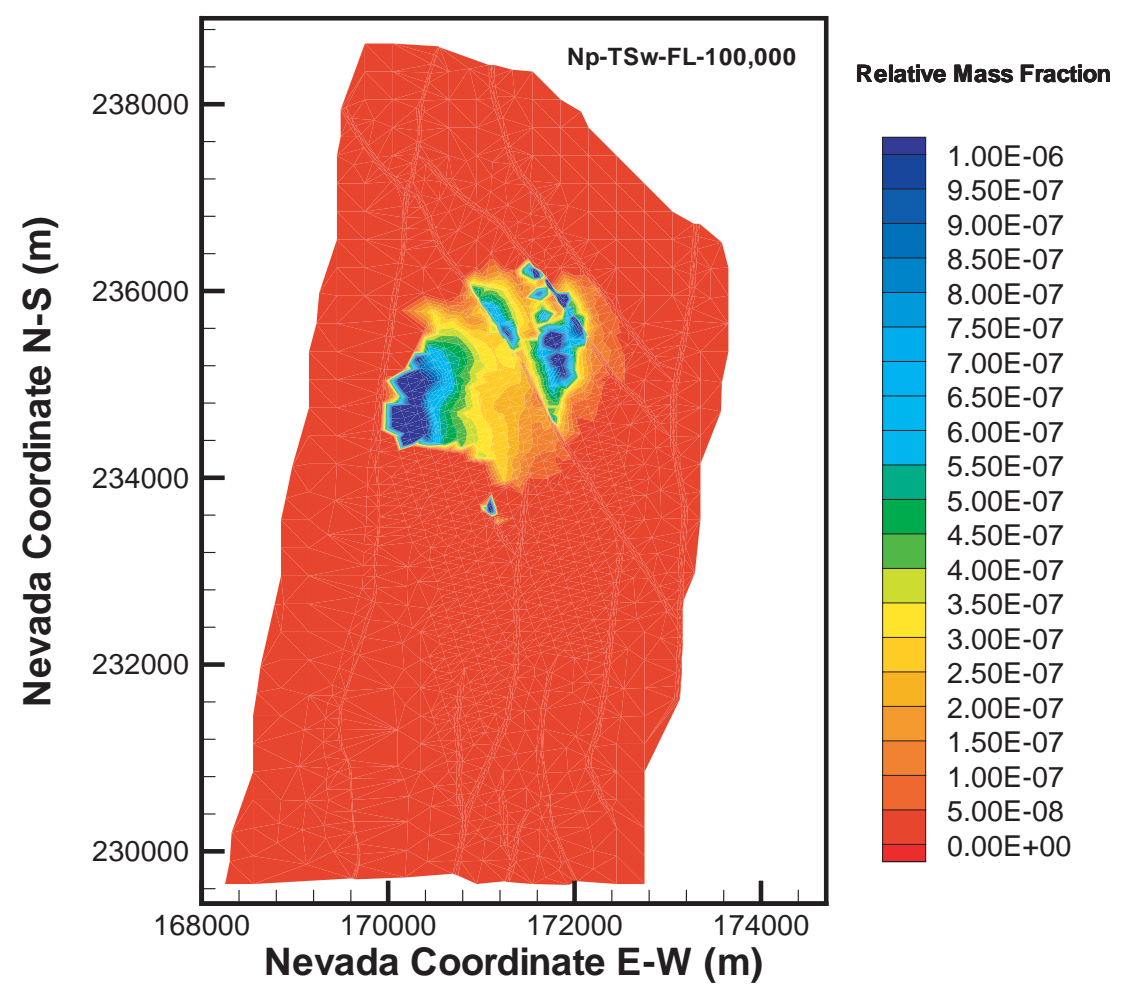

Output DTN: LB0307MR0060R1.002.

Figure D-9. Distribution of the Relative Mass Fraction $X_{R}$ of ${ }^{237} \mathrm{~Np}$ in the Fractures of the tsw39 Layer at $t=100,000$ Years for Mean Present-Day Infiltration and Instantaneous Release 


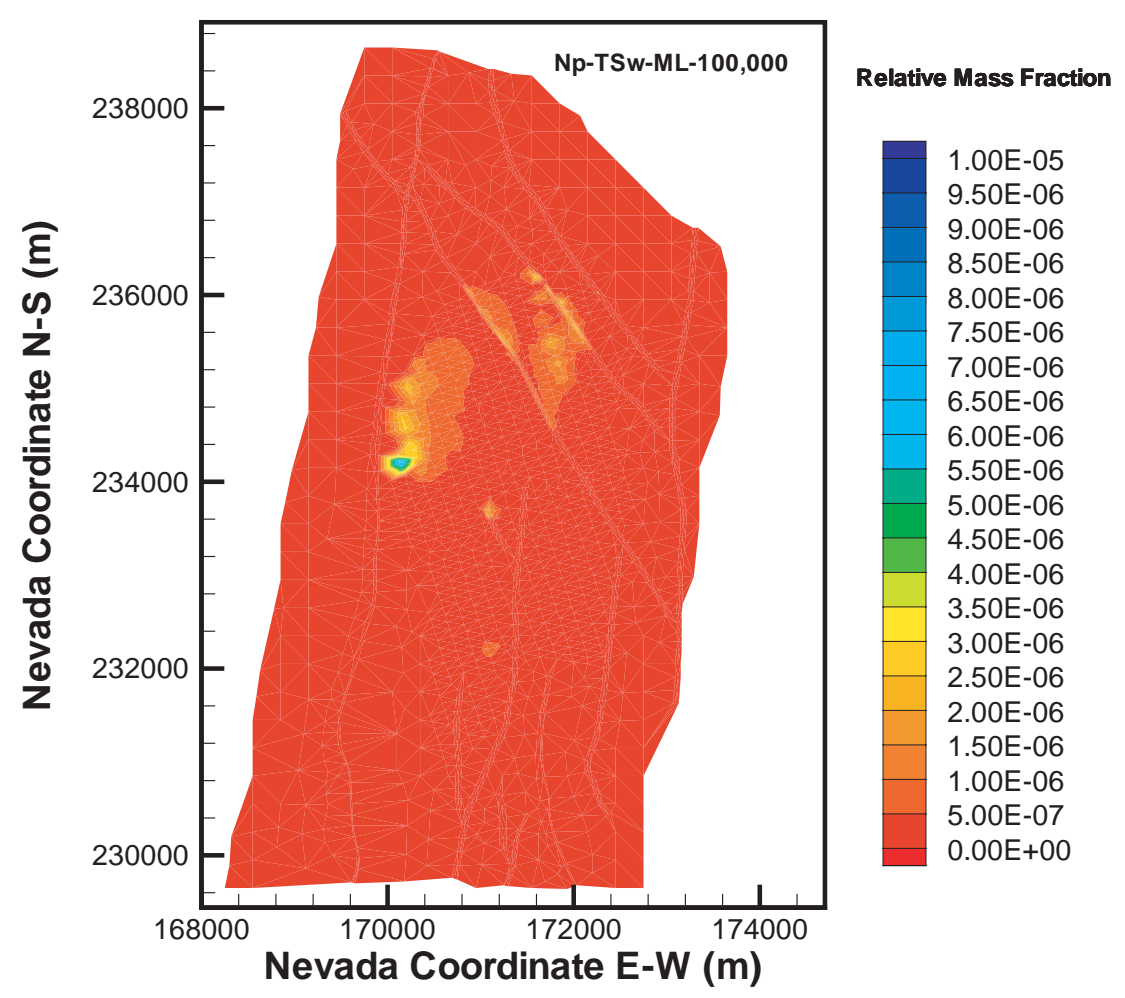

Output DTN: LB0307MR0060R1.002.

Figure D-10. Distribution of the Relative Mass Fraction $X_{R}$ of ${ }^{237} \mathrm{~Np}$ in the Matrix of the tsw39 Layer at $t=100,000$ Years for Mean Present-Day Infiltration and Instantaneous Release 


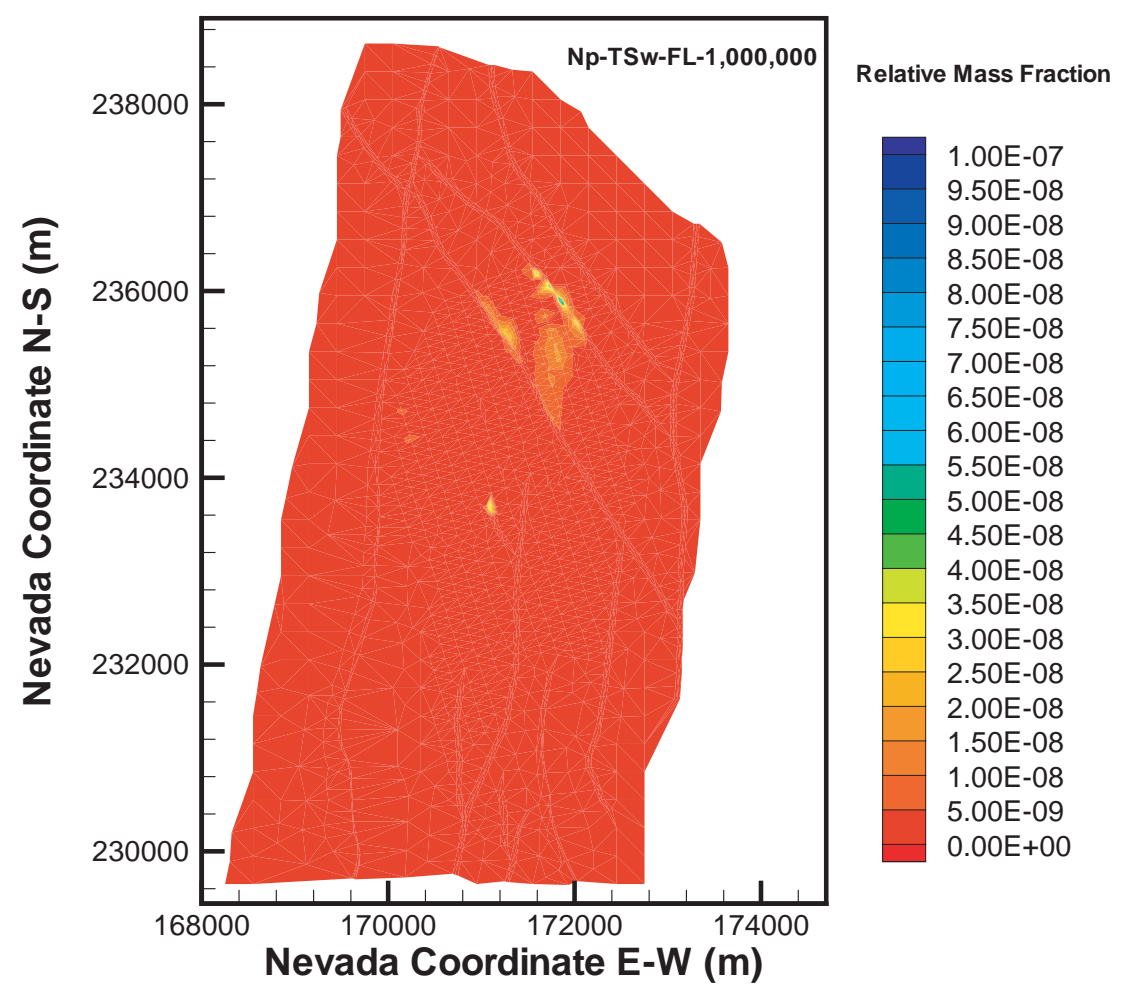

Output DTN: LB0307MR0060R1.002.

Figure D-11. Distribution of the Relative Mass Fraction $X_{R}$ of ${ }^{237} \mathrm{~Np}$ in the Fractures of the tsw39 Layer at $t=1,000,000$ Years for Mean Present-Day Infiltration and Instantaneous Release 


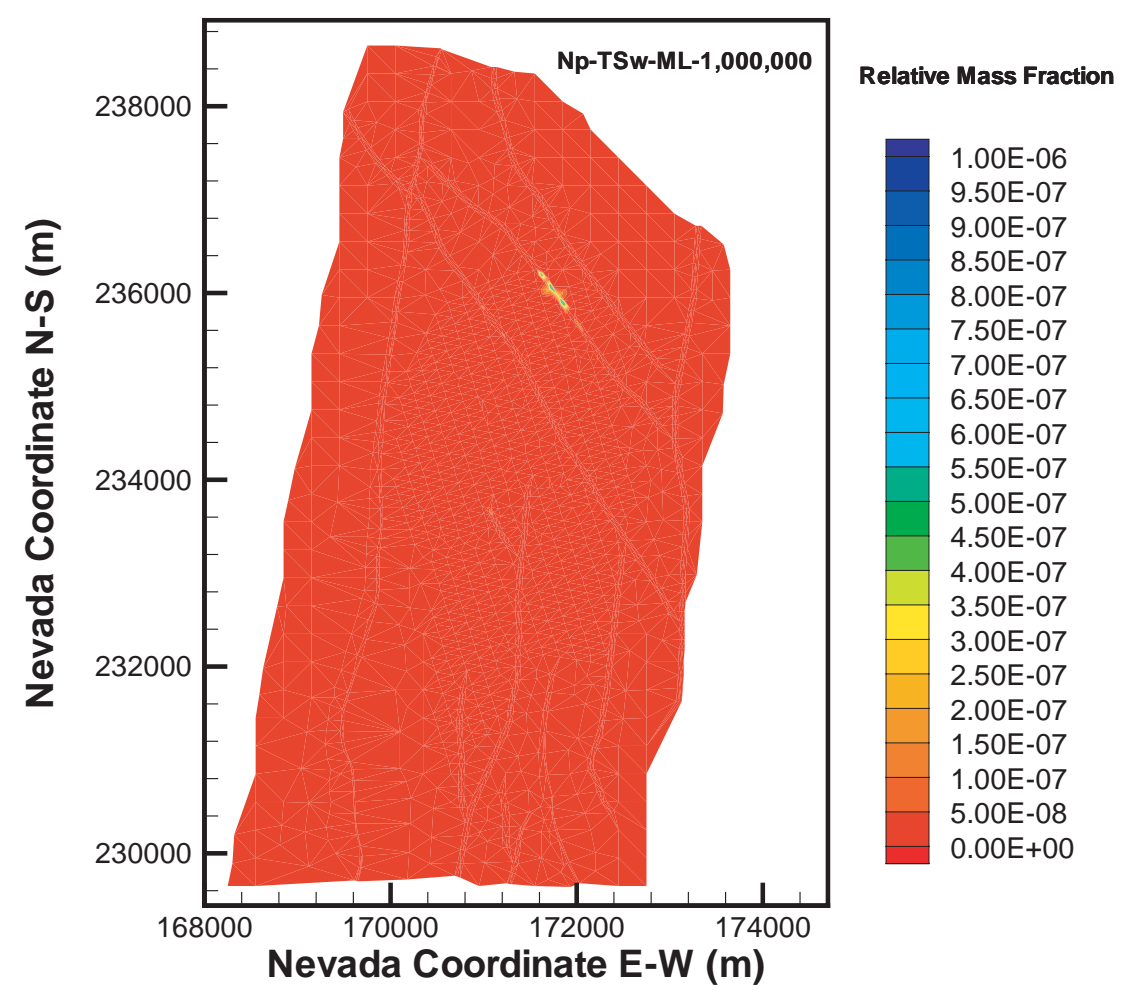

Output DTN: LB0307MR0060R1.002.

Figure D-12. Distribution of the Relative Mass Fraction $X_{R}$ of ${ }^{237} \mathrm{~Np}$ in the Matrix of the tsw39 Layer at $t=1,000,000$ Years for Mean Present-Day Infiltration and Instantaneous Release 


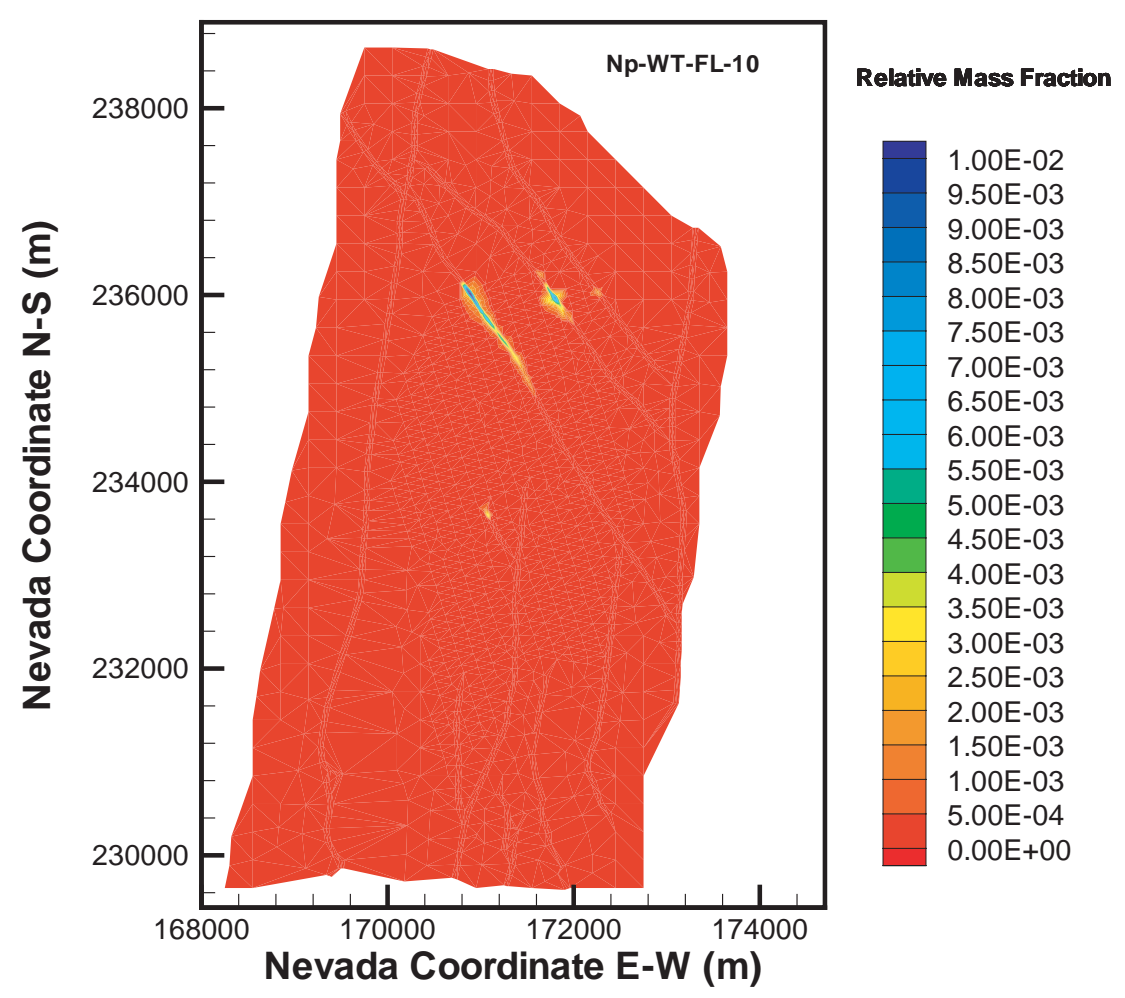

Output DTN: LB0307MR0060R1.002.

Figure D-13. Distribution of the Relative Mass Fraction $X_{R}$ of ${ }^{237} \mathrm{~Np}$ in the Fractures Immediately above the Groundwater Table at $\mathrm{t}=10$ Years for Mean Present-Day Infiltration and Instantaneous Release 


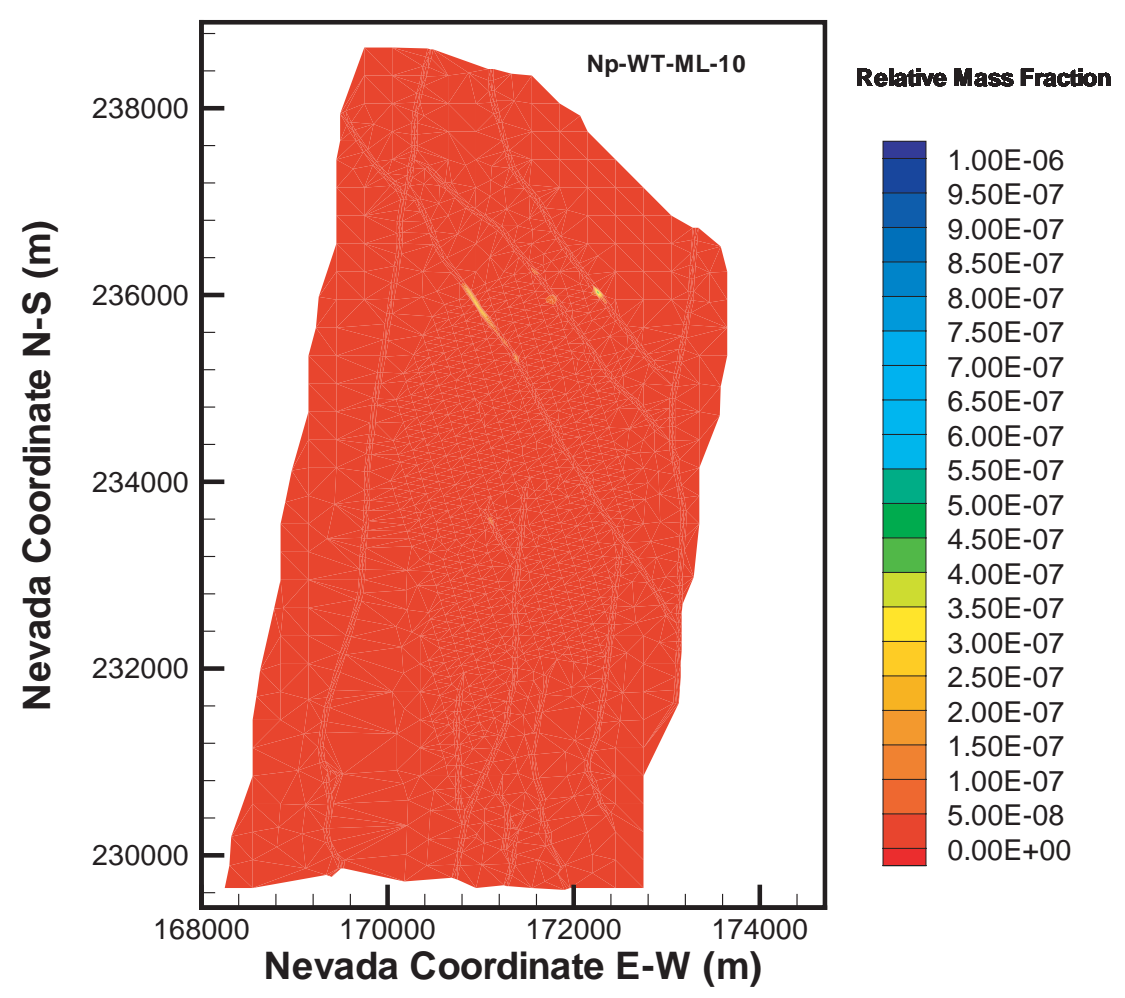

Output DTN: LB0307MR0060R1.002.

Figure D-14. Distribution of the Relative Mass Fraction $X_{R}$ of ${ }^{237} \mathrm{~Np}$ in the Matrix Immediately above the Groundwater at $t=10$ Years for Mean Present-Day Infiltration and Instantaneous Release 


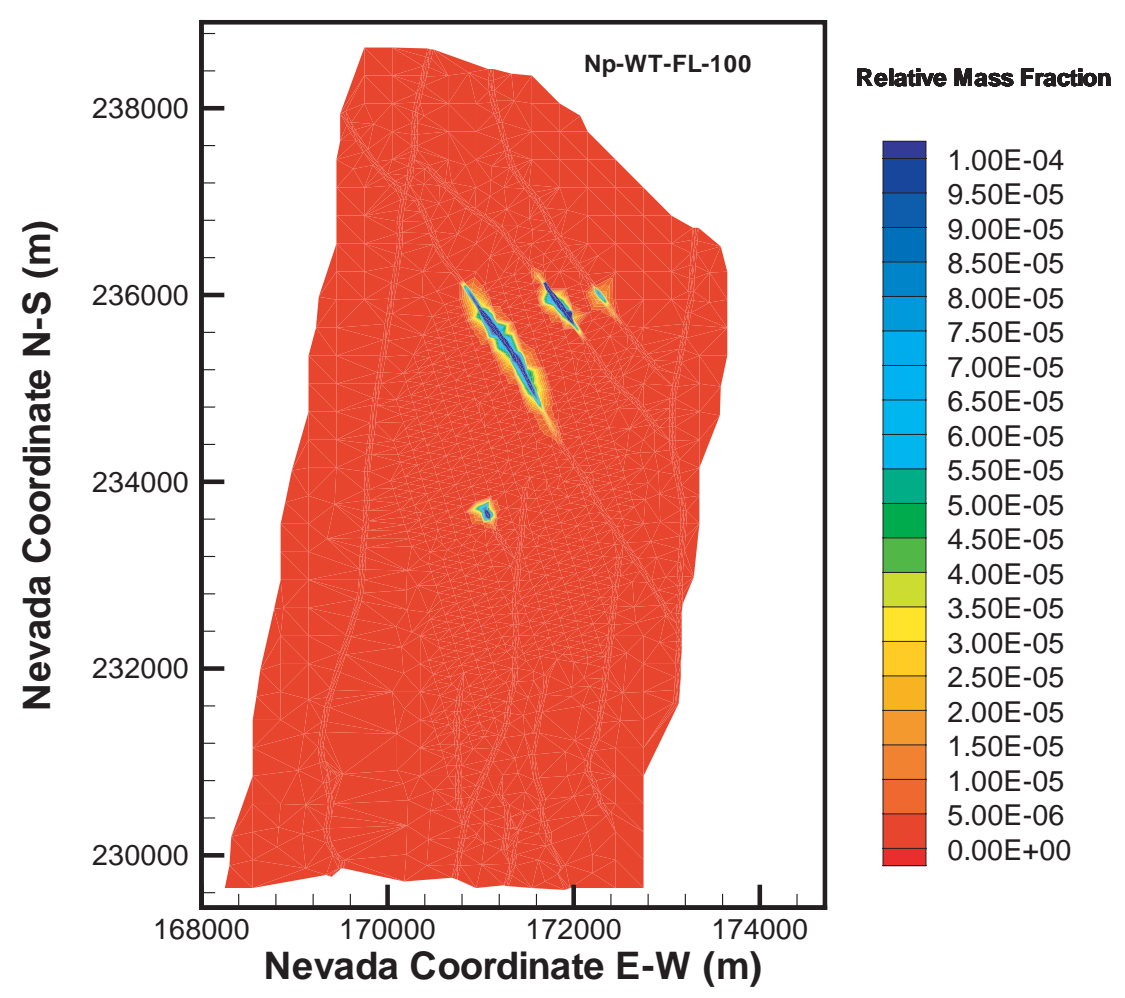

Output DTN: LB0307MR0060R1.002.

Figure D-15. Distribution of the Relative Mass Fraction $X_{R}$ of ${ }^{237} \mathrm{~Np}$ in the Fractures Immediately above the Groundwater at $t=100$ Years for Mean Present-Day Infiltration and Instantaneous Release 


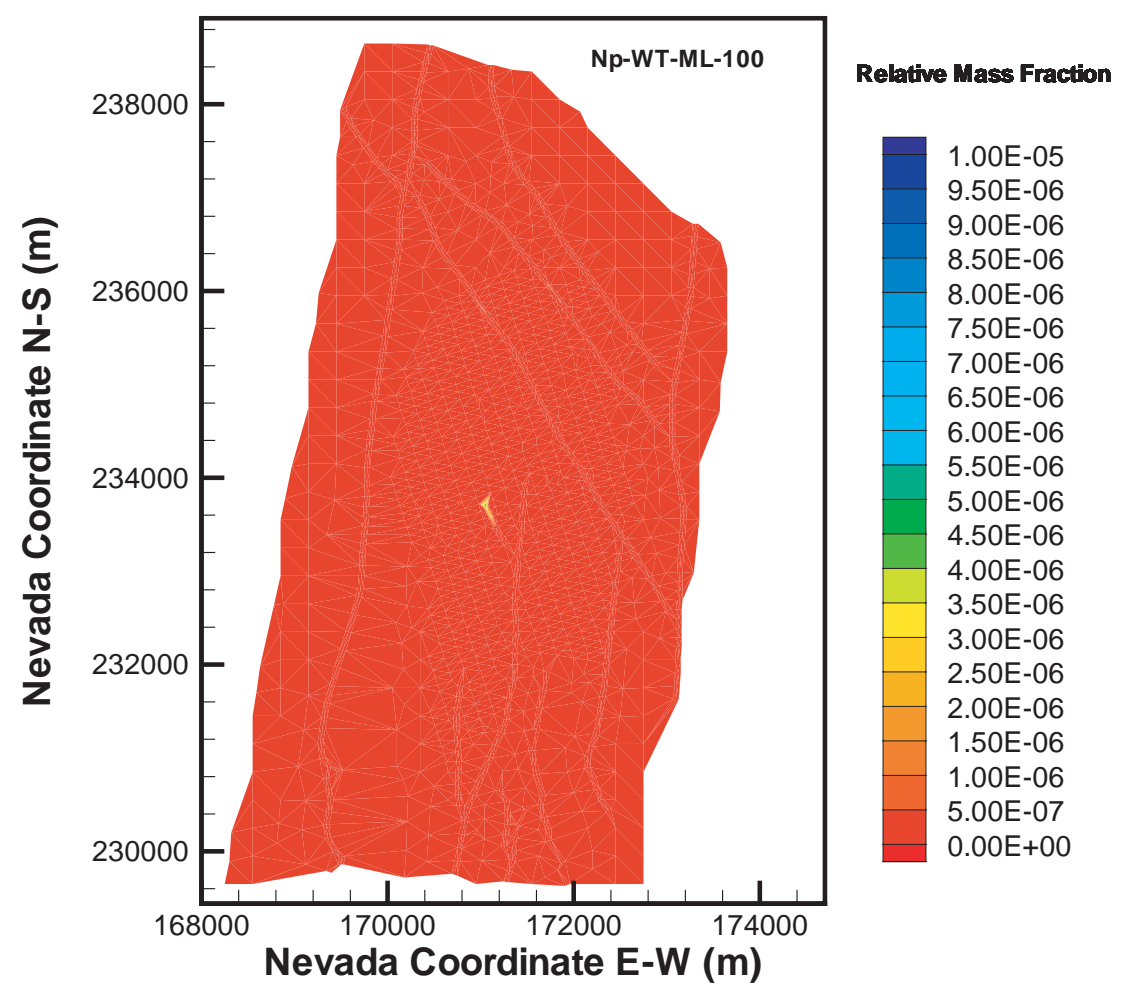

Output DTN: LB0307MR0060R1.002.

Figure D-16. Distribution of the Relative Mass Fraction $X_{R}$ of ${ }^{237} \mathrm{~Np}$ in the Matrix Immediately above the Groundwater at $t=100$ Years for Mean Present-Day Infiltration and Instantaneous Release 


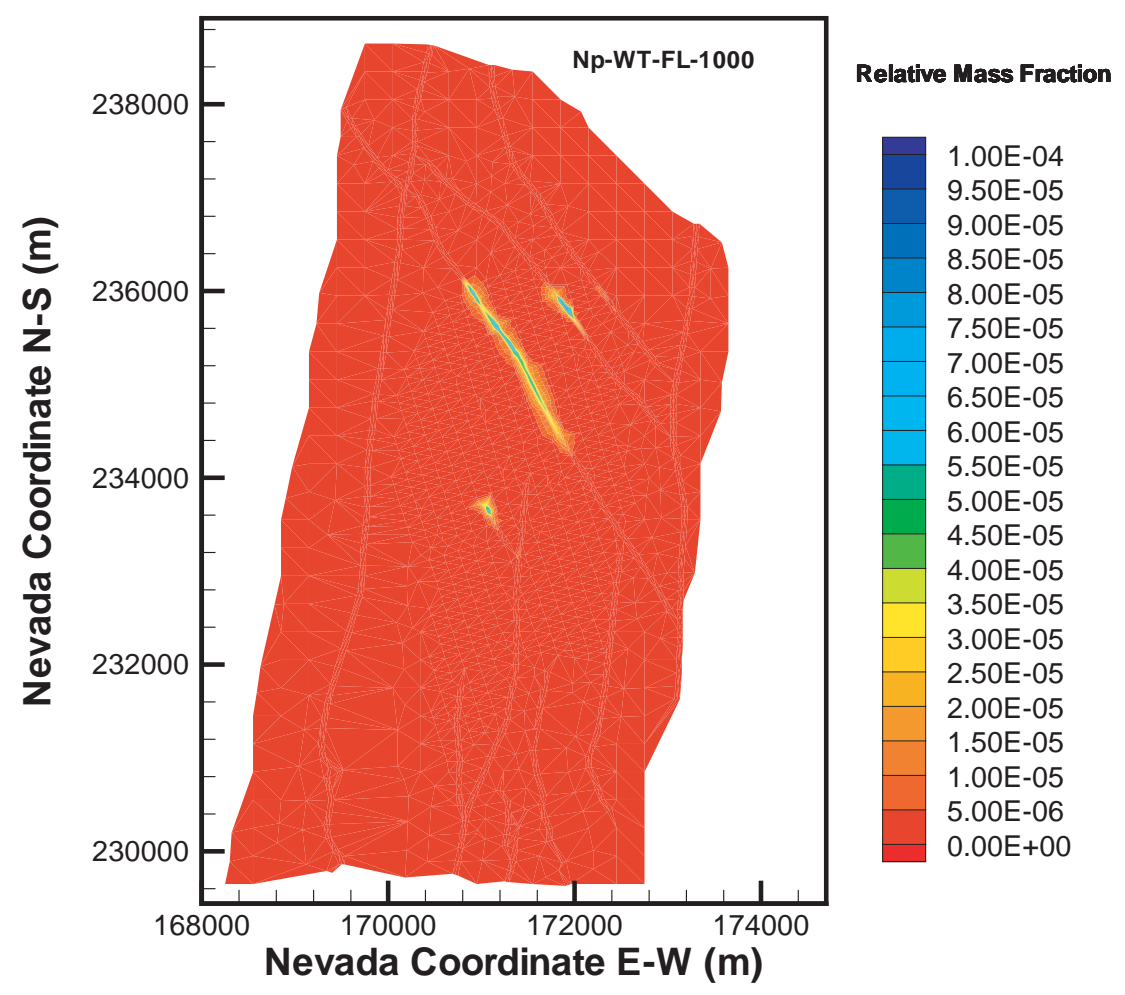

Output DTN: LB0307MR0060R1.002.

Figure D-17. Distribution of the Relative Mass Fraction $X_{R}$ of ${ }^{237} \mathrm{~Np}$ in the Fractures Immediately above the Groundwater at $t=1,000$ Years for Mean Present-Day Infiltration and Instantaneous Release 


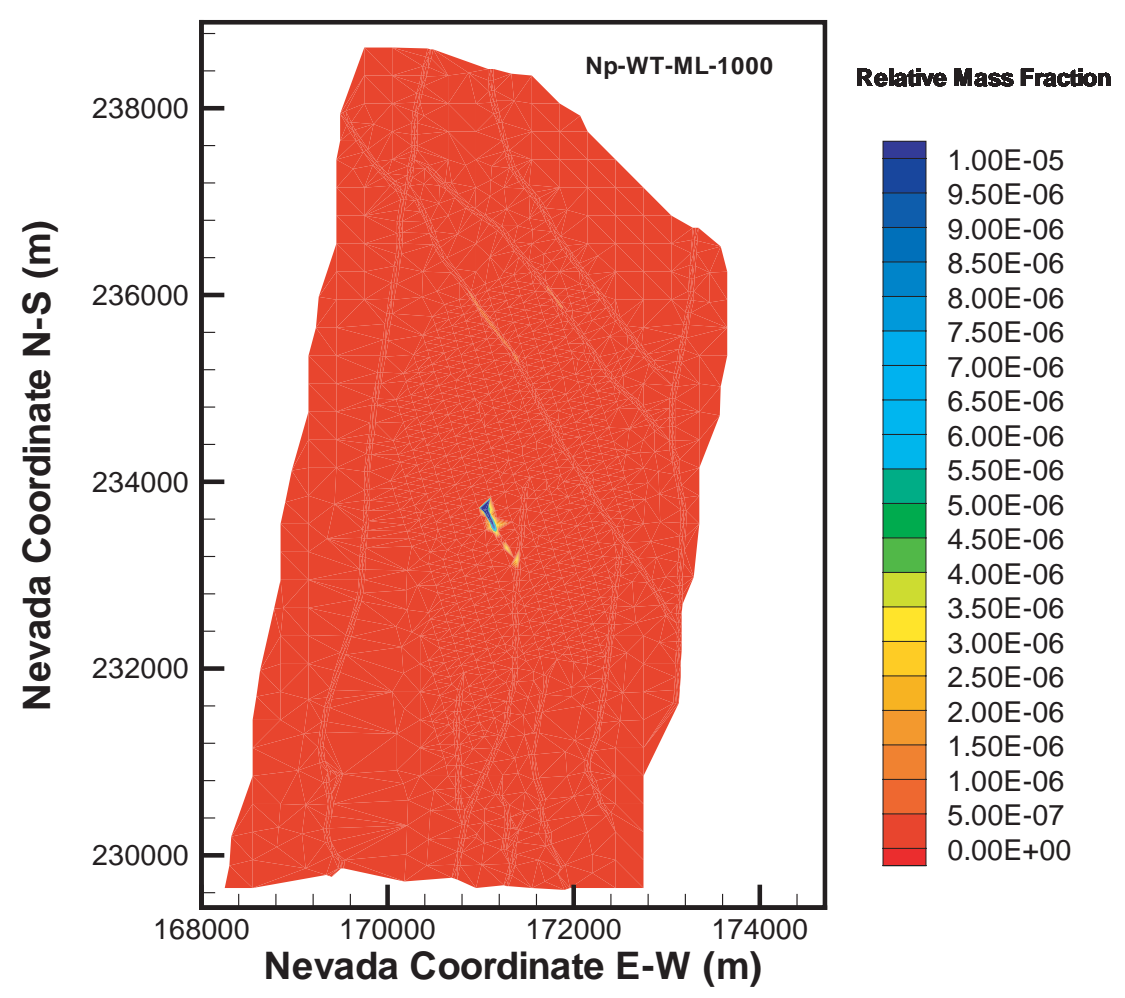

Output DTN: LB0307MR0060R1.002.

Figure D-18. Distribution of the Relative Mass Fraction $X_{R}$ of ${ }^{237} \mathrm{~Np}$ in the Matrix Immediately above the Groundwater at $t=1,000$ Years for Mean Present-Day Infiltration and Instantaneous Release 


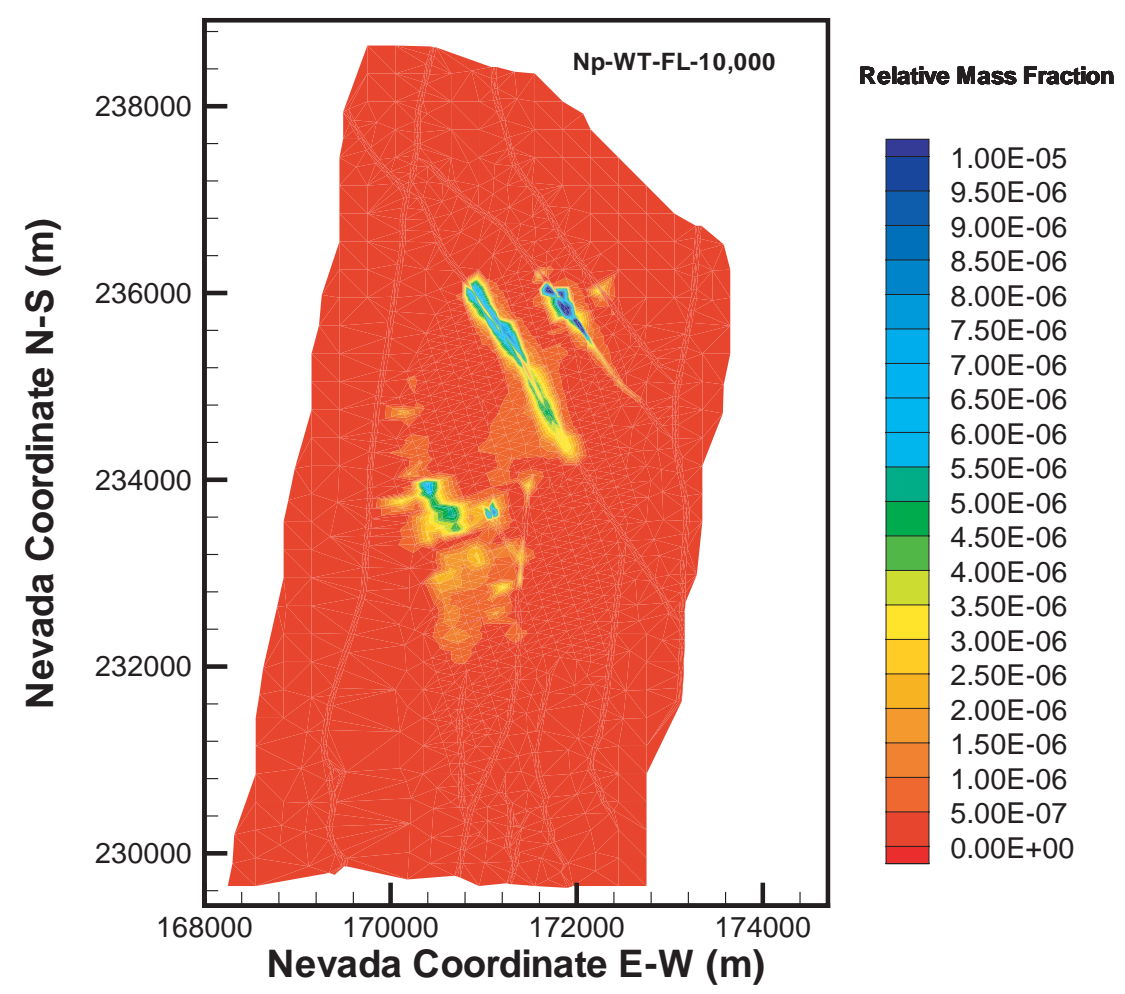

Output DTN: LB0307MR0060R1.002.

Figure D-19. Distribution of the Relative Mass Fraction $X_{R}$ of ${ }^{237} \mathrm{~Np}$ in the Fractures Immediately above the Groundwater at $t=10,000$ Years for Mean Present-Day Infiltration and Instantaneous Release 


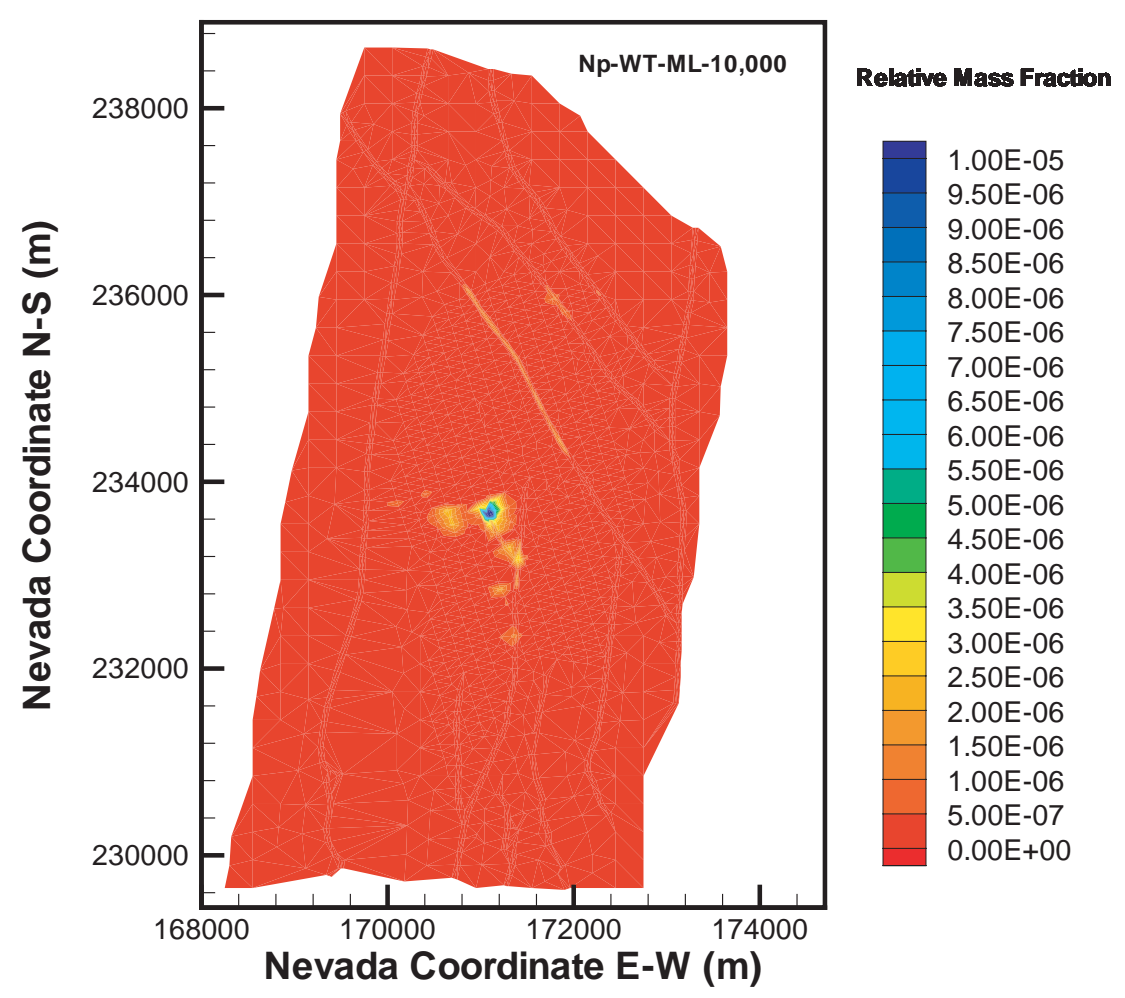

Output DTN: LB0307MR0060R1.002.

Figure D-20. Distribution of the Relative Mass Fraction $X_{R}$ of ${ }^{237} \mathrm{~Np}$ in the Matrix Immediately above the Groundwater at $t=10,000$ Years for Mean Present-Day Infiltration and Instantaneous Release 


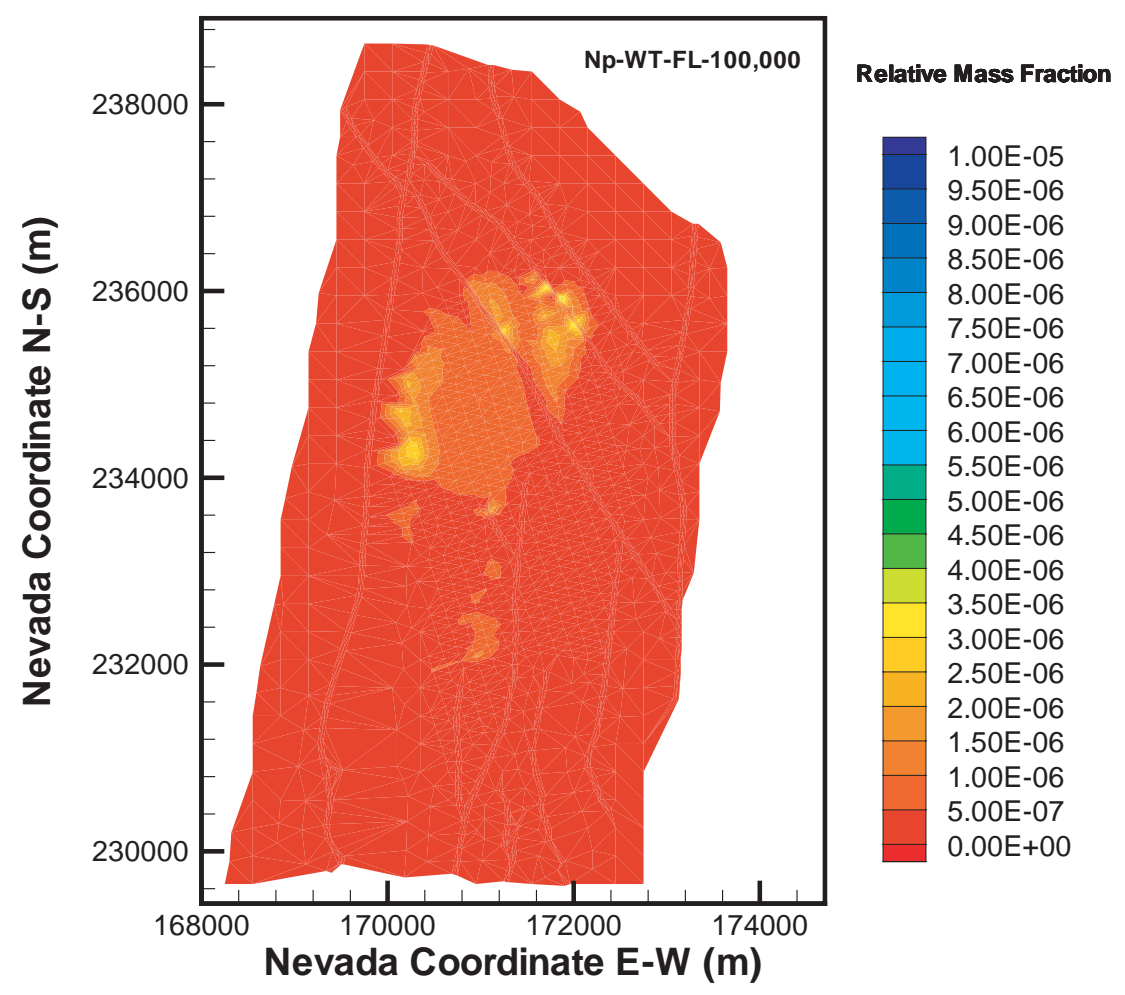

Output DTN: LB0307MR0060R1.002.

Figure D-21. Distribution of the Relative Mass Fraction $X_{R}$ of ${ }^{237} \mathrm{~Np}$ in the Fractures Immediately above the Groundwater at $t=100,000$ Years for Mean Present-Day Infiltration and Instantaneous Release 


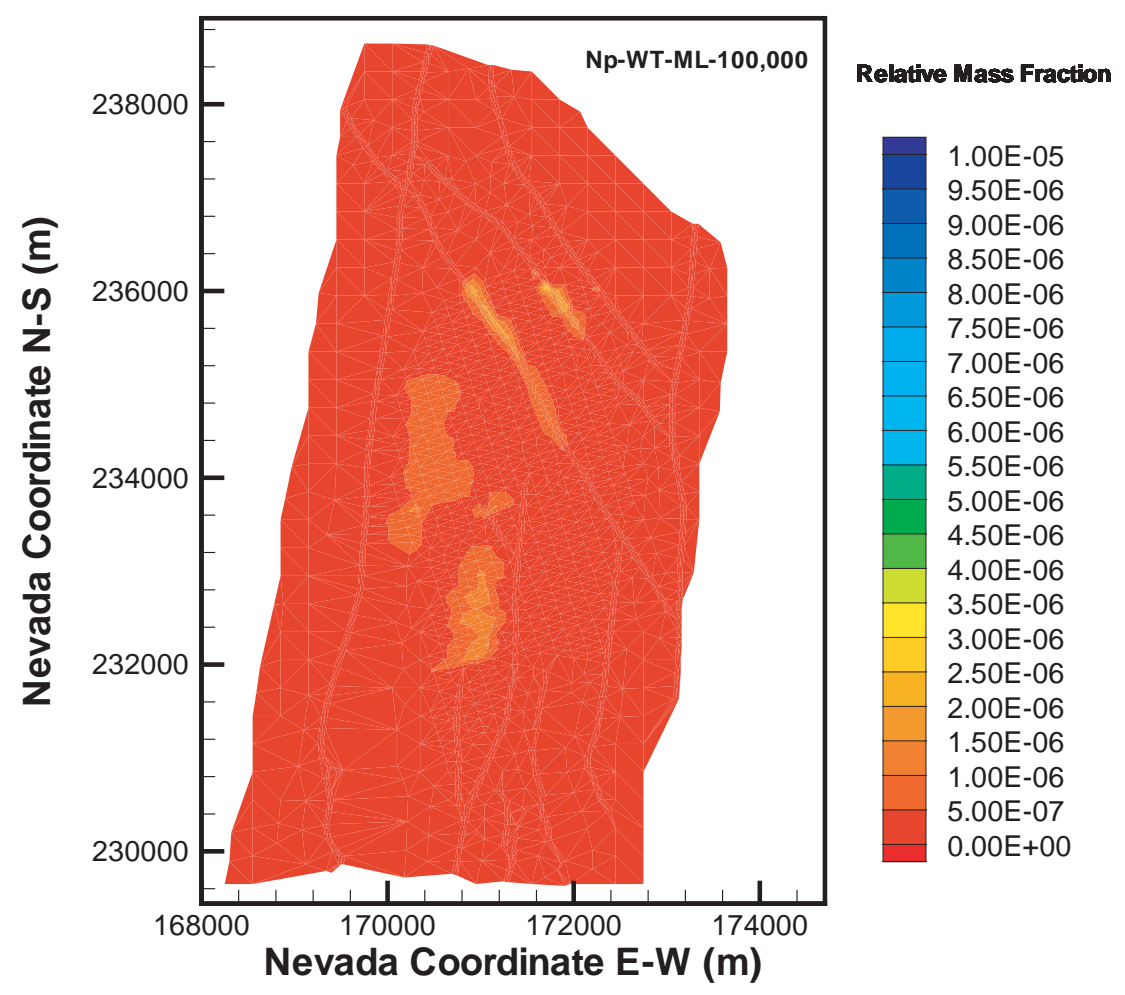

Output DTN: LB0307MR0060R1.002.

Figure D-22. Distribution of the Relative Mass Fraction $X_{R}$ of ${ }^{237} \mathrm{~Np}$ in the Matrix Immediately above the Groundwater at $t=100,000$ Years for Mean Present-Day Infiltration and Instantaneous Release 


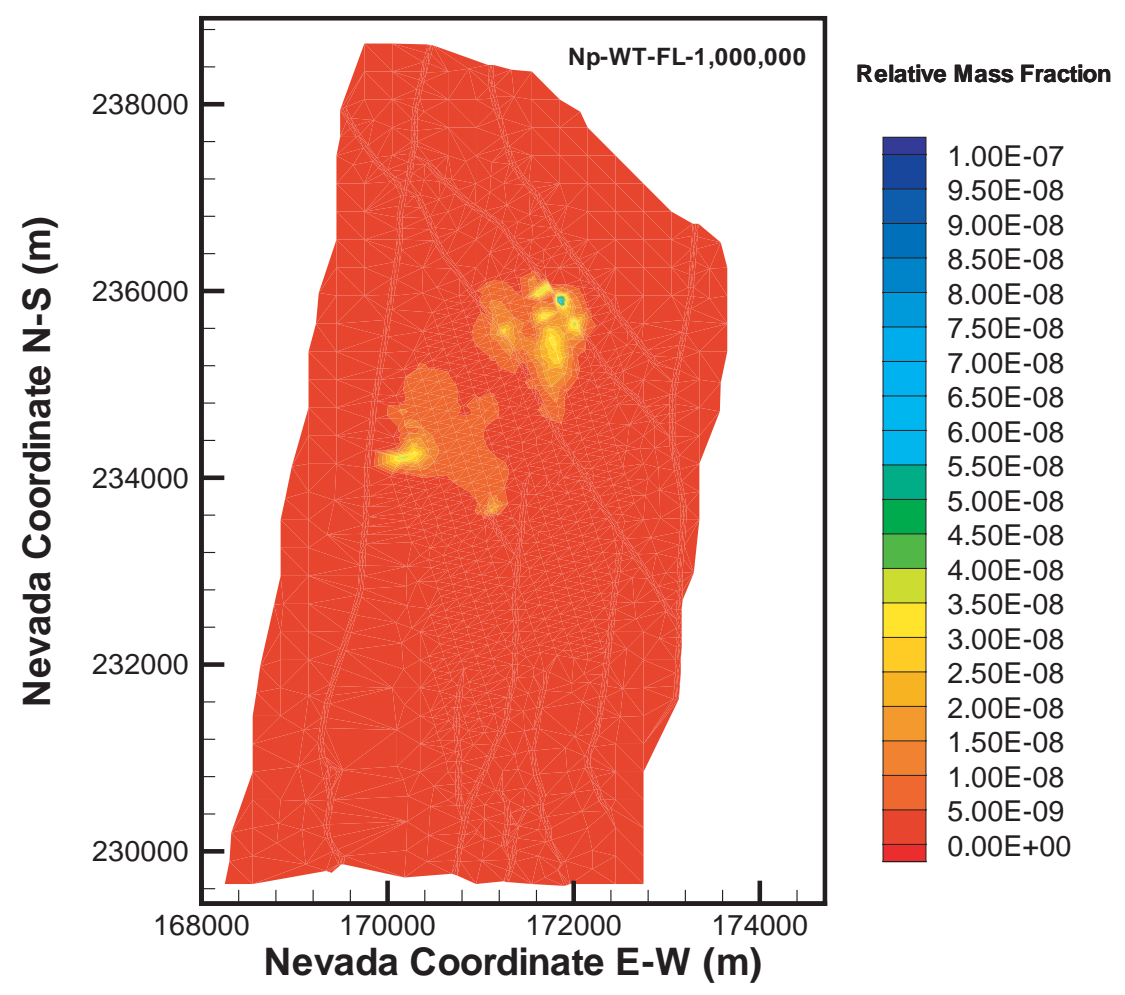

Output DTN: LB0307MR0060R1.002.

Figure D-23. Distribution of the Relative Mass Fraction $X_{R}$ of ${ }^{237} \mathrm{~Np}$ in the Fractures Immediately above the Groundwater at $t=1,000,000$ Years for Mean Present-Day Infiltration and Instantaneous Release 


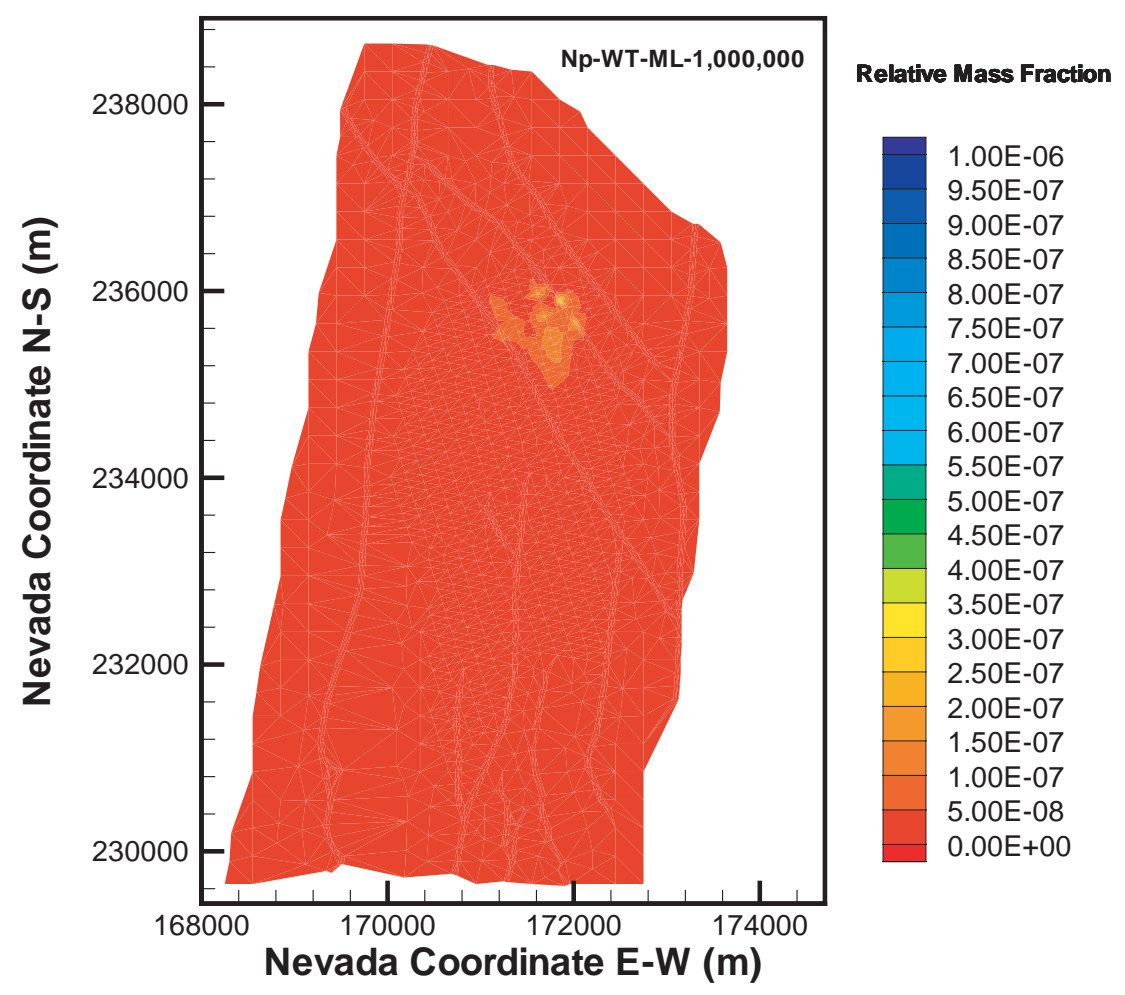

Output DTN: LB0307MR0060R1.002.

Figure D-24. Distribution of the Relative Mass Fraction $X_{R}$ of ${ }^{237} \mathrm{~Np}$ in the Matrix Immediately above the Groundwater at $t=1,000,000$ Years for Mean Present-Day Infiltration and Instantaneous Release 


\section{APPENDIX E}

FIGURES FROM THE ${ }^{99}$ Tc THREE-DIMENSIONAL TRANSPORT STUDIES (CONTINUOUS RELEASE, MEAN PRESENT-DAY INFILTRATION) 


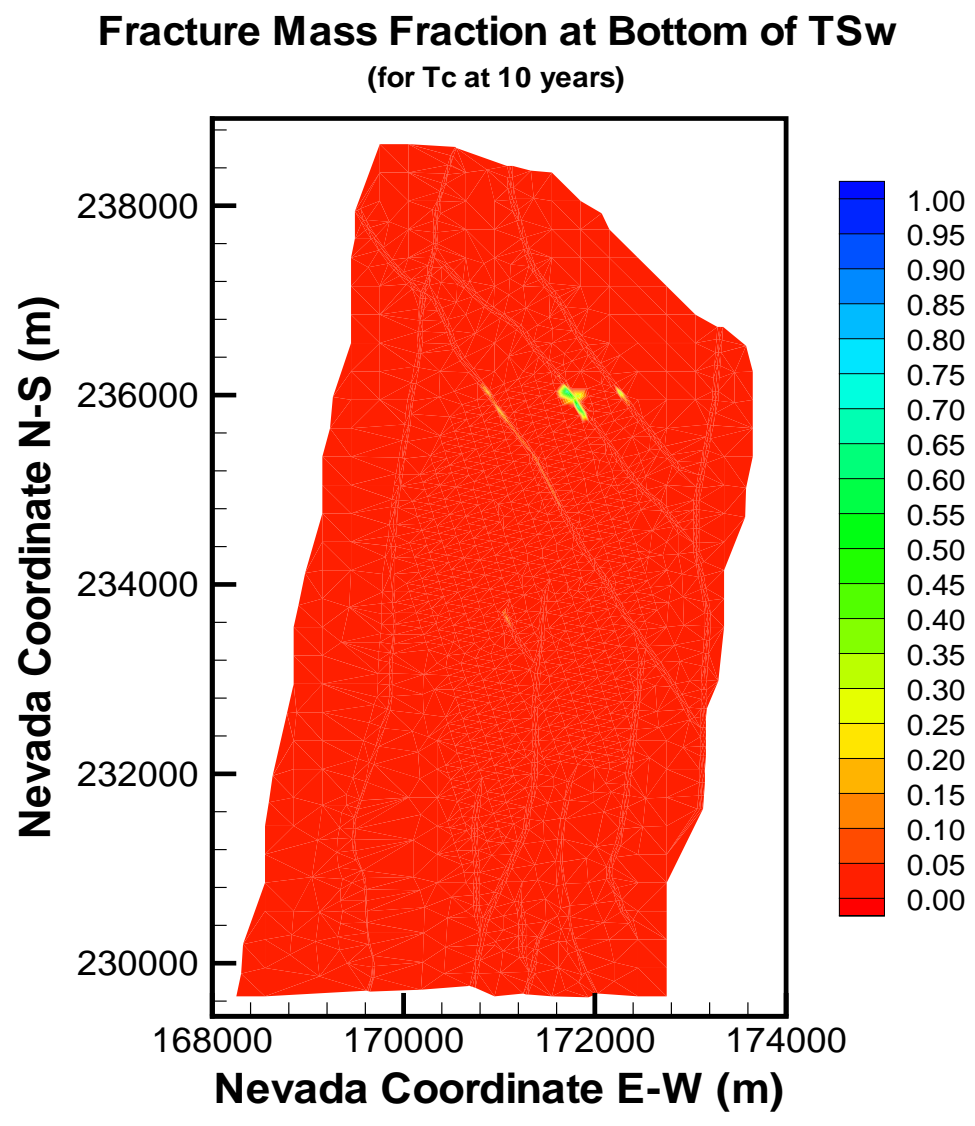

Output DTN: LB0307MR0060R1.004.

Figure E-1. Distribution of the Relative Mass Fraction $X_{R}$ of ${ }^{99} \mathrm{Tc}$ in the Fractures of the tsw39 Layer at $t=10$ Years for Mean Present-Day Infiltration and Continuous Release

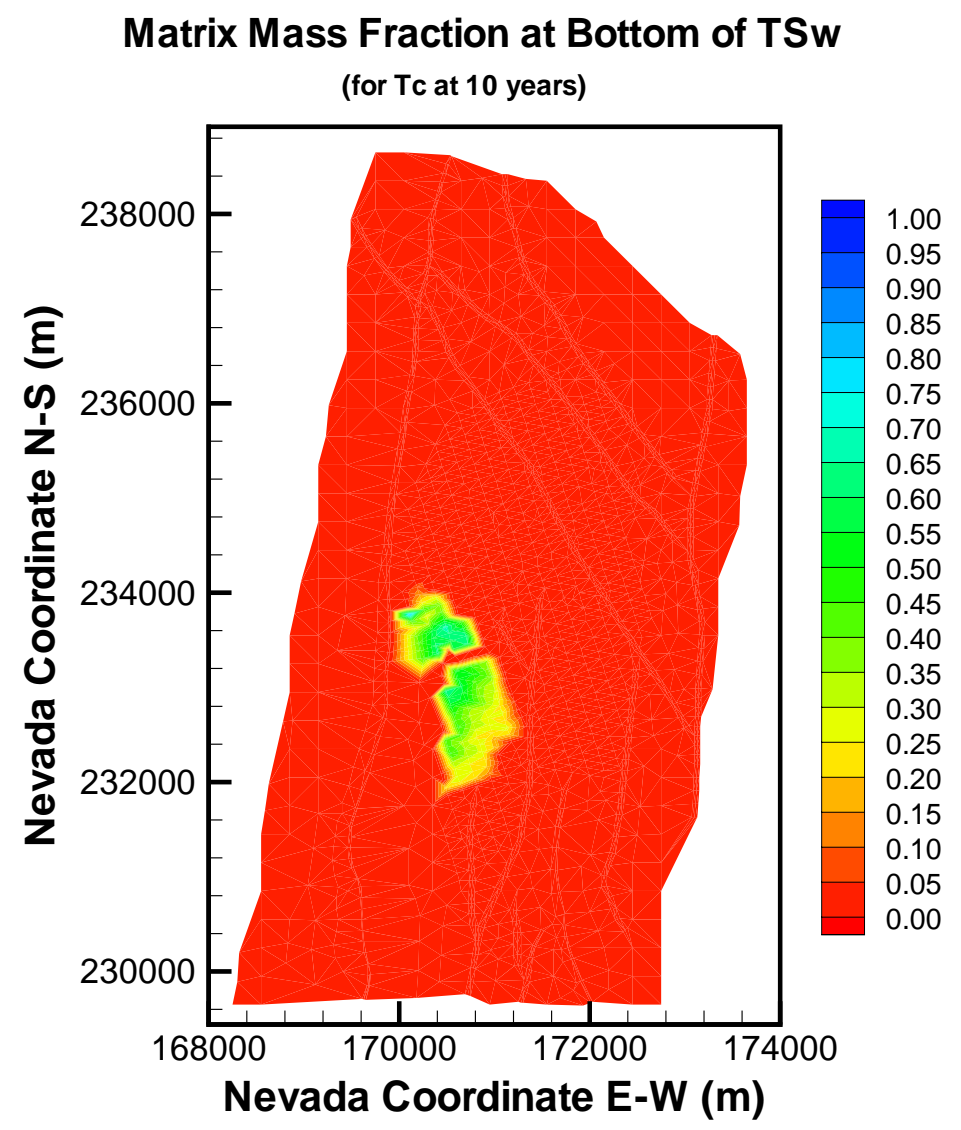

Output DTN: LB0307MR0060R1.004.

Figure E-2. Distribution of the Relative Mass Fraction $X_{R}$ of ${ }^{99} T c$ in the Matrix of the tsw39 Layer at $t=10$ Years for Mean Present-Day Infiltration and Continuous Release 
Fracture Mass Fraction at Bottom of TSw (for Tc at 100 years)

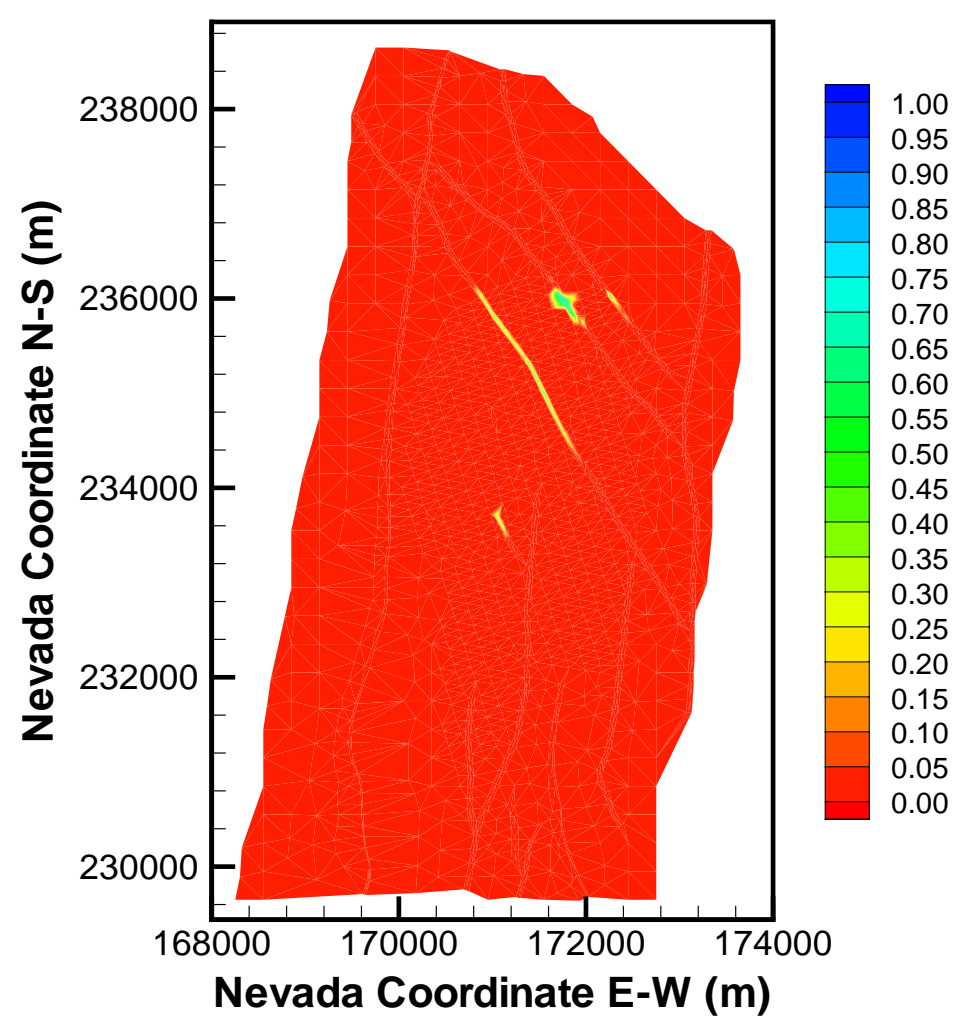

Output DTN: LB0307MR0060R1.004.

Figure E-3. Distribution of the Relative Mass Fraction $X_{R}$ of ${ }^{99} \mathrm{Tc}$ in the Fractures of the tsw39 Layer at $t=100$ Years for Mean Present-Day Infiltration and Continuous Release
Matrix Mass Fraction at Bottom of TSw (for Tc at 100 years)

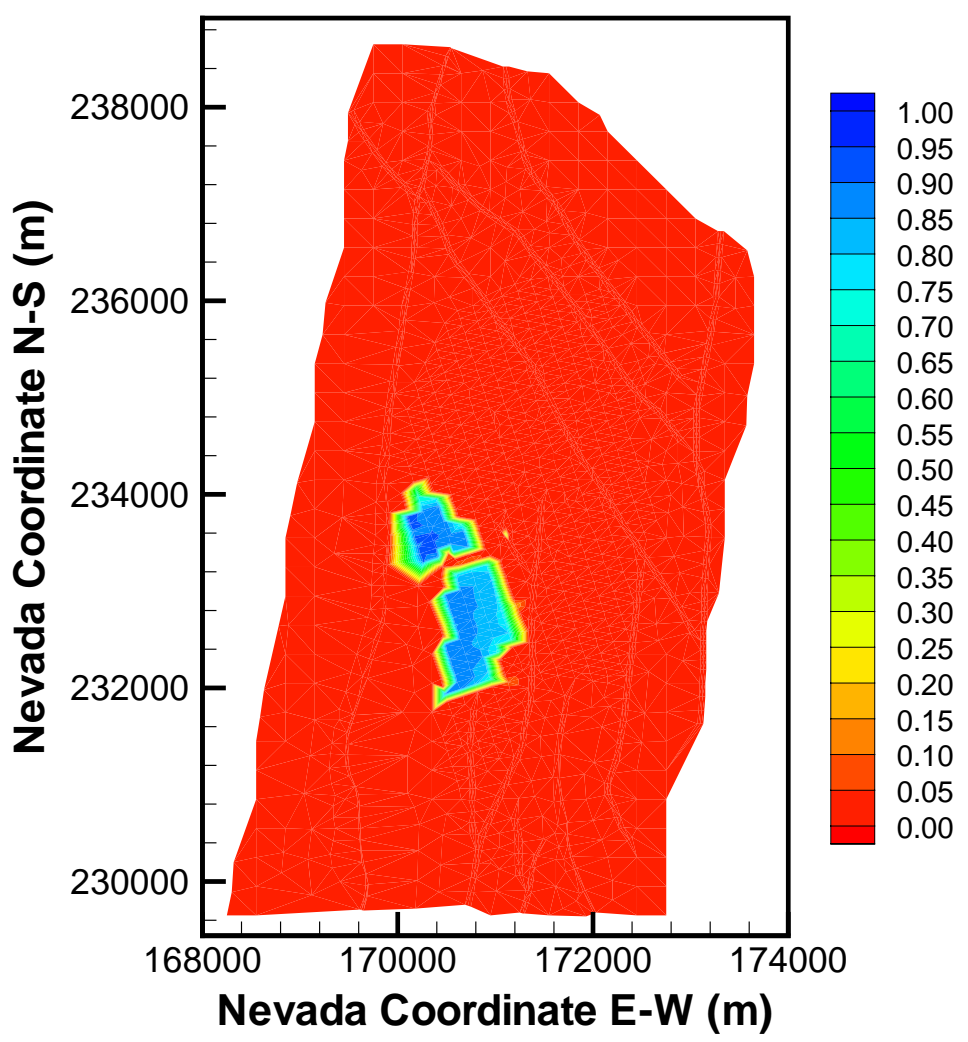

Output DTN: LB0307MR0060R1.004.

Figure E-4. Distribution of the Relative Mass Fraction $X_{R}$ of ${ }^{99} T c$ in the Matrix of the tsw39 Layer at $t=100$ Years for Mean Present-Day Infiltration and Continuous Release 
Fracture Mass Fraction at Bottom of TSw

(for Tc at 1000 years)

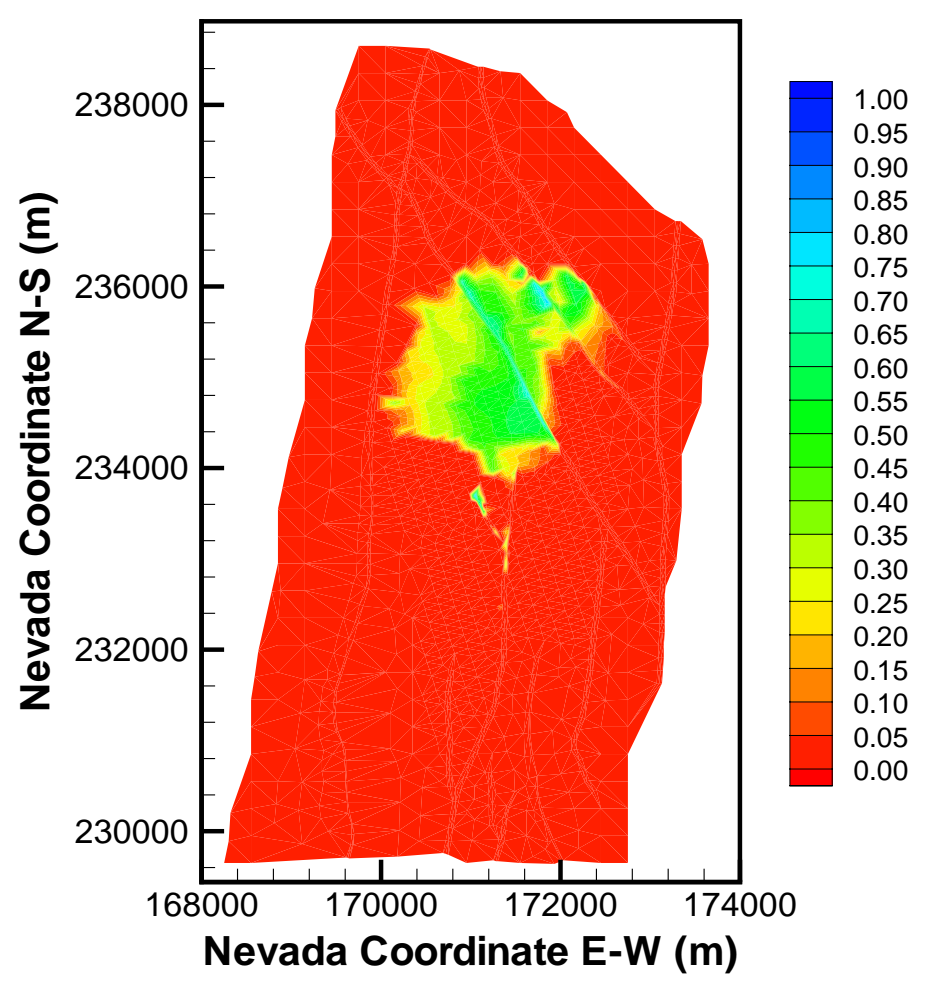

Output DTN: LB0307MR0060R1.004.

Figure E-5. Distribution of the Relative Mass Fraction $X_{R}$ of ${ }^{99} T c$ in the Fractures of the tsw39 Layer at $t=1,000$ Years for Mean Present-Day Infiltration and Continuous Release
Matrix Mass Fraction at Bottom of TSw (for Tc at 1000 years)

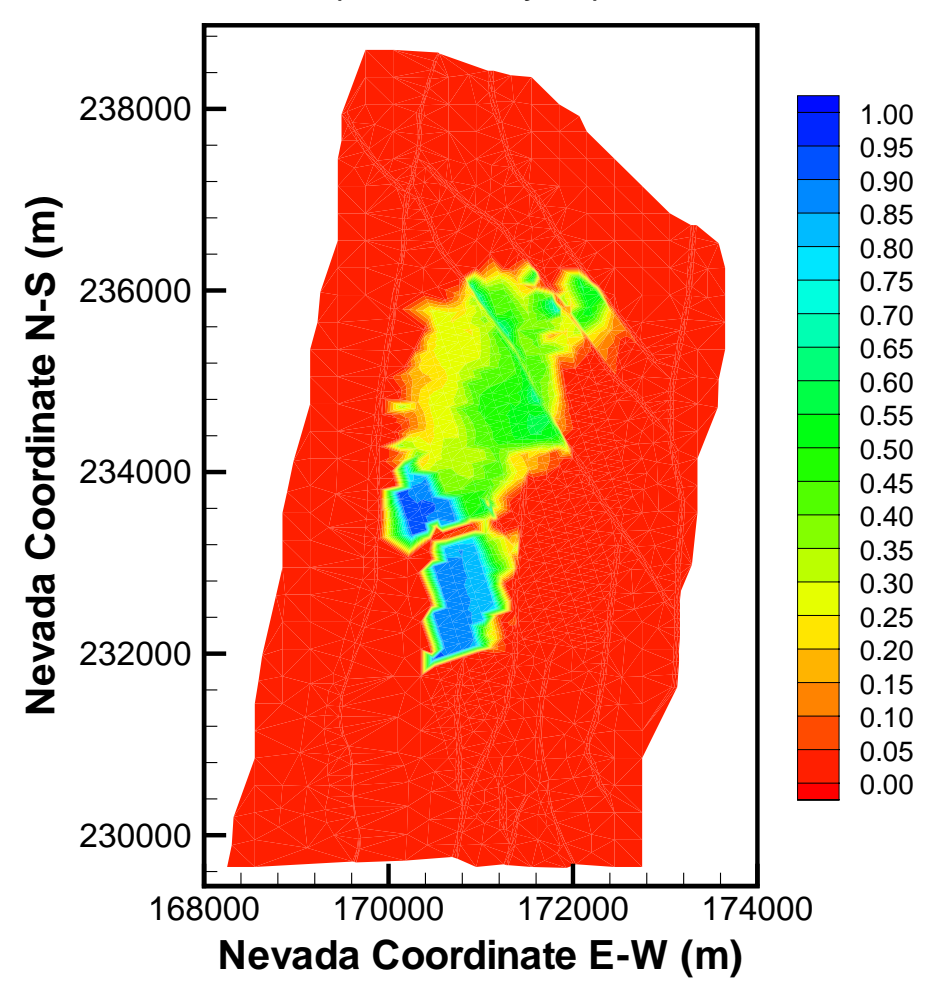

Output DTN: LB0307MR0060R1.004.

Figure E-6. Distribution of the Relative Mass Fraction $X_{R}$ of ${ }^{99} T c$ in the Matrix of the tsw39 Layer at $t=1,000$ Years for Mean Present-Day Infiltration and Continuous Release 
Fracture Mass Fraction at Bottom of TSw (for Tc at 10000 years)



Output DTN: LB0307MR0060R1.004.

Figure E-7. Distribution of the Relative Mass Fraction $X_{R}$ of ${ }^{99} \mathrm{Tc}$ in the Fractures of the tsw39 Layer at $t=10,000$ Years for Mean Present-Day Infiltration and Continuous Release
Matrix Mass Fraction at Bottom of TSw

(for Tc at 10000 years)

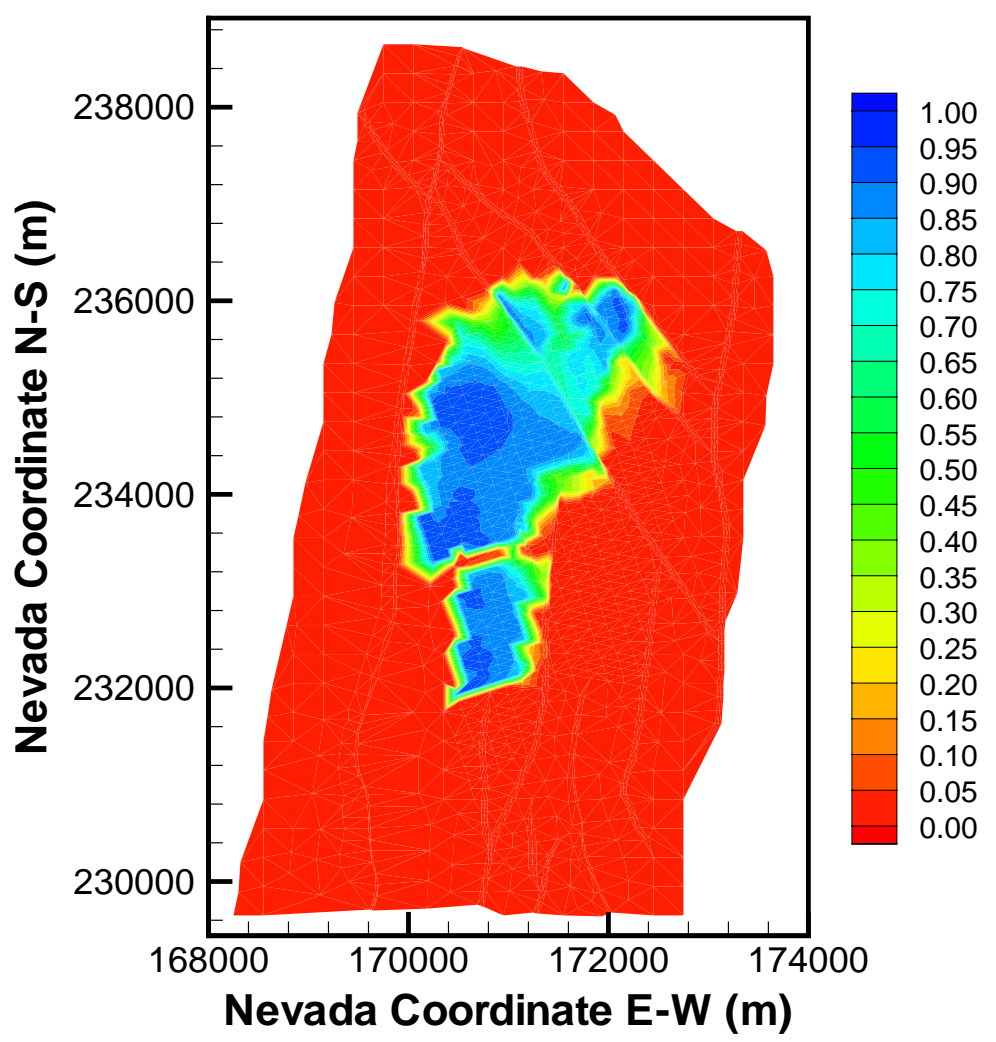

Output DTN: LB0307MR0060R1.004.

Figure E-8. Distribution of the Relative Mass Fraction $X_{R}$ of ${ }^{99} T c$ in the Matrix of the tsw39 Layer at $t=10,000$ Years for Mean Present-Day Infiltration and Continuous Release 
Fracture Mass Fraction at Bottom of TSw

(for Tc at 100000 years)

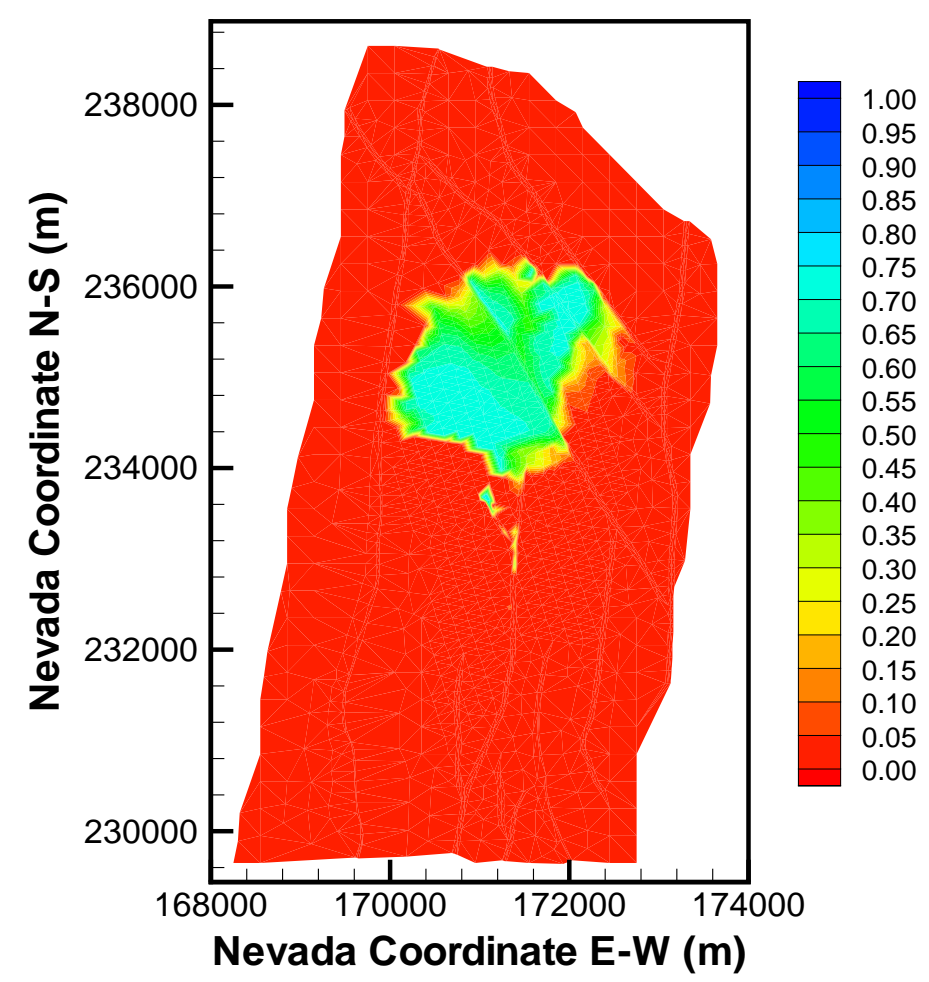

Output DTN: LB0307MR0060R1.004.

Figure E-9. Distribution of the Relative Mass Fraction $X_{R}$ of ${ }^{99} T c$ in the Fractures of the tsw39 Layer at $t=100,000$ Years for Mean Present-Day Infiltration and Continuous Release

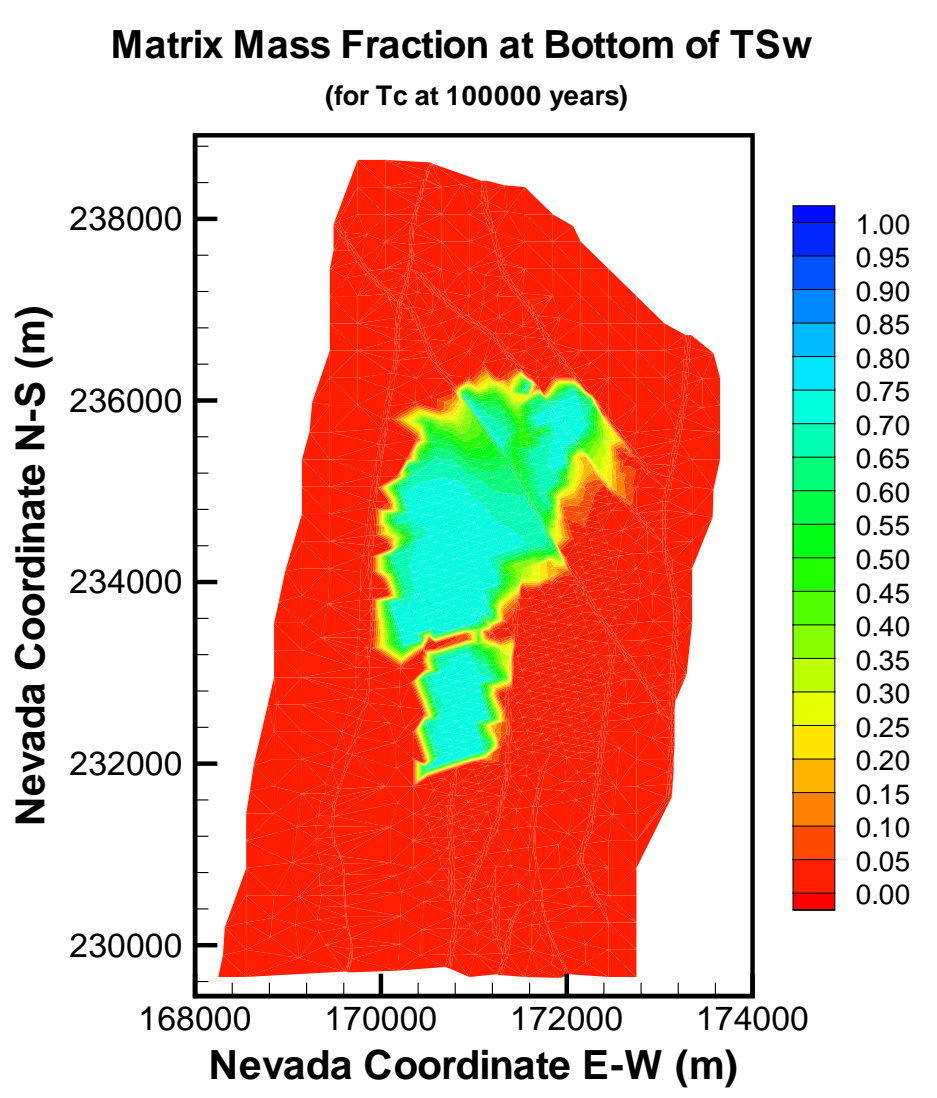

Output DTN: LB0307MR0060R1.004.

Figure E-10. Distribution of the Relative Mass Fraction $X_{R}$ of ${ }^{99} T c$ in the Matrix of the tsw39 Layer at $t=100,000$ Years for Mean Present-Day Infiltration and Continuous Release 
Fracture Mass Fraction at Water Table

(for Tc at 10 years)

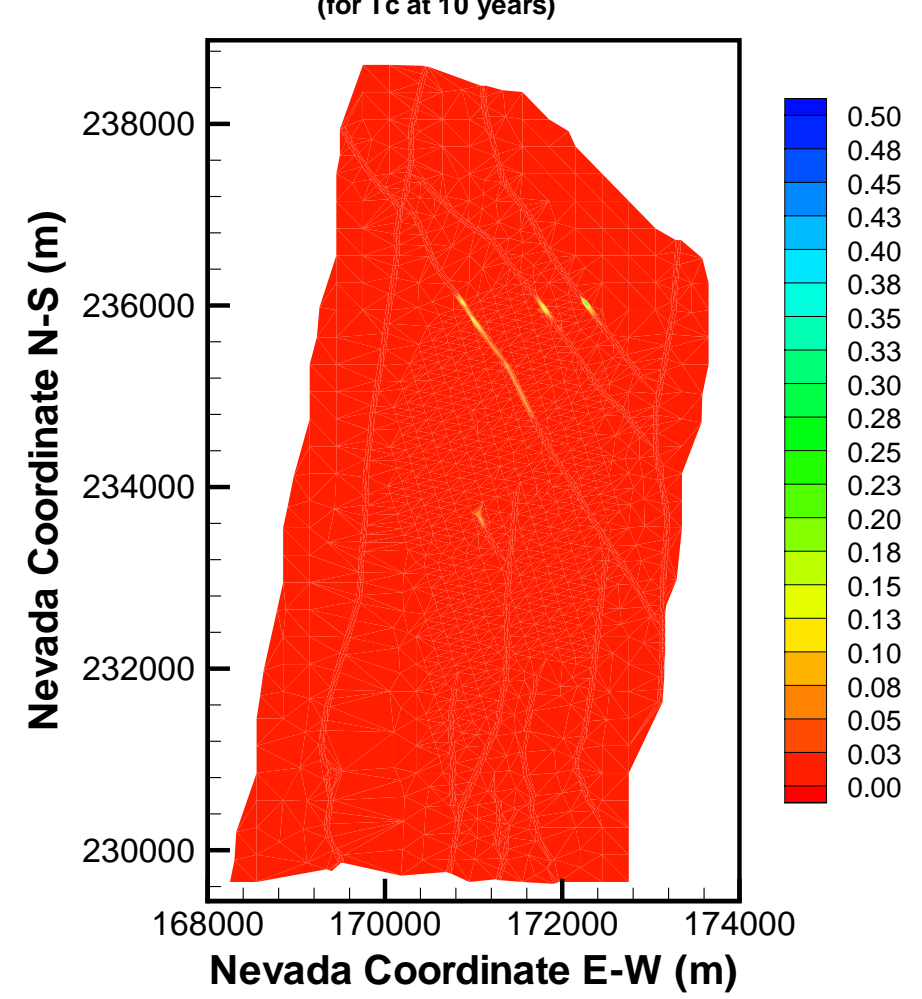

Output DTN: LB0307MR0060R1.004.

Figure E-11. Distribution of the Relative Mass Fraction $X_{R}$ of ${ }^{99} \mathrm{TC}$ in the Fractures Immediately above the Groundwater Table at $t=10$ Years for Mean Present-Day Infiltration and Continuous Release

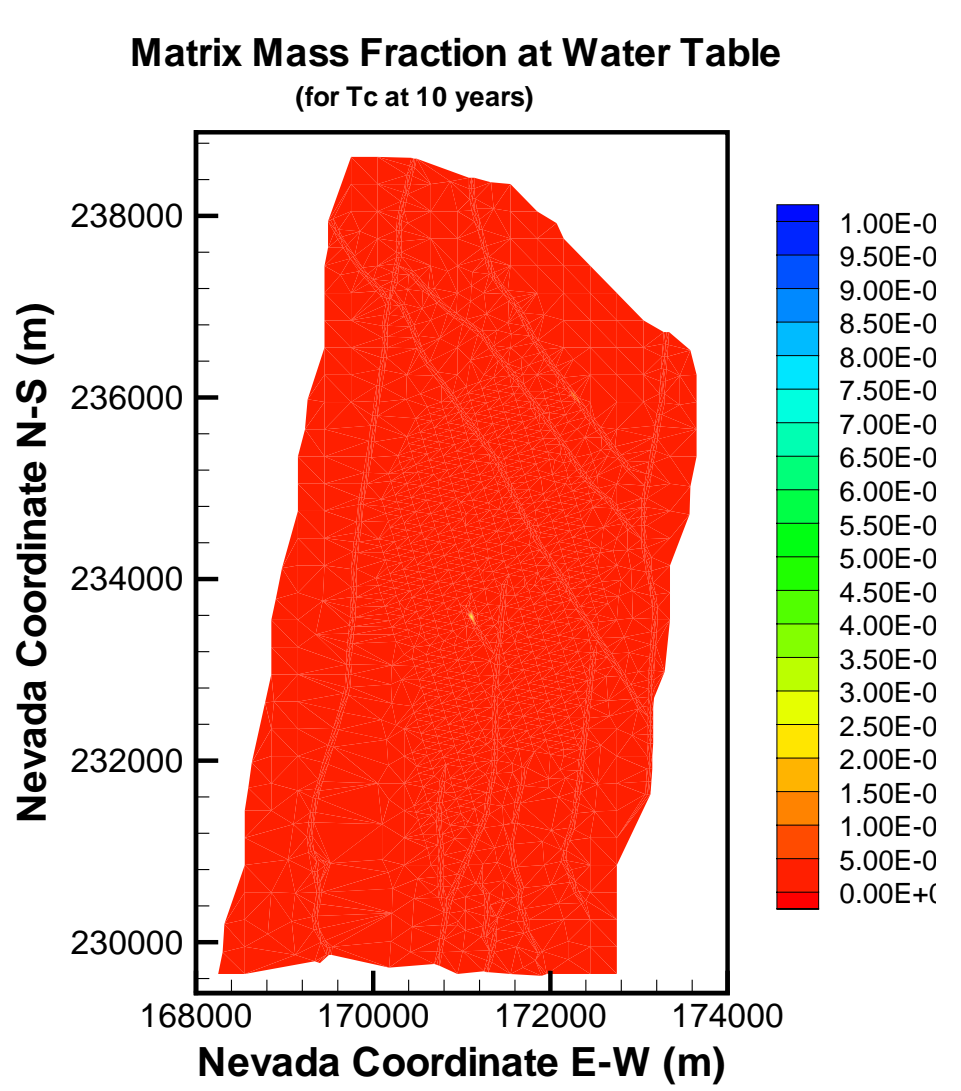

Output DTN: LB0307MR0060R1.004.

Figure E-12. Distribution of the Relative Mass Fraction $X_{R}$ of ${ }^{99} \mathrm{TC}$ in the Matrix Immediately above the Groundwater at $t=10$ Years for Mean Present-Day Infiltration and Continuous Release 
Fracture Mass Fraction at Water Table

(for Tc at 100 years)

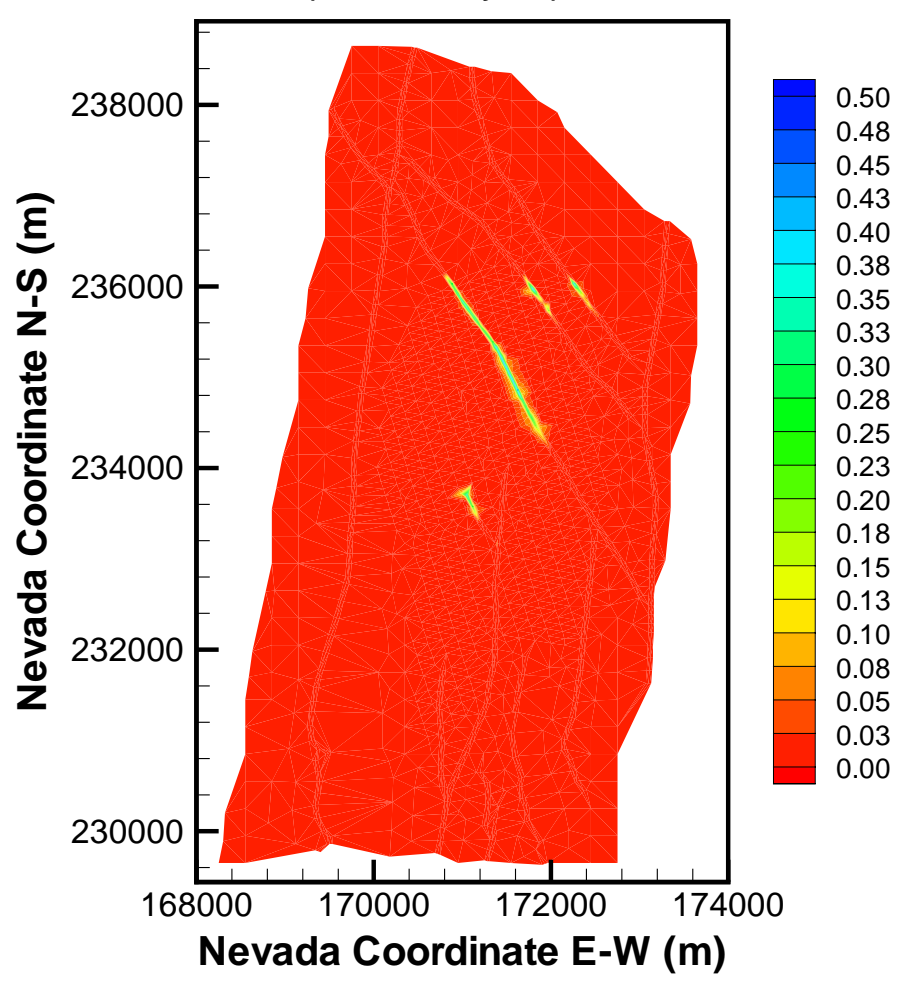

Output DTN: LB0307MR0060R1.004.

Figure E-13. Distribution of the Relative Mass Fraction $X_{R}$ of ${ }^{99} \mathrm{TC}$ in the Fractures Immediately above the Groundwater at $t=100$ Years for Mean Present-Day Infiltration and Continuous Release

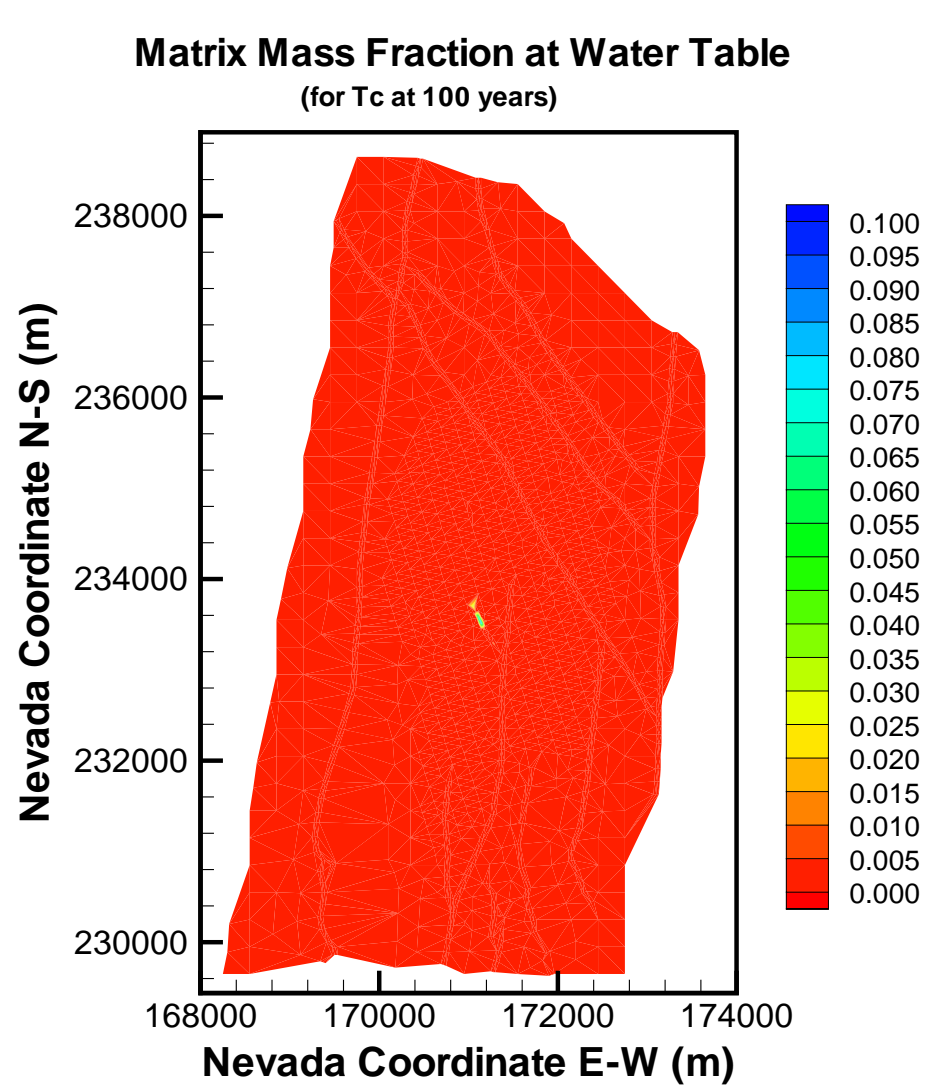

Output DTN: LB0307MR0060R1.004.

Figure E-14. Distribution of the Relative Mass Fraction $X_{R}$ of ${ }^{99} \mathrm{TC}$ in the Matrix Immediately above the groundwater at $t=100$ Years for Mean Present-Day Infiltration and Continuous Release 
Fracture Mass Fraction at Water Table

(for Tc at 1000 years)

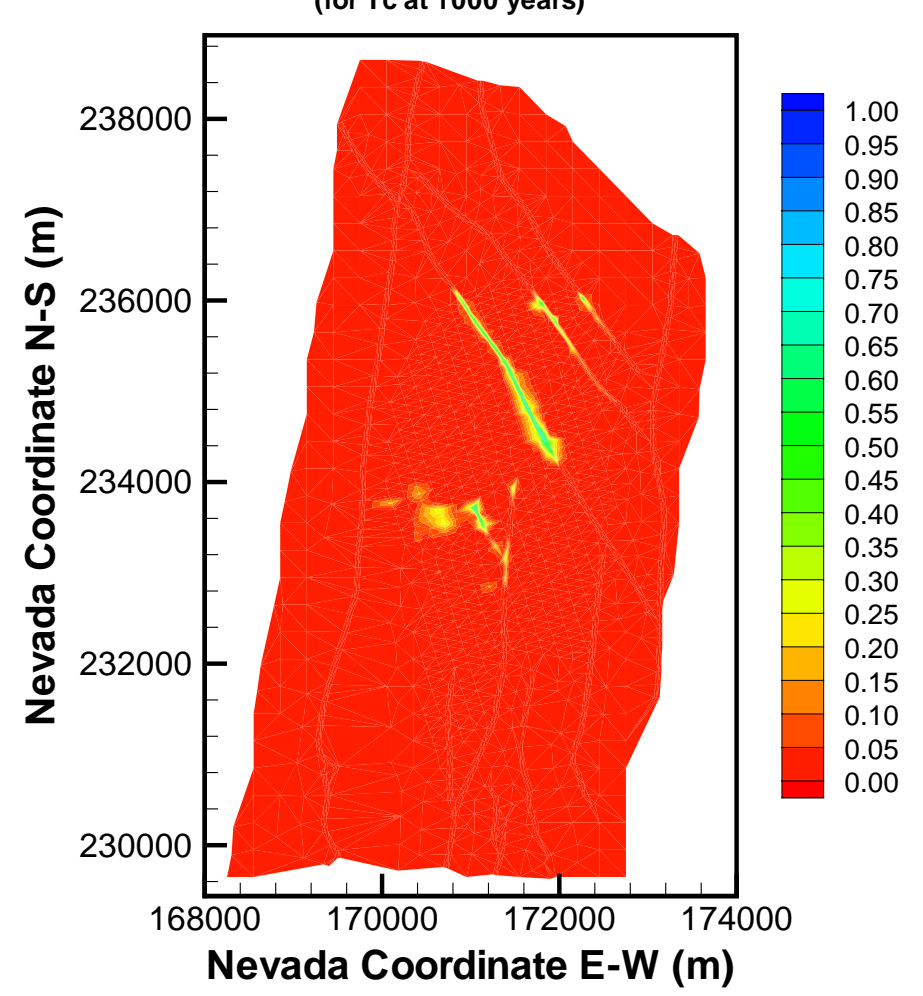

Output DTN: LB0307MR0060R1.004.

Figure E-15. Distribution of the Relative Mass Fraction $X_{R}$ of ${ }^{99} T c$ in the Fractures Immediately above the Groundwater at $T=1,000$ Years for Mean Present-Day Infiltration and Continuous Release

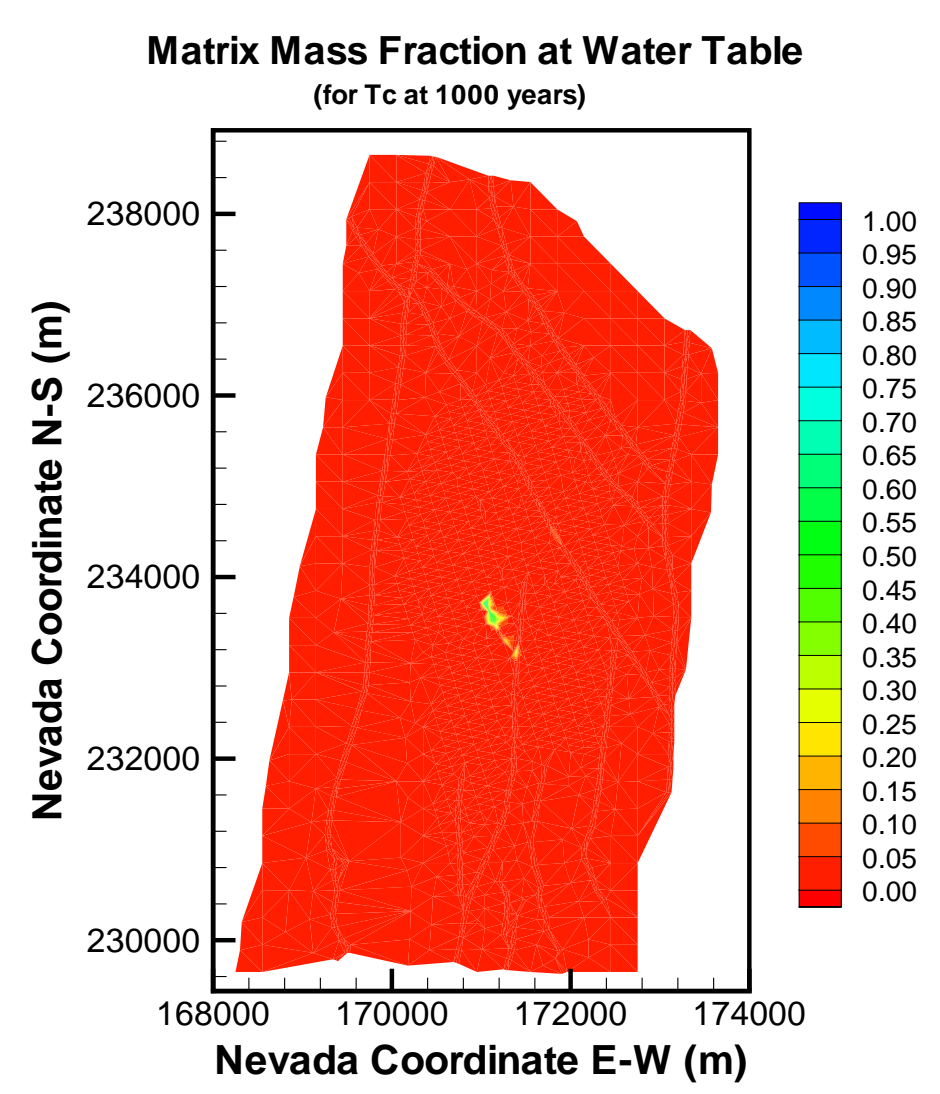

Output DTN: LB0307MR0060R1.004.

Figure E-16. Distribution of the Relative Mass Fraction $X_{R}$ of ${ }^{99} T c$ in the Matrix Immediately above the Groundwater at $t=$ 1,000 Years for Mean Present-Day Infiltration and Continuous Release 
Fracture Mass Fraction at Water Table

(for Tc at 10000 years)

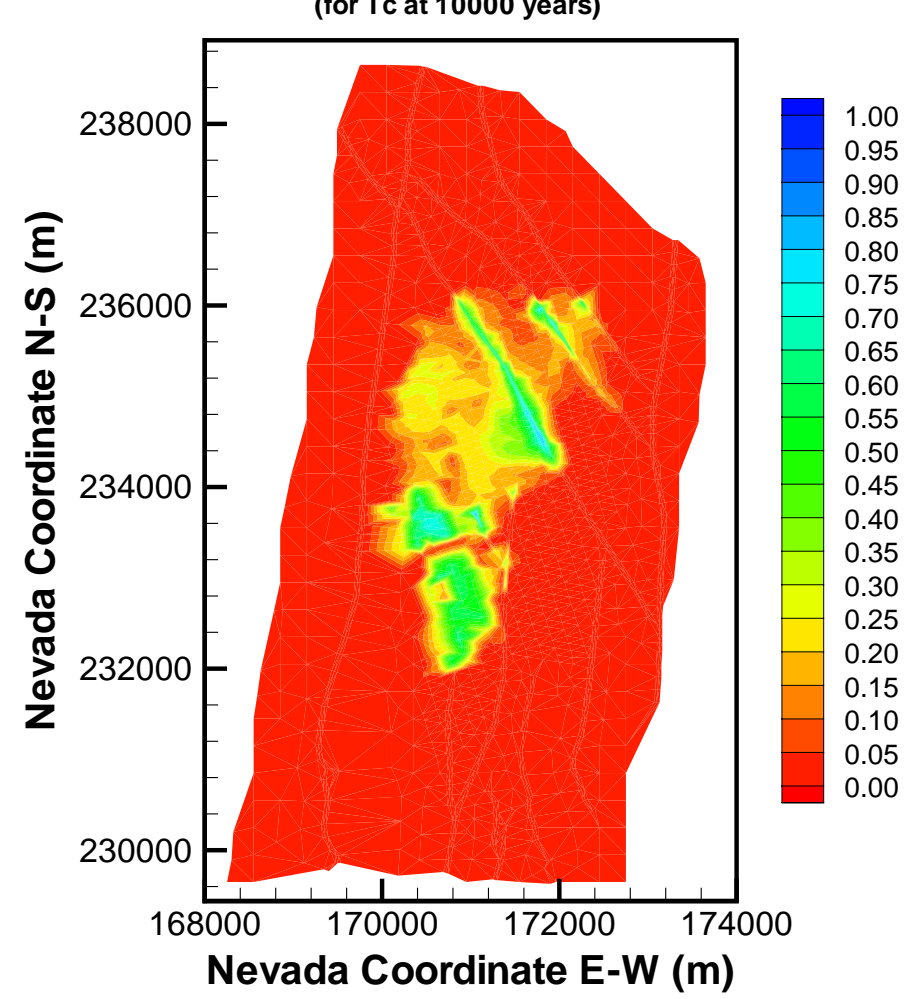

Output DTN: LB0307MR0060R1.004.

Figure E-17. Distribution of the Relative Mass Fraction $X_{R}$ of ${ }^{99} T c$ in the Fractures Immediately above the Groundwater at $t=10,000$ Years for Mean Present-Day Infiltration and Continuous Release

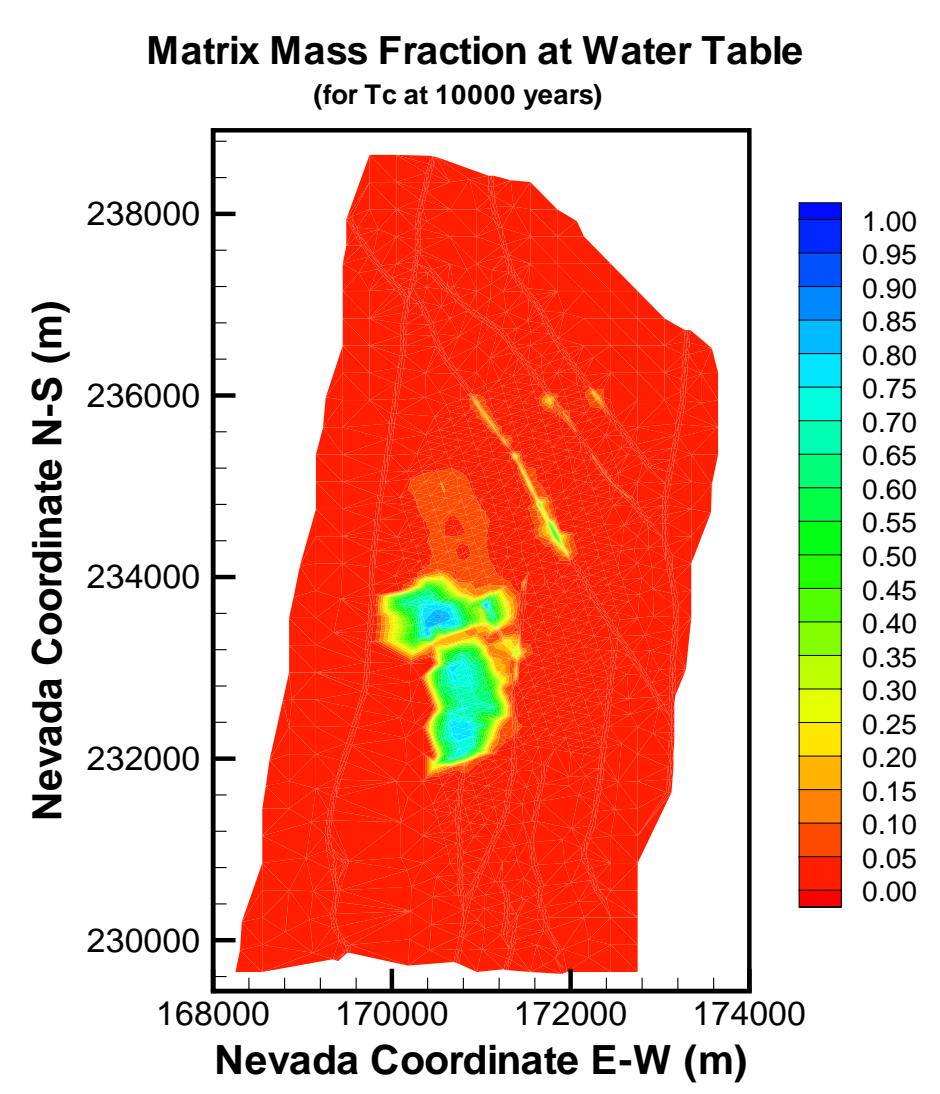

Output DTN: LB0307MR0060R1.004.

Figure E-18. Distribution of the Relative Mass Fraction $X_{R}$ of ${ }^{99} T c$ in the Matrix Immediately above the Groundwater at $t=$ 10,000 Years for Mean Present-Day Infiltration and Continuous Release 
Fracture Mass Fraction at Water Table

(for Tc at 100000 years)

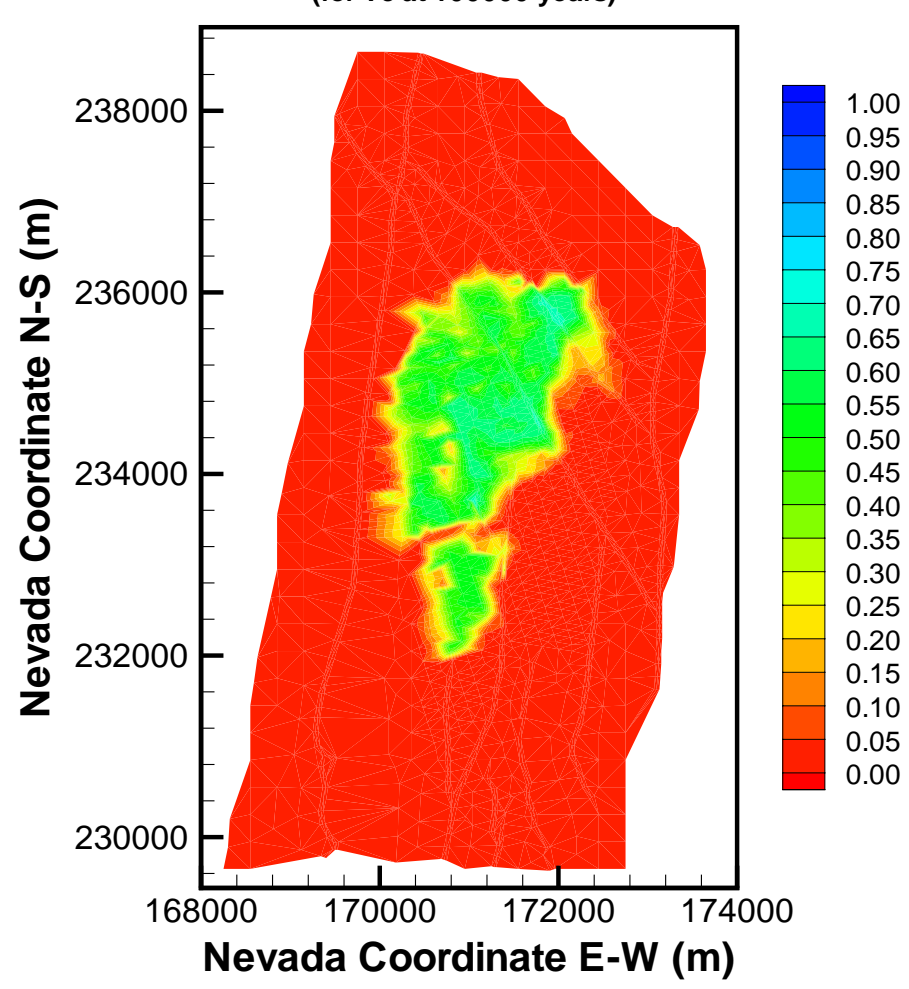

Output DTN: LB0307MR0060R1.004.

Figure E-19. Distribution of the Relative Mass Fraction $X_{R}$ of ${ }^{99} T c$ in the Fractures Immediately above the Groundwater at $t=100,000$ Years for Mean Present-Day Infiltration and Continuous Release

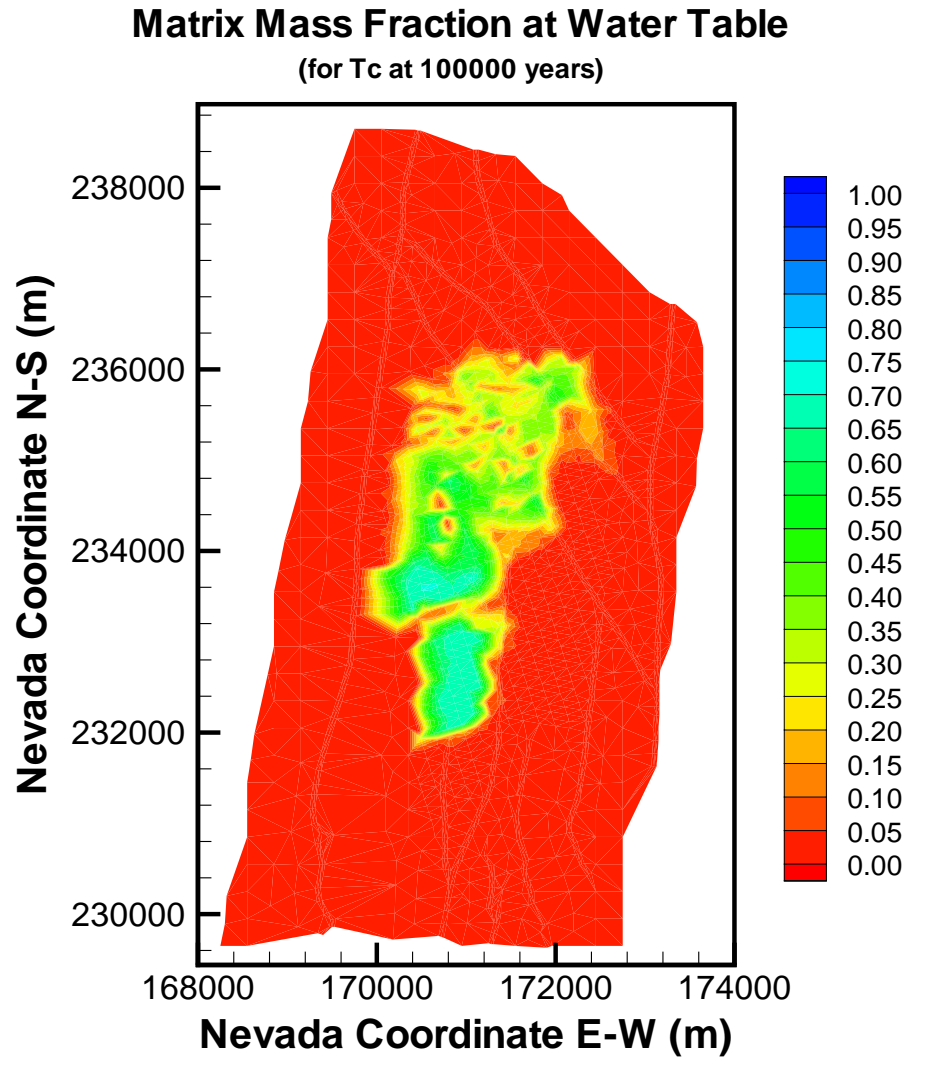

Output DTN: LB0307MR0060R1.004.

Figure E-20. Distribution of the Relative Mass Fraction $X_{R}$ of ${ }^{99} T c$ in the Matrix Immediately above the Groundwater at $\mathrm{t}=$ 100,000 Years for Mean Present-Day Infiltration and Continuous Release 


\section{APPENDIX F \\ FIGURES FROM THE THREE-DIMENSIONAL TRANSPORT STUDIES OF THE $6 \mathrm{NM}^{239} \mathrm{PUO}_{2}$ COLLOID IN CASE 2 (CONTINUOUS RELEASE, MEAN PRESENT-DAY INFILTRATION)}


Fracture Mass Fraction at Bottom of TSw

(for $\mathrm{Co006}$ at 10 years)

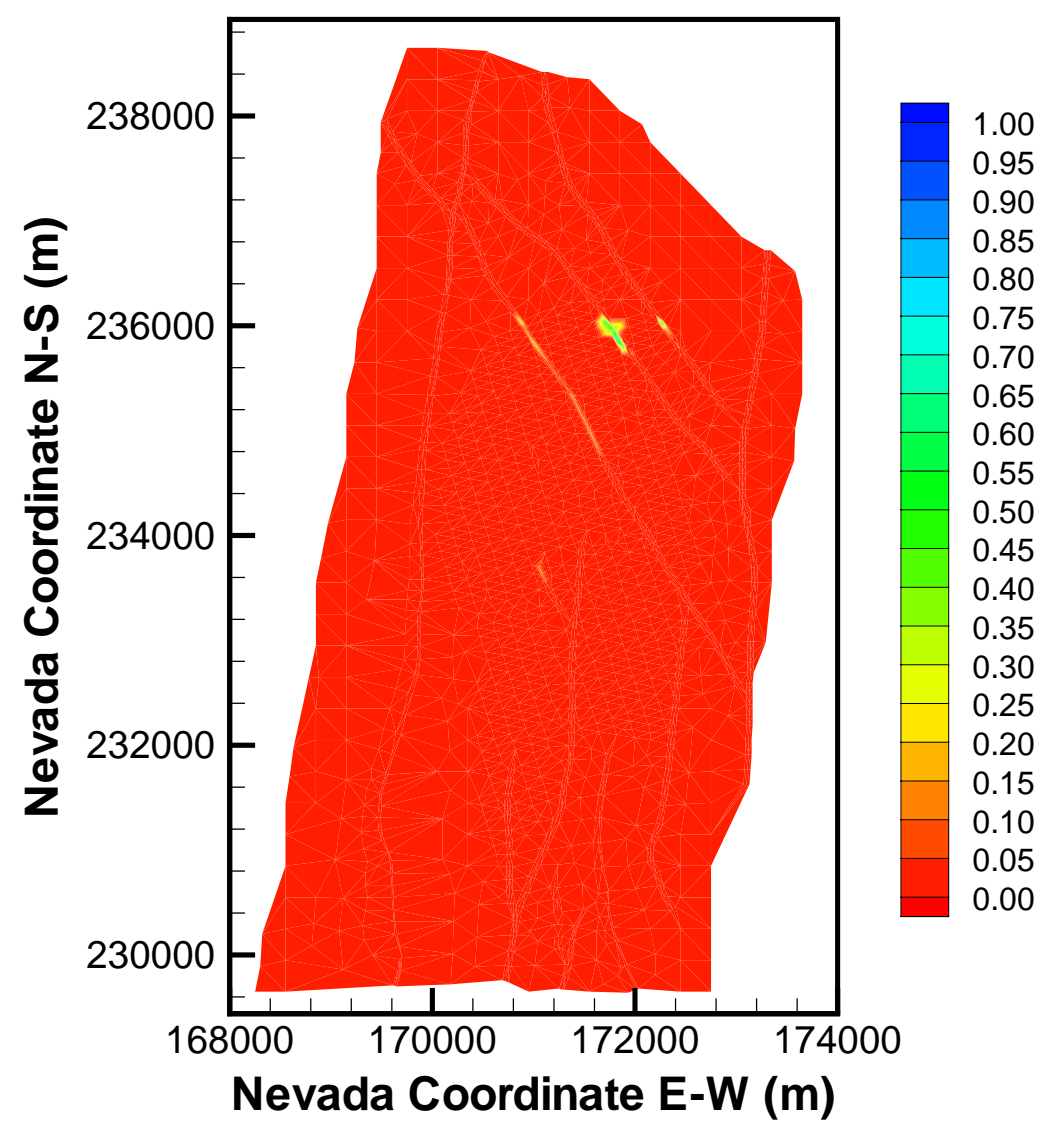

Output DTN: LB0307MR0060R1.004.

Figure F-1. Distribution of the Relative Mass Fraction $X_{R}$ of the $6 \mathrm{~nm}{ }^{239} \mathrm{PuO}_{2}$ Colloid in the Fractures of the tsw39 Layer at $t=10$ Years for Mean Present-Day Infiltration and Continuous Release

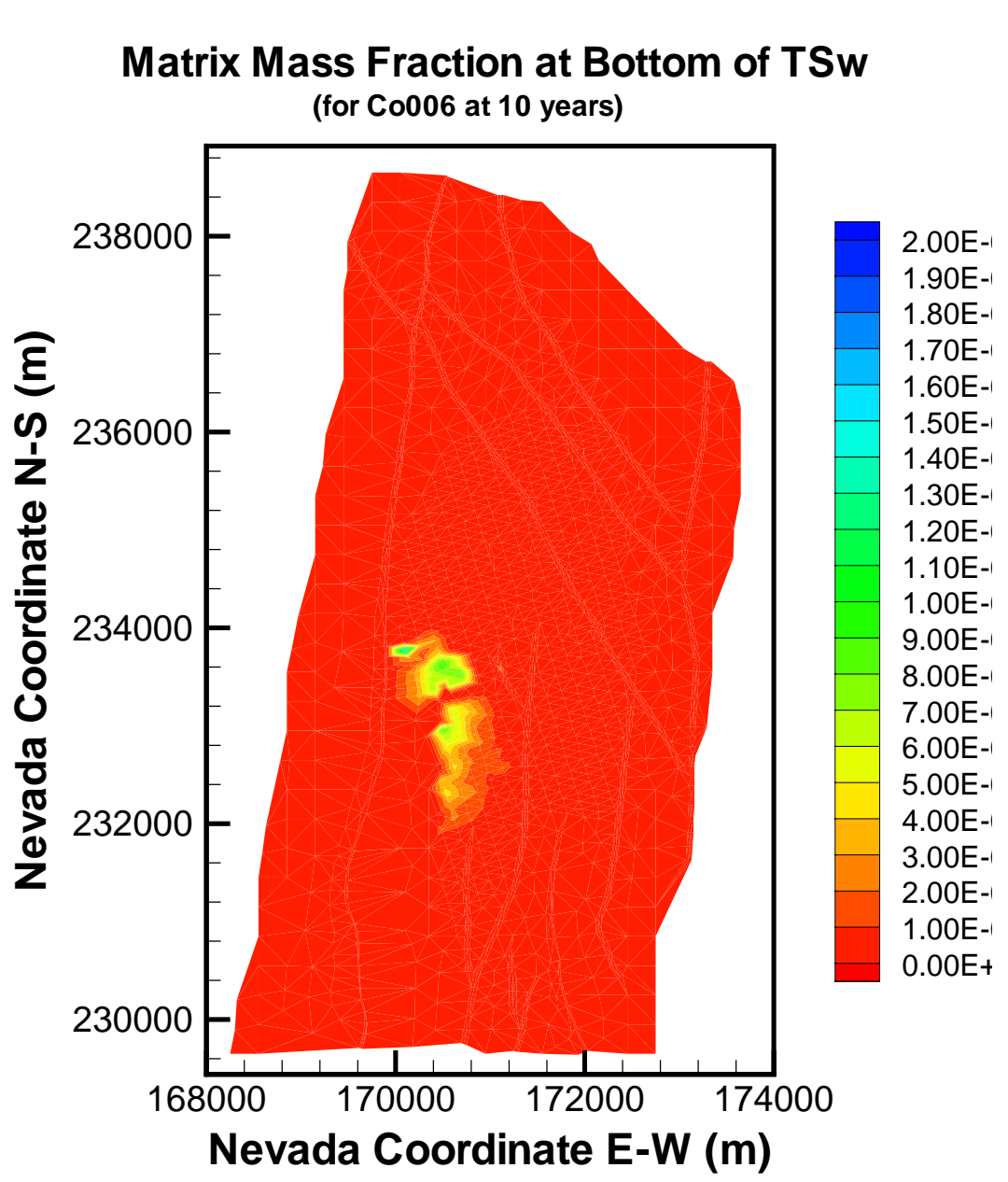

Output DTN: LB0307MR0060R1.004.

Figure F-2. Distribution of the Relative Mass Fraction $X_{R}$ of the $6 \mathrm{~nm}{ }^{239} \mathrm{Pu} \mathrm{O} \mathrm{O}_{2}$ Colloid in the Matrix of the tsw39 Layer at $t=10$ Years for Mean Present-Day Infiltration and Continuous Release 


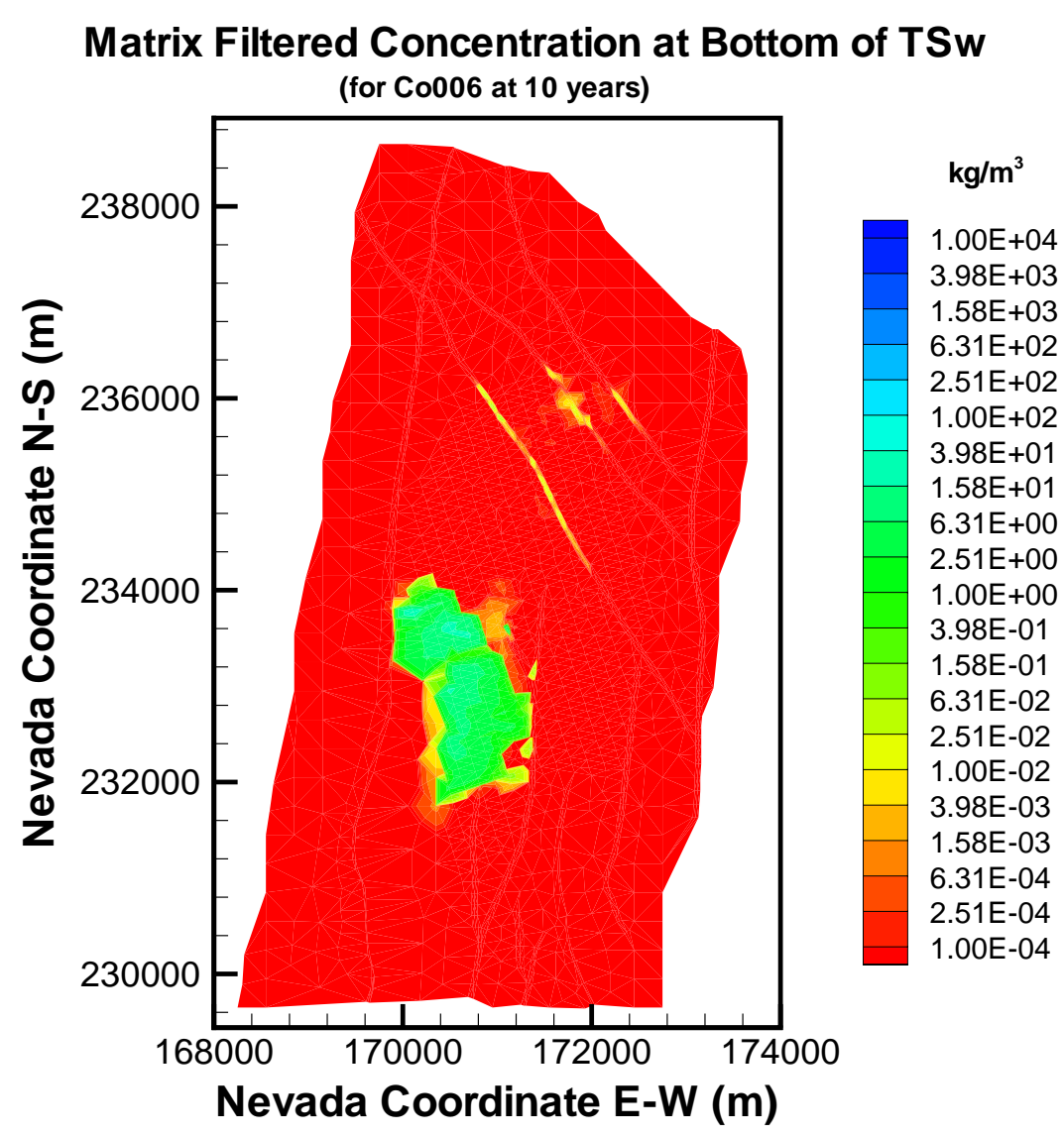

Output DTN: LB0307MR0060R1.004.

Figure F-3. Distribution of the Relative Filtered Concentration $X_{R}$ of the $6 \mathrm{~nm}{ }^{239} \mathrm{PuO}_{2}$ Colloid in the Matrix of the tsw39 Layer at $t=10$ Years for Mean Present-Day Infiltration and Continuous Release

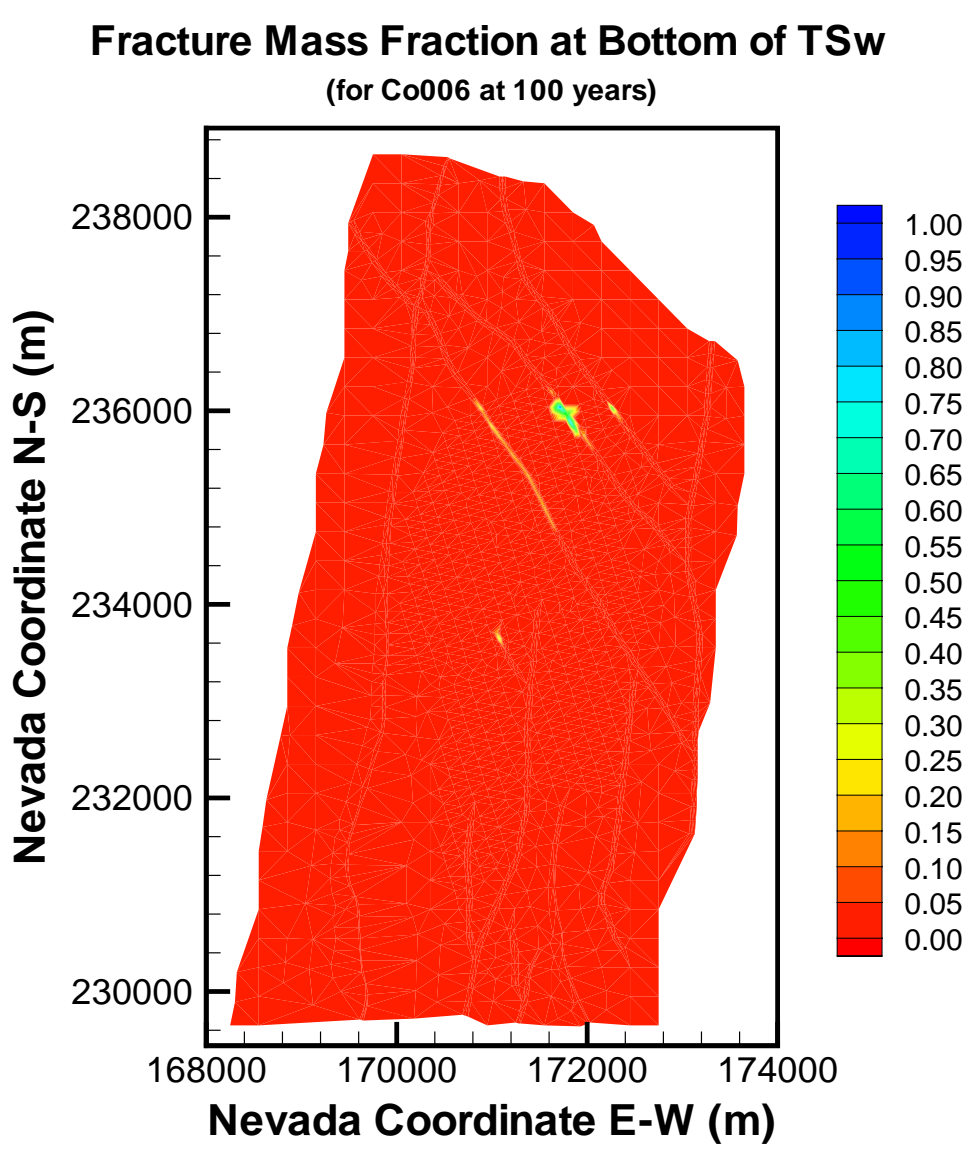

Output DTN: LB0307MR0060R1.004.

Figure F-4. Distribution of the Relative Mass Fraction $X_{R}$ of the $6 \mathrm{~nm}{ }^{239} \mathrm{PuO}_{2}$ Colloid in the Fractures of the tsw39 Layer at $\mathrm{t}=100$ Years for Mean Present-Day Infiltration and Continuous Release 


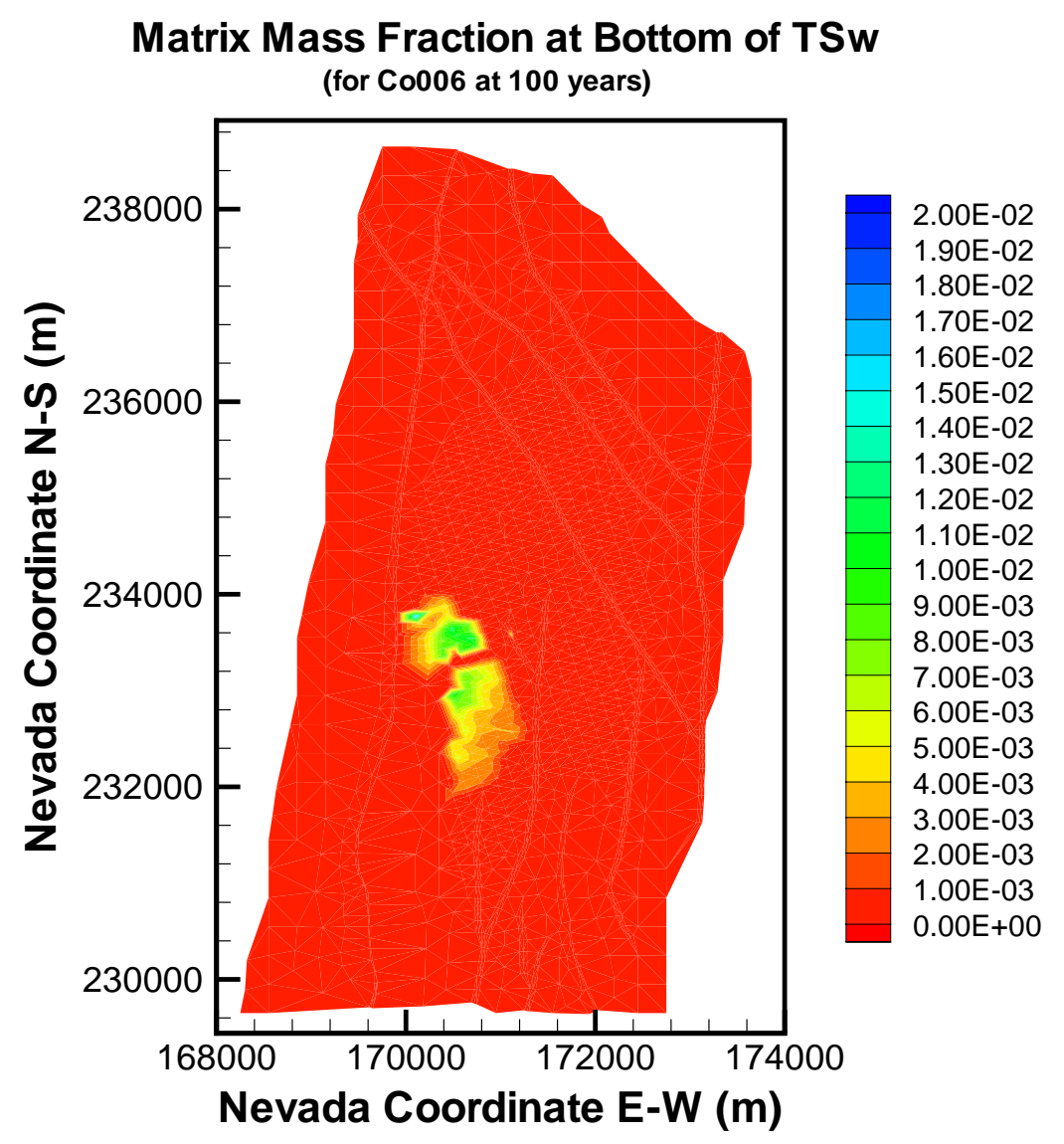

Output DTN: LB0307MR0060R1.004.

Figure F-5. Distribution of the Relative Mass Fraction $X_{R}$ of the $6 \mathrm{~nm}{ }^{239} \mathrm{PuO}_{2}$ Colloid in the Matrix of the tsw39 Layer at $\mathrm{t}=100$ Years for Mean Present-Day Infiltration and Continuous Release

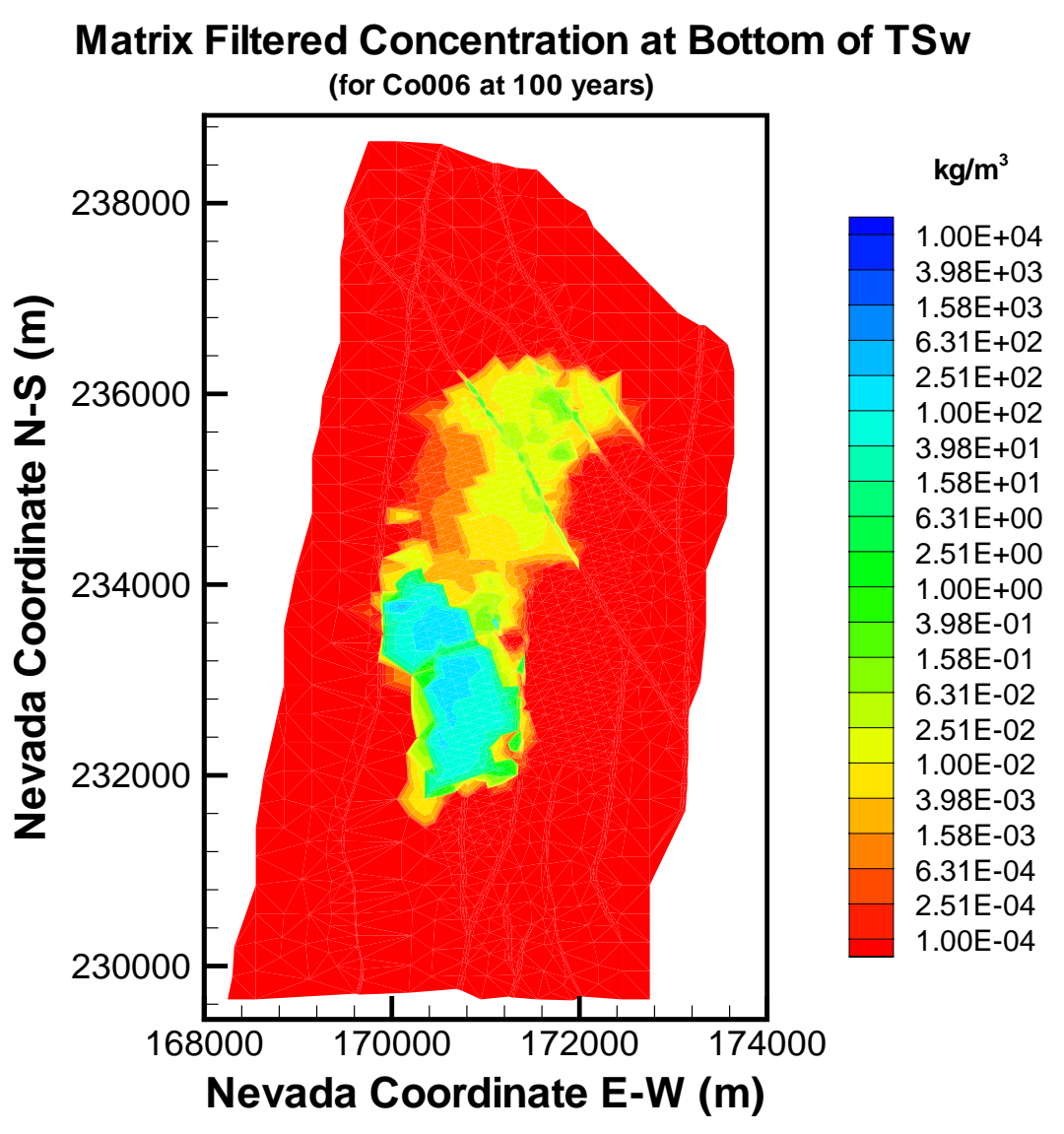

Output DTN: LB0307MR0060R1.004.

Figure F-6. Distribution of the Relative Filtered Concentration $\mathrm{X}_{R}$ of the $6 \mathrm{~nm}{ }^{239} \mathrm{PuO}_{2}$ Colloid in the Matrix of the tsw39 Layer at $t=100$ Years for Mean Present-Day Infiltration and Continuous Release 


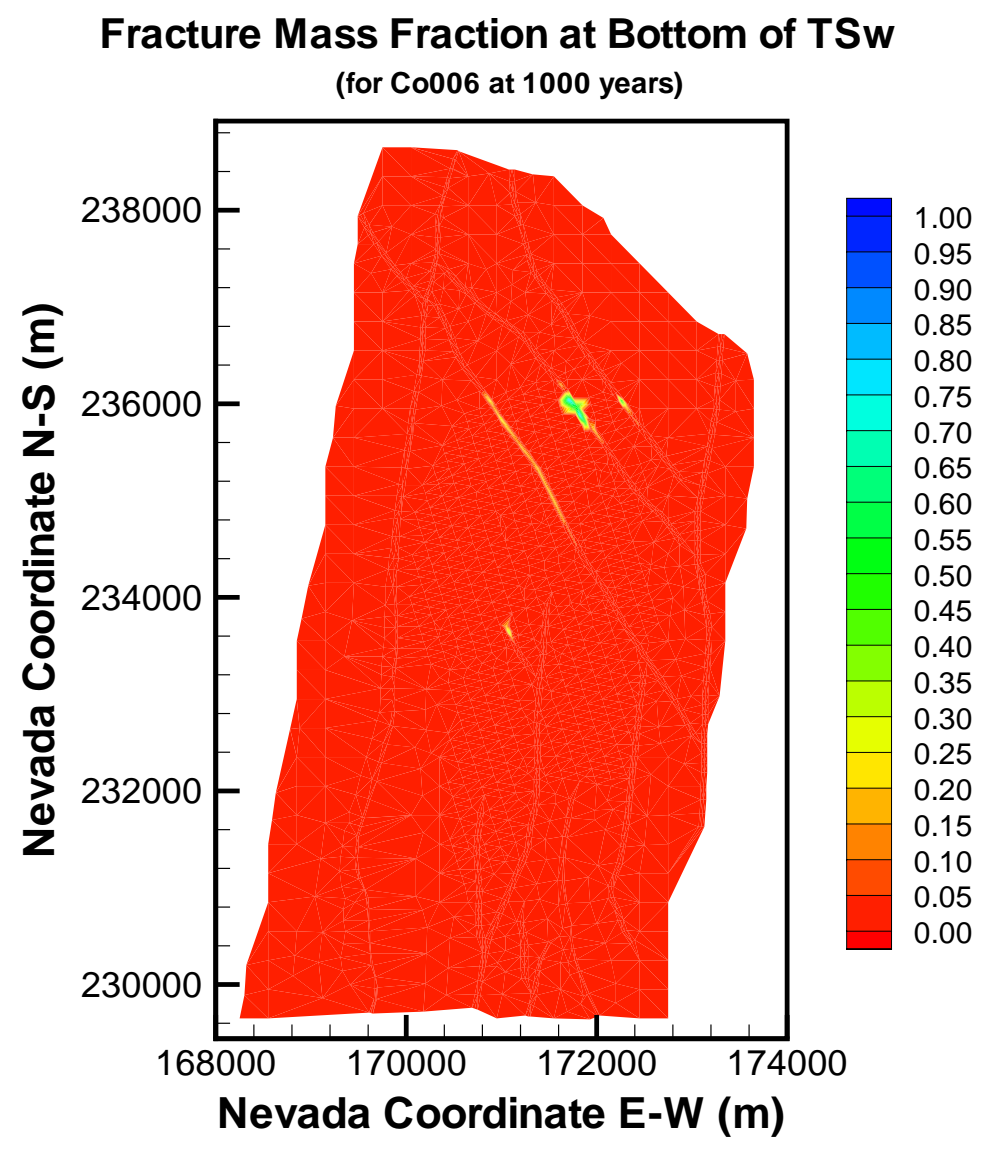

Output DTN: LB0307MR0060R1.004.

Figure F-7. Distribution of the Relative Mass Fraction $X_{R}$ of the $6 \mathrm{~nm}{ }^{239} \mathrm{PuO}_{2}$ Colloid in the Fractures of the tsw39 Layer at $t=1,000$ Years for Mean Present-Day Infiltration and Continuous Release

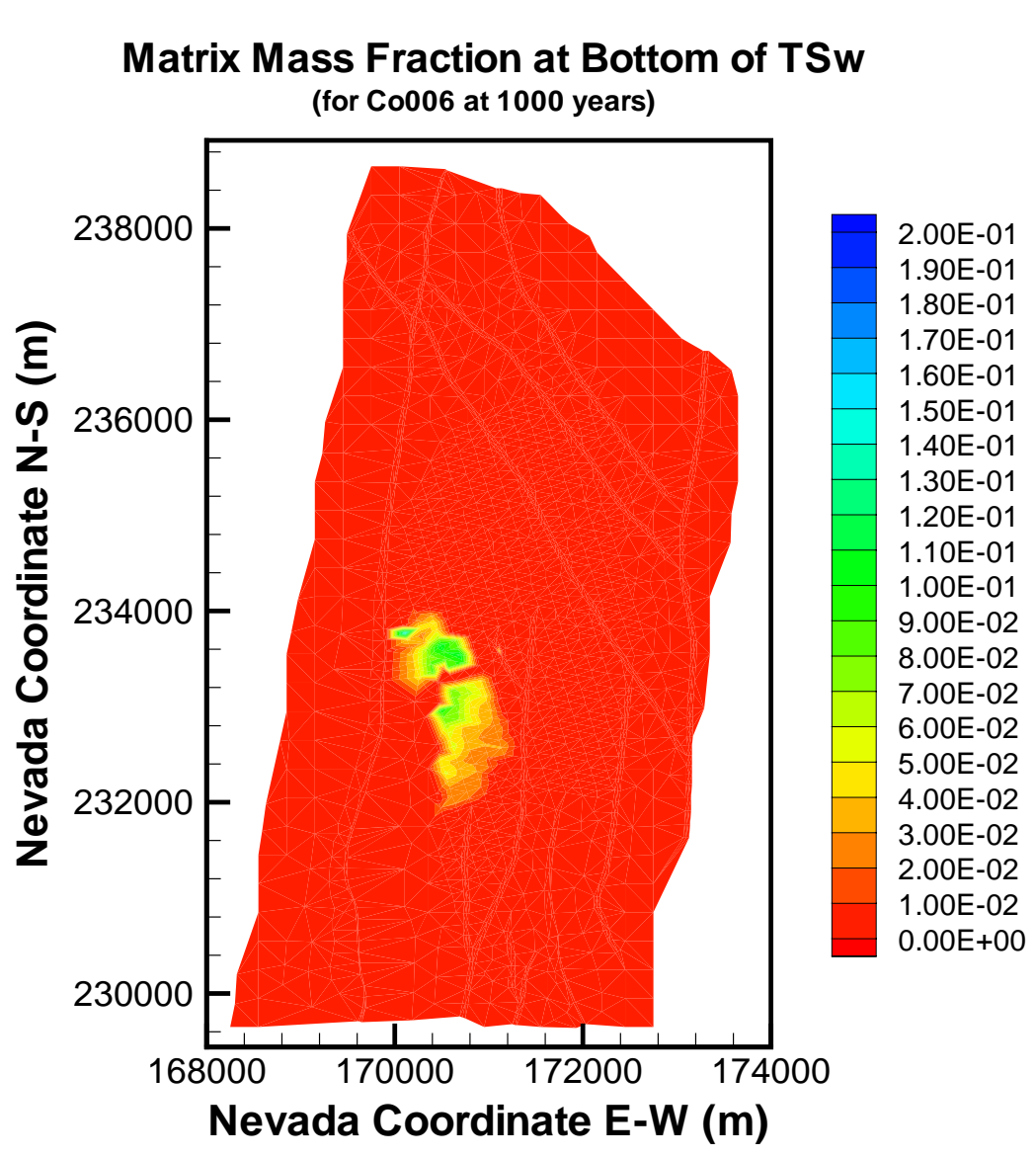

Output DTN: LB0307MR0060R1.004.

Figure F-8. Distribution of the Relative Mass Fraction $\mathrm{X}_{\mathrm{R}}$ of the $6 \mathrm{~nm}{ }^{239} \mathrm{PuO}_{2}$ Colloid in the Matrix of the tsw39 Layer at $t=1,000$ Years for Mean Present-Day Infiltration and Continuous Release 


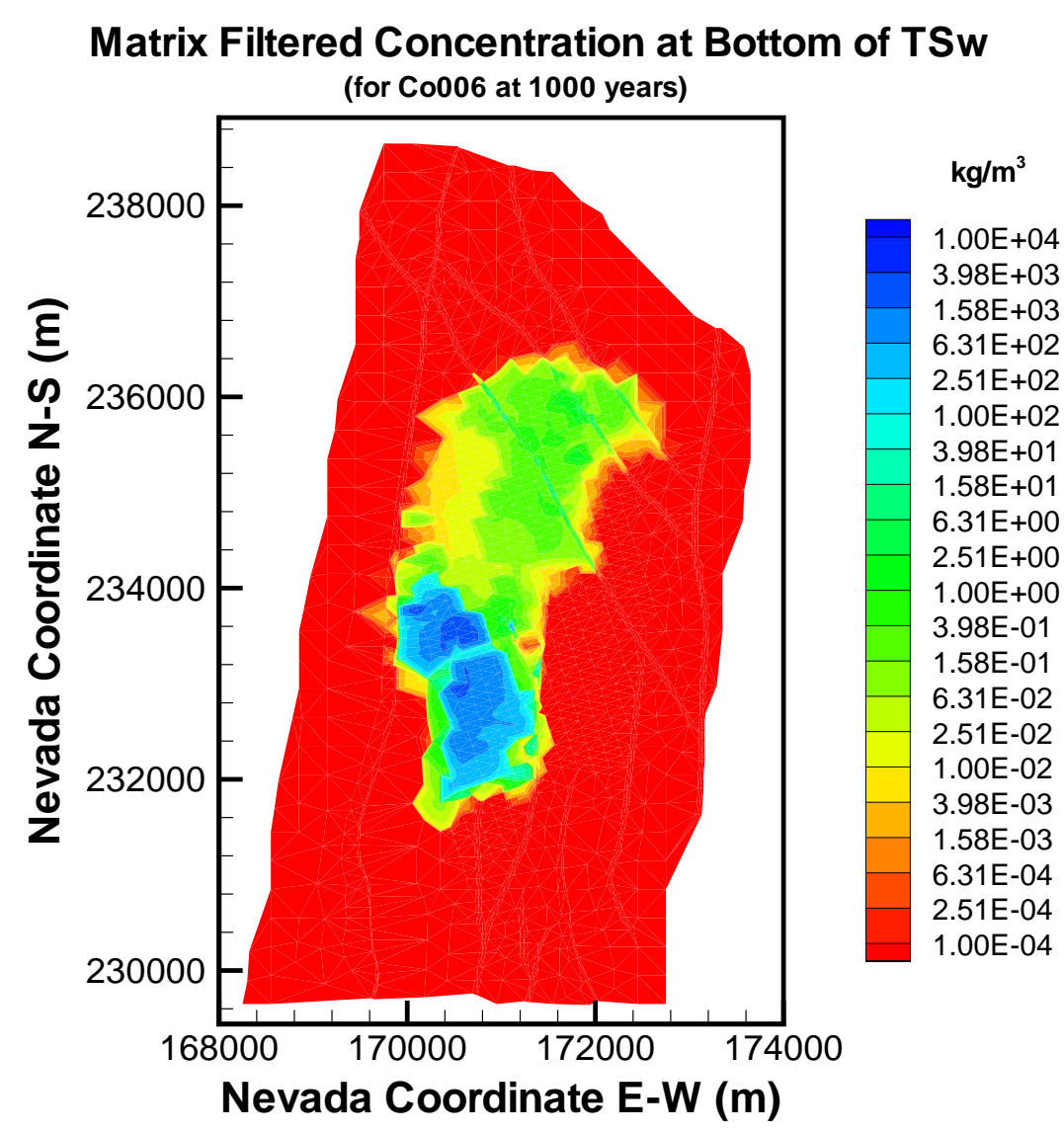

Output DTN: LB0307MR0060R1.004.

Figure F-9. Distribution of the Relative Filtered Concentration $X_{R}$ of the $6 \mathrm{~nm}{ }^{239} \mathrm{PuO}_{2}$ Colloid in the Matrix of the tsw39 Layer at $t=1,000$ Years for Mean Present-Day Infiltration and Continuous Release
Fracture Mass Fraction at Bottom of TSw

(for $\mathrm{C} 0006$ at 10000 years)

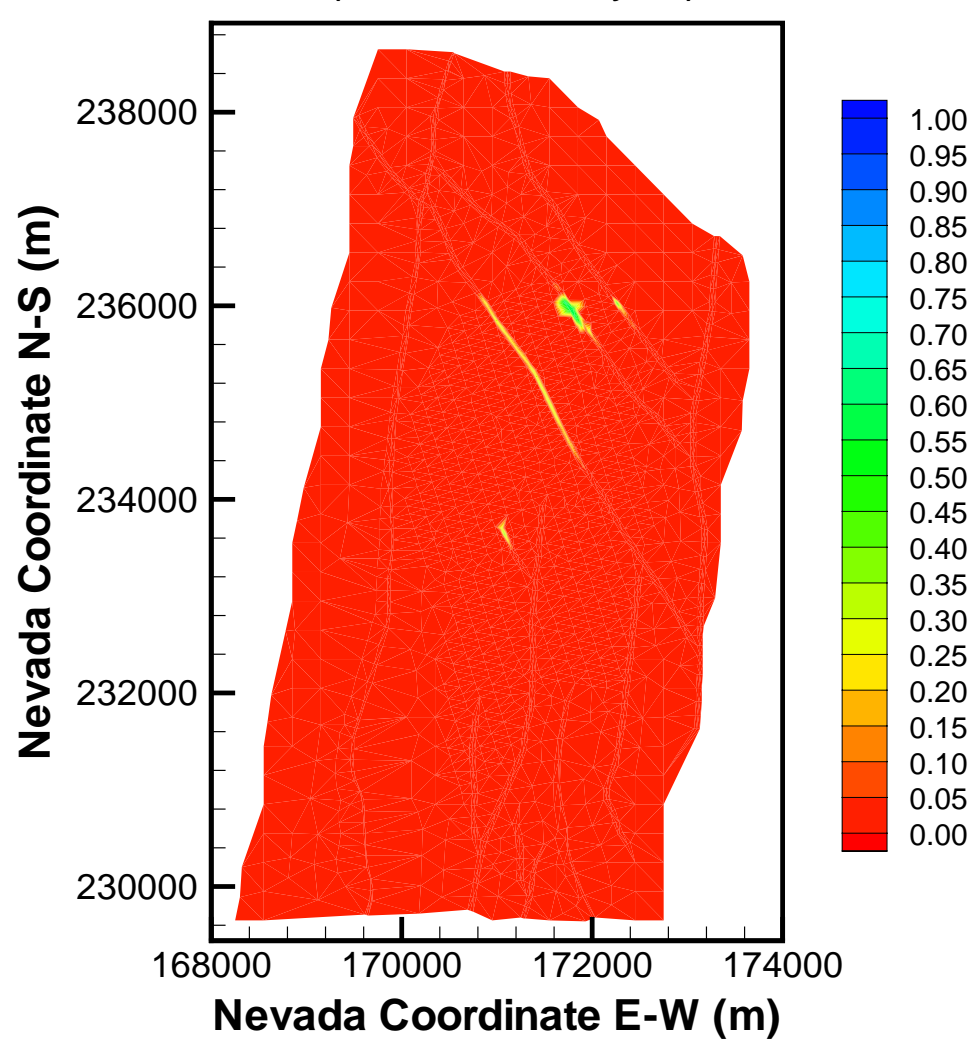

Output DTN: LB0307MR0060R1.004.

Figure F-10. Distribution of the Relative Mass Fraction $X_{R}$ of the $6 \mathrm{~nm}{ }^{239} \mathrm{PuO}_{2}$ Colloid in the Fractures of the tsw39 Layer at $\mathrm{t}=10,000$ Years for Mean Present-Day Infiltration and Continuous Release 


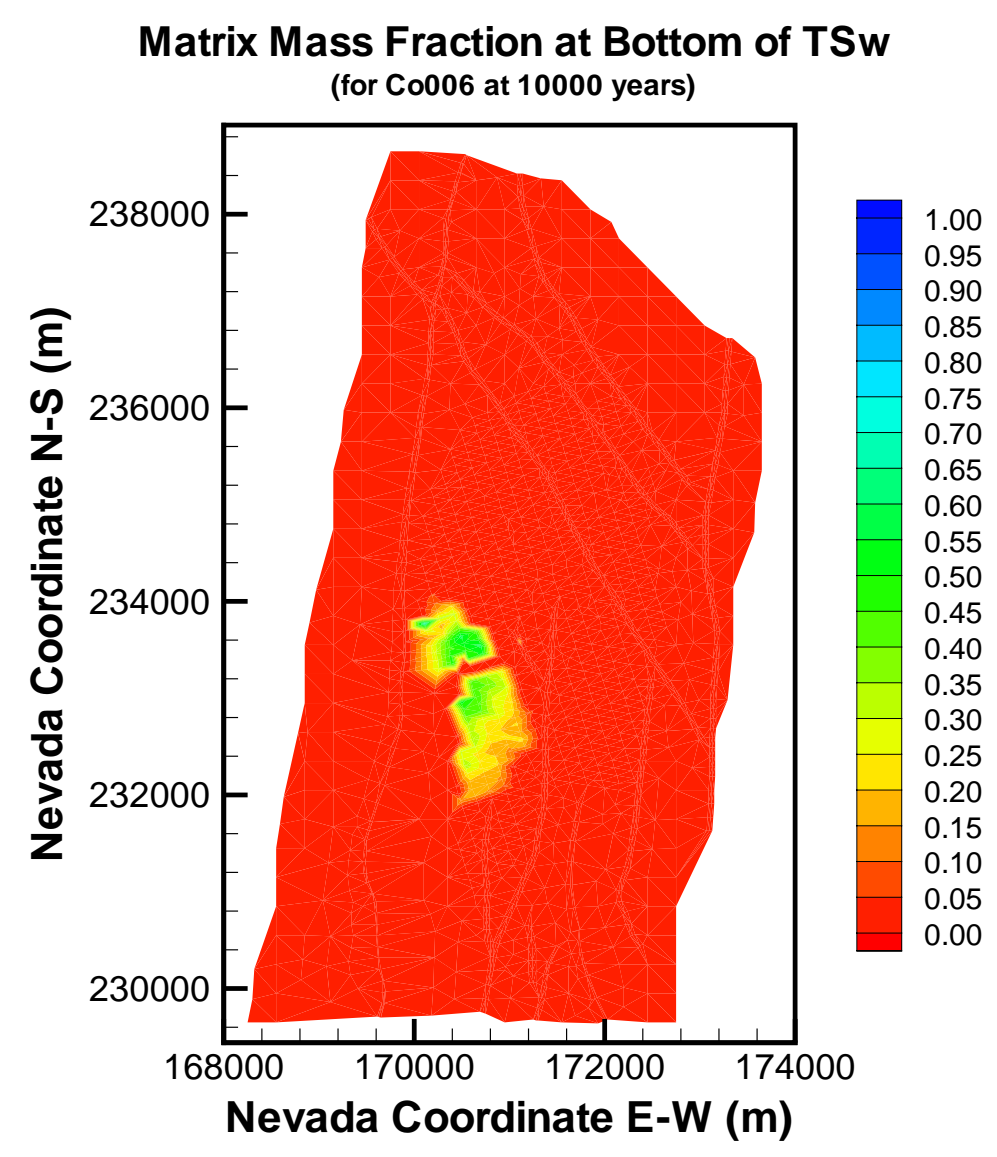

Output DTN: LB0307MR0060R1.004.

Figure F-11. Distribution of the Relative Mass Fraction $X_{R}$ of the $6 \mathrm{~nm}^{239} \mathrm{PuO}_{2}$ Colloid in the Matrix of the tsw39 Layer at $\mathrm{t}=10,000$ Years for Mean Present-Day Infiltration and Continuous Release

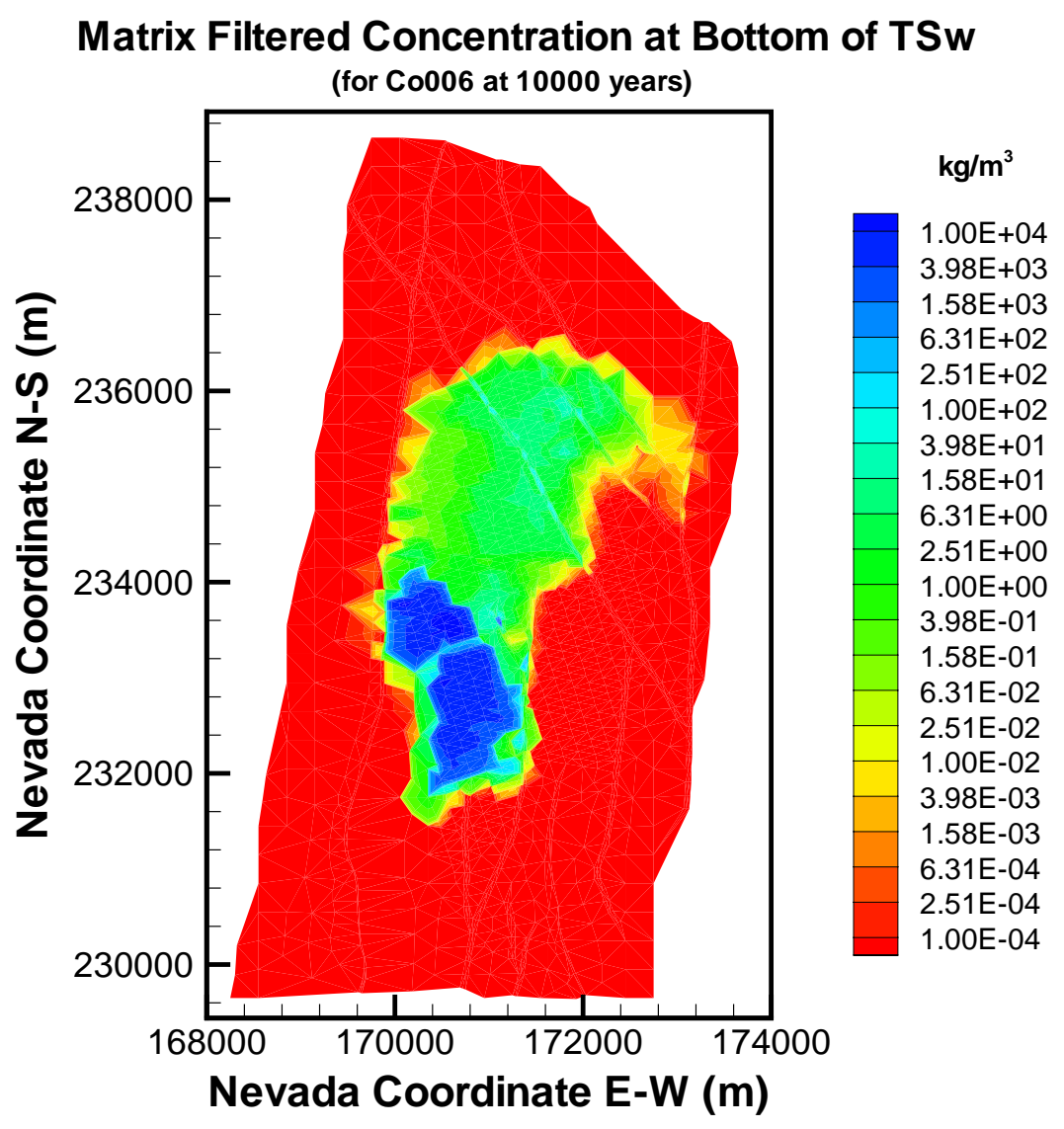

Output DTN: LB0307MR0060R1.004.

Figure F-12. Distribution of the Relative Filtered Concentration $F_{R}$ of the $6 \mathrm{~nm}{ }^{239} \mathrm{PuO}_{2}$ Colloid in the Matrix of the tsw39 Layer at $\mathrm{t}=10,000$ Years for Mean Present-Day Infiltration and Continuous Release 


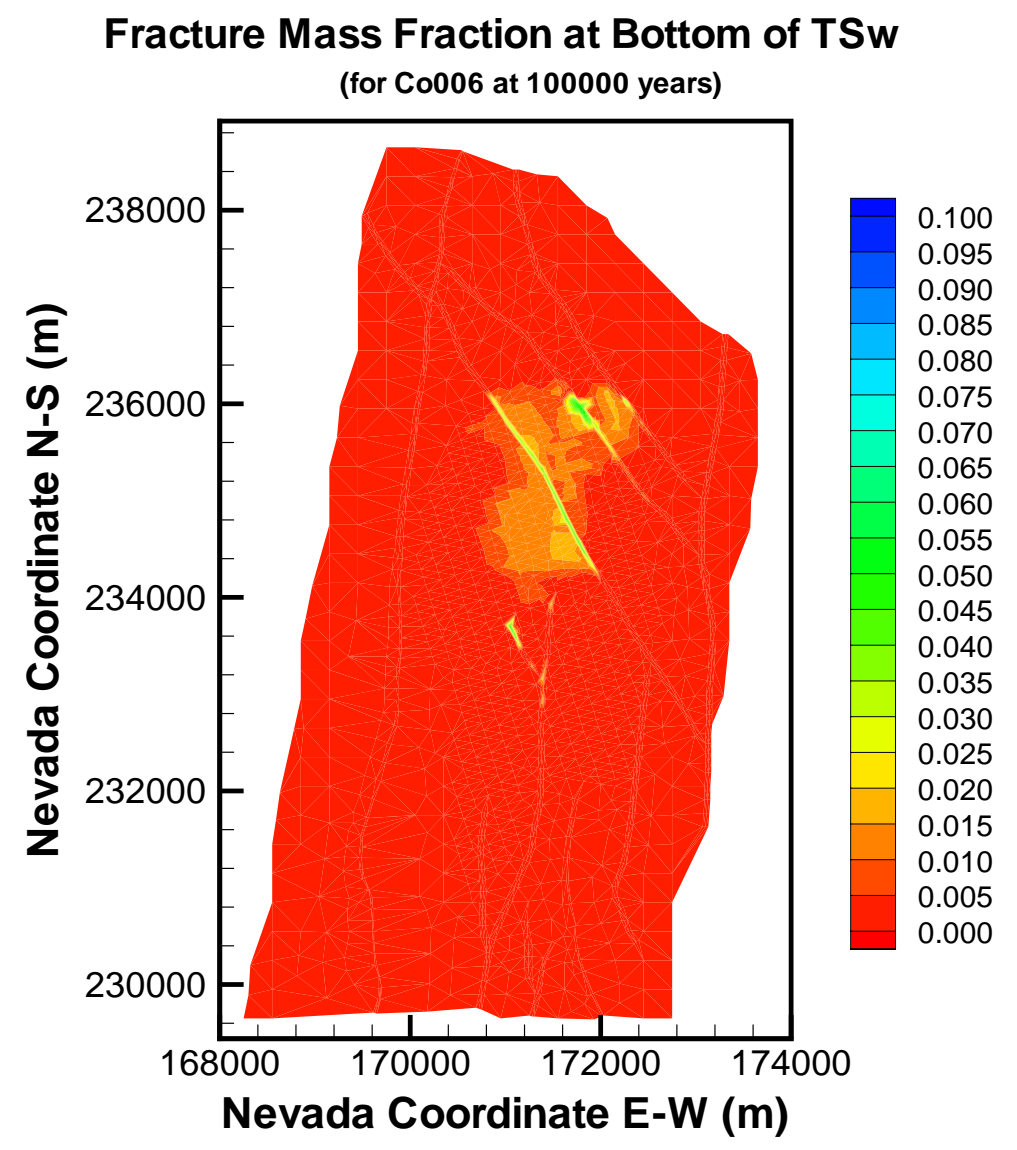

Output DTN: LB0307MR0060R1.004.

Figure F-13. Distribution of the Relative Mass Fraction $X_{R}$ of the $6 \mathrm{~nm}{ }^{239} \mathrm{PuO}_{2}$ Colloid in the Fractures of the tsw39 Layer at $\mathrm{t}=100,000$ Years for Mean Present-Day Infiltration and Continuous Release

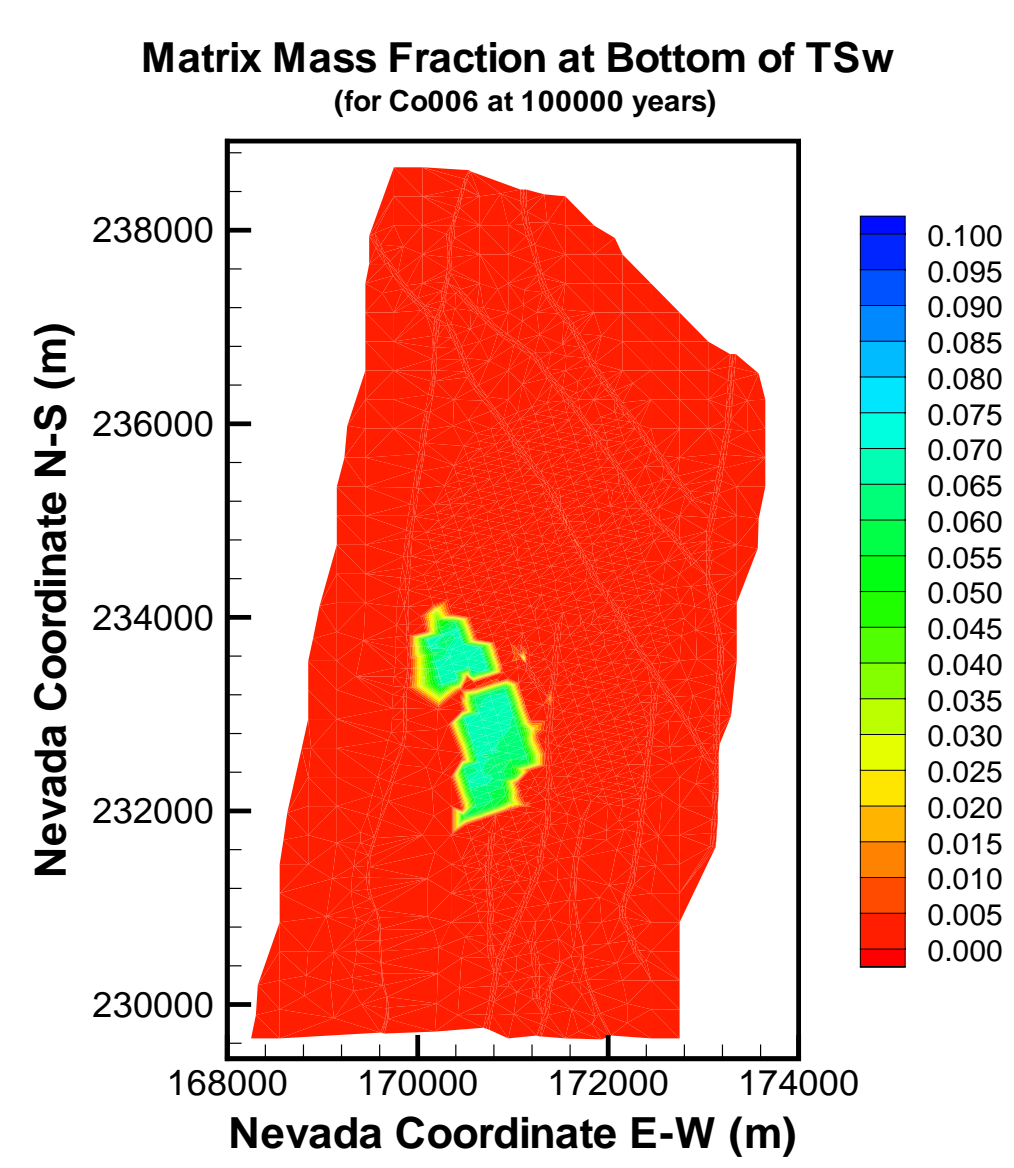

Output DTN: LB0307MR0060R1.004.

Figure F-14. Distribution of the Relative Mass Fraction $X_{R}$ of the $6 \mathrm{~nm}{ }^{239} \mathrm{PuO}_{2}$ Colloid in the Matrix of the tsw39 Layer at $t=100,000$ Years for Mean Present-Day Infiltration and Continuous Release 


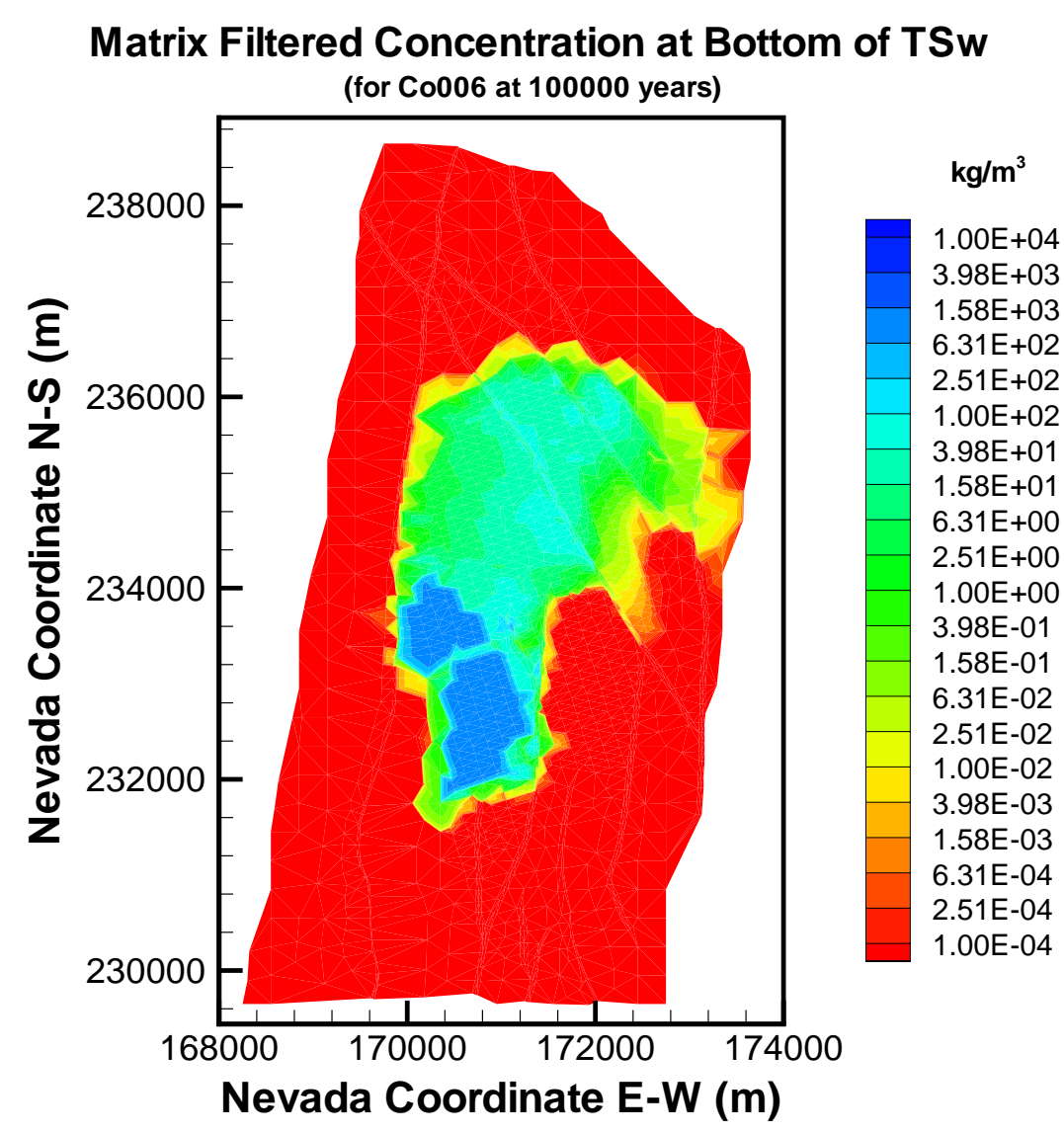

Output DTN: LB0307MR0060R1.004.

Figure F-15. Distribution of the Relative Filtered Concentration $F_{R}$ of the $6 \mathrm{~nm}^{239} \mathrm{PuO}_{2}$ Colloid in the Matrix of the tsw39 Layer at $t=100,000$ Years for Mean Present-Day Infiltration and Continuous Release

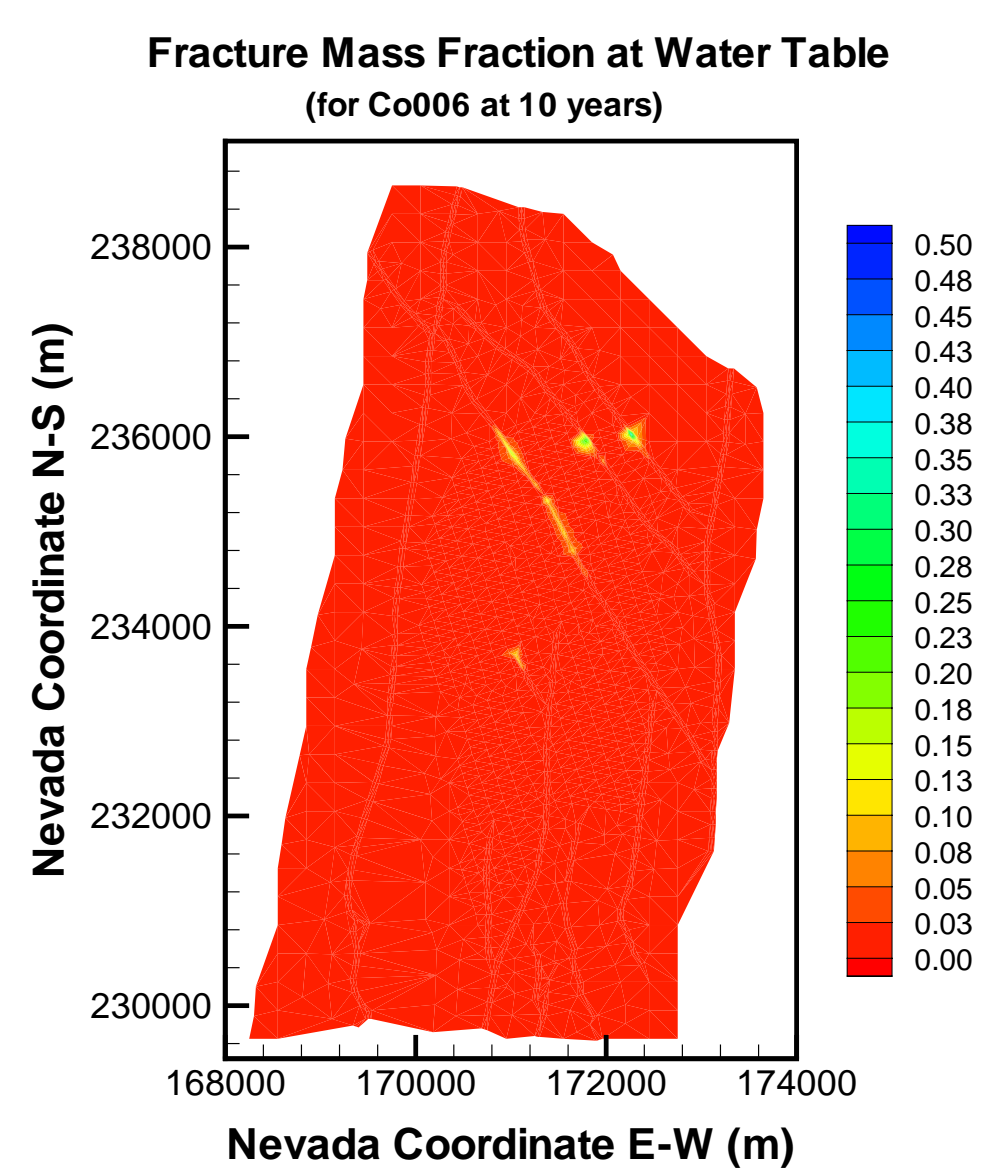

Output DTN: LB0307MR0060R1.004.

Figure F-16. Distribution of the Relative Mass Fraction $\mathrm{X}_{R}$ of the $6 \mathrm{~nm}^{239} \mathrm{PuO}_{2}$ Colloid in the Fractures Immediately above the Groundwater Table at $t=10$ Years for Mean Present-Day Infiltration and Continuous Release 


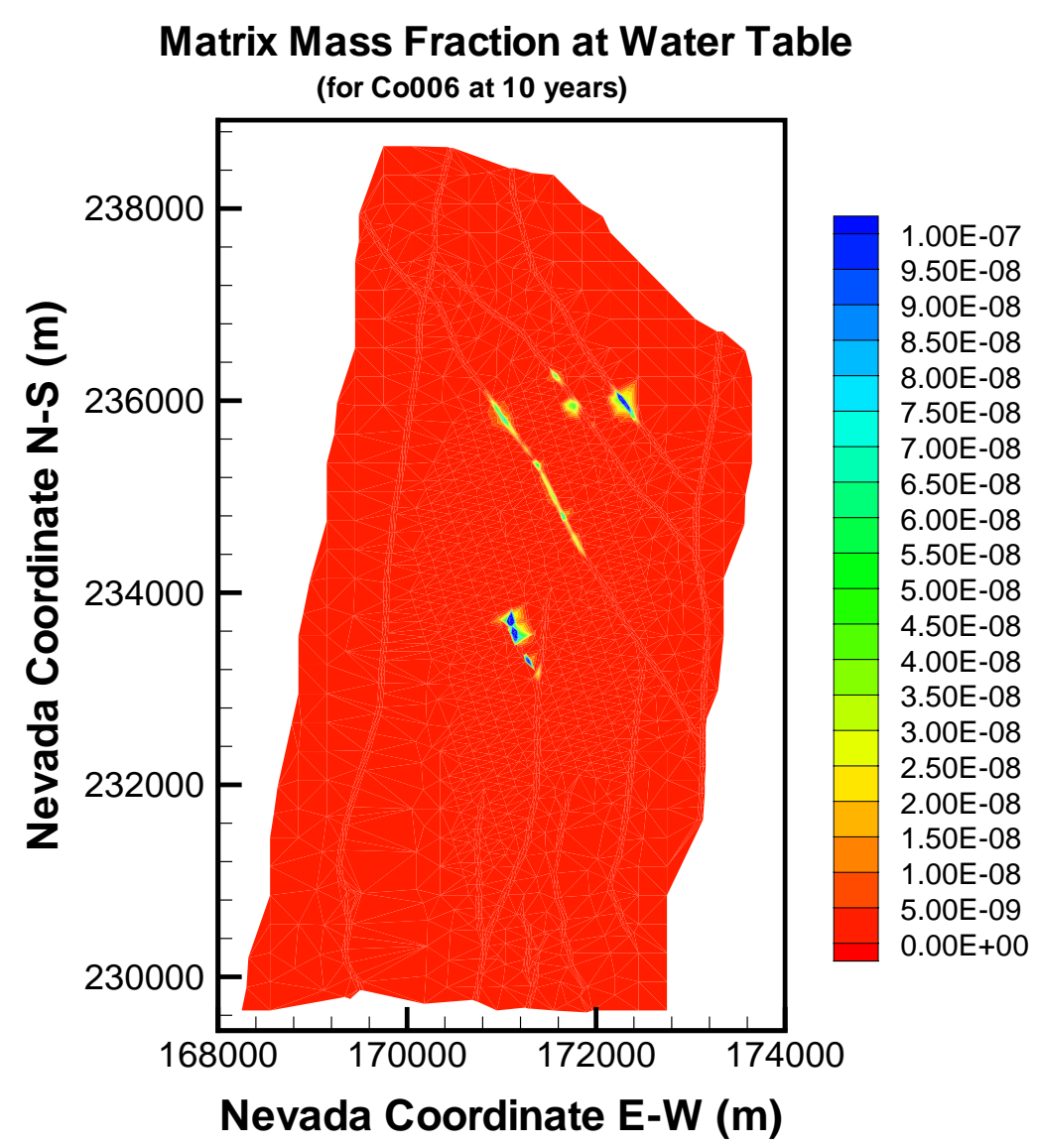

Output DTN: LB0307MR0060R1.004.

Figure $\mathrm{F}-17$. Distribution of the Relative Mass Fraction $\mathrm{X}_{\mathrm{R}}$ of the $6 \mathrm{~nm}^{239} \mathrm{PuO}_{2}$ Colloid in the Matrix immediately above the Groundwater at $t=10$ Years for Mean PresentDay Infiltration and Continuous Release

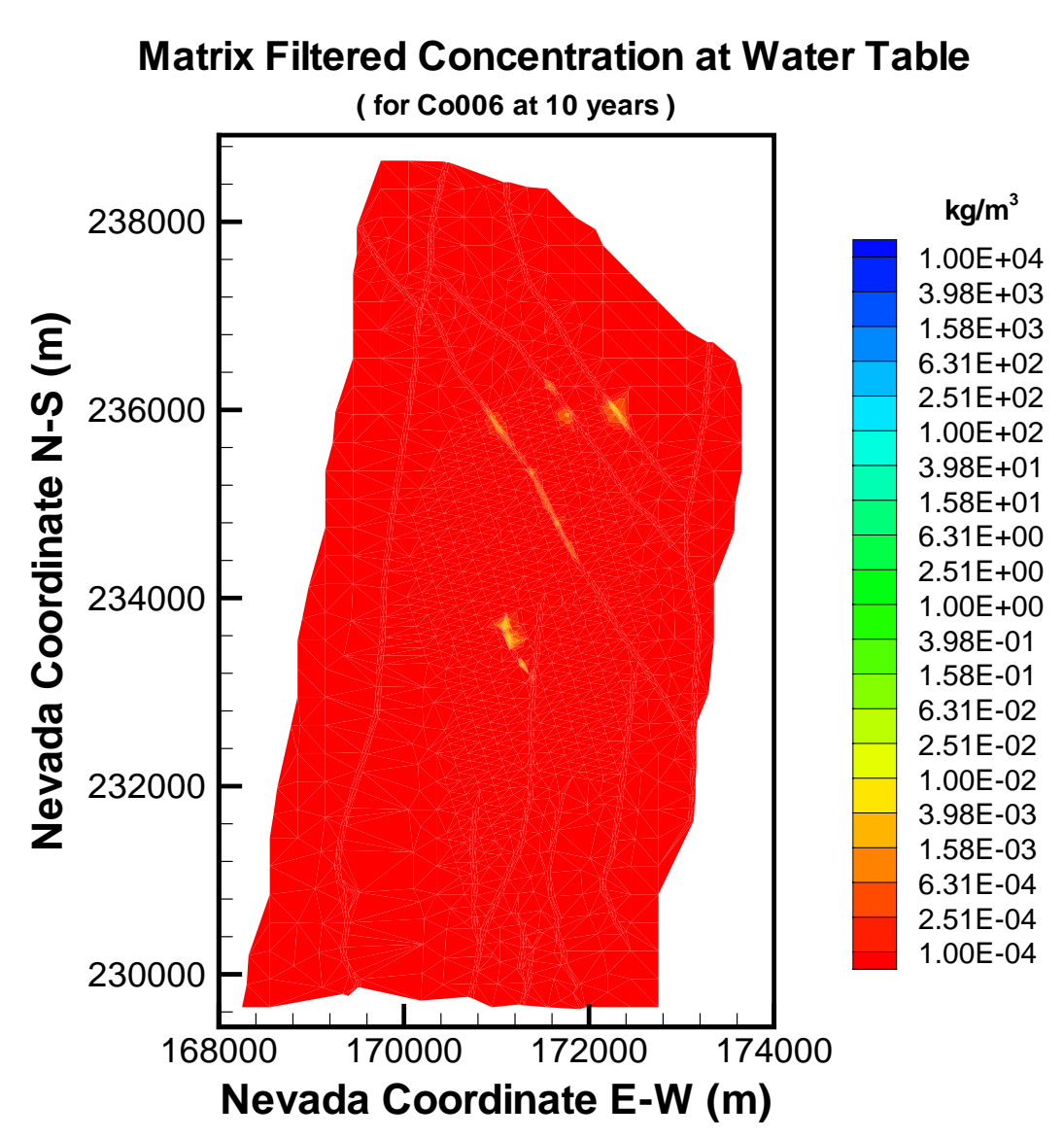

Output DTN: LB0307MR0060R1.004.

Figure F-18. Distribution of the Relative Filtered Concentration $F_{R}$ of the $6 \mathrm{~nm}^{239} \mathrm{PuO}_{2}$ Colloid in the Matrix immediately above the Groundwater at $t=10$ Years for Mean Present-Day Infiltration and Continuous Release 
Fracture Mass Fraction at Water Table (for Co006 at 100 years)

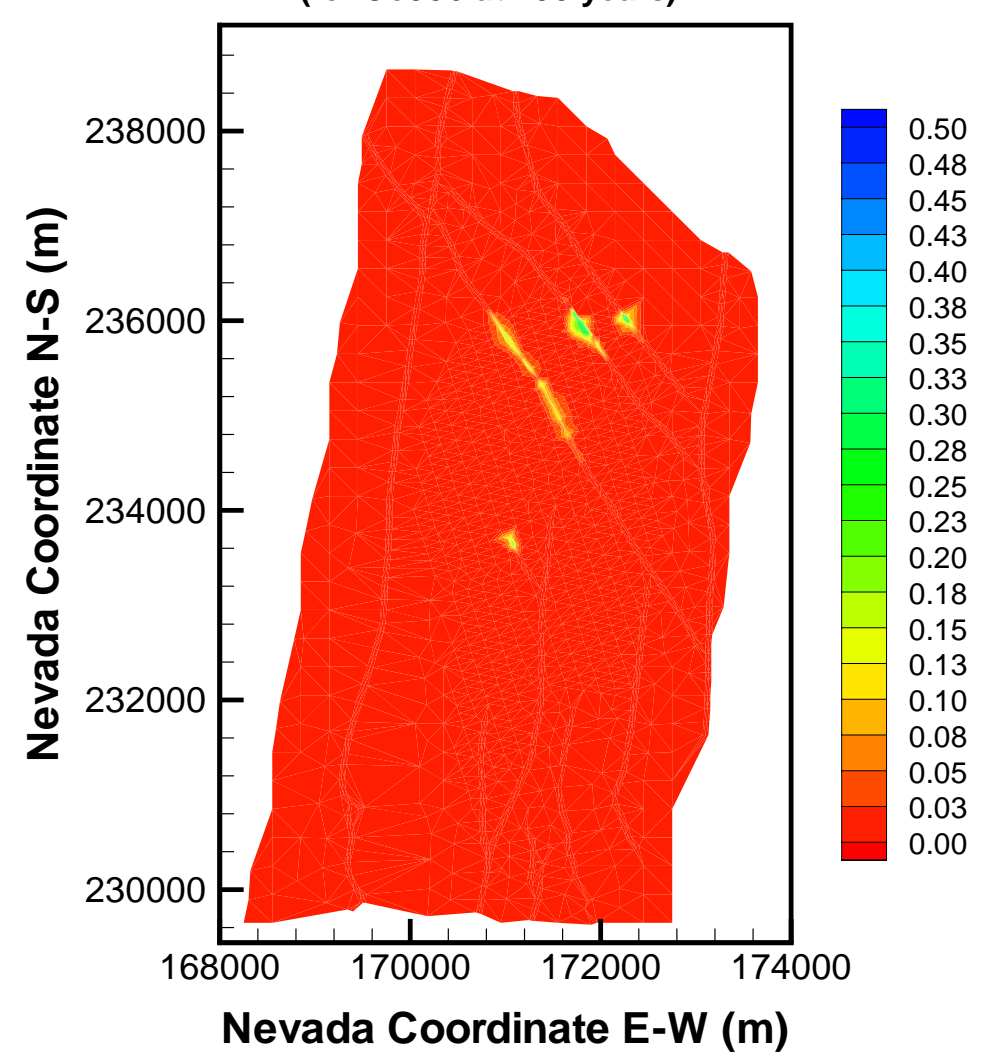

Output DTN: LB0307MR0060R1.004.

Figure F-19. Distribution of the Relative Mass Fraction $X_{R}$ of the $6 \mathrm{~nm}^{239} \mathrm{PuO}_{2}$ Colloid in the Fractures Immediately above the Groundwater at $t=100$ Years for Mean Present-Day Infiltration and Continuous Release

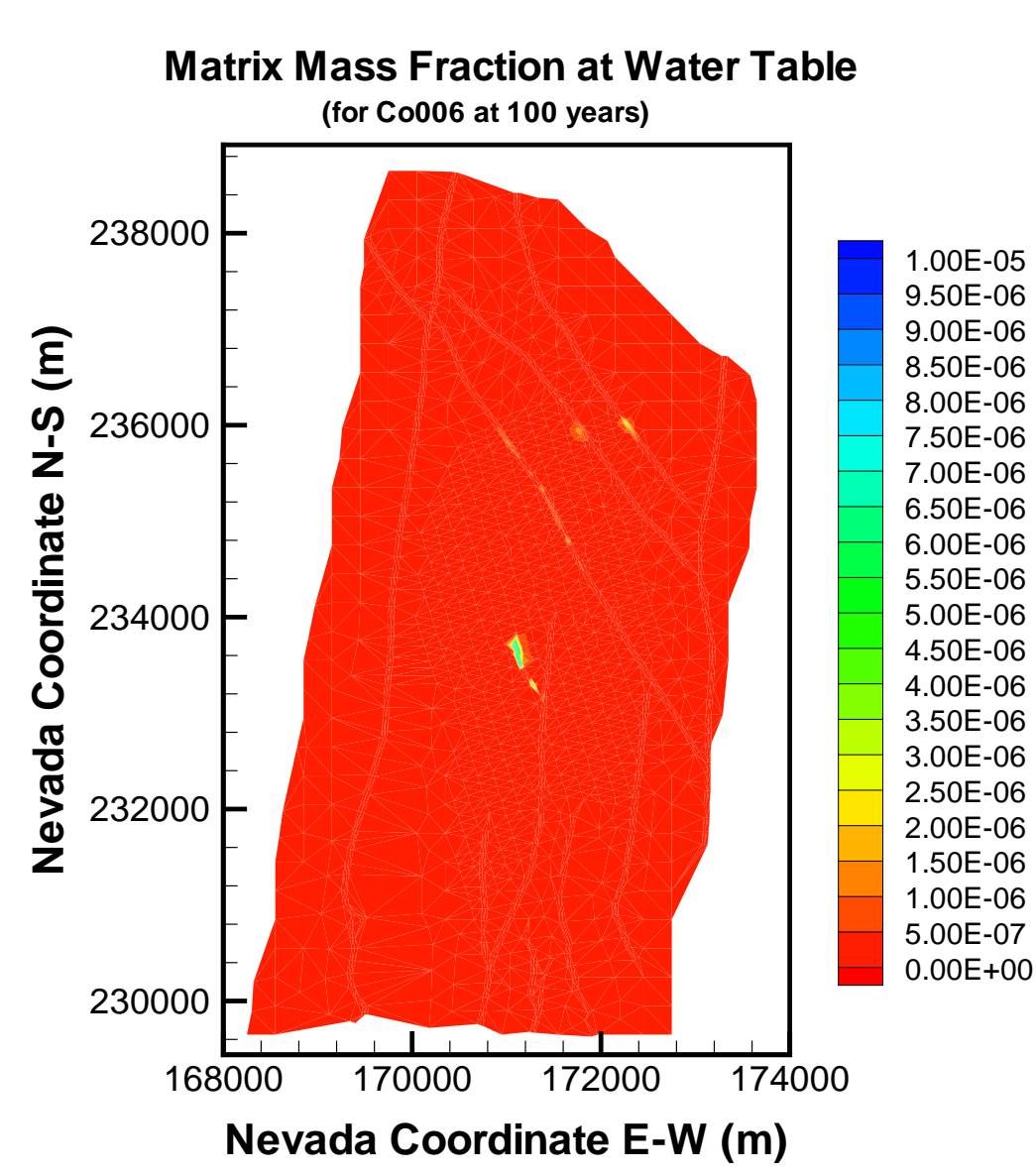

Output DTN: LB0307MR0060R1.004.

Figure F-20. Distribution of the Relative Mass Fraction $\mathrm{X}_{\mathrm{R}}$ of the $6 \mathrm{~nm}{ }^{239} \mathrm{PuO}_{2}$ Colloid in the Matrix Immediately above the Groundwater at $\mathrm{t}=100$ Years for Mean PresentDay Infiltration and Continuous Release 


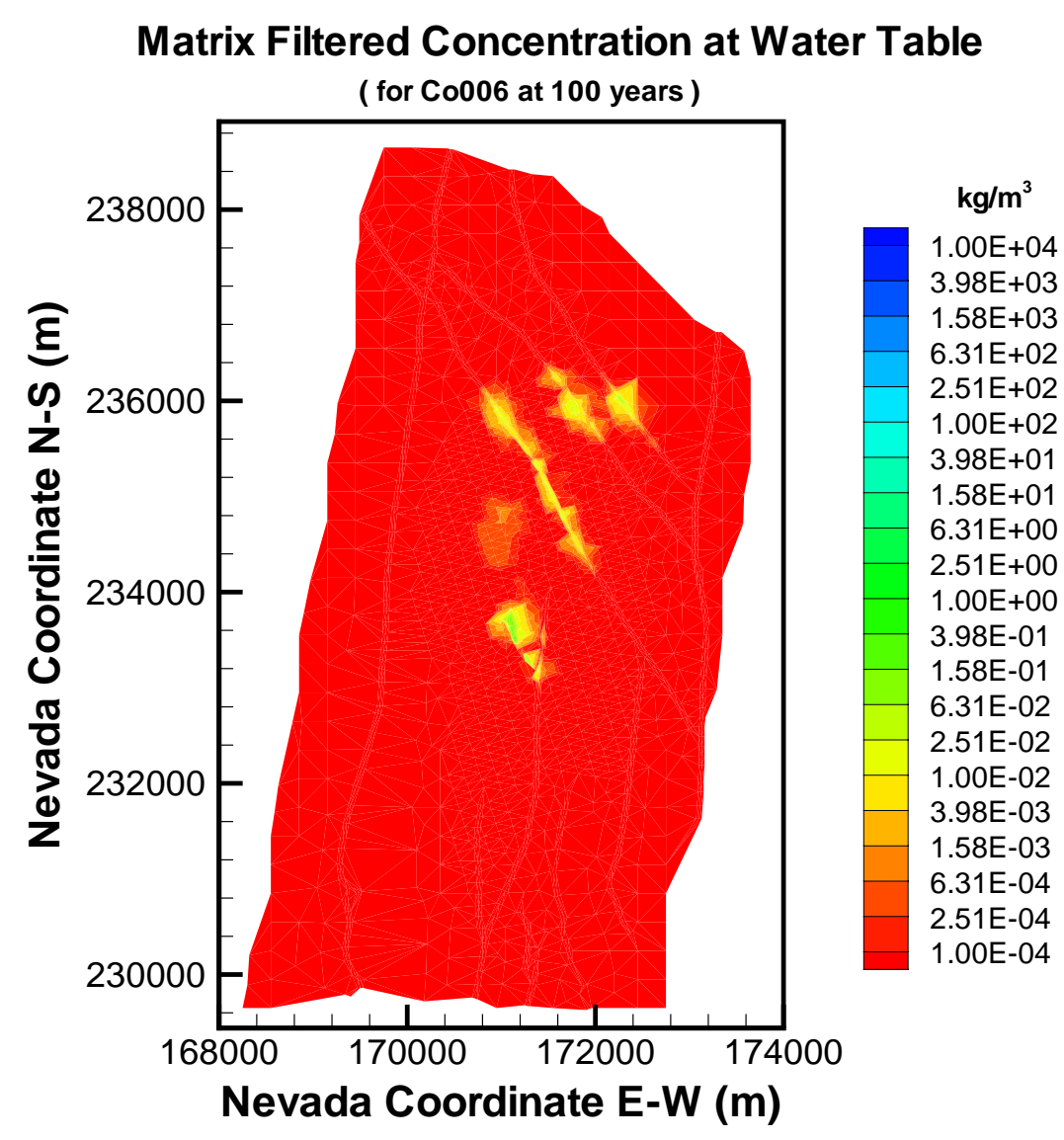

Output DTN: LB0307MR0060R1.004.

Figure F-21. Distribution of the Relative Filtered Concentration $F_{R}$ of the $6 \mathrm{~nm}^{239} \mathrm{PuO}_{2}$ Colloid in the Matrix Immediately above the Groundwater at $t=100$ Years for Mean Present-Day Infiltration and Continuous Release

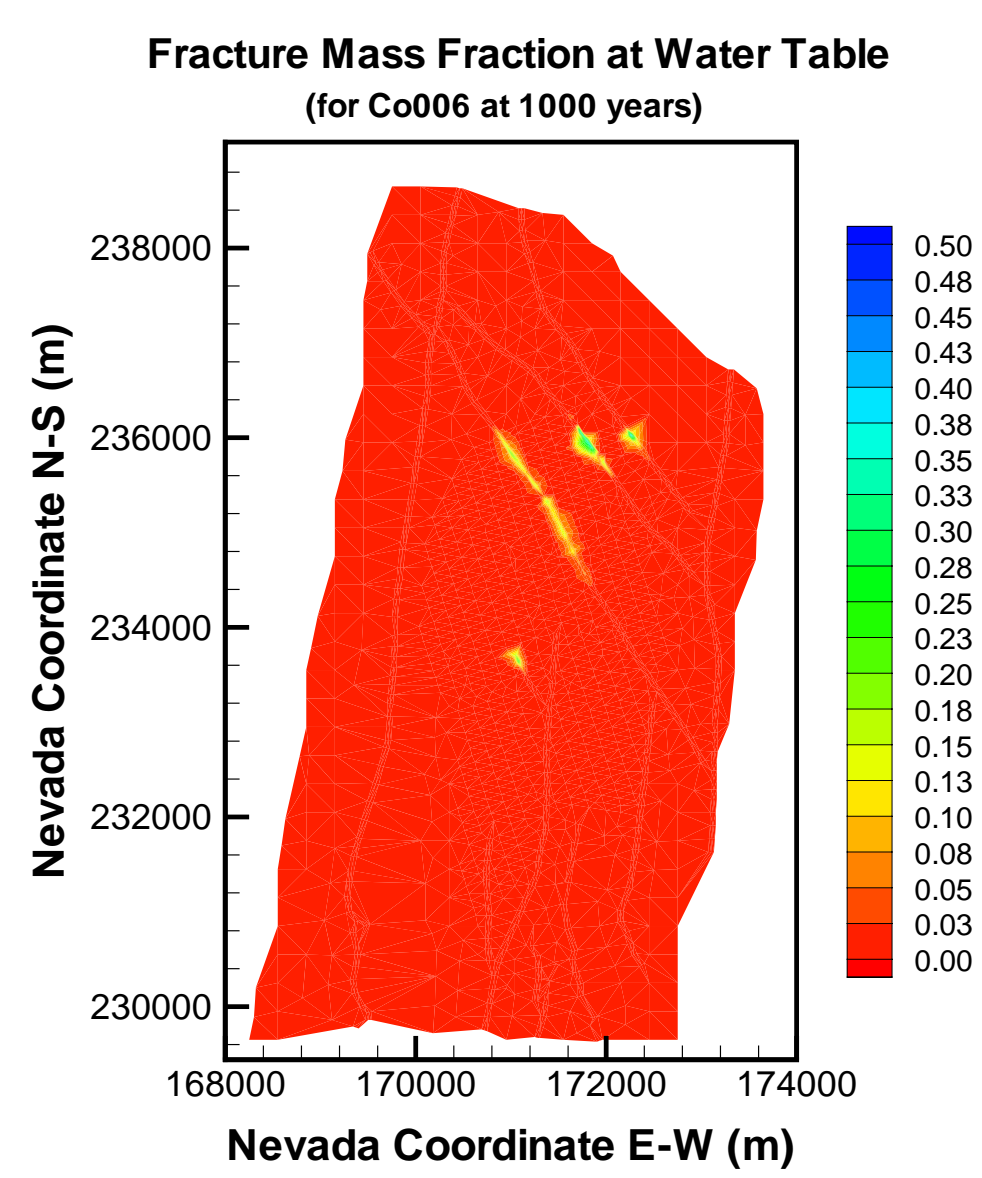

Output DTN: LB0307MR0060R1.004.

Figure F-22. Distribution of the Relative Mass Fraction $\mathrm{X}_{R}$ of the $6 \mathrm{~nm}^{239} \mathrm{PuO}_{2}$ Colloid in the Fractures Immediately above the Groundwater at $t=1,000$ Years for Mean Present-Day Infiltration and Continuous Release 


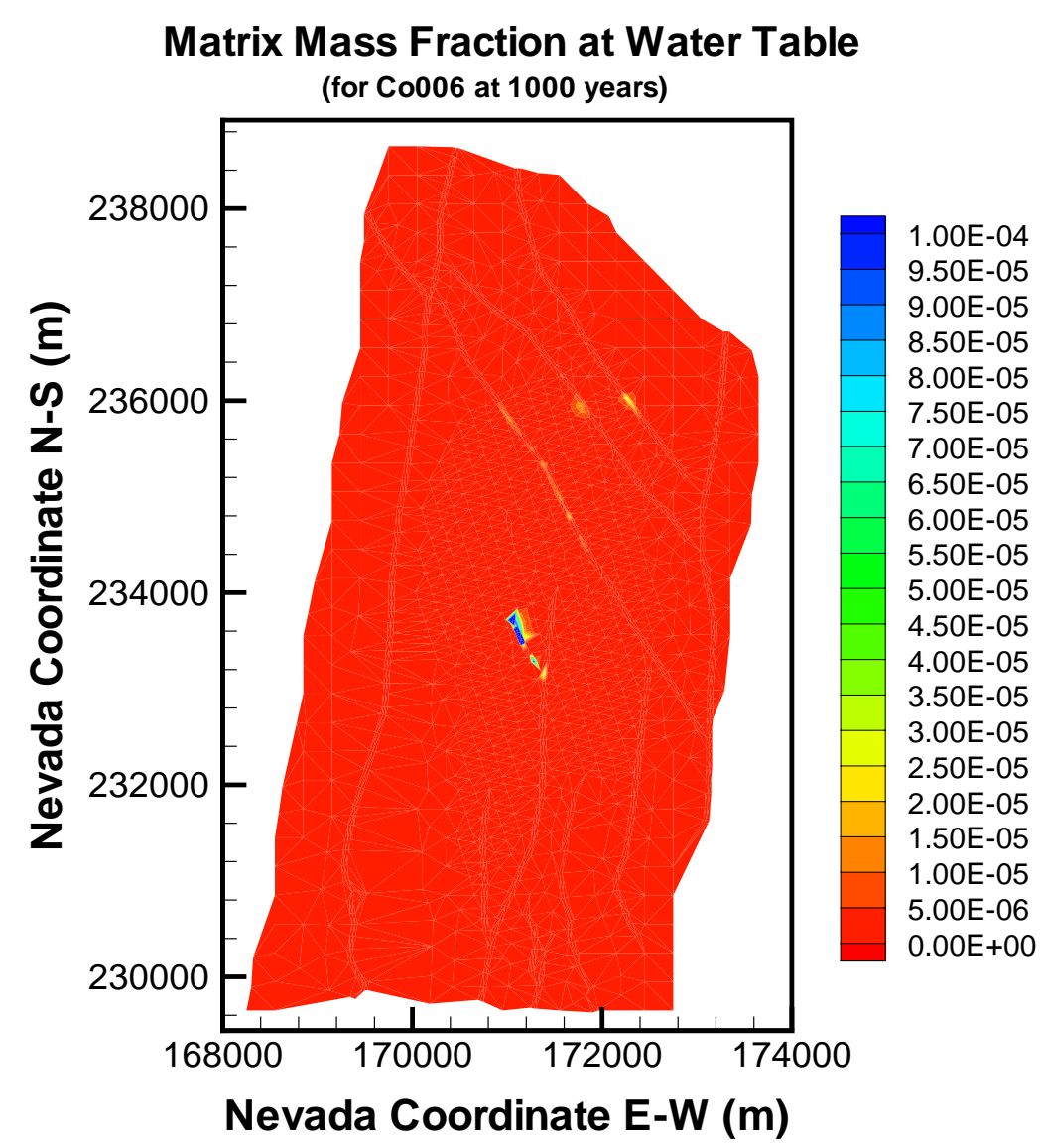

Output DTN: LB0307MR0060R1.004.

Figure F-23. Distribution of the Relative Mass Fraction $X_{R}$ of the $6 \mathrm{~nm}{ }^{239} \mathrm{PuO}_{2}$ Colloid in the Matrix Immediately above the Groundwater at $t=1,000$ Years for Mean PresentDay Infiltration and Continuous Release

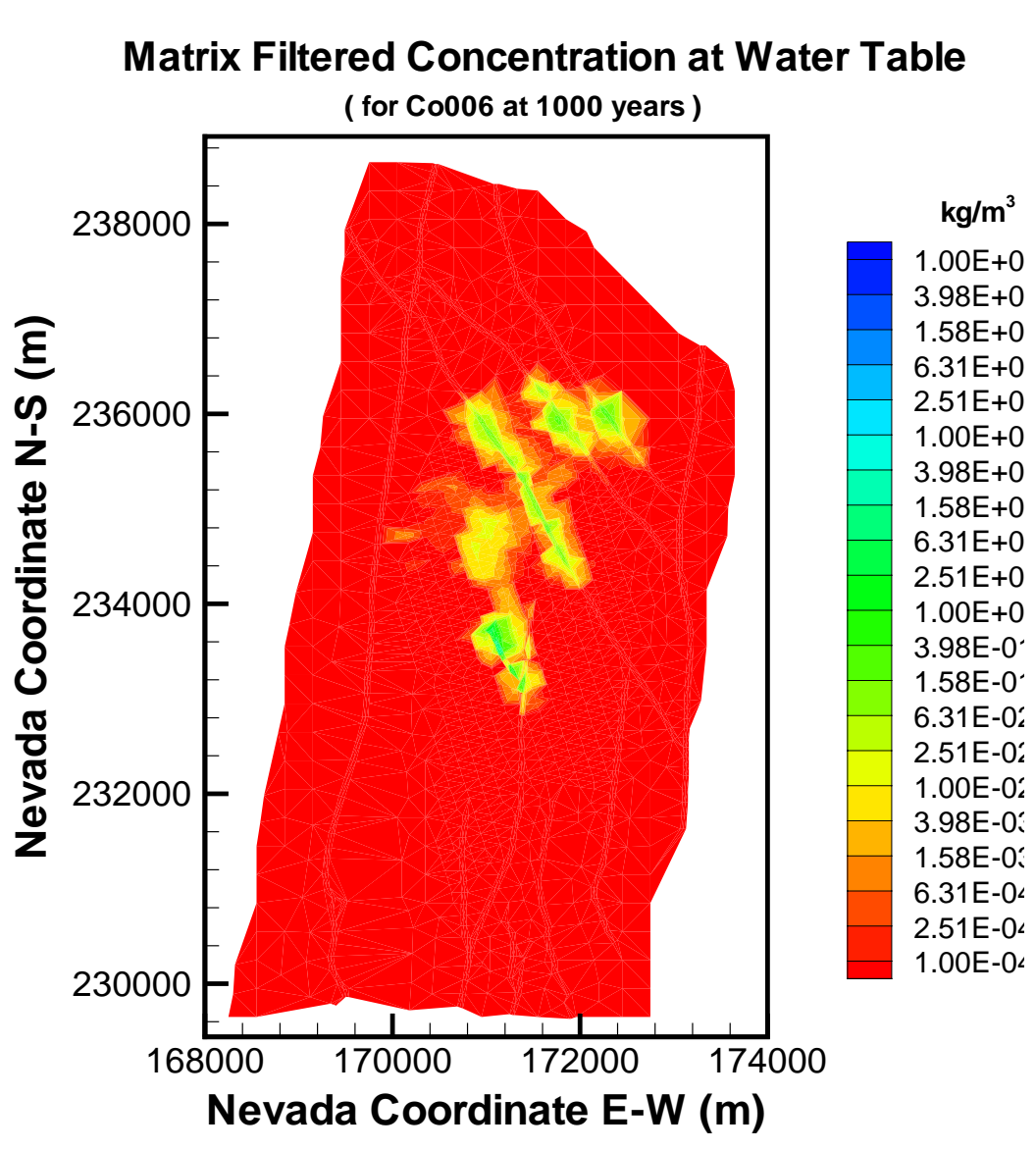

Output DTN: LB0307MR0060R1.004.

Figure F-24. Distribution of the Relative Filtered Concentration $F_{R}$ of the $6 \mathrm{~nm}^{239} \mathrm{PuO}_{2}$ Colloid in the Matrix Immediately above the Groundwater at $t=1,000$ Years for Mean Present-Day Infiltration and Continuous Release 


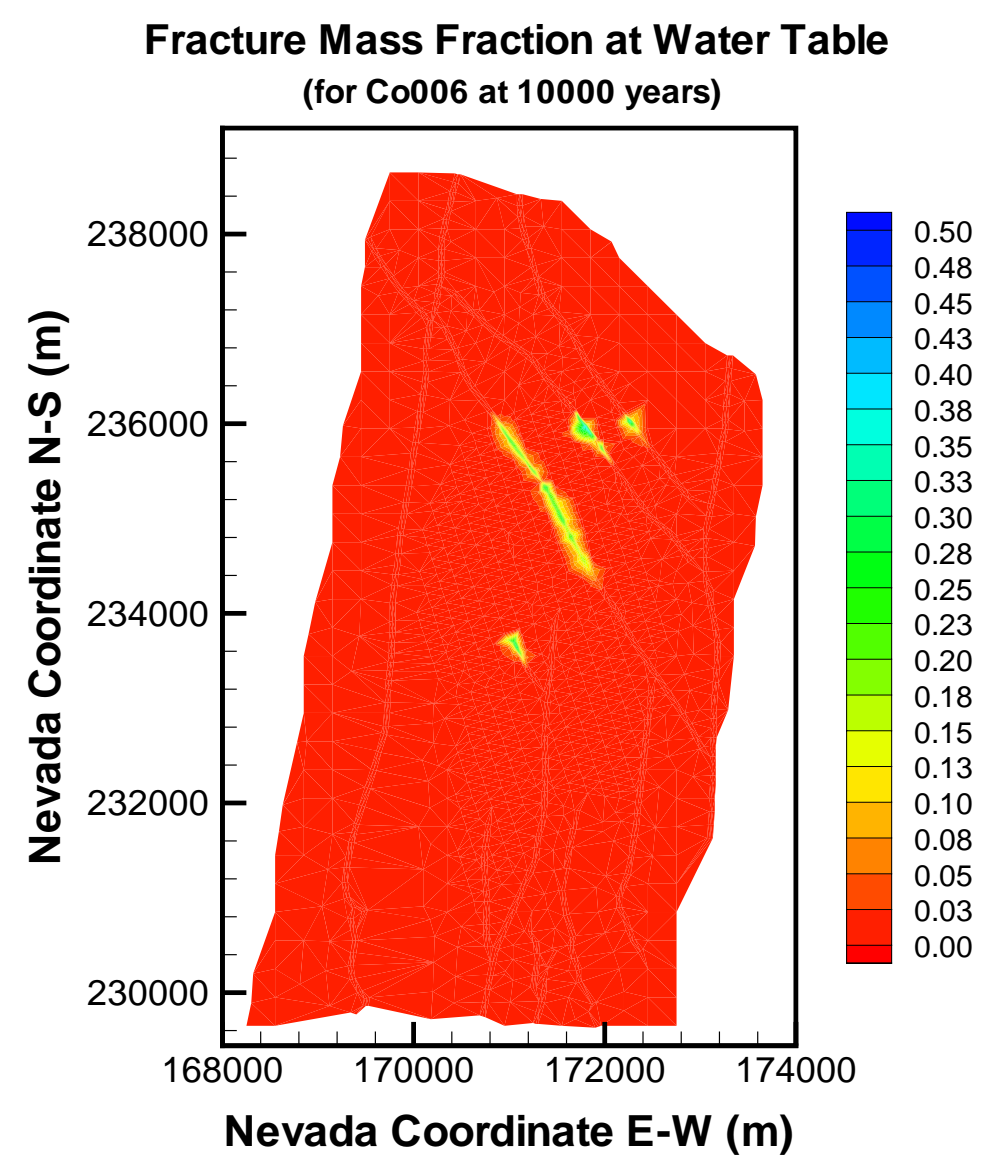

Output DTN: LB0307MR0060R1.004.

Figure F-25. Distribution of the Relative Mass Fraction $X_{R}$ of the $6 \mathrm{~nm}^{239} \mathrm{PuO}_{2}$ Colloid in the Fractures Immediately above the Groundwater at $\mathrm{t}=10,000$ Years for Mean Present-Day Infiltration and Continuous Release

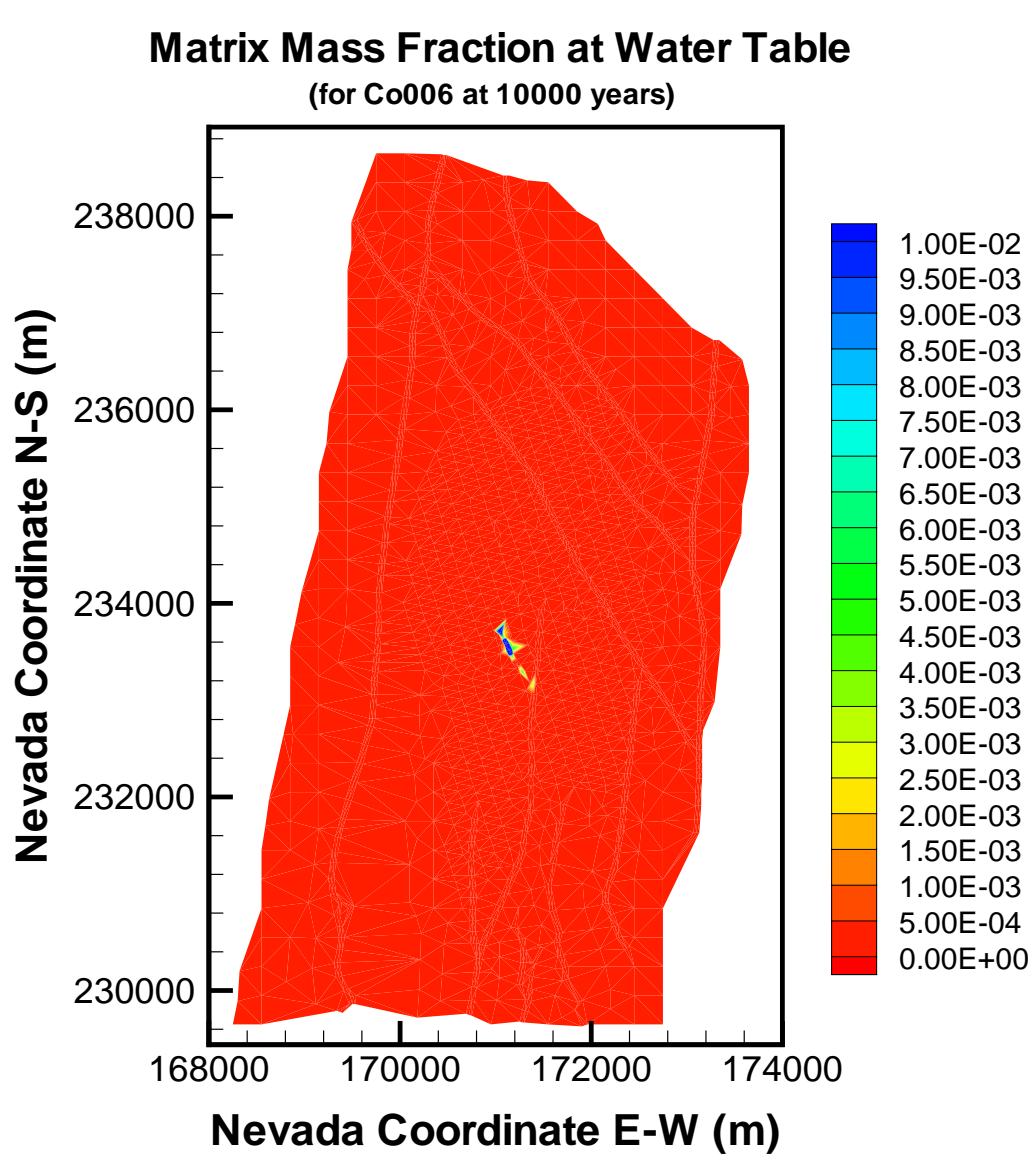

Output DTN: LB0307MR0060R1.004.

Figure F-26. Distribution of the Relative Mass Fraction $\mathrm{X}_{\mathrm{R}}$ of the $6 \mathrm{~nm}{ }^{239} \mathrm{PuO}_{2}$ Colloid in the Matrix Immediately above the Groundwater at $t=10,000$ Years for Mean Present-Day Infiltration and Continuous Release 


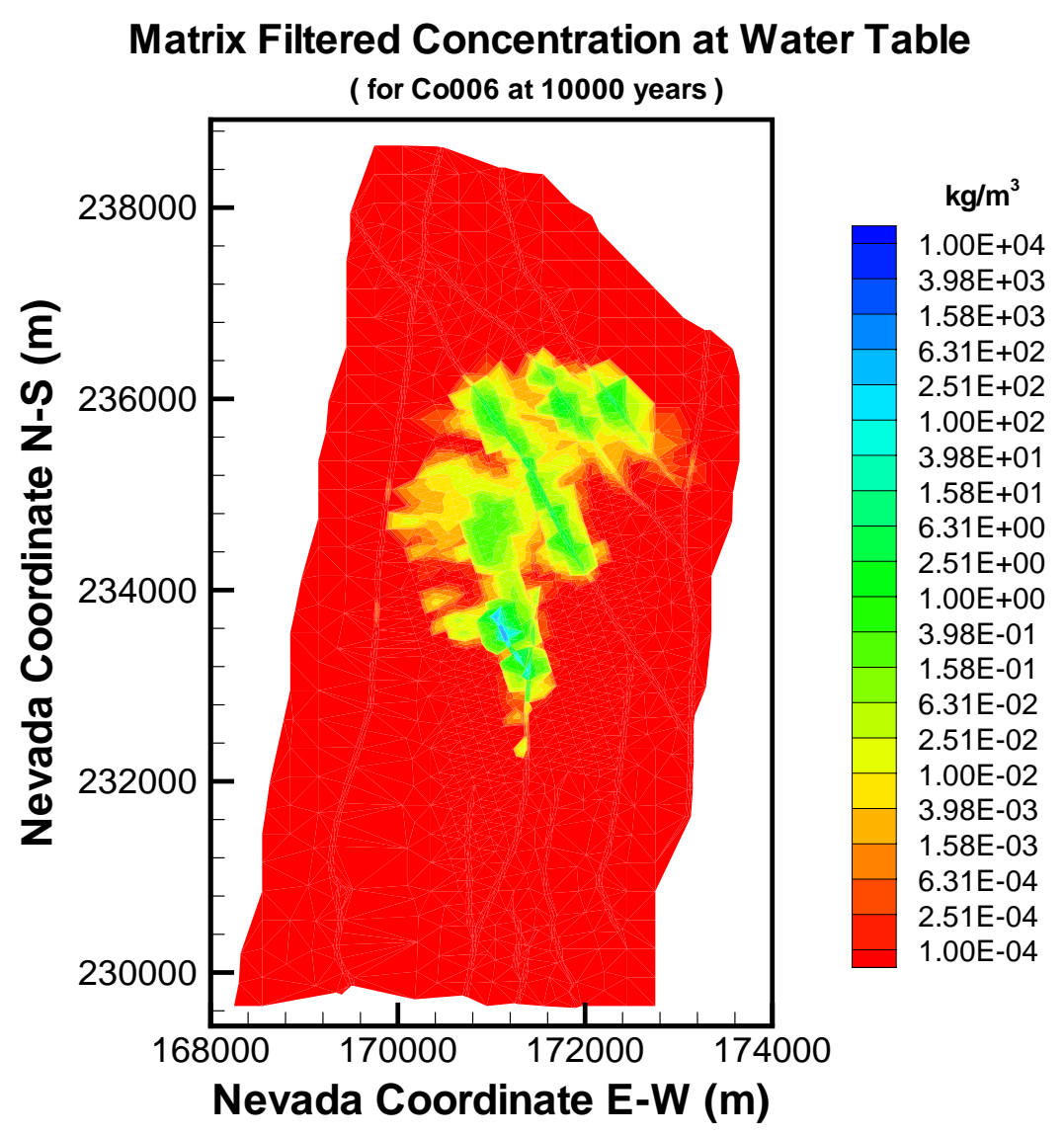

Output DTN: LB0307MR0060R1.004.

Figure F-27. Distribution of the Relative Filtered Concentration $F_{R}$ of the $6 \mathrm{~nm}^{239} \mathrm{PuO}_{2}$ Colloid in the Matrix Immediately above the Groundwater at $t=10,000$ Years for Mean Present-Day Infiltration and Continuous Release

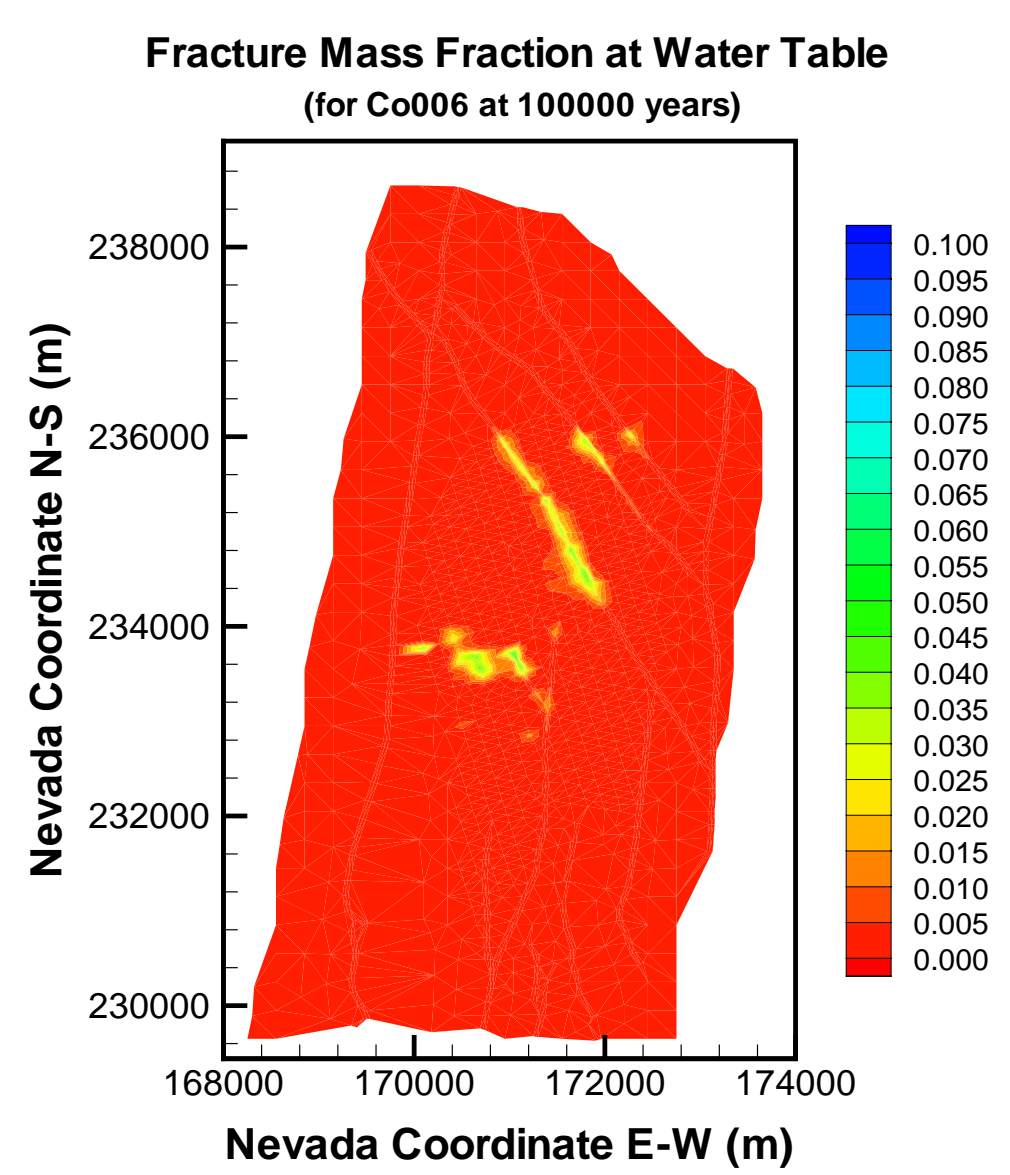

Output DTN: LB0307MR0060R1.004.

Figure F-28. Distribution of the Relative Mass Fraction $\mathrm{X}_{\mathrm{R}}$ of the $6 \mathrm{~nm}{ }^{239} \mathrm{PuO}_{2}$ Colloid in the Fractures Immediately above the Groundwater at $t=100,000$ Years for Mean Present-Day Infiltration and Continuous Release 


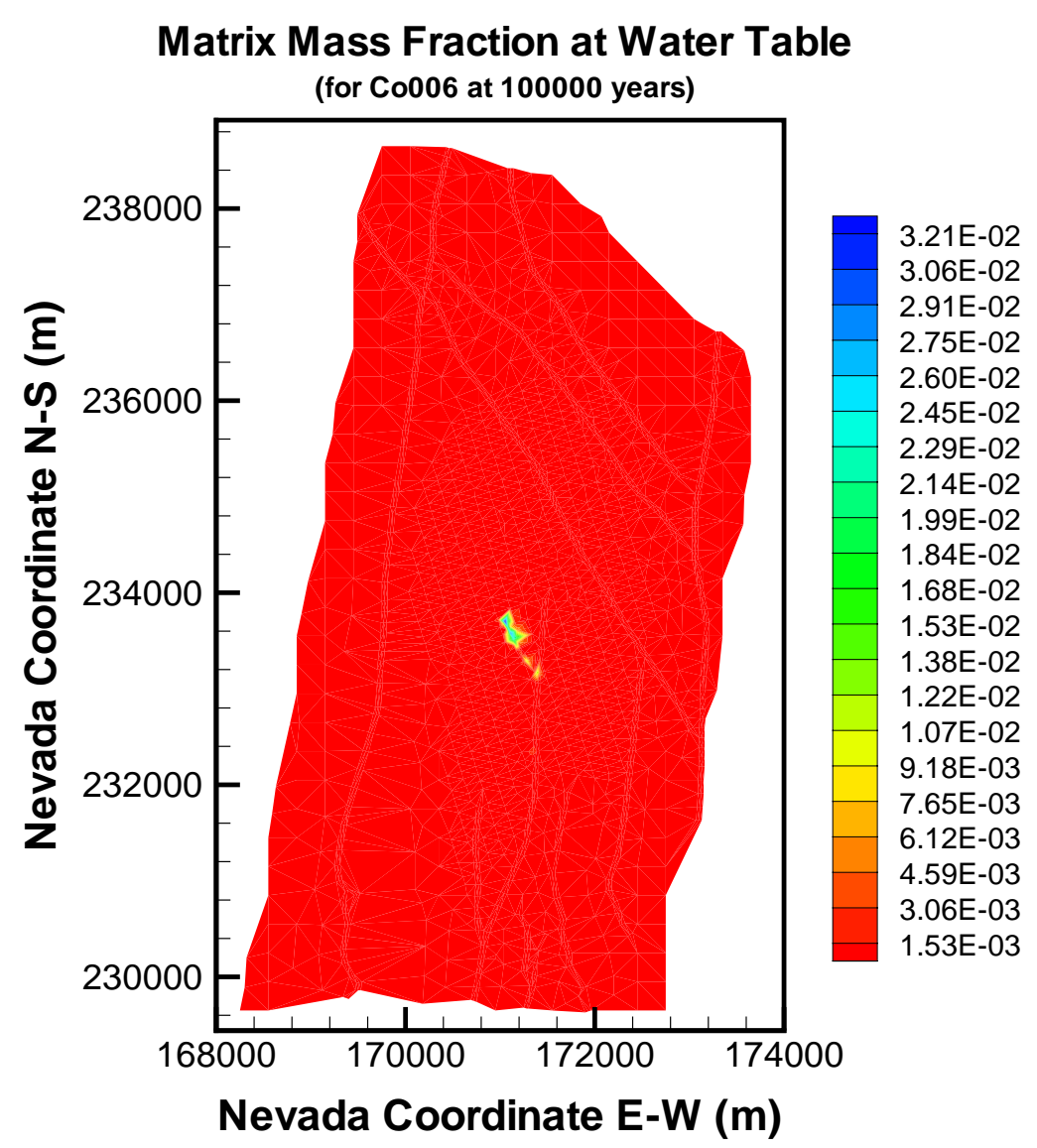

Output DTN: LB0307MR0060R1.004.

Figure F-29. Distribution of the Relative Mass Fraction $X_{R}$ of the $6 \mathrm{~nm}{ }^{239} \mathrm{PuO}_{2}$ Colloid in the Matrix Immediately above the Groundwater at $t=100,000$ Years for Mean Present-Day Infiltration and Continuous Release

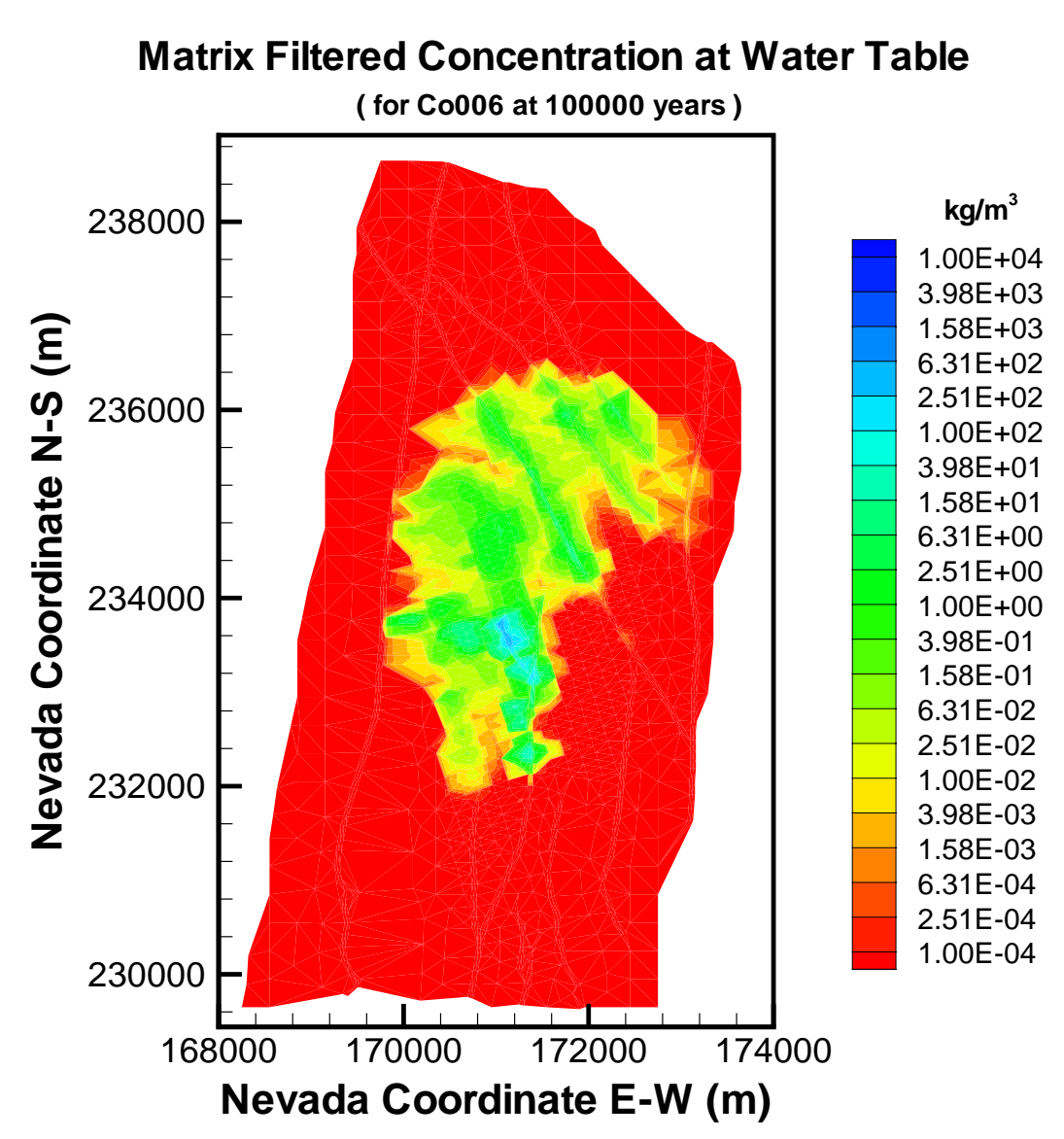

Output DTN: LB0307MR0060R1.004.

Figure F-30. Distribution of the Relative Filtered Concentration $F_{R}$ of the $6 \mathrm{~nm}^{239} \mathrm{PuO}_{2}$ Colloid in the Matrix Immediately above the Groundwater at $t=100,000$ Years for Mean Present-Day Infiltration and Continuous Release 


\section{INTENTIONALLY LEFT BLANK}




\section{APPENDIX G \\ FIGURES FROM THE THREE-DIMENSIONAL TRANSPORT STUDIES OF THE $450 \mathrm{NM}^{239} \mathrm{PUO}_{2}$ COLLOID IN CASE 2 (CONTINUOUS RELEASE, MEAN PRESENT-DAY INFILTRATION)}




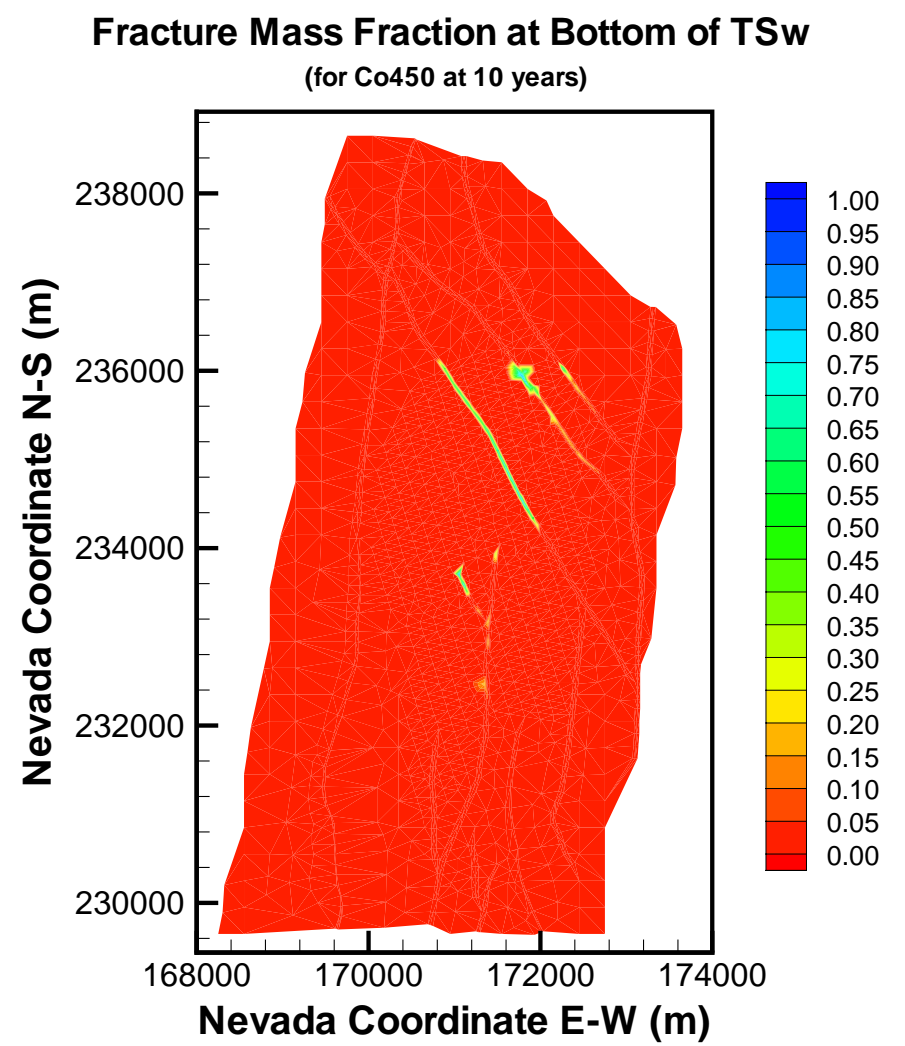

Output DTN: LB0307MR0060R1.004.

Figure G-1. Distribution of the Relative Mass Fraction $\mathrm{X}_{\mathrm{R}}$ of the $450 \mathrm{~nm}{ }^{239} \mathrm{PuO}_{2}$ Colloid in the Fractures of the tsw39 Layer at $t=10$ Years for Mean Present-Day Infiltration and Continuous Release

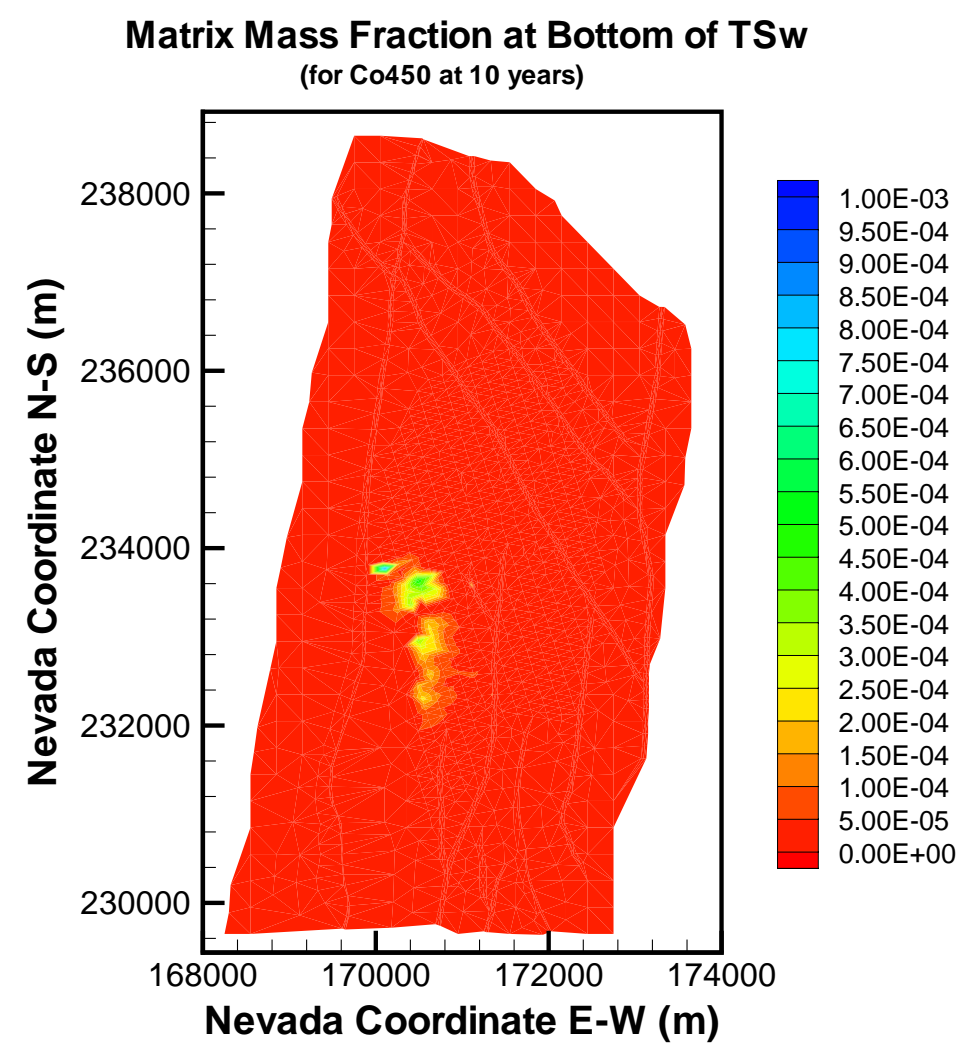

Output DTN: LB0307MR0060R1.004.

Figure G-2. Distribution of the Relative Mass Fraction $X_{R}$ of the $450 \mathrm{~nm}{ }^{239} \mathrm{PuO}_{2}$ Colloid in the Matrix of the tsw39 Layer at $t=10$ Years for Mean Present-Day Infiltration and Continuous Release 


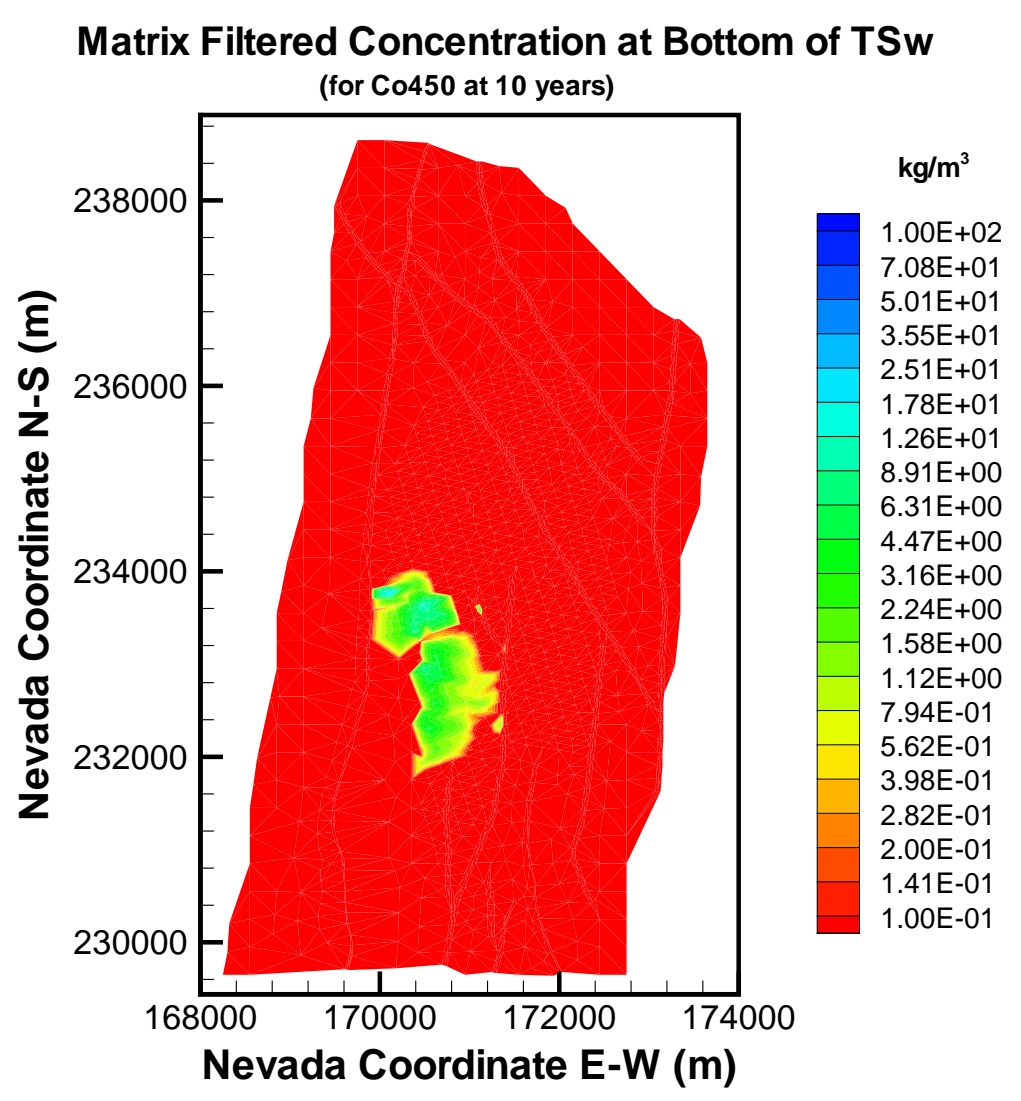

Output DTN: LB0307MR0060R1.004.

Figure G-3. Distribution of the Relative Filtered Concentration $X_{R}$ of the $450 \mathrm{~nm}^{239} \mathrm{PuO}_{2}$ Colloid in the Matrix of the tsw39 Layer at $t=10$ Years for Mean Present-Day Infiltration and Continuous Release

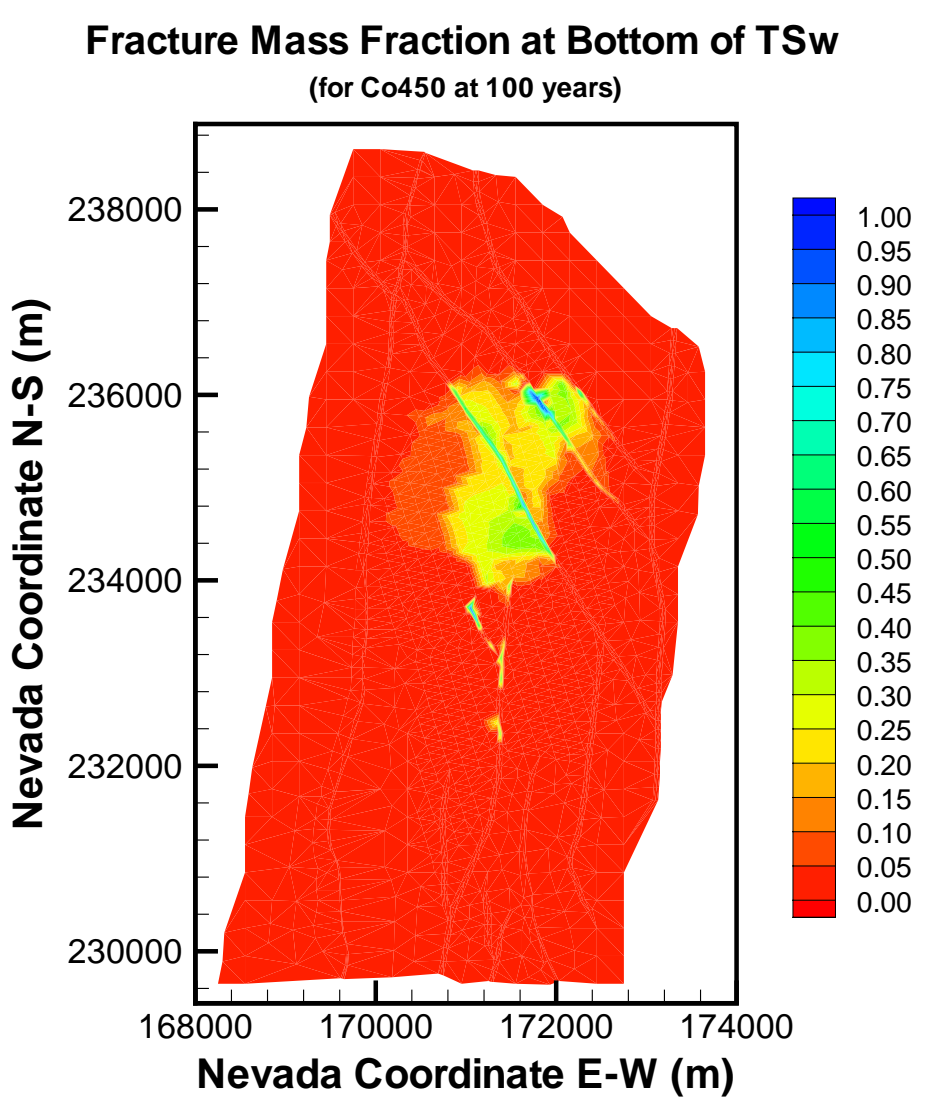

Output DTN: LB0307MR0060R1.004.

Figure G-4. Distribution of the Relative Mass Fraction $X_{R}$ of the $450 \mathrm{~nm}{ }^{239} \mathrm{PuO}_{2}$ Colloid in the Fractures of the tsw39 Layer at $t=100$ Years for Mean Present-Day Infiltration and Continuous Release 




Output DTN: LB0307MR0060R1.004.

Figure G-5. Distribution of the Relative Mass Fraction $X_{R}$ of the $450 \mathrm{~nm}{ }^{239} \mathrm{PuO}_{2}$ Colloid in the Matrix of the tsw39 Layer at $t=100$ Years for Mean Present-Day Infiltration and Continuous Release

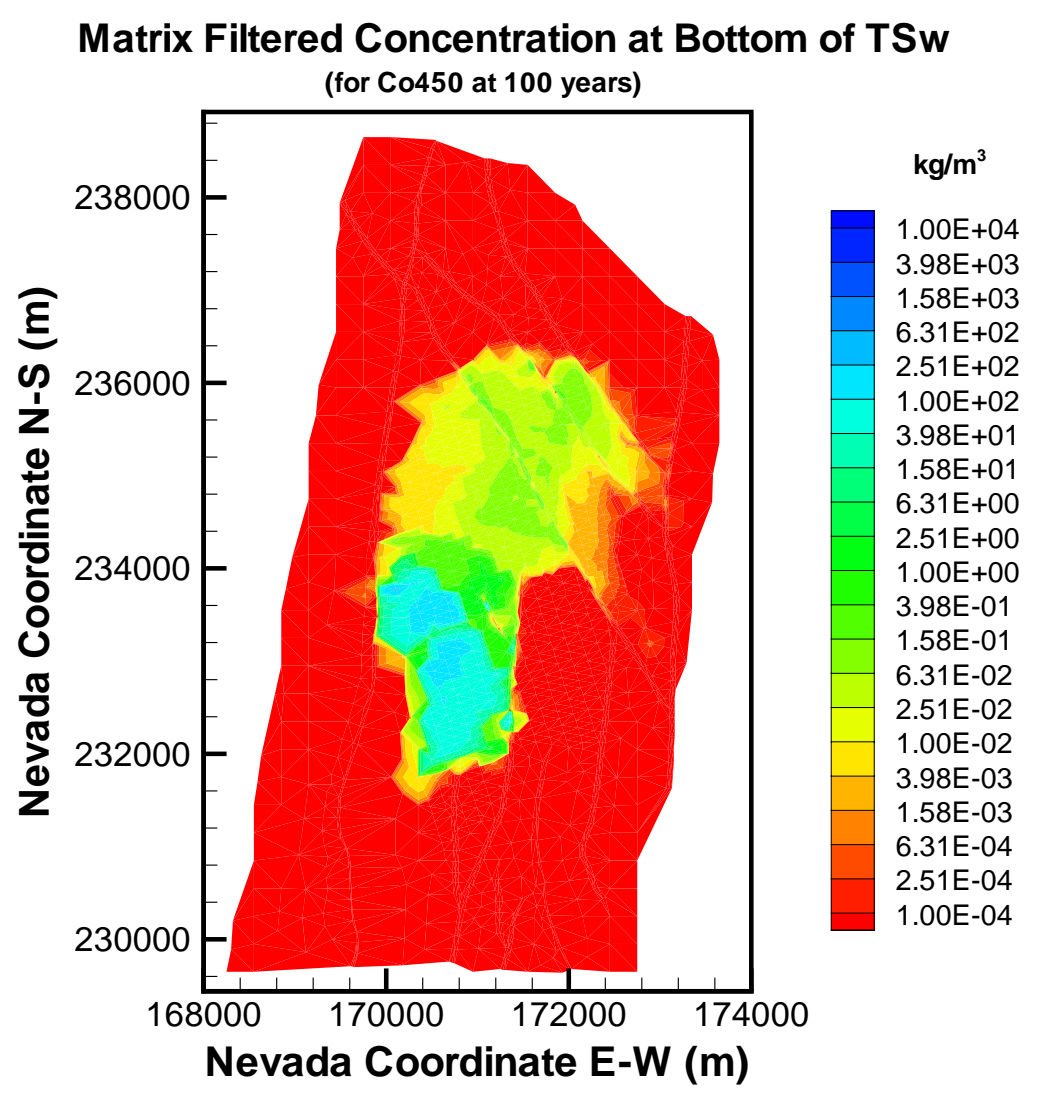

Output DTN: LB0307MR0060R1.004.

Figure G-6. Distribution of the Relative Filtered Concentration $X_{R}$ of the $450 \mathrm{~nm}^{239} \mathrm{PuO}_{2}$ Colloid in the Matrix of the tsw39 Layer at $t=100$ Years for Mean Present-Day Infiltration and Continuous Release 
Fracture Mass Fraction at Bottom of TSw (for Co450 at 1000 years)

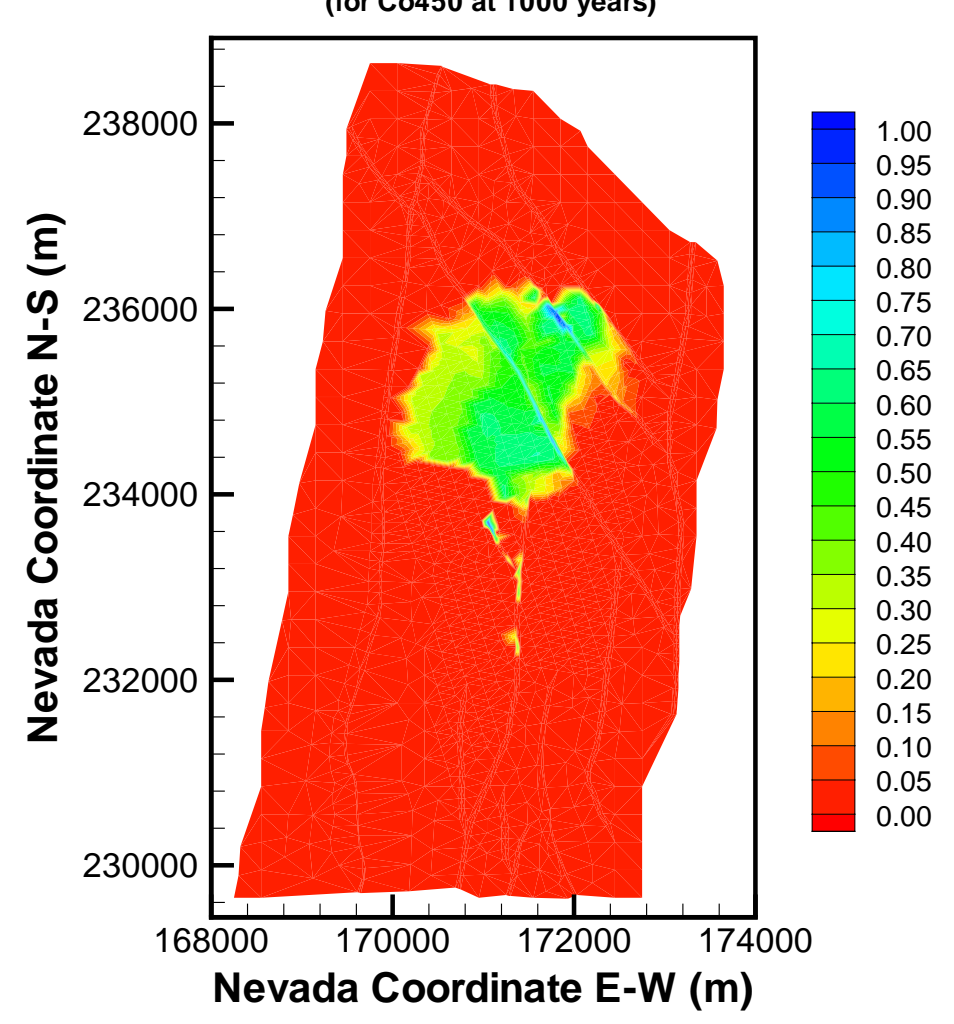

Output DTN: LB0307MR0060R1.004.

Figure G-7. Distribution of the Relative Mass Fraction $X_{R}$ of the $450 \mathrm{~nm}{ }^{239} \mathrm{PuO}_{2}$ Colloid in the Fractures of the tsw39 Layer at $t=1,000$ Years for Mean Present-Day Infiltration and Continuous Release

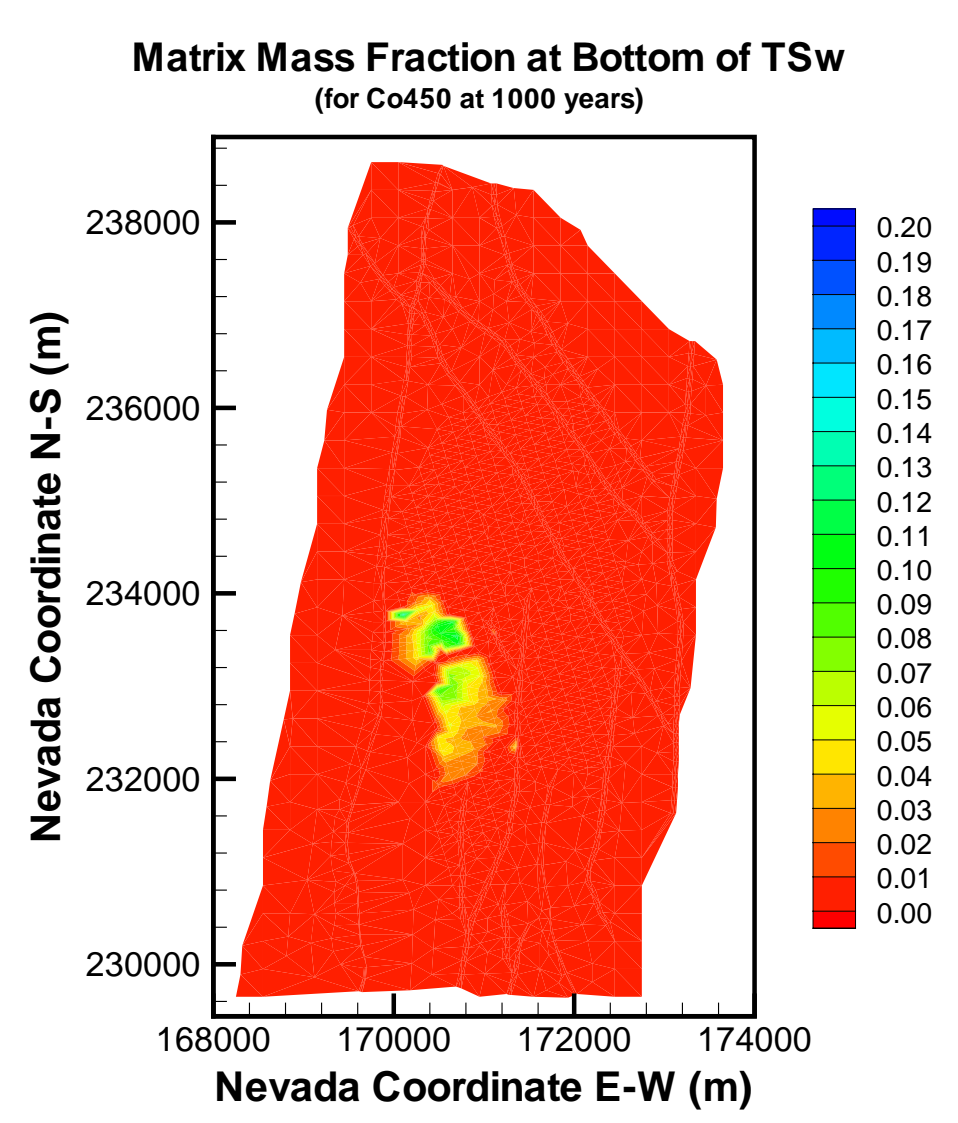

Output DTN: LB0307MR0060R1.004.

Figure G-8. Distribution of the Relative Mass Fraction $X_{R}$ of the $450 \mathrm{~nm}{ }^{239} \mathrm{PuO}_{2}$ Colloid in the Matrix of the tsw39 Layer at $t=1,000$ Years for Mean Present-Day Infiltration and Continuous Release 


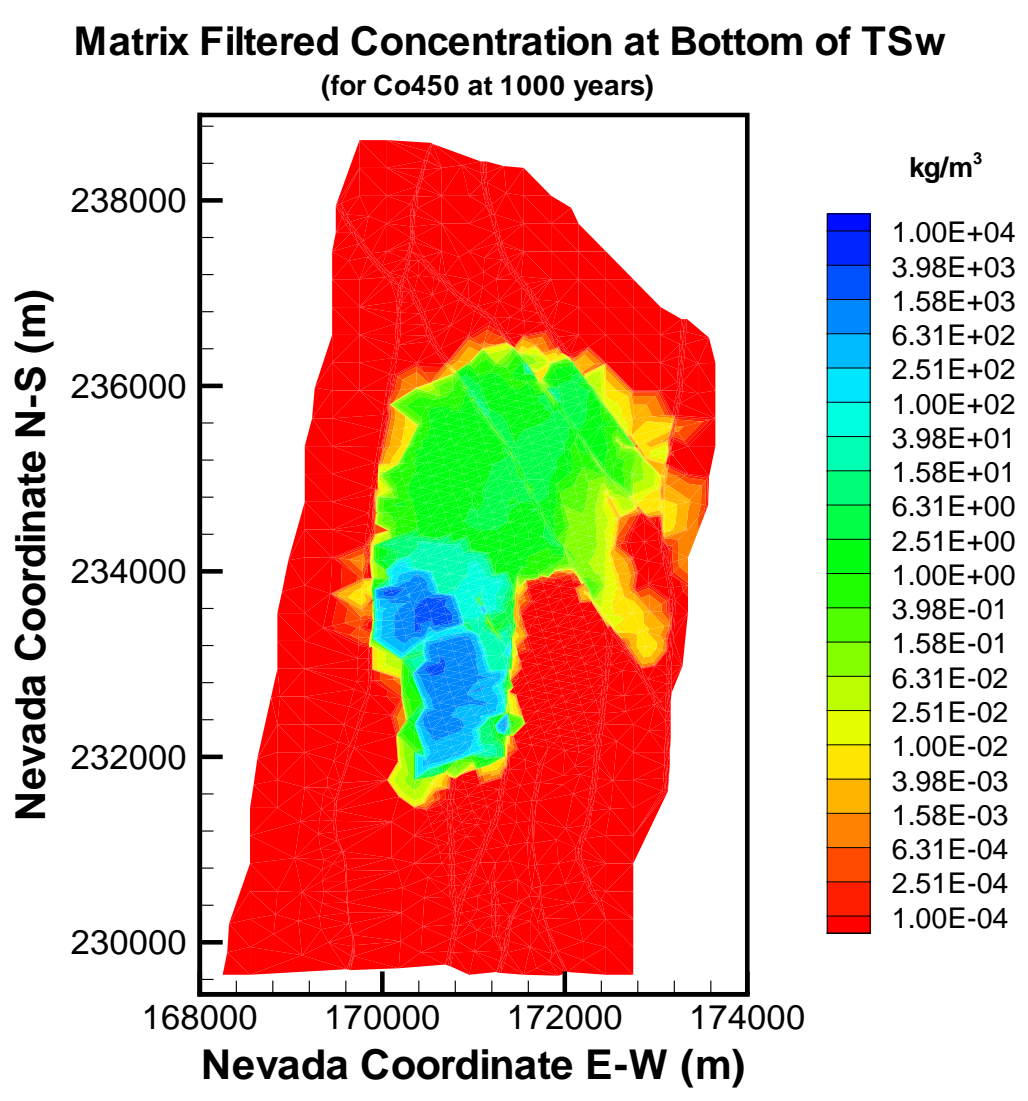

Output DTN: LB0307MR0060R1.004.

Figure G-9. Distribution of the Relative Filtered Concentration $X_{R}$ of the $450 \mathrm{~nm}^{239} \mathrm{PuO}_{2}$ Colloid in the Matrix of the tsw39 Layer at $t=1,000$ Years for Mean Present-Day Infiltration and Continuous Release

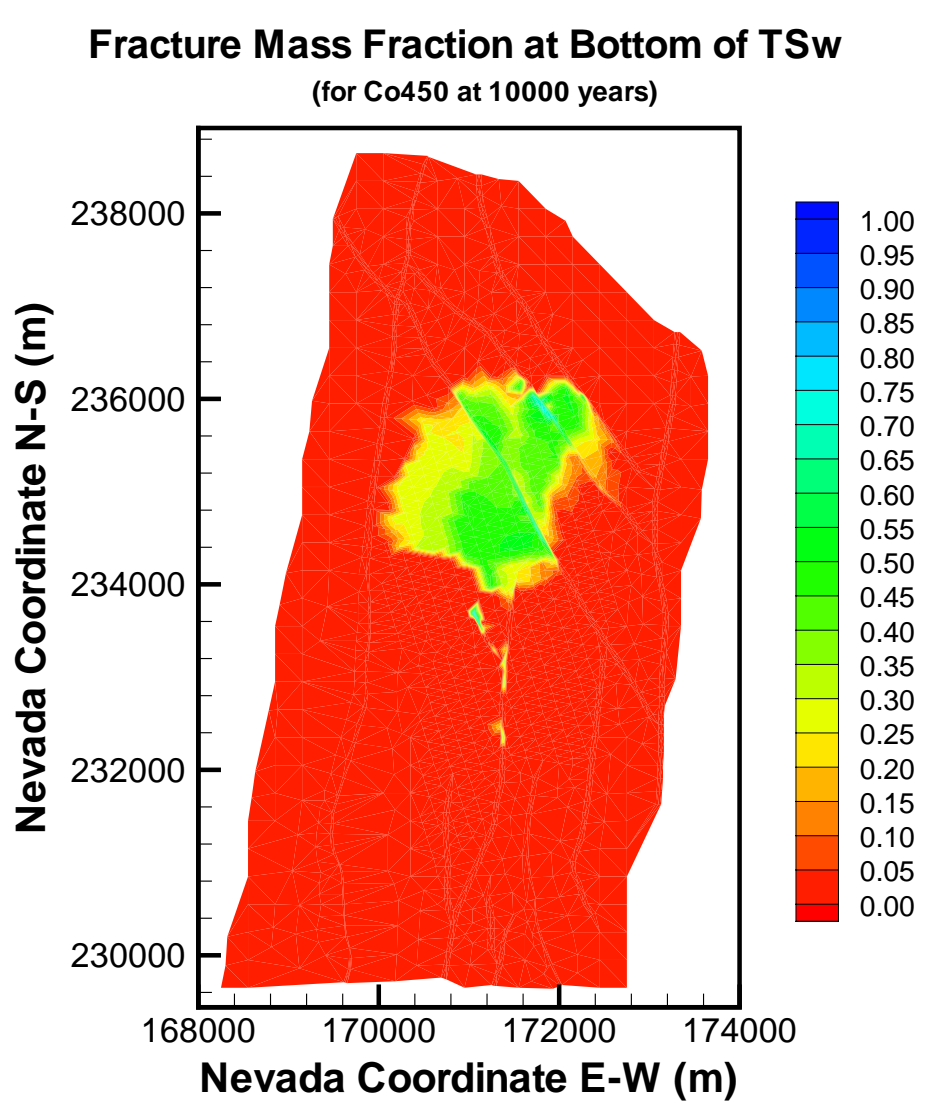

Output DTN: LB0307MR0060R1.004.

Figure $\mathrm{G}-10$. Distribution of the Relative Mass Fraction $\mathrm{X}_{\mathrm{R}}$ of the $450 \mathrm{~nm}{ }^{239} \mathrm{PuO}_{2}$ Colloid in the Fractures of the tsw39 Layer at $\mathrm{t}=10,000$ Years for Mean Present-Day Infiltration and Continuous Release 


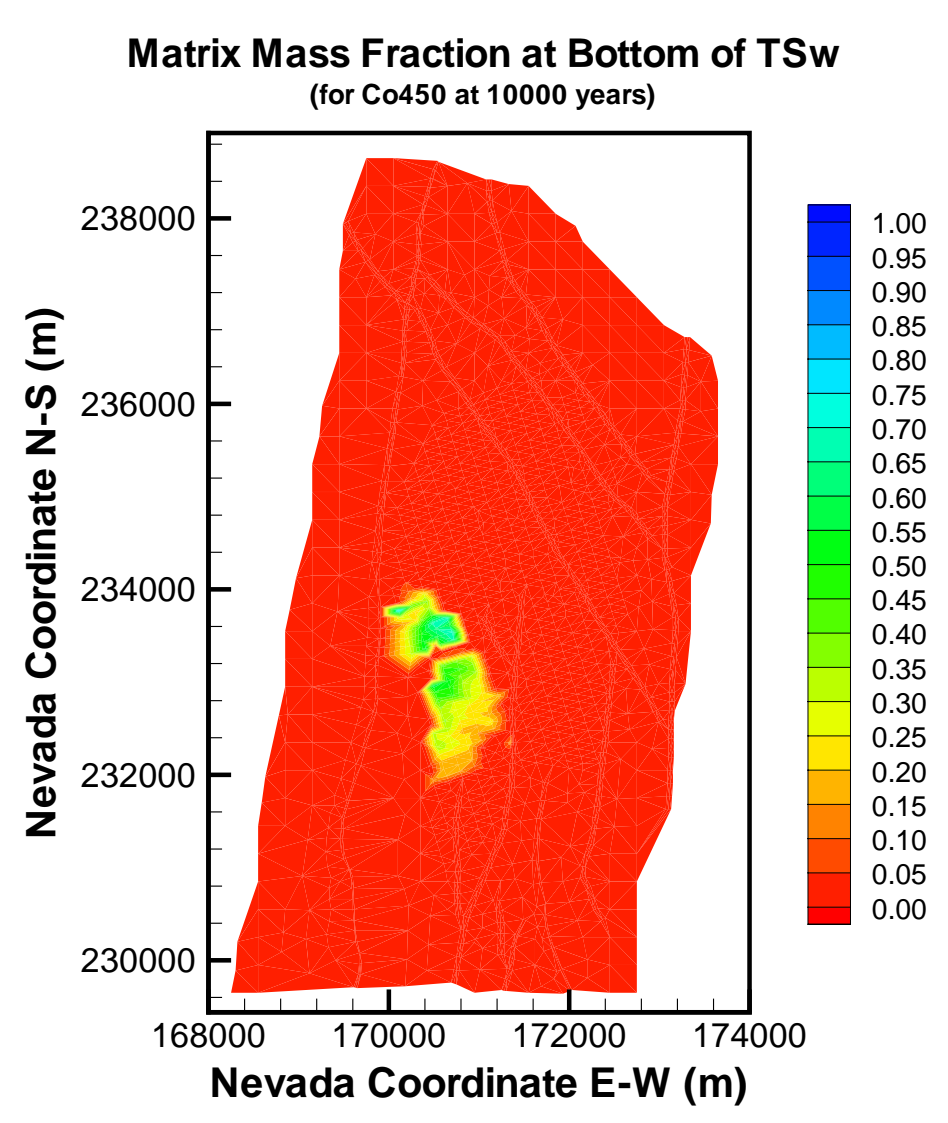

Output DTN: LB0307MR0060R1.004.

Figure G-11. Distribution of the Relative Mass Fraction $X_{R}$ of the $450 \mathrm{~nm}{ }^{239} \mathrm{PuO}_{2}$ Colloid in the Matrix of the tsw39 Layer at $t=10,000$ Years for Mean Present-Day Infiltration and Continuous Release

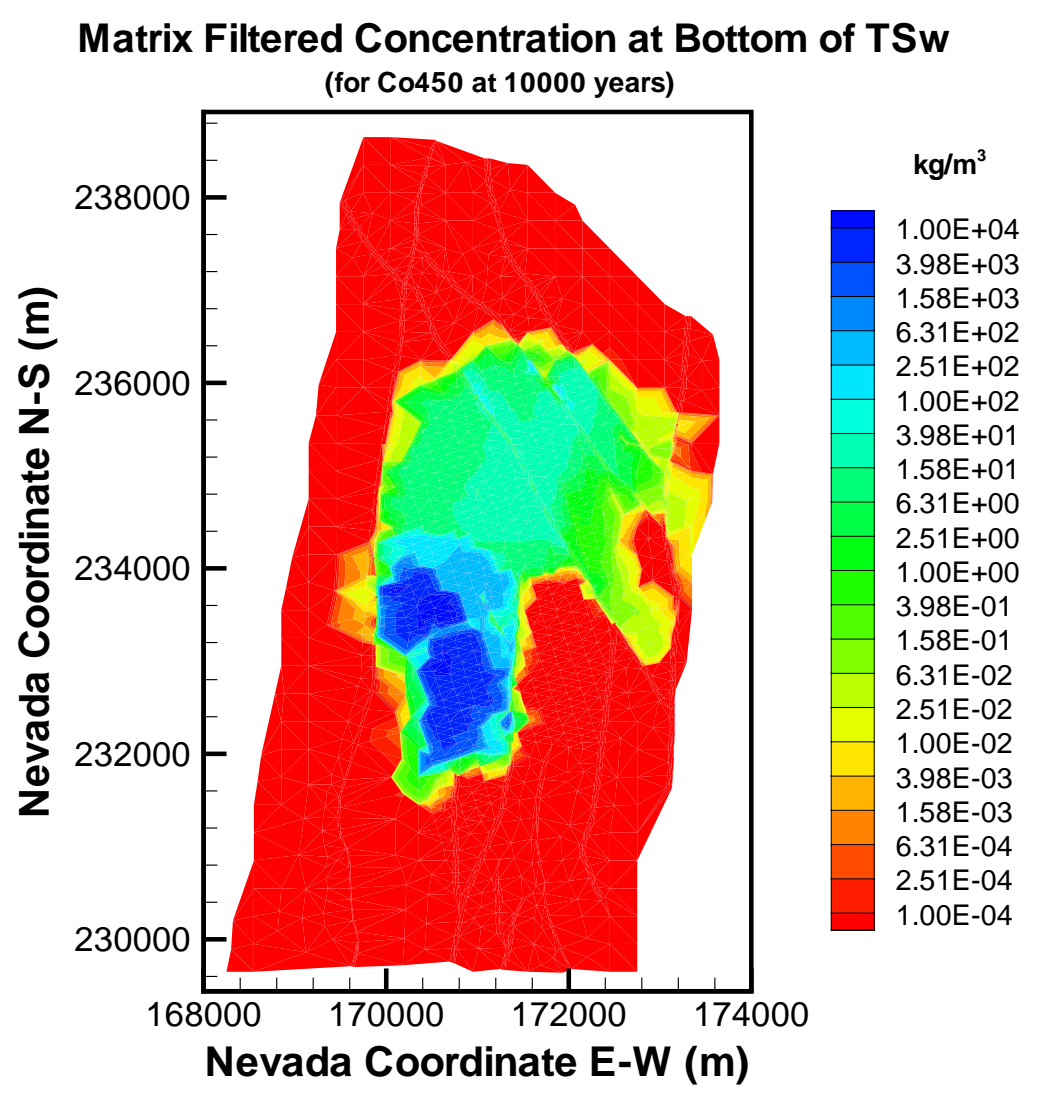

Output DTN: LB0307MR0060R1.004.

Figure G-12. Distribution of the Relative Filtered Concentration $X_{R}$ of the $450 \mathrm{~nm}{ }^{239} \mathrm{PuO} 2$ Colloid in the Matrix of the tsw39 Layer at $t=10,000$ Years for Mean Present-Day Infiltration and Continuous Release 
Fracture Mass Fraction at Bottom of TSw

(for $\mathrm{Co} 450$ at 100000 years)

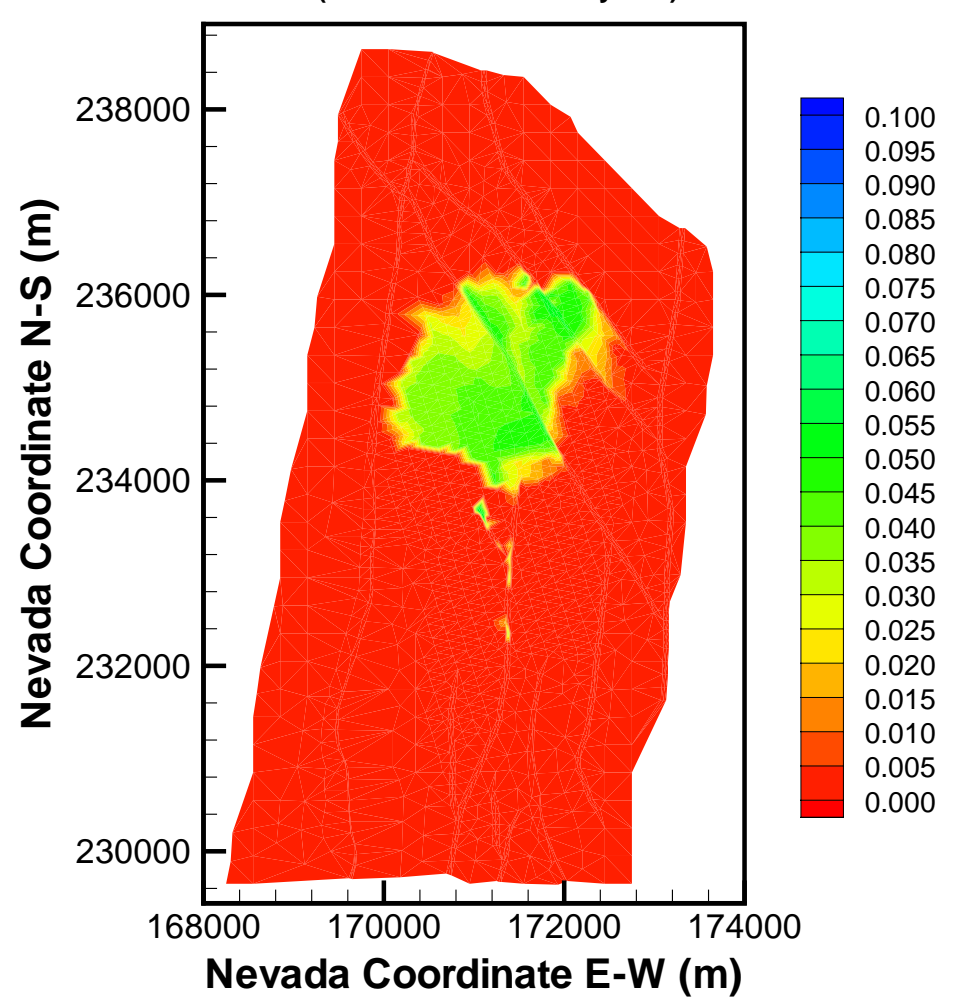

Output DTN: LB0307MR0060R1.004.

Figure G-13. Distribution of the Relative Mass Fraction $X_{R}$ of the $450 \mathrm{~nm}{ }^{239} \mathrm{PuO} 2$ Colloid in the Fractures of the tsw39 Layer at $t=100,000$ Years for Mean Present-Day Infiltration and Continuous Release

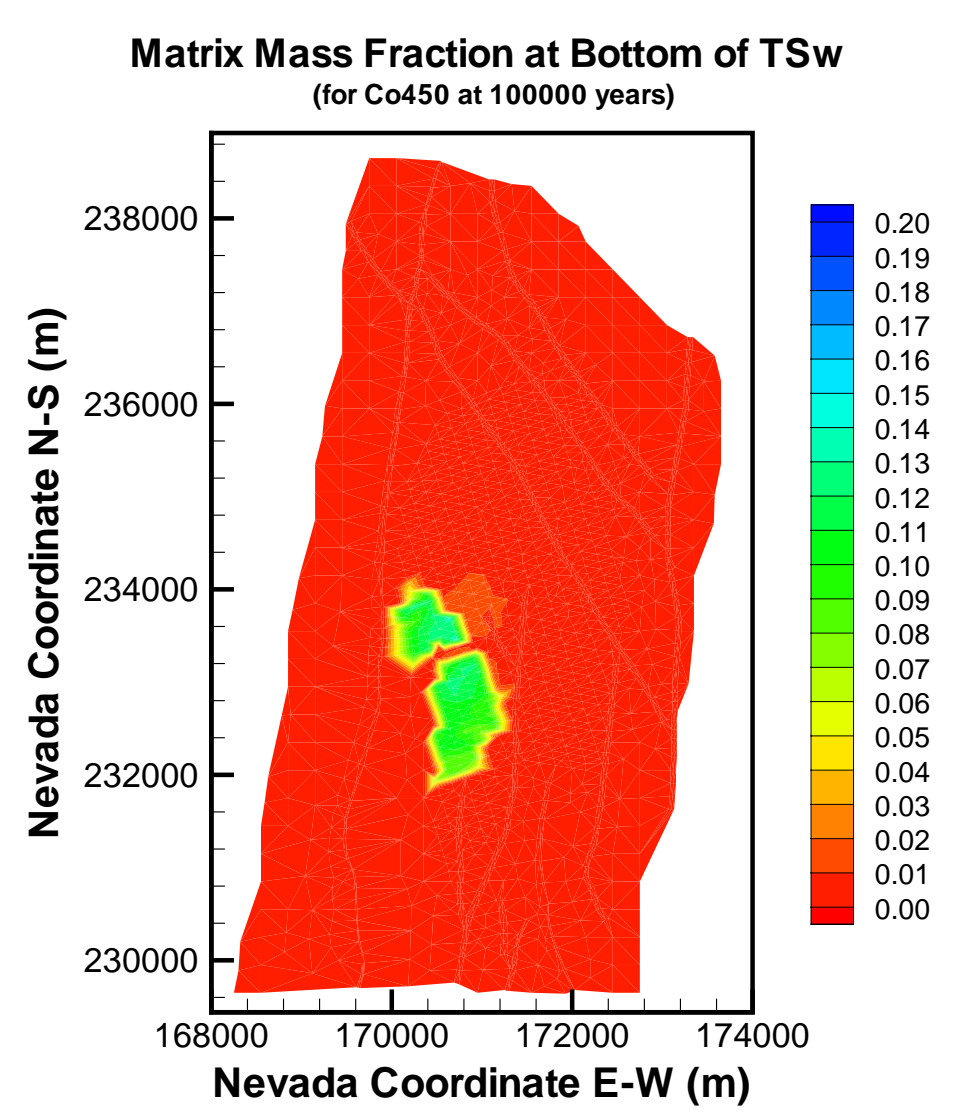

Output DTN: LB0307MR0060R1.004.

Figure G-14. Distribution of the Relative Mass Fraction $X_{R}$ of the $450 \mathrm{~nm}{ }^{239} \mathrm{PuO} 2$ Colloid in the Matrix of the tsw39 Layer at $t=100,000$ Years for Mean Present-Day Infiltration and Continuous Release 


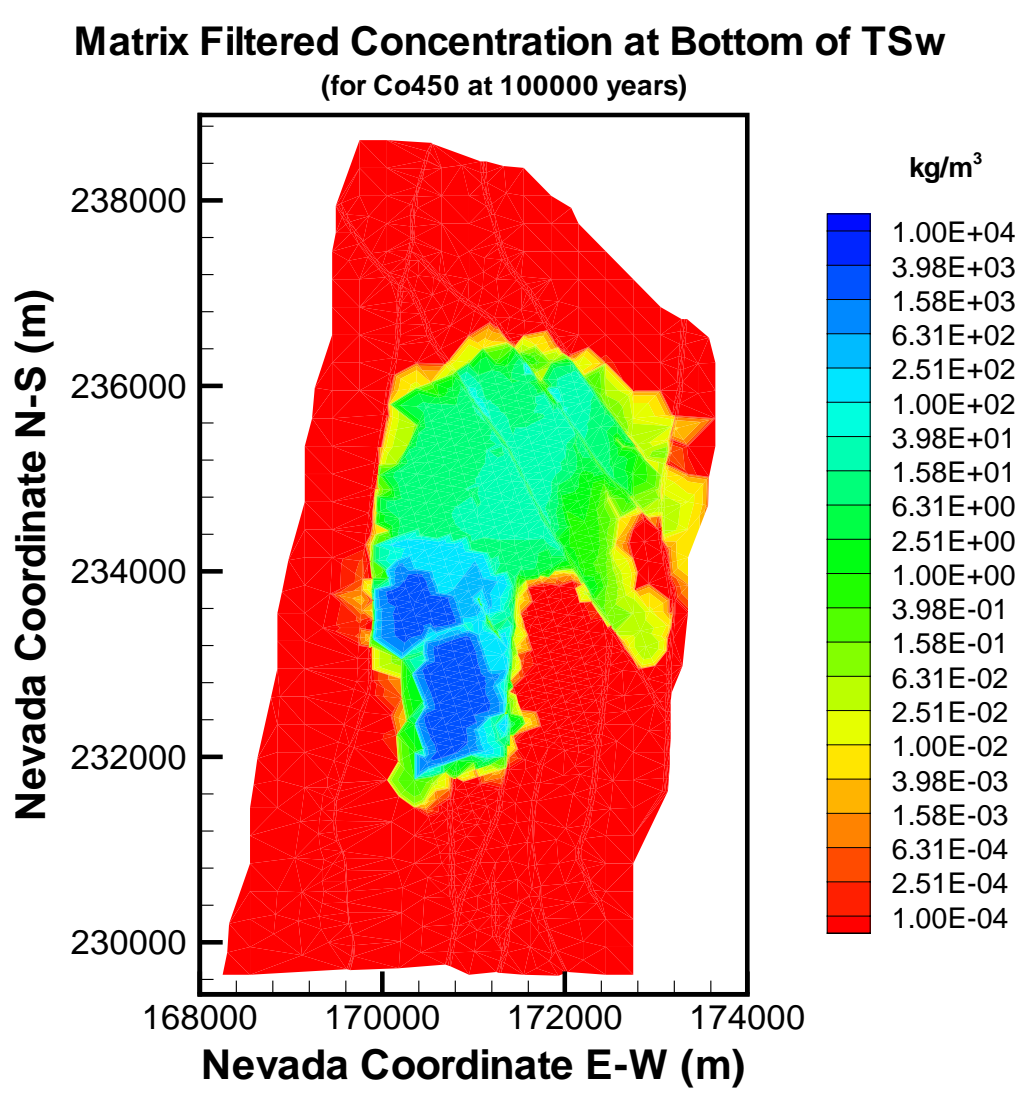

Output DTN: LB0307MR0060R1.004.

Figure G-15. Distribution of the Relative Filtered Concentration $X_{R}$ of the $450 \mathrm{~nm}{ }^{239} \mathrm{PuO} 2$ Colloid in the Matrix of the tsw39 Layer at $t=100,000$ Years for Mean PresentDay Infiltration and Continuous Release

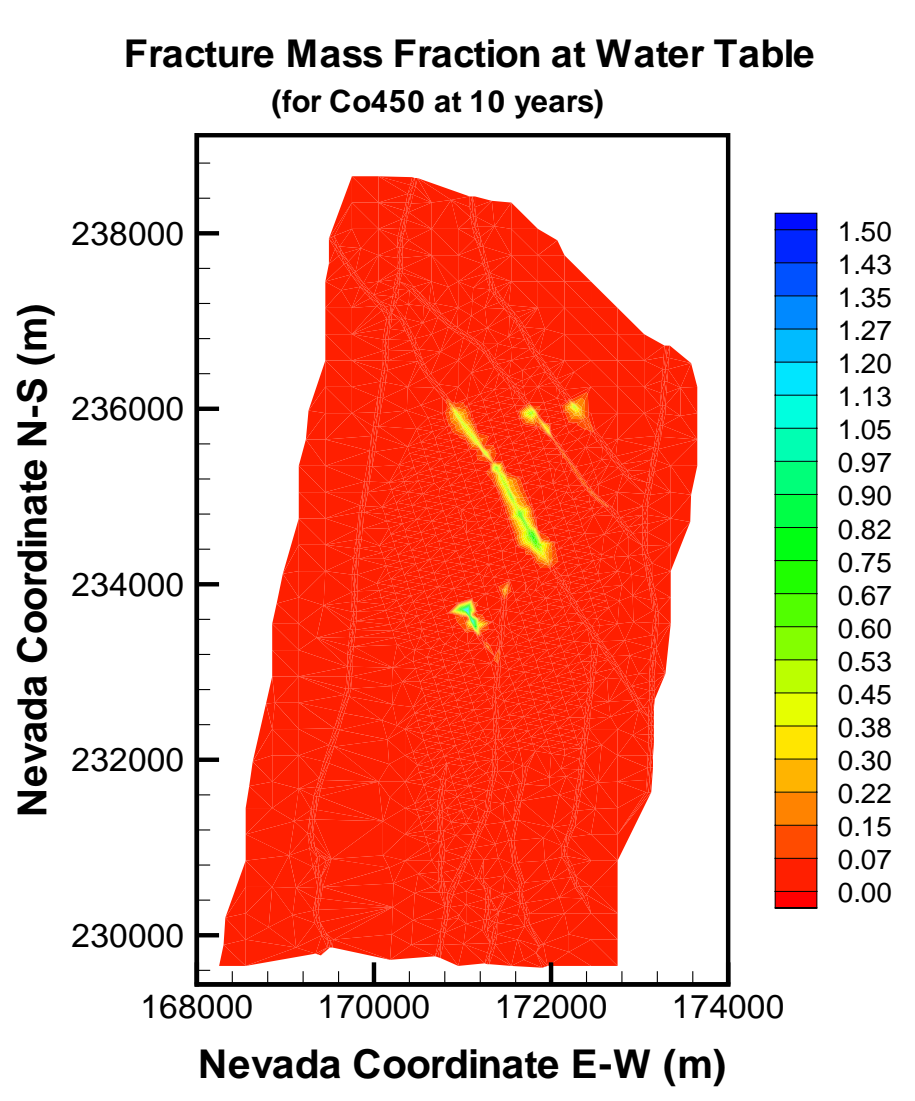

Output DTN: LB0307MR0060R1.004.

Figure G-16. Distribution of the Relative Mass Fraction $X_{R}$ of the $450 \mathrm{~nm}{ }^{239} \mathrm{PuO} 2$ Colloid in the Fractures Immediately above the Groundwater Table at $t=10$ Years for Mean Present-Day Infiltration and Continuous Release 


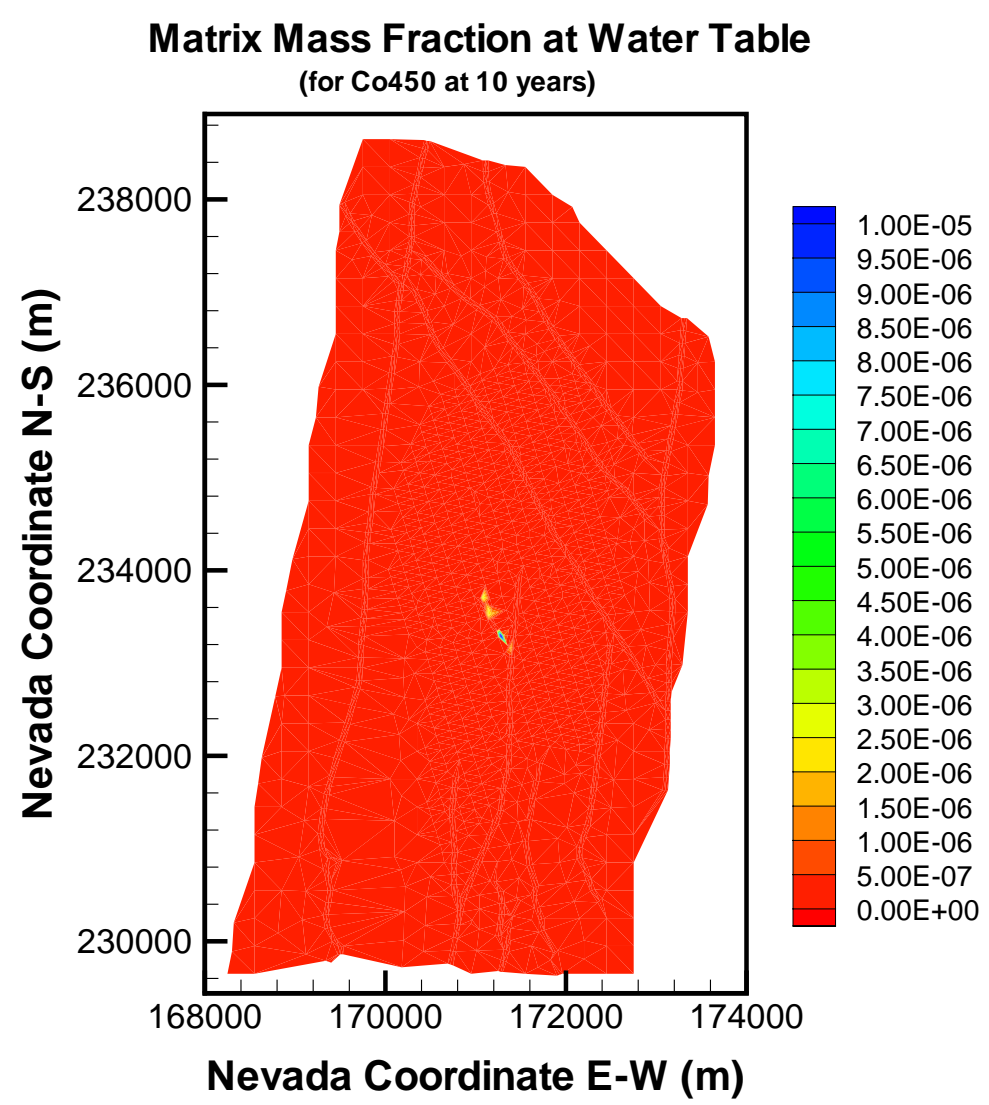

Output DTN: LB0307MR0060R1.004.

Figure G-17. Distribution of the Relative Mass Fraction $X_{R}$ of the $450 \mathrm{~nm}{ }^{239} \mathrm{PuO} 2$ Colloid in the Matrix Immediately above the Groundwater at $\mathrm{t}=10$ Years for Mean Present-Day Infiltration and Continuous Release

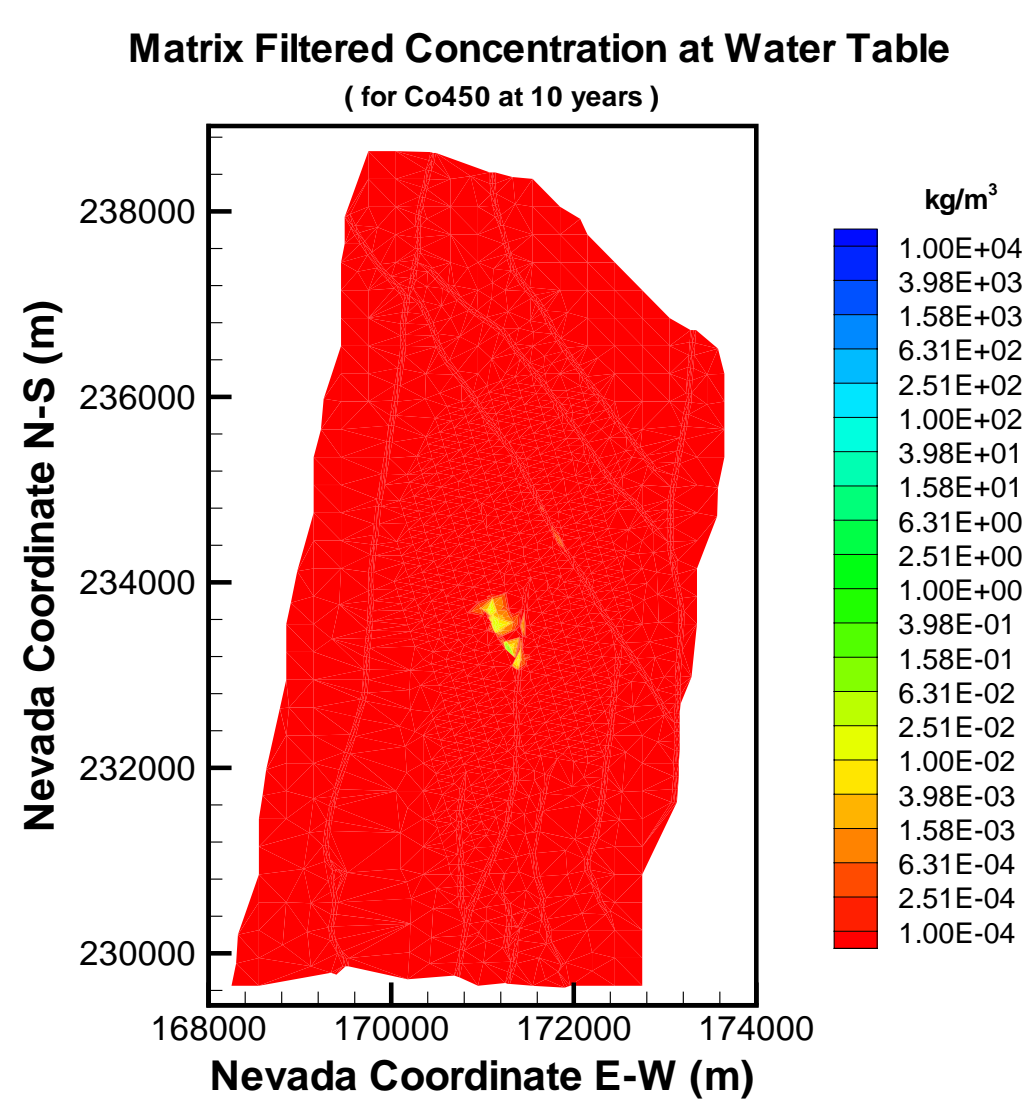

Output DTN: LB0307MR0060R1.004.

Figure G-18. Distribution of the Relative Filtered Concentration $X_{R}$ of the $450 \mathrm{~nm}{ }^{239} \mathrm{PuO} 2$ Colloid in the Matrix

Immediately above the Groundwater at $\mathrm{t}=10$ Years for Mean Present-Day Infiltration and Continuous Release 
Fracture Mass Fraction at Water Table

(for Co450 at 100 years)

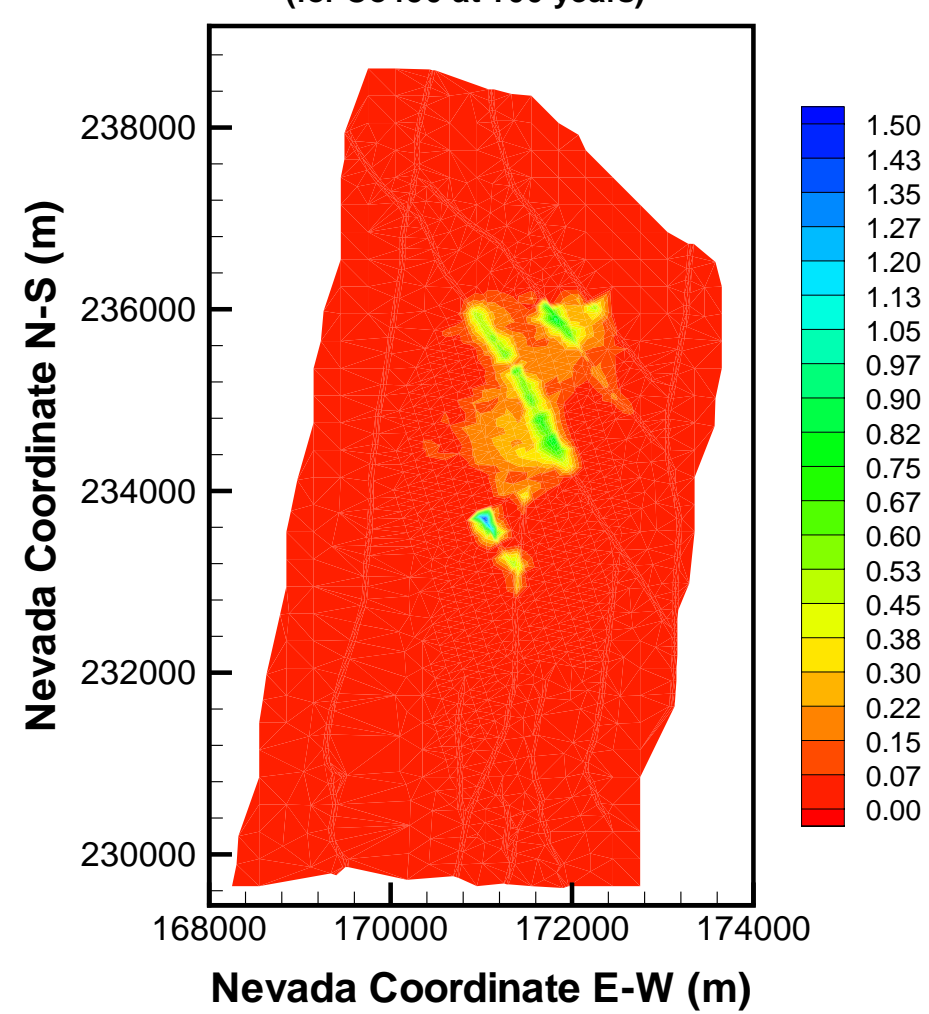

Output DTN: LB0307MR0060R1.004.

Figure G-19. Distribution of the Relative Mass Fraction $\mathrm{X}_{\mathrm{R}}$ of the $450 \mathrm{~nm}{ }^{239} \mathrm{PuO} 2$ Colloid in the Fractures Immediately above the Groundwater at $t=100$ Years for Mean Present-Day Infiltration and Continuous Release

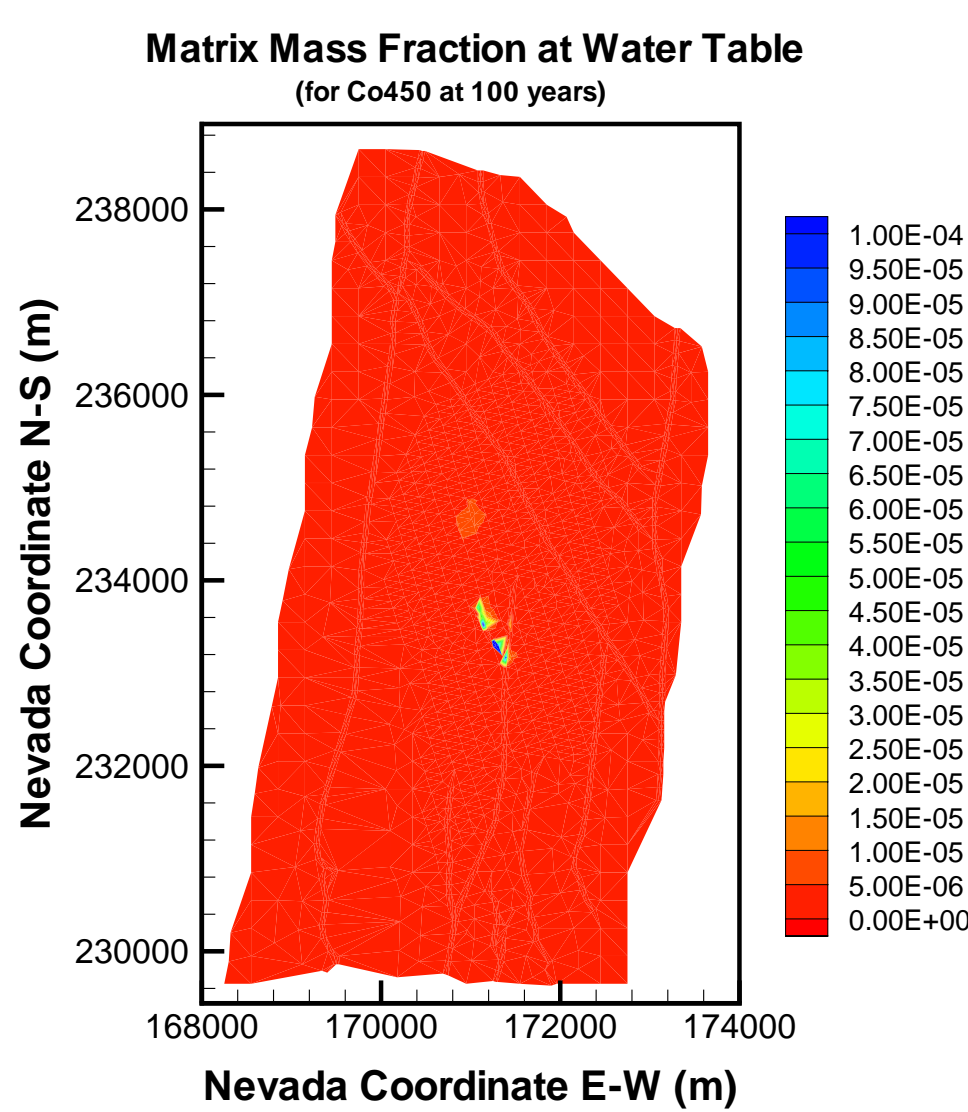

Output DTN: LB0307MR0060R1.004.

Figure G-20. Distribution of the Relative Mass Fraction $X_{R}$ of the $450 \mathrm{~nm}{ }^{239} \mathrm{PuO} 2$ Colloid in the Matrix Immediately above the Groundwater at $t=100$ Years for Mean Present-Day Infiltration and Continuous Release 


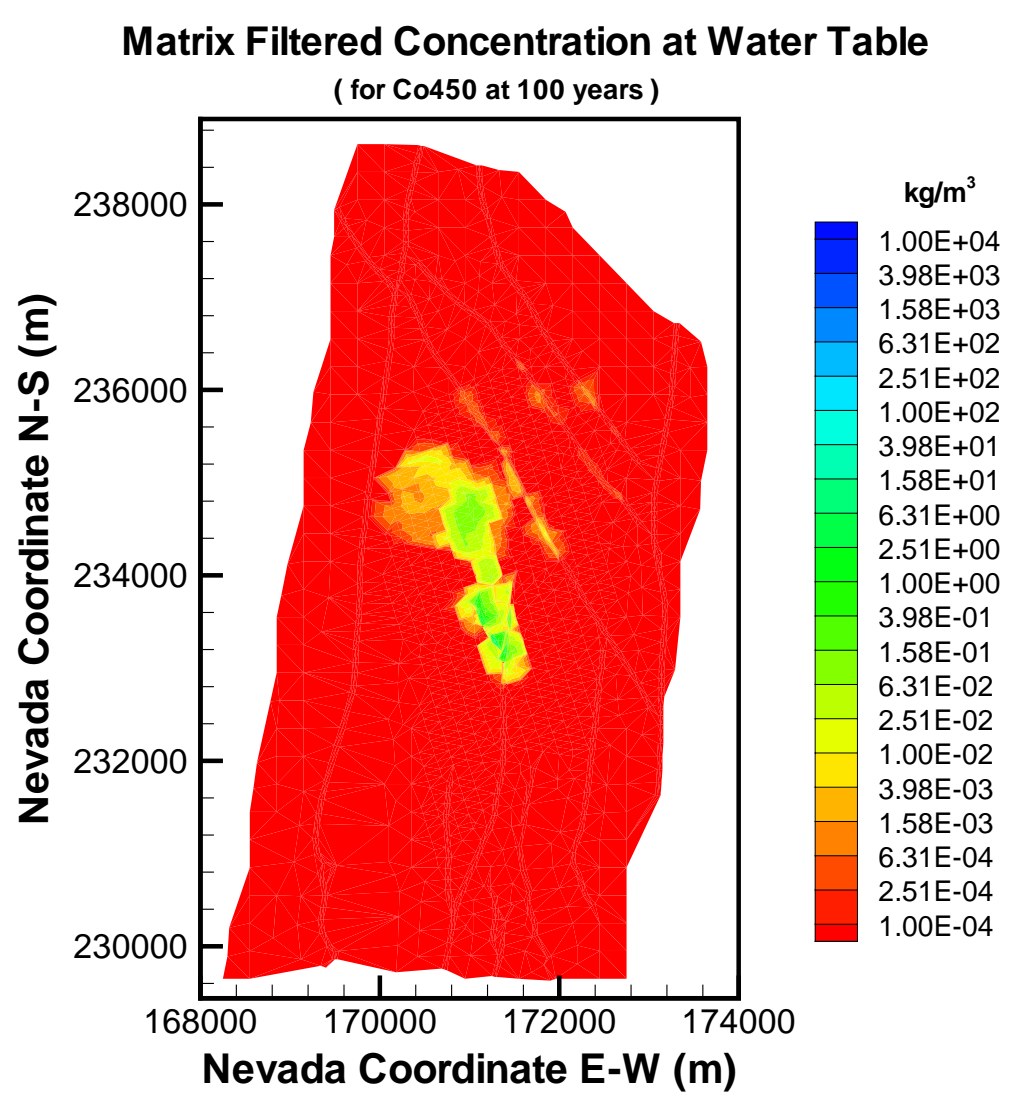

Output DTN: LB0307MR0060R1.004.

Figure G-21. Distribution of the Relative Filtered Concentration $X_{R}$ of the $450 \mathrm{~nm}{ }^{239} \mathrm{PuO} 2$ Colloid in the Matrix

Immediately above the Groundwater at $\mathrm{t}=100$ Years for Mean Present-Day Infiltration and Continuous

Release

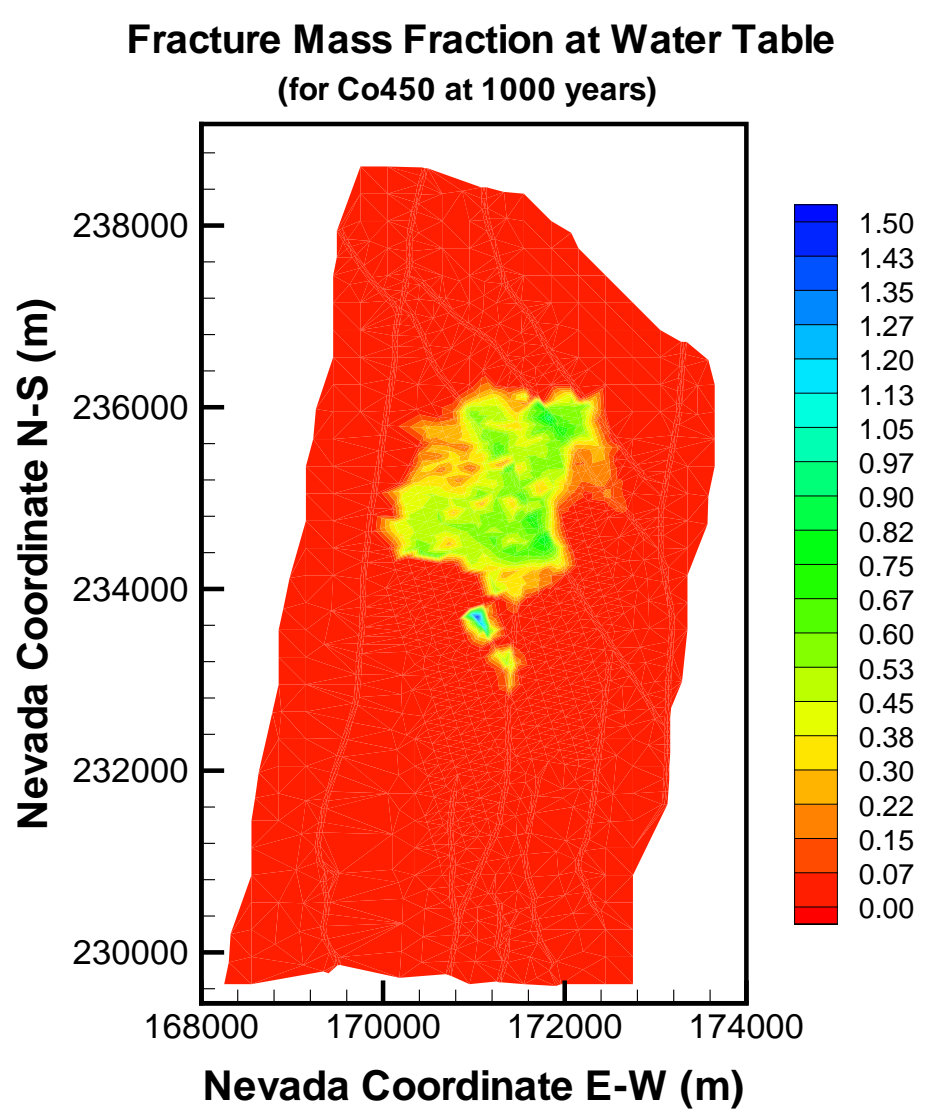

Output DTN: LB0307MR0060R1.004.

Figure G-22. Distribution of the Relative Mass Fraction $X_{R}$ of the $450 \mathrm{~nm}{ }^{239} \mathrm{PuO} 2$ Colloid in the Fractures Immediately above the Groundwater at $t=1,000$ Years for Mean Present-Day Infiltration and Continuous Release 
Matrix Mass Fraction at Water Table

(for Co450 at 1000 years)

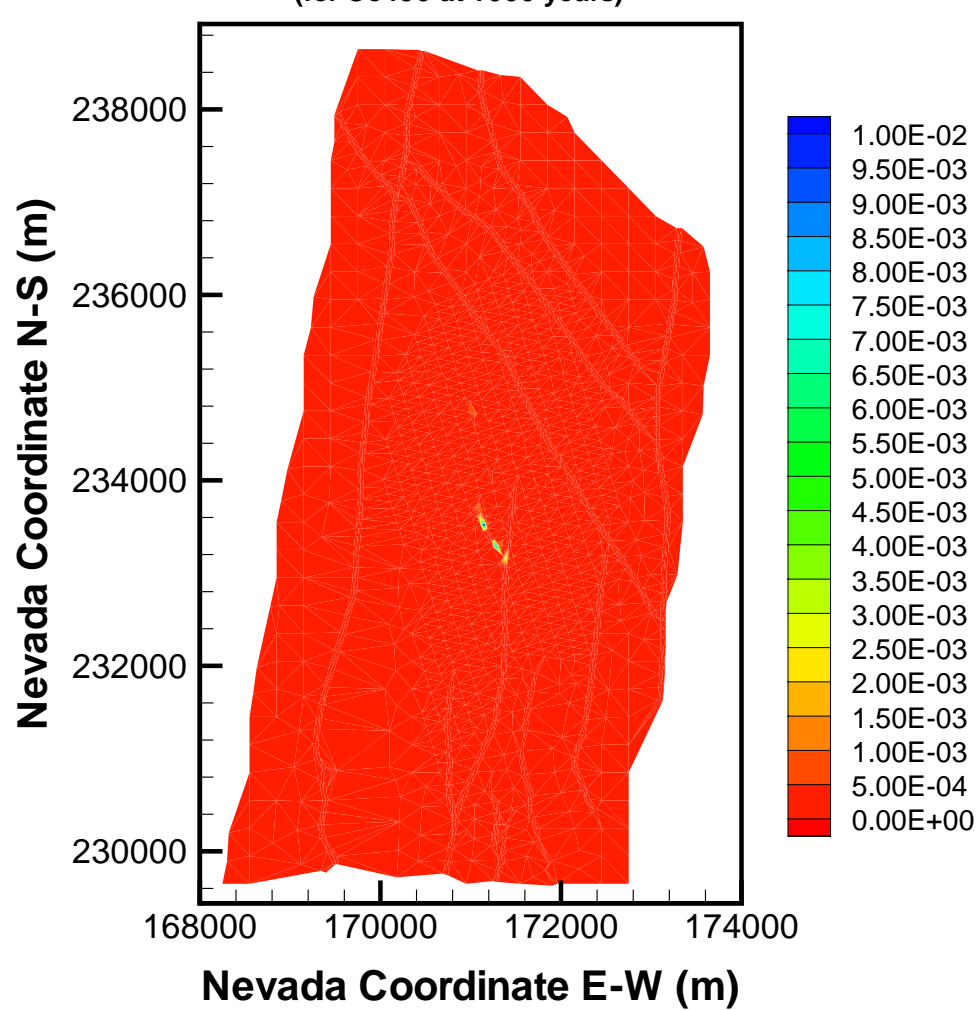

Output DTN: LB0307MR0060R1.004.

Figure G-23. Distribution of the Relative Mass Fraction $X_{R}$ of the $450 \mathrm{~nm}{ }^{239} \mathrm{PuO} 2$ Colloid in the Matrix Immediately above the Groundwater at $t=1,000$ Years for Mean Present-Day Infiltration and Continuous Release

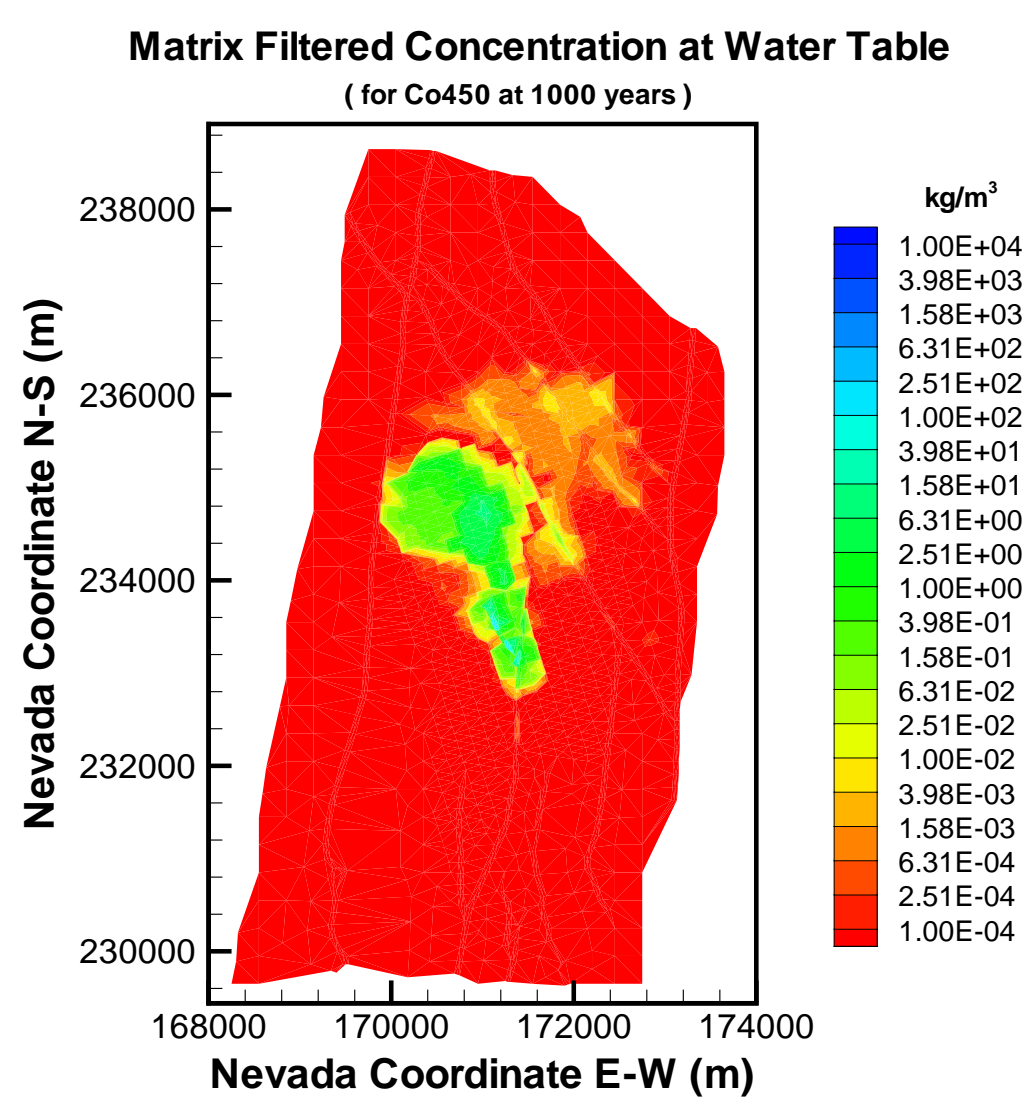

Output DTN: LB0307MR0060R1.004.

Figure G-24. Distribution of the Relative Filtered Concentration $X_{R}$ of the $450 \mathrm{~nm}{ }^{239} \mathrm{PuO} 2$ Colloid in the Matrix

Immediately above the Groundwater at $\mathrm{t}=1,000$

Years for Mean Present-Day Infiltration and

Continuous Release 
Fracture Mass Fraction at Water Table (for Co450 at 10000 years)

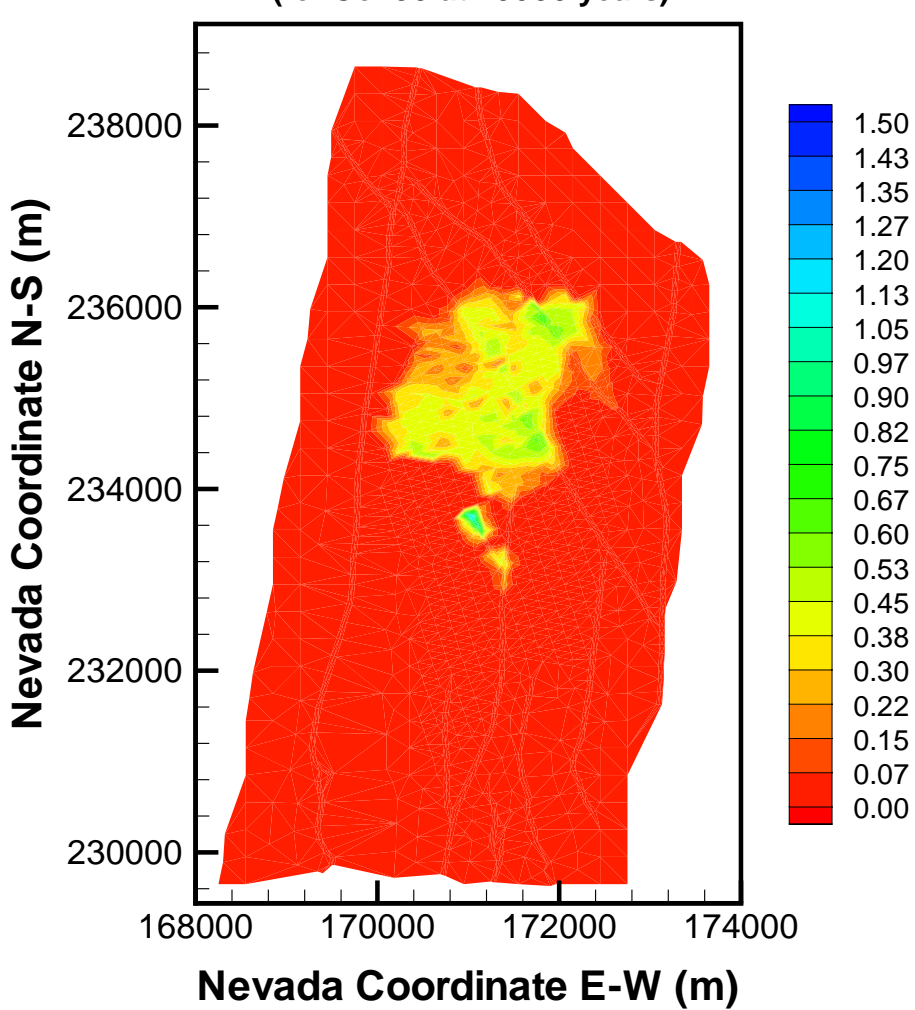

Output DTN: LB0307MR0060R1.004.

Figure G-25. Distribution of the Relative Mass Fraction $X_{R}$ of the $450 \mathrm{~nm}{ }^{239} \mathrm{PuO} 2$ Colloid in the Fractures Immediately above the Groundwater at $t=10,000$ Years for Mean Present-Day Infiltration and Continuous Release

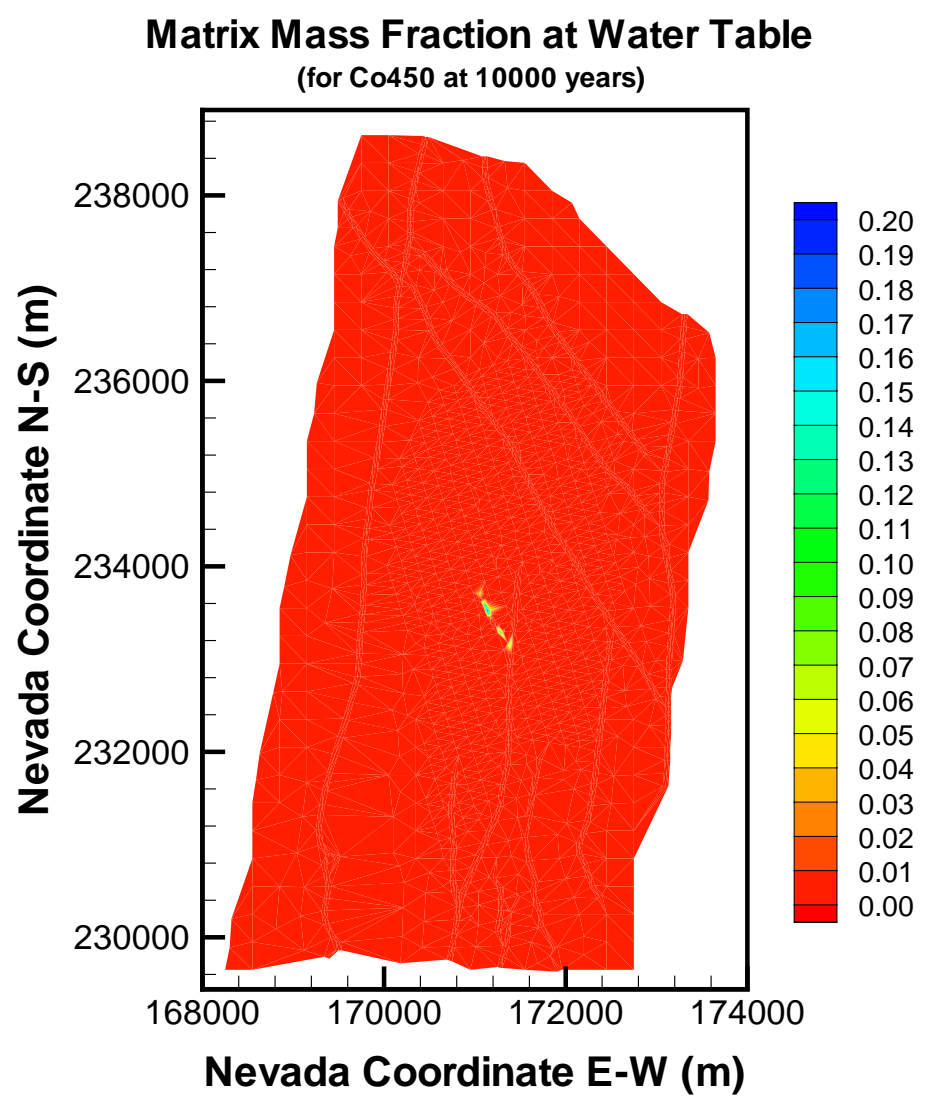

Output DTN: LB0307MR0060R1.004.

Figure G-26. Distribution of the Relative Mass Fraction $X_{R}$ of the $450 \mathrm{~nm}{ }^{239} \mathrm{PuO} 2$ Colloid in the Matrix Immediately above the Groundwater at $t=10,000$ Years for Mean Present-Day Infiltration and Continuous Release 


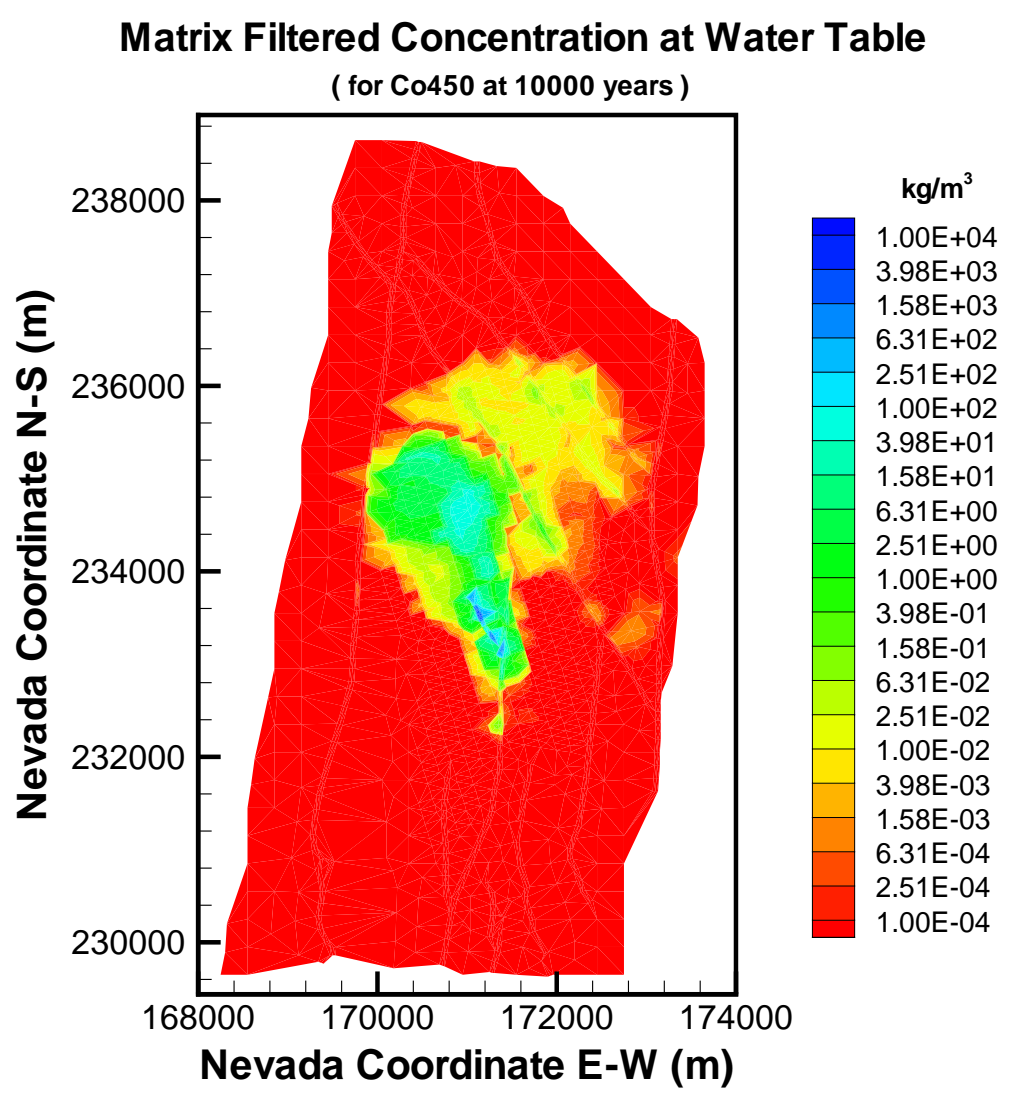

Output DTN: LB0307MR0060R1.004.

Figure G-27. Distribution of the Relative Filtered Concentration $X_{R}$ of the $450 \mathrm{~nm}{ }^{239} \mathrm{PuO} 2$ Colloid in the Matrix

Immediately above the Groundwater at $t=10,000$

Years for Mean Present-Day Infiltration and

Continuous Release

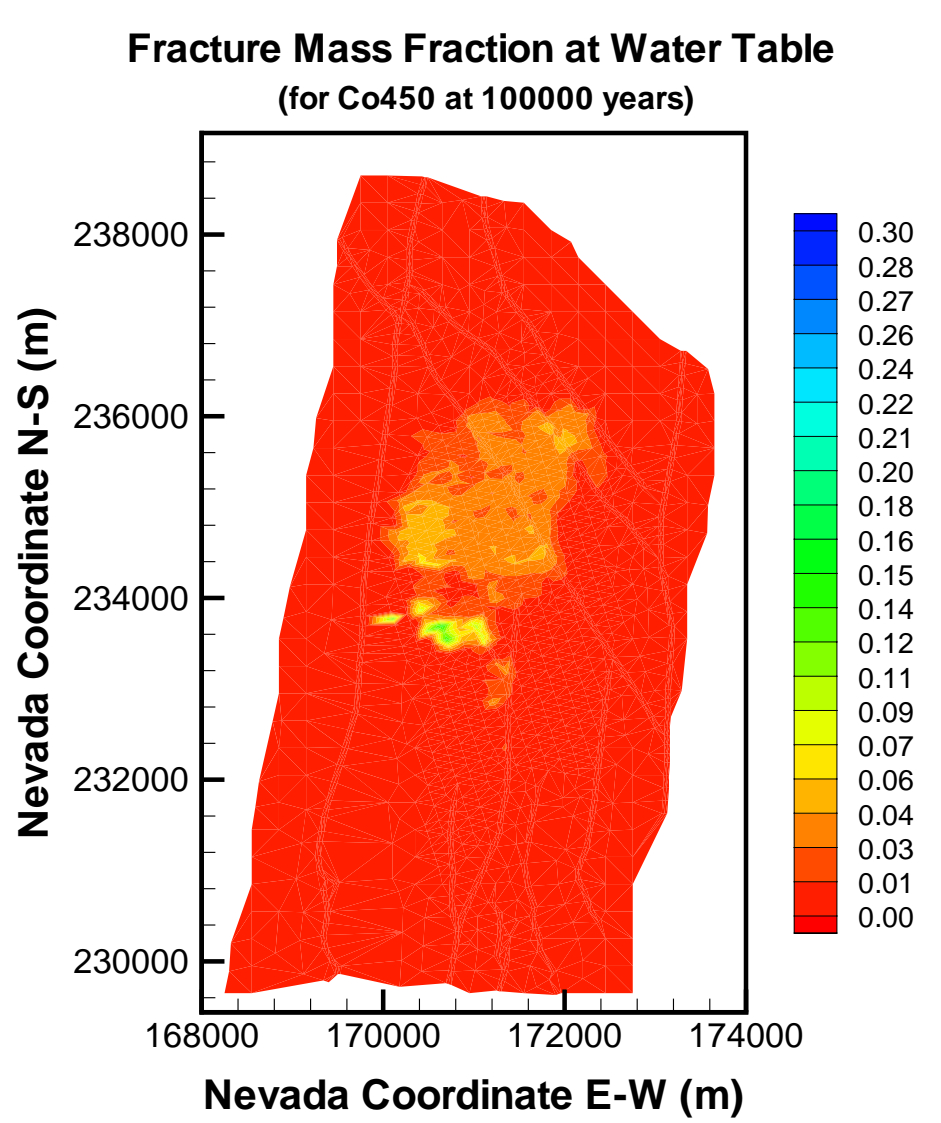

Output DTN: LB0307MR0060R1.004.

Figure G-28. Distribution of the Relative Mass Fraction $X_{R}$ of the $450 \mathrm{~nm}{ }^{239} \mathrm{PuO} 2$ Colloid in the Fractures Immediately above the Groundwater at $t=100,000$ Years for Mean Present-Day Infiltration and Continuous Release 


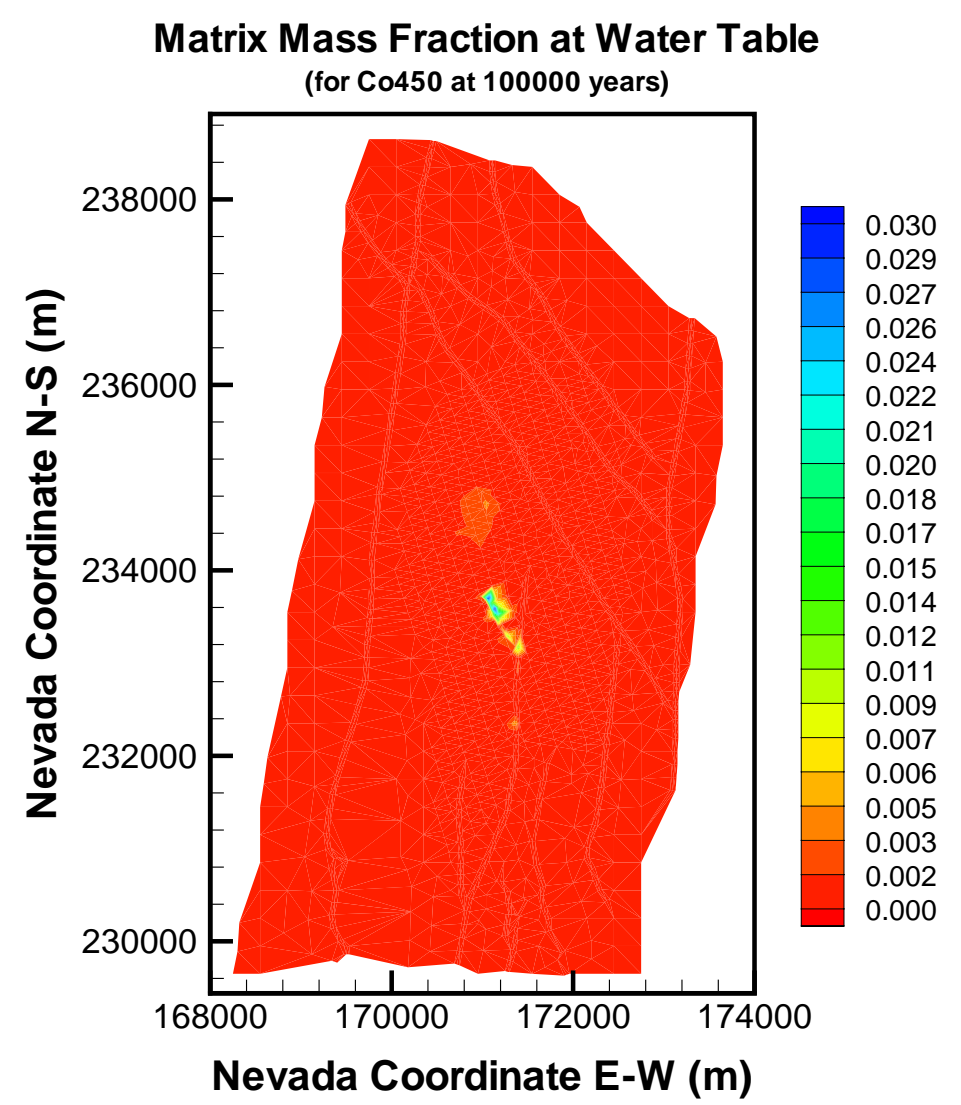

Output DTN: LB0307MR0060R1.004.

Figure G-29. Distribution of the Relative Mass Fraction $\mathrm{X}_{\mathrm{R}}$ of the $450 \mathrm{~nm}{ }^{239} \mathrm{PuO} 2$ Colloid in the Matrix Immediately above the Groundwater at $t=100,000$ Years for Mean Present-Day Infiltration and Continuous Release

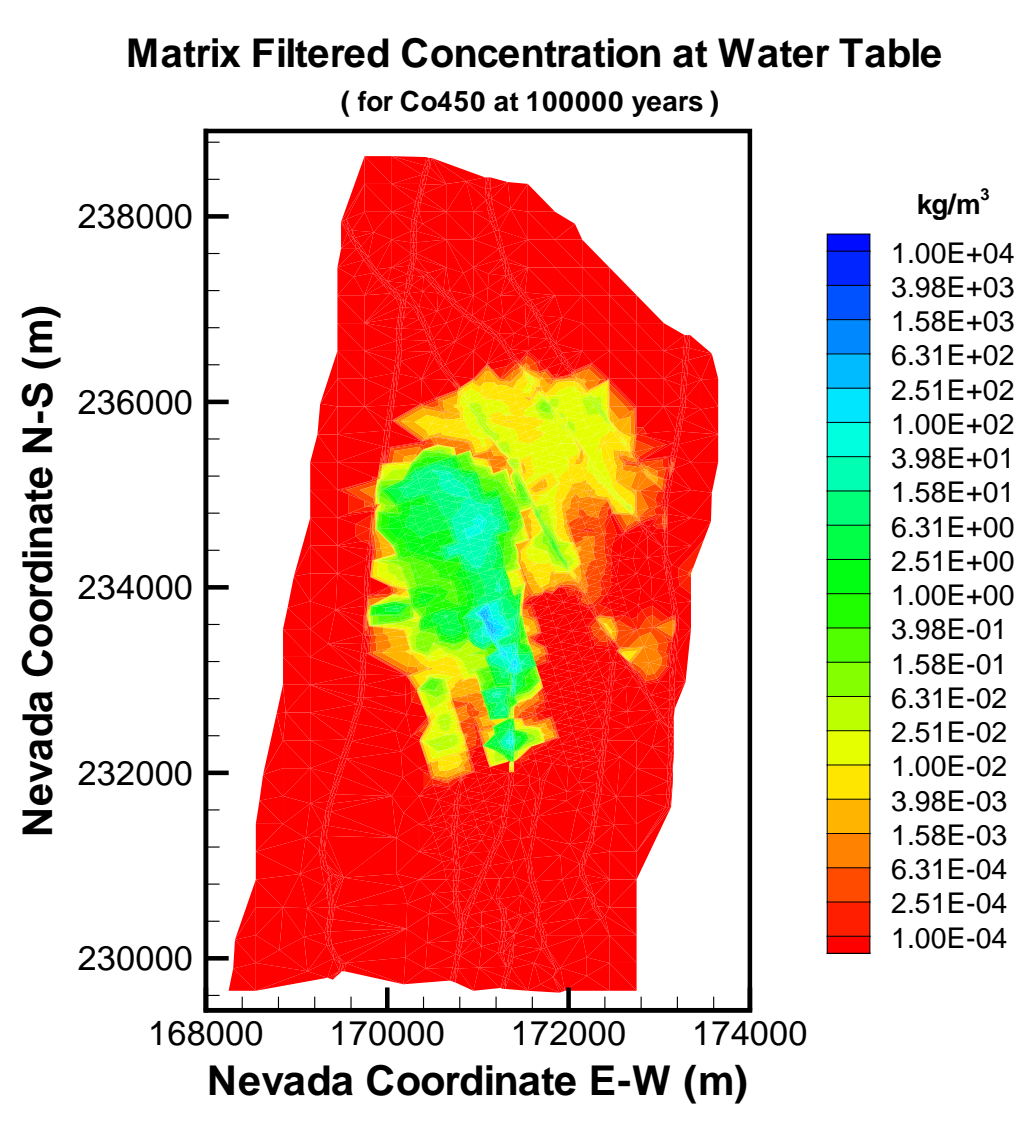

Output DTN: LB0307MR0060R1.004.

Figure G-30. Distribution of the Relative Filtered Concentration $X_{R}$ of the $450 \mathrm{~nm}{ }^{239} \mathrm{PuO} 2$ Colloid in the Matrix Immediately above the Groundwater at $t=100,000$ Years for Mean Present-Day Infiltration and Continuous Release 


\section{INTENTIONALLY LEFT BLANK}




\section{APPENDIX H}

FIGURES FROM THE ${ }^{99}$ Tc THREE-DIMENSIONAL TRANSPORT STUDIES (INSTANTANEOUS RELEASE ONLY FROM NONFAULTED DOMAIN, MEAN PRESENT-DAY INFILTRATION) 


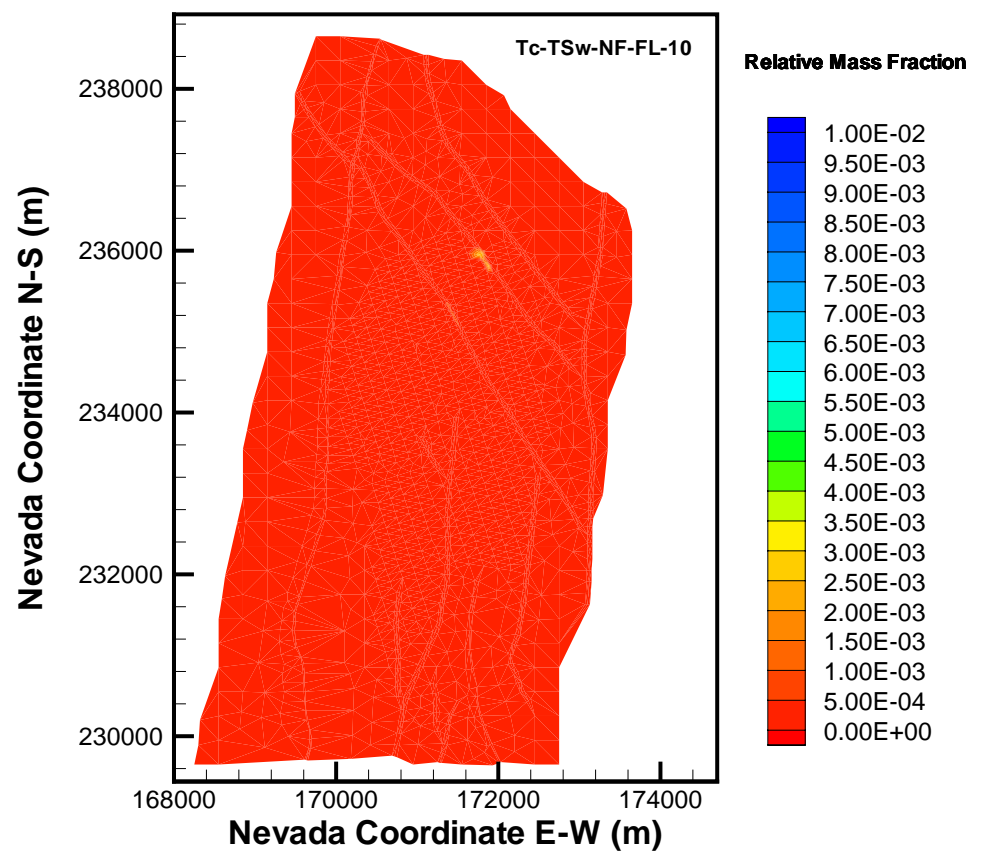

Output DTN: LB0307MR0060R1.006.

Figure $\mathrm{H}-1$. Distribution of the Relative Mass Fraction $\mathrm{X}_{\mathrm{R}}$ of ${ }^{99} \mathrm{Tc}$ in the Fractures of the TSw39 Layer at $t=10$ Years for Mean Present-Day Infiltration and Instantaneous Release Only from Nonfaulted Domain

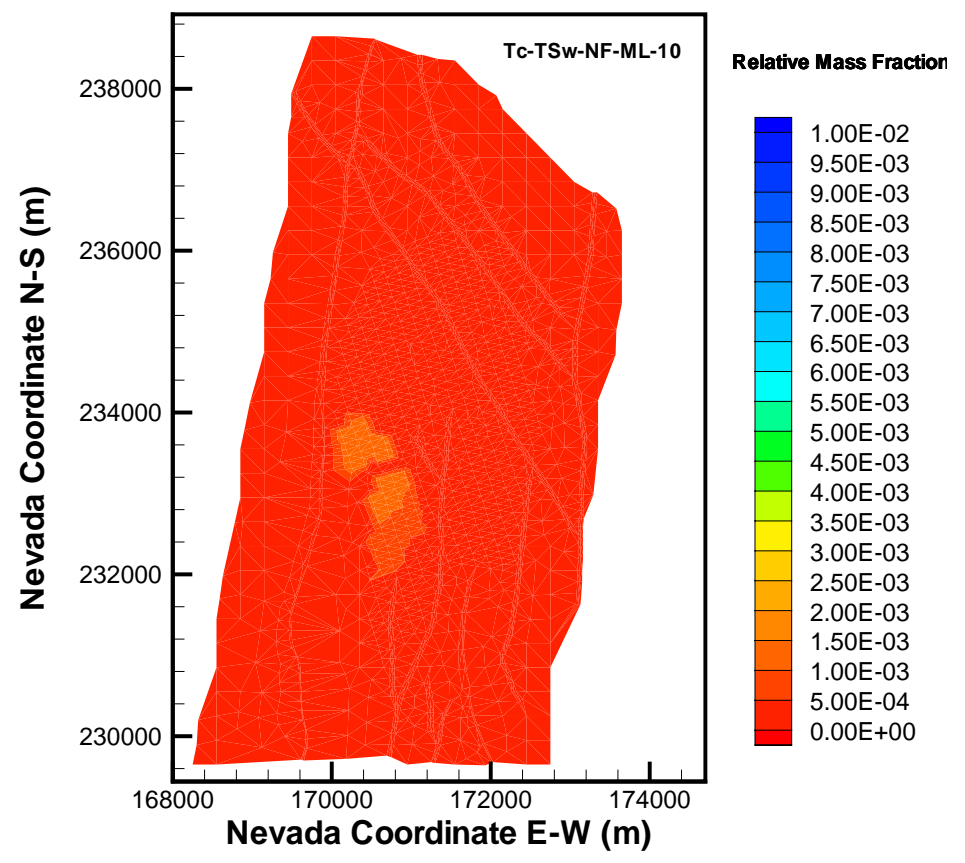

Output DTN: LB0307MR0060R1.006.

Figure H-2. Distribution of the Relative Mass Fraction $X_{R}$ of ${ }^{99} T c$ in the Matrix of the TSw39 Layer at $t=10$ Years for Mean Present-Day Infiltration and Instantaneous Release Only from Nonfaulted Domain 


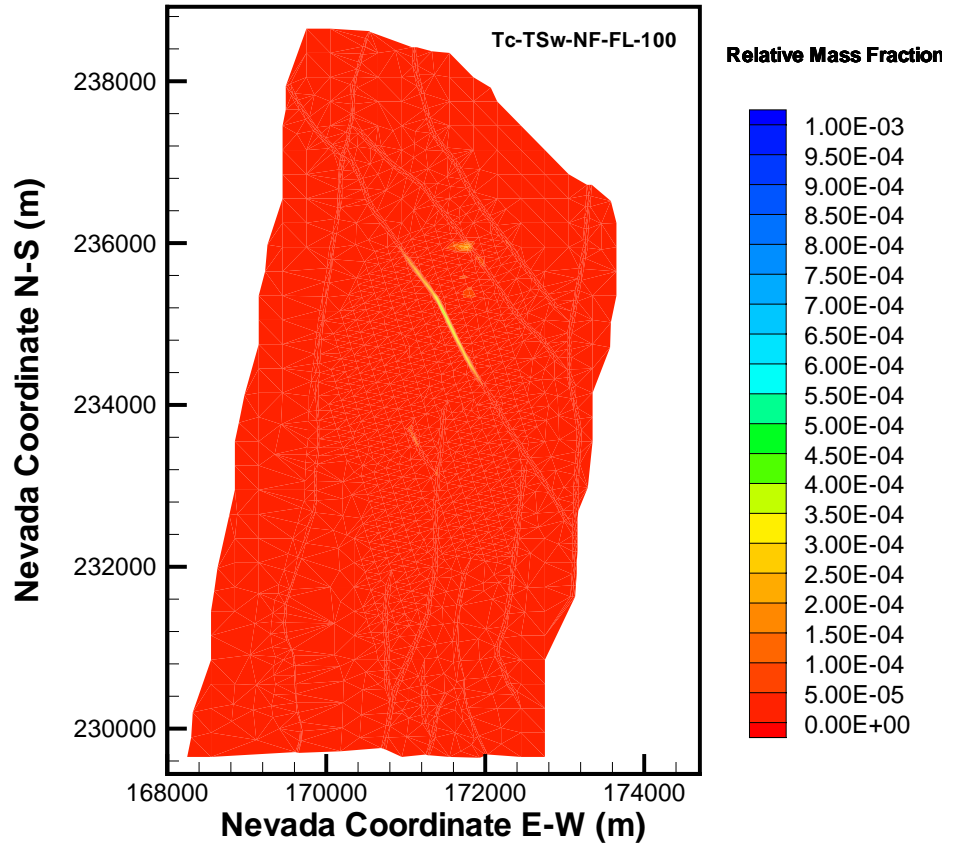

Output DTN: LB0307MR0060R1.006.

Figure H-3. Distribution of the Relative Mass Fraction $\mathrm{X}_{\mathrm{R}}$ of ${ }^{99} \mathrm{Tc}$ in the Fractures of the TSw39 Layer at $t=100$ Years for Mean Present-Day Infiltration and Instantaneous Release Only from Nonfaulted Domain

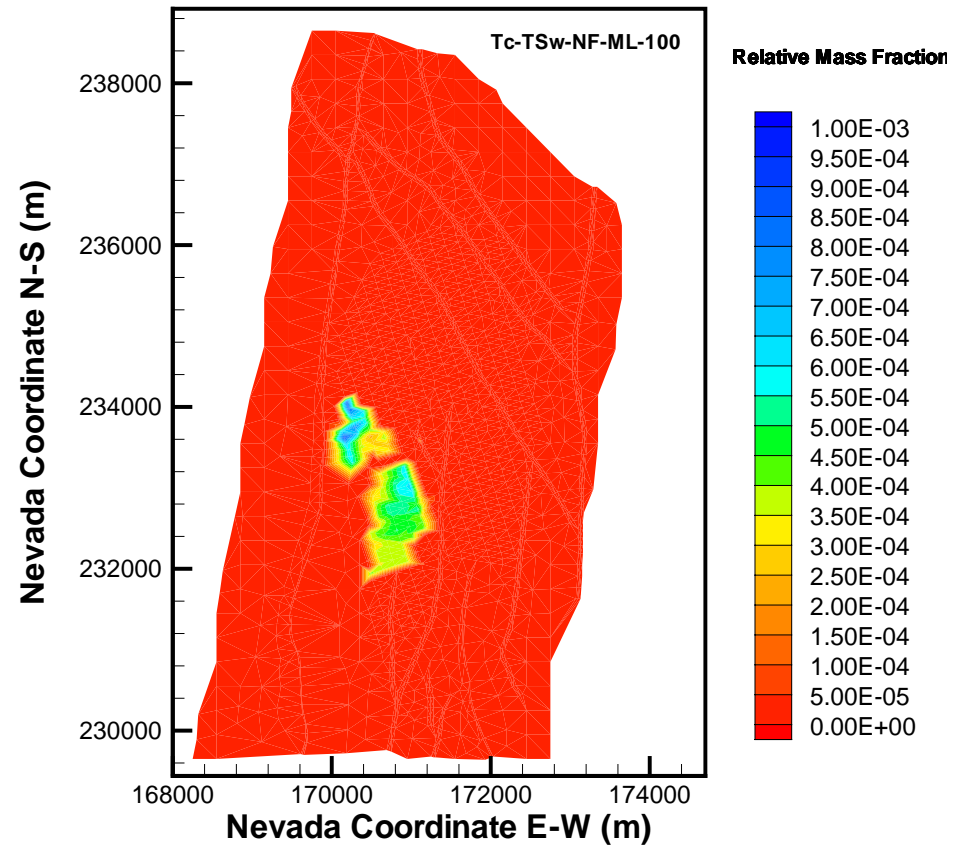

Output DTN: LB0307MR0060R1.006.

Figure H-4. Distribution of the Relative Mass Fraction $X_{R}$ of ${ }^{99} \mathrm{TC}$ in the Matrix of the TSw39 Layer at $t=100$ Years for Mean Present-Day Infiltration and Instantaneous Release Only from Nonfaulted Domain 


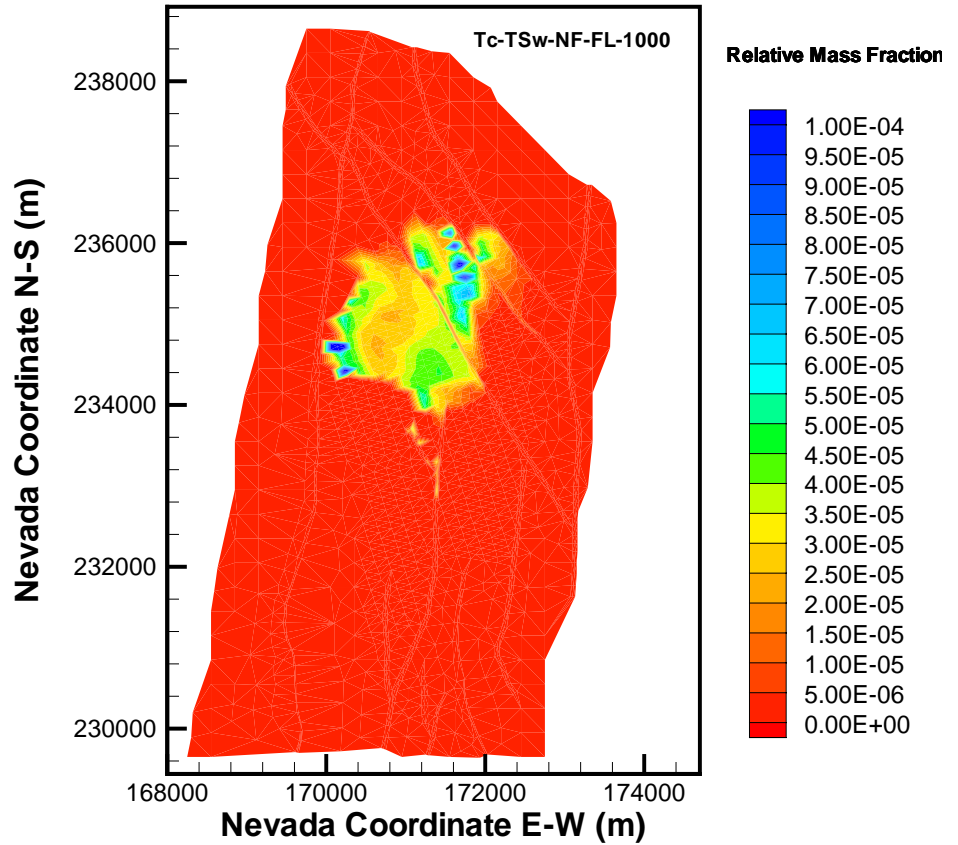

Output DTN: LB0307MR0060R1.006.

Figure H-5. Distribution of the Relative Mass Fraction $\mathrm{X}_{\mathrm{R}}$ of ${ }^{99} \mathrm{Tc}$ in the Fractures of the TSw39 Layer at $t=1,000$ Years for Mean Present-Day Infiltration and Instantaneous Release Only from Nonfaulted Domain

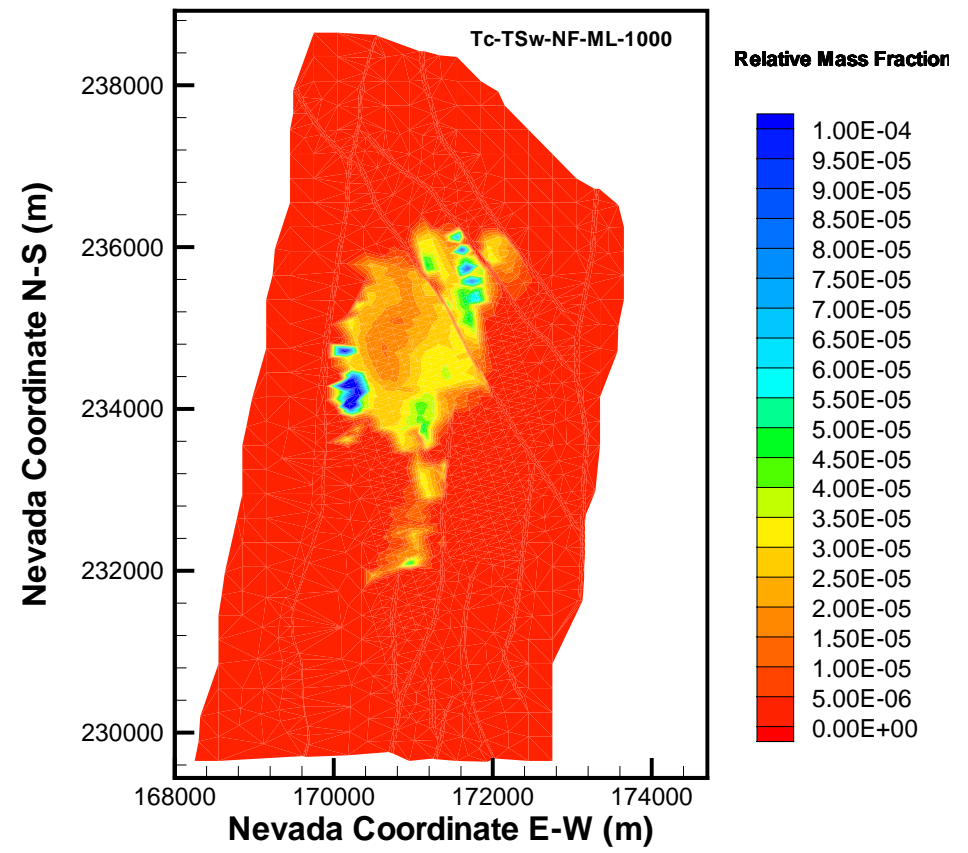

Output DTN: LB0307MR0060R1.006.

Figure H-6. Distribution of the Relative Mass Fraction $X_{R}$ of ${ }^{99} \mathrm{Tc}$ in the Matrix of the TSw39 Layer at $t=1,000$ Years for Mean Present-Day Infiltration and Instantaneous Release Only from Nonfaulted Domain 


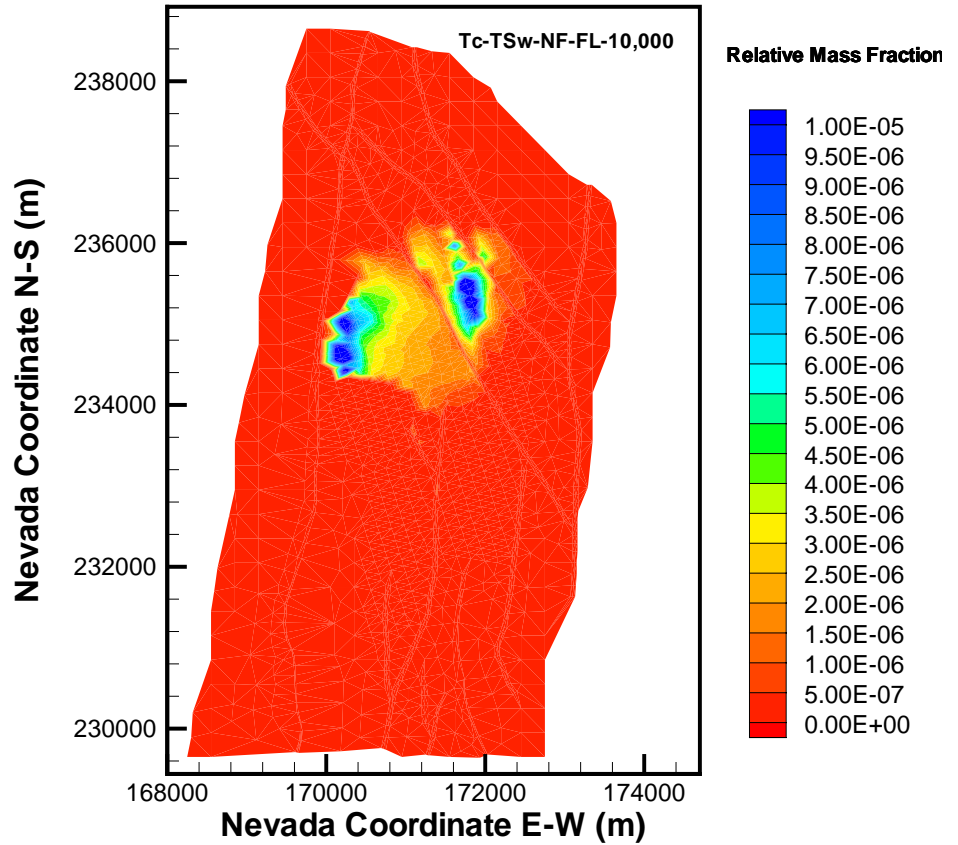

Output DTN: LB0307MR0060R1.006.

Figure H-7. Distribution of the Relative Mass Fraction $\mathrm{X}_{\mathrm{R}}$ of ${ }^{99} \mathrm{Tc}$ in the Fractures of the TSw39 Layer at $t=10,000$ Years for Mean Present-Day Infiltration and Instantaneous Release Only from Nonfaulted Domain

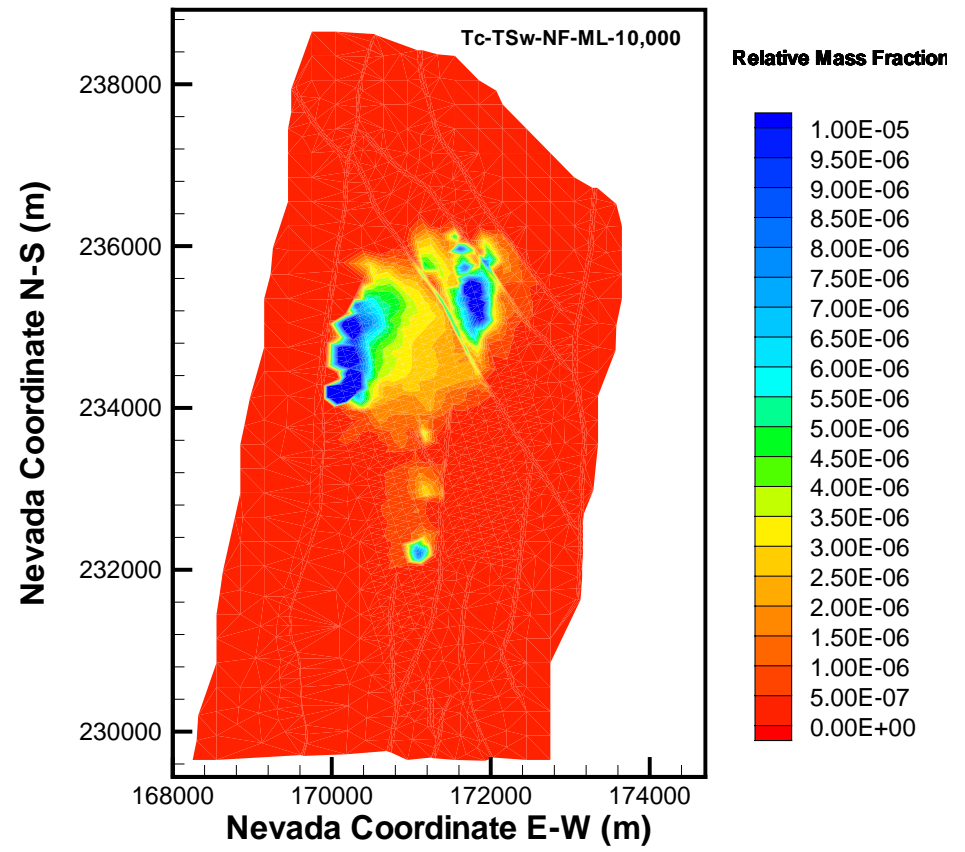

Output DTN: LB0307MR0060R1.006.

Figure H-8. Distribution of the Relative Mass Fraction $\mathrm{X}_{\mathrm{R}}$ of ${ }^{99} \mathrm{Tc}$ in the Matrix of the TSw39 Layer at $t=10,000$ Years for Mean Present-Day Infiltration and Instantaneous Release Only from Nonfaulted Domain 


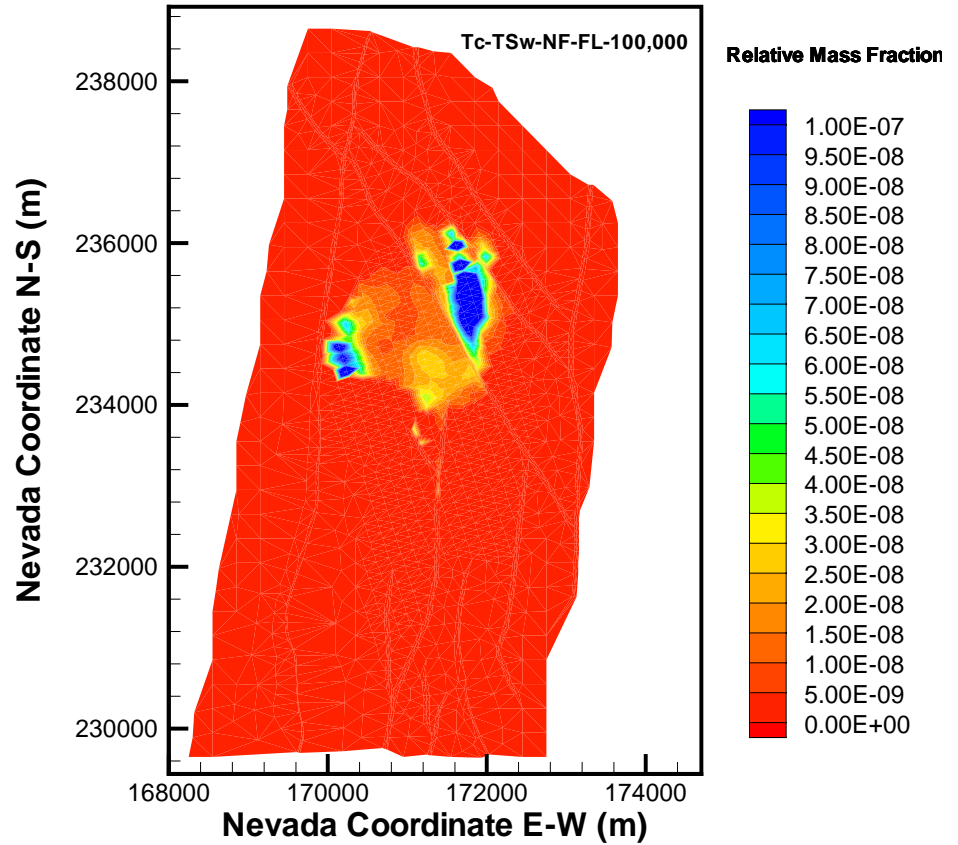

Output DTN: LB0307MR0060R1.006.

Figure H-9. Distribution of the Relative Mass Fraction $X_{R}$ of ${ }^{99} T c$ in the Fractures of the TSw39 Layer at $t=100,000$ Years for Mean Present-Day Infiltration and Instantaneous Release Only from Nonfaulted Domain

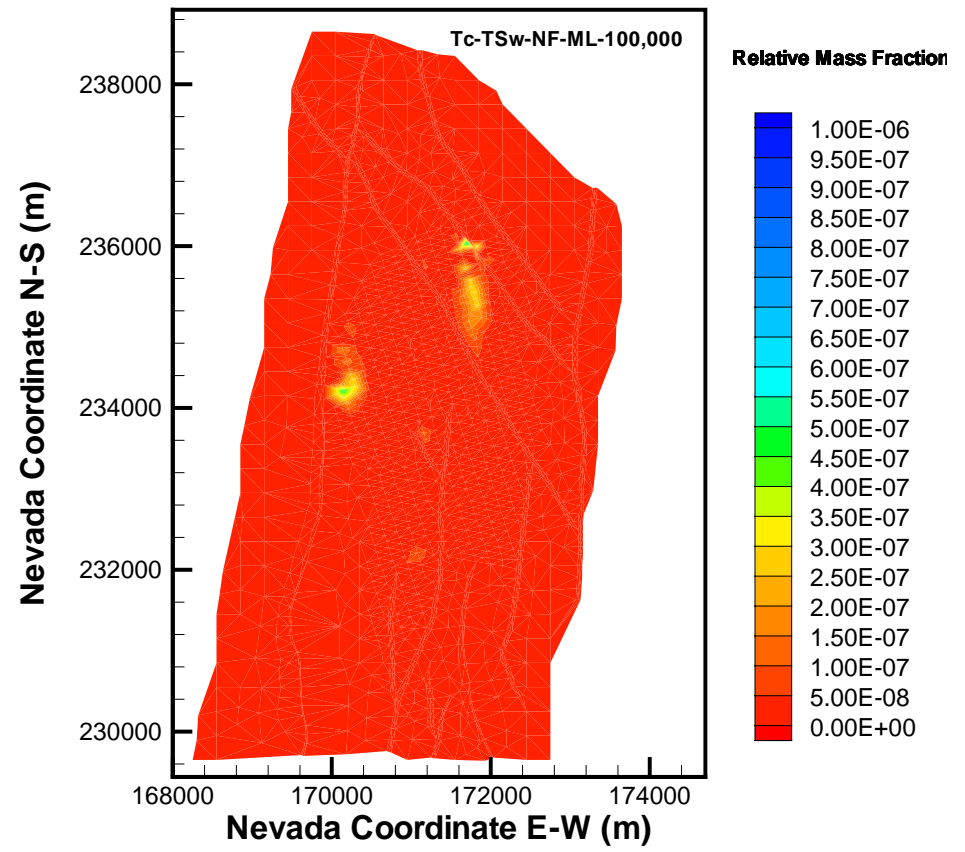

Output DTN: LB0307MR0060R1.006.

Figure $\mathrm{H}-10$. Distribution of the Relative Mass Fraction $\mathrm{X}_{\mathrm{R}}$ of ${ }^{99} \mathrm{TC}$ in the Matrix of the TSw39 Layer at $t=100,000$ Years for Mean Present-Day Infiltration and Instantaneous Release Only from Nonfaulted Domain 


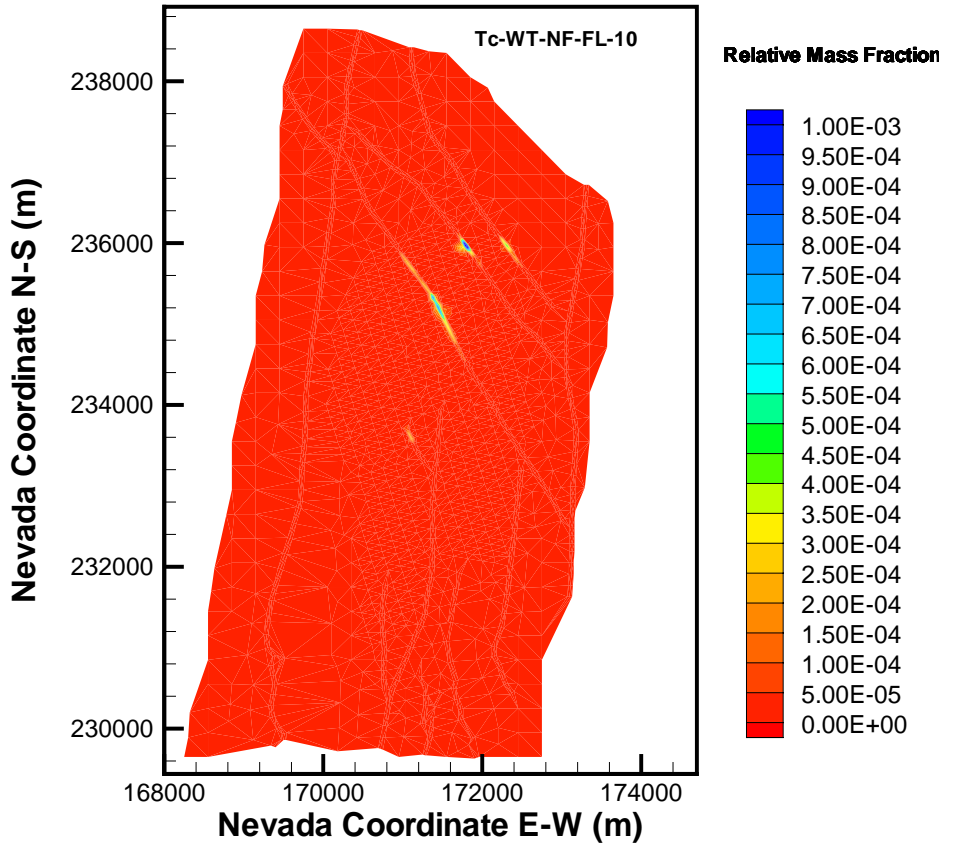

Output DTN: LB0307MR0060R1.006.

Figure $\mathrm{H}-11$. Distribution of the Relative Mass Fraction $\mathrm{X}_{\mathrm{R}}$ of ${ }^{99} \mathrm{TC}$ in the Fractures Immediately above the Groundwater Table at $\mathrm{t}=10$ Years for Mean Present-Day

Infiltration and Instantaneous Release Only from Nonfaulted Domain

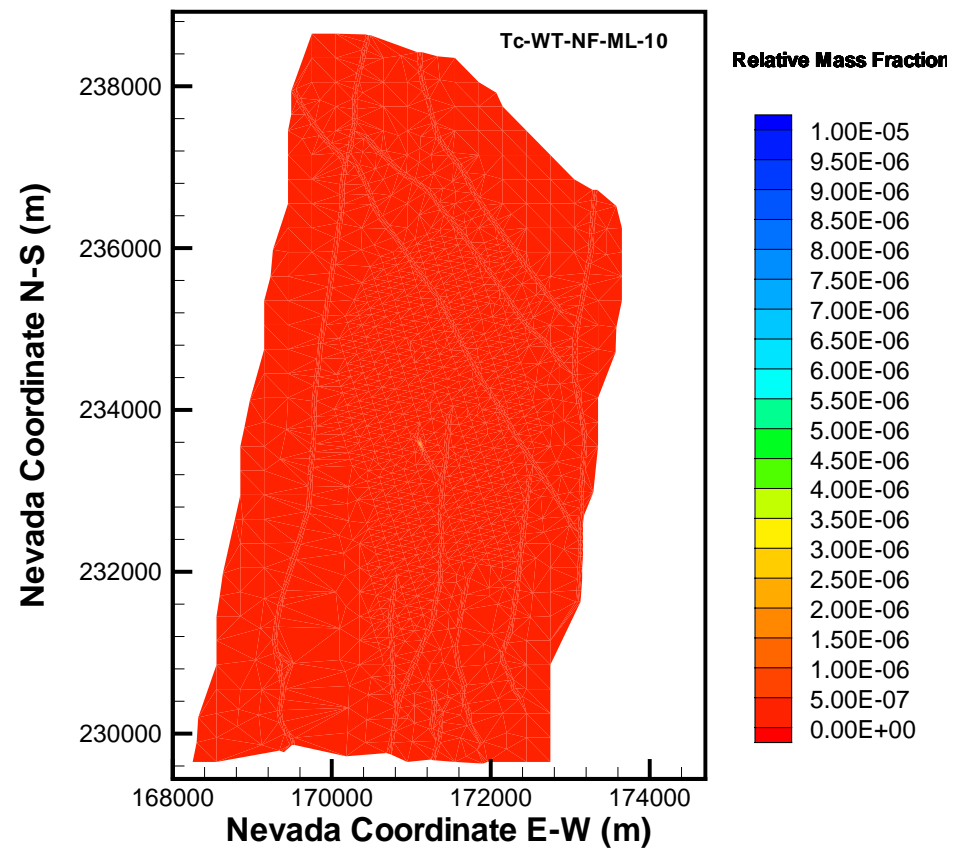

Output DTN: LB0307MR0060R1.006.

Figure $\mathrm{H}-12$. Distribution of the Relative Mass Fraction $\mathrm{X}_{\mathrm{R}}$ of ${ }^{99} \mathrm{TC}$ in the Matrix Immediately above the Groundwater at $t$ $=10$ Years for Mean Present-Day Infiltration and Instantaneous Release Only from Nonfaulted Domain 


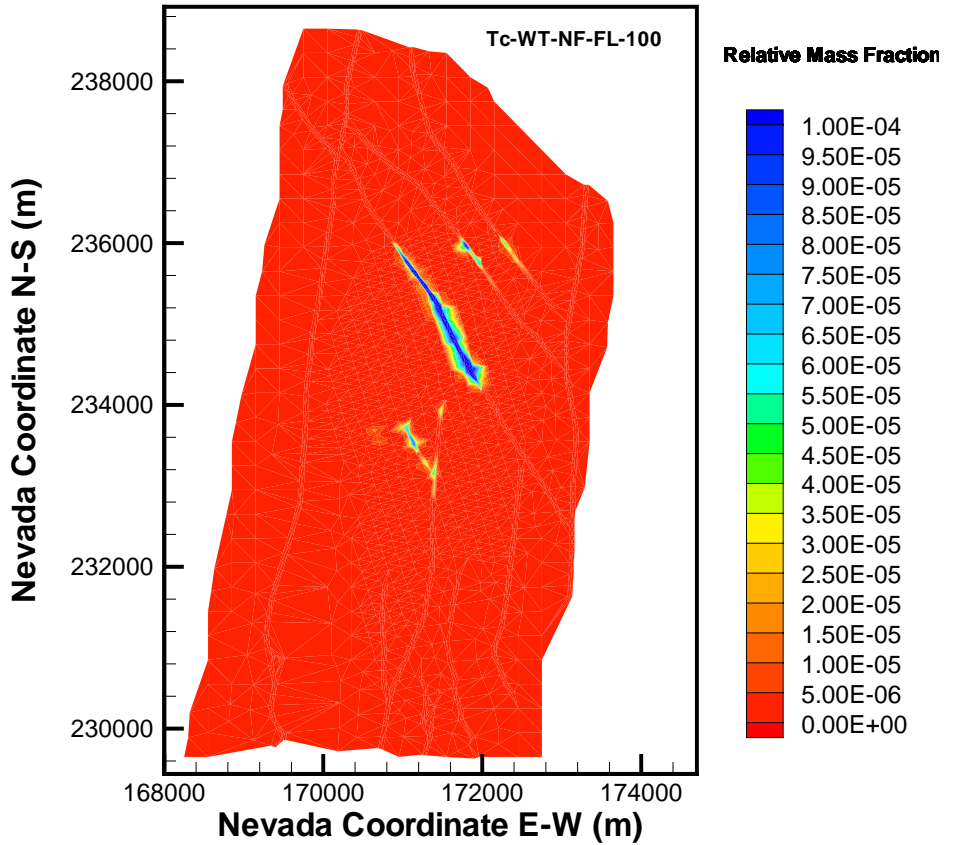

Output DTN: LB0307MR0060R1.006.

Figure $\mathrm{H}-13$. Distribution of the Relative Mass Fraction $\mathrm{X}_{\mathrm{R}}$ of ${ }^{99} \mathrm{Tc}$ in the Fractures Immediately above the Groundwater at $t=100$ Years for Mean Present-Day Infiltration and Instantaneous Release Only from Nonfaulted Domain

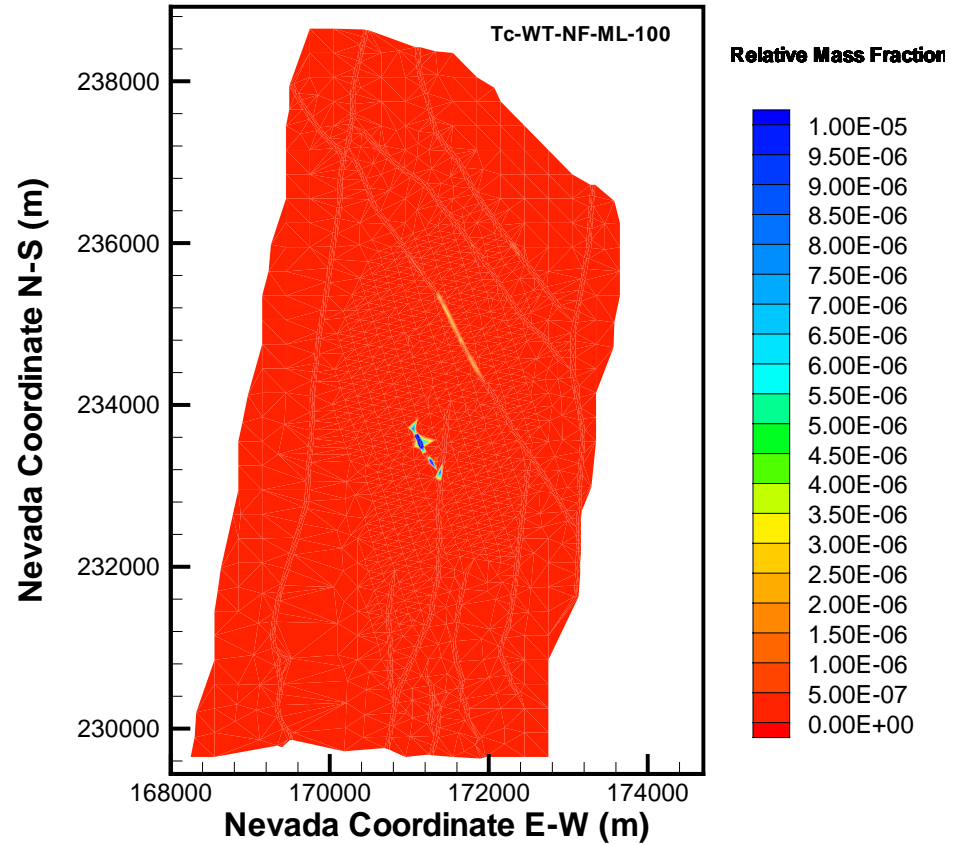

Output DTN: LB0307MR0060R1.006.

Figure H-14. Distribution of the Relative Mass Fraction $X_{R}$ of ${ }^{99} \mathrm{Tc}$ in the Matrix Immediately above the Groundwater at $t$ $=100$ Years for Mean Present-Day Infiltration and Instantaneous Release Only from Nonfaulted Domain 


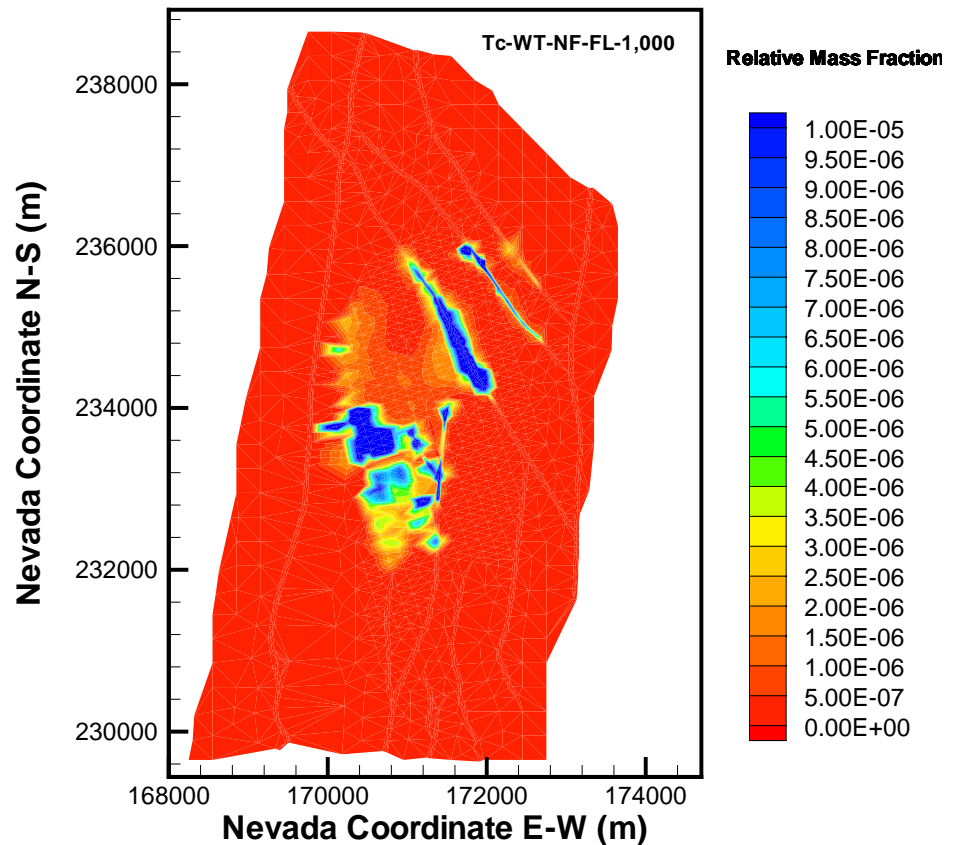

Output DTN: LB0307MR0060R1.006.

Figure $\mathrm{H}-15$. Distribution of the Relative Mass Fraction $\mathrm{X}_{\mathrm{R}}$ of ${ }^{99} \mathrm{TC}$ in the Fractures Immediately above the Groundwater at $t=1,000$ Years for Mean Present-Day Infiltration and Instantaneous Release Only from Nonfaulted Domain

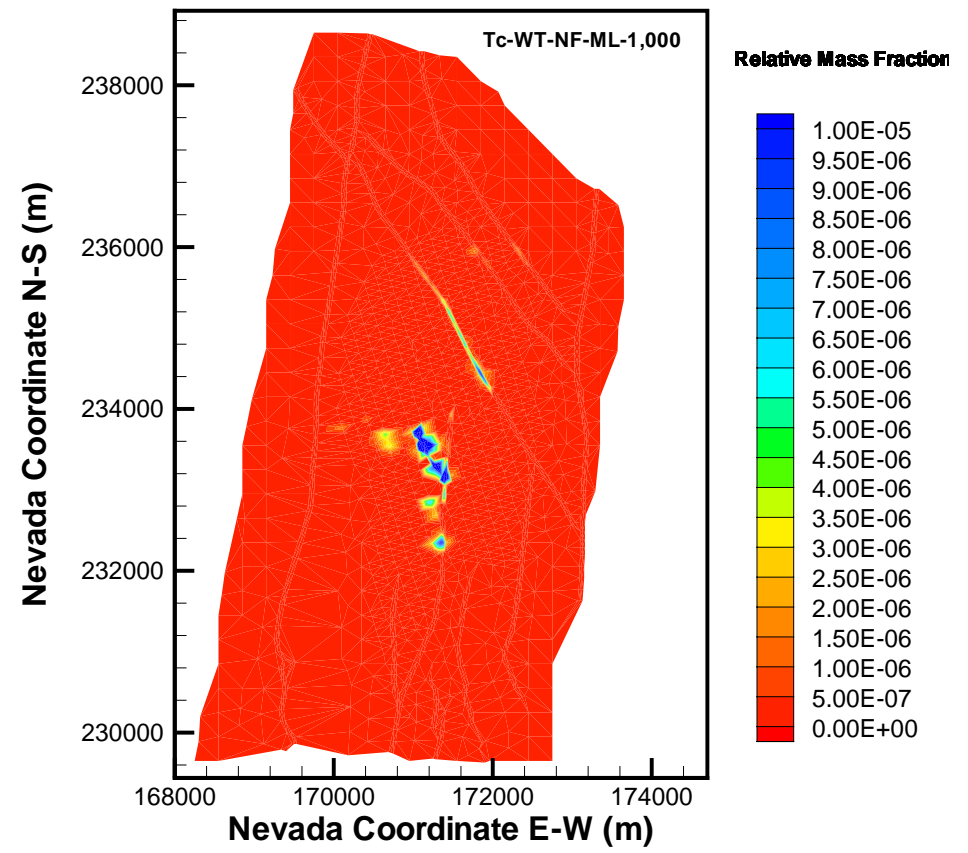

Output DTN: LB0307MR0060R1.006

Figure $\mathrm{H}-16$. Distribution of the Relative Mass Fraction $\mathrm{X}_{\mathrm{R}}$ of ${ }^{99} \mathrm{TC}$ in the Matrix Immediately above the Groundwater at $t$ $=1,000$ Years for Mean Present-Day Infiltration and Instantaneous Release Only from Nonfaulted Domain 


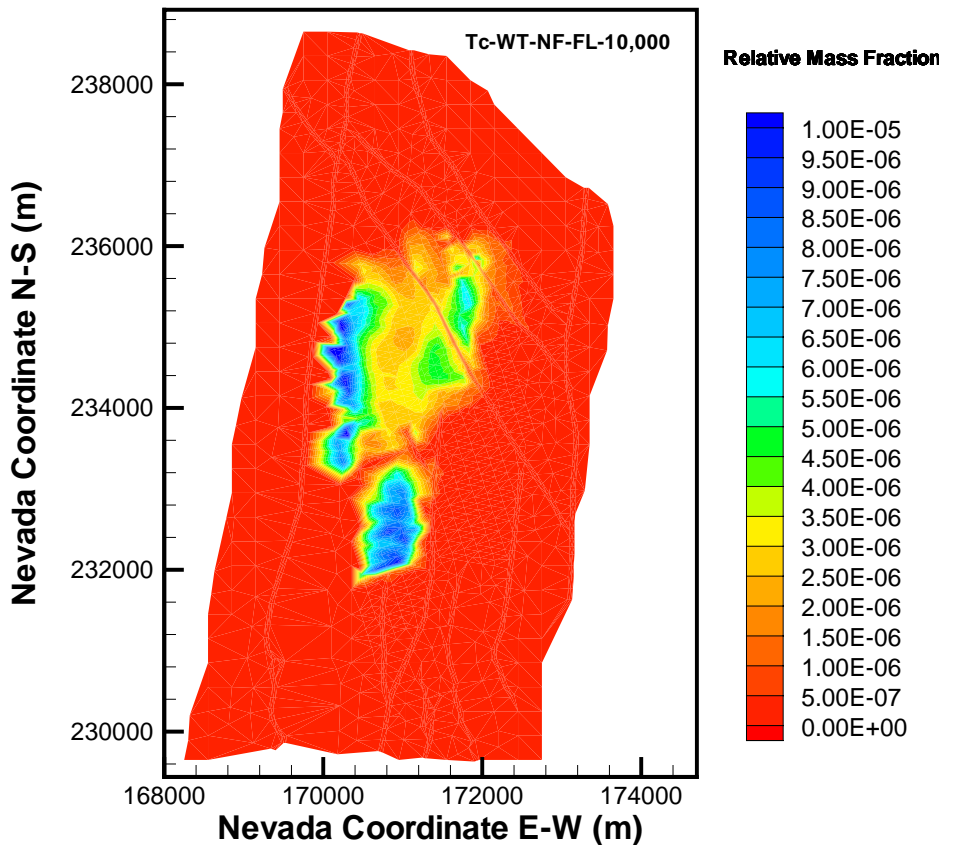

Output DTN: LB0307MR0060R1.006.

Figure $\mathrm{H}-17$. Distribution of the Relative Mass Fraction $\mathrm{X}_{\mathrm{R}}$ of ${ }^{99} \mathrm{Tc}$ in the Fractures Immediately above the Groundwater at $t=10,000$ Years for Mean Present-Day Infiltration and Instantaneous Release Only from Nonfaulted Domain

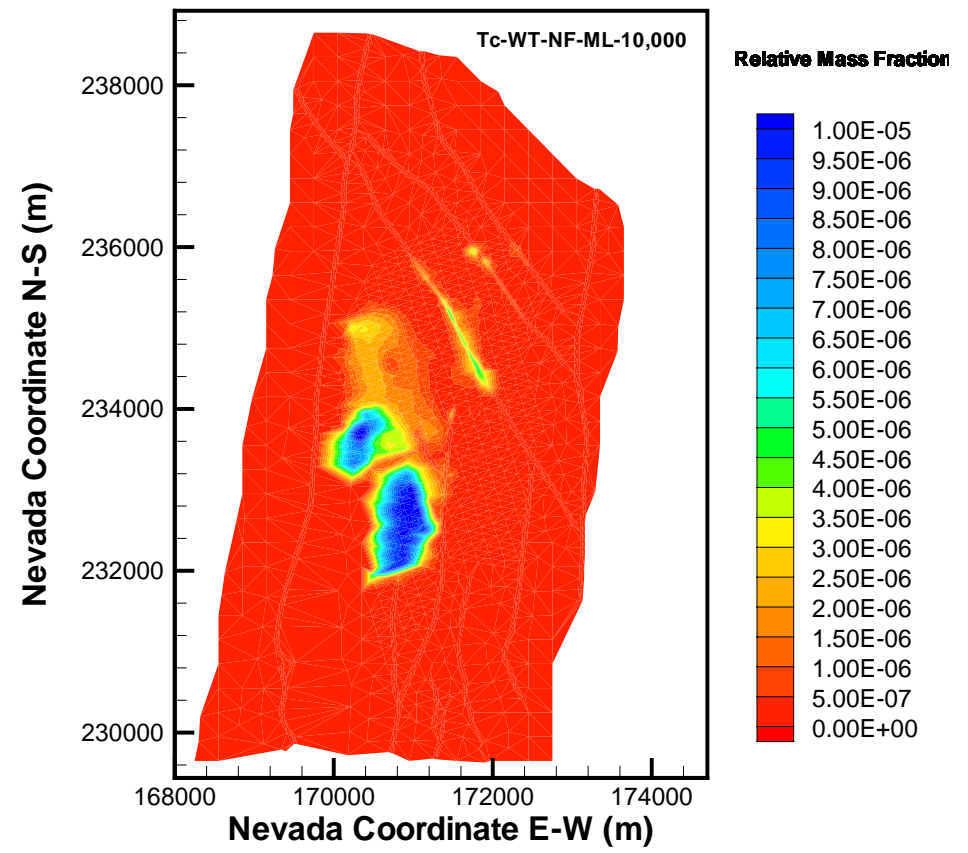

Output DTN: LB0307MR0060R1.006.

Figure $\mathrm{H}-18$. Distribution of the Relative Mass Fraction $\mathrm{X}_{\mathrm{R}}$ of ${ }^{99} \mathrm{Tc}$ in the Matrix Immediately above the Groundwater at $t$ $=10,000$ Years for Mean Present-Day Infiltration and Instantaneous Release Only from Nonfaulted Domain 


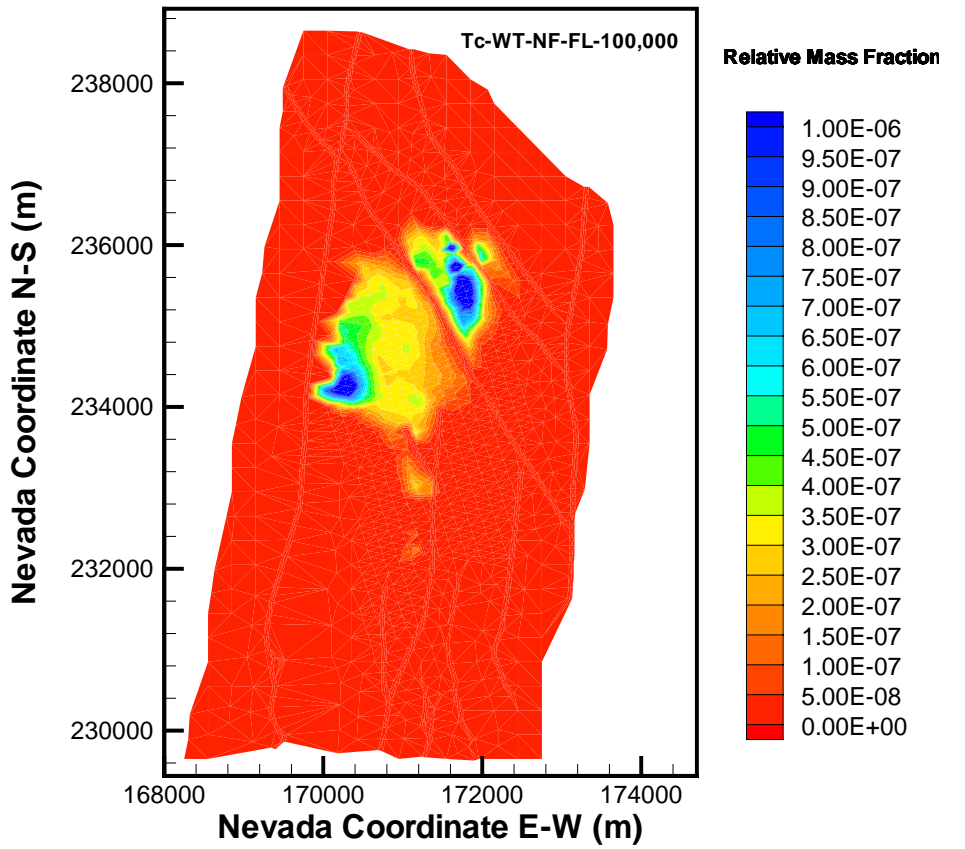

Output DTN: LB0307MR0060R1.006.

Figure $\mathrm{H}-19$. Distribution of the Relative Mass Fraction $\mathrm{X}_{\mathrm{R}}$ of ${ }^{99} \mathrm{TC}$ in the Fractures Immediately above the Groundwater at $t=100,000$ Years for Mean Present-Day Infiltration and Instantaneous Release Only from Nonfaulted Domain

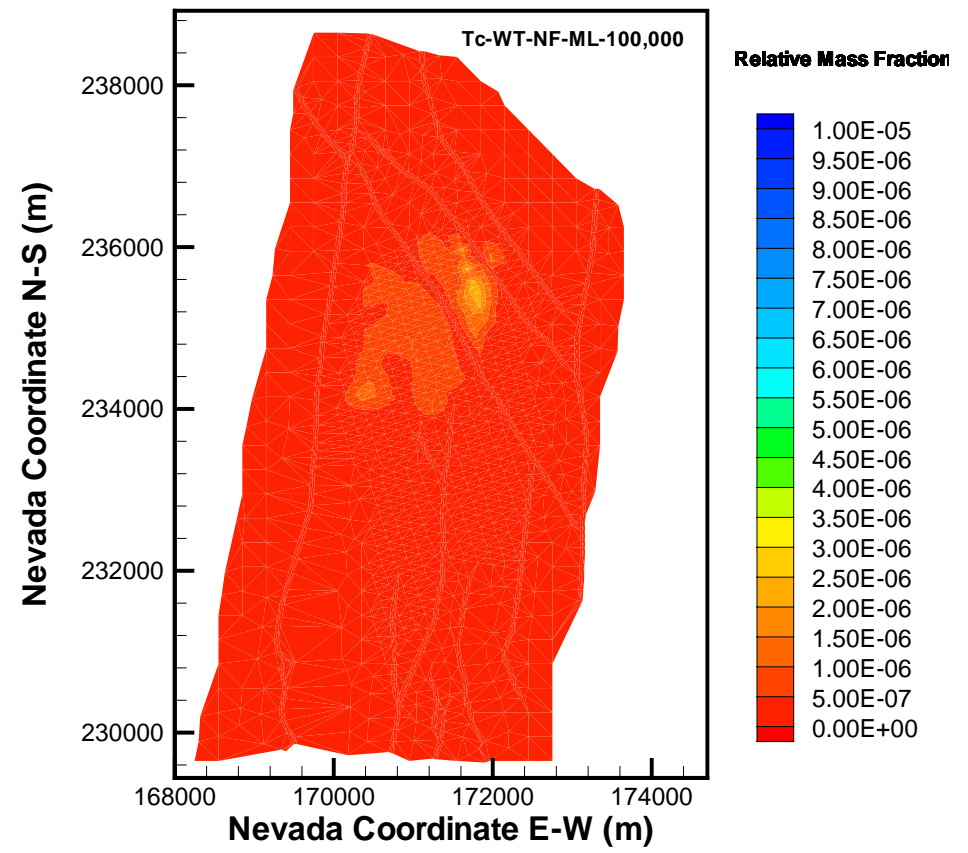

Output DTN: LB0307MR0060R1.006.

Figure $\mathrm{H}-20$. Distribution of the Relative Mass Fraction $X_{R}$ of ${ }^{99} \mathrm{Tc}$ in the Matrix Immediately above the Groundwater at $\mathrm{t}=$ 100,000 Years for Mean Present-Day Infiltration and Instantaneous Release Only from Nonfaulted Domain 


\section{APPENDIX I}

TEMPERATURE EFFECT ON RADIONUCLIDE SORPTION 


\section{I1. TEMPERATURE EFFECT ON RADIONUCLIDE SORPTION}

Potential variations with temperature in the value of the sorption (distribution) coefficient, $\mathrm{K}_{\mathrm{d}}$, for a given radionuclide with respect to a host rock substrate could be important when modeling radionuclide transport in the near field of the nuclear waste repository at Yucca Mountain, Nevada. The near field is considered that region around the repository subject to temperature perturbations caused by radioactive decay of the waste. At Yucca Mountain, radionuclide transport in the unsaturated zone is restricted above about $95^{\circ} \mathrm{C}$ by the absence of a mobile liquid aqueous phase. Therefore, the temperature range of interest would normally range between approximately 0 and $100^{\circ} \mathrm{C}$. Another potential reason for understanding variations in $\mathrm{K}_{\mathrm{d}}$ with temperature would be the need for making precise corrections in $\mathrm{K}_{\mathrm{d}}$ for temperature differences between laboratory experimental conditions and ambient conditions in the far field. However, as examined further below, such corrections would be small, and in any case, differences in physico-chemical conditions between laboratory and the field would probably introduce uncertainties larger than corrections for temperature.

\section{I1.1 CONCEPTUAL MODEL}

Radionuclide sorption involves the uptake of ionic or molecular species of that radionuclide (associated in most cases with nonradioactive isotopes of the same element) from an aqueous solution onto a solid phase. Sorption, $\mathrm{R}_{\mathrm{s}}$, is usually presented as the ratio of the radionuclide partitioned between the aqueous and solid phases, expressed as the percentage sorbed, thus,

$$
R_{s}=100\left(A_{i}-A_{f}\right) / A_{f}
$$

where $\mathrm{A}$ is measured in disintegration counts/second or $\mathrm{Bq}$, and the subscripts, $\mathrm{i}$ and $\mathrm{f}$, refer to the initial and final concentrations, respectively. Because a given concentration of a radioelement in solution is normally spiked with a known activity of one of its corresponding radionuclides, the final concentration of the radioelement in solution can easily be calculated. Thus, $A_{i} \propto C_{0}$, where $C_{o}$ is the initial concentration, usually given as the molarity, $\mathrm{mol} / \mathrm{mL}$, and $A_{i}-A_{f} \propto\left(C_{o}-C_{f}\right)$, equivalent to the moles sorbed on a given mass of solid. The ratio is then expressed as:

$$
\mathrm{R}_{\mathrm{d}}=\left(\mathrm{C}_{\mathrm{o}}-\mathrm{C}_{\mathrm{f}}\right) / \mathrm{C}_{\mathrm{f}} \cdot \mathrm{V} / \mathrm{m}(\mathrm{mL} / \mathrm{g})
$$

where $\mathrm{V}$ is the volume of solution in $\mathrm{mL}$, and $\mathrm{m}$ is the mass of sorbent in $\mathrm{g}$. Use of the term $\mathrm{Rd}$ implies that thermodynamic equilibrium has not necessarily been achieved. Upon achievement of equilibrium, $\mathrm{Rd}$ can be equated with $\mathrm{Kd}$, the sorption (or distribution) coefficient, which is also expressed in units of $\mathrm{mL} / \mathrm{g}$.

If measurements to determine $\mathrm{Kd}$ are made at different temperatures, then the dependence of $\mathrm{Kd}$ on temperature can be evaluated. This dependence has a thermodynamic basis, which is defined further under Section I1.3.2.

Simple models incorporating the Kd term have the advantage of economical data acquisition and convenient incorporation in hydrological computer codes. However, their ability to replicate radionuclide transport in the near field of a nuclear waste repository is less than optimal. Experience with the environmental remediation of contaminated aquifers has demonstrated that 
transport models incorporating $\mathrm{Kd}$ seriously underestimate the length of time required for cleanup and grossly overestimate the maximum concentration at the time of breakthrough when compared with a model incorporating site complexation (Bethke and Brady 2000 [DIRS 154437]). However, the objective of aquifer remediation is opposite to that of using a geologic formation as a natural barrier to radionuclide migration. In the former, the goal is to lower groundwater contamination permanently to some mandated standard acceptable for drinking water, whereas in the latter, the goal is to ensure that the radionuclide activity penetrating the geologic barrier never rises above specified limits. The use of $\mathrm{Kd}$ formulations to predict radionuclide transport is therefore inherently conservative, because it tends to over-predict the maximum concentration in solution upon breakthrough to the so-called "accessible environment" when compared with site complexation models for a given set of conditions. Thus, such modeling would be consistent with regulatory guidelines requiring conservatism in predicting radionuclide release, that is, at earlier times and at higher concentrations than would be predicted using more sophisticated site complexation models as illustrated by Bethke and Brady (2000 [DIRS 154437]).

In the following analysis, the temperature dependence of radionuclide sorption is based on an evaluation of empirically determined $\mathrm{Kd}$ values at discrete temperatures. This approach is taken for the following technical and pragmatic reasons:

- The application of a simple linear isotherm for a given radionuclide, that is, experimentally determined $\mathrm{K}_{\mathrm{d}}$ value, in computing radionuclide transport is inherently conservative in relation to more sophisticated site complexation models, because the breakthrough concentration to the accessible environment for a given set of conditions is over-predicted. Therefore, if an analysis using $\mathrm{K}_{d} \mathrm{~S}$ demonstrates acceptable confinement of radionuclides by the repository natural barriers, the use of linear isotherms can be justified as being adequate for their intended purpose.

- An initial examination of the available qualified (and unqualified) data pertaining to the sorption of radionuclides on tuff samples from Yucca Mountain and used in the following analysis did not indicate $a$ priori that the data were of sufficient quality or sufficiently detailed to permit their use in more sophisticated models. The primary reason is that the temperature intervals at which $\mathrm{K}_{\mathrm{d}}$ measurements were made are insufficient in number, and too widely spaced over the temperature interval of interest to permit identification of possible changes in the enthalpy of sorption with temperature (reflecting changes in sorption mechanism). Furthermore, unequivocal evidence of sorption reaction reversibility, necessary for any analysis where an equilibrium state is assumed, was not clearly demonstrated.

- Time constraints were such that no more than an exploratory evaluation could be conducted in which the adequacy and limitations of the temperature dependence of the linear isotherm could be assessed for those radionuclides for which data were available.

- Section 5 of this report assumes the validity of the linear isotherm for modeling radionuclide transport in matrix and fractures of the unsaturated zone at Yucca Mountain. The evaluation conducted in this appendix is, therefore, consistent with that assumption in Section 5 of this report. 


\section{I1.2 ALTERNATIVE CONCEPTUAL MODELS}

Models describing sorption range from the most primitive, for example that represented by the $\mathrm{K}_{\mathrm{d}}$ term discussed above, to complex multisite surface complexation models. The choice of model for a particular application depends on the nature of the application and the degree of accuracy required. In the following paragraphs, some alternative models are briefly described that could be used, with potential improvements in the accuracy of predictive radionuclide transport simulations.

In Section I1.1, it is implicitly assumed that a single $\mathrm{K}_{\mathrm{d}}$ value is constant for all radioelement concentrations at a given temperature. This assumption is based on an analysis of an isothermal series of measurements with differing initial aqueous concentrations of a given sorbent, sometimes over many decades of concentration. The resulting logarithms of the sorbed concentration can be plotted in relation to the corresponding logarithms of the equilibrium concentration in solution. Various equations expressing this relationship have been developed, and are referred to as isotherms. The most commonly assumed relationship is the linear isotherm:

$$
\log C_{e}=\log Q+k
$$

where $\mathrm{C}_{\mathrm{e}}$ is the equilibrium concentration of the sorbent in mol/L, usually equivalent to $\mathrm{C}_{\mathrm{f}}$, and $\mathrm{Q}$ is the amount adsorbed in mol/g. Rearranging Equation I-3 yields $\log \left(\mathrm{C}_{\mathrm{e}} / \mathrm{Q}\right)=\mathrm{k}$ and $\mathrm{k}=\log$ $\mathrm{K}_{\mathrm{d}}$. This relationship is the basis for applications using $\mathrm{K}_{\mathrm{d}}$ values in determinations of radionuclide transport at Yucca Mountain, as discussed above. In practice, experimentally determined isotherms are better fitted to more complex terms, the most common being the Freundlich isotherm (Freundlich 1926 [DIRS 165848]):

$$
\log \mathrm{C}_{\mathrm{e}}=\mathrm{nLog} \mathrm{Q}+\mathrm{k}
$$

where $\mathrm{n}$ and $\mathrm{k}$ are fitting parameters, and the Langmuir isotherm is (Langmuir 1918 [DIRS 126305]):

$$
\mathrm{C}_{\mathrm{e}} / \mathrm{Q}=1 /\left(\mathrm{BC}_{\mathrm{m}}\right)+\left(1 / \mathrm{C}_{\mathrm{m}}\right) \cdot \mathrm{C}_{\mathrm{e}}
$$

where $\mathrm{C}_{\mathrm{m}}$ is the sorption capacity in $\mathrm{mol} / \mathrm{g}$, and $\mathrm{B}$ is a constant related to the heat of sorption. The applicability of each isotherm varies with the nature of the sorption process under investigation. It can be associated with specific sorption mechanisms, although model fitting is usually empirical. The nature of the sorption process, whether chemical or physical adsorption, ion exchange, or even precipitation, is commonly ignored. Thus, the term "sorption" is used in this model report rather than adsorption, unless the process has been specifically identified as the latter. The Freundlich isotherm, in particular, has enjoyed a large measure of success in fitting sorption data, and was long thought to have no theoretical basis. However, Sposito (1980 [DIRS 127235]) determined that the Freundlich isotherm reflects the competitive adsorption of a tracer species in the presence of another species at much higher concentration. The theoretical basis for the Langmuir isotherm involves adsorption of a sorbent monolayer. 
Other isotherms also have been formulated, particularly in relation to separation technology, such as the adsorption of gaseous and aqueous organic and inorganic species on activated carbon, or the adsorption of fatty acids on mineral surfaces for the purpose of selective flotation. These isotherms, such as the Flory-Huggins, Frumkin, and Dubinin-Radushkevich (D-R) equations (Yehia et al. 1993 [DIRS 164922]; Atun et al. 1996 [DIRS 164865]; Sabah et al. 2002 [DIRS 164909]; Kara et al. 2003 [DIRS 164880]) could be potentially useful in describing radioelement adsorption on Yucca Mountain host rock minerals. Evaluation as to their suitability for that purpose is, however, beyond the scope of this model report. Finally, it should be noted that composite sorption models, invoking additive combinations of various sorption isotherms to describe sorption behavior, have been applied to describe adequately some experimental data (e.g., see Weber et al. 1992 [DIRS 165228]).

The applicability of various isotherms is restricted to the experimental conditions under which they are tested. Models that permit predictions of sorptive behavior over a range of conditions, taking into account the effect of varying $\mathrm{pH}$, competition between aqueous species, variations in ionic strength, and multiple adsorption sites, require much more sophisticated models. To address this problem, a variety of surface complexation models have been developed. These models require the precise measurement of more parameters and a more rigorous definition of the conditions to be modeled than isotherm models. The basis for these models is recognition that mineral surfaces are invariably electrically charged, the charge arising from unsatisfied valences caused by the discontinuity in a crystal structure, or from polarization of molecules or atoms at the interface. Minerals in sediments and soils are usually negatively charged. The charge, which is intrinsic to the mineral, is referred to as a permanent structural charge. In the aqueous phase, this charge is partially satisfied by a tightly coordinated layer of so-called potential determining ions, which bind to the exposed functional groups on the mineral. The surface charge resulting from this coordinated layer of ions may be either negative or positive. To preserve electrical neutrality, a diffuse layer of counter-ions must accumulate in the aqueous phase adjacent to the surface. The resulting interfacial structure containing the surface layer with its attendant tightly coordinated ions and the diffuse layer of counter-ions is referred to as the electrical double layer (EDL). The thickness of the EDL varies with ionic strength of the aqueous phase. The distribution of ionic species in the coordinated and diffuse layers is some function of the surface properties of the mineral and the activities of competing solutes in the aqueous phase.

To predict quantitatively the adsorption of a given solute, an electrostatic model is required that reproduces the behavior of the electrical double layer in relation to the solute species. The history of the development of surface complexation models incorporating the EDL spans a period of more than 80 years. Bolt (1991 [DIRS 165056]), Bolt and Van Riemsdijk (1991 [DIRS 165188]), Sposito (1984 [DIRS 127253]), Westall (1987 [DIRS 127323]), and Davis and Kent (1990 [DIRS 143280]) give historical reviews. Presently, three such surface complexation models have found widespread use, and are referred to respectively as the "constant capacitance," "double layer," and "triple layer" models. Detailed discussion of their formulation and theoretical basis can be found in the above-cited references.

Despite the promise of such models in predicting the adsorption behavior of aqueous species on mineral surfaces, they have serious limitations. The large majority of studies to quantify the surface complexation constants of aqueous species have been conducted on a limited set of 
substrates, such as "hydrous ferric oxide," $\alpha$-alumina, aluminum hydroxide (usually gibbsite), and amorphous silica. Studies involving minerals of relevance to radioelement transport in soils and rocks have been undertaken only to a limited extent. Clays, zeolites, and carbonates, for example, have not been studied in relation to their surface complexation properties in any detail. Furthermore, even had the surface complexation properties of the mineral been investigated, it is not feasible to use measurements on individual minerals to predict the sorption behavior of mineral aggregates such as soils or rocks.

The formulation of a surface complexation model assumes experimental characterization of the adsorbent properties under conditions in which the substrate is a dilute suspension in the aqueous phase. However, with increasing concentration of the suspension, particles come into contact with each other and modify the apparent surface properties of the adsorbent. Still greater effects have been observed when two different adsorbents are mixed. It would have been advantageous if the sorption properties of an aggregate of different adsorbents had been additive, obeying the so-called linear adsorptivity model. However, experimental studies reported by Honeyman (1984 [DIRS 164878]) reveal that major nonlinear effects on adsorption are sometimes observed with mixtures. The linear adsorptivity model may therefore be an exception rather than the rule.

To overcome the practical limitations of surface complexation models, Davis et al. (1998 [DIRS 154436]) formulated a semi-empirical version of a site complexation model with limited parameterization, which represents a considerable advance over empirical isotherm formulations. Their approach could, with further refinement, finally realize a suitable model for predicting species adsorption under field conditions.

\section{I1.3 MATHEMATICAL MODEL}

\section{I1.3.1 Approximations and Simplifications}

The advantage in assuming a linear isotherm for a given radioelement lies in its simplicity of measurement, and in the ease with which the term can be incorporated into hydrological models, where it is assumed that all other geochemical parameters except the concentration of the sorbent remain invariant. Under these conditions, experimentally determined $\mathrm{K}_{\mathrm{d}}$ values from the laboratory can be used for approximate predictions of radionuclide migration, provided that the host rocks have identical composition and properties per unit mass, and thus temperature and aqueous phase composition are identical and remain invariant. Without such constraints, predictions of radionuclide transport in the natural environment will be subject to large and unpredictable errors (Reardon 1981 [DIRS 154434]). Table I-1 modified after Apps et al. (1977 [DIRS 164864]) and Apps (1992 [DIRS 165225]), provides a very general estimate of potential uncertainties introduced through the application of experimental laboratory $\mathrm{K}_{\mathrm{d}}$ values to radionuclide sorption in the field. The values given are only estimates, actual deviations being almost impossible to quantify. 
Table I-1. Factors Influencing $K_{d}$ for an Adsorbent, Based on Conditions Expected in the Subsurface

\begin{tabular}{|c|c|c|c|}
\hline Parameters & Principal Effect & Field Conditions & Potential Variation in $K_{d}$ \\
\hline \multicolumn{4}{|l|}{ 1. Solution Chemistry } \\
\hline a. Major components & lonic strength, complexing & $\begin{array}{l}\text { Determined by host rock chemistry and by } \\
\text { other factors including the leaching } \\
\text { chemistry of the waste product. }\end{array}$ & Difficult to predict, probably $10^{+3}$ \\
\hline b. Minor components & Complexing & Same as above & Same as above \\
\hline c. $\mathrm{pH}$ & Complexing, hydrolysis & $\begin{array}{l}\text { 5.0-8.5 Buffering by heterogeneous and } \\
\text { homogeneous reactions keep the } \mathrm{pH} \text { range } \\
\text { within narrow limits. }\end{array}$ & Up to $10^{+4}$ \\
\hline d. Eh & Change in redox state & $\begin{array}{l}\text { Variable, over a narrow range, usually } \\
\text { reducing. }\end{array}$ & $\begin{array}{l}\text { Up to } 10^{10} \text { or even more. Effect likely to be } \\
\text { small in Yucca Mountain UZ. Localized } \\
\text { effects near the waste containers could be } \\
\text { significant. }\end{array}$ \\
\hline $\begin{array}{l}\text { 2. Radioelement } \\
\text { Concentration }\end{array}$ & $\begin{array}{l}\text { Supersaturation, polymerization, } \\
\text { colloid formation, metastable } \\
\text { equilibrium. }\end{array}$ & $\begin{array}{l}\text { Very variable concentration. Could range } \\
\text { from } 0 \text { to } 10,000 \mathrm{mg} / \mathrm{L}\end{array}$ & $\begin{array}{l}\text { Difficult to estimate, but could be very large for } \\
\text { inorganic amphoteric species near the } \\
\text { isoelectric point (approx. } 10^{6} \text { ). }\end{array}$ \\
\hline 3. Flow rate & $\begin{array}{l}\text { Impact on transport rate, metastable } \\
\text { equilibrium, colloid transport. } \\
\text { Changes in apparent surface area } \\
\text { contacted. }\end{array}$ & $10^{-3}$ to $10^{-7} \mathrm{~cm} / \mathrm{s}$ & $\begin{array}{l}\text { Slow flow rates could lead to different rate } \\
\text { controlling transport mechanisms (e.g. ionic or } \\
\text { molecular diffusion) and lead to different } \\
\text { thermodynamic controls }\left(0 \text { to } 10^{6}\right)\end{array}$ \\
\hline 4. Permeability & Flow rate (see above) & $1-10^{-5}$ millidarcy & Same as above \\
\hline 5. Surface area & Adsorption & $\begin{array}{l}\text { Approx. } 10^{-2} \mathrm{~cm}^{2} / \mathrm{g} \text { Fractures, } \\
\text { microfractures, intergranular pores. }\end{array}$ & Approx. $10^{3}$ \\
\hline 6. Temperature & Complexing, solubility, adsorption & 25 to $95^{\circ} \mathrm{C}$ & Up to $10^{1}$ \\
\hline
\end{tabular}

Source: Modified after Apps et al. 1977 (DIRS 164864); Apps 1992 (DIRS 165225).

UZ=unsaturated zone 
The presumption is also made that the $\mathrm{K}_{\mathrm{d}}$ measurements reflect a process that has achieved stable or metastable thermodynamic equilibrium. In many studies, scant consideration is given to the question of reversibility, although some measurements by Los Alamos National Laboratory (LANL) staff took into account both sorption and desorption (Daniels et al. 1982 [DIRS 105803]; Thomas 1987 [DIRS 101361]). If a temporal variation in $\mathrm{K}_{\mathrm{d}}$ is observed, and no basis exists for temporal extrapolation to an equilibrium state, then no correlation between a $\mathrm{K}_{\mathrm{d}}$ measurement and a thermodynamic model can be established. Nonattainment of equilibrium invalidates any true or quasi-thermodynamic treatment for the temperature dependence of $\mathrm{K}_{\mathrm{d}}$ measurements given in Section I1.3.2 below.

The nonattainment of thermodynamic equilibrium can be attributed to many factors, depending on the nature of the sample and its preparation. Crushed tuff samples, for example, could be actively dissolving, with concurrent precipitation of secondary products that entrain and coprecipitate radionuclides. Diffusional processes could also be active, either through intergranular pores or through "volume" diffusion or ion exchange, including diffusion through altered surface layers. In the presence of organic matter, field evidence suggests that long-term structural reorganization can lead to so-called "irreversible" adsorption, indicating that kinetically controlled processes are at play.

Measurements are commonly made on materials where several sorption mechanisms might be operative, either concurrently or over different time domains. Because $\mathrm{K}_{\mathrm{d}}$ determinations do not discriminate as to the mechanism of adsorption, ion-exchange processes in clays and zeolites can be confounded with adsorption on the exterior crystal faces. The thermodynamic treatment of ion exchange processes is entirely different from that of adsorption on surfaces. Many ion exchange models have been formulated and are described in the literature (Sposito 1981 [DIRS 129861]). If a $K_{d}$ determination has been conducted on a zeolitized vitroclastic tuff, such as one from the Calico Hills formation at Yucca Mountain, none of the foregoing arguments pertaining to corrections for surface area or the formulation of EDL models involving site surface complexation is relevant. With such measurements, both surface adsorption and ion exchange may be acting interdependently, requiring the formulation of mixed process models (Meyer et al. 1990 [DIRS 165040]).

The surfaces of different minerals also have different adsorptive properties for different sorbents. Thus, for example, $\mathrm{Np}$ adsorbs weakly on silicate surfaces, but quite strongly on iron oxides and carbonates under field conditions. At Yucca Mountain, both iron oxides and carbonates are present in the silicic vitroclastics in only minor quantities. Yet they could have a significant impact on the sorptive properties of the rock as a whole ${ }^{1}$. Although a doubling of the concentration of either mineral would have only a minor impact on the bulk mineralogy and composition of the host rock, the sorptivity of $\mathrm{Np}$ could increase substantially. In such situations, the representativeness of the sample chosen for experimental determination of $\mathrm{Np} \mathrm{K}_{\mathrm{d}}$ values becomes critical. However, the selection of isolated intervals of drill core for testing calls into question whether a representative sampling of rocks that could host potential transport paths at Yucca Mountain has been achieved.

\footnotetext{
${ }^{1}$ The evaluation in this model report of the temperature dependence of neptunium sorption on two samples of Yucca Mountain Tuff, and on natural calcite and synthetic hematite (Fig 6.4-1d) suggests that neptunium sorption behavior on tuffs is dominated by the presence of small quantities of hematite.
} 
Another problem concerns the actual characterization of the mineral surface in relation to those surfaces encountered in the field. Both surface complexation and $\mathrm{K}_{\mathrm{d}}$ characterization studies commonly are conducted on materials that have been synthesized, chemically treated, or subjected to grinding or comminution. Such preparatory work can result in exposed mineral surfaces that bear little relation to surfaces exposed under field conditions. Furthermore, it is well established that groundwaters exposed to rocks weathering under near-surface conditions tend to attain a quasi equilibrium state with a metastable hydrated aluminosilicate phase of uncertain composition and structure (Paces 1978 [DIRS 164891]). The field evidence suggests that this phase coats aluminosilicate minerals.

Mineral surfaces in soils and shallow sediments also respond to adsorbents in quite different ways, depending on the presence of swelling clays (i.e., smectites) and the quantity of organic material present - for example, see "Organic Pollutant Sorption in Aquatic Systems." Journal of Hydraulic Engineering (Karickhoff 1984 [DIRS 164881]) regarding the sorption of biquinoline and pyrene. Soil scientists believe that the organic content of soils and sediments is present as a coating on mineral grains forming the matrix of the soil or sediment. The concentration of this organic coating is in direct proportion to the specific surface area of the soil and suggests the presence of a "biofilm." There is circumstantial evidence that a biofilm may be present throughout the vadose zone at Yucca Mountain (Kieft et al. 1997 [DIRS 100767]). However, the organic content is sufficiently low such that the mineral surfaces are probably exposed to the adsorbate with little hindrance from a biofilm.

The organic coating responds quite differently to sorbing species than does the uncoated mineral surface; consequently, a bimodal sorptive behavior is sometimes observed. It has become common practice among soil scientists, therefore, to consider sorption by soils and sediments to be a function of both the organic coating and the mineral surface. Neither surface complexation models nor laboratory $\mathrm{K}_{\mathrm{d}}$ measurements would be relevant to soil and vadose zones unless the significance of these film coatings was evaluated.

\section{I1.3.2 Thermodynamic Basis for $K_{d}$ Determinations}

The gross feature of sorption, as reflected by empirical $\mathrm{K}_{\mathrm{d}}$ determinations, can be treated thermodynamically, as with any other equilibrium process, provided that the limitations cited in Section I1.3.1 are recognized and taken into account. Consider any chemical reaction involving surface sorption:

$$
S-(\mathrm{s})+i(\mathrm{aq})=S-i(\mathrm{~s})
$$

Where $S$-(s) is the solid, $i(\mathrm{aq})$ is the sorbent, and $S$ - $i(\mathrm{~s})$ is the sorbed species. Here, $S$-(s) and $S-i(s)$ are expressed as mole fractions, normalized to that surface occupied by one mole of sorbent at full site occupancy, and $i(\mathrm{aq})$ is in $\mathrm{mol} /(\mathrm{kg}$ solvent). The Gibbs free energy of the reaction is

$$
\Delta \mathrm{G}_{\mathrm{r}}=\Delta \mathrm{G}_{\mathrm{r}}^{\mathrm{o}}+\mathrm{RT} \ln \mathrm{K}_{\mathrm{r}}
$$


At equilibrium, $\Delta \mathrm{G}_{\mathrm{r}}=0$, and

$$
\Delta \mathrm{G}_{\mathrm{r}}^{\mathrm{o}}=-\mathrm{RT} \ln \mathrm{K}_{\mathrm{r}}
$$

where $\mathrm{K}_{\mathrm{r}}=[S-i(\mathrm{~s})] /\{[S-(\mathrm{s})][i(\mathrm{aq})]\}$, the brackets representing the activities of the participating species.

$\mathrm{K}_{\mathrm{r}}$ and the molal activities are all dimensionless. In terms of measurable concentrations:

$$
\mathrm{K}_{\mathrm{r}}=\lambda_{S-i(\mathrm{~s})} \mathrm{X}_{S-i(\mathrm{~s})} /\left\{\lambda_{S-(\mathrm{s})} \mathrm{X}_{S-(\mathrm{s})} \cdot \gamma_{i(\mathrm{aq})} m_{i(\mathrm{aq})}\right\}
$$

where $\mathrm{X}$ stands for mole fraction, $\mathrm{m}$ for molality, and $\lambda$ and $\gamma$ are the activity coefficients of the solid and aqueous species, respectively. The relationship between the empirical $\mathrm{K}_{\mathrm{d}}$ measurement and $\mathrm{K}_{\mathrm{r}}$ is complex. It may be expressed as:

$$
\mathrm{K}_{\mathrm{r}}=f\left(x_{1}, x_{2}, x_{3}, \ldots, x_{n}\right) \mathrm{K}_{\mathrm{d}}
$$

where $f\left(x_{1}, x_{2}, x_{3}, \ldots, x_{n}\right)$ are correction functions required to reconcile the experimental $\mathrm{K}_{\mathrm{d}}$ with the thermodynamic $\mathrm{K}_{\mathrm{r}}$. Thus, $\mathrm{K}_{\mathrm{r}}$ is dimensionless, in contrast to the conventional $\mathrm{K}_{\mathrm{d}}$ value, which has the dimensions of $[\mathrm{L}]^{3} /[\mathrm{M}]$, expressed as $\mathrm{mL} / \mathrm{g}$. $\mathrm{K}_{\mathrm{d}}$ can be corrected to the dimensionless form by converting the measured aqueous concentration from molarity (mol/L) to molality $(\mathrm{mol} / \mathrm{kg})$, which involves the density term, $\rho(\mathrm{aq}),\left([\mathrm{M}] /[\mathrm{L}]^{3}\right)$. Thus,

$$
\mathrm{K}_{\mathrm{r}} \propto \mathrm{K}_{\mathrm{d}} \cdot \rho(\mathrm{aq})
$$

In making $\mathrm{K}_{\mathrm{d}}$ determinations, the nature of the sorption reaction is normally ignored, and the specification of a reactive surface area is not considered. However, normalization of measurements to a fixed reactive surface area can be accomplished through incorporation of a dimensionless coefficient, $\sigma$, expressed as the ratio of the measured surface area per unit mass of sorbent, divided by a specified standard surface area per unit mass of sorbent. Taking this correction into account yields:

$$
\mathrm{K}_{\mathrm{r}} \propto \mathrm{K}_{\mathrm{d}} \cdot \sigma
$$

$\sigma$ is not easily defined, because it depends on the method of measuring surface area in relation to the adsorption of a given sorbent, which can vary with mineral substrate and other factors affecting its interaction with the liquid aqueous phase. A common method is the Brunauer, Emmett, Teller (B.E.T.) method (Brunauer et al. 1938 [DIRS 156646]) in which a gaseous species, usually nitrogen, is allowed to adsorb on the sorbate under evacuated conditions. This method, however, does not replicate conditions in which the reactive surface area of a mineral is in contact with the aqueous phase. Nevertheless, Pabalan et al. (1998 [DIRS 162987]) determined that quartz, alpha-alumina, clinoptilolite, montmorillonite, amorphous silica, kaolinite, and hydrous titanium dioxide all adsorbed the uranyl ion, $\mathrm{UO}_{2}{ }^{2+}$, to the same extent when the $K_{d}$ values for each mineral were corrected for their respective B.E.T. surface areas. This observation suggests a consistency between B.E.T. determinations and adsorption by aqueous species, which do not participate in ion-exchange processes in clays or zeolites. 
Computer codes such as EQ3/6 (Wolery 1983 [DIRS 108808]; Wolery and Daveler 1992 [DIRS 100097]), PHREEQC (Parkhurst and Appelo 1999 [DIRS 159511]) or SOLVEQ/CHILLER (Reed 1982 [DIRS 117901]; Spycher and Reed 1992 [DIRS 165846]) can be used to estimate $\gamma$, the species activity.

Correction for the surface activity coefficients, $\lambda$, is more difficult and implies some knowledge of the thermodynamic behavior of the sorption sites in competition with other species, as well as the nature of the sorption process itself. Parks (1990 [DIRS 165849]) points out that there are two broad classes of adsorbate, each distinguished by characteristic behavior.

1. Sorption is nonspecific: There is negligible adsorption at the point of zero net proton charge and isoelectric point, and both are coincident, suggesting that adsorption occurs primarily through coulombic attraction to an oppositely charged surface. Adsorption is assumed to take place through weak interactions, and the adsorbed ions remain solvated. The surface structure also remains essentially unaltered. Because no true chemical bond is created, the term "physical adsorption" is sometimes applied.

2. Sorption is specific: This class of sorption is characterized by reversal of charge at the surface, and adsorption can occur even though the surface charge and adsorbate charge are of the same sign. Such adsorption must be noncoulombic in character, suggesting that chemical bonding takes place. Evidence indicates that the adsorbate bonds close to the surface as an inner sphere complex, with disruption of both the solvation sheath around the sorbing ion and the surface functional groups. The term "chemical bonding" is sometimes applied to this form of adsorption.

\section{I1.3.3 Representation of $K_{d}$ Measurements as a Function of Temperature}

The temperature dependence of $\mathrm{K}_{\mathrm{r}}$ can be established by invoking the thermodynamic relation:

$$
\Delta \mathrm{G}_{\mathrm{r}}(\mathrm{P}, \mathrm{T})=\Delta \mathrm{H}_{\mathrm{r}}(\mathrm{P}, \mathrm{T})-\mathrm{T} \Delta \mathrm{S}_{\mathrm{r}}(\mathrm{P}, \mathrm{T})
$$

Assuming equilibrium, substituting Equation I-8 into Equation I-12, and converting to base 10 logarithms, yields:

$$
\log \mathrm{K}_{\mathrm{r}}=\Delta \mathrm{S}_{\mathrm{r}} / 2.303 \mathrm{R}-\left[\Delta \mathrm{H}_{\mathrm{r}} / 2.303 \mathrm{R}\right](1 / \mathrm{T})
$$

Over small temperature intervals, such as between 0 and $100^{\circ} \mathrm{C}$, it can be assumed that the heat capacity of reaction, $\Delta \mathrm{C}_{\mathrm{P}, \mathrm{r}} \approx 0$, which means that $\Delta \mathrm{H}_{\mathrm{r}}$ and $\Delta \mathrm{S}_{\mathrm{r}}$ are constant over the specified temperature interval. Thus, Equation I-13a is a good approximation of the variation of $\log \mathrm{K}_{\mathrm{r}}$ with temperature ${ }^{2}$. A plot of $\log \mathrm{K}_{\mathrm{r}}$ data versus $1 / \mathrm{T}$ would yield, upon linear regression, a slope

\footnotetext{
${ }^{2}$ In reality, measurement of the heat capacity of sorption would be difficult. Relevant experimental data for this parameter was searched for in the literature, but no useful information was located. Precise $\mathrm{K}_{\mathrm{d}}$ measurements as a function of temperature so far examined in the published literature indicate that the assumption, $\Delta \mathrm{C}_{\mathrm{P}, \mathrm{r}} \approx 0$, would be a valid approximation. The approximation is consistent with the small variations in $\Delta \mathrm{S}_{\mathrm{r}}$ for $\mathrm{Co}$ (II) sorption on sepiolite at different temperatures reported by Kara et al. (2003 [DIRS 164880]), and similar data for Cs(I) on KCNF (Ishfaq et al. 1997 [DIRS 164879]) and Ag(I) on bentonite (Zafar et al. 2002 [DIRS 165052]). [See a summary of these data in Table 6.4-10]. In general, the quality of the data is insufficient to justify other than a linear approximation.
} 
of $-\Delta \mathrm{H}_{\mathrm{r}} / 2.303 \mathrm{R}$ and a value of $\Delta \mathrm{S}_{\mathrm{r}} / 2.303 \mathrm{R}$ on the $\mathrm{y}$-axis where $(1 / \mathrm{T})=0$. Depending on the nature of the sorption process, $\Delta \mathrm{H}^{\mathrm{o}}{ }_{\mathrm{r}}$ can be either negative or positive, leading respectively to either decreasing or increasing sorption with temperature.

No attempt is made in this model report to consider either corrections to $K_{d}$ measurements at ambient temperature or the effect of such corrections on $\Delta \mathrm{H}^{\mathrm{o}}{ }_{\mathrm{r}}$. While such corrections could be made, or at least estimated in some cases, the effort involved is scarcely commensurate with the resulting increase in purported accuracy, given the known degree of uncertainty in existing measurements. Instead, the assumption is that corrections will have a small effect on $\Delta \mathrm{H}_{\mathrm{r}}$, and that corrections will predominantly affect $\log \mathrm{K}_{\mathrm{d}}$ and the apparent value of $\Delta \mathrm{S}$. Therefore, the assumption is that, by substituting Equation I-10 into Equation I-13a, the equation is

$$
\log \mathrm{K}_{\mathrm{d}}+\mathrm{k}^{\prime}=\Delta \mathrm{S}_{\mathrm{r}} / 2.303 \mathrm{R}-\left[\Delta \mathrm{H}_{\mathrm{r}} / 2.303 \mathrm{R}\right](1 / \mathrm{T})
$$

where $\mathrm{k}^{\prime}$ is $\log \left[f\left(x_{1}, x_{2}, x_{3}, \ldots, x_{n}\right)\right]$. Included in these corrections is that for correcting $\mathrm{K}_{\mathrm{d}}[\mathrm{mL} / \mathrm{g}]$ to $\mathrm{K}_{\mathrm{r}}[-]$, the dimensionless form. The corrections embedded in this parameter would result in differing intercepts of the $\mathrm{y}$ axis for differing experimental conditions for a given aqueous species sorbing on a given solid phase. However, the slope of Equation I-13b, $-\Delta \mathrm{H}_{\mathrm{r}} / 2.303 \mathrm{R}$, will remain essentially unchanged. Because of the practical difficulty in calculating $\mathrm{k}^{\prime}$, it will be ignored, and the uncorrected $\mathrm{K}_{\mathrm{d}}$ will be plotted versus $1 / \mathrm{T}\left[\mathrm{K}^{-1}\right]$.

The temperature dependence of $\mathrm{K}_{\mathrm{d}}$ is somewhat more difficult to evaluate accurately. If the $\mathrm{K}_{\mathrm{d}}$ measurements have demonstrated reversibility, as is the case with some measurements conducted under the auspices of the U.S. Department of Energy Yucca Mountain Project at Los Alamos National Laboratory (LANL) (Daniels et al. 1982 [DIRS 105803]; Thomas 1987 [DIRS 101361]), a thermo-dynamic basis for the sorption process can be tentatively accepted, and the relationship between $\mathrm{K}_{\mathrm{r}}$ and $\mathrm{K}_{\mathrm{d}}$ accepted, as specified in Equation I-10. The question as to how to treat $f\left(x_{1}, x_{2}, x_{3}, \ldots . x_{n}\right)$ remains. Many of the corrections necessary to correlate differing $\mathrm{K}_{\mathrm{d}}$ measurements would affect both the Gibbs free energy, $\Delta \mathrm{G}_{\mathrm{r}}{ }_{\mathrm{r}}$, and the enthalpy of sorption, $\Delta \mathrm{H}_{\mathrm{r}}^{\mathrm{o}}$. However, if such corrections were to be temperature independent, they would merely change the y intercept of a $\log \mathrm{K}_{d}$ versus $1 / \mathrm{T}$ plot without affecting the slope. Most of the cited parameters required to correlate $K_{d} s$ are functionally dependent on temperature, and as a consequence, the enthalpy of sorption will vary depending on the sorption model used, the concentration of the sorbent in solution, the degree of complexation, oxidation state, and so on. These variations are illustrated in Table I-10 by data presented by Kara et al. (2003 [DIRS 164880]), where Co(II) sorption on sepiolite at different temperatures is interpreted using four different isotherms, each yielding quite different values of $\Delta \mathrm{H}_{\mathrm{r}}$. Similarly, in Table I-10, Rauf and Tahir (2000 [DIRS 164893]) show an aqueous concentration dependence of $\Delta \mathrm{H}_{\mathrm{r}}$ for both $\mathrm{Fe}(\mathrm{II})$ and $\mathrm{Mn}(\mathrm{II})$ sorption on bentonite. This functional dependence probably reflects uncorrected effects of $\lambda_{i}$ and $\gamma_{i}$ respectively. Because the temperature dependence of most $\mathrm{K}_{\mathrm{d}}$ measurements is small in many cases, such corrections, if they could be applied, might easily result in a change from negative to positive temperature dependence or vice versa, as will be illustrated below with examples from the published literature. 


\section{I1.4 EVALUATION OF THE TEMPERATURE DEPENDENCE OF SELECTED KD DATA}

Temperature dependent $\mathrm{K}_{\mathrm{d}}$ data evaluated in this section are derived from three sources. The first two sources represent "qualified" (Sections 4.1 of this report and Section I1.4.1) and "unqualified" (Section I1.4.2) measurements, respectively, conducted LANL over the last 20 or more years. The third source consists of data from the refereed literature (Section I1.4.3). These three sources of data are evaluated below in three separate subsections. The purpose in assembling the three cited sources of information is first, to augment the otherwise relatively sparse qualified temperature dependent $\mathrm{K}_{\mathrm{d}}$ measurements with similar quality, but unqualified, measurements to provide a better statistical basis for the reported results of the analysis of this evaluation, and, second, to allow for a corroboration of the results using independent information obtained from the literature.

The LANL measurements were conducted primarily on natural rock samples obtained from Yucca Mountain and its vicinity at the U.S. Nevada Test Site, and on various synthetic and natural mineral samples. The rock samples were taken from drill cores penetrating the volcaniclastic stratigraphy of Yucca Mountain, and almost all were tuffaceous, including vitrophyre, devitrified welded tuff, and altered nonwelded (zeolitized) tuff. Experiments conducted on these samples employed intact core, or crushed and sized material in batch or column percolation studies. The tests were conducted using radionuclide tracers of the elements Sr, Cs, Ba, Ce, Eu, Np(V), Am, U(VI), and Pu in J-13 well water. A listing of the rock samples used, and radioelements investigated for their sorption properties, are given in Table I- 2 . The mineralogical composition of these rocks is presented in Table I-3.

The literature data were obtained from six independent literature searches on Chemical Abstracts of the American Chemical Society, using various combinations of key words involving sorption of inorganic constituents, including radionuclides on natural mineral substrates in the aqueous phase. The results of these searches were further screened for relevance and a subset of abstracts was selected for printing. The abstracts were reviewed, and a final selection of papers was copied from locally available journals in libraries of the University of California, Berkeley Campus and Lawrence Berkeley National Laboratory, or downloaded electronically. 
Table I-2. Rock Samples Used for Radioelement Sorption and Radioelements Sorbed

\begin{tabular}{|c|c|c|c|c|c|c|c|c|c|c|}
\hline Sorption Sample & Rock Type & $\mathrm{Sr}$ & Cs & $\mathrm{Ba}$ & $\mathrm{Ce}$ & Eu & $\mathrm{Np}$ & $\mathrm{Am}$ & $\mathrm{Pu}$ & $\mathbf{U}$ \\
\hline $\mathrm{J}-13(18)^{\mathrm{a}}$ & GLASS2 & $\sqrt{ }$ & $\sqrt{ }$ & $\sqrt{ }$ & & & & & & $\sqrt{ }$ \\
\hline $\mathrm{J}-13(32)^{a}$ & DEVIT1 & $\sqrt{ }$ & $\sqrt{ }$ & $\sqrt{ }$ & $\sqrt{ }$ & $\sqrt{ }$ & & $\sqrt{ }$ & & $\sqrt{ }$ \\
\hline $\mathrm{J}-13(37)^{\mathrm{a}}$ & CLAYS & $\sqrt{ }$ & $\sqrt{ }$ & $\sqrt{ }$ & & $\sqrt{ }$ & & $\sqrt{ }$ & $\sqrt{ }$ & $\sqrt{ }$ \\
\hline USW-GU3 (0433 ft) ${ }^{b}$ & DEVITRIFIED & & & & & & & $\sqrt{ }$ & $\sqrt{ }$ & \\
\hline USW-GU3 (1203ft) ${ }^{b}$ & GLASS & & & & & & & $\sqrt{ }$ & $\sqrt{ }$ & \\
\hline USW-GU3 (1301 ft) ${ }^{b}$ & GLASS & & & & & & & $\sqrt{ }$ & $\sqrt{ }$ & \\
\hline USW-GU3 (1407 ft) ${ }^{b}$ & GLASS & $\sqrt{ }$ & $\sqrt{ }$ & $\sqrt{ }$ & & & & & & \\
\hline USW-G4 (0270 ft) ${ }^{b}$ & ZEOLITIZED & $\sqrt{ }$ & $\sqrt{ }$ & $\sqrt{ }$ & & & $\sqrt{ }$ & & & \\
\hline USW-G4 $(1506 \mathrm{ft})^{\mathrm{b}}$ & ZEOLITIZED & $\sqrt{ }$ & $\sqrt{ }$ & $\sqrt{ }$ & & & & & & \\
\hline Nat. Calcite ${ }^{\mathrm{c}}$ & & & & & & & $\sqrt{ }$ & & & \\
\hline Syn. Hematite ${ }^{c}$ & & & & & & & $\sqrt{ }$ & & & \\
\hline
\end{tabular}

Source: Thomas 1987 (DIRS 101361), pp. 14 to 16.

a Daniels et al. 1982 (DIRS 105803).

b Bish and Vaniman 1985 (DIRS 101196), Appendix A.

c DTN: LA0010JC831341.007 (DIRS 153319). 
Table I-3. Mineralogical Composition of Rock Samples, by X-Ray Analysis

\begin{tabular}{|c|c|c|c|c|c|c|c|c|c|c|c|c|}
\hline \multirow[b]{2}{*}{ Sorption Sample } & \multirow[b]{2}{*}{$\begin{array}{c}\text { Hole } \\
\text { depth, } \mathrm{ft}^{\text {a }}\end{array}$} & \multicolumn{11}{|c|}{ Mineral Composition, Wt. \% } \\
\hline & & Calcite & Hematite & Smectite & Micas` & Clinoptilolite & Mordenite & Quartz & Cristobalite & $\begin{array}{c}\text { Alkali } \\
\text { Feldspar }\end{array}$ & Tridymite & Glass \\
\hline$J-13(18)^{b}$ & 1420 & & & 5 & 5 & $5-10$ & & & $15-25$ & $15-25$ & & $\approx 50$ \\
\hline $\mathrm{J}-13(32)^{\mathrm{b}}$ & 2533 & & & 5 & $5-15$ & & & $30-50$ & & $30-50$ & & \\
\hline $\mathrm{J}-13(37)^{\mathrm{b}}$ & 3497 & & & $20-40$ & 5 & $\approx 5$ & & $30-60$ & & $15-30$ & & \\
\hline USW-GU3 (0433 ft) ${ }^{c}$ & 430.5 & & & & $2-4$ & & & & $10-15$ & $75-85$ & $2-6$ & \\
\hline USW-GU3 (1203 ft) & 1195.7 & & & $4-6$ & & & & $2-4$ & $25-30$ & $30-40$ & & $20-40$ \\
\hline USW-GU3 $(1301 \mathrm{ft})^{c}$ & 1302.4 & & & $1-3$ & & & & $2-6$ & $10-15$ & $35-45$ & & $30-60$ \\
\hline USW-GU3 $(1407 \mathrm{ft})^{c}$ & 1415.5 & & & & & & & $2-8$ & $2-10$ & $30-40$ & & $40-70$ \\
\hline USW-G4 (0270 ft) ${ }^{c}$ & 268 & & & & $\approx 1$ & & & & $6 \pm 2$ & $73 \pm 10$ & $20 \pm 10$ & \\
\hline USW-G4 (1506 ft) ${ }^{c}$ & 1470 & & & $3 \pm 2$ & & $77 \pm 10$ & & $6 \pm 2$ & $14 \pm 4$ & & & \\
\hline & 1544 & & & $3 \pm 2$ & & $50 \pm 10$ & $20 \pm 10$ & $6 \pm 2$ & $12 \pm 4$ & & & \\
\hline
\end{tabular}

a Hole depth is the depth closest to the depth from which a sample was taken for which a mineralogical analysis is available.

b Source of X-ray analyses: Daniels et al. 1982 (DIRS 105803), Table XXIV.

${ }^{c}$ Source of X-ray analyses: Bish and Vaniman 1985 (DIRS 101196), Appendix A. 


\section{I1.4.1 Qualified LANL Data}

The qualified data were generated and compiled subsequent to 1987 and are recorded under data tracking numbers shown in Section 4.1, Table 4-2. These data contain $\mathrm{K}_{\mathrm{d}}$ values for $\mathrm{Cs}$ and $\mathrm{Sr}$, collected at $20^{\circ} \mathrm{C}, 60^{\circ} \mathrm{C}$, and $80^{\circ} \mathrm{C}$; for $\mathrm{Ba}$ at $20^{\circ} \mathrm{C}$ and at $60^{\circ} \mathrm{C}$; and data for $\mathrm{Np}$ collected at $20^{\circ} \mathrm{C}, 25^{\circ} \mathrm{C}, 60^{\circ} \mathrm{C}, 80^{\circ} \mathrm{C}$, and $90^{\circ} \mathrm{C}$ (Table I-4).

Table I-4. Batch Sorption Ratios for Pulverized Tuff and Mineral Samples* from Sorption Experiments at Room Temperature $\left(20 \pm 4^{\circ} \mathrm{C}\right), 60^{\circ} \mathrm{C}$, and $80^{\circ} \mathrm{C}$ or $90^{\circ} \mathrm{C}$ with $\mathrm{J}-13$ Well Water

\begin{tabular}{|l|l|c|c|c|c|}
\hline \multicolumn{1}{|c|}{ MATERIAL } & \multicolumn{1}{|c|}{ SAMPLE DESCRIPTION } & $\mathbf{T}^{\circ} \mathbf{C}$ & $\mathbf{K}_{\mathbf{d}}, \mathbf{m L} / \mathbf{g}$ & $\mathbf{1 / T} \mathbf{~ X 1 0 0 0 , \mathbf { K } ^ { - 1 }}$ & Log $_{\mathbf{d}}$ \\
\hline Strontium & & & & & \\
\hline G4-270 & J-13 G4-270-C.955-20 & 20 & 31.6 & 3.411 & 1.500 \\
\hline G4-270 & J-13 G4-270-C.956-20 & 20 & 30.3 & 3.411 & 1.481 \\
\hline G4-270 & J-13 G4-270-C.961-20 & 20 & 26.4 & 3.411 & 1.422 \\
\hline G4-270 & J-13 G4-270-C.962-20 & 20 & 27.4 & 3.411 & 1.438 \\
\hline G4-270 & J-13 G4-270-C.967-20 & 20 & 23.8 & 3.411 & 1.377 \\
\hline G4-270 & J-13 G4-270-C.968-20 & 20 & 25.1 & 3.411 & 1.400 \\
\hline G4-270 & J-13 G4-270-C.1021-60 & 60 & 32.5 & 3.002 & 1.512 \\
\hline G4-270 & J-13 G4-270-C.1022-60 & 60 & 33.5 & 3.002 & 1.525 \\
\hline G4-270 & J-13 G4-270-C.1029-80 & 80 & 40 & 2.832 & 1.602 \\
\hline G4-270 & J-13 G4-270-C.1030-80 & 80 & 37.8 & 2.832 & 1.577 \\
\hline G4-1506 & J-13 G4-1506-C.957-20 & 20 & 25615.4 & 3.411 & 4.409 \\
\hline G4-1506 & J-13 G4-1506-C.958-20 & 20 & 12941.8 & 3.411 & 4.112 \\
\hline G4-1506 & J-13 G4-1506-C.963-20 & 20 & 53434.8 & 3.411 & 4.728 \\
\hline G4-1506 & J-13 G4-1506-C.964-20 & 20 & 38473.4 & 3.411 & 4.585 \\
\hline G4-1506 & J-13 G4-1506-C.969-20 & 20 & 147540.9 & 3.411 & 5.169 \\
\hline G4-1506 & J-13 G4-1506-C.970-20 & 20 & 142538.9 & 3.411 & 5.154 \\
\hline G4-1506 & J-13 G4-1506-C.1019-60 & 60 & 75289 & 3.002 & 4.877 \\
\hline G4-1506 & J-13 G4-1506-C.1020-60 & 60 & 207934.7 & 3.002 & 5.318 \\
\hline G4-1506 & J-13 G4-1506-C.1027-80 & 80 & 151746.5 & 2.832 & 5.181 \\
\hline G4-1506 & J-13 G4-1507-C.1028-80 & 80 & 245084.5 & 2.832 & 5.389 \\
\hline GU3-1407 & J-13 GU3-1407-C.959-20 & 20 & 132.2 & 3.411 & 2.121 \\
\hline GU3-1407 & J-13 GU3-1407-C.960-20 & 20 & 168.7 & 3.411 & 2.227 \\
\hline GU3-1407 & J-13 GU3-1407-C.965-20 & 20 & 188.2 & 3.411 & 2.275 \\
\hline GU3-1407 & J-13 GU3-1407-C.966-20 & 20 & 186.5 & 3.411 & 2.271 \\
\hline GU3-1407 & J-13 GU3-1407-C.971-20 & 20 & 169.7 & 3.411 & 2.230 \\
\hline GU3-1407 & J-13 GU3-1407-C.972-20 & 20 & 175.6 & 3.411 & 2.245 \\
\hline GU3-1407 & J-13 GU3-1407-C.1023-60 & 60 & 183 & 3.002 & 2.262 \\
\hline GU3-1407 & J-13 GU3-1407-C.1024-60 & 60 & 183.3 & 3.002 & 2.263 \\
\hline GU3-1407 & J-13 GU3-1407-C.1031-80 & 80 & 175.5 & 2.832 & 2.244 \\
\hline GU3-1407 & J-13 GU3-1407-C.1032-80 & 80 & 198.4 & 2.832 & 2.298 \\
\hline DTN LA0010J & & & \\
\hline
\end{tabular}

DTN: LA0010JC831341.003 (DIRS 153322).

NOTE: *Fractions do not contain less than $75 \mu \mathrm{m}$ diameter particles (TWS-INC-DP-63, R1). 
Table I-4. Batch Sorption Ratios for Pulverized Tuff and Mineral Samples* from Sorption Experiments at Room Temperature $\left(20 \pm 4^{\circ} \mathrm{C}\right), 60^{\circ} \mathrm{C}$, and 80 or $90^{\circ} \mathrm{C}$ with $\mathrm{J}-13$ Well Water (Continued)

\begin{tabular}{|c|c|c|c|c|c|}
\hline MATERIAL & SAMPLE DESCRIPTION & $\mathbf{T},{ }^{\circ} \mathrm{C}$ & $\mathrm{K}_{\mathrm{d}}, \mathrm{mL} / \mathrm{g}$ & $1 / \mathrm{T} \times 1000, \mathrm{~K}^{-1}$ & $\log K_{d}$ \\
\hline \multicolumn{6}{|l|}{ Barium } \\
\hline G4-270 & J-13 G4 -270-C.955-20 & 20 & 384.2 & 3.411 & 2.585 \\
\hline G4-270 & J-13 G4-270-C.956-20 & 20 & 349.7 & 3.411 & 2.544 \\
\hline G4-270 & J-13 G4 -270-C.961-20 & 20 & 356 & 3.411 & 2.551 \\
\hline G4-270 & J-13 G4-270-C.962-20 & 20 & 362.3 & 3.411 & 2.559 \\
\hline G4-270 & J-13 G4 -270-C.967-20 & 20 & 324.8 & 3.411 & 2.512 \\
\hline G4-270 & J-13 G4-270-C.968-20 & 20 & 322.7 & 3.411 & 2.509 \\
\hline G4-270 & J-13 G4-270-C.1021-60 & 60 & 516.9 & 3.002 & 2.713 \\
\hline G4-270 & $\mathrm{J}-13$ G4-270-C.1022-60 & 60 & 517.7 & 3.002 & 2.714 \\
\hline G4-1506 & $\mathrm{J}-13$ G4-1506-C.957-20 & 20 & 30828.8 & 3.411 & 4.489 \\
\hline G4-1506 & J-13 G4-1506-C.958-20 & 20 & 12959.9 & 3.411 & 4.113 \\
\hline G4-1506 & J-13 G4-1506-C.963-20 & 20 & 100657.6 & 3.411 & 5.003 \\
\hline G4-1506 & J-13 G4-1506-C.964-20 & 20 & 42781.2 & 3.411 & 4.631 \\
\hline G4-1506 & J-13 G4-1506-C.1019-60 & 60 & 106252 & 3.002 & 5.026 \\
\hline GU3-1407 & J-13 GU3-1407-C.959-20 & 20 & 2662.8 & 3.411 & 3.425 \\
\hline GU3-1407 & J-13 GU3-1407-C.960-20 & 20 & 4187 & 3.411 & 3.622 \\
\hline GU3-1407 & J-13 GU3-1407-C.965-20 & 20 & 3820.4 & 3.411 & 3.582 \\
\hline GU3-1407 & J-13 GU3-1407-C.966-20 & 20 & 4144.8 & 3.411 & 3.618 \\
\hline GU3-1407 & J-13 GU3-1407-C.971-20 & 20 & 4674.9 & 3.411 & 3.670 \\
\hline GU3-1407 & J-13 GU3-1407-C.972-20 & 20 & 4739.8 & 3.411 & 3.676 \\
\hline GU3-1407 & J-13 GU3-1407-C.1023-60 & 60 & 8177 & 3.002 & 3.913 \\
\hline GU3-1407 & J-13 GU3-1407-C.1024-60 & 60 & 7366.2 & 3.002 & 3.867 \\
\hline
\end{tabular}

DTN: LA0010JC831341.001 (DIRS 162476).

NOTE: *Fractions do not contain less than $75 \mu \mathrm{m}$ diameter particles (TWS-INC-DP-63, R1). 
Table I-4. Batch Sorption Ratios for Pulverized Tuff and Mineral Samples* from Sorption Experiments at Room Temperature $\left(20 \pm 4^{\circ} \mathrm{C}\right), 60^{\circ} \mathrm{C}$, and 80 or $90^{\circ} \mathrm{C}$ with $\mathrm{J}-13$ Well Water (Continued)

\begin{tabular}{|c|c|c|c|c|c|}
\hline MATERIAL & SAMPLE DESCRIPTION & $\mathbf{T},{ }^{\circ} \mathrm{C}$ & $\mathrm{K}_{\mathrm{d}}, \mathrm{mL} / \mathrm{g}$ & $1 / \mathrm{T} \times 1000, \mathrm{~K}^{-1}$ & $\log K_{d}$ \\
\hline \multicolumn{6}{|l|}{ Cesium } \\
\hline G4-270 & J-13 G4-270-C.955-20 & 20 & 220.3 & 3.411 & 2.343 \\
\hline G4-270 & J-13 G4-270-C.956-20 & 20 & 212.8 & 3.411 & 2.328 \\
\hline G4-270 & J-13 G4-270-C.961-20 & 20 & 218 & 3.411 & 2.338 \\
\hline G4-270 & J-13 G4-270-C.962-20 & 20 & 126.4 & 3.411 & 2.102 \\
\hline G4-270 & J-13 G4-270-C.967-20 & 20 & 123.7 & 3.411 & 2.092 \\
\hline G4-270 & J-13 G4-270-C.968-20 & 20 & 125.06 & 3.411 & 2.097 \\
\hline G4-270 & J-13 G4-270-C.1021-60 & 60 & 239.4 & 3.002 & 2.379 \\
\hline G4-270 & J-13 G4-270-C.1022-60 & 60 & 244.6 & 3.002 & 2.388 \\
\hline G4-270 & J-13 G4-270-C.1029-80 & 80 & 165.7 & 2.832 & 2.219 \\
\hline G4-270 & J-13 G4-270-C.1030-80 & 80 & 170.9 & 2.832 & 2.233 \\
\hline G4-1506 & J-13 G4-1506-C.957-20 & 20 & 7381.5 & 3.411 & 3.868 \\
\hline G4-1506 & J-13 G4-1506-C.958-20 & 20 & 42321.6 & 3.411 & 4.627 \\
\hline G4-1506 & J-13 G4-1506-C.963-20 & 20 & 10206.3 & 3.411 & 4.009 \\
\hline G4-1506 & J-13 G4-1506-C.964-20 & 20 & 8271.6 & 3.411 & 3.918 \\
\hline G4-1506 & J-13 G4-1506-C.969-20 & 20 & 36364.24 & 3.411 & 4.561 \\
\hline G4-1506 & J-13 G4-1506-C.970-20 & 20 & 29730.22 & 3.411 & 4.473 \\
\hline G4-1506 & J-13 G4-1506-C.1019-60 & 60 & 19553.2 & 3.002 & 4.291 \\
\hline G4-1506 & J-13 G4-1506-C.1020-60 & 60 & 35154.3 & 3.002 & 4.546 \\
\hline G4-1506 & J-13 G4-1506-C.1027-80 & 80 & 16468 & 2.832 & 4.217 \\
\hline G4-1506 & J-13 G4-1507-C.1028-80 & 80 & 17518.1 & 2.832 & 4.243 \\
\hline GU3-1407 & J-13 GU3-1407-C.959-20 & 20 & 767.4 & 3.411 & 2.885 \\
\hline GU3-1407 & J-13 GU3-1407-C.960-20 & 20 & 950.2 & 3.411 & 2.978 \\
\hline GU3-1407 & J-13 GU3-1407-C.965-20 & 20 & 525.8 & 3.411 & 2.721 \\
\hline GU3-1407 & J-13 GU3-1407-C.966-20 & 20 & 516.7 & 3.411 & 2.713 \\
\hline GU3-1407 & J-13 GU3-1407-C.971-20 & 20 & 1052 & 3.411 & 3.022 \\
\hline GU3-1407 & J-13 GU3-1407-C.972-20 & 20 & 1041.13 & 3.411 & 3.018 \\
\hline GU3-1407 & J-13 GU3-1407-C.1023-60 & 60 & 1061.4 & 3.002 & 3.026 \\
\hline GU3-1407 & J-13 GU3-1407-C.1024-60 & 60 & 1026.7 & 3.002 & 3.011 \\
\hline GU3-1407 & J-13 GU3-1407-C.1031-80 & 80 & 672.3 & 2.832 & 2.828 \\
\hline GU3-1407 & J-13 GU3-1407-C.1032-80 & 80 & 767.9 & 2.832 & 2.885 \\
\hline
\end{tabular}

DTN: LA0010JC831341.002 (DIRS 153321).

NOTE: *Fractions do not contain less than $75 \mu \mathrm{m}$ diameter particles (TWS-INC-DP-63, R1). 
Table I-4. Batch Sorption Ratios for Pulverized Tuff and Mineral Samples* from Sorption Experiments at Room Temperature $\left(20 \pm 4^{\circ} \mathrm{C}\right), 60^{\circ} \mathrm{C}$, and 80 or $90^{\circ} \mathrm{C}$ with $\mathrm{J}-13$ Well Water (Continued)

\begin{tabular}{|c|c|c|c|c|c|}
\hline MATERIAL & SAMPLE DESCRIPTION & $\mathbf{T},{ }^{\circ} \mathbf{C}$ & $\mathrm{K}_{\mathrm{d}}, \mathrm{mL} / \mathrm{g}$ & $1 / \mathrm{T} \times 1000, \mathrm{~K}^{-1}$ & $\log K_{d}$ \\
\hline \multicolumn{6}{|l|}{ Neptunium } \\
\hline G4-270 & J-13-G-4 270-K-01-20 & 20 & -0.5 & & \\
\hline G4-270 & J-13-G-4 270-K-02-20 & 20 & 0.2 & 3.411 & -0.699 \\
\hline G4-270 & J-13-G-4 270-K-01D-20 & 20 & 1.1 & 3.411 & 0.041 \\
\hline G4-270 & J-13-G-4 270-K-02D-20 & 20 & 0.9 & 3.411 & -0.046 \\
\hline G4-270 & J-13-G-4 270-C.15-20 & 20 & 0.4 & 3.411 & -0.398 \\
\hline G4-270 & J-13-G-4 270-C.16-20 & 20 & 0.8 & 3.411 & -0.097 \\
\hline G4-270 & J-13-G-4 270-C.33-60 & 60 & 0.9 & 3.002 & -0.046 \\
\hline G4-270 & J-13-G-4 270-C.51-90 & 90 & 1 & 2.754 & 0.000 \\
\hline G4-270 & J-13-G-4 270-C.52-90 & 90 & 1.1 & 2.754 & 0.041 \\
\hline G4-270 & J-13-G-4 270-C.67-20 & 20 & 0.2 & 3.411 & -0.699 \\
\hline G4-270 & J-13-G-4 270-C.68-20 & 20 & 0.5 & 3.411 & -0.301 \\
\hline G4-270 & J-13-G-4 270-C.70-60 & 60 & 12.9 & 3.002 & 1.111 \\
\hline G4-270 & J-13-G-4 270-C.171-20 & 20 & 1 & 3.411 & 0.000 \\
\hline G4-270 & J-13-G-4 270-C.172-20 & 20 & 1.1 & 3.411 & 0.041 \\
\hline G4-270 & J-13-G-4 270-C.189-60 & 60 & 1.1 & 3.002 & 0.041 \\
\hline G4-270 & J-13-G-4 270-C.190-60 & 60 & 0.8 & 3.002 & -0.097 \\
\hline G4-270 & J-13-G-4 270-C.223-20 & 20 & 0.1 & 3.411 & -1.000 \\
\hline G4-270 & J-13-G-4 270-C.227-90 & 90 & 1.7 & 2.754 & 0.230 \\
\hline G4-270 & J-13-G-4 270-C.228-90 & 90 & 2.7 & 2.754 & 0.431 \\
\hline G4-270 & J-13-G4 270-C.341-20 & 20 & 0.7 & 3.411 & -0.155 \\
\hline G4-270 & J-13-G4 270-C.342-20 & 20 & 0.8 & 3.411 & -0.097 \\
\hline G4-270 & J-13-G4 270-C.395-20 & 20 & 0.1 & 3.411 & -1.000 \\
\hline G4-270 & J-13-G4 270-C.396-20 & 20 & 0.6 & 3.411 & -0.222 \\
\hline G4-270 & J13-G4-270-C.409-80 & 80 & 0.6 & 2.832 & -0.222 \\
\hline G4-270 & J13-G4-270-C.410-80 & 80 & 0.9 & 2.832 & -0.046 \\
\hline G4-270 & J-13 G4-270-C.437-20 & 20 & 0.1 & 3.411 & -1.000 \\
\hline G4-270 & J-13 G4-270-C.438-20 & 20 & 0.2 & 3.411 & -0.699 \\
\hline G4-270 & J-13 G4-270-C.459-60 & 60 & 0.7 & 3.002 & -0.155 \\
\hline G4-270 & J-13 G4-270-C.460-60 & 60 & 0.2 & 3.002 & -0.699 \\
\hline G4-270 & J-13 G4-270-C.973-20 & 20 & 0.4 & 3.411 & -0.398 \\
\hline G4-270 & J-13 SYN. G4-270-C.994-20 & 20 & 0.5 & 3.411 & -0.301 \\
\hline G4-270 & $\begin{array}{l}\text { J-13 SYN. G4-270-C.2047- } \\
20\end{array}$ & 20 & 0.4 & 3.411 & -0.398 \\
\hline G4-270 & $\begin{array}{l}\text { J-13 SYN. G4-270-C.2048- } \\
20\end{array}$ & 20 & 0.3 & 3.411 & -0.523 \\
\hline G4-270 & J-13 G4-270-C.2251-25 & 25 & 0.3 & 3.354 & -0.523 \\
\hline G4-270 & J-13-G4 270 C.2295-25 & 25 & 0.2 & 3.354 & -0.699 \\
\hline G4-270 & J-13-G4 270 C.2296-25 & 25 & 0.5 & 3.354 & -0.301 \\
\hline G4-270 & J-13 G4-270-C.6000-20 & 20 & 0.9 & 3.411 & -0.046 \\
\hline G4-270 & J-13 G4-270-C.6001-20 & 20 & 0.5 & 3.411 & -0.301 \\
\hline G4-270 & J-13 G4-270-C.6006-20 & 20 & 0.5 & 3.411 & -0.301 \\
\hline G4-270 & J-13 G4-270-C.6007-20 & 20 & 3.5 & 3.411 & 0.544 \\
\hline
\end{tabular}

DTN: LA0010JC831341.007 (DIRS 153319).

NOTE: *Fractions do not contain less than $75 \mu \mathrm{m}$ diameter particles (TWS-INC-DP-63, R1). 
Table I-4. Batch Sorption Ratios for Pulverized Tuff and Mineral Samples* from Sorption Experiments at Room Temperature $\left(20 \pm 4^{\circ} \mathrm{C}\right), 60^{\circ} \mathrm{C}$, and 80 or $90^{\circ} \mathrm{C}$ with $\mathrm{J}-13$ Well Water (Continued)

\begin{tabular}{|c|c|c|c|c|c|}
\hline MATERIAL & SAMPLE DESCRIPTION & $\mathrm{T},{ }^{\circ} \mathrm{C}$ & $\mathrm{K}_{\mathrm{d}}, \mathrm{mL} / \mathrm{g}$ & $1 / \mathrm{T} \times 1000, \mathrm{~K}^{-1}$ & $\log K_{d}$ \\
\hline \multicolumn{6}{|l|}{ Neptunium } \\
\hline G4-1506 & J-13-G-4 1506-K-03-20 & 20 & 3.7 & 3.411 & 0.568 \\
\hline G4-1506 & J-13-G-4 1506-K-04-20 & 20 & 4.3 & 3.411 & 0.633 \\
\hline G4-1506 & J-13-G-4 1506-C.17-20 & 20 & 4.4 & 3.411 & 0.643 \\
\hline G4-1506 & J-13-G-4 1506-C.18-20 & 20 & 5.1 & 3.411 & 0.708 \\
\hline G4-1506 & J-13-G-4 1506-C.35-60 & 60 & 6.8 & 3.002 & 0.833 \\
\hline G4-1506 & J-13-G-4 1506-C.36-60 & 60 & 1.5 & 3.002 & 0.176 \\
\hline G4-1506 & J-13-G-4 1506-C.53-90 & 90 & 5.4 & 2.754 & 0.732 \\
\hline G4-1506 & J-13-G-4 1506-C.54-90 & 90 & 10 & 2.754 & 1.000 \\
\hline G4-1506 & J-13-G-4 1506-C.73-20 & 20 & 2.9 & 3.411 & 0.462 \\
\hline G4-1506 & J-13-G-4 1506-C.74-20 & 20 & 2.4 & 3.411 & 0.380 \\
\hline G4-1506 & J-13-G-4 1506-C.76-60 & 60 & 3660.5 & 3.002 & 3.564 \\
\hline G4-1506 & J-13-G-4 1506-C.173-20 & 20 & 6.1 & 3.411 & 0.785 \\
\hline G4-1506 & J-13-G-4 1506-C.174-20 & 20 & 6.4 & 3.411 & 0.806 \\
\hline G4-1506 & J-13-G-4 1506-C.191-60 & 60 & 8.5 & 3.002 & 0.929 \\
\hline G4-1506 & J-13-G-4 1506-C.192-60 & 60 & 8.4 & 3.002 & 0.924 \\
\hline G4-1506 & J-13-G-4 1506-C.229-20 & 20 & 4.1 & 3.411 & 0.613 \\
\hline G4-1506 & J-13-G-4 1506-C.230-20 & 20 & 4 & 3.411 & 0.602 \\
\hline G4-1506 & J-13-G-4 1506-C.233-90 & 90 & 1.9 & 2.754 & 0.279 \\
\hline G4-1506 & J-13-G-4 1506-C.234-90 & 90 & 7.8 & 2.754 & 0.892 \\
\hline G4-1506 & J-13-G4 1506-C.343-20 & 20 & 3.7 & 3.411 & 0.568 \\
\hline G4-1506 & J-13-G4 1506-C.344-20 & 20 & 4 & 3.411 & 0.602 \\
\hline G4-1506 & J-13-G4 1506-C.397-20 & 20 & 1.9 & 3.411 & 0.279 \\
\hline G4-1506 & J-13-G4 1506-C.398-20 & 20 & 2.2 & 3.411 & 0.342 \\
\hline G4-1506 & J-13 G4-1506-C.439-20 & 20 & 1.9 & 3.411 & 0.279 \\
\hline G4-1506 & J-13 G4-1506-C.440-20 & 20 & 2 & 3.411 & 0.301 \\
\hline G4-1506 & J-13 G4-1506-C.461-60 & 60 & 3.2 & 3.002 & 0.505 \\
\hline G4-1506 & J-13 G4-1506-C.462-60 & 60 & 3.1 & 3.002 & 0.491 \\
\hline G4-1506 & J-13 G4-1506-C.975-20 & 20 & 2.3 & 3.411 & 0.362 \\
\hline G4-1506 & J-13 G4-1506-C.976-20 & 20 & 2.2 & 3.411 & 0.342 \\
\hline G4-1506 & J-13 G4-1506-C.987-20 & 20 & 2.9 & 3.411 & 0.462 \\
\hline G4-1506 & J-13 G4-1506-C.988-20 & 20 & 2.5 & 3.411 & 0.398 \\
\hline G4-1506 & J-13 SYN. G4-1506-C.991-20 & 20 & 3.7 & 3.411 & 0.568 \\
\hline G4-1506 & J-13 SYN. G4-1506-C.992-20 & 20 & 3.4 & 3.411 & 0.531 \\
\hline G4-1506 & $\begin{array}{l}\text { J-13 SYN. G4-1506-C.2045- } \\
20\end{array}$ & 20 & 5.2 & 3.411 & 0.716 \\
\hline G4-1506 & $\begin{array}{l}\text { J-13 SYN. G4-1506-C.2046- } \\
20\end{array}$ & 20 & 5.4 & 3.411 & 0.732 \\
\hline G4-1506 & J-13-G-4 1506-C.209X-90 & 90 & 8.7 & 2.754 & 0.940 \\
\hline G4-1506 & J-13-G-4 1506-C.210X-90 & 90 & 10.4 & 2.754 & 1.017 \\
\hline G4-1506 & J-13-G-4 1506-C.231-60 & 60 & 5.7 & 3.002 & 0.756 \\
\hline G4-1506 & J-13-G-4 1506-C.232-60 & 60 & 6.4 & 3.002 & 0.806 \\
\hline G4-1506 & J-13 G4-1506-C.571-20 & 20 & 2.5 & 3.411 & 0.398 \\
\hline G4-1506 & J-13 G4-1506-C.572-20 & 20 & 2.6 & 3.411 & 0.415 \\
\hline G4-1506 & J-13 G4-1506-C.981-20 & 20 & 2.2 & 3.411 & 0.342 \\
\hline G4-1506 & J-13 G4-1506-C.571-20 & 20 & 2.5 & 3.411 & 0.398 \\
\hline G4-1506 & J-13 G4-1506-C.2002-20 & 20 & 3.5 & 3.411 & 0.544 \\
\hline G4-1506 & J-13 G4-1506-C.2003-20 & 20 & 3.2 & 3.411 & 0.505 \\
\hline
\end{tabular}

DTN: LA0010JC831341.007 (DIRS 153319).

NOTE: *Fractions do not contain less than $75 \mu \mathrm{m}$ diameter particles (TWS-INC-DP-63, R1). 
Table I-4. Batch Sorption Ratios for Pulverized Tuff and Mineral Samples* from Sorption Experiments at Room Temperature $\left(20 \pm 4^{\circ} \mathrm{C}\right), 60^{\circ} \mathrm{C}$, and 80 or $90^{\circ} \mathrm{C}$ with $\mathrm{J}-13$ Well Water (Continued)

\begin{tabular}{|c|c|c|c|c|c|}
\hline MATERIAL & SAMPLE DESCRIPTION & $\mathbf{T},{ }^{\circ} \mathrm{C}$ & $\mathrm{K}_{\mathrm{d}}, \mathrm{mL} / \mathrm{g}$ & $1 / T \times 1000, K^{-1}$ & $\log K_{c}$ \\
\hline \multicolumn{6}{|l|}{ Neptunium } \\
\hline Natural Calcite & J-13-B-C.3-20 & 20 & 979.5 & 3.411 & 2.991 \\
\hline Natural Calcite & J-13-B-C.4-20 & 20 & 883.4 & 3.411 & 2.946 \\
\hline Natural Calcite & J-13-B-C.21-60 & 60 & 154.5 & 3.002 & 2.189 \\
\hline Natural Calcite & J-13-B-C.22-60 & 60 & 136.9 & 3.002 & 2.136 \\
\hline Natural Calcite & J-13-B-C.40-90 & 90 & 66.5 & 2.754 & 1.823 \\
\hline Natural Calcite & J-13-B-C.55-20 & 20 & 96.9 & 3.411 & 1.986 \\
\hline Natural Calcite & J-13-B-C.56-20 & 20 & 73.1 & 3.411 & 1.864 \\
\hline Natural Calcite & J-13-B-C.57-60 & 60 & 30 & 3.002 & 1.477 \\
\hline Natural Calcite & J-13-B-C.58-60 & 60 & 28.8 & 3.002 & 1.459 \\
\hline Natural Calcite & J-13-B-C.159-20 & 20 & 640.5 & 3.411 & 2.807 \\
\hline Natural Calcite & J-13-B-C.160-20 & 20 & 543.9 & 3.411 & 2.736 \\
\hline Natural Calcite & J-13-B-C.177-60 & 60 & 95.8 & 3.002 & 1.981 \\
\hline Natural Calcite & J-13-B-C.178-60 & 60 & 77.1 & 3.002 & 1.887 \\
\hline Natural Calcite & J-13-B-C.195-90 & 90 & 38.8 & 2.754 & 1.589 \\
\hline Natural Calcite & J-13-B-C.196-90 & 90 & 93.5 & 2.754 & 1.971 \\
\hline Natural Calcite & J-13-B-C.211-20 & 20 & 54.6 & 3.411 & 1.737 \\
\hline Natural Calcite & J-13-B-C.212-20 & 20 & 56.4 & 3.411 & 1.751 \\
\hline Natural Calcite & J-13-B-C.213-60 & 60 & 9 & 3.002 & 0.954 \\
\hline Natural Calcite & J-13-B-C.214-60 & 60 & 9 & 3.002 & 0.954 \\
\hline Natural Calcite & J-13-B-C.215-90 & 90 & 3.1 & 2.754 & 0.491 \\
\hline Natural Calcite & J-13-B-C.216-90 & 90 & 3.6 & 2.754 & 0.556 \\
\hline Natural Calcite & J-13-B-C.331-20 & 20 & 51.5 & 3.411 & 1.712 \\
\hline Natural Calcite & J-13-B-C.332-20 & 20 & 53 & 3.411 & 1.724 \\
\hline Natural Calcite & J-13 B-C.575-20 & 20 & 2.4 & 3.411 & 0.380 \\
\hline Natural Calcite & J-13 B-C.576-20 & 20 & 3.9 & 3.411 & 0.591 \\
\hline Natural Calcite & J-13 SYN. B-C.997-20 & 20 & 1.9 & 3.411 & 0.279 \\
\hline Natural Calcite & J-13 SYN. B-C.998-20 & 20 & 2.1 & 3.411 & 0.322 \\
\hline Natural Calcite & J-13 SYN. B-C.2051-20 & 20 & 4.3 & 3.411 & 0.633 \\
\hline Natural Calcite & J-13 SYN. B-C.2052-20 & 20 & 5 & 3.411 & 0.699 \\
\hline Natural Calcite & SYN.J13.B B-C.2127-20 & 20 & 36.1 & 3.411 & 1.558 \\
\hline Natural Calcite & SYN.J13.B B-C.2128-20 & 20 & 38.7 & 3.411 & 1.588 \\
\hline Natural Calcite & J-13 B-C.3882-20 (3 Days) & 20 & 680.3 & 3.411 & 2.833 \\
\hline Natural Calcite & J-13 B-C.3883-20 (3 Days) & 20 & 650.9 & 3.411 & 2.814 \\
\hline Natural Calcite & J-13 B-C.3886-20 (30 Days) & 20 & 6492.9 & 3.411 & 3.812 \\
\hline Natural Calcite & J-13 B-C.3887-20 (30 Days) & 20 & 7808.9 & 3.411 & 3.893 \\
\hline
\end{tabular}

DTN: LA0010JC831341.007 (DIRS 153319).

NOTE: Fractions do not contain less than $75 \mu \mathrm{m}$ diameter particles (TWS-INC-DP-63, R1). 
Table 1-4. Batch Sorption Ratios for Pulverized Tuff and Mineral Samples* from Sorption Experiments at Room Temperature $\left(20 \pm 4^{\circ} \mathrm{C}\right), 60^{\circ} \mathrm{C}$, and 80 or $90^{\circ} \mathrm{C}$ with $\mathrm{J}-13$ Well Water (Continued)

\begin{tabular}{|c|c|c|c|c|c|}
\hline MATERIAL & SAMPLE DESCRIPTION & $\mathbf{T},{ }^{\circ} \mathbf{C}$ & $\mathrm{K}_{\mathrm{d}}, \mathrm{mL} / \mathrm{g}$ & $1 / T \times 1000, K^{-1}$ & $\log K_{d}$ \\
\hline \multicolumn{6}{|l|}{ Neptunium } \\
\hline Synthetic Hematite & J-13-C-C.42-90 & 90 & 2662.8 & 2.754 & 3.425 \\
\hline Synthetic Hematite & J-13-C-C.161-20 & 20 & 2003.7 & 3.411 & 3.302 \\
\hline Synthetic Hematite & J-13-C-C.162-20 & 20 & 1749.1 & 3.411 & 3.243 \\
\hline Synthetic Hematite & J-13-C-C.179-60 & 60 & 696.8 & 3.002 & 2.843 \\
\hline Synthetic Hematite & J-13-C-C.180-60 & 60 & 848.1 & 3.002 & 2.928 \\
\hline Synthetic Hematite & J-13-C-C.197-90 & 90 & 5898.5 & 2.754 & 3.771 \\
\hline Synthetic Hematite & J-13-C-C.198-90 & 90 & 18360.3 & 2.754 & 4.264 \\
\hline Synthetic Hematite & J-13-C-C.333-20 & 20 & 149.4 & 3.411 & 2.174 \\
\hline Synthetic Hematite & J-13-C-C.334-20 & 20 & 567.5 & 3.411 & 2.754 \\
\hline Synthetic Hematite & J-13-C-C.387-20 & 20 & 233.5 & 3.411 & 2.368 \\
\hline Synthetic Hematite & J-13-C-C.388-20 & 20 & 199.2 & 3.411 & 2.299 \\
\hline Synthetic Hematite & J13-C-C.403-80 & 80 & 561.5 & 2.832 & 2.749 \\
\hline Synthetic Hematite & J13-C-C.404-80 & 80 & 462.5 & 2.832 & 2.665 \\
\hline Synthetic Hematite & J-13-C-C.433-20 & 20 & 67.8 & 3.411 & 1.831 \\
\hline Synthetic Hematite & J-13-C-C.434-20 & 20 & 74.8 & 3.411 & 1.874 \\
\hline Synthetic Hematite & J-13-C-C.451-60 & 60 & 199.9 & 3.002 & 2.301 \\
\hline Synthetic Hematite & J-13-C-C.452-60 & 60 & 215.5 & 3.002 & 2.333 \\
\hline Synthetic Hematite & J-13 C-C.577-20 & 20 & 732.8 & 3.411 & 2.865 \\
\hline Synthetic Hematite & $\mathrm{J}-13$ C-C.578-20 & 20 & 539.1 & 3.411 & 2.732 \\
\hline Synthetic Hematite & J-13 SYN. C-C.999-20 & 20 & 432 & 3.411 & 2.635 \\
\hline Synthetic Hematite & J-13 SYN. C-C.1000-20 & 20 & 330.6 & 3.411 & 2.519 \\
\hline Synthetic Hematite & J-13 SYN. C-C.2053-20 & 20 & 782.4 & 3.411 & 2.893 \\
\hline Synthetic Hematite & J-13 SYN. C-C.2054-20 & 20 & 1022.2 & 3.411 & 3.010 \\
\hline Synthetic Hematite & SYN.J13.B C-C.2129-20 & 20 & 1973.3 & 3.411 & 3.295 \\
\hline Synthetic Hematite & SYN.J13.B C-C.2130-20 & 20 & 2720.7 & 3.411 & 3.435 \\
\hline
\end{tabular}

DTN: LA0010JC831341.007 (DIRS 153319).

NOTE: *Fractions do not contain less than $75 \mu \mathrm{m}$ diameter particles (TWS-INC-DP-63, R1).

These $K_{d}$ measurements were plotted as Log $K_{d}$ versus $1 / T\left[K^{-1}\right]$. The plots are illustrated in Figures I-1(a) to I-1(d). Data were regressed to obtain linear equations, and the slopes and calculated enthalpies of sorption were summarized in Table I-5. 
(a)

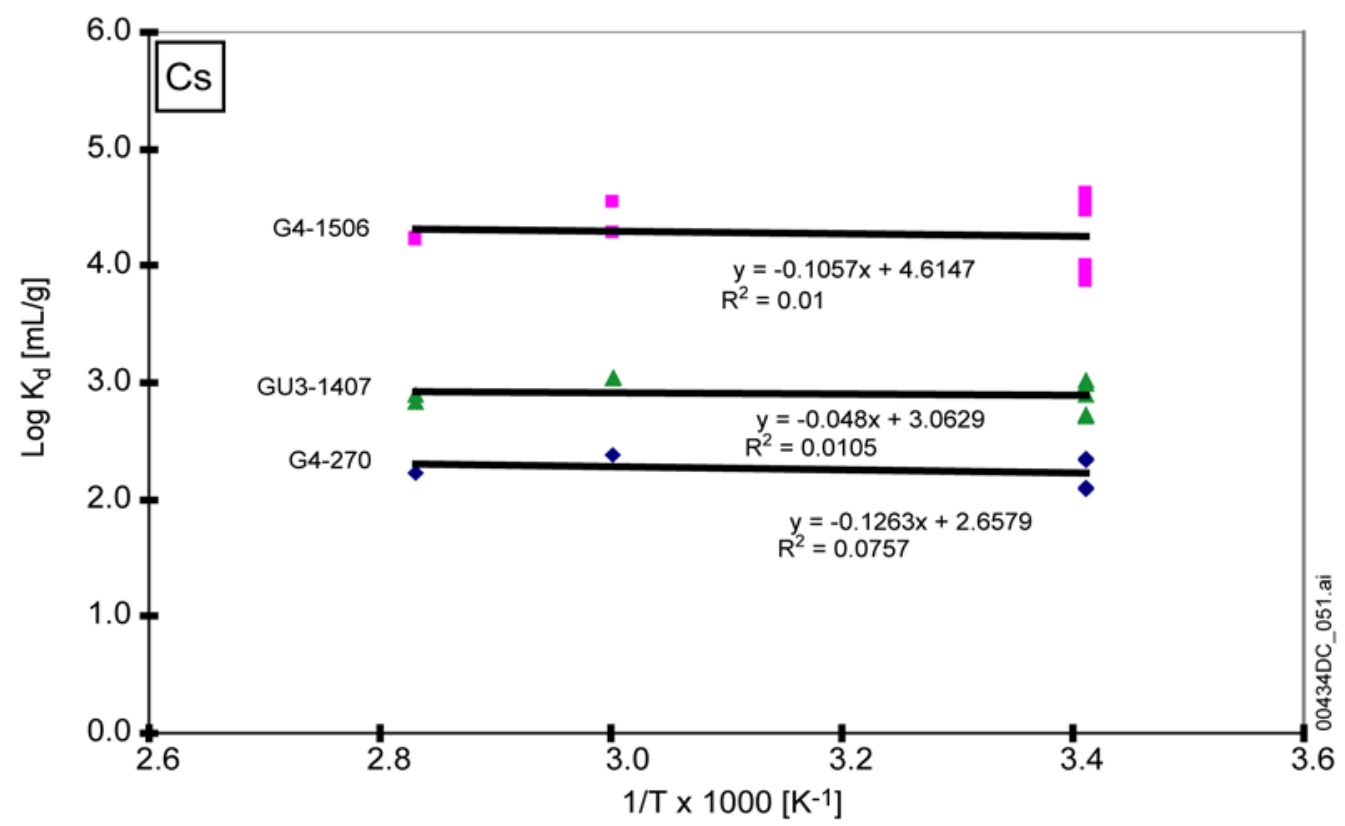

(b)

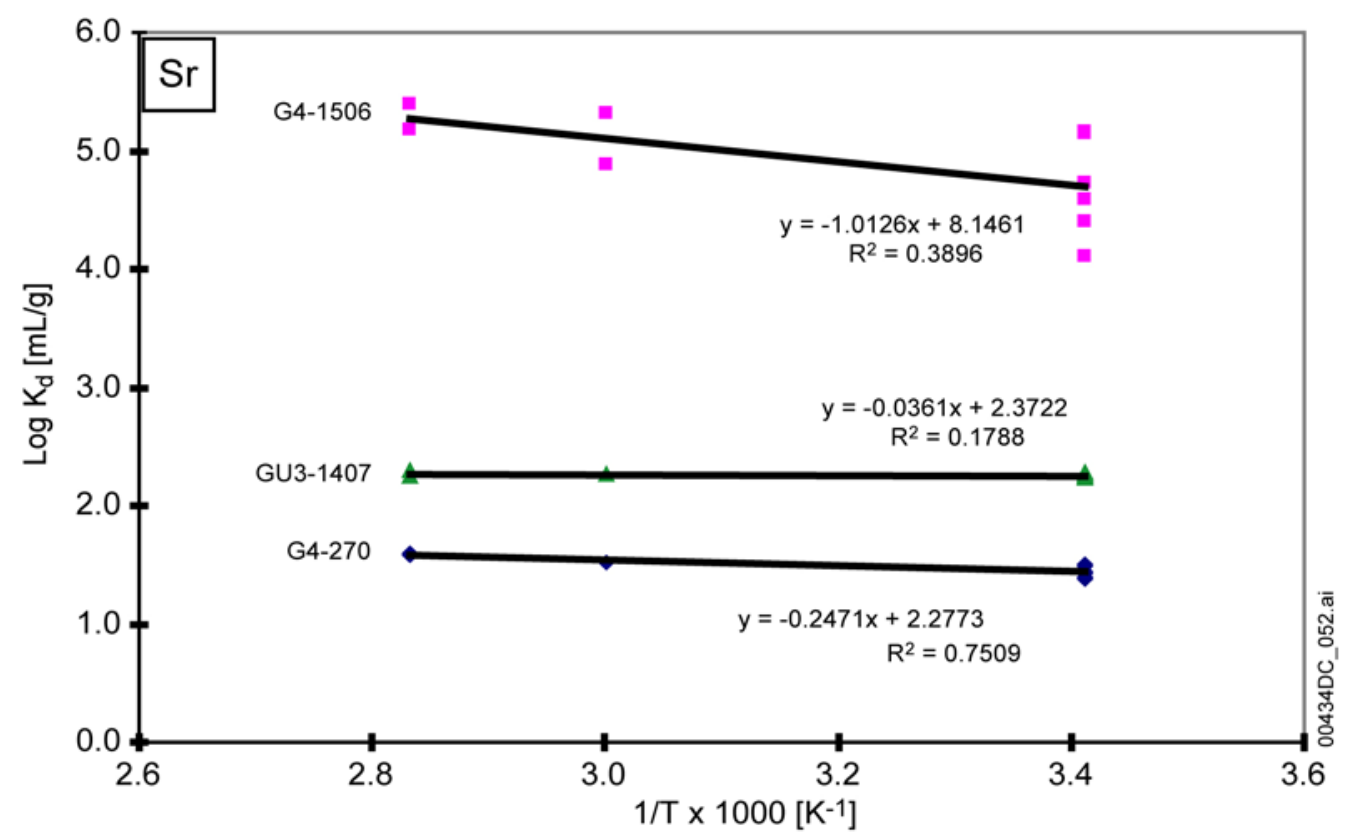

Input DTNs: LA0010JC831341.001 (DIRS 162476); LA0010JC831341.002 (DIRS 153321).

LA0010JC831341.003 (DIRS 153322); LA0010JC831341.007 (DIRS 153319).

Output DTN: LB0311ABSTHCR2.004.

Figure I-1(a)-(b). Calculated Values of $\log \mathrm{K}_{d}$ as a Function of Reciprocal Absolute Temperature, Based on Batch Sorption of Specified Radioelements on Crushed Yucca Mountain Tuff and Mineral Samples 
(c)

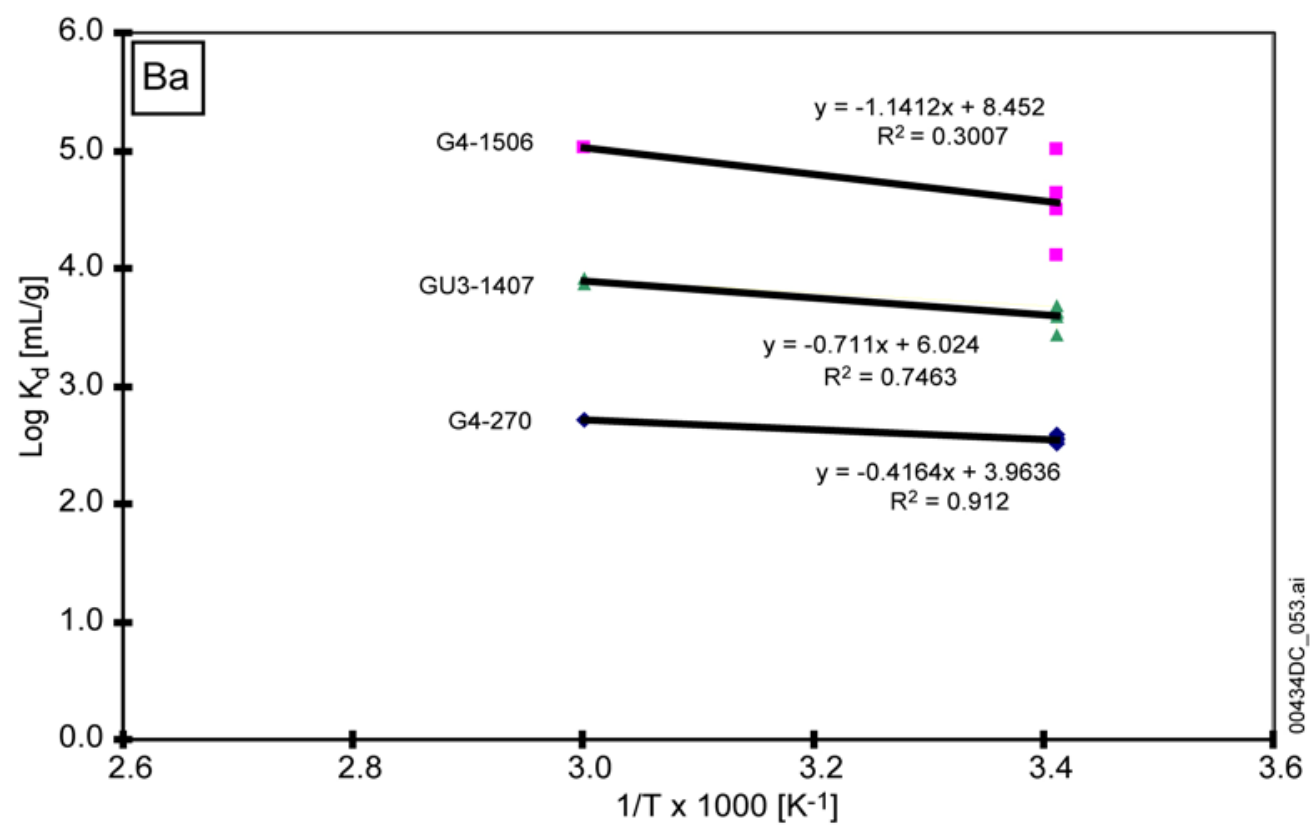

(d)

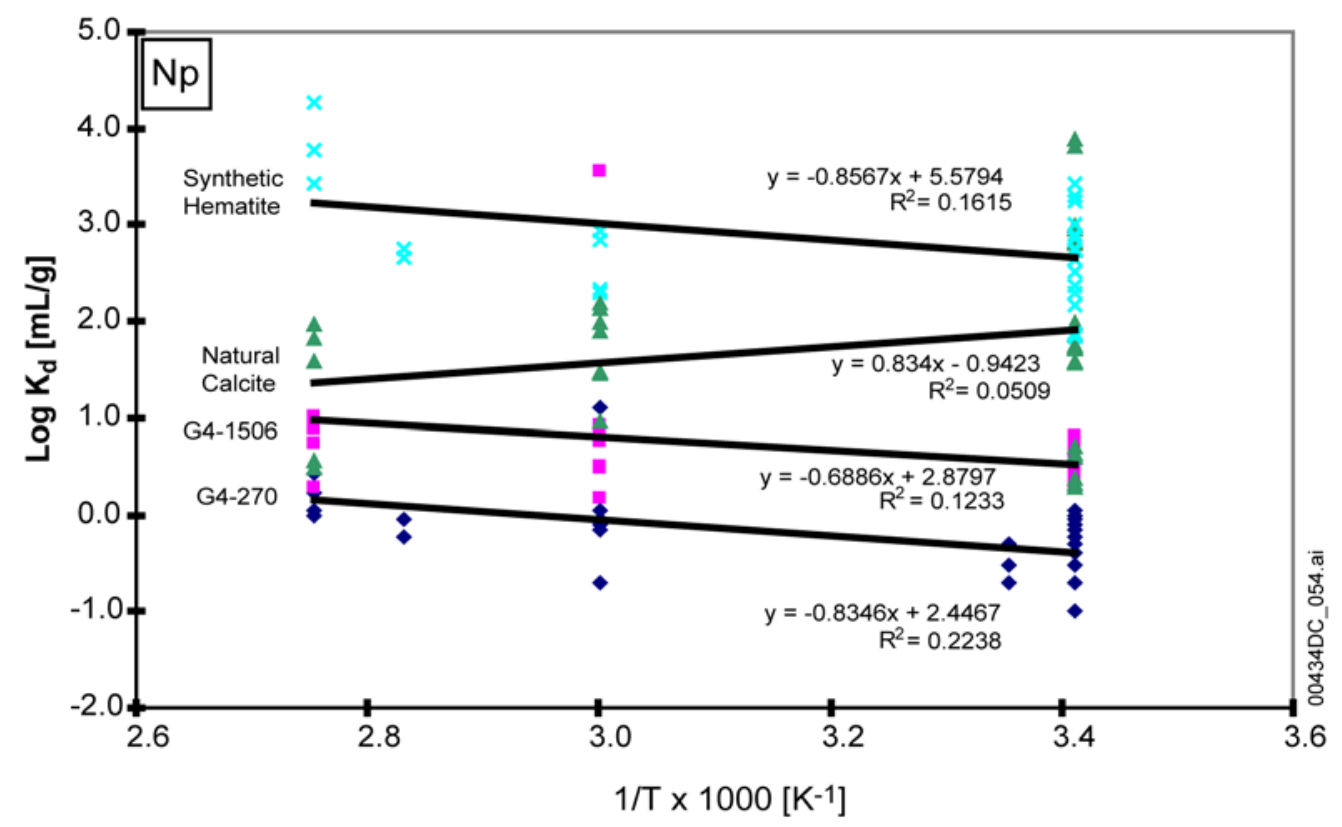

Source DTNs: LA0010JC831341.001 (DIRS 162476); LA0010JC831341.002 (DIRS 153321). LA0010JC831341.003 (DIRS 153322); LA0010JC831341.007 (DIRS 153319). Output DTN: LB0311ABSTHCR2.004.

NOTE: The $\mathrm{K}_{\mathrm{d}}$ measurements are listed in Table $\mathrm{l}-4$.

Figure I-1(c)-(d). Calculated Values of log $K_{d}$ as a Function of Reciprocal Absolute Temperature, Based on Batch Sorption of Specified Radioelements on Crushed Yucca Mountain Tuff and Mineral Samples (Continued) 
Table I-5. Calculation of the Enthalpy of Sorption of Aqueous Species on Various Sorbents ${ }^{a}$

\begin{tabular}{|c|c|c|c|c|c|c|}
\hline Aq. Species & Sorbent & $\begin{array}{c}\text { Temperature. } \\
\text { Range, }{ }^{\circ} \mathrm{C}\end{array}$ & Slope & $\mathbf{R}^{2}$ & $\begin{array}{c}\Delta \mathbf{H}_{\mathrm{r}}, \\
\mathrm{kcal} / \mathrm{mol}\end{array}$ & Notes \\
\hline \multirow[t]{3}{*}{$\mathrm{Sr}$} & G4-270 & $20,60,80$ & -0.2471 & 0.751 & 1.13 & sorption \\
\hline & G4-1506 & & -1.0126 & 0.390 & 4.63 & \\
\hline & GU3-1407 & & -0.0361 & 0.179 & 0.17 & \\
\hline \multirow[t]{3}{*}{ Cs } & G4-270 & $20,60,80$ & -0.1263 & 0.076 & 0.58 & sorption \\
\hline & G4-1506 & & -0.1057 & 0.010 & 0.48 & \\
\hline & GU3-1407 & & -0.0480 & 0.011 & 0.22 & \\
\hline \multirow[t]{3}{*}{$\mathrm{Ba}$} & G4-270 & 20,60 & -0.4164 & 0.912 & 1.91 & sorption \\
\hline & G4-1506 & & -1.1412 & 0.301 & 5.22 & \\
\hline & GU3-1407 & & -0.7110 & 0.746 & 3.25 & \\
\hline \multirow[t]{4}{*}{$\mathrm{Np}$} & G4-270 & $\begin{array}{l}20,25,60,80 \\
90\end{array}$ & -0.8346 & 0.224 & 3.82 & sorption \\
\hline & G4-1506 & & -0.6886 & 0.123 & 3.15 & \\
\hline & Natural calcite & & 0.8340 & 0.051 & -3.82 & \\
\hline & Synthetic hematite & $20,60,80,90$ & -0.8567 & 0.162 & 3.92 & \\
\hline
\end{tabular}

Source DTNs: LA0010JC831341.001 (DIRS 162476); LA0010JC831341.002 (DIRS 153321); LA0010JC831341.003 (DIRS 153322); LA0010JC831341.007 (DIRS 153319).

Output DTN: LB0311ABSTHCR2.004.

a Summarized from Table I-4 and Figure I-1.

\subsubsection{Unqualified LANL Data}

These data consist of $K_{d}$ measurements made before 1987, compiled in LANL Report No. LA-10960-MS (Thomas 1987 [DIRS 101361]) (Table I-7), and those averaged at discrete temperatures summarized in LANL Report No. LA-9328-MS (Daniels et al. 1982 [DIRS 105803]) (Table I-6). 
Table I-6. Average Sorption and Desorption Ratios for Pulverized Tuff ${ }^{a}$ from Sorption and Desorption Experiments at Room Temperature $\left(20 \pm 4^{\circ} \mathrm{C}\right)$ and at $70^{\circ} \mathrm{C}$ with $\mathrm{J}-13$ Well Water

\begin{tabular}{|c|c|c|c|c|c|c|c|c|c|c|c|}
\hline \multirow[b]{2}{*}{ Sample } & \multirow{2}{*}{$\begin{array}{c}\text { Depth, } \\
\mathrm{ft}\end{array}$} & \multirow{2}{*}{$\begin{array}{l}\text { USW-G1 } \\
\text { depth }^{\mathrm{b}}, \mathrm{ft} .\end{array}$} & \multicolumn{9}{|c|}{$\mathrm{K}_{\mathrm{d}}, \mathrm{mL} / \mathrm{g}$} \\
\hline & & & $\mathrm{Sr}$ & Cs & $\mathrm{Ba}$ & $\mathrm{Ce}$ & Eu & Am & $\mathbf{P u}$ & $\mathbf{U}$ & $\mathrm{Np}$ \\
\hline \multicolumn{12}{|c|}{ Room Temperature $\left(20 \pm 4^{\circ} \mathrm{C}\right)$} \\
\hline \multicolumn{12}{|l|}{ Sorption } \\
\hline$J A-18$ & 1420 & 1339 & $\begin{array}{l}17000 \\
(3000)^{c}\end{array}$ & $\begin{array}{l}16000 \\
(1000)\end{array}$ & $\begin{array}{l}38000 \\
(18000)\end{array}$ & $\begin{array}{l}2800 \\
(1400)\end{array}$ & $\begin{array}{l}1400 \\
(200)\end{array}$ & $180(30)$ & $120(20)$ & $2.5(0.4)$ & \\
\hline $\mathrm{JA}-32$ & 2533 & 2467 & $57(3)$ & $123(4)$ & $380(30)$ & $82(14)$ & $90(20)$ & $130(30)$ & 110 & $2.2(0.9)$ & \\
\hline JA-37 & 3497 & 3286 & $287(14)$ & $610(40)$ & $760(150)$ & & $\begin{array}{l}6000 \\
(800)\end{array}$ & $\begin{array}{l}28000 \\
(10000)\end{array}$ & $400(70)$ & $4.6(0.3)$ & $28(7)$ \\
\hline \multicolumn{12}{|c|}{ Desorption } \\
\hline$J A-18$ & 1420 & 1339 & $\begin{array}{l}15000 \\
(2000) \\
\end{array}$ & $\begin{array}{l}17500 \\
(700)\end{array}$ & $\begin{array}{l}280000 \\
(50000)\end{array}$ & $\begin{array}{l}1600 \\
(500)\end{array}$ & $\begin{array}{l}2400 \\
(300)\end{array}$ & $\begin{array}{l}1100 \\
(300)\end{array}$ & $350(140)$ & $9.4(1.4)$ & \\
\hline JA-32 & 2533 & 2467 & $53(3)$ & $175(11)$ & $490(40)$ & $530(120)$ & $850(130)$ & $\begin{array}{l}2200 \\
(600)\end{array}$ & & $8(2)$ & \\
\hline JA-37 & 3497 & 3286 & $312(9)$ & $850(50)$ & $920(40)$ & & $\begin{array}{l}11000 \\
(2000)\end{array}$ & $\begin{array}{l}32000 \\
(10000)\end{array}$ & $\begin{array}{l}1400 \\
(300)\end{array}$ & $9.9(0.4)$ & $170(50)$ \\
\hline
\end{tabular}

Source: Daniels et al. 1982 ([DIRS 105803], Tables XXI, XXII).

a Fractions do not contain less than $75 \mu \mathrm{m}$ diameter particles (TWS-INC-DP-63, R1).

bepth equivalent in Drill Hole USW-G1 according to position in geologic unit.

${ }^{c}$ Values in parentheses are the absolute value standard deviations of the means. 
Table I-6. Average Sorption and Desorption Ratios for Pulverized Tuff ${ }^{a}$ from Sorption and Desorption Experiments at Room Temperature (20 \pm 4 ${ }^{\circ} \mathrm{C}$ ) and at $70^{\circ} \mathrm{C}$ with $\mathrm{J}-13$ Well Water (Continued)

\begin{tabular}{|c|c|c|c|c|c|c|c|c|c|c|c|}
\hline \multirow[b]{2}{*}{ Sample } & \multirow[b]{2}{*}{ Depth, ft } & \multirow{2}{*}{$\begin{array}{c}\text { USW-G1 } \\
\text { depth }^{\mathrm{b}} \\
\mathrm{ft.}\end{array}$} & \multicolumn{9}{|c|}{$\mathrm{K}_{\mathrm{d}}, \mathrm{mL} / \mathrm{g}$} \\
\hline & & & $\mathrm{Sr}$ & Cs & $\mathrm{Ba}$ & $\mathrm{Ce}$ & $\mathrm{Eu}$ & $\mathrm{Am}$ & $\mathrm{Pu}$ & U (VI) & $\mathrm{Np}$ \\
\hline \multicolumn{12}{|l|}{$70^{\circ} \mathrm{C}$} \\
\hline \multicolumn{12}{|l|}{ Sorption } \\
\hline JA-18 & 1420 & 1339 & $\begin{array}{l}18000 \\
(2000)^{\mathrm{c,d}}\end{array}$ & $\begin{array}{l}18000 \\
(1000)\end{array}$ & $\begin{array}{l}49000 \\
(7000)\end{array}$ & & & & & $4.0(0.2)$ & \\
\hline JA-32 & 2533 & 2467 & $113(9)$ & $97(6)$ & $110(120)$ & $80(20)$ & $140(30)$ & $110(20)$ & & $11.7(1.7)$ & \\
\hline JA-37 & 3497 & 3286 & $\begin{array}{l}1050 \\
(130)\end{array}$ & $1360(85)$ & $\begin{array}{l}3670 \\
(700)\end{array}$ & & $\begin{array}{l}4200 \\
(400)\end{array}$ & $\begin{array}{l}1000 \\
(200)\end{array}$ & 240 & $16(2)$ & \\
\hline \multicolumn{12}{|c|}{ Desorption } \\
\hline JA-18 & 1420 & 1339 & $\begin{array}{l}21000 \\
(2000) \\
\end{array}$ & $\begin{array}{l}19300 \\
(1300) \\
\end{array}$ & $\begin{array}{l}108000 \\
(13000)\end{array}$ & & & & & $14(3)$ & \\
\hline JA-32 & 2533 & 2467 & $100(8)$ & $108(4)$ & $\begin{array}{l}1160 \\
(100)\end{array}$ & $640(8)$ & $\begin{array}{l}1800 \\
(300)\end{array}$ & & & $21.1(1)$ & \\
\hline JA-37 & 3497 & 3286 & $\begin{array}{l}1340 \\
(110)\end{array}$ & $\begin{array}{l}2700 \\
(500)\end{array}$ & $\begin{array}{l}5900 \\
(900)\end{array}$ & & $\begin{array}{l}14000 \\
(1000) \\
\end{array}$ & & & $47(6)$ & \\
\hline
\end{tabular}

Source: Daniels et al. 1982 ([DIRS 105803], Table XXVII).

${ }^{a}$ Fractions do not contain less than $75 \mu \mathrm{m}$ diameter particles (TWS-INC-DP-63, R1).

Depth equivalent in Drill Hole USW-G1 according to position in geologic unit.

c Nonweighted average.

${ }^{d}$ Values in parentheses are the absolute value standard deviations of the means. 
Table 1-7. Sorption and Desorption Ratios for Plutonium and Americium on Tuffaceous Core from Sorption and Desorption Experiments at Room Temperature $\left(20 \pm 4^{\circ} \mathrm{C}\right)$ and at $85^{\circ} \mathrm{C}$ with $\mathrm{J}$ 13 Well Water

\begin{tabular}{|c|c|c|c|c|c|c|c|}
\hline Core Sample & $\begin{array}{c}\text { Approach } \\
\text { to } \\
\text { equilibrium }\end{array}$ & $\begin{array}{c}\text { Temperature, } \\
{ }^{\circ} \mathbf{C}\end{array}$ & $\begin{array}{c}\text { Duration, } \\
\text { day }\end{array}$ & $\begin{array}{c}\text { Concentration } \\
\text { of sorbent, } \\
\mathrm{mol} / \mathrm{L}\end{array}$ & $\mathrm{K}_{\mathrm{d}}, \mathrm{mL} / \mathrm{g}$ & $1 / \mathrm{T} \times 1000, \mathrm{~K}^{-1}$ & $\log K_{d}$ \\
\hline \multicolumn{8}{|l|}{ Plutonium } \\
\hline \multirow[t]{12}{*}{ GU3-0433 } & Sorption & 20 & 42 & $2.60 \times 10^{-8}$ & 340 & 3.411 & 2.531 \\
\hline & & 20 & & & 500 & 3.411 & 2.699 \\
\hline & & 20 & & & 240 & 3.411 & 2.380 \\
\hline & & 20 & & & 240 & 3.411 & 2.380 \\
\hline & & 85 & & & 1700 & 2.792 & 3.230 \\
\hline & & 85 & & & 1800 & 2.792 & 3.255 \\
\hline & Desorption & 20 & 42 & $2.60 \times 10^{-8}$ & 960 & 3.411 & 2.982 \\
\hline & & 20 & & & 810 & 3.411 & 2.908 \\
\hline & & 20 & & & 100 & 3.411 & 2.000 \\
\hline & & 20 & & & 890 & 3.411 & 2.949 \\
\hline & & 85 & & & 4500 & 2.792 & 3.653 \\
\hline & & 85 & & & 7800 & 2.792 & 3.892 \\
\hline \multirow[t]{12}{*}{ GU-1203 } & Sorption & 20 & 42 & $2.40 \times 10^{-8}$ & 330 & 3.411 & 2.519 \\
\hline & & 20 & & & 480 & 3.411 & 2.681 \\
\hline & & 20 & & & 300 & 3.411 & 2.477 \\
\hline & & 20 & & & 340 & 3.411 & 2.531 \\
\hline & & 85 & & & 440 & 2.792 & 2.643 \\
\hline & & 85 & & & 940 & 2.792 & 2.973 \\
\hline & Desorption & 20 & 42 & $2.40 \times 10^{-8}$ & 920 & 3.411 & 2.964 \\
\hline & & 20 & & & 880 & 3.411 & 2.944 \\
\hline & & 20 & & & 920 & 3.411 & 2.964 \\
\hline & & 20 & & & 950 & 3.411 & 2.978 \\
\hline & & 85 & & & 620 & 2.792 & 2.792 \\
\hline & & 85 & & & 830 & 2.792 & 2.919 \\
\hline \multirow[t]{12}{*}{ GU3-1301 } & Sorption & 20 & 42 & $4.60 \times 10^{-8}$ & 380 & 3.411 & 2.580 \\
\hline & & 20 & & & 330 & 3.411 & 2.519 \\
\hline & & 20 & & & 200 & 3.411 & 2.301 \\
\hline & & 20 & & & 250 & 3.411 & 2.398 \\
\hline & & 85 & & & 2500 & 2.792 & 3.398 \\
\hline & & 85 & & & 7600 & 2.792 & 3.881 \\
\hline & Desorption & 20 & 42 & $4.60 \times 10^{-8}$ & 1800 & 3.411 & 3.255 \\
\hline & & 20 & & & 2400 & 3.411 & 3.380 \\
\hline & & 20 & & & 560 & 3.411 & 2.748 \\
\hline & & 20 & & & 600 & 3.411 & 2.778 \\
\hline & & 85 & & & 1300 & 2.792 & 3.114 \\
\hline & & 85 & & & 1600 & 2.792 & 3.204 \\
\hline
\end{tabular}

NOTE: $\quad$ *Fractions do not contain less than $75 \mu \mathrm{m}$ diameter particles (TWS-INC-DP-63, R1). Unqualified data from Thomas (1987 [DIRS 101361], pp. 58 to 62, Appendix). 
Table 1-7. Sorption and Desorption Ratios for Plutonium and Americium on Tuffaceous Core from Sorption and Desorption Experiments at Room Temperature $\left(20 \pm 4^{\circ} \mathrm{C}\right)$ and at $85^{\circ} \mathrm{C}$ with $\mathrm{J}-13$ Well Water (Continued)

\begin{tabular}{|c|c|c|c|c|c|c|c|}
\hline Core Sample & $\begin{array}{c}\text { Approach to } \\
\text { equilibrium }\end{array}$ & $\begin{array}{c}\text { Temperature, } \\
{ }^{\circ} \mathrm{C} \\
\end{array}$ & $\begin{array}{c}\text { Duration, } \\
\text { day }\end{array}$ & $\begin{array}{c}\text { Concentration } \\
\text { of sorbent, } \\
\text { mol/L }\end{array}$ & $\mathrm{K}_{\mathrm{d}}, \mathrm{mL} / \mathrm{g}$ & $1 / \mathrm{T} \times 1000, \mathrm{~K}^{-1}$ & $\log K_{d}$ \\
\hline \multicolumn{8}{|l|}{ Americium } \\
\hline \multirow[t]{12}{*}{ GU3-0433 } & Sorption & 20 & 42 & $2.10 \times 10^{-7}$ & 3300 & 3.411 & 3.519 \\
\hline & & 20 & & & 2900 & 3.411 & 3.462 \\
\hline & & 20 & & & 3500 & 3.411 & 3.544 \\
\hline & & 20 & & & 3800 & 3.411 & 3.580 \\
\hline & & 85 & & & 2100 & 2.792 & 3.322 \\
\hline & & 85 & & & 4200 & 2.792 & 3.623 \\
\hline & Desorption & 20 & 42 & $2.10 \times 10^{-7}$ & 9500 & 3.411 & 3.978 \\
\hline & & 20 & & & 6000 & 3.411 & 3.778 \\
\hline & & 20 & & & 7800 & 3.411 & 3.892 \\
\hline & & 20 & & & 14000 & 3.411 & 4.146 \\
\hline & & 85 & & & 6700 & 2.792 & 3.826 \\
\hline & & 85 & & & 3500 & 2.792 & 3.544 \\
\hline \multirow[t]{12}{*}{ GU3-1203 } & Sorption & 20 & 42 & $2.10 \times 10^{-7}$ & 1400 & 3.411 & 3.146 \\
\hline & & 20 & & & 1200 & 3.411 & 3.079 \\
\hline & & 20 & & & 860 & 3.411 & 2.934 \\
\hline & & 20 & & & 970 & 3.411 & 2.987 \\
\hline & & 85 & & & 4200 & 2.792 & 3.623 \\
\hline & & 85 & & & 4300 & 2.792 & 3.633 \\
\hline & Desorption & 20 & 42 & $2.10 \times 10^{-7}$ & 900 & 3.411 & 2.954 \\
\hline & & 20 & & & 1800 & 3.411 & 3.255 \\
\hline & & 20 & & & 990 & 3.411 & 2.996 \\
\hline & & 20 & & & 1400 & 3.411 & 3.146 \\
\hline & & 85 & & & 7200 & 2.792 & 3.857 \\
\hline & & 85 & & & 10500 & 2.792 & 4.021 \\
\hline \multirow[t]{12}{*}{ GU3-1301 } & Sorption & 20 & 42 & $2.40 \times 10^{-7}$ & 1600 & 3.411 & 3.204 \\
\hline & & 20 & & & 1400 & 3.411 & 3.146 \\
\hline & & 20 & & & 2000 & 3.411 & 3.301 \\
\hline & & 20 & & & 2050 & 3.411 & 3.311 \\
\hline & & 85 & & & 8600 & 2.792 & 3.934 \\
\hline & & 85 & & & 11000 & 2.792 & 4.041 \\
\hline & Desorption & 20 & 42 & $2.40 \times 10^{-7}$ & 3800 & 3.411 & 3.580 \\
\hline & & 20 & & & 3200 & 3.411 & 3.505 \\
\hline & & 20 & & & 1500 & 3.411 & 3.176 \\
\hline & & 20 & & & 1500 & 3.411 & 3.176 \\
\hline & & 85 & & & 23000 & 2.792 & 4.362 \\
\hline & & 85 & & & 19000 & 2.792 & 4.279 \\
\hline
\end{tabular}

NOTE: *Fractions do not contain less than $75 \mu \mathrm{m}$ diameter particles (TWS-INC-DP-63, R1). Unqualified data from Thomas (1987 [DIRS 101361], pp. 58 to 62, Appendix). 
The data from Summary Report on the Geochemistry of Yucca Mountain and Environs (Daniels et al. 1982 [DIRS 105803]) are tabulated for each given radioelement averaged at "room temperature" (estimated at $20^{\circ} \mathrm{C} \pm 4^{\circ} \mathrm{C}$ ) and a smaller set of measurements averaged at $70^{\circ} \mathrm{C}$ in Tables XXI, XXII and XXVII, respectively, in the subject reference. These data are summarized in Table I-6. The radioelements for which there are $\mathrm{K}_{\mathrm{d}}$ data include $\mathrm{Cs}, \mathrm{Sr}, \mathrm{Ba}, \mathrm{Ce}$, $\mathrm{Eu}, \mathrm{Am}, \mathrm{Pu}$, and $\mathrm{U}(\mathrm{VI})$. Advantage was taken of this compilation and the data plotted as Log $\mathrm{K}_{\mathrm{d}}$ versus $1 / T\left[\mathrm{~K}^{-1}\right]$, as illustrated in Figures I-2(a) to I-2(h). Because only two previously averaged values were entered, no statistical fit can be obtained for a line passing through two points. In addition, Thomas (1987 [DIRS 101361]) also includes data on Pu and Am adsorption on core material at 20 and $85^{\circ} \mathrm{C}$, which are summarized in Table I-7. The corresponding Log $\mathrm{K}_{\mathrm{d}}$ values were plotted and regressed to obtain linear equations, as illustrated in Figures I-3(a) to I-3(b). The calculated enthalpies of sorption are summarized in Table I-8. 


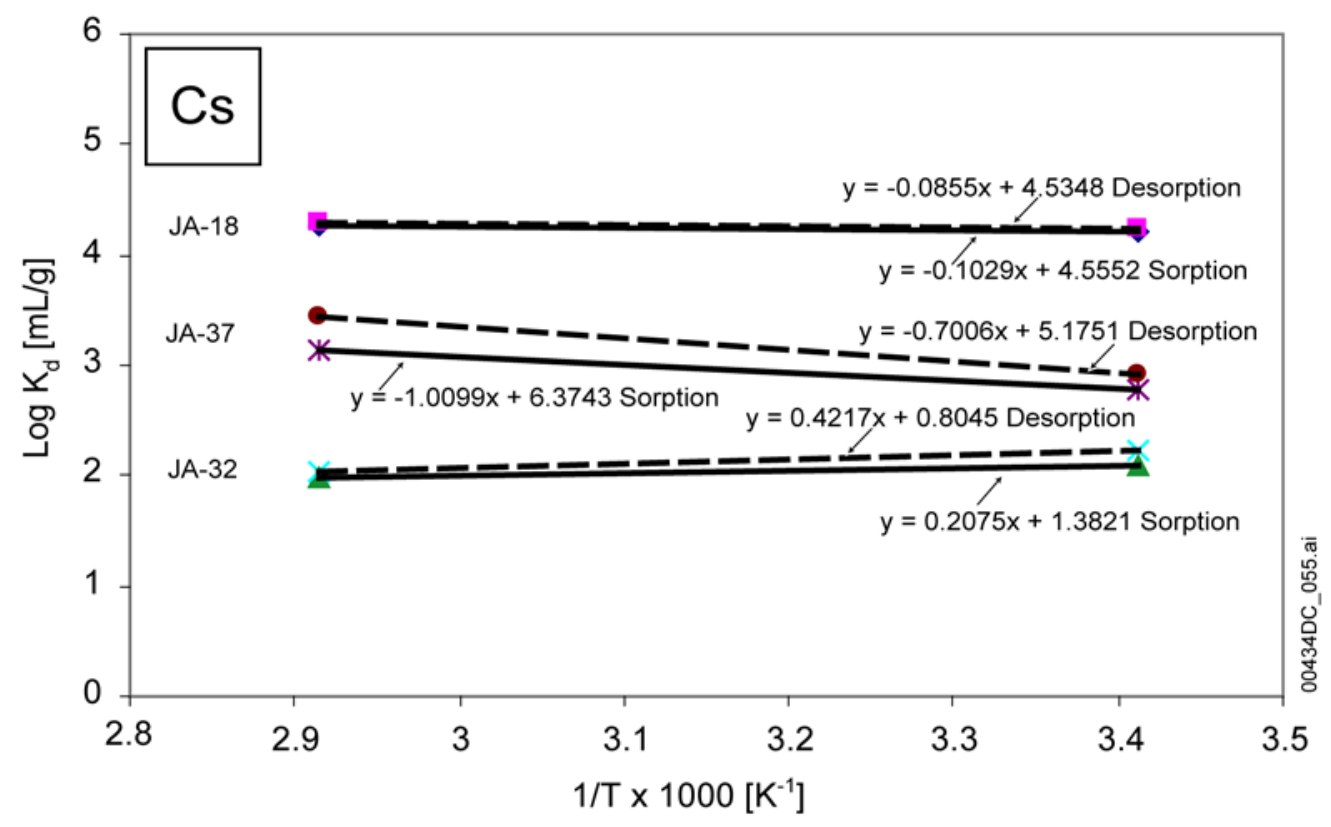

(a)

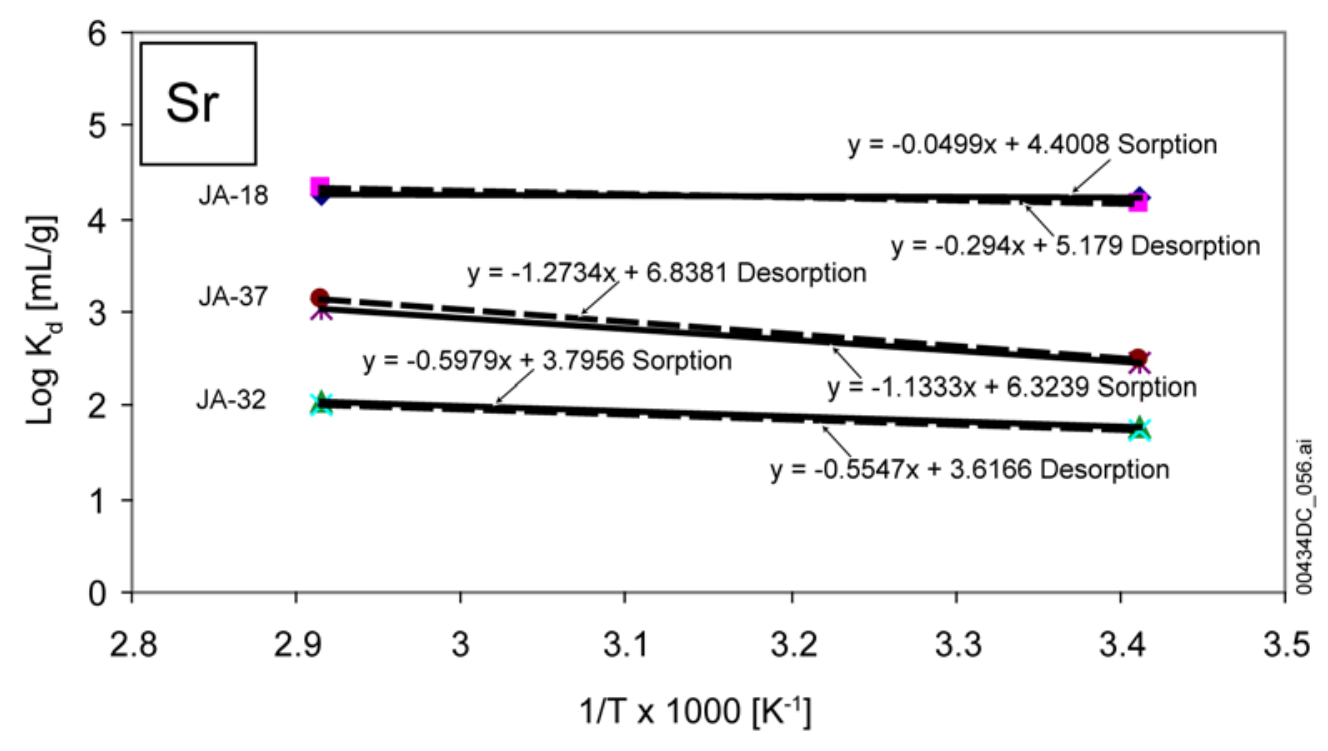

Output DTN: LB0311ABSTHCR2.004.

Source: Daniels et al. 1982 ([DIRS 105803], pp. 78 to 79, 111, Tables XXI, XXII, and XXVII).

NOTE: Data listed in Table I-6; data summarized in Table I-8.

Figure I-2(a)-(b). Calculated Values of log $\mathrm{K}_{d}$ as a Function of Reciprocal Absolute Temperature, Based on Both Sorption and Desorption Radioelements on Crushed Yucca Mountain Tuff 


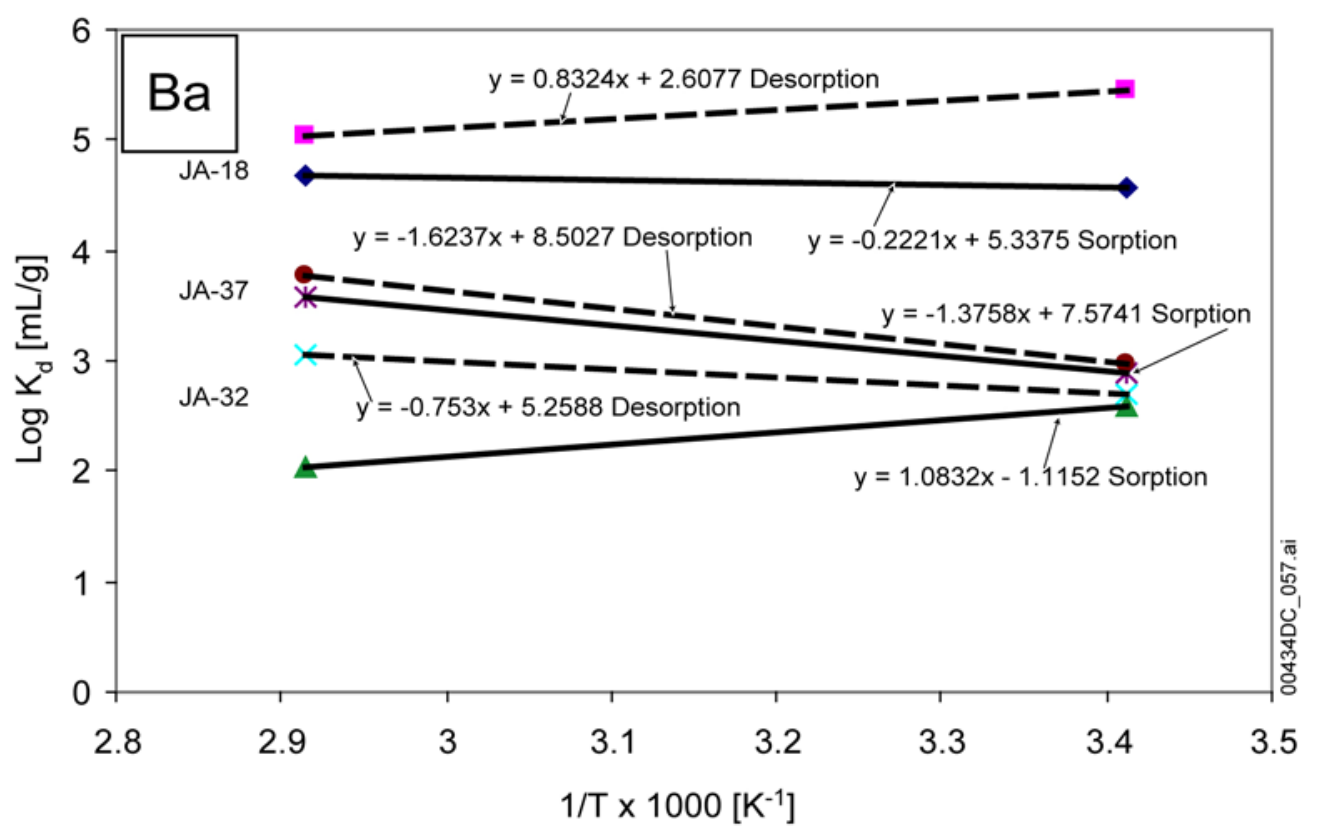

(c)

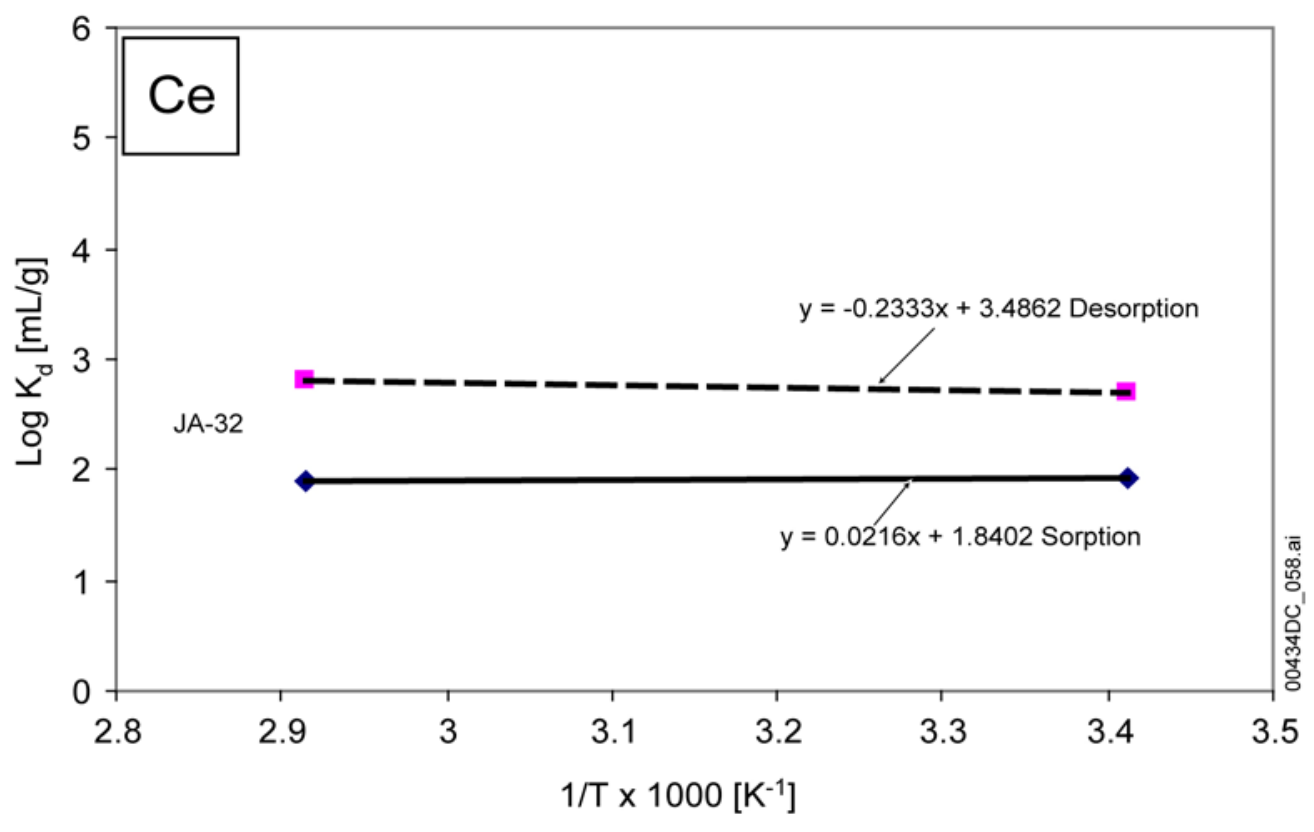

(d)

Output DTN: LB0311ABSTHCR2.004.

Source: Daniels et al. 1982 ([DIRS 105803], pp. 78, 79, 111,Tables XXI, XXII, and XXVII).

NOTE: Data listed in Table I-6; data summarized in Table I-8.

Figure I-2(c)-(d). Calculated Values of $\log K_{d}$ as a Function of Reciprocal Absolute Temperature Based on Both Sorption and Desorption Radioelements on Crushed Yucca Mountain Tuff (Continued) 


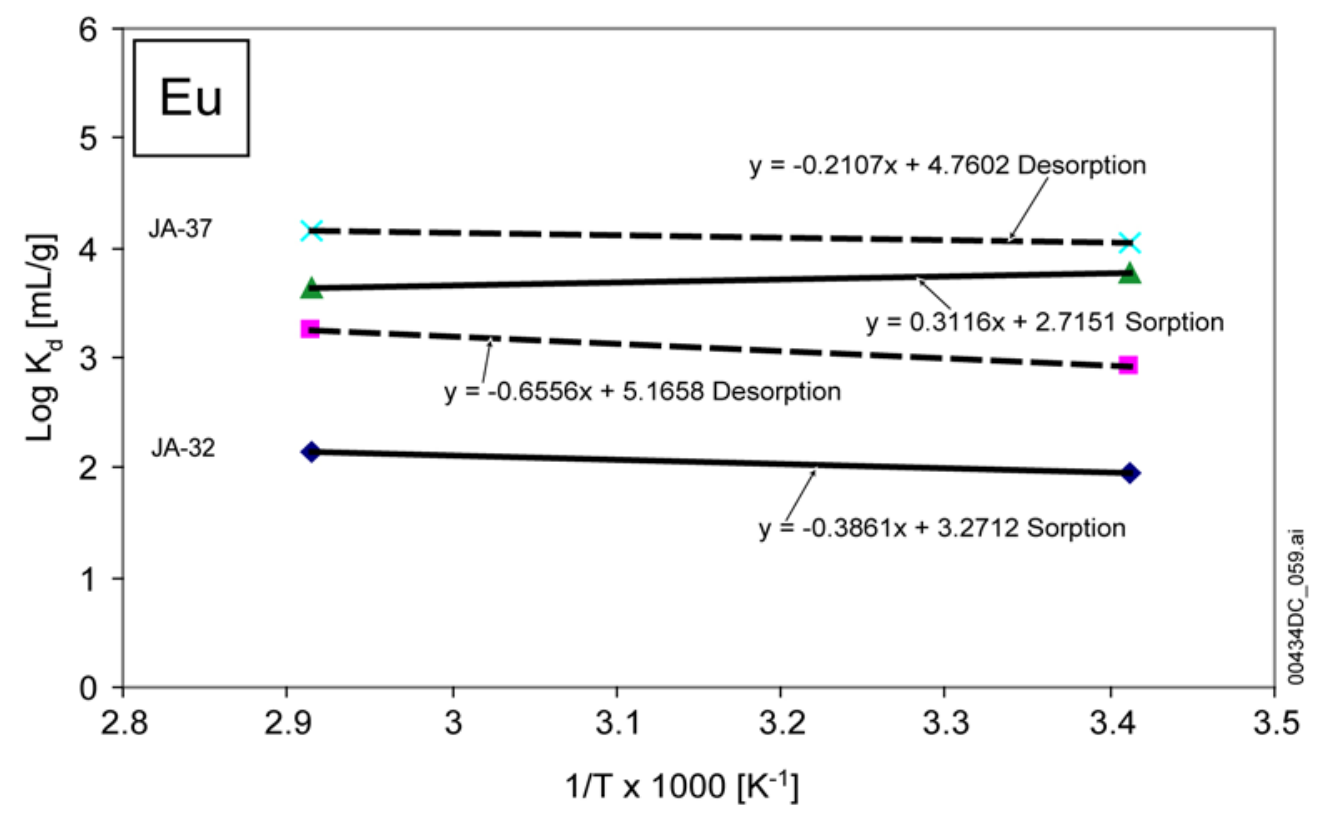

(e)

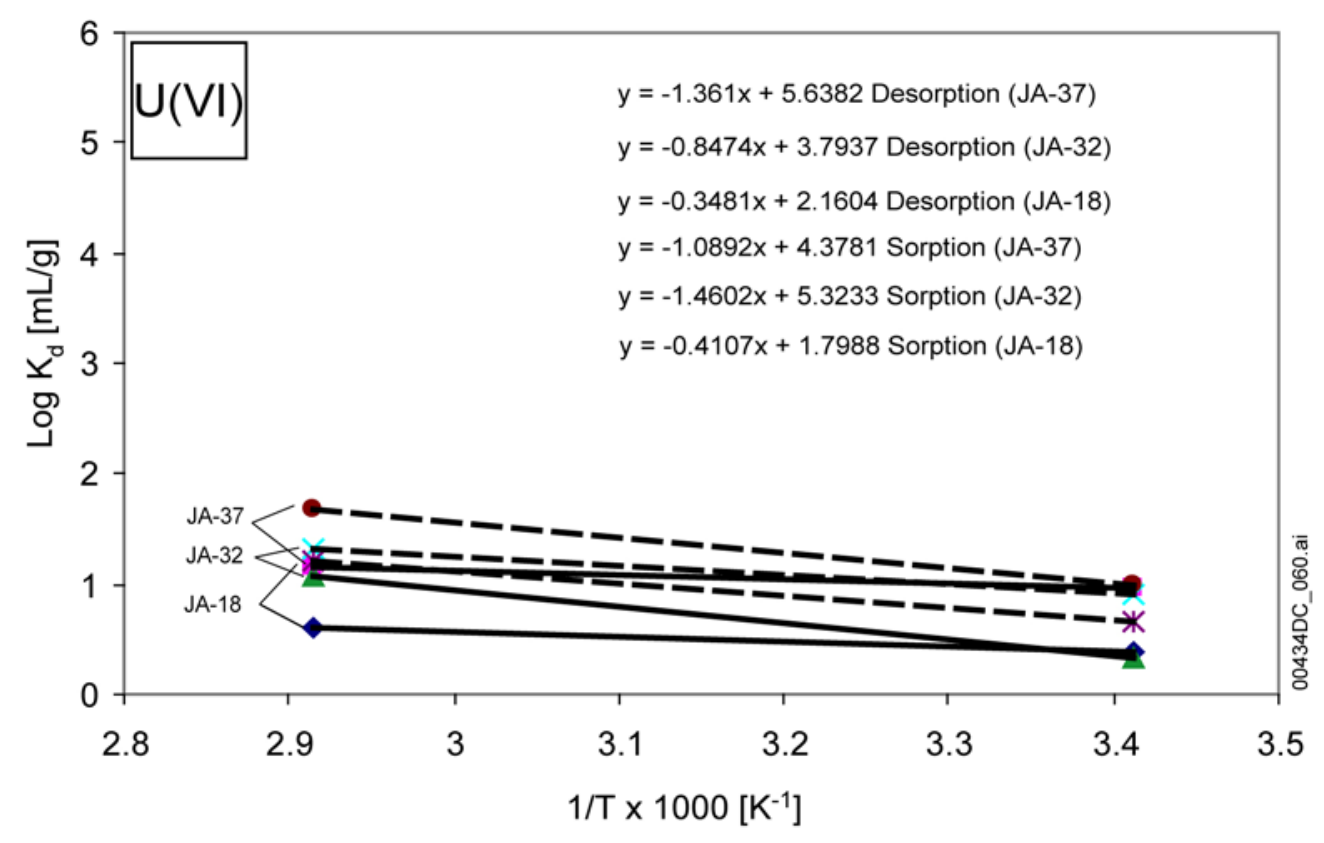

Output DTN: LB0311ABSTHCR2.004.

Source: Daniels et al. 1982 ([DIRS 105803], pp. 78, 79, 111, Tables XXI, XXII, and XXVII).

NOTE: Data listed in Table I-6; data summarized in Table I-8.

Figure I-2(e)-(f). Calculated Values of log $\mathrm{K}_{d}$ as a Function of Reciprocal Absolute Temperature, Based on Both Sorption and Desorption Radioelements on Crushed Yucca Mountain Tuff (Continued) 


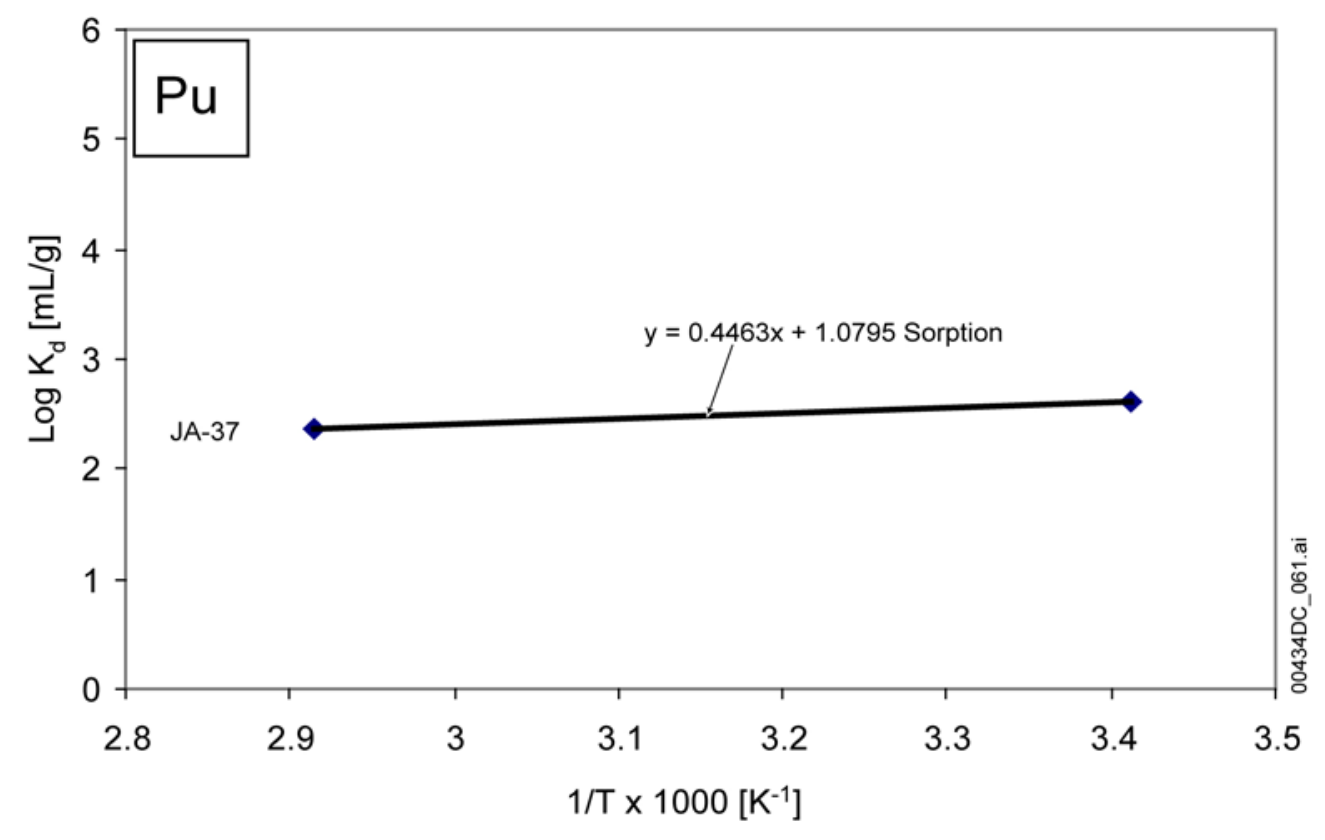

(g)

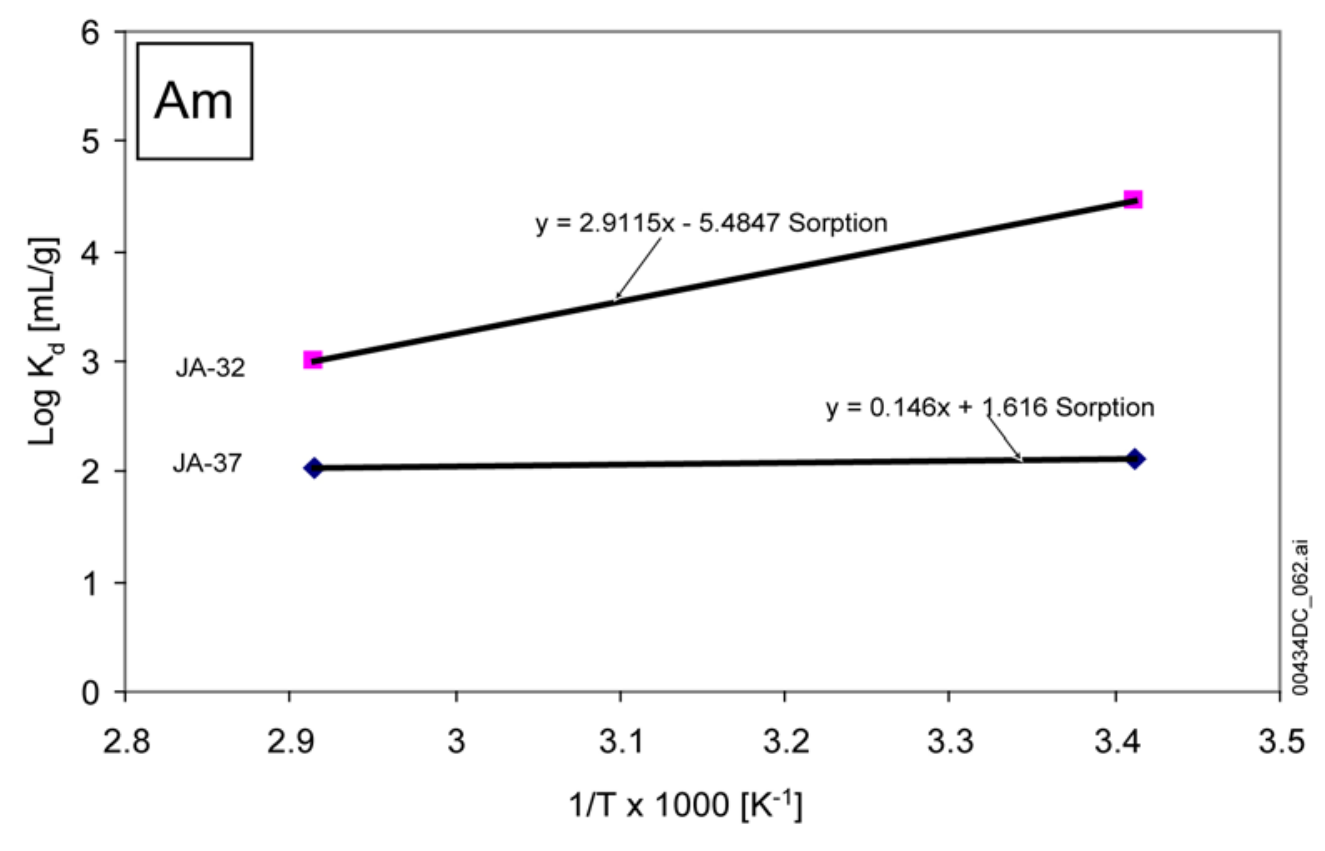

(h)

Output DTN: LB0311ABSTHCR2.004

Source: Daniels et al. 1982 ([DIRS 105803], pp. 78, 79, 111, Tables XXI, XXII, and XXVII).

NOTE: Data listed in Table I-6; data summarized in Table I-8.

Figure I-2(g)-(h). Calculated Values of $\log \mathrm{K}_{d}$ as a Function of Reciprocal Absolute Temperature, Based on Both Sorption and Desorption Radioelements on Crushed Yucca Mountain Tuff (Continued) 
(a)
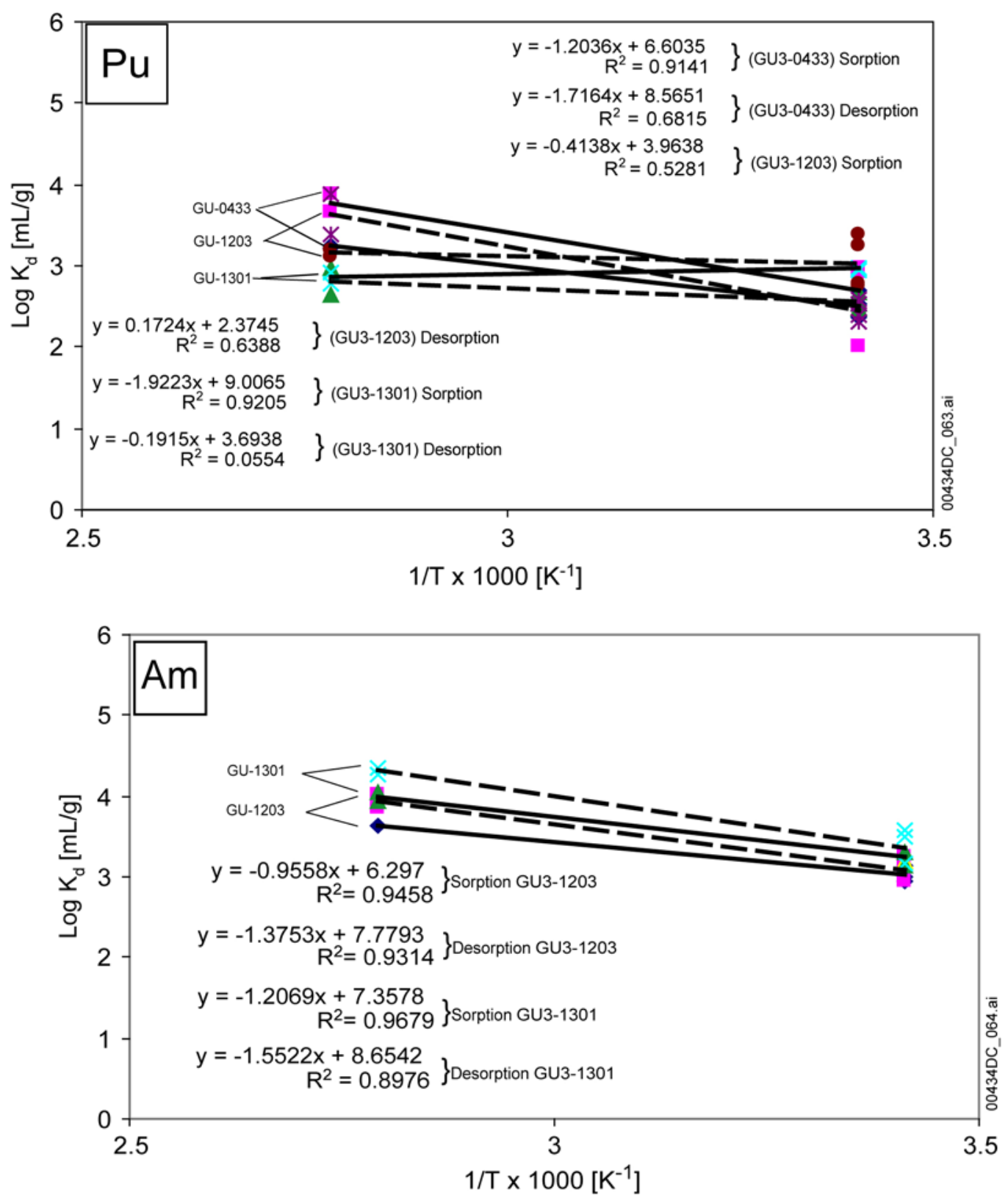

(b)

Output DTN: LB0311ABSTHCR2.004.

Source: Thomas 1987 ([DIRS 101361], pp. 58 to 62, Appendix), listed in Table I-7. Data summarized in Table I-8.

Figure I-3(a)-(b). Calculated Value of log $\mathrm{K}_{d}$ as a Function of Reciprocal Absolute Temperature, Based on Both Sorption and Desorption of Specified Radioelements on Tuff Core Samples from Yucca Mountain 
Table I-8. Calculation of the Enthalpy of Sorption of Aqueous Species on Various Sorbents ${ }^{a}$

\begin{tabular}{|c|c|c|c|c|c|c|}
\hline Aq. Species & Sorbent & $\begin{array}{c}\text { Temperature } \\
\text { Range }{ }^{\circ} \mathrm{C}\end{array}$ & Slope & R2 & $\begin{array}{c}\Delta \mathrm{Hr} \\
\mathrm{kcal} / \mathrm{mol}\end{array}$ & Notes \\
\hline \multirow[t]{6}{*}{$\mathrm{Sr}$} & $\mathrm{JA}-18$ & $20-70$ & -0.0499 & & +0.23 & Sorption \\
\hline & & & -0.294 & & +1.35 & Desorption \\
\hline & JA-32 & & -0.5979 & & +2.74 & Sorption \\
\hline & & & -0.5547 & & +2.54 & Desorption \\
\hline & JA-37 & & -1.1333 & & +5.19 & Sorption \\
\hline & & & -1.2753 & & +5.83 & Desorption \\
\hline \multirow[t]{6}{*}{$\mathrm{Cs}$} & JA-18 & & -0.1029 & & +0.47 & Sorption \\
\hline & & & -0.0855 & & +0.39 & Desorption \\
\hline & JA-32 & & +0.2075 & & -0.95 & Sorption \\
\hline & & & +0.4217 & & -1.93 & Desorption \\
\hline & JA-37 & & -0.7006 & & +3.21 & Sorption \\
\hline & & & -1.0099 & & +4.62 & Desorption \\
\hline \multirow[t]{6}{*}{$\mathrm{Ba}$} & JA-18 & & -0.2221 & & +1.02 & Sorption \\
\hline & & & +0.8324 & & -3.81 & Desorption \\
\hline & JA-32 & & +1.0832 & & -4.96 & Sorption \\
\hline & & & -0.7530 & & +3.45 & Desorption \\
\hline & JA-37 & & -1.3758 & & +6.30 & Sorption \\
\hline & & & -1.6237 & & +7.43 & Desorption \\
\hline \multirow[t]{2}{*}{$\mathrm{Ce}$} & JA-32 & & +0.0216 & & -0.10 & Sorption \\
\hline & & & -0.2333 & & +1.07 & Desorption \\
\hline \multirow[t]{4}{*}{$\mathrm{Eu}$} & $\mathrm{JA}-32$ & & -0.3861 & & +1.77 & Sorption \\
\hline & & & -0.6556 & & +3.00 & Desorption \\
\hline & JA-37 & & +0.3116 & & -1.43 & Sorption \\
\hline & & & -0.2107 & & +0.96 & Desorption \\
\hline \multirow[t]{2}{*}{$\mathrm{Am}$} & JA-32 & & +0.146 & & -0.67 & Sorption \\
\hline & JA-37 & & +2.9115 & & -13.32 & Sorption \\
\hline $\mathrm{Pu}$ & JA-37 & & +0.4463 & & -2.04 & Sorption \\
\hline \multirow[t]{6}{*}{$\mathrm{U}(\mathrm{VI})$} & JA-18 & & -0.4107 & & +1.88 & Sorption \\
\hline & & & -0.3481 & & +1.59 & Desorption \\
\hline & JA-32 & & -1.4602 & & +6.68 & Sorption \\
\hline & & & -0.8474 & & +3.88 & Desorption \\
\hline & JA-37 & & -1.0892 & & +4.98 & Sorption \\
\hline & & & -1.3610 & & +6.23 & Desorption \\
\hline \multirow[t]{6}{*}{$\mathrm{Pu}$} & GU3-0433 & $20-85$ & -1.2036 & 0.914 & +5.51 & Sorption \\
\hline & & & -1.7164 & 0.682 & +7.85 & Desorption \\
\hline & GU3-1203 & & -0.4138 & 0.528 & +1.89 & Sorption \\
\hline & & & 0.1724 & 0.639 & -0.79 & Desorption \\
\hline & GU3-1301 & & -1.9223 & 0.921 & +8.80 & Sorption \\
\hline & & & -0.1915 & 0.055 & +0.88 & Desorption \\
\hline \multirow[t]{4}{*}{ Am } & GU3-1203 & & -0.9558 & 0.946 & +4.37 & Sorption \\
\hline & & & -1.3753 & 0.931 & +6.29 & Desorption \\
\hline & GU3-1301 & & -1.2069 & 0.968 & +5.52 & Sorption \\
\hline & & & -1.5522 & 0.898 & +7.10 & Desorption \\
\hline
\end{tabular}

Output DTN: LB0311ABSTHCR2.004.

Source: Data from Daniels et al. 1982 ([DIRS 105803], Tables XXI, XXII, and XXVII) and Thomas 1987 ([DIRS 101361], pp. 58 to 62, Appendix 0).

a Summarized from Figure I-2, which plots data in Tables I-6 and I-7. 


\section{I1.4.3 Data from the Refereed Literature}

Only a few papers report on the temperature dependence of radionuclide sorption on natural substrates. Several other papers containing useful information in the general subject area were also reviewed and data extracted for comparative purposes. A summary of information contained in journal articles considered for further review is given in Tables I-9 and I-10. The refereed data fall into two classifications: (1) (Table I-9), raw data, where $K_{d}$ values at various temperatures are provided without further evaluation of sorption thermodynamic properties in relation to temperature; and (2) (Table I-10), evaluated data, where $\Delta \mathrm{G}_{\mathrm{r}}(\mathrm{T}), \Delta \mathrm{H}_{\mathrm{r}}$, and $\Delta \mathrm{S}_{\mathrm{r}}$ of the sorption reaction under consideration have been determined. The latter data sources commonly omit tabulated sorption coefficient data, data, instead, being presented in graphical form. Note that the data summarized in these two tables were collected using various concentrations of sorbent and $\mathrm{pH}$ values, as well as using aqueous solutions containing differing supporting electrolytes at various specified concentrations. Details concerning these study conditions can be found in the cited sources. Direct comparison between data sets from these sources is, therefore, not feasible, as will be discussed further.

Table I-9. Sorption Coefficients, $K_{d}$, for Various Metal Species, Reported in the Literature (Unqualified Data)

\begin{tabular}{|c|c|c|c|c|}
\hline Sorbate and Sorbent & $\mathrm{T},{ }^{\circ} \mathrm{C}$ & $\mathrm{K}_{\mathrm{d}}, \mathrm{mL} / \mathrm{g}$ & $\begin{array}{c}1 / \mathrm{T} \times 1000, \\
\mathrm{~K}^{-1}\end{array}$ & $\log K_{d}$ \\
\hline \multicolumn{5}{|c|}{ van Geldermalsen and Duursma 1984 (DIRS 165087) } \\
\hline \multirow[t]{6}{*}{ Cd, Estuarine Sediments } & 4 & 1765 & 3.608 & 3.247 \\
\hline & 20 & 1150 & 3.411 & 3.061 \\
\hline & 37 & 941 & 3.224 & 2.974 \\
\hline & 50 & 624 & 3.095 & 2.795 \\
\hline & 75 & 246 & 2.872 & 2.391 \\
\hline & 90 & 111 & 2.754 & 2.045 \\
\hline \multirow[t]{4}{*}{ Cd, Marine Sediments } & 4 & 56 & 3.608 & 1.748 \\
\hline & 37 & 45.9 & 3.224 & 1.662 \\
\hline & 60 & 28.6 & 3.002 & 1.456 \\
\hline & 90 & 17.6 & 2.754 & 1.246 \\
\hline \multirow{3}{*}{\begin{tabular}{|l} 
Pu-239, Estuarine \\
Sediments
\end{tabular}} & 4 & & 3.608 & 5.945 \\
\hline & 20 & & 3.411 & 5.624 \\
\hline & 80 & & 2.832 & 5.038 \\
\hline \multirow[t]{4}{*}{ Pu-239, Marine Sediments } & 4 & & 3.608 & 6.360 \\
\hline & 20 & & 3.411 & 6.101 \\
\hline & 80 & & 2.832 & 5.904 \\
\hline & 140 & & 2.420 & 6.027 \\
\hline \multirow{2}{*}{$\begin{array}{l}\text { Eu-152, Estuarine } \\
\text { Sediments }\end{array}$} & 4 & & 3.608 & 4.284 \\
\hline & 80 & & 2.832 & 4.277 \\
\hline \multirow[t]{2}{*}{ Eu-152, Marine Sediments } & 4 & & 3.608 & 4.678 \\
\hline & 80 & & 2.832 & 4.827 \\
\hline
\end{tabular}


Table I-9. Sorption Coefficients, $K_{d}$, for Various Metal Species, Reported in the Literature (Unqualified Data) (Continued)

\begin{tabular}{|c|c|c|c|c|}
\hline Sorbate and Sorbent & $\mathbf{T},{ }^{\circ} \mathrm{C}$ & $\mathrm{K}_{\mathrm{d}}, \mathrm{mL} / \mathrm{g}$ & $\begin{array}{c}1 / \mathrm{T} \times 1000 \\
\mathrm{~K}^{-1}\end{array}$ & $\log K_{d}$ \\
\hline \multicolumn{5}{|c|}{ Kenna 1981 (DIRS 165049); Erickson 1979 (DIRS 164876) } \\
\hline \multirow[t]{3}{*}{ Cs, Seabed Smectite Clay } & 4 & 5012 & 3.608 & 3.700 \\
\hline & 20 & 1180 & 3.411 & 3.072 \\
\hline & 60 & 343 & 3.002 & 2.535 \\
\hline \multirow[t]{3}{*}{ Sr, Seabed Smectite Clay } & 4 & 200 & 3.608 & 2.301 \\
\hline & 20 & 126 & 3.411 & 2.100 \\
\hline & 60 & 170 & 3.002 & 2.230 \\
\hline \multirow[t]{3}{*}{ Eu, Seabed Smectite Clay } & 4 & 2500 & 3.608 & 3.398 \\
\hline & 20 & $1.00 \mathrm{E}+07$ & 3.411 & 7.000 \\
\hline & 60 & $1.00 \mathrm{E}+07$ & 3.002 & 7.000 \\
\hline \multirow[t]{3}{*}{$\mathrm{Ba}$, Seabed Smectite Clay } & 4 & $1.12 \mathrm{E}+05$ & 3.608 & 5.049 \\
\hline & 20 & $9.30 \mathrm{E}+04$ & 3.411 & 4.968 \\
\hline & 60 & $5.50 \mathrm{E}+04$ & 3.002 & 4.740 \\
\hline \multicolumn{5}{|c|}{ Aksyuk et al. (1995 [DIRS 165054]) } \\
\hline \multirow[t]{23}{*}{ Sr, porphyrite } & 20 & 308 & 3.411 & 2.489 \\
\hline & & 280 & 3.411 & 2.447 \\
\hline & & 60 & 3.411 & 1.778 \\
\hline & & 33.2 & 3.411 & 1.521 \\
\hline & & 852 & 3.411 & 2.930 \\
\hline & & 25.2 & 3.411 & 1.401 \\
\hline & & 368 & 3.411 & 2.566 \\
\hline & & 1192 & 3.411 & 3.076 \\
\hline & & 280 & 3.411 & 2.447 \\
\hline & & 596 & 3.411 & 2.775 \\
\hline & & 2548 & 3.411 & 3.406 \\
\hline & & 1880 & 3.411 & 3.274 \\
\hline & & 1824 & 3.411 & 3.261 \\
\hline & & 138.4 & 3.411 & 2.141 \\
\hline & & 38.4 & 3.411 & 1.584 \\
\hline & & 118.4 & 3.411 & 2.073 \\
\hline & & 86.4 & 3.411 & 1.937 \\
\hline & 100 & 11.96 & 2.680 & 1.078 \\
\hline & & 1.86 & 2.680 & 0.271 \\
\hline & & 5.86 & 2.680 & 0.768 \\
\hline & & 4.03 & 2.680 & 0.606 \\
\hline & & 17.87 & 2.680 & 1.252 \\
\hline & & 18.82 & 2.680 & 1.275 \\
\hline
\end{tabular}


Table I-9. Sorption Coefficients, $K_{d}$, for Various Metal Species, Reported in the Literature (Unqualified Data) (Continued)

\begin{tabular}{|c|c|c|c|c|}
\hline Sorbate and Sorbent & $\mathrm{T},{ }^{\circ} \mathrm{C}$ & $\mathrm{K}_{\mathrm{d}}, \mathrm{mL} / \mathrm{g}$ & $\begin{array}{c}1 / \mathrm{T} \times 1000, \\
\mathrm{~K}^{-1}\end{array}$ & $\log K_{d}$ \\
\hline \multicolumn{5}{|c|}{ Aksyuk et al. (1995 [DIRS 165054]) (Continued) } \\
\hline & 200 & 38.63 & 2.113 & 1.587 \\
\hline & & 17.76 & 2.113 & 1.249 \\
\hline & & 29.32 & 2.113 & 1.467 \\
\hline & & 4.53 & 2.113 & 0.656 \\
\hline & & 16.66 & 2.113 & 1.222 \\
\hline & & 16.36 & 2.113 & 1.214 \\
\hline & 250 & 8.89 & 1.911 & 0.949 \\
\hline & & 13.95 & 1.911 & 1.145 \\
\hline & & 1.37 & 1.911 & 0.137 \\
\hline & & 13.05 & 1.911 & 1.116 \\
\hline & & 6.00 & 1.911 & 0.778 \\
\hline & & 2891.4 & 1.911 & 3.461 \\
\hline & & 2916.1 & 1.911 & 3.465 \\
\hline
\end{tabular}


Table I-10. Enthalpy, Entropy, and Gibbs Free Energy of Adsorption of Aqueous Species on Various Sorbents (Unqualified Data from the Published Literature)

\begin{tabular}{|c|c|c|c|c|c|c|c|c|}
\hline Sorbate & $\begin{array}{c}\text { Sorbate Concentration, } \\
\mathrm{mmol} / \mathrm{dm}^{3}\end{array}$ & Sorbent & Model & $\begin{array}{c}\text { Temperature, } \\
{ }^{\circ} \mathrm{C} \\
\end{array}$ & $\begin{array}{c}\Delta \mathrm{H}^{\circ}{ }_{\text {ads }} \\
\mathrm{kcal} / \mathrm{mol}\end{array}$ & $\begin{array}{l}\Delta \mathbf{S}^{\circ} \text { ads, } \\
\mathrm{cal} / \mathrm{mol} \cdot \mathrm{K}\end{array}$ & $\begin{array}{r}\Delta \mathbf{G}^{\circ}{ }_{\text {ads }} \\
\mathrm{kcal} / \mathrm{mol}\end{array}$ & Reference \\
\hline \multirow[t]{5}{*}{$\mathrm{Cs}(\mathrm{I})$} & 3.7 & $\mathrm{KCNF}$ & Assumed linear ${ }^{a}$ & 20 & 11.13 & 57.36 & -6.01 & $\begin{array}{l}\text { Ishfaq et al. 1997 } \\
\text { (DIRS 164879) }\end{array}$ \\
\hline & & & & 30 & & 56.88 & -6.33 & \\
\hline & & & & 40 & & 56.41 & -6.51 & \\
\hline & & & & 50 & & 56.41 & -7.08 & \\
\hline & & & & 60 & & 56.88 & -7.82 & \\
\hline \multirow[t]{12}{*}{$\mathrm{Co}(\mathrm{II})$} & $5 \times 10^{-4}-10.0$ & sepiolite & Frumkin & 20 & 0.98 & 16.13 & -3.75 & $\begin{array}{l}\text { Kara et al. } 2003 \\
\text { (DIRS 164880) }\end{array}$ \\
\hline & & & & 30 & & 16.23 & -3.93 & \\
\hline & & & & 40 & & 16.13 & -4.07 & \\
\hline & & & Modified Frumkin & 20 & 0.89 & 15.82 & -3.74 & \\
\hline & & & & 30 & & 15.89 & -3.92 & \\
\hline & & & & 40 & & 15.82 & -4.06 & \\
\hline & & & Langmuir & 20 & -1.37 & 8.91 & -3.27 & \\
\hline & & & & 30 & & 6.29 & -3.28 & \\
\hline & & & & 40 & & 6.48 & -3.40 & \\
\hline & & & Flory-Higgins & 20 & 0.22 & 12.52 & -3.45 & \\
\hline & & & & 30 & & 12.52 & -3.58 & \\
\hline & & & & 40 & & 11.76 & -3.47 & \\
\hline \multirow[t]{12}{*}{$\mathrm{Fe}(\mathrm{II})$} & 1.79 & bentonite & Assumed linear ${ }^{b}$ & 25 & 8.14 & 42.30 & -5.52 & $\begin{array}{l}\text { Rauf and Tahir } 2000 \\
\text { (DIRS 164893) }\end{array}$ \\
\hline & & & & 30 & & & -5.09 & \\
\hline & & & & 40 & & & -4.67 & \\
\hline & & & & 50 & & & -4.46 & \\
\hline & 3.58 & & & 25 & 16.20 & 67.29 & -5.54 & \\
\hline & & & & 30 & & & -4.87 & \\
\hline & & & & 40 & & & -4.19 & \\
\hline & & & & 50 & & & -3.86 & \\
\hline & 5.37 & & & 25 & 16.03 & 67.29 & -5.70 & \\
\hline & & & & 30 & & & -5.03 & \\
\hline & & & & 40 & & & -4.36 & \\
\hline & & & & 50 & & & -4.02 & \\
\hline
\end{tabular}


Table I-10. Enthalpy, Entropy, and Gibbs Free Energy of Adsorption of Aqueous Species on Various Sorbents (Unqualified Data from the Published Literature) (Continued)

\begin{tabular}{|c|c|c|c|}
\hline Sorbate & $\begin{array}{c}\text { Sorbate } \\
\text { Concentration, } \\
\text { mmol/dm }\end{array}$ & Sorbent & Model \\
\hline $\mathrm{Mn}(\mathrm{II})$ & 1.82 & & \\
\hline & & & \\
\hline & & & \\
\hline & 3.64 & & \\
\hline & & & \\
\hline & & & \\
\hline & 5.46 & & \\
\hline & & & \\
\hline & & & \\
\hline $\mathrm{Cu}(\mathrm{II})$ & 0.157 & $\alpha$-Al hydroxide & \multirow{2}{*}{$\begin{array}{l}\text { Calculation of the apparent } \\
\text { stability constant, } \mathrm{K}_{\mathrm{s}} \text {, for the } \\
\text { surface complexation reaction: } \\
\mathrm{AlO}^{-}+\mathrm{M}^{2+}=\mathrm{AlOM}^{+}\end{array}$} \\
\hline & & & \\
\hline $\mathrm{Zn}(\mathrm{II})$ & 0.153 & & \\
\hline & & & \\
\hline & & & \\
\hline $\mathrm{Co}(\mathrm{II})$ & 0.170 & & \\
\hline & & & \\
\hline & & & \\
\hline $\mathrm{Ni}(\mathrm{II})$ & 0.170 & & \\
\hline & & & \\
\hline & & & \\
\hline $\mathrm{Ag}(\mathrm{I})$ & $1-200$ & bentonite & \multirow{2}{*}{$\begin{array}{l}\text { Binding constant, } \mathrm{K} \text {, for } \\
\text { Langmuir isotherm }^{\mathrm{c}}\end{array}$} \\
\hline & & & \\
\hline
\end{tabular}

\begin{tabular}{|c|c|c|c|c|}
\hline $\begin{array}{c}\text { Temperature, } \\
{ }^{\circ} \mathrm{C}\end{array}$ & $\begin{array}{l}\Delta \mathrm{H}^{\circ} \text { ads, } \\
\mathrm{kJ} / \mathrm{mol}\end{array}$ & $\begin{array}{l}\Delta \mathrm{S}^{\circ}{ }_{\text {ads }} \\
\mathrm{J} / \mathrm{mol} \cdot \mathrm{K}\end{array}$ & $\begin{array}{l}\Delta \mathbf{G}^{\circ}{ }_{\text {ads }} \\
\mathrm{kJ} / \mathrm{mol}\end{array}$ & Reference \\
\hline 25 & -3.13 & 0.12 & -3.16 & $\begin{array}{l}\text { Rauf and Tahir } 2000 \\
\text { (DIRS 164893) }\end{array}$ \\
\hline 30 & & & -3.16 & \\
\hline 40 & & & -3.16 & \\
\hline 50 & & & -3.16 & \\
\hline 25 & -2.32 & 0.63 & -2.51 & \\
\hline 30 & & & -2.51 & \\
\hline 40 & & & -2.52 & \\
\hline 50 & & & -2.52 & \\
\hline 25 & -0.62 & 2.21 & -2.29 & \\
\hline 30 & & & -2.30 & \\
\hline 40 & & & -2.32 & \\
\hline 50 & & & -2.34 & \\
\hline 20 & 6.87 & 27.96 & -1.31 & $\begin{array}{l}\text { Mustafa et al. } 1991 \\
\text { (DIRS 165048) }\end{array}$ \\
\hline 30 & & & -1.59 & \\
\hline 40 & & & -1.87 & \\
\hline 50 & & & -2.15 & \\
\hline 20 & 14.97 & 53.54 & -0.69 & \\
\hline 30 & & & -1.22 & \\
\hline 40 & & & -1.75 & \\
\hline 50 & & & -2.29 & \\
\hline 20 & 11.82 & 37.52 & 0.83 & \\
\hline 30 & & & 0.45 & \\
\hline 40 & & & -0.08 & \\
\hline 50 & & & -0.30 & \\
\hline 20 & 10.45 & 29.16 & 1.90 & \\
\hline 30 & & & 1.61 & \\
\hline 40 & & & 1.32 & \\
\hline 50 & & & 1.03 & \\
\hline 10 & -0.87 & 5.74 & 0.78 & Zafar et al. 2002 (DIRS 165052) \\
\hline 25 & & 5.74 & 0.86 & \\
\hline 45 & & 5.98 & 0.99 & \\
\hline
\end{tabular}




\begin{tabular}{|c|c|c|c|c|c|c|c|c|}
\hline Sorbate & $\begin{array}{c}\text { Sorbate Concentration, } \\
\mathrm{mmol} / \mathrm{dm}^{3}\end{array}$ & Sorbent & Model & $\begin{array}{c}\text { Temperature, } \\
{ }^{\circ} \mathrm{C}\end{array}$ & $\Delta \mathrm{H}^{\circ}$ ads, $\mathrm{kJ} / \mathrm{mol}$ & $\begin{array}{l}\Delta \mathrm{S}_{\text {ads, }}^{\circ} \\
\mathrm{J} / \mathrm{mol} \cdot \mathrm{K}\end{array}$ & $\Delta \mathbf{G}^{\circ}{ }_{\text {ads }}, \mathbf{k J} / \mathrm{mol}$ & Reference \\
\hline $\mathrm{U}(\mathrm{VI})$ & $10-100$ & halloysite & $\begin{array}{l}\text { Assumed } \\
\text { linear }^{d}\end{array}$ & 20 & 1.55 & 15.61 & -3.02 & $\begin{array}{l}\text { Kilislioglu and Bilgin } 2002 \\
\text { (DIRS 165050) }\end{array}$ \\
\hline & & & & 30 & & & -3.18 & \\
\hline & & & & 40 & & & -3.33 & \\
\hline & & & & 50 & & & -3.49 & \\
\hline & & & & 60 & & & -3.64 & \\
\hline
\end{tabular}

${ }^{a}$ Freundlich, Langmuir, and Dubinin-Radushkevich (D-R) isotherms tested.

${ }^{b}$ Freundlich and Langmuir isotherms tested.

c Freundlich isotherm also tested.

${ }^{d}$ Freundlich, Langmuir, and Dubinin-Radushkevich (D-R) isotherms tested. 
Note also that considerable study was devoted to the sorption behavior of radioelements (Tc, I, $\mathrm{Np}, \mathrm{Ra}, \mathrm{Se}, \mathrm{Pu}, \mathrm{U}, \mathrm{Cs}, \mathrm{Am}$, and $\mathrm{Sr}$ ) on basalt, secondary alteration minerals, and interbed materials under the former Basalt Waste Isolation Project at the Hanford Site near Richland, Washington. A significant body of sorption measurements was conducted at elevated temperatures. Some of the data collected during the project were abstracted and summarized by Kelmers (1984 [DIRS 165041]). However, the summary is too cursory for suitable analysis, and cited original sources were neither located nor consulted for this evaluation.

Data from reports by Aksyuk et al. (1995 [DIRS 165054]), Kenna (1981 [DIRS 165049]), and van Geldermalsen and Duursma (1984 [DIRS 165087]) were manipulated to obtain values of log $\mathrm{K}_{\mathrm{d}}$ as a function of $1 / \mathrm{T}\left[\mathrm{K}^{-1}\right]$, which were plotted graphically as illustrated in Figures I-4(a) to I-4(c). The data were further subjected to linear regression analysis to obtain the calculated enthalpies of sorption summarized in Table I-11. 


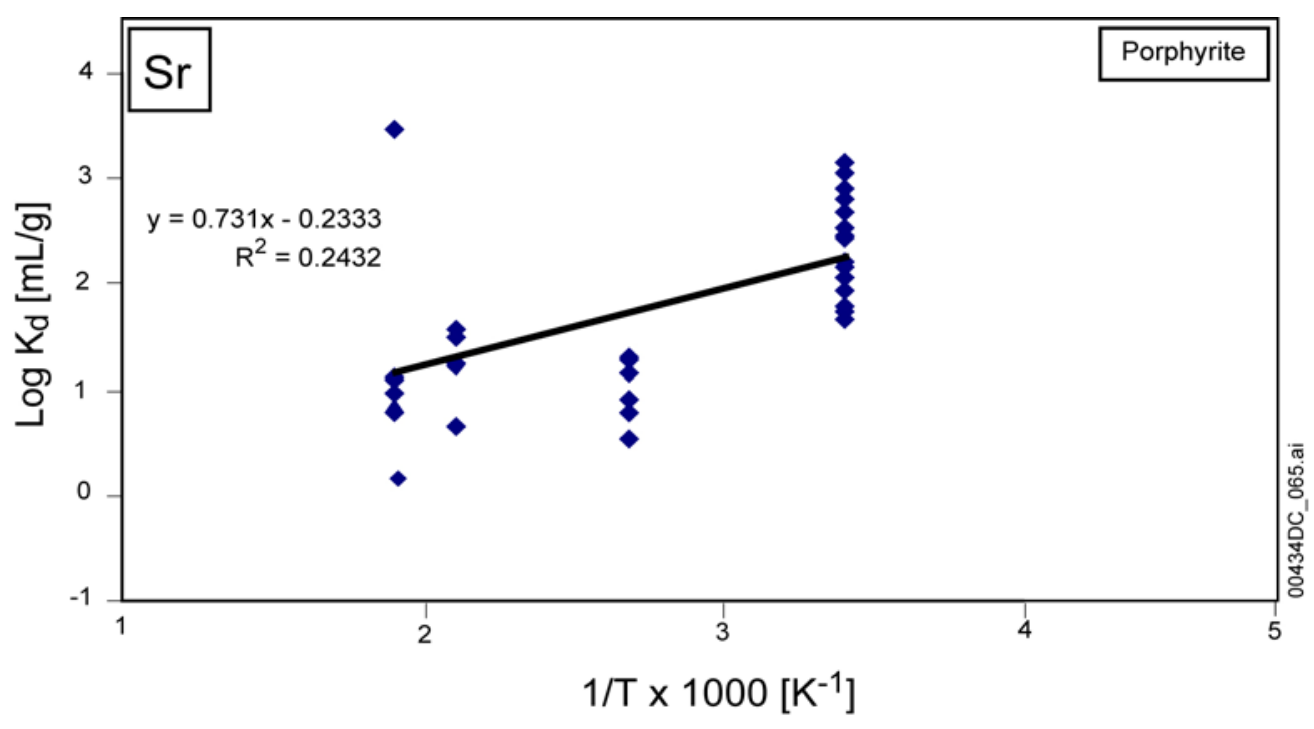

(a)

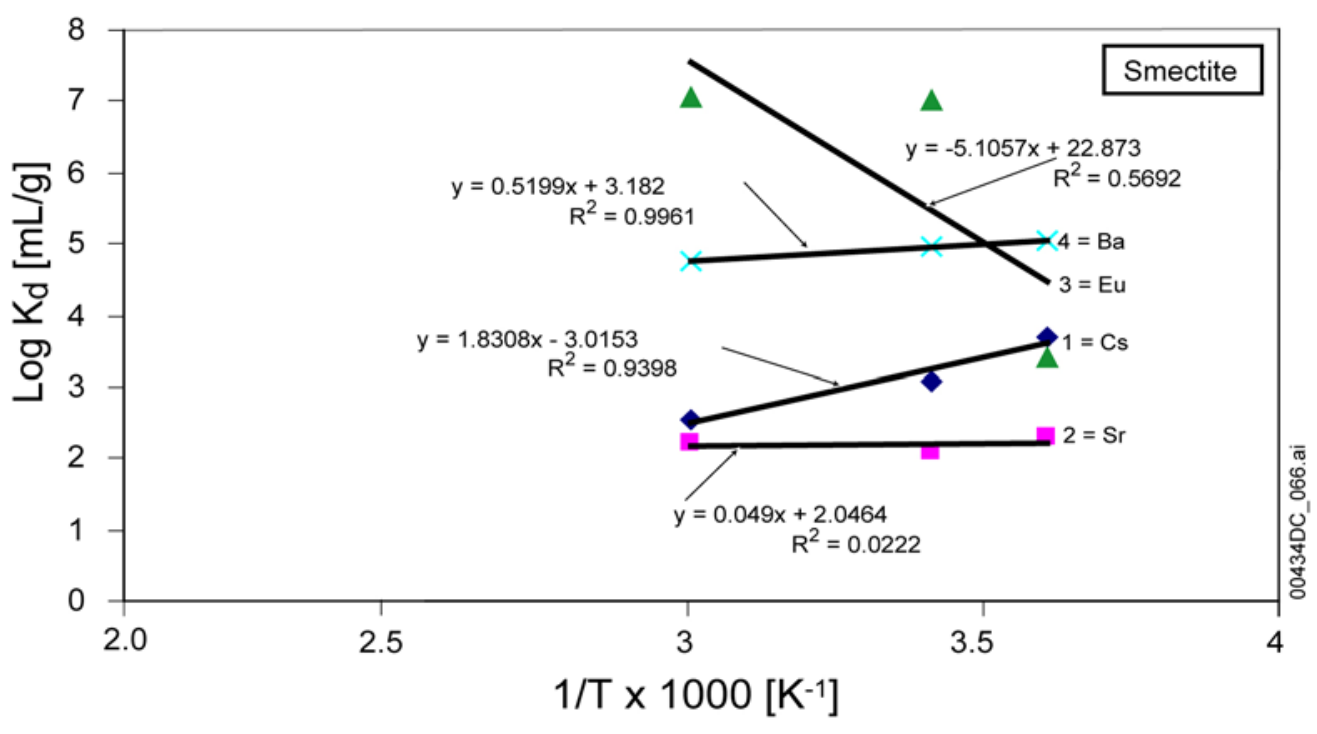

(b)

Output DTN: LB0311ABSTHCR2.003.

Source: (a) Aksyuk et al. 1995 [DIRS 165054], (b) Kenna 1981 [DIRS 165049], (c) van Geldermalsen and Duursma 1984 [DIRS 165087].

NOTE: Data are listed in Table I-9.

Figure I-4(a)-(b). Log $\mathrm{K}_{d}$ Values as a Function of the Reciprocal Absolute Temperature for Specified Elements on Various Sorbents 


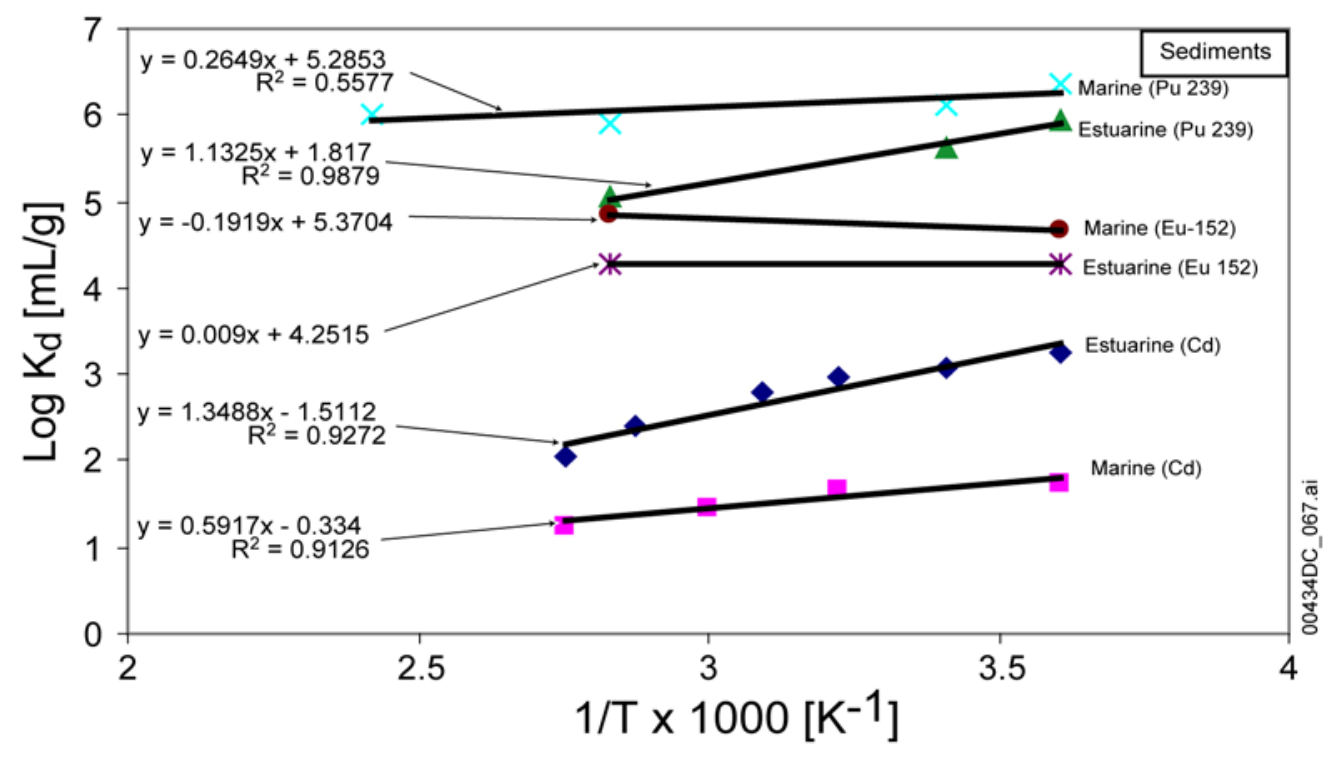

(c)

Output DTN: LB0311ABSTHCR23.004.

Source: (a) Aksyuk et al. 1995 [DIRS 165054], (b) Kenna 1981 [DIRS 165049], (c) van Geldermalsen and Duursma 1984 [DIRS 165087].

NOTE: Data are listed in Table I-9.

Figure I-4(c). Log $\mathrm{K}_{d}$ Values as a Function of the Reciprocal Absolute Temperature for Specified Elements on Various Sorbents (Continued)

Table I-11. Calculated Enthalpy of Sorption of Aqueous Species on Various Sorbents (from the Literature)

\begin{tabular}{|c|c|c|c|c|c|c|c|}
\hline $\begin{array}{c}\text { Aq. } \\
\text { Species }\end{array}$ & Sorbent & $\begin{array}{c}\text { Temperature } \\
\text { Range, }{ }^{\circ} \mathrm{C} \\
\end{array}$ & Slope & $\mathbf{R}^{2}$ & $\begin{array}{c}\Delta \mathrm{H}_{\mathrm{r}} \\
\text { (kcal/mol) }\end{array}$ & Source & Notes \\
\hline Cs & $\begin{array}{l}\text { Seabed smectite } \\
\text { clay }\end{array}$ & $4-60$ & +1.8308 & 0.940 & -8.38 & $\begin{array}{l}\text { Kenna 1981 } \\
\text { (DIRS 165049); } \\
\text { Erickson 1980 } \\
\text { (DIRS 164876) }\end{array}$ & \\
\hline $\mathrm{Sr}$ & & & +0.0490 & 0.022 & -0.22 & & \\
\hline $\mathrm{Ba}$ & & & +0.5199 & 0.996 & -2.38 & & \\
\hline $\mathrm{Eu}$ & & & -5.1057 & 0.569 & +23.4 & & \\
\hline $\mathrm{Sr}$ & Porphyrite & $20-250$ & +0.7310 & 0.243 & -3.35 & $\begin{array}{l}\text { Aksyuk et al. } 1995 \\
\text { (DIRS 165054) }\end{array}$ & \\
\hline $\mathrm{Cd}$ & Estuarine sediment & $4-90$ & +1.3488 & 0.927 & -6.17 & $\begin{array}{l}\text { van Geldermalsen } \\
\text { and Duursma } 1984 \\
\text { (DIRS 165087) }\end{array}$ & In seawater \\
\hline $\mathrm{Cd}$ & Marine sediment & $4-90$ & +0.5917 & 0.913 & -2.71 & & In seawater \\
\hline Pu-239 & Estuarine sediment & $4-80$ & +1.1325 & 0.988 & -5.18 & & In seawater \\
\hline Pu-239 & Marine sediment & $4-140$ & +0.2649 & 0.558 & -1.21 & & In seawater \\
\hline Eu-152 & Estuarine sediment & 4,80 & +0.0090 & - & -0.04 & & In seawater \\
\hline Eu-152 & Marine sediment & 4,80 & -0.1919 & - & +0.88 & & In seawater \\
\hline
\end{tabular}

Output DTN: LB0311ABSTHCR2.004.

NOTE: Data were illustrated in Figures I-4(a) to I-4(c). 


\section{I1.4.4 Evaluation of Results}

The calculated enthalpies of sorption on Yucca Mountain tuffs, $\Delta \mathrm{H}^{\circ}$, based on linear regression of the $\mathrm{K}_{\mathrm{d}}$ data with respect to the reciprocal of absolute temperature, are plotted in Figure I-5 for the radioelements $\mathrm{Cs}, \mathrm{Sr}, \mathrm{Ba}$ (a proxy for $\mathrm{Ra}$ ), Ce, Eu (a proxy for trivalent actinides), U(VI), Np, $\mathrm{Pu}$, and $\mathrm{Am}$, as given in Tables I-5, I-8, I-10, and I-11. The $\Delta \mathrm{H}^{\circ}{ }_{\mathrm{r}}$ values include those from both qualified and unqualified data generated by LANL. Both data sets are used because the qualified data set alone is too limited in scope to allow meaningful analysis of the distribution of $\Delta \mathrm{H}^{\circ}{ }_{\mathrm{r}}$ values. $\Delta \mathrm{H}^{\circ}{ }_{\mathrm{r}}$ values for $\mathrm{Np}$ adsorption on natural calcite and synthetic hematite are omitted from this analysis because they are not necessarily representative of Np sorption on tuffaceous rocks. The mean value and standard deviation at the 95 percent confidence level for each radioelement is also included and summarized separately in Table I-12. All radioelements are characterized by a positive mean $\Delta \mathrm{H}^{\circ}{ }_{\mathrm{r}}$. The range for all but $\mathrm{Sr}, \mathrm{U}(\mathrm{VI})$, and $\mathrm{Np}$ is sufficiently large that the assumption of a zero enthalpy of sorption might be conveniently assumed. Any variation in the sorption behavior of the radioelements with temperature can, therefore, be ignored in modeling radionuclide transport in the near field of the proposed Yucca Mountain geologic waste repository. A positive $\Delta \mathrm{H}^{\circ}{ }_{\mathrm{r}}$ for $\mathrm{Sr}, \mathrm{U}(\mathrm{VI})$, and $\mathrm{Np}$ is indicative of increasing sorption with temperature; thus, for the range of temperatures of interest to those modeling radionuclide release scenarios from the repository at Yucca Mountain, that is, approximately $25^{\circ} \mathrm{C}$ to $95^{\circ} \mathrm{C}$, $\mathrm{K}_{\mathrm{d}}$ increases by approximately 2.3, 3.9, and 2.3 times, respectively (Wang 2003 [DIRS 165562], SN-LBNL-SCI-005-V1, p. 283).

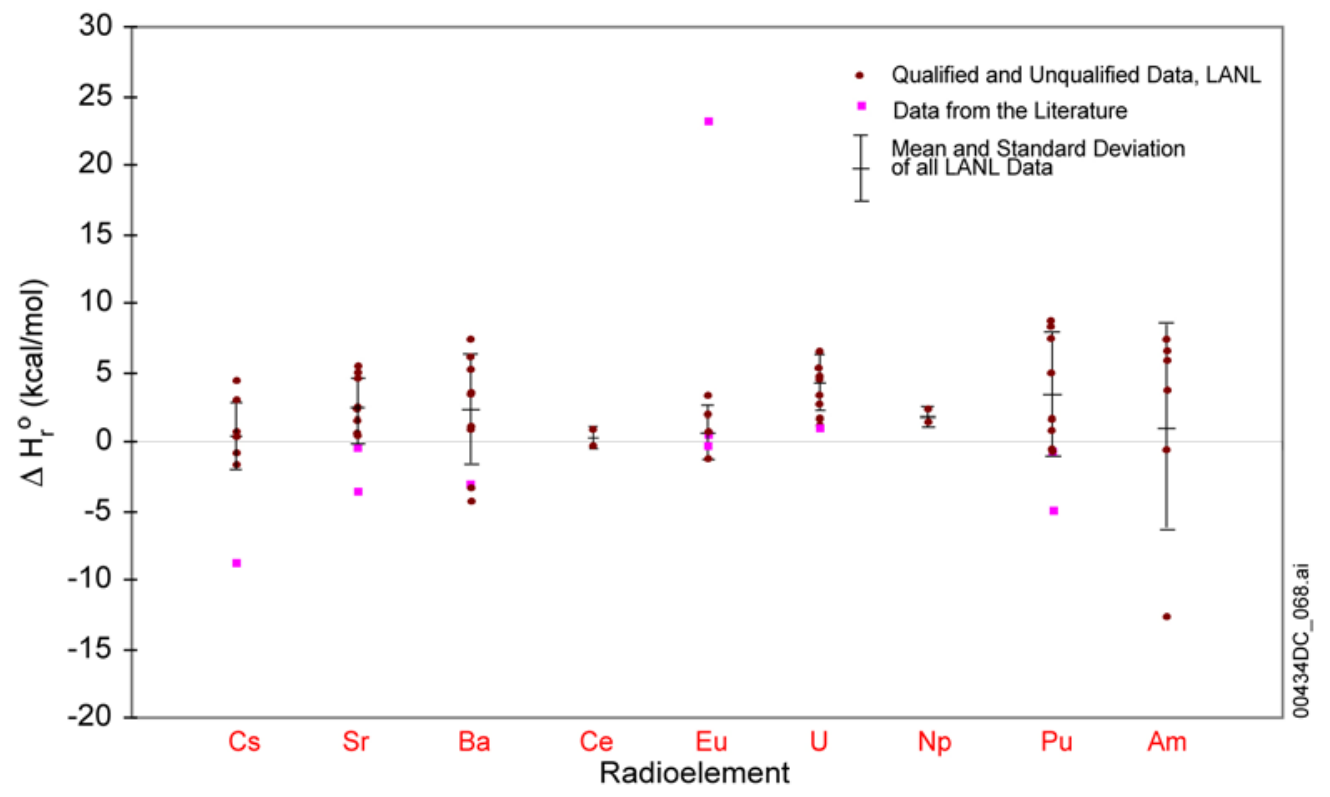

Output DTN: LB0311ABSTHCR2.004

(See Wang 2003 [DIRS 165562], SN-LBNL-SCI-005-V1, pp. 249 to 285 for analyses details.)

Figure I-5. Range of $\Delta \mathrm{H}_{\mathrm{r}}^{\circ}$ for the Sorption of Radioelements on Natural Substrates 
Table I-12. Radioelement Enthalpies of Sorption, $\Delta \mathrm{H}_{\mathrm{r}}$, Based on Qualified and Unqualified Data from LANL

\begin{tabular}{|l|c|c|c|}
\hline \multirow{2}{*}{ Radioelement } & \multirow{2}{*}{ Count } & \multicolumn{2}{c|}{$\Delta \mathbf{H}_{\mathbf{r}}, \mathbf{k c a l} / \mathbf{m o l}$} \\
\cline { 3 - 4 } & 9 & Mean value & Standard Deviation (95\%) \\
\hline $\mathrm{Cs}$ & 9 & +0.788 & 1.990 \\
\hline $\mathrm{Sr}$ & 9 & +2.646 & 2.136 \\
\hline $\mathrm{Ba}$ & 2 & +2.201 & 4.255 \\
\hline $\mathrm{Ce}$ & 4 & +0.485 & 0.827 \\
\hline $\mathrm{Eu}$ & 6 & +1.075 & 1.869 \\
\hline $\mathrm{U}(\mathrm{VI})$ & 2 & +4.207 & 2.153 \\
\hline $\mathrm{Np}$ & 7 & +2.640 & 0.721 \\
\hline $\mathrm{Pu}$ & 6 & +3.157 & 4.258 \\
\hline $\mathrm{Am}$ & & +1.548 & 7.785 \\
\hline
\end{tabular}

Output DTN: LB0311ABSTHCR2.004.

For comparison, Figure I-5 also includes $\mathrm{K}_{\mathrm{d}}$ values derived from literatures studies and summarized in Tables I-9 and I-11. The substrates upon which these radioelements sorbed differ from tuffs at Yucca Mountain, and the corresponding aqueous solutions differ in composition from Yucca Mountain pore waters. With the exception of one questionable value for Eu $\left(\Delta \mathrm{H}^{\circ}{ }_{\mathrm{r}}=27.4 \mathrm{kcal} / \mathrm{mol}\right)$, all literature $\Delta \mathrm{H}^{\circ}{ }_{\mathrm{r}}$ values are small and comparable in magnitude with those derived from Yucca Mountain data. However, the extent of overlap of the literature and Yucca Mountain data is limited, and suggests that either different sorption processes are operative, or that the uncertainties are larger than determined statistically for the Yucca Mountain data sets. Intercomparison is not generally possible between differing sorbents, or similar sorbents where radioelement sorption takes place under differing conditions (e.g., with differing temperatures, aqueous solution compositions, or sorption mechanisms). Thus, it is to be expected that radioelement $\mathrm{K}_{\mathrm{d}}$ values for Yucca Mountain sorbents are poorly correlated with independent data from the literature

The analysis presented in this model report is preliminary. An evaluation was not made of the effects of various cited parameters on computed $\mathrm{K}_{\mathrm{d}}$ values, and, therefore, a refinement that could lead to a reduction in the uncertainty of $\Delta \mathrm{H}_{\mathrm{r}}$ for each radioelement was not accomplished. Such a refinement would include consideration of the experimental conditions under which the sorption tests were conducted, for example, whether initial conditions indicated supersaturation of the radioelement with respect to a solid phase, and the extent to which thermodynamic equilibrium had been attained. Any such refinement has the potential for creating more complex and unwieldy equations relating radionuclide sorption to the rock physical, chemical, and spatial properties, and to the groundwater composition, $\mathrm{pH}$, and redox state. Furthermore, considerable uncertainty would attend the outcome of such an endeavor. Given the difficulty in making these refinements, the writer believes that the present analysis suffices for its intended purpose. 


\section{I1.5 SUMMARY AND DISCUSSION}

The evaluation of specific radioelement $\mathrm{K}_{\mathrm{d}}$ values to determine their temperature dependence was conducted with the aid of standard thermodynamic relations that are well established and accepted in the refereed literature, and, therefore, require no further validation (see Mustafa et al. 1991 [DIRS 165048]; Ishfaq et al. 1997 [DIRS 164879]; Rauf and Tahir 2000 [DIRS 164893]; Zafar et al. 2002 [DIRS 165052]; and Kara et al. 2003 [DIRS 164880]). These relations constitute the "model," together with assumptions and approximations inherent in the use of experimentally determined laboratory sorption measurements to compute $K_{d}$ values (see Section I1.3.1).

Published studies concerning the temperature dependence of metal ion sorption on various substrates with respect to temperature show that the sorption coefficient for a given dissolved constituent, that is, an ionic species of some metal, can show variation as a function of temperature, but that this functionality will vary depending on a number of experimental variables as discussed under Section I1.3.1 An example of this variability is illustrated by Rauf and Tahir (2000 [DIRS 164893]), in which it is shown that $\Delta \mathrm{H}_{\mathrm{r}}^{\circ}$ of sorption of Mn(II) can vary by a factor of five depending on the sorbate concentration. Another example, (Kara et al. 2003 [DIRS 164880]), shows that alternative sorption models will lead to reversals in the computed $\Delta \mathrm{H}_{\mathrm{r}}^{\circ}$ of $\mathrm{Co}(\mathrm{II})$ sorption from negative to positive.

The analysis of the temperature dependence of $K_{d}$ values of various radioelements sorbed on tuffaceous materials recovered from vitroclastic horizons at Yucca Mountain reveal that $\mathrm{K}_{\mathrm{d}}$ changes over the temperature range of interest are small and positive for all studied radioelements, but no statistically significant $\mathrm{K}_{\mathrm{d}}$ temperature dependence can be discerned, with the possible exception of $\mathrm{Sr}, \mathrm{U}(\mathrm{VI})$, and $\mathrm{Np}$ (Figure I-5). A positive $\Delta \mathrm{H}^{\circ}{ }_{\mathrm{r}}$ for $\mathrm{Sr}, \mathrm{U}(\mathrm{VI})$, and $\mathrm{Np}$ is indicative of increasing sorption with temperature. For the range of temperatures expected in the near field of the Yucca Mountain repository, that is, approximately $25^{\circ} \mathrm{C}$ to $95^{\circ} \mathrm{C}, \mathrm{K}_{\mathrm{d}}$ increases by approximately $2.3,3.9$, and 2.3 times, respectively for $\mathrm{Sr}, \mathrm{U}(\mathrm{VI})$, and $\mathrm{Np}$. These variations are modest, and given the overall model uncertainties, are too small to justify inclusion of $\mathrm{K}_{\mathrm{d}}$ temperature dependence in modeling radionuclide waste release scenarios. Ignoring temperature dependence of all studied radionuclides in simulations predicting radionuclide release from the near field would mean that sorption would be less than if temperature dependent corrections had been made; a conservative assumption.

Independent evaluations of transport of a sorbing metal show that using a linear isotherm would over-predict the maximum concentration of that metal upon release into the accessible environment when compared with more sophisticated site complexation models. The application of the linear isotherm, represented by a $\mathrm{K}_{\mathrm{d}}$ value, to radionuclide transport is, therefore, inherently conservative. 
Finally, it should be noted that the techniques used in the evaluation of $\mathrm{K}_{\mathrm{d}}$ data reported in this appendix are also very conservative. The intent of this appendix is to address a broader range of issues for the total system performance assessment of the Yucca Mountain Repository. These issues, which include anticipated modifications of the repository design through incorporation of anthropogenic and anthropogenically modified natural barrier and ground support materials, dictate a substantially more conservative approach to the estimation of radionuclide $\mathrm{K}_{\mathrm{d}}$ values than would otherwise be mandated.

To summarize, radionuclide transport modeling through the near field includes three levels of conservatism: (1) use of a linear isotherm in transport modeling, (2) an analysis of $K_{d}$ values that leads to the selection of overly conservative, that is low $\mathrm{K}_{\mathrm{d}}$ values, and (3) omission of temperature dependence of $\mathrm{K}_{\mathrm{d}}$ values, as recommended in this appendix. 
APPENDIX J

QUALIFICATION OF SORPTION DATA

DTNs: LA0305AM831341.001 AND LA0310AM831341.001 


\section{J1. DATA QUALIFICATION}

The qualification of DTNs: LA0305AM831341.001 (DIRS 163789) and LA0310AM831341.001 (DIRS 165865) is documented here in accordance with AP-SIII.2Q Rev. 1, ICN 2. These data are unqualified because they were developed before the quality assurance program was established. This qualification provides a desired level of confidence that the data are suitable for their intended use, which is limited to the subject of this work product (unsaturated zone radionuclide transport) for use in total system performance assessment. The qualification is based on corroboration of data and technical assessment.

\section{J1.1 QUALIFICATION TEAM}

The qualification team consisted of Arend Meijer, John Collins, and Chunhong Li.

Arend Meijer is the qualification chairperson for the data qualification team and is the principal investigator for the analyses of sorption documented in this report. He has been involved with the YMP sorption program carried out at Los Alamos National Laboratories since 1984. He has detailed familiarity with the methods and results of laboratory sorption coefficient experiments carried out at LANL since the inception of the YMP/Nevada Nuclear Waste Storage Investigations (NNWSI) programs. He holds a Ph.D. in Geology/Geochemistry from the University of California at Santa Barbara.

John Collins is a technical data specialist with several years experience in data qualification on the Yucca Mountain Project, including extensive experience with qualification of sorption data. In addition, he has 20 years experience in the areas of management and supervision, project engineering, regulatory and licensing compliance, root cause analysis, quality assurance (audits and surveillances), training, procedure writing, and startup testing for commercial nuclear power plants, 2 years regulatory compliance and quality assurance experience in the oil pipeline industry, and naval nuclear power operations and maintenance. He holds an A. B. in mathematics from LaGrange College, LaGrange, Georgia and an M. S. in mathematics from Auburn University, Auburn, Alabama.

Chunhong $\mathrm{Li}$ is the technical checker of this report. He is a geohydrologist with several years experience in subjects of flow and transport in geologic environments. He has conducted numerical modeling studies of groundwater flow and mass transport at the Yucca Mountain site to assist system performance analysis at the proposed high level nuclear waste repository site. In additional to numerical modeling, he has conducted field hydrogeological investigations, field pumping tests and tracer tests, and data analysis and has designed and carried out laboratory experiments to study solute transport behavior in fractures. He holds a B.S. in hydrogeology from Changchun University of Earth Sciences, Changchun, China, an M.S. in geophysics from the Institute of Geology, State Seismological Bureau of China, Beijing, China, and a Ph.D. in hydrology from the New Mexico Institute of Mining and Technology, Socorro, New Mexico.

None of the team members were involved in the collection of the data being qualified in this appendix. 


\section{J1.2 QUALIFICATION CRITERIA}

Four evaluation criteria are presented in the data qualification plan (Appendix K, Figure K-1). The qualification is to be considered adequate if:

1. The procedures and equipment are shown to be reasonably similar to those used for data already qualified.

Discussion: Given that sorption data are determined directly from experimental measurements, a demonstration that the experimental procedures and equipment are similar to those implemented for determining sorption data under the quality assurance program adds confidence in the results of the unqualified data.

2. The data are shown to provide the information required.

Discussion: In this case, the data directly provide the information required and do not require manipulation through correlations or theoretical models to be implemented. This direct relationship between the data and the information needed adds confidence in the relevance of the data for its intended use.

3. The quality and reliability are shown to be reasonably similar to the sorption data already qualified.

Discussion: A demonstration that processes work planning and personnel training and qualification were carried out in a manner that is consistent with the processes implemented for the collection of qualified sorption data adds confidence that the results are adequate.

4. The subject data and corroborating data are found to span the same range, or if not, why these differences are expected based on the variations in experimental methods and numbers of measurements.

Discussion: Comparisons of measured sorption coefficients for radionuclides under similar conditions can be used to establish that the data are equivalent. Due to the uncontrolled variables in sorption measurements using natural geologic materials, such as mineral content of rock samples, sorption values are only expected to fall within a similar range of values; exact comparisons are not possible. However, this is sufficient because the uncertainty inherent in the measurements are captured and propagated into the downstream models representing sorption processes.

\section{J2. CORROBORATING DATA}

One method used here for the data qualification process is corroboration of unqualified data with qualified data. If it can be shown that unqualified sorption coefficient experiments on a given water/rock combination and under a specified set of experimental parameters produced sorption coefficient data equivalent to data obtained under a qualified quality assurance program using a similar set of rock/water combinations and experimental parameters, the concordance of the two data sets can be used to support the qualification of the unqualified data set. 
Sorption coefficients are subject to a range of factors including some associated with the solid phase, some associated with the liquid phase (groundwater or pore water), and some associated with the element-of-interest. The two main factors associated with the solid phase include mineralogy and surface area. Mineralogy includes mineral type and mineral composition, particularly for minerals that sorb primarily by ion exchange. The factors in the liquid phase that can influence sorption behavior are different for different elements-of-interest. For example, for alkali and alkaline earth elements of interest (i.e., $\mathrm{Ba}, \mathrm{Cs}, \mathrm{Sr}, \mathrm{Ra}$ ), the concentrations of alkali and alkali earth elements in solution (e.g., $\mathrm{Ca}, \mathrm{K}, \mathrm{Na}, \mathrm{Mg}$ ) are important factors. For an element such as uranium, $\mathrm{pH}$ and the alkalinity are important factors. Factors associated with the element-of-interest include concentration in solution and complexation behavior.

In comparing an unqualified dataset with a qualified dataset for a given element-of-interest, it is important that the factors that can control sorption behavior are at the same or similar values in both datasets unless it can be shown that a given factor does not control sorption behavior for the element-of-interest.

Two examples are cited of comparisons of qualified and unqualified datasets for elements-of-interest. The first example is for barium sorption coefficients on zeolitic tuff in J-13 water. Qualified and unqualified data points for sorption coefficients and final solution concentrations are shown in Figure J-1. The same water composition was used in both the unqualified and the qualified experiments. Thus, differences in water composition are not an issue in comparisons of the two datasets. The differences in final solution concentrations did not influence the sorption coefficient ranges for the two datasets because the isotherms for barium sorption on zeolitic tuff in J-13 water are essentially linear (Appendix A, Figure A-45). Differences in experimental duration did not influence the sorption coefficient ranges because the barium sorption reactions are fast (Appendix A, Section A8.6). The fact that the ranges in sorption coefficient values are very similar for the two datasets provides confidence that the experimental procedures used to obtain the unqualified data were appropriate and equivalent to those used to obtain the qualified dataset.

The second example of a comparison of qualified and unqualified datasets involves uranium sorption on devitrified tuff in J-13 water. Qualified and unqualified data points for sorption coefficients and $\mathrm{pH}$ are shown in Figure J-2. The same water composition was used in both the unqualified and the qualified experiments. Thus, differences in water composition are not an issue in comparisons of the two datasets. The differences in final solution concentrations did not influence the sorption coefficient ranges for the two datasets because the uranium sorption coefficients are not sensitive to final solution concentrations (Appendix A, Figure A-60). Differences in experimental duration did not influence the sorption coefficient ranges because the uranium sorption reactions on devitrified tuff are fast (Appendix A, Section A8.9). The fact that the ranges in uranium sorption coefficient values are similar for the two datasets, excluding the outlier with a $\mathrm{K}_{\mathrm{d}}=10 \mathrm{~mL} / \mathrm{g}$, provides confidence that the experimental procedures used to obtain the unqualified data were appropriate and equivalent to those used to obtain the qualified dataset. 
Based on this information, the following conclusion can be made relative to qualification Criterion 4:

4. The subject data and corroborating data are found to span the same range, or if not, why these differences are expected based on the variations in experimental methods and numbers of measurements.

Discussion: The data for both $\mathrm{Ba}$ and $\mathrm{U}$ are found to lie within the same ranges. Therefore, the data from either data set would result in the derivation of similar $\mathrm{K}_{\mathrm{d}}$ distributions for modeling radionuclide transport in TSPA. This supports the qualification of the old data sets, DTN: LA0305AM831341.001 (DIRS 163789) and DTN: LA0310AM831341.001 (DIRS 165865), as being adequate for their intended use.

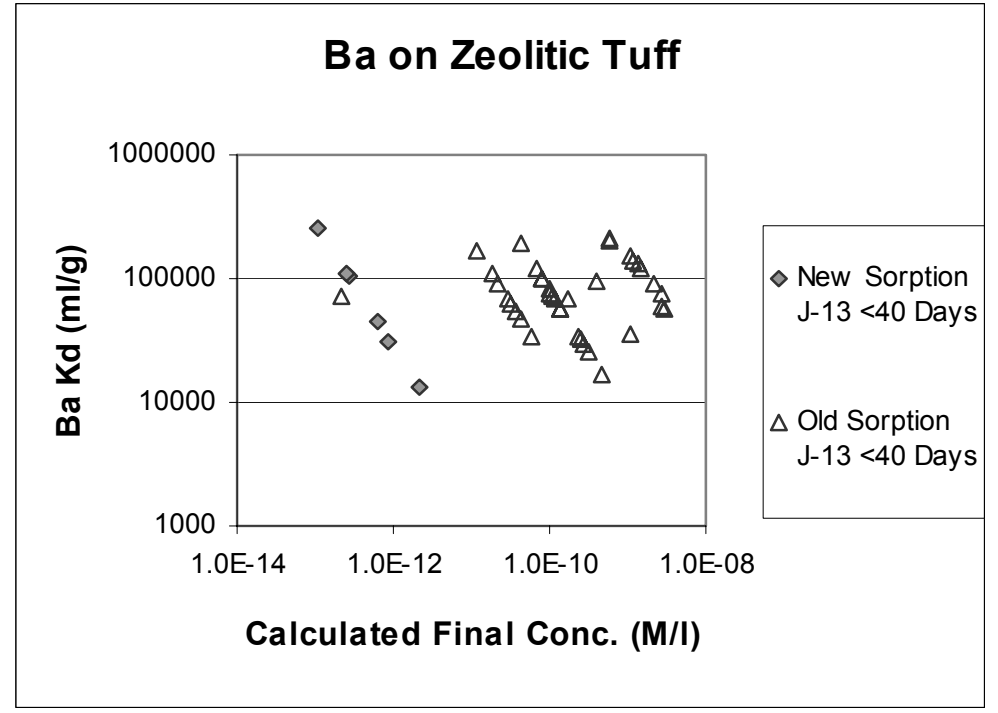

Source: DTN: LA0305AM831341.001 (DIRS 163789); DTN: LA0407AM831341.001 (DIRS 170623).

Figure J-1 Barium Sorption Coefficient on Zeolitic Tuff in J-13 Water versus Calculated Final Barium-133 Solution Concentration

\section{J3. TECHNICAL ASSESSMENT}

The Los Alamos National Laboratory studies of the sorptive behavior of tuff and transport of radionuclides through tuff were performed under the NNWSI project managed by the Nevada Operations Office of the U.S. Department of Energy. These investigations were performed under the Los Alamos quality assurance program for the NNWSI, with the Materials Science and Technology Division quality assurance organization responsible for the planning and implementation of the Los Alamos quality assurance program. 


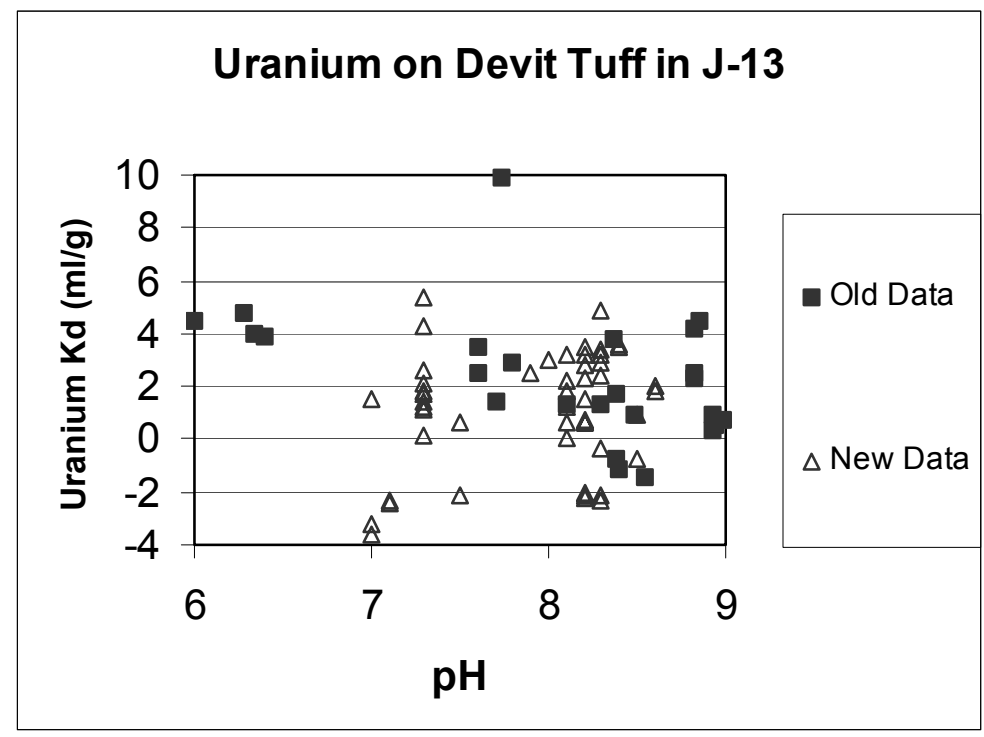

Source: DTN: LA0305AM831341.001 (DIRS 163789); DTN: LA0407AM831341.006 (DIRS 170628).

Figure J-2 Uranium Sorption Coefficient on Devitrified Tuff in J-13 Water versus pH

The Los Alamos National Laboratory Quality Assurance Plan And Procedures For The Nevada Nuclear Waste Storage Investigations is a collection of the quality assurance documents that provides a detailed account of the Los Alamos NNWSI quality assurance program, including the Quality Assurance Program Plan for Nevada Nuclear Waste Investigations (QAPP) (LANL 1982 [DIRS 172031]), the Quality Assurance Program Index, and procedures, work plans, and the detailed procedures developed for the project.

The quality assurance program developed for the NNWSI at Los Alamos was outlined in the QAPP and was structured to meet the requirements of NQA-1 and 10 CFR 50 ([DIRS 107619], Appendix B) as applied to the evaluation of major geologic formations with regard to their suitability as locations of permanent repositories for high-level radioactive wastes.

The Quality Assurance Program Index is a cross-reference between the basic requirements standards NQA-1 and 10 CFR 50 (DIRS 107619), showing where these requirements are addressed in the Los Alamos Quality Assurance Manual (QAM) and project implementing procedures. The QAM was the primary compliance document for this work unless otherwise stated in the QAPP or in the specific procedure documents written for NNWSI.

Work plans were the primary planning documents covering the Los Alamos technical activities for the NNWSI. These work plans were written to provide an adequate description of the scope and purpose of the task. They include, directly or by reference, the quality assurance requirements with regard to data validity and documentation. Review boards that consisted of, at a minimum, a management member, a quality assurance member, and an independent technical reviewer experienced and competent in the field under review, but with no direct program responsibility were used to approve the work plans as well as the acceptance of final documents. The work plan containing the sorption experiments was Tuff Experiments-Sorption Ratios and Migration Measurements. This work plan contains the scope of the work, an overview of how 
the batch sorption studies were to be performed, what procedure would be used to control the experiments, how the measurement of radioactivity would be performed, type of equipment to be used, quality assurance requirements, and documentation of work requirements. The procedure used to control the experiments was Tuff Experiments-Sorption Ratios and Migration Measurements. The process used in this procedure is the same process as given in procedure Sorption and Desorption Determinations by a Batch Sample Technique for the Dynamic Transport Task - that is, the procedure used for the qualified $\mathrm{K}_{\mathrm{d}}$ data. This latter procedure only provides more detailed guidance.

Training requirements for personnel were delineated in the QAM by procedure QMR 1, Quality Assurance Management and Planning and QMI 1-1, Quality Program Management.

Measuring and testing equipment that require calibration was controlled in accordance with the applicable sections of the QAM (QMR 9 and QMI 9-1). The only equipment used for these experiments that required calibration were the balances and gamma counters. These were controlled in accordance with procedures QMR 9, Control and Calibration of Standards and Measuring and Testing Equipment, and QMI 9-1, Acquisition and Calibration Control of Standards and Measuring and Testing Equipment. These procedures required, among other things, that all measuring and testing equipment used in QA work was labeled for a fixed calibration schedule or operator-to-calibrate. If an instrument was found to be out of calibration, any evaluations and examinations based on measurements using this instrument since the last calibration were repeated or documented to record the condition (QMI 9-1, Sections 7.2 and 8.1). Quality Assurance Audit of Los Alamos National Laboratories (LANL) Nevada Nuclear Waste Storage Investigations (NNWSI) Project performed July 16-18, 1984 (LANL 1984 [DIRS 171444]), verified that the control of measuring and test equipment was adequate. There were no problems found with the measuring and test equipment program. Audit of Los Alamos Scientific Laboratories Quality Assurance Plan for Nevada Nuclear Waste Storage Investigations conducted May 1-3, 1979 (LANL 1979 [DIRS 171445]), also found that the Measuring and Test Equipment program was satisfactory.

Corrective actions for significant conditions adverse to quality were provided in accordance with QMR 12, Corrective Action, of the QAM. Compliance with the quality assurance program was verified by periodic audits that were planned, documented, and carried out in accordance with procedure QMI 15-1, Quality Audits, of the QAM.

Based on this information, the following conclusions can be made relative to qualification criteria 1) through 3):

1. The procedures and equipment are shown to be reasonably similar to those used for data already qualified.

Discussion: The procedure used to control the experiments was Tuff Experiments-Sorption Ratios and Migration Measurements, which may be compared with the process used for qualified $\mathrm{K}_{\mathrm{d}}$ data, Sorption and Desorption Determinations by a Batch Sample Technique for the Dynamic Transport Task. Both of these procedures include similar steps for pre-treatment of water in contact with crushed rock materials by shaking, determination of sample weights, centrifugation or filtration 
separation, analytical determination of initial solution tracer concentrations (e.g., gamma counting), contact of ground rock with tracer solution by shaking, centrifugation or filtration separation after contact with traced solution, post-contact analytical determination of solution concentrations, and post-contact $\mathrm{pH}$ determination of the solution. Both procedures identify the same types of equipment, such as balances, centrifuges, shakers, filters. The similarity of the processes and kinds of equipment used provides confidence in the data.

\section{The data are shown to provide the information required.}

Discussion: The data obtained in these experiments are the same as the information required; the amount of radionuclide sorbed to rock from an aqueous solution is directly measured. Therefore, the observations can be directly implemented in transport models providing confidence in the application of the results.

\section{The quality and reliability are shown to be reasonably similar to the sorption data already qualified.}

Discussion: Compliance with the processes discussed above were demonstrated through audits of the experimental program on two occasions during the development of the subject sorption data. This leads to confidence in the results given that the processes themselves have been shown to be standard practice by comparison with processes implemented under the quality assurance program.

\section{J3. SUMMARY}

The programs in place at the time these sorption experiments were performed and the fact that the audits mentioned above did not identify anything that would affect the quality of the data lend support to the conclusion that the data collection methodology and equipment used were appropriate for the type of data under consideration. Corroborating information available for barium and uranium also show the equivalence of the unqualified and qualified data, supporting the adequacy of the unqualified data set. Furthermore, the uncertainty distributions for sorption coefficients generally span a range that is one order of magnitude or more (see Table 6-3). The corroborating data comparisons suggest that differences between the unqualified and qualified data are much less than the inherent uncertainty. Therefore, the unqualified data are justified for use in supporting the sorption coefficient distributions to be used in total system performance assessment. 
INTENTIONALLY LEFT BLANK 


\section{APPENDIX K \\ PLAN FOR QUALIFICATION OF SORPTION DATA \\ DTNs: LA0305AM831341.001 AND LA0310AM831341.001}




\begin{tabular}{|l|l|l|}
\hline \multirow{2}{*}{ BSC } & \multirow{2}{*}{ DATA QUALIFICATION PLAN } & QA: QA \\
\cline { 3 - 3 } & Page 1 of $/ 2 / 2 / 1 / 3 / 04$ \\
\hline
\end{tabular}

\begin{tabular}{|l|}
\hline Section I. Organizational Information \\
\hline Qualification Title \\
Qualification of Sorption Data \\
\hline Requesting Organization \\
Regulatory Integration Team/Integration Team/Natural System Team \\
\hline Section II. Process Planning Requirements \\
\hline
\end{tabular}

1. List of Unqualified Data to be Evaluated

LA0305AM831341.001 and LA0310AM831341.001

2. Type of Data Qualification Method(s) [Including rationale for selection of method(s) (Attachment 3) and qualification attributes (Attachment 4)] The types of data qualification methods to be used are Corroborating Data and Technical Assessment. Corroborating data are available for some of the elements from other DTNs currently being qualified with road map assessments. Technical assessment will also be used to show that the employed methodology for measuring sorption is acceptable.

The following qualification attributes will be used:

2. The technical adequacy of equipment and procedures used to collect and analyze the data.

3. The extent to which the data demonstrate the properties of interest (e.g., physical, chemical, geologic, mechanical).

5. The quality and reliability of the measurement control program under which the data were generated.

10. Extent and quality of corroborating data or confirmatary testing results.

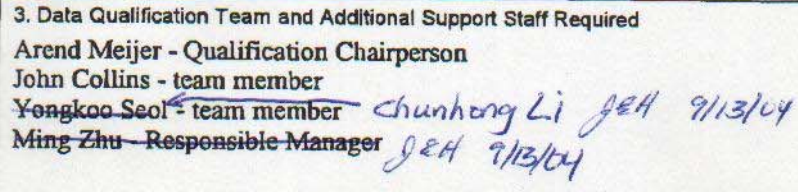

Figure K-1. Plan for Qualification of Sorption Data 


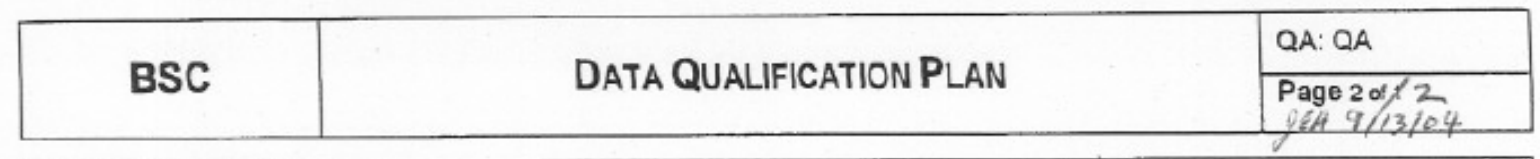

\begin{tabular}{|l|l|l|l|}
\hline Section III. Approval \\
Qualification Chairperson Printed Name \\
Arend Meijer \\
Responaible Manager Printed Name \\
Ming Zhu
\end{tabular}

Figure K-1. Plan for Qualification of Sorption Data (Continued) 


\section{APPENDIX L}

QUALIFICATION OF COMPOSITIONS OF WATERS FROM UE-25 J-13 AND UE-25 P\#1(CARBONATE) 


\section{L1. PURPOSE}

This report documents the findings of the data qualification team assembled to evaluate selected unqualified hydrology data that were used in the characterization of transport processes in the saturated zone (SZ) at Yucca Mountain, Nevada. These data are being qualified for inclusion in this model report. This activity has been planned and implemented in accordance with AP-SIII.2Q, Qualification of Unqualified Data.

\section{L1.1 QUALIFICATION TEAM}

Chairperson Sharad Kelkar has 25 years experience in the area of flow and transport through geological media. He has been a member of the Hydrology, Geochemistry, and Geology group at the Los Alamos National Laboratory (LANL) since 1985. In 2002, Mr. Kelkar began his ongoing role as a principal scientist with the Yucca Mountain Project, where he is responsible for documentation in support of the license application for the repository. Mr. Kelkar has contributed to the Yucca Mountain Project in the development of the code FEHM that forms a basis for the flow and transport modeling for the SZ. He has also worked on geothermal energy programs at LANL. Before his association with LANL, he worked on laboratory and field testing and engineering analysis in support of petroleum production (Terra Tek Inc., Salt Lake City, Utah, 1982 to 1985) and laboratory and field testing in support of gas recovery programs (Institute of Gas Technology, Chicago, Illinois, 1979 to 1982). Mr. Kelkar received his M.S. degree in Petroleum Engineering from the University of Texas at Austin (1979) for measurements of poro-elastic properties of rocks at elevated pressures and temperatures. Mr. Kelkar received his M.S. degree in Nuclear Physics from the University of Bombay (1974). In addition, Mr. Kelkar has done extensive course work in hydrology and minerals engineering at the University of California, Berkeley.

Team member: Dr. James Houseworth's professional experience includes groundwater and surface water hydrology and petroleum reservoir engineering. He has provided, over a twelve-year period, technical and management support for performance assessment and site characterization efforts at Yucca Mountain, Nevada, which is being studied by the DOE as a disposal site for high-level radioactive waste. This includes unsaturated zone flow and transport analyses in fractured rock to support performance assessment of the potential repository system and analyses to support construction and testing activities at Yucca Mountain. In addition, he has eight years experience in characterization of petroleum reservoirs including laboratory core analysis methods to determine single and multiphase flow properties and reservoir simulation. Other experience includes numerical modeling of mixing and stratification in surface water reservoirs and the design of outfalls for the discharge of waste heat from nuclear power plants and municipal sewage effluent. Dr. Houseworth received his Ph.D. in 1984 in Environmental Engineering Science from California Institute of Technology, his M.S. in 1978 in Environmental Engineering Science from California Institute of Technology and his B.S. in 1977 in Environmental Engineering Science from California Institute of Technology

\section{L1.2 DESCRIPTION OF THE DATA TO BE QUALIFIED}

The data that were evaluated and documented in this qualification report are published in the report by Ogard and Kerrisk (1984 [DIRS 100783]). These data are shown in Table L-1. 
Table L-1 Compositions of Waters from UE-25 J-13 and UE-25 p\#1

\begin{tabular}{|l|l|l|}
\hline \multicolumn{1}{|c|}{ Chemical Constituent } & \multicolumn{1}{|c|}{$\begin{array}{c}\text { UE-25 } \\
\text { J-13 Water }\end{array}$} & $\begin{array}{c}\text { UE-25 p\#1 } \\
\text { (Carbonate) Water } \\
\text { (mg/L) }\end{array}$ \\
\hline $\mathrm{Ca}^{2+}$ & 11.5 & 87.8 \\
\hline $\mathrm{Mg}^{2+}$ & 1.76 & 31.9 \\
\hline $\mathrm{Na}^{+}$ & 45 & 171 \\
\hline $\mathrm{K}^{+}$ & 5.3 & 13.4 \\
\hline $\mathrm{SiO}_{2}$ & 64.2 & 64.2 \\
\hline $\mathrm{Cl}^{-}$ & 6.4 & 37 \\
\hline $\mathrm{F}^{-}$ & 2.1 & 3.5 \\
\hline $\mathrm{SO}_{4}{ }^{2-}$ & 18.1 & 129 \\
\hline $\mathrm{HCO}_{3}{ }^{-}$ & 128 & 666 \\
\hline $\mathrm{CO}_{3}{ }^{2-}$ & 0.0 & 0 \\
\hline $\mathrm{pH}$ & 6.9 & 6.7 \\
\hline
\end{tabular}

Source: Ogard and Kerrisk 1984 (DIRS 100783).

\section{L2. QUALIFICATION METHOD AND EVALUATION CRITERIA}

The method used to qualify the data in this report is corroborating data (AP-SIII.2Q, Attachment 3, Item \#1). The confidence in the data is in question, because data collection procedures are unavailable for review.

A comparison of the unqualified data and available qualified corroborating data will be conducted to determine that the unqualified data is acceptable and confidence in the data is warranted.

The team will conduct the comparison by considering the importance of the data to showing that the proposed U.S. Department of Energy repository design meets the performance objectives.

The following data evaluation criteria will be used:

- Extent and quality of corroborating data

- Agreement between the data being qualified and the corroborating data

- The importance of the data to showing that the proposed U.S. Department of Energy repository design meets the performance objectives of 10 CFR 60.

\section{L3. EVALUATION RESULTS}

The compositions reported by Ogard and Kerrisk (1984 [DIRS 100783]) for J-13 and p\#1 waters were used in the analysis presented in Appendix A because many of the sorption coefficient experiments used in this analysis were performed in the early 1980s. Thus, to make the comparisons of experimental data and model results more direct, the Ogard and Kerrisk data were used in the analysis. The Ogard and Kerrisk data were used as limiting cases to set bounding ranges for concentrations of various constituents, which were then used as inputs for 
the PHREEQC modeling for extrapolating soprtion coefficient data (described in Appendix A) to $\mathrm{pH}$ and geochemical compositions for which sufficient laboratory measurements were not available. The results of this modeling, in conjunction with the experimental data, inform the process of expert judgment to arrive at the uncertainty distributions for sorption coefficients for Am (Appendix A8.1), Np (Appendix A8.3), Pu (Appendix A8.4), and U (Appendix A8.9). As such, the recommended distributions for sorption coefficient are expected to be weakly to moderately sensitive to the limits of compositional values that were input to the surface complexation modeling.

The composition of water from J-13 as reported by Ogard and Kerrisk (1984 [DIRS 100783]) and the corroborating data from DTN: MO0006J13WTRCM.000 [DIRS 151029] are given in Table L-2. The corroborating data set is a verified and qualified data set per AP-SIII.2Q. The uncertainty in concentration of major anions and cations is on the order of 10 percent for most species and 15 percent for $\mathrm{F}^{-}$(BSC 2004 [DIRS 170037], Section A7.3.1). Thus, the data from the two sets can be expected to agree within 20 percent of each other for all the constituents except for $\mathrm{F}^{-}$, which should agree within 30 percent of each other. As seen in Table L-2, there is remarkably good agreement between the two data sets. It is seen that for number of constituents, the two data sets agree within the experimental uncertainty given in Column 3. For those constituents that the data differences fall somewhat outside the data uncertainty $\left(\mathrm{Ca}^{2+}, \mathrm{Mg}^{2+}, \mathrm{K}^{+}\right.$, $\mathrm{SiO}_{2}$ ), the differences are still within the 20 percent limit. Thus, the Ogard and Kerrisk data for the composition of the water sample from UE-25 J-13 meet the qualification requirements.

Table L-2. Comparison of Composition of Water from UE-25 J-13

\begin{tabular}{|l|c|c|c|}
\hline \multicolumn{1}{|c|}{ Chemical Constituent } & $\begin{array}{c}\text { Ogard and Kerrisk } \\
\text { data }^{\text {a }} \\
\text { Concentration (mg/L) }\end{array}$ & $\begin{array}{c}\text { Corroborating Data } \\
\text { Concentration +- } \\
\text { Uncertainty (mg/L) }\end{array}$ & $\begin{array}{c}\text { \% Difference } \\
\text { ABS(100*(Column2- } \\
\text { Column3)/Column2) }\end{array}$ \\
\hline $\mathrm{Ca}^{2+}$ & 11.5 & $13 .+-0.99$ & 13 \\
\hline $\mathrm{Mg}^{2+}$ & 1.76 & $2.01+-0.21$ & 14 \\
\hline $\mathrm{Na}^{+}$ & 45 & $45.8+-2.29$ & 2 \\
\hline $\mathrm{K}^{+}$ & 5.3 & $5.04+-0.61$ & 5 \\
\hline $\mathrm{SiO}_{2}$ & 64.2 & $60.97+-1.85$ & 5 \\
\hline $\mathrm{Cl}^{-}$ & 6.4 & $7.14+-0.61$ & 4 \\
\hline $\mathrm{F}^{-}$ & 2.1 & $2.18+0.29$ & 2 \\
\hline $\mathrm{SO}_{4}{ }^{2-}$ & 18.1 & $18.4+-1.03$ & 1 \\
\hline $\mathrm{HCO}_{3}{ }^{-}$ & $128^{(\mathrm{b})}$ & $129.8+-8.6$ & $\mathrm{~N} / \mathrm{A}$ \\
\hline $\mathrm{CO}_{3}{ }^{2-}$ & 0.0 & - & 7 \\
\hline $\mathrm{pH}$ & 6.9 & $7.41+-0.44$ & 2 \\
\hline
\end{tabular}

a Ogard and Kerrisk 1984 (DIRS 100783).

b DTN: MO0006J13WTRCM.000 (DIRS 151029).

The composition of water from UE-25 p\#1 (carbonate) as reported by Ogard and Kerrisk (1984 [DIRS 100783]) and the corroborating data from DTN: MO0006J13WTRCM.000 [DIRS 151029] are given in Table L-3. The corroborating data set is verified and qualified per AP-SIII.2Q. The uncertainty in concentration of major anions and cations is on the order of 10 percent for most species and 15 percent for $\mathrm{F}^{-}$(BSC 2004 ([DIRS 170037], Section A7.3.1). Thus, the data from the two sets can be expected to agree within 20 percent of each other. As shown in column 4 of the Table L-3, this holds for all the constituents except $\mathrm{Cl}^{-}$. The Ogard and Kerrisk value for $\mathrm{Cl}^{-}$is somewhat higher than this range. However, since the data are used for 
setting limits on the range compositional values, this is considered acceptable. Thus, the Ogard and Kerrisk data for the composition of the water sample from UE-25 p\#1 (carbonate) meet the qualification requirements.

Table L-3. Comparison of Compositions of Water from UE-25 p\#1(Carbonate)

\begin{tabular}{|c|c|c|c|}
\hline Chemical Constituent & $\begin{array}{c}\text { Corroborating Data }{ }^{a} \\
(\mathrm{mg} / \mathrm{L})\end{array}$ & $\begin{array}{c}\text { Ogard and Kerrisk } \\
\text { Data }^{\mathrm{b}} \\
(\mathrm{mg} / \mathrm{L})\end{array}$ & $\begin{array}{c}\% \text { Difference } \\
\text { ABS }\left(100^{*}(\text { Column2- }\right. \\
\text { Column3)/Column2) }\end{array}$ \\
\hline $\mathrm{Ca}^{2+}$ & 100. & 87.8 & 12 \\
\hline $\mathrm{Mg}^{2+}$ & 39. & 31.9 & 18 \\
\hline $\mathrm{Na}^{+}$ & 150 & 171 & 14 \\
\hline $\mathrm{K}^{+}$ & 12 & 13.4 & 12 \\
\hline $\mathrm{SiO}_{2}$ & 64.2 & 64.2 & 0 \\
\hline $\mathrm{Cl}^{-}$ & 28 & 37 & 32 \\
\hline $\mathrm{F}^{-}$ & $\mathrm{N} / \mathrm{D}$ & 3.5 & $\mathrm{~N} / \mathrm{A}$ \\
\hline $\mathrm{SO}_{4}{ }^{2-}$ & 160 & 129 & 19 \\
\hline $\mathrm{HCO}_{3}^{-}$ & 694 & 666 & 4 \\
\hline $\mathrm{CO}_{3}{ }^{2-}$ & 0.0 & 0 & N/A \\
\hline $\mathrm{pH}$ & 6.6 & 6.7 & 6 \\
\hline
\end{tabular}

a DTN: MO0007MAJIONPH.011 (DIRS 151524).

b Ogard and Kerrisk 1984 (DIRS 100783).

\section{L4. RESULTS}

The qualification team determined, based on the discussions above, that the referenced data are both reasonable and appropriate for the application. This evaluation did not produce any new data.

\section{L5. CONCLUSION}

The data qualification effort documented in this report was performed in accordance with the requirements of the approved data qualification plan in Figure L-1. The approved qualification method, corroborating data, was implemented as required. As a result of this qualification evaluation, these data are qualified for inclusion in the model report Radionuclide Transport Models Under Ambient Conditions. 


\begin{tabular}{|c|c|c|}
\hline BSC & DATA QUALIFICATION PLAN & $\frac{\text { QA: QA }}{\text { Page } 1 \text { of } 1}$ \\
\hline \multicolumn{3}{|c|}{ Section I. Organizational Information } \\
\hline \multicolumn{3}{|l|}{ Qualification Title } \\
\hline \multicolumn{3}{|c|}{ QUALIFICATION OF COMPOSITIONS OF WATERS FROM UE-25 J-13 AND UE-25 P\#1(CARBONATE) } \\
\hline \multicolumn{3}{|l|}{ Requesting Organization } \\
\hline \multicolumn{3}{|c|}{ Regulatory Integration Team - Saturated Zone. } \\
\hline \multicolumn{3}{|c|}{ Section II. Process Planning Requirements } \\
\hline \multicolumn{3}{|c|}{ 1. List of Unqualified Data to be Evaluated } \\
\hline \multicolumn{3}{|c|}{$\begin{array}{l}\text { The data that were evaluated and documented in this qualification report are published in the report Ogard, A.E. and Kerrisk, J.F. } \\
\text { 1984. Groundwater Chemistry Along Flow Paths Between a Proposed Repository Site and the Accessible Environment. LA-10188- } \\
\text { MS. Los Alamos, New Mexico: Los Alamos National Laboratory. ACC: HQS.19880517.2031. [DIRS 100783]. These data are shown } \\
\text { in Table below. }\end{array}$} \\
\hline Chemical Constituent & UE-25 J-13 Water UE-25 p\#1 (Carbonate) Water (mg/ & \\
\hline $\mathrm{Ca} 2+$ & 87.8 & \\
\hline $\mathrm{Mg} 2+$ & 1.76 & \\
\hline $\mathrm{Na}+$ & 171 & \\
\hline $\mathrm{K}+$ & 13.4 & \\
\hline $\mathrm{SiO} 2$ & 64.2 & \\
\hline $\mathrm{Cl}-$ & 6.4 & \\
\hline $\mathrm{F}-$ & 3.5 & \\
\hline SO42- & 18.1 & \\
\hline $\mathrm{HCO}_{3}-$ & 128 & \\
\hline $\mathrm{CO} 32-$ & 0.0 & \\
\hline \multicolumn{3}{|c|}{$\begin{array}{l}\text { A comparison of the unqualified data and available qualified corroborating data will be conducted to determine that the unqualified } \\
\text { data is acceptable and confidence in the data is warranted. } \\
\text { The team will conduct the comparison by considering the importance of the data to showing that the proposed U.S. Department of } \\
\text { Energy repository design meets the performance objectives. } \\
\text { The following data evaluation criteria will be used: } \\
\text { 1. Extent and quality of corroborating data } \\
\text { 2. Agreement between the data being qualified and the corroborating data. } \\
\text { 3. The importance of the data to showing that the proposed U.S. Department of Energy repository design meets the performance } \\
\text { objectives of } 10 \text { CFR } 60 \text {. }\end{array}$} \\
\hline \multicolumn{3}{|c|}{ 3. Data Qualification Team and Additional Support Staff Required } \\
\hline \multicolumn{3}{|c|}{$\begin{array}{l}\text { Qualification Chairperson: Sharad Kelkar, LANL, } 5056674639 \\
\text { Team Member: James Houseworth, LBL, } 7022957632\end{array}$} \\
\hline
\end{tabular}

Figure L-1. Plan for Qualification of Compositions of Waters from UE-25 J-13 and UE-25 P\#1 


\begin{tabular}{|l|l|l|}
\hline \multirow{2}{*}{ BSC } & DATA Qualification PLAN & QA: QA \\
\cline { 3 - 3 } & Page 2 of 1 \\
\hline
\end{tabular}

1. The following data evaluation criteria will be used:

1. Extent and quality of corroborating data- the corroborating data should be verified and qualified per AP-SIII.2Q

2. Agreement between the data being qualified and the corroborating data should be within the experimental uncertainty.

3. The importance of the data to showing that the proposed U.S. Department of Energy repository design meets the performance objectives of 10 CFR 60 will be considered in evaluating the criteria 1 and 2 above.

5. Identification of Procedures Used

AP-SIII.10Q, Models

Section III. Approval

\begin{tabular}{|l|l|l|}
\hline Qualification Chairperson Printed Name & Qualification Chairperson Signature, & Date
\end{tabular}

Sharad Kelkar

Responsible Manager Printed Name

Shara o the Kar

Responsible Manager Signature

Ahmed Monib

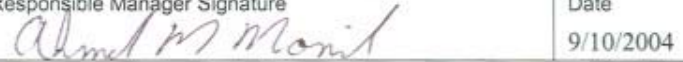

Figure L-1. Plan for Qualification of Compositions of Waters from UE-25 J-13 and UE-25 P\#1 (Continued) 